Future Wave and Wind Projections for United States and United States-Affiliated Pacific Islands

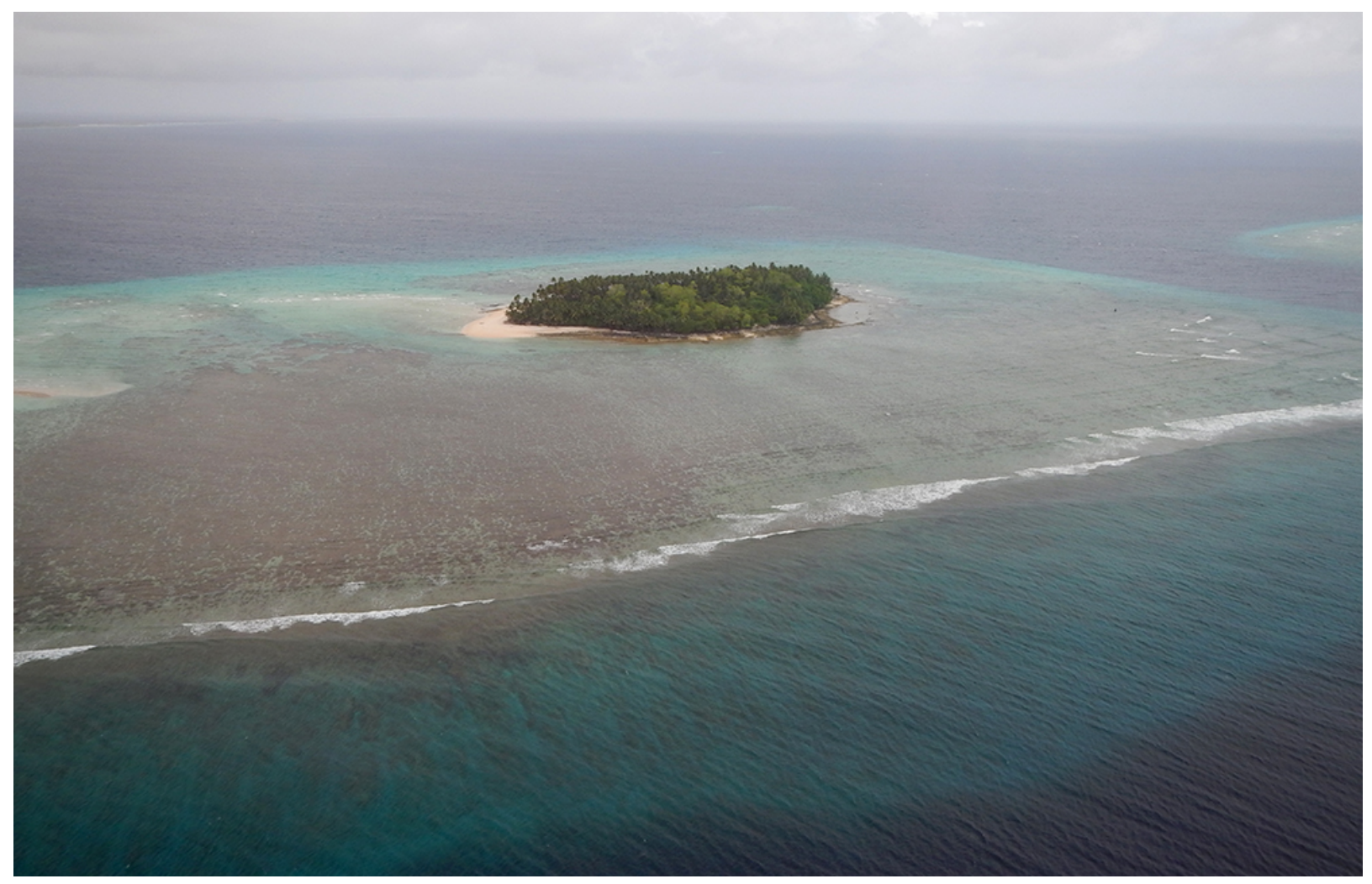

Open-File Report 2015-1001

U.S. Department of the Interior

U.S. Geological Survey 
Cover image:

Aerial photograph of waves breaking on the fringing reef off Ennuebing Island, Kwajalein Atoll, Republic of the Marshall Islands 


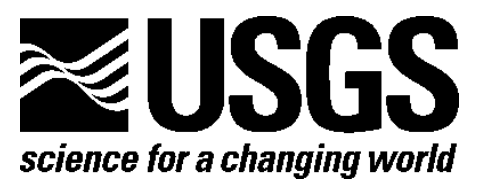

\section{Future Wave and Wind Projections for United States and United States-Affiliated Pacific Islands}

By Curt D. Storlazzi, James B. Shope, Li H. Erikson, Christie A. Hegermiller, and Patrick L. Barnard

Open-File Report 2015-1001

U.S. Department of the Interior

U.S. Geological Survey 


\section{U.S. Department of the Interior}

SALLY JEWELL, Secretary

U.S. Geological Survey

Suzette M. Kimball, Acting Director

U.S. Geological Survey, Reston, Virginia: 2015

For more information on the USGS—-the Federal source for science about the Earth, its natural and living resources, natural hazards, and the environment-visit http://www.usgs.gov or call 1-888-ASK-USGS (1-888-275-8747)

For an overview of USGS information products, including maps, imagery, and publications, visit http://www.usgs.gov/pubprod

To order this and other USGS information products, visit $h$ ttp://store.usgs.gov

Any use of trade, firm, or product names is for descriptive purposes only and does not imply endorsement by the U.S. Government.

Although this information product, for the most part, is in the public domain, it also may contain copyrighted materials as noted in the text. Permission to reproduce copyrighted items must be secured from the copyright owner.

Suggested citation:

Storlazzi, C.D., Shope, J.B., Erikson, L.H., Hegermiller, C.A., and Barnard, P.L., 2015, Future wave and wind projections for United States and United States-affiliated Pacific Islands: U.S. Geological Survey Open-File Report 2015-1001, 426 p., http://dx.doi.org/10.3133/ofr20151001. 


\section{Contents}

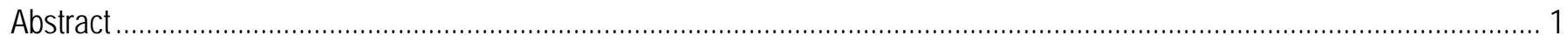

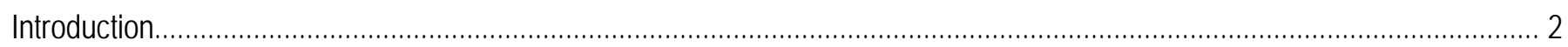

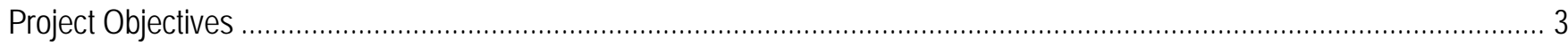

Study Area.

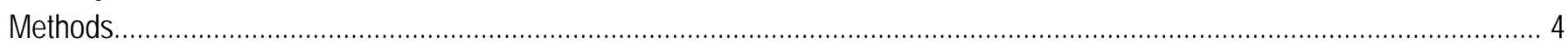

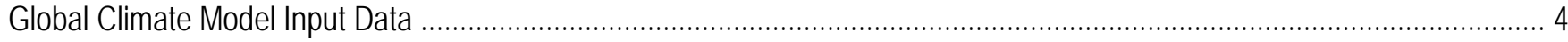

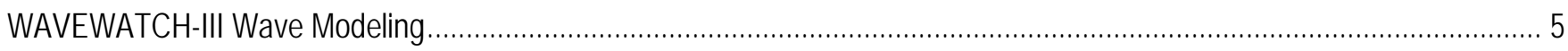

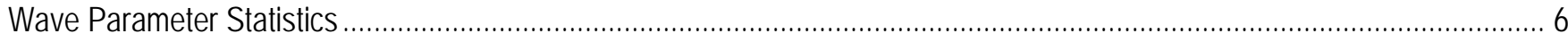

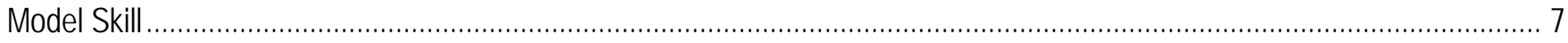

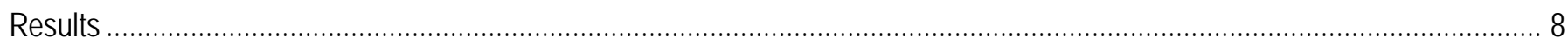

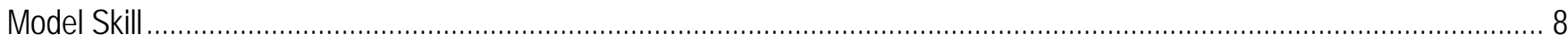

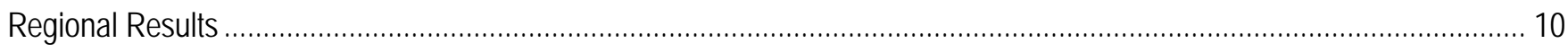

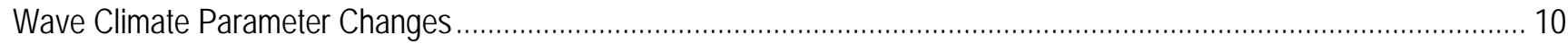

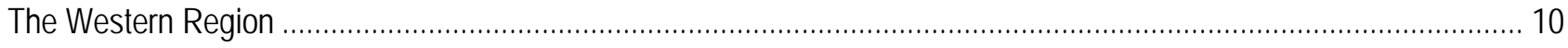

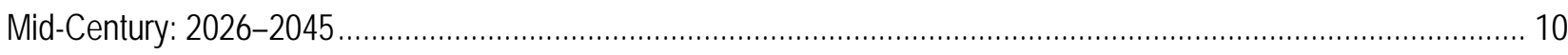

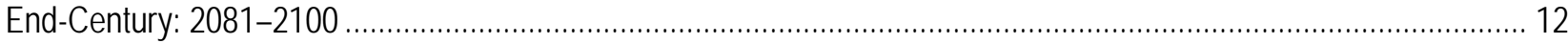

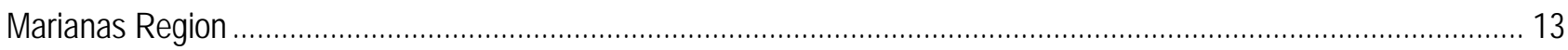

Mid-Century: 2026-2045

End-Century: 2081-2100

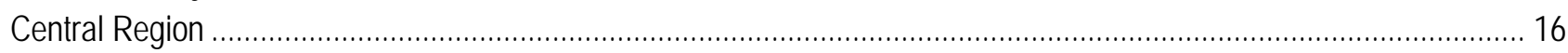

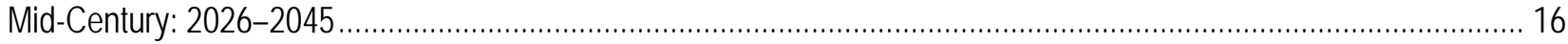

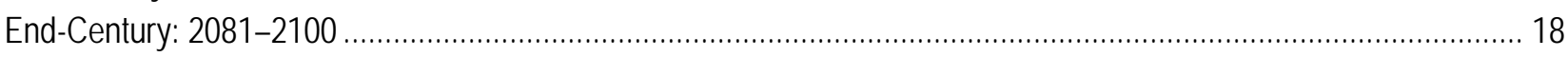

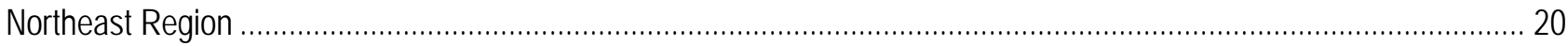

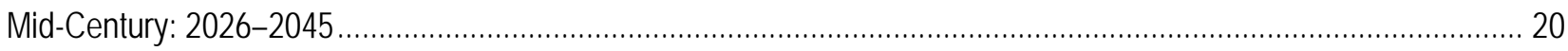

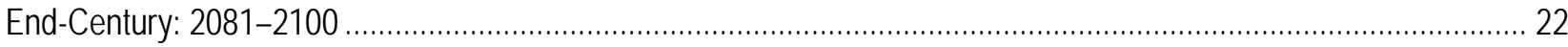

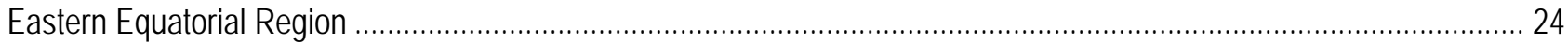

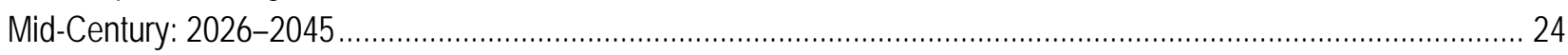

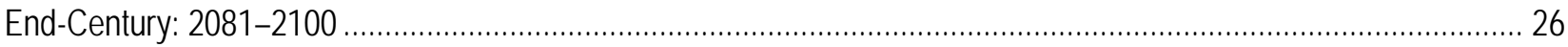

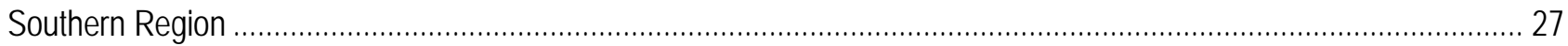

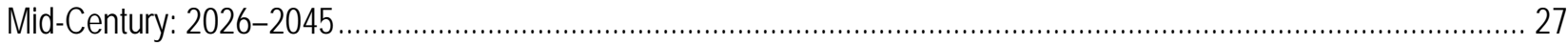

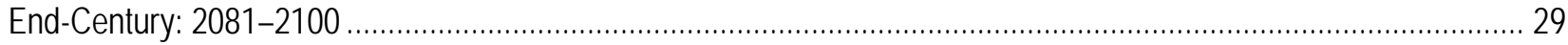

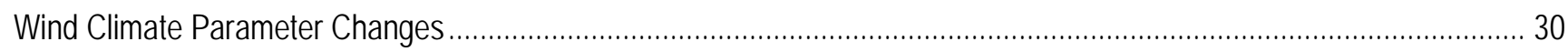

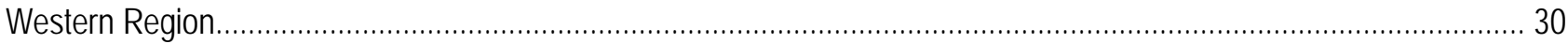

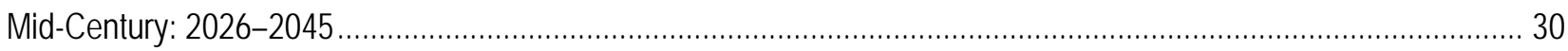

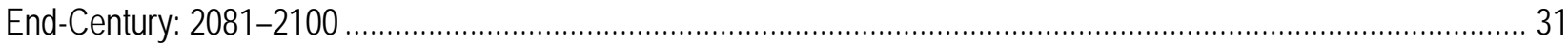

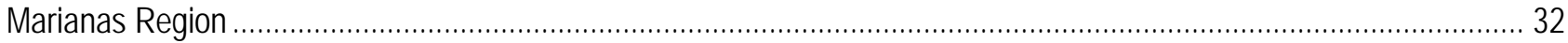

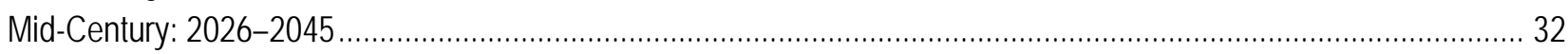

End-Century: 2081-2100

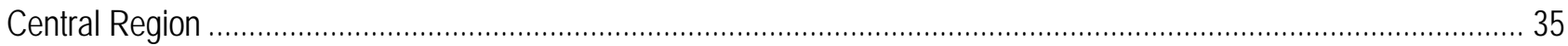

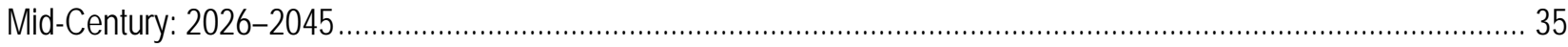

End-Century: 2081-2100 


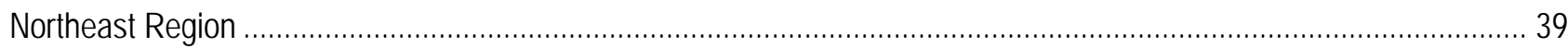

Mid-Century: 2026-2045

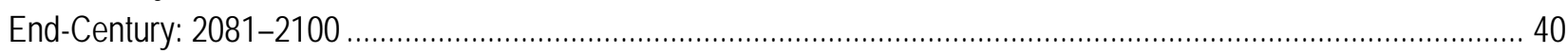

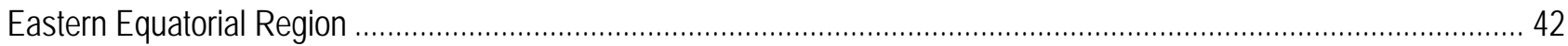

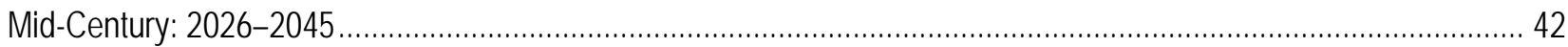

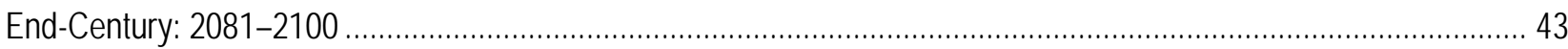

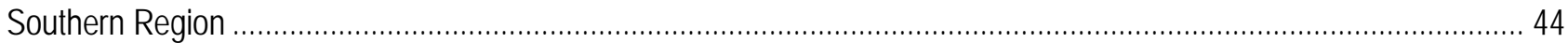

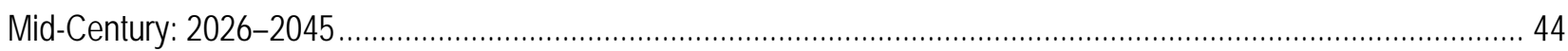

End-Century: 2081-2100

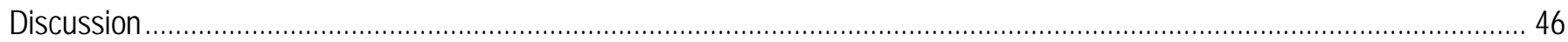

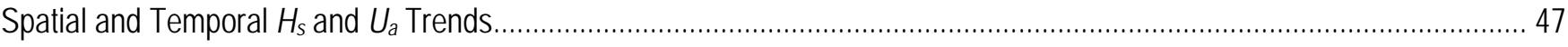

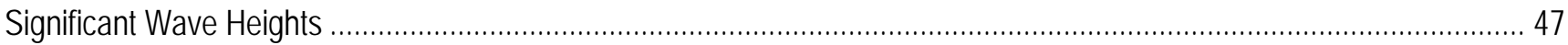

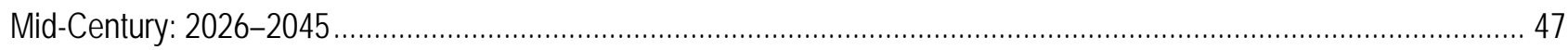

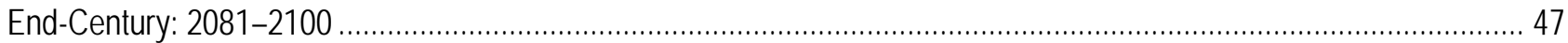

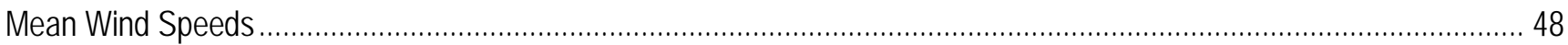

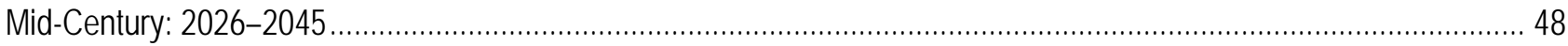

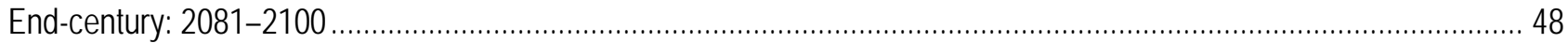

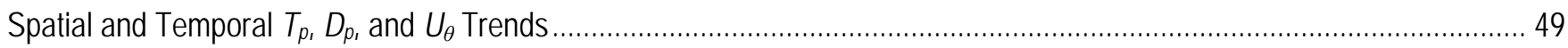

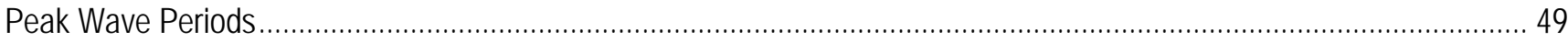

Mid-Century: 2026-2045

End Century: 2081-2100

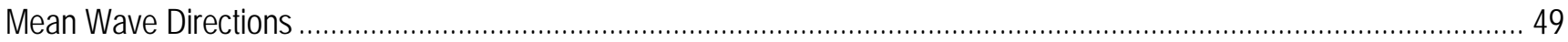

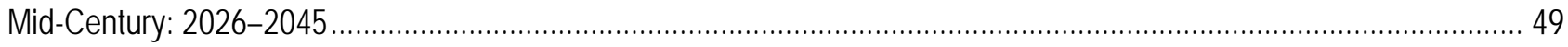

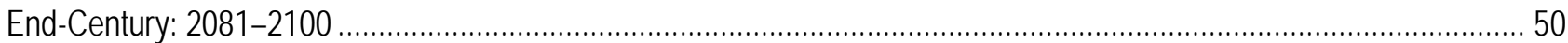

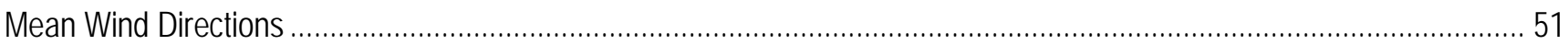

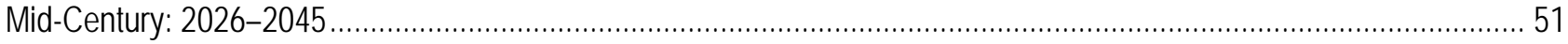

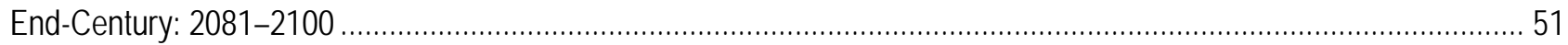

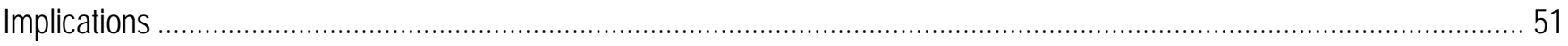

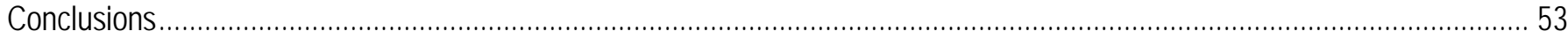

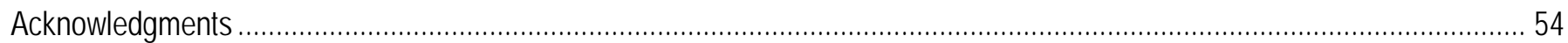

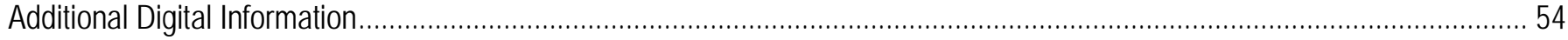

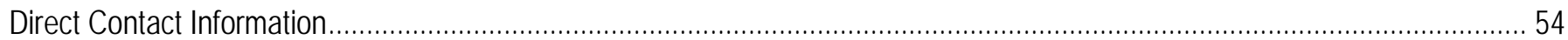

References Cited.

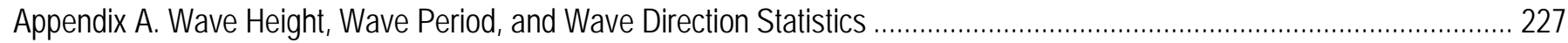

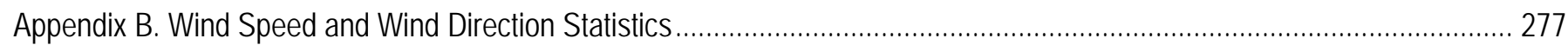

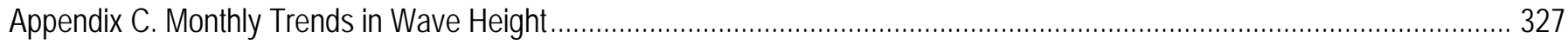

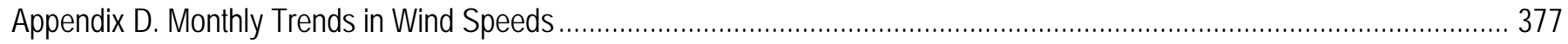




\section{Tables}

1. Table of names and locations of WAVEWATCH III model points within the mid-to-western tropical Pacific

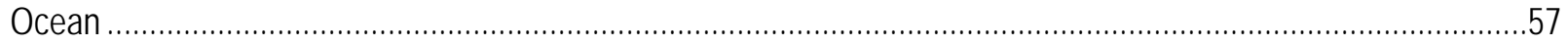

2. List of Global Climate Models used, wave model used, and the model resolutions ..................................................57

3. Table showing comparisons between hindcast significant wave height data, in meters, at the Molokai point and historical significant wave height data from selected NDBC stations throughout the Hawaiian Islands by months

4. Table showing comparisons between hindcast significant wave height data, in meters, at the Big Island of Hawaii point and historical significant wave height data from selected NDBC stations throughout the Hawaiian Islands by months.

5. Table showing comparisons between hindcast significant wave height data, in meters, at the Kauai point and historical significant wave height data from selected NDBC stations throughout the Hawaiian Islands by months.

6. Table showing comparisons between hindcast top 5 percent of significant wave height data, in meters, at the Molokai point and historical significant wave height data from selected NDBC stations throughout the Hawaiian Islands by months.

7. Table showing comparisons between hindcast top 5 percent of significant wave height data, in meters, at the Big Island of Hawaii point and historical significant wave height data from selected NDBC stations throughout the Hawaiian Islands by months.

8. Table showing comparisons between hindcast top 5 percent of significant wave height data, in meters, at the Kauai point and historical significant wave height data from selected NDBC stations throughout the Hawaiian Islands by months.

\section{Figures}

1. Map showing the locations of the 25 modeled points within the tropical Pacific Ocean used in this study......

2. Map showing the locations of selected National Data Buoy Center stations, in purple, and modeled points, in red, within in the Hawaiian Island Chain.

3. Plots showing comparisons of the empirical cumulative density functions of hindcasted (1986-2005) Molokai point significant wave heights $\left(H_{s}\right)$ in meters, from each GCM-driven WW3 model run with the observed historical cumulative density function at each NDBC station..

4. Plots showing comparisons of the empirical cumulative density functions of hindcasted (1986-2005) Big Island of Hawaii point significant wave heights $\left(H_{s}\right)$ in meters from each GCM-driven WW3 model run with the observed historical cumulative density function at each NDBC station.

5. Plots showing comparisons of the empirical cumulative density functions of hindcasted (1986-2005) Kauai point significant wave heights $\left(H_{s}\right)$ in meters from each GCM-driven WW3 model run with the observed historical cumulative density function at each NDBC station.

6. Map showing forecasted differences in mean significant wave height and variance in significant wave height for the years 2026-2045 from hindcasted values during the December-February season under the RCP4.5 future climatic scenario.

7. Map showing forecasted differences in mean significant wave height and variance in significant wave height for the years 2026-2045 from hindcasted values during the March-May season under the RCP4.5 future climatic scenario. 
8. Map showing forecasted differences in mean significant wave height and variance in significant wave height for the years 2026-2045 from hindcasted values during the June-August season under the

RCP4.5 future climatic scenario.

9. Map showing forecasted differences in mean significant wave height and variance in significant wave height for the years 2026-2045 from hindcasted values during the September-November season under the RCP4.5 future climatic scenario.

10. Map showing forecasted differences in the mean of the top 5 percent of significant wave heights and variance in the top 5 percent of significant wave heights for the years 2026-2045 from hindcasted values during the December-February season under the RCP4.5 future climatic scenario.

11. Map showing forecasted differences in the mean of the top 5 percent of significant wave heights and variance in the top 5 percent of significant wave heights for the years 2026-2045 from hindcasted values during the March-May season under the RCP4.5 future climatic scenario.

12. Map showing forecasted differences in the mean of the top 5 percent of significant wave heights and variance in the top 5 percent of significant wave heights for the years 2026-2045 from hindcasted values during the June-August season under the RCP4.5 future climatic scenario.

13. Map showing forecasted differences in the mean of the top 5 percent of significant wave heights and variance in the top 5 percent of significant wave heights for the years 2026-2045 from hindcasted values during the September-November season under the RCP4.5 future climatic scenario.

14. Map showing forecasted differences in mean significant wave height and variance in significant wave height for the years 2026-2045 from hindcasted values during the December-February season under the RCP8.5 future climatic scenario.

15. Map showing forecasted differences in mean significant wave height and variance in significant wave height for the years 2026-2045 from hindcasted values during the March-May season under the RCP8.5 future climatic scenario.

16. Map showing forecasted differences in mean significant wave height and variance in significant wave height for the years 2026-2045 from hindcasted values during the June-August season under the RCP8.5 future climatic scenario.

17. Map showing forecasted differences in mean significant wave height and variance in significant wave height for the years 2026-2045 from hindcasted values during the September-November season under the RCP8.5 future climatic scenario.

18. Map showing forecasted differences in the mean of the top 5 percent of significant wave heights and variance in the top 5 percent of significant wave heights for the years 2026-2045 from hindcasted values during the December-February season under the RCP8.5 future climatic scenario..

19. Map showing forecasted differences in the mean of the top 5 percent of significant wave heights and variance in the top 5 percent of significant wave heights for the years 2026-2045 from hindcasted values during the March-May season under the RCP8.5 future climatic scenario.

20. Map showing forecasted differences in the mean of the top 5 percent of significant wave heights and variance in the top 5 percent of significant wave heights for the years 2026-2045 from hindcasted values during the June-August season under the RCP8.5 future climatic scenario.

21. Map showing forecasted differences in the mean of the top 5 percent of significant wave heights and variance in the top 5 percent of significant wave heights for the years 2026-2045 from hindcasted values during the September-November season under the RCP8.5 future climatic scenario.

22. Map showing forecasted differences in mean significant wave height and variance in significant wave height for the years 2081-2100 from hindcasted values during the December-February season under the RCP4.5 future climatic scenario. 
23. Map showing forecasted differences in mean significant wave height and variance in significant wave height for the years 2081-2100 from hindcasted values during the March-May season under the RCP4.5

future climatic scenario.

24. Map showing forecasted differences in mean significant wave height and variance in significant wave height for the years 2081-2100 from hindcasted values during the June-August season under the RCP4.5 future climatic scenario.

25. Map showing forecasted differences in mean significant wave height and variance in significant wave height for the years 2081-2100 from hindcasted values during the September-November season under the RCP4.5 future climatic scenario.

26. Map showing forecasted differences in the mean of the top 5 percent of significant wave heights and variance in the top 5 percent of significant wave heights for the years 2081-2100 from hindcasted values during the December-February season under the RCP4.5 future climatic scenario.

27. Map showing forecasted differences in the mean of the top 5 percent of significant wave heights and variance in the top 5 percent of significant wave heights for the years 2081-2100 from hindcasted values during the March-May season under the RCP4.5 future climatic scenario.

28. Map showing forecasted differences in the mean of the top 5 percent of significant wave heights and variance in the top 5 percent of significant wave heights for the years 2081-2100 from hindcasted values during the June-August season under the RCP4.5 future climatic scenario.

29. Map showing forecasted differences in the mean of the top 5 percent of significant wave heights and variance in the top 5 percent of significant wave heights for the years 2081-2100 from hindcasted values during the September-November season under the RCP4.5 future climatic scenario.

30. Map showing forecasted differences in mean significant wave height and variance in significant wave height for the years 2081-2100 from hindcasted values during the December-February season under the RCP8.5 future climatic scenario.

31. Map showing forecasted differences in mean significant wave height and variance in significant wave height for the years 2081-2100 from hindcasted values during the March-May season under the RCP8.5 future climatic scenario

32. Map showing forecasted differences in mean significant wave height and variance in significant wave height for the years 2081-2100 from hindcasted values during the June-August season under the RCP8.5 future climatic scenario. The colors correspond to the magnitude of change in modeled mean significant wave heights during 2081-2100 from those hindcasted for 1976-2005. The shapes correspond to the magnitude of change in modeled variance in significant wave height during 2081-2100 from those hindcasted for 1976-2005. Units are in meters.

33. Map showing forecasted differences in mean significant wave height and variance in significant wave height for the years 2081-2100 from hindcasted values during the September-November season under the RCP8.5 future climatic scenario.

34. Map showing forecasted differences in the mean of the top 5 percent of significant wave heights and variance in the top 5 percent of significant wave heights for the years 2081-2100 from hindcasted values during the December-February season under the RCP8.5 future climatic scenario.

35. Map showing forecasted differences in the mean of the top 5 percent of significant wave heights and variance in the top 5 percent of significant wave heights for the years 2081-2100 from hindcasted values during the March-May season under the RCP8.5 future climatic scenario.

36. Map showing forecasted differences in the mean of the top 5 percent of significant wave heights and variance in the top 5 percent of significant wave heights for the years 2081-2100 from hindcasted values during the June-August season under the RCP8.5 future climatic scenario. 
37. Map showing forecasted differences in the mean of the top 5 percent of significant wave heights and variance in the top 5 percent of significant wave heights for the years 2081-2100 from hindcasted values during the September-November season under the RCP8.5 future climatic scenario.

38. Map showing forecasted differences in mean peak wave period and variance in peak wave period for the years 2026-2045 from hindcasted values during the December-February season under the RCP4.5 future climatic scenario

39. Map showing forecasted differences in mean peak wave period and variance in peak wave period for the years 2026-2045 from hindcasted values during the March-May season under the RCP4.5 future climatic scenario.

40. Map showing forecasted differences in mean peak wave period and variance in peak wave period for the years 2026-2045 from hindcasted values during the June-August season under the RCP4.5 future climatic scenario.

41. Map showing forecasted differences in mean peak wave period and variance in peak wave period for the years 2026-2045 from hindcasted values during the September-November season under the RCP4.5 future climatic scenario.

42. Map showing forecasted differences in the mean peak wave period of the top 5 percent of significant wave heights and variance in the peak wave period of top 5 percent of significant wave heights for the years 2026-2045 from hindcasted values during the December-February season under the RCP4.5 future climatic scenario.

43. Map showing forecasted differences in the mean peak wave period of the top 5 percent of significant wave heights and variance in the peak wave period of top 5 percent of significant wave heights for the years 2026-2045 from hindcasted values during the March-May season under the RCP4.5 future climatic scenario.

44. Map showing forecasted differences in the mean peak wave period of the top 5 percent of significant wave heights and variance in the peak wave period of top 5 percent of significant wave heights for the years 2026-2045 from hindcasted values during the June-August season under the RCP4.5 future climatic scenario.

45. Map showing forecasted differences in the mean peak wave period of the top 5 percent of significant wave heights and variance in the peak wave period of top 5 percent of significant wave heights for the years 2026-2045 from hindcasted values during the September-November season under the RCP4.5 future climatic scenario.

46. Map showing forecasted differences in mean peak wave period and variance in peak wave period for the years 2026-2045 from hindcasted values during the December-February season under the RCP8.5 future climatic scenario

47. Map showing forecasted differences in mean peak wave period and variance in peak wave period for the years 2026-2045 from hindcasted values during the March-May season under the RCP8.5 future climatic scenario.

48. Map showing forecasted differences in mean peak wave period and variance in peak wave period for the years 2026-2045 from hindcasted values during the June-August season under the RCP8.5 future climatic scenario.

49. Map showing forecasted differences in mean peak wave period and variance in peak wave period for the years 2026-2045 from hindcasted values during the September-November season under the RCP8.5 future climatic scenario.

50. Map showing forecasted differences in the mean peak wave period of the top 5 percent of significant wave heights and variance in the peak wave period of top 5 percent of significant wave heights for the years 2026-2045 from hindcasted values during the December-February season under the RCP8.5 future climatic scenario. 
51. Map showing forecasted differences in the mean peak wave period of the top 5 percent of significant wave heights and variance in the peak wave period of top 5 percent of significant wave heights for the years 2026-2045 from hindcasted values during the March-May season under the RCP8.5 future climatic scenario.

52. Map showing forecasted differences in the mean peak wave period of the top 5 percent of significant wave heights and variance in the peak wave period of top 5 percent of significant wave heights for the years 2026-2045 from hindcasted values during the June-August season under the RCP8.5 future climatic scenario.

53. Map showing forecasted differences in the mean peak wave period of the top 5 percent of significant wave heights and variance in the peak wave period of top 5 percent of significant wave heights for the years 2026-2045 from hindcasted values during the September-November season under the RCP8.5 future climatic scenario.

54. Map showing forecasted differences in mean peak wave period and variance in peak wave period for the years 2081-2100 from hindcasted values during the December-February season under the RCP4.5 future climatic scenario.

55. Map showing forecasted differences in mean peak wave period and variance in peak wave period for the years 2081-2100 from hindcasted values during the March-May season under the RCP4.5 future climatic scenario.

56. Map showing forecasted differences in mean peak wave period and variance in peak wave period for the years 2081-2100 from hindcasted values during the June-August season under the RCP4.5 future climatic scenario..

57. Map showing forecasted differences in mean peak wave period and variance in peak wave period for the years 2081-2100 from hindcasted values during the September-November season under the RCP4.5 future climatic scenario

58. Map showing forecasted differences in the mean peak wave period of the top 5 percent of significant wave heights and variance in the peak wave period of top 5 percent of significant wave heights for the years 2081-2100 from hindcasted values during the December-February season under the RCP4.5 future climatic scenario.

59. Map showing forecasted differences in the mean peak wave period of the top 5 percent of significant wave heights and variance in the peak wave period of top 5 percent of significant wave heights for the years 2081-2100 from hindcasted values during the March-May season under the RCP4.5 future climatic scenario.

60. Map showing forecasted differences in the mean peak wave period of the top 5 percent of significant wave heights and variance in the peak wave period of top 5 percent of significant wave heights for the years 2081-2100 from hindcasted values during the June-August season under the RCP4.5 future climatic scenario.

61. Map showing forecasted differences in the mean peak wave period of the top 5 percent of significant wave heights and variance in the peak wave period of top 5 percent of significant wave heights for the years 2081-2100 from hindcasted values during the September-November season under the RCP4.5 future climatic scenario.

62. Map showing forecasted differences in mean peak wave period and variance in peak wave period for the years 2081-2100 from hindcasted values during the December-February season under the RCP8.5 future climatic scenario.

63. Map showing forecasted differences in mean peak wave period and variance in peak wave period for the years 2081-2100 from hindcasted values during the March-May season under the RCP8.5 future climatic scenario. 
64. Map showing forecasted differences in mean peak wave period and variance in peak wave period for the years 2081-2100 from hindcasted values during the June-August season under the RCP8.5 future climatic scenario.

65. Map showing forecasted differences in mean peak wave period and variance in peak wave period for the years 2081-2100 from hindcasted values during the September-November season under the RCP8.5 future climatic scenario

66. Map showing forecasted differences in the mean peak wave period of the top 5 percent of significant wave heights and variance in the peak wave period of top 5 percent of significant wave heights for the years 2081-2100 from hindcasted values during the December-February season under the RCP8.5 future climatic scenario.

67. Map showing forecasted differences in the mean peak wave period of the top 5 percent of significant wave heights and variance in the peak wave period of top 5 percent of significant wave heights for the years 2081-2100 from hindcasted values during the March-May season under the RCP8.5 future climatic scenario.

68. Map showing forecasted differences in the mean peak wave period of the top 5 percent of significant wave heights and variance in the peak wave period of top 5 percent of significant wave heights for the years 2081-2100 from hindcasted values during the June-August season under the RCP8.5 future climatic scenario.

69. Map showing forecasted differences in the mean peak wave period of the top 5 percent of significant wave heights and variance in the peak wave period of top 5 percent of significant wave heights for the years 2081-2100 from hindcasted values during the September-November season under the RCP8.5 future climatic scenario.

70. Map showing forecasted differences in the mean wave directions of significant wave heights and the standard deviation of wave directions of significant wave heights for the years 2026-2045 from hindcasted values during the December-February season under the RCP4.5 future climatic scenario.

71. Map showing forecasted differences in the mean wave directions of significant wave heights and the standard deviation of wave directions of significant wave heights for the years 2026-2045 from hindcasted values during the March-May season under the RCP4.5 future climatic scenario.

72. Map showing forecasted differences in the mean wave directions of significant wave heights and the standard deviation of wave directions of significant wave heights for the years 2026-2045 from hindcasted values during the June-July season under the RCP4.5 future climatic scenario.

73. Map showing forecasted differences in the mean wave directions of significant wave heights and the standard deviation of wave directions of significant wave heights for the years 2026-2045 from hindcasted values during the September-November season under the RCP4.5 future climatic scenario.

74. Map showing forecasted differences in the mean wave directions of the top 5 percent of significant wave heights and the standard deviation of wave directions of the top 5 percent of significant wave heights for the years 2026-2045 from hindcasted values during the December-February season under the RCP4.5 future climatic scenario.

75. Map showing forecasted differences in the mean wave directions of the top 5 percent of significant wave heights and the standard deviation of wave directions of the top 5 percent of significant wave heights for the years 2026-2045 from hindcasted values during the March-May season under the RCP4.5 future climatic scenario.

76. Map showing forecasted differences in the mean wave directions of the top 5 percent of significant wave heights and the standard deviation of wave directions of the top 5 percent of significant wave heights for the years 2026-2045 from hindcasted values during the June-August season under the RCP4.5 future climatic scenario. 
77. Map showing forecasted differences in the mean wave directions of the top 5 percent of significant wave heights and the standard deviation of wave directions of the top 5 percent of significant wave heights for the years 2026-2045 from hindcasted values during the September-November season under the RCP4.5 future climatic scenario.

78. Map showing forecasted differences in the mean wave directions of significant wave heights and the standard deviation of wave directions of significant wave heights for the years 2026-2045 from hindcasted values during the December-February season under the RCP8.5 future climatic scenario.

79. Map showing forecasted differences in the mean wave directions of significant wave heights and the standard deviation of wave directions of significant wave heights for the years 2026-2045 from hindcasted values during the March-May season under the RCP8.5 future climatic scenario.

80. Map showing forecasted differences in the mean wave directions of significant wave heights and the standard deviation of wave directions of significant wave heights for the years 2026-2045 from hindcasted values during the June-July season under the RCP8.5 future climatic scenario.

81. Map showing forecasted differences in the mean wave directions of significant wave heights and the standard deviation of wave directions of significant wave heights for the years 2026-2045 from hindcasted values during the September-November season under the RCP8.5 future climatic scenario.

82. Map showing forecasted differences in the mean wave directions of the top 5 percent of significant wave heights and the standard deviation of wave directions of the top 5 percent of significant wave heights for the years 2026-2045 from hindcasted values during the December-February season under the RCP8.5 future climatic scenario.

83. Map showing forecasted differences in the mean wave directions of the top 5 percent of significant wave heights and the standard deviation of wave directions of the top 5 percent of significant wave heights for the years 2026-2045 from hindcasted values during the March-May season under the RCP8.5 future climatic scenario.

84. Map showing forecasted differences in the mean wave directions of the top 5 percent of significant wave heights and the standard deviation of wave directions of the top 5 percent of significant wave heights for the years 2026-2045 from hindcasted values during the June-August season under the RCP8.5 future climatic scenario..

85. Map showing forecasted differences in the mean wave directions of the top 5 percent of significant wave heights and the standard deviation of wave directions of the top 5 percent of significant wave heights for the years 2026-2045 from hindcasted values during the September-November season under the RCP8.5 future climatic scenario.

86. Map showing forecasted differences in the mean wave directions of significant wave heights and the standard deviation of wave directions of significant wave heights for the years 2081-2100 from hindcasted values during the December-February season under the RCP4.5 future climatic scenario.

87. Map showing forecasted differences in the mean wave directions of significant wave heights and the standard deviation of wave directions of significant wave heights for the years 2081-2100 from hindcasted values during the March-May season under the RCP4.5 future climatic scenario.

88. Map showing forecasted differences in the mean wave directions of significant wave heights and the standard deviation of wave directions of significant wave heights for the years 2081-2100 from hindcasted values during the June-July season under the RCP4.5 future climatic scenario.

89. Map showing forecasted differences in the mean wave directions of significant wave heights and the standard deviation of wave directions of significant wave heights for the years 2081-2100 from hindcasted values during the September-November season under the RCP4.5 future climatic scenario. 150

90. Map showing forecasted differences in the mean wave directions of the top 5 percent of significant wave heights and the standard deviation of wave directions of the top 5 percent of significant wave heights for 
the years 2081-2100 from hindcasted values during the December-February season under the RCP4.5 future climatic scenario.

91. Map showing forecasted differences in the mean wave directions of the top 5 percent of significant wave heights and the standard deviation of wave directions of the top 5 percent of significant wave heights for the years 2081-2100 from hindcasted values during the March-May season under the RCP4.5 future climatic scenario.

92. Map showing forecasted differences in the mean wave directions of the top 5 percent of significant wave heights and the standard deviation of wave directions of the top 5 percent of significant wave heights for the years 2081-2100 from hindcasted values during the June-August season under the RCP4.5 future climatic scenario.

93. Map showing forecasted differences in the mean wave directions of the top 5 percent of significant wave heights and the standard deviation of wave directions of the top 5 percent of significant wave heights for the years 2081-2100 from hindcasted values during the September-November season under the RCP4.5 future climatic scenario.

94. Map showing forecasted differences in the mean wave directions of significant wave heights and the standard deviation of wave directions of significant wave heights for the years 2081-2100 from hindcasted values during the December-February season under the RCP8.5 future climatic scenario

95. Map showing forecasted differences in the mean wave directions of significant wave heights and the standard deviation of wave directions of significant wave heights for the years 2081-2100 from hindcasted values during the March-May season under the RCP8.5 future climatic scenario.

96. Map showing forecasted differences in the mean wave directions of significant wave heights and the standard deviation of wave directions of significant wave heights for the years 2081-2100 from hindcasted values during the June-July season under the RCP8.5 future climatic scenario.

97. Map showing forecasted differences in the mean wave directions of significant wave heights and the standard deviation of wave directions of significant wave heights for the years 2081-2100 from hindcasted values during the September-November season under the RCP8.5 future climatic scenario.

98. Map showing forecasted differences in the mean wave directions of the top 5 percent of significant wave heights and the standard deviation of wave directions of the top 5 percent of significant wave heights for the years 2081-2100 from hindcasted values during the December-February season under the RCP8.5 future climatic scenario.

99. Map showing forecasted differences in the mean wave directions of the top 5 percent of significant wave heights and the standard deviation of wave directions of the top 5 percent of significant wave heights for the years 2081-2100 from hindcasted values during the March-May season under the RCP8.5 future climatic scenario.

100. Map showing forecasted differences in the mean wave directions of the top 5 percent of significant wave heights and the standard deviation of wave directions of the top 5 percent of significant wave heights for the years 2081-2100 from hindcasted values during the June-August season under the RCP8.5 future climatic scenario.

101. Map showing forecasted differences in the mean wave directions of the top 5 percent of significant wave heights and the standard deviation of wave directions of the top 5 percent of significant wave heights for the years 2081-2100 from hindcasted values during the September-November season under the RCP8.5 future climatic scenario.

102. Map showing forecasted differences in mean wind speed and variance in wind speed for the years 20262045 from hindcasted values during the December-February season under the RCP4.5 future climatic scenario.

103. Map showing forecasted differences in mean wind speed and variance in wind speed for the years 20262045 from hindcasted values during the March-May season under the RCP4.5 future climatic scenario. 
104. Map showing forecasted differences in mean wind speed and variance in wind speed for the years 20262045 from hindcasted values during the June-August season under the RCP4.5 future climatic scenario.

105. Map showing forecasted differences in mean wind speed and variance in wind speed for the years 20262045 from hindcasted values during the September-November season under the RCP4.5 future climatic scenario.

106. Map showing forecasted differences in the mean of the top 5 percent of wind speeds and variance in the top 5 percent of wind speeds for the years 2026-2045 from hindcasted values during the December-

February season under the RCP4.5 future climatic scenario.......

107. Map showing forecasted differences in the mean of the top 5 percent of wind speeds and variance in the top 5 percent of wind speeds for the years 2026-2045 from hindcasted values during the March-May season under the RCP4.5 future climatic scenario.

108. Map showing forecasted differences in the mean of the top 5 percent of wind speeds and variance in the top 5 percent of wind speeds for the years 2026-2045 from hindcasted values during the June-August season under the RCP4.5 future climatic scenario..

109. Map showing forecasted differences in the mean of the top 5 percent of wind speeds and variance in the top 5 percent of wind speeds for the years 2026-2045 from hindcasted values during the SeptemberNovember season under the RCP4.5 future climatic scenario.

110. Map showing forecasted differences in mean wind speed and variance in wind speed for the years 20262045 from hindcasted values during the December-February season under the RCP8.5 future climatic scenario.

111. Map showing forecasted differences in mean wind speed and variance in wind speed for the years 20262045 from hindcasted values during the March-May season under the RCP8.5 future climatic scenario.

112. Map showing forecasted differences in mean wind speed and variance in wind speed for the years 20262045 from hindcasted values during the June-August season under the RCP8.5 future climatic scenario.

113. Map showing forecasted differences in mean wind speed and variance in wind speed for the years 20262045 from hindcasted values during the September-November season under the RCP8.5 future climatic scenario.

114. Map showing forecasted differences in the mean of the top 5 percent of wind speeds and variance in the top 5 percent of wind speeds for the years 2026-2045 from hindcasted values during the DecemberFebruary season under the RCP8.5 future climatic scenario......

115. Map showing forecasted differences in the mean of the top 5 percent of wind speeds and variance in the top 5 percent of wind speeds for the years 2026-2045 from hindcasted values during the March-May season under the RCP8.5 future climatic scenario..

116. Map showing forecasted differences in the mean of the top 5 percent of wind speeds and variance in the top 5 percent of wind speeds for the years 2026-2045 from hindcasted values during the June-August season under the RCP8.5 future climatic scenario.

117. Map showing forecasted differences in the mean of the top 5 percent of wind speeds and variance in the top 5 percent of wind speeds for the years 2026-2045 from hindcasted values during the SeptemberNovember season under the RCP8.5 future climatic scenario.

118. Map showing forecasted differences in mean wind speed and variance in wind speed for the years 20812100 from hindcasted values during the December-February season under the RCP4.5 future climatic scenario.

119. Map showing forecasted differences in mean wind speed and variance in wind speed for the years 20812100 from hindcasted values during the March-May season under the RCP4.5 future climatic scenario.

120. Map showing forecasted differences in mean wind speed and variance in wind speed for the years 20812100 from hindcasted values during the June-August season under the RCP4.5 future climatic scenario. 
121. Map showing forecasted differences in mean wind speed and variance in wind speed for the years 20812100 from hindcasted values during the September-November season under the RCP4.5 future climatic scenario.

122. Map showing forecasted differences in the mean of the top 5 percent of wind speeds and variance in the top 5 percent of wind speeds for the years 2081-2100 from hindcasted values during the December-

February season under the RCP4.5 future climatic scenario.

123. Map showing forecasted differences in the mean of the top 5 percent of wind speeds and variance in the top 5 percent of wind speeds for the years 2081-2100 from hindcasted values during the March-May season under the RCP4.5 future climatic scenario.

124. Map showing forecasted differences in the mean of the top 5 percent of wind speeds and variance in the top 5 percent of wind speeds for the years 2081-2100 from hindcasted values during the June-August season under the RCP4.5 future climatic scenario.

125. Map showing forecasted differences in the mean of the top 5 percent of wind speeds and variance in the top 5 percent of wind speeds for the years 2081-2100 from hindcasted values during the SeptemberNovember season under the RCP4.5 future climatic scenario.

126. Map showing forecasted differences in mean wind speed and variance in wind speed for the years 20812100 from hindcasted values during the December-February season under the RCP8.5 future climatic scenario.

127. Map showing forecasted differences in mean wind speed and variance in wind speed for the years 20812100 from hindcasted values during the March-May season under the RCP8.5 future climatic scenario.

128. Map showing forecasted differences in mean wind speed and variance in wind speed for the years 20812100 from hindcasted values during the June-August season under the RCP8.5 future climatic scenario.

129. Map showing forecasted differences in mean wind speed and variance in wind speed for the years 20812100 from hindcasted values during the September-November season under the RCP8.5 future climatic scenario.

130. Map showing forecasted differences in the mean of the top 5 percent of wind speeds and variance in the top 5 percent of wind speeds for the years 2081-2100 from hindcasted values during the December-

February season under the RCP8.5 future climatic scenario.

131. Map showing forecasted differences in the mean of the top 5 percent of wind speeds and variance in the top 5 percent of wind speeds for the years 2081-2100 from hindcasted values during the March-May season under the RCP8.5 future climatic scenario.

132. Map showing forecasted differences in the mean of the top 5 percent of wind speeds and variance in the top 5 percent of wind speeds for the years 2081-2100 from hindcasted values during the June-August season under the RCP8.5 future climatic scenario.

133. Map showing forecasted differences in the mean of the top 5 percent of wind speeds and variance in the top 5 percent of wind speeds for the years 2081-2100 from hindcasted values during the SeptemberNovember season under the RCP8.5 future climatic scenario.

134. Map showing forecasted differences in mean wind directions and the standard deviation of wind directions for the years 2026-2045 from hindcasted values during the December-February season under the RCP4.5 future climatic scenario.

135. Map showing forecasted differences in mean wind directions and the standard deviation of wind directions for the years 2026-2045 from hindcasted values during the March-May season under the RCP4.5 future climatic scenario...

136. Map showing forecasted differences in mean wind directions and the standard deviation of wind directions for the years 2026-2045 from hindcasted values during the June-August season under the RCP4.5 future climatic scenario. 
137. Map showing forecasted differences in mean wind directions and the standard deviation of wind directions for the years 2026-2045 from hindcasted values during the September-November season under the RCP4.5 future climatic scenario.

138. Map showing forecasted differences in the mean wind directions of the top 5 percent of wind speeds and the standard deviation of wind directions of the top 5 percent of wind speeds for the years 2026-2045 from hindcasted values during the December-February season under the RCP4.5 future climatic scenario.

139. Map showing forecasted differences in the mean wind directions of the top 5 percent of wind speeds and the standard deviation of wind directions of the top 5 percent of wind speeds for the years 2026-2045 from hindcasted values during the March-May season under the RCP4.5 future climatic scenario.

140. Map showing forecasted differences in the mean wind directions of the top 5 percent of wind speeds and the standard deviation of wind directions of the top 5 percent of wind speeds for the years 2026-2045 from hindcasted values during the June-August season under the RCP4.5 future climatic scenario. 201

141. Map showing forecasted differences in the mean wind directions of the top 5 percent of wind speeds and the standard deviation of wind directions of the top 5 percent of wind speeds for the years 2026-2045 from hindcasted values during the September-November season under the RCP4.5 future climatic scenario.

142. Map showing forecasted differences in mean wind directions and the standard deviation of wind directions for the years 2026-2045 from hindcasted values during the December-February season under the RCP8.5 future climatic scenario.

143. Map showing forecasted differences in mean wind directions and the standard deviation of wind directions for the years 2026-2045 from hindcasted values during the March-May season under the RCP8.5 future climatic scenario.

144. Map showing forecasted differences in mean wind directions and the standard deviation of wind directions for the years 2026-2045 from hindcasted values during the June-August season under the RCP8.5 future climatic scenario.

145. Map showing forecasted differences in mean wind directions and the standard deviation of wind directions for the years 2026-2045 from hindcasted values during the September-November season under the RCP8.5 future climatic scenario.

146. Map showing forecasted differences in the mean wind directions of the top 5 percent of wind speeds and the standard deviation of wind directions of the top 5 percent of wind speeds for the years 2026-2045 from hindcasted values during the December-February season under the RCP8.5 future climatic scenario. 207

147. Map showing forecasted differences in the mean wind directions of the top 5 percent of wind speeds and the standard deviation of wind directions of the top 5 percent of wind speeds for the years 2026-2045 from hindcasted values during the March-May season under the RCP8.5 future climatic scenario.

148. Map showing forecasted differences in the mean wind directions of the top 5 percent of wind speeds and the standard deviation of wind directions of the top 5 percent of wind speeds for the years 2026-2045 from hindcasted values during the June-August season under the RCP8.5 future climatic scenario. 209

149. Map showing forecasted differences in the mean wind directions of the top 5 percent of wind speeds and the standard deviation of wind directions of the top 5 percent of wind speeds for the years 2026-2045 from hindcasted values during the September-November season under the RCP8.5 future climatic scenario.

150. Map showing forecasted differences in mean wind directions and the standard deviation of wind directions for the years 2081-2100 from hindcasted values during the December-February season under the RCP4.5 future climatic scenario. 
151. Map showing forecasted differences in mean wind directions and the standard deviation of wind directions for the years 2081-2100 from hindcasted values during the March-May season under the RCP4.5 future climatic scenario.

152. Map showing forecasted differences in mean wind directions and the standard deviation of wind directions for the years 2081-2100 from hindcasted values during the June-August season under the RCP4.5 future climatic scenario.

153. Map showing forecasted differences in mean wind directions and the standard deviation of wind directions for the years 2081-2100 from hindcasted values during the September-November season under the RCP4.5 future climatic scenario.

154. Map showing forecasted differences in the mean wind directions of the top 5 percent of wind speeds and the standard deviation of wind directions of the top 5 percent of wind speeds for the years 2081-2100 from hindcasted values during the December-February season under the RCP4.5 future climatic scenario.

155. Map showing forecasted differences in the mean wind directions of the top 5 percent of wind speeds and the standard deviation of wind directions of the top 5 percent of wind speeds for the years 2081-2100 from hindcasted values during the March-May season under the RCP4.5 future climatic scenario. 216

156. Map showing forecasted differences in the mean wind directions of the top 5 percent of wind speeds and the standard deviation of wind directions of the top 5 percent of wind speeds for the years 2081-2100 from hindcasted values during the June-August season under the RCP4.5 future climatic scenario.

157. Map showing forecasted differences in the mean wind directions of the top 5 percent of wind speeds and the standard deviation of wind directions of the top 5 percent of wind speeds for the years 2081-2100 from hindcasted values during the September-November season under the RCP4.5 future climatic scenario.

158. Map showing forecasted differences in mean wind directions and the standard deviation of wind directions for the years 2081-2100 from hindcasted values during the December-February season under the RCP8.5 future climatic scenario.

159. Map showing forecasted differences in mean wind directions and the standard deviation of wind directions for the years 2081-2100 from hindcasted values during the March-May season under the RCP8.5 future climatic scenario.

160. Map showing forecasted differences in mean wind directions and the standard deviation of wind directions for the years 2081-2100 from hindcasted values during the June-August season under the RCP8.5 future climatic scenario.

161. Map showing forecasted differences in mean wind directions and the standard deviation of wind directions for the years 2081-2100 from hindcasted values during the September-November season under the RCP8.5 future climatic scenario.

162. Map showing forecasted differences in the mean wind directions of the top 5 percent of wind speeds and the standard deviation of wind directions of the top 5 percent of wind speeds for the years 2081-2100 from hindcasted values during the December-February season under the RCP8.5 future climatic scenario.

163. Map showing forecasted differences in the mean wind directions of the top 5 percent of wind speeds and the standard deviation of wind directions of the top 5 percent of wind speeds for the years 2081-2100 from hindcasted values during the March-May season under the RCP8.5 future climatic scenario.

164. Map showing forecasted differences in the mean wind directions of the top 5 percent of wind speeds and the standard deviation of wind directions of the top 5 percent of wind speeds for the years 2081-2100 from hindcasted values during the June-August season under the RCP8.5 future climatic scenario. 225

165. Map showing forecasted differences in the mean wind directions of the top 5 percent of wind speeds and the standard deviation of wind directions of the top 5 percent of wind speeds for the years 2081-2100 
from hindcasted values during the September-November season under the RCP8.5 future climatic scenario.

\section{Appendixes}

\section{Appendix A. Wave Height, Wave Period, and Wave Direction Statistics}

A1. Table showing American Samoa monthly means and mean of the top 5 percent for significant wave height, peak wave period, and peak wave direction.

A2. Table showing return values of ensemble-average significant wave heights of hindcast and forecast scenarios, including lower and higher 95 percent confidence intervals, at the American Samoa location.

A3. Table showing Kauai monthly means and mean of the top 5 percent for significant wave height, peak wave period, and peak wave direction.

A4. Table showing return values of ensemble-average significant wave heights of hindcast and forecast scenarios, including lower and higher 95 percent confidence intervals, at the Kauai location.

A5. Table showing Big Island of Hawaii monthly means and mean of the top 5 percent for significant wave height, peak wave period, and peak wave direction.

A6. Table showing return values of ensemble-average significant wave heights of hindcast and forecast scenarios, including lower and higher 95 percent confidence intervals, at the Big Island of Hawaii location.

A7. Table showing Midway monthly means and mean of the top 5 percent for significant wave height, peak wave period, and peak wave direction.

A8. Table showing return values of ensemble-average significant wave heights of hindcast and forecast scenarios, including lower and higher 95 percent confidence intervals, at the Midway location.

A9. Table showing Chuuk monthly means and mean of the top 5 percent for significant wave height, peak wave period, and peak wave direction.

A10. Table showing return values of ensemble-average significant wave heights of hindcast and forecast scenarios, including lower and higher 95 percent confidence intervals, at the Chuuk location.

A11. Table showing Saipan monthly means and mean of the top 5 percent for significant wave height, peak wave period, and peak wave direction.

A12. Table showing return values of ensemble-average significant wave heights of hindcast and forecast scenarios, including lower and higher 95 percent confidence intervals, at the Saipan location.

A13. Table showing Asuncion monthly means and mean of the top 5 percent for significant wave height, peak wave period, and peak wave direction.

A14. Table showing return values of ensemble-average significant wave heights of hindcast and forecast scenarios, including lower and higher 95 percent confidence intervals, at the Asuncion location.

A15. Table showing Kosrae monthly means and mean of the top 5 percent for significant wave height, peak wave period, and peak wave direction.

A16. Table showing return values of ensemble-average significant wave heights of hindcast and forecast scenarios, including lower and higher 95 percent confidence intervals, at the Kosrae location.

A17. Table showing Palau monthly means and mean of the top 5 percent for significant wave height, peak wave period, and peak wave direction.

A18. Table showing return values of ensemble-average significant wave heights of hindcast and forecast scenarios, including lower and higher 95 percent confidence intervals, at the Palau location. 
A19. Table showing Pohnpei monthly means and mean of the top 5 percent for significant wave height, peak wave period, and peak wave direction.

A20. Table showing return values of ensemble-average significant wave heights of hindcast and forecast scenarios, including lower and higher 95 percent confidence intervals, at the Pohnpei location.

A21. Table showing Yap monthly means and mean of the top 5 percent for significant wave height, peak wave period, and peak wave direction.

A22. Table showing return values of ensemble-average significant wave heights of hindcast and forecast scenarios, including lower and higher 95 percent confidence intervals, at the Yap location.

A23. Table showing Majuro monthly means and mean of the top 5 percent for significant wave height, peak wave period, and peak wave direction.

A24. Table showing return values of ensemble-average significant wave heights of hindcast and forecast scenarios, including lower and higher 95 percent confidence intervals, at the Majuro location.

A25. Table showing Enewetak monthly means and mean of the top 5 percent for significant wave height, peak wave period, and peak wave direction.

A26. Table showing return values of ensemble-average significant wave heights of hindcast and forecast scenarios, including lower and higher 95 percent confidence intervals, at the Enewetak location.

A27. Table showing Bikini monthly means and mean of the top 5 percent for significant wave height, peak wave period, and peak wave direction.

A28. Table showing return values of ensemble-average significant wave heights of hindcast and forecast scenarios, including lower and higher 95 percent confidence intervals, at the Bikini location...

A29. Table showing Molokai monthly means and mean of the top 5 percent for significant wave height, peak wave period, and peak wave direction.

A30. Table showing return values of ensemble-average significant wave heights of hindcast and forecast scenarios, including lower and higher 95 percent confidence intervals, at the Molokai location.

A31. Table showing Northwest Hawaiian Islands monthly means and mean of the top 5 percent for significant wave height, peak wave period, and peak wave direction.

A32. Table showing return values of ensemble-average significant wave heights of hindcast and forecast scenarios, including lower and higher 95 percent confidence intervals, at the Northwest Hawaiian Islands location.

A33. Table showing Guam monthly means and mean of the top 5 percent for significant wave height, peak wave period, and peak wave direction.

A34. Table showing return values of ensemble-average significant wave heights of hindcast and forecast scenarios, including lower and higher 95 percent confidence intervals, at the Guam location.

A35. Table showing Kwajalein monthly means and mean of the top 5 percent for significant wave height, peak wave period, and peak wave direction.

A36. Table showing return values of ensemble-average significant wave heights of hindcast and forecast scenarios, including lower and higher 95 percent confidence intervals, at the Kwajalein location.

A37. Table showing Wake monthly means and mean of the top 5 percent for significant wave height, peak wave period, and peak wave direction.

A38. Table showing return values of ensemble-average significant wave heights of hindcast and forecast scenarios, including lower and higher 95 percent confidence intervals, at the Wake location.

A39. Table showing Johnston Atoll monthly means and mean of the top 5 percent for significant wave height, peak wave period, and peak wave direction.

A40. Table showing return values of ensemble-average significant wave heights of hindcast and forecast scenarios, including lower and higher 95 percent confidence intervals, at the Johnston Atoll location. 
A41. Table showing Kingman Reef monthly means and mean of the top 5 percent for significant wave height, peak wave period, and peak wave direction.

A42. Table showing return values of ensemble-average significant wave heights of hindcast and forecast scenarios, including lower and higher 95 percent confidence intervals, at the Kingman Reef location.

A43. Table showing Palmyra monthly means and mean of the top 5 percent for significant wave height, peak wave period, and peak wave direction.

A44. Table showing return values of ensemble-average significant wave heights of hindcast and forecast scenarios, including lower and higher 95 percent confidence intervals, at the Palmyra location.

A45. Table showing Rose Atoll monthly means and mean of the top 5 percent for significant wave height, peak wave period, and peak wave direction.

A46. Table showing return values of ensemble-average significant wave heights of hindcast and forecast scenarios, including lower and higher 95 percent confidence intervals, at the Rose Atoll location.

A47. Table showing Howland monthly means and mean of the top 5 percent for significant wave height, peak wave period, and peak wave direction.

A48. Table showing return values of ensemble-average significant wave heights of hindcast and forecast scenarios, including lower and higher 95 percent confidence intervals, at the Howland location.

A49. Table showing Jarvis monthly means and mean of the top 5 percent for significant wave height, peak wave period, and peak wave direction.

A50. Table showing return values of ensemble-average significant wave heights of hindcast and forecast scenarios, including lower and higher 95 percent confidence intervals, at the Jarvis location.

\section{Appendix B. Wind Speed and Wind Direction Statistics}

B1. Table showing American Samoa monthly means and mean of the top 5 percent for wind speed and mean wind direction.

B2. Table showing return values of ensemble-average wind speeds of hindcast and forecast scenarios, including lower and higher 95 percent confidence intervals, at the American Samoa location.

B3. Table showing Kauai monthly means and mean of the top 5 percent for wind speed and mean wind direction.

B4. Table showing return values of ensemble-average wind speeds of hindcast and forecast scenarios, including lower and higher 95 percent confidence intervals, at the Kauai location.

B5. Table showing Big Island of Hawaii monthly means and mean of the top 5 percent for wind speed and mean wind direction.

B6. Table showing return values of ensemble-average wind speeds of hindcast and forecast scenarios, including lower and higher 95 percent confidence intervals, at the Big Island of Hawaii location.

B7. Table showing Midway monthly means and mean of the top 5 percent for wind speed and mean wind direction.

B8. Table showing return values of ensemble-average wind speeds of hindcast and forecast scenarios, including lower and higher 95 percent confidence intervals, at the Midway location.

B9. Table showing Chuuk monthly means and mean of the top 5 percent for wind speed and mean wind direction.

B10. Table showing return values of ensemble-average wind speeds of hindcast and forecast scenarios, including lower and higher 95 percent confidence intervals, at the Chuuk location.

B11. Table showing Saipan monthly means and mean of the top 5 percent for wind speed and mean wind direction. 
B12. Table showing return values of ensemble-average wind speeds of hindcast and forecast scenarios, including lower and higher 95 percent confidence intervals, at the Saipan location.

B13. Table showing Asuncion monthly means and mean of the top 5 percent for wind speed and mean wind direction.

B14. Table showing return values of ensemble-average wind speeds of hindcast and forecast scenarios, including lower and higher 95 percent confidence intervals, at the Asuncion location.

B15. Table showing Kosrae monthly means and mean of the top 5 percent for wind speed and mean wind direction.

B16. Table showing return values of ensemble-average wind speeds of hindcast and forecast scenarios, including lower and higher 95 percent confidence intervals, at the Kosrae location.

B17. Table showing Palau monthly means and mean of the top 5 percent for wind speed and mean wind direction.

B18. Table showing return values of ensemble-average wind speeds of hindcast and forecast scenarios, including lower and higher 95 percent confidence intervals, at the Palau location.

B19. Table showing Pohnpei monthly means and mean of the top 5 percent for wind speed and mean wind direction.

B20. Table showing return values of ensemble-average wind speeds of hindcast and forecast scenarios, including lower and higher 95 percent confidence intervals, at the Pohnpei location.

B21. Table showing Yap monthly means and mean of the top 5 percent for wind speed and mean wind direction.

B22. Table showing return values of ensemble-average wind speeds of hindcast and forecast scenarios, including lower and higher 95 percent confidence intervals, at the Yap location.

B23. Table showing Majuro monthly means and mean of the top 5 percent for wind speed and mean wind direction.

B24. Table showing return values of ensemble-average wind speeds of hindcast and forecast scenarios, including lower and higher 95 percent confidence intervals, at the Majuro location.

B25. Table showing Enewetak monthly means and mean of the top 5 percent for wind speed and mean wind direction.

B26. Table showing return values of ensemble-average wind speeds of hindcast and forecast scenarios, including lower and higher 95 percent confidence intervals, at the Enewetak location.

B27. Table showing Bikini monthly means and mean of the top 5 percent for wind speed and mean wind direction.

B28. Table showing return values of ensemble-average wind speeds of hindcast and forecast scenarios, including lower and higher 95 percent confidence intervals, at the Bikini location......

B29. Table showing Molokai monthly means and mean of the top 5 percent for wind speed and mean wind direction.

B30. Table showing return values of ensemble-average wind speeds of hindcast and forecast scenarios, including lower and higher 95 percent confidence intervals, at the Molokai location.

B31. Table showing Northwest Hawaiian Islands monthly means and mean of the top 5 percent for wind speed and mean wind direction.

B32. Table showing return values of ensemble-average wind speeds of hindcast and forecast scenarios, including lower and higher 95 percent confidence intervals, at the Northwest Hawaiian Islands location.

B33. Table showing Guam monthly means and mean of the top 5 percent for wind speed and mean wind direction.

B34. Table showing return values of ensemble-average wind speeds of hindcast and forecast scenarios, including lower and higher 95 percent confidence intervals, at the Guam location... 
B35. Table showing Kwajalein monthly means and mean of the top 5 percent for wind speed and mean wind direction.

B36. Table showing return values of ensemble-average wind speeds of hindcast and forecast scenarios, including lower and higher 95 percent confidence intervals, at the Kwajalein location.

B37. Table showing Wake monthly means and mean of the top 5 percent for wind speed and mean wind direction.

B38. Table showing return values of ensemble-average wind speeds of hindcast and forecast scenarios, including lower and higher 95 percent confidence intervals, at the Wake location.

B39. Table showing Johnston Atoll monthly means and mean of the top 5 percent for wind speed and mean wind direction

B40. Table showing return values of ensemble-average wind speeds of hindcast and forecast scenarios, including lower and higher 95 percent confidence intervals, at the Johnston Atoll location.

B41. Table showing Kingman Reef monthly means and mean of the top 5 percent for wind speed and mean wind direction.

B42. Table showing return values of ensemble-average wind speeds of hindcast and forecast scenarios, including lower and higher 95 percent confidence intervals, at the Kingman Reef location.

B43. Table showing Palmyra monthly means and mean of the top 5 percent for wind speed and mean wind direction.

B44. Table showing return values of ensemble-average wind speeds of hindcast and forecast scenarios, including lower and higher 95 percent confidence intervals, at the Palmyra location.

B45. Table showing Rose Atoll monthly means and mean of the top 5 percent for wind speed and mean wind direction.

B46. Table showing return values of ensemble-average wind speeds of hindcast and forecast scenarios, including lower and higher 95 percent confidence intervals, at the Rose Atoll location.

B47. Table showing Howland monthly means and mean of the top 5 percent for wind speed and mean wind direction.

B48. Table showing return values of ensemble-average wind speeds of hindcast and forecast scenarios, including lower and higher 95 percent confidence intervals, at the Howland location.

B49. Table showing Jarvis monthly means and mean of the top 5 percent for wind speed and mean wind direction.

B50. Table showing return values of ensemble-average wind speeds of hindcast and forecast scenarios, including lower and higher 95 percent confidence intervals, at the Jarvis location.

\section{Appendix C. Monthly Trends in Wave Heights}

C1. Plots showing trends in monthly mean significant wave height $\left(H_{s}\right)$, in meters, at the American Samoa location.

C2. Plots showing trends in monthly mean of the top 5 percent of significant wave heights $\left(H_{s}\right)$, in meters, at the American Samoa location.

C3. Plots showing trends in monthly mean significant wave height $\left(H_{s}\right)$, in meters, at the Kauai location.

C4. Plots showing trends in monthly mean of the top 5 percent of significant wave heights $\left(H_{s}\right)$, in meters, at the Kauai location.

C5. Plots showing trends in monthly mean significant wave height $\left(H_{s}\right)$, in meters, at the Big Island of Hawaii location.

C6. Plots showing trends in monthly mean of the top 5 percent of significant wave heights $\left(H_{s}\right)$, in meters, at the Big Island of Hawaii location. 
C7. Plots showing trends in monthly mean significant wave height $\left(H_{s}\right)$, in meters, at the Midway location. ...............333

C8. Plots showing trends in monthly mean of the top 5 percent of significant wave heights $\left(H_{s}\right)$, in meters, at the Midway location.

C9. Plots showing trends in monthly mean significant wave height $\left(H_{s}\right)$, in meters, at the Chuuk location.

C10. Plots showing trends in monthly mean of the top 5 percent of significant wave heights $\left(H_{s}\right)$, in meters, at the Chuuk location.

C11. Plots showing trends in monthly mean significant wave height $\left(H_{s}\right)$, in meters, at the Saipan location..

C12. Plots showing trends in monthly mean of the top 5 percent of significant wave heights $\left(H_{s}\right)$, in meters, at the Saipan location.

C13. Plots showing trends in monthly mean significant wave height $\left(H_{s}\right)$, in meters, at the Asuncion location......

C14. Plots showing trends in monthly mean of the top 5 percent of significant wave heights $\left(H_{s}\right)$, in meters, at the Asuncion location

C15. Plots showing trends in monthly mean significant wave height $\left(H_{s}\right)$, in meters, at the Kosrae location......

C16. Plots showing trends in monthly mean of the top 5 percent of significant wave heights $\left(H_{s}\right)$, in meters, at the Kosrae location.

C17. Plots showing trends in monthly mean significant wave height $\left(H_{s}\right)$, in meters, at the Palau location.

C18. Plots showing trends in monthly mean of the top 5 percent of significant wave heights $\left(H_{\mathrm{s}}\right)$, in meters, at the Palau location.

C19. Plots showing trends in monthly mean significant wave height $\left(H_{s}\right)$, in meters, at the Pohnpei location.

C20. Plots showing trends in monthly mean of the top 5 percent of significant wave heights $\left(H_{s}\right)$, in meters, at the Pohnpei location..

C21. Plots showing trends in monthly mean significant wave height $\left(H_{s}\right)$, in meters, at the Yap location......

C22. Plots showing trends in monthly mean of the top 5 percent of significant wave heights $\left(H_{s}\right)$, in meters, at the Yap location.

C23. Plots showing trends in monthly mean significant wave height $\left(H_{s}\right)$, in meters, at the Majuro location.

C24. Plots showing trends in monthly mean of the top 5 percent of significant wave heights $\left(H_{s}\right)$, in meters, at the Majuro location.

C25. Plots showing trends in monthly mean significant wave height $\left(H_{s}\right)$, in meters, at the Enewetak location.

C26. Plots showing trends in monthly mean of the top 5 percent of significant wave heights $\left(H_{s}\right)$, in meters, at the Enewetak location.

C27. Plots showing trends in monthly mean significant wave height $\left(H_{s}\right)$, in meters, at the Bikini location......

C28. Plots showing trends in monthly mean of the top 5 percent of significant wave heights $\left(H_{\mathrm{s}}\right)$, in meters, at the Bikini location.

C29. Plots showing trends in monthly mean significant wave height $\left(H_{s}\right)$, in meters, at the Molokai location.

C30. Plots showing trends in monthly mean of the top 5 percent of significant wave heights $\left(H_{s}\right)$, in meters, at the Molokai location.

C31. Plots showing trends in monthly mean significant wave height $\left(H_{s}\right)$, in meters, at the Northwest Hawaiian Islands location.

C32. Plots showing trends in monthly mean of the top 5 percent of significant wave heights $\left(H_{\mathrm{s}}\right)$, in meters, at the Northwest Hawaiian Islands location.

C33. Plots showing trends in monthly mean significant wave height $\left(H_{s}\right)$, in meters, at the Guam location......

C34. Plots showing trends in monthly mean of the top 5 percent of significant wave heights $\left(H_{s}\right)$, in meters, at the Guam location.

C35.

Plots showing trends in monthly mean significant wave height $\left(H_{s}\right)$, in meters, at the Kwajalein location. 
C36. Plots showing trends in monthly mean of the top 5 percent of significant wave heights $\left(H_{\mathrm{s}}\right)$, in meters, at the Kwajalein location.

C37. Plots showing trends in monthly mean significant wave height $\left(H_{s}\right)$, in meters, at the Wake location..

C38. Plots showing trends in monthly mean of the top 5 percent of significant wave heights $\left(H_{s}\right)$, in meters, at the Wake location.

C39. Plots showing trends in monthly mean significant wave height $\left(H_{s}\right)$, in meters, at the Johnston Atoll location.

C40. Plots showing trends in monthly mean of the top 5 percent of significant wave heights $\left(H_{s}\right)$, in meters, at the Johnston Atoll location.

C41. Plots showing trends in monthly mean significant wave height $\left(H_{s}\right)$, in meters, at the Kingman Reef location.

C42. Plots showing trends in monthly mean of the top 5 percent of significant wave heights $\left(H_{s}\right)$, in meters, at the Kingman Reef location.

C43. Plots showing trends in monthly mean significant wave height $\left(H_{s}\right)$, in meters, at the Palmyra Atoll location.

C44. Plots showing trends in monthly mean of the top 5 percent of significant wave heights $\left(H_{s}\right)$, in meters, at the Palmyra Atoll location.

C45. Plots showing trends in monthly mean significant wave height $\left(H_{s}\right)$, in meters, at the Rose Atoll location.

C46. Plots showing trends in monthly mean of the top 5 percent of significant wave heights $\left(H_{s}\right)$, in meters, at the Rose Atoll location.

C47. Plots showing trends in monthly mean significant wave height $\left(H_{s}\right)$, in meters, at the Howland location.

C48. Plots showing trends in monthly mean of the top 5 percent of significant wave heights $\left(H_{s}\right)$, in meters, at the Howland location.

C49. Plots showing trends in monthly mean significant wave height $\left(H_{s}\right)$, in meters, at the Jarvis location......

C50. Plots showing trends in monthly mean of the top 5 percent of significant wave heights $\left(H_{s}\right)$, in meters, at the Jarvis location.

\section{Appendix D. Monthly Trends in Wind Speeds}

D1. Plots showing trends in monthly mean wind speed, in meters per second, at the American Samoa location.

D2. Plots showing trends in monthly mean of the top 5 percent of wind speeds, in meters, at the American Samoa location.

D3. Plots showing trends in monthly mean wind speed, in meters per second, at the Kauai location.

D4. Plots showing trends in monthly mean of the top 5 percent of wind speeds, in meters, at the Kauai location.

D5. Plots showing trends in monthly mean wind speed, in meters per second, at the Big Island of Hawaii location.

D6. Plots showing trends in monthly mean of the top 5 percent of wind speeds, in meters, at the Big Island of Hawaii location.

D7. Plots showing trends in monthly mean wind speed, in meters per second, at the Midway location

D8. Plots showing trends in monthly mean of the top 5 percent of wind speeds, in meters, at the Midway location.

D9. Plots showing trends in monthly mean wind speed, in meters per second, at the Chuuk location. 
D10. Plots showing trends in monthly mean of the top 5 percent of wind speeds, in meters, at the Chuuk location.

D11. Plots showing trends in monthly mean wind speed, in meters per second, at the Saipan location.

D12. Plots showing trends in monthly mean of the top 5 percent of wind speeds, in meters, at the Saipan location.

D13. Plots showing trends in monthly mean wind speed, in meters per second, at the Asuncion location.

D14. Plots showing trends in monthly mean of the top 5 percent of wind speeds, in meters, at the Asuncion location.

D15. Plots showing trends in monthly mean wind speed, in meters per second, at the Kosrae location.

D16. Plots showing trends in monthly mean of the top 5 percent of wind speeds, in meters, at the Kosrae location.

D17. Plots showing trends in monthly mean wind speed, in meters per second, at the Palau location.

D18. Plots showing trends in monthly mean of the top 5 percent of wind speeds, in meters, at the Palau location.

D19. Plots showing trends in monthly mean wind speed, in meters per second, at the Pohnpei location........

D20.

Plots showing trends in monthly mean of the top 5 percent of wind speeds, in meters, at the Pohnpei location.

D24. Plots showing trends in monthly mean of the top 5 percent of wind speeds, in meters, at the Majuro location.

D25.

Plots showing trends in monthly mean wind speed, in meters per second, at the Enewetak location.

D27. Plots showing trends in monthly mean wind speed, in meters per second, at the Bikini location.

D29. Plots showing trends in monthly mean of the top 5 percent of wind speeds, in meters, at the Bikini location.

D31. Plots showing trends in monthly mean wind speed, in meters per second, at the Northwest Hawaiian Islands location.

D32. Plots showing trends in monthly mean of the top 5 percent of wind speeds, in meters, at the Northwest Hawaiian Islands location.

D33. Plots showing trends in monthly mean wind speed, in meters per second, at the Guam location.

D34. Plots showing trends in monthly mean of the top 5 percent of wind speeds, in meters, at the Guam location.

D35. Plots showing trends in monthly mean wind speed, in meters per second, at the Kwajalein location.

D36. Plots showing trends in monthly mean of the top 5 percent of wind speeds, in meters, at the Kwajalein location.

D37.

D38.

Plots showing trends in monthly mean wind speed, in meters per second, at the Wake location.

Plots showing trends in monthly mean of the top 5 percent of wind speeds, in meters, at the Wake location.

D39. 
D40. Plots showing trends in monthly mean of the top 5 percent of wind speeds, in meters, at the Johnston Atoll location

D41. Plots showing trends in monthly mean wind speed, in meters per second, at the Kingman Reef location.

D42. Plots showing trends in monthly mean of the top 5 percent of wind speeds, in meters, at the Kingman Reef location.

D43. Plots showing trends in monthly mean wind speed, in meters per second, at the Palmyra Atoll location

D44. Plots showing trends in monthly mean of the top 5 percent of wind speeds, in meters, at the Palmyra Atoll location.

D45. Plots showing trends in monthly mean wind speed, in meters per second, at the Rose Atoll location.

D46. Plots showing trends in monthly mean of the top 5 percent of wind speeds, in meters, at the Rose Atoll location.

D47. Plots showing trends in monthly mean wind speed, in meters per second, at the Howland location.

D48. Plots showing trends in monthly mean of the top 5 percent of wind speeds, in meters, at the Howland location.

D49. Plots showing trends in monthly mean wind speed, in meters per second, at the Jarvis location.

D50. Plots showing trends in monthly mean of the top 5 percent of wind speeds, in meters, at the Jarvis location. 


\begin{tabular}{ll} 
Abbreviations \\
AORI & Atmosphere and Ocean Research Institute, University of Tokyo \\
BCC & Beijing Climate Center, China \\
CDF & cumulative density function \\
CMIP & Coupled Model Inter-Comparison Project \\
D & mean wave direction \\
DBDB2 & Digital Bathymetric Data Base \\
DJF & December to February time period \\
DOD & U.S. Department of Defense \\
DOI & U.S. Department of the Interior \\
GCM & global climate model \\
GFDL & Geophysical Fluid Dynamics Laboratory, NOAA \\
GIS & geographic information system \\
GPD & generalized Pareto distribution \\
GSHHS & Global Self-consistent Hierarchical High-resolution Geography Database \\
$H_{S}$ & significant wave height \\
INMCM & Institute of Numerical Mathematics (Russia) climate model \\
JAMSTEC & Japan Agency for Marine-Earth Science and Technology \\
JJA & June to August time period \\
MAE & mean absolute error \\
MAM & March to May time period \\
MIROC & Model for Interdisciplinary Research on Climate \\
NDBC & National Data Buoy Center \\
NGDC & National Geophysical Data Center \\
NIES & National Institute for Environmental Studies, Japan \\
NRL & Naval Research Laboratory \\
\hline
\end{tabular}




$\begin{array}{ll}\text { nVar } & \text { normalized variance } \\ \text { NWW3 } & \text { a near-global domain for the wave model } \\ R_{V} & \text { return value } \\ \text { RCP } & \text { representative concentration pathways } \\ \text { SON } & \text { September to November time period } \\ \text { SRES } & \text { Special Report on Emission Scenarios } \\ \text { SWAN } & \text { Simulating WAves Nearshore, a wave model } \\ T_{p} & \text { peak wave period } \\ U_{a} & \text { wind speed } \\ U_{\theta} & \text { wind direction } \\ \text { WTP } & \text { western tropical Pacific } \\ \text { WW3 } & \text { WAVEWATCH-III wave model }\end{array}$




\title{
Future Wave and Wind Projections for United States and United States-Affiliated Pacific Islands
}

\author{
By Curt D. Storlazzi ${ }^{1}$, James B. Shope ${ }^{2}$, Li H. Erikson ${ }^{1}$, Christie A. Hegermiller ${ }^{2}$, and Patrick L. Barnard ${ }^{1}$
}

\begin{abstract}
Changes in future wave climates in the tropical Pacific Ocean from global climate change are not well understood. Spatially and temporally varying waves dominate coastal morphology and ecosystem structure of the islands throughout the tropical Pacific. Waves also impact coastal infrastructure, natural and cultural resources, and coastal-related economic activities of the islands. Wave heights, periods, and directions were forecast through the year 2100 using wind parameter outputs from four atmosphere-ocean global climate models from the Coupled Model Inter-Comparison Project, Phase 5, for Representative Concentration Pathways (RCP) scenarios 4.5 and 8.5 that correspond to moderately mitigated and unmitigated greenhouse gas emissions, respectively. Wind fields from the global climate models were used to drive a global WAVEWATCH-III wave model and generate hourly time-series of bulk wave parameters for 25 islands in the mid to western tropical Pacific for the years 1976-2005 (historical), 2026-2045 (mid-century projection), and 2085-2100 (end-of-century projection). Although the results show some spatial heterogeneity, overall the December-February extreme significant wave heights, defined as the mean of the top 5 percent of significant wave height time-series data modeled within a specific period, increase from present to mid-century and then decrease toward the end of the century; June-August extreme wave heights increase throughout the century within the Central region of the study area; and September-November wave heights decrease strongly throughout the 21st century, displaying the largest and most widespread decreases of any season. Peak wave periods increase east of the International Date Line during the December-February and JuneAugust seasons under RCP4.5. Under the RCP8.5 scenario, wave periods decrease west of the International Date Line during December-February but increase in the eastern half of the study area. Otherwise, wave periods decrease throughout the study area during other seasons. Extreme wave directions in equatorial Micronesia during June-August undergo an approximate $30^{\circ}$ clockwise rotation from primarily west to northwest. September-November RCP4.5 extreme mean wave directions rotate counterclockwise by approximately 30 to $45^{\circ}$ in equatorial Micronesia; September-November RCP8.5 extreme mean wave directions within equatorial Micronesia rotate
\end{abstract}

\footnotetext{
${ }^{1}$ U.S. Geological Survey.

${ }^{2}$ University of California at Santa Cruz.
} 
clockwise by approximately 20 to $30^{\circ}$. Extreme wind speeds decreased within both scenarios, with the largest decreases occurring in the September-November season. Extreme wind directions under RCP4.5 rotated clockwise by more than $60^{\circ}$ in equatorial Micronesia during the SeptemberNovember season and by approximately $30^{\circ}$ during June-August. RCP8.5 extreme wind directions rotated counterclockwise during September-November within the same region by 30 to $50^{\circ}$ and clockwise by 30 to $40^{\circ}$ at one island. The spatial patterns and trends are similar between the two different greenhouse gas emission scenarios, with the magnitude and extent of the trends generally greater for the higher (RCP8.5) scenario.

\section{Introduction}

The oceanographic processes that disturb coasts and insular shelves include the actions of surface waves, internal waves, and currents (tidal, density, wave-driven, wind-driven, and geostrophic). The Pacific Ocean can generate extremely large ocean surface waves, and the resulting near-bed wave-orbital velocities on insular shelves are generally much larger than velocities due to currents and internal waves (see, for example, Storlazzi and Reid, 2010). Although a few studies have investigated the wave climate in Hawaii (for example, Vitousek and Fletcher, 2008; Hoeke and others, 2011), there is little information on the wind and wave climate for most of the western Pacific Ocean because of a lack of observational platforms (such as National Data Buoy Center buoys). Whereas scientific understanding of the dominant processes controlling coastal morphology and coastal and marine ecosystem structure on islands has improved over the past decade, our understanding of the linkages between these factors and variations in the wave climate across the Pacific and U.S.-managed assets is limited.

Furthermore, the influence of global climate change on wind and wave conditions is not well understood. Stationary statistical approaches (such as return values) have typically been used to predict future extreme and mean wind and wave conditions, but with the changing climate this may not be a valid approach. Although some nonstationary statistical approaches (for example, nonstationary generalized extreme values) may sufficiently capture the variations and changes, recent work seems to point in the direction that the current climate alone cannot be used to estimate future conditions. It has been shown that calculated return values from different decades are not compatible with each other in the North Pacific (for example, Caires and others, 2006) and that under different climate scenarios for the 21st century, the rate of projected future wave height changes is not constant and depends on variations in the greenhouse-gas forcings (see, for example, Wang and Swail, 2006). Applying downscaling methods, such as making causal relations between winds and sea-level pressures with wave heights, may be appropriate for areas of the Atlantic and other smaller water bodies, but for the Pacific Ocean, where swell of periods in excess of 15 seconds generated by very distant storms is of concern, it is more difficult to make such empirical relations. An alternative option is then to obtain wave conditions through analytical or numerical wave models forced with projected climate conditions from global climate models (GCMs). 
Some work has been done to estimate future wave conditions in the Pacific using wave models and projected winds from GCMs (for example, Cayan and others, 2008; Mori and others, 2010; Hemer and others, 2013). However, the data are sparse and not freely available for trendand region-specific analysis. Furthermore, the recent and continued update of GCM outputs warranted the development of a new mean and extreme wave climatology for U.S. assets in the Pacific Basin. This climatology is important because wave- and wind-driven processes drive inundation (for example, Stockdon and others, 2006) that results in coastal erosion and damage to infrastructure and fresh-water supplies (for example, in the Federated States of Micronesia in 2008; Fletcher and Richmond, 2010) and can damage natural resources and kill U.S.-Federally protected species (for example, in the Northwestern Hawaiian Islands in January and February 2011; unpublished data). Such impacts may only be exacerbated in the future with projected trends in sea-level rise (for example, Storlazzi and others, 2011). Furthermore, numerical modeling efforts have shown how wave energy constrains marine species' distribution in the Pacific Ocean (for example, Storlazzi and others, 2005). Information on potential changes in wind and wave climate under future global climate change scenarios is therefore crucial to understanding not only the sustainability of existing infrastructure, natural, and cultural resources, but also planning for future investments in infrastructure and the viability of coastal-related economic activities such as fishing and tourism.

\section{Project Objectives}

The goal of this effort was to provide hourly data and statistical measures (mean and mean of top 5 percent values) of wave height, wave period, wave direction, wind speed, and wind direction for Department of the Interior (DOI)- and Department of Defense (DOD)-managed assets in the Pacific Ocean for the historical (1976-2005) time period and future time periods (out to the year 2100). The goal was accomplished by forcing the WAVEWATCH-III numerical wave model with winds computed by global climate models (GCMs) for two climate scenarios, Representative Concentration Pathways (RCP) RCP4.5 and RCP8.5 (see section on "Methods" for further descriptions). Resulting model data are summarized with wave and wind statistics at 25 islands (table 1) comprising U.S. National Parks, National Wildlife Refuges, U.S. National Monuments, areas managed by the U.S. Office of Insular Affairs, and U.S. Department of Defense facilities.

The data presented herein provide information required for adaptive conservation planning by providing a better understanding of potential climate change impacts. This effort investigated trends and variability, on an island and archipelago scale, of geophysical variables that are expected to respond to global-scale forcing. Furthermore, the data generated by this effort are expected to be crucial in projecting future transient sea level extremes on coasts and small islands, because winds and waves are the key processes driving extreme water levels and inundation. 


\section{Study Area}

Wave and wind statistics were generated for 25 locations across the western tropical Pacific (WTP) at a number of United States territories and U.S.-affiliated islands (fig. 1). The modeled area encompasses a number of varying regions subject to varying climates. The trade winds dominate in the region, blowing from the east. However, despite the main winds being the trades, the stronger winds, and thus the larger waves, result from storm systems within the region. The northeast quadrant of the study area receives strong winds and large waves from higher latitude storms during the boreal winter (Semedo and others, 2011). Winds in the rest of the eastern half of the study area remain dominated by the trade winds, though larger swell waves are likely due to storms from the South Pacific and Southern Ocean. The western half of the study area's extreme winds and waves are dominated by typhoons, which are generated in the center of the region and proceed to the west, and by extratropical cyclones from the North Pacific (Mori and others, 2010). Therefore, islands such as Guam receive larger, more destructive waves because it is subject to larger typhoons. However, this study focused primarily on swell waves from extratropical regions and how they may change in the future. These swell waves, while likely smaller than typhoon-generated waves, still pose a hazard to the western Pacific islands throughout the 21st century.

\section{Methods}

\section{Global Climate Model Input Data}

A dynamical downscaling (Wang and others, 2009) approach was used to generate recent and future wave climates for the study areas by using updated climate scenarios and models as input for the numerical wave model WAVEWATCH-III (WW3; Tolman, 2009). For the purpose of assessing recent past and projected wave climates, a suite of atmosphere-ocean coupled GCM simulations of near-surface (10-m height) wind fields were chosen on the basis of the availability of (1) "historical" runs for evaluation of how realistic the models are in simulating the recent past, (2) projections out to the year 2100, (3) GCM simulations completed by spring 2012, and (4) frequency of synoptic (nonaveraged) outputs (3-hr interval). Based on these requirements, the following set of experiments from four modeling centers were chosen: (a) Beijing Climate Center, Meteorological Administration, China, model BCC-CSM1.1; (b) Institute for Numerical Mathematics, Russia, model INM-CM4; (c) Model for Interdisciplinary Research on ClimateAOEI, NIES, JAMSTEC, Japan, model MIROC5; and (d) Geophysical Fluid Dynamics Laboratory, United States, GFDL-ESM2M. These GCM model outputs were generated in support of the Coupled Model Inter-Comparison Project, Phase 5 (CMIP5; World Climate Research Programme, 2013). GCM model resolutions are described in table 2. CMIP5 was proposed, conceived, and developed by the climate modeling community with the intent of providing a framework for coordinated climate change experiments conducted at various institutions around the globe (Taylor and others, 2009). Although hundreds of model simulations are available as part of this effort, this work focused on a few select simulations, as described above. 
Future emission scenarios differed slightly from past CMIP work, in which SRES scenarios (named after their publication "Special Report on Emission Scenarios") provided six alternative scenarios based on narrative storylines (for example, van Vuuren and others, 2011). The "new" scenarios or so-called "representative concentration pathways" (RCPs) are labeled according to the approximate target radiative forcing at year 2100 (for example, RCP4.5 identifies a concentration pathway that approximately results in a radiative forcing of 4.5 watts per square meter $\left(\mathrm{W} / \mathrm{m}^{2}\right.$ ) by year 2100 relative to preindustrial conditions). In this study, GCM results from two climate scenarios were used, one representing a medium mitigation (RCP4.5; Thomson and others, 2011) and one a high-emissions scenario of $8.5 \mathrm{~W} / \mathrm{m}^{2}$ by year 2100 (RCP8.5; Riahi and others, 2010). Projections for scenarios RCP4.5 and RCP8.5 are available for two separate time periods in the 21st century: years 2026-2045 and 2085-2100, as prescribed by the CMIP5 modeling framework. The historical (1976-2005) simulations were used to assess model skill and determine the magnitude of change in the future scenarios.

\section{WAVEWATCH-III Wave Modeling}

To reduce uncertainty in model variability, wind speed and direction from the four different GCMs were used as boundary conditions to the third-generation physics-based WAVEWATCH-III (WW3, ver. 3.14, Tolman, 2009) numerical wave model. WW3 solves the random phase spectral action density balance equation for wave number-direction spectra. The implicit assumption of this equation is that properties of the medium (water depth and current) as well as the wave field itself vary on time and space scales that are much larger than the variation scales of a single wave. The model was implemented over a near-global domain (NWW3, latitude $80^{\circ} \mathrm{S}$ to $80^{\circ} \mathrm{N}$ ) at a $1^{\circ} \mathrm{x} 1.25^{\circ}$ spatial resolution. Bathymetry and shoreline positions were populated with the Naval Research Laboratory’s (NRL) Digital Bathymetric Data Base (DBDB2) version 3.0 (National Research Laboratory, 2013) and the National Geophysical Data Center's (NGDC) Global Self-consistent Hierarchical High-resolution Geography Database (GSHHS) version 2.2.2 (National Geophysical Data Center, 2013), respectively. Wave spectra were computed at a directional resolution of $15^{\circ}$ and using 25 frequency bands ranging nonlinearly from 0.04 to $0.50 \mathrm{~Hz}$. Parameterizations of physical processes (source terms) include wave growth and decay due to the actions of wind, nonlinear resonant interactions, dissipation, bottom friction, surf-breaking (for example, depth-induced breaking) and scattering due to wave-bottom interactions.

Map files of wave heights, periods, and directions and wind speeds and directions were saved on a daily basis, while time series of wind and wave parameters at deep-water observation (output) points in the model were saved on a 3-hourly and hourly basis, respectively. WW3 was run at a global resolution scale to capture the Pacific Ocean in a computationally efficient manner (table 1). A few output points (coincident with NDBC measurement buoys) were used for model/measurement comparisons; comparisons were done in a probabilistic sense (for example, cumulative density functions), not in a time-series sense, as the goal was to understand the overall climate and not the details of potential future modeled storm events, which were not possible 
without major downscaling of winds. The deep-water output points are intended to serve as points for analysis of wave climate trends, which can then be used as inputs to nearshore wave models (for example, Deltares' SWAN) investigating impacts to infrastructure, natural, and cultural resources.

\section{Wave Parameter Statistics}

Modeled output data comprised (1) significant wave height $\left(H_{s}\right)$, peak wave period $\left(T_{p}\right)$, and mean wave direction $\left(D_{p}\right)$ values saved at hourly intervals and (2) wind speed $\left(U_{a}\right)$ and wind direction $\left(U_{\theta}\right)$ values saved at 3-hour intervals. $H_{s}$ is defined as 4 times the standard deviation of the wave displacement time series during a modeled time period, which approximates the mean of the top third of measured wave heights within that period, with units in meters $(\mathrm{m}) . T_{p}$ is defined as the wave period with the largest energy within a modeled timestep, with units of seconds (s). $U_{a}$ units are in meters per second $(\mathrm{m} / \mathrm{s}) . D_{p}$ and $U_{\theta}$ values are defined within this report as the direction in degrees clockwise from North $\left(^{\circ}\right)$ in which the waves and winds are travelling. Outputs from each GCM-driven WW3 model run were combined into a multimodel ensemble. An ensemble of models has been shown to more accurately represent observed data than any one model for dynamic GCMs and regional climate models (RCMs) with similar parameters (Donat and others, 2010).

Averages and variances (or standard deviations) for monthly and seasonal extreme $H_{S}$ $\left(\sigma_{H s}^{2}\right), T_{p}\left(\sigma_{T p}^{2}\right), D_{p}\left(\sigma_{D p}\right), U_{a}\left(\sigma_{U a}^{2}\right)$, and $U_{\theta}\left(\sigma_{U \theta}\right)$ values were calculated for hindcast, mid-century, and end-century periods, with averages for each RCP emission scenario being calculated for the latter two periods. Angular standard deviation values were calculated for wave directional data because the standard deviation values produced were more easily interpretable compared to variances, which, when calculated, produced an extremely large range to visualize, as the angular standard deviation values were often more than $10^{\circ}$, resulting in variances that ranged from 100 to more than 1,000 variance being the square of the standard deviation. Variance values for other variables were more comparable with one another. The process was repeated for extreme $H_{s}$ scenarios, which use the variables relating to the the mean of the top 5 percent of $H_{s}$ or $U_{a}$ timeseries values modeled within each modeled time period. For example, extreme $H_{s}$ values are calculated as the mean of the top 5 percent of $H_{s}$ values within a time period, whereas extreme $T_{p}$ values are defined as the mean of the $T_{p}$ values associated with the top 5 percent of $H_{s}$ values within a given time period. Changes in bulk parameters were derived by subtracting hindcast means from forecast averages. The climate variables for the December-February (DJF), MarchMay (MAM), June-August (JJA), and September-November (SON) seasons were mapped using the ArcGIS geographic information systems software developed by the Environmental Systems Research Institute with the expectation that the largest or most important changes in $H_{s}$ would occur during those seasons. For example, $H_{s}$ during the northern hemisphere winter in Hawaii is typically larger than during other seasons (Vitousek and Fletcher, 2008). Therefore, changes in 
these wave heights may have a stronger impact on overtopping and erosional events as witnessed throughout the tropical Pacific Ocean in 2009 (Hoeke and others. 2013).

Return values $\left(R_{v}\right)$ for each modeled point for $H_{s}$ and $U_{a}$ were generated for 2-, 5-, 10-, 20 , 50-, and 100-year events for the hindcast, RCP4.5 projections, and RCP8.5 projections following the methods of Caires and Sterl (2005) and Caires and others (2006). The ensemble $H_{s}$ and $U_{a}$ data were declustered so that the largest events were selected, but consecutive exceedances of individual storms were not selected. Individual events were declustered to be at least 3 days apart, to ensure that each selected large wave or wind event was from a separate storm. The largest declustered 150 events (30 years of 5 events per year on average) from the hindcast ensemble and declustered 100 events (20 years of 5 events per year on average) from each forecast ensemble were fitted to a generalized Pareto distribution (GPD), which can be used approximate $H_{s}$ and $U_{a}$ extrema. The GPD method is a peak-over-threshold method. In each calculation, the threshold value selected was the minimum value of the selected declustered extreme modeled events. Once fitted, the $R_{v}$ trend is extrapolated from the 20 or 30 years of data modeled to a 100-year event. Therefore, it is possible to estimate an $R_{v}$ for a 100 -year event without 100 years of data, contrary to more traditional methods. The 95-percent confidence intervals of $R_{v}$ values were also calculated at each point.

\section{Model Skill}

Ensemble model hindcasts (1976-2005) were compared to buoy measurements from the National Data Buoy Center (NDBC) platforms in the Hawaiian Island Chain for the same period to evaluate how well the model approximated observed climate trends (National Data Buoy Center, 2013). The Pacific Ocean historical buoy dataset is largely scattered; until recently, only a small areal extent of the ocean basin was buoyed. Only the Hawaiian Island Chain has observation platforms with sufficiently long datasets that corresponded to selected island locations of Molokai, Kauai, and the Big Island; these are NDBC stations 51001, 51002, 51003, and 51004 (fig. 2). The selected buoys collected deep-water wave and meteorological measurements at hourly intervals. This dataset, however, was limited to $H_{s}, T_{p}, U_{a}$, and $U_{\theta}$, with $D_{p}$ not recorded. The WAVEWATCH-III model resolution limited the spatial overlap of output points and buoy locations. Therefore, model output points were compared to historical buoy measurements instead of comparing virtual buoy output to physical buoys. The model resolution of $1.25^{\circ}$ latitude and $1.00^{\circ}$ longitude limited the ability to place an output point coincident to physical buoy locations.

Model performance was assessed by the mean absolute error (MAE) and normalized variance (nVar) calculated from hindcasted and observed values of $H_{s}$. The MAE is a good indication of model accuracy when model and observed values are at approximately the same scale (Hyndman and Koehler, 2006). The MAE is given by:

$$
M A E=\frac{1}{N} \sum_{i=1}^{N}\left|o b s_{i}-\bmod _{i}\right|
$$


where $o b s_{i}=$ the observed value at the $i^{\text {th }}$ value and $\bmod _{i}=$ the modeled value at the $i^{\text {th }}$ value.

The MAE was calculated probabilistically from the empirical cumulative density functions (CDFs) of the seasonal and annual ensemble model and observed datasets after the CDFs were interpolated to the same number of points within cumulative frequency space. That is, the CDFs were interpolated to the same number of points in terms of the y-axis. This interpolation enabled functions to be input into the MAE equation, which required both inputs to be the same length, and preserved the curves' positioning along the $H_{s}$ (x-axis) and the $\mathrm{x}$-axis distance between the two curves. The MAE formula then returned a mean of the distance in terms of $H_{s}$ (x-axis) position calculated at each interpolated y-axis point in the function. The MAE was calculated for each season (DJF, MAM, JJA, and SON) by computing the $H_{S}$ CDF curves for each season within the historical and model data after isolating the season time-series within each dataset. The result was that individual seasons could be compared for accuracy. Much of the large-wave activity in the tropical Pacific is due to DJF storms at higher latitudes. By comparing seasons, the wave activity of a season that was typically more responsible for larger waves could be evaluated for accuracy, despite another season (such as JJA, which typically delivered less wave energy to the islands) possibly being more inaccurate. The entire time-series of both historical and model hindcast data were also compared and the resultant CDF comparisons were plotted (figs. 3-5). The MAE values of the complete time-series was also calculated and labeled as "Yearly" in tables 3-8. The $n V a r$ is the ratio of modeled to observed variance where a value of 1 indicates the variances are identical and is given by:

$$
n V a r=\frac{\gamma_{m o d}^{2}}{\gamma_{o b s}^{2}}
$$

where $\gamma_{m o d}^{2}=$ the variance of the model dataset and $\gamma_{o b s}^{2}=$ the variance of the observed dataset.

\section{Results}

\section{Model Skill}

The individual models compared favorably to the NDBC datasets based on the shape of CDFs alone (figs. 3-5). However, there was much variation between models, resulting in a wider spread of potential approximations for the data, most notably in the tails of each distribution. The ensemble hindcast dataset showed relatively small error values when compared to the observed dataset for the Hawaiian sites (tables 3-5). The Molokai point approximates all four stations around Hawaii well, with the largest yearly MAE value across all stations being $0.22 \mathrm{~m}$ (table 3, fig. 3). The point it best approximates, however, is station 51001 in the northwest sector of the islands, with both DJF and yearly errors lower than $0.14 \mathrm{~m}$. Similarly, the nVar of this station 
tends to be closer to 1 , which indicates that the ensemble and observed variances in the data are similar.

A similar trend is found for the Big Island dataset (table 4) with Stations 51004 and 51002, which are spatially closer to the modeled point, showing smallest annual MAE values across all stations of $0.08 \mathrm{~m}$ and $0.06 \mathrm{~m}$, respectively (fig. 4). Station 51003 also approximated the Big Island dataset; it has a very small DJF MAE and reveals a smaller nVar and standard deviation (Std). The Kauai point best represented station 51001 with an annual MAE of $0.18 \mathrm{~m}$ (table 5, fig. 5). This point did not approximate the remaining points as well compared to the other modeled points, likely because of the effect of the islands on wave propagation at stations 51003, 51002, and 51004 that is unresolved in the coarse-resolution GCMs. The yearly-normalized variance ratio was 1.01, indicating that variances at these modeled point were captured well.

In summary, the ensemble hindcast $H_{s}$ dataset approximated the NDBC buoy stations well. Generally, the modeled points represented the stations closest to their positions, but each served as a good representation for each station when compared to the complete observed dataset. The points collectively reflected the broad Hawaiian wave climate for the hindcast period, indicating that while individual trends are captured well, the larger wave trends of the Hawaiian chain are also captured within the model. Deviations from the observed dataset in figures 3-5 reflected the position of the modeled points relative to the island. For example, the modeled Molokai point over-predicted the waves present at 51004, which was expected because the station is to the southeast of the Big Island, which serves to block many of the larger waves that come from the northwest during the boreal winter.

Tables 6-8 show the MAE, Std, and $n V a r$ for the top 5 percent of $H_{s}$. As expected, the extreme $H_{s}$ showed a larger model deviation from the observed values. The annual MAE for all points was approximately 0.40 to $0.60 \mathrm{~m}$, indicating that the models did not represent the extremes as closely as the entire observed dataset. Additionally, the MAE for each station did not necessarily represent the nearest modeled point. For example, the modeled Kauai did not best approximate Station 51001 for all seasons, producing a value of $0.58 \mathrm{~m}$, but showed similar annual error for stations 51003 and 51004, 0.52-0.54 m (table 8). However, for the boreal winter season, when wave heights in the area are largest, the modeled points displayed small error approximating the nearest stations, with Kauai having an error of $0.08 \mathrm{~m}$ compared to Station 51001, for example. Additionally, the nVar for each location indicated that the variance in the dataset was also not as well reflected in the observed dataset, with the observed values often varying more than modeled values. The processes that govern the formation of extreme wave events are difficult to capture, and it is therefore more likely that there were a greater number of variations that altered observed wave trends that the model was not able to emulate.

The modeled points in Hawaii represented the historical datasets well, often having a yearly MAE of less than $0.20 \mathrm{~m}$. However, the deviation of modeled extreme $H_{s}$ events demonstrates that the models do not necessarily predict extrema as accurately. The larger 
deviation in extreme $H_{s}$ was expected, and although the error was larger, the modeled points still represented the Hawaiian Islands' wave climates well. The accuracy of the remainder of the modeled points for the hindcast period could not be ascertained because of a lack of complete datasets in the remainder of the study area. However, given the accuracy of the Hawaiian points, it is more likely that the model ensembles of other locations simulated the hindcasted period accurately as well.

\section{Regional Results}

The results for each of the model points are listed in appendixes A-B and their monthly trends are displayed in appendixes $\mathrm{C}-\mathrm{D}$. The modeled points were grouped into one of six regions based on proximity and apparent similarity of wave climate trends within the "Results" and "Discussion" sections to more easily describe basinwide trends. These areas are the Western, Marianas, Central, Northeast, Eastern Equatorial, and Southern regions. The Western region contains the islands of Palau and Yap. The Marianas region comprises Asuncion, Saipan, and Guam. The Central region is the largest, with Chuuk, Kosrae, Enewetak, Bikini, Pohnpei, Wake, and Majuro. The Northeast region contains the Big Island of Hawaii, Molokai, Kauai, Midway, Johnston Atoll, and the Northwest Hawaiian Islands points. The Eastern Equatorial region contains Palmyra, Kingman Reef, Howland, and Jarvis. Finally, the Southern region encompasses two locations: American Samoa and Rose Atoll.

Wave Climate Parameter Changes

The Western Region

Mid-Century: 2026-2045

\section{RCP8.4}

\section{Mean}

The average DJF and SON $H_{s}$ of the Western region decreased by 0.10 to $0.20 \mathrm{~m}$ (fig. 6, fig. 9). MAM and JJA mean $H_{s}$ values did not change significantly (fig. 7, fig. 8). $\sigma_{H S}^{2}$ values did not change significantly.

Mean $T_{p}$, associated $\sigma_{T p}^{2}$ values, and $D_{p}$ values did not change significantly within the region (figs. 38-41, figs. 70-73).

\section{Top 5 Percent}

DJF mean $H_{s}$ values dropped by 0.20 to $0.30 \mathrm{~m}$ (fig. 10). MAM values did not change significantly, while JJA values decreased by 0.10 to $0.20 \mathrm{~m}$ (fig. 11, fig. 12). The largest decrease 
was during the SON season, where values decreased by $>0.30 \mathrm{~m}$ (fig. 13). Extreme $\sigma_{H s}^{2}$ did not change significantly.

Mean $T_{p}$ values decreased at Palau during the DJF and SON seasons by 0.25 to $0.50 \mathrm{~s}$ (fig. 42, fig. 45). JJA values similarly decreased by 0.25 to $0.50 \mathrm{~s}$ (fig. 43). MAM $T_{p}$ means did not change significantly, nor did $\sigma_{T p}^{2}$ values during any season (fig. 44). DJF and MAM mean $D_{p}$ values rotated clockwise by 5 to $10^{\circ}$ at Palau (figs. $74-75$ ). JJA mean $D_{p}$ values rotated counterclockwise by 10 to $20^{\circ}$ (fig. 76). SON $\sigma_{D p}$ values increased by $>15.0^{\circ}$ and mean $D_{p}$ values rotated counterclockwise by 20 to $30^{\circ}$ (fig. 77).

\section{RCP8.5}

\section{Mean}

The region displayed a decrease of 0.10-0.20 m during the DJF and SON seasons (fig. 14, fig. 17). Mean $H_{s}$ did not change significantly in the remaining seasons (figs. 15-16). $\sigma_{H S}^{2}$ did not change significantly.

Mean $T_{p}$ and associated $\sigma_{T p}^{2}$ values did not change significantly within the region (figs. 46-49). $\sigma_{D p}$ values did not change significantly throughout all seasons, and mean wave directions rotated clockwise by 5 to $10^{\circ}$ during the JJA season (figs. 78-81).

\section{Top 5 Percent}

Extreme mean $H_{s}$ values decreased by 0.10 to $0.30 \mathrm{~m}$ for the DJF season and decreased by $>0.30 \mathrm{~m}$ during the SON season (fig. 18, fig. 21). Extreme values for the remaining seasons did not change significantly (figs. 19-20). Extreme $\sigma_{H S}^{2}$ values did not change significantly.

$T_{p}$ means decreased by 0.25 to $0.50 \mathrm{~s}$ at Palau during the DJF and JJA seasons (fig. 50, fig. 52). SON $T_{p}$ means also decreased by 0.25 to $0.50 \mathrm{~s}$ (fig. 53). MAM $T_{p}$ means did not change significantly, nor did $\sigma_{T p}^{2}$ in any season (fig. 51). DJF $\sigma_{D p}$ values increased by 5.0 to $10.0^{\circ}$ at Palau and decreased by the same amount at Yap (fig. 82). $D_{p}$ rotated clockwise by $5-10^{\circ}$. The $D_{p}$ values rotated clockwise by $5-10^{\circ}$ during the MAM season (fig. 83). JJA $\sigma_{D p}$ values decreased by 10.0 to $15.0^{\circ}$, and $D_{p}$ rotated by $5-15^{\circ}$ counterclockwise (fig. 84). SON $\sigma_{D p}$ values increased by $5.0-10.0^{\circ}$ at Yap (fig. 85). 


\section{RCP4.5}

\section{Mean}

The mean $H_{s}$ values of the DJF and SON seasons decreased by 0.10 to $0.20 \mathrm{~cm}$ (fig. 22, fig. 25). Mean values did not change significantly during the other seasons (figs. 23-24). $\sigma_{H s}^{2}$ values did not change significantly.

Mean $T_{p}$ and associated $\sigma_{T p}^{2}$ values did not change significantly within the region (figs. 54-57). The $D_{p}$ of Palau rotated clockwise by 5 to $10^{\circ}$ during the JJA season but otherwise did not change throughout the region during other seasons (figs. 86-89). $\sigma_{D p}$ values increased at Yap by 5.0 to $10.0^{\circ}$ during the SON season but did not change significantly during other seasons.

\section{Top 5 Percent}

The DJF extreme mean values decreased by 0.20 to $0.30 \mathrm{~m}$ (fig. 26). MAM and SON extreme values decreased by a smaller range of 0.10 to $0.20 \mathrm{~m}$ (fig. 27, fig. 29). Values did not change significantly during the boreal summer months (fig. 28). Extreme $\sigma_{H s}^{2}$ values did not change significantly.

The extreme JJA $T_{p}$ mean decreased by 0.25 to $0.50 \mathrm{~s}$ at Yap (fig. 60 ). $T_{p}$ means did not change significantly during the remaining seasons and $\sigma_{T p}^{2}$ values did not significantly change throughout all seasons (figs. 58-59, fig. 61). DJF $\sigma_{D p}$ values decreased by 5.0 to $10.0^{\circ}$ at Palau and increased by 5.0 to $10.0^{\circ}$ at Yap (fig. 90). Palau's $D_{p}$ rotated clockwise by 5 to $10^{\circ} . D_{p}$ at Palau rotated by 10 to $20^{\circ}$ counterclockwise during the JJA season (fig. 92). $\sigma_{D p}$ values increased by $>15.0^{\circ}$ within the region during the SON season and mean directions rotated $20-30^{\circ}$ counterclockwise at Palau and $100-110^{\circ}$ counterclockwise at Yap (fig. 93). MAM $D_{p}$ values did not change significantly (fig. 91).

\section{RCP8.5}

\section{Mean}

The region's DJF mean $H_{s}$ values decreased by 0.20 to $0.30 \mathrm{~m}$, while the MAM values dropped by a smaller, 0.10 to $0.20 \mathrm{~m}$ (figs. 30-31). The SON season values decreased by a mix of small and larger values, ranging from 0.10 to $0.30 \mathrm{~m}$ (fig. 33). The JJA values did not change significantly (fig. 32 ). $\sigma_{H S}^{2}$ values decreased by 0.20 to 0.40 during the SON season. Values did not change during other seasons. 
Mean $T_{p}$ values decreased by 0.25 to $0.50 \mathrm{~s}$ during both the MAM and SON seasons (fig. 63, fig. 65). $T_{p}$ means did not decrease during the remaining seasons (fig. 62, fig. 64). $\sigma_{T p}^{2}$ did not change throughout all seasons. $D_{p}$ values rotated clockwise by 5 to $10^{\circ}$ during the JJA and SON seasons (figs. 96-97). $D_{p}$ values did not change significantly during the DJF and MAM seasons (figs. 94-95). $\sigma_{D p}$ values did not change significantly throughout all seasons.

\section{Top 5 Percent}

Extreme $H_{s}$ values decreased by 0.10 to $>0.30 \mathrm{~m}$ for the DJF season (fig. 34). MAM values decreased by 0.20 to $>0.30 \mathrm{~m}$, and JJA values decreased by 0.10 to $0.20 \mathrm{~m}$ (figs. 35-36). The SON values had the strongest decrease in the region with each point decreasing by $>0.30 \mathrm{~m}$ (fig. 37). Extreme $\sigma_{H S}^{2}$ values did not change significantly from hindcast values.

Mean $T_{p}$ values decreased by 0.25 to 0.50 s during the DJF, MAM, and SON seasons (figs. 66-67, fig. 69). JJA $T_{p}$ values did not change significantly (fig. 68). $\sigma_{T p}^{2}$ did not change significantly throughout all seasons. Palau's $D_{p}$ rotated by $5-10^{\circ}$ clockwise during the DJF season (fig. 98). MAM $\sigma_{D p}$ values decreased by 5.0 to $10.0^{\circ}$, and $D_{p}$ rotated clockwise by 5 to $10^{\circ}$ (fig. 99). JJA $\sigma_{D p}$ values decreased by the same amount, but directions rotated $15-20^{\circ}$ counterclockwise (fig. 100). SON $\sigma_{D p}$ values increased by $10-15^{\circ}$, and directions rotated 30 to $40^{\circ}$ counterclockwise at Palau and 80 to $90^{\circ}$ clockwise at Yap (fig. 101).

Marianas Region

Mid-Century: 2026-2045

\section{RCP4.5}

\section{Mean}

MAM and SON values decreased by 0.10 to $0.20 \mathrm{~m}$ (fig. 7, fig. 9). The boreal summer and winter means did not change significantly from hindcast data (fig. 6, fig. 8). $\sigma_{H S}^{2}$ values did not change significantly.

Mean $T_{p}$, associated $\sigma_{T p}^{2}$ values, and $D_{p}$ values did not change significantly within the region (figs. 38-41, figs. 70-73).

\section{Top 5 Percent}

Extreme wave heights increased by 0.10 to $0.20 \mathrm{~m}$ in Asuncion for the DJF season (fig. 10). Conversely, MAM extreme $H_{s}$ means decreased by 0.10 to $0.20 \mathrm{~m}$ (fig. 11). JJA values decreased by more than $0.30 \mathrm{~m}$, but in SON, the values decreased by $>0.30 \mathrm{~m}$ at each point (figs. 
$12-13)$. JJA $\sigma_{H s}^{2}$ values decreased by 0.20 to 0.40 . SON $\sigma_{H s}^{2}$ values increased at Asuncion by 0.20 to 0.40 .

Mean $T_{p}$ values decreased by 0.25 to $0.50 \mathrm{~s}$ during the DJF season at Asuncion and during JJA at Guam (fig. 42, fig. 44). The mean $T_{p}$ values did not significantly change during other seasons (fig. 43, fig. 45). $\sigma_{T p}^{2}$ did not significantly change during all seasons. The $D_{p}$ of Asuncion rotated clockwise by 5 to $10^{\circ}$ during DJF (fig. 74). MAM $\sigma_{D p}$ values decreased by 5.0 to $10.0^{\circ}$ (fig. 75). JJA $\sigma_{D p}$ values increased by 10.0 to $15.0^{\circ}$, and SON values increased by 10.0 to $>15.0^{\circ}$ (figs. 76-77).

\section{RCP8.5}

\section{Mean}

Mean SON $H_{s}$ values at each point decreased by 0.10 to $0.20 \mathrm{~m}$, while the means for the remaining seasons did not change significantly (figs. 14-17). $\sigma_{H S}^{2}$ values did not change significantly.

Mean $T_{p}$ and associated $\sigma_{T p}^{2}$ values did not change significantly within the region (figs. 46-49). Saipan's $\sigma_{D p}$ increased by 5.0 to $10.0^{\circ}$ during the SON season (fig. 81). $\sigma_{D p}$ values did not change significantly throughout the remaining seasons, and directions did not change noticeably throughout all seasons (figs. 78-80).

\section{Top 5 Percent}

Extreme $H_{s}$ values increased by 0.10 to $0.20 \mathrm{~m}$ during the MAM and JJA seasons (figs. 19-20). The SON values decreased by 0.10 to $0.20 \mathrm{~m}$ (fig. 21). The boreal winter season did not change significantly (fig. 18). $\sigma_{H S}^{2}$ values increased during the SON season by 0.20 to 0.40 at Saipan and by $>0.40$ at Asuncion.

Mean $T_{p}$ values decreased by 0.25 to 0.50 at Asuncion during DJF and at Guam during SON (fig. 50, fig. 53). $T_{p}$ values did not significantly change during the remaining seasons (figs. 51-52). $\sigma_{T p}^{2}$ did not significantly change during all seasons. $D_{p}$ at Asuncion rotated 10 to $20^{\circ}$ clockwise during the DJF season (fig. 82). $\sigma_{D p}$ values decreased by 5.0 to $15.0^{\circ}$ over the region during MAM (fig. 83). The JJA $\sigma_{D p}$ value at Guam decreased by 5.0 to $10.0^{\circ}$ and $D_{p}$ rotated 20$30^{\circ}$ clockwise at Asuncion and 5 to $10^{\circ}$ at Saipan (fig. 84). SON $\sigma_{D p}$ values increased by 10.0 to $>15.0^{\circ}$ and directions rotated clockwise by 5 to $20^{\circ}$ (fig. 85 ). 


\section{RCP4.5}

\section{Mean}

SON means decreased by 0.10 to $0.20 \mathrm{~m}$ (fig. 25). The means of the remaining seasons did not significantly deviate from hindcast values (figs. 22-24). $\sigma_{H s}^{2}$ values did not change within the modeled region.

Mean $T_{p}$ and associated $\sigma_{T p}^{2}$ values did not change significantly within the region (figs. 54-57). The $\sigma_{D p}$ value decreased at Guam by 5.0 to $10.0^{\circ}$ during the SON season (fig. 89). $\sigma_{D p}$ values did not change significantly during other seasons (figs. 86-88). $D_{p}$ at each point did not change noticeably.

\section{Top 5 Percent}

MAM and JJA extreme $H_{s}$ means decreased by 0.20 to $0.30 \mathrm{~m}$ (figs. 27-28). The SON mean decreased by more than $0.30 \mathrm{~m}$ from the hindcast mean (fig. 29). The DJF mean did not change significantly form the hindcast mean (fig. 26). MAM $\sigma_{H S}^{2}$ values decreased by 0.20 to 0.40 , except at the Guam point, which decreased by $>0.40$. Values did not change significantly during the remaining seasons.

$T_{p}$ means decreased by 0.25 to $0.50 \mathrm{~s}$ during the DJF season (fig. 58). $T_{p}$ means did not change significantly during other seasons (figs. 59-61). $\sigma_{T p}^{2}$ did not change significantly throughout all seasons. The DJF $D_{p}$ of Asuncion rotated 5 to $10^{\circ}$ clockwise (fig. 90). MAM $\sigma_{D p}$ values decreased by 5.0 to $15.0^{\circ}$ throughout the region and directions rotated by 5 to $10^{\circ}$ counterclockwise as Asuncion (fig. 91). JJA $\sigma_{D p}$ values increased by 5.0 to $1.0^{\circ}$, and $D_{p}$ values at Saipan and Guam rotated clockwise by 5 to $10^{\circ}$ (fig. 92). SON $\sigma_{D p}$ valued increased by 5.0 to $15.0^{\circ}$ throughout the region, and $D_{p}$ values at Saipan and Guam rotated counterclockwise by 5 to $10^{\circ}$ (fig. 93).

\section{RCP8.5}

\section{Mean}

DJF and MAM means decreased by 0.10 to 0.20 m (figs. 30-31). SON values decreased by a wider range of 0.10 to $0.30 \mathrm{~m}$ (fig. 33). The JJA season mean did not change significantly (fig. 32). $\sigma_{H s}^{2}$ values did not change significantly. 
Mean $T_{p}$ values decreased by 0.25 to $0.50 \mathrm{~s}$ during the DJF, MAM, and SON seasons (figs. 62-63, fig. 65). JJA $T_{p}$ means didn't change significantly (fig. 64). $\sigma_{T p}^{2}$ did not change significantly throughout all seasons. $\sigma_{D p}$ values increased during the SON season by 5.0 to $1.0^{\circ}$ (fig. 97). $\sigma_{D p}$ values did not change significantly during other seasons and directions did not change noticeably (figs. 94-96).

\section{Top 5 Percent}

DJF extreme $H_{s}$ means decreased by 0.10 to $0.30 \mathrm{~m}$, while JJA values decreased by 0.10 to $0.20 \mathrm{~m}$ (fig. 34, fig. 36). Both MAM and SON means decreased by more than $0.30 \mathrm{~m}$ (fig. 35, fig. 37). DJF $\sigma_{H S}^{2}$ values increased by 0.20 to 0.40 at Guam. MAM values decreased by 0.20 to 0.40 , with values decreasing by $>0.40$ at Guam. JJA $\sigma_{H S}^{2}$ values increased by 0.20 to 0.40 at Guam and decreased by the same amount at Asuncion. SON values did not change significantly.

DJF $T_{p}$ means decreased over a wide range. $T_{p}$ mean decreased by 0.25 to $0.50 \mathrm{~s}$ at Guam, 0.50 to $0.75 \mathrm{~s}$ at Saipan, and $>0.75 \mathrm{~s}$ at Asuncion (fig. 66). MAM and SON $T_{p}$ means decreased by 0.25 to $0.50 \mathrm{~s}$ (fig. 67 , fig. 69). JJA mean $T_{p}$ values did not change significantly (fig. 68 ). $\sigma_{T p}^{2}$ did not change significantly throughout all seasons. The $D_{p}$ of Asuncion rotated 10 to $20^{\circ}$ clockwise during the DJF season and 5 to $10^{\circ}$ clockwise during the JJA season (fig. 98, fig. 100). $\sigma_{D p}$ values decreased by 5.0 to 15.0 during MAM (fig. 99). SON $\sigma_{D p}$ values increased by 5.0 to 15.0 across the region and directions rotated 10 to $20^{\circ}$ clockwise (fig. 101).

\section{Central Region}

Mid-Century: 2026-2045

\section{RCP4.5}

\section{Mean}

The SON mean $H_{s}$ value decreased by 0.10 to $0.20 \mathrm{~m}$ within the center of the island cluster (fig. 9). The mean values from other seasons did not change significantly (figs. 6-8). $\sigma_{H S}^{2}$ values did not change significantly.

Mean $T_{p}$ and associated $\sigma_{T p}^{2}$ values did not change significantly within the region (figs. 38-41). The $D_{p}$ of Majuro and Pohnpei rotated by 5 to $10^{\circ}$ clockwise and of Kosrae by 10 to $20^{\circ}$ clockwise during the SON season (fig. 73). The $D_{p}$ of all points did not change significantly during remaining seasons, and $\sigma_{D p}$ values did not significantly change across all seasons (figs. 70-72). 


\section{Top 5 Percent}

The region experienced decreases in extreme mean wave heights during most seasons. The DJF mean decreased by 0.20 to $0.30 \mathrm{~m}$, and the MAM season decreased by a smaller 0.10 to 0.20 m (figs. 10-11). The SON mean extreme $H_{s}$ decreased by more than $0.30 \mathrm{~cm}$ (fig. 13). There was no significant change in the JJA means (fig. 12). Extreme $\sigma_{H s}^{2}$ values did not change significantly from hindcast values.

The MAM mean $T_{p}$ value at Kosrae decreased by 0.25 to $0.50 \mathrm{~s}$ (fig. 43 ). The JJA and SON $T_{p}$ mean at Chuuk decreased by 0.25 to $0.50 \mathrm{~s}$. additionally, the $\sigma_{T p}^{2}$ during SON at Kwajalein increased by 1.5 to 3.0 (figs. 44-45). $T_{p}$ means did not change significantly during the DJF season and $\sigma_{T p}^{2}$ values did not significantly change during the remaining seasons (fig. 42). DJF $\sigma_{D p}$ values increased by $>15.0^{\circ}$ but lowered to 5.0 to $15.0^{\circ}$ closer to the Equator and the $D_{p}$ of Wake rotated counterclockwise by 5 to $10^{\circ}$ (fig. 74). The $D_{p}$ at Kosrae rotated 5 to $10^{\circ}$ clockwise and the regions $\sigma_{D p}$ values decreased by 5.0 to $>15.0^{\circ}$, with the largest decrease at Kosrae, during the MAM season (fig. 75). $\sigma_{D p}$ values decreased by 5.0 to $10.0^{\circ}$ during the JJA season (fig. 76). Directions rotated 10 to $20^{\circ}$ counterclockwise in the southwest half of the region and 5 to $10^{\circ}$ clockwise in the northeast half. SON $\sigma_{D p}$ values decreased by 10.0 to $>15.0$ in the northwest half of the region (fig. 77). The $D_{p}$ rotated 40 to $50^{\circ}$ clockwise at Kosrae and 5 to $10^{\circ}$ counterclockwise at Kwajalein.

\section{RCP8.5}

\section{Mean}

The SON mean $H_{s}$ decreased by 0.10-0.20 m (fig. 17). The remaining seasons' mean did not change significantly (figs. 14-16). $\sigma_{H S}^{2}$ values did not change significantly.

Mean $T_{p}$ and associated $\sigma_{T p}^{2}$ values did not change significantly within the region (figs. 46-49). $D_{p}$ at Kosrae and Pohnpei rotated clockwise by 5 to $10^{\circ}$ during SON and $\sigma_{D p}$ values did not change significantly throughout all seasons (figs. 78-81).

\section{Top 5 Percent}

DJF extreme $H_{s}$ means decreased by 0.10 to 0.20 at the Enewetak, Wake, and Bikini points (fig. 18). Conversely, the JJA means at Enewetak, Bikini, and Kosrae increased by 0.10 to $0.20 \mathrm{~m}$ (fig. 20). Extreme $H_{s}$ means for the SON season decreased by 0.20 to more than $0.30 \mathrm{~m}$ across the entire region (fig. 21). The boreal spring season averages did not change significantly 
(fig. 19). Extreme $\sigma_{H s}^{2}$ values increased by 0.20 to 0.40 at the Wake, Enewetak, and Bikini points. Values did not significantly change in other seasons.

The $T_{p}$ mean at Kosrae increased by 0.50 to $0.75 \mathrm{~s}$ during the DJF season (fig. 50 ). $T_{p}$ means during MAM decreased by 0.25 to $0.50 \mathrm{~s}$ at Pohnpei and Kosrae (fig. 51). Mean $T_{p}$ values did not change significantly during other seasons (figs. 52-53). $\sigma_{T p}^{2}$ did not significantly change during all seasons. DJF $\sigma_{D p}$ values increased by 5.0 to $10.0^{\circ}$ at Kosrae, Chuuk, Pohnpei, and Wake (fig. 82). The MAM $\sigma_{D p}$ value at Chuuk decreased by 10.0 to $15.0^{\circ}$, and directions at Kosrae and Pohnpei rotated clockwise by 5 to $10^{\circ}$ (fig. 83). JJA $\sigma_{D p}$ values decreased by 5.0 to $15.0^{\circ}$ in the west half of the region, and islands' directions rotated 5 to $10^{\circ}$ clockwise at Majuro, Enewetak, Bikini, and Wake (fig. 84). Kosrae's $D_{p}$ in the rotated by 20 to $30^{\circ}$ clockwise and the $\sigma_{D p}$ value at Wake increased by 10.0 to $15.0^{\circ}$. The $\sigma_{D p}$ values increased by 5.0 to $15.0^{\circ}$ throughout. The SON $D_{p}$ values of Kosrae, Pohnpei, and Kwajalein rotated counterclockwise by 10 to $20^{\circ}$ and at Majuro by 5 to $10^{\circ}$ (fig. 85).

End-Century: 2081-2100

\section{RCP4.5}

\section{Mean}

The DJF mean $H_{s}$ values decreased by 0.10 to $0.20 \mathrm{~m}$ across the entire region, and the SON values decreased by the same amount but only in the western half of the region (fig. 22, fig. 25). The remaining seasons did not display significant changes form hindcast means (figs. 23-24). $\sigma_{H S}^{2}$ values did not change significantly.

Mean $T_{p}$ and associated $\sigma_{T p}^{2}$ values did not change significantly within the region (figs. 54-57). The SON $\sigma_{D p}$ value at Enewetak increased by 5.0 to $1.0^{\circ}$, and the $D_{p}$ of Kosrae rotated 5 to $10^{\circ}$ clockwise (fig. 89). Directions and $\sigma_{D p}$ values did not change significantly through the other seasons (figs. 86-88).

\section{Top 5 Percent}

DJF, MAM, and SON extreme means decrease by 0.10 to $0.30 \mathrm{~m}$, with the larger decreases occurring at the western points in the former two seasons and at the central islands in the latter (figs. 26-27, fig. 29). JJA means increase by 0.10 to greater than $0.30 \mathrm{~m}$, with the largest deviations from hindcast means occurring at Enewetak and Bikini (fig. 28). MAM $\sigma_{H s}^{2}$ values decreased by 0.20 to 0.40 at the Enewetak point. JJA values increased by 0.20 to 0.40 at Enewetak, Bikini, and Wake. Values did not significantly change during other seasons. 
The $T_{p}$ mean decreased by 0.25 to $0.50 \mathrm{~s}$ at Pohnpei during the MAM season (fig. 59). $T_{p}$ means increased during the JJA season at Enewetak, Bikini, and Wake by 0.25 to $0.50 \mathrm{~s}$ (fig. 60). Means did not change significantly during other seasons (fig. 58, fig. 61). $\sigma_{T p}^{2}$ did not change significantly throughout all seasons. DJF $\sigma_{D p}$ values increased by 5.0 to $15.0^{\circ}$ at Chuuk, Pohnpei, and Kosrae and the $D_{p}$ of Wake rotated 5 to $10^{\circ}$ counterclockwise (fig. 90). MAM $\sigma_{D p}$ values decreased by 5.0 to $15.0^{\circ}$, and directions rotated clockwise by 5 to $10^{\circ}$ at Chuuk, Kosrae, and Wake (fig. 91). JJA trends were split by west and east within the region (fig. 92). The west half of the area displayed a decrease of $\sigma_{D p}$ values by 5.0 to $>15.0^{\circ}$, and directions rotated clockwise by 5 to $30^{\circ}$. The eastern half had an increase of $\sigma_{D p}$ values by 5.0 to $>15.0^{\circ}$ and directions rotated clockwise by 30 to $60^{\circ}$. The SON seasons displayed an increase of $\sigma_{D p}$ values by 5.0 to $>15.0^{\circ}$, and directions shifted counterclockwise by 20 to $30^{\circ}$ at Chuuk, 50 to $60^{\circ}$ at Pohnpei, 80 to $90^{\circ}$ at Kosrae, and 10 to $20^{\circ}$ at Kwajalein (fig. 93).

\section{RCP8.5}

\section{Mean}

Mean DJF values decreased by 0.10 to $0.20 \mathrm{~m}$ (fig. 30). SON values decreased by a larger 0.10 to $0.30 \mathrm{~m}$ (fig. 33). The remaining seasons' means did not change significantly (figs. 31-32). SON $\sigma_{H S}^{2}$ values decreased by 0.20 to 0.40 at Bikini, but values did not change significantly during other seasons.

Mean $T_{p}$ values decreased by 0.25 to $0.50 \mathrm{~s}$ at Bikini through the DJF season and at Kosrae during JJA (fig. 62, fig. 64). $T_{p}$ means decreased through the entire region by 0.25 to $0.50 \mathrm{~s}$ during the SON season (fig. 65). There were no significant changes in $T_{p}$ means during MAM (fig. 63). Additionally, $\sigma_{T p}^{2}$ values did not significantly change throughout all seasons. MAM $\sigma_{D p}$ values decreased by 5.0 to $10.0^{\circ}$ at Chuuk (fig. 95). SON $\sigma_{D p}$ values decreased by 5.0 to $10.0^{\circ}$ at Kosrae and directions rotated 5 to $10^{\circ}$ clockwise at Kwajalein, Majuro, and Chuuk, and by 10 to $20^{\circ}$ at Kosrae and Pohnpei (fig. 97). No significant changes were observed during other seasons (fig. 94, fig. 96).

\section{Top 5 Percent}

DJF means decreased by 0.10 to $0.30 \mathrm{~m}$ over the region (fig. 34). MAM mean values decreased over a wide range, from 0.10 to more than $0.30 \mathrm{~m}$ (fig. 35). JJA values increased from 0.10 to more than $0.30 \mathrm{~m}$, with the largest increases occurring at Enewetak and Bikini (fig. 36). The strongest change was during the SON season, where the majority of the region's values decreased by $>0.30 \mathrm{~m}$ (fig. 37). $\sigma_{H S}^{2}$ values decreased by 0.20 to 0.40 at Wake during the DJF season. Values decreased by the same amount at Enewetak and Bikini during the MAM season. 
JJA values decreased by $>0.40$ at Enewetak, Bikini, and Wake. SON valued increased by 0.20 to 0.40 at Enewetak and Wake.

DJF $T_{p}$ means decreased by 0.25 to $0.50 \mathrm{~s}$ within the region, while the $\sigma_{T p}^{2}$ at Kwajalein increased by 1.5 to 3.0 (fig. 66). $\sigma_{T p}^{2}$ values did not significantly change in the other seasons. MAM $T_{p}$ means decreased by 0.25 to $0.50 \mathrm{~s}$ except at Chuuk and Pohnpei, where values decreased by 0.50 to $0.75 \mathrm{~s}$ (fig. 67). JJA $T_{p}$ means decreased at Chuuk by 0.25 to $0.50 \mathrm{~s}$ and increased by the same amount at Wake (fig. 68). SON $T_{p}$ means decreased by 0.25 to $0.50 \mathrm{~s}$, except at Chuuk, where the decrease was 0.50 to $0.75 \mathrm{~s}$ (fig. 69). The $D_{p}$ of Wake during DJF rotated counterclockwise by 5 to $10^{\circ}$ (fig. 98). MAM $\sigma_{D p}$ values decreased by 5.0 to $15.0^{\circ}$ at Chuuk, Majuro, and Kwajalien (fig. 99). The $D_{p}$ of Chuuk, Kosrae, and Pohnpei rotated clockwise by 5 to $10^{\circ}$. The JJA $\sigma_{D p}$ values decreased at Enewetak by 5.0 to $10.0^{\circ}$ and its direction rotated clockwise by 30 to $40^{\circ}$ (fig. 100). The other islands' directions in the western half of the region rotated 5 to $20^{\circ}$ counterclockwise. The east half of the region's $\sigma_{D p}$ values increased by 5.0 to $>15.0^{\circ}$ and directions rotated 5 to $20^{\circ}$ clockwise, and 20 to $30^{\circ}$ at Wake and Bikini. SON $\sigma_{D p}$ values increased by 5.0 to $>15.0^{\circ}$ and directions rotated clockwise by 5 to $20^{\circ}$ at Enewetak and Pohnpei, by 20 to $30^{\circ}$ at Chuuk, and by 40 to $50^{\circ}$ at Kosrae (fig. 101). There were no significant changes during the DJF season.

Northeast Region

Mid-Century: 2026-2045

\section{RCP4.5}

\section{Mean}

The mean $H_{s}$ values of the Northeast region did not change significantly throughout all seasons (figs. 6-9). $\sigma_{H S}^{2}$ values did not significantly change from hindcast values.

Mean $T_{p}$ and associated $\sigma_{T p}^{2}$ values did not change significantly within the region (figs. 38-41). The $D_{p}$ of Midway rotated clockwise by 5 to $10^{\circ}$ during the SON season (fig. 73). Other $D_{p}$ and associated $\sigma_{D p}$ values did not change significantly throughout all seasons (figs. 70-72).

\section{Top 5 Percent}

The main Hawaiian Island points' extreme mean $H_{s}$ decreased by 0.10 to $0.20 \mathrm{~m}$ during the JJA season (fig. 12). The values of the remaining seasons did not change significantly (figs. $10-11$, fig. 13). SON extreme $\sigma_{H S}^{2}$ values decreased by 0.20 to 0.40 at Midway and by $>0.40$ at the Northwest Hawaiian Islands point. Values did not change significantly during the other seasons. 
DJF $T_{p}$ mean values increased by 0.25 to $0.50 \mathrm{~s}$ at Kauai and Johnston Atoll (fig. 42). The Big Island $T_{p}$ mean increased by 0.50 to $0.75 \mathrm{~s}$. The MAM $T_{p}$ mean of Kauai increased by 0.25 to $0.50 \mathrm{~s}$ and its $\sigma_{T p}^{2}$ increased by 1.5 to 3.0 (fig. 43). JJA means remain unchanged but the $\sigma_{T p}^{2}$ of Kauai increased by 1.5 to 3.0 (fig. 44). SON values did not significantly change (fig. 45 ). $\sigma_{T p}^{2}$ values during the boreal winter and fall seasons did not change significantly. The DJF $D_{p}$ of the Big Island and Johnston Atoll rotated counterclockwise by 5 to $10^{\circ}$ (fig. 74). The MAM $D_{p}$ of Kauai rotated counterclockwise by 5 to $10^{\circ}$ (fig. 75). The JJA $\sigma_{D p}$ value at Johnston Atoll increased by 10.0 to $15.0^{\circ}$, and the $D_{p}$ at the Northwest Hawaiian Islands rotated clockwise by 5 to $10^{\circ}$ (fig. 76). SON $\sigma_{D p}$ values increased in the western half of the region by 5.0 to $10.0^{\circ}$, and wave directions in the Northwest Hawaiian Islands and Midway rotated 5 to $20^{\circ}$ clockwise (fig. 77). The $D_{p}$ of Kauai rotated clockwise by 5 to $10^{\circ}$.

\section{RCP8.5}

\section{Mean}

Mean valued decreased by 0.10 to $0.20 \mathrm{~m}$ during the SON season throughout the region (fig. 17). There were no significant changes observed in other seasons (figs. 14-16). $\sigma_{H S}^{2}$ values did not change significantly.

Mean $T_{p}$ values increased at Kauai during the JJA season and the Big Island point during SON by 0.25 to $0.50 \mathrm{~s}$ (figs. 48-49). Means did not change significantly during other seasons (figs. 46-47). $\sigma_{T p}^{2}$ values did not significantly change throughout all seasons. MAM $D_{p}$ rotated by 5 to $10^{\circ}$ counterclockwise at the Northwest Hawaiian Islands point and by 10 to $20^{\circ}$ at the Midway point (fig. 79). Midway's $\sigma_{D p}$ increased by 5.0 to $10.0^{\circ}$ during the JJA season (fig. 80). Midway's $D_{p}$ rotated clockwise by 5 to $10^{\circ}$ during the SON season (fig. 81). DJF $D_{p}$ values did not change significantly (fig. 78).

\section{Top 5 Percent}

Extreme values over most seasons decreased at all points within the region. DJF values decreased by 0.10 to $0.30 \mathrm{~m}$ at all points except the Big Island of Hawaii point, which increased by 0.10 to $0.20 \mathrm{~m}$ (fig. 18). MAM means decreased by 0.10 to $0.20 \mathrm{~m}$ at the Big Island of Hawaii point, but the remainder of the study area did not change significantly (fig. 19). The JJA and SON seasons' means decreased irregularly over the region by 0.10 to $0.20 \mathrm{~m}$ (figs. 20-21). Extreme $\sigma_{H S}^{2}$ values did not change significantly.

Mean $T_{p}$ increased at Johnston Atoll and Kauai during DJF by 0.25 to 0.50 (fig. 50). The Big Island point's $T_{p}$ mean increased by 0.50 to $0.75 \mathrm{~s}$, and its $\sigma_{T p}^{2}$ decreased by 1.5 to 3.0. The JJA $\sigma_{T p}^{2}$ of Kauai increased by 1.5 to 3.0 and the SON mean $T_{p}$ values of Kauai increased by 0.25 
to $0.50 \mathrm{~s}$ (figs. 52-53). The MAM season had not significant changes (fig. 51). DJF $\sigma_{D p}$ values increased by 5.0 to $10.0^{\circ}$ except at the Big island point, which decreased by the 5.0 to $10.0^{\circ}$ (fig. 82). Johnston Atoll Molokai's $D_{p}$ rotated 10 to $20^{\circ}$ clockwise. Kauai's $D_{p}$ rotated clockwise by 5 to $10^{\circ}$. The MAM $\sigma_{D p}$ of Midway decreased by 5.0 to $10.0^{\circ}$ and the Northwest Hawaiian Islands, Kauai, and Molokai points' mean $D_{p}$ values rotated counterclockwise by 5 to $20^{\circ}$ (fig. 83). JJA $\sigma_{D p}$ values increased by 5.0 to $15.0^{\circ}$ across the region and Northwest Hawaiian Islands, and Midway's mean $D_{p}$ rotated clockwise by 5 to $10^{\circ}$ (fig. 84). The SON $\sigma_{D p}$ value of Kauai decreased by 5.0 to $10.0^{\circ}$, and the mean $D_{p}$ of the Northwest Hawaiian Islands rotated clockwise by 10 to $20^{\circ}$ (fig. 85 ). Midway's $D_{p}$ rotated counterclockwise by 20 to $30^{\circ}$.

End-Century: 2081-2100

\section{RCP4.5}

\section{Mean}

The Midway and Northwest Hawaii points' $H_{s}$ means decreased by 0.10 to $0.20 \mathrm{~m}$ from hindcast means during the SON season, but the remainder of the region during this season did not change significantly (figs. 22-25). Additionally, the means of these points did not deviate significantly from hindcast means during other seasons. $\sigma_{H S}^{2}$ values did not change significantly.

Mean $T_{p}$ values increased by 0.25 to $0.50 \mathrm{~s}$ at Molokai and the Big Island points during MAM and at Molokai and Kauai during JJA (figs. 55-56). $T_{p}$ means didn't change significantly during other seasons, and $\sigma_{T p}^{2}$ values did not significantly change throughout all seasons (fig. 54, fig. 57). The mean MAM $D_{p}$ at Midway, Kauai, Molokai, and the Northwest Hawaiian Islands rotated counterclockwise by 5 to $20^{\circ}$ (fig. 87). $D_{p}$ means at Midway and the Northwest Hawaiian Islands rotated clockwise by 5 to $20^{\circ}$. $D_{p}$ values did not change significantly during the remaining seasons (fig. 86, figs. 88-89).

\section{Top 5 Percent}

The top 5 percent of mean $H_{s}$ values decreased during the SON season by 0.10 to $0.30 \mathrm{~m}$ (fig. 29). The extreme $H_{s}$ means did not change significantly for other seasons (figs. 26-28). JJA

$\sigma_{H S}^{2}$ values increased by $>0.40$ within the region except at the Northwest Hawaiian Islands and Kauai points, which increased by 0.20 to $0.40 . H_{s}$ values did not change significantly during other seasons.

Mean $T_{p}$ values increased by 0.25 to $0.50 \mathrm{~s}$ at Johnston Atoll, Kauai, and Molokai during the DJF season (fig. 58). MAM $T_{p}$ means increased by 0.25 to $0.50 \mathrm{~s}$ at Kauai and Johnston Atoll, and the Big Island's $T_{p}$ mean increased by 0.50 to $0.75 \mathrm{~s}$ (fig. 59). JJA means increased by 0.25 to 
$0.50 \mathrm{~s}$, except at Kauai, which increased by 0.50 to $0.75 \mathrm{~s}$ (fig. 60). $\sigma_{T p}^{2}$ at the Big Island increased by 1.5 to 3.0 and at Kauai by >3.0. SON means decreased by 0.25 to $0.50 \mathrm{~s}$ at Johnston Atoll, Kauai, and Molokai (fig. 61). DJF $\sigma_{D p}$ values increased by 5.0 to $10.0^{\circ}$ at Midway and the Northwest Hawaiian Islands. Johnston Atoll's $D_{p}$ rotated counterclockwise by 5 to $10^{\circ}$ (fig. 90). The MAM mean $D_{p}$ at Kauai and Molokai rotated counterclockwise by 5 to $20^{\circ}$ (fig. 91). JJA $\sigma_{D p}$ values increased by 5.0 to $>15.0^{\circ}$, and mean $D_{p}$ rotated clockwise at all islands except Johnston Atoll by 5 to $20^{\circ}$ (fig. 92). Similarly, the mean $D_{p}$ of Kauai, Johnston Atoll, Midway, and the Northwest Hawaiian Islands rotated 5 to $20^{\circ}$ clockwise during the SON season (fig. 93). $\sigma_{D p}$ values increased by 5.0 to $10.0^{\circ}$ throughout the region.

\section{RCP8.5}

\section{Mean}

DJF, MAM, and JJA mean $H_{s}$ values decreased by 0.10 to 0.20 m over the region (figs. 30-32). SON $H_{s}$ means decreased by a larger range of 0.10 to $0.30 \mathrm{~m}$ (fig. 33). SON extreme $\sigma_{H s}^{2}$ values decreased by 0.20 to 0.40 at Midway. $H_{s}$ values did not change significantly during the remaining seasons.

JJA $T_{p}$ means increased by 0.25 to $0.50 \mathrm{~s}$ at the Big Island and Northwest Hawaiian Islands points and by 0.502 to 0.75 at the Molokai and Kauai points (fig. 64). $\sigma_{T p}^{2}$ increased by 1.5 to 3.0 at the Big Island and Northwest Hawaiian Islands points. $\sigma_{T p}^{2}$ increased by $>3.0$ at Molokai and Kauai. The SON Big Island $T_{p}$ mean increased by 0.25 to $0.50 \mathrm{~s}$ (fig. 65). $\sigma_{T p}^{2}$ values increased by 1.5 to 3.0 at the Kauai and the Big Island points. $T_{p}$ means and associated $\sigma_{T p}^{2}$ values did not change significantly during other seasons (figs. 62-63). The MAM and JJA mean $D_{p}$ values of Midway rotated 5 to $10^{\circ}$ counterclockwise and clockwise, respectively (figs. 95-96). The SON mean $D_{p}$ at the Northwest Hawaiian Islands and Midway rotated clockwise by 10 to $20^{\circ}$ and at Kauai and Johnston Atoll by 5 to $10^{\circ}$ (fig. 97). The $D_{p}$ values of the DJF season did not significantly change (fig. 94).

\section{Top 5 Percent}

Extreme DJF $H_{s}$ means decreased by 0.10 to $>0.30 \mathrm{~m}$, with the largest decrease occurring at the Midway point (fig. 34). MAM $H_{s}$ values decreased by 0.10 to $0.30 \mathrm{~m}$ (fig. 35). The Northwest Hawaii, Midway, and Johnston Atoll points decreased by 0.20 to $>0.30 \mathrm{~m}$. The whole region decreased by the same amount during the SON season (fig. 37). Extreme $\sigma_{H s}^{2}$ values increased by 0.20 to 0.40 at the Big Island of Hawaii point during the DJF period. JJA values increased by 0.20 to 0.40 except at Midway, which increased by $>0.40$ (fig. 36). 
DJF extreme $T_{p}$ means increased by 0.25 to $0.50 \mathrm{~s}$ (fig. 66). MAM means increased by 0.25 to $0.50 \mathrm{~s}$ at Johnston Atoll, 0.50 to $0.75 \mathrm{~s}$ at Kauai, and $>0.75 \mathrm{~s}$ at Molokai (fig. 67). JJA mean $T_{p}$ values increased by 0.25 to $0.50 \mathrm{~s}$, except at Molokai, which increased by 0.50 to $0.75 \mathrm{~s}$

(fig. 68). $\sigma_{T p}^{2}$ values increased by 1.5 to 3.0, but Molokai and Kauai's $\sigma_{T p}^{2}$ valuess increased by >3.0. Mean SON $T_{p}$ values decreased by 0.50 to $0.75 \mathrm{~s}$, except at Johnston Atoll, which decreased by 0.25 to $0.50 \mathrm{~s}$ (fig. 69). DJF $\sigma_{D p}$ values increased by 5.0 to $10.0^{\circ}$, and the $D_{p}$ values of Kauai and Molokai rotated clockwise by 5 to $10^{\circ}$ (fig. 98). MAM $\sigma_{D p}$ values increased by 5.0 to $10.0^{\circ}$ at Johnston Atoll and the Big Island, while values decreased by 5.0 to $10.0^{\circ}$ at Midway and Kauai (fig. 99). Mean $D_{p}$ at the Northwest Hawaiian Islands, Kauai, and Molokai rotated counterclockwise by 5 to $30^{\circ}$. JJA $\sigma_{D p}$ values decreased by 5.0 to $10.0^{\circ}$, except at Midway, which decreased by 10.0 to $15.0^{\circ}$ (fig. 100). The $D_{p}$ values of the Big Island, the Northwest Hawaiian Islands, and Midway points rotated clockwise by 5 to $20^{\circ}$. SON $\sigma_{D p}$ values decreased by 5.0 to $10.0^{\circ}$ at the Big Island point, and $D_{p}$ at Kauai, Midway, and the Northwest Hawaiian Islands rotated clockwise by 10 to $30^{\circ}$ (fig. 101).

\section{Eastern Equatorial Region}

Mid-Century: 2026-2045

\section{RCP4.5}

\section{Mean}

There was no significant change in mean $H_{s}$ values from hindcast means over all seasons (figs. 6-9). $\sigma_{H S}^{2}$ values did not change significantly.

Mean $T_{p}$ and associated $\sigma_{T p}^{2}$ values did not change significantly within the region (figs. 38-41). The $D_{p}$ of Jarvis rotated counterclockwise by 5 to $10^{\circ}$ during the JJA season (fig. 72). The $D_{p}$ of all islands within the region rotated clockwise by 5 to $10^{\circ}$ during the SON season (fig. 73). $\sigma_{D p}$ values did not change significantly throughout all seasons (figs. 70-71).

\section{Top 5 Percent}

Extreme mean $H_{s}$ values decreased by 0.10 to $0.20 \mathrm{~m}$ at the Howland point during the DJF season, but the other points remain essentially unchanged (fig. 10). MAM means at Palmyra and Kingman Reef increased by 0.10 to $0.20 \mathrm{~m}$, while the remaining points were unchanged (fig. 11). Means decreased by 0.20 to $0.30 \mathrm{~m}$ during the SON season (fig. 13). The JJA season means did not change significantly from hindcast means (fig. 12). Extreme $\sigma_{H S}^{2}$ values decreased by 0.20 to 0.40 during the SON season. 
DJF extreme $T_{p}$ means increased by 0.25 to $0.50 \mathrm{~s}$ at Palmyra and Kingman Reef, and Howland's mean values increased by 0.50 to $0.75 \mathrm{~s}$ (fig. 42). MAM and JJA mean $T_{p}$ values increased at Jarvis by 0.25 to $0.50 \mathrm{~s}$ (figs. 43-44). SON $T_{p}$ means increased by a larger 0.50 to $0.75 \mathrm{~s}$ range (fig. 45 ). $\sigma_{T p}^{2}$ values did not significantly change throughout all seasons. The MAM and JJA $\sigma_{D p}$ value at Howland decreased by 5.0 to $10.0^{\circ}$ and the $D_{p}$ of the latter season rotated 5 to $10^{\circ}$ counterclockwise (figs. 75-76). The SON $\sigma_{D p}$ value at Jarvis decreased by 5.0 to $10.0^{\circ}$; it and Howland's $D_{p}$ rotated clockwise by 10 to $20^{\circ}$ and Palmyra and Kingman Reef's $D_{p}$ rotated counterclockwise by 5 to $10^{\circ}$ (fig. 77). The DJF $D_{p}$ values did not change significantly (fig. 74).

\section{RCP8.5}

\section{Mean}

Mean $H_{s}$ values did not deviate significantly from hindcast means during this period (figs. 14-17). $\sigma_{H S}^{2}$ values did not change significantly throughout the region.

Mean $T_{p}$ and associated $\sigma_{T p}^{2}$ values did not change significantly within the region (figs. 46-49). The SON mean $D_{p}$ values rotated 5 to $10^{\circ}$ clockwise within the region but did not significantly change throughout other seasons (fig. 81). $\sigma_{D p}$ values did not change significantly (figs. 78-80).

\section{Top 5 Percent}

Palmyra and Kingman Reef extreme $\mathrm{SON} H_{s}$ means decreased by $>0.30 \mathrm{~m}$ compared to hindcast mean values, while the rest of the region did not change (figs. 18-21). Additionally, means did not change significantly during the other seasons. SON $\sigma_{H S}^{2}$ values decreased by 0.20 to 0.40 , but remain unchanged throughout all other seasons.

Mean $T_{p}$ values increased during the DJF season at the Palmyra and Kingman Reef points by 0.25 to $0.50 \mathrm{~s}$ (fig. 50). Howland's mean $T_{p}$ decreased by 0.25 to $0.50 \mathrm{~s}$ during the MAM season, whereas Palmyra and Kingman Reef's values decreased by 0.50 to $0.75 \mathrm{~s}$ (fig. 51). The $\sigma_{T p}^{2}$ at Palmyra and Kingman Reef decreased by 1.5 to 3.0. The SON season's mean $T_{p}$ values increased by 0.25 to $0.50 \mathrm{~s}$ throughout the region (fig. 53). The $\sigma_{T p}^{2}$ during this season at Palmyra and Kingman Reef increased by 1.5 to 3.0. JJA $T_{p}$ values did not change significantly (fig. 52). MAM $\sigma_{D p}$ values decreased by 5.0 to $10.0^{\circ}$ and the $D_{p}$ of Jarvis rotated counterclockwise by 5 to $10^{\circ}$ (fig. 83). SON $\sigma_{D p}$ values increased by 5.0 to $10.0^{\circ}$, except at Jarvis, where it's $\sigma_{D p}$ decreased by 10.0 to 15.0 and the $D_{p}$ of all islands rotated 5 to $30^{\circ}$ clockwise (fig. 85). The $D_{p}$ values of the remaining seasons did not change significantly (fig. 84, fig. 82). 


\section{RCP4.5}

\section{Mean}

Mean end-century $H_{s}$ values and associated $\sigma_{H S}^{2}$ values did not change significantly across all seasons (figs. 22-25).

Mean $T_{p}$ and associated $\sigma_{T p}^{2}$ values did not change significantly within the region (figs. 54-57). The mean $D_{p}$ of Jarvis rotated counterclockwise by 5 to $10^{\circ}$ during MAM (fig. 87). SON $D_{p}$ values rotated clockwise by 5 to $10^{\circ}$ across all islands (fig. 89). $\sigma_{D p}$ values did not change significantly throughout all seasons (fig. 86, fig. 88).

\section{Top 5 Percent}

Extreme $H_{s}$ means decreased by 0.10 to $0.20 \mathrm{~m}$ during the SON season (fig. 29). $H_{s}$ means did not change significantly during other seasons (figs. 26-28). Extreme $\sigma_{H s}^{2}$ values did not change significantly.

DJF $T_{p}$ means increased by 0.25 to 0.50 s at the Palmyra and Kingman Reef points and decreased by the same amount during the MAM season (figs. 58-59). The mean $T_{p}$ increased at Jarvis by 0.25 to 0.50 and the $\sigma_{T p}^{2}$ values increased at Palmyra and Kingman Reef by 1.5 to 3.0 during the JJA season (fig. 60). Not significant $T_{p}$ changes were observed during the SON season, and no significant $\sigma_{T p}^{2}$ changes were observed in other seasons (fig. 61). The $\sigma_{D p}$ value at Jarvis increased by 5.0 to $1.0^{\circ}$ during the DJF season (fig. 90). MAM displayed an increase of 5.0 to $10.0^{\circ}$ in the $\sigma_{D p}$ of Jarvis as well as a 30 to 40 degree counterclockwise shift of the island's $D_{p}$ (fig. 91). JJA $\sigma_{D p}$ values increased by 5.0 to 10.0 throughout the region and the mean $D_{p}$ of Jarvis rotated clockwise by 5 to $10^{\circ}$ (fig. 92). The $\sigma_{D p}$ of Jarvis increased by 5.0 to $10.0^{\circ}$; it and Howland's $D_{p}$ rotated clockwise by 5 to $20^{\circ}$ during the SON season (fig. 93).

\section{RCP8.5}

\section{Mean}

The DJF $H_{s}$ means decreased by 0.10 to $0.20 \mathrm{~m}$ (fig. 30). Similarly, the Palmyra and Kingman Reef points decreased by 0.10 to $0.20 \mathrm{~m}$ for the SON season (fig. 33). The region's means did not change significantly during the other modeled seasons (figs. $31-32$ ). $\sigma_{H s}^{2}$ values did not change significantly. 
The SON $\sigma_{T p}^{2}$ value at Jarvis increased by 1.5 to 3.0 (fig. 65). However, significant $\sigma_{T p}^{2}$ changes were not observed in the remaining seasons, and $T_{p}$ values did not change significantly across all seasons (figs. 62-64). The SON $\sigma_{D p}$ values decreased at Howland and Jarvis by 5.0 to $10.0^{\circ}$, and the $D_{p}$ values throughout the region rotated clockwise by 10 to $20^{\circ}$ (fig. 97 ). $D_{p}$ values did not change significantly during other seasons (figs. 94-96).

\section{Top 5 Percent}

Extreme DJF mean $H_{s}$ values decreased by 0.10 to $0.30 \mathrm{~m}$ within the region (fig. 34). MAM $H_{s}$ means decreased by 0.10 to $0.20 \mathrm{~m}$, and SON $H_{s}$ values decreased by 0.20 to $>0.30 \mathrm{~m}$ (fig. 35, fig. 37). The means of the JJA season did not change significantly (fig. 36). $\sigma_{H S}^{2}$ values decreased by 0.20 to 0.40 during the SON season. Values did not significantly change from hindcast values in the other seasons.

DJF $T_{p}$ values increased by 0.25 to $0.50 \mathrm{~s}$, except Palmyra and Kingman Reef, which increased by 0.50 to $0.75 \mathrm{~s}$ (fig. 66). Palmyra and Kingman Reef's mean $T_{p}$ values decreased by 0.25 to $0.50 \mathrm{~s}$ and Howland's decreased by 0.50 to $0.75 \mathrm{~s}$ during the MAM season (fig. 67). JJA's mean $T_{p}$ values decreased by 0.25 to $0.50 \mathrm{~s}$ at Howland, but increased by the same amount at Jarvis (fig. 68). Additionally, $\sigma_{T p}^{2}$ values at Palmyra and Kingman reef increased by 1.5 to 3.0 during this season. The SON season trends were similar to the JJA trends, but Howland had a stronger mean $T_{p}$ decrease of 0.50 to $0.75 \mathrm{~s}$ (fig. 69). The DJF $\sigma_{D p}$ value at Jarvis decreased by 5.0 to $10.0^{\circ}$ (fig. 98). JJA $\sigma_{D p}$ values increased by 5.0 to $10.0^{\circ}$ within the region (fig. 100). The $D_{p}$ of Jarvis rotated counterclockwise by 5 to $10^{\circ}$ during the MAM season (fig. 99). $\sigma_{D p}$ values increased by 5.0 to $15.0^{\circ}$ at Kingman Reef, Palmyra, and Howland, and the $\sigma_{D p}$ at Jarvis decreased by 10.0 to $15.0^{\circ}$ during SON (fig. 101). $D_{p}$ at Jarvis and Howland rotated clockwise by 20 to $40^{\circ}$.

Southern Region

Mid-Century: 2026-2045

\section{RCP4.5}

\section{Mean}

There were no significant changes in the Southern islands' mean $H_{s}$ values during the throughout all seasons (figs. 6-9). $\sigma_{H S}^{2}$ values did not significantly change. 
$T_{p}$ means did not change significantly across all seasons within the region (figs. 38-41). $\sigma_{T p}^{2}$ values did not change significantly across all seasons. $D_{p}$ and $\sigma_{D p}$ values did not change significantly throughout all seasons (figs. 70-73).

\section{Top 5 Percent}

The mean of the top 5 percent of $H_{s}$ values decreased at the American Samoa and the Rose point by 0.20 to $0.30 \mathrm{~m}$ during the DJF season (fig. 10). SON $H_{s}$ values increased by 0.10 to 0.20 $\mathrm{m}$ at both islands within the region (fig. 13). Extreme $\sigma_{H s}^{2}$ values decreased by 0.20 to 0.40 at the American Samoa point during the DJF season. Values did not change significantly throughout the remaining seasons (fig. 11-12).

Mean $T_{p}$ values increased within the region by 0.25 to $0.50 \mathrm{~s}$ during the DJF period (fig. 42). The $\sigma_{T p}^{2}$ at both islands increased by 1.5 to 3.0 during the same season. The mean $T_{p}$ values during other seasons did not change significantly (figs. 43-45). The SON American Samoa point's $\sigma_{T p}^{2}$ decreased by 1.5 to 3.0. The DJF $\sigma_{D p}$ at American Samoa decreased by 5.0 to $10.0^{\circ}$ (fig. 74). The MAM $D_{p}$ of Rose and American Samoa rotated clockwise by 5 to $10^{\circ}$ (fig. 75 ). $D_{p}$ values during the JJA and SON did not change significantly (figs. 76-77).

\section{RCP8.5}

\section{Mean}

There was not a significant change in mean $H_{s}$ values from hindcast means over all seasons (figs. 14-17). $\sigma_{H s}^{2}$ values did not change significantly.

Mean $T_{p}$ values did not change significantly (figs. 46-49). $\sigma_{T p}^{2}$ values did not change significantly across all seasons. The DJF $D_{p}$ Rose rotated by 5 to $10^{\circ}$ clockwise (fig. 78). $D_{p}$ and $\sigma_{D p}$ values did not change significantly during other seasons (figs. 79-81).

\section{Top 5 Percent}

Extreme mean $H_{s}$ values increased by 0.10 to $0.20 \mathrm{~m}$ at the American Samoa point and 0.20 to $0.30 \mathrm{~m}$ at the Rose Atoll point for the DJF period (fig. 18). During the SON season, the region's mean $H_{s}$ values increased by 0.10 to $0.20 \mathrm{~m}$ (fig. 21). JJA mean $H_{s}$ values increased at Rose by 0.10 to $0.20 \mathrm{~m}$ (fig. 20). DJF $\sigma_{H S}^{2}$ values decreased by 0.20 to 0.40 at the American Samoa point. Extreme $H_{s}$ values did not significantly change during the MAM or JJA seasons (fig. 19).

Mean $T_{p}$ values increased at Rose by 0.25 to $0.50 \mathrm{~s}$ during the DJF season (fig. 50). During the same season, the region's $\sigma_{T p}^{2}$ decreased by 1.5 to 3.0 at Rose and by $>3.0$ at American Samoa. MAM $T_{p}$ means decreased by 0.25 to $0.50 \mathrm{~s}$ (fig. 51 ). The SON mean $T_{p}$ increased by 0.25 
to $0.50 \mathrm{~s}$ (fig. 53). The JJA $T_{p}$ means did not change significantly (fig. 52). Rose and American Samoa's DJF $D_{p}$ rotated clockwise by 10 to $20^{\circ}$ at Rose and by 20 to $30^{\circ}$ at American Samoa (fig. 82). $D_{p}$ and $\sigma_{D p}$ values did not change significantly during other seasons (figs. 83-85).

End-Century: 2081-2100

\section{RCP4.5}

\section{Mean}

There was no significant change from hindcast mean $H_{s}$ values across all seasons (figs. 22-25). $\sigma_{H S}^{2}$ values did not change significantly.

The mean $T_{p}$ and $\sigma_{T p}^{2}$ values did not change significantly throughout all seasons (figs. 5457). $D_{p}$ and $\sigma_{D p}$ values did not change significantly throughout all seasons (figs. 86-89).

\section{Top 5 Percent}

Extreme MAM and SON $H_{s}$ means increased by 0.10 to $0.20 \mathrm{~m}$ (fig. 27, fig. 29). DJF and JJA means did not change significantly (fig. 26, fig. 28). DJF extreme $\sigma_{H s}^{2}$ values decreased at American Samoa by $>0.40$ and extreme MAM $\sigma_{H S}^{2}$ values increased by 0.20 to 0.40 .

The DJF $\sigma_{T p}^{2}$ at American Samoa decreased by 1.5 to 3.0 (fig. 58). The SON mean $T_{p}$ values did not change significantly (fig. 61). The MAM and JJA mean $T_{p}$ values did not change significantly (figs. 59-60). During the DJF season, both points' $D_{p}$ rotated by 10 to $20^{\circ}$ clockwise (fig. 90). The $\sigma_{D p}$ value at Rose decreased by 5.0 to $10.0^{\circ}$. Values did not change significantly during other seasons (figs. 91-93).

\section{RCP8.5}

\section{Mean}

There was no significant change from hindcast mean $H_{s}$ values across all seasons at American Samoa and Rose Atoll (figs. 30-33).

Mean $T_{p}$ values did not change significantly across all seasons (figs. 62-65). $\sigma_{T p}^{2}$ values did not change significantly throughout all seasons. The mean $D_{p}$ in the southern islands rotated by 5 to $10^{\circ}$ clockwise during the DJF season (fig. 94). SON $\sigma_{D p}$ values decreased by 5.0 to $10.0^{\circ}$ (fig. 97). Values did not change significantly during MAM and JJA (figs. 95-96). 


\section{Top 5 Percent}

Extreme mean $H_{s}$ values increased by 0.10 to 0.20 at Rose Atoll during the DJF season (fig. 34). JJA $H_{s}$ values increased by 0.10 to $0.20 \mathrm{~m}$ at Rose (fig. 36). Extreme mean values did not change significantly for the MAM and SON seasons (fig. 35, fig. 37). DJF $\sigma_{H s}^{2}$ values decreased by 0.10 to 0.20 at American Samoa. $\sigma_{H S}^{2}$ values did not change significantly during other seasons.

The SON mean $T_{p}$ values increased by 0.25 to $0.50 \mathrm{~s}$ (fig. 69). $T_{p}$ values did not change significantly during other seasons (figs. 66-68). MAM $\sigma_{D p}$ values increased by 5.0 to $15.0^{\circ}$ (fig. 99). The SON $\sigma_{D p}$ values decreased by 5.0 to $10.0^{\circ}$ and Rose's and its $D_{p}$ rotated 5 to $10^{\circ}$ clockwise (fig. 101). Values did not change significantly during DJF and JJA (fig. 98, fig. 100).

\section{Wind Climate Parameter Changes}

Western Region

Mid-Century: 2026-2045

\section{RCP4.5}

\section{Mean}

The mean $U_{a}$ of the Western region decreased by 0.25 to $0.50 \mathrm{~m} / \mathrm{s}$ during the DJF season (fig. 102). Mean wind speeds did not change significantly during the other three seasons (figs. $103-105) . \sigma_{U a}^{2}$ decreased at the Yap point by 0.75 to 1.50 during the SON season. $\sigma_{U a}^{2}$ values did not change significantly during other seasons.

MAM $\sigma_{U \theta}$ values decreased by 5.0 to $10.0^{\circ}$ (fig. 135). Yap's $U_{\theta}$ values rotated counterclockwise during the JJA season and clockwise during the SON season by 5 to $10^{\circ}$ (figs. 136-137). DJF values did not significantly change during other seasons (fig. 134).

\section{Top 5 percent}

The means of top 5 percent of wind speeds did not change significantly except at the Yap point during the DJF season, which decreased by 0.25 to $0.50 \mathrm{~m} / \mathrm{s}$ (figs. 106-109). Extreme $\sigma_{U a}^{2}$ increased by 0.75 to 1.50 at Palau during the SON season. $\sigma_{U a}^{2}$ values did not change significantly during the other seasons.

DJF $\sigma_{U \theta}$ values increased by 5.0 to $10.0^{\circ}$ (fig. 138). JJA $\sigma_{U \theta}$ values increased by 5.0 to $10.0^{\circ}$ and Palau's $U_{\theta}$ rotated counterclockwise by 5 to $10^{\circ}$ (fig. 140). MAM $\sigma_{U \theta}$ values decreased 
by 5.0 to $10.0^{\circ}$ (fig. 139). SON $\sigma_{U \theta}$ values increased by 5.0 to $10.0^{\circ}$ (fig. 141). During this season, Palau's $U_{\theta}$ rotated by 5 to $10^{\circ}$ counterclockwise and Yap's $U_{\theta}$ rotated by 20 to $30^{\circ}$ counterclockwise.

\section{RCP8.5}

\section{Mean}

Mean wind speeds decreased by 0.25 to $0.50 \mathrm{~m} / \mathrm{s}$ during the DJF season (fig. 110). Mean values did not change significantly during the remaining seasons (figs. 111-113). $\sigma_{U a}^{2}$ decreased during the SON season at Yap. $\sigma_{U a}^{2}$ values did not change significantly during other seasons

MAM $U \theta \sigma_{U \theta}$ values decreased by 5.0 to 10.0 (fig. 143). JJA $U_{\theta}$ values rotated clockwise at Palau by 5 to $10^{\circ}$. SON mean wave directions rotated 5 to $20^{\circ}$ clockwise (fig. 144). Values did not change significantly during other seasons (fig. 142, fig. 145).

\section{Top 5 percent}

DJF extreme wind speed means decreased by 0.25 to $0.50 \mathrm{~m} / \mathrm{s}$ (fig. 114). SON means decreased by 0.50 to $0.75 \mathrm{~m} / \mathrm{s}$ (fig. 117). The remaining seasons displayed no significant changes (figs. 115-116). Extreme $\sigma_{U a}^{2}$ values did not change in the region throughout all seasons.

The DJF $\sigma_{U \theta}$ value at Palau increased by 5.0 to $10.0^{\circ}$ and $U_{\theta}$ through the region rotated 10 to $20^{\circ}$ clockwise (fig. 146). $\sigma_{U \theta}$ values decreased by 5.0 to $15.0^{\circ}$ during the JJA season (fig. 148). SON $\sigma_{U \theta}$ values decreased by 5.0 to $10.0^{\circ}$ (fig. 149). The MAM season's values did not change significantly (fig. 147).

End-Century: 2081-2100

\section{RCP4.5}

\section{Mean}

There were no significant changes in $U_{a}$ means observed during all seasons (figs. 118121). SON $\sigma_{U a}^{2}$ values decreased by 0.75 to 1.50 at all points. $\sigma_{U a}^{2}$ values did not change significantly during the remaining seasons.

Yap's $U_{\theta}$ rotated counterclockwise by 5 to $10^{\circ}$ during JJA (fig. 152). $U_{\theta}$ s rotated by 10 to $20^{\circ}$ clockwise during the SON, but values did not change significantly during other seasons (figs. 150-151, fig. 153). 


\section{Top 5 percent}

The DJF and SON extreme wind speeds decreased by 0.25 to $0.50 \mathrm{~m} / \mathrm{s}$ (fig. 122, fig. 125). Other seasons showed no significant change in wind speeds (figs. 123-124). Extreme $\sigma_{U a}^{2}$ values did not significantly change within the region.

SON $\sigma_{U \theta}$ values decreased by 10.0 to $15.0^{\circ}$ (fig. 157). Direction values did not significantly change during other seasons (figs. 154-156).

\section{RCP8.5}

\section{Mean}

Mean wind speeds decreased by 0.50 to $0.75 \mathrm{~m} / \mathrm{s}$ during the DJF season but increased by 0.25 to $0.50 \mathrm{~m} / \mathrm{s}$ during the JJA season (fig. 126, fig. 128). The remaining seasons displayed no significant change in mean wind speeds (fig. 127, fig. 129). MAM $\sigma_{U a}^{2}$ decreased by 0.75 to 1.50 at Yap. SON $\sigma_{U a}^{2}$ values decreased by 0.75 to 1.50 throughout the region.

The JJA $U_{\theta}$ of Yap rotated by 10 to $20^{\circ}$ counterclockwise (fig. 160). $\sigma_{U \theta}$ values decreased by 5.0 to $10.0^{\circ}$ during the MAM and SON seasons (fig. 159, fig. 161). The $U_{\theta}$ of Yap rotated 10 to $20^{\circ}$ clockwise during the SON seasons. DJF values did not change significantly (fig. 158).

\section{Top 5 percent}

DJF and SON mean wind speed values decreased by 0.50 to $0.75 \mathrm{~m} / \mathrm{s}$ (fig. 130, fig. 133). MAM values decreased by 0.25 to $0.50 \mathrm{~m} / \mathrm{s}$ (fig. 131). The JJA season values did not change significantly (fig. 132). JJA $\sigma_{U a}^{2}$ increased at Yap by 0.75 to 1.50 . SON $\sigma_{U a}^{2}$ values increased by 0.75 to 1.50 at both islands within the region. $U_{a}$ values did not change significantly during the other seasons.

DJF $\sigma_{U \theta}$ values increased by 5.0 to $10.0^{\circ}$ and mean wave directions rotated by 5 to $10^{\circ}$ clockwise at Palau (fig. 162). Similarly, the JJA $U_{\theta}$ of Yap rotated by 10 to $20^{\circ}$ counterclockwise (fig. 164). MAM $\sigma_{U \theta}$ values decreased by 5.0 to $10.0^{\circ}$ as did SON values (fig. 163, fig. 165). The $U_{\theta}$ of Palau rotated counterclockwise by 5 to $10^{\circ}$ during the JJA season. SON $U_{\theta}$ rotated counterclockwise by 5 to $20^{\circ}$.

\section{Marianas Region}

Mid-Century: 2026-2045 


\section{RCP4.5}

\section{Mean}

Mean wind speeds decreased by 0.25 to $0.50 \mathrm{~m} / \mathrm{s}$ in the Marianas region during the SON season, but values did not change significantly during the other seasons (figs. 102-105). JJA $\sigma_{U a}^{2}$ decreased by 0.75 to 1.50 at Asuncion. Guam $\sigma_{U a}^{2}$ values decreased by the same amount during the SON season.

SON $\sigma_{U \theta}$ values increase by 5.0 to $10.0^{\circ}$ (fig. 137). $U_{\theta}$ values did not significantly change during other seasons (figs. 134-136).

\section{Top 5 percent}

JJA extreme $U_{a}$ means decreased by 0.25 to $0.50 \mathrm{~m} / \mathrm{s}$ at Saipan and Guam, with speeds decreasing my $>0.75 \mathrm{~m} / \mathrm{s}$ at the Asuncion point (fig. 108). Similarly, SON values decreased by 0.25 to $0.50 \mathrm{~m} / \mathrm{s}$ at Saipan and Asuncion but decreased by $>0.75 \mathrm{~m} / \mathrm{s}$ at Guam (fig. 109). The DJF and MAM means did not change significantly (figs. 106-107). MAM extreme $\sigma_{U a}^{2}$ decreased by 0.75 to 1.50 at Guam. JJA $\sigma_{U a}^{2}$ values decreased by 0.75 to 1.50 at Asuncion.

The JJA $\sigma_{U \theta}$ value increased by 10.0 to $15.0^{\circ}$ at Saipan and rotated counterclockwise by 5 to $10^{\circ}$ at the Saipan and Guam points (fig. 140). $\sigma_{U \theta}$ values decreased by 5.0 to $10.0^{\circ}$ during MAM and increased by 5.0 to $10.0^{\circ}$ during SON (fig. 139, fig. 141). The $U_{\theta}$ of Guam rotated clockwise by 10 to $20^{\circ}$ during the SON season. DJF direction values did not change significantly (fig. 138).

\section{RCP8.5}

\section{Mean}

Mean $U_{a}$ values decreased by 0.25 to $0.50 \mathrm{~m} / \mathrm{s}$ during the MAM and SON seasons (fig. 111, fig. 113). The remaining seasons' mean values did not change significantly (fig. 110, fig. 112). $\sigma_{U a}^{2}$ decreased by 0.75 to 1.50 at Guam during the SON season.

$\sigma_{U \theta}$ values decreased at the Guam point by 5.0 to $10.0^{\circ}$ during the JJA season and increased by 5.0 to $15.0^{\circ}$ during the SON season (figs. 144-145). $\sigma_{U \theta}$ values did not change significantly during other seasons (figs. 142-143).

\section{Top 5 percent}

MAM mean extreme $U_{a}$ values decreased by 0.25 to $0.50 \mathrm{~m} / \mathrm{s}$ (fig. 115). JJA changes were mixed. $U_{a}$ mean increased at the Saipan point by 0.25 to $0.50 \mathrm{~m} / \mathrm{s}$ and decreased at the Asuncion point by the same amount (fig. 116). SON values decreased by 0.25 to $0.75 \mathrm{~m} / \mathrm{s}$, with the largest 
change occurring at the Guam point (fig. 117). DJF values did not change significantly (fig. 114). MAM $\sigma_{U a}^{2}$ values decreased by 0.75 to 1.50 , except at Guam, where the $\sigma_{U a}^{2}$ decreased by $>1.50$. SON $\sigma_{U a}^{2}$ values increased by 0.75 to 1.50 at Saipan and by $>1.50$ at Asuncion.

JJA $\sigma_{U \theta}$ values decreased at Guam by 10.0 to $15.0^{\circ}$ and increased at Asuncion by 5.0 to $10.0^{\circ}$ (fig. 148). Saipan and Asuncion's $U_{\theta}$ rotated clockwise by 10 to $20^{\circ}$. MAM $\sigma_{U \theta}$ valued decreased by 5.0 to $10.0^{\circ}$ (fig. 147). SON $\sigma_{U \theta}$ values increased by 10.0 to $>15.0^{\circ}$ and $U_{\theta}$ s rotated clockwise by 5 to $30^{\circ}$, with the larger deviation from hindcast means occurring at Guam (fig. 149). DJF direction values did not change significantly (fig. 146).

End-Century: 2081-2100

\section{RCP4.5}

\section{Mean}

Mean $U_{a}$ values decreased by 0.25 to $0.50 \mathrm{~m} / \mathrm{s}$ during the SON season but did not change significantly during the remaining seasons (figs. 118-121). $\sigma_{U a}^{2}$ decreased by 0.75 to 1.50 at Asuncion during the JJA season. SON $\sigma_{U a}^{2}$ values decreased by 0.75 to 1.50 at all islands.

The JJA $\sigma_{U \theta}$ value at Guam decreased by 5.0 to $10.0^{\circ}$ (fig. 152). SON $\sigma_{U \theta}$ values increased by 5.0 to $10.0^{\circ}$ (fig. 153). $\sigma_{U \theta}$ values did not change significantly during other seasons (figs. 150-151).

\section{Top 5 percent}

MAM extreme $U_{a}$ means decreased by 0.25 to $0.50 \mathrm{~m} / \mathrm{s}$ (fig. 123). JJA values decreased by 0.25 to $>0.75 \mathrm{~m} / \mathrm{s}$, with the largest change occurring at Asuncion (fig. 124). SON values decreased by 0.50 to $>0.75 \mathrm{~m} / \mathrm{s}$ (fig. 125). No significant change in extreme $U_{a}$ means occurred during the DJF season (fig. 122). MAM $\sigma_{U a}^{2}$ values decreased by 0.75 to 1.50 at Saipan and $>1.50$ at Guam. Values did not change significantly during other seasons.

MAM $\sigma_{U \theta}$ values decreased by 5.0 to $15.0^{\circ}$ (fig. 155). Saipan's $\sigma_{U \theta}$ value increased by 10.0 to $15.0^{\circ}$ and it and Guam's $U_{\theta}$ s rotated clockwise by 10 to $20^{\circ}$ during the JJA season (fig. 156). The SON $\sigma_{U \theta}$ at Guam increased by 10.0 to $15.0^{\circ}$ and its $U_{\theta}$ rotated clockwise by 10 to $20^{\circ}$ (fig. 157). DJF direction values did not change significantly (fig. 154). 


\section{RCP8.5}

Mean

Mean $U_{a}$ values decreased during the DJF and SON seasons (fig. 126, fig. 129). No significant changes were observed during the other seasons (figs. 127-128). $\sigma_{U a}^{2}$ values decreased by 0.75 to 1.50 during the MAM and SON seasons. The JJA $\sigma_{U a}^{2}$ at Asuncion decreased by 0.75 to 1.50. $\sigma_{U a}^{2}$ values did not change significantly during the DJF season.

JJA $\sigma_{U \theta}$ values decreased by 5.0 to $10.0^{\circ}$, and SON $\sigma_{U \theta}$ values increased by 5.0 to $10.0^{\circ}$ (fig. 160). $U_{\theta}$ values did not significantly change during other seasons (figs. 158-159, fig. 161).

\section{Top 5 percent}

MAM $U_{a}$ values decreased by 0.50 to $>0.75 \mathrm{~m} / \mathrm{s}$ while JJA values decreased by a smaller 0.25 to $0.50 \mathrm{~m} / \mathrm{s}$ (figs. $131-132$ ). SON values decreased by 0.25 to $0.75 \mathrm{~m} / \mathrm{s}$, with the largest change occurring at the Guam point (fig. 133). DJF values did not change significantly (fig. 130). The $\sigma_{U a}^{2}$ of the Guam point increased by $>1.50$ during the DJF season. $\sigma_{U a}^{2}$ values increased by a smaller amount of 0.75 to 1.50 during the JJA and SON seasons for the Guam and Asuncion points, respectively. MAM values decreased by 0.75 to 1.50 , except at Guam where the $\sigma_{U a}^{2}$ decreased by $>1.50$.

The DJF $\sigma_{U \theta}$ at Asuncion decrease by 5.0 to $10.0^{\circ}$ (fig. 162). MAM $\sigma_{U \theta}$ values decreased by 5.0 to $15.0^{\circ}$ (fig. 163). $U_{\theta}$ s during JJA rotated by 5 to $20^{\circ}$ clockwise within the region (fig. 164). SON $\sigma_{U \theta}$ values increased by 5.0 to $15.0^{\circ}$, and the $U_{\theta}$ of Guam rotated 10 to $20^{\circ}$ clockwise (fig. 165).

Central Region

Mid-Century: 2026-2045

\section{RCP4.5}

Mean

SON mean $U_{a}$ values decreased at the Enewetak, Bikini, and Kwajalein points by 0.25 to $0.50 \mathrm{~m} / \mathrm{s}$ (fig. 105). There were no significant changes observed during the remaining seasons (figs. 102-104). SON $\sigma_{U a}^{2}$ values decreased by 0.75 to 1.50 at Pohnpei and Kwajalein. $\sigma_{U a}^{2}$ values did not change significantly throughout the other seasons. 
MAM $\sigma_{U \theta}$ values decreased by 5.0 to $10.0^{\circ}$ (fig. 135). The JJA value at Bikini decreased by 5.0 to $10.0^{\circ}$ (fig. 136). The $U \theta$ values of Chuuk and Kwajalein rotated counterclockwise by 5 to $10^{\circ}$. $U \theta$ values did not significantly change in other seasons (fig. 134, fig. 137).

\section{Top 5 percent}

MAM $U_{a}$ values decreased by 0.25 to $0.50 \mathrm{~m} / \mathrm{s}$ at Enewetak and Bikini (fig. 107). Means increased by the same amount throughout the region during the JJA season (fig. 108). SON means decreased by 0.25 to $>0.75 \mathrm{~m} / \mathrm{s}$, with the largest change occurring at Pohnpei (fig. 109). Mean $U_{a}$ values did not change significantly during the DJF season (fig. 106). MAM $\sigma_{U a}^{2}$ values decreased by 0.75 to 1.50 at Enewetak, Bikini, and Kosrae. Conversely, $\sigma_{U a}^{2}$ values increased by 0.75 to 1.50 at Enewtok, Bikini, and Pohnpei during the SON season.

DJF $\sigma_{U \theta}$ values decreased by 5.0 to 10.0 at Pohnpei and Kosrae, but by 10.0 to 15.0 at Chuuk (fig. 138). MAM $\sigma_{U \theta}$ decreased by 5.0 to $15.0^{\circ}$ through the region (fig. 139). JJA $\sigma_{U \theta}$ values increased at Pohnpei by 5.0 to $10.0^{\circ}$ and decreased at Majuro by 5.0 to $10.0^{\circ}$ (fig. 140). $\sigma_{U \theta}$ values through the region increased by 5.0 to $10.0^{\circ}$ during the SON season, except at Chuuk and Kosrae where they decreased by 10.0 to $>15.0^{\circ}$ (fig. 141). The $U_{\theta}$ of Bikini and Enewetak rotated clockwise by 5 to $20^{\circ}$ and counterclockwise at Kosrae by the same amount. The $U_{\theta}$ of Pohnpei and Chuuk rotated clockwise by 60 to $70^{\circ}$.

\section{RCP8.5}

\section{Mean}

$U_{a}$ means decreased during the SON season at Enewetak, Bikini, and Kwajalein (fig. 113). Values did not change significantly during the remaining seasons (figs. 110-112). The MAM $\sigma_{U a}^{2}$ decreased by 0.75 to 1.50 at Bikini. SON values decreased at Pohnpei and Kwajalein by 0.75 to 1.50 .

$\sigma_{U \theta}$ values decreased during the MAM season at Chuuk and Pohnpei by 5.0 to $10.0^{\circ}$ and at Enewetak and Bikini during JJA by the same amount (figs. 143-144). The $U_{\theta} \mathrm{s}$ of Chuuk and Kwajalein rotated clockwise by 5 to $10^{\circ}$ in the SON season, and values did not change significantly during DJF (fig. 142, fig. 145).

\section{Top 5 percent}

Extreme $U_{a}$ values decreased in the eastern half of the region during the JJA season by 0.25 to $0.50 \mathrm{~m} / \mathrm{s}$ (fig. 116). SON values decreased by the same amount, though the sites of decrease were sparse, not displaying any observable clustering (fig. 117). JJA $\sigma_{U a}^{2}$ values increased by 0.75 to 1.50 at Kwajalein and $>1.50$ at Wake. The SON $\sigma_{U a}^{2}$ at the Majuro point 
decreased by 0.75 to 1.50 . $U_{a}$ values did not change significantly during other seasons (figs. 114115).

DJF $\sigma_{U \theta}$ values at Chuuk and Pohnpei increased by 5.0 to $10.0^{\circ}$ and decreased by 5.0 to $10.0^{\circ}$ at Majuro (fig. 146). Chuuk's $\sigma_{U \theta}$ value during MAM decreased by 5.0 to $10.0^{\circ}$ (fig. 147). JJA $\sigma_{U \theta}$ values decreased by 5.0 to $10.0^{\circ}$ at Pohnpei and increased by the same amount at Wake and Kosrae (fig. 148). The $U_{\theta}$ of Pohnpei and Kosrae rotated clockwise by 20 to 30 and 40 to $50^{\circ}$, respectively. The $U_{\theta}$ of Chuuk, Wake, and Kwajalein rotated clockwise by 5 to $20^{\circ}$. SON $\sigma_{U \theta}$

values decreased at Chuuk by 5.0 to $10.0^{\circ}$ and increased throughout the rest of the region by 5.0 to $>15.0^{\circ}$ (fig. 149). The $U_{\theta}$ at Chuuk and Pohnpei rotated by 40 to $60^{\circ}$ clockwise and at Majuro and Kosrae by 10 to $20^{\circ}$ clockwise.

End-Century: 2081-2100

\section{RCP4.5}

\section{Mean}

DJF means decreased by 0.25 to $0.50 \mathrm{~m} / \mathrm{s}$ at Chuuk, Pohnpei, and Majuro (fig. 118). Similarly, values decreased by 0.25 to 0.50 at Enewetak, Bikini, and Kwajalein during the SON season (fig. 121). JJA $U_{a}$ means increased by 0.25 to $0.50 \mathrm{~m} / \mathrm{s}$ throughout the region (fig. 120). MAM values did not change significantly (fig. 119). The MAM $\sigma_{U a}^{2}$ at Majuro decreased by 0.75 to 1.50 and the $\mathrm{SON} \sigma_{U a}^{2}$ at Kwajalein decreased by 0.75 to 1.50 .

MAM $\sigma_{U \theta}$ values decreased by 5.0 to $10.0^{\circ}$ at Chuuk, Pohnpei, and Kosrae (fig. 151). JJA $\sigma_{U \theta}$ values decreased by 5.0 to $10.0^{\circ}$ at Bikini and Enewetak (fig. 152). SON $\sigma_{U \theta}$ values increased by 5.0 to $10.0^{\circ}$ at Enewetak, Bikini, and Kwajalein (fig. 153). $U_{\theta}$ of Chuuk, Pohnpei, Majuro, and Kwajalein rotated clockwise by 5 to $10^{\circ}$ during SON. DJF direction values did not change significantly (fig. 150).

\section{Top 5 percent}

Extreme $U_{a}$ means decreased by 0.25 to $0.50 \mathrm{~m} / \mathrm{s}$ during the DJF and SON seasons and specifically at the Chuuk, Pohnpei, and Kosrae points during the MAM season (figs. 122-123, fig. 125). There was an increase in JJA $U_{a}$ values by 0.25 to $0.75 \mathrm{~m} / \mathrm{s}$ across the region, with the largest changes displayed at Enewetak, Bikini, and Kosrae (fig. 124). $\sigma_{U a}^{2}$ values decreased by 0.75 to 1.50 at Bikini and Kosrae during the MAM season. JJA values increased throughout the region by 0.75 to 1.50 , except at Chuuk, where values decreased by 0.75 to 1.50 . SON $\sigma_{U a}^{2}$ values increased by 0.75 to 1.50 at Enewetak, Bikini, and Majuro. Values did not change significantly during the DJF season. 
DJF $\sigma_{U \theta}$ values increased at Chuuk and Kosrae by 5.0 to $10.0^{\circ}$ (fig. 154). MAM $\sigma_{U \theta}$ values decreased by 5.0 to $>15.0$ at Kosrae, Chuuk, Pohnpei, and Kwajalein (fig. 155). JJA $\sigma_{U \theta}$ values decreased by 5.0 to $>15.0^{\circ}$ at Majuro and Chuuk, but values increased by 5.0 to $>15.0$ at Wake, Kosrae, Enewtok, and Bikini (fig. 156). The $U_{\theta}$ of Chuuk, Bikini, and Kwajalein rotated clockwise by 5 to $20^{\circ}$ and the directions of Majuro, Pohnpei, and Kosrae rotated by 20 to $40^{\circ}$ clockwise. SON $\sigma_{U \theta}$ values decreased at Chuuk, Pohnpei, and Kosrae by $>15.0^{\circ}$, but values increased by 10.0 to $>15.0^{\circ}$ at Enewetak, Bikini, Kwajalein, and Majuro (fig. 157). $U_{\theta}$ rotated clockwise by 70 to $90^{\circ}$ at Chuuk and Pohnpei, 60 to $70^{\circ}$ at Majuro, and 5 to $30^{\circ}$ at Kosrae, Enewetak, Bikini, and Kwajalein.

\section{RCP8.5}

\section{Mean}

DJF $U_{a}$ means decreased by 0.25 to $0.50 \mathrm{~m} / \mathrm{s}$, and $\mathrm{SON}$ means decreased by the same amount at Enewetak, Bikini, and Kwajalein (fig. 126, fig. 129). MAM means increased by 0.25 to $0.50 \mathrm{~m} / \mathrm{s}$ at Pohnpei, Kwajalein, and Majuro (fig. 127). JJA values increased by 0.25 to $0.50 \mathrm{~m} / \mathrm{s}$ across the entire region (fig. 128). The $\sigma_{U a}^{2}$ decreased at the Majuro point by 0.75 to 1.50 during the DJF and MAM seasons. Similarly, the $\sigma_{U a}^{2}$ at the Wake point decreased by 0.75 to 1.50 during the JJA season. $\sigma_{U a}^{2}$ values decreased across the entire region by 0.75 to 1.50 during SON.

MAM $\sigma_{U \theta}$ values decreased by 5.0 to $10.0^{\circ}$ at Chuuk, Pohnpei, and Majuro (fig. 159). JJA $\sigma_{U \theta}$ values decreased by 5.0 to $15.0^{\circ}$, and the mean directions of Chuuk, Kosrae, Majuro, and Pohnpei rotated counterclockwise by 5 to $10^{\circ}$ (fig. 160). Wind direction values did not change significantly during the DJF and SON seasons (fig. 158, fig. 161).

\section{Top 5 percent}

The top 5 percent of $U_{a}$ values decreased by 0.25 to $0.75 \mathrm{~m} / \mathrm{s}$ during the DJF, MAM, and SON, with the largest boreal winter values in the center of the region and fall values at Pohnpei (figs. 130-131, fig. 133). Conversely, JJA $U_{a}$ values increased by 0.25 to $0.75 \mathrm{~m} / \mathrm{s}$, with the largest increases occurring at Enewetak, Bikini, and Pohnpei (fig. 132). MAM extreme $\sigma_{U a}^{2}$ at Bikini decreased by 0.75 to 1.50 . JJA $\sigma_{U a}^{2}$ values increased by 0.75 to 1.50 at Pohnpei and Kwajalein and by $>1.50$ at Enewetak, Bikini, and Wake. SON season $\sigma_{U a}^{2}$ values increased by 0.75 to 1.50 at Pohnpei and by $>1.50$ at Enewetak, Bikini, and Chuuk.

MAM $\sigma_{U \theta}$ values decreased by 5.0 to $15.0^{\circ}$ (fig. 163). JJA mean wave directions rotated 5 to $20^{\circ}$ clockwise at Enewetak, Bikini, Wake, and Kwajalein, but rotated 5 to $10^{\circ}$ counterclockwise at Majuro and Kosrae (fig. 164). SON $\sigma_{U \theta}$ values decreased by 1.0 to $>15.0^{\circ}$ at Chuuk, Pohnpei, and Kosrae (fig. 165). $U_{\theta}$ rotated 30 to $40^{\circ}$ clockwise at Chuuk and 20 to $40^{\circ}$ counterclockwise at 
Pohnpei and Kosrae during the SON season. DJF direction values did not change significantly (fig. 162).

Northeast Region

Mid-Century: 2026-2045

\section{RCP4.5}

Mean

Mean $U_{a}$ values did not change significantly across all seasons (figs. 102-105). The DJF $\sigma_{U a}^{2}$ decreased at Johnston Atoll by 0.75 to 1.560 .

The $\sigma_{U \theta}$ value at the Northwest Hawaiian Islands increased by 5.0 to $10.0^{\circ}$ during the DJF season (fig. 134). Wind direction values did not change significantly during other seasons (figs. 135-137).

\section{Top 5 percent}

Extreme $U_{a}$ means decreased by 0.25 to $0.50 \mathrm{~m} / \mathrm{s}$ at the Northwest Hawaii and Midway points during the DJF season and at the main Hawaiian Islands points during the SON season (fig. 106, fig. 109). $U_{a}$ values did not change significantly during the remaining seasons (figs. 107108). The $\sigma_{U a}^{2}$ of the Northwest Hawaiian point increased by 0.75 to 1.50 during the SON season.

The DJF $U_{\theta}$ of Midway rotated by 5 to $10^{\circ}$ clockwise and its $\sigma_{U \theta}$ value increased by 5.0 to $10.0^{\circ}$ (fig. 138). Midway's $U_{\theta}$ rotated 10 to $20^{\circ}$ clockwise during the MAM season and by 5 to $10^{\circ}$ clockwise during the SON season (fig. 139, fig. 141). Johnston Atoll's $\sigma_{U \theta}$ value increased by 5.0 to $10.0^{\circ}$ during the JJA and SON seasons (fig. 140).

\section{RCP8.5}

\section{Mean}

$U_{a}$ means decreased by 0.25 to $0.50 \mathrm{~m} / \mathrm{s}$ at the Big Island of Hawaii point during SON (fig. 113). The other seasons' means did not change significantly (figs. 110-112). The $\sigma_{U a}^{2}$ at the Northwest Hawaiian point decreased by 0.75 to 1.50 during the DJF season.

The DJF $U_{\theta}$ of the Northwest Hawaiian Islands rotated clockwise by 20 to $30^{\circ}$ (fig. 142). Wind direction values did not change significantly during other seasons (figs. 143-145). 


\section{Top 5 percent}

DJF changes were mixed. The $U_{a}$ values at the Big Island point increased by 0.25 to 0.50 $\mathrm{m} / \mathrm{s}$, but the Northwest Hawaii and Midway means decreased by 0.25 to $0.50 \mathrm{~m} / \mathrm{s}$ (fig. 114). JJA means decreased by 0.25 to $0.50 \mathrm{~m} / \mathrm{s}$ at the main Hawaiian Islands points (fig. 116). SON means decreased by 0.25 to $0.50 \mathrm{~m} / \mathrm{s}$ across the whole region (fig. 117). MAM means did not change significantly (fig. 115). $\sigma_{U a}^{2}$ values within the region did not change significantly.

The DJF $\sigma_{U \theta}$ values increased by 5.0 to $10.0^{\circ}$ at Midway and the Northwest Hawaiian Islands and decreased by 5.0 to $15.0^{\circ}$ at Molokai, Kauai, and Johnston Atoll (fig. 146). The $U_{\theta}$ of Midway rotated clockwise by 5 to $10^{\circ}$ at Midway during the MAM season (fig. 147). JJA $\sigma_{U \theta}$ values increased by 5.0 to $10.0^{\circ}$ at the Big Island point (fig. 148). The $\sigma_{U \theta}$ values during SON decreased by 5.0 to $15.0^{\circ}$ at Midway and the Northwest Hawaiian Islands and increased by 5.0 to $10.0^{\circ}$ at Johnston Atoll (fig. 149).

End-Century: 2081-2100

\section{RCP4.5}

\section{Mean}

There were no significant changes in $U_{a}$ means across all seasons. DJF $\sigma_{U a}^{2}$ values decreased by 0.75 to 1.50 at Johnston Atoll, the Northwest Hawaiian Islands point, and Midway (fig. 118). SON $\sigma_{U a}^{2}$ values decreased by 0.75 to 1.50 at the Northwest Hawaiian Islands and Midway points (fig. 121). $U_{a}$ Values did not change significantly during the other seasons (figs. 119-120).

The $\sigma_{U \theta}$ of the Northwest Hawaiian Islands increased by 5.0 to $10.0^{\circ}$, and its $U_{\theta}$ rotated 5 to $10^{\circ}$ clockwise during the DJF season (fig. 150). $\sigma_{U \theta}$ values decreased by 5.0 to $10.0^{\circ}$ at Midway and the Northwest Hawaiian Islands during SON (fig. 153). Wind direction values did not change significantly during other seasons (figs. 151-152).

\section{Top 5 percent}

Extreme $U_{a}$ means decreased by 0.25 to $0.75 \mathrm{~m} / \mathrm{s}$ during the DJF season at the Northwest Hawaii, Midway, and Johnston Atoll points (fig. 122). The Molokai and Johnston Atoll's means decreased by 0.25 to $0.50 \mathrm{~m} / \mathrm{s}$ during the MAM season (fig. 123). The JJA season means decreased at the Northwest Hawaii point by 0.25 to 0.50 , and the SON season $U_{a}$ values decreased by 0.25 to $0.50 \mathrm{~m} / \mathrm{s}$ at the Northwest Hawaii and Midway points (figs. 124-125). JJA $\sigma_{U a}^{2}$ values 
increased by 0.75 to 1.50 at the Northwest Hawaii point and by $>1.50$ at the Big Island and Midway points.

The DJF $\sigma_{U \theta}$ values at Kauai and Molokai decreased by 5.0 to $10.0^{\circ}$ (fig. 154). The $U_{\theta}$ at Midway during MAM rotated by 5 to $10^{\circ}$ clockwise (fig. 155). $\sigma_{U \theta}$ values increased by 5.0 to $15.0^{\circ}$ during JJA (fig. 156). SON wind directions $\sigma_{U \theta}$ values decreased by 5.0 to $15.0^{\circ}$ at Midway and the Northwest Hawaiian Islands while the rest of the region's values increased by 5.0 to $10.0^{\circ}$ (fig. 157).

\section{RCP8.5}

\section{Mean}

$U_{a}$ means decreased by 0.25 to $0.50 \mathrm{~m} / \mathrm{s}$, except at the midway point, which decreased by 0.50 to $0.75 \mathrm{~m} / \mathrm{s}$ during the DJF (fig. 126). JJA means decreased by 0.25 to $0.50 \mathrm{~m} / \mathrm{s}$ (fig. 128). SON values at the Big Island of Hawaii decreased by 0.25 to $0.50 \mathrm{~m} / \mathrm{s}$ (fig. 129). The MAM season means did not change significantly (fig. 127). $\sigma_{U a}^{2}$ values decreased by 0.75 to 1.50 at Johnston Atoll and by $>1.50$ at the Northwest Hawaii and Midway points during the DJF season. JJA values decreased by 0.75 to 1.50 at Northwest Hawaii, Kauai, and Johnston Atoll. SON values decreased by 0.75 to 1.50 at Kauai and the Northwest Hawaii points, but decreased further by $>1.50$ at Johnston atoll. The MAM season's $\sigma_{U a}^{2}$ values did not change significantly.

DJF $\sigma_{U \theta}$ values decreased by 5.0 to $10.0^{\circ}$ at the Big Island, Kauai, and Johnston Atoll points, and the $U_{\theta}$ of the Northwest Hawaiian Islands rotated clockwise 10 to $20^{\circ}$ (fig. 158). The SON $\sigma_{U \theta}$ values decreased by 5.0 to $15.0^{\circ}$ at the Northwest Hawaiian Islands, Midway, and Kauai (fig. 161). Wind direction values did not change significantly during other seasons (figs. 159160).

\section{Top 5 percent}

DJF extreme mean $U_{a}$ values decreased by 0.50 to $>0.75 \mathrm{~m} / \mathrm{s}$ (fig. 130). MAM values decreased by 0.25 to $0.75 \mathrm{~m} / \mathrm{s}$ (fig. 131). JJA means decreased over a wide range of 0.25 to $>0.75$ $\mathrm{m} / \mathrm{s}$ (fig. 132). Extreme means decreased by 0.25 to $0.50 \mathrm{~m} / \mathrm{s}$ at the Big Island of Hawaii point for the SON season (fig. 133). Kauai's $\sigma_{U a}^{2}$ increased by 0.75 to 1.50 during the SON season.

DJF $\sigma_{U \theta}$ values increased by 5.0 to $10.0^{\circ}$ at the Northwest Hawaiian Islands and decreased by $>15.0^{\circ}$ at Molokai and Kauai (fig. 162). MAM direction $\sigma_{U \theta}$ values at the Northwest Hawaiian Islands and Kauai decreased by 5.0 to $10.0^{\circ}$, and the $U_{\theta}$ of Midway rotated by 20 to $30^{\circ}$ clockwise (fig. 163). SON $\sigma_{U \theta}$ values decreased by 5.0 to $>15.0^{\circ}$ at the Northwest Hawaiian Islands and Midway (fig. 165). Midway's $U_{\theta}$ rotated 5 to $10^{\circ}$ clockwise. JJA wind direction values did not change significantly (fig. 164). 
Eastern Equatorial Region

Mid-Century: 2026-2045

\section{RCP4.5}

\section{Mean}

MAM mean $U_{a}$ values increased by 0.25 to $0.50 \mathrm{~m} / \mathrm{s}$ at the Palmyra and Kingman Reef points (fig. 103). Means did not significantly change during the other seasons through the region (fig. 102, figs. 104-105).

The MAM $\sigma_{U \theta}$ of Jarvis increased by 5.0 to $10.0^{\circ}$ (fig. 135). The $U_{\theta}$ of Kingman Reef rotated counterclockwise by 5 to $10^{\circ}$ during JJA (fig. 136). Wind direction values did not change significantly during other seasons (fig. 134, fig. 137).

\section{Top 5 percent}

The Palmyra and Kingman Reef points' extreme mean $U_{a}$ values increased by 0.25 to 0.50 $\mathrm{m} / \mathrm{s}$ during the MAM and JJA seasons (figs. 107-108). The SON $U_{a}$ values decreased by 0.25 to $0.50 \mathrm{~m} / \mathrm{s}$ throughout the entire region (fig. 109). The DJF values did not change significantly (fig. 106). SON $\sigma_{U a}^{2}$ values decreased by 0.75 to 1.50 across the entire region. However, $\sigma_{U a}^{2}$ values did not significantly change during other seasons.

DJF $\sigma_{U \theta}$ values decreased by 5.0 to $15.0^{\circ}$ at Howland, Palmyra (fig. 138). Jarvis and Howland's $U_{\theta}$ rotated clockwise by 5 to $20^{\circ}$. The MAM $\sigma_{U \theta}$ value at Jarvis increased by 5.0 to $10.0^{\circ}$ (fig. 139). The JJA wind direction values decreased by 5.0 to $10.0^{\circ}$ at Howland and Kingman Reef, with Palmyra and Kingman Reef's $U_{\theta}$ rotating counterclockwise by 10 to $20^{\circ}$ (fig. 140). The $\sigma_{U \theta}$ values of Jarvis and Howland decreased by 10.0 to $15.0^{\circ}$ during the SON season (fig. 141). Palmyra's $U_{\theta}$ rotated counterclockwise by 5 to $10^{\circ}$.

\section{RCP8.5}

\section{Mean}

Mean $U_{a}$ values increased during the MAM season at the Palmyra and Kingman Reef points (fig. 111). Otherwise, means did not change significantly at other points or during the other seasons (fig. 110, figs. 112-113). $\sigma_{U a}^{2}$ values did not change within the region.

MAM $\sigma_{U \theta}$ values at Palmyra and Kingman Reef decreased by 5.0 to $10.0^{\circ}$ (fig. 143). The $U_{\theta}$ of Kingman Reef within the JJA season rotated counterclockwise by 5 to $10^{\circ}$ (fig. 144). Wind direction values did not change significantly during other seasons (fig. 142, fig. 145). 


\section{Top 5 percent}

Extreme $U_{a}$ values decreased by 0.20 to $0.50 \mathrm{~m} / \mathrm{s}$ at the Jarvis point during the MAM season, while the other islands remain relatively unchanged (fig. 115). The SON extreme $U_{a}$ values decreased by 0.25 to $0.50 \mathrm{~m} / \mathrm{s}$, except for the Howland point, which did not change significantly (fig. 117). Similarly, there were not significant changes observed in the remaining seasons (fig. 114, fig. 116). SON $\sigma_{U a}^{2}$ values decreased by 0.75 to 1.50 . Values did not change significantly during the remaining seasons.

DJF $\sigma_{U \theta}$ values decreased at Howland and Palmyra by 5.0 to $10.0^{\circ}$ (fig. 146). The MAM $\sigma_{U \theta}$ value at Howland decreased by 10.0 to $15.0^{\circ}$ (fig. 147). The JJA $\sigma_{U \theta}$ values decreased by 5.0 to $15.0^{\circ}$ at Howland, Palmyra, and Kingman Reef (fig. 148). The $U_{\theta}$ of Palmyra and Kingman Reef rotated counterclockwise by 10 to $20^{\circ}$. SON $\sigma_{U \theta}$ values decreased by $>15.0^{\circ}$ at Jarvis and increased by 5.0 to $10.0^{\circ}$ at Kingman Reef (fig. 149). Kingman Reef's $U_{\theta}$ rotated counterclockwise by 10 to $20^{\circ}$.

End-Century: 2081-2100

\section{RCP4.5}

\section{Mean}

Mean $U_{a}$ values increased by 0.25 to $0.50 \mathrm{~m} / \mathrm{s}$ at the Palmyra and Kingman Reef points during the MAM season (fig. 119). The remaining seasons had not significant changes observed (fig. 118, figs. 120-121). $\sigma_{U a}^{2}$ values did not change significantly within the region.

The MAM $\sigma_{U \theta}$ values at Palmyra and Kingman Reef decreased by 5.0 to $10.0^{\circ}$ (fig. 151). Wind direction values did not change significantly during other seasons (figs. 150, 152-153).

\section{Top 5 percent}

Extreme $U_{a}$ values increased by 0.25 to $0.50 \mathrm{~m} / \mathrm{s}$ throughout the region during the JJA season (fig. 124). SON values decreased by 0.25 to $0.50 \mathrm{~m} / \mathrm{s}$ at Jarvis, but did not change significantly at the other points (fig. 125). $U_{a}$ values did not significantly change during the remaining seasons (figs. 122-123). Extreme $\sigma_{U a}^{2}$ values did not change significantly within the region.

The $U_{\theta}$ within the region rotated counterclockwise by 5 to $10^{\circ}$ during the DJF season (fig. 154). The MAM $\sigma_{U \theta}$ at Jarvis increased by 5.0 to $10.0^{\circ}$, and Howland's $U_{\theta}$ rotated counterclockwise by 5 to $10^{\circ}$ (fig. 155). JJA $U_{\theta}$ of Palmyra and Kingman Reef rotated 
counterclockwise by 10 to $20^{\circ}$ (fig. 156). SON $\sigma_{U \theta}$ values increased by 5.0 to $15.0^{\circ}$, and the $U_{\theta}$ of Palmyra rotated counterclockwise by 5 to $10^{\circ}$ (fig. 157).

\section{RCP8.5}

\section{Mean}

MAM $U_{a}$ means increased by 0.50 to $>0.75 \mathrm{~m} / \mathrm{s}$ throughout the region (fig. 127). Means did not significantly deviate from hindcast means during other seasons (fig. 126, figs. 128-129). $\sigma_{U a}^{2}$ values decreased by 0.75 to 1.50 at Palmyra and Kingman Reef during the MAM season.

The DJF $\sigma_{U \theta}$ values at Palmyra and Kingman Reef decreased by 5.0 to $10.0^{\circ}$ (fig. 158). The MAM $\sigma_{U \theta}$ at Jarvis increased by 5.0 to $10.0^{\circ}$, and the $U_{\theta}$ of Palmyra rotated counterclockwise by 5 to $10^{\circ}$ (fig. 159). JJA mean wave directions at Palmyra and Kingman Reef rotated counterclockwise by 5 to $20^{\circ}$ (fig. 160). SON wind direction values did not change significantly (fig. 161).

\section{Top 5 percent}

DJF extreme $U_{a}$ means decreased by 0.25 to $0.50 \mathrm{~m} / \mathrm{s}$ (fig. 130). SON values also decreased by 0.25 to $0.50 \mathrm{~m} / \mathrm{s}$, except the Howland point remain unchanged (fig. 133). JJA values intensified throughout the study area by 0.25 to $0.50 \mathrm{~m} / \mathrm{s}$ (fig. 132). MAM values did not change significantly (fig. 131). SON $\sigma_{U a}^{2}$ values decreased by 0.75 to 1.50 across the region.

DJF $\sigma_{U \theta}$ values decreased at Howland and Palmyra by 5.0 to $15.0^{\circ}$, and the $U_{\theta}$ of Jarvis rotated counterclockwise by 5 to $10^{\circ}$ (fig. 162). MAM $\sigma_{U \theta}$ values increased by 5.0 to $10.0^{\circ}$ at Palmyra and Jarvis (fig. 163). JJA mean wave directions at Palmyra and Kingman Reef rotated counterclockwise by 10 to $30^{\circ}$ and by 5 to $10^{\circ}$ counterclockwise at Howland (fig. 164). SON $\sigma_{U \theta}$ values increased by 5.0 to $10.0^{\circ}$ at Palmyra and Kingman Reef and decreased by 5.0 to $15.0^{\circ}$ at Howland and Jarvis (fig. 165). Palmyra's $U_{\theta}$ rotated counterclockwise by 10 to $20^{\circ}$.

Southern Region

Mid-Century: 2026-2045

\section{RCP4.5}

\section{Mean}


Mean $U_{a}$ values did not change significantly across all seasons within the region (figs. $102-105) . \sigma_{U a}^{2}$ values did not change significantly within the region.

$U_{\theta}$ and associated $\sigma_{U \theta}$ values did not change significantly (figs. 134-137).

\section{Top 5 percent}

DJF and JJA extreme mean $U_{a}$ values decreased by 0.25 to $0.50 \mathrm{~m} / \mathrm{s}$ (fig. 106, fig. 108). Values did not change significantly in the MAM and SON seasons (fig. 107, fig. 109). Extreme $\sigma_{U a}^{2}$ values did not change significantly within the region.

Mean wave directions of the DJF season rotated by 5 to $20^{\circ}$ counterclockwise, and $\sigma_{U \theta}$ decreased by 5.0 to $10.0^{\circ}$ (fig. 138). MAM $\sigma_{U \theta}$ values increased by 5.0 to $10.0^{\circ}$, and $U_{\theta}$ rotated clockwise by 5 to $10^{\circ}$ (fig. 139). Wind direction values did not change significantly during the JJA and SON seasons (figs. 140-141).

\section{RCP8.5}

Mean

Mean $U_{a}$ and associated $\sigma_{U a}^{2}$ values did not change significantly within the region (figs. 110-113).

The $U_{\theta}$ of Rose rotated clockwise by 5 to $10^{\circ}$ during the MAM season (fig. 143). $U_{\theta}$ and associated $\sigma_{U \theta}$ values during other seasons did not change significantly (fig. 142, figs. 144-145).

\section{Top 5 percent}

The means of the top 5 percent of $U_{a}$ values did not change significantly. The MAM $\sigma_{U a}^{2}$ of Rose increased by 0.75 to 1.50 (fig. 115). Values did not change significantly during the remaining seasons (fig. 114, figs. 116-117).

$\sigma_{U \theta}$ values decreased by 5.0 to $10.0^{\circ}$ during the DJF and MAM seasons, and $U_{\theta}$ rotated clockwise by 10 to 20 and 5 to $10^{\circ}$, respectively (figs. 146-147). Wind direction values did not change significantly during other seasons (figs. 148-149).

End-Century: 2081-2100

\section{RCP4.5}

\section{Mean}


Mean $U_{a}$, associated $\sigma_{U a}^{2}$ values, and wind direction values did not change significantly throughout the modeled region (figs. 118-121, figs. 150-153).

\section{Top 5 percent}

Extreme $U_{a}$ means did not change significantly throughout the region. DJF $\sigma_{U a}^{2}$ values decreased by $>0.75$ (fig. 122). MAM values increased by 0.75 to 1.50 , but did not change significantly during the remaining seasons (figs. 123-125).

The DJF $\sigma_{U \theta}$ values decreased by 5.0 to $10.0^{\circ}$, and $U_{\theta}$ rotated clockwise by 20 to $30^{\circ}$ (fig. 154). SON $\sigma_{U \theta}$ values increased by 5.0 to $10.0^{\circ}$ (fig. 157). Wind direction values did not change significantly during the MAM and JJA seasons (figs. 155-156).

\section{RCP8.5}

\section{Mean}

Mean $U_{a}$ and associated $\sigma_{U a}^{2}$ values did not change significantly within the region (figs. 126-129).

DJF $\sigma_{U \theta}$ values decreased by 5.0 to $15.0^{\circ}$, and $\mathrm{SON} \sigma_{U \theta}$ values decreased by 5.0 to $10.0^{\circ}$ (fig. 158, fig. 161). The $U_{\theta}$ of Rose rotated clockwise by 5 to $10^{\circ}$ during DJF. Wind direction values did not change significantly during the MAM and JJA seasons (figs. 159-160).

\section{Top 5 percent}

Extreme $U_{a}$ means did not change significantly within the region. MAM $\sigma_{U a}^{2}$ values increased by 0.75 to 1.50 within the region (fig. 131). $\sigma_{U a}^{2}$ values did not significantly change during the other seasons (fig. 130, figs. 132-133).

DJF $\sigma_{U \theta}$ values decreased by 5.0 to $10.0^{\circ}$, and $U_{\theta}$ values rotated clockwise by 20 to $30^{\circ}$ (fig. 162). The MAM $\sigma_{U \theta}$ of Rose decreased by 5.0 to $10.0^{\circ}$, and both islands' $U_{\theta}$ rotated 5 to $10^{\circ}$ counterclockwise (fig. 163). SON $\sigma_{U \theta}$ values decreased by 5.0 to $10.0^{\circ}$ (fig. 165). Wind direction values during the JJA season did not change significantly from hindcast values (fig. 164).

\section{Discussion}

This section will primarily focus on the trends found present in the top 5 percent of $H_{s}$ values, because trends within unfiltered values were small to insignificant. Extreme $H_{s}$ values will likely be more important within these regions for planning purposes and understanding the changing nature of wave hazards in the future. Thus, it is more practical and important that these extreme trends are well understood. 


\title{
Spatial and Temporal $H_{s}$ and $U_{a}$ Trends
}

\author{
Significant Wave Heights
}

Mid-Century: 2026-2045

RCP4.5 mean $H_{s}$ values decreased primarily west of latitude $180^{\circ}$ within the study area. These changes were concentrated in the Central region during the DJF, MAM, and SON seasons. The largest decrease occurred during the JJA season within the Marianas region; however, the other main observed change was a small decrease in $H_{s}$ values in the Hawaiian Islands.

As with the RCP4.5 scenario, the RCP8.5 mean $H_{s}$ values decreased primarily west of $180^{\circ}$. However, the largest areas of decrease during the SON season were within the Western and Marianas regions. JJA values increased within the Central region, and there were decreases in the eastern half of the study area. Additionally, during seasons when the Northern Hemisphere points decreased in either intensity or breadth, the Southern region's mean $H_{s}$ values increased.

These trends suggest that within both scenarios, wave heights are decreasing mainly in the western regions of the study area by 0.20 to $0.30 \mathrm{~m}$. The RCP4.5 scenario seems to have larger decreasing trends at the mid-century compared to the 8.5 scenario. These trends suggest that the extratropical cyclones that cause large swell waves within the Pacific seemed to decrease in frequency, decrease in strength, or change their storm tracks. The strongest decrease trends within the Northern Hemisphere occurred during the DJF and SON seasons, but during these periods the Southern region's $H_{s}$ values increased, particularly at American Samoa, where the $H_{s}$ increased by $>0.30 \mathrm{~m}$.

End-Century: 2081-2100

The $H_{s}$ trends throughout the end-century scenarios were similar to those of mid-century, but they were larger in aerial extent and intensity. Most of the changes occurred in the western half of the modeled area, with the majority happening in the Central and Marianas regions. The SON extreme $H_{s}$ means of the RCP4.5 scenario decreased widely across the study area and had the largest decreases of any season. As opposed to the mid-century JJA season, there was an increase in $H_{s}$ values within the Central region, with the largest increases at Enewetak.

The RCP8.5 trends were similar to the RCP4.5 trends but tended to be more widespread and stronger. The SON decreases were widespread, affecting every region except the Southern region. The mean $H_{s}$ values of the majority of the points within the Northern Hemisphere decreased by more than $0.30 \mathrm{~m}$, the strongest trend observed through all seasons and scenarios. JJA patterns exhibited a stronger increase in Central $H_{s}$ values of 0.10 to more than $0.30 \mathrm{~m}$, but the surrounding regions' values decreased by 0.10 to more than $0.30 \mathrm{~m}$. DJF and MAM $H_{s}$ values decreased primarily in the western half of the study area. Also, the Southern region islands' $H_{s}$ generally increased, with American Samoa's usually being larger, in all but the MAM season. The 
similarity between the two end-century scenarios was in contrast to the mid-century values where the trends were similar but the stronger decreases were present in the RCP4.5 scenario. Boreal fall values remained the largest changes observed throughout all seasons, with the DJF values being the next largest.

\section{Mean Wind Speeds}

Mid-Century: 2026-2045

RCP4.5 SON $U_{a}$ values decreased by 0.25 to more than $0.75 \mathrm{~m} / \mathrm{s}$ in the Eastern Equatorial region, the Marianas region, and the southern islands of the Central region. Conversely, southern Central region islands increased by 0.25 to $0.50 \mathrm{~m} / \mathrm{s}$, while the Marianas islands' $U_{a}$ values decreased, as did the Southern and Northeast regions' values.

As with the RCP4.5 scenario, the RCP8.5 SON $U_{a}$ values decreased at the points west of $180^{\circ}$ near the equator, though the decreases were smaller in intensity, 0.25 to $0.75 \mathrm{~m} / \mathrm{s}$, and aerial extent. JJA $U_{a}$ values increased by 0.25 to $0.50 \mathrm{~m} / \mathrm{s}$ within the Central region, similar to those of RCP4.5, but the affected points were fewer, and the $U_{a}$ decreases present within other regions were not as clearly defined. Changes in mid-century $U_{a}$ values were more intense and widespread during the RCP4.5 scenario.

End-century: 2081-2100

RCP4.5 extreme $U_{a}$ values decreased widely during the SON season west of $180^{\circ}$. The largest decrease was within the Marians region, $>0.75 \mathrm{~m} / \mathrm{s}$ in the southern two islands. Within the MAM season, $U_{a}$ values decreased in the southern Central islands and the Marianas by 0.25 to $0.50 \mathrm{~m} / \mathrm{s}$. This trend followed the general decrease in SON values of the mid-century.

RCP8.5 SON $U_{a}$ values decreased strongly throughout the study area, with decreases of more than $0.75 \mathrm{~m} / \mathrm{s}$ in the far western islands and southern Central region and the western islands within the Northeast region. Similarly, MAM $U_{a}$ values decreased throughout the study area, with the larger decreases of 0.50 to more than $0.75 \mathrm{~m}$ occurring north of $10^{\circ} \mathrm{N}$. JJA values increased within the Central and Eastern Equatorial regions, by 0.25 to $0.75 \mathrm{~m} / \mathrm{s}$, and the Northeast's $U_{a}$ values decreased, mimicking the end-century RCP8.5 JJA $H_{s}$ values. DJF $U_{a}$ values decreased throughout most regions, with the western Northeast points displaying the largest decreases.

Throughout all seasons, the end century $U_{a}$ values exhibited mainly the same trends as the mid-century scenarios, but the trends were intensified by the end of the century. The mid-century RCP4.5 $U_{a}$ changes were generally stronger and more widespread than the RCP8.5 changes. However, these trends reversed by the end of the century, where the RCP8.5 changes were generally stronger, more widespread, and well defined. 


\section{Spatial and Temporal $T_{p}, D_{p}$, and $U_{\theta}$ Trends}

\section{Peak Wave Periods}

Mid-Century: 2026-2045

RCP4.5 $T_{p}$ values increased in the eastern half of the study region by 0.25 to $0.75 \mathrm{~s}$ during the SON and DJF seasons. Values decreased by 0.25 to $0.50 \mathrm{~s}$ in the western islands that are close to the equator during the JJA season.

The RCP8.5 scenario $T_{p}$ values decreased in the westernmost islands by 0.25 to $0.50 \mathrm{~s}$ during SON and by 0.25 to $0.75 \mathrm{~s}$ in the Central equatorial and Eastern Equatorial islands during the MAM season. However, there was a 0.25 to $0.75 \mathrm{~s}$ increase in the eastern half of the study area during the DJF season.

End Century: 2081-2100

RCP4.5 SON $T_{p}$ values decreased by 0.25 to $0.50 \mathrm{~s}$ in the Northeast region. DJF and JJA values increased in the east half of the study region and the Central region.

The RCP8.5 SON season had widespread decreases across the Northern Hemisphere points. DJF values are split between the west and east half of the study area. Points west of $180^{\circ}$ displayed decreasing $T_{p}$ values, while the east had 0.25 to $0.50 \mathrm{~s}$ increases. MAM and JJA values increased in the Northeast region by 0.25 to $>0.75 \mathrm{~s}$. The end-century scenario represented an increase of some basic trends observed during the mid-century period.

As with the $H_{s}$ values, RCP8.5, while showing similar or weaker trends in the mid-century period, displayed a stronger change during each season than the 4.5 scenario. By the end of the century, the 4.5 scenario displayed little deviation from hindcast values. The end-century trends of 4.5 are generally weak, such that there were no significant trends discernable. The sole trend observed in both scenarios was an increase in JJA $T_{p}$ values in the Northeast region. The changes in mean $T_{p}$ were small; therefore, the changes observed and their relation to wave generation areas or storm changes were tenuous.

\section{Mean Wave Directions}

Mid-Century: 2026-2045

RCP4.5 $D_{p}$ changes were mainly relegated to regions west of $180^{\circ}$, with the majority of the changes occurring in the Central region. The $\sigma_{D p}$ values increased by 10.0 to more than $15.0^{\circ}$ within the west and were accompanied with the largest observed directional shifts for the time period and scenario. There were 5.0 to $15.0^{\circ} \sigma_{D p}$ increases within the Central and Marianas regions during the DJF and JJA, respectively. 
The majority of the RCP8.5 changes occurred during the SON season. $\sigma_{D p}$ values generally increased west of $180^{\circ}$ and had the most widespread directional shifts, occurring primarily in the Marianas region and the Central region, closer to the equator. There was an east and west trend present during the JJA, where the Northeast $\sigma_{D p}$ values increased by 0.50 to 15.0 degree and the equatorial islands west of $180^{\circ}$ decreased by 0.50 to $15.0^{\circ}$. The two scenarios differed primarily within the SON season. The RCP4.5 scenario showed a larger and more widespread increase of $\sigma_{D p}$ values and directional shifts within the west islands compared to the RCP8.5 scenario. Otherwise, the RCP8.5 showed more complex JJA patterns in $\sigma_{D p}$ values, reflecting changes in dominant JJA extreme wave generation.

End-Century: 2081-2100

The RCP4.5 SON $\sigma_{D p}$ values displayed a large increase west of $180^{\circ}$ of generally more than $15.0^{\circ}$. This increase was accompanied by large counterclockwise shifts in the islands near the equator within these west regions. The JJA season's $\sigma_{D p}$ values increased in the east half of the study area and within the eastern islands of the Central region.

The RCP8.5 JJA $\sigma_{D p}$ values followed a similar pattern of increasing within the Northern hemisphere islands east of $165^{\circ}$, but the values generally increased by a weaker 0.50 to $10.0^{\circ}$ compared to the stronger values observed in the RCP4.5 scenario. However, the Islands in the Central region displayed a clockwise $D_{p}$ shift, though smaller than the RCP4.5 scenario; Enewetak remained the largest $D_{p}$ shift and, similarly, its $\sigma_{D p}$ value decreased. MAM $\sigma_{D p}$ values generally decreased within the west half of the study area, similar to those of RCP4.5. The SON $\sigma_{D p}$ values in the western half of the study region increased, but the increases were weaker than in the RCP4.5 scenario.

The RCP4.5 end-century scenario displayed generally stronger SON and JJA trends in $D_{p}$ values. Both JJA values seemed to indicate a weakening of the trade wind's influence on extreme $D_{p}$ values within the central region. Enewetak appeared to go through a transition from being dominated by trade wind swell waves to Southern ocean swell waves, causing the reorientation of extreme wave directions towards the north. The increase of $\sigma_{D p}$ values indicated that the extreme wave heights during this season, probably more composed of trade wind swell during the hindcast period, have become subject to increased large swell from other regions. Similarly, the trade winds seem to become more dominant during the SON seasons, because in the Central region, directions rotated clockwise closer to the equator and within the Marianas region during RCP8.5, but they rotated counterclockwise during RCP4.5. 


\section{Mean Wind Directions}

Mid-Century: 2026-2045

RCP4.5 SON $\sigma_{U \theta}$ values increased west of $180^{\circ}$, except for Chuuk and Kosrae, which decreased by 10.0 to more than $15.0^{\circ}$. MAM $\sigma_{U \theta}$ values decreased in the west half of the study area, but the other seasons did not show a significant trend.

RCP8.5 values showed larger changes in $\sigma_{U \theta}$ and direction values during the SON season than RCP4.5. Points west of $180^{\circ}$ increased by 5.0 to more than $15.0^{\circ}$, and many points experienced significant clockwise rotations in $U_{\theta}$. DJF $\sigma_{U \theta}$ values decreased throughout the eastern half of the study region by 5.0 to $10.0^{\circ}$.

End-Century: 2081-2100

The end-century RCP4.5 SON trends were much more complex than those present in the mid-century. The largest changes were still within the Central region. The $\sigma_{U \theta}$ of the southern islands within the region decreased by more than $15.0^{\circ}$, and their mean direction values rotated clockwise from the northwest to the northeast. The $\sigma_{U \theta}$ of the eastern islands within the Central region increased by more than $15.0^{\circ}$.

RCP8.5 SON trends change dramatically from mid-century values. The southern Central region islands rotated counterclockwise. MAM $\sigma_{U \theta}$ values decreased across the study area, though directions did not change significantly. There were not many significant deviations from hindcast values during other seasons. RCP8.5 end-century SON wind directions did not match the RCP4.5 scenario or represent an intensification of the trends displayed within RCP4.5. Instead, RCP4.5 end-century wind direction changes more closely resembled the changes present within the midcentury RCP8.5 scenario. Therefore, wind directions within the RCP8.5 scenario may have represented the end member of changes, whereby changes were much larger until the mid-century, but over a longer period, these $U_{\theta}$ values returned to approximately hindcast directions.

\section{Implications}

The end-century RCP8.5 scenario's trends appear to be an intensification of the trends of the RCP4.5 scenario, except for $D_{p}$ and $U_{\theta}$ values. This pattern suggests that the intensification of the radiative forcing will result in similar, but stronger variable changes by $2100 . H_{s}$ changes over the next century are forecasted to be a spectrum of large decreases across the tropical Pacific, with the strength of the decrease scaling with the intensity of the radiative forcing. This trend was broadly observed by Hemer and others (2013), who projected $H_{s}$ values to decrease by 5 to 10 percent of hindcast values throughout the northern Pacific during the January-March and JulySeptember periods by the end of the 21st century. However, Hemer and others (2013) did not address the SON season, therefore not describing the large decreases in $H_{s}$ values forecasted in this study. The decrease in $H_{s}$ values may be linked to a decrease in extratropical cyclone frequency, strength, or storm tracks. Ulbrich and others (2008), using an ensemble of models, 
project storm tracks within the North Pacific to shift poleward, which would have a direct effect on the extreme swell waves produced. Changes in storm tracks would explain the large decrease of $H_{s}$ values projected throughout most periods, except JJA, when North Pacific storm activity is reduced. It is unlikely that these changes are due to tropical cyclone or El Niño Southern Oscillation activity, as neither phenomenon is captured well within the selected global climate models (GCMs).

Wave periods are related to frequency as 1 /frequency. Therefore, as wave periods increase, the frequency of the spectrum decreases. Lower frequency spectra indicate a greater distance between swell wave generation region and the affected area. The end-century RCP8.5 scenario displays a DJF difference between $T_{p}$ trends for the west and east halves of the study area. These changes in $T_{p}$ are similar to the trends observed by Hemer and others (2013) for January-March, where the eastern half of the study regions values increased and those in the west decreased. However, July-September values show a clear decrease, but within this study, JJA values do not suggest any significantly comparable trends. Eastern $T_{p}$ values increase while western values decrease, possibly indicating that the DJF waves will be generated closer to the western half of the study area and farther from the eastern half. This trend assumes that DJF extreme waves throughout the study area are generated by extratropical storms in the North Pacific.

The RCP4.5 end-century scenario display generally stronger SON and JJA trends in $D_{p}$ values but little to no change during the DJF season. The changes in wave directions that are present during JJA differ from the general directions presented by Hemer and others (2013) whereby the Central, Western, and Marianas regions' directions are predominantly oriented from south to north. This difference is likely due to the fact that these directions were the result of averaging the directions of the top 5 percent of wave heights. Both values seemed to indicate a weakening of the trade winds' influence on extreme $D_{p}$ values within the Central region. Merrifield (2011) described historical increases in Pacific trade-wind intensity as tracking signatures of global warming trends, and inferred that this trend should persist into the future unless other processes began to override these tendencies. Therefore, it would be unlikely, as radiative forcing increased within both scenarios, for the trade winds themselves to weaken. Instead, it appears that changes in the Central region's wave directions were subject to strong, extratropical forcing.

Enewetak is projected to go through a transition from being dominated by trade-wind waves to Southern Ocean swell. The increase of $\sigma_{U \theta}$ values indicates that the extreme east-towest-trending wave heights during the JJA season, possibly more composed of trade wind waves during the hindcast period, have become subject to increased large swell from other regions. During RCP8.5, it seemed that the trade-wind waves become more dominant, influencing Central islands, whereas during RCP4.5, Southern Hemisphere swell seems to dominate the Central equatorial islands. Under the assumption that the trend in trade wind intensity will continue to scale with increased radiative forcing, it would appear that a larger portion of extreme wave 
heights will be generated by trade winds during the end-century RCP8.5 scenario, resulting in the stronger east-to-west shift in wave directions opposed to mid-century directions.

Overall, increases in wind speeds during the JJA season within the Central region corresponded with increases in wave heights within the region. However, the cause of the decreasing strength of $U_{a}$ values throughout other seasons remains uncertain. Unlike other variables, end-century RCP8.5 directions more closely matched hindcast means. It is possible that over the short term or weaker radiative forcing, new processes may affect local wind conditions, exacerbated by the degree of climate change, but under extended forcing, previous trends will once again become dominate. However, this supposition will need to be studied further.

\section{Conclusions}

Extreme wave climates within the tropical Pacific Ocean were forecasted to change dramatically during the 2026-2045 and 2081-2100 periods. $H_{s}$ trends predicted by both RCP4.5 and RCP8.5 show marked decreases in $H_{s}$ magnitudes by the end of the 21 st century. The endcentury trends of the two scenarios were similar, with the magnitude and breadth of the $H_{s}$ values within RCP8.5 generally being larger. This difference suggests that over the next century, the response of wave climate parameters within the tropical Pacific may be a spectrum of change, with the magnitude of the change dependent on the magnitude of the radiative forcing. $T_{p}$ and $U_{a}$ values respond similarly. The forecasted region to experience the most change is the Central region, where $H_{s}$ values generally decrease dramatically and $D_{p}$ values rotated often more than $40^{\circ}$ within the southern portion of the region. Clearly these islands will be subject to new conditions that will need to be studied more and in greater depth. Additionally, past work has mainly focused on the boreal winter and summer seasons; however, it was found within this study that $\mathrm{SON} H_{s}$ values changed the most, suggesting that future studies would benefit from describing the MAM and SON seasons as well. 


\section{Acknowledgments}

This work was carried out under the USGS's Pacific Coral Reef Project as part of an effort in the United States and its trust territories to better understand the affect of geologic and oceanographic processes on coral reef systems and the USGS's Climate Change Impacts to the U.S. Pacific and Arctic Coasts Project to understand the impact of climate change on U.S. and U.S.-affiliated island shorelines. This project was funded by the USGS's Coastal and Marine Geology Program and the Pacific Islands Climate Change Cooperative (PICCC). Sean Vitousek (USGS) and Patrick Limber (USGS) contributed numerous excellent suggestions and a timely review of our work. Peter Stauffer (USGS) provided the editorial review and Susan Cochran (USGS) performed all of the formatting and layout for this report. Use of trademark names does not imply USGS endorsement of products.

\section{Additional Digital Information}

For an online PDF version of this report, please see:

http://pubs.usgs.gov/of/2015/1001/

For more information on the U.S. Geological Survey’s Pacific Coastal and Marine Science Center, please see:

http://walrus.wr.usgs.gov/

For more information on the U.S. Geological Survey’s Pacific Coral Reef Project, please see: http://coralreefs.wr.usgs.gov/

\section{Direct Contact Information}

Regarding this report

Curt D. Storlazzi (Project Chief): cstorlazzi@usgs.gov 


\section{References Cited}

Caires, S., and Sterl, A., 2005, 100-year return value estimates for ocean wind speed and significant wave height from the ERA-40 data: Journal of Climate, v. 18, p. 1032-1048.

Caires, S., Swail, V.R., and Wang, X.L., 2006, Projections and analysis of extreme wave climate: Journal of Climate, v. 19, p. 5581-5605.

Cayan, D.R., Bromirski, P.D., Hayhoe, K., Tyree, M., Dettinger, M.D., and Flick, R.E., 2008, Climate change projections of sea level extremes along the California Coast: Climatic Change, v. 87, no. S1, p. S57-S73.

Donat, M.G., Leckebusch, G.C., Wild, S., and Ulbrich, U., 2010, Benefits and limitations of regional multi-model ensembles for storm loss estimations: Climate Research, v. 44, p. 211225.

Fletcher, C.H., and Richmond, B.M., 2010, Climate change in the Federated States of Micronesia; Food and water security, climate risk management, and adaptive strategies: University of Hawaii Sea Grant Report TT-10-02, 32 p.

Hemer, M.A., Fan, Y., Mori, N., Semedo, A., and Wang X.L., 2013, Projected changes in wave climate from a multi-model ensemble: Nature Climate Change, v. 3, p. 471-476.

Hoeke, R.K., Storlazzi, C.D., and Ridd, P.V., 2011, Hydrodynamics of a bathymetrically complex fringing coral reef embayment-wave climate, in situ observations and wave prediction: Journal of Geophysical Research-Oceans, v. 116, C04018

Hoeke, R.K., McInnes, K.L., Kruger, J.C., McNaught, R.J., Hunter, J.R., and Smithers, S.G., 2013, Widespread inundation of Pacific islands triggered by distant-source wind-waves: Global and Planetary Change, v. 108, p. 128-138.

Hyndman, R.J., and Koehler, A.B., 2006, Another look at measures of forecast accuracy: Journal of Forecasting, v. 22, p. 679-688.

Merrifield, M.A., 2011, A shift in western tropical Pacific sea level trends during the 1990s: Journal of Climate, v. 24, p. 4126-4138.

Mori, N., Yasuda, T., Mase, H., Tom, T., and Oku, Y., 2010, Projection of extreme wave climate change under global warming: Hydrological Research Letters, v. 4, p. 15-19

National Data Buoy Center, 2013, Pacific Ocean historical buoy dataset: National Oceanic and Atmospheric Administration, accessed online at http://www.ndbc.noaa.gov/.

National Geophysical Data Center, 2013, Global self-consistent hierarchical high-resolution geography database version 2.2.2: National Geophysical Data Center online data, accessed online at http://www.ngdc.noaa.gov/mgg/shorelines/gshhs.html.

Naval Research Laboratory, 2013, Digital bathymetric database version 3.0: Naval Research Laboratory online data, accessed online at http://www7320.nrlssc.navy.mil/DBDB2_WWW/v3.0/NRLCOM_dbdb2.html.

Riahi, K., Krey, V., Rao, S., Chirkov, V., Fischer, G., Kolp, P., Kindermann, G., Nakicenovic, N., and Rafai, P., 2010, RCP-8.5; Exploring the consequence of high emission trajectories: Climatic Change, v. 109 no. 1-2, p. 33-57.

Semedo, A., Sušelj, K., Rutgersson, A., and Sterl, A., 2011, A global view on the wind sea and swell climate and variability from ERA-40: Journal of Climate, v. 24, p. 1461-1479.

Stockdon, H.F., Holman, R.A., Howd, P.A., and Sallenger, A.H., 2006, Empirical parameterization of setup, swash, and runup: Coastal Engineering, v. 53, p. 573-588. 
Storlazzi, C.D., and Reid, J.A., 2010, The influence of ENSO cycles on wave-driven sea-floor sediment mobility along central California continental margin: Continental Shelf Research, v. 30, p. 1582-1599.

Storlazzi, C.D., Brown, E., Field, M.E., Rogers, K., and Jokiel, P.L., 2005, A model for wave control on coral breakage and species distribution in the Hawaiian Islands: Coral Reefs, v. 24, p. 43-55.

Storlazzi, C.D., Elias, E., Field, M.E., and Presto, M.K., 2011, Numerical modeling of the impact of sea-level rise on fringing coral reef hydrodynamics and sediment transport: Coral Reefs, v. 30, p. 23-38.

Taylor, K.E., Stouffer, R.J., and Meehl, G.A., 2009, A summary of the CMIP5 experiment design: Program for Climate Model Diagnosis and Intercomparison Report, 33 p., accessed online at http://cmip-pcmdi.llnl.gov/cmip5/docs/Taylor_CMIP5_design.pdf.

Thomson, A.M., Calvin, K.V., Smith, S.J., Kyle, G.P., Volke, A., Patel, P., Delgado-Arias, S., Bond-Lamberty, B., Wise, M.A., Clarke, L.E., and Edmonds, J.A., 2011, RCP4.5; a pathway for stabilization of radiative forcing by 2100: Climatic Change, v. 109, no. 1-2, p. 77-94.

Tolman, H.L., 2009, User manual and system documentation of WAVEWATCH III ${ }^{\mathrm{TM}}$ version 3.14: National Oceanic and Atmospheric Administration, National Weather Service, National Centers for Environmental Prediction Technical Note 276, 194 p.

Ulbrich, U., Pinto, J.G., Kupfer, H., Leckebusch, G.C., Spangehl, T., and Reyers, M., 2008, Changing Northern Hemisphere storm tracks in an ensemble of IPCC climate change simulations: Journal of Climate, v. 21, p. 1669-1679.

van Vuuren, D.P., Edmonds, J.A., Kainuma, M., Riahi, K., Thomson, A.M., Hibbard, K., Hurtt, G.C., Kram, T., Krey, V., Lamarque, J-F., Masui, T., Meinshausen, M., Nakicenovic, N., Smith, S.J., and Rose, S., 2011, The representative concentration pathways—an overview: Climatic Change, v. 109, p. 5-31.

Vitousek, S., and Fletcher, C.H., 2008, Maximum annually recurring wave heights in Hawaii: Pacific Science, v. 62, no. 4, p. 541-555.

World Climate Research Programme, 2013, CMIP5—Coupled Model Intercomparison Project Phase 5-overview: World Climate Research Programme, accessed online at http://cmippcmdi.llnl.gov/cmip5/.

Wang, X.L., and Swail, V.R., 2006, Climate change signal and uncertainty in projections of ocean wave height: Climate Dynamics, v. 26, p. 109-126.

Wang, X.L., Swail, V.R., and Cox, A., 2009, Dynamical versus statistical downscaling methods for ocean wave heights: International Journal of Climatology, v. 30, no. 3, p. 317-332, DOI: 10.1002/joc. 1899 . 
Table 1. Table of names and locations of WAVEWATCH III model points within the mid-to-western tropical Pacific Ocean.

[Negative latitudes are degrees south; negative longitudes are degrees west]

\begin{tabular}{|c|c|c|}
\hline Modeled location name & Latitude ( $\left.{ }^{\circ} \mathrm{N}\right)$ & Longitude $\left({ }^{\circ} \mathrm{E}\right)$ \\
\hline American Samoa & -14.25 & -170.00 \\
\hline Kauai & 22.00 & -160.00 \\
\hline Big Island of Hawaii & 19.00 & -154.00 \\
\hline Midway & 28.20 & -177.40 \\
\hline Chuuk & 7.40 & 151.85 \\
\hline Saipan & 15.25 & 145.75 \\
\hline Asuncion & 19.70 & 145.40 \\
\hline Kosrae & 5.20 & 163.00 \\
\hline Palau & 7.30 & 134.50 \\
\hline Pohnpei & 6.75 & 158.00 \\
\hline Yap & 9.50 & 138.10 \\
\hline Majuro & 7.10 & 171.30 \\
\hline Enewetak & 11.50 & 162.20 \\
\hline Bikini & 11.60 & 165.40 \\
\hline Molokai & 21.25 & -157.00 \\
\hline Northwest Hawaiian Islands & 25.75 & -171.75 \\
\hline Guam & 13.50 & 144.75 \\
\hline Kwajalein & 8.75 & 167.75 \\
\hline Wake & 19.25 & 166.75 \\
\hline Johnston Atoll & 16.75 & -169.50 \\
\hline Kingman Reef & 6.50 & -162.50 \\
\hline Palmyra Atoll & 5.83 & -162.08 \\
\hline Rose Atoll & -14.50 & -168.25 \\
\hline Howland & 0.75 & -176.50 \\
\hline Jarvis & -0.50 & -160.00 \\
\hline
\end{tabular}


Table 2. List of Global Climate Models used, wave model used, and the model resolutions.

\begin{tabular}{|l|c|}
\hline Global Climate Model & Resolution (longitude $\mathrm{x}$ latitude) in Degrees \\
\hline BCC-CSM1.1 & $2.8 \times 2.8$ \\
INM-CM4 & $2.0 \times 1.5$ \\
MIROC5 & $1.4 \times 1.4$ \\
GFDL-ESM2M & $2.5 \times 1.5$ \\
\hline Global Wave Model & Resolution (longitude x latitude) in Degrees \\
\hline WAVEWATCHIII & $1.25 \times 1.00$ \\
\hline
\end{tabular}

Table 3. Table showing comparisons between hindcast significant wave height data, in meters, at the Molokai point and historical significant wave height data from selected NDBC stations throughout the Hawaiian Islands by months.

[DJF=December-February, MAM=March-May, JJA=June-August, and SON=September-November; NDBC, National Data Buoy Center; statistics shown are mean absolute error (MAE), standard deviation (Std), and normalized variance (nVar)]

\begin{tabular}{|l|l|c|c|c|c|c|c|c|c|c|c|c|}
\hline \multirow{2}{*}{$\begin{array}{c}\text { Time } \\
\text { Period }\end{array}$} & \multicolumn{3}{|c|}{ NDBC 51001 } & \multicolumn{3}{c|}{ NDBC 51002 } & \multicolumn{3}{c|}{ NDBC 51003 } & \multicolumn{3}{c|}{ NDBC 51004 } \\
\cline { 2 - 12 } & MAE & Std & nVar & MAE & Std & nVar & MAE & Std & nVar & MAE & Std & nVar \\
\hline DJF & 0.14 & 0.17 & 0.68 & 0.40 & 0.15 & 1.46 & 0.44 & 0.14 & 1.40 & 0.39 & 0.18 & 1.55 \\
MAM & 0.08 & 0.09 & 0.81 & 0.12 & 0.05 & 1.22 & 0.07 & 0.08 & 1.24 & 0.13 & 0.08 & 1.38 \\
JJA & 0.10 & 0.08 & 1.40 & 0.15 & 0.04 & 1.23 & 0.15 & 0.14 & 1.79 & 0.12 & 0.07 & 1.42 \\
SON & 0.19 & 0.17 & 0.85 & 0.23 & 0.17 & 1.71 & 0.33 & 0.16 & 1.65 & 0.19 & 0.16 & 1.75 \\
Yearly & 0.10 & 0.12 & 0.81 & 0.15 & 0.16 & 1.65 & 0.22 & 0.15 & 1.51 & 0.16 & 0.16 & 1.73 \\
\hline
\end{tabular}


Table 4. Table showing comparisons between hindcast significant wave height data, in meters, at the Big Island of Hawaii point and historical significant wave height data from selected NDBC stations throughout the Hawaiian Islands by months.

[DJF=December-February, MAM=March-May, JJA=June-August, and SON=September-November; NDBC,

National Data Buoy Center; statistics shown are mean absolute error (MAE), standard deviation (Std), and normalized variance (nVar)]

\begin{tabular}{|l|l|c|c|c|c|c|c|c|c|c|c|c|}
\hline \multirow{2}{*}{$\begin{array}{c}\text { Time } \\
\text { Period }\end{array}$} & \multicolumn{3}{|c|}{ NDBC 51001 } & \multicolumn{3}{c|}{ NDBC 51002 } & \multicolumn{3}{c|}{ NDBC 51003 } & \multicolumn{3}{c|}{ NDBC 51004 } \\
\cline { 2 - 12 } & MAE & Std & nVar & MAE & Std & nVar & MAE & Std & nVar & MAE & Std & nVar \\
\hline DJF & 0.34 & 0.34 & 0.50 & 0.09 & 0.04 & 1.07 & 0.11 & 0.03 & 0.97 & 0.10 & 0.07 & 1.18 \\
MAM & 0.11 & 0.13 & 0.65 & 0.08 & 0.03 & 0.98 & 0.09 & 0.03 & 1.03 & 0.07 & 0.04 & 1.14 \\
JJA & 0.28 & 0.10 & 1.52 & 0.05 & 0.06 & 1.34 & 0.32 & 0.16 & 1.95 & 0.09 & 0.07 & 1.53 \\
SON & 0.18 & 0.12 & 0.83 & 0.22 & 0.17 & 1.68 & 0.33 & 0.17 & 1.63 & 0.19 & 0.16 & 1.73 \\
Yearly & 0.17 & 0.20 & 0.59 & 0.07 & 0.06 & 1.21 & 0.21 & 0.04 & 1.09 & 0.09 & 0.08 & 1.31 \\
\hline
\end{tabular}

Table 5. Table showing comparisons between hindcast significant wave height data, in meters, at the Kauai point and historical significant wave height data from selected NDBC stations throughout the Hawaiian Islands by months.

[DJF=December-February, MAM=March-May, JJA=June-August, and SON=September-November; NDBC, National Data Buoy Center; statistics shown are mean absolute error (MAE), standard deviation (Std), and normalized variance (nVar)]

\begin{tabular}{|l|l|c|c|c|c|c|c|c|c|c|c|c|}
\hline \multirow{2}{*}{$\begin{array}{c}\text { Time } \\
\text { Period }\end{array}$} & \multicolumn{3}{|c|}{ NDBC 51001 } & \multicolumn{3}{c|}{ NDBC 51002 } & \multicolumn{3}{c|}{ NDBC 51003 } & \multicolumn{3}{c|}{ NDBC 51004 } \\
\cline { 2 - 12 } & MAE & Std & nVar & MAE & Std & nVar & MAE & Std & nVar & MAE & Std & nVar \\
\hline DJF & 0.22 & 0.05 & 0.92 & 0.63 & 0.30 & 1.95 & 0.67 & 0.29 & 1.88 & 0.61 & 0.32 & 2.05 \\
MAM & 0.05 & 0.03 & 1.00 & 0.12 & 0.11 & 1.52 & 0.18 & 0.17 & 1.52 & 0.16 & 0.17 & 1.72 \\
JJA & 0.18 & 0.13 & 1.72 & 0.10 & 0.07 & 1.53 & 0.22 & 0.19 & 2.17 & 0.11 & 0.07 & 1.71 \\
SON & 0.29 & 0.15 & 0.93 & 0.34 & 0.20 & 1.86 & 0.44 & 0.20 & 1.80 & 0.29 & 0.20 & 1.91 \\
Yearly & 0.18 & 0.07 & 1.01 & 0.26 & 0.28 & 2.05 & 0.36 & 0.25 & 1.88 & 0.26 & 0.27 & 2.13 \\
\hline
\end{tabular}


Table 6. Table showing comparisons between hindcast top 5 percent of significant wave height data, in meters, at the Molokai point and historical significant wave height data from selected NDBC stations throughout the Hawaiian Islands by months.

[DJF=December-February, MAM=March-May, JJA=June-August, and SON=September-November; NDBC,

National Data Buoy Center; statistics shown are mean absolute error (MAE), standard deviation (Std), and normalized variance (nVar)]

\begin{tabular}{|l|l|c|c|c|c|c|c|c|c|c|c|c|}
\hline \multirow{2}{*}{$\begin{array}{c}\text { Time } \\
\text { Period }\end{array}$} & \multicolumn{3}{|c|}{ NDBC 51001 } & \multicolumn{3}{c|}{ NDBC 51002 } & \multicolumn{3}{c|}{ NDBC 51003 } & \multicolumn{3}{c|}{ NDBC 51004 } \\
\cline { 2 - 12 } & MAE & Std & nVar & MAE & Std & nVar & MAE & Std & nVar & MAE & Std & nVar \\
\hline DJF & 0.67 & 0.24 & 0.51 & 0.76 & 0.18 & 2.02 & 0.74 & 0.16 & 1.52 & 0.76 & 0.09 & 1.28 \\
MAM & 0.37 & 0.06 & 0.82 & 0.12 & 0.03 & 1.02 & 0.28 & 0.09 & 0.85 & 0.33 & 0.07 & 0.44 \\
JJA & 0.35 & 0.27 & 3.71 & 0.06 & 0.05 & 1.29 & 0.51 & 0.13 & 1.52 & 0.08 & 0.09 & 0.81 \\
SON & 0.46 & 0.44 & 0.33 & 0.64 & 0.14 & 1.77 & 0.65 & 0.08 & 1.43 & 0.54 & 0.05 & 1.19 \\
Yearly & 0.49 & 0.03 & 1.06 & 0.49 & 0.02 & 1.06 & 0.46 & 0.04 & 1.07 & 0.44 & 0.02 & 1.03 \\
\hline
\end{tabular}

Table 7. Table showing comparisons between hindcast top 5 percent of significant wave height data, in meters, at the Big Island of Hawaii point and historical significant wave height data from selected NDBC stations throughout the Hawaiian Islands by months.

[DJF=December-February, MAM=March-May, JJA=June-August, and SON=September-November; NDBC, National Data Buoy Center; statistics shown are mean absolute error (MAE), standard deviation (Std), and normalized variance (nVar)]

\begin{tabular}{|l|l|l|l|l|l|l|l|l|l|l|l|l|l|}
\hline \multirow{2}{*}{$\begin{array}{c}\text { Time } \\
\text { Period }\end{array}$} & \multicolumn{3}{|c|}{ NDBC 51001 } & \multicolumn{3}{c|}{ NDBC 51002 } & \multicolumn{3}{c|}{ NDBC 51003 } & \multicolumn{3}{c|}{ NDBC 51004 } \\
\cline { 2 - 11 } & MAE & Std & nVar & MAE & Std & nVar & MAE & Std & nVar & MAE & Std & nVar \\
\hline DJF & 1.36 & 0.39 & 0.31 & 0.12 & 0.08 & 1.26 & 0.04 & 0.04 & 0.93 & 0.15 & 0.05 & 0.69 \\
MAM & 0.52 & 0.12 & 0.61 & 0.03 & 0.02 & 0.96 & 0.17 & 0.05 & 0.68 & 0.20 & 0.05 & 0.37 \\
JJA & 0.56 & 0.22 & 3.51 & 0.22 & 0.10 & 1.36 & 0.72 & 0.10 & 1.31 & 0.30 & 0.08 & 0.71 \\
SON & 0.41 & 0.35 & 0.41 & 0.71 & 0.20 & 2.10 & 0.74 & 0.16 & 1.79 & 0.64 & 0.09 & 1.37 \\
Yearly & 0.42 & 0.03 & 1.07 & 0.44 & 0.05 & 1.14 & 0.39 & 0.03 & 1.06 & 0.38 & 0.04 & 1.12 \\
\hline
\end{tabular}


Table 8. Table showing comparisons between hindcast top 5 percent of significant wave height data, in meters, at the Kauai point and historical significant wave height data from selected NDBC stations throughout the Hawaiian Islands by months.

[DJF=December-February, MAM=March-May, JJA=June-August, and SON=September-November; NDBC, National Data Buoy Center; statistics shown are mean absolute error (MAE), standard deviation (Std), and normalized variance (nVar)]

\begin{tabular}{|l|l|c|c|c|c|c|c|c|c|c|c|c|}
\hline \multirow{2}{*}{$\begin{array}{c}\text { Time } \\
\text { Period }\end{array}$} & \multicolumn{3}{|c|}{ NDBC 51001 } & \multicolumn{3}{c|}{ NDBC 51002 } & \multicolumn{3}{c|}{ NDBC 51003 } & \multicolumn{3}{c|}{ NDBC 51004 } \\
\cline { 2 - 12 } & MAE & Std & nVar & MAE & Std & nVar & MAE & Std & nVar & MAE & Std & nVar \\
\hline DJF & 0.08 & 0.07 & 0.78 & 1.39 & 0.33 & 3.10 & 1.39 & 0.28 & 2.29 & 1.39 & 0.22 & 1.90 \\
MAM & 0.05 & 0.02 & 1.07 & 0.44 & 0.08 & 1.38 & 0.58 & 0.14 & 1.10 & 0.66 & 0.12 & 0.54 \\
JJA & 0.53 & 0.28 & 4.14 & 0.23 & 0.08 & 1.39 & 0.67 & 0.15 & 1.74 & 0.23 & 0.03 & 0.84 \\
SON & 0.33 & 0.36 & 0.32 & 0.84 & 0.19 & 1.76 & 0.83 & 0.17 & 1.39 & 0.72 & 0.07 & 1.07 \\
Yearly & 0.59 & 0.02 & 1.05 & 0.59 & 0.03 & 1.05 & 0.54 & 0.02 & 1.05 & 0.52 & 0.02 & 1.02 \\
\hline
\end{tabular}




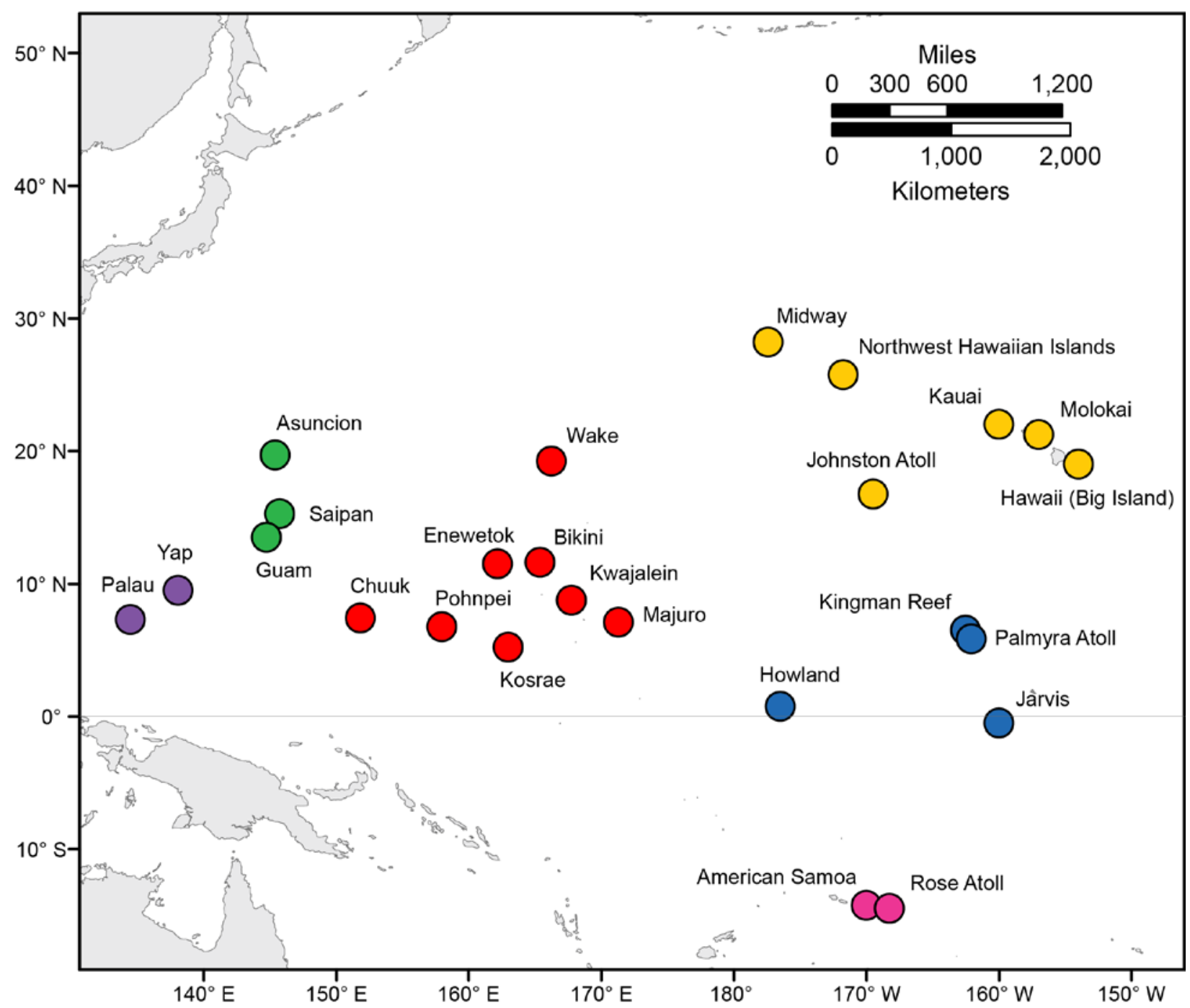

\section{Regional Divisons}

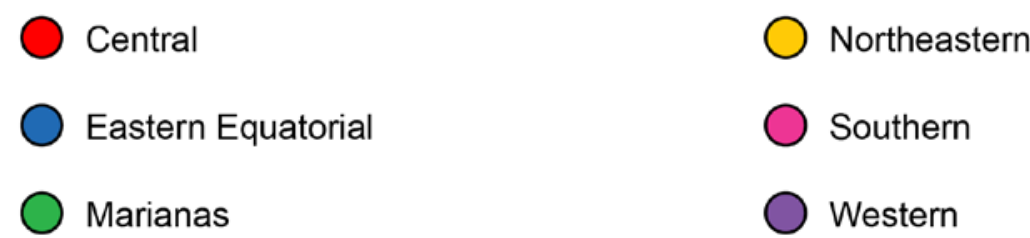

Figure 1. Map showing the locations of the 25 modeled points within the tropical Pacific Ocean used in this study. 


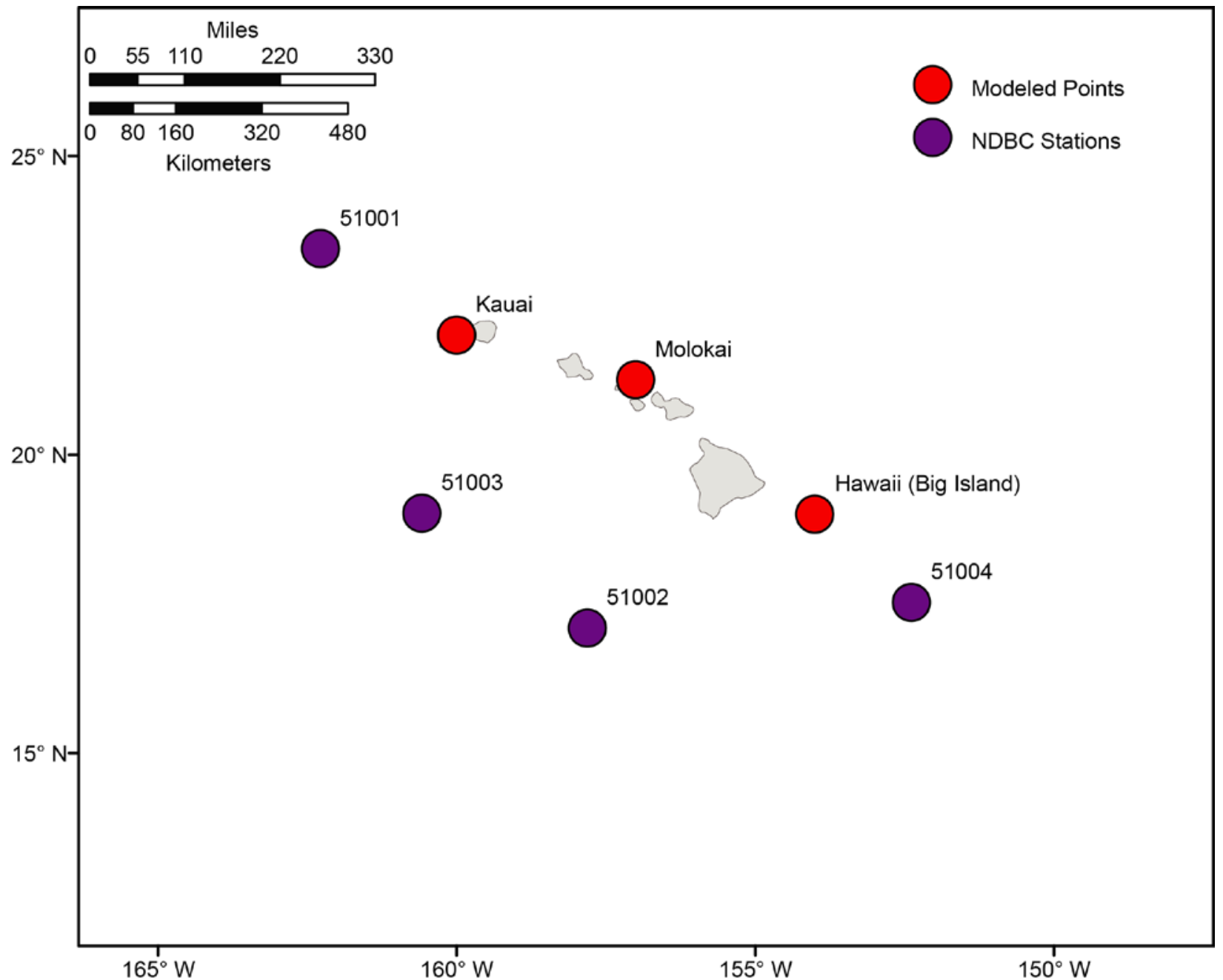

Figure 2. Map showing the locations of selected National Data Buoy Center stations, in purple, and modeled points, in red, within in the Hawaiian Island Chain. 

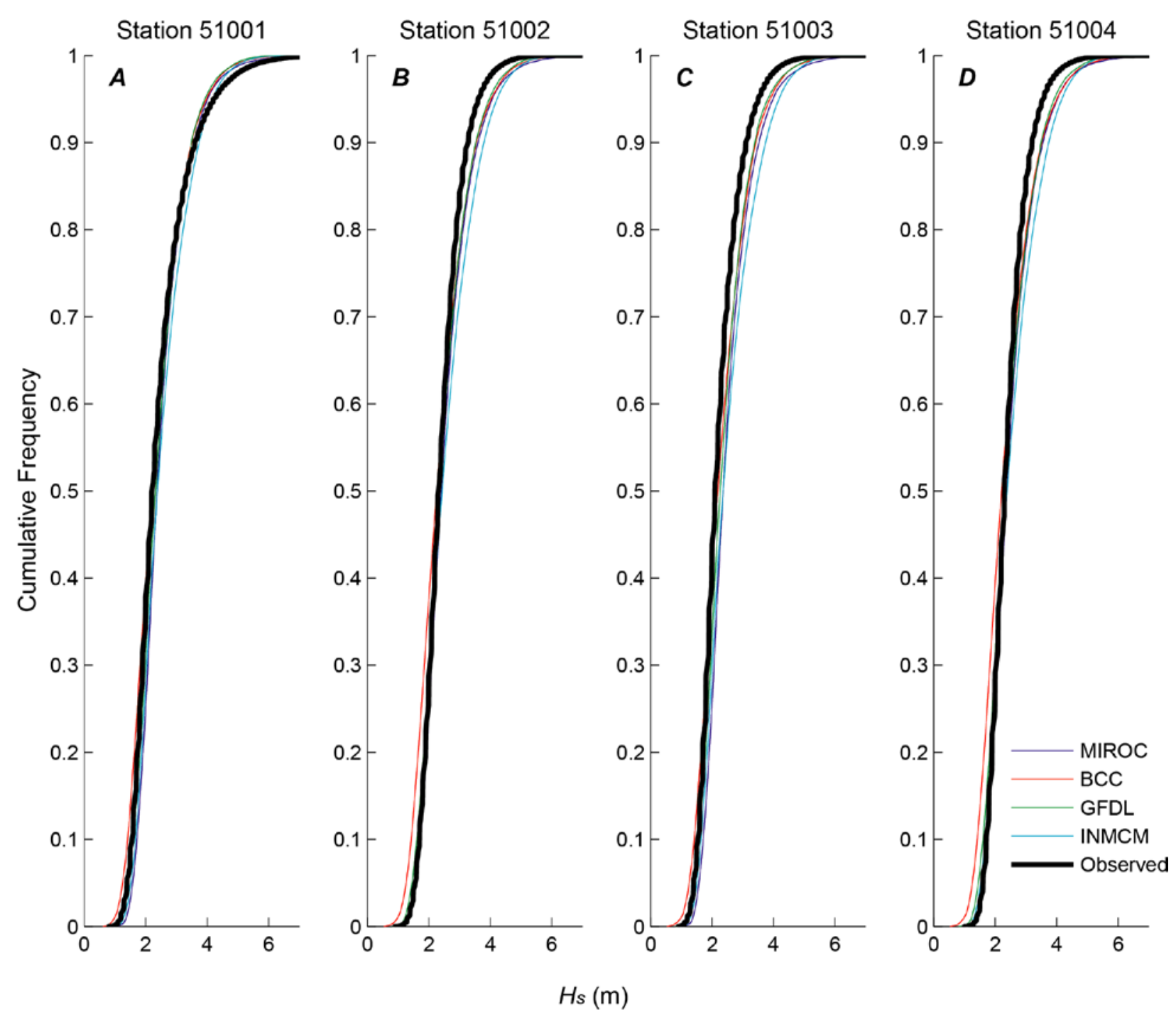

Figure 3. Plots showing comparisons of the empirical cumulative density functions of hindcasted (19862005) Molokai point significant wave heights $\left(H_{s}\right)$ in meters, from each GCM-driven WW3 model run with the observed historical cumulative density function at each NDBC station. A. Comparison of station 51001 significant wave height cumulative density function with hindcasted Molokai WW3 generated cumulative density functions. B. Comparison of station 51002 significant wave height cumulative density function with hindcasted Molokai WW3 generated cumulative density functions. C. Comparison of station 51003 significant wave height cumulative density function with hindcasted Molokai WW3 generated cumulative density functions. D. Comparison of station 51004 significant wave height cumulative density function with hindcasted Molokai WW3 generated cumulative density functions. 

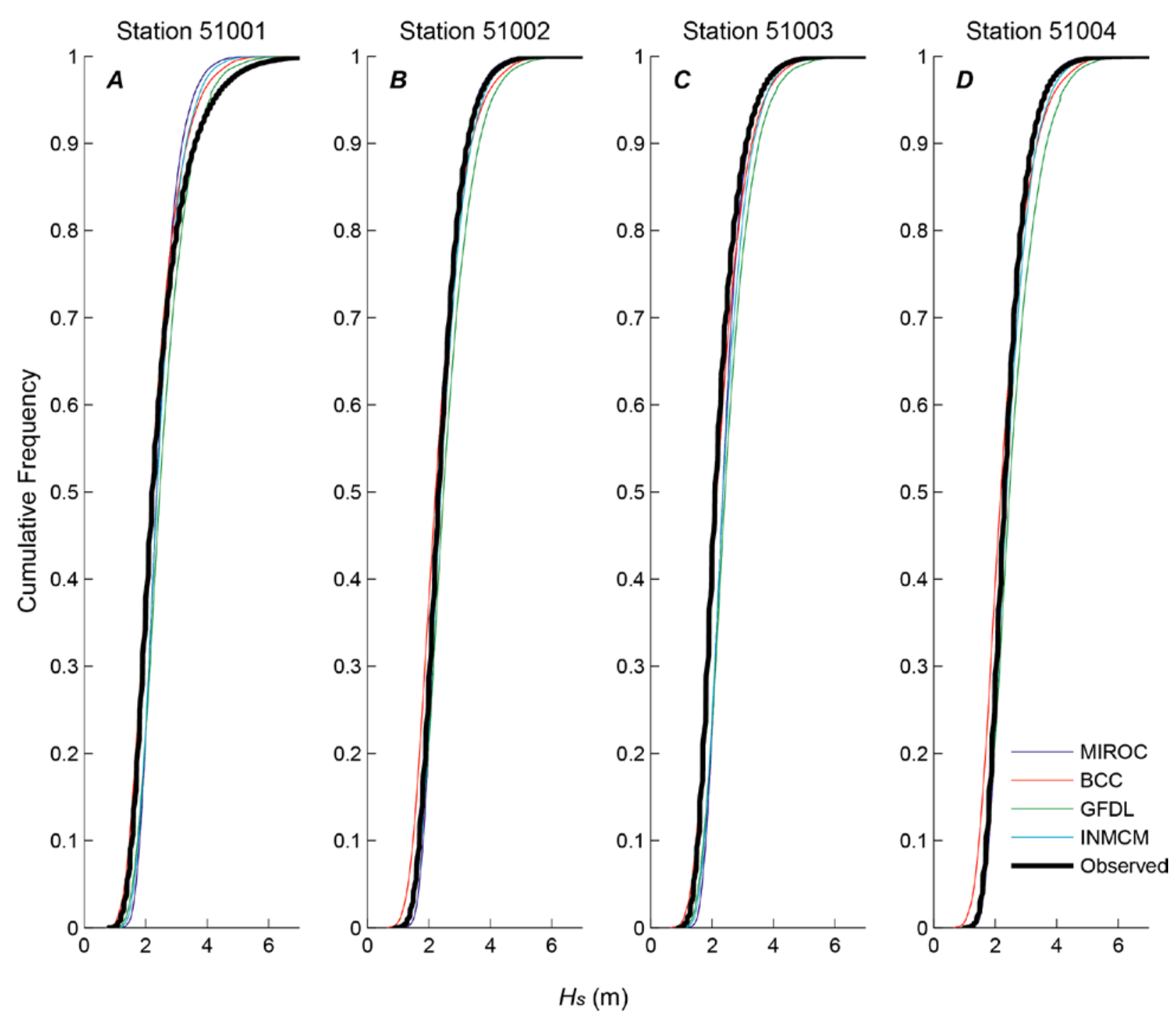

Figure 4. Plots showing comparisons of the empirical cumulative density functions of hindcasted (19862005) Big Island of Hawaii point significant wave heights $\left(H_{s}\right)$ in meters from each GCM-driven WW3 model run with the observed historical cumulative density function at each NDBC station. A. Comparison of station 51001 significant wave height cumulative density function with hindcasted Big Island of Hawaii WW3 generated cumulative density functions. $B$. Comparison of station 51002 significant wave height cumulative density function with hindcasted Big Island of Hawaii WW3 generated cumulative density functions. C. Comparison of station 51003 significant wave height cumulative density function with hindcasted Big Island of Hawaii WW3 generated cumulative density functions. D. Comparison of station 51004 significant wave height cumulative density function with hindcasted Big Island of Hawaii WW3 generated cumulative density functions. 

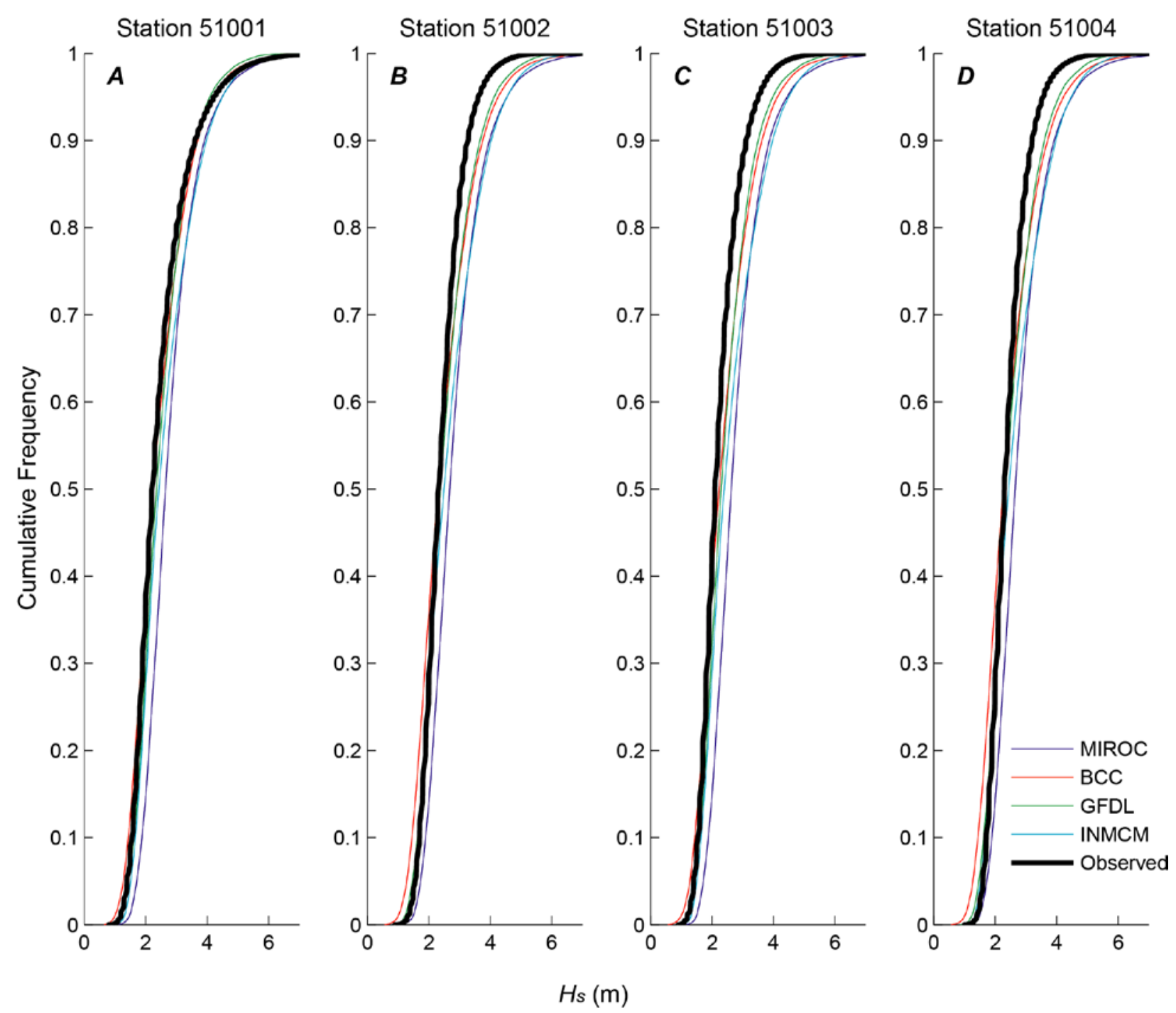

Figure 5. Plots showing comparisons of the empirical cumulative density functions of hindcasted (19862005) Kauai point significant wave heights $\left(H_{s}\right)$ in meters from each GCM-driven WW3 model run with the observed historical cumulative density function at each NDBC station. $A$. Comparison of station 51001 significant wave height cumulative density function with hindcasted Kauai WW3 generated cumulative density functions. B. Comparison of station 51002 significant wave height cumulative density function with hindcasted Kauai WW3 generated cumulative density functions. C. Comparison of station 51003 significant wave height cumulative density function with hindcasted Kauai WW3 generated cumulative density functions. D. Comparison of station 51004 significant wave height cumulative density function with hindcasted Kauai WW3 generated cumulative density functions. 


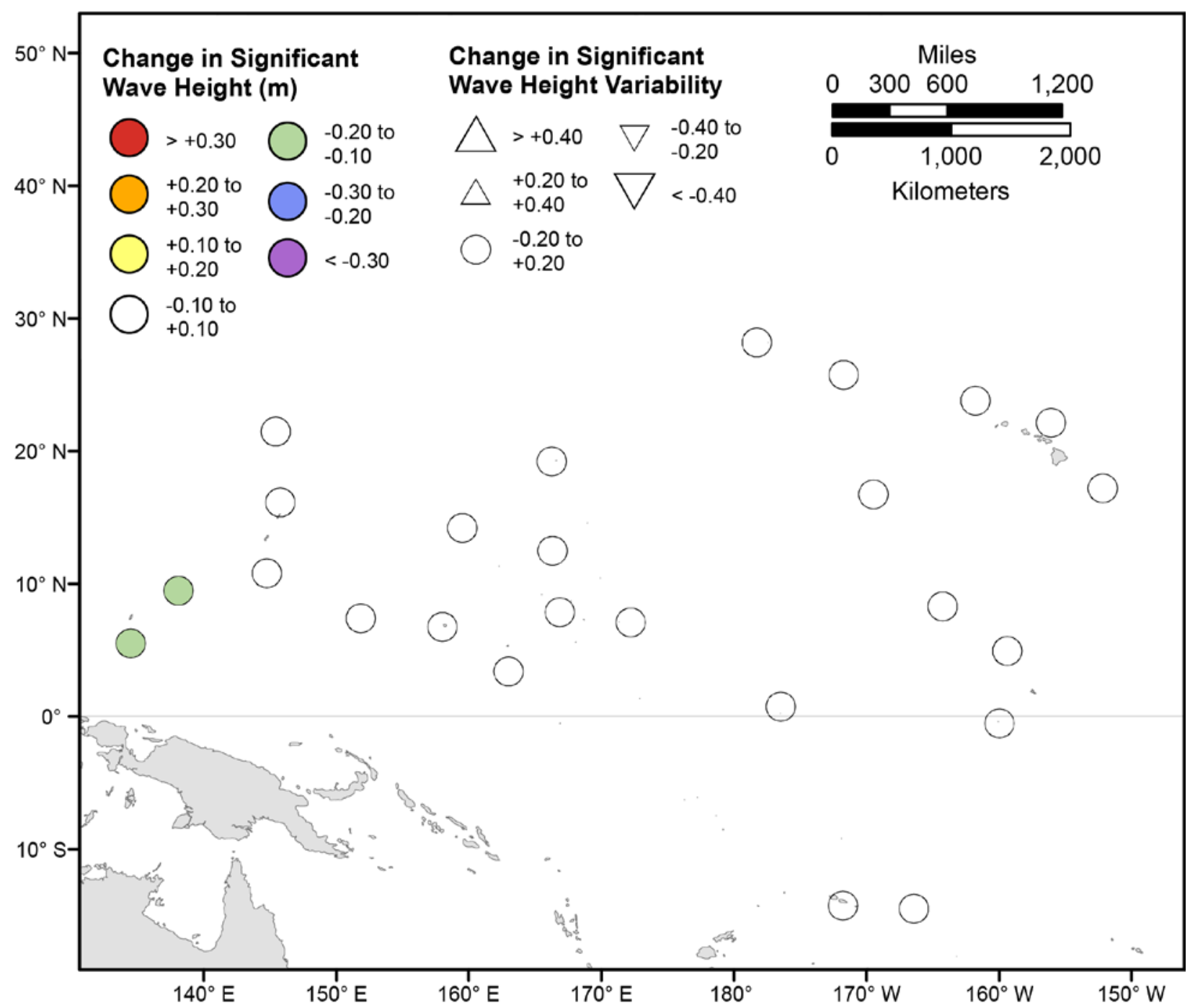

Figure 6. Map showing forecasted differences in mean significant wave height and variance in significant wave height for the years 2026-2045 from hindcasted values during the December-February season under the RCP4.5 future climatic scenario. The colors correspond to the magnitude of change in modeled mean significant wave heights during 2026-2045 from those hindcasted for 1976-2005. The shapes correspond to the magnitude of change in modeled variance in significant wave height during 2026-2045 from those hindcasted for 1976-2005. Units are in meters. 


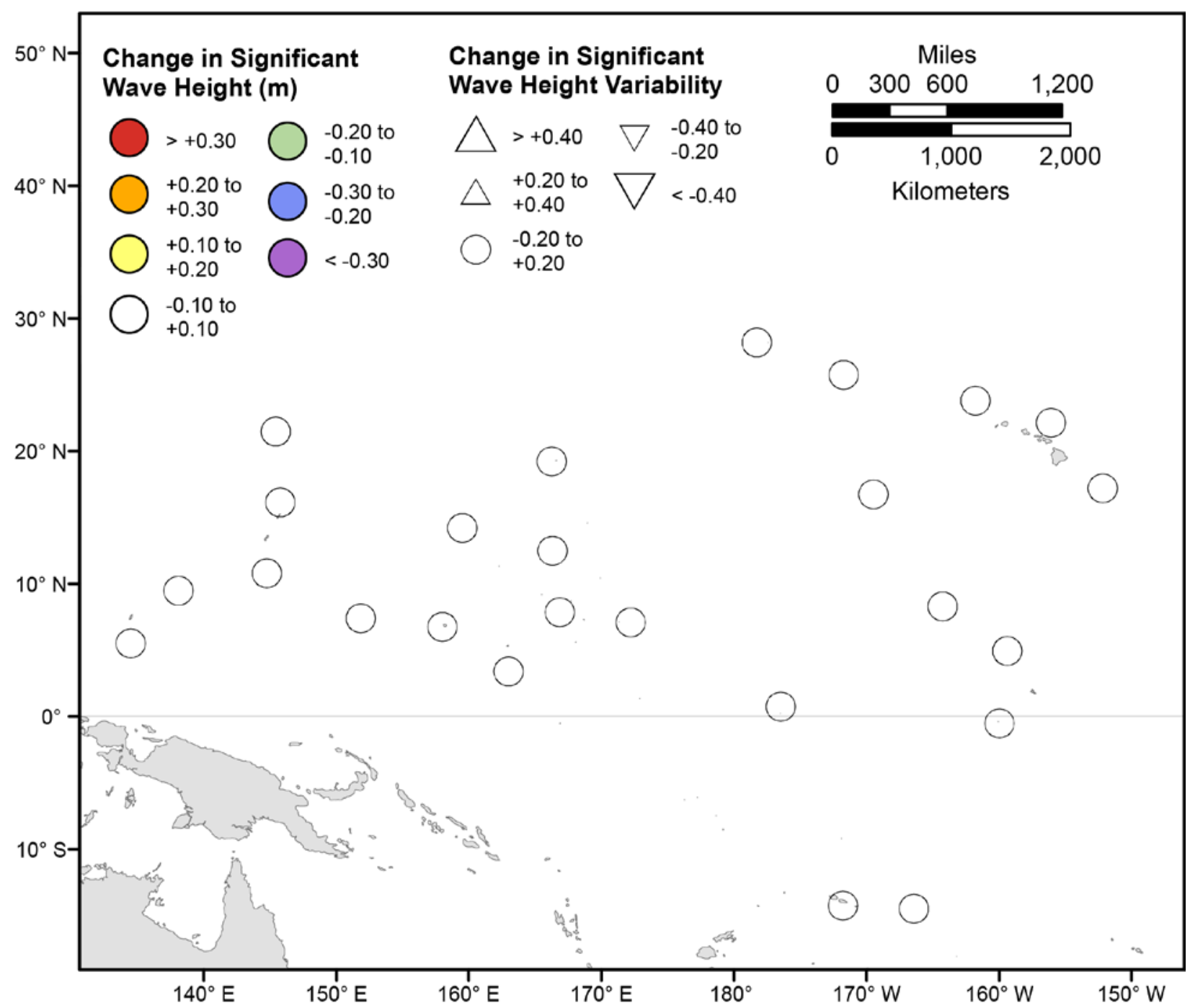

Figure 7. Map showing forecasted differences in mean significant wave height and variance in significant wave height for the years 2026-2045 from hindcasted values during the March-May season under the RCP4.5 future climatic scenario. The colors correspond to the magnitude of change in modeled mean significant wave heights during 2026-2045 from those hindcasted for 1976-2005. The shapes correspond to the magnitude of change in modeled variance in significant wave height during 20262045 from those hindcasted for 1976-2005. Units are in meters. 


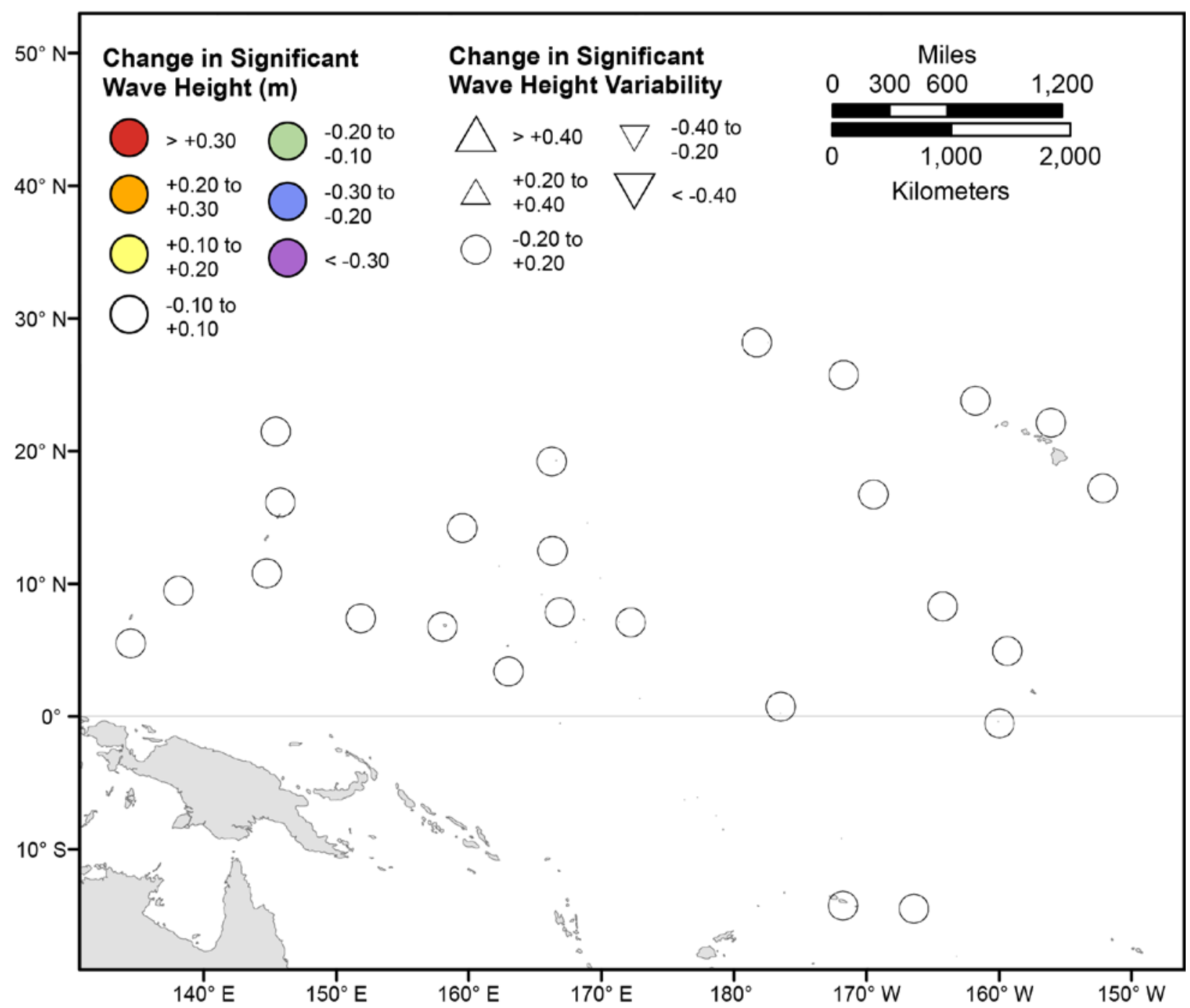

Figure 8. Map showing forecasted differences in mean significant wave height and variance in significant wave height for the years 2026-2045 from hindcasted values during the June-August season under the RCP4.5 future climatic scenario. The colors correspond to the magnitude of change in modeled mean significant wave heights during 2026-2045 from those hindcasted for 1976-2005. The shapes correspond to the magnitude of change in modeled variance in significant wave height during 20262045 from those hindcasted for 1976-2005. Units are in meters. 


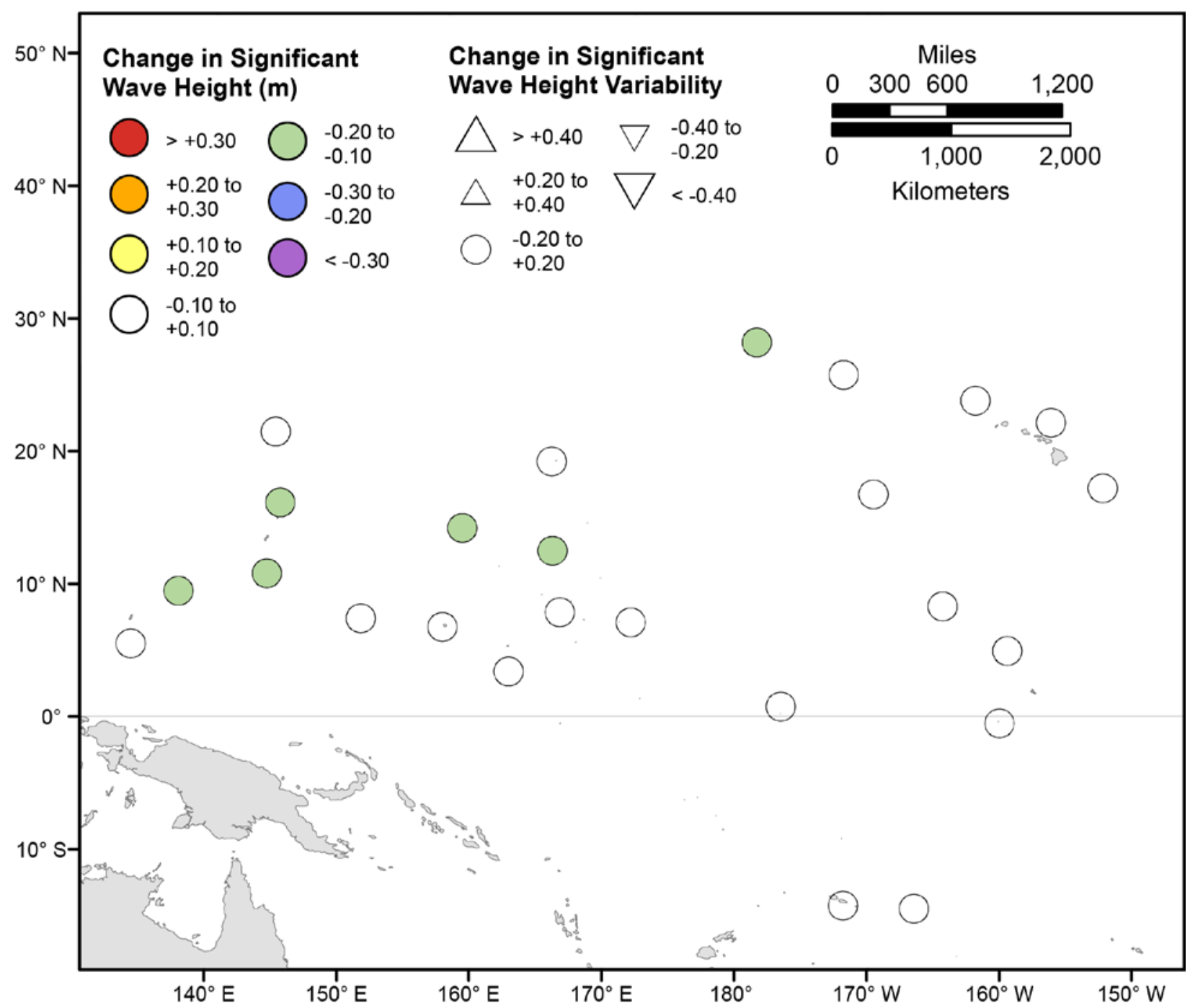

Figure 9. Map showing forecasted differences in mean significant wave height and variance in significant wave height for the years 2026-2045 from hindcasted values during the September-November season under the RCP4.5 future climatic scenario. The colors correspond to the magnitude of change in modeled mean significant wave heights during 2026-2045 from those hindcasted for 1976-2005. The shapes correspond to the magnitude of change in modeled variance in significant wave height during 2026-2045 from those hindcasted for 1976-2005. Units are in meters. 


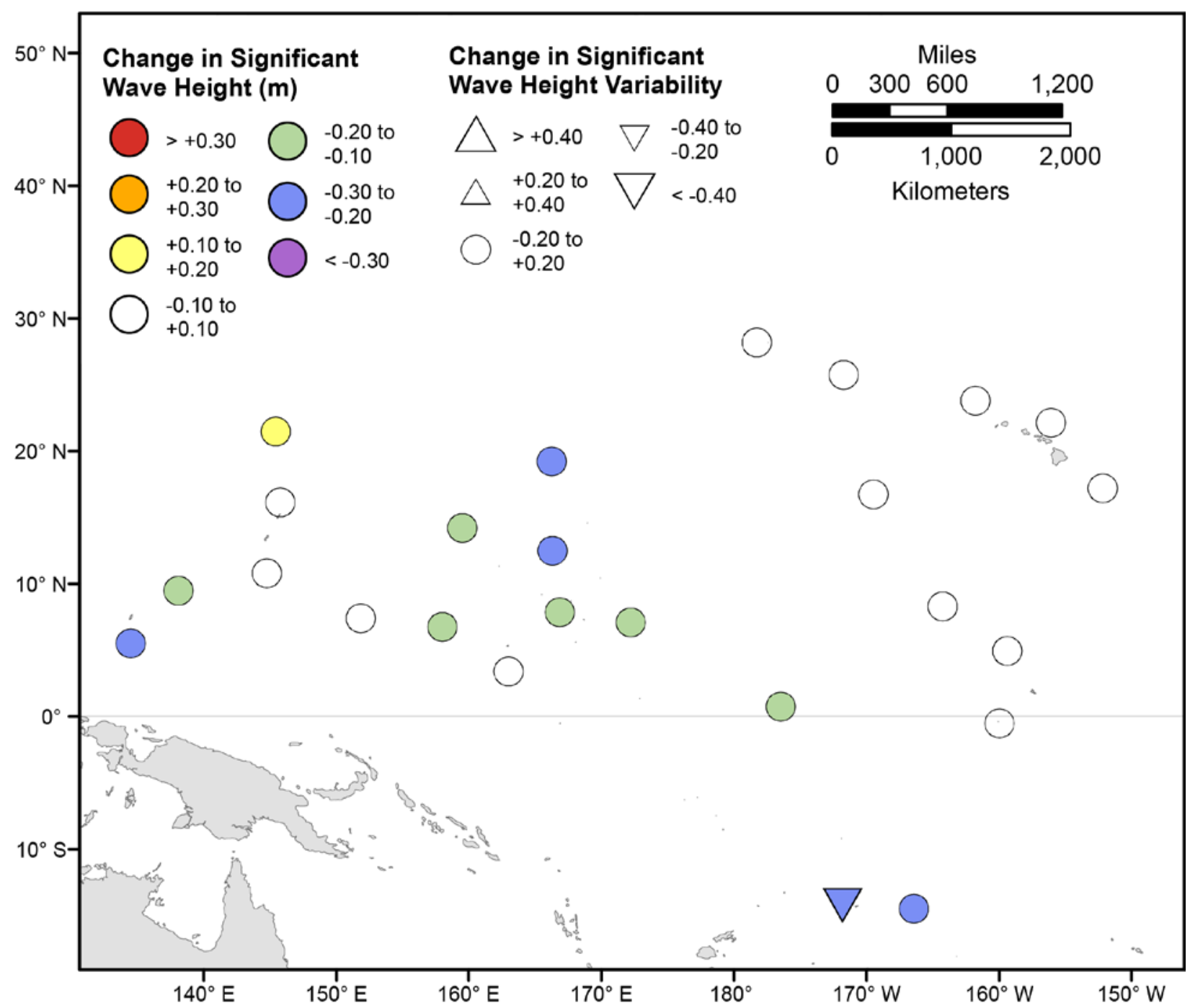

Figure 10. Map showing forecasted differences in the mean of the top 5 percent of significant wave heights and variance in the top 5 percent of significant wave heights for the years 2026-2045 from hindcasted values during the December-February season under the RCP4.5 future climatic scenario. The colors correspond to the magnitude of change in modeled mean significant wave heights during 2026-2045 from those hindcasted for 1976-2005. The shapes correspond to the magnitude of change in modeled variance in significant wave height during 2026-2045 from those hindcasted for 1976-2005. Units are in meters. 


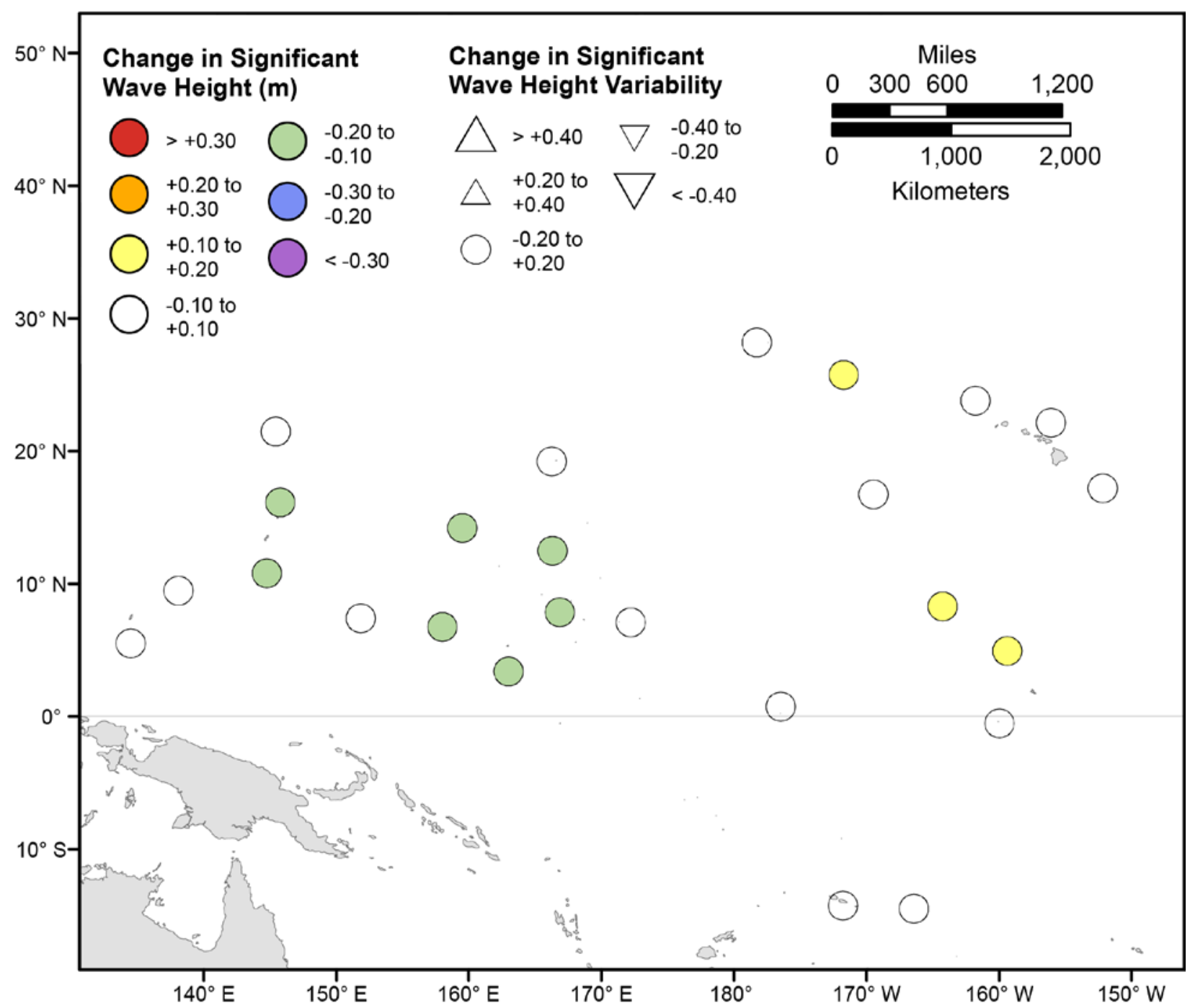

Figure 11. Map showing forecasted differences in the mean of the top 5 percent of significant wave heights and variance in the top 5 percent of significant wave heights for the years 2026-2045 from hindcasted values during the March-May season under the RCP4.5 future climatic scenario. The colors correspond to the magnitude of change in modeled mean significant wave heights during 2026-2045 from those hindcasted for 1976-2005. The shapes correspond to the magnitude of change in modeled variance in significant wave height during 2026-2045 from those hindcasted for 1976-2005. Units are in meters. 


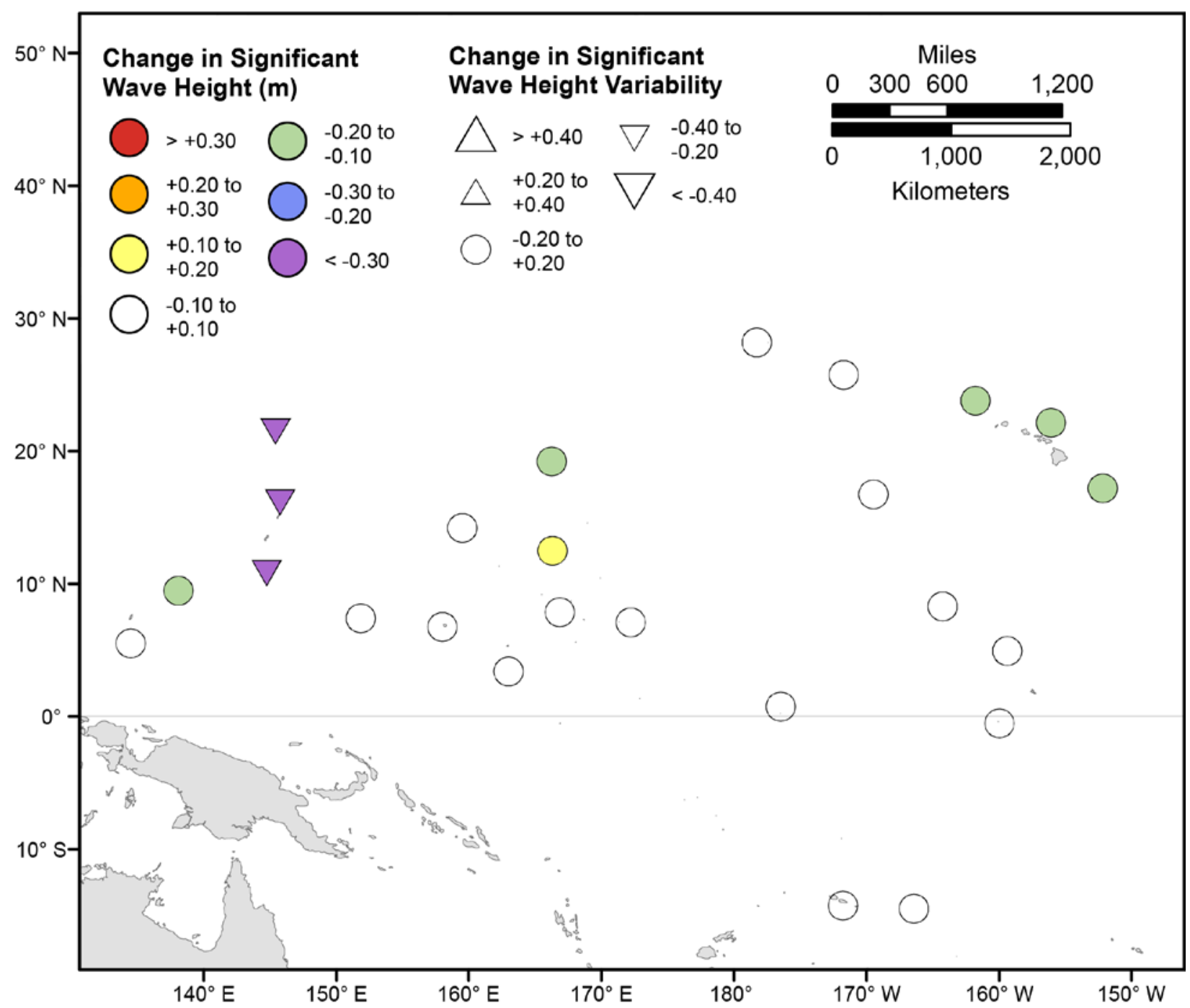

Figure 12. Map showing forecasted differences in the mean of the top 5 percent of significant wave heights and variance in the top 5 percent of significant wave heights for the years 2026-2045 from hindcasted values during the June-August season under the RCP4.5 future climatic scenario. The colors correspond to the magnitude of change in modeled mean significant wave heights during 2026-2045 from those hindcasted for 1976-2005. The shapes correspond to the magnitude of change in modeled variance in significant wave height during 2026-2045 from those hindcasted for 1976-2005. Units are in meters. 


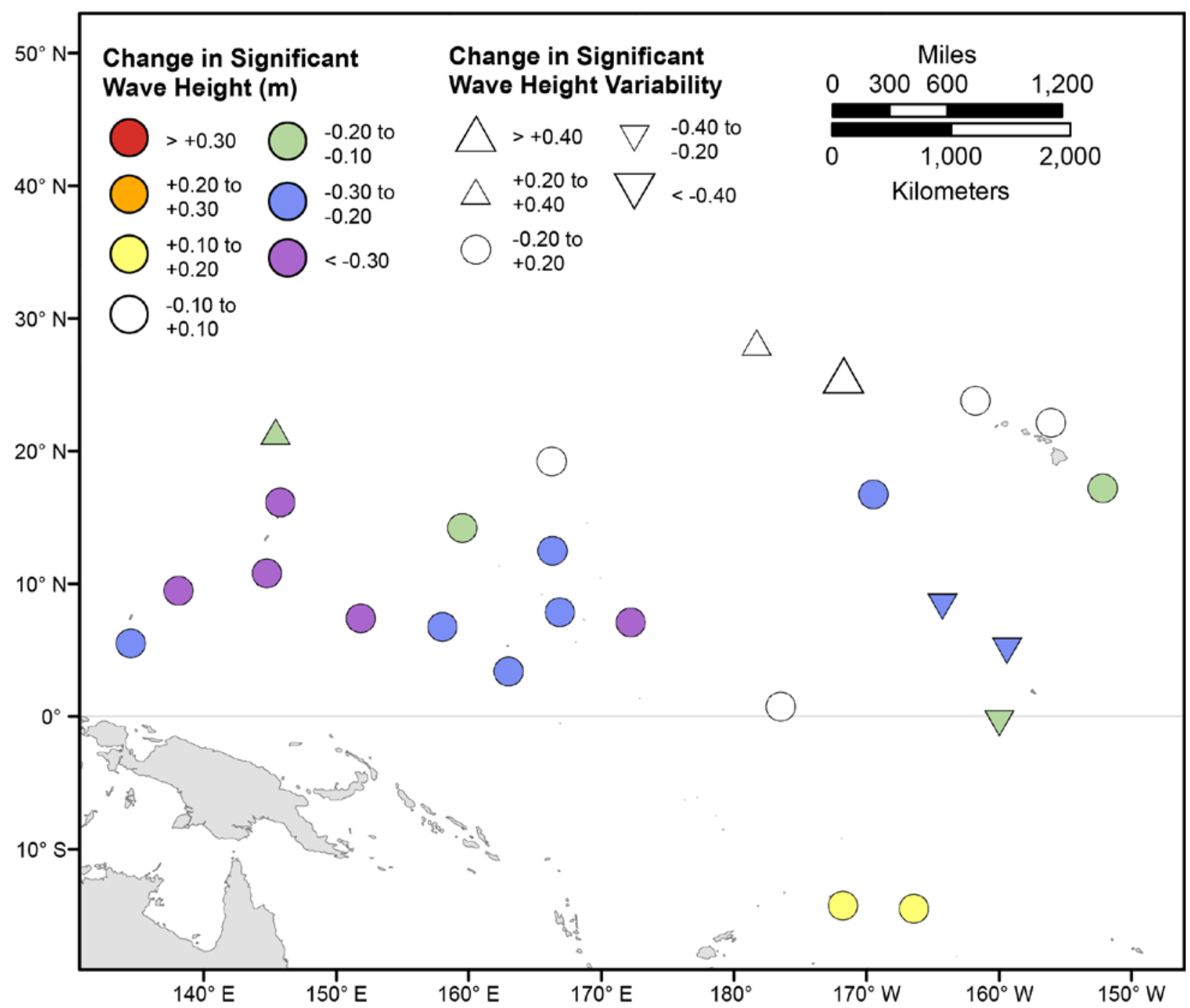

Figure 13. Map showing forecasted differences in the mean of the top 5 percent of significant wave heights and variance in the top 5 percent of significant wave heights for the years 2026-2045 from hindcasted values during the September-November season under the RCP4.5 future climatic scenario. The colors correspond to the magnitude of change in modeled mean significant wave heights during 2026-2045 from those hindcasted for 1976-2005. The shapes correspond to the magnitude of change in modeled variance in significant wave height during 2026-2045 from those hindcasted for 1976-2005. Units are in meters. 


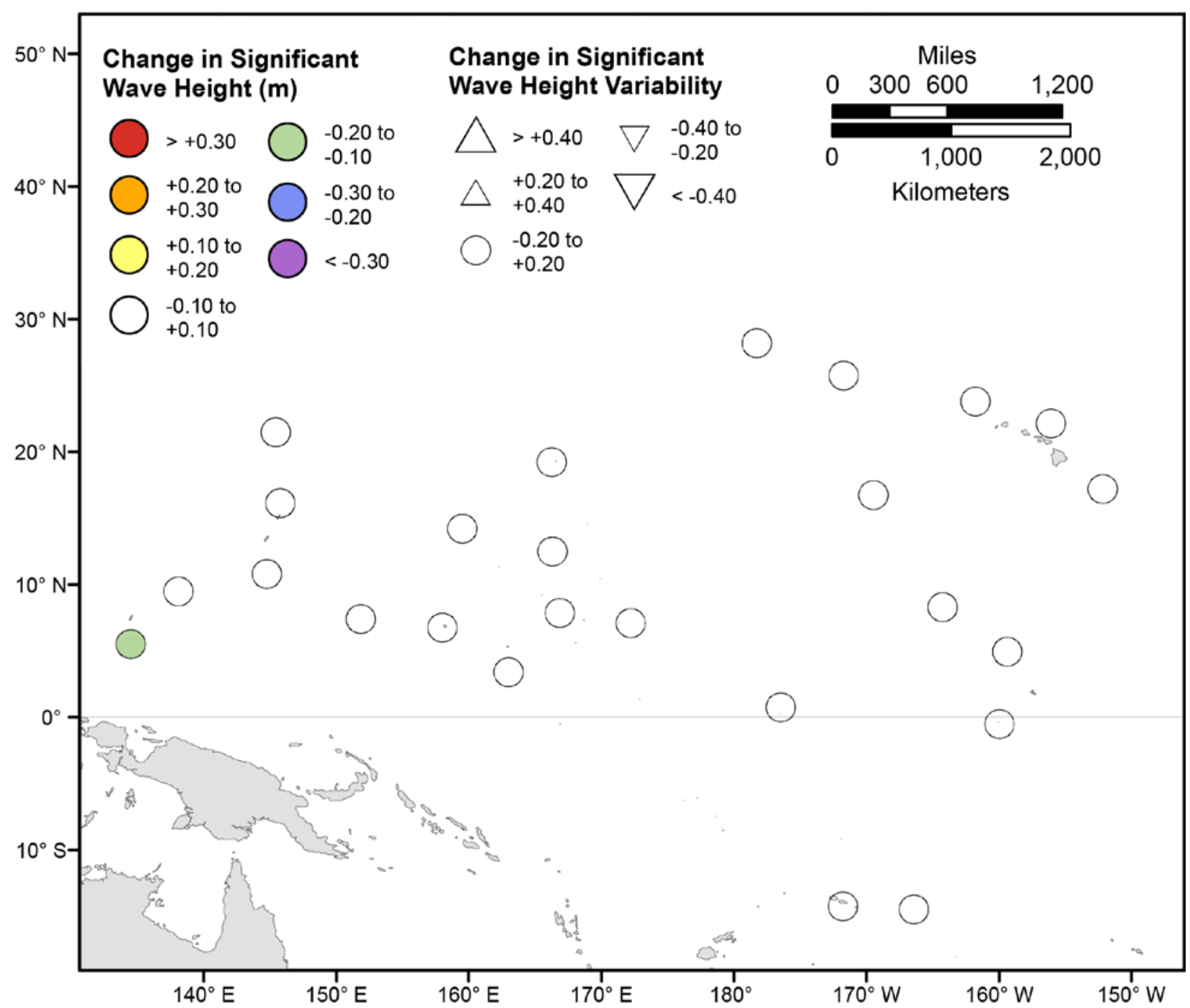

Figure 14. Map showing forecasted differences in mean significant wave height and variance in significant wave height for the years 2026-2045 from hindcasted values during the December-February season under the RCP8.5 future climatic scenario. The colors correspond to the magnitude of change in modeled mean significant wave heights during 2026-2045 from those hindcasted for 1976-2005. The shapes correspond to the magnitude of change in modeled variance in significant wave height during 2026-2045 from those hindcasted for 1976-2005. Units are in meters. 


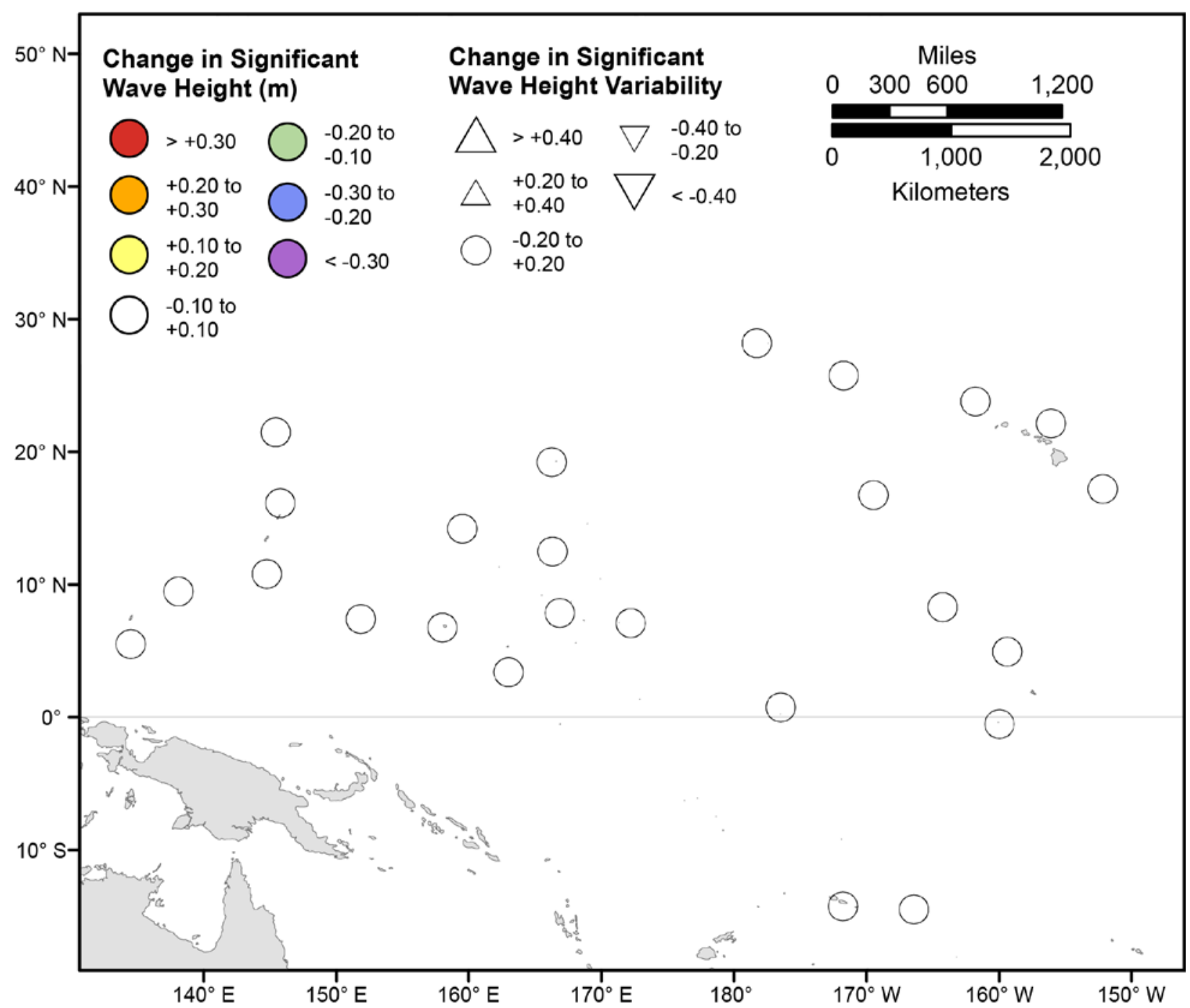

Figure 15. Map showing forecasted differences in mean significant wave height and variance in significant wave height for the years 2026-2045 from hindcasted values during the March-May season under the RCP8.5 future climatic scenario. The colors correspond to the magnitude of change in modeled mean significant wave heights during 2026-2045 from those hindcasted for 1976-2005. The shapes correspond to the magnitude of change in modeled variance in significant wave height during 20262045 from those hindcasted for 1976-2005. Units are in meters. 


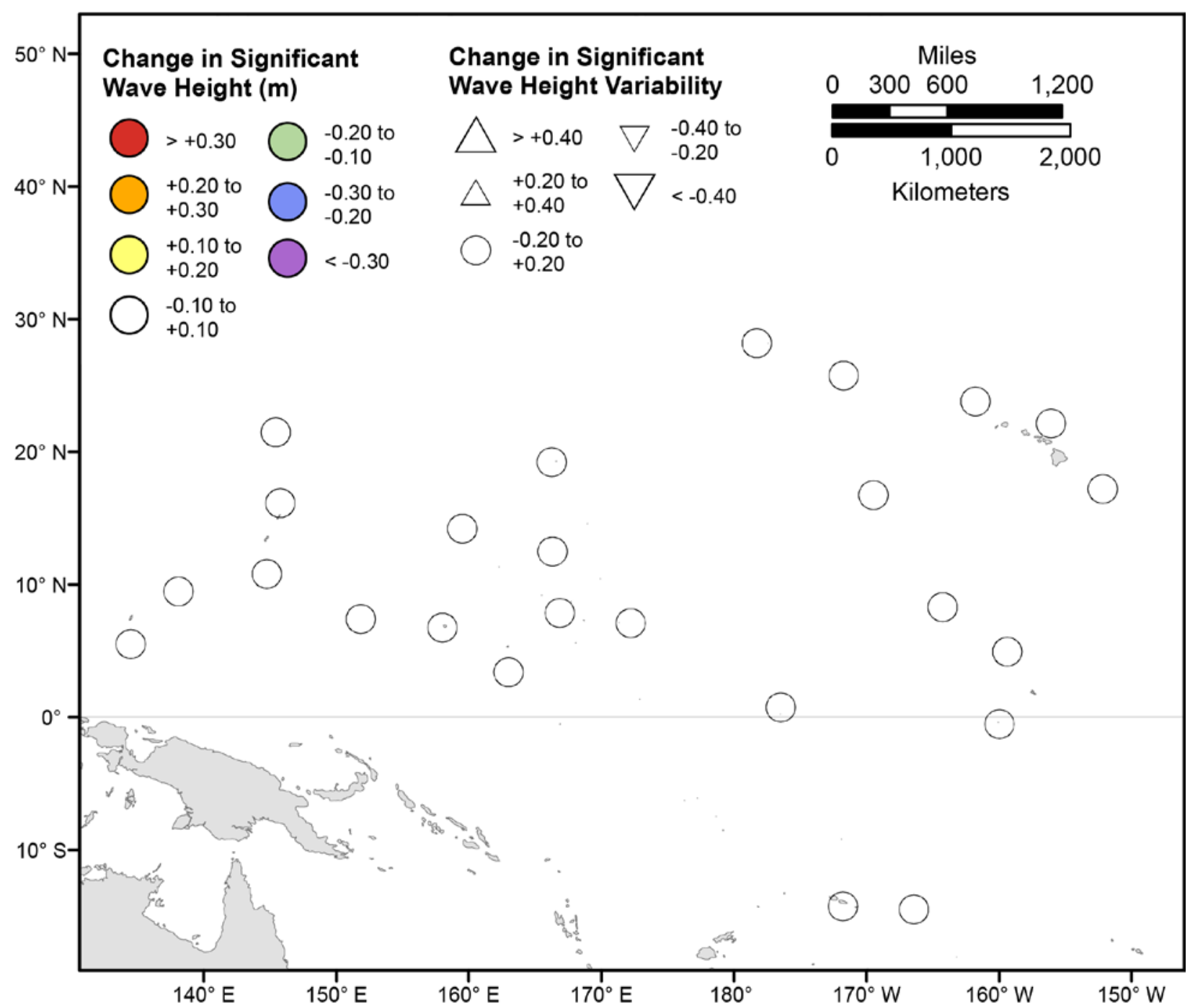

Figure 16. Map showing forecasted differences in mean significant wave height and variance in significant wave height for the years 2026-2045 from hindcasted values during the June-August season under the RCP8.5 future climatic scenario. The colors correspond to the magnitude of change in modeled mean significant wave heights during 2026-2045 from those hindcasted for 1976-2005. The shapes correspond to the magnitude of change in modeled variance in significant wave height during 20262045 from those hindcasted for 1976-2005. Units are in meters. 


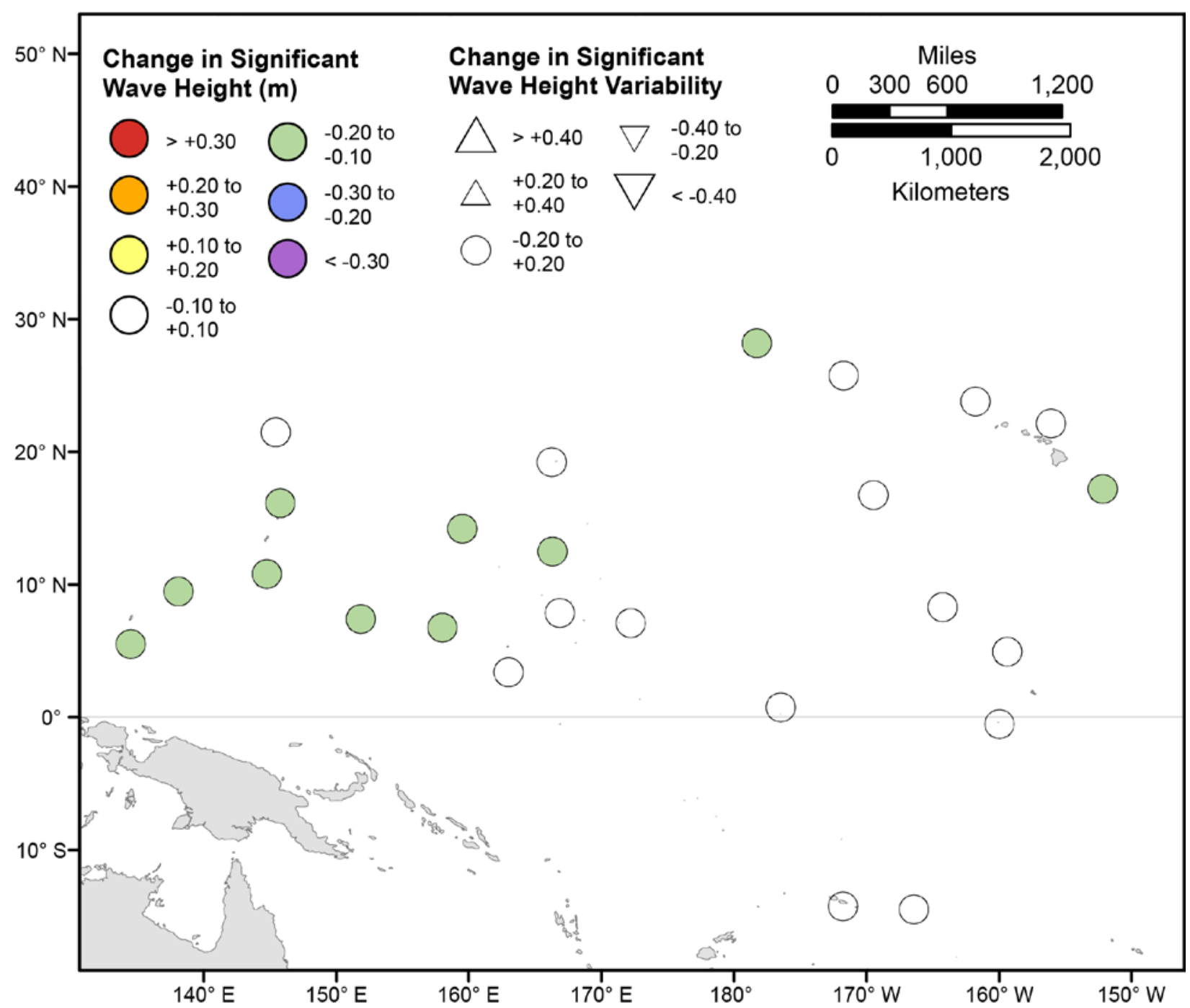

Figure 17. Map showing forecasted differences in mean significant wave height and variance in significant wave height for the years 2026-2045 from hindcasted values during the September-November season under the RCP8.5 future climatic scenario. The colors correspond to the magnitude of change in modeled mean significant wave heights during 2026-2045 from those hindcasted for 1976-2005. The shapes correspond to the magnitude of change in modeled variance in significant wave height during 2026-2045 from those hindcasted for 1976-2005. Units are in meters. 


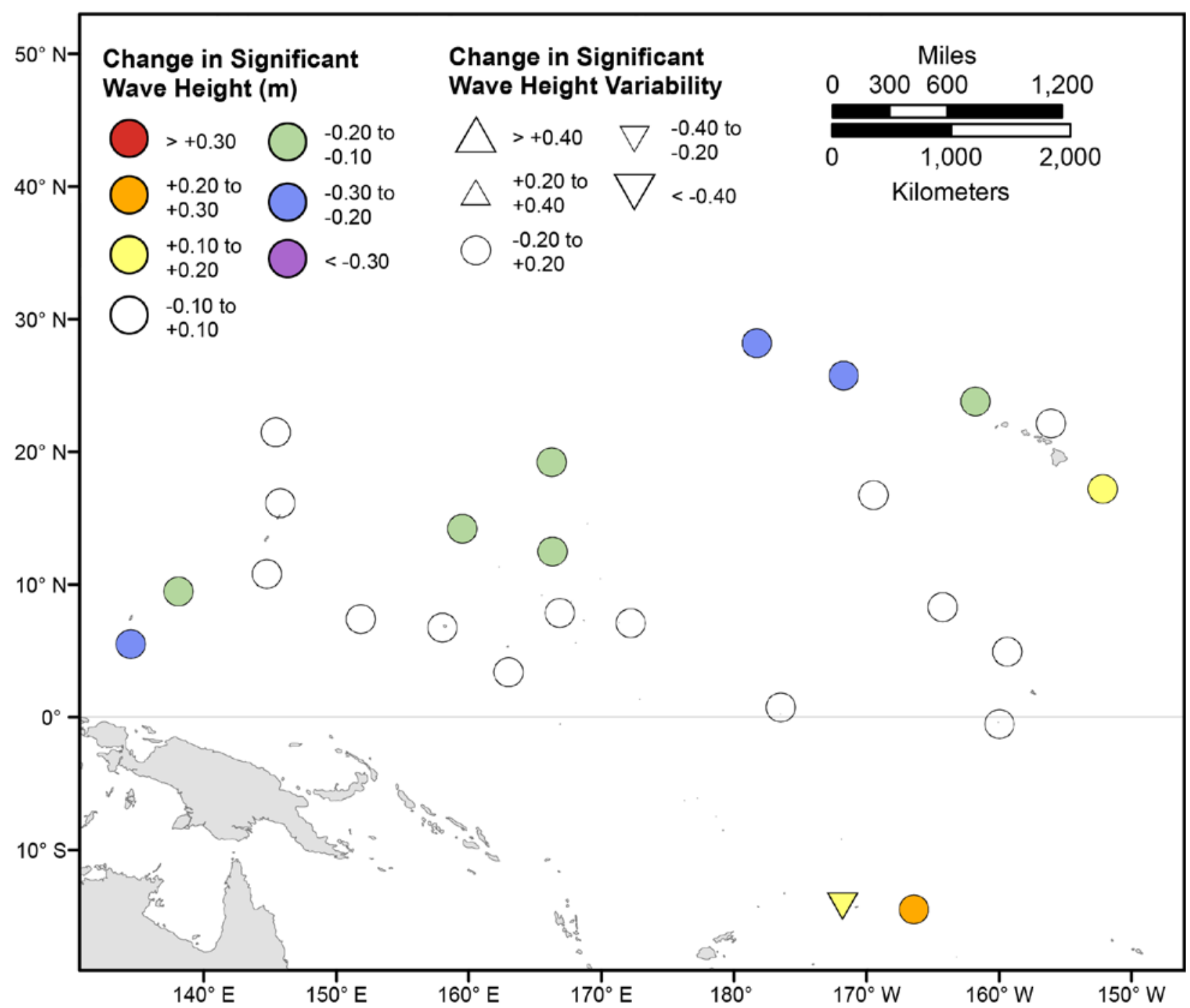

Figure 18. Map showing forecasted differences in the mean of the top 5 percent of significant wave heights and variance in the top 5 percent of significant wave heights for the years 2026-2045 from hindcasted values during the December-February season under the RCP8.5 future climatic scenario. The colors correspond to the magnitude of change in modeled mean significant wave heights during 2026-2045 from those hindcasted for 1976-2005. The shapes correspond to the magnitude of change in modeled variance in significant wave height during 2026-2045 from those hindcasted for 1976-2005. Units are in meters. 


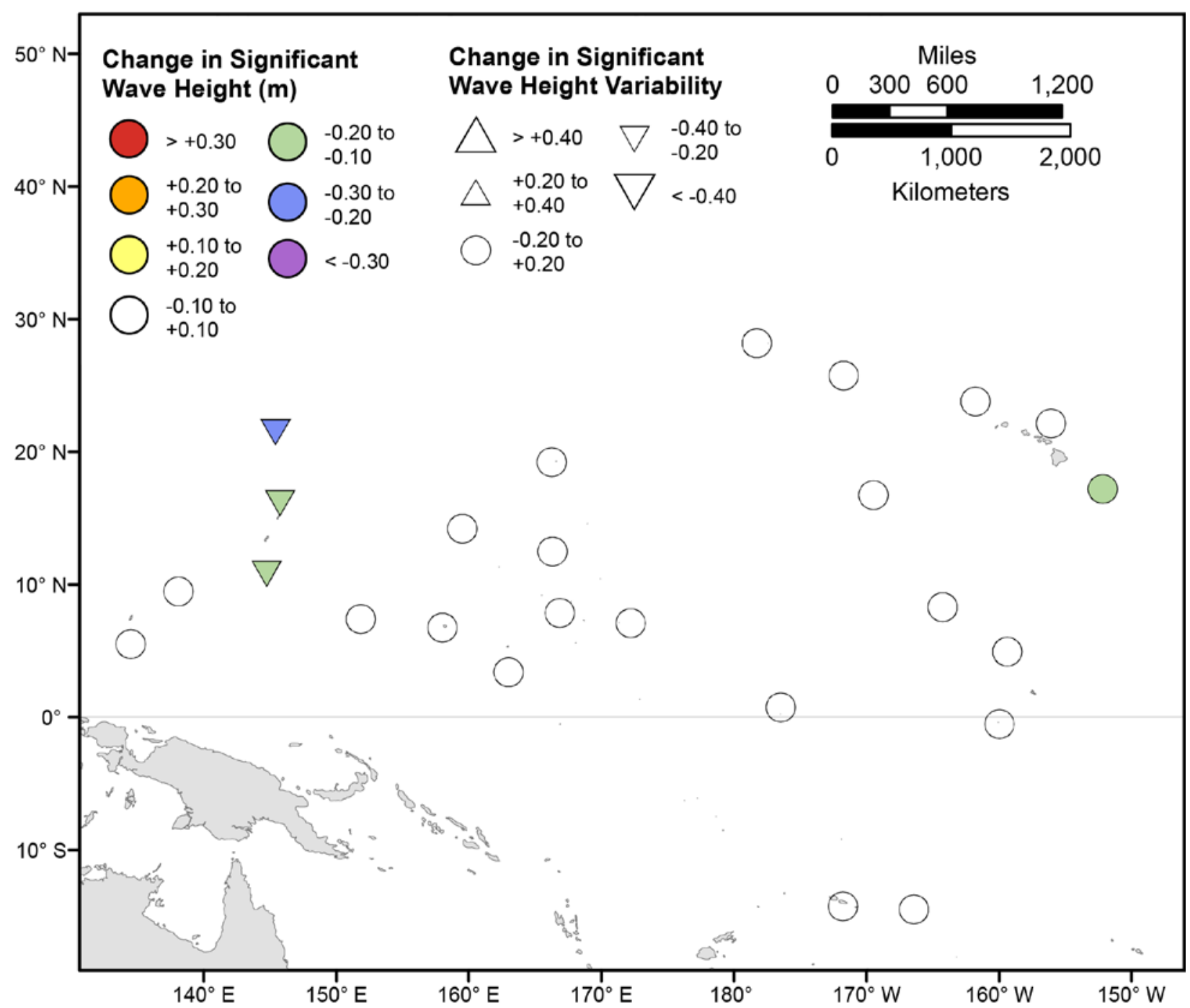

Figure 19. Map showing forecasted differences in the mean of the top 5 percent of significant wave heights and variance in the top 5 percent of significant wave heights for the years 2026-2045 from hindcasted values during the March-May season under the RCP8.5 future climatic scenario. The colors correspond to the magnitude of change in modeled mean significant wave heights during 2026-2045 from those hindcasted for 1976-2005. The shapes correspond to the magnitude of change in modeled variance in significant wave height during 2026-2045 from those hindcasted for 1976-2005. Units are in meters. 


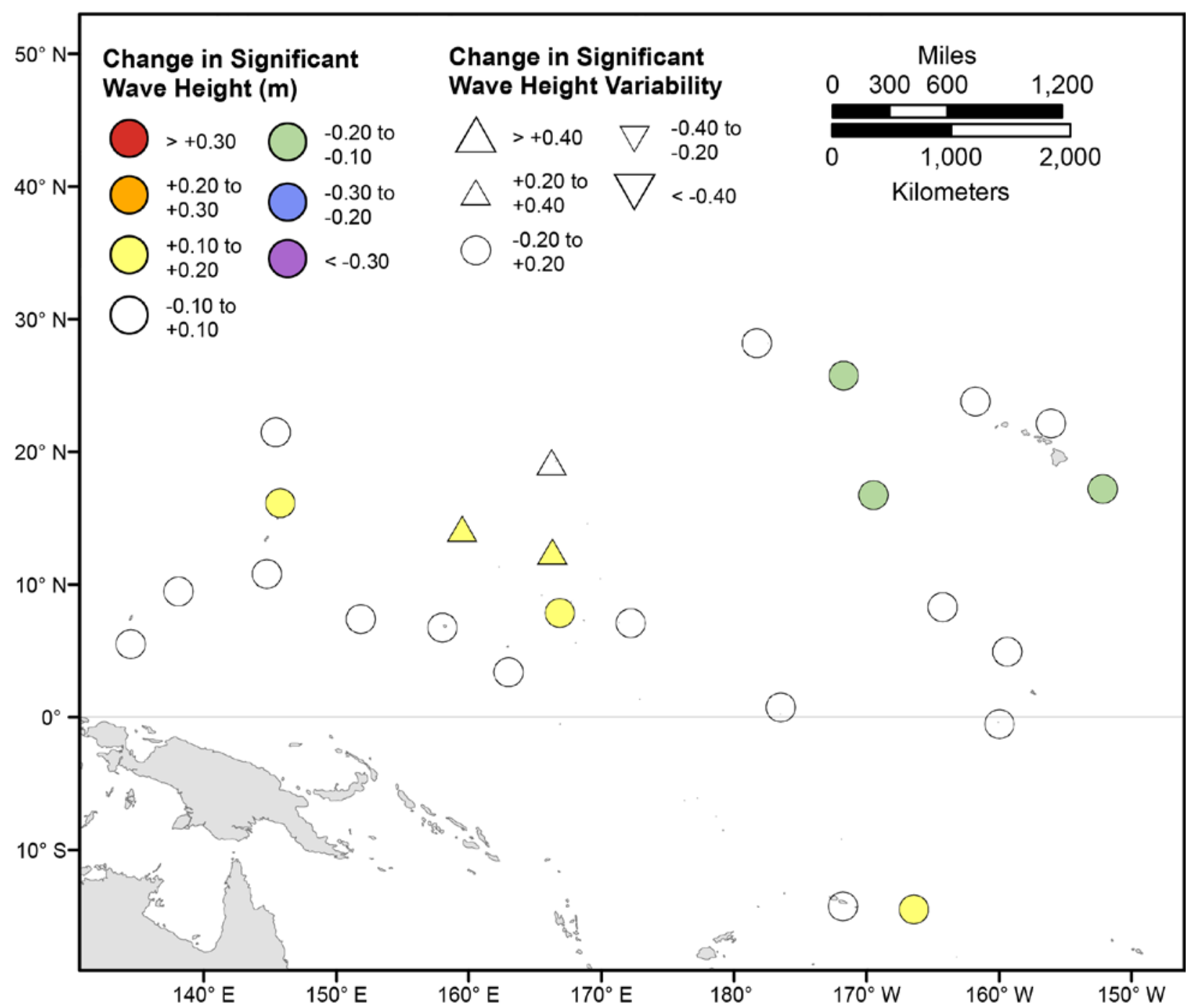

Figure 20. Map showing forecasted differences in the mean of the top 5 percent of significant wave heights and variance in the top 5 percent of significant wave heights for the years 2026-2045 from hindcasted values during the June-August season under the RCP8.5 future climatic scenario. The colors correspond to the magnitude of change in modeled mean significant wave heights during 2026-2045 from those hindcasted for 1976-2005. The shapes correspond to the magnitude of change in modeled variance in significant wave height during 2026-2045 from those hindcasted for 1976-2005. Units are in meters. 


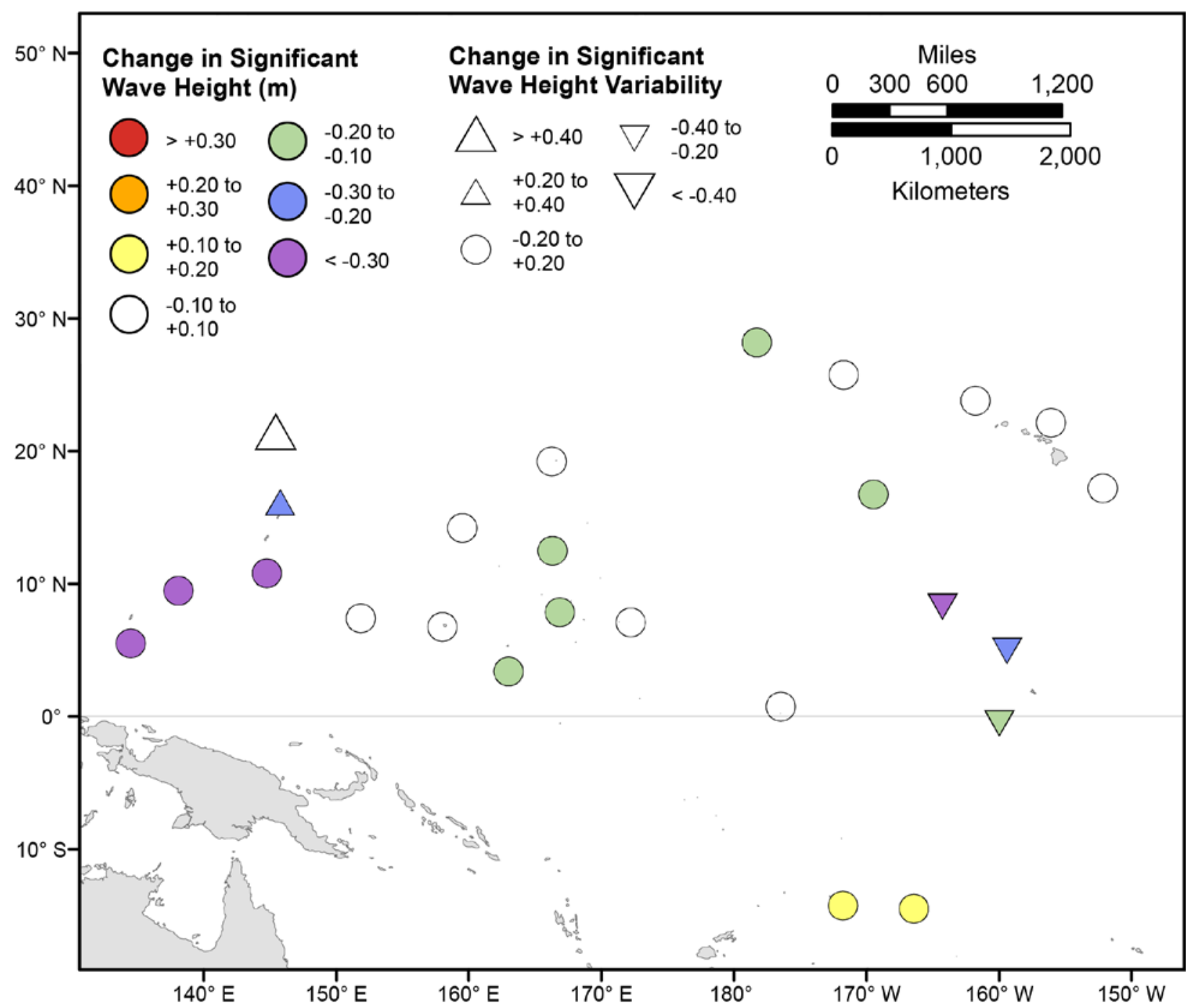

Figure 21. Map showing forecasted differences in the mean of the top 5 percent of significant wave heights and variance in the top 5 percent of significant wave heights for the years 2026-2045 from hindcasted values during the September-November season under the RCP8.5 future climatic scenario. The colors correspond to the magnitude of change in modeled mean significant wave heights during 2026-2045 from those hindcasted for 1976-2005. The shapes correspond to the magnitude of change in modeled variance in significant wave height during 2026-2045 from those hindcasted for 1976-2005. Units are in meters. 


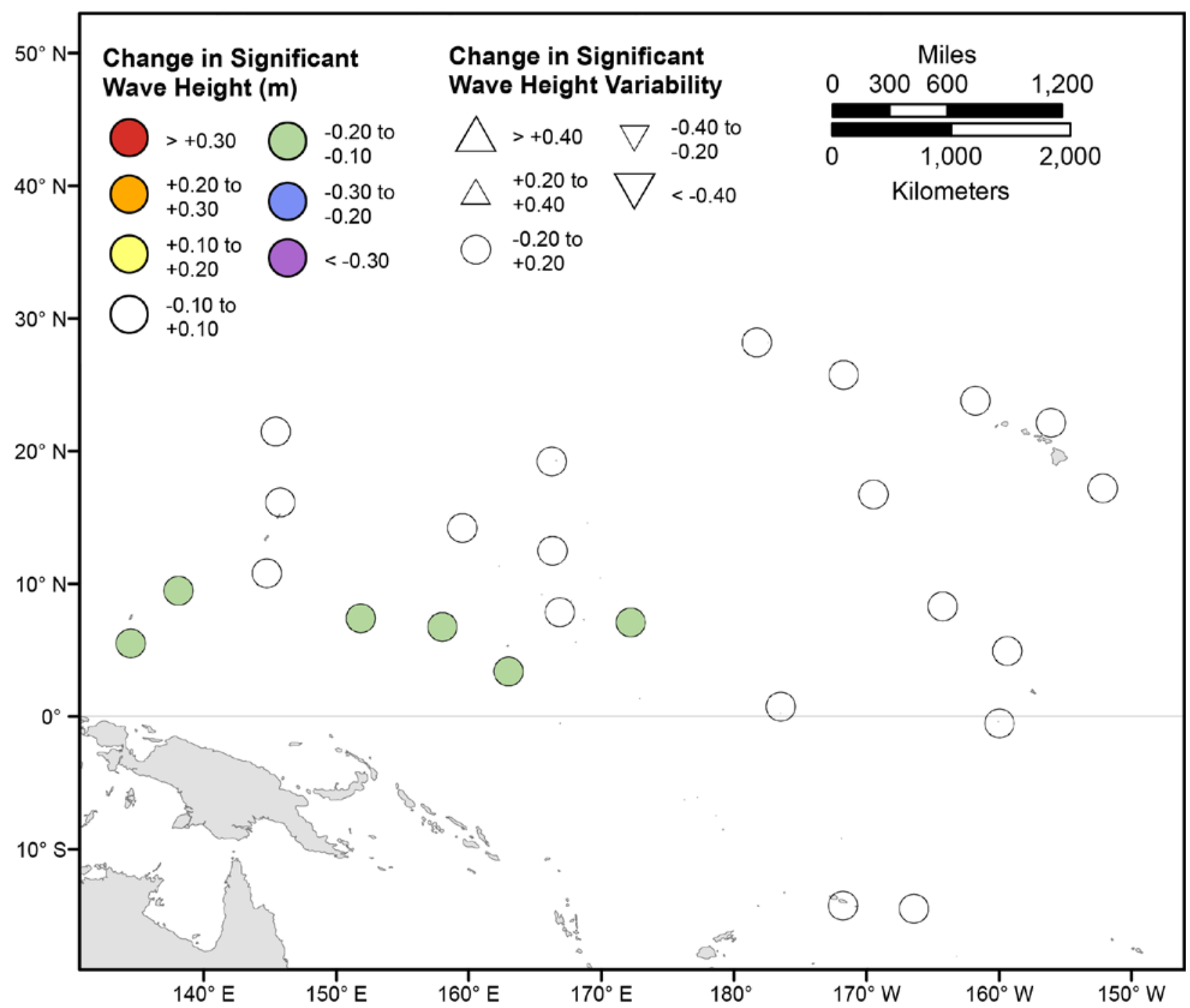

Figure 22. Map showing forecasted differences in mean significant wave height and variance in significant wave height for the years 2081-2100 from hindcasted values during the December-February season under the RCP4.5 future climatic scenario. The colors correspond to the magnitude of change in modeled mean significant wave heights during 2081-2100 from those hindcasted for 1976-2005. The shapes correspond to the magnitude of change in modeled variance in significant wave height during 2081-2100 from those hindcasted for 1976-2005. Units are in meters. 


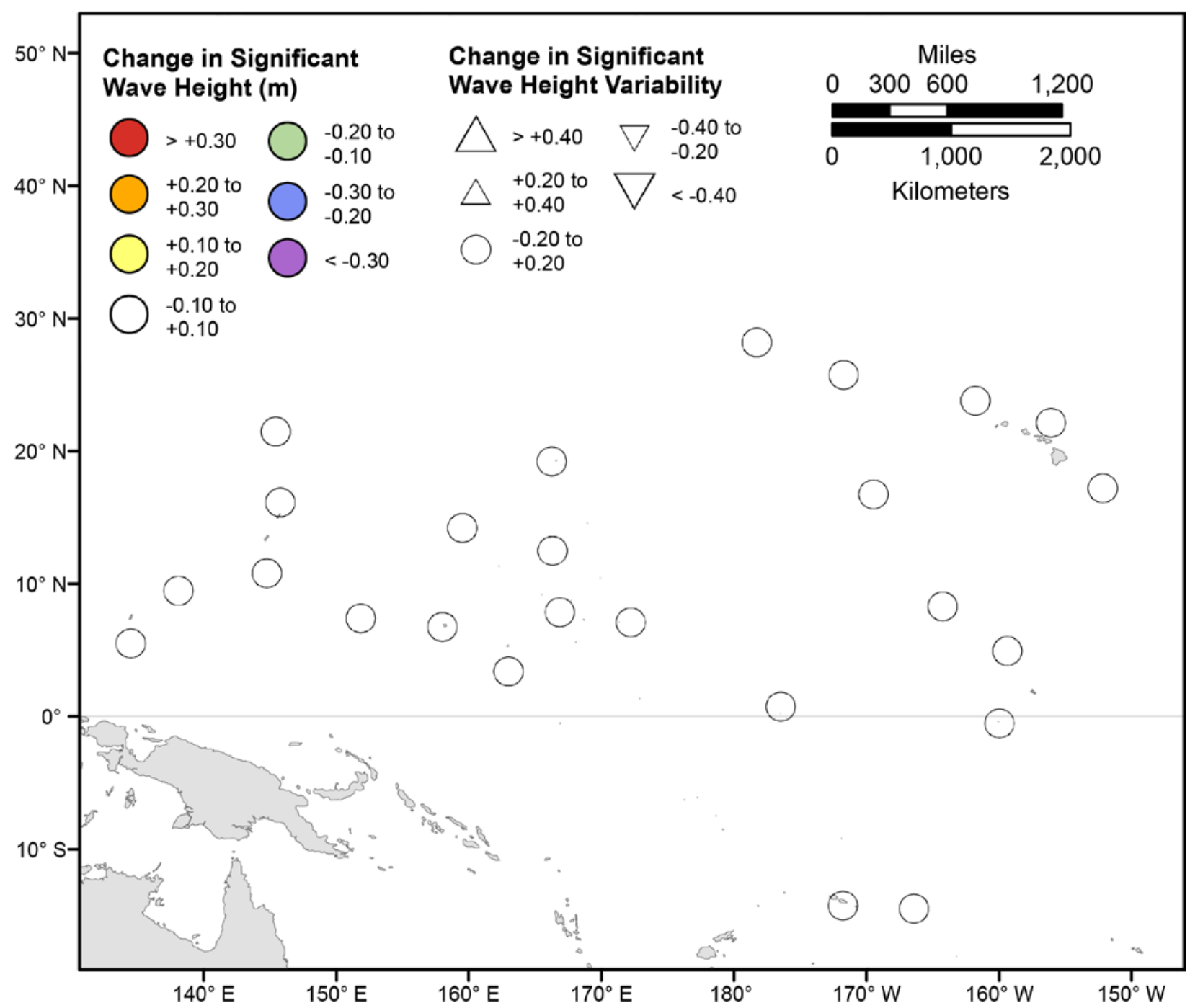

Figure 23. Map showing forecasted differences in mean significant wave height and variance in significant wave height for the years 2081-2100 from hindcasted values during the March-May season under the RCP4.5 future climatic scenario. The colors correspond to the magnitude of change in modeled mean significant wave heights during 2081-2100 from those hindcasted for 1976-2005. The shapes correspond to the magnitude of change in modeled variance in significant wave height during 20812100 from those hindcasted for 1976-2005. Units are in meters. 


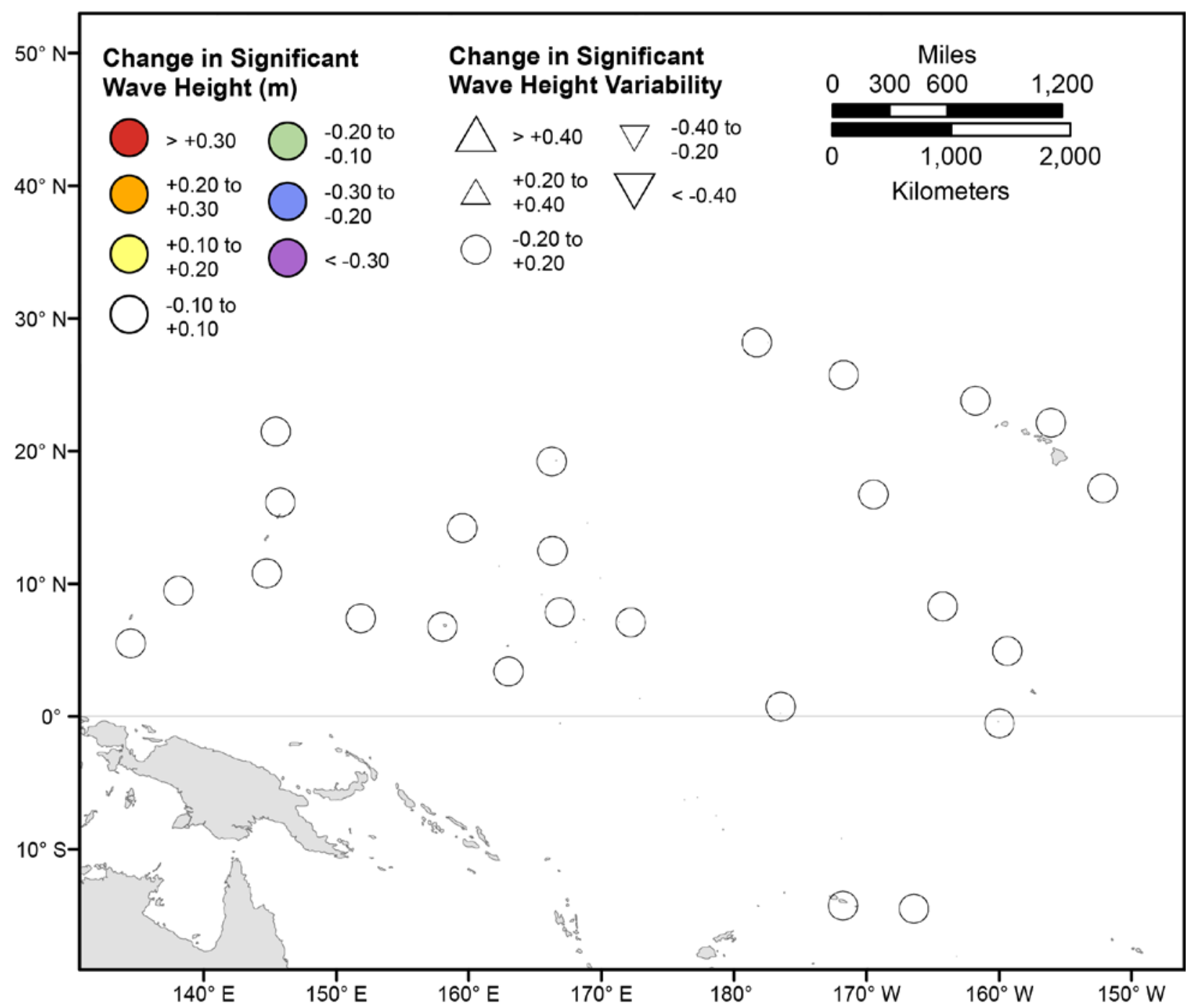

Figure 24. Map showing forecasted differences in mean significant wave height and variance in significant wave height for the years 2081-2100 from hindcasted values during the June-August season under the RCP4.5 future climatic scenario. The colors correspond to the magnitude of change in modeled mean significant wave heights during 2081-2100 from those hindcasted for 1976-2005. The shapes correspond to the magnitude of change in modeled variance in significant wave height during 20812100 from those hindcasted for 1976-2005. Units are in meters. 


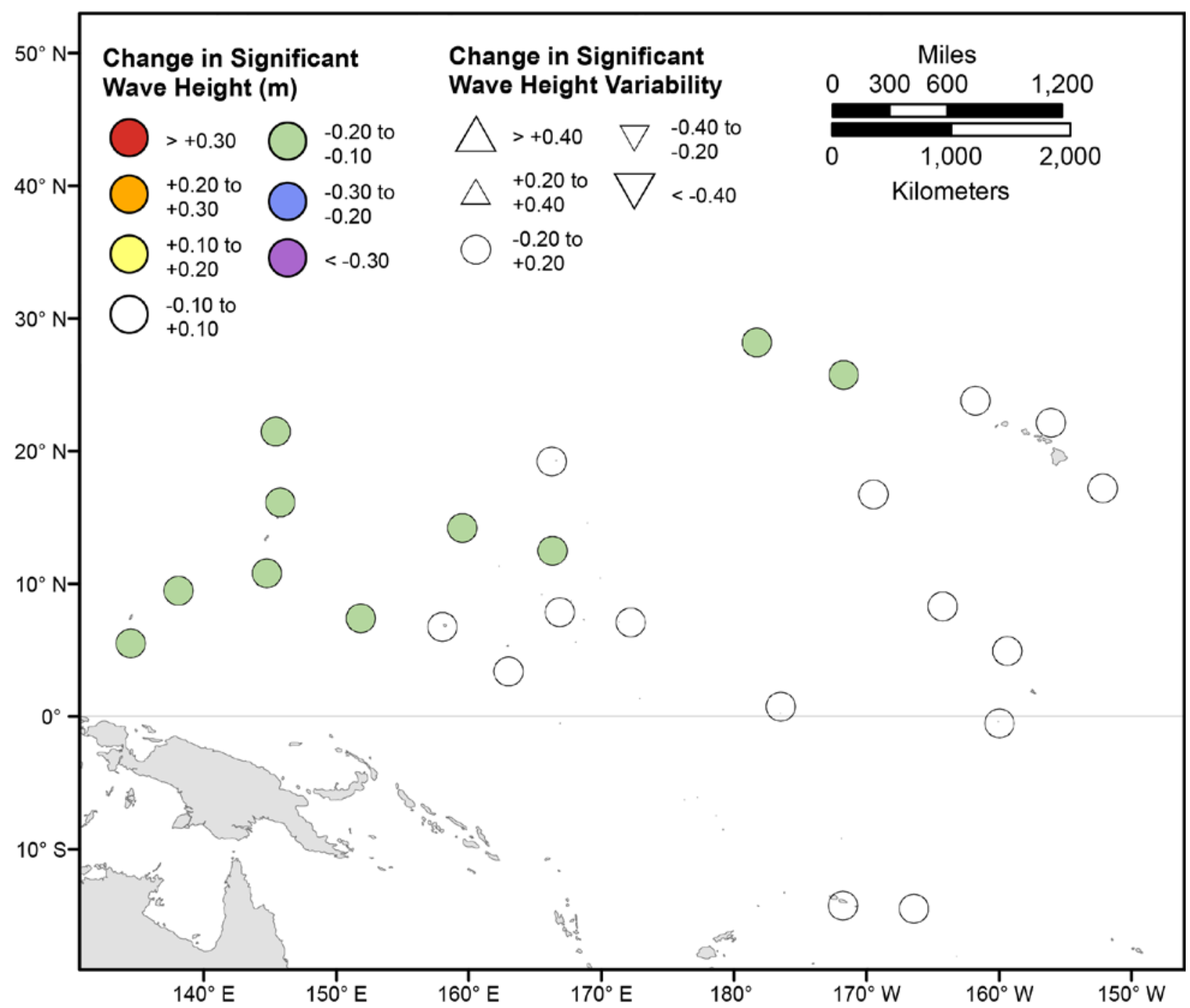

Figure 25. Map showing forecasted differences in mean significant wave height and variance in significant wave height for the years 2081-2100 from hindcasted values during the September-November season under the RCP4.5 future climatic scenario. The colors correspond to the magnitude of change in modeled mean significant wave heights during 2081-2100 from those hindcasted for 1976-2005. The shapes correspond to the magnitude of change in modeled variance in significant wave height during 2081-2100 from those hindcasted for 1976-2005. Units are in meters. 


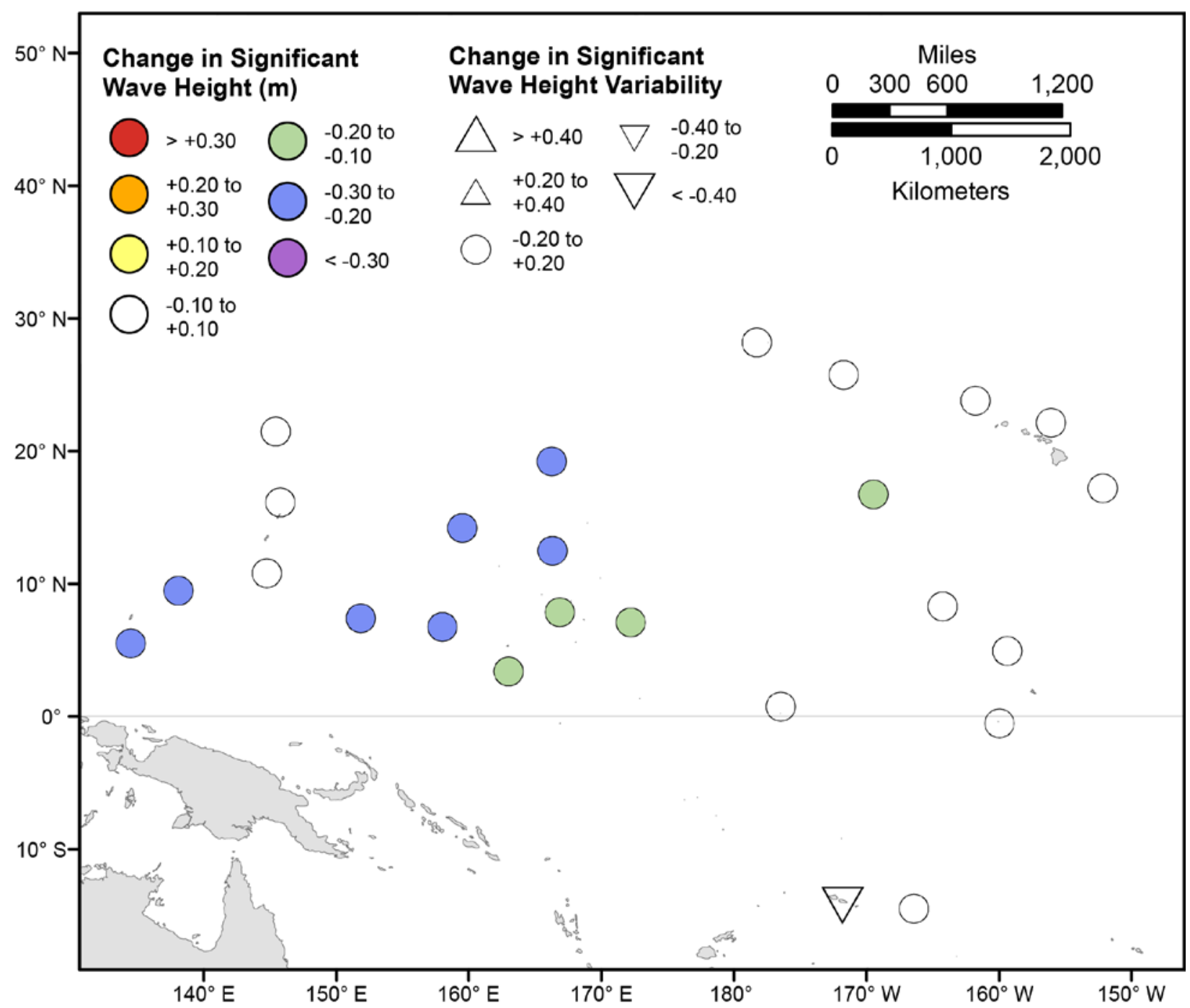

Figure 26. Map showing forecasted differences in the mean of the top 5 percent of significant wave heights and variance in the top 5 percent of significant wave heights for the years 2081-2100 from hindcasted values during the December-February season under the RCP4.5 future climatic scenario. The colors correspond to the magnitude of change in modeled mean significant wave heights during 2081-2100 from those hindcasted for 1976-2005. The shapes correspond to the magnitude of change in modeled variance in significant wave height during 2081-2100 from those hindcasted for 1976-2005. Units are in meters. 


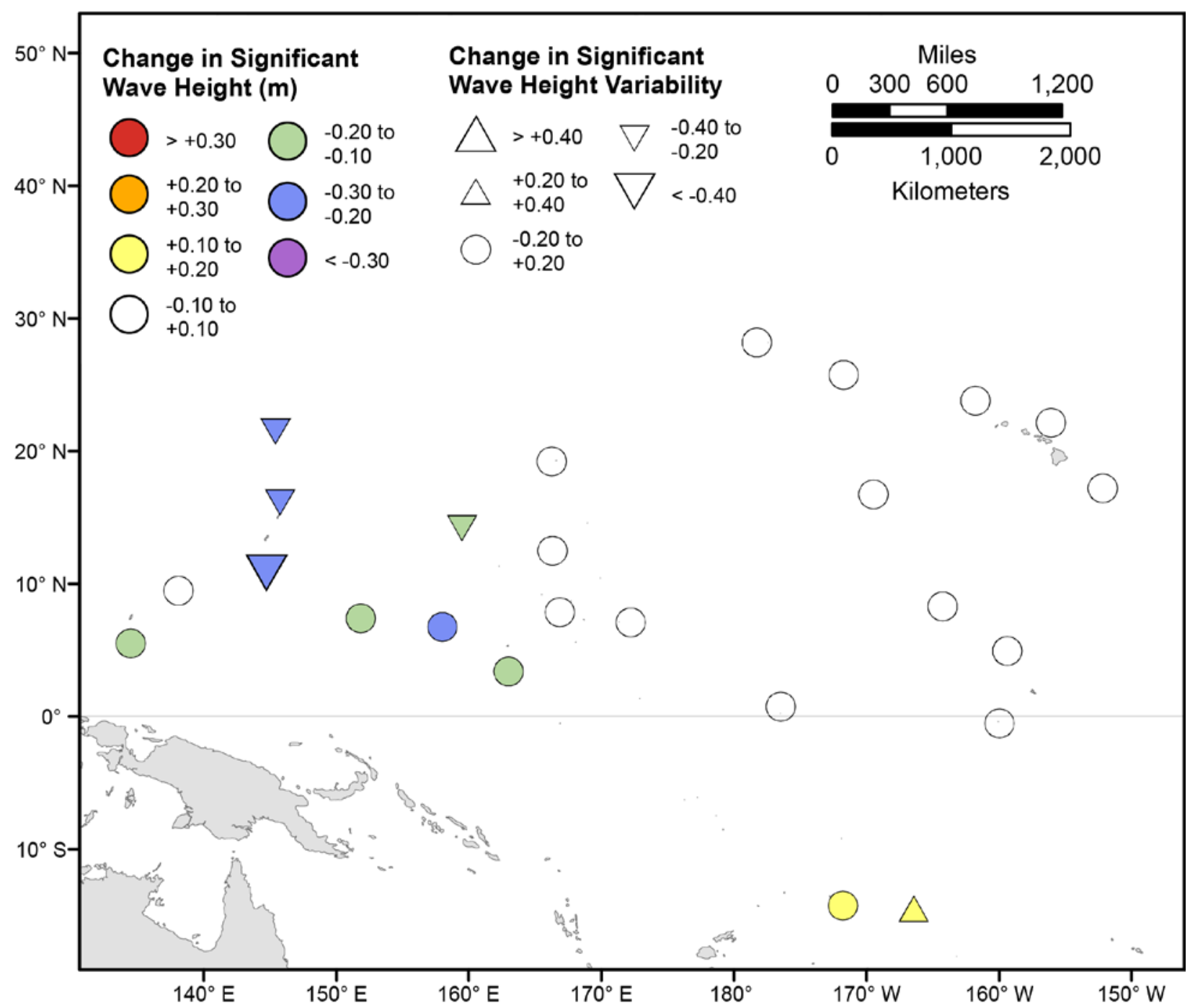

Figure 27. Map showing forecasted differences in the mean of the top 5 percent of significant wave heights and variance in the top 5 percent of significant wave heights for the years 2081-2100 from hindcasted values during the March-May season under the RCP4.5 future climatic scenario. The colors correspond to the magnitude of change in modeled mean significant wave heights during 2081-2100 from those hindcasted for 1976-2005. The shapes correspond to the magnitude of change in modeled variance in significant wave height during 2081-2100 from those hindcasted for 1976-2005. Units are in meters. 


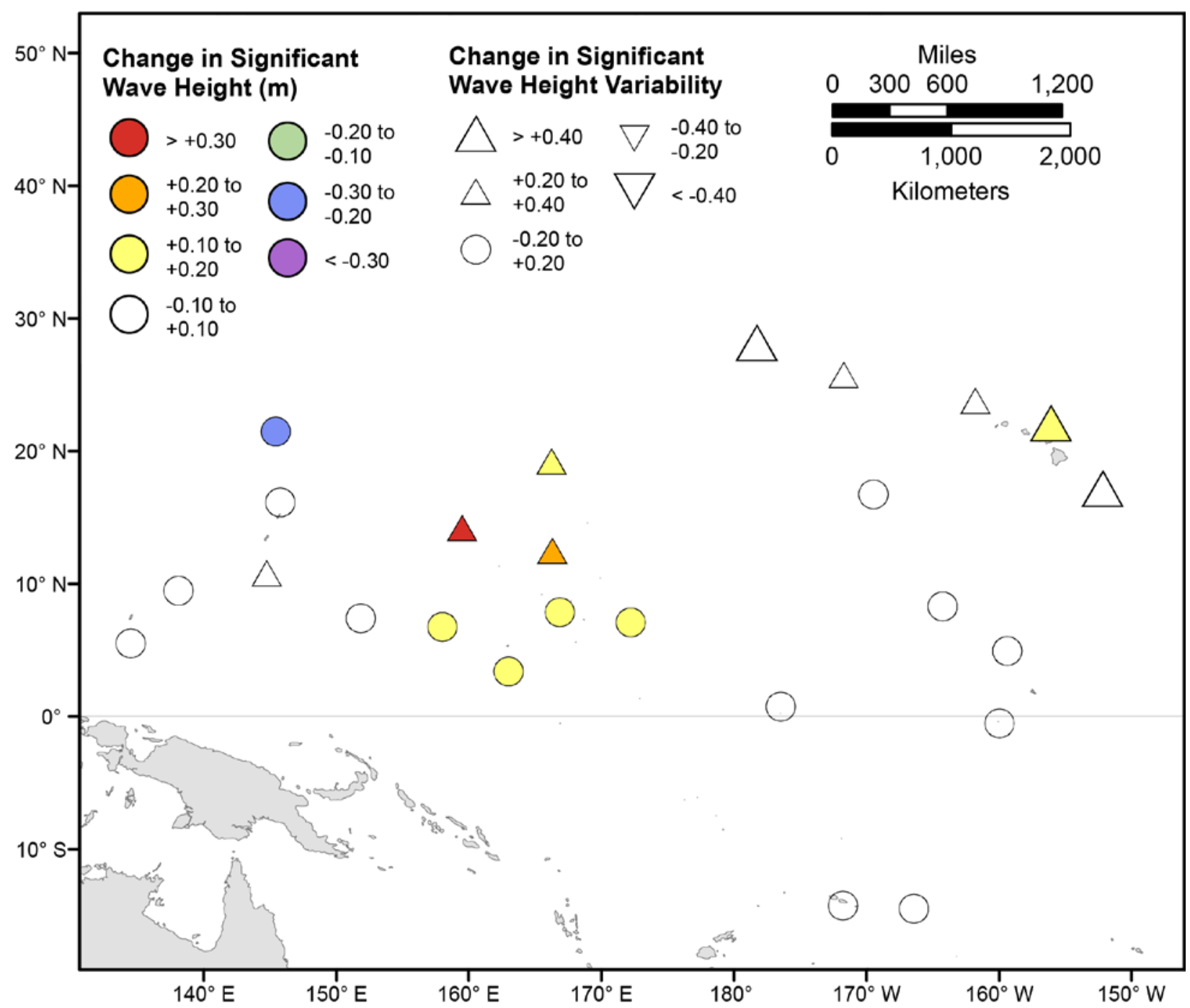

Figure 28. Map showing forecasted differences in the mean of the top 5 percent of significant wave heights and variance in the top 5 percent of significant wave heights for the years 2081-2100 from hindcasted values during the June-August season under the RCP4.5 future climatic scenario. The colors correspond to the magnitude of change in modeled mean significant wave heights during 2081-2100 from those hindcasted for 1976-2005. The shapes correspond to the magnitude of change in modeled variance in significant wave height during 2081-2100 from those hindcasted for 1976-2005. Units are in meters. 


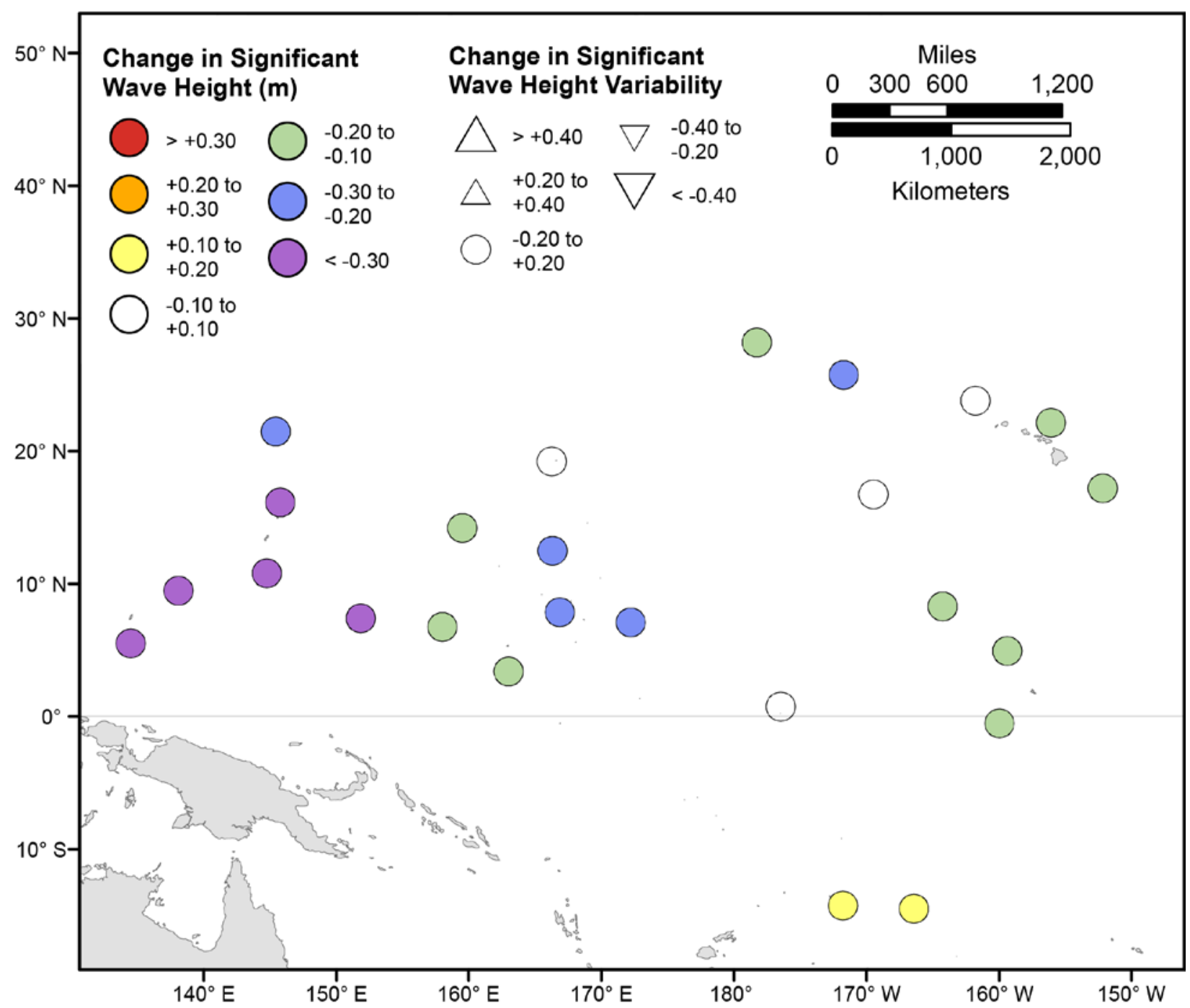

Figure 29. Map showing forecasted differences in the mean of the top 5 percent of significant wave heights and variance in the top 5 percent of significant wave heights for the years 2081-2100 from hindcasted values during the September-November season under the RCP4.5 future climatic scenario. The colors correspond to the magnitude of change in modeled mean significant wave heights during 2081-2100 from those hindcasted for 1976-2005. The shapes correspond to the magnitude of change in modeled variance in significant wave height during 2081-2100 from those hindcasted for 1976-2005. Units are in meters. 


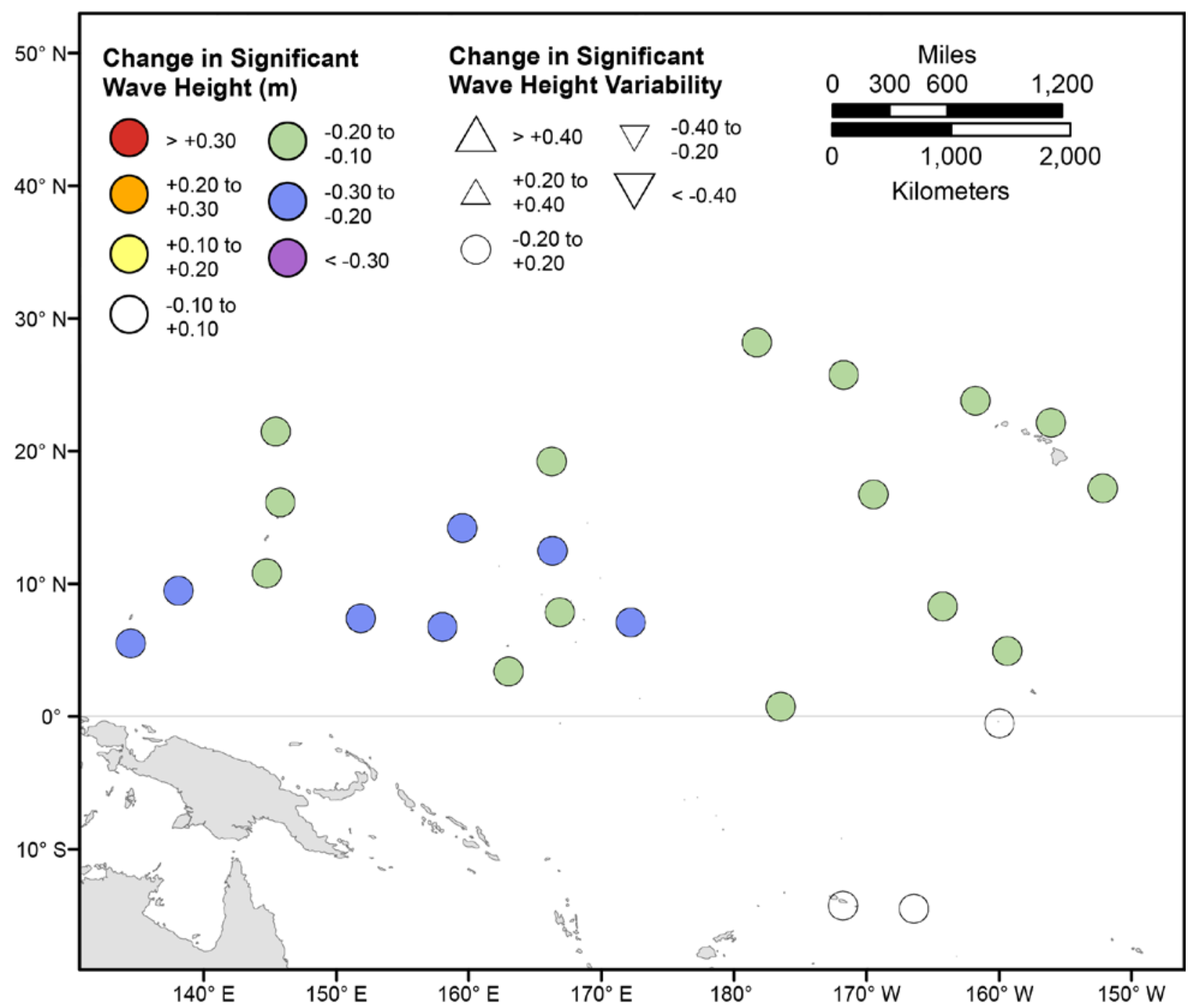

Figure 30. Map showing forecasted differences in mean significant wave height and variance in significant wave height for the years 2081-2100 from hindcasted values during the December-February season under the RCP8.5 future climatic scenario. The colors correspond to the magnitude of change in modeled mean significant wave heights during 2081-2100 from those hindcasted for 1976-2005. The shapes correspond to the magnitude of change in modeled variance in significant wave height during 2081-2100 from those hindcasted for 1976-2005. Units are in meters. 


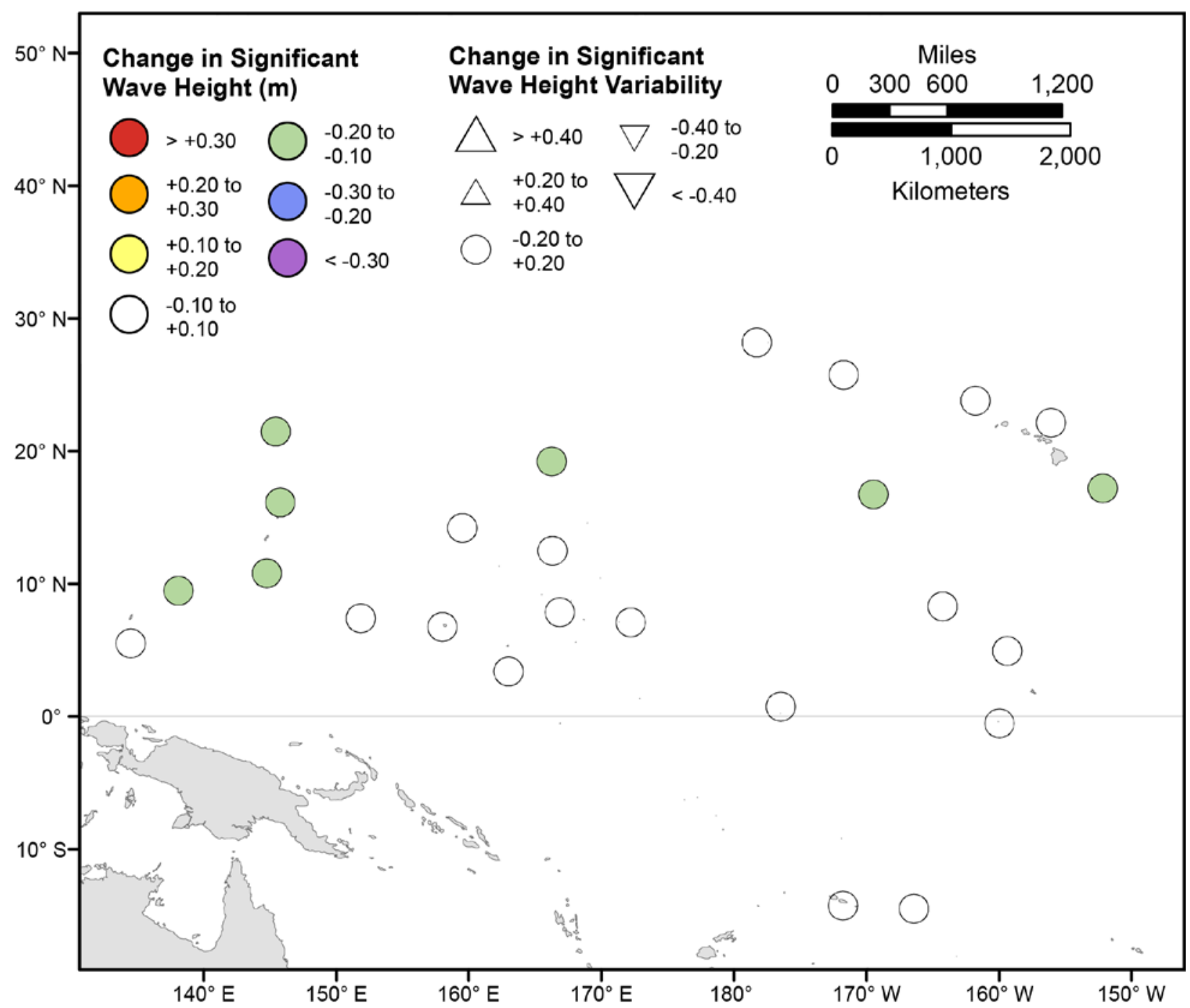

Figure 31. Map showing forecasted differences in mean significant wave height and variance in significant wave height for the years 2081-2100 from hindcasted values during the March-May season under the RCP8.5 future climatic scenario. The colors correspond to the magnitude of change in modeled mean significant wave heights during 2081-2100 from those hindcasted for 1976-2005. The shapes correspond to the magnitude of change in modeled variance in significant wave height during 20812100 from those hindcasted for 1976-2005. Units are in meters. 


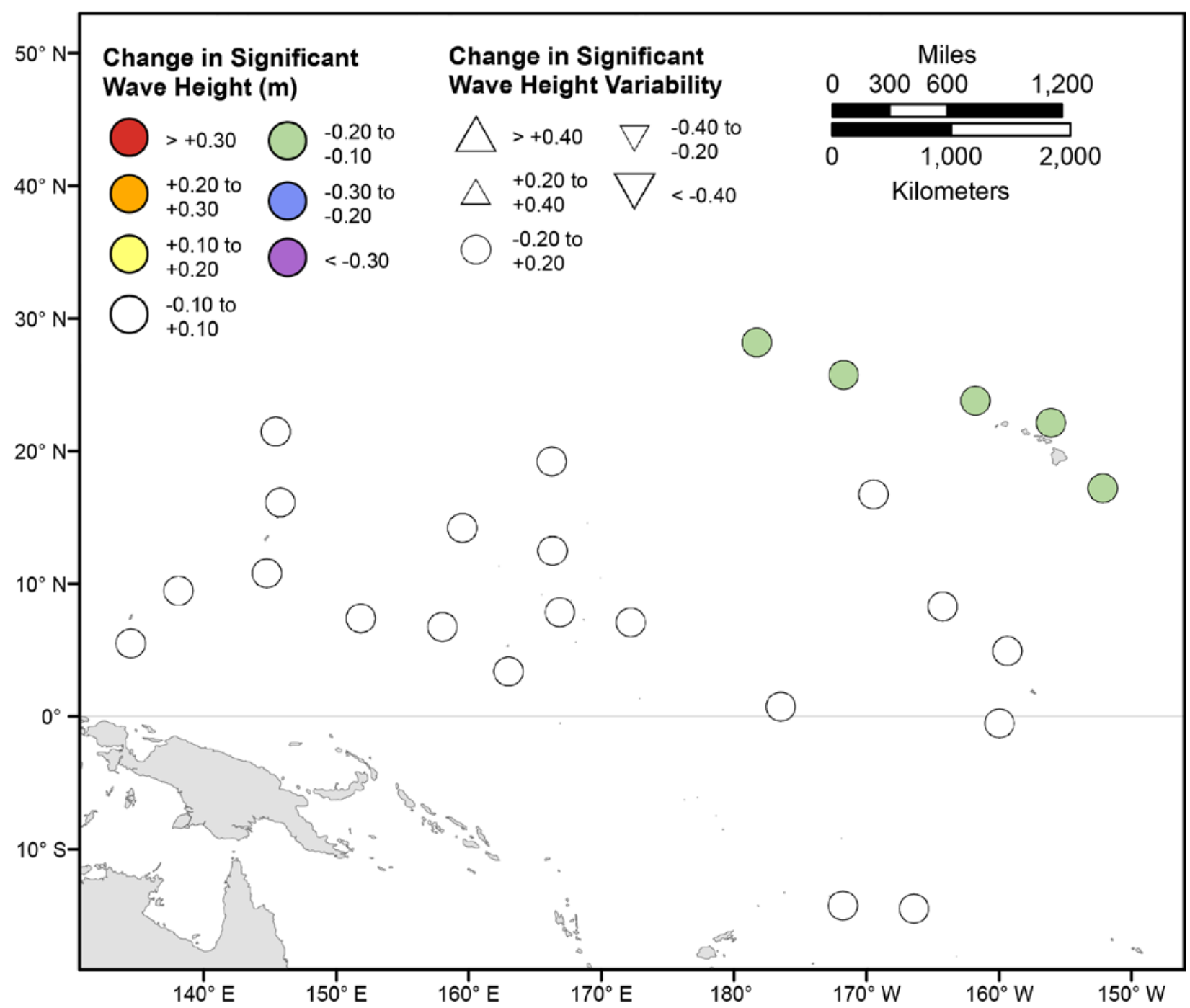

Figure 32. Map showing forecasted differences in mean significant wave height and variance in significant wave height for the years 2081-2100 from hindcasted values during the June-August season under the RCP8.5 future climatic scenario. The colors correspond to the magnitude of change in modeled mean significant wave heights during 2081-2100 from those hindcasted for 1976-2005. The shapes correspond to the magnitude of change in modeled variance in significant wave height during 20812100 from those hindcasted for 1976-2005. Units are in meters. 


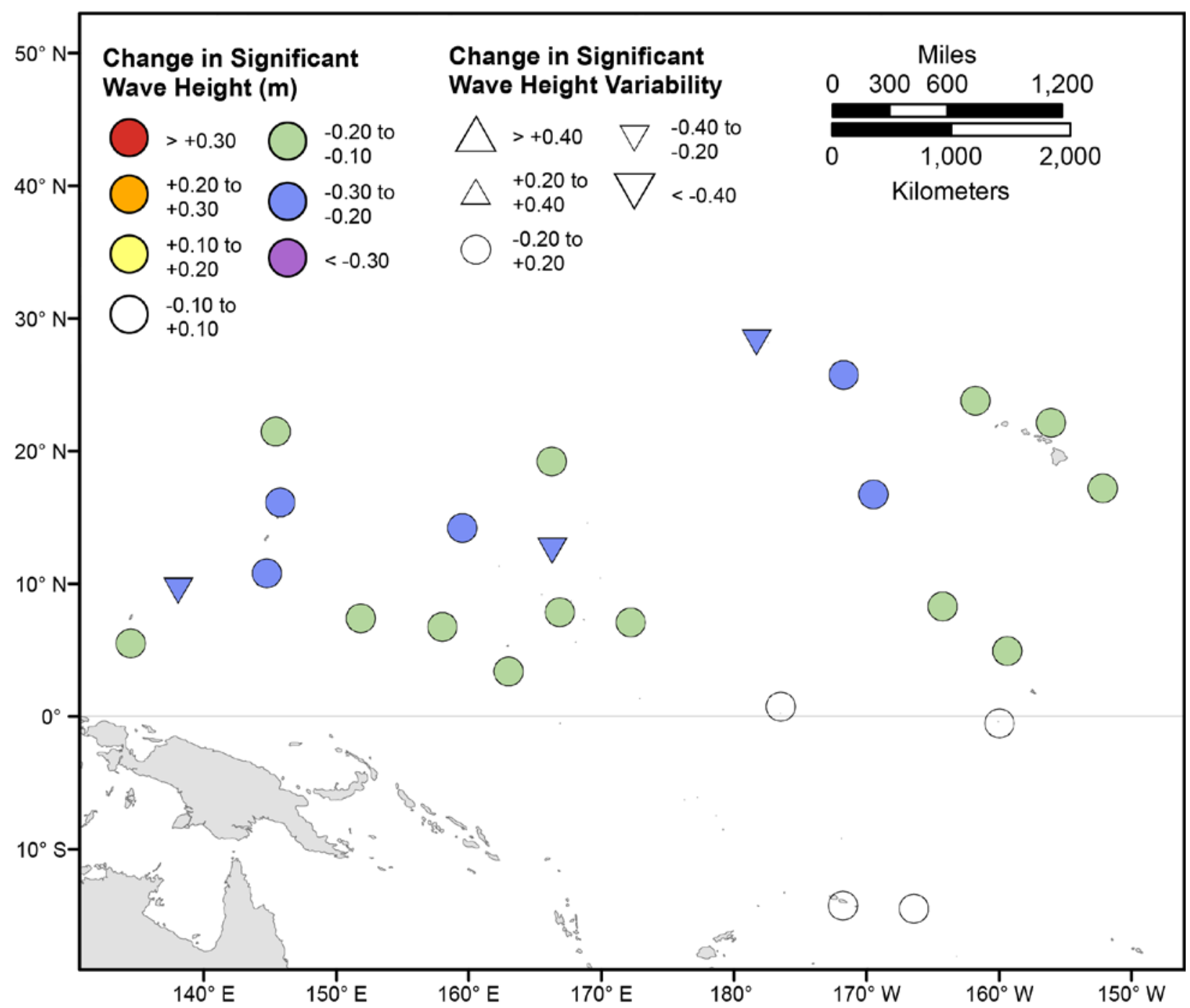

Figure 33. Map showing forecasted differences in mean significant wave height and variance in significant wave height for the years 2081-2100 from hindcasted values during the September-November season under the RCP8.5 future climatic scenario. The colors correspond to the magnitude of change in modeled mean significant wave heights during 2081-2100 from those hindcasted for 1976-2005. The shapes correspond to the magnitude of change in modeled variance in significant wave height during 2081-2100 from those hindcasted for 1976-2005. Units are in meters. 


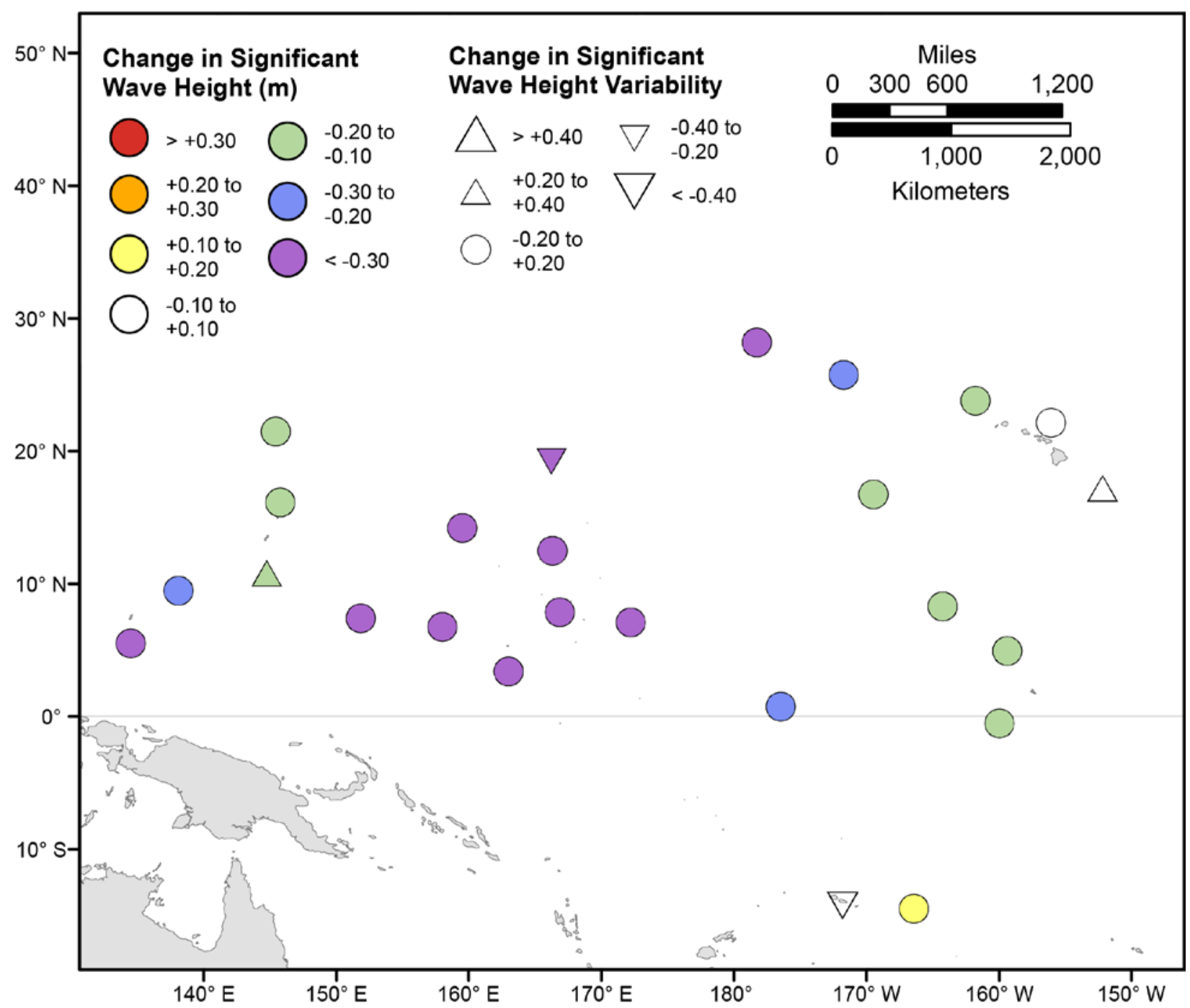

Figure 34. Map showing forecasted differences in the mean of the top 5 percent of significant wave heights and variance in the top 5 percent of significant wave heights for the years 2081-2100 from hindcasted values during the December-February season under the RCP8.5 future climatic scenario. The colors correspond to the magnitude of change in modeled mean significant wave heights during 2081-2100 from those hindcasted for 1976-2005. The shapes correspond to the magnitude of change in modeled variance in significant wave height during 2081-2100 from those hindcasted for 1976-2005. Units are in meters. 


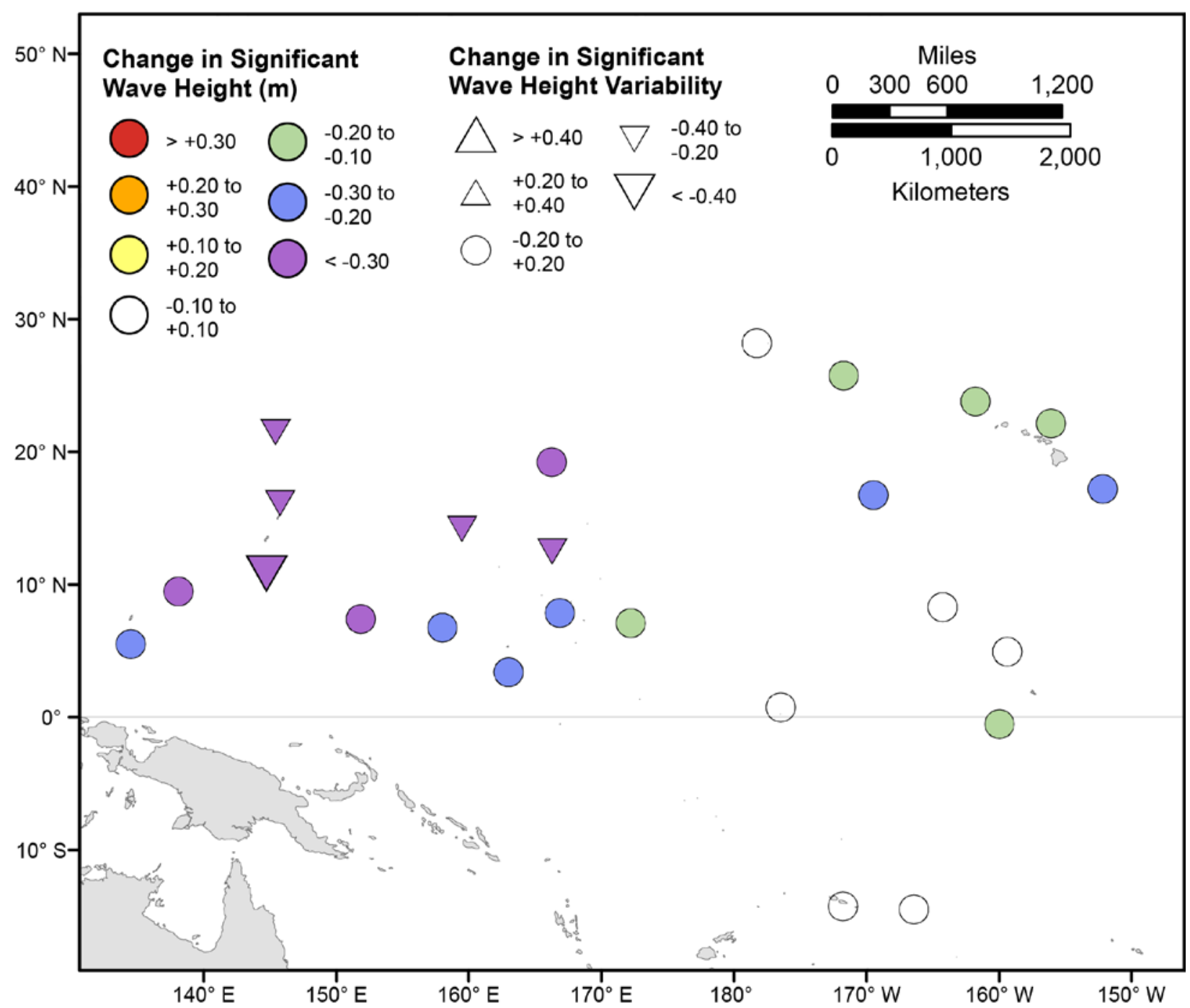

Figure 35. Map showing forecasted differences in the mean of the top 5 percent of significant wave heights and variance in the top 5 percent of significant wave heights for the years 2081-2100 from hindcasted values during the March-May season under the RCP8.5 future climatic scenario. The colors correspond to the magnitude of change in modeled mean significant wave heights during 2081-2100 from those hindcasted for 1976-2005. The shapes correspond to the magnitude of change in modeled variance in significant wave height during 2081-2100 from those hindcasted for 1976-2005. Units are in meters. 


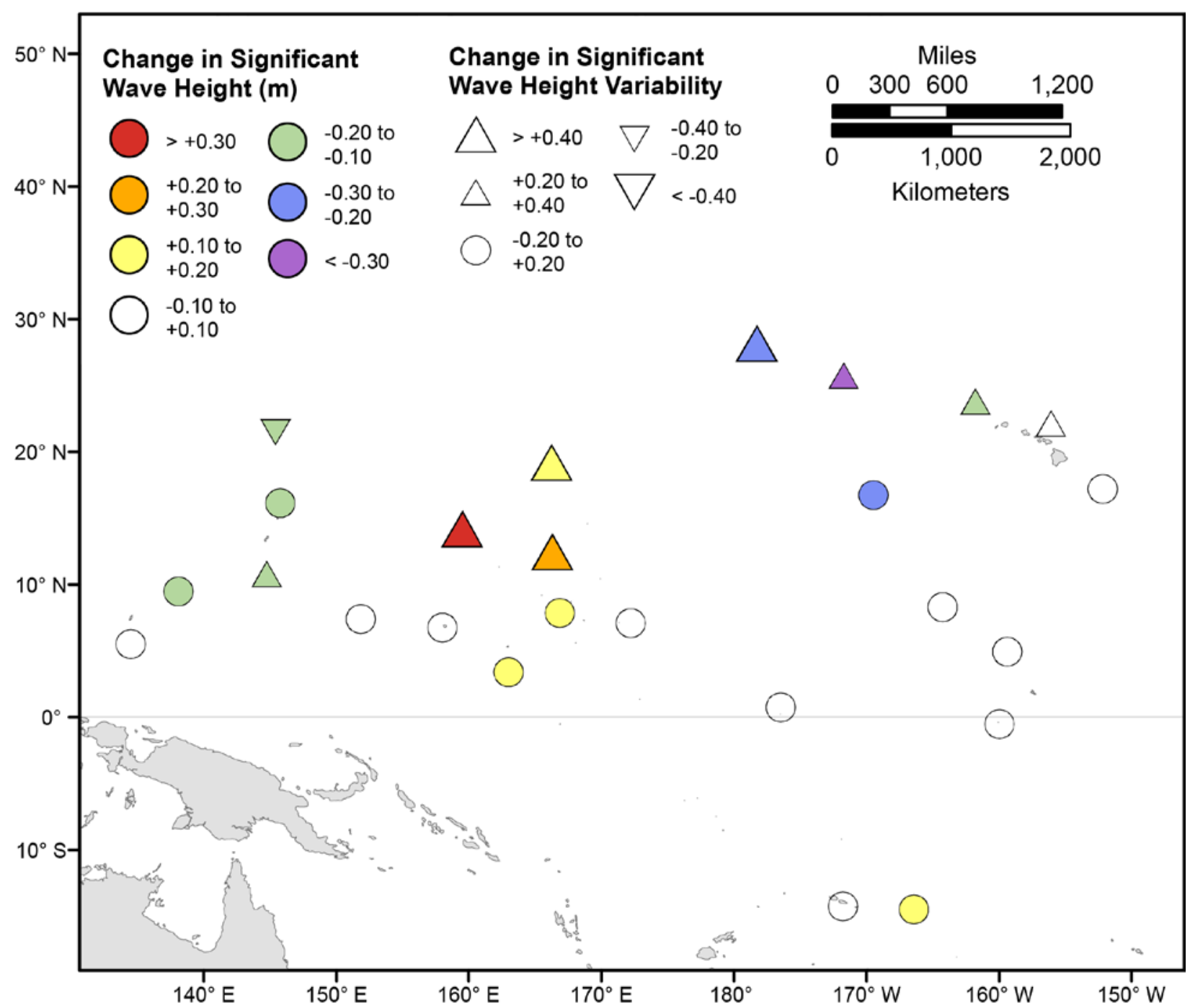

Figure 36. Map showing forecasted differences in the mean of the top 5 percent of significant wave heights and variance in the top 5 percent of significant wave heights for the years 2081-2100 from hindcasted values during the June-August season under the RCP8.5 future climatic scenario. The colors correspond to the magnitude of change in modeled mean significant wave heights during 2081-2100 from those hindcasted for 1976-2005. The shapes correspond to the magnitude of change in modeled variance in significant wave height during 2081-2100 from those hindcasted for 1976-2005. Units are in meters. 


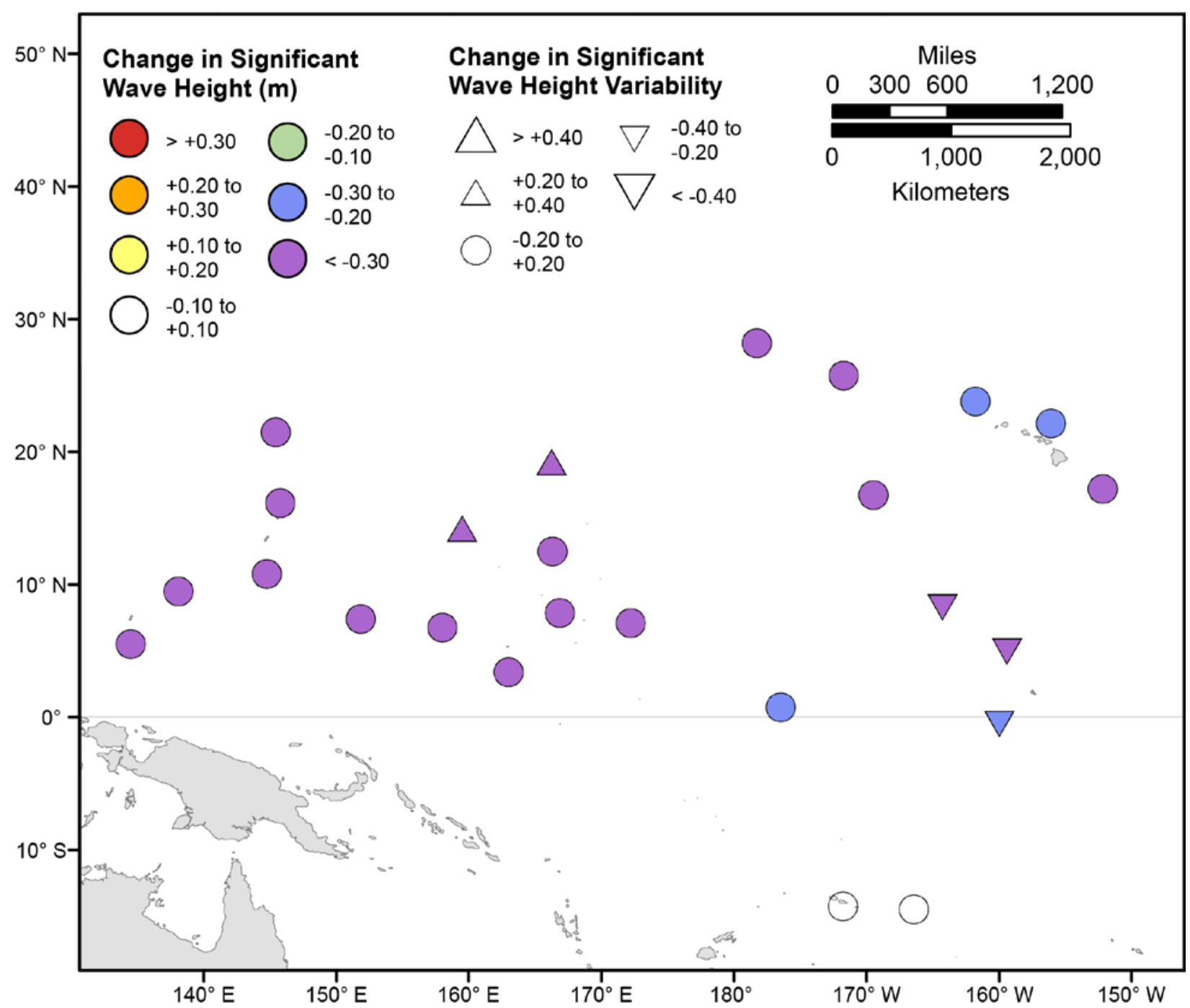

Figure 37. Map showing forecasted differences in the mean of the top 5 percent of significant wave heights and variance in the top 5 percent of significant wave heights for the years 2081-2100 from hindcasted values during the September-November season under the RCP8.5 future climatic scenario. The colors correspond to the magnitude of change in modeled mean significant wave heights during 2081-2100 from those hindcasted for 1976-2005. The shapes correspond to the magnitude of change in modeled variance in significant wave height during 2081-2100 from those hindcasted for 1976-2005. Units are in meters. 


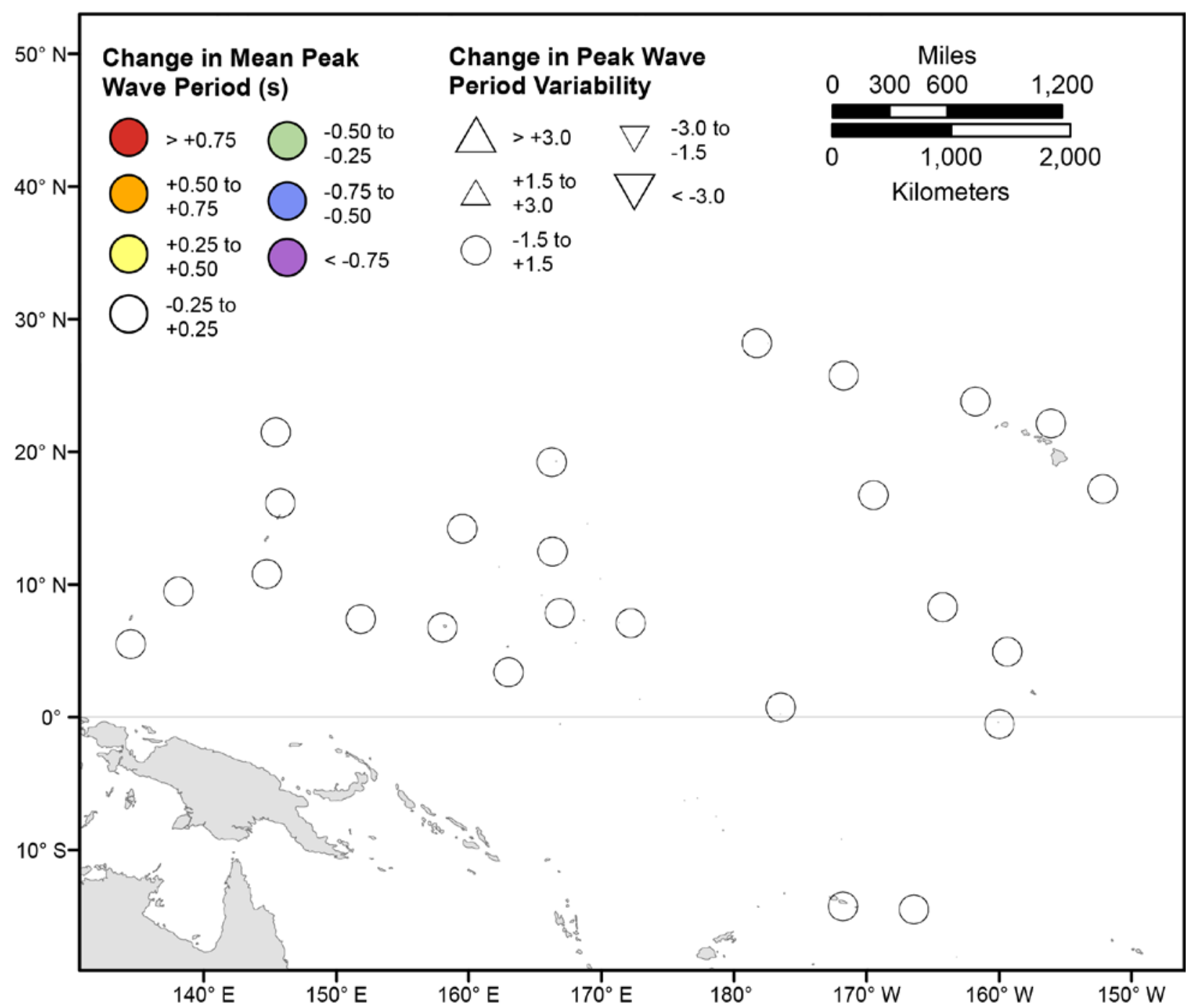

Figure 38. Map showing forecasted differences in mean peak wave period and variance in peak wave period for the years 2026-2045 from hindcasted values during the December-February season under the RCP4.5 future climatic scenario. The colors correspond to the magnitude of change in modeled mean peak wave periods during 2026-2045 from those hindcasted for 1976-2005. The shapes correspond to the magnitude of change in modeled variance in peak wave period during 2026-2045 from those hindcasted for 1976-2005. Units are in seconds. 


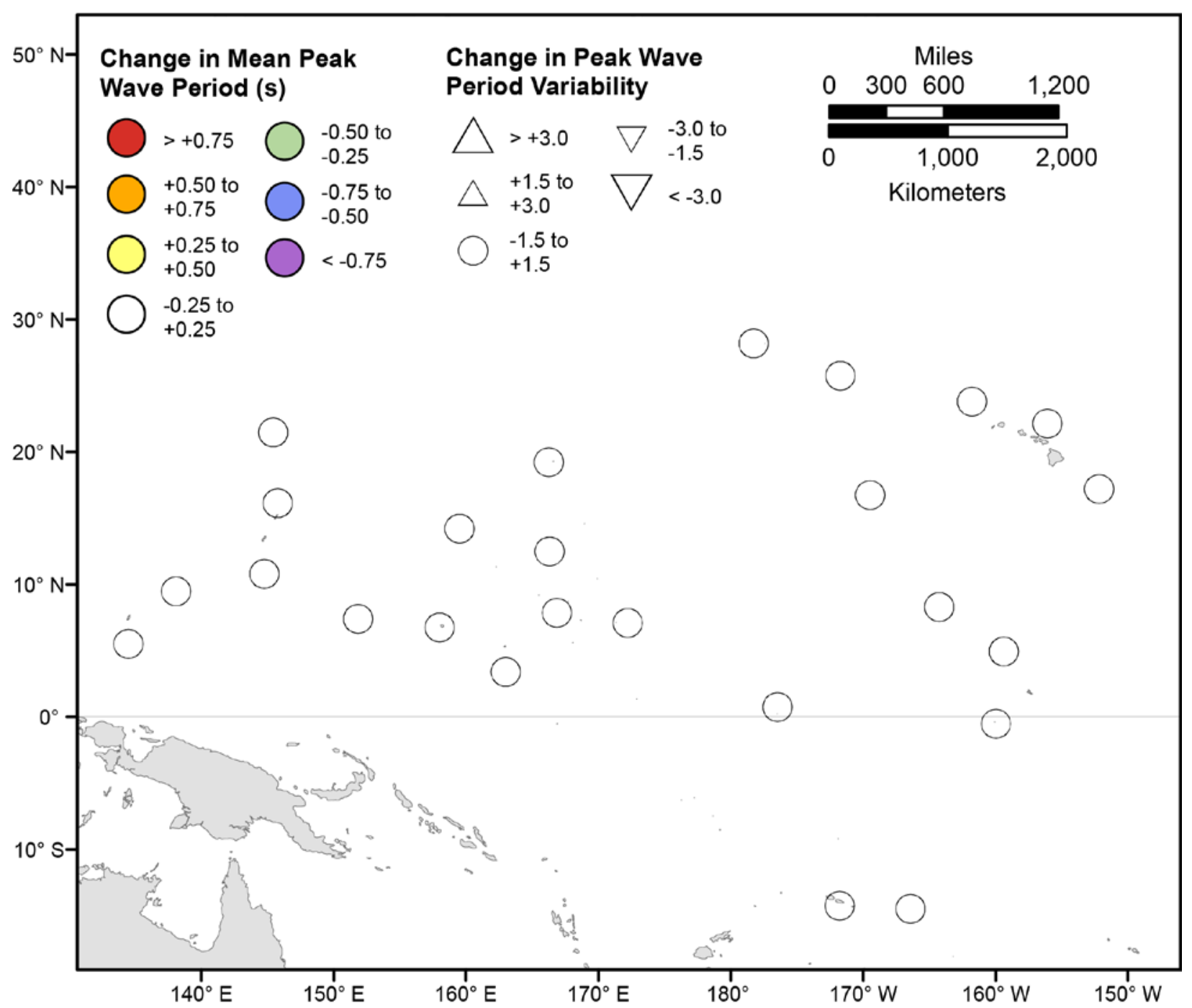

Figure 39. Map showing forecasted differences in mean peak wave period and variance in peak wave period for the years 2026-2045 from hindcasted values during the March-May season under the RCP4.5 future climatic scenario. The colors correspond to the magnitude of change in modeled mean peak wave periods during 2026-2045 from those hindcasted for 1976-2005. The shapes correspond to the magnitude of change in modeled variance in peak wave period during 2026-2045 from those hindcasted for 1976-2005. Units are in seconds. 


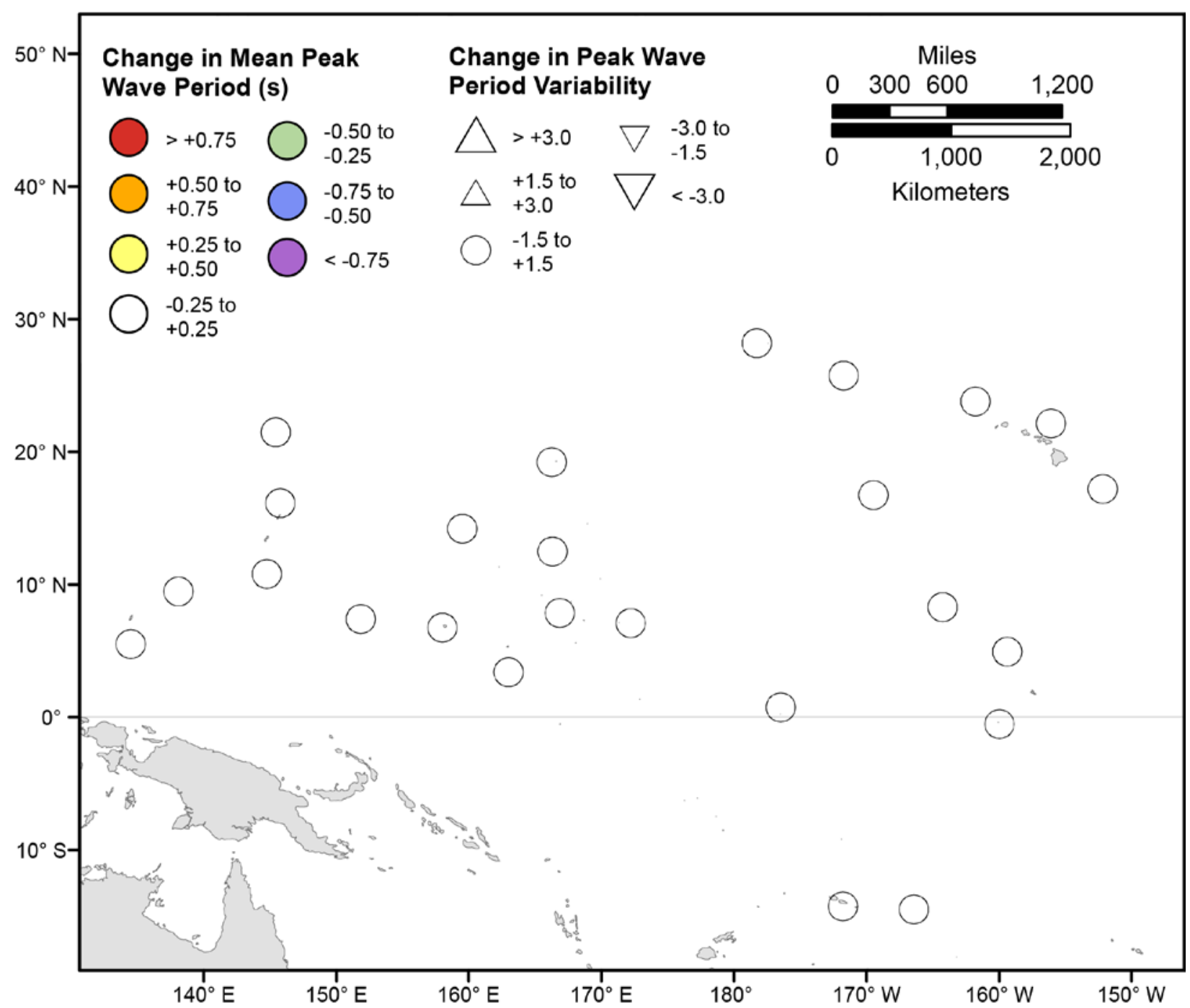

Figure 40. Map showing forecasted differences in mean peak wave period and variance in peak wave period for the years 2026-2045 from hindcasted values during the June-August season under the RCP4.5 future climatic scenario. The colors correspond to the magnitude of change in modeled mean peak wave periods during 2026-2045 from those hindcasted for 1976-2005. The shapes correspond to the magnitude of change in modeled variance in peak wave period during 2026-2045 from those hindcasted for 1976-2005. Units are in seconds. 


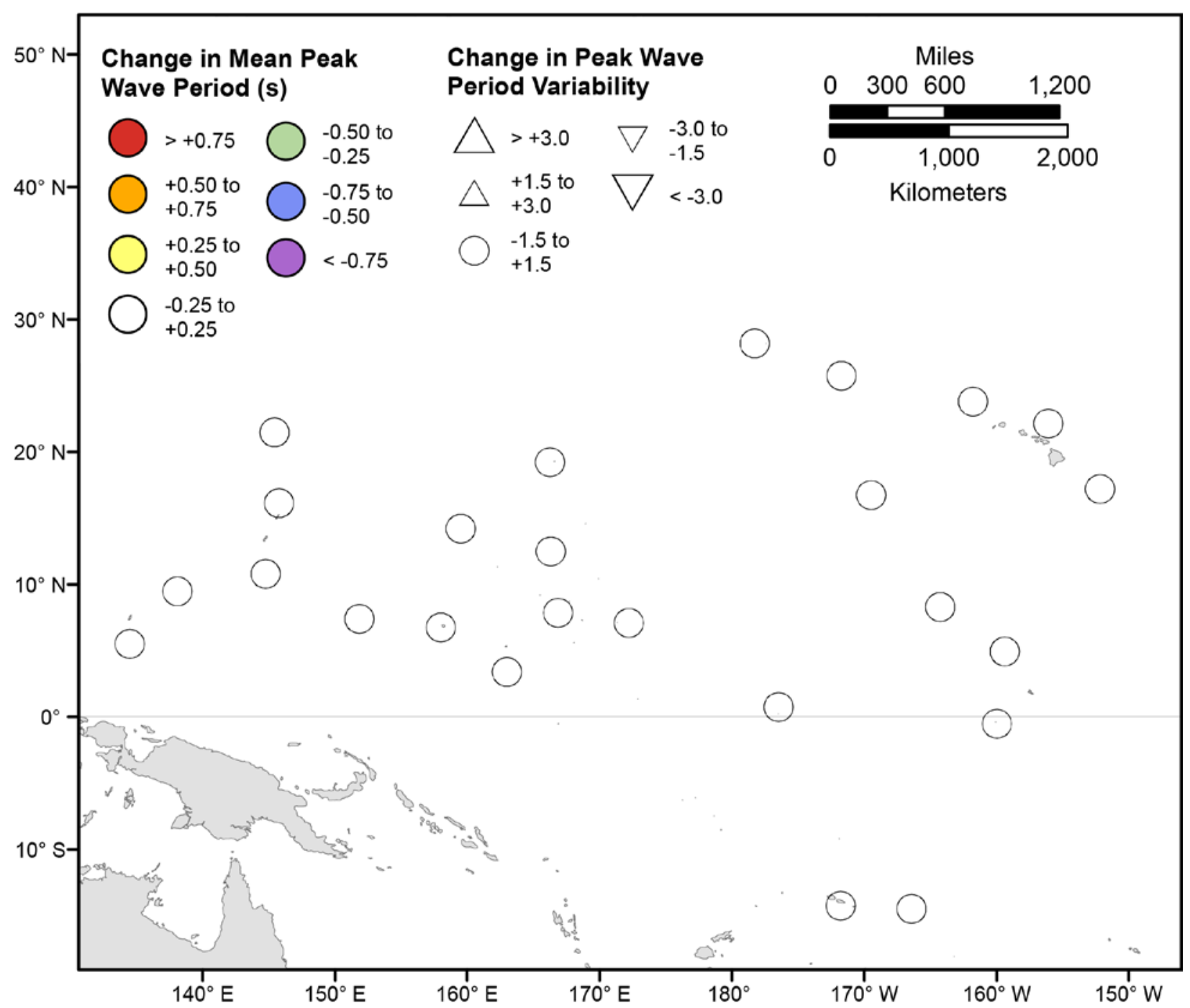

Figure 41. Map showing forecasted differences in mean peak wave period and variance in peak wave period for the years 2026-2045 from hindcasted values during the September-November season under the RCP4.5 future climatic scenario. The colors correspond to the magnitude of change in modeled mean peak wave periods during 2026-2045 from those hindcasted for 1976-2005. The shapes correspond to the magnitude of change in modeled variance in peak wave period during 2026-2045 from those hindcasted for 1976-2005. Units are in seconds. 


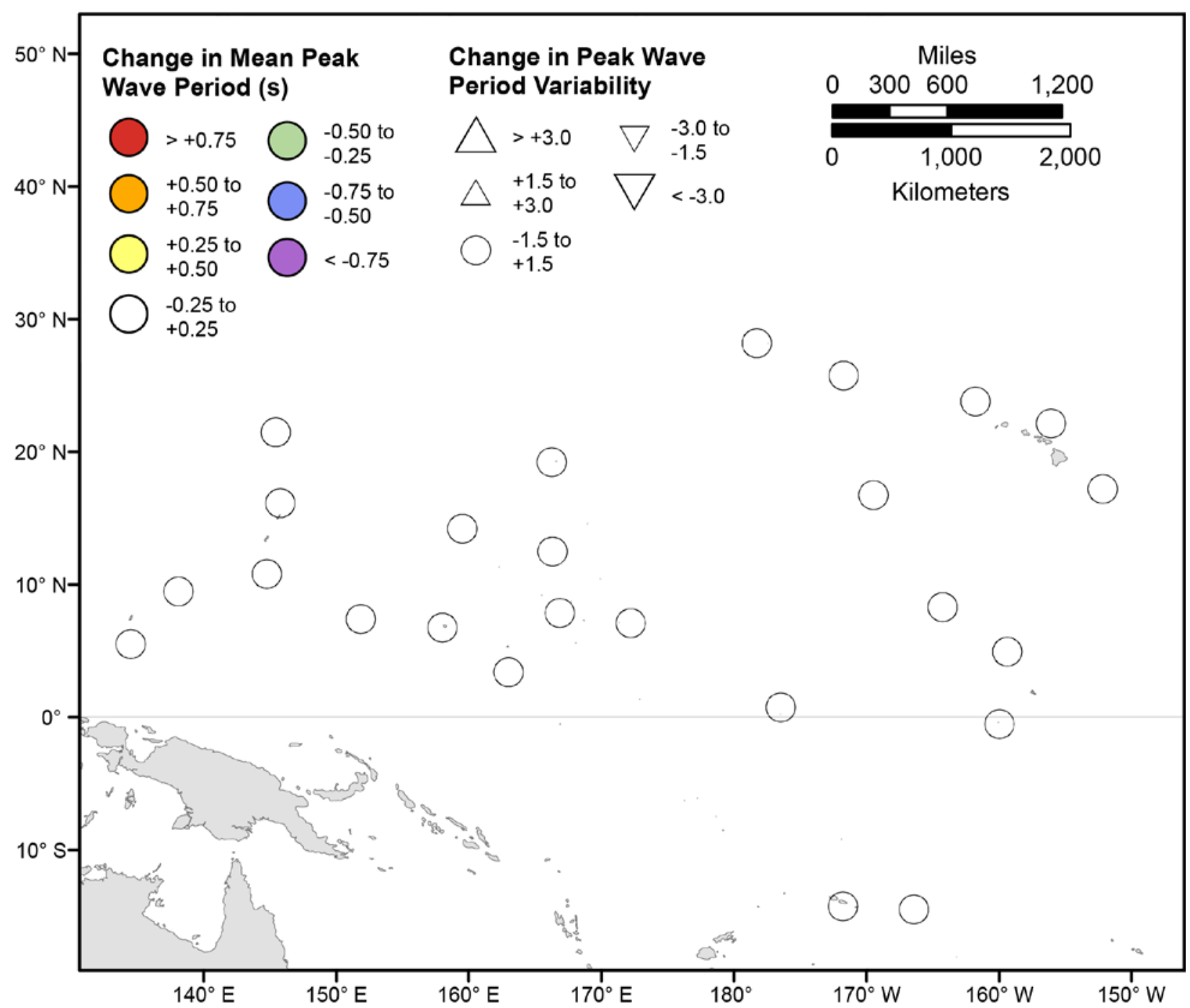

Figure 42. Map showing forecasted differences in the mean peak wave period of the top 5 percent of significant wave heights and variance in the peak wave period of top 5 percent of significant wave heights for the years 2026-2045 from hindcasted values during the December-February season under the RCP4.5 future climatic scenario. The colors correspond to the magnitude of change in modeled mean peak wave periods during 2026-2045 from those hindcasted for 1976-2005. The shapes correspond to the magnitude of change in modeled variance in peak wave period during 2026-2045 from those hindcasted for 1976-2005. Units are in seconds. 


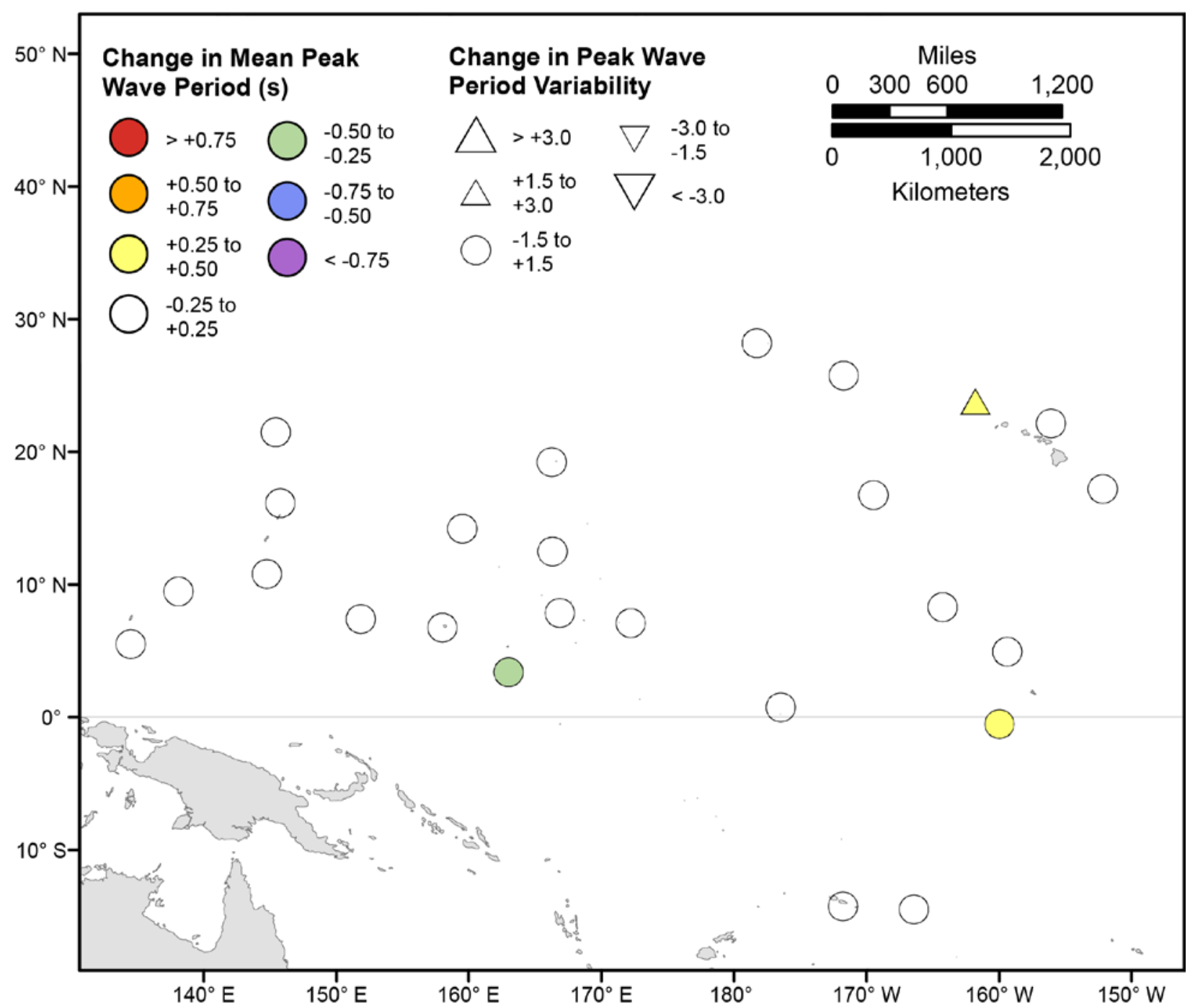

Figure 43. Map showing forecasted differences in the mean peak wave period of the top 5 percent of significant wave heights and variance in the peak wave period of top 5 percent of significant wave heights for the years 2026-2045 from hindcasted values during the March-May season under the RCP4.5 future climatic scenario. The colors correspond to the magnitude of change in modeled mean peak wave periods during 2026-2045 from those hindcasted for 1976-2005. The shapes correspond to the magnitude of change in modeled variance in peak wave period during 2026-2045 from those hindcasted for 1976-2005. Units are in seconds. 


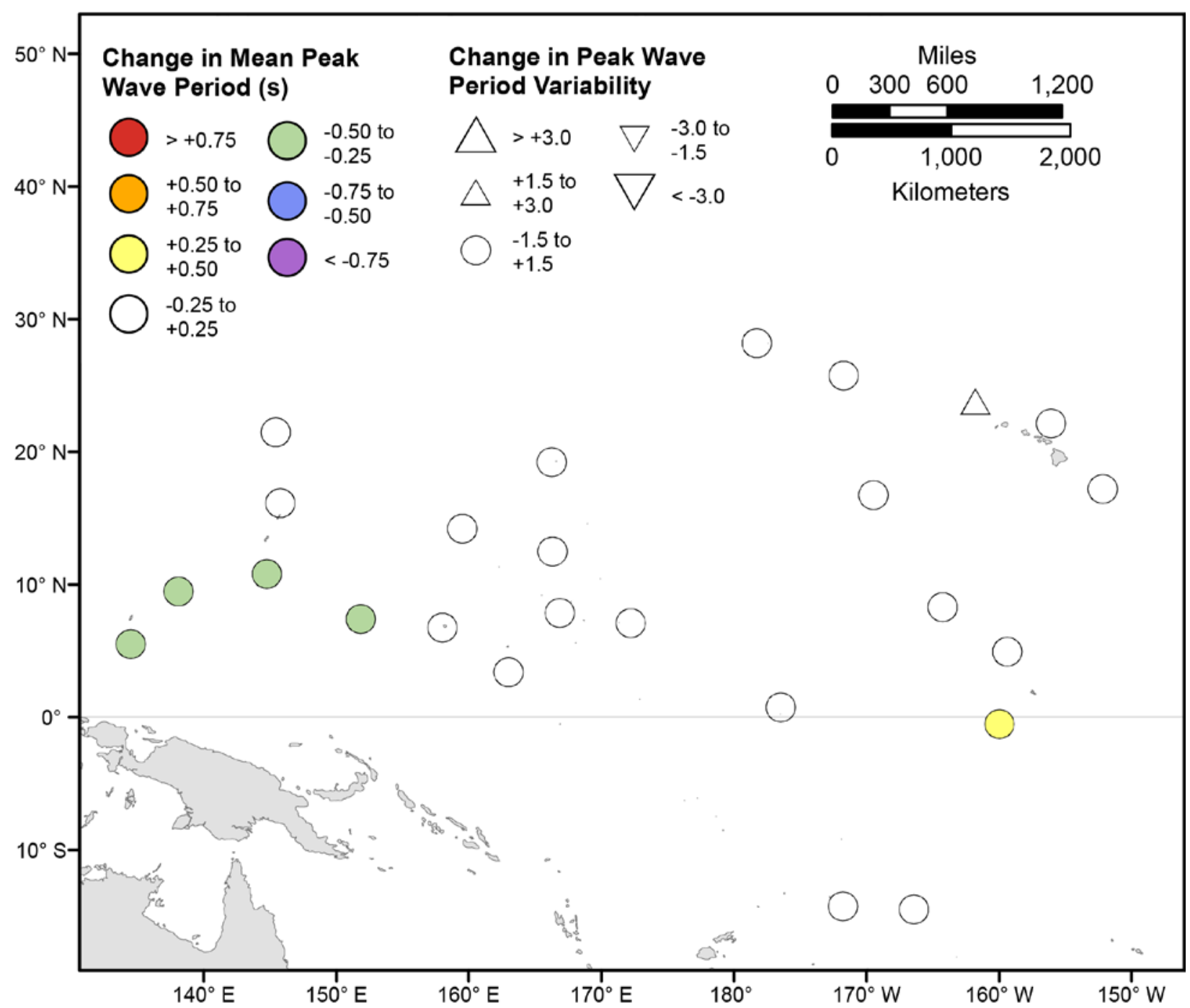

Figure 44. Map showing forecasted differences in the mean peak wave period of the top 5 percent of significant wave heights and variance in the peak wave period of top 5 percent of significant wave heights for the years 2026-2045 from hindcasted values during the June-August season under the RCP4.5 future climatic scenario. The colors correspond to the magnitude of change in modeled mean peak wave periods during 2026-2045 from those hindcasted for 1976-2005. The shapes correspond to the magnitude of change in modeled variance in peak wave period during 2026-2045 from those hindcasted for 1976-2005. Units are in seconds. 


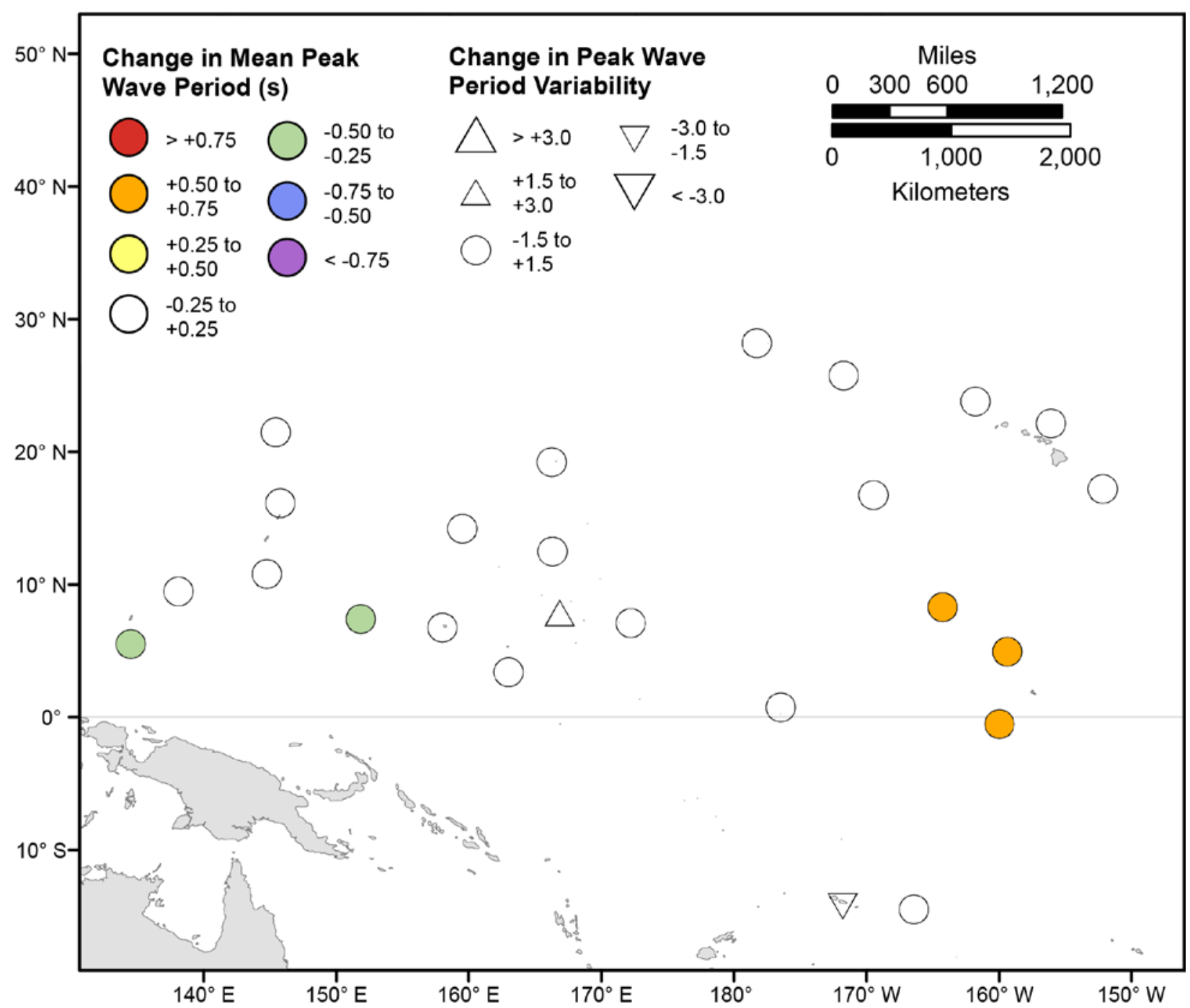

Figure 45. Map showing forecasted differences in the mean peak wave period of the top 5 percent of significant wave heights and variance in the peak wave period of top 5 percent of significant wave heights for the years 2026-2045 from hindcasted values during the September-November season under the RCP4.5 future climatic scenario. The colors correspond to the magnitude of change in modeled mean peak wave periods during 2026-2045 from those hindcasted for 1976-2005. The shapes correspond to the magnitude of change in modeled variance in peak wave period during 2026-2045 from those hindcasted for 1976-2005. Units are in seconds. 


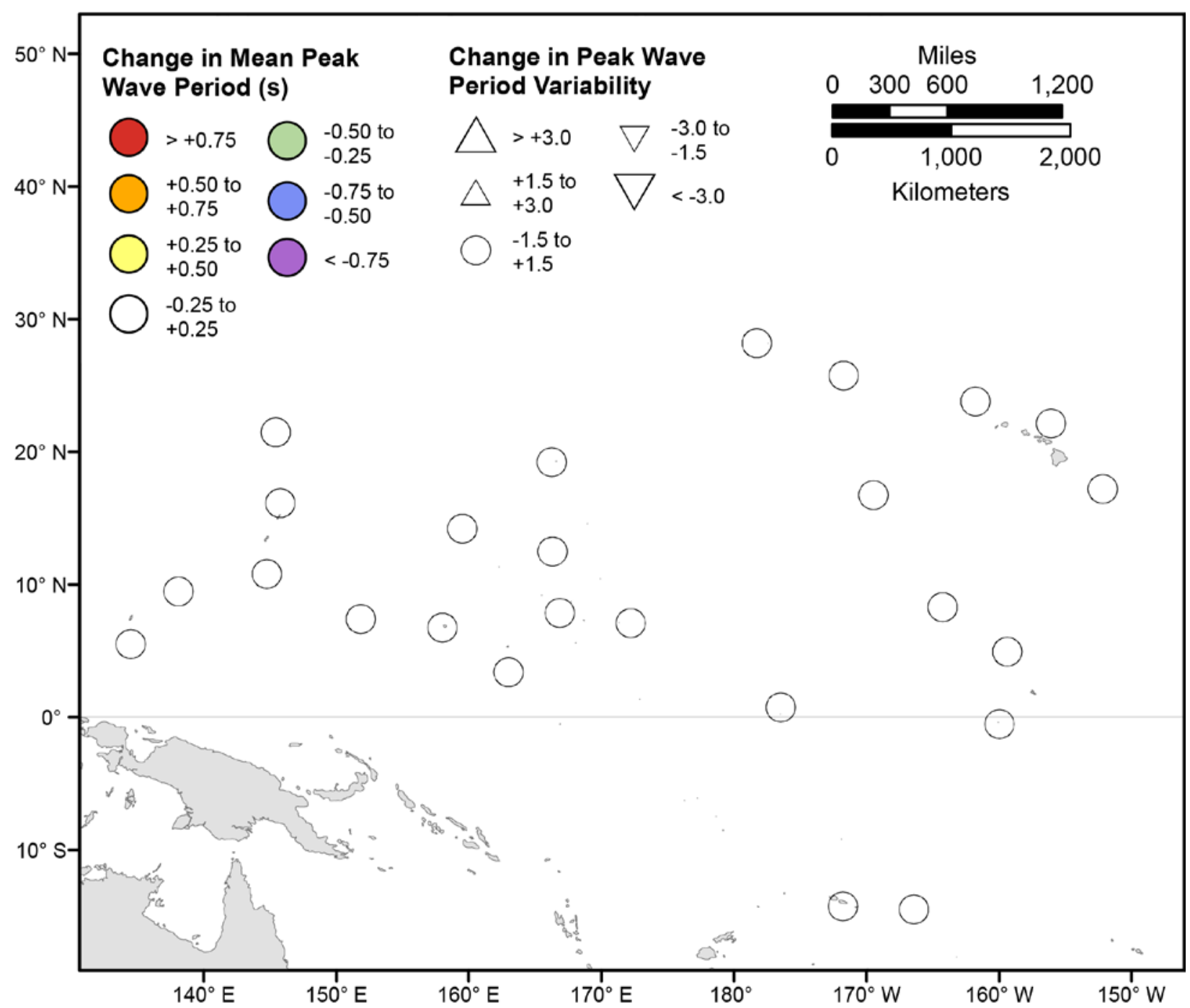

Figure 46. Map showing forecasted differences in mean peak wave period and variance in peak wave period for the years 2026-2045 from hindcasted values during the December-February season under the RCP8.5 future climatic scenario. The colors correspond to the magnitude of change in modeled mean peak wave periods during 2026-2045 from those hindcasted for 1976-2005. The shapes correspond to the magnitude of change in modeled variance in peak wave period during 2026-2045 from those hindcasted for 1976-2005. Units are in seconds. 


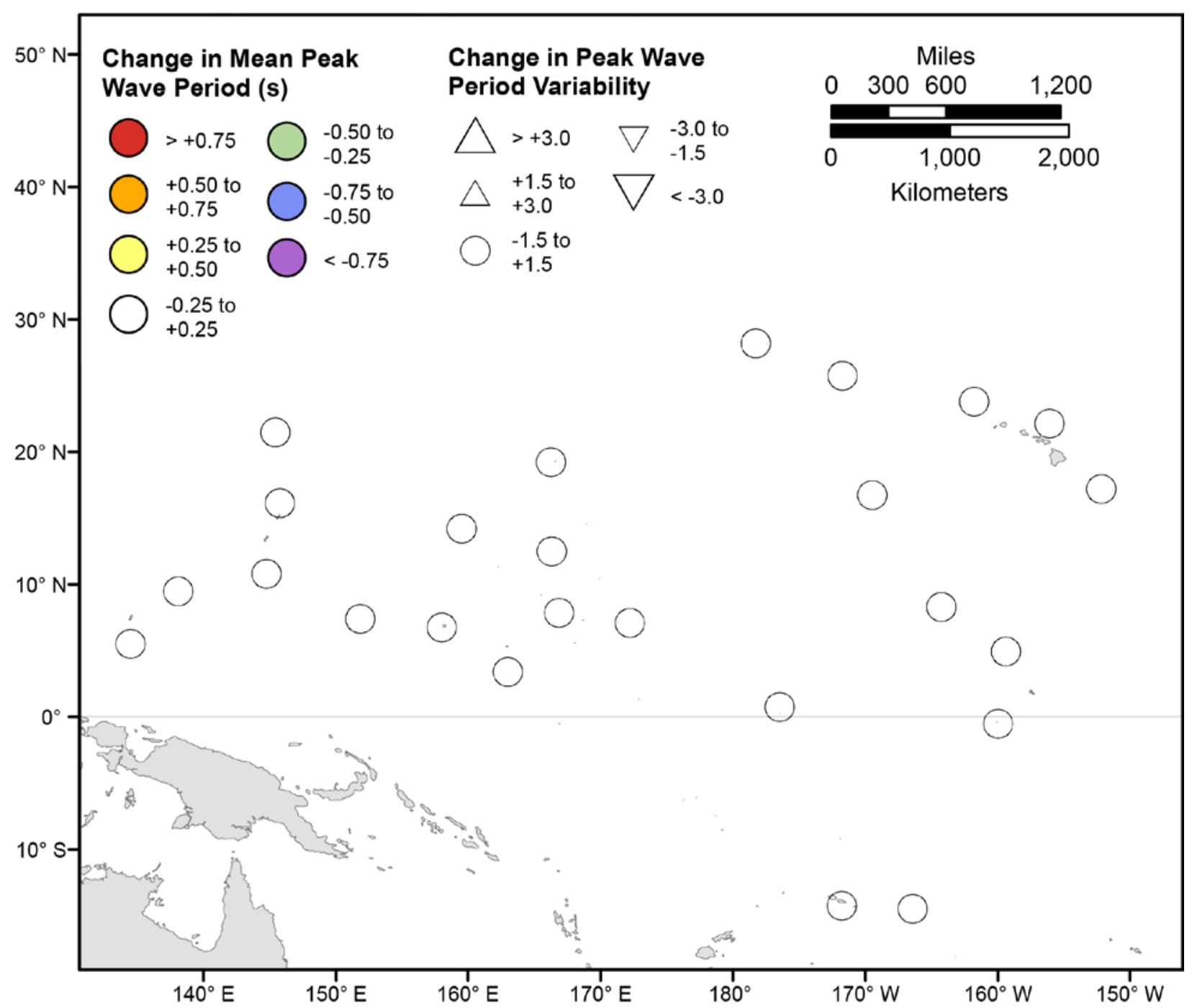

Figure 47. Map showing forecasted differences in mean peak wave period and variance in peak wave period for the years 2026-2045 from hindcasted values during the March-May season under the RCP8.5 future climatic scenario. The colors correspond to the magnitude of change in modeled mean peak wave periods during 2026-2045 from those hindcasted for 1976-2005. The shapes correspond to the magnitude of change in modeled variance in peak wave period during 2026-2045 from those hindcasted for 1976-2005. Units are in seconds. 


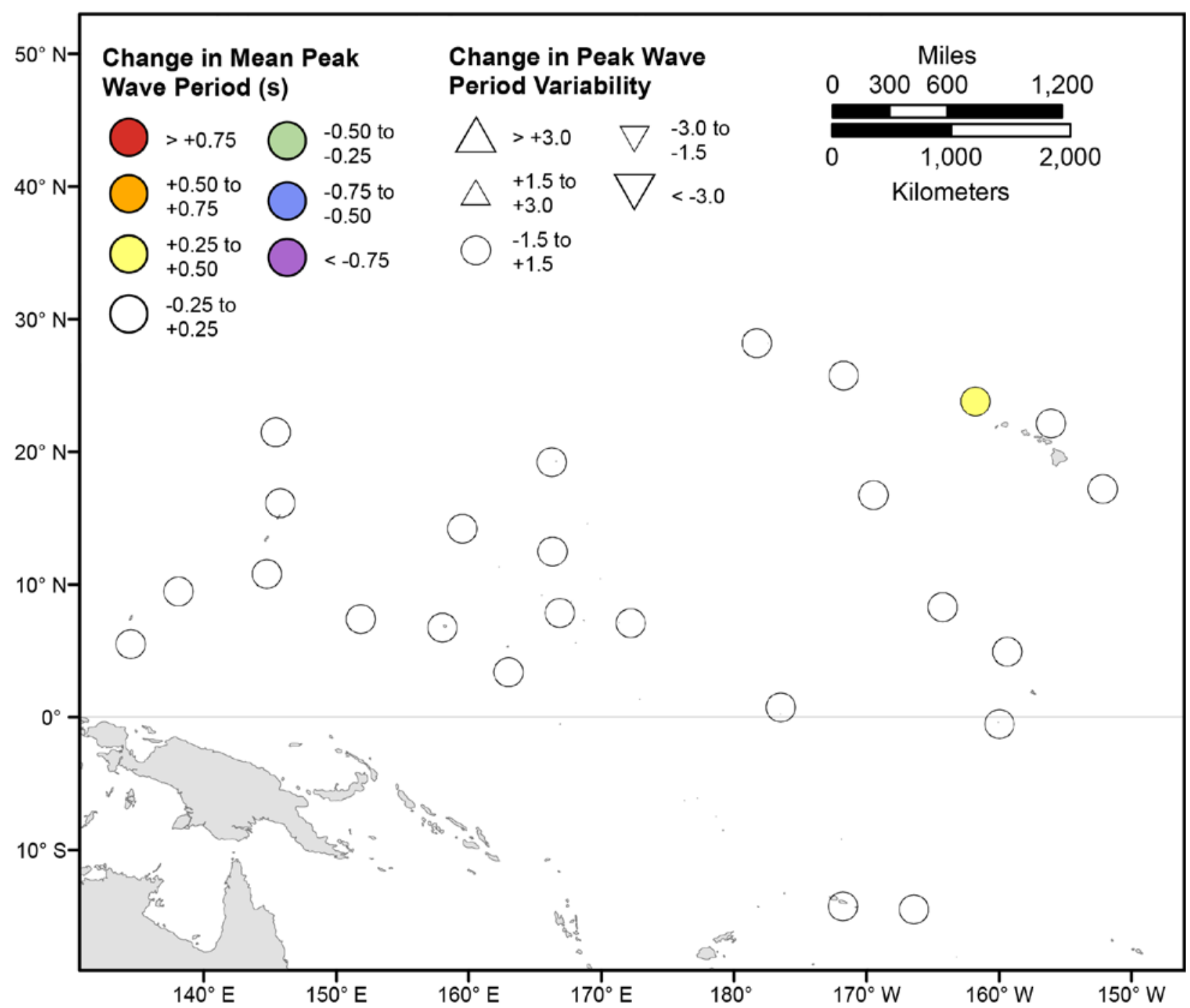

Figure 48. Map showing forecasted differences in mean peak wave period and variance in peak wave period for the years 2026-2045 from hindcasted values during the June-August season under the RCP8.5 future climatic scenario. The colors correspond to the magnitude of change in modeled mean peak wave periods during 2026-2045 from those hindcasted for 1976-2005. The shapes correspond to the magnitude of change in modeled variance in peak wave period during 2026-2045 from those hindcasted for 1976-2005. Units are in seconds. 


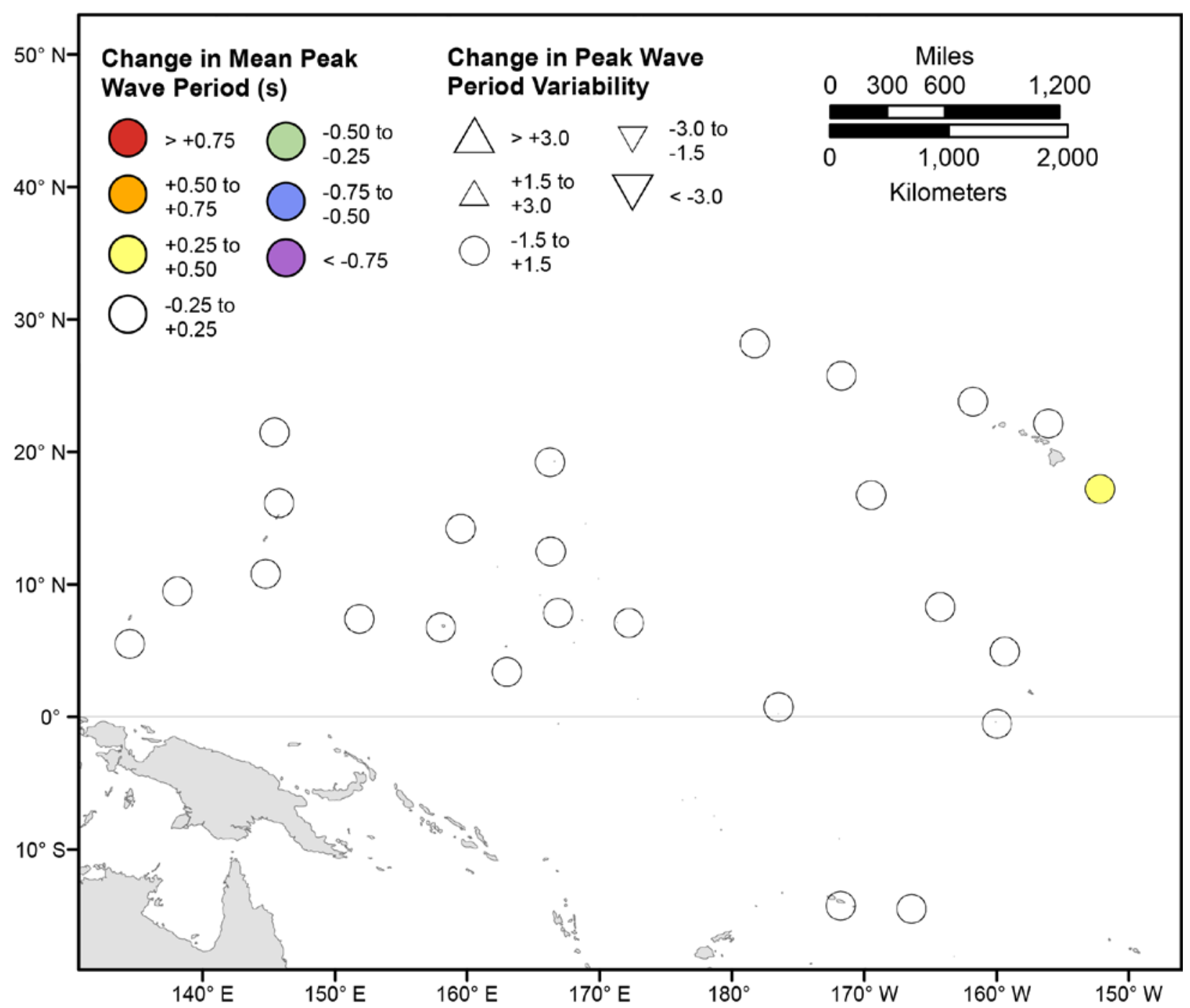

Figure 49. Map showing forecasted differences in mean peak wave period and variance in peak wave period for the years 2026-2045 from hindcasted values during the September-November season under the RCP8.5 future climatic scenario. The colors correspond to the magnitude of change in modeled mean peak wave periods during 2026-2045 from those hindcasted for 1976-2005. The shapes correspond to the magnitude of change in modeled variance in peak wave period during 2026-2045 from those hindcasted for 1976-2005. Units are in seconds. 


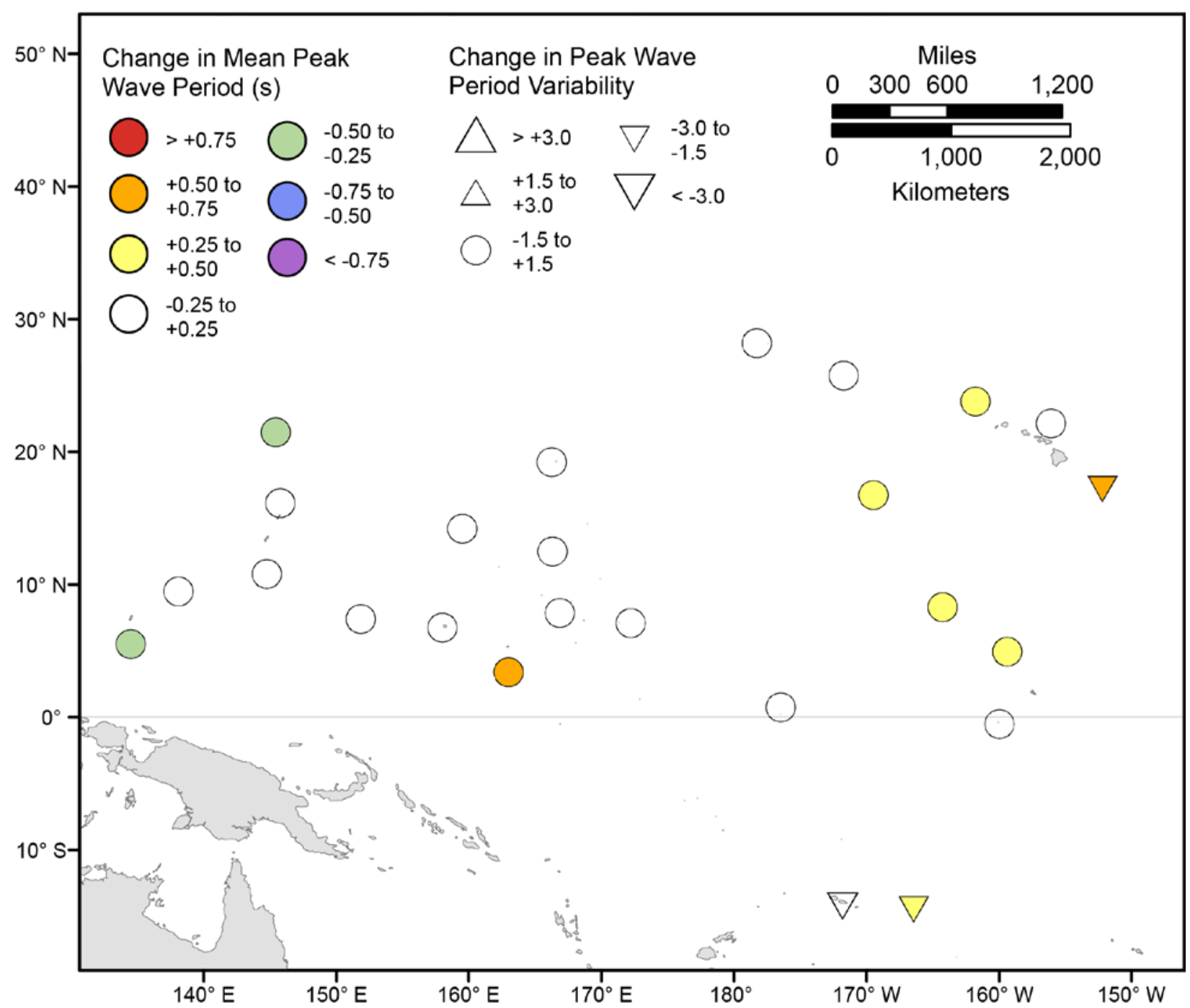

Figure 50. Map showing forecasted differences in the mean peak wave period of the top 5 percent of significant wave heights and variance in the peak wave period of top 5 percent of significant wave heights for the years 2026-2045 from hindcasted values during the December-February season under the RCP8.5 future climatic scenario. The colors correspond to the magnitude of change in modeled mean peak wave periods during 2026-2045 from those hindcasted for 1976-2005. The shapes correspond to the magnitude of change in modeled variance in peak wave period during 2026-2045 from those hindcasted for 1976-2005. Units are in seconds. 


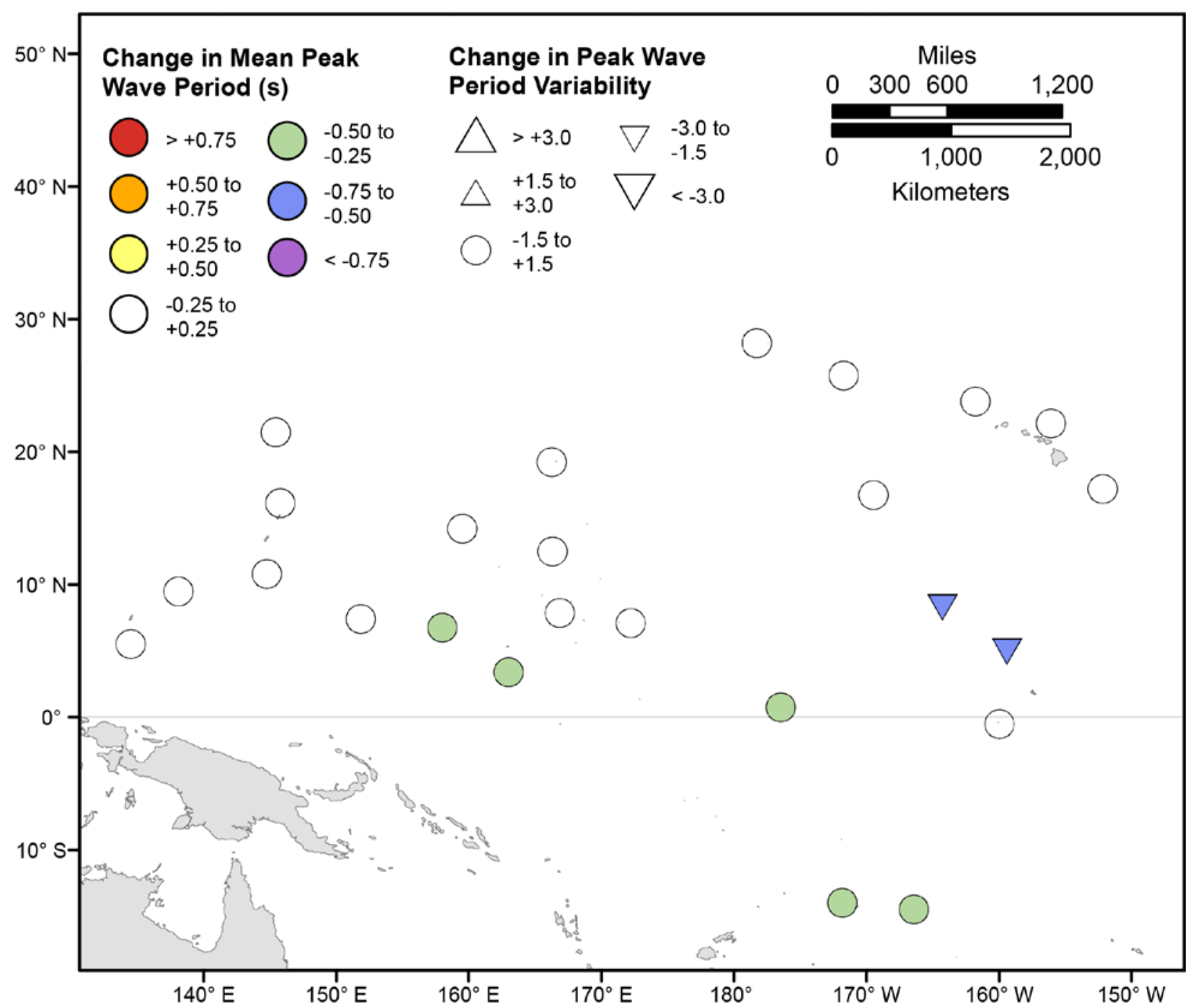

Figure 51. Map showing forecasted differences in the mean peak wave period of the top 5 percent of significant wave heights and variance in the peak wave period of top 5 percent of significant wave heights for the years 2026-2045 from hindcasted values during the March-May season under the RCP8.5 future climatic scenario. The colors correspond to the magnitude of change in modeled mean peak wave periods during 2026-2045 from those hindcasted for 1976-2005. The shapes correspond to the magnitude of change in modeled variance in peak wave period during 2026-2045 from those hindcasted for 1976-2005. Units are in seconds. 


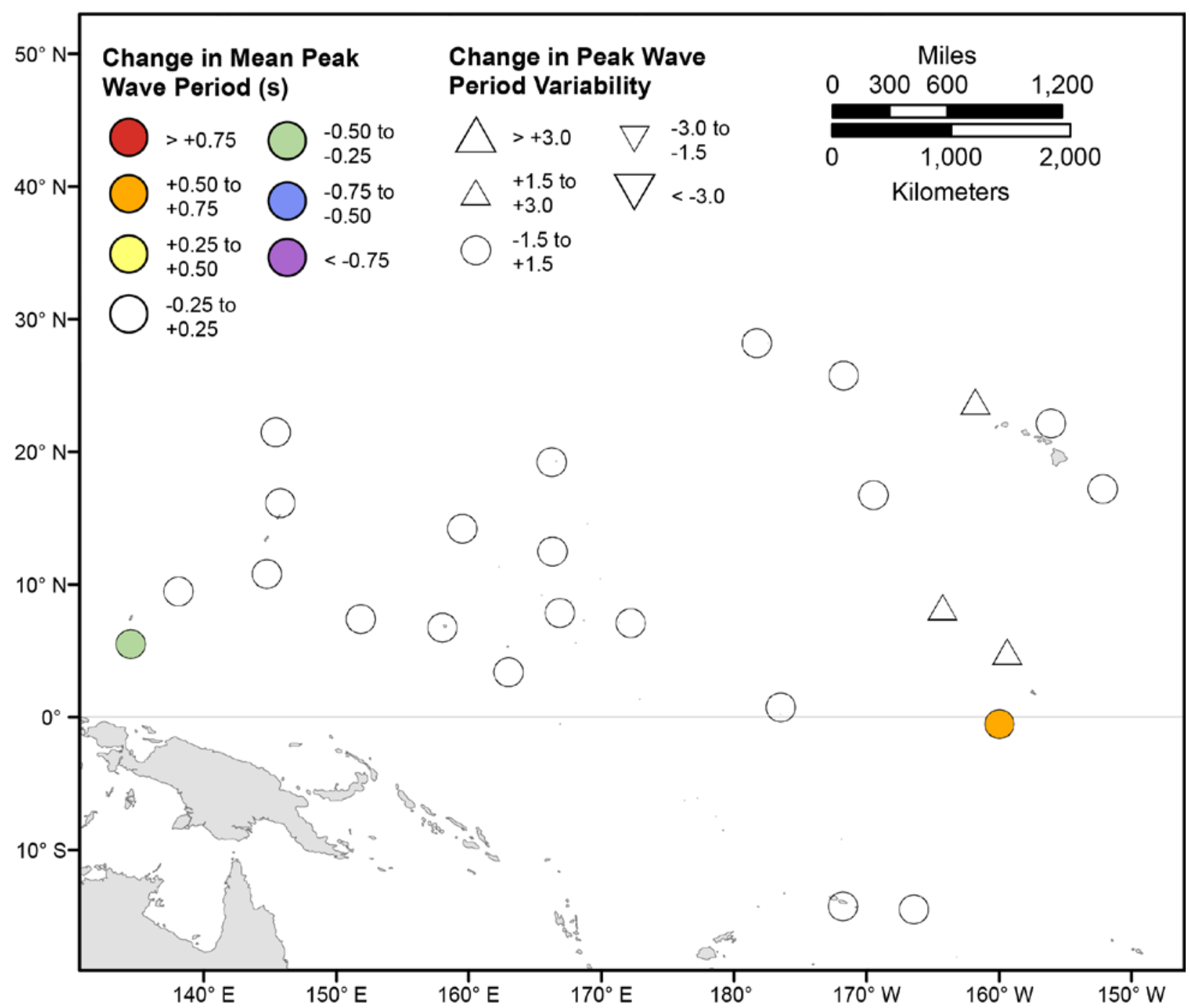

Figure 52. Map showing forecasted differences in the mean peak wave period of the top 5 percent of significant wave heights and variance in the peak wave period of top 5 percent of significant wave heights for the years 2026-2045 from hindcasted values during the June-August season under the RCP8.5 future climatic scenario. The colors correspond to the magnitude of change in modeled mean peak wave periods during 2026-2045 from those hindcasted for 1976-2005. The shapes correspond to the magnitude of change in modeled variance in peak wave period during 2026-2045 from those hindcasted for 1976-2005. Units are in seconds. 


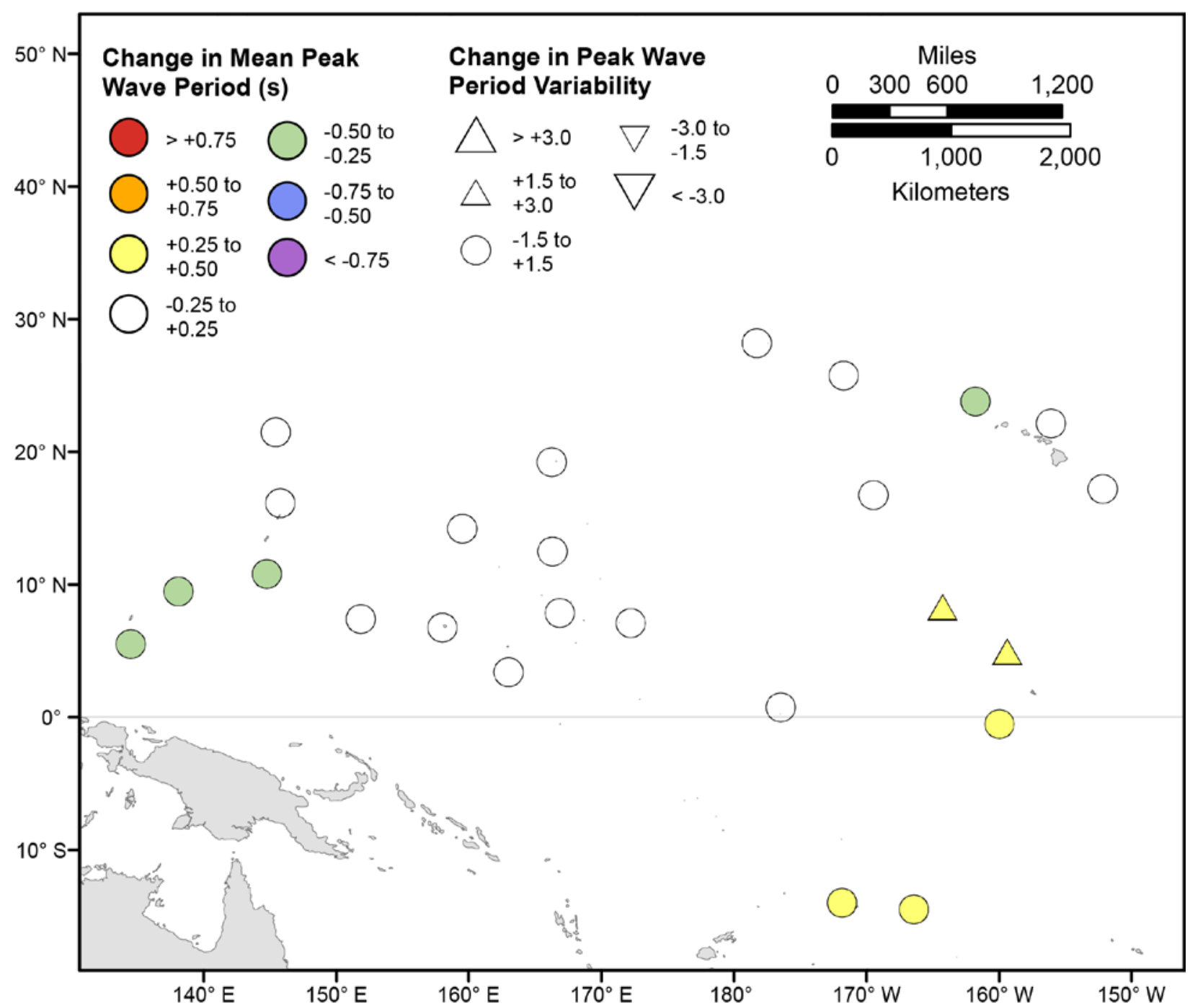

Figure 53. Map showing forecasted differences in the mean peak wave period of the top 5 percent of significant wave heights and variance in the peak wave period of top 5 percent of significant wave heights for the years 2026-2045 from hindcasted values during the September-November season under the RCP8.5 future climatic scenario. The colors correspond to the magnitude of change in modeled mean peak wave periods during 2026-2045 from those hindcasted for 1976-2005. The shapes correspond to the magnitude of change in modeled variance in peak wave period during 2026-2045 from those hindcasted for 1976-2005. Units are in seconds. 


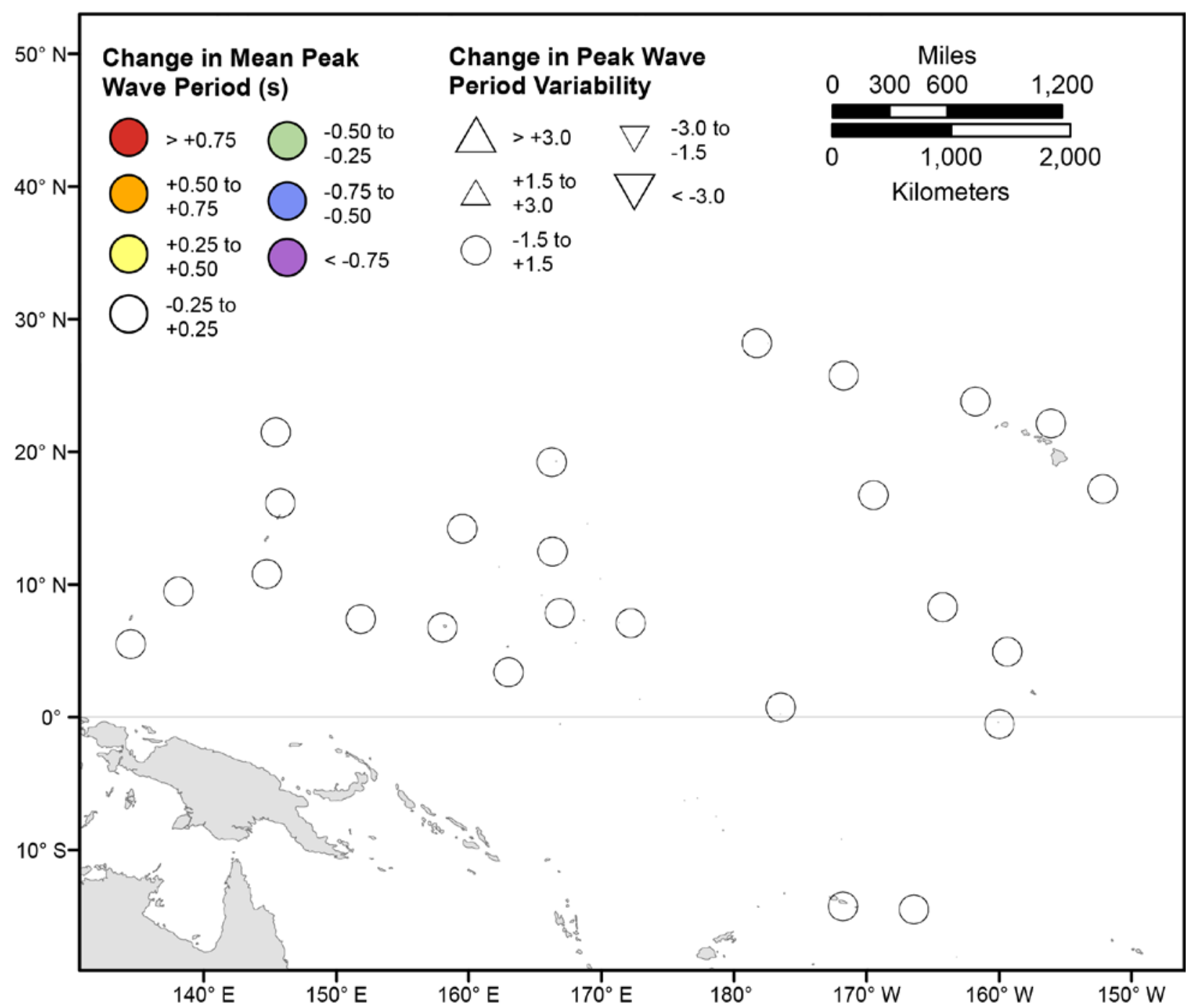

Figure 54. Map showing forecasted differences in mean peak wave period and variance in peak wave period for the years 2081-2100 from hindcasted values during the December-February season under the RCP4.5 future climatic scenario. The colors correspond to the magnitude of change in modeled mean peak wave periods during 2081-2100 from those hindcasted for 1976-2005. The shapes correspond to the magnitude of change in modeled variance in peak wave period during 2081-2100 from those hindcasted for 1976-2005. Units are in seconds. 


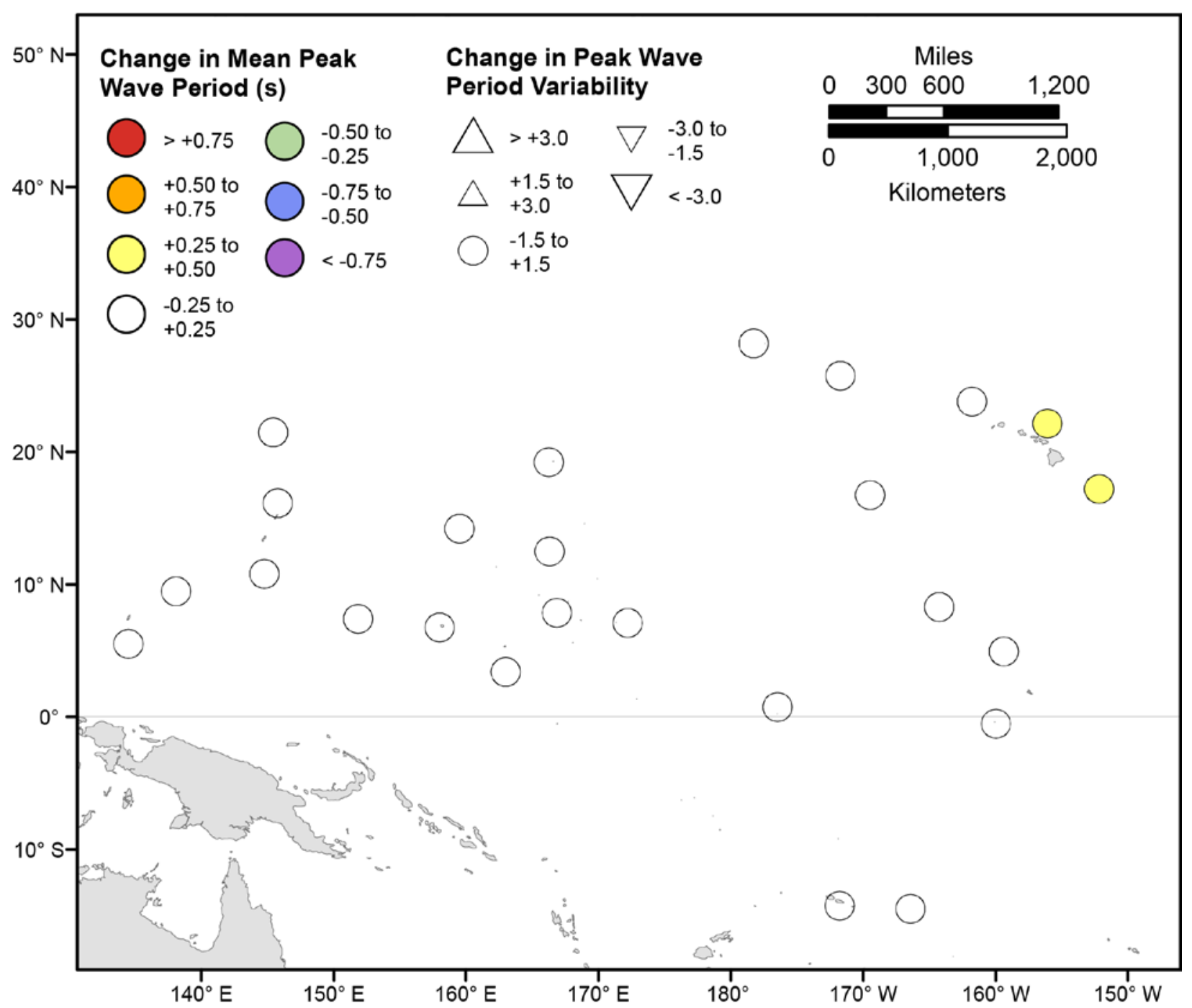

Figure 55. Map showing forecasted differences in mean peak wave period and variance in peak wave period for the years 2081-2100 from hindcasted values during the March-May season under the RCP4.5 future climatic scenario. The colors correspond to the magnitude of change in modeled mean peak wave periods during 2081-2100 from those hindcasted for 1976-2005. The shapes correspond to the magnitude of change in modeled variance in peak wave period during 2081-2100 from those hindcasted for 1976-2005. Units are in seconds. 


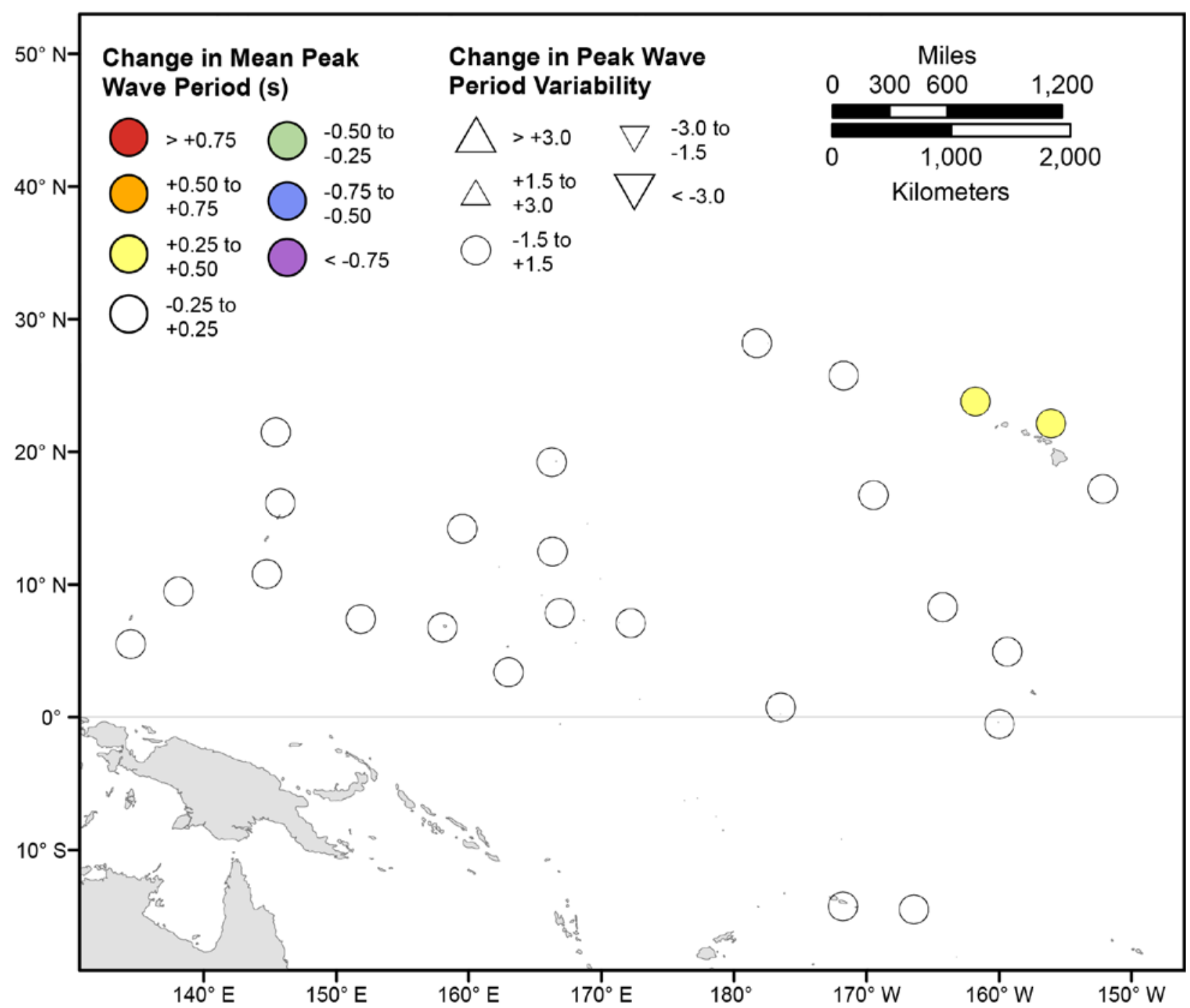

Figure 56. Map showing forecasted differences in mean peak wave period and variance in peak wave period for the years 2081-2100 from hindcasted values during the June-August season under the RCP4.5 future climatic scenario. The colors correspond to the magnitude of change in modeled mean peak wave periods during 2081-2100 from those hindcasted for 1976-2005. The shapes correspond to the magnitude of change in modeled variance in peak wave period during 2081-2100 from those hindcasted for 1976-2005. Units are in seconds. 


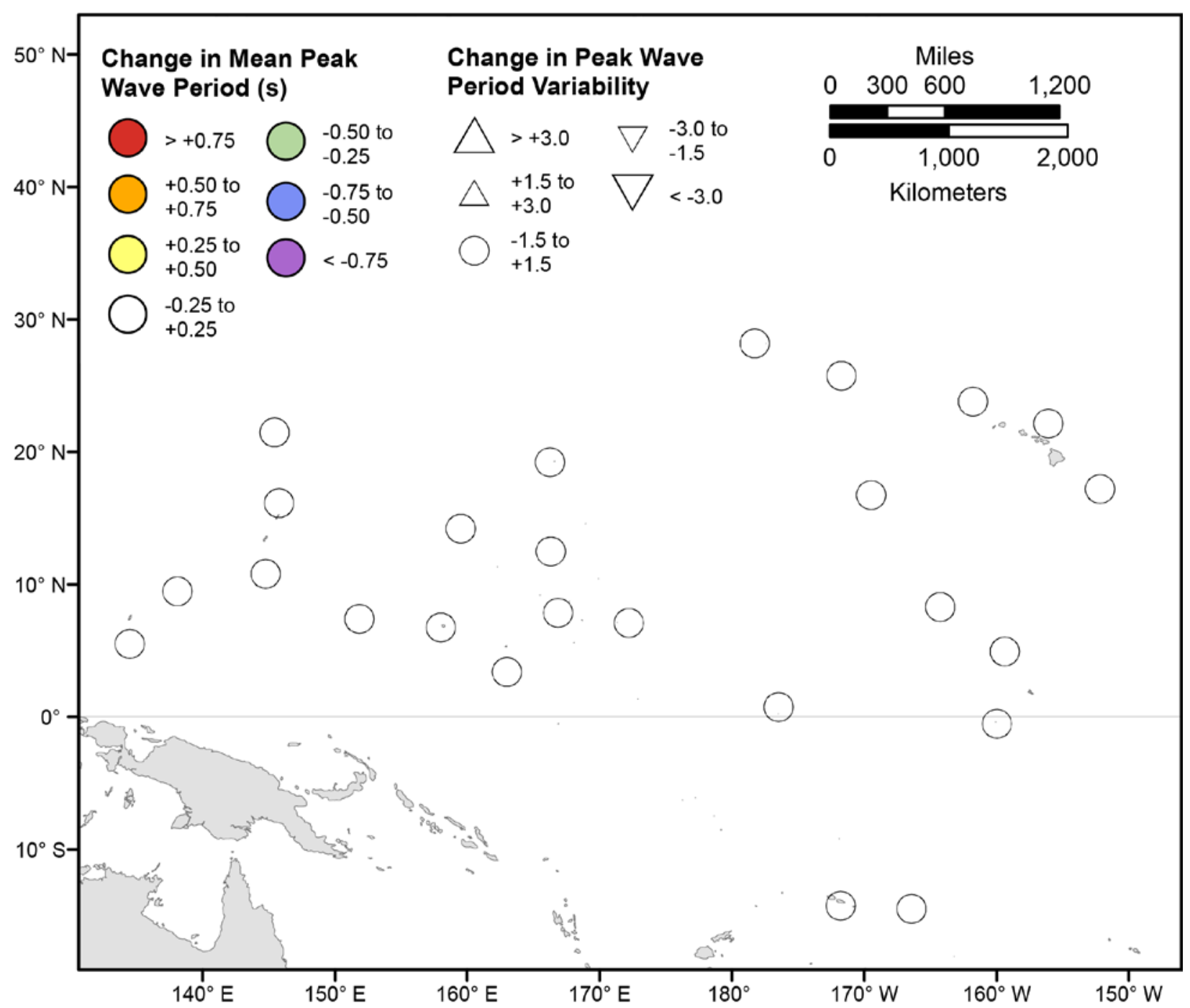

Figure 57. Map showing forecasted differences in mean peak wave period and variance in peak wave period for the years 2081-2100 from hindcasted values during the September-November season under the RCP4.5 future climatic scenario. The colors correspond to the magnitude of change in modeled mean peak wave periods during 2081-2100 from those hindcasted for 1976-2005. The shapes correspond to the magnitude of change in modeled variance in peak wave period during 2081-2100 from those hindcasted for 1976-2005. Units are in seconds. 


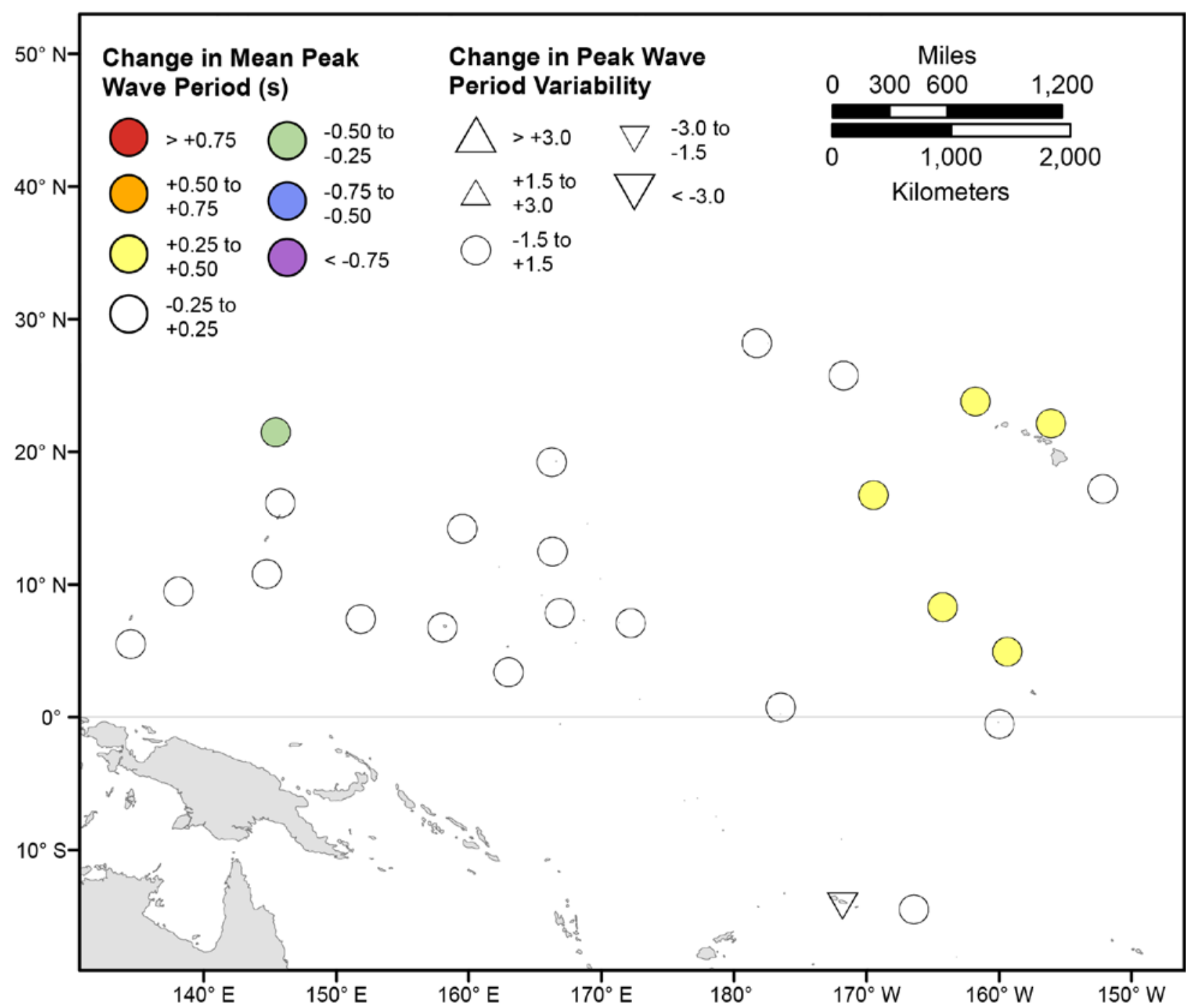

Figure 58. Map showing forecasted differences in the mean peak wave period of the top 5 percent of significant wave heights and variance in the peak wave period of top 5 percent of significant wave heights for the years 2081-2100 from hindcasted values during the December-February season under the RCP4.5 future climatic scenario. The colors correspond to the magnitude of change in modeled mean peak wave periods during 2081-2100 from those hindcasted for 1976-2005. The shapes correspond to the magnitude of change in modeled variance in peak wave period during 2081-2100 from those hindcasted for 1976-2005. Units are in seconds. 


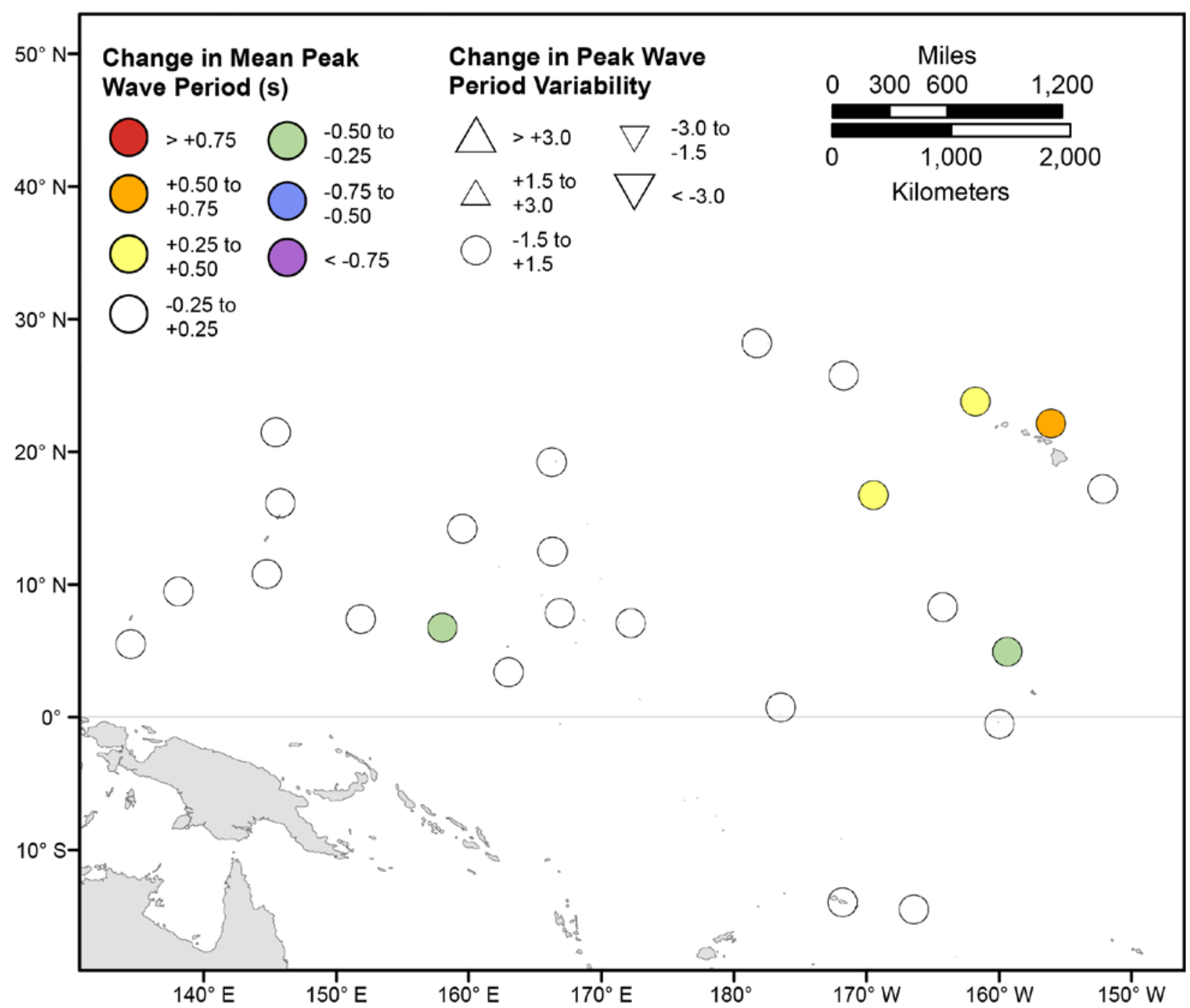

Figure 59. Map showing forecasted differences in the mean peak wave period of the top 5 percent of significant wave heights and variance in the peak wave period of top 5 percent of significant wave heights for the years 2081-2100 from hindcasted values during the March-May season under the RCP4.5 future climatic scenario. The colors correspond to the magnitude of change in modeled mean peak wave periods during 2081-2100 from those hindcasted for 1976-2005. The shapes correspond to the magnitude of change in modeled variance in peak wave period during 2081-2100 from those hindcasted for 1976-2005. Units are in seconds. 


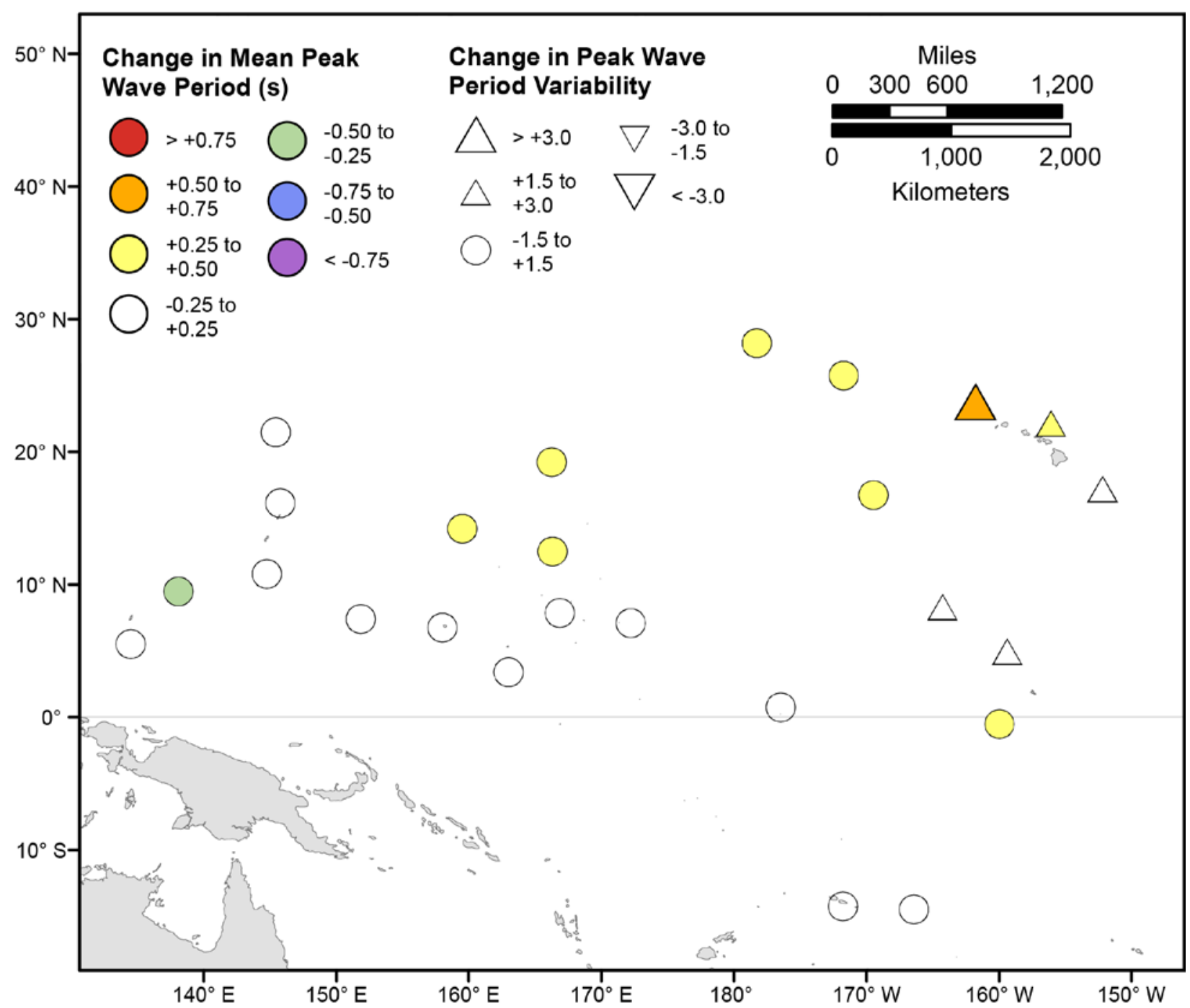

Figure 60. Map showing forecasted differences in the mean peak wave period of the top 5 percent of significant wave heights and variance in the peak wave period of top 5 percent of significant wave heights for the years 2081-2100 from hindcasted values during the June-August season under the RCP4.5 future climatic scenario. The colors correspond to the magnitude of change in modeled mean peak wave periods during 2081-2100 from those hindcasted for 1976-2005. The shapes correspond to the magnitude of change in modeled variance in peak wave period during 2081-2100 from those hindcasted for 1976-2005. Units are in seconds. 


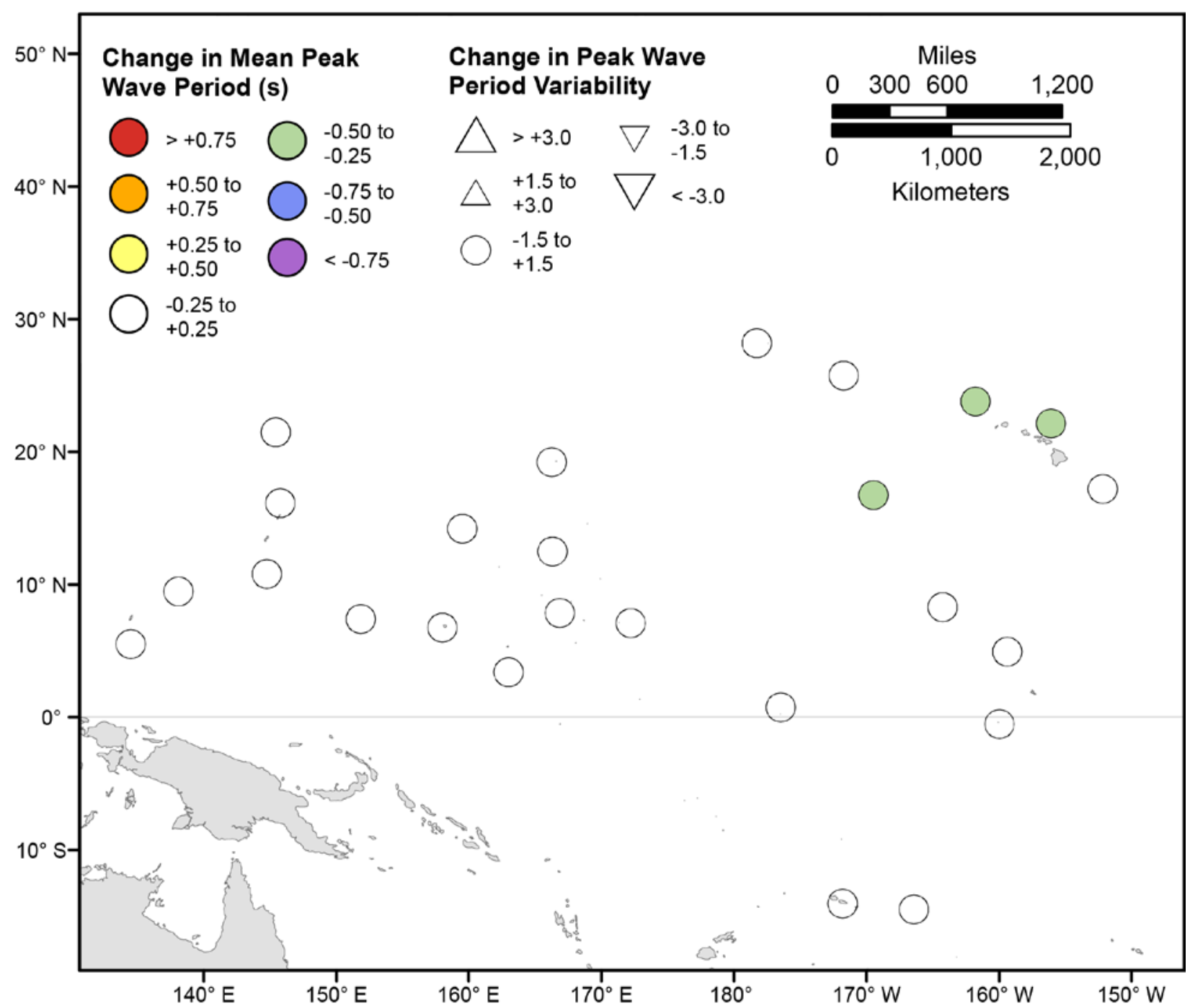

Figure 61. Map showing forecasted differences in the mean peak wave period of the top 5 percent of significant wave heights and variance in the peak wave period of top 5 percent of significant wave heights for the years 2081-2100 from hindcasted values during the September-November season under the RCP4.5 future climatic scenario. The colors correspond to the magnitude of change in modeled mean peak wave periods during 2081-2100 from those hindcasted for 1976-2005. The shapes correspond to the magnitude of change in modeled variance in peak wave period during 2081-2100 from those hindcasted for 1976-2005. Units are in seconds. 


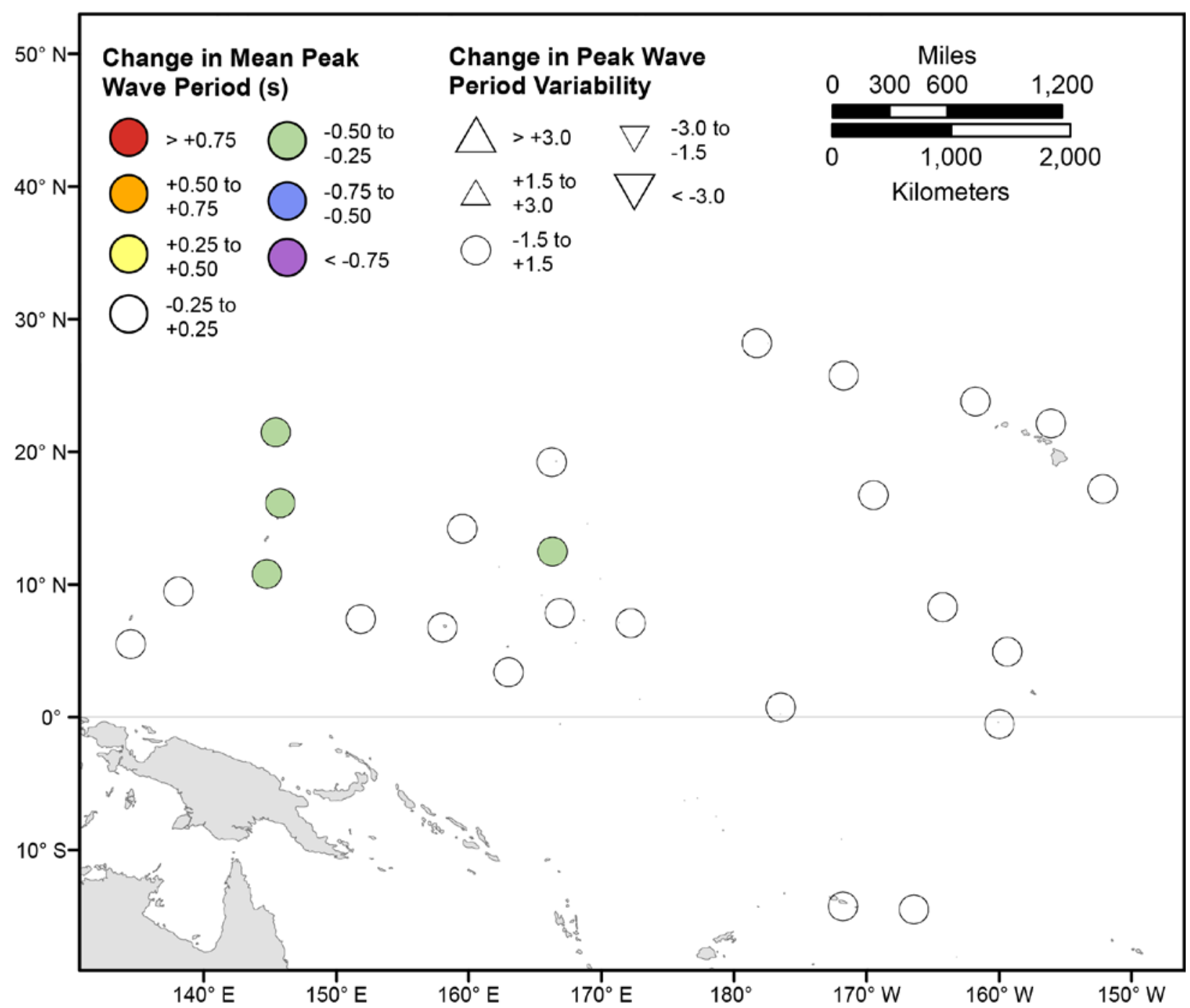

Figure 62. Map showing forecasted differences in mean peak wave period and variance in peak wave period for the years 2081-2100 from hindcasted values during the December-February season under the RCP8.5 future climatic scenario. The colors correspond to the magnitude of change in modeled mean peak wave periods during 2081-2100 from those hindcasted for 1976-2005. The shapes correspond to the magnitude of change in modeled variance in peak wave period during 2081-2100 from those hindcasted for 1976-2005. Units are in seconds. 


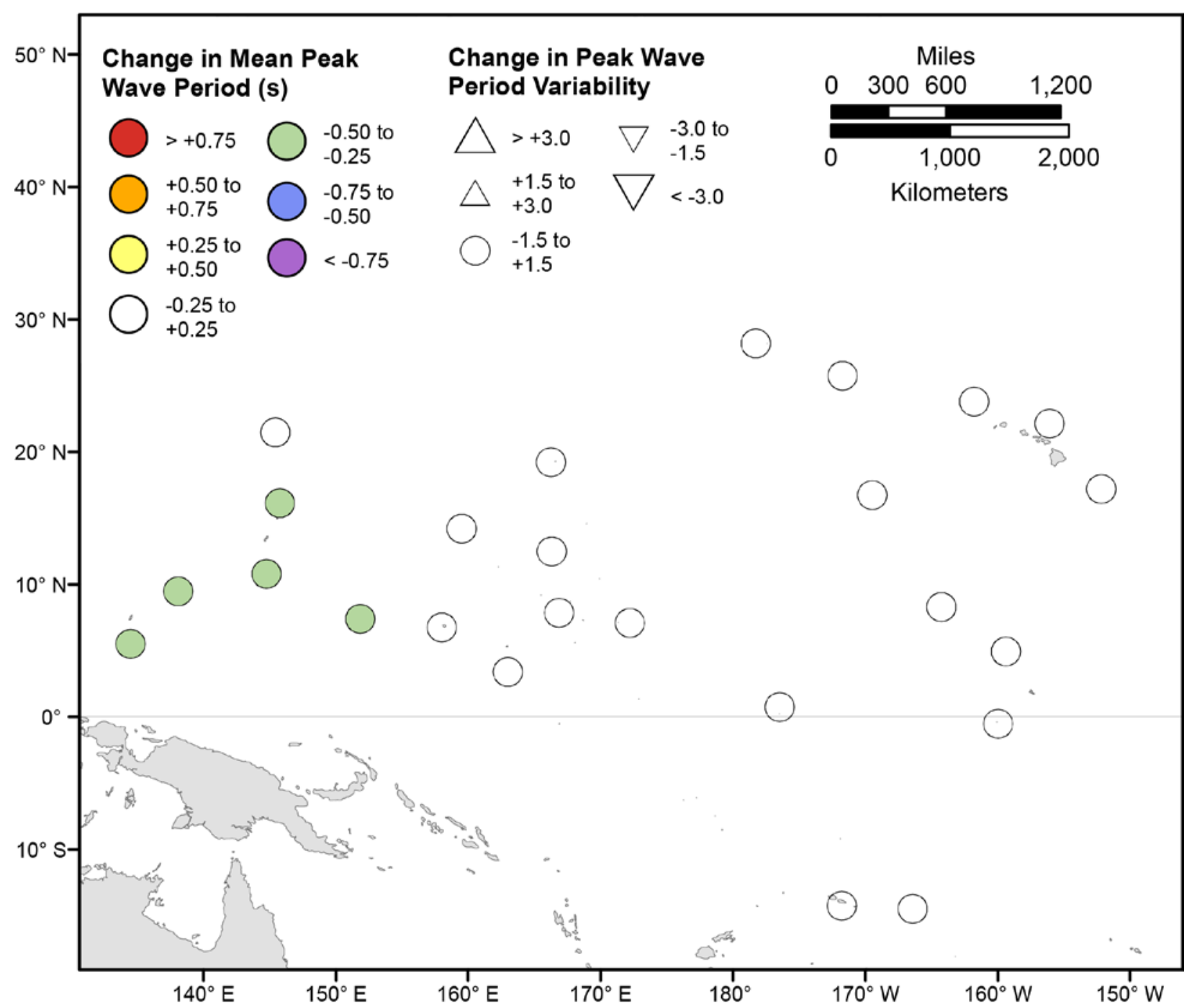

Figure 63. Map showing forecasted differences in mean peak wave period and variance in peak wave period for the years 2081-2100 from hindcasted values during the March-May season under the RCP8.5 future climatic scenario. The colors correspond to the magnitude of change in modeled mean peak wave periods during 2081-2100 from those hindcasted for 1976-2005. The shapes correspond to the magnitude of change in modeled variance in peak wave period during 2081-2100 from those hindcasted for 1976-2005. Units are in seconds. 


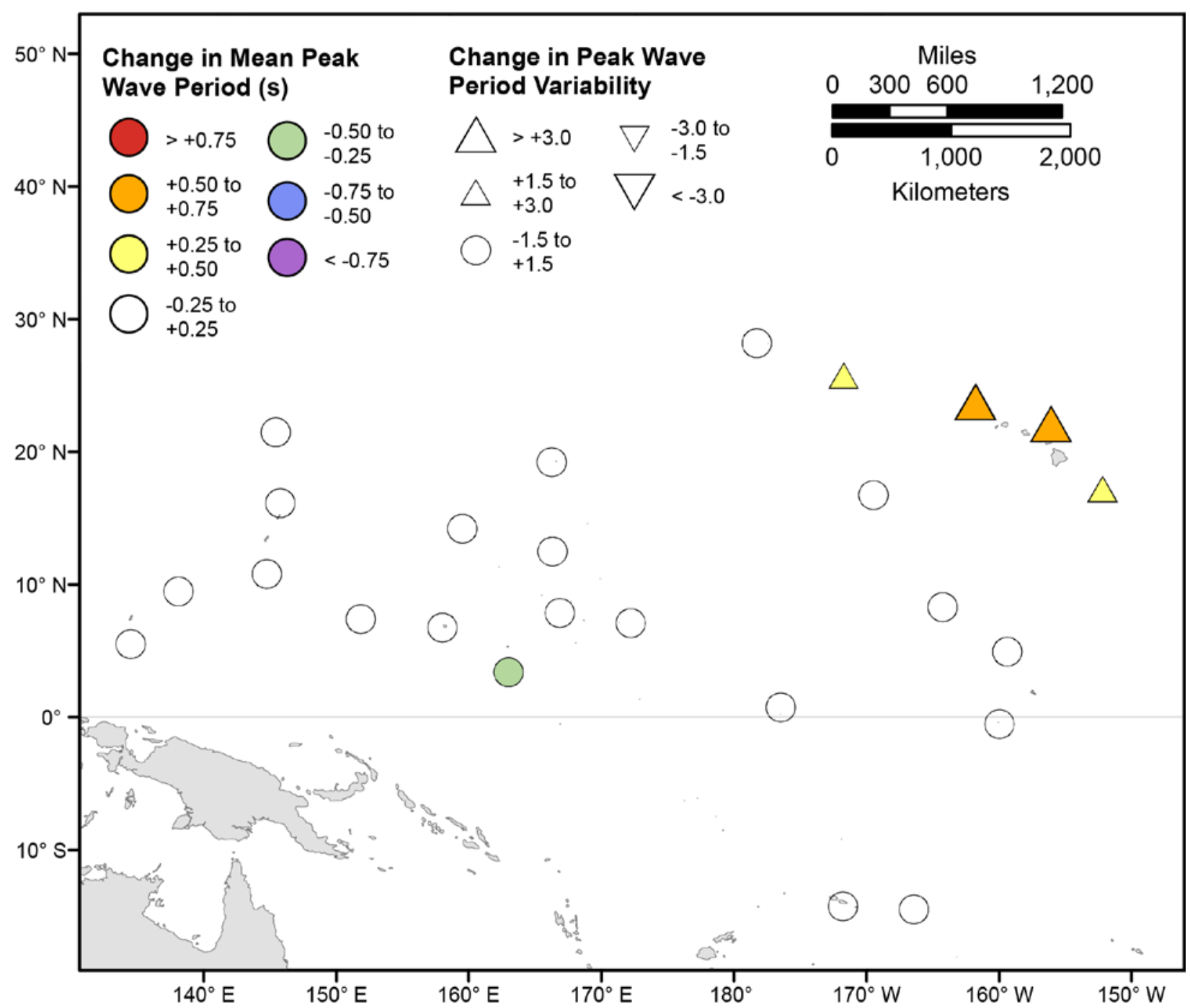

Figure 64. Map showing forecasted differences in mean peak wave period and variance in peak wave period for the years 2081-2100 from hindcasted values during the June-August season under the RCP8.5 future climatic scenario. The colors correspond to the magnitude of change in modeled mean peak wave periods during 2081-2100 from those hindcasted for 1976-2005. The shapes correspond to the magnitude of change in modeled variance in peak wave period during 2081-2100 from those hindcasted for 1976-2005. Units are in seconds. 


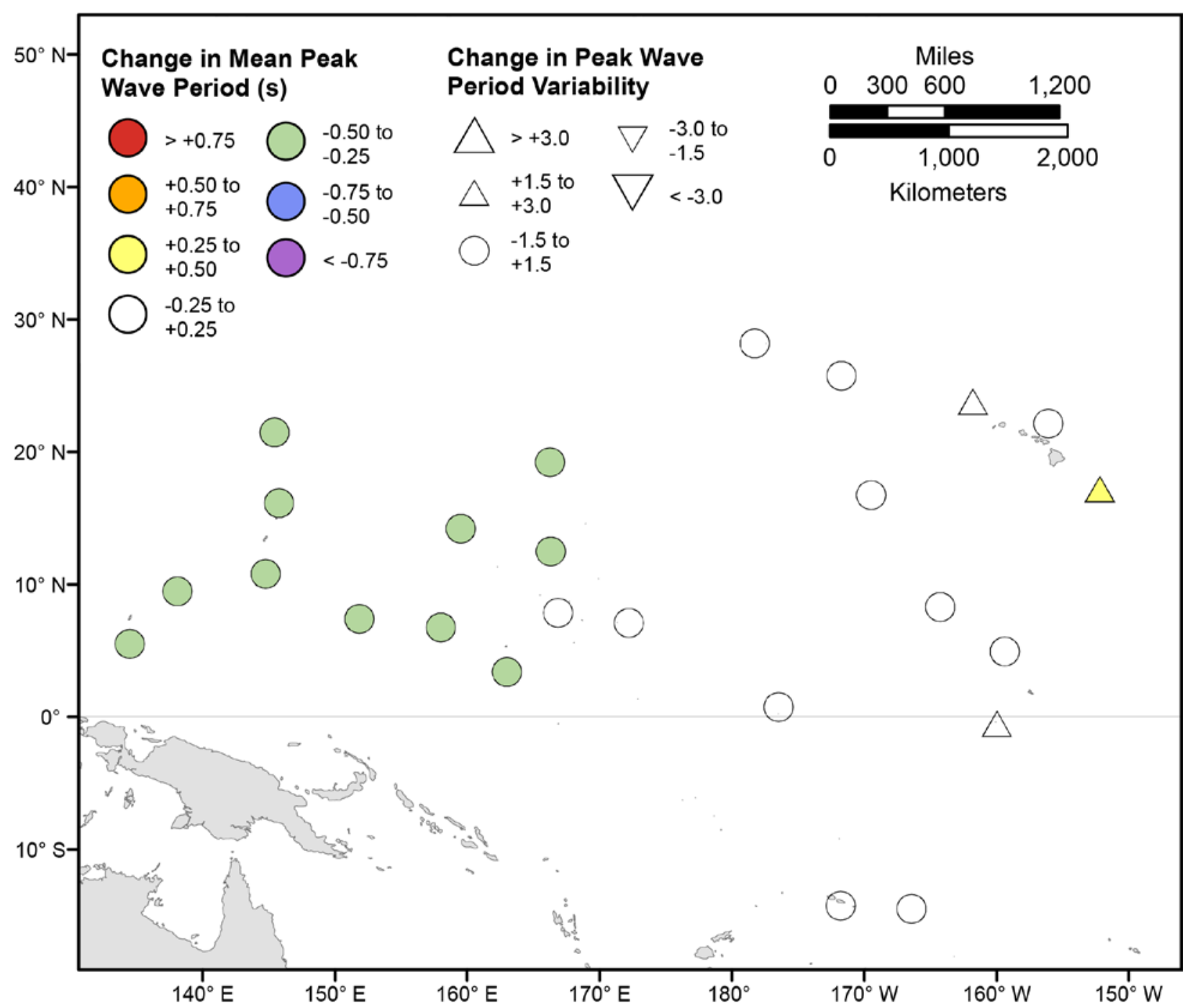

Figure 65. Map showing forecasted differences in mean peak wave period and variance in peak wave period for the years 2081-2100 from hindcasted values during the September-November season under the RCP8.5 future climatic scenario. The colors correspond to the magnitude of change in modeled mean peak wave periods during 2081-2100 from those hindcasted for 1976-2005. The shapes correspond to the magnitude of change in modeled variance in peak wave period during 2081-2100 from those hindcasted for 1976-2005. Units are in seconds. 


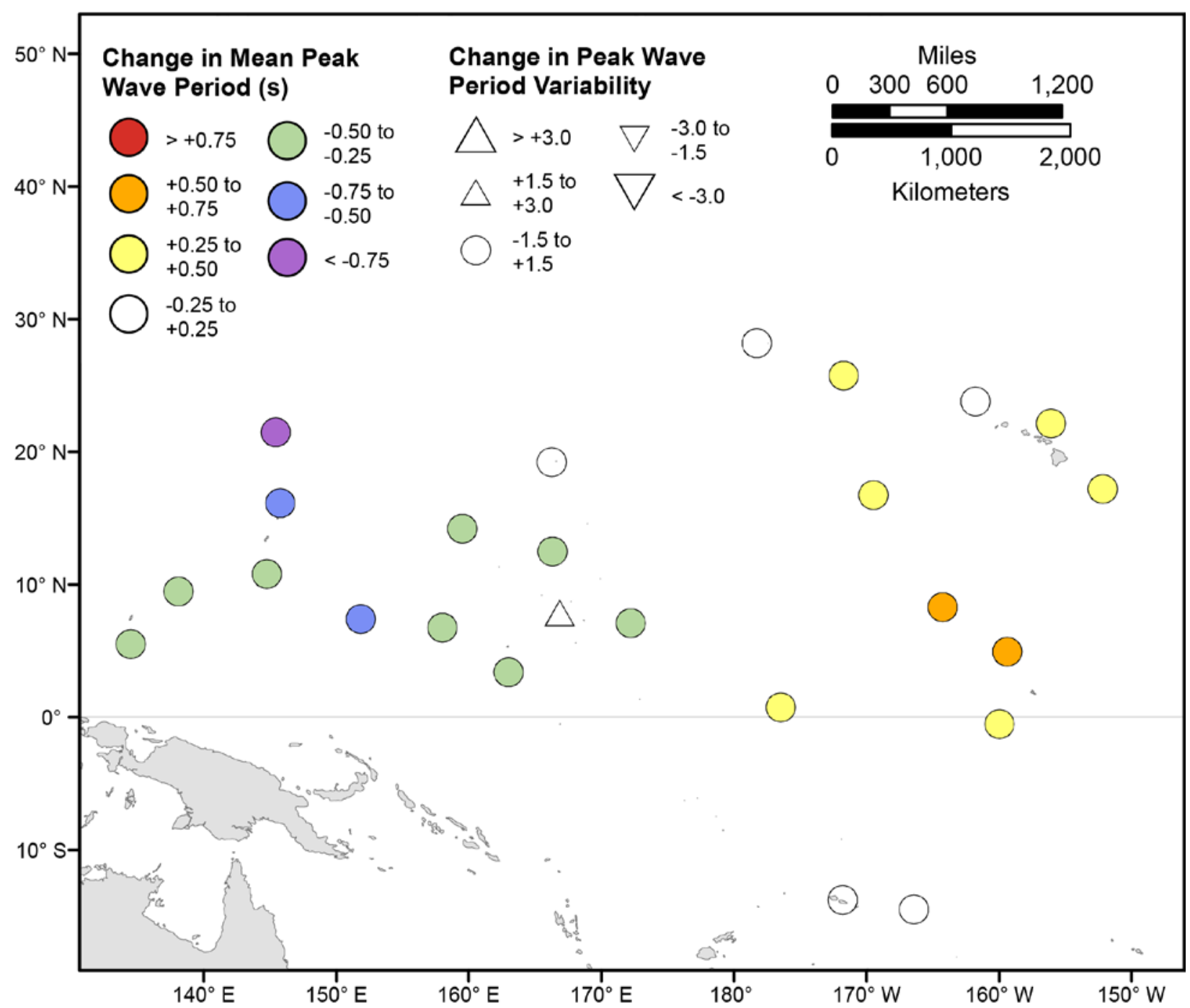

Figure 66. Map showing forecasted differences in the mean peak wave period of the top 5 percent of significant wave heights and variance in the peak wave period of top 5 percent of significant wave heights for the years 2081-2100 from hindcasted values during the December-February season under the RCP8.5 future climatic scenario. The colors correspond to the magnitude of change in modeled mean peak wave periods during 2081-2100 from those hindcasted for 1976-2005. The shapes correspond to the magnitude of change in modeled variance in peak wave period during 2081-2100 from those hindcasted for 1976-2005. Units are in seconds. 


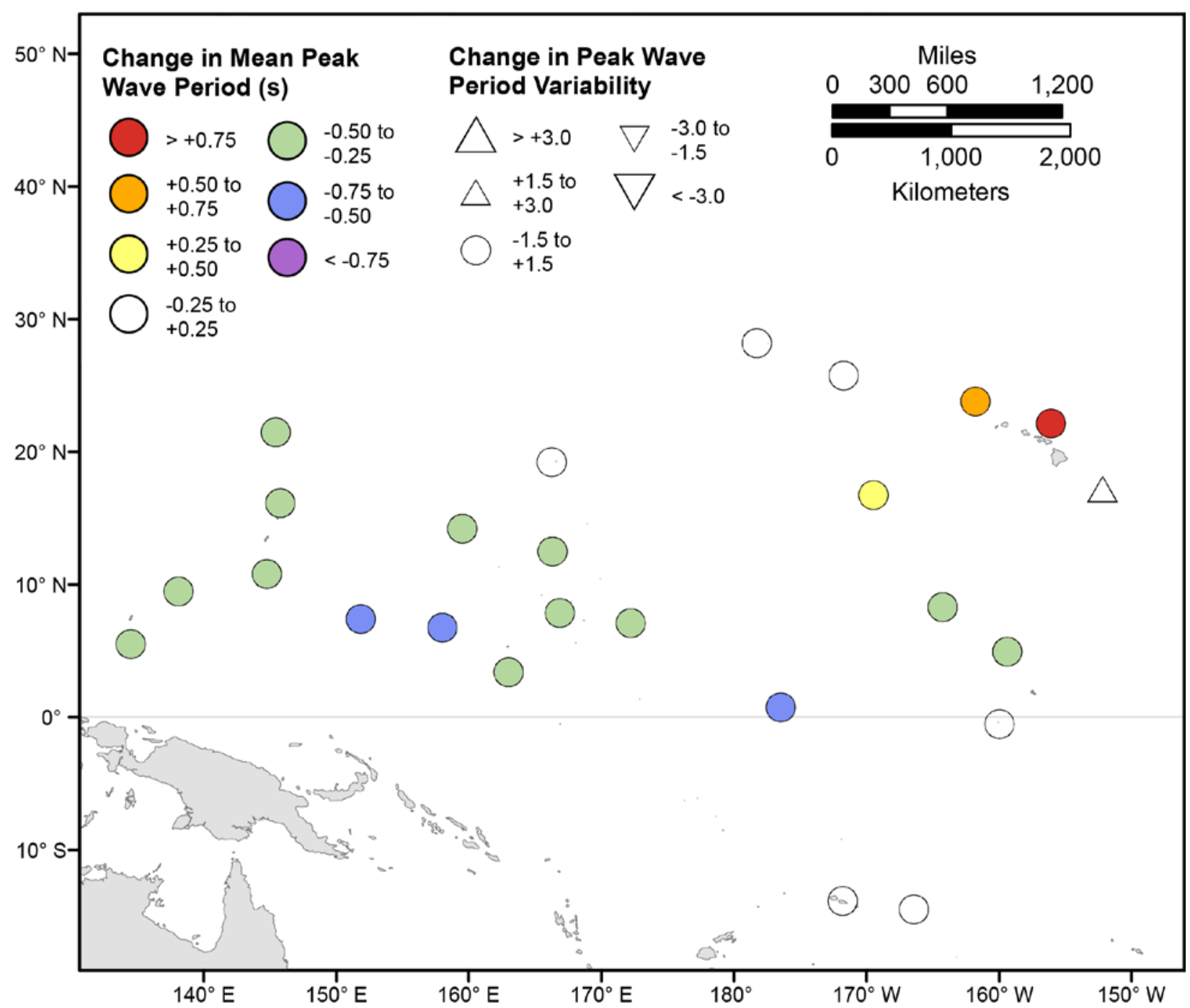

Figure 67. Map showing forecasted differences in the mean peak wave period of the top 5 percent of significant wave heights and variance in the peak wave period of top 5 percent of significant wave heights for the years 2081-2100 from hindcasted values during the March-May season under the RCP8.5 future climatic scenario. The colors correspond to the magnitude of change in modeled mean peak wave periods during 2081-2100 from those hindcasted for 1976-2005. The shapes correspond to the magnitude of change in modeled variance in peak wave period during 2081-2100 from those hindcasted for 1976-2005. Units are in seconds. 


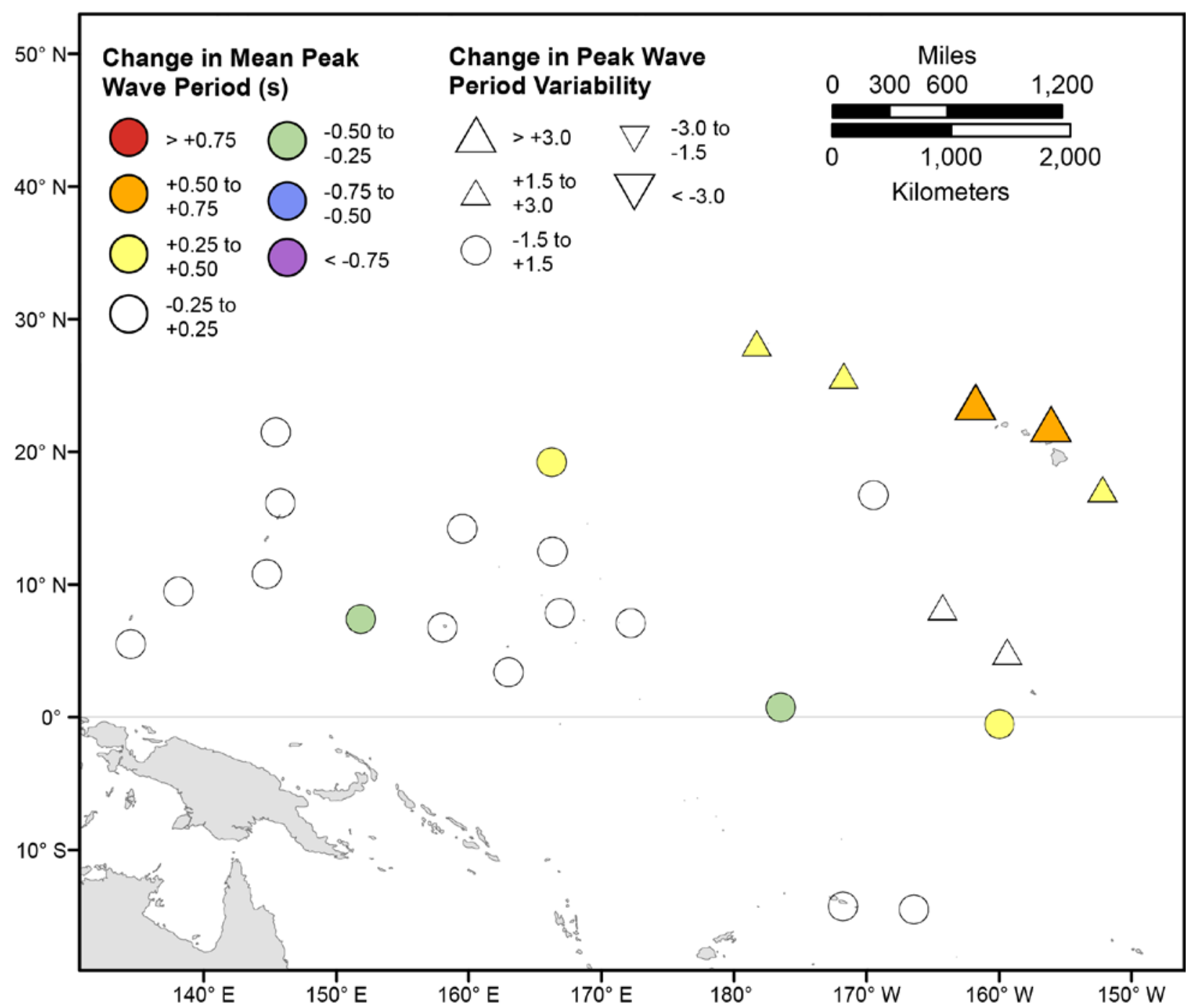

Figure 68. Map showing forecasted differences in the mean peak wave period of the top 5 percent of significant wave heights and variance in the peak wave period of top 5 percent of significant wave heights for the years 2081-2100 from hindcasted values during the June-August season under the RCP8.5 future climatic scenario. The colors correspond to the magnitude of change in modeled mean peak wave periods during 2081-2100 from those hindcasted for 1976-2005. The shapes correspond to the magnitude of change in modeled variance in peak wave period during 2081-2100 from those hindcasted for 1976-2005. Units are in seconds. 


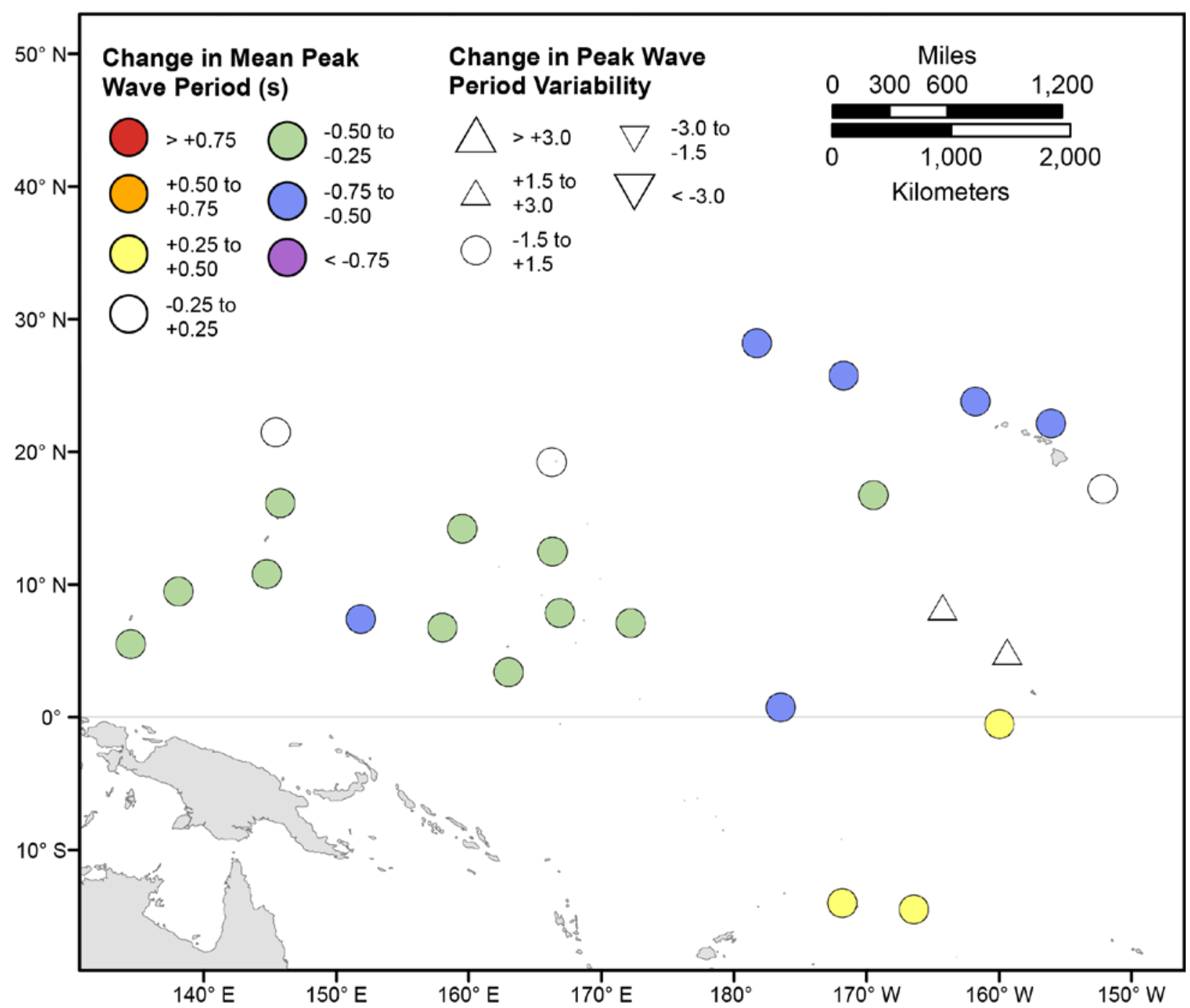

Figure 69. Map showing forecasted differences in the mean peak wave period of the top 5 percent of significant wave heights and variance in the peak wave period of top 5 percent of significant wave heights for the years 2081-2100 from hindcasted values during the September-November season under the RCP8.5 future climatic scenario. The colors correspond to the magnitude of change in modeled mean peak wave periods during 2081-2100 from those hindcasted for 1976-2005. The shapes correspond to the magnitude of change in modeled variance in peak wave period during 2081-2100 from those hindcasted for 1976-2005. Units are in seconds. 


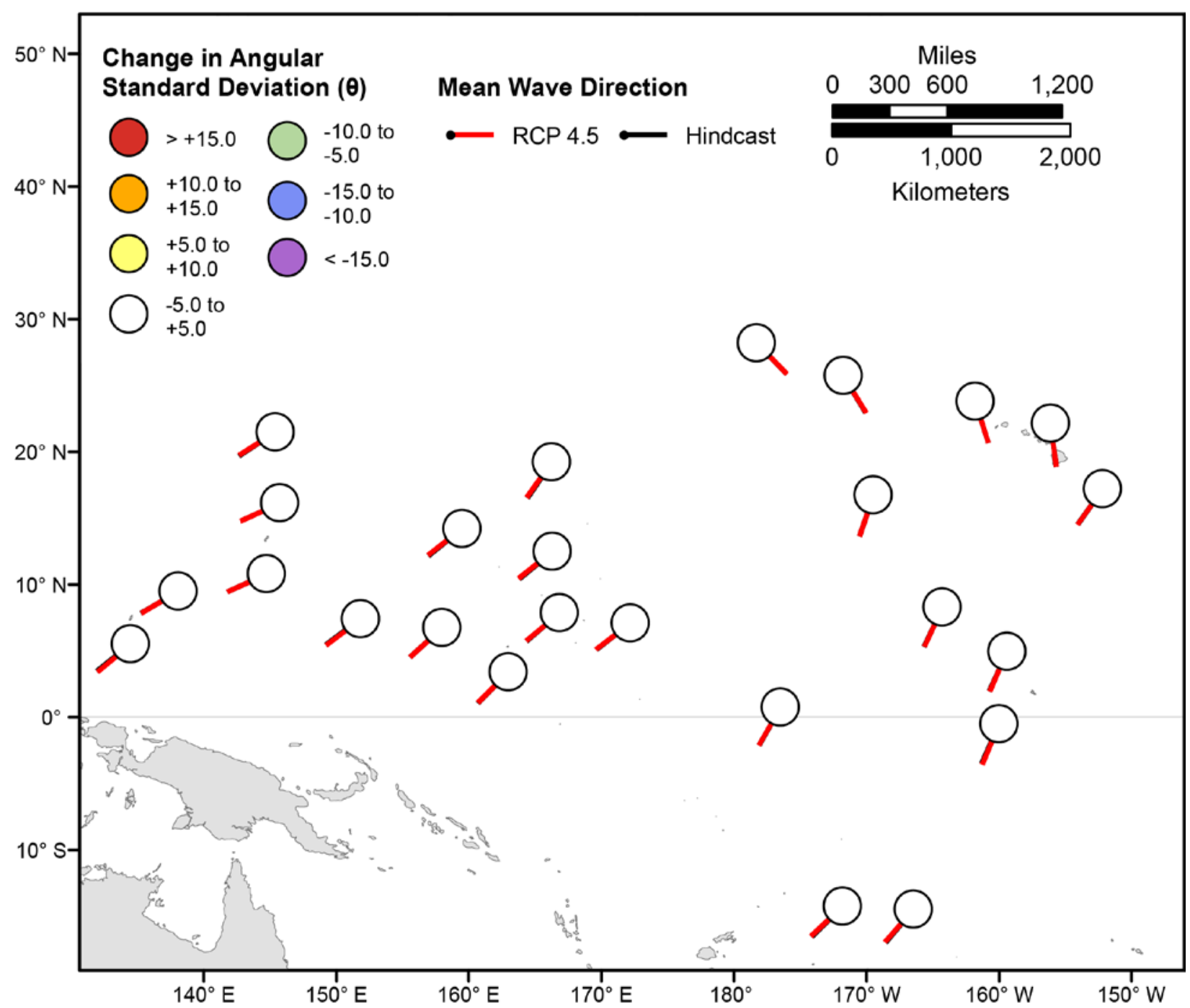

Figure 70. Map showing forecasted differences in the mean wave directions of significant wave heights and the standard deviation of wave directions of significant wave heights for the years 2026-2045 from hindcasted values during the December-February season under the RCP4.5 future climatic scenario. Mean wave directions at each point are indicated by lines radiating from the center of each point where RCP 2026-2045 mean wave directions are red and 1976-2005 hindcasted mean wave directions are black. The colors correspond to the magnitude of change in modeled mean wave direction standard deviation during 2026-2045 from those hindcasted for 1976-2005. Angular standard deviation units are in degrees. Mean wave directions are "heading towards". 


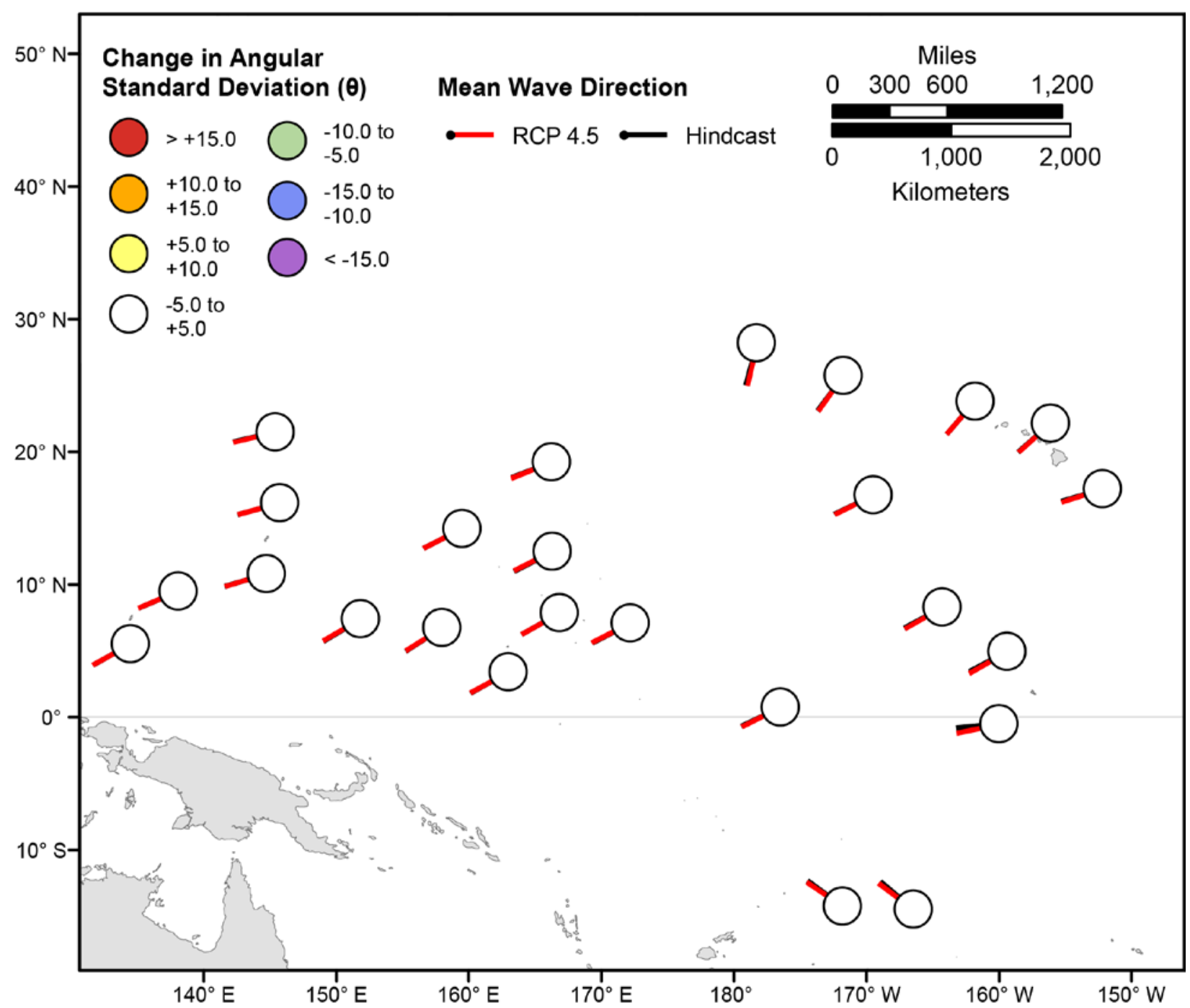

Figure 71. Map showing forecasted differences in the mean wave directions of significant wave heights and the standard deviation of wave directions of significant wave heights for the years 2026-2045 from hindcasted values during the March-May season under the RCP4.5 future climatic scenario. Mean wave directions at each point are indicated by lines radiating from the center of each point where RCP 20262045 mean wave directions are red and 1976-2005 hindcasted mean wave directions are black. The colors correspond to the magnitude of change in modeled mean wave direction standard deviation during 2026-2045 from those hindcasted for 1976-2005. Angular standard deviation units are in degrees. Mean wave directions are "heading towards". 


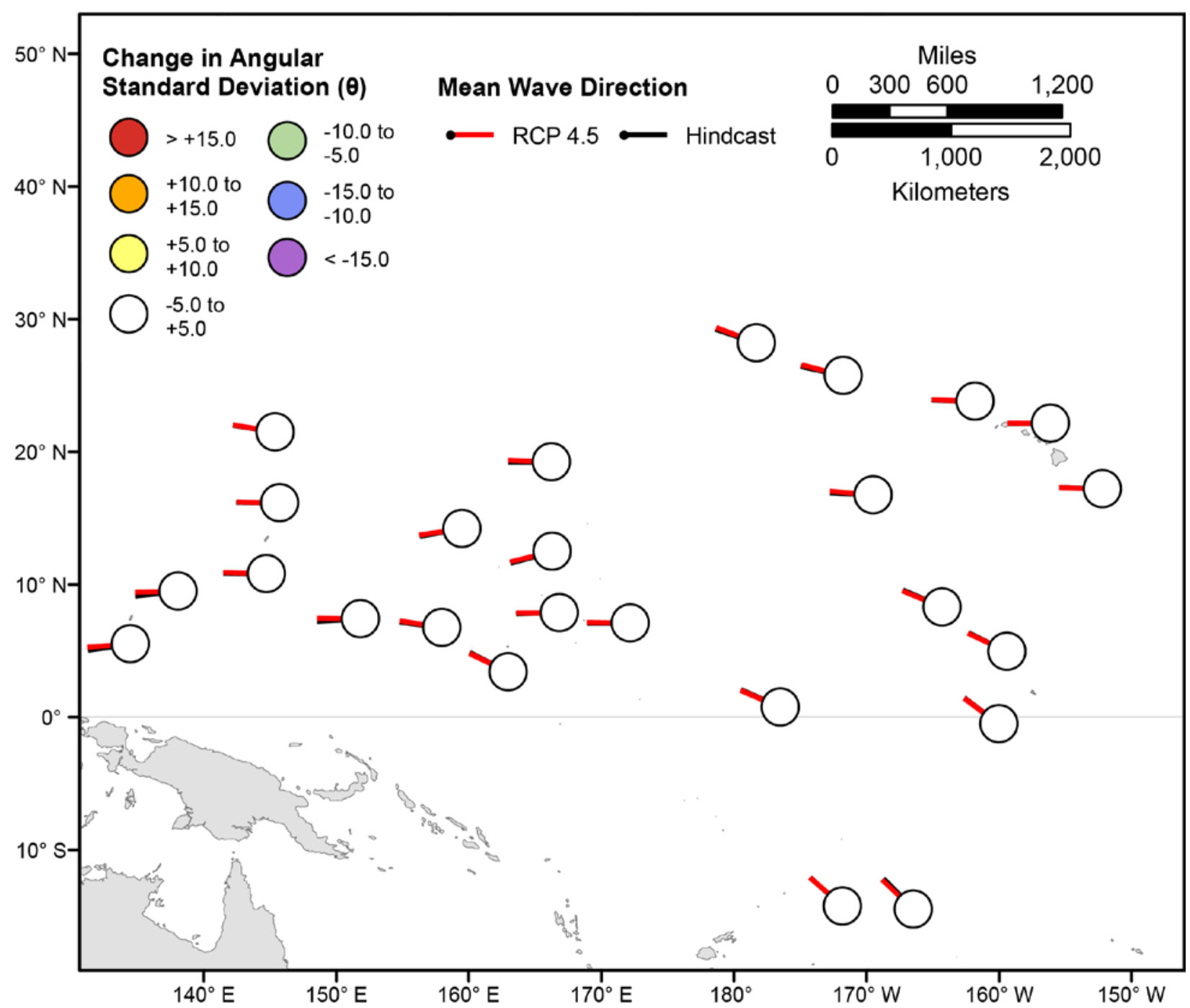

Figure 72. Map showing forecasted differences in the mean wave directions of significant wave heights and the standard deviation of wave directions of significant wave heights for the years 2026-2045 from hindcasted values during the June-July season under the RCP4.5 future climatic scenario. Mean wave directions at each point are indicated by lines radiating from the center of each point where RCP 20262045 mean wave directions are red and 1976-2005 hindcasted mean wave directions are black. The colors correspond to the magnitude of change in modeled mean wave direction standard deviation during 2026-2045 from those hindcasted for 1976-2005. Angular standard deviation units are in degrees. Mean wave directions are "heading towards". 


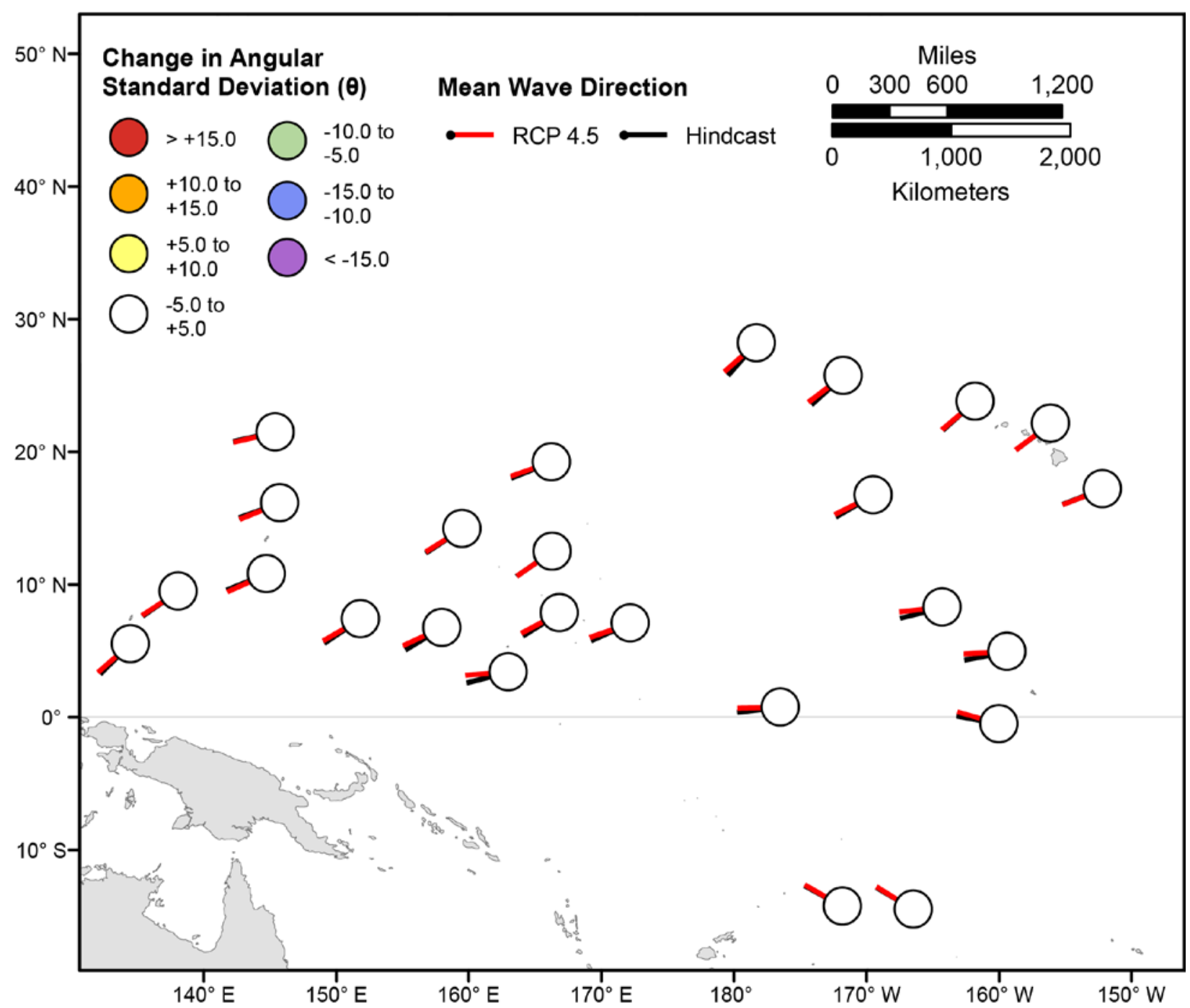

Figure 73. Map showing forecasted differences in the mean wave directions of significant wave heights and the standard deviation of wave directions of significant wave heights for the years 2026-2045 from hindcasted values during the September-November season under the RCP4.5 future climatic scenario. Mean wave directions at each point are indicated by lines radiating from the center of each point where RCP 2026-2045 mean wave directions are red and 1976-2005 hindcasted mean wave directions are black. The colors correspond to the magnitude of change in modeled mean wave direction standard deviation during 2026-2045 from those hindcasted for 1976-2005. Angular standard deviation units are in degrees. Mean wave directions are "heading towards". 


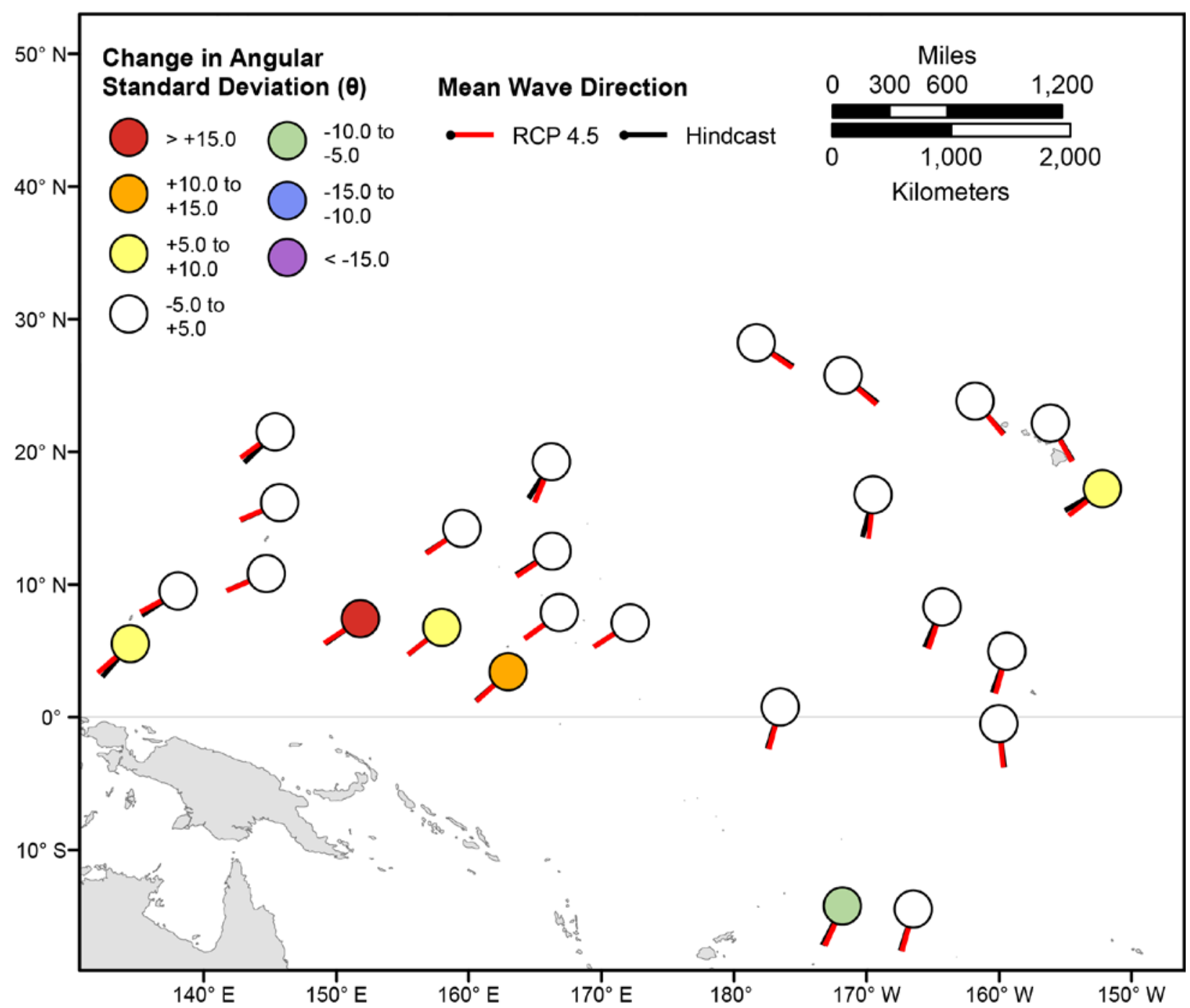

Figure 74. Map showing forecasted differences in the mean wave directions of the top 5 percent of significant wave heights and the standard deviation of wave directions of the top 5 percent of significant wave heights for the years 2026-2045 from hindcasted values during the December-February season under the RCP4.5 future climatic scenario. Mean wave directions at each point are indicated by lines radiating from the center of each point where RCP 2026-2045 mean wave directions are red and 19762005 hindcasted mean wave directions are black. The colors correspond to the magnitude of change in modeled mean wave direction standard deviation during 2026-2045 from those hindcasted for 19762005. Angular standard deviation units are in degrees. Mean wave directions are "heading towards". 


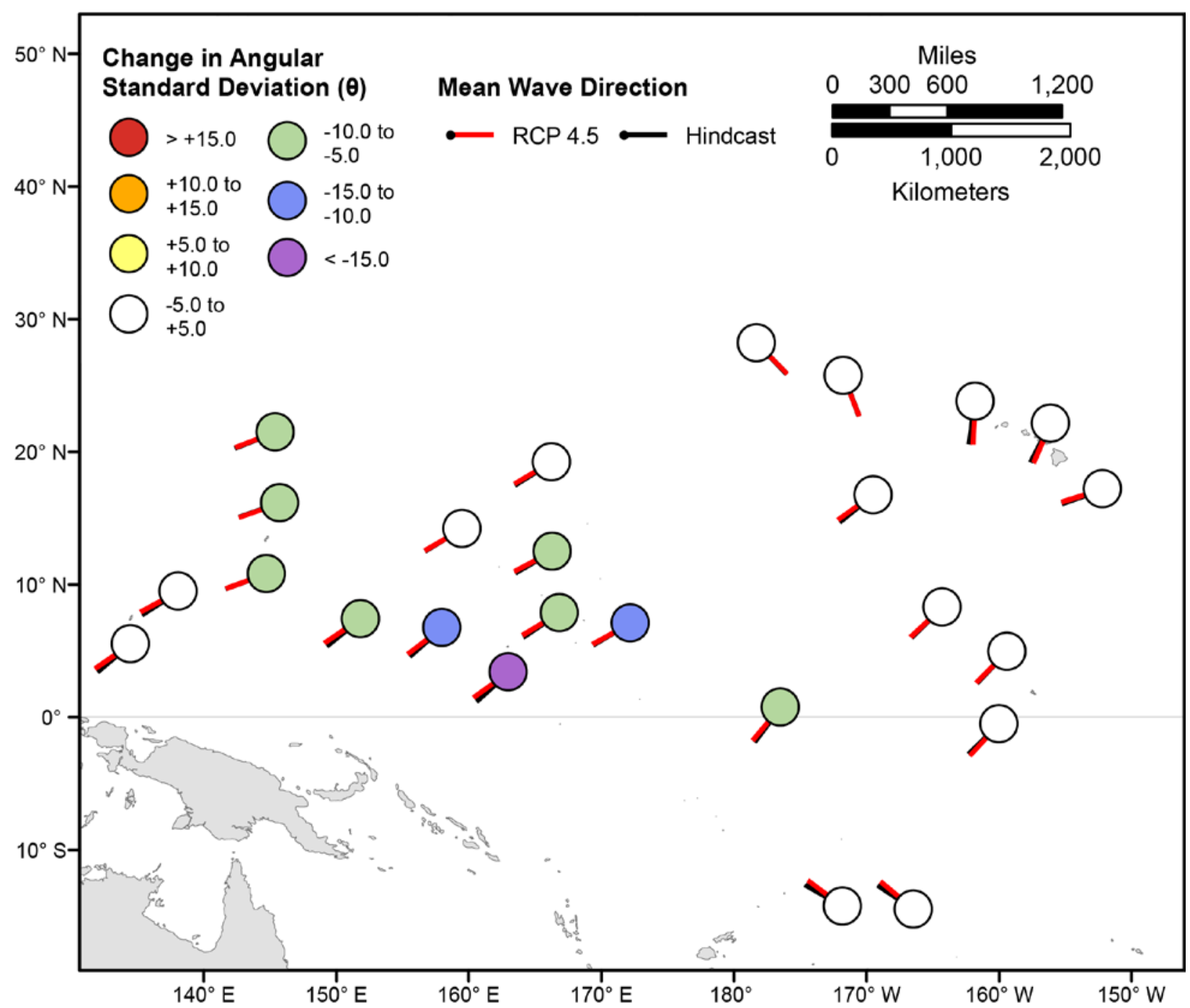

Figure 75. Map showing forecasted differences in the mean wave directions of the top 5 percent of significant wave heights and the standard deviation of wave directions of the top 5 percent of significant wave heights for the years 2026-2045 from hindcasted values during the March-May season under the RCP4.5 future climatic scenario. Mean wave directions at each point are indicated by lines radiating from the center of each point where RCP 2026-2045 mean wave directions are red and 1976-2005 hindcasted mean wave directions are black. The colors correspond to the magnitude of change in modeled mean wave direction standard deviation during 2026-2045 from those hindcasted for 19762005. Angular standard deviation units are in degrees. Mean wave directions are "heading towards". 


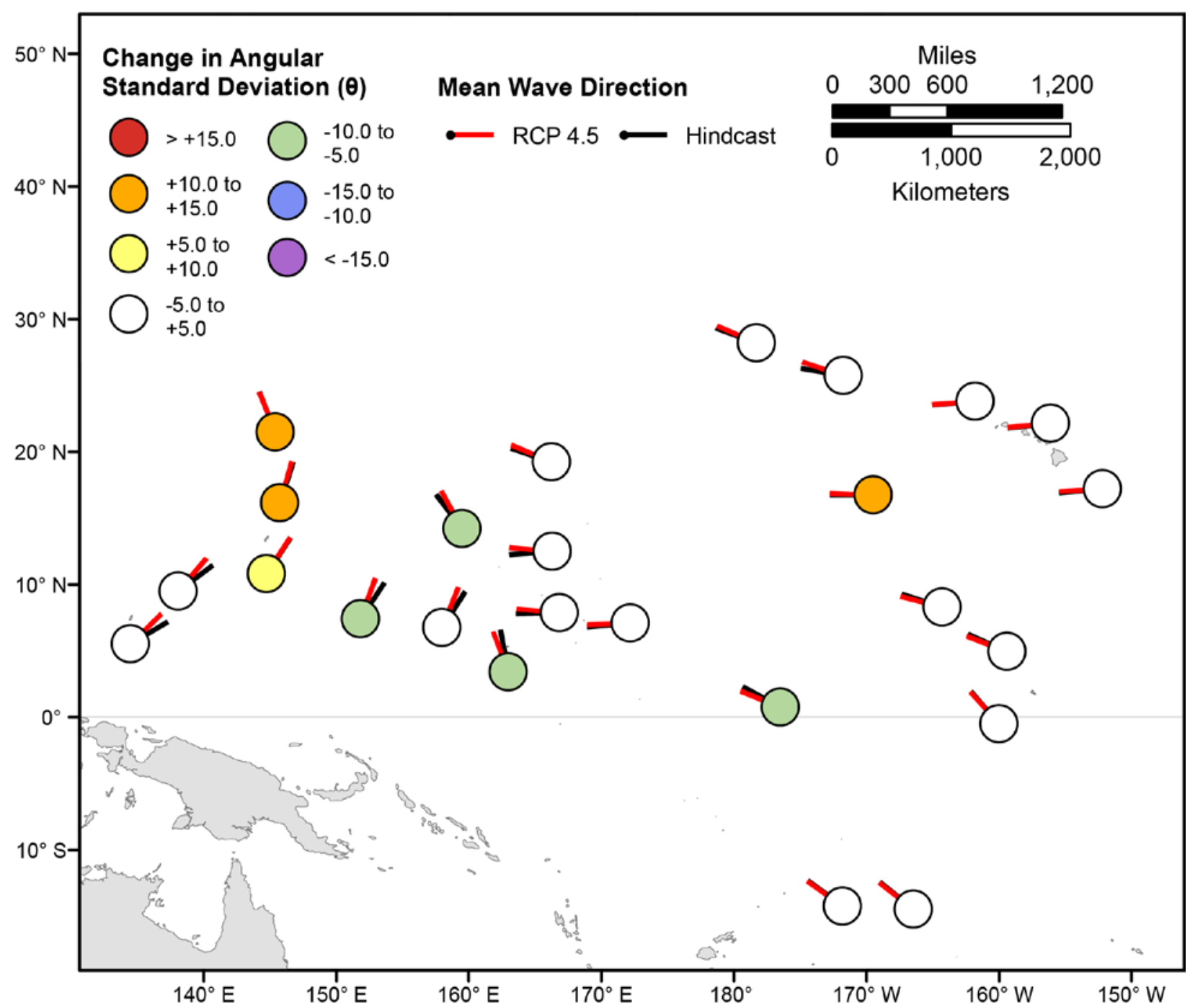

Figure 76. Map showing forecasted differences in the mean wave directions of the top 5 percent of significant wave heights and the standard deviation of wave directions of the top 5 percent of significant wave heights for the years 2026-2045 from hindcasted values during the June-August season under the RCP4.5 future climatic scenario. Mean wave directions at each point are indicated by lines radiating from the center of each point where RCP 2026-2045 mean wave directions are red and 1976-2005 hindcasted mean wave directions are black. The colors correspond to the magnitude of change in modeled mean wave direction standard deviation during 2026-2045 from those hindcasted for 19762005. Angular standard deviation units are in degrees. Mean wave directions are "heading towards". 


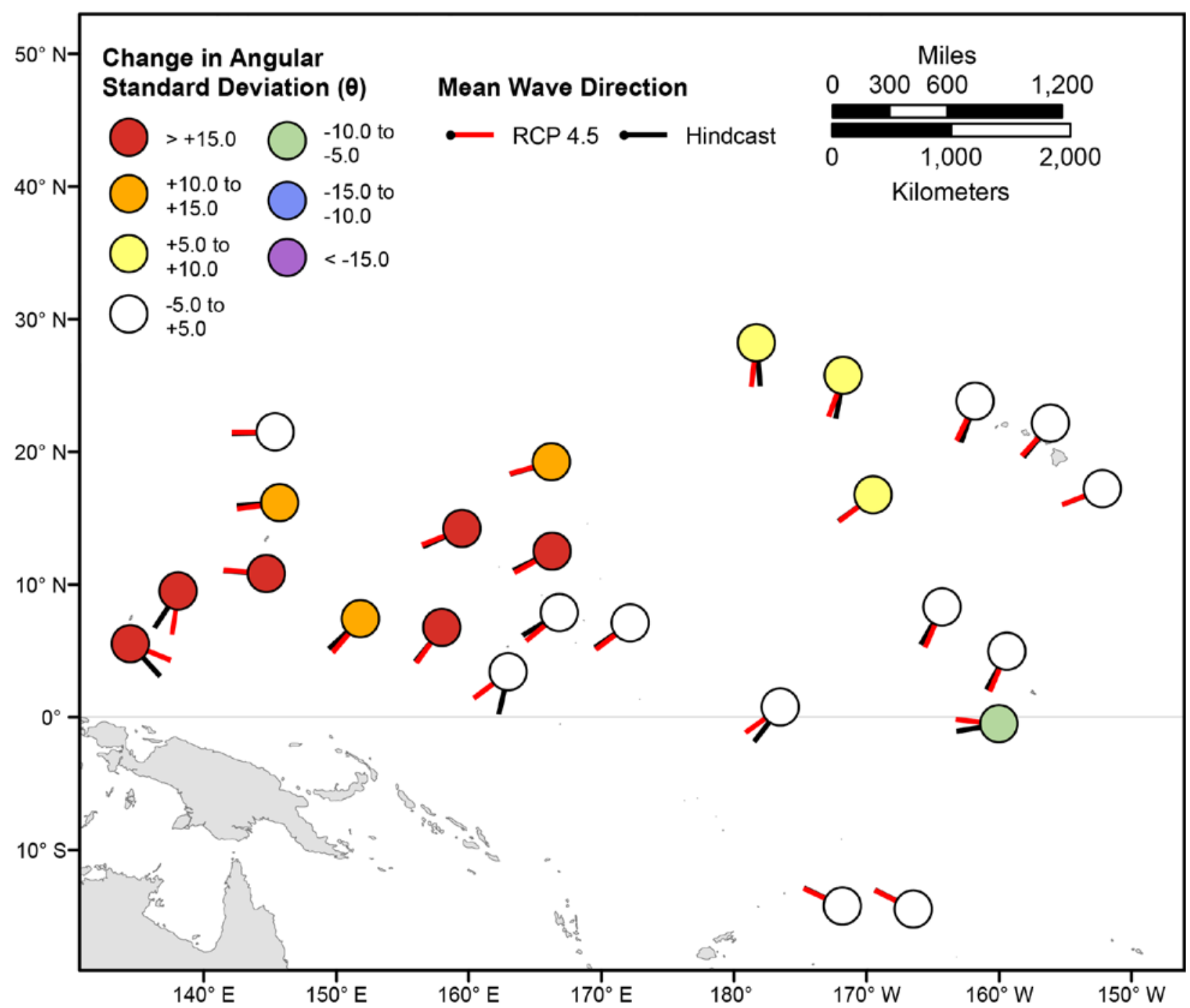

Figure 77. Map showing forecasted differences in the mean wave directions of the top 5 percent of significant wave heights and the standard deviation of wave directions of the top 5 percent of significant wave heights for the years 2026-2045 from hindcasted values during the September-November season under the RCP4.5 future climatic scenario. Mean wave directions at each point are indicated by lines radiating from the center of each point where RCP 2026-2045 mean wave directions are red and 19762005 hindcasted mean wave directions are black. The colors correspond to the magnitude of change in modeled mean wave direction standard deviation during 2026-2045 from those hindcasted for 19762005. Angular standard deviation units are in degrees. Mean wave directions are "heading towards". 


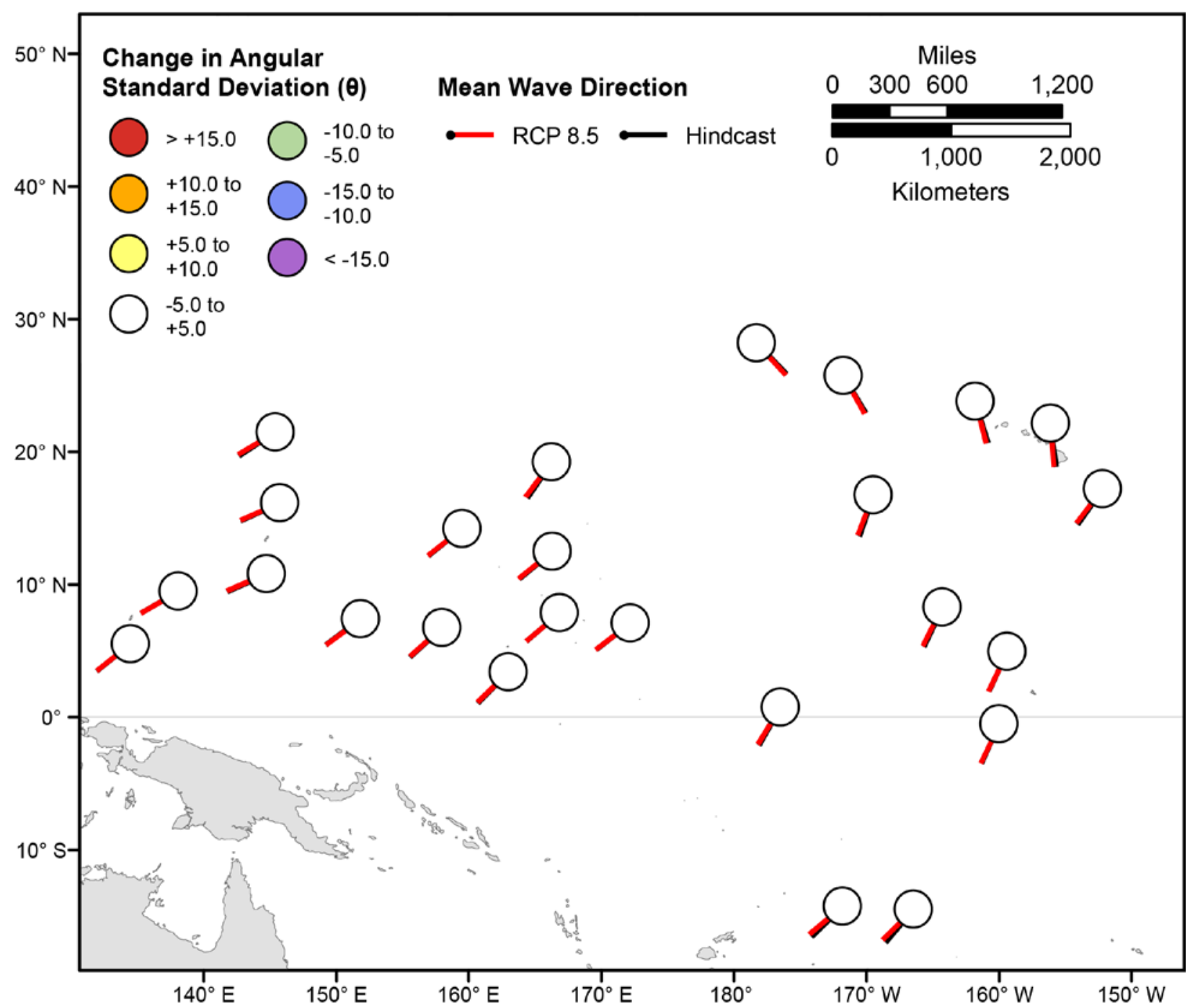

Figure 78. Map showing forecasted differences in the mean wave directions of significant wave heights and the standard deviation of wave directions of significant wave heights for the years 2026-2045 from hindcasted values during the December-February season under the RCP8.5 future climatic scenario. Mean wave directions at each point are indicated by lines radiating from the center of each point where RCP 2026-2045 mean wave directions are red and 1976-2005 hindcasted mean wave directions are black. The colors correspond to the magnitude of change in modeled mean wave direction standard deviation during 2026-2045 from those hindcasted for 1976-2005. Angular standard deviation units are in degrees. Mean wave directions are "heading towards". 


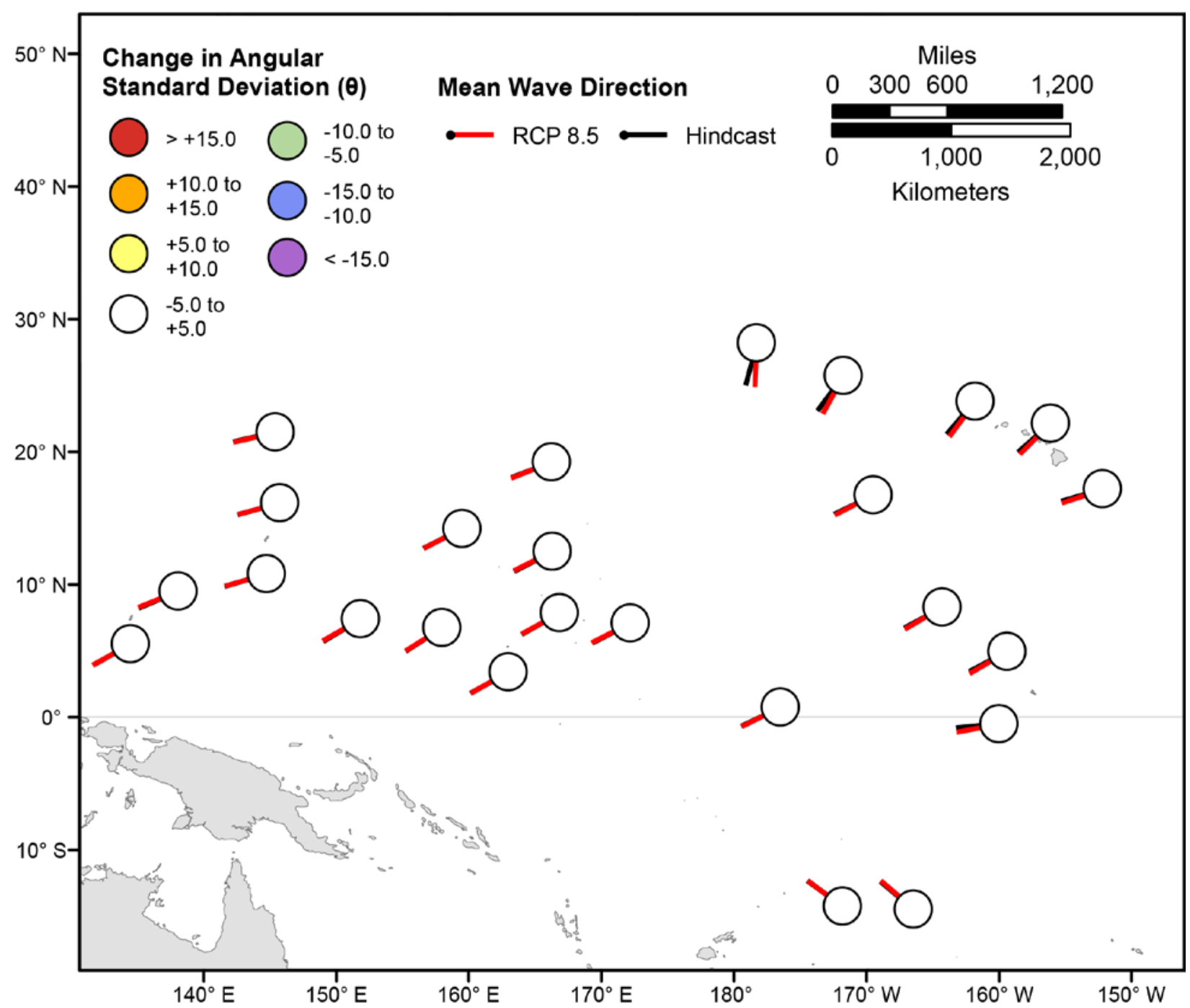

Figure 79. Map showing forecasted differences in the mean wave directions of significant wave heights and the standard deviation of wave directions of significant wave heights for the years 2026-2045 from hindcasted values during the March-May season under the RCP8.5 future climatic scenario. Mean wave directions at each point are indicated by lines radiating from the center of each point where RCP 20262045 mean wave directions are red and 1976-2005 hindcasted mean wave directions are black. The colors correspond to the magnitude of change in modeled mean wave direction standard deviation during 2026-2045 from those hindcasted for 1976-2005. Angular standard deviation units are in degrees. Mean wave directions are "heading towards". 


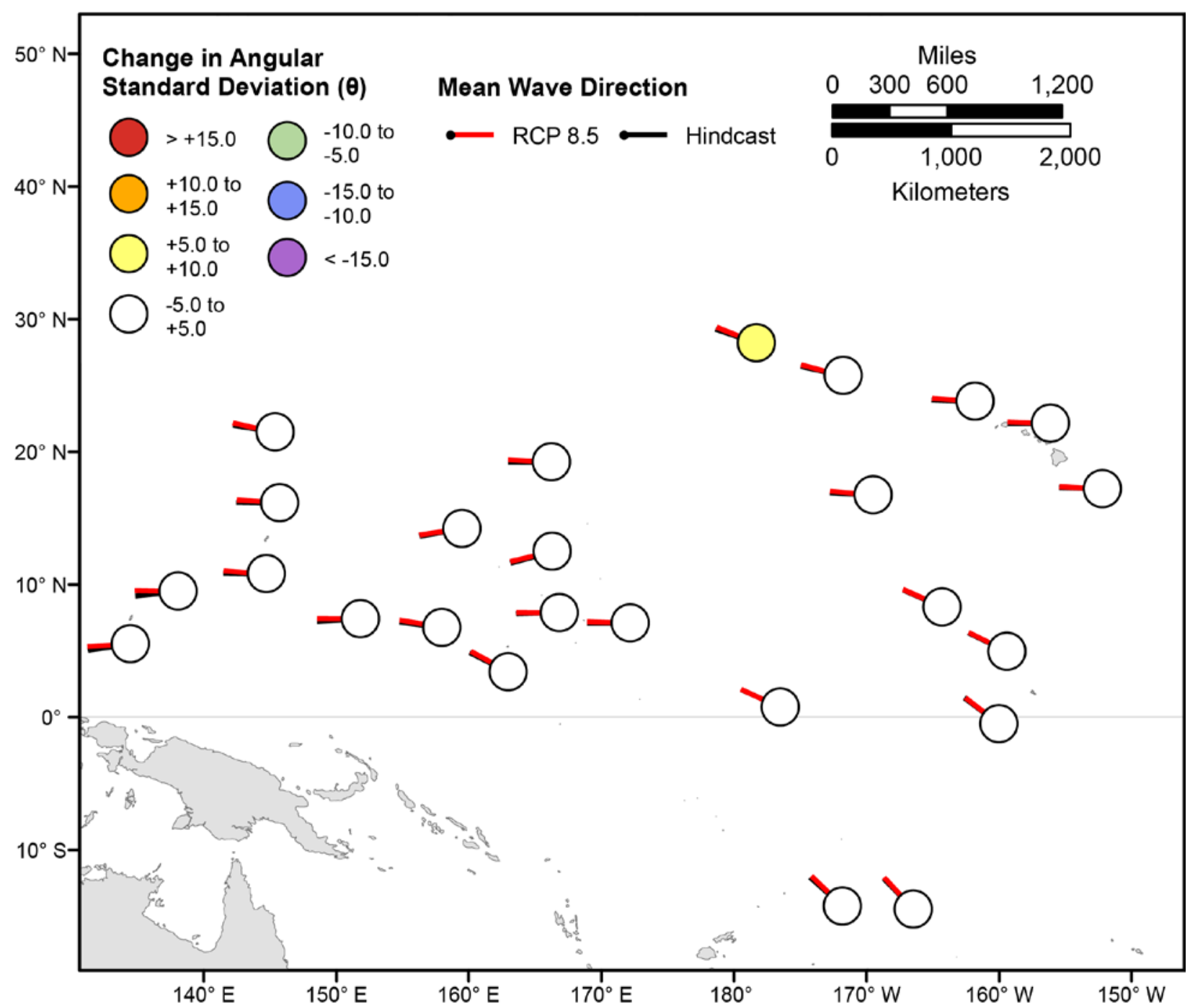

Figure 80. Map showing forecasted differences in the mean wave directions of significant wave heights and the standard deviation of wave directions of significant wave heights for the years 2026-2045 from hindcasted values during the June-July season under the RCP8.5 future climatic scenario. Mean wave directions at each point are indicated by lines radiating from the center of each point where RCP 20262045 mean wave directions are red and 1976-2005 hindcasted mean wave directions are black. The colors correspond to the magnitude of change in modeled mean wave direction standard deviation during 2026-2045 from those hindcasted for 1976-2005. Angular standard deviation units are in degrees. Mean wave directions are "heading towards". 


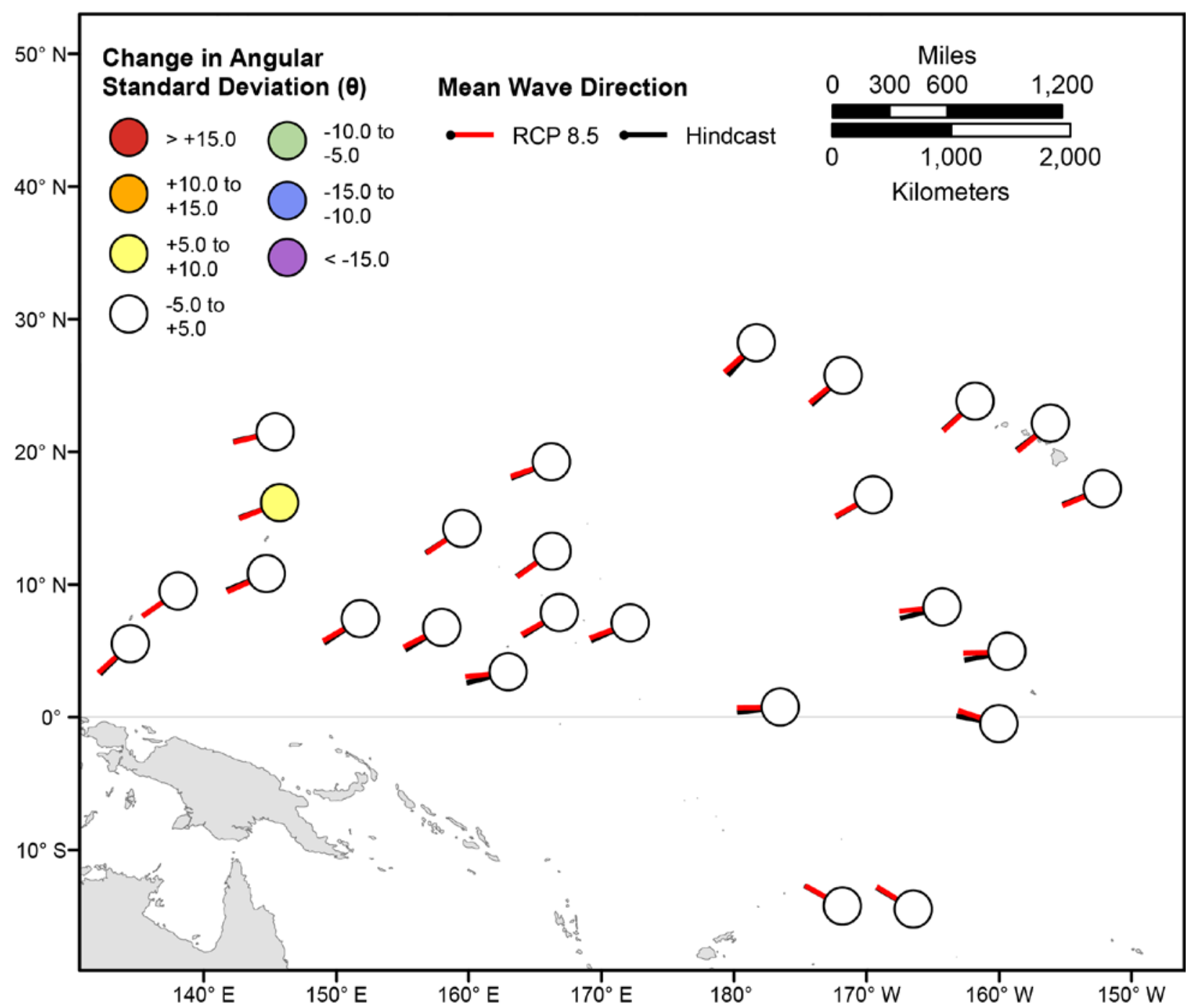

Figure 81. Map showing forecasted differences in the mean wave directions of significant wave heights and the standard deviation of wave directions of significant wave heights for the years 2026-2045 from hindcasted values during the September-November season under the RCP8.5 future climatic scenario. Mean wave directions at each point are indicated by lines radiating from the center of each point where RCP 2026-2045 mean wave directions are red and 1976-2005 hindcasted mean wave directions are black. The colors correspond to the magnitude of change in modeled mean wave direction standard deviation during 2026-2045 from those hindcasted for 1976-2005. Angular standard deviation units are in degrees. Mean wave directions are "heading towards". 


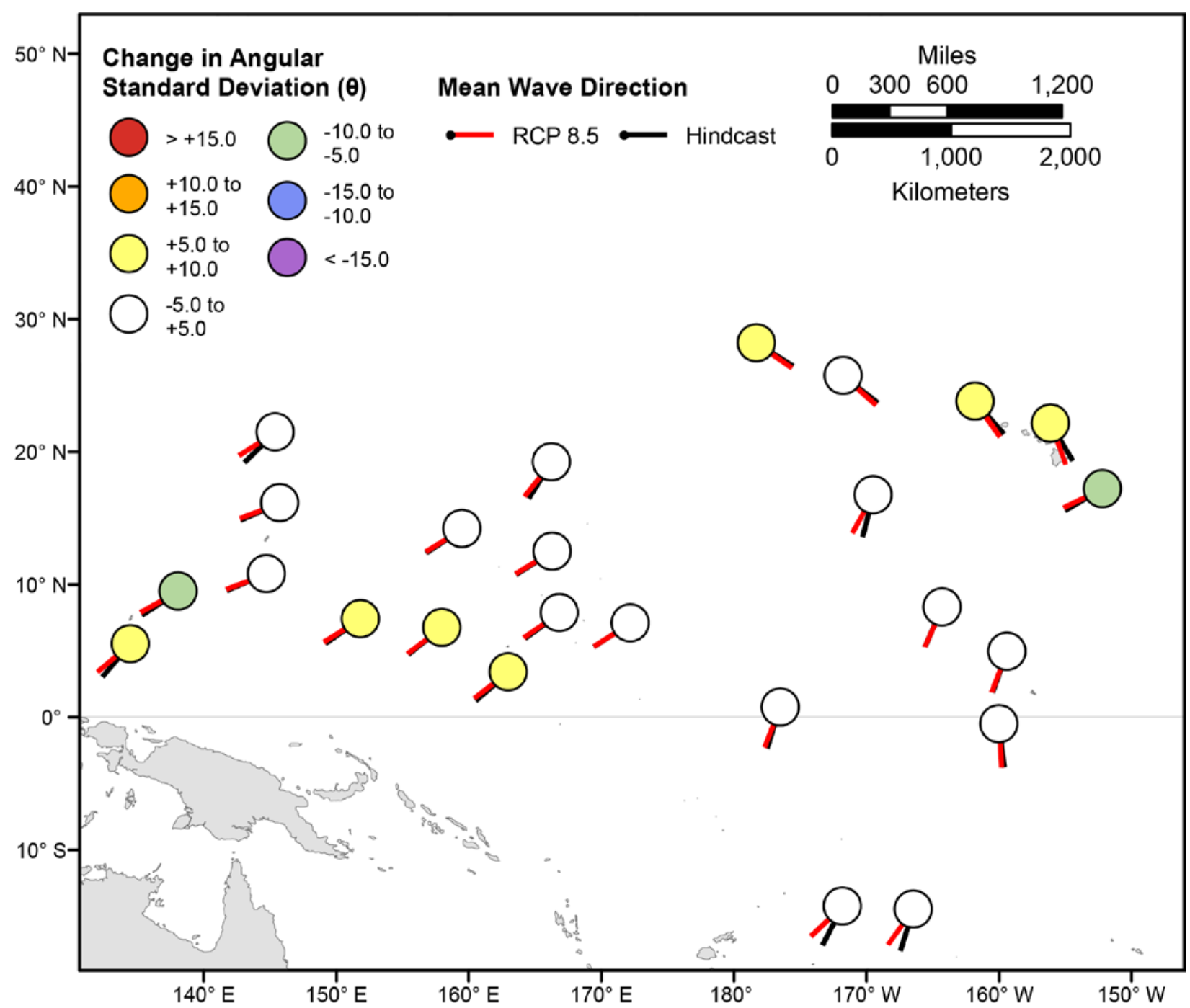

Figure 82. Map showing forecasted differences in the mean wave directions of the top 5 percent of significant wave heights and the standard deviation of wave directions of the top 5 percent of significant wave heights for the years 2026-2045 from hindcasted values during the December-February season under the RCP8.5 future climatic scenario. Mean wave directions at each point are indicated by lines radiating from the center of each point where RCP 2026-2045 mean wave directions are red and 19762005 hindcasted mean wave directions are black. The colors correspond to the magnitude of change in modeled mean wave direction standard deviation during 2026-2045 from those hindcasted for 19762005. Angular standard deviation units are in degrees. Mean wave directions are "heading towards". 


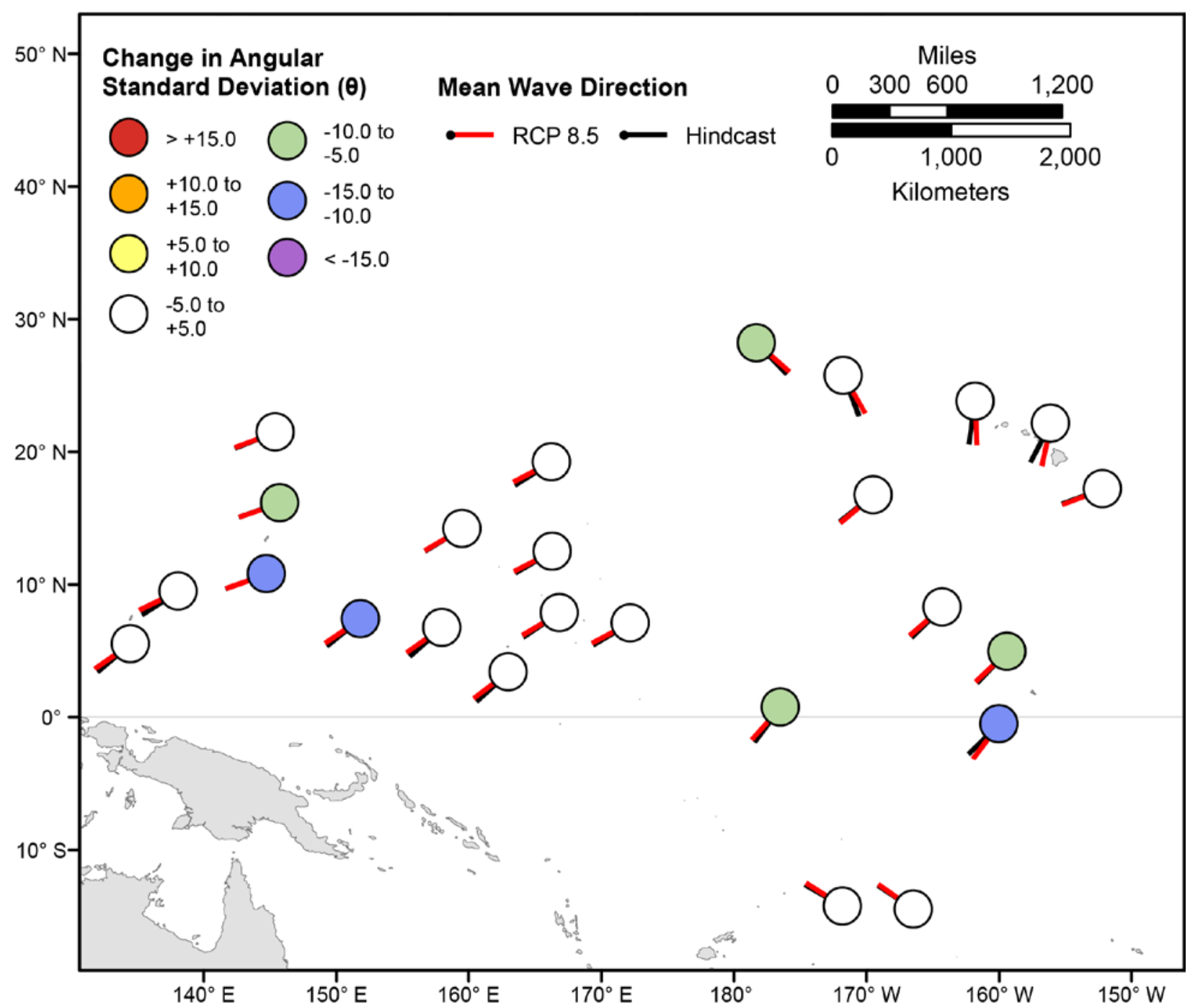

Figure 83. Map showing forecasted differences in the mean wave directions of the top 5 percent of significant wave heights and the standard deviation of wave directions of the top 5 percent of significant wave heights for the years 2026-2045 from hindcasted values during the March-May season under the RCP8.5 future climatic scenario. Mean wave directions at each point are indicated by lines radiating from the center of each point where RCP 2026-2045 mean wave directions are red and 1976-2005 hindcasted mean wave directions are black. The colors correspond to the magnitude of change in modeled mean wave direction standard deviation during 2026-2045 from those hindcasted for 19762005. Angular standard deviation units are in degrees. Mean wave directions are "heading towards". 


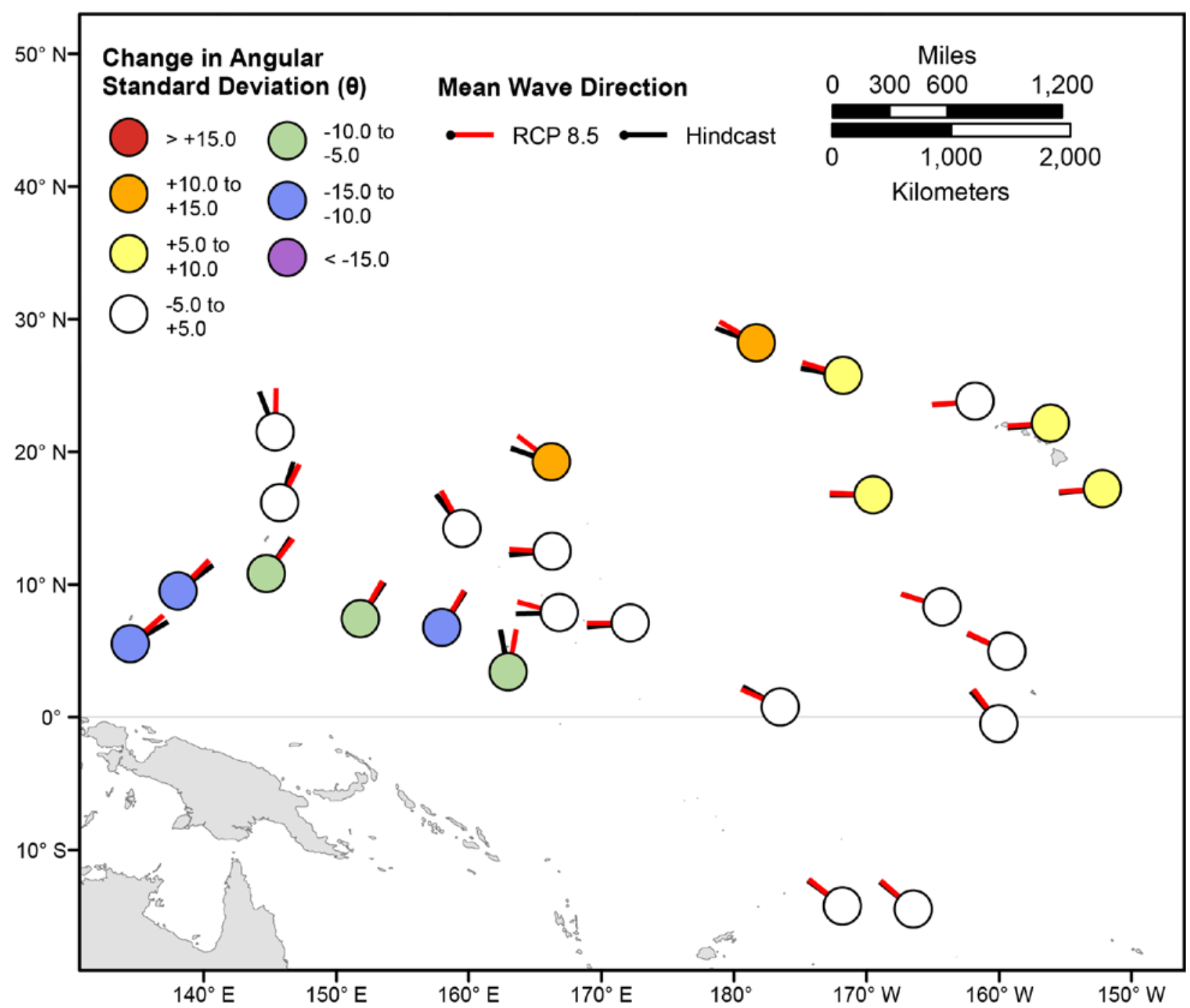

Figure 84. Map showing forecasted differences in the mean wave directions of the top 5 percent of significant wave heights and the standard deviation of wave directions of the top 5 percent of significant wave heights for the years 2026-2045 from hindcasted values during the June-August season under the RCP8.5 future climatic scenario. Mean wave directions at each point are indicated by lines radiating from the center of each point where RCP 2026-2045 mean wave directions are red and 1976-2005 hindcasted mean wave directions are black. The colors correspond to the magnitude of change in modeled mean wave direction standard deviation during 2026-2045 from those hindcasted for 19762005. Angular standard deviation units are in degrees. Mean wave directions are "heading towards". 


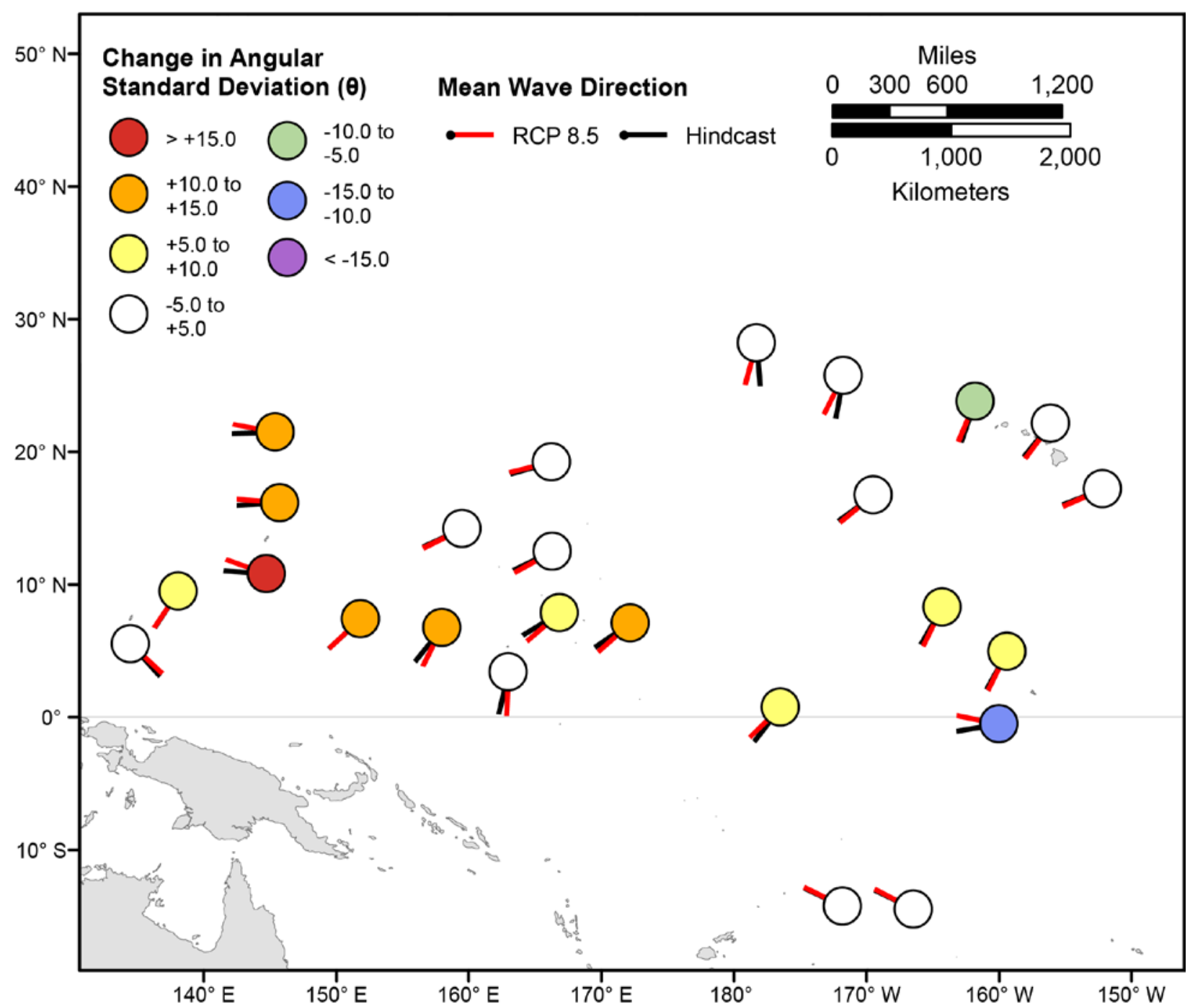

Figure 85. Map showing forecasted differences in the mean wave directions of the top 5 percent of significant wave heights and the standard deviation of wave directions of the top 5 percent of significant wave heights for the years 2026-2045 from hindcasted values during the September-November season under the RCP8.5 future climatic scenario. Mean wave directions at each point are indicated by lines radiating from the center of each point where RCP 2026-2045 mean wave directions are red and 19762005 hindcasted mean wave directions are black. The colors correspond to the magnitude of change in modeled mean wave direction standard deviation during 2026-2045 from those hindcasted for 19762005. Angular standard deviation units are in degrees. Mean wave directions are "heading towards". 


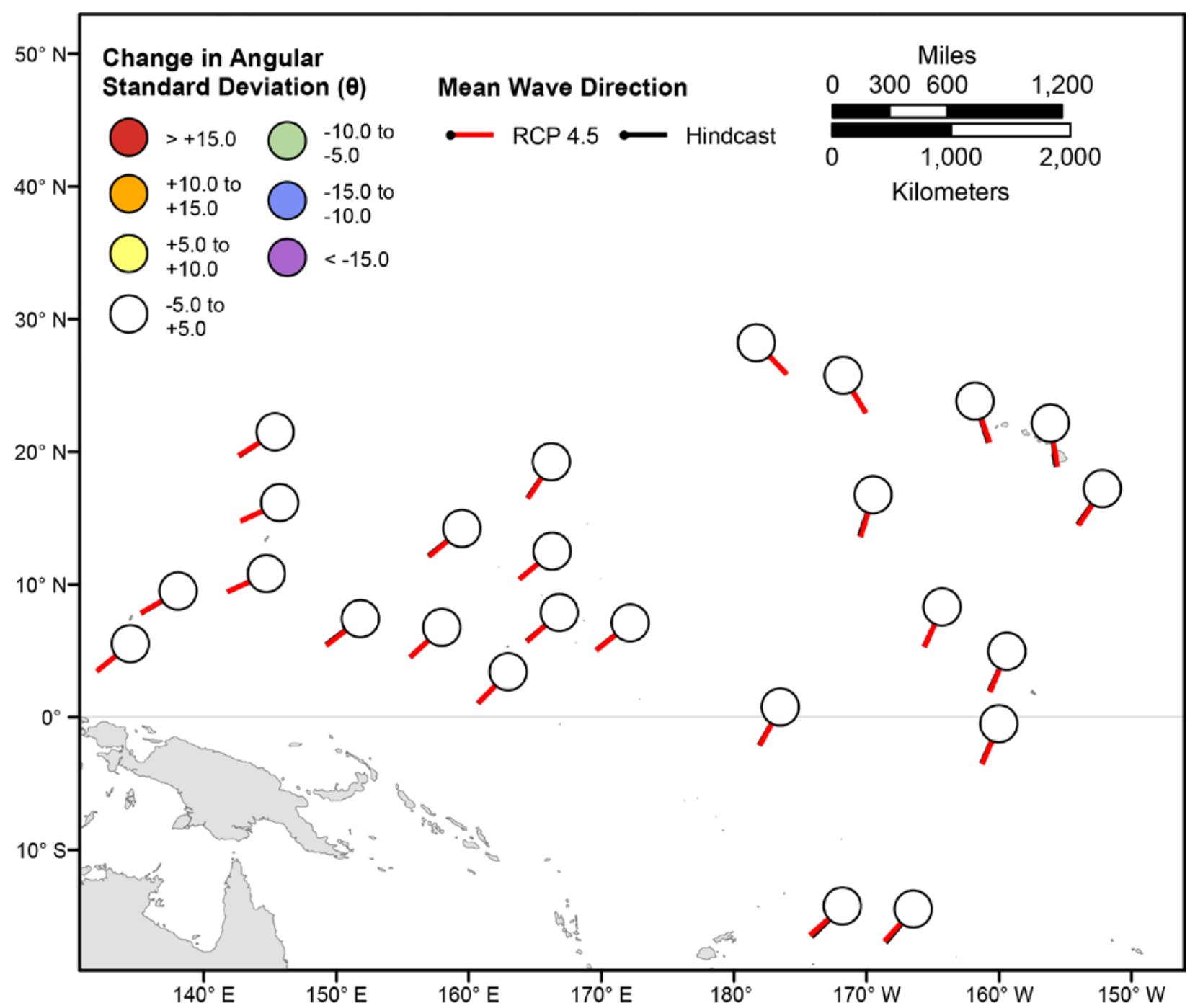

Figure 86. Map showing forecasted differences in the mean wave directions of significant wave heights and the standard deviation of wave directions of significant wave heights for the years 2081-2100 from hindcasted values during the December-February season under the RCP4.5 future climatic scenario. Mean wave directions at each point are indicated by lines radiating from the center of each point where RCP 2081-2100 mean wave directions are red and 1976-2005 hindcasted mean wave directions are black. The colors correspond to the magnitude of change in modeled mean wave direction standard deviation during 2081-2100 from those hindcasted for 1976-2005. Angular standard deviation units are in degrees. Mean wave directions are "heading towards". 


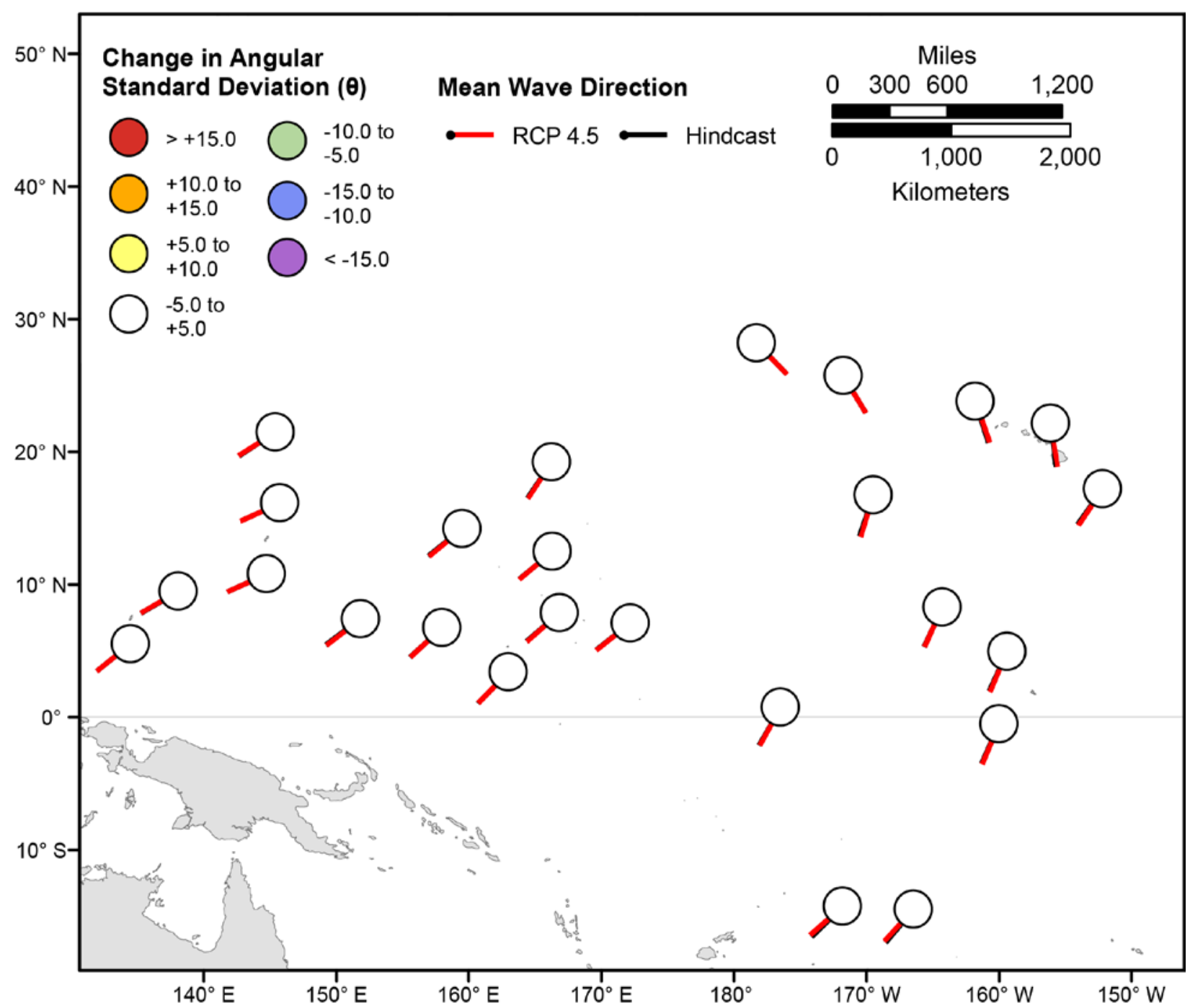

Figure 87. Map showing forecasted differences in the mean wave directions of significant wave heights and the standard deviation of wave directions of significant wave heights for the years 2081-2100 from hindcasted values during the March-May season under the RCP4.5 future climatic scenario. Mean wave directions at each point are indicated by lines radiating from the center of each point where RCP 20812100 mean wave directions are red and 1976-2005 hindcasted mean wave directions are black. The colors correspond to the magnitude of change in modeled mean wave direction standard deviation during 2081-2100 from those hindcasted for 1976-2005. Angular standard deviation units are in degrees. Mean wave directions are "heading towards". 


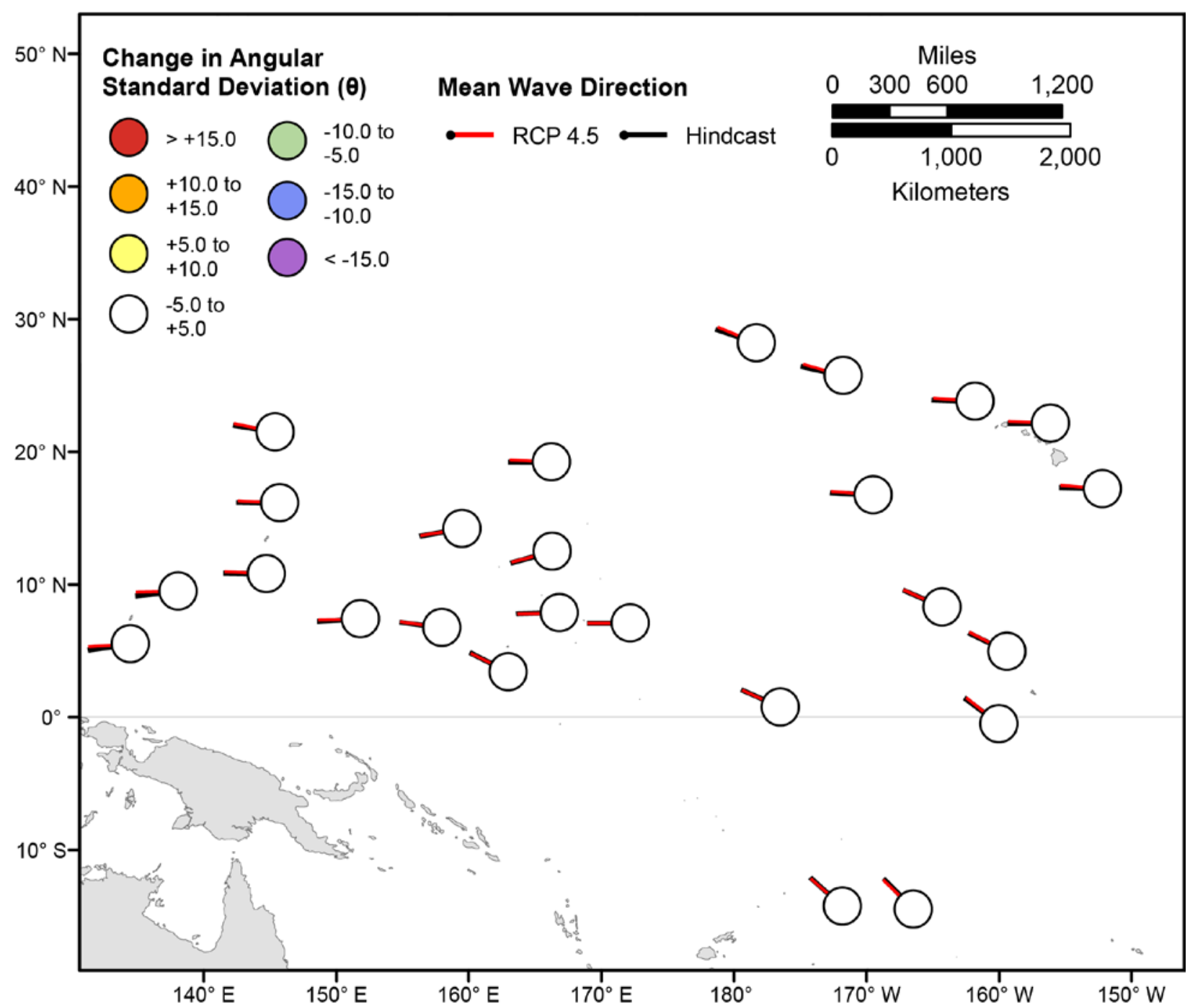

Figure 88. Map showing forecasted differences in the mean wave directions of significant wave heights and the standard deviation of wave directions of significant wave heights for the years 2081-2100 from hindcasted values during the June-July season under the RCP4.5 future climatic scenario. Mean wave directions at each point are indicated by lines radiating from the center of each point where RCP 20812100 mean wave directions are red and 1976-2005 hindcasted mean wave directions are black. The colors correspond to the magnitude of change in modeled mean wave direction standard deviation during 2081-2100 from those hindcasted for 1976-2005. Angular standard deviation units are in degrees. Mean wave directions are "heading towards". 


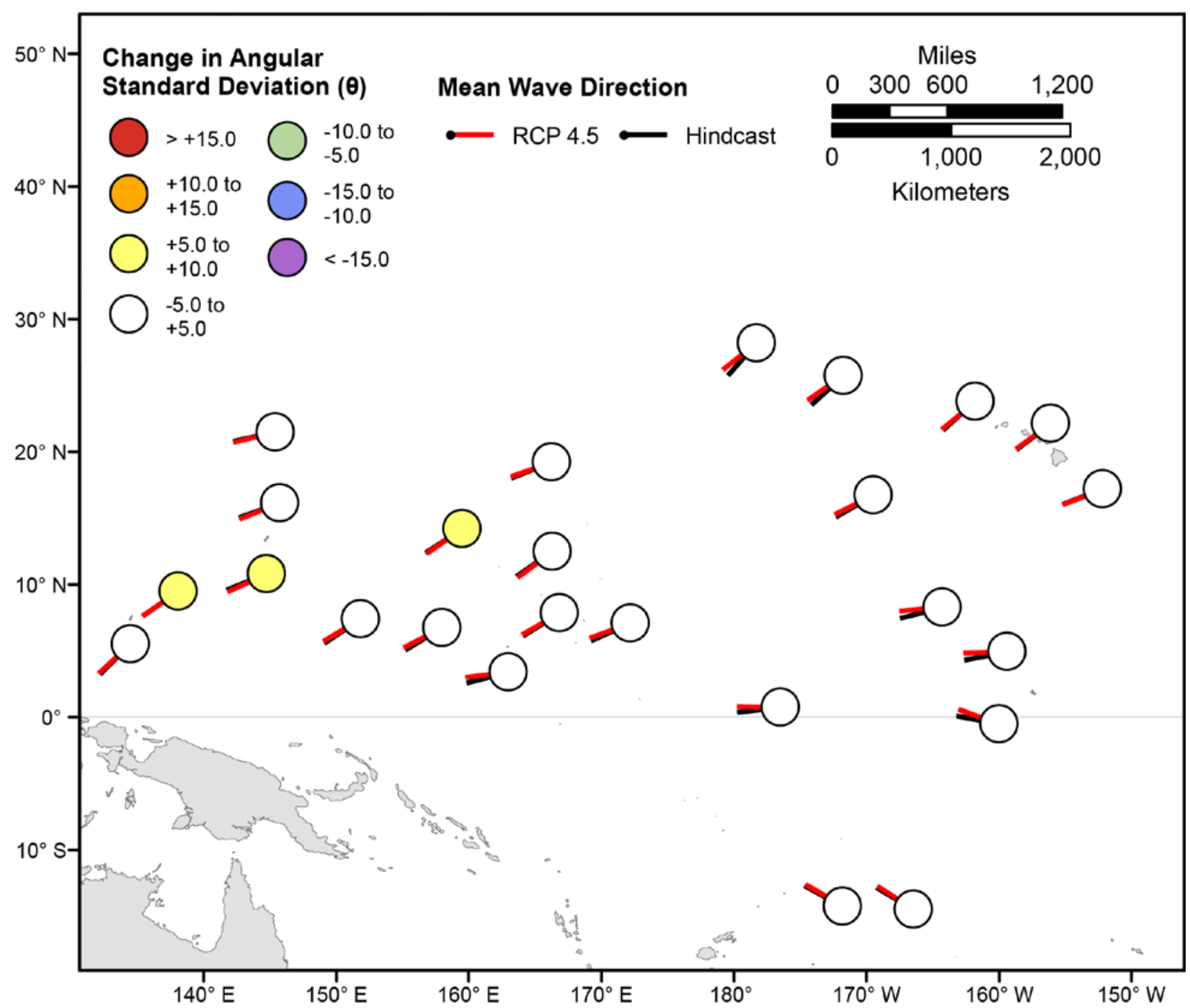

Figure 89. Map showing forecasted differences in the mean wave directions of significant wave heights and the standard deviation of wave directions of significant wave heights for the years 2081-2100 from hindcasted values during the September-November season under the RCP4.5 future climatic scenario. Mean wave directions at each point are indicated by lines radiating from the center of each point where RCP 2081-2100 mean wave directions are red and 1976-2005 hindcasted mean wave directions are black. The colors correspond to the magnitude of change in modeled mean wave direction standard deviation during 2081-2100 from those hindcasted for 1976-2005. Angular standard deviation units are in degrees. Mean wave directions are "heading towards". 


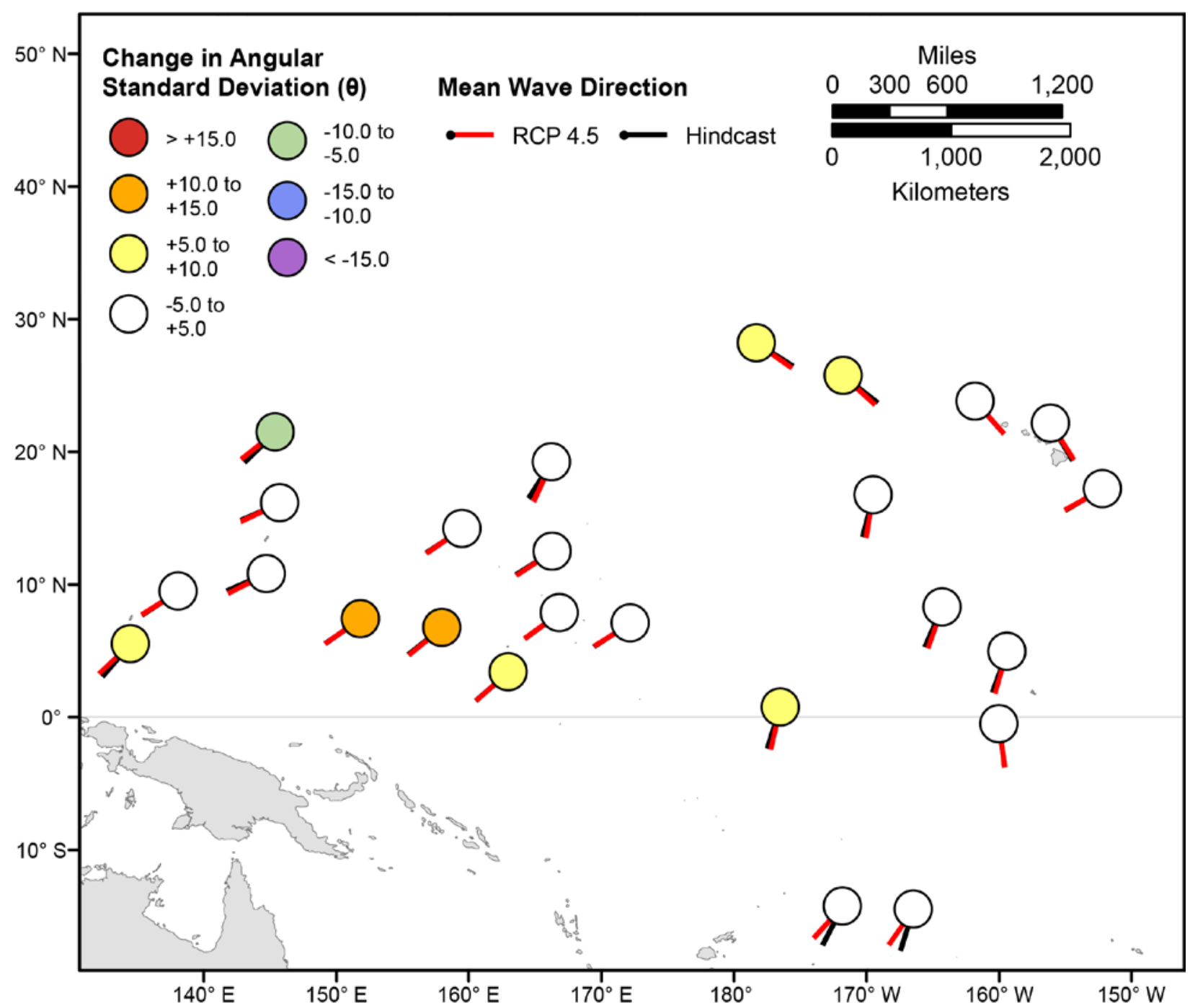

Figure 90. Map showing forecasted differences in the mean wave directions of the top 5 percent of significant wave heights and the standard deviation of wave directions of the top 5 percent of significant wave heights for the years 2081-2100 from hindcasted values during the December-February season under the RCP4.5 future climatic scenario. Mean wave directions at each point are indicated by lines radiating from the center of each point where RCP 2081-2100 mean wave directions are red and 19762005 hindcasted mean wave directions are black. The colors correspond to the magnitude of change in modeled mean wave direction standard deviation during 2081-2100 from those hindcasted for 19762005. Angular standard deviation units are in degrees. Mean wave directions are "heading towards". 


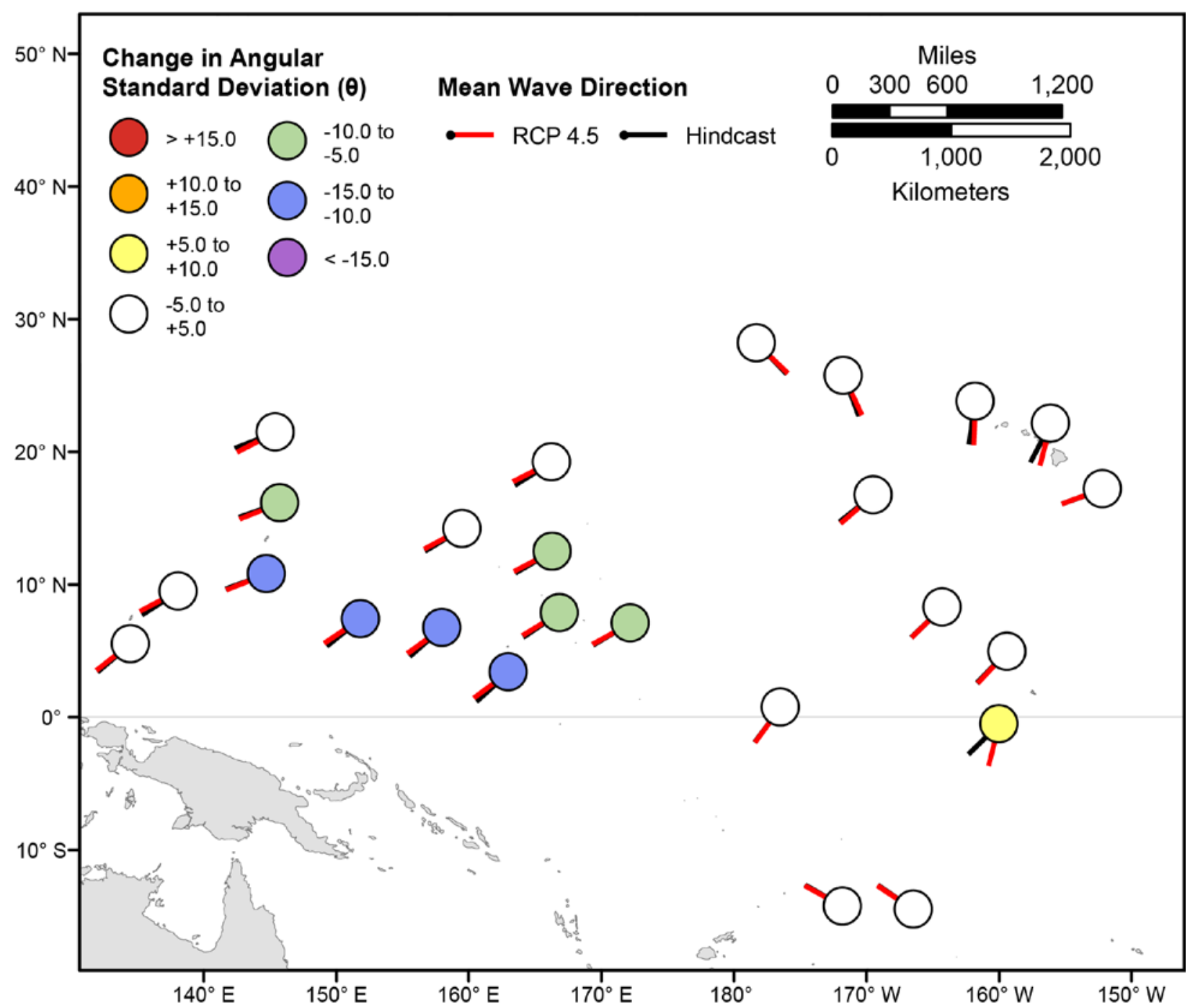

Figure 91. Map showing forecasted differences in the mean wave directions of the top 5 percent of significant wave heights and the standard deviation of wave directions of the top 5 percent of significant wave heights for the years 2081-2100 from hindcasted values during the March-May season under the RCP4.5 future climatic scenario. Mean wave directions at each point are indicated by lines radiating from the center of each point where RCP 2081-2100 mean wave directions are red and 1976-2005 hindcasted mean wave directions are black. The colors correspond to the magnitude of change in modeled mean wave direction standard deviation during 2081-2100 from those hindcasted for 19762005. Angular standard deviation units are in degrees. Mean wave directions are "heading towards". 


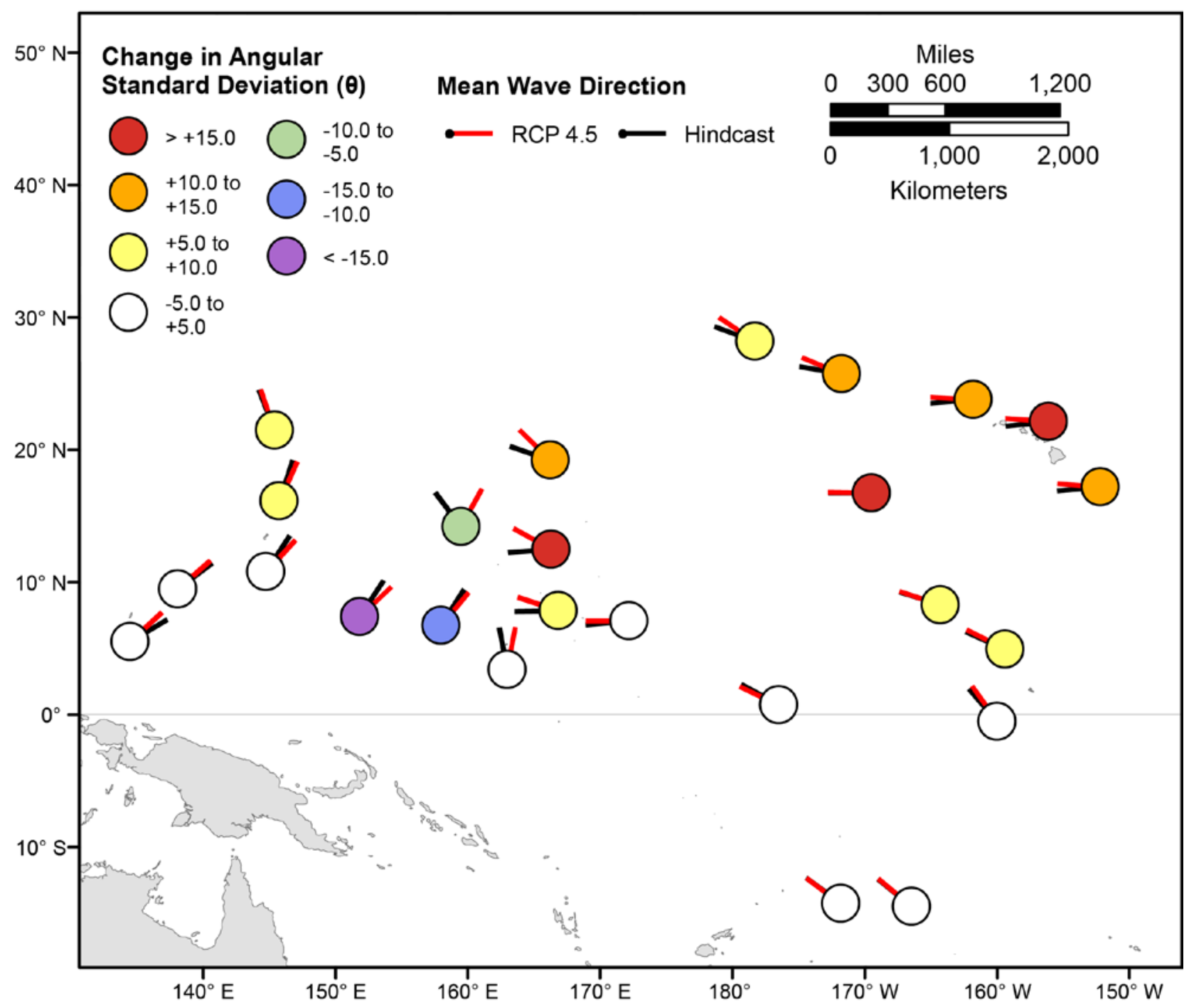

Figure 92. Map showing forecasted differences in the mean wave directions of the top 5 percent of significant wave heights and the standard deviation of wave directions of the top 5 percent of significant wave heights for the years 2081-2100 from hindcasted values during the June-August season under the RCP4.5 future climatic scenario. Mean wave directions at each point are indicated by lines radiating from the center of each point where RCP 2081-2100 mean wave directions are red and 1976-2005 hindcasted mean wave directions are black. The colors correspond to the magnitude of change in modeled mean wave direction standard deviation during 2081-2100 from those hindcasted for 19762005. Angular standard deviation units are in degrees. Mean wave directions are "heading towards". 


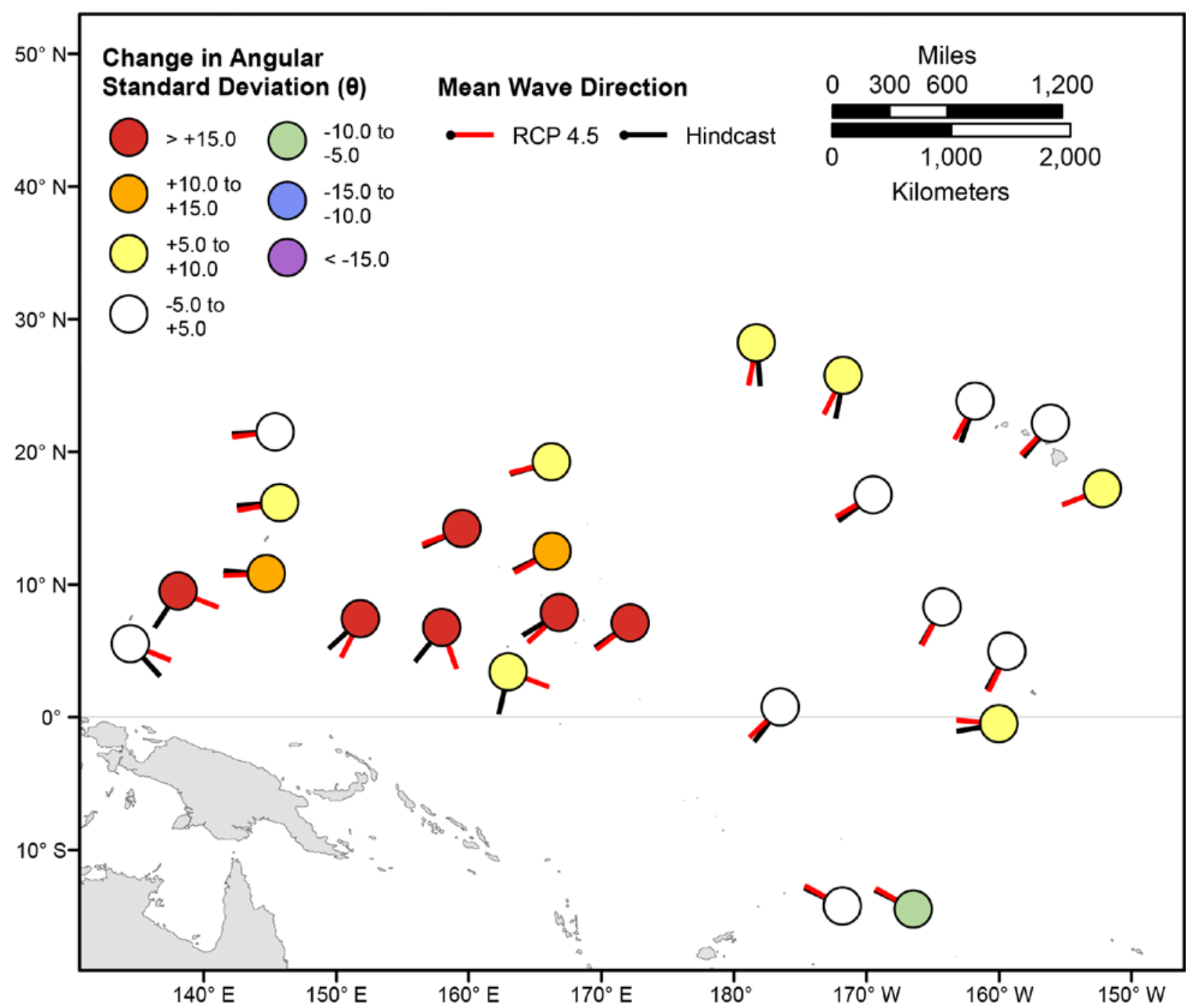

Figure 93. Map showing forecasted differences in the mean wave directions of the top 5 percent of significant wave heights and the standard deviation of wave directions of the top 5 percent of significant wave heights for the years 2081-2100 from hindcasted values during the September-November season under the RCP4.5 future climatic scenario. Mean wave directions at each point are indicated by lines radiating from the center of each point where RCP 2081-2100 mean wave directions are red and 19762005 hindcasted mean wave directions are black. The colors correspond to the magnitude of change in modeled mean wave direction standard deviation during 2081-2100 from those hindcasted for 19762005. Angular standard deviation units are in degrees. Mean wave directions are "heading towards". 


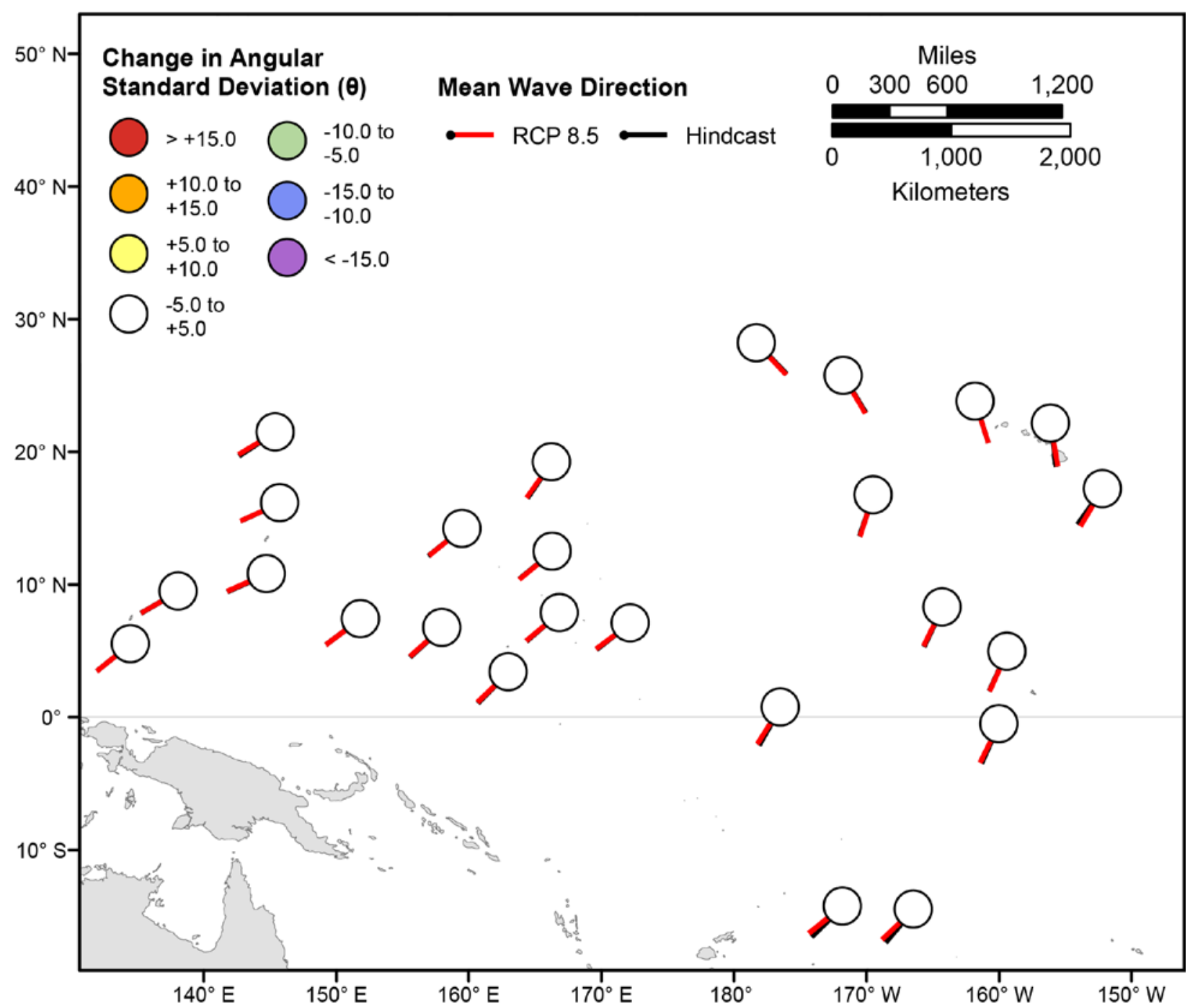

Figure 94. Map showing forecasted differences in the mean wave directions of significant wave heights and the standard deviation of wave directions of significant wave heights for the years 2081-2100 from hindcasted values during the December-February season under the RCP8.5 future climatic scenario. Mean wave directions at each point are indicated by lines radiating from the center of each point where RCP 2081-2100 mean wave directions are red and 1976-2005 hindcasted mean wave directions are black. The colors correspond to the magnitude of change in modeled mean wave direction standard deviation during 2081-2100 from those hindcasted for 1976-2005. Angular standard deviation units are in degrees. Mean wave directions are "heading towards". 


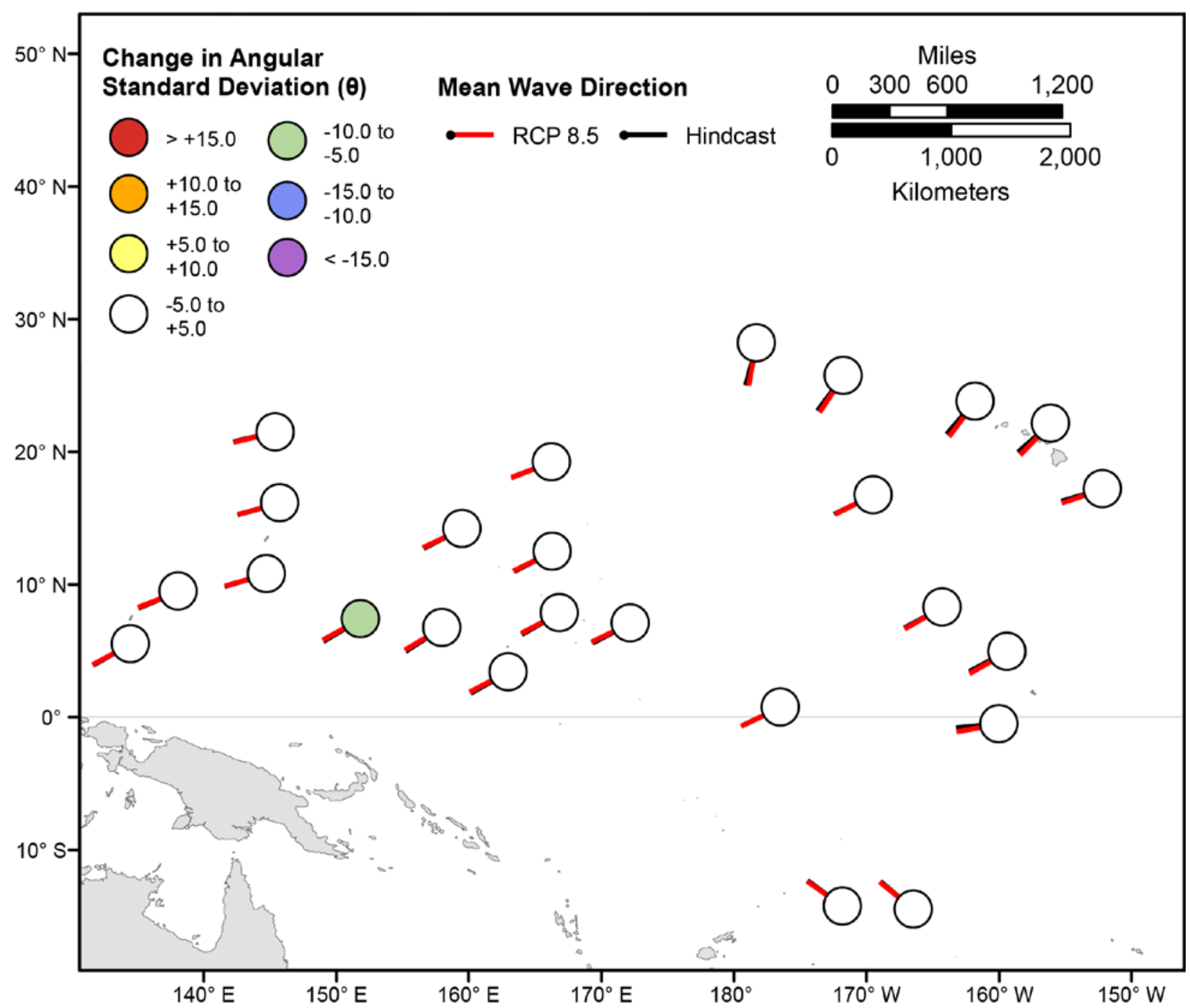

Figure 95. Map showing forecasted differences in the mean wave directions of significant wave heights and the standard deviation of wave directions of significant wave heights for the years 2081-2100 from hindcasted values during the March-May season under the RCP8.5 future climatic scenario. Mean wave directions at each point are indicated by lines radiating from the center of each point where RCP 20812100 mean wave directions are red and 1976-2005 hindcasted mean wave directions are black. The colors correspond to the magnitude of change in modeled mean wave direction standard deviation during 2081-2100 from those hindcasted for 1976-2005. Angular standard deviation units are in degrees. Mean wave directions are "heading towards". 


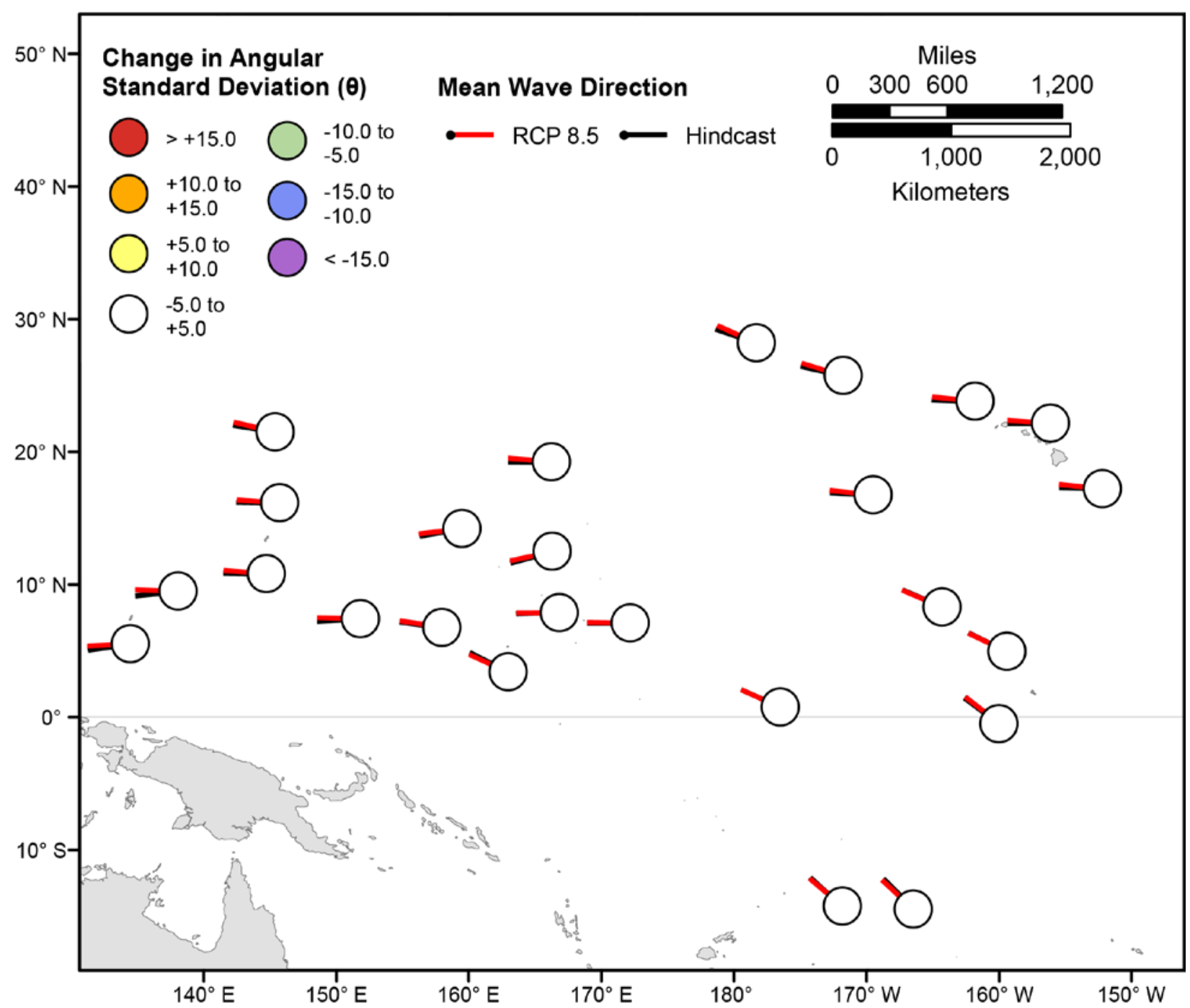

Figure 96. Map showing forecasted differences in the mean wave directions of significant wave heights and the standard deviation of wave directions of significant wave heights for the years 2081-2100 from hindcasted values during the June-July season under the RCP8.5 future climatic scenario. Mean wave directions at each point are indicated by lines radiating from the center of each point where RCP 20812100 mean wave directions are red and 1976-2005 hindcasted mean wave directions are black. The colors correspond to the magnitude of change in modeled mean wave direction standard deviation during 2081-2100 from those hindcasted for 1976-2005. Angular standard deviation units are in degrees. Mean wave directions are "heading towards". 


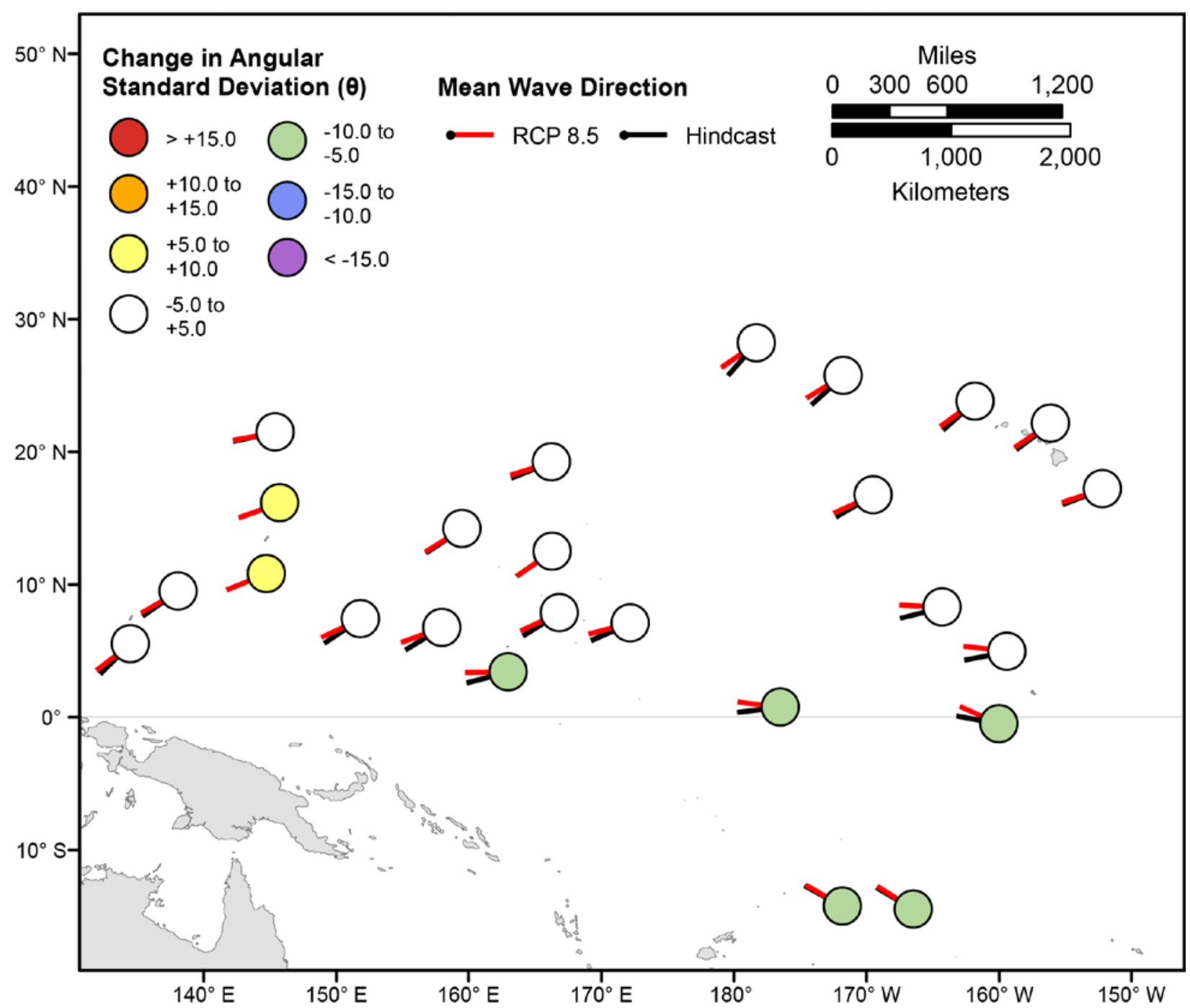

Figure 97. Map showing forecasted differences in the mean wave directions of significant wave heights and the standard deviation of wave directions of significant wave heights for the years 2081-2100 from hindcasted values during the September-November season under the RCP8.5 future climatic scenario. Mean wave directions at each point are indicated by lines radiating from the center of each point where RCP 2081-2100 mean wave directions are red and 1976-2005 hindcasted mean wave directions are black. The colors correspond to the magnitude of change in modeled mean wave direction standard deviation during 2081-2100 from those hindcasted for 1976-2005. Angular standard deviation units are in degrees. Mean wave directions are "heading towards". 


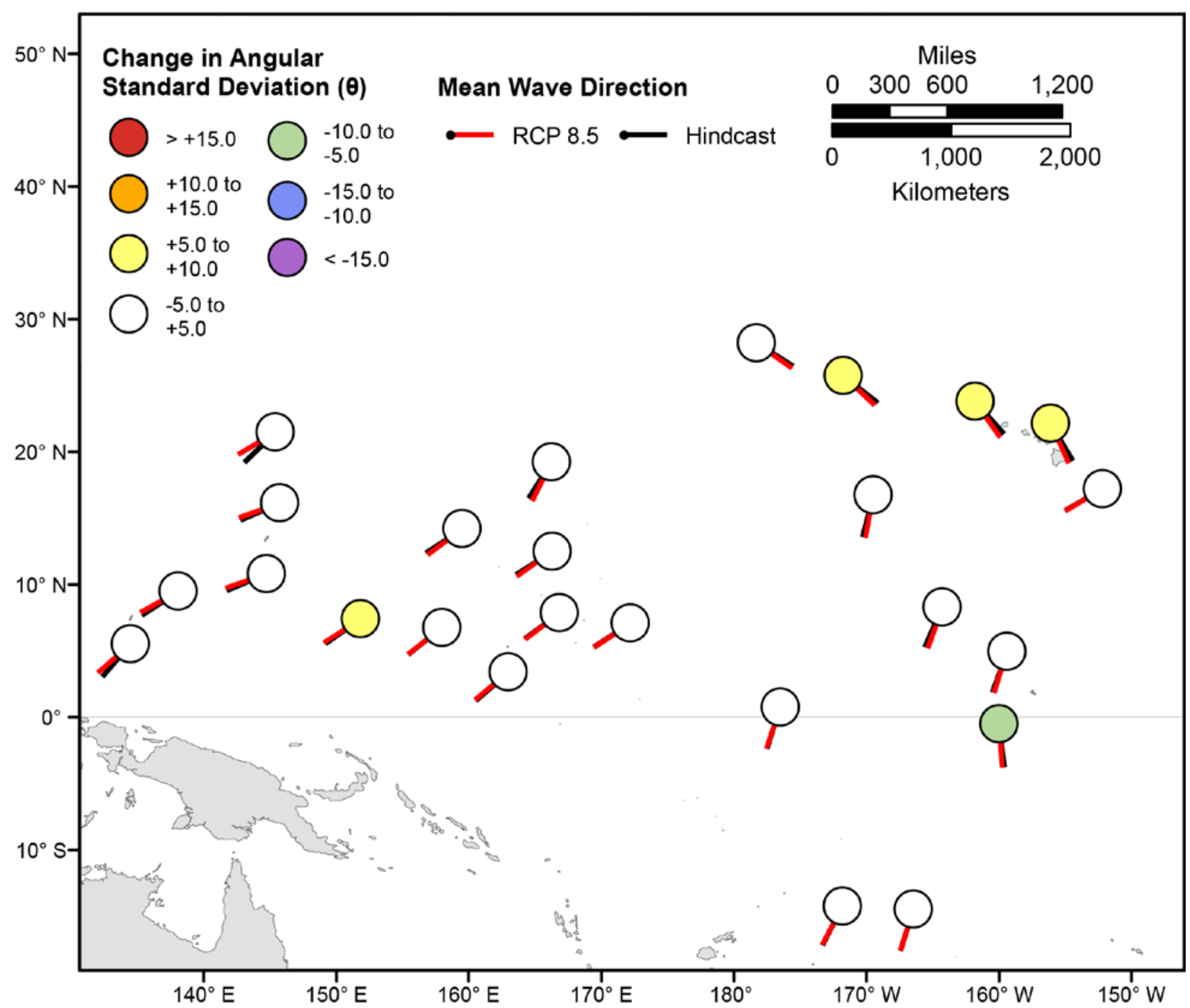

Figure 98. Map showing forecasted differences in the mean wave directions of the top 5 percent of significant wave heights and the standard deviation of wave directions of the top 5 percent of significant wave heights for the years 2081-2100 from hindcasted values during the December-February season under the RCP8.5 future climatic scenario. Mean wave directions at each point are indicated by lines radiating from the center of each point where RCP 2081-2100 mean wave directions are red and 19762005 hindcasted mean wave directions are black. The colors correspond to the magnitude of change in modeled mean wave direction standard deviation during 2081-2100 from those hindcasted for 19762005. Angular standard deviation units are in degrees. Mean wave directions are "heading towards". 


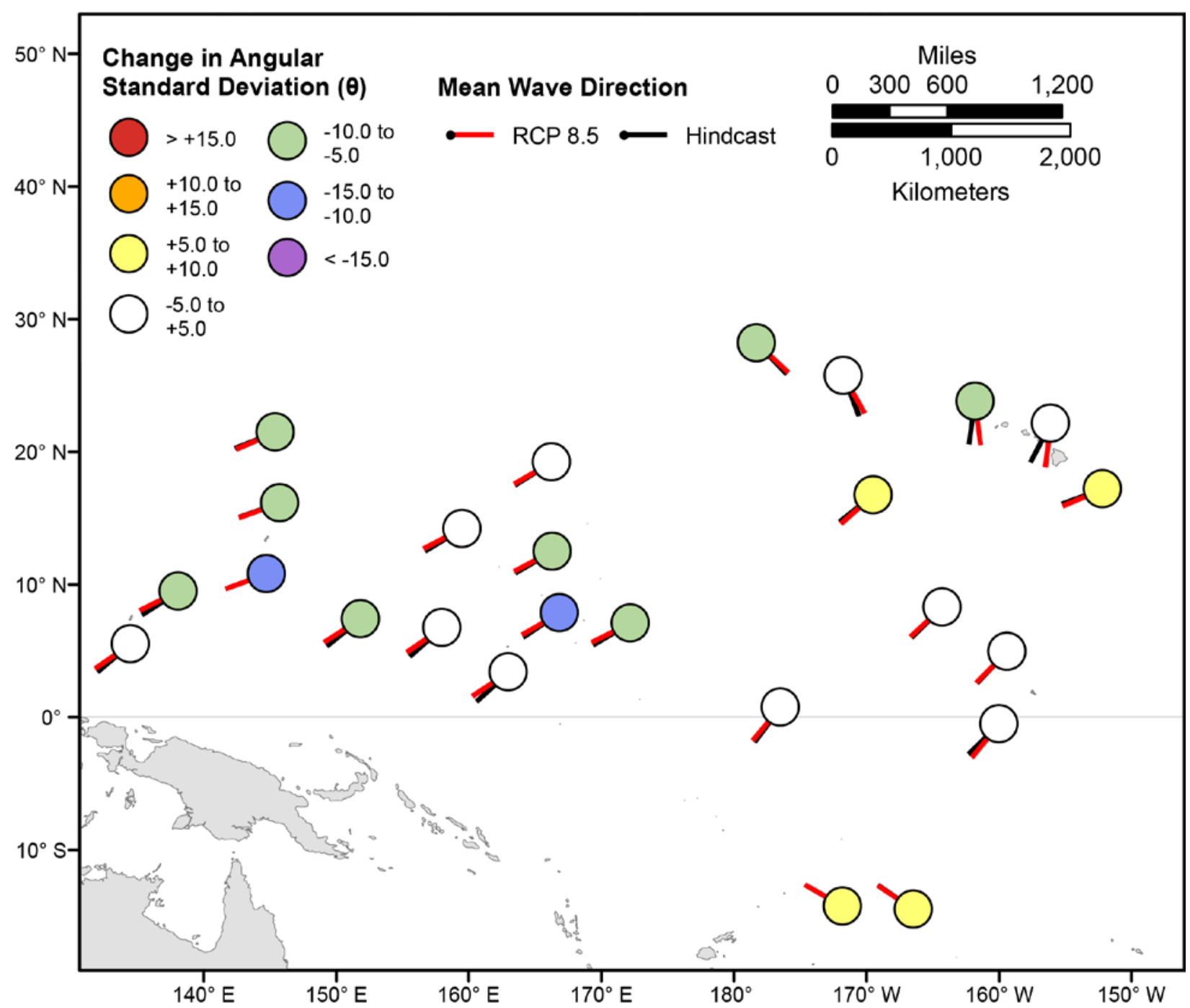

Figure 99. Map showing forecasted differences in the mean wave directions of the top 5 percent of significant wave heights and the standard deviation of wave directions of the top 5 percent of significant wave heights for the years 2081-2100 from hindcasted values during the March-May season under the RCP8.5 future climatic scenario. Mean wave directions at each point are indicated by lines radiating from the center of each point where RCP 2081-2100 mean wave directions are red and 1976-2005 hindcasted mean wave directions are black. The colors correspond to the magnitude of change in modeled mean wave direction standard deviation during 2081-2100 from those hindcasted for 19762005. Angular standard deviation units are in degrees. Mean wave directions are "heading towards". 


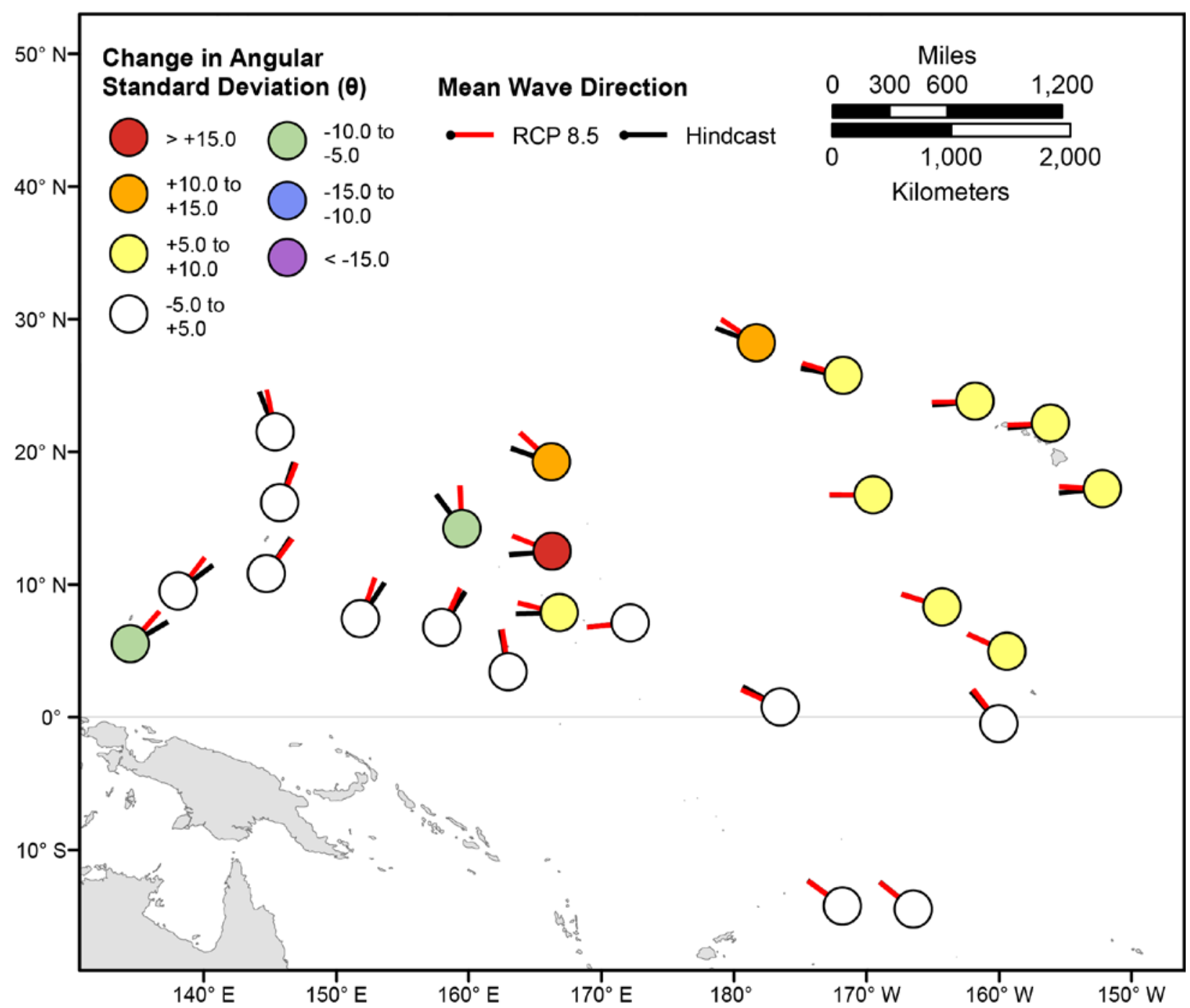

Figure 100. Map showing forecasted differences in the mean wave directions of the top 5 percent of significant wave heights and the standard deviation of wave directions of the top 5 percent of significant wave heights for the years 2081-2100 from hindcasted values during the June-August season under the RCP8.5 future climatic scenario. Mean wave directions at each point are indicated by lines radiating from the center of each point where RCP 2081-2100 mean wave directions are red and 1976-2005 hindcasted mean wave directions are black. The colors correspond to the magnitude of change in modeled mean wave direction standard deviation during 2081-2100 from those hindcasted for 19762005. Angular standard deviation units are in degrees. Mean wave directions are "heading towards". 


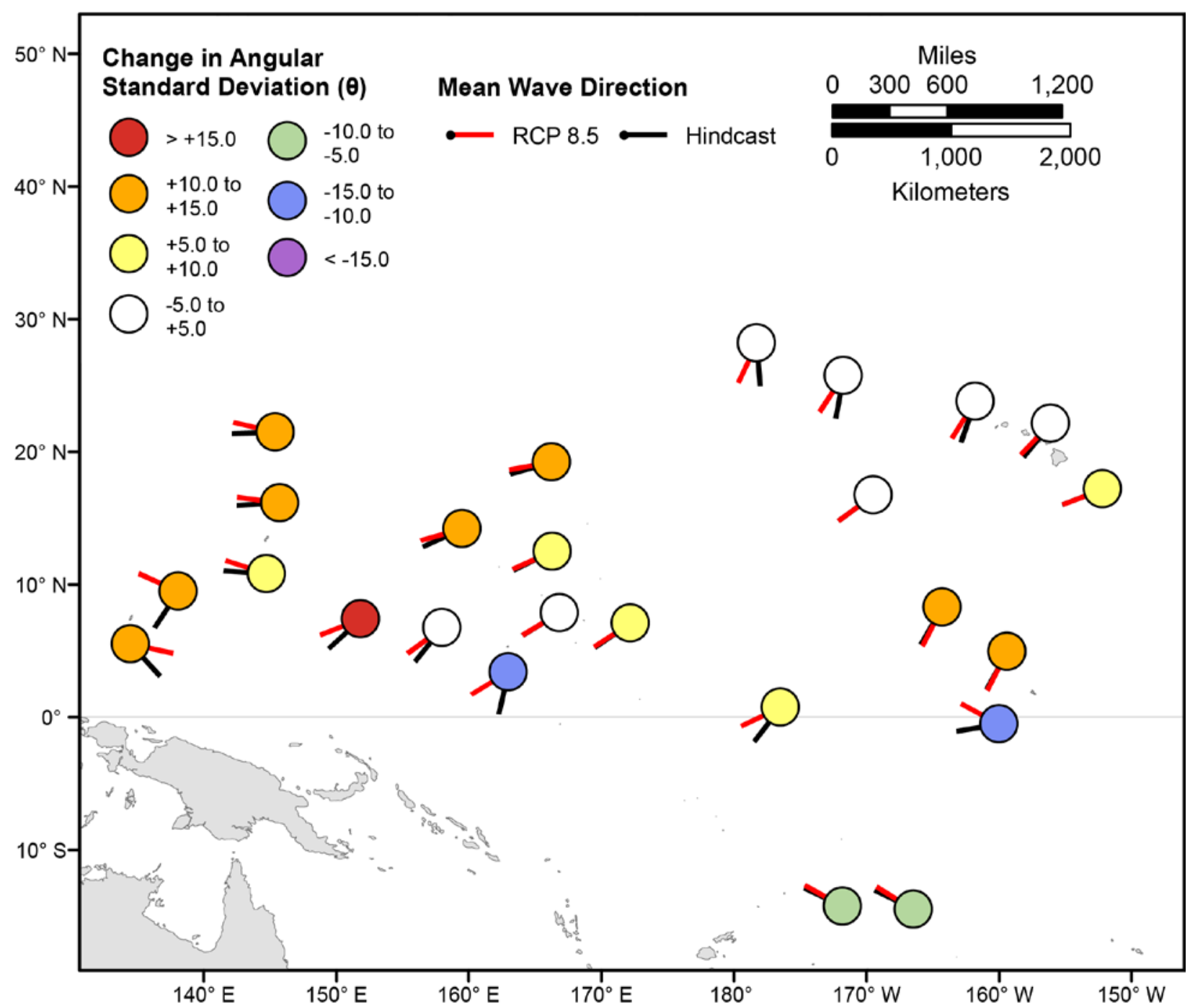

Figure 101. Map showing forecasted differences in the mean wave directions of the top 5 percent of significant wave heights and the standard deviation of wave directions of the top 5 percent of significant wave heights for the years 2081-2100 from hindcasted values during the September-November season under the RCP8.5 future climatic scenario. Mean wave directions at each point are indicated by lines radiating from the center of each point where RCP 2081-2100 mean wave directions are red and 19762005 hindcasted mean wave directions are black. The colors correspond to the magnitude of change in modeled mean wave direction standard deviation during 2081-2100 from those hindcasted for 19762005. Angular standard deviation units are in degrees. Mean wave directions are "heading towards". 


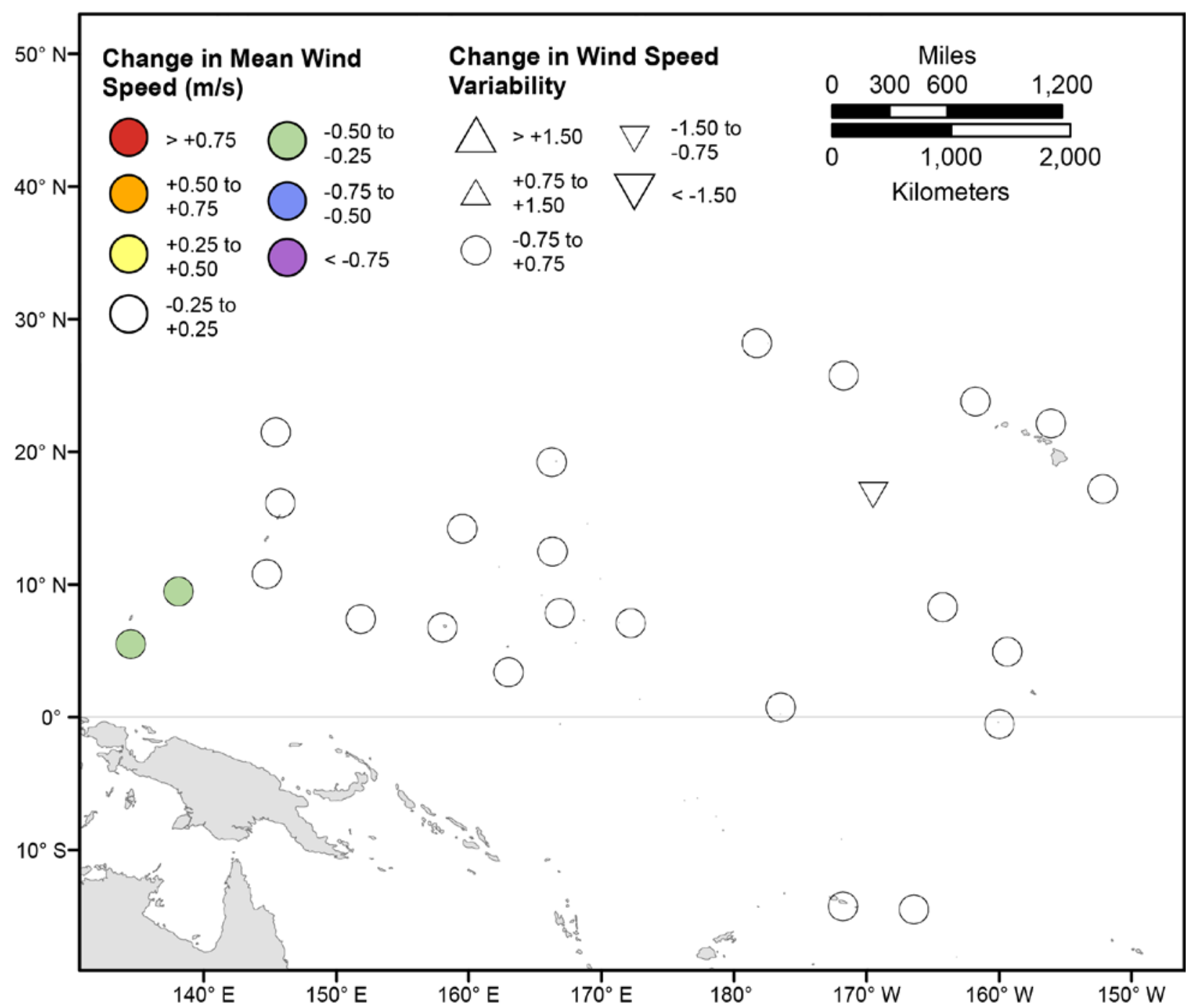

Figure 102. Map showing forecasted differences in mean wind speed and variance in wind speed for the years 2026-2045 from hindcasted values during the December-February season under the RCP4.5 future climatic scenario. The colors correspond to the magnitude of change in modeled mean wind speeds during 2026-2045 from those hindcasted for 1976-2005. The shapes correspond to the magnitude of change in modeled variance in wind speed during 2026-2045 from those hindcasted for 1976-2005. Units are in meters per second. 


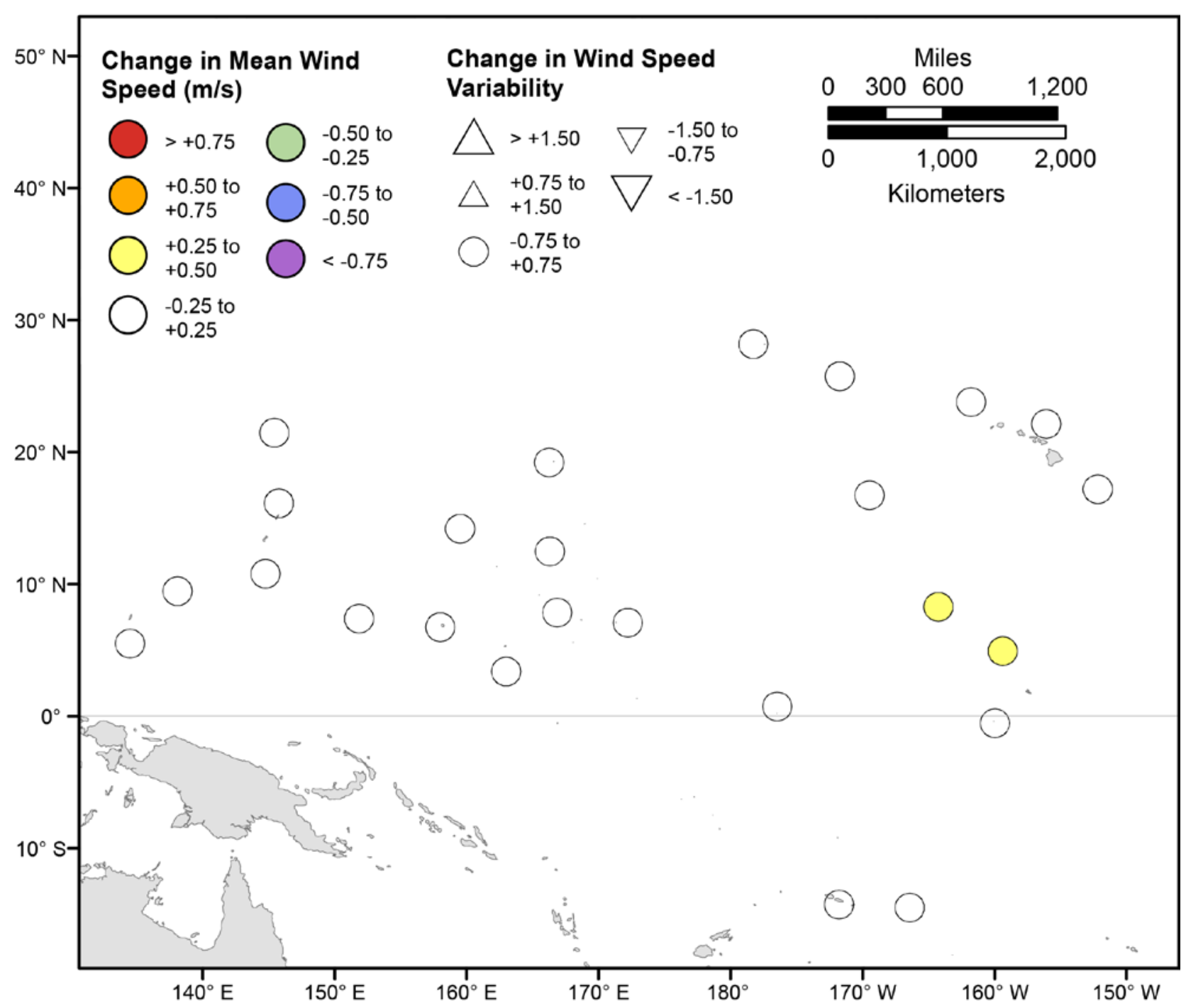

Figure 103. Map showing forecasted differences in mean wind speed and variance in wind speed for the years 2026-2045 from hindcasted values during the March-May season under the RCP4.5 future climatic scenario. The colors correspond to the magnitude of change in modeled mean wind speeds during 2026-2045 from those hindcasted for 1976-2005. The shapes correspond to the magnitude of change in modeled variance in wind speed during 2026-2045 from those hindcasted for 1976-2005. Units are in meters per second. 


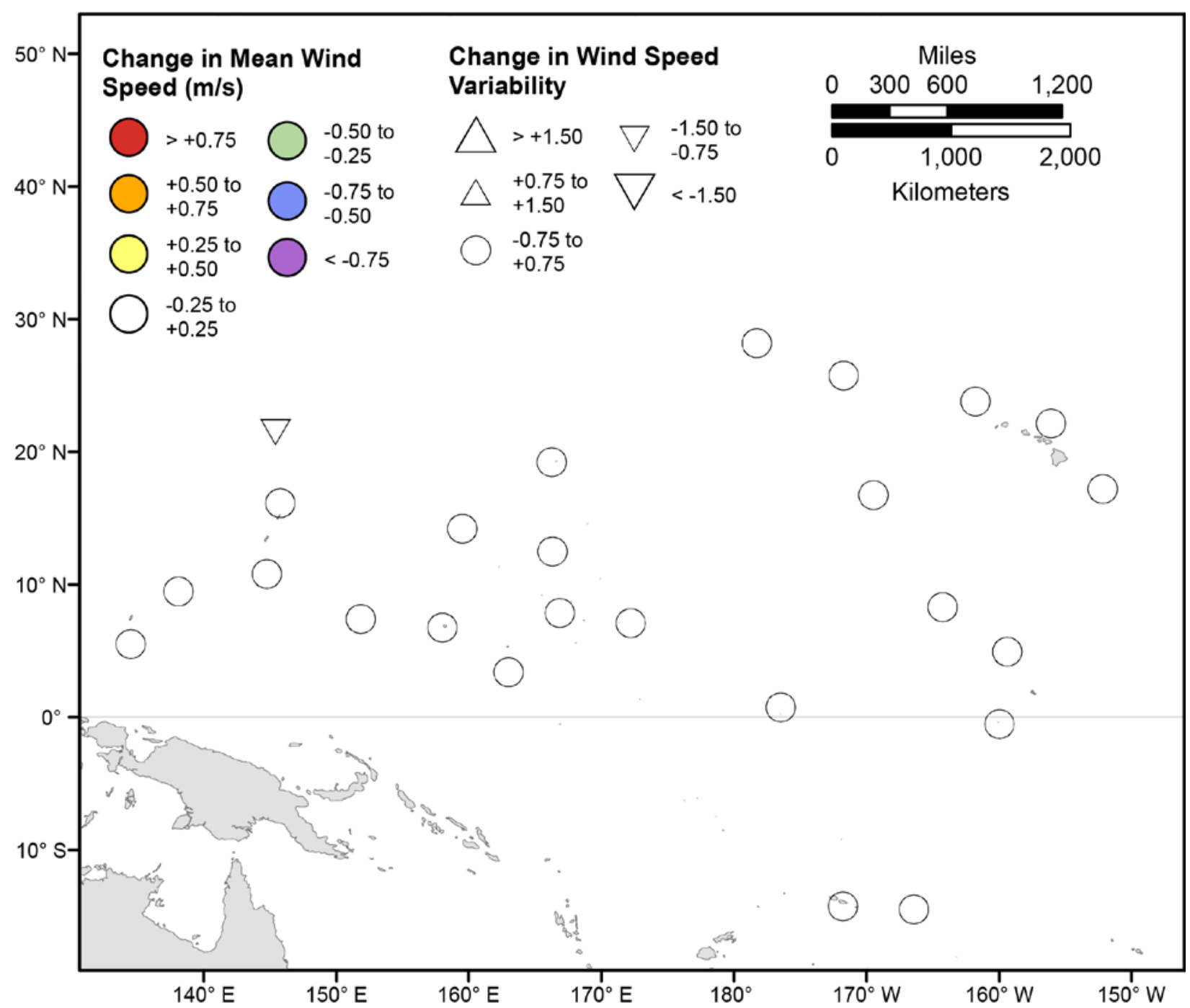

Figure 104. Map showing forecasted differences in mean wind speed and variance in wind speed for the years 2026-2045 from hindcasted values during the June-August season under the RCP4.5 future climatic scenario. The colors correspond to the magnitude of change in modeled mean wind speeds during 2026-2045 from those hindcasted for 1976-2005. The shapes correspond to the magnitude of change in modeled variance in wind speed during 2026-2045 from those hindcasted for 1976-2005. Units are in meters per second. 


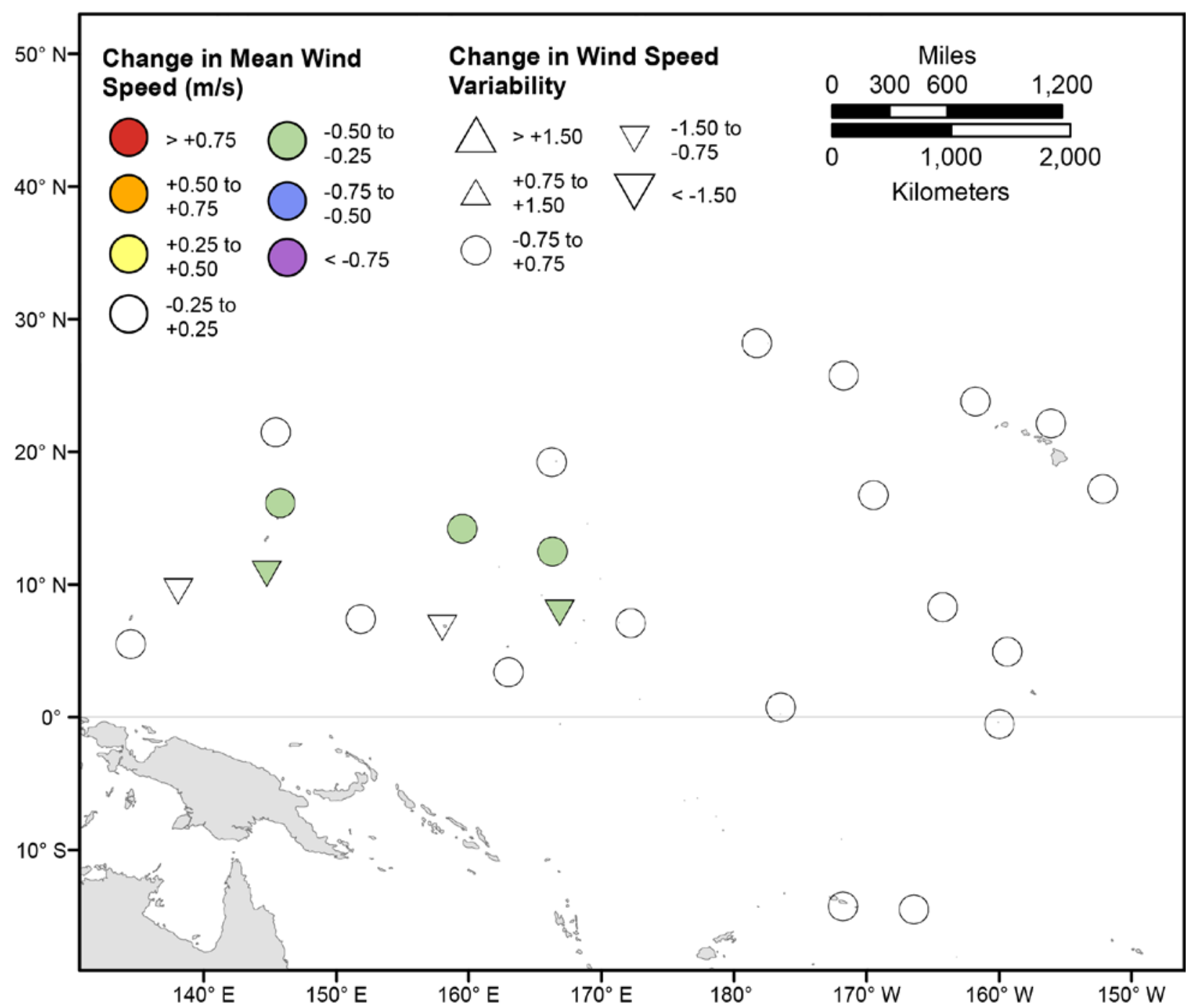

Figure 105. Map showing forecasted differences in mean wind speed and variance in wind speed for the years 2026-2045 from hindcasted values during the September-November season under the RCP4.5 future climatic scenario. The colors correspond to the magnitude of change in modeled mean wind speeds during 2026-2045 from those hindcasted for 1976-2005. The shapes correspond to the magnitude of change in modeled variance in wind speed during 2026-2045 from those hindcasted for 1976-2005. Units are in meters per second. 


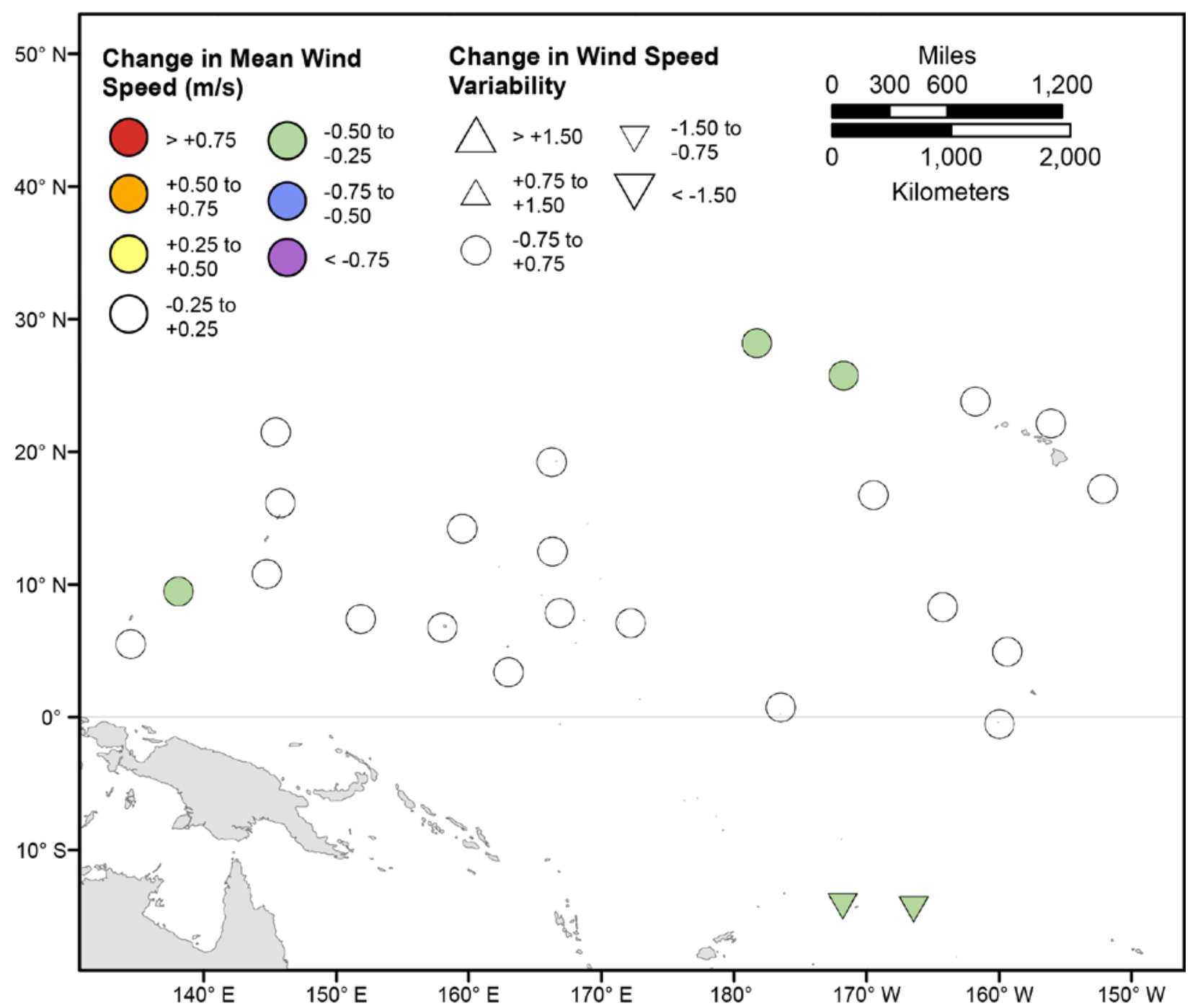

Figure 106. Map showing forecasted differences in the mean of the top 5 percent of wind speeds and variance in the top 5 percent of wind speeds for the years 2026-2045 from hindcasted values during the December-February season under the RCP4.5 future climatic scenario. The colors correspond to the magnitude of change in modeled mean wind speeds during 2026-2045 from those hindcasted for 19762005. The shapes correspond to the magnitude of change in modeled variance in wind speed during 2026-2045 from those hindcasted for 1976-2005. Units are in meters per second. 


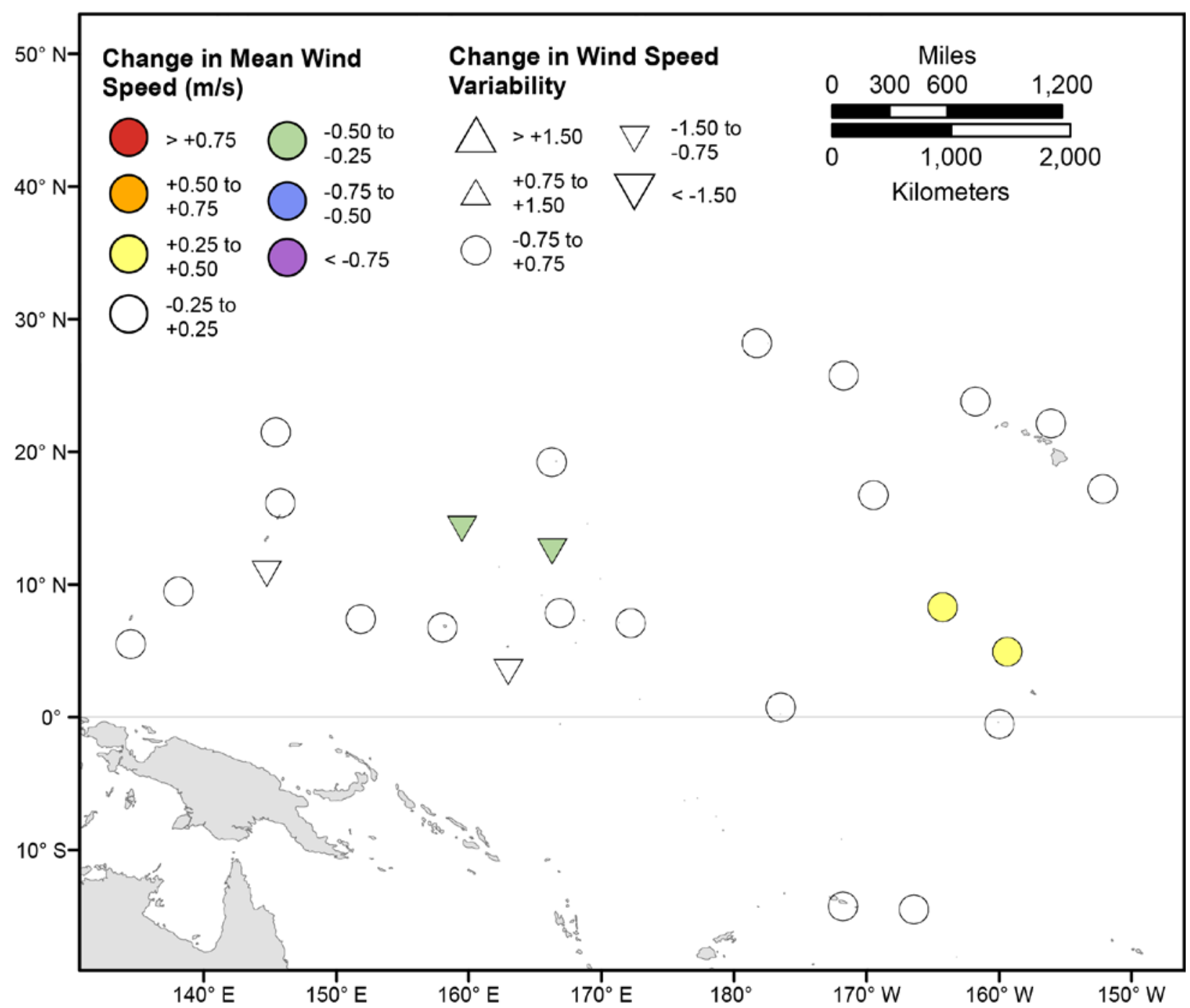

Figure 107. Map showing forecasted differences in the mean of the top 5 percent of wind speeds and variance in the top 5 percent of wind speeds for the years 2026-2045 from hindcasted values during the March-May season under the RCP4.5 future climatic scenario. The colors correspond to the magnitude of change in modeled mean wind speeds during 2026-2045 from those hindcasted for 1976-2005. The shapes correspond to the magnitude of change in modeled variance in wind speed during 2026-2045 from those hindcasted for 1976-2005. Units are in meters per second. 


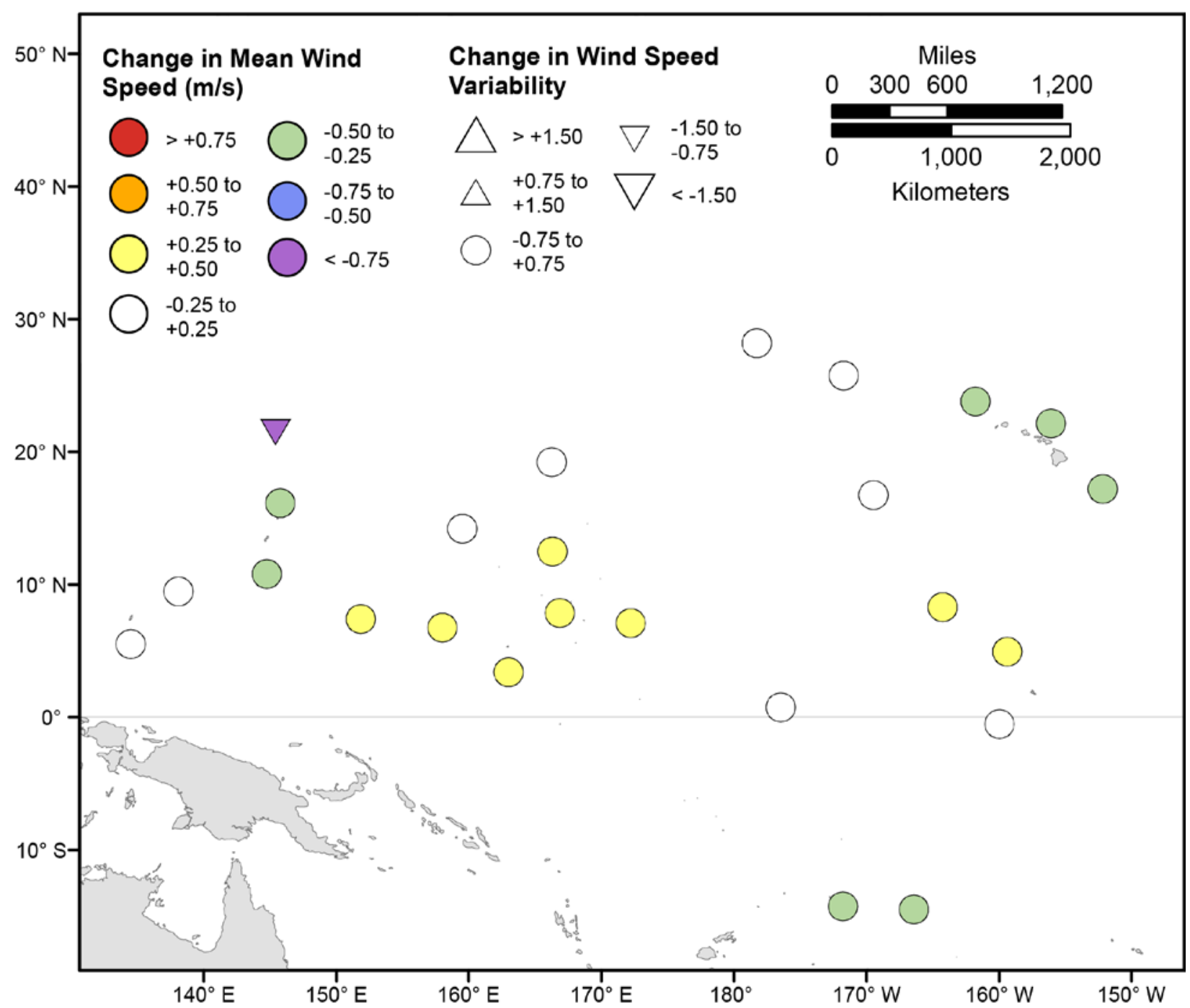

Figure 108. Map showing forecasted differences in the mean of the top 5 percent of wind speeds and variance in the top 5 percent of wind speeds for the years 2026-2045 from hindcasted values during the June-August season under the RCP4.5 future climatic scenario. The colors correspond to the magnitude of change in modeled mean wind speeds during 2026-2045 from those hindcasted for 1976-2005. The shapes correspond to the magnitude of change in modeled variance in wind speed during 2026-2045 from those hindcasted for 1976-2005. Units are in meters per second. 


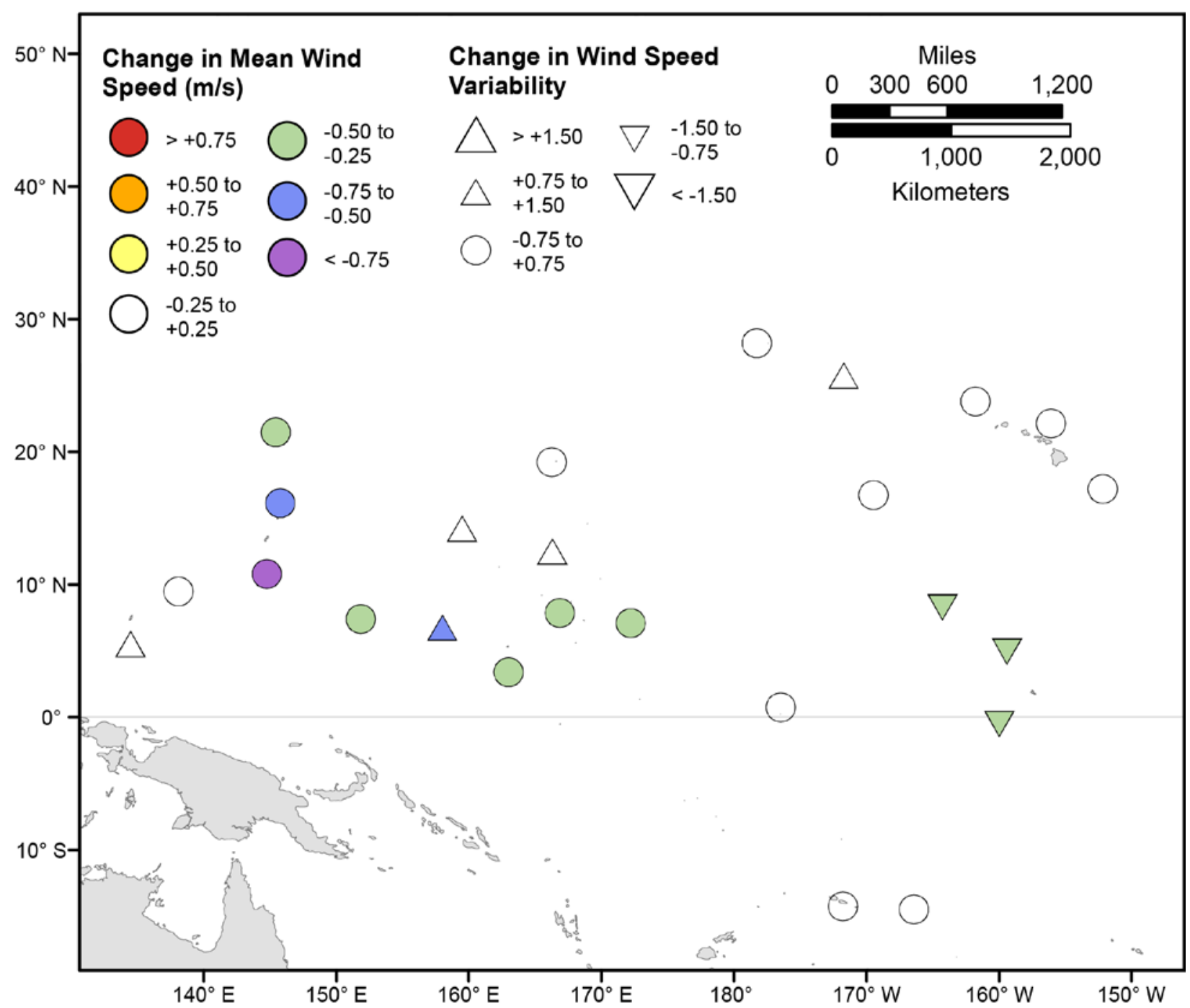

Figure 109. Map showing forecasted differences in the mean of the top 5 percent of wind speeds and variance in the top 5 percent of wind speeds for the years 2026-2045 from hindcasted values during the September-November season under the RCP4.5 future climatic scenario. The colors correspond to the magnitude of change in modeled mean wind speeds during 2026-2045 from those hindcasted for 19762005. The shapes correspond to the magnitude of change in modeled variance in wind speed during 2026-2045 from those hindcasted for 1976-2005. Units are in meters per second. 


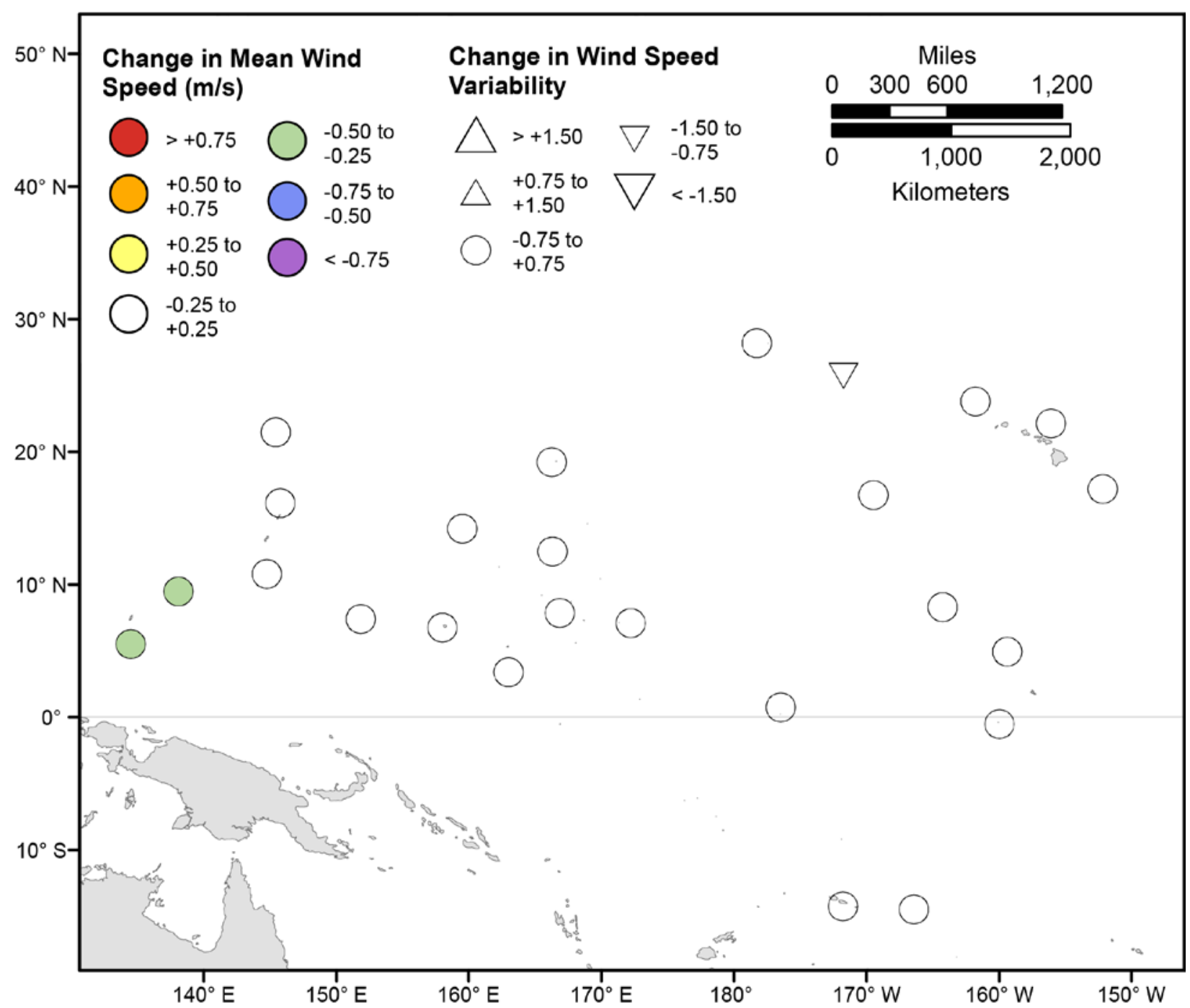

Figure 110. Map showing forecasted differences in mean wind speed and variance in wind speed for the years 2026-2045 from hindcasted values during the December-February season under the RCP8.5 future climatic scenario. The colors correspond to the magnitude of change in modeled mean wind speeds during 2026-2045 from those hindcasted for 1976-2005. The shapes correspond to the magnitude of change in modeled variance in wind speed during 2026-2045 from those hindcasted for 1976-2005. Units are in meters per second. 


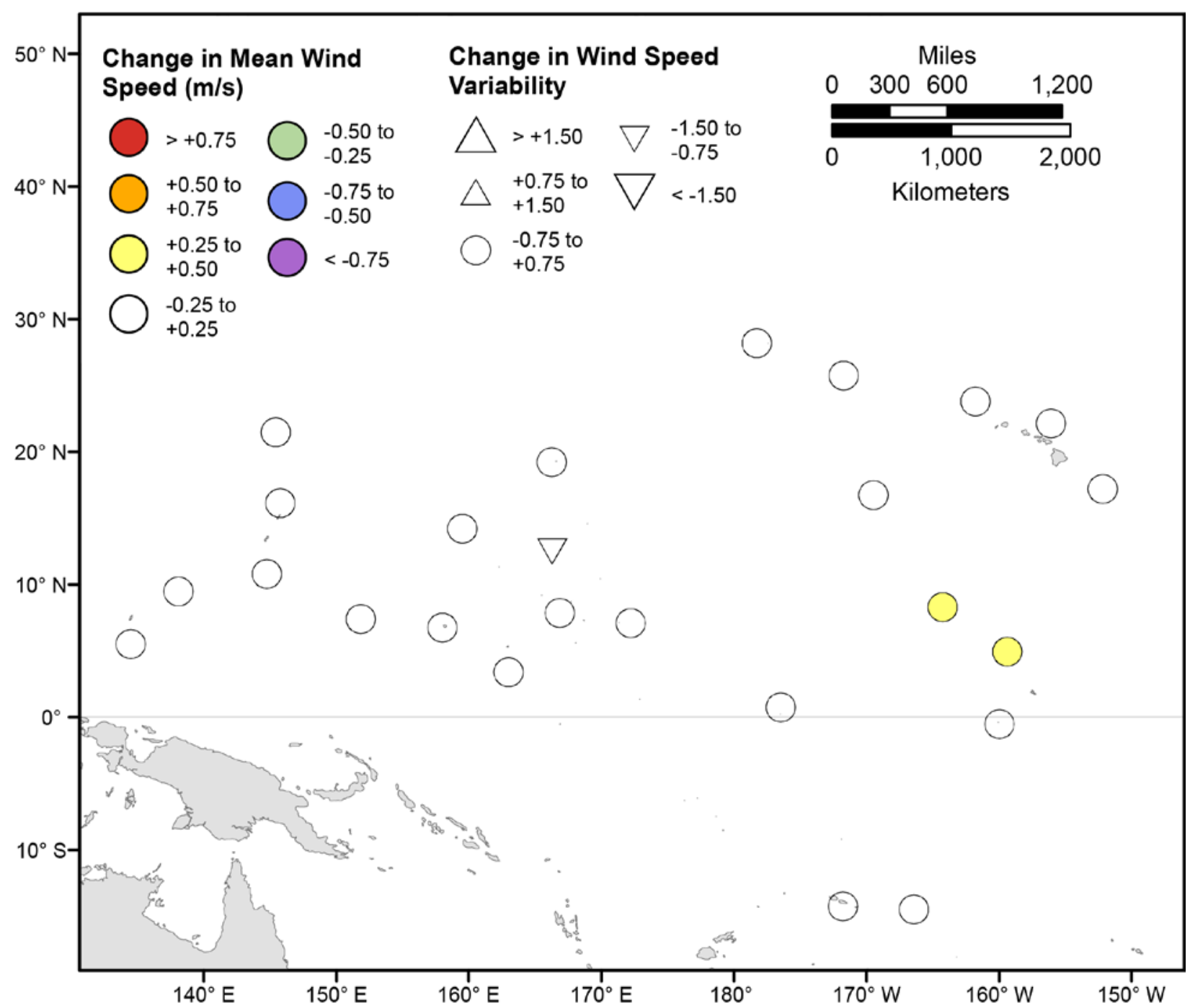

Figure 111. Map showing forecasted differences in mean wind speed and variance in wind speed for the years 2026-2045 from hindcasted values during the March-May season under the RCP8.5 future climatic scenario. The colors correspond to the magnitude of change in modeled mean wind speeds during 2026-2045 from those hindcasted for 1976-2005. The shapes correspond to the magnitude of change in modeled variance in wind speed during 2026-2045 from those hindcasted for 1976-2005. Units are in meters per second. 


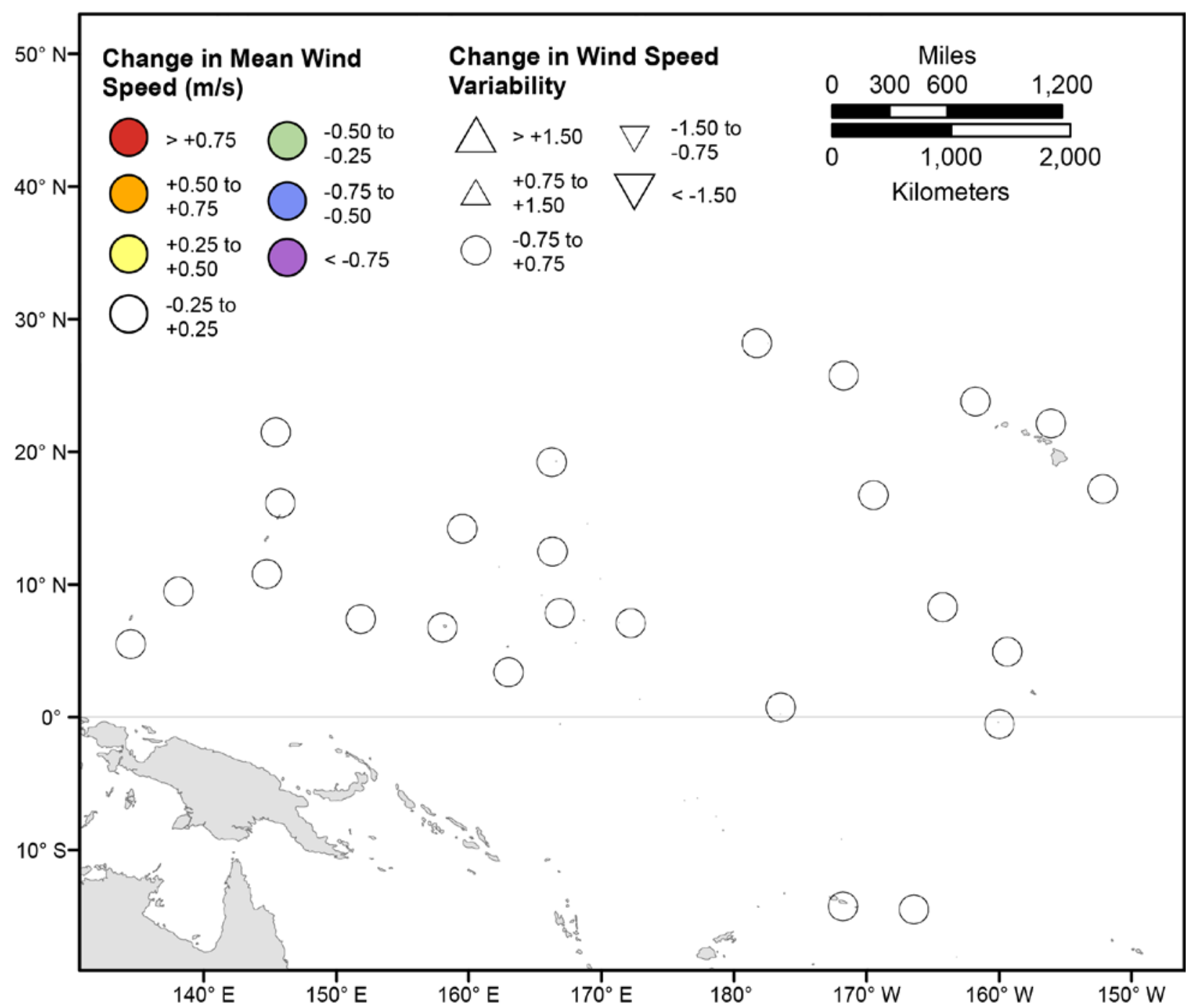

Figure 112. Map showing forecasted differences in mean wind speed and variance in wind speed for the years 2026-2045 from hindcasted values during the June-August season under the RCP8.5 future climatic scenario. The colors correspond to the magnitude of change in modeled mean wind speeds during 2026-2045 from those hindcasted for 1976-2005. The shapes correspond to the magnitude of change in modeled variance in wind speed during 2026-2045 from those hindcasted for 1976-2005. Units are in meters per second. 


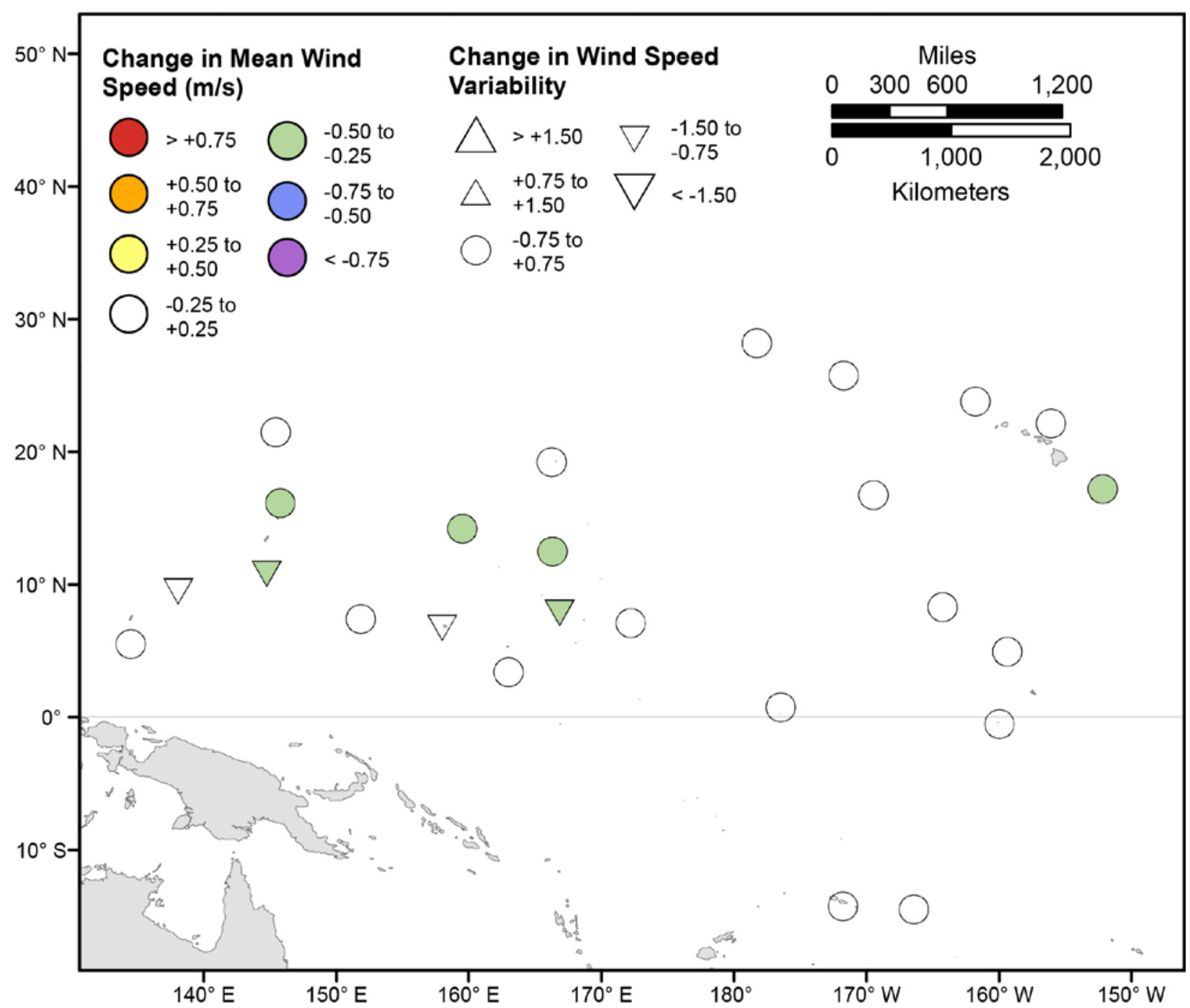

Figure 113. Map showing forecasted differences in mean wind speed and variance in wind speed for the years 2026-2045 from hindcasted values during the September-November season under the RCP8.5 future climatic scenario. The colors correspond to the magnitude of change in modeled mean wind speeds during 2026-2045 from those hindcasted for 1976-2005. The shapes correspond to the magnitude of change in modeled variance in wind speed during 2026-2045 from those hindcasted for 1976-2005. Units are in meters per second. 


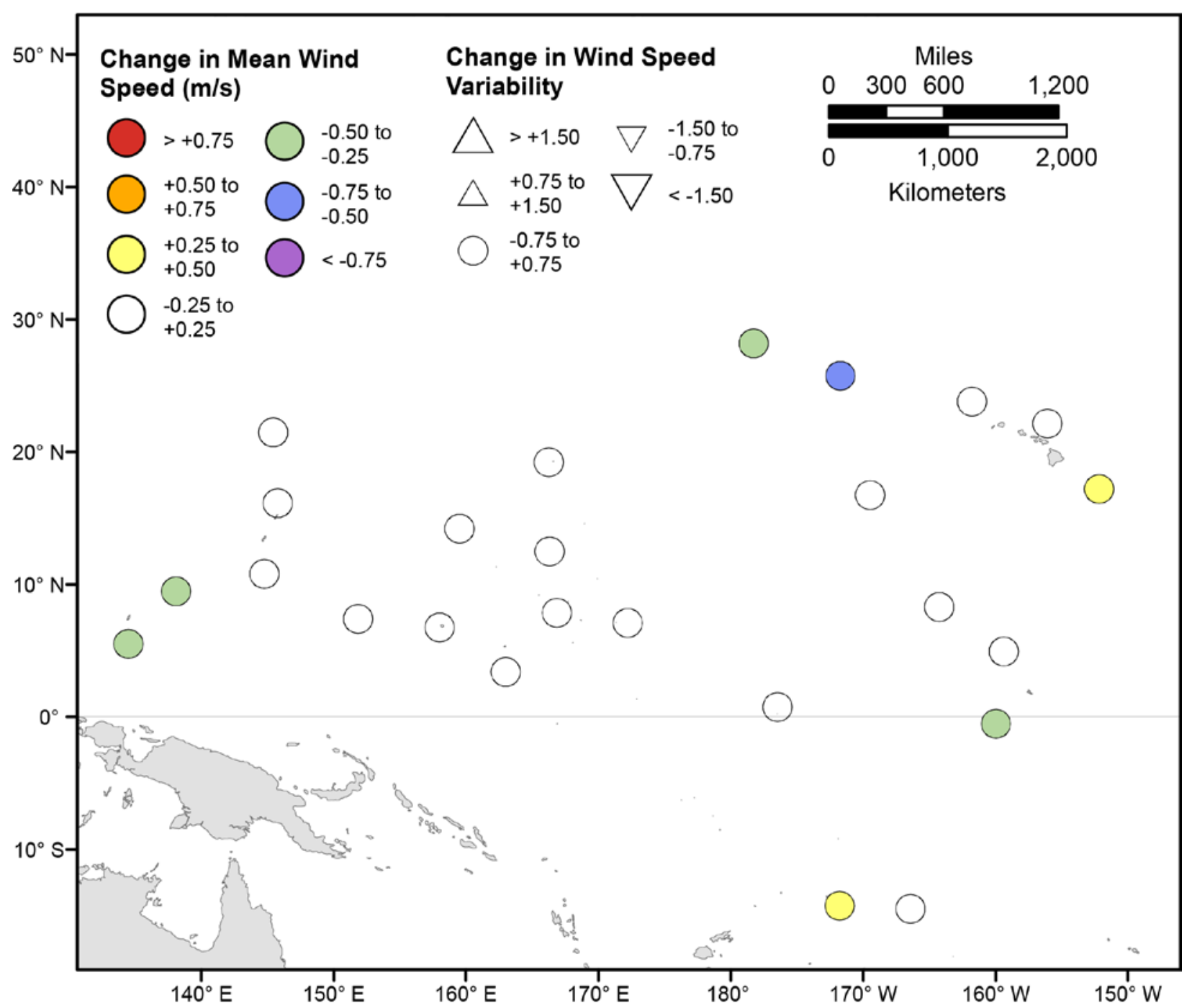

Figure 114. Map showing forecasted differences in the mean of the top 5 percent of wind speeds and variance in the top 5 percent of wind speeds for the years 2026-2045 from hindcasted values during the December-February season under the RCP8.5 future climatic scenario. The colors correspond to the magnitude of change in modeled mean wind speeds during 2026-2045 from those hindcasted for 19762005. The shapes correspond to the magnitude of change in modeled variance in wind speed during 2026-2045 from those hindcasted for 1976-2005. Units are in meters per second. 


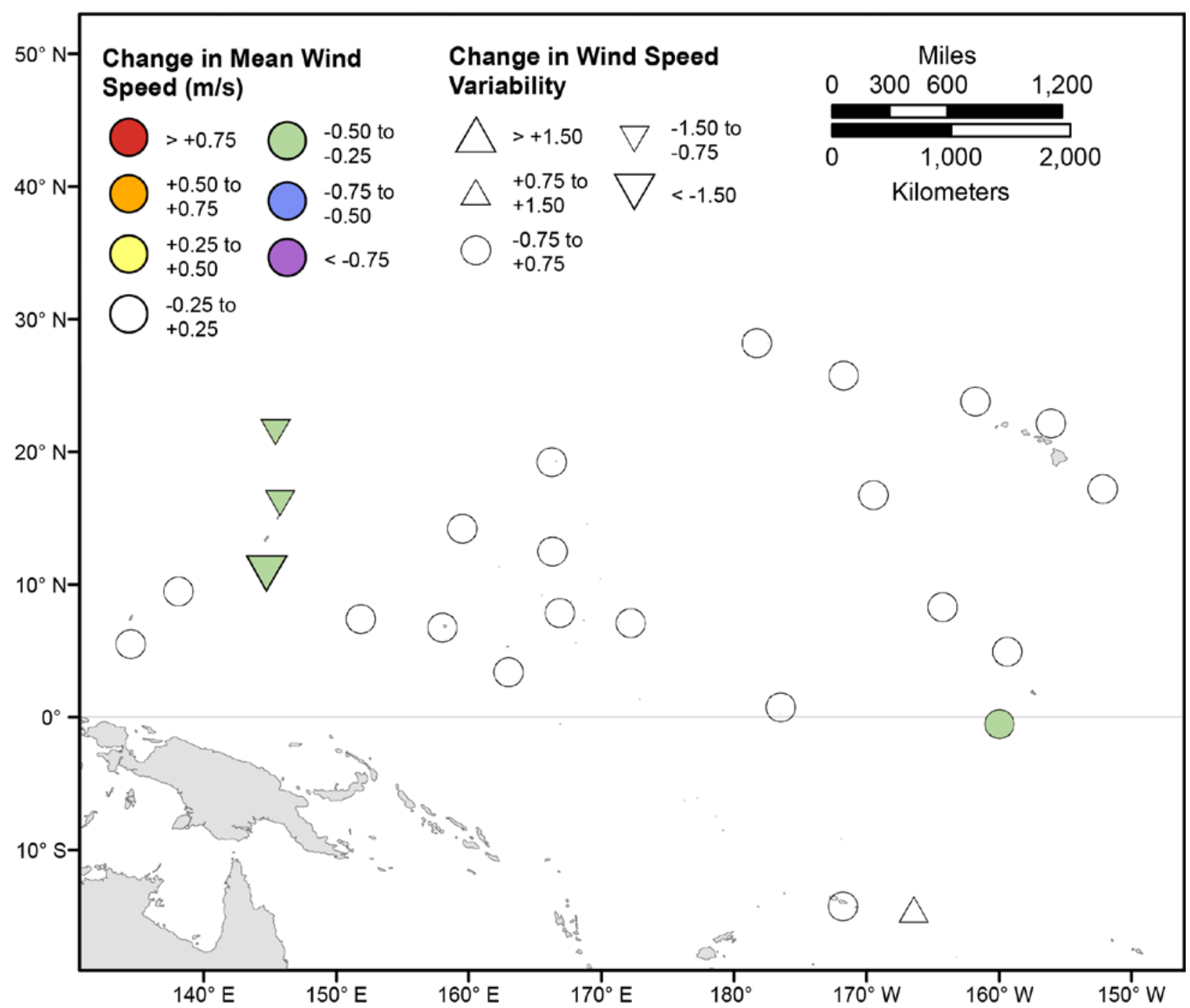

Figure 115. Map showing forecasted differences in the mean of the top 5 percent of wind speeds and variance in the top 5 percent of wind speeds for the years 2026-2045 from hindcasted values during the March-May season under the RCP8.5 future climatic scenario. The colors correspond to the magnitude of change in modeled mean wind speeds during 2026-2045 from those hindcasted for 1976-2005. The shapes correspond to the magnitude of change in modeled variance in wind speed during 2026-2045 from those hindcasted for 1976-2005. Units are in meters per second. 


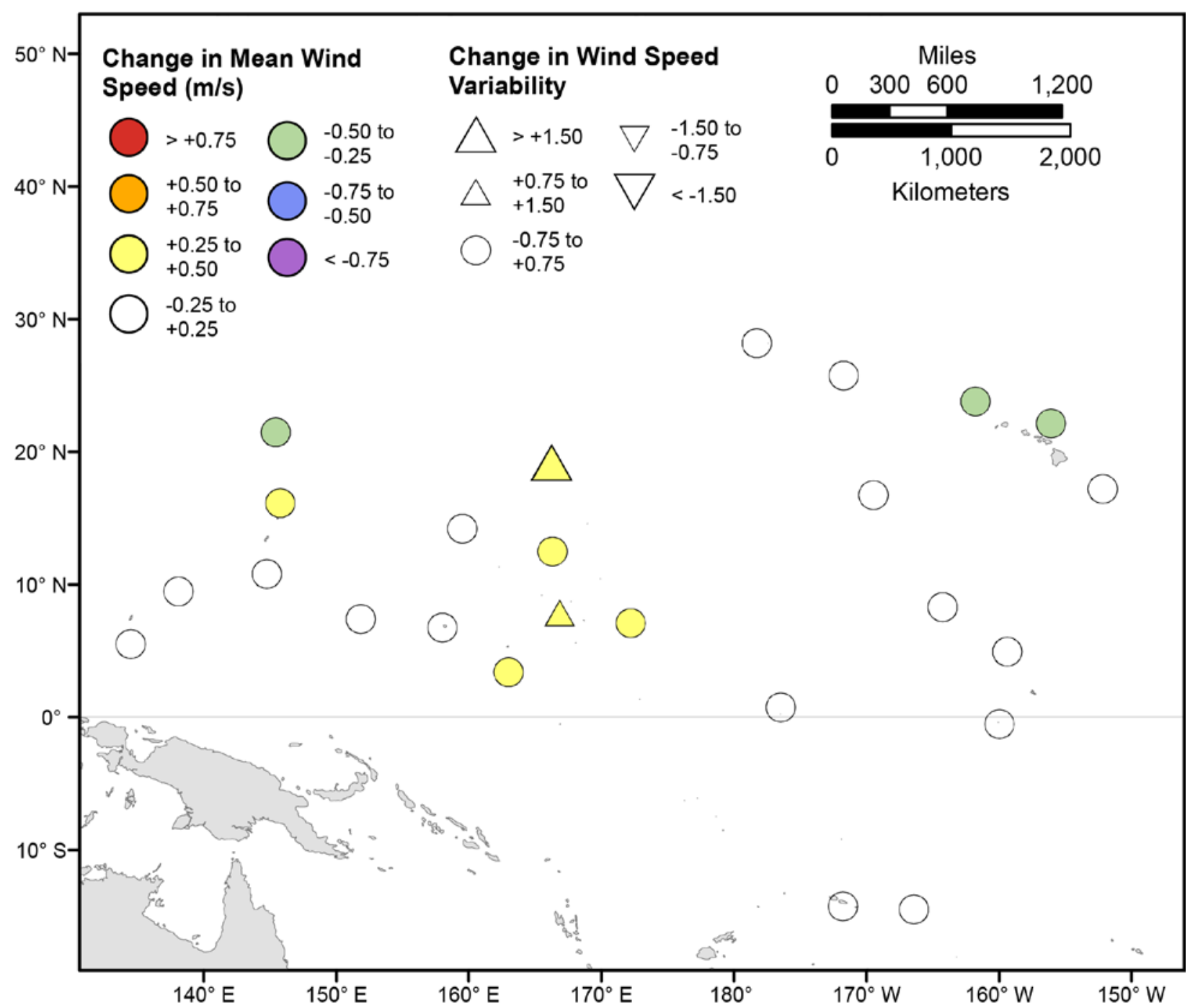

Figure 116. Map showing forecasted differences in the mean of the top 5 percent of wind speeds and variance in the top 5 percent of wind speeds for the years 2026-2045 from hindcasted values during the June-August season under the RCP8.5 future climatic scenario. The colors correspond to the magnitude of change in modeled mean wind speeds during 2026-2045 from those hindcasted for 1976-2005. The shapes correspond to the magnitude of change in modeled variance in wind speed during 2026-2045 from those hindcasted for 1976-2005. Units are in meters per second. 


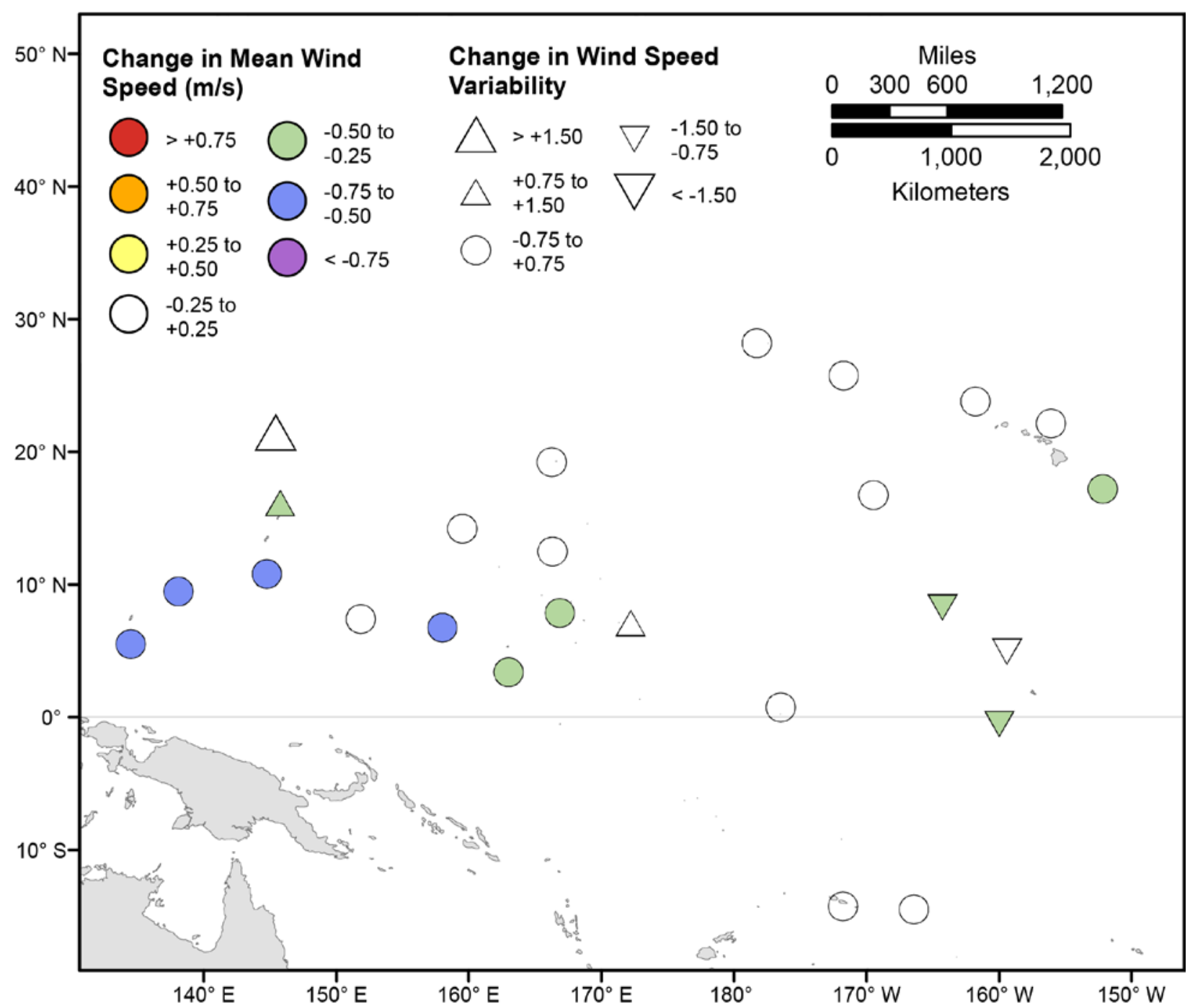

Figure 117. Map showing forecasted differences in the mean of the top 5 percent of wind speeds and variance in the top 5 percent of wind speeds for the years 2026-2045 from hindcasted values during the September-November season under the RCP8.5 future climatic scenario. The colors correspond to the magnitude of change in modeled mean wind speeds during 2026-2045 from those hindcasted for 19762005. The shapes correspond to the magnitude of change in modeled variance in wind speed during 2026-2045 from those hindcasted for 1976-2005. Units are in meters per second. 


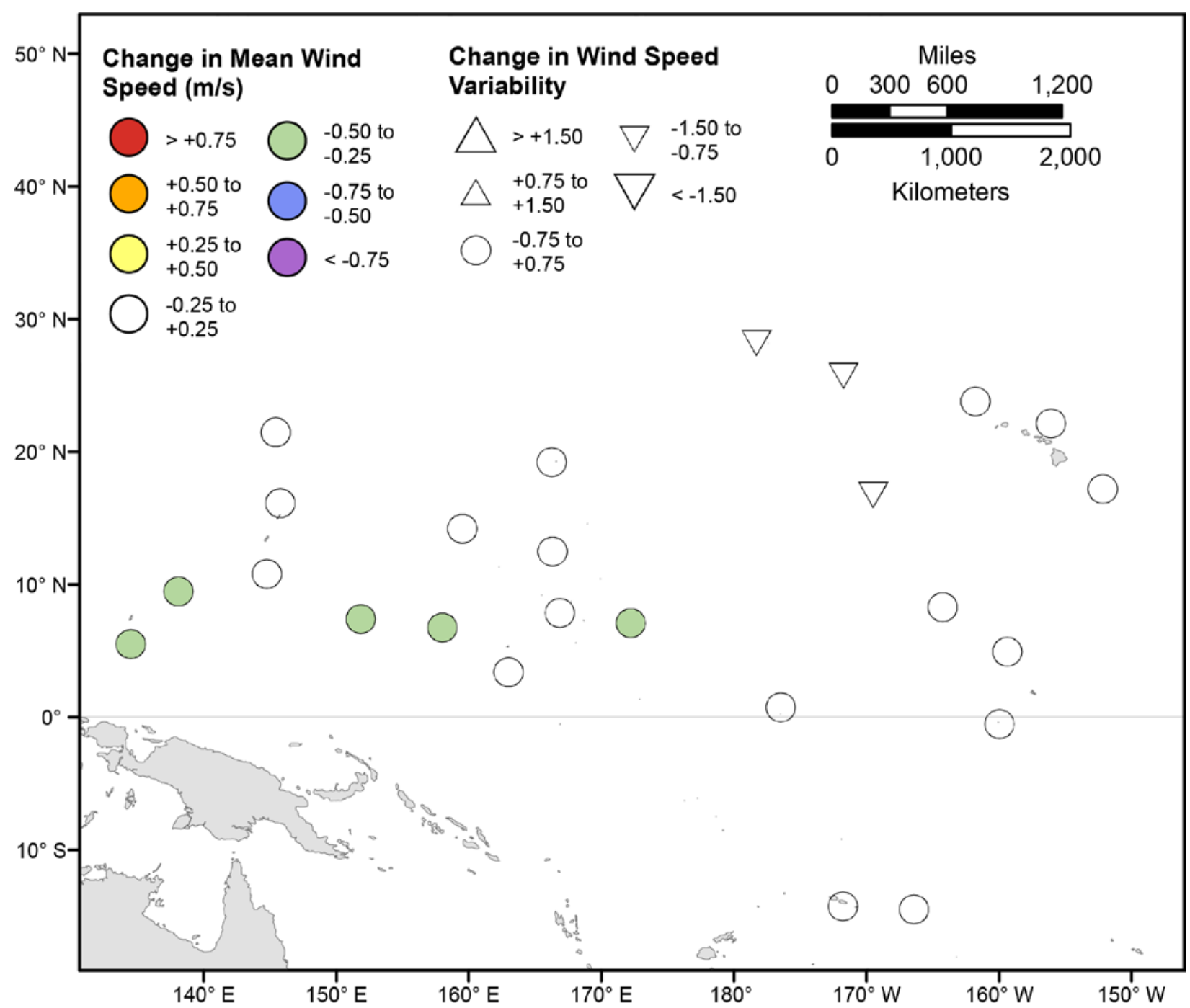

Figure 118. Map showing forecasted differences in mean wind speed and variance in wind speed for the years 2081-2100 from hindcasted values during the December-February season under the RCP4.5 future climatic scenario. The colors correspond to the magnitude of change in modeled mean wind speeds during 2081-2100 from those hindcasted for 1976-2005. The shapes correspond to the magnitude of change in modeled variance in wind speed during 2081-2100 from those hindcasted for 1976-2005. Units are in meters per second. 


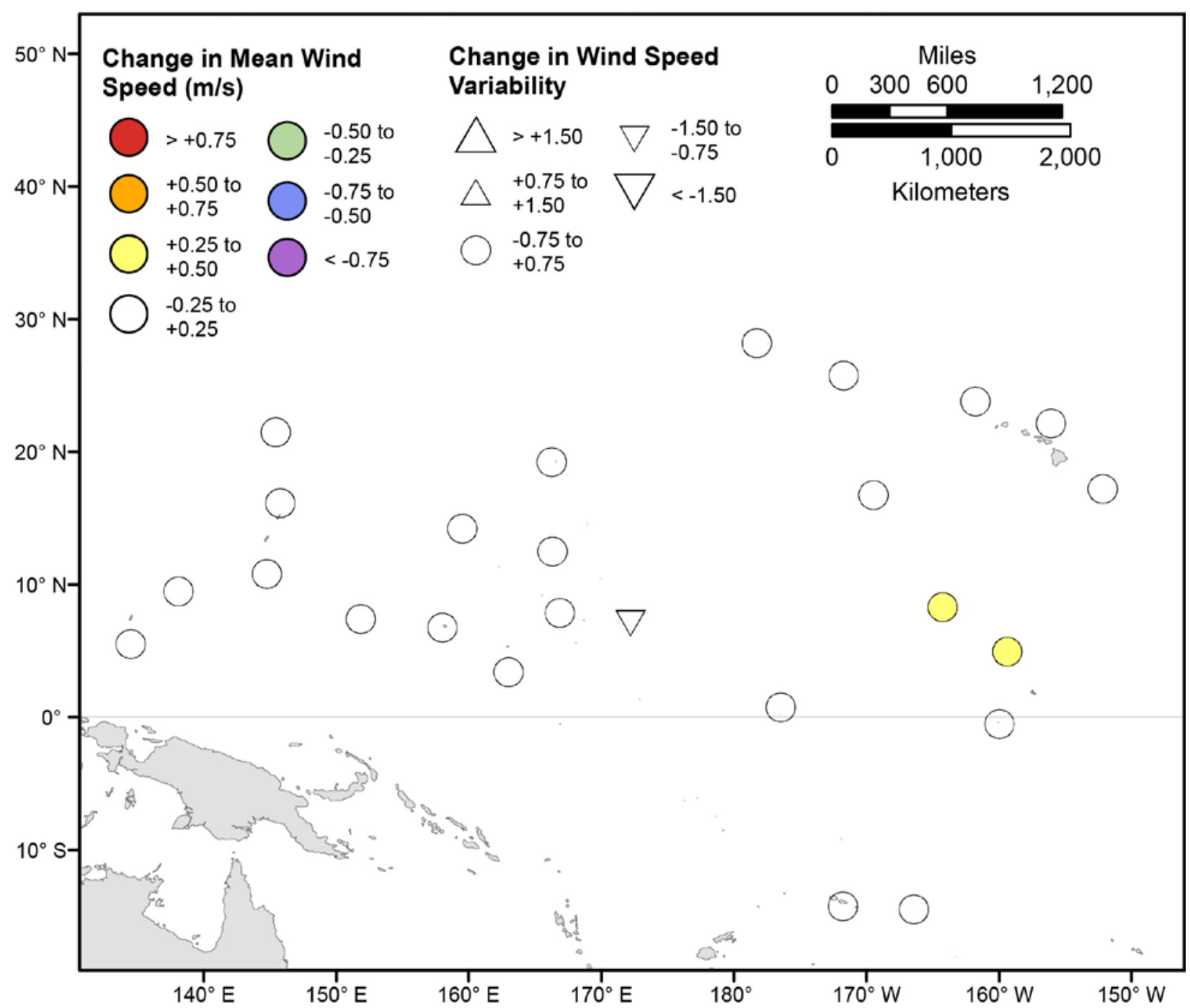

Figure 119. Map showing forecasted differences in mean wind speed and variance in wind speed for the years 2081-2100 from hindcasted values during the March-May season under the RCP4.5 future climatic scenario. The colors correspond to the magnitude of change in modeled mean wind speeds during 2081-2100 from those hindcasted for 1976-2005. The shapes correspond to the magnitude of change in modeled variance in wind speed during 2081-2100 from those hindcasted for 1976-2005. Units are in meters per second. 


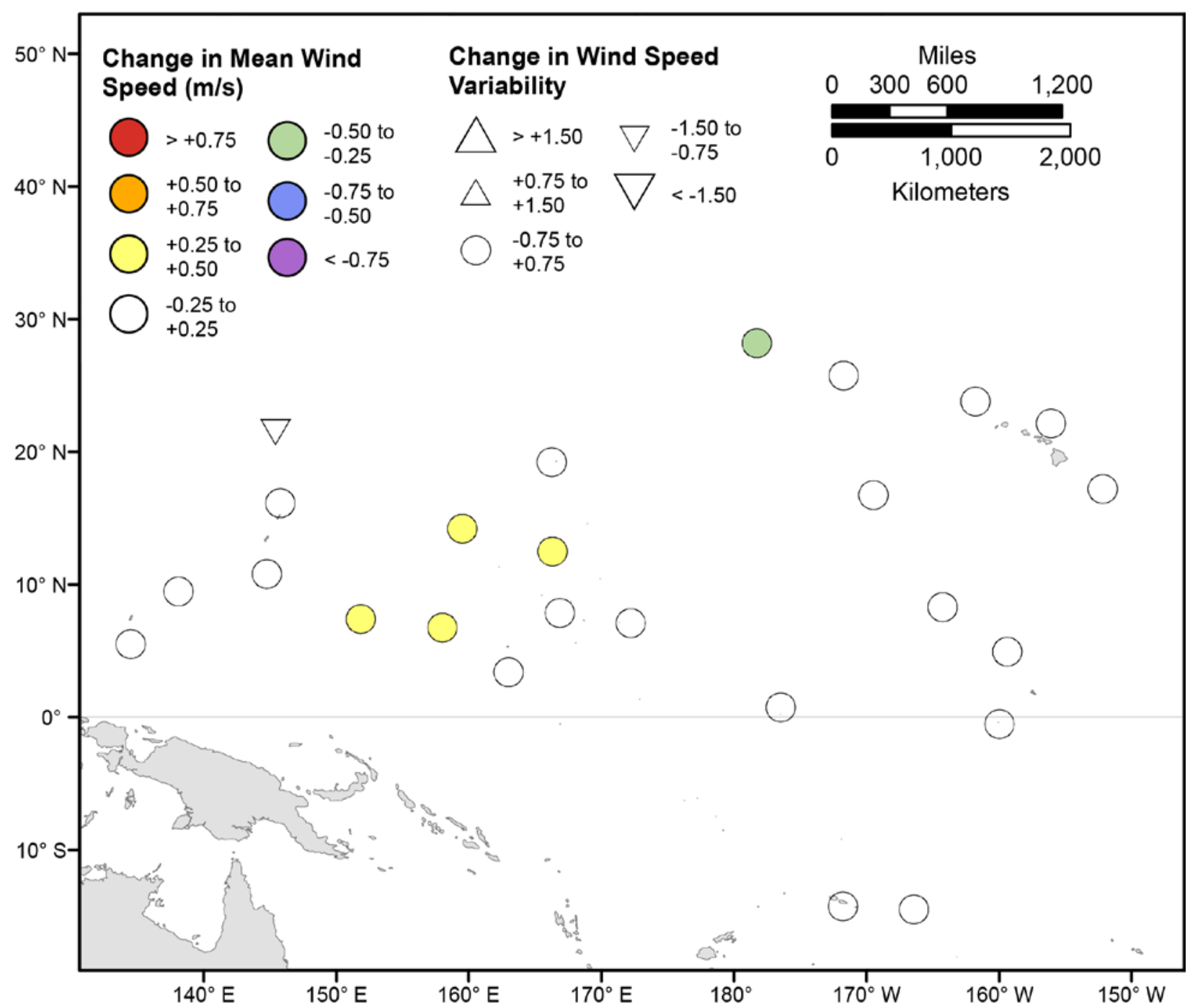

Figure 120. Map showing forecasted differences in mean wind speed and variance in wind speed for the years 2081-2100 from hindcasted values during the June-August season under the RCP4.5 future climatic scenario. The colors correspond to the magnitude of change in modeled mean wind speeds during 2081-2100 from those hindcasted for 1976-2005. The shapes correspond to the magnitude of change in modeled variance in wind speed during 2081-2100 from those hindcasted for 1976-2005. Units are in meters per second. 


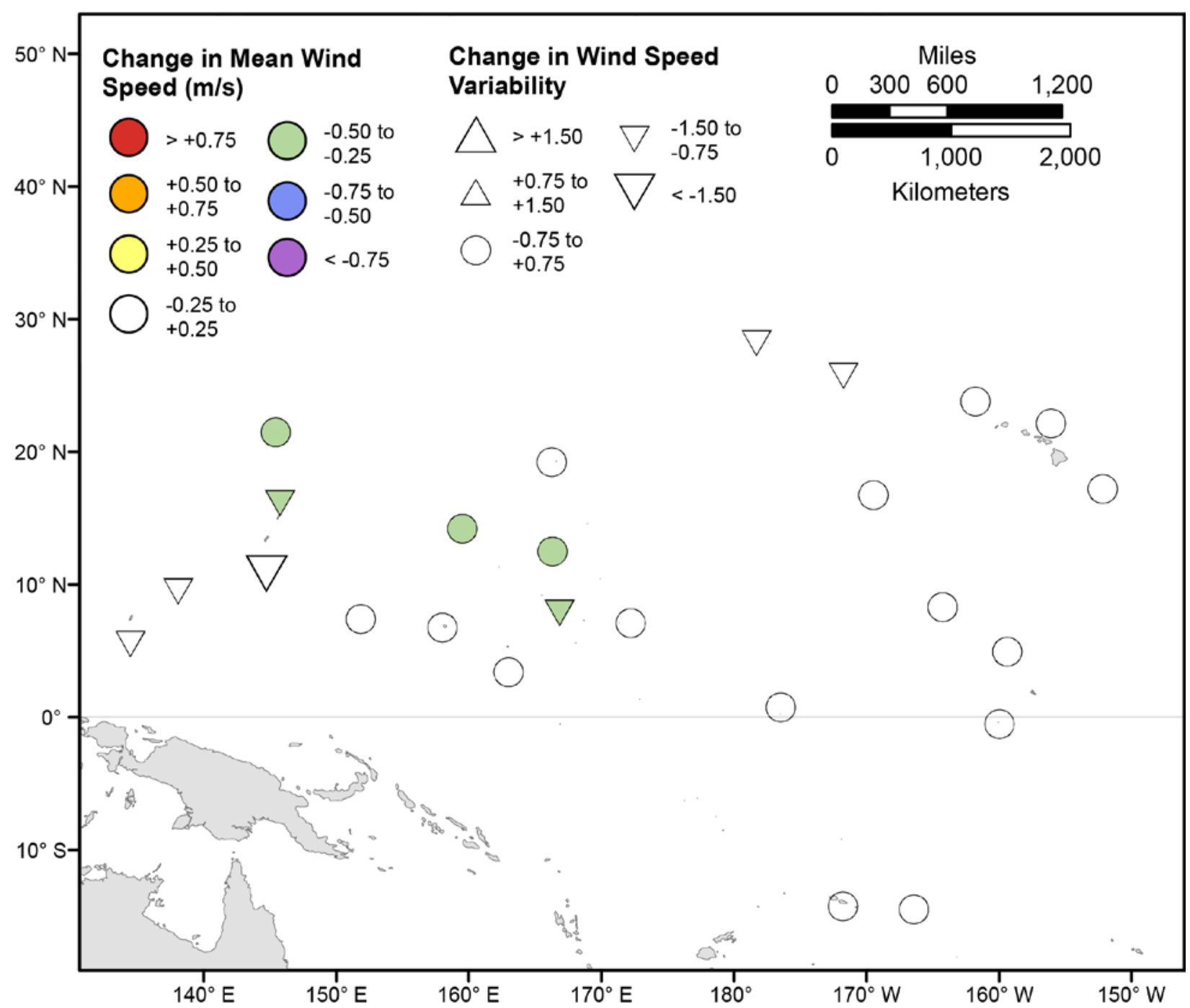

Figure 121. Map showing forecasted differences in mean wind speed and variance in wind speed for the years 2081-2100 from hindcasted values during the September-November season under the RCP4.5 future climatic scenario. The colors correspond to the magnitude of change in modeled mean wind speeds during 2081-2100 from those hindcasted for 1976-2005. The shapes correspond to the magnitude of change in modeled variance in wind speed during 2081-2100 from those hindcasted for 1976-2005. Units are in meters per second. 


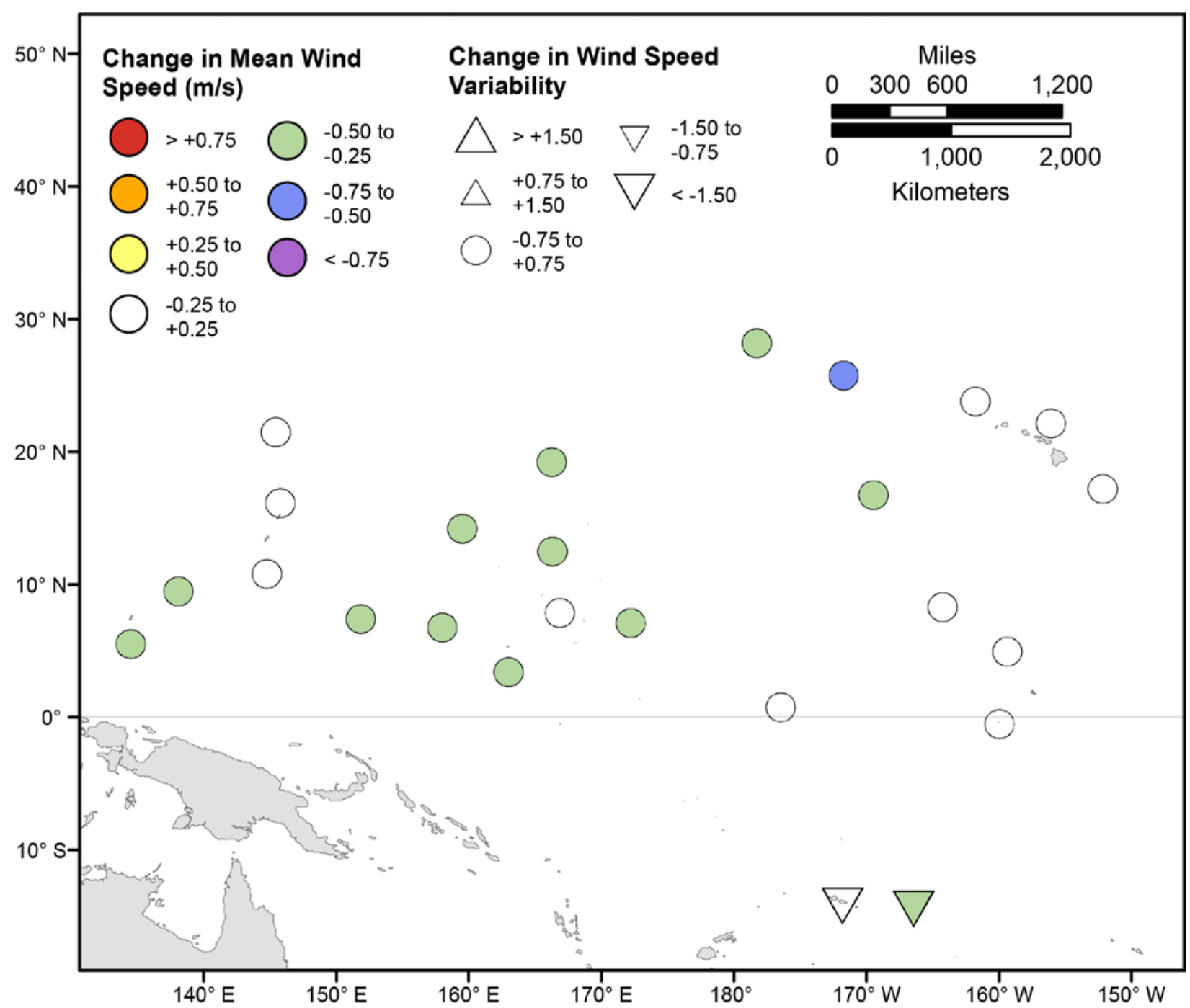

Figure 122. Map showing forecasted differences in the mean of the top 5 percent of wind speeds and variance in the top 5 percent of wind speeds for the years 2081-2100 from hindcasted values during the December-February season under the RCP4.5 future climatic scenario. The colors correspond to the magnitude of change in modeled mean wind speeds during 2081-2100 from those hindcasted for 19762005. The shapes correspond to the magnitude of change in modeled variance in wind speed during 2081-2100 from those hindcasted for 1976-2005. Units are in meters per second. 


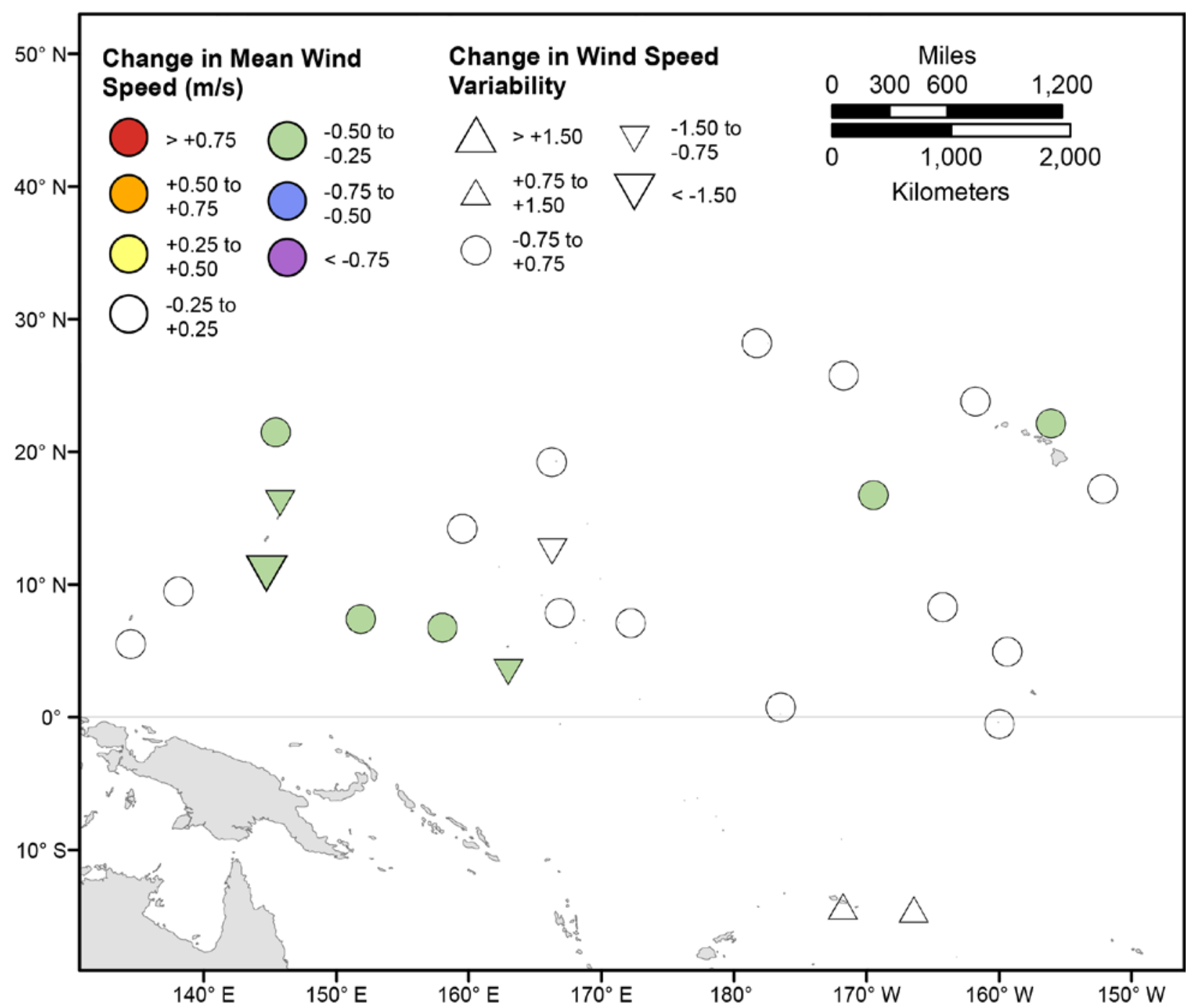

Figure 123. Map showing forecasted differences in the mean of the top 5 percent of wind speeds and variance in the top 5 percent of wind speeds for the years 2081-2100 from hindcasted values during the March-May season under the RCP4.5 future climatic scenario. The colors correspond to the magnitude of change in modeled mean wind speeds during 2081-2100 from those hindcasted for 1976-2005. The shapes correspond to the magnitude of change in modeled variance in wind speed during 2081-2100 from those hindcasted for 1976-2005. Units are in meters per second. 


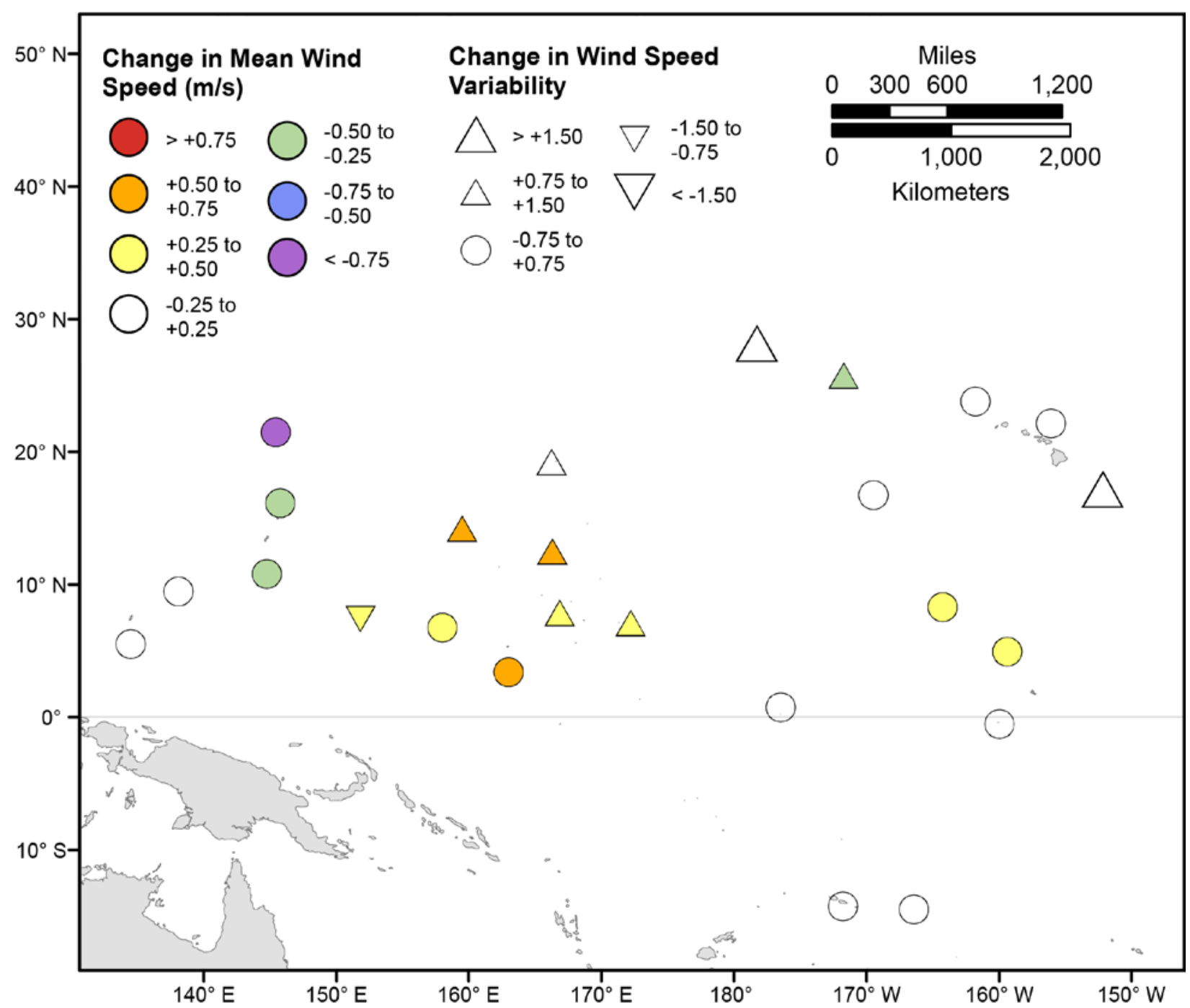

Figure 124. Map showing forecasted differences in the mean of the top 5 percent of wind speeds and variance in the top 5 percent of wind speeds for the years 2081-2100 from hindcasted values during the June-August season under the RCP4.5 future climatic scenario. The colors correspond to the magnitude of change in modeled mean wind speeds during 2081-2100 from those hindcasted for 1976-2005. The shapes correspond to the magnitude of change in modeled variance in wind speed during 2081-2100 from those hindcasted for 1976-2005. Units are in meters per second. 


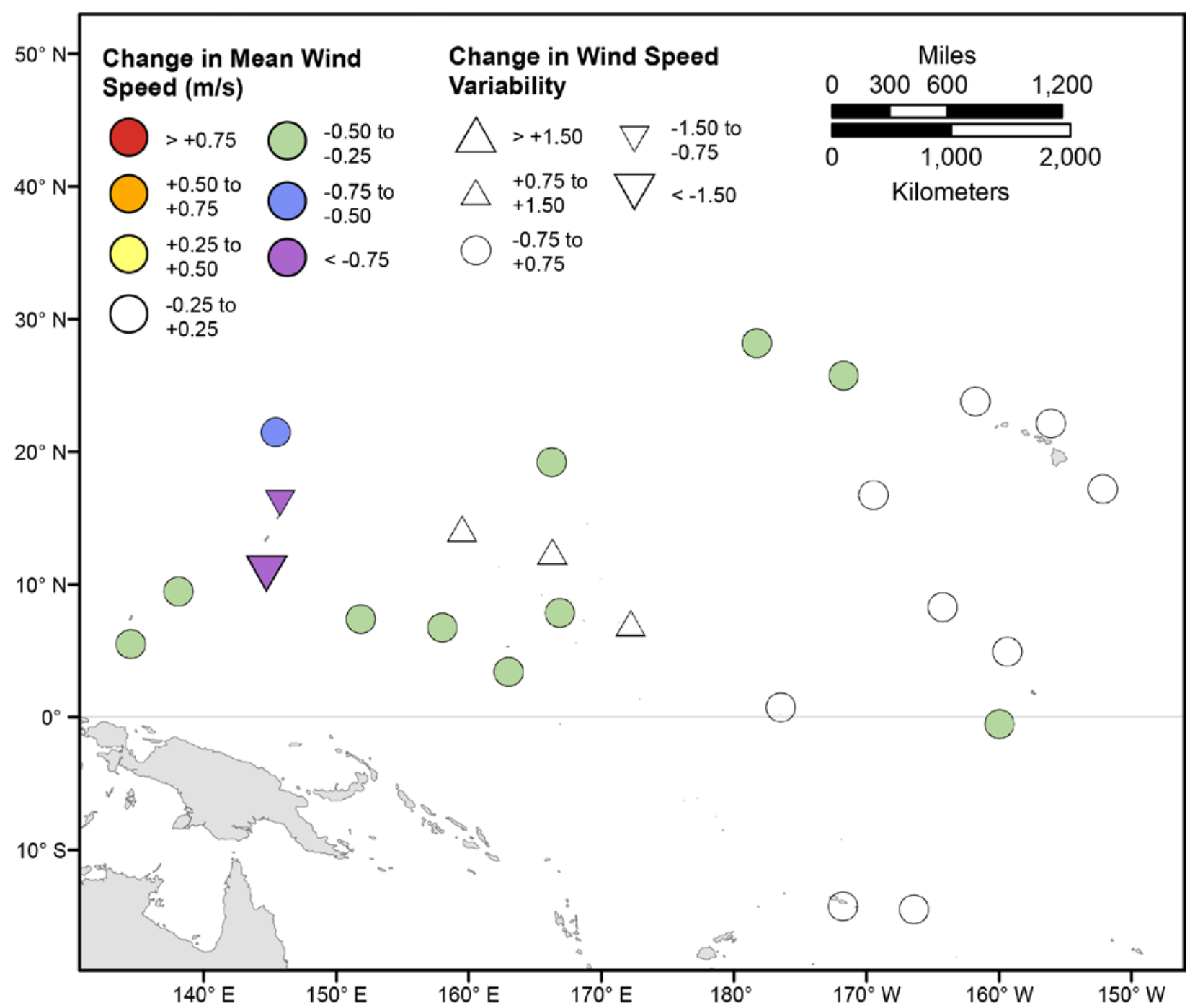

Figure 125. Map showing forecasted differences in the mean of the top 5 percent of wind speeds and variance in the top 5 percent of wind speeds for the years 2081-2100 from hindcasted values during the September-November season under the RCP4.5 future climatic scenario. The colors correspond to the magnitude of change in modeled mean wind speeds during 2081-2100 from those hindcasted for 19762005. The shapes correspond to the magnitude of change in modeled variance in wind speed during 2081-2100 from those hindcasted for 1976-2005. Units are in meters per second. 


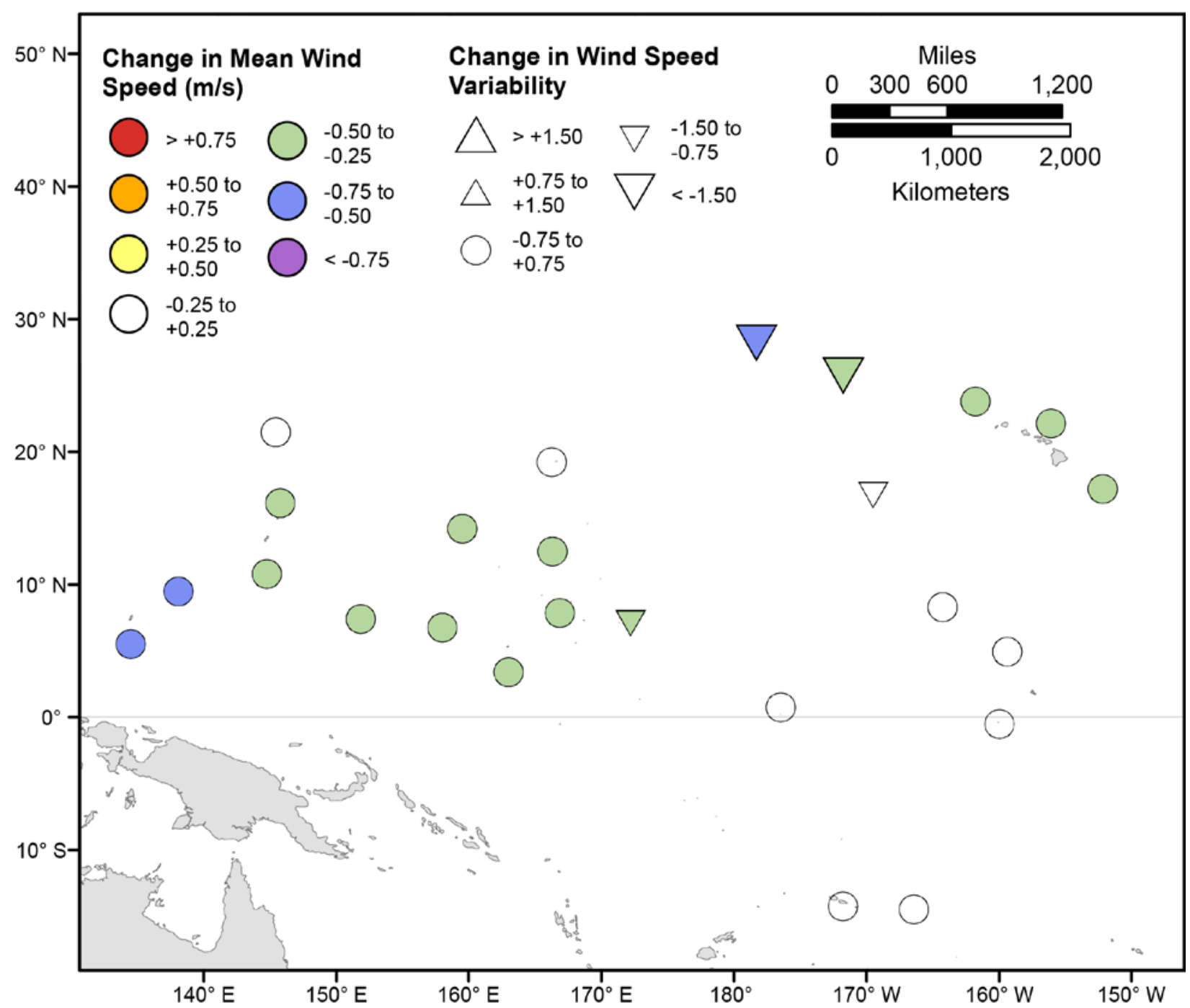

Figure 126. Map showing forecasted differences in mean wind speed and variance in wind speed for the years 2081-2100 from hindcasted values during the December-February season under the RCP8.5 future climatic scenario. The colors correspond to the magnitude of change in modeled mean wind speeds during 2081-2100 from those hindcasted for 1976-2005. The shapes correspond to the magnitude of change in modeled variance in wind speed during 2081-2100 from those hindcasted for 1976-2005. Units are in meters per second. 


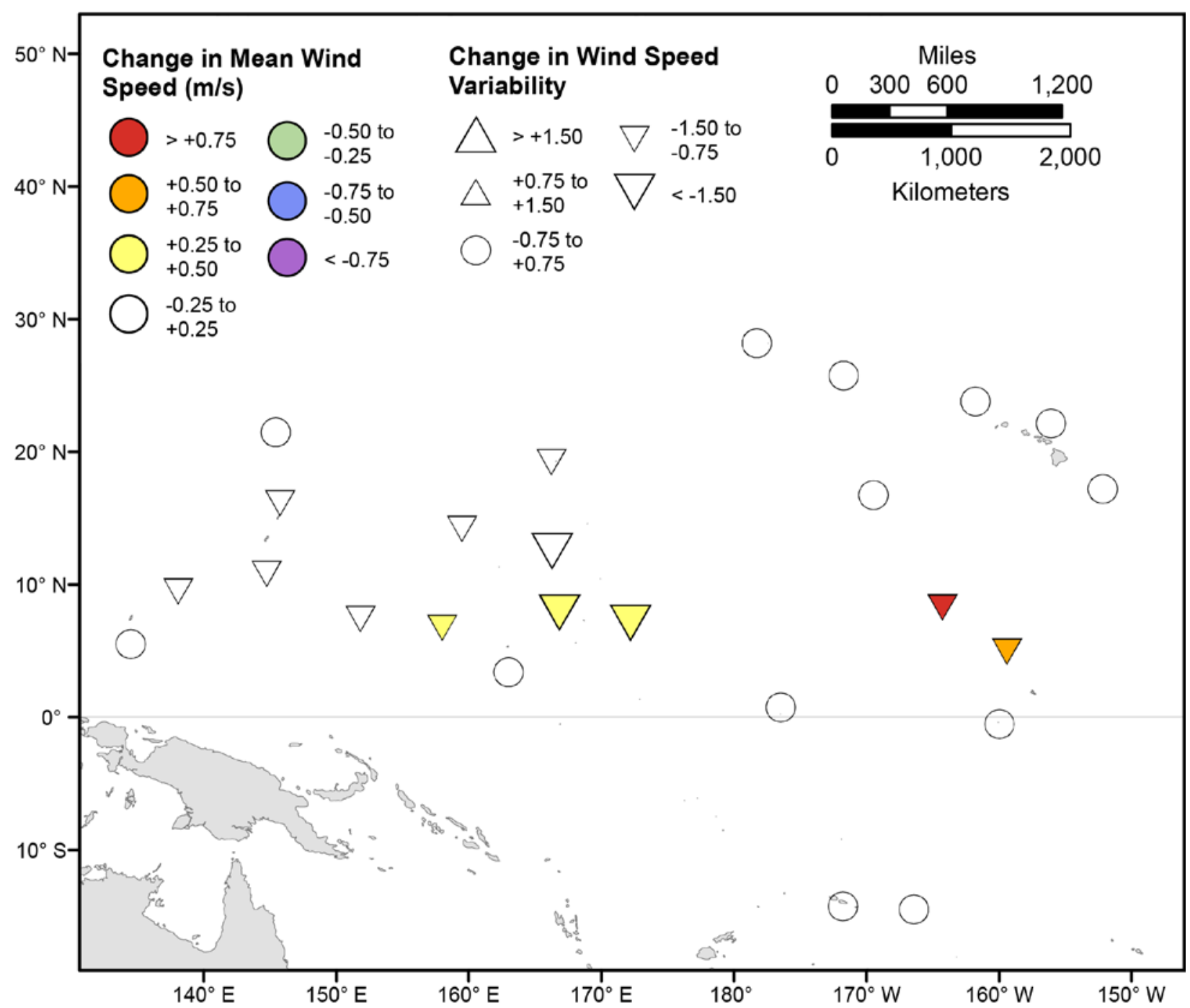

Figure 127. Map showing forecasted differences in mean wind speed and variance in wind speed for the years 2081-2100 from hindcasted values during the March-May season under the RCP8.5 future climatic scenario. The colors correspond to the magnitude of change in modeled mean wind speeds during 2081-2100 from those hindcasted for 1976-2005. The shapes correspond to the magnitude of change in modeled variance in wind speed during 2081-2100 from those hindcasted for 1976-2005. Units are in meters per second. 


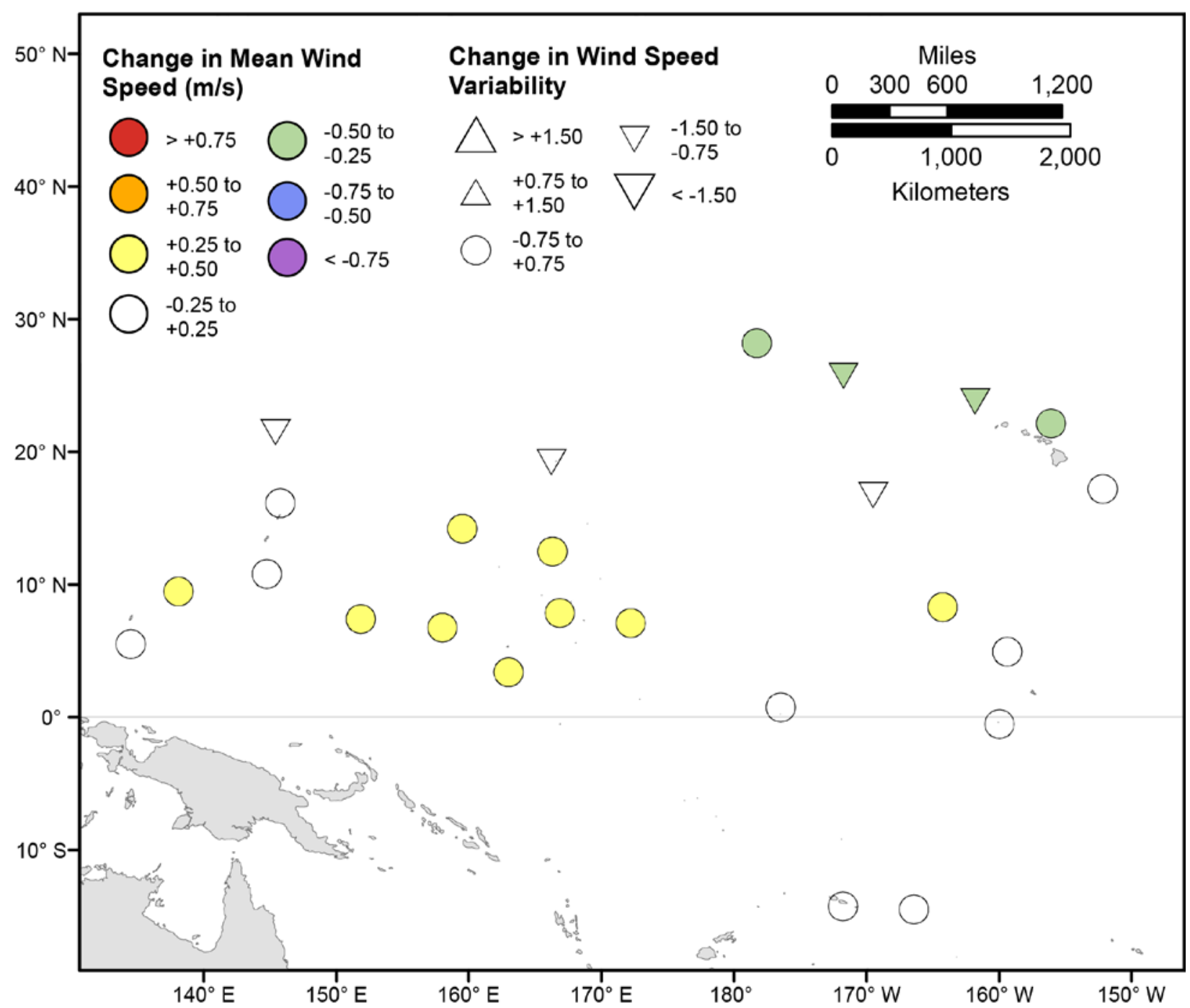

Figure 128. Map showing forecasted differences in mean wind speed and variance in wind speed for the years 2081-2100 from hindcasted values during the June-August season under the RCP8.5 future climatic scenario. The colors correspond to the magnitude of change in modeled mean wind speeds during 2081-2100 from those hindcasted for 1976-2005. The shapes correspond to the magnitude of change in modeled variance in wind speed during 2081-2100 from those hindcasted for 1976-2005. Units are in meters per second. 


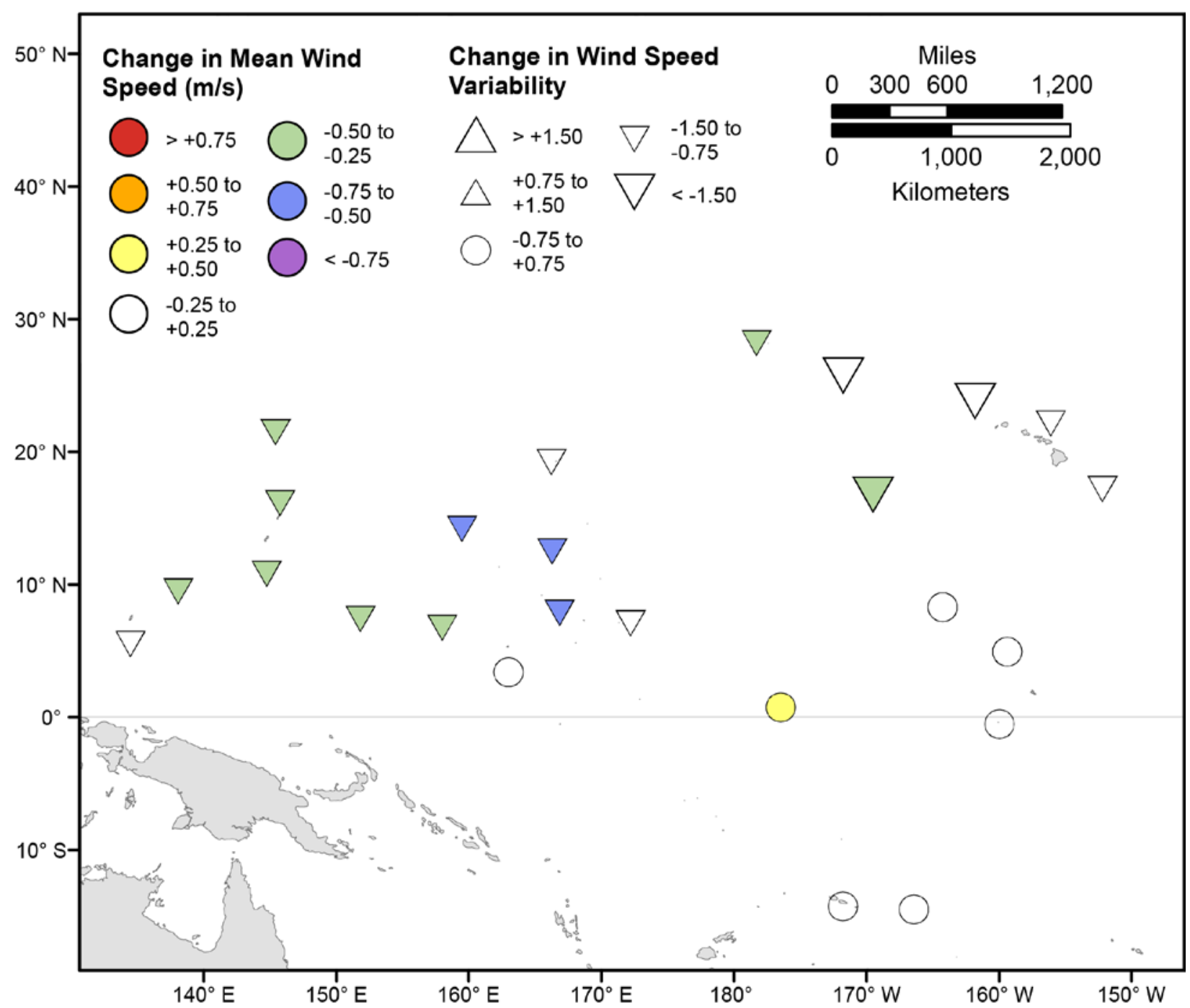

Figure 129. Map showing forecasted differences in mean wind speed and variance in wind speed for the years 2081-2100 from hindcasted values during the September-November season under the RCP8.5 future climatic scenario. The colors correspond to the magnitude of change in modeled mean wind speeds during 2081-2100 from those hindcasted for 1976-2005. The shapes correspond to the magnitude of change in modeled variance in wind speed during 2081-2100 from those hindcasted for 1976-2005. Units are in meters per second. 


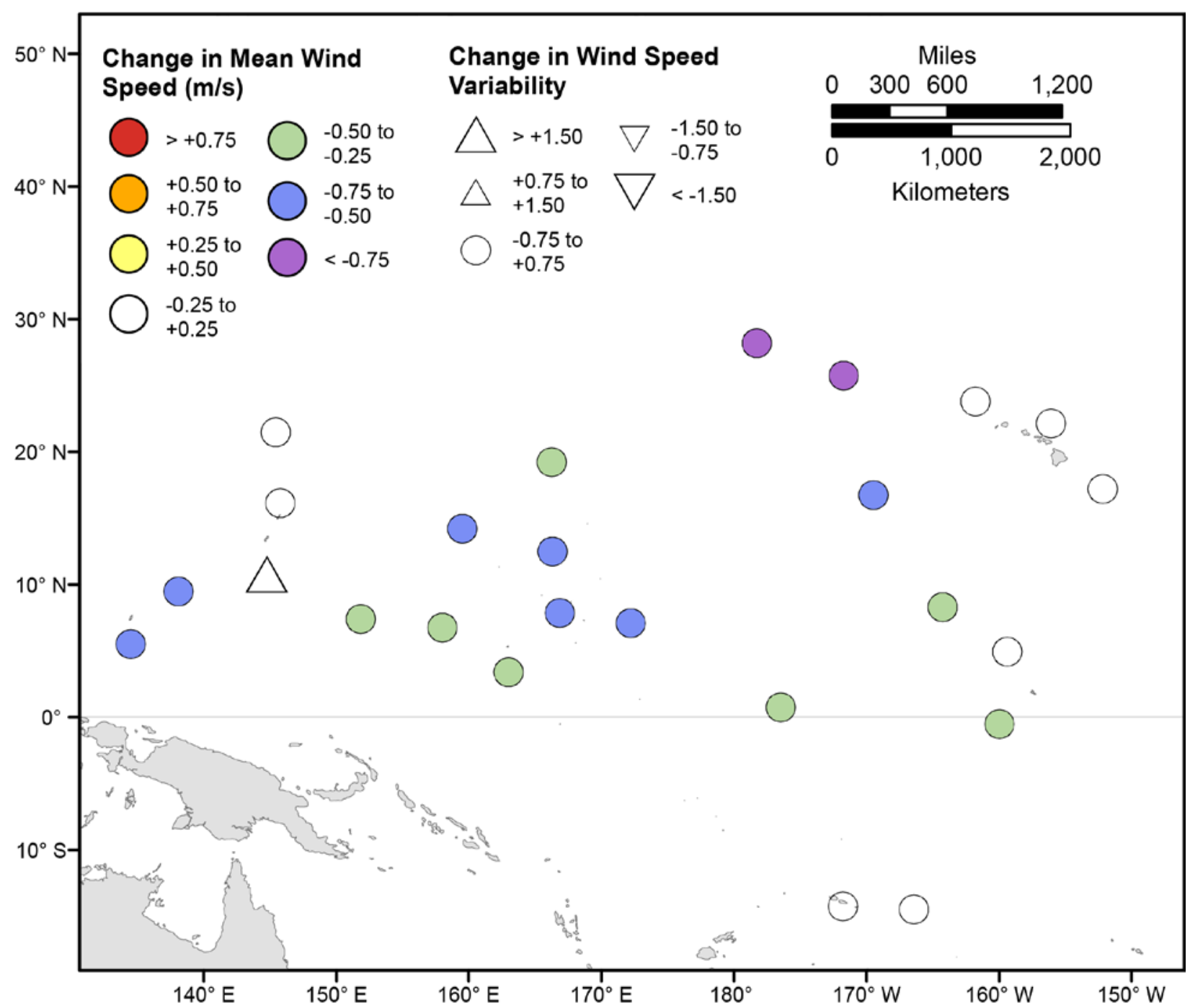

Figure 130. Map showing forecasted differences in the mean of the top 5 percent of wind speeds and variance in the top 5 percent of wind speeds for the years 2081-2100 from hindcasted values during the December-February season under the RCP8.5 future climatic scenario. The colors correspond to the magnitude of change in modeled mean wind speeds during 2081-2100 from those hindcasted for 19762005. The shapes correspond to the magnitude of change in modeled variance in wind speed during 2081-2100 from those hindcasted for 1976-2005. Units are in meters per second. 


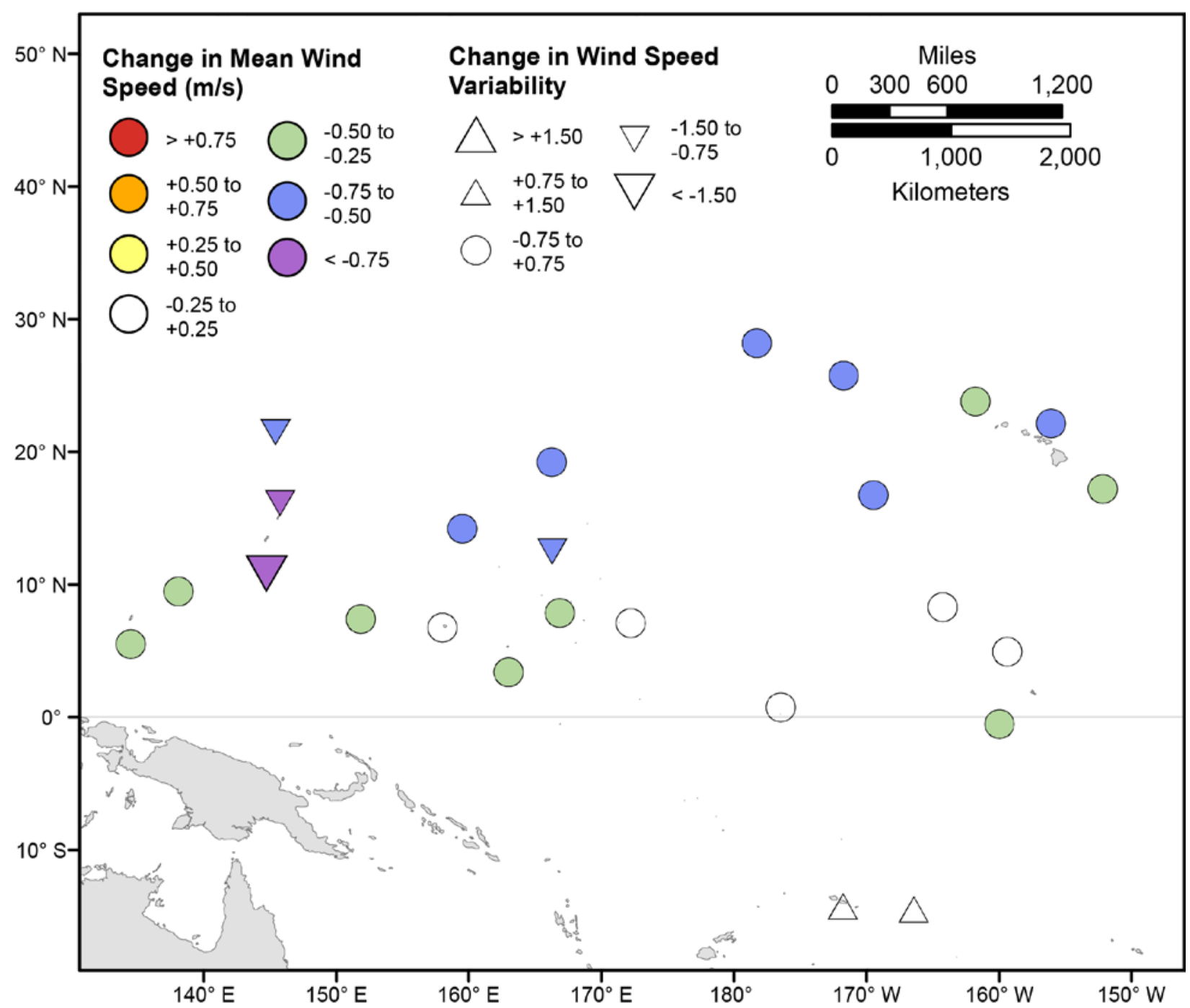

Figure 131. Map showing forecasted differences in the mean of the top 5 percent of wind speeds and variance in the top 5 percent of wind speeds for the years 2081-2100 from hindcasted values during the March-May season under the RCP8.5 future climatic scenario. The colors correspond to the magnitude of change in modeled mean wind speeds during 2081-2100 from those hindcasted for 1976-2005. The shapes correspond to the magnitude of change in modeled variance in wind speed during 2081-2100 from those hindcasted for 1976-2005. Units are in meters per second. 


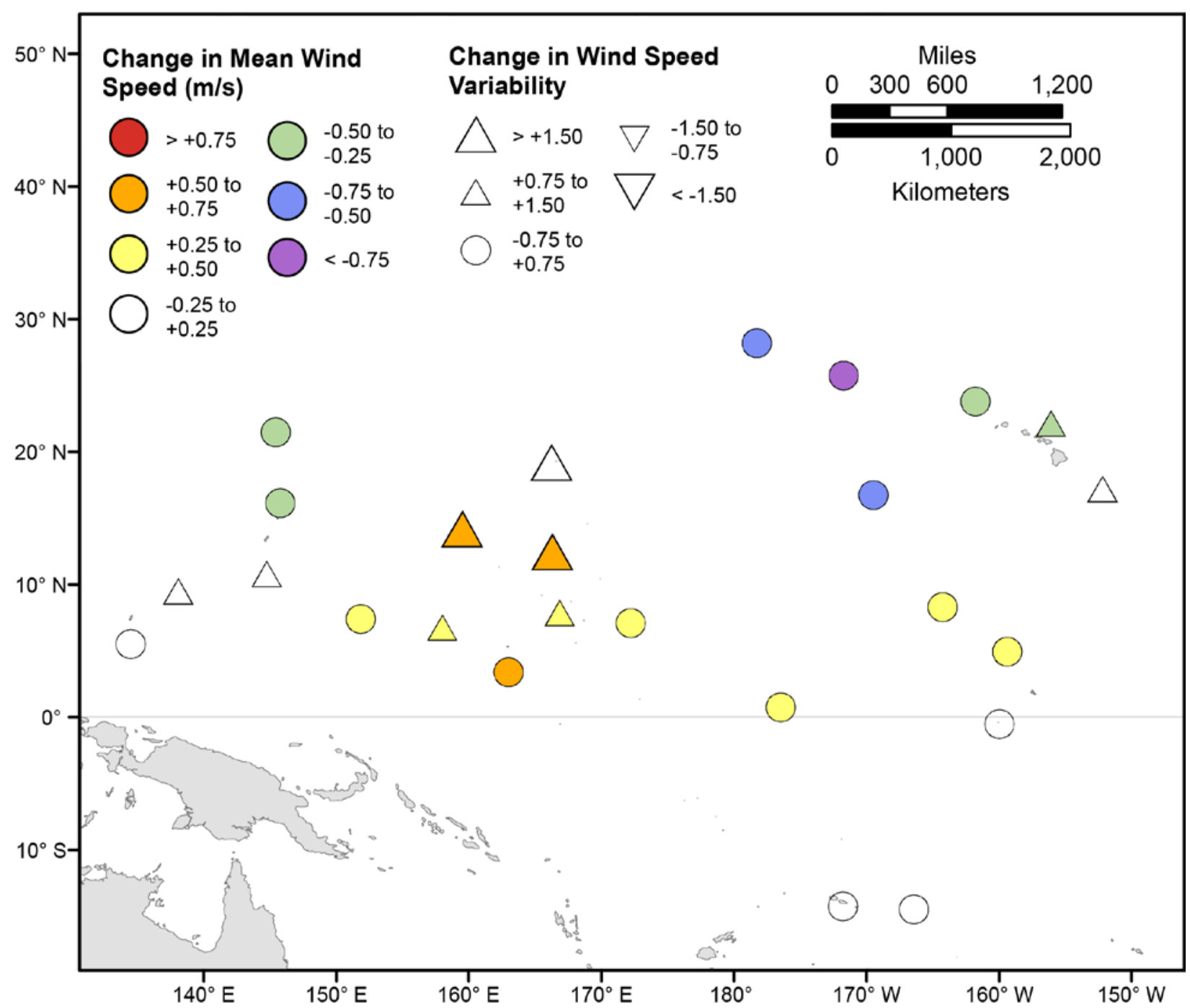

Figure 132. Map showing forecasted differences in the mean of the top 5 percent of wind speeds and variance in the top 5 percent of wind speeds for the years 2081-2100 from hindcasted values during the June-August season under the RCP8.5 future climatic scenario. The colors correspond to the magnitude of change in modeled mean wind speeds during 2081-2100 from those hindcasted for 1976-2005. The shapes correspond to the magnitude of change in modeled variance in wind speed during 2081-2100 from those hindcasted for 1976-2005. Units are in meters per second. 


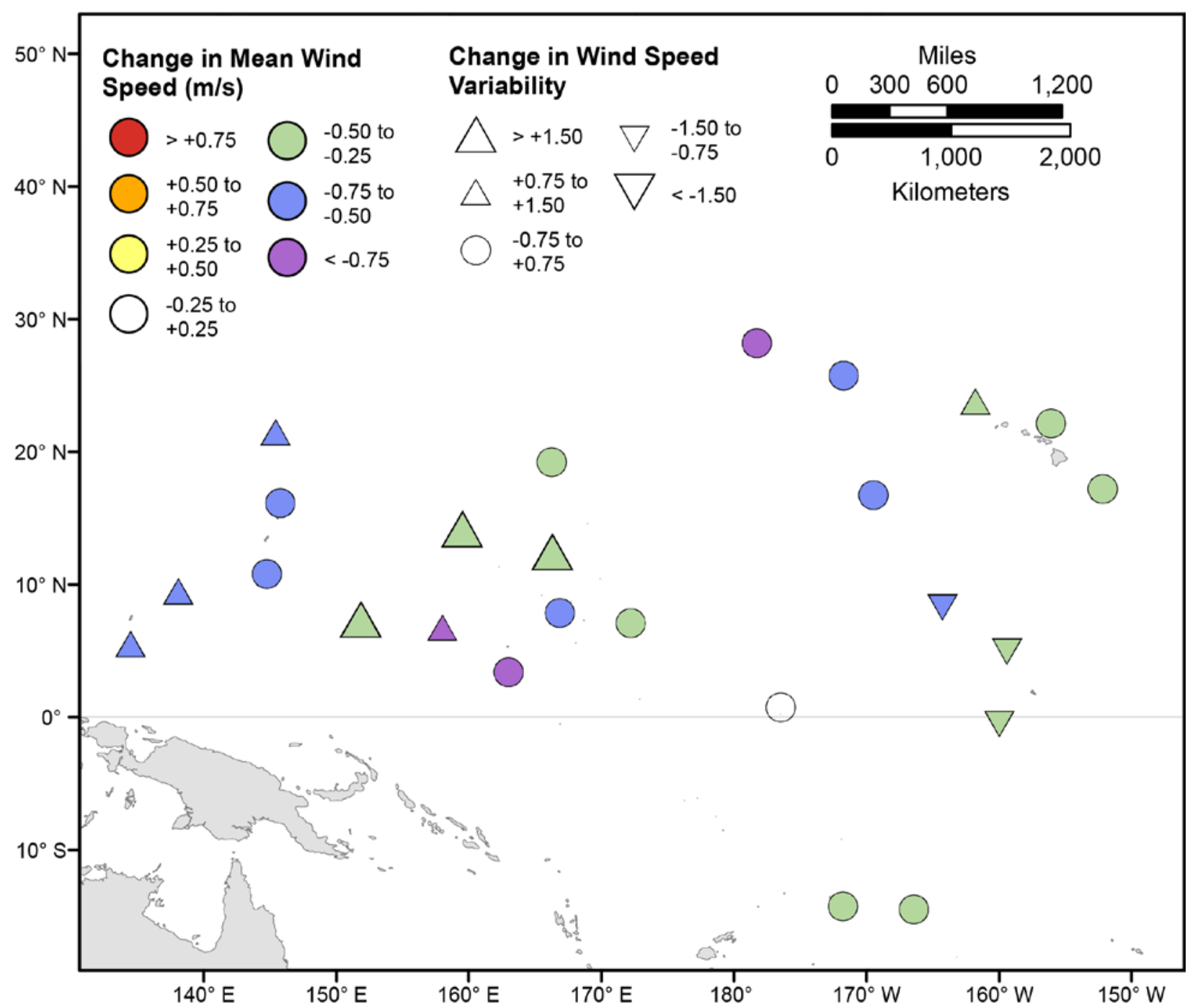

Figure 133. Map showing forecasted differences in the mean of the top 5 percent of wind speeds and variance in the top 5 percent of wind speeds for the years 2081-2100 from hindcasted values during the September-November season under the RCP8.5 future climatic scenario. The colors correspond to the magnitude of change in modeled mean wind speeds during 2081-2100 from those hindcasted for 19762005. The shapes correspond to the magnitude of change in modeled variance in wind speed during 2081-2100 from those hindcasted for 1976-2005. Units are in meters per second. 


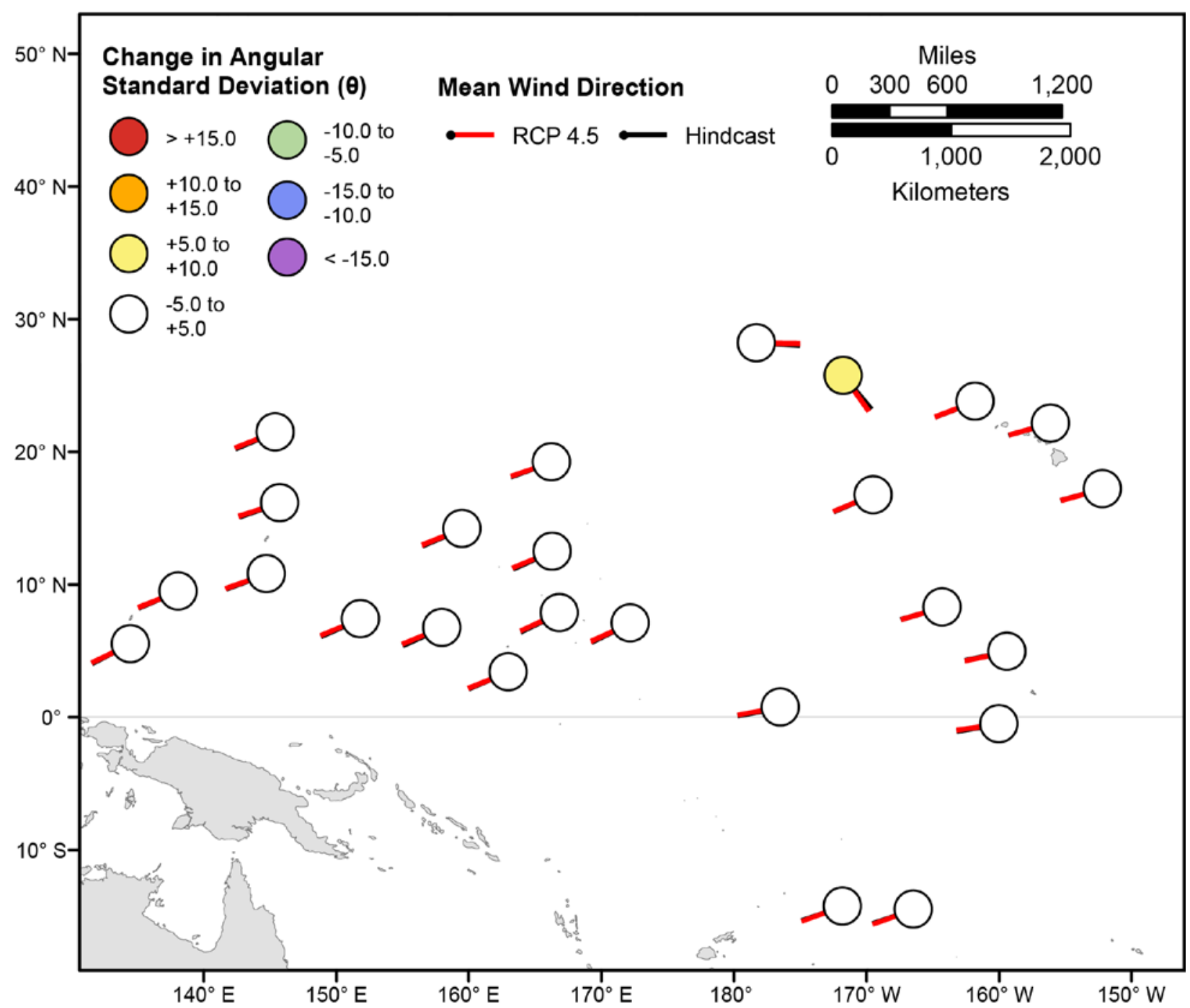

Figure 134. Map showing forecasted differences in mean wind directions and the standard deviation of wind directions for the years 2026-2045 from hindcasted values during the December-February season under the RCP4.5 future climatic scenario. Mean wind directions at each point are indicated by lines radiating from the center of each point where RCP4.5 2026-2045 mean wind directions are red and 1976-2005 hindcasted mean wind directions are black. The colors correspond to the magnitude of change in modeled mean wind direction standard deviation during 2026-2045 from those hindcasted for 19762005. Angular standard deviation units are in degrees. Mean wind directions are "heading towards". 


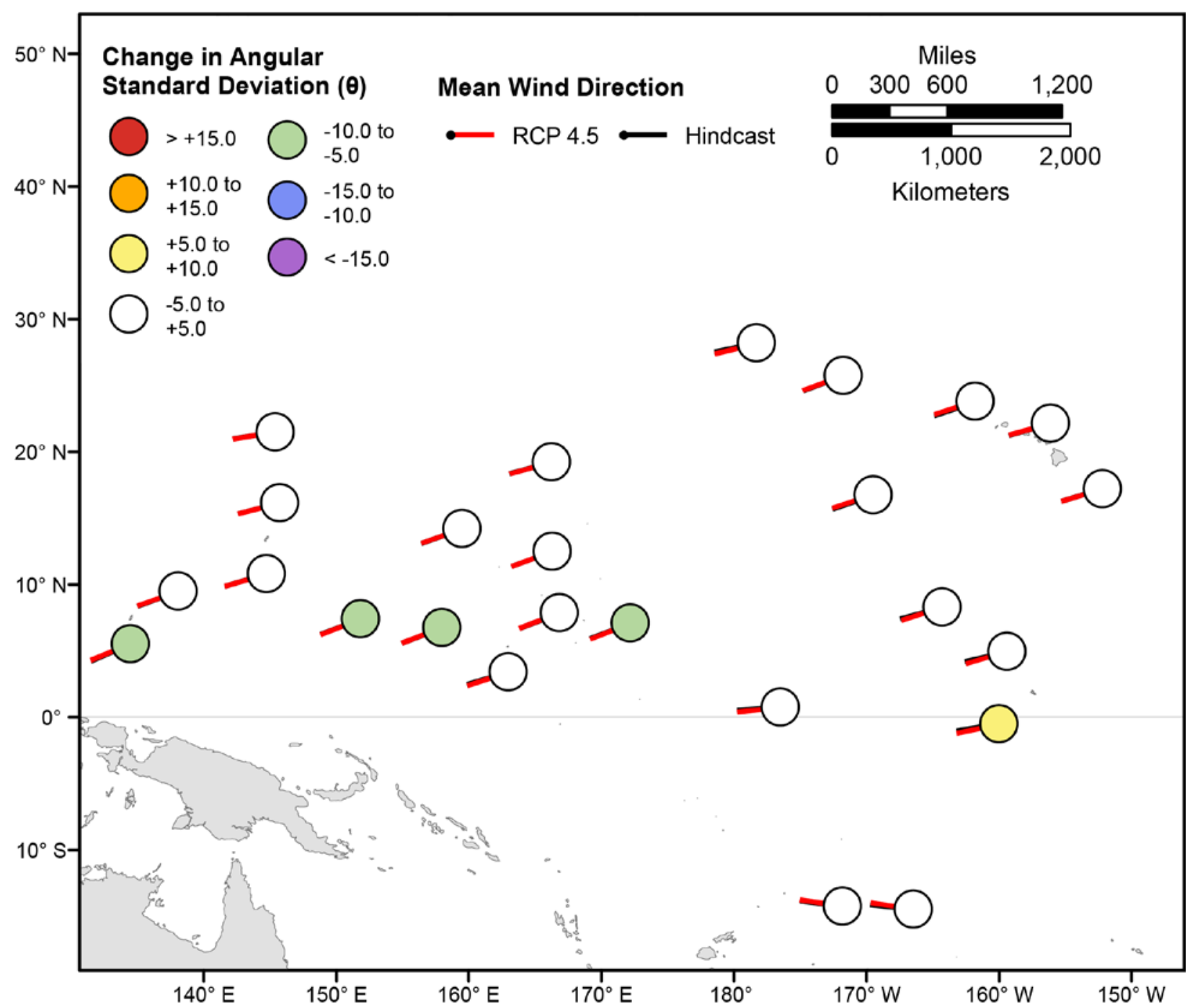

Figure 135. Map showing forecasted differences in mean wind directions and the standard deviation of wind directions for the years 2026-2045 from hindcasted values during the March-May season under the RCP4.5 future climatic scenario. Mean wind directions at each point are indicated by lines radiating from the center of each point where RCP4.5 2026-2045 mean wind directions are red and 1976-2005 hindcasted mean wind directions are black. The colors correspond to the magnitude of change in modeled mean wind direction standard deviation during 2026-2045 from those hindcasted for 19762005. Angular standard deviation units are in degrees. Mean wind directions are "heading towards". 


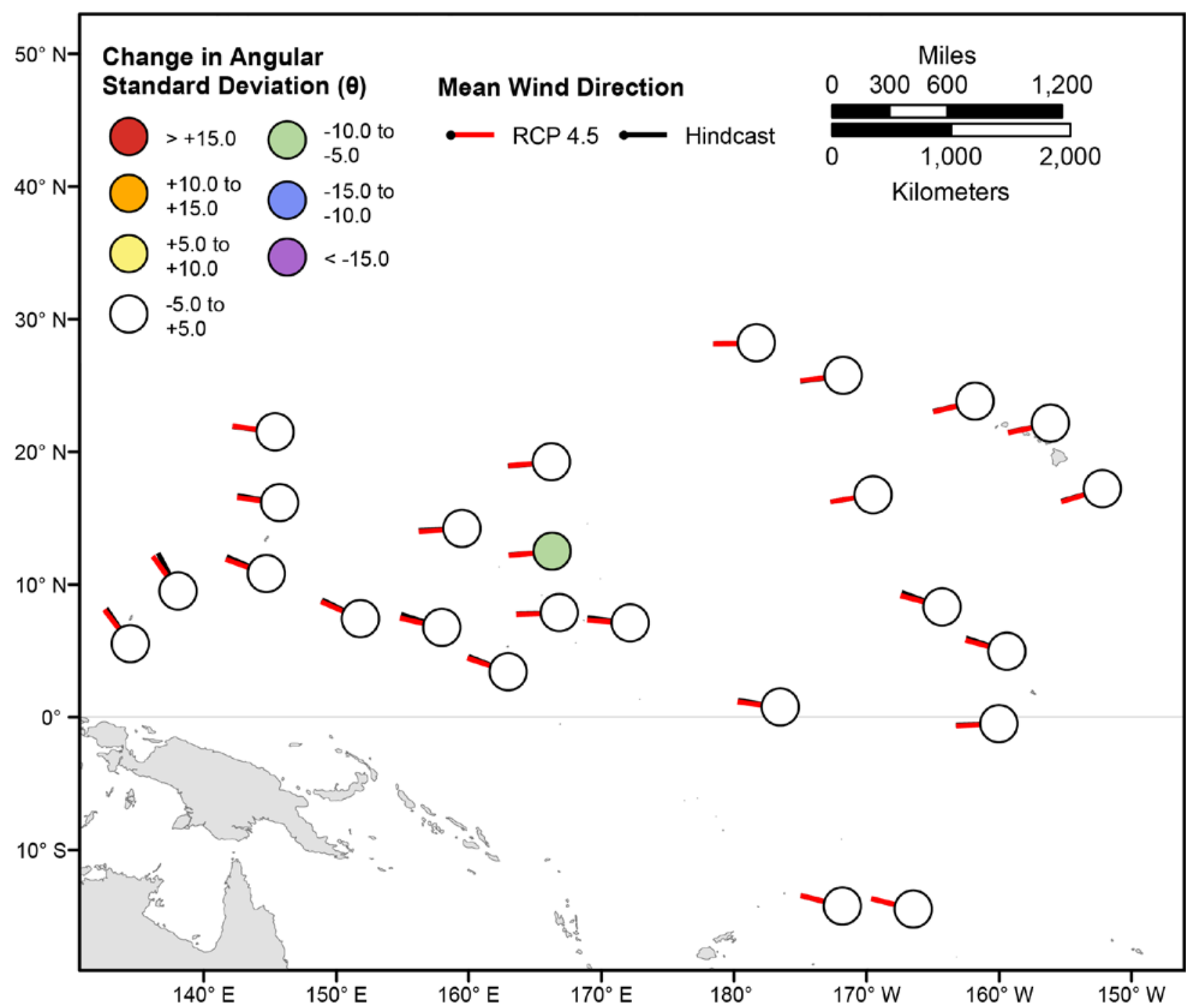

Figure 136. Map showing forecasted differences in mean wind directions and the standard deviation of wind directions for the years 2026-2045 from hindcasted values during the June-August season under the RCP4.5 future climatic scenario. Mean wind directions at each point are indicated by lines radiating from the center of each point where RCP4.5 2026-2045 mean wind directions are red and 1976-2005 hindcasted mean wind directions are black. The colors correspond to the magnitude of change in modeled mean wind direction standard deviation during 2026-2045 from those hindcasted for 19762005. Angular standard deviation units are in degrees. Mean wind directions are "heading towards". 


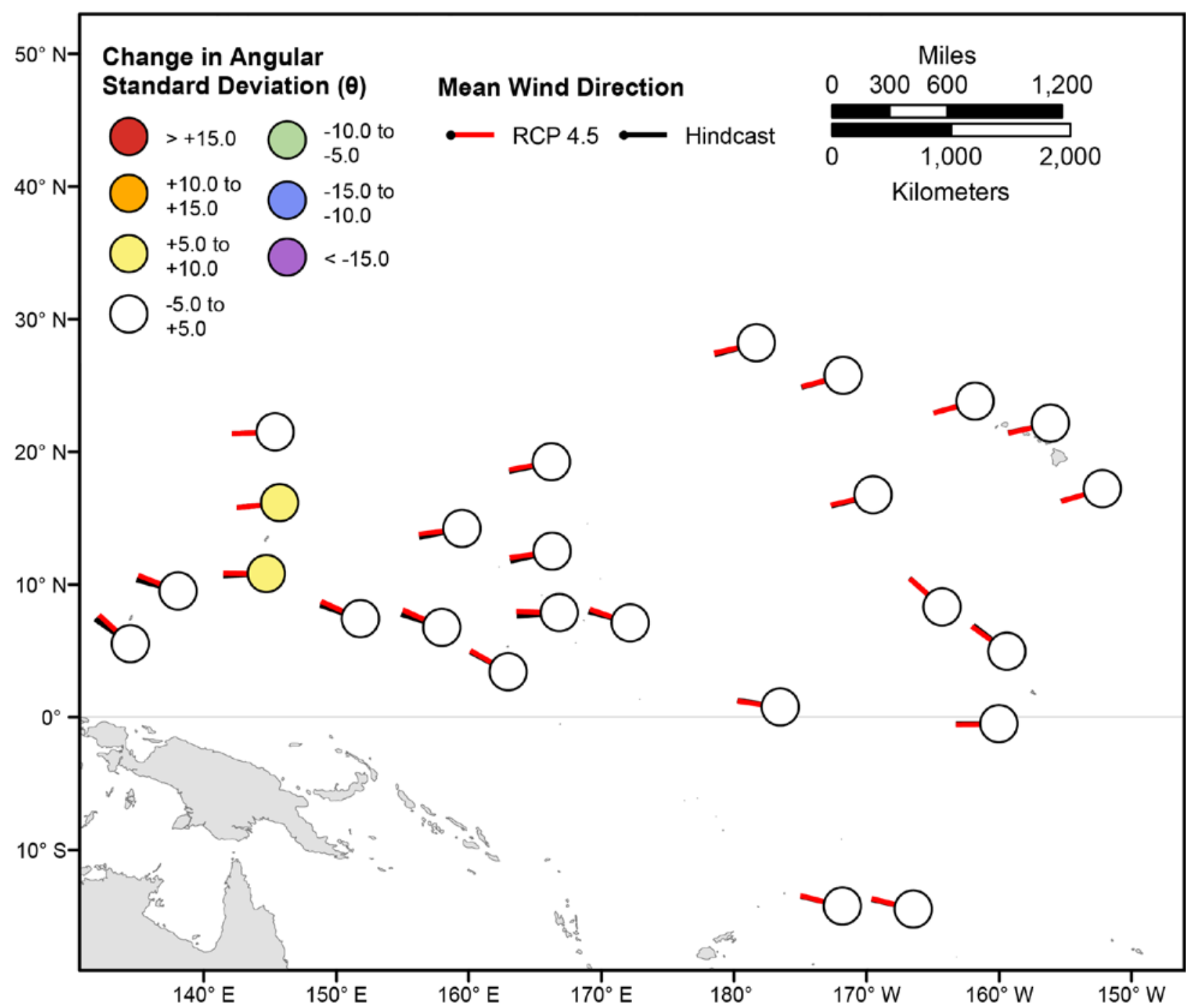

Figure 137. Map showing forecasted differences in mean wind directions and the standard deviation of wind directions for the years 2026-2045 from hindcasted values during the September-November season under the RCP4.5 future climatic scenario. Mean wind directions at each point are indicated by lines radiating from the center of each point where RCP4.5 2026-2045 mean wind directions are red and 1976-2005 hindcasted mean wind directions are black. The colors correspond to the magnitude of change in modeled mean wind direction standard deviation during 2026-2045 from those hindcasted for 1976-2005. Angular standard deviation units are in degrees. Mean wind directions are "heading towards". 


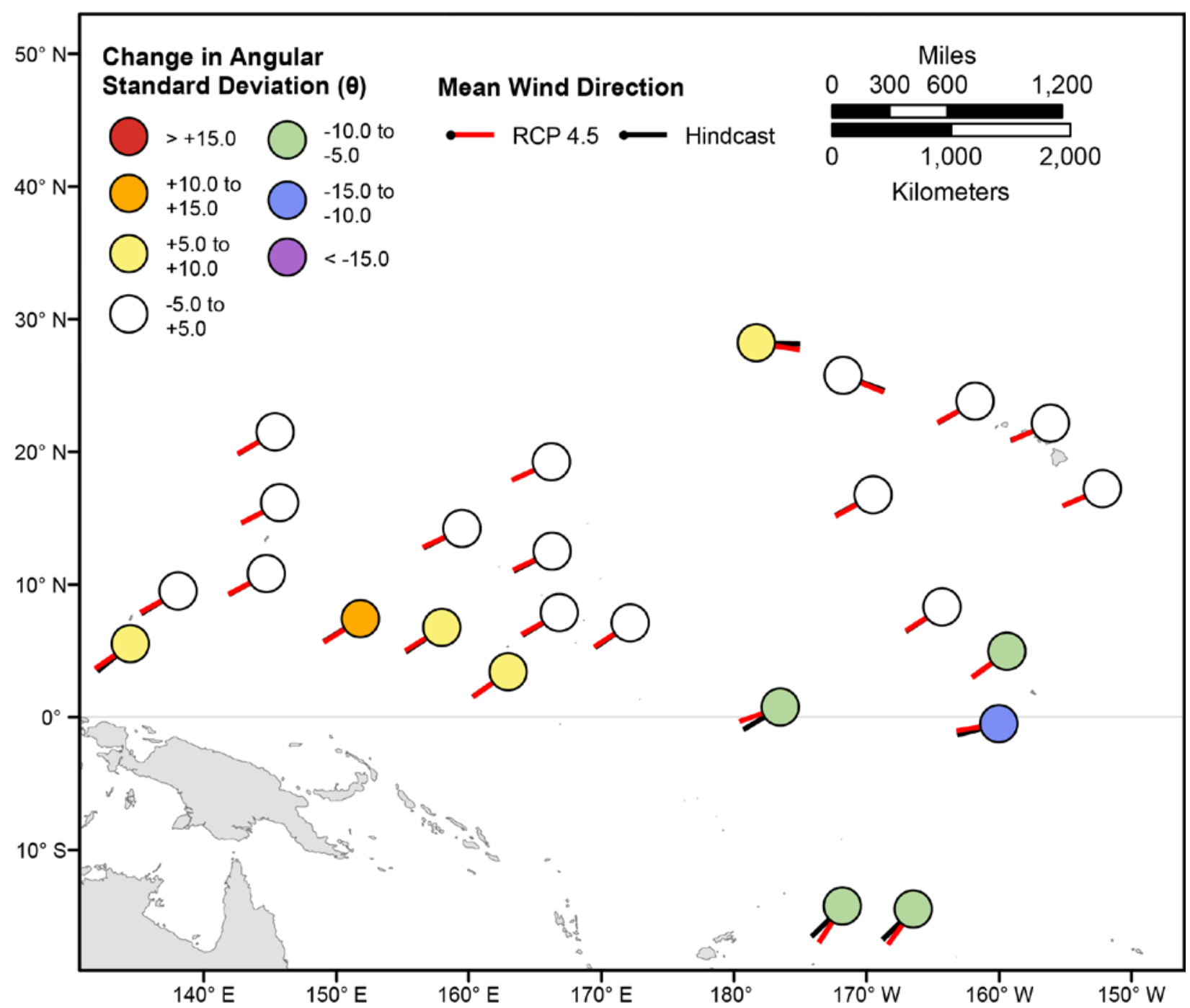

Figure 138. Map showing forecasted differences in the mean wind directions of the top 5 percent of wind speeds and the standard deviation of wind directions of the top 5 percent of wind speeds for the years 2026-2045 from hindcasted values during the December-February season under the RCP4.5 future climatic scenario. Mean wind directions at each point are indicated by lines radiating from the center of each point where RCP4.5 2026-2045 mean wind directions are red and 1976-2005 hindcasted mean wind directions are black. The colors correspond to the magnitude of change in modeled mean wind direction standard deviation during 2026-2045 from those hindcasted for 1976-2005. Angular standard deviation units are in degrees. Mean wind directions are "heading towards". 


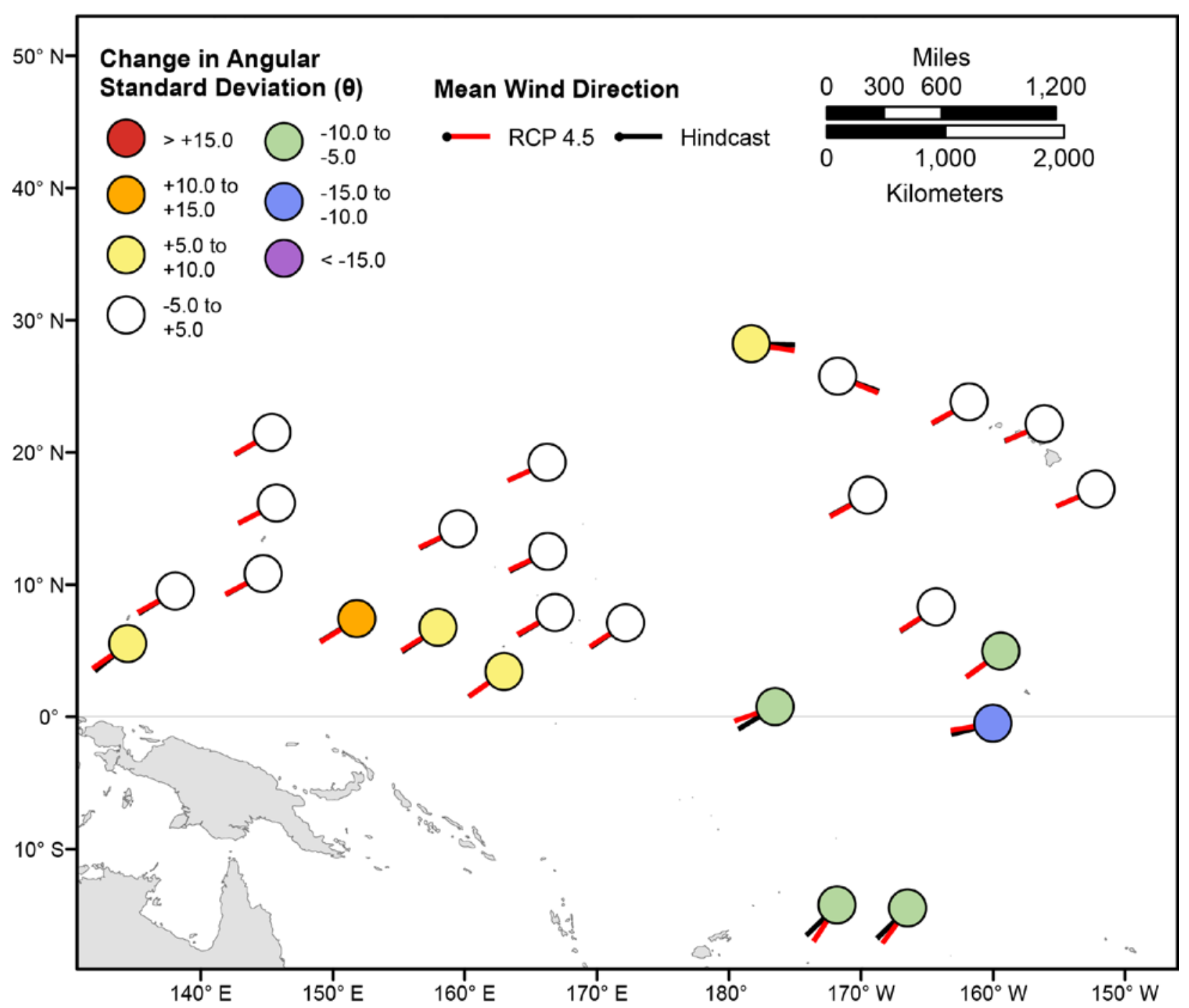

Figure 139. Map showing forecasted differences in the mean wind directions of the top 5 percent of wind speeds and the standard deviation of wind directions of the top 5 percent of wind speeds for the years 2026-2045 from hindcasted values during the March-May season under the RCP4.5 future climatic scenario. Mean wind directions at each point are indicated by lines radiating from the center of each point where RCP4.5 2026-2045 mean wind directions are red and 1976-2005 hindcasted mean wind directions are black. The colors correspond to the magnitude of change in modeled mean wind direction standard deviation during 2026-2045 from those hindcasted for 1976-2005. Angular standard deviation units are in degrees. Mean wind directions are "heading towards". 


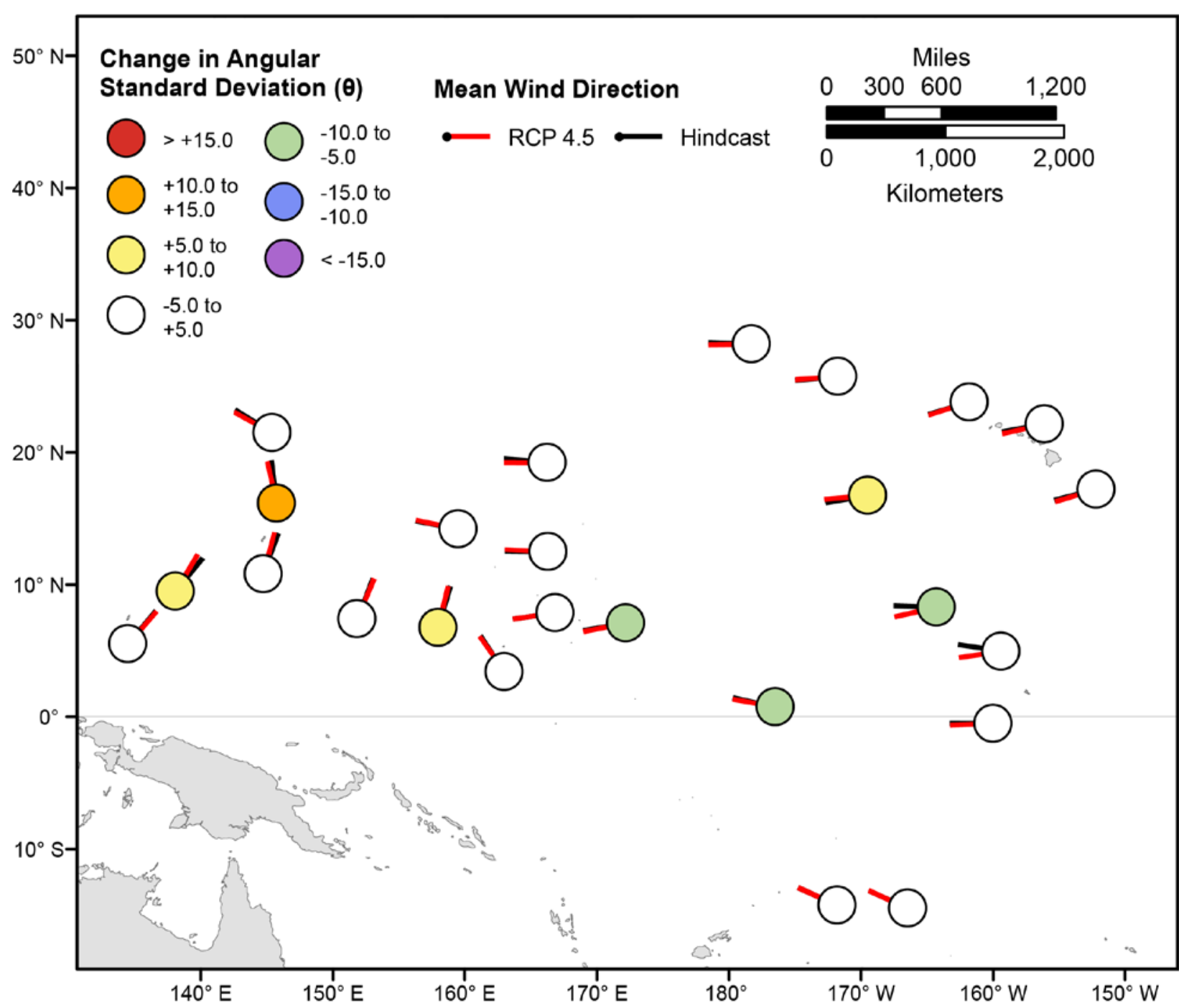

Figure 140. Map showing forecasted differences in the mean wind directions of the top 5 percent of wind speeds and the standard deviation of wind directions of the top 5 percent of wind speeds for the years 2026-2045 from hindcasted values during the June-August season under the RCP4.5 future climatic scenario. Mean wind directions at each point are indicated by lines radiating from the center of each point where RCP4.5 2026-2045 mean wind directions are red and 1976-2005 hindcasted mean wind directions are black. The colors correspond to the magnitude of change in modeled mean wind direction standard deviation during 2026-2045 from those hindcasted for 1976-2005. Angular standard deviation units are in degrees. Mean wind directions are "heading towards". 


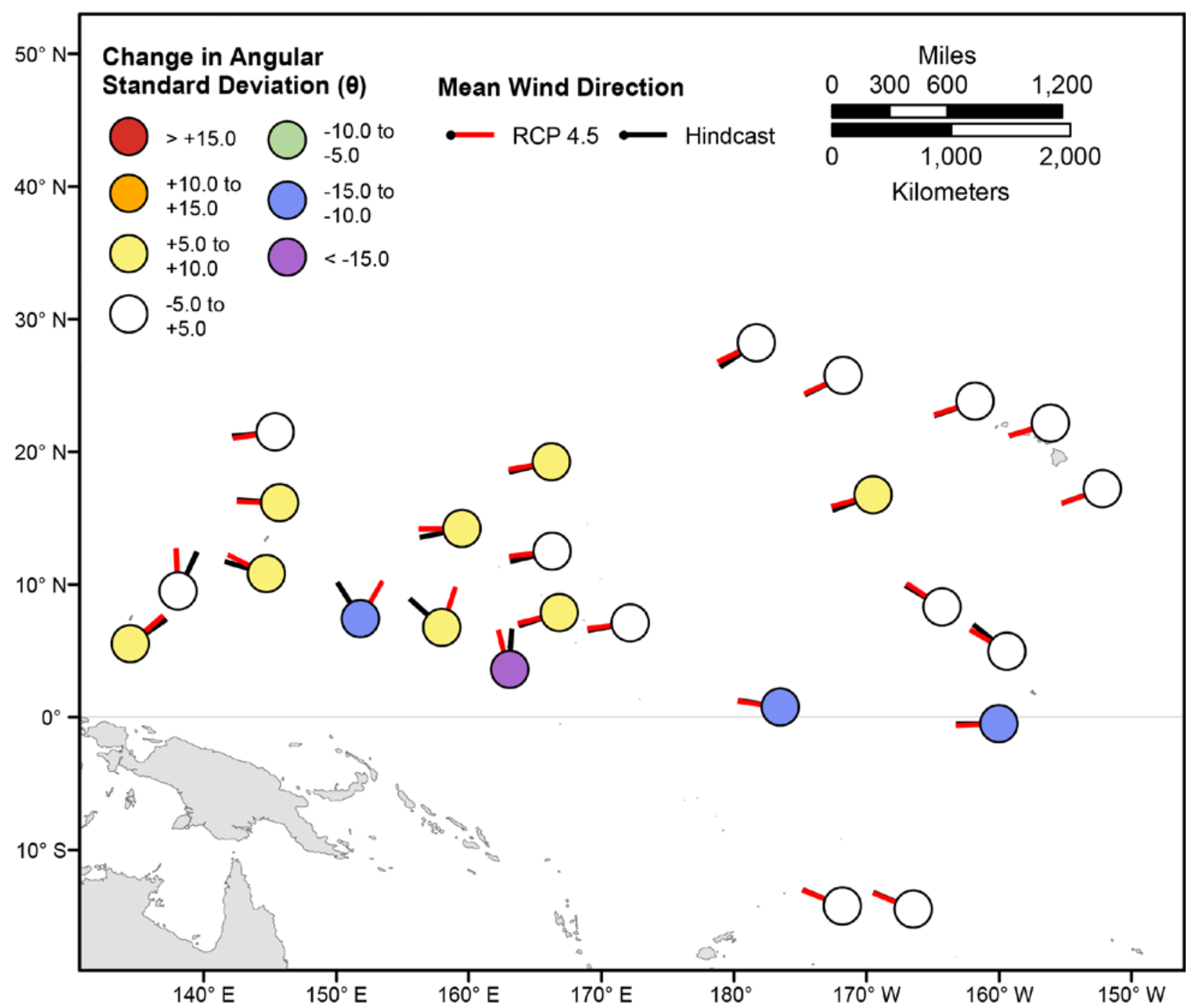

Figure 141. Map showing forecasted differences in the mean wind directions of the top 5 percent of wind speeds and the standard deviation of wind directions of the top 5 percent of wind speeds for the years 2026-2045 from hindcasted values during the September-November season under the RCP4.5 future climatic scenario. Mean wind directions at each point are indicated by lines radiating from the center of each point where RCP4.5 2026-2045 mean wind directions are red and 1976-2005 hindcasted mean wind directions are black. The colors correspond to the magnitude of change in modeled mean wind direction standard deviation during 2026-2045 from those hindcasted for 1976-2005. Angular standard deviation units are in degrees. Mean wind directions are "heading towards". 


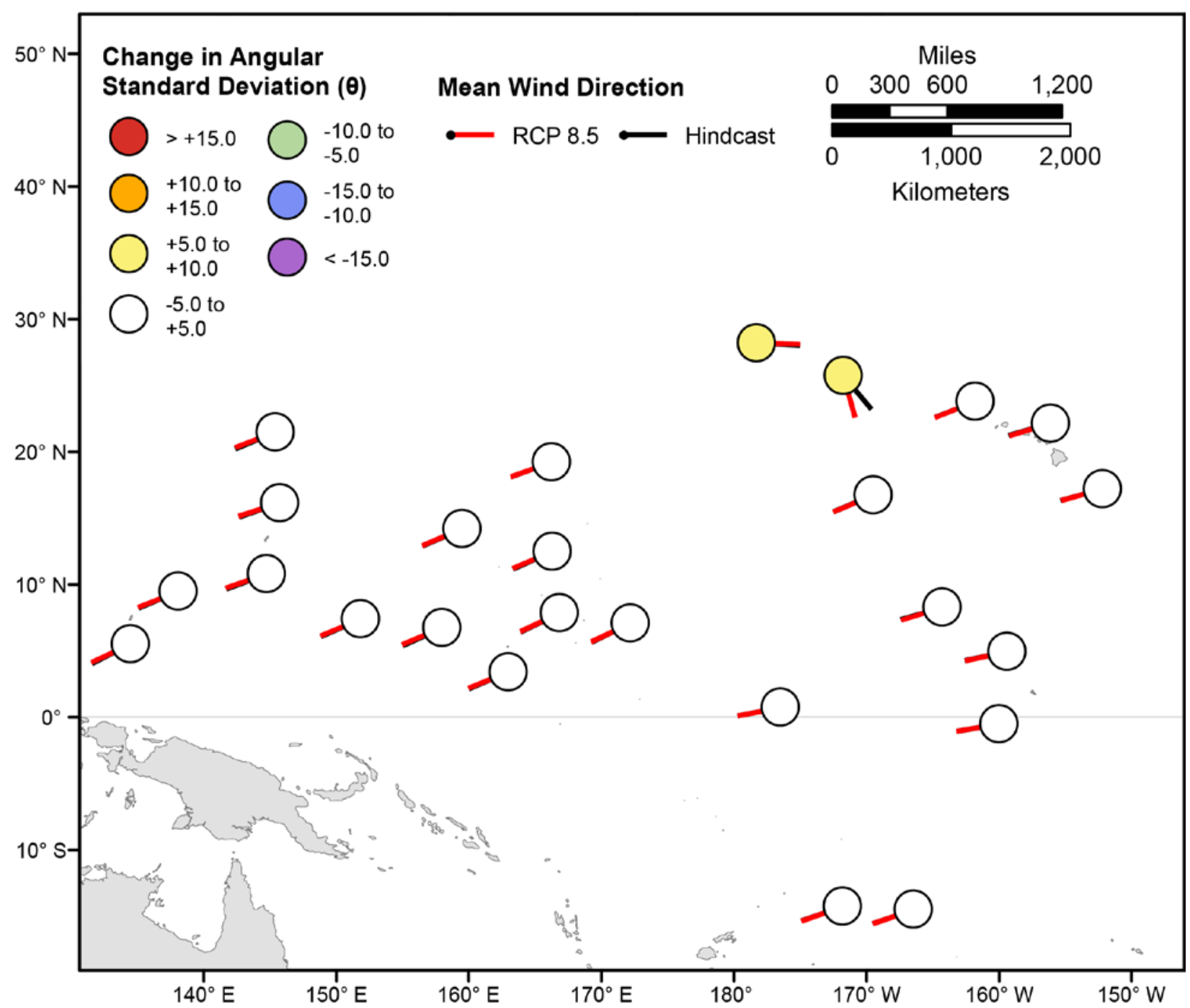

Figure 142. Map showing forecasted differences in mean wind directions and the standard deviation of wind directions for the years 2026-2045 from hindcasted values during the December-February season under the RCP8.5 future climatic scenario. Mean wind directions at each point are indicated by lines radiating from the center of each point where RCP8.5 2026-2045 mean wind directions are red and 1976-2005 hindcasted mean wind directions are black. The colors correspond to the magnitude of change in modeled mean wind direction standard deviation during 2026-2045 from those hindcasted for 19762005. Angular standard deviation units are in degrees. Mean wind directions are "heading towards". 


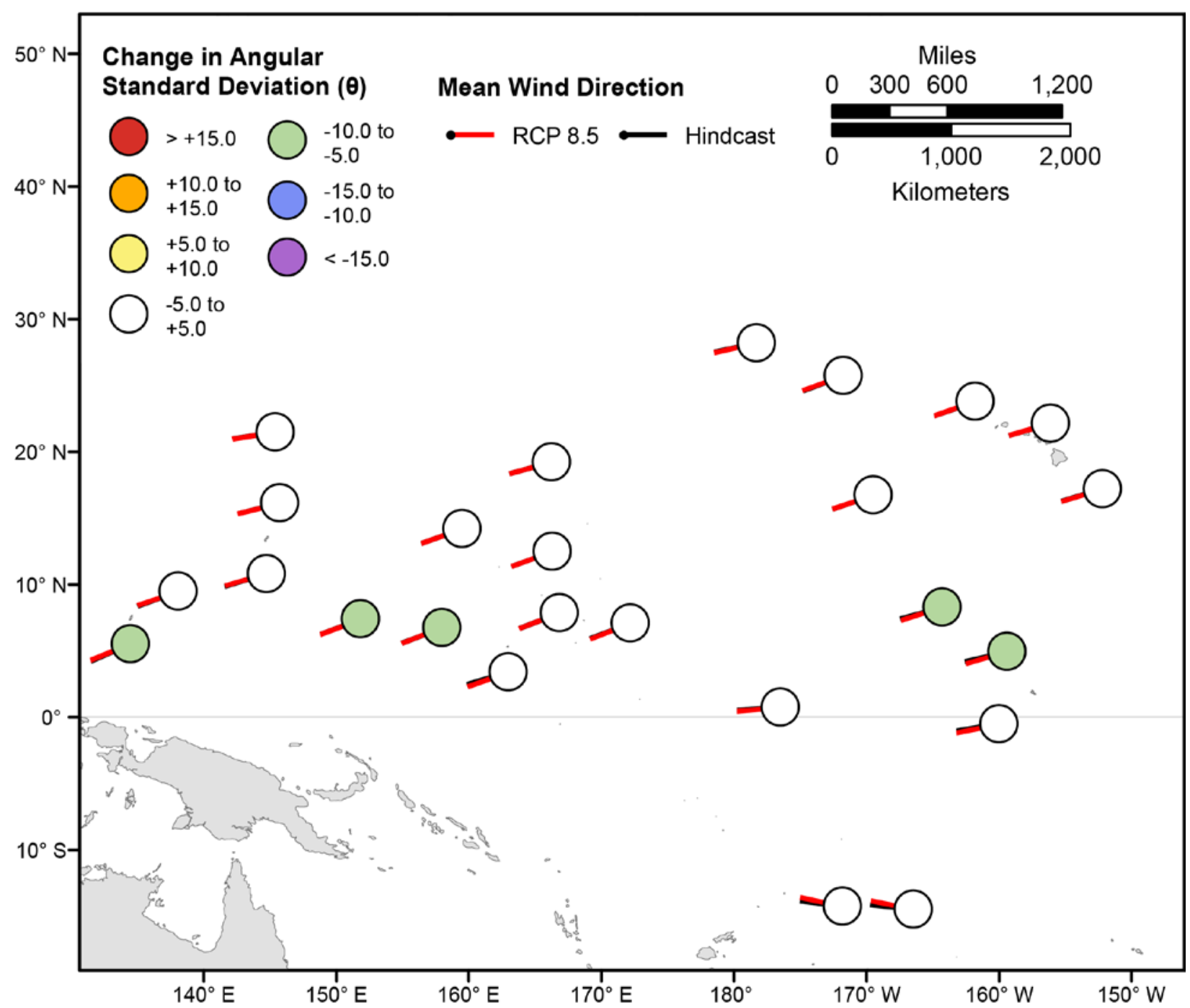

Figure 143. Map showing forecasted differences in mean wind directions and the standard deviation of wind directions for the years 2026-2045 from hindcasted values during the March-May season under the RCP8.5 future climatic scenario. Mean wind directions at each point are indicated by lines radiating from the center of each point where RCP8.5 2026-2045 mean wind directions are red and 1976-2005 hindcasted mean wind directions are black. The colors correspond to the magnitude of change in modeled mean wind direction standard deviation during 2026-2045 from those hindcasted for 19762005. Angular standard deviation units are in degrees. Mean wind directions are "heading towards". 


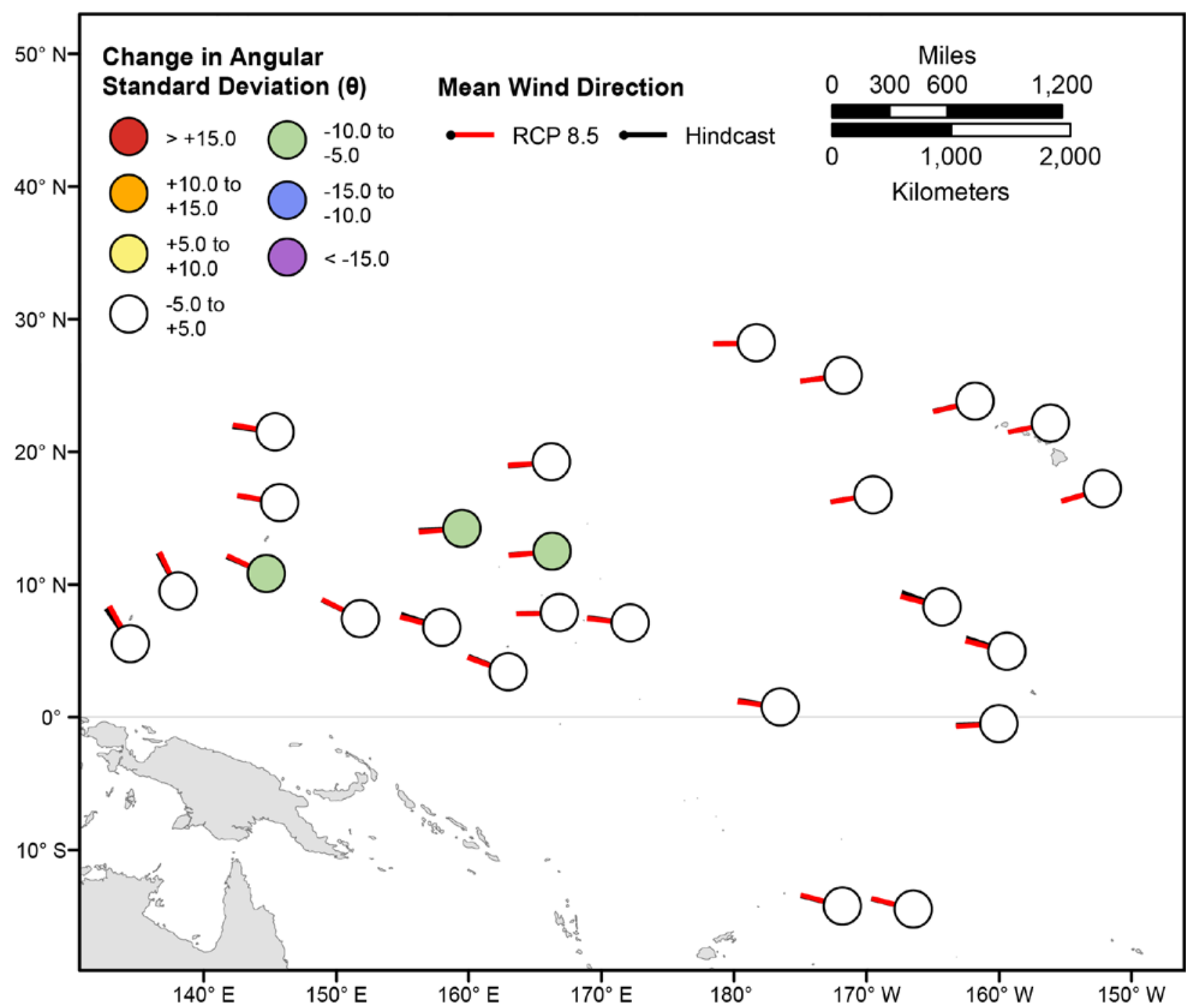

Figure 144. Map showing forecasted differences in mean wind directions and the standard deviation of wind directions for the years 2026-2045 from hindcasted values during the June-August season under the RCP8.5 future climatic scenario. Mean wind directions at each point are indicated by lines radiating from the center of each point where RCP8.5 2026-2045 mean wind directions are red and 1976-2005 hindcasted mean wind directions are black. The colors correspond to the magnitude of change in modeled mean wind direction standard deviation during 2026-2045 from those hindcasted for 19762005. Angular standard deviation units are in degrees. Mean wind directions are "heading towards". 


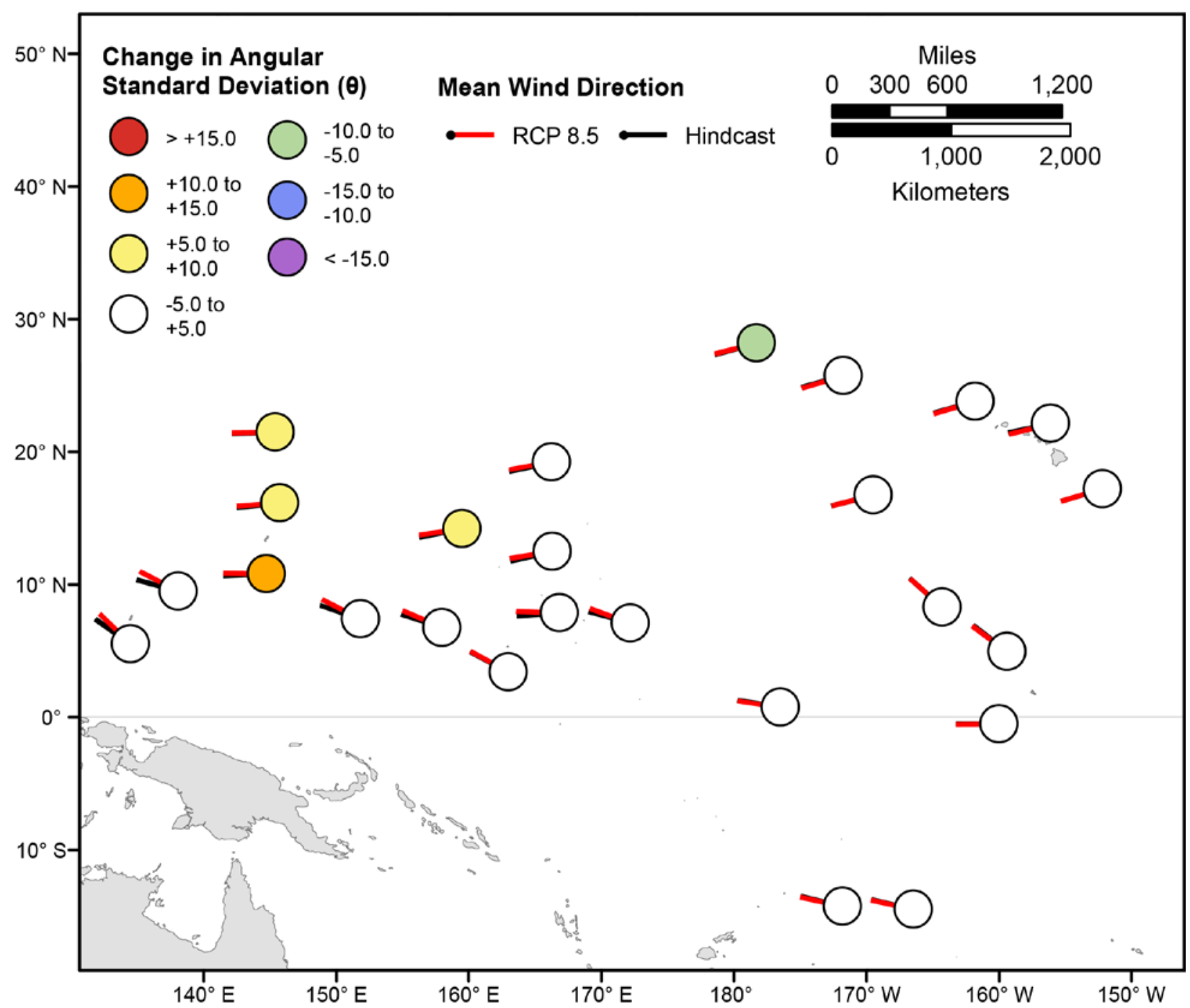

Figure 145. Map showing forecasted differences in mean wind directions and the standard deviation of wind directions for the years 2026-2045 from hindcasted values during the September-November season under the RCP8.5 future climatic scenario. Mean wind directions at each point are indicated by lines radiating from the center of each point where RCP8.5 2026-2045 mean wind directions are red and 1976-2005 hindcasted mean wind directions are black. The colors correspond to the magnitude of change in modeled mean wind direction standard deviation during 2026-2045 from those hindcasted for 1976-2005. Angular standard deviation units are in degrees. Mean wind directions are "heading towards". 


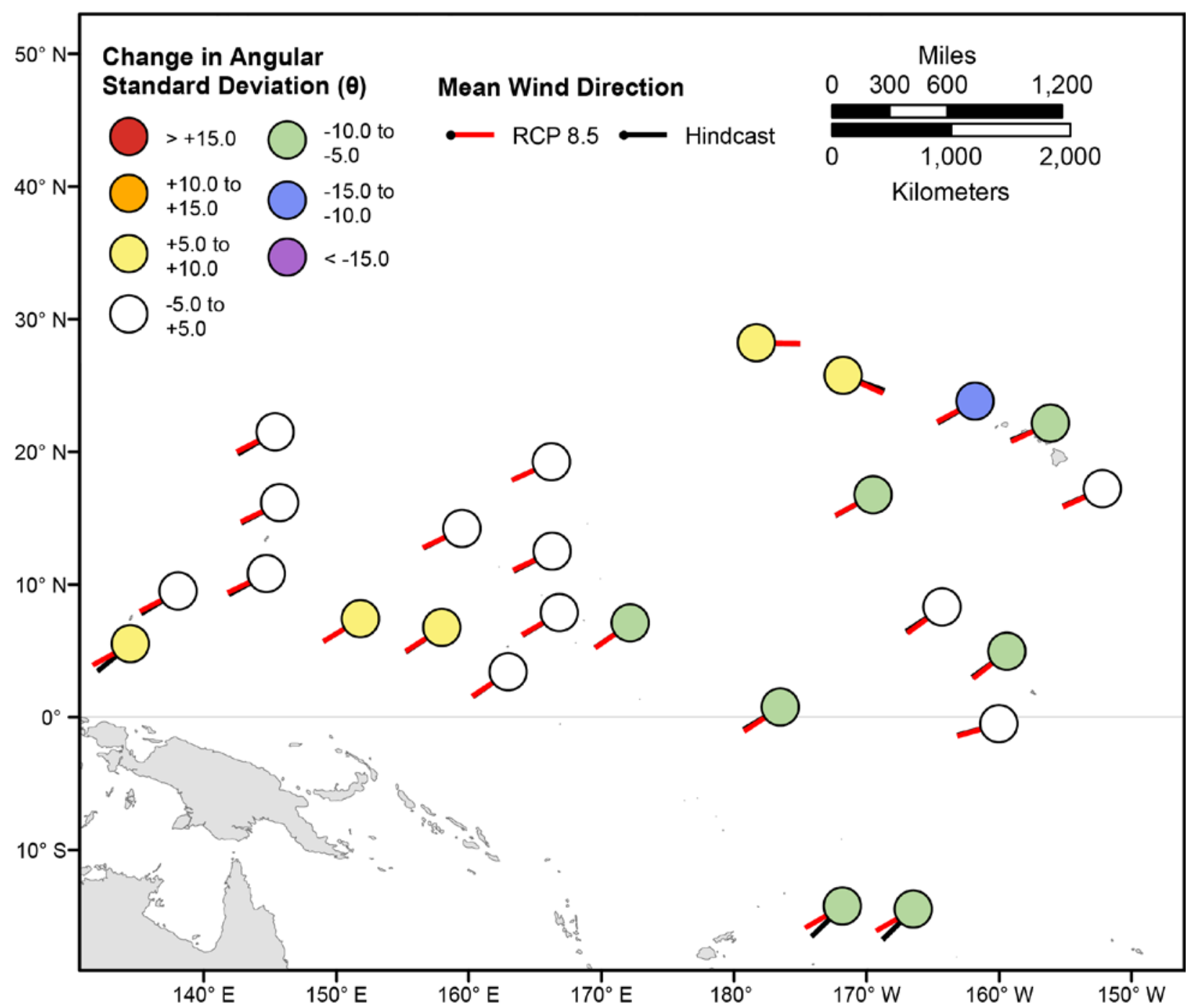

Figure 146. Map showing forecasted differences in the mean wind directions of the top 5 percent of wind speeds and the standard deviation of wind directions of the top 5 percent of wind speeds for the years 2026-2045 from hindcasted values during the December-February season under the RCP8.5 future climatic scenario. Mean wind directions at each point are indicated by lines radiating from the center of each point where RCP8.5 2026-2045 mean wind directions are red and 1976-2005 hindcasted mean wind directions are black. The colors correspond to the magnitude of change in modeled mean wind direction standard deviation during 2026-2045 from those hindcasted for 1976-2005. Angular standard deviation units are in degrees. Mean wind directions are "heading towards". 


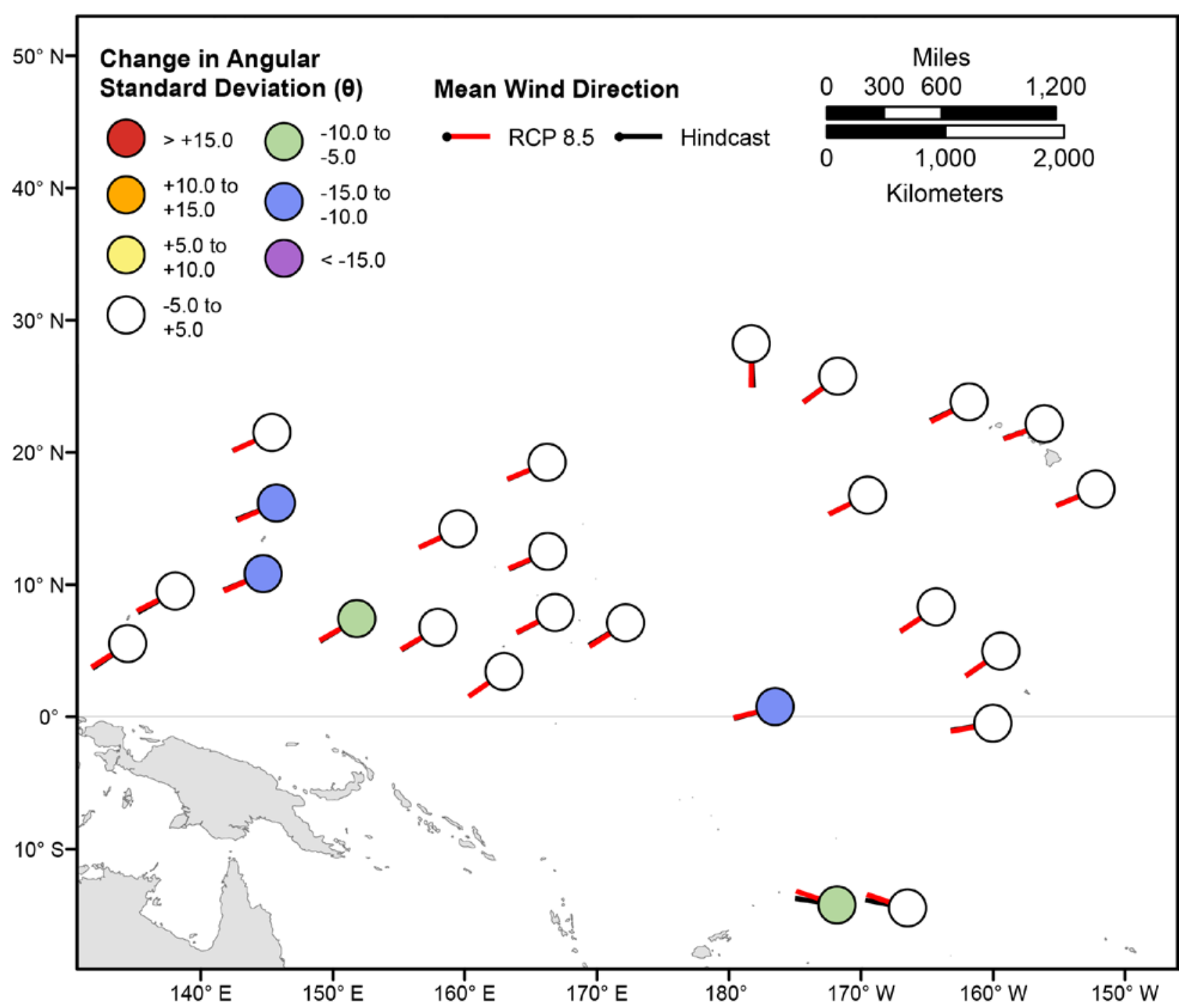

Figure 147. Map showing forecasted differences in the mean wind directions of the top 5 percent of wind speeds and the standard deviation of wind directions of the top 5 percent of wind speeds for the years 2026-2045 from hindcasted values during the March-May season under the RCP8.5 future climatic scenario. Mean wind directions at each point are indicated by lines radiating from the center of each point where RCP8.5 2026-2045 mean wind directions are red and 1976-2005 hindcasted mean wind directions are black. The colors correspond to the magnitude of change in modeled mean wind direction standard deviation during 2026-2045 from those hindcasted for 1976-2005. Angular standard deviation units are in degrees. Mean wind directions are "heading towards". 


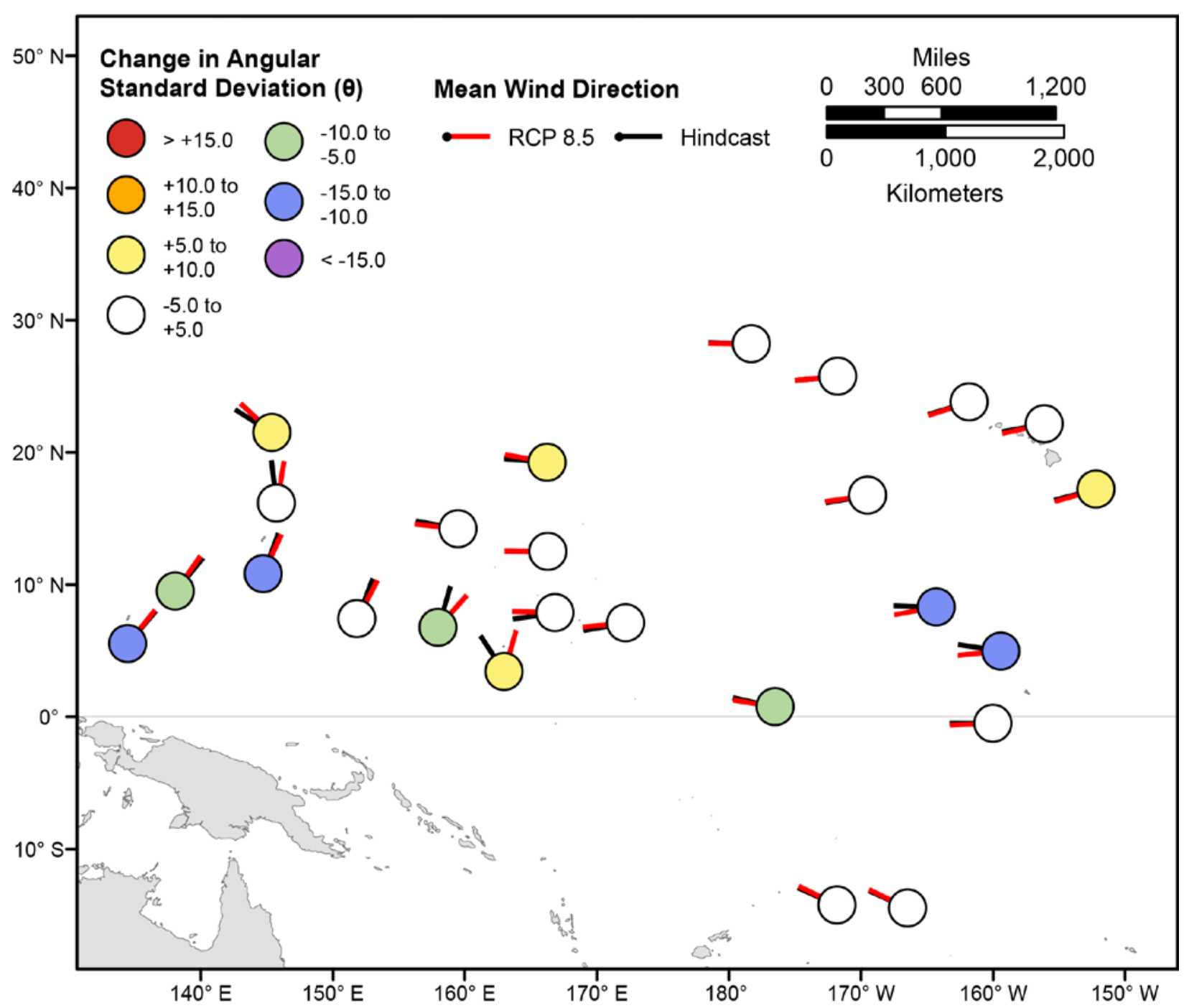

Figure 148. Map showing forecasted differences in the mean wind directions of the top 5 percent of wind speeds and the standard deviation of wind directions of the top 5 percent of wind speeds for the years 2026-2045 from hindcasted values during the June-August season under the RCP8.5 future climatic scenario. Mean wind directions at each point are indicated by lines radiating from the center of each point where RCP8.5 2026-2045 mean wind directions are red and 1976-2005 hindcasted mean wind directions are black. The colors correspond to the magnitude of change in modeled mean wind direction standard deviation during 2026-2045 from those hindcasted for 1976-2005. Angular standard deviation units are in degrees. Mean wind directions are "heading towards". 


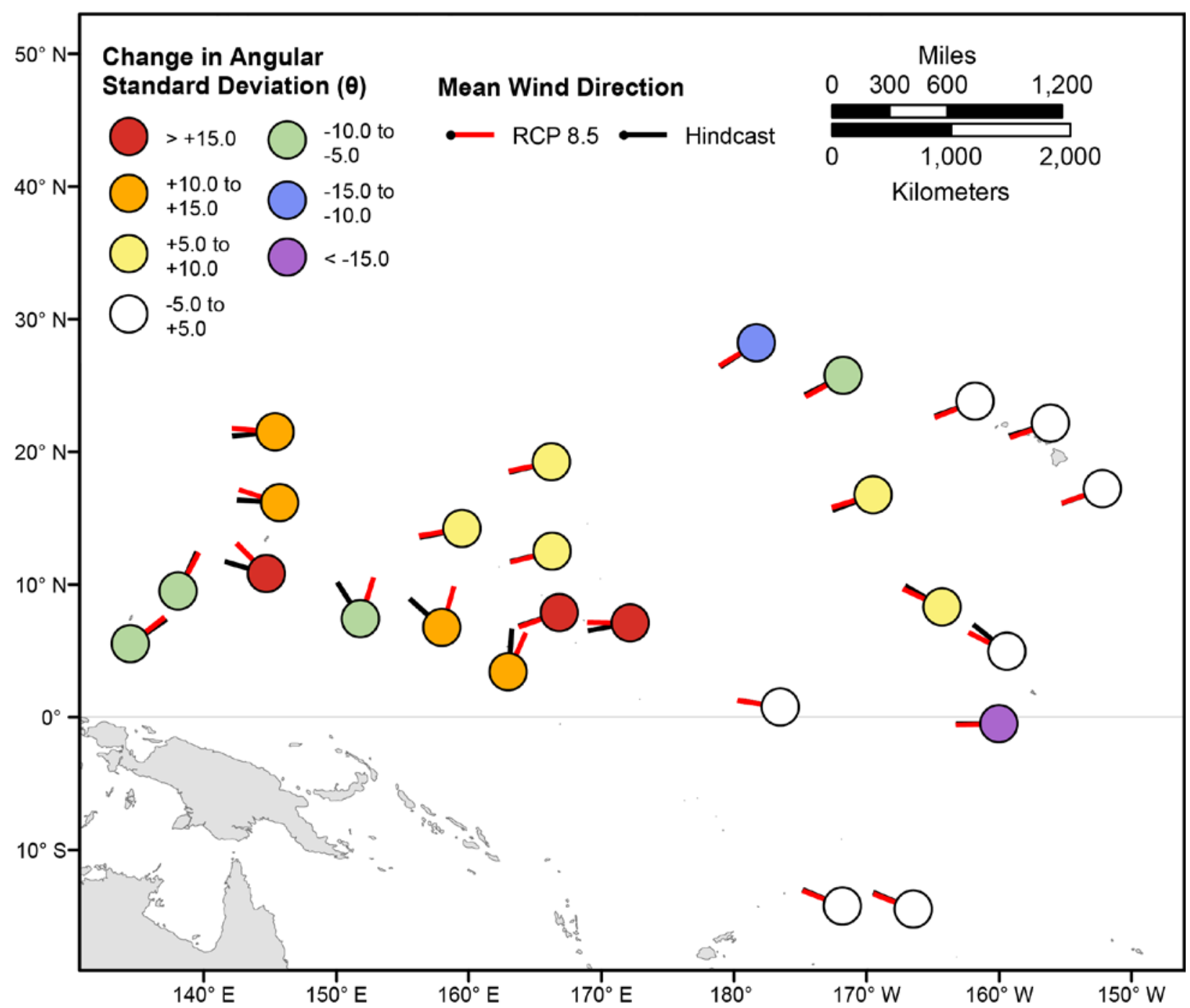

Figure 149. Map showing forecasted differences in the mean wind directions of the top 5 percent of wind speeds and the standard deviation of wind directions of the top 5 percent of wind speeds for the years 2026-2045 from hindcasted values during the September-November season under the RCP8.5 future climatic scenario. Mean wind directions at each point are indicated by lines radiating from the center of each point where RCP8.5 2026-2045 mean wind directions are red and 1976-2005 hindcasted mean wind directions are black. The colors correspond to the magnitude of change in modeled mean wind direction standard deviation during 2026-2045 from those hindcasted for 1976-2005. Angular standard deviation units are in degrees. Mean wind directions are "heading towards". 


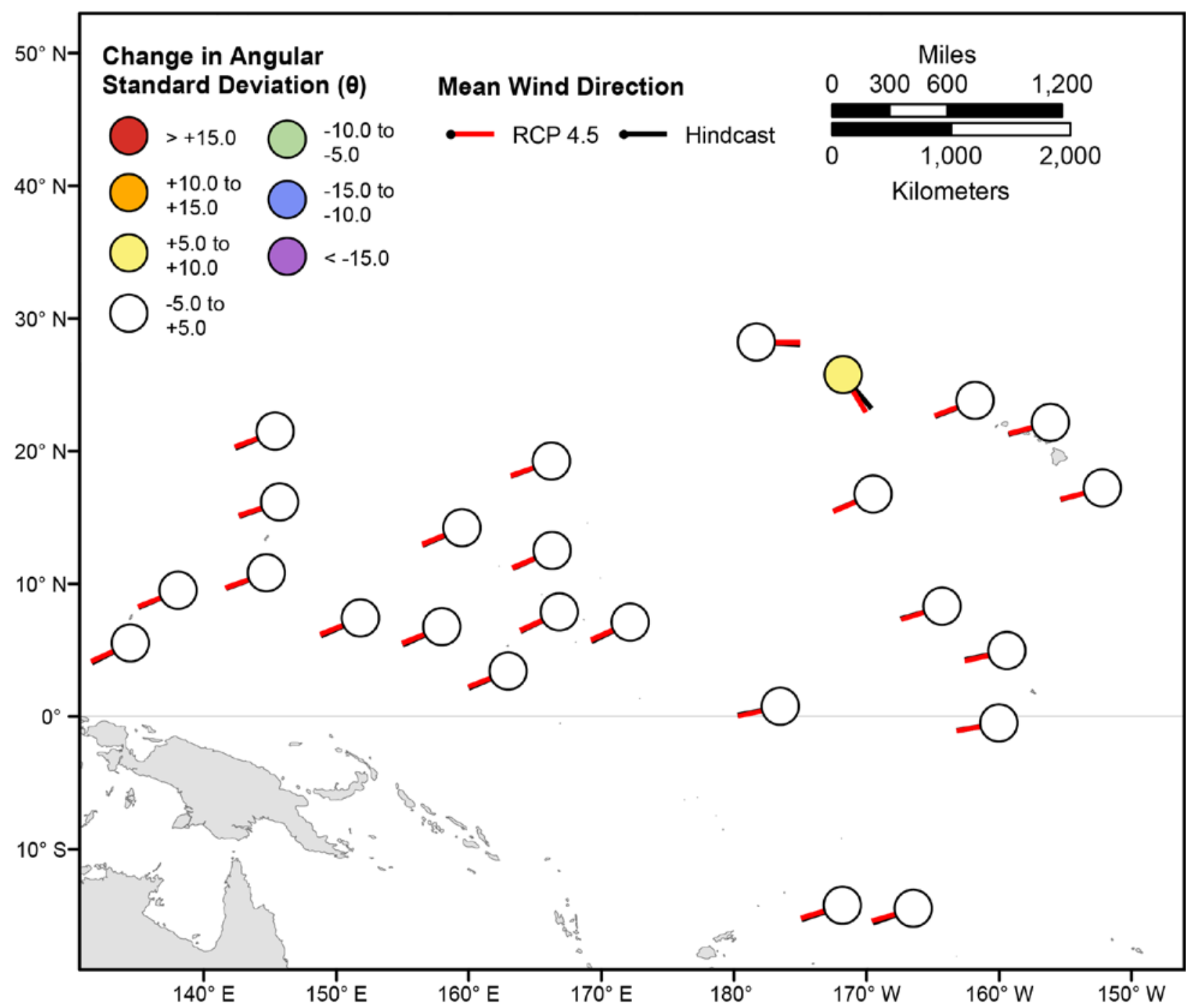

Figure 150. Map showing forecasted differences in mean wind directions and the standard deviation of wind directions for the years 2081-2100 from hindcasted values during the December-February season under the RCP4.5 future climatic scenario. Mean wind directions at each point are indicated by lines radiating from the center of each point where RCP4.5 2081-2100 mean wind directions are red and 1976-2005 hindcasted mean wind directions are black. The colors correspond to the magnitude of change in modeled mean wind direction standard deviation during 2081-2100 from those hindcasted for 19762005. Angular standard deviation units are in degrees. Mean wind directions are "heading towards". 


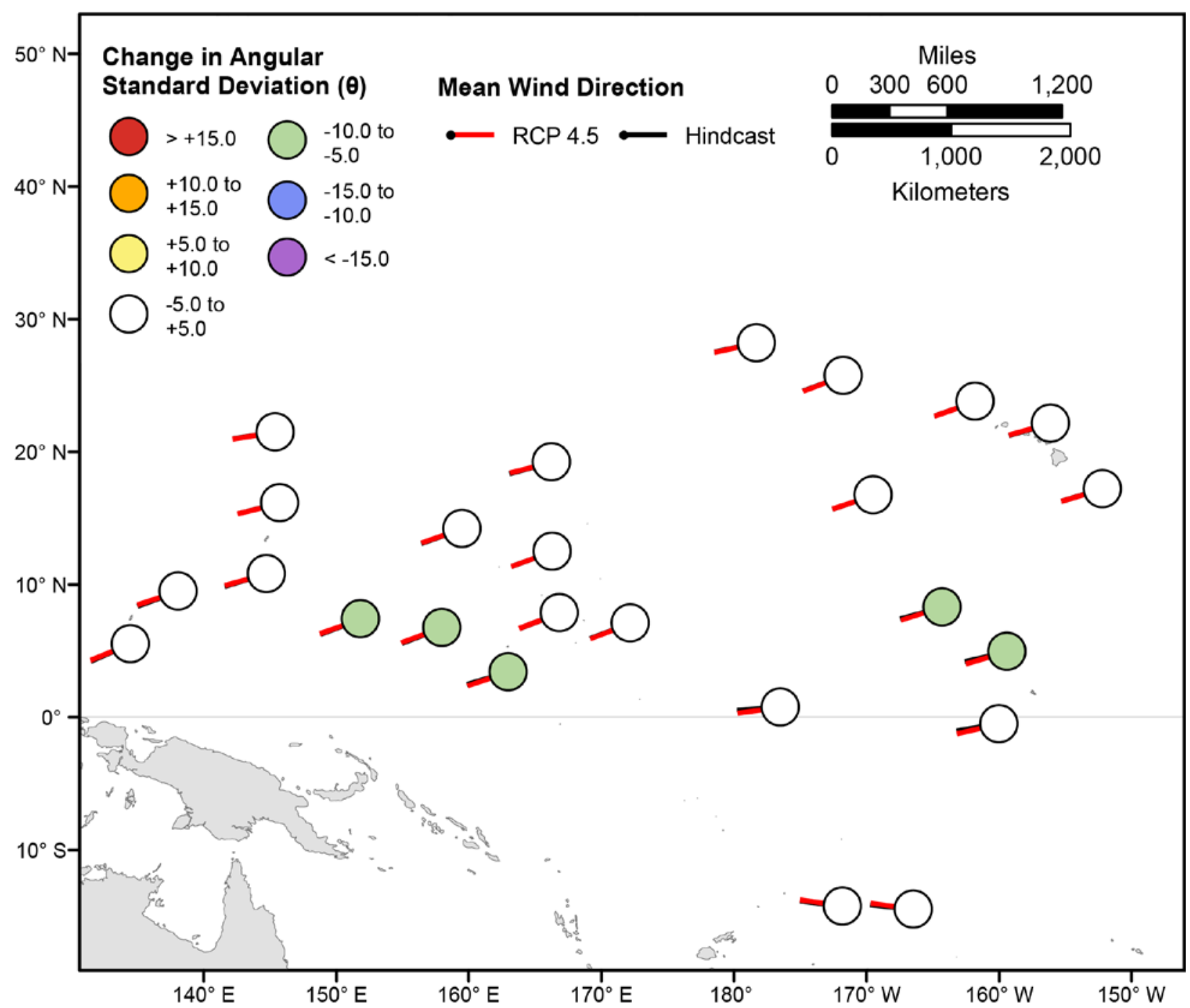

Figure 151. Map showing forecasted differences in mean wind directions and the standard deviation of wind directions for the years 2081-2100 from hindcasted values during the March-May season under the RCP4.5 future climatic scenario. Mean wind directions at each point are indicated by lines radiating from the center of each point where RCP4.5 2081-2100 mean wind directions are red and 1976-2005 hindcasted mean wind directions are black. The colors correspond to the magnitude of change in modeled mean wind direction standard deviation during 2081-2100 from those hindcasted for 19762005. Angular standard deviation units are in degrees. Mean wind directions are "heading towards". 


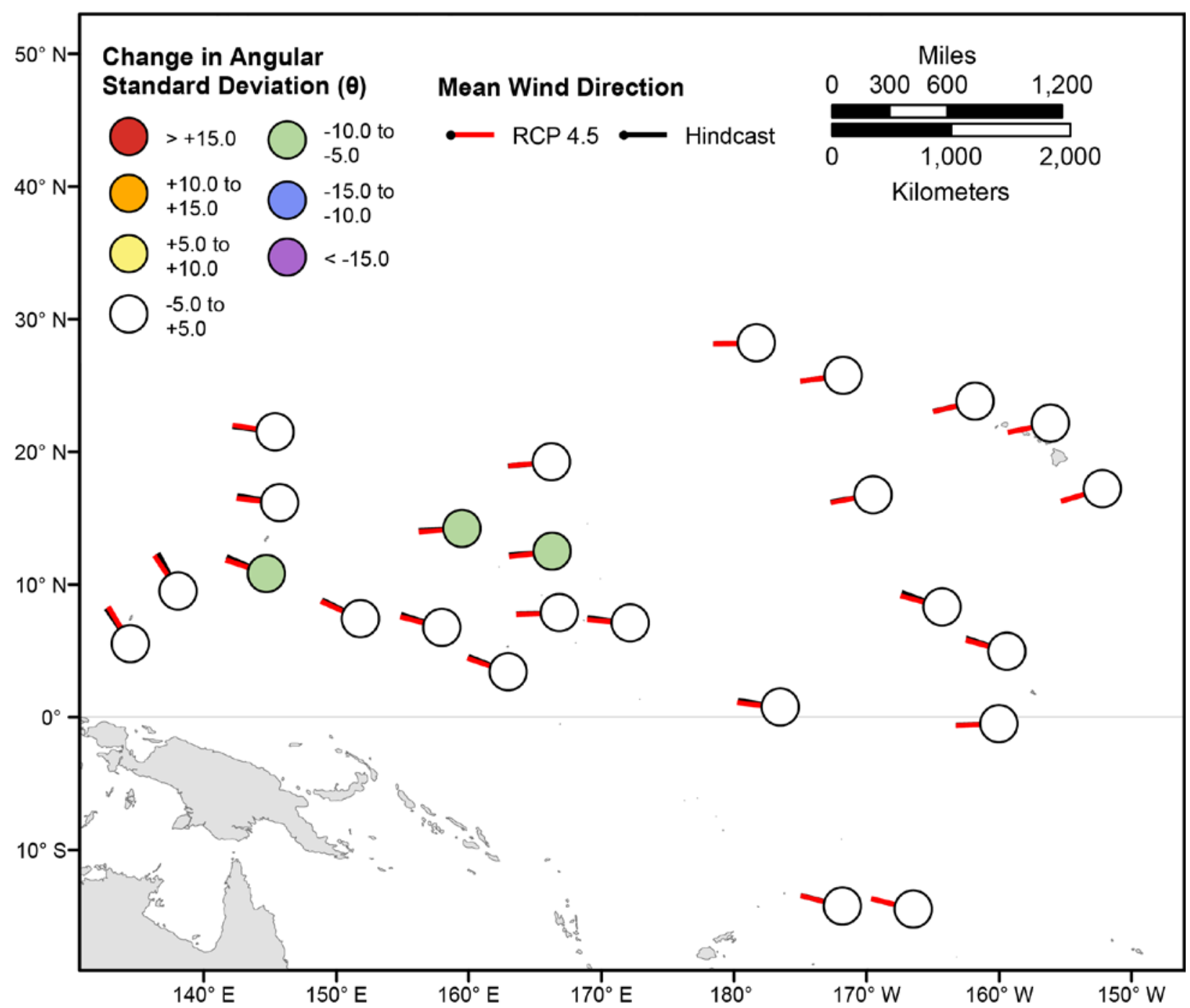

Figure 152. Map showing forecasted differences in mean wind directions and the standard deviation of wind directions for the years 2081-2100 from hindcasted values during the June-August season under the RCP4.5 future climatic scenario. Mean wind directions at each point are indicated by lines radiating from the center of each point where RCP4.5 2081-2100 mean wind directions are red and 1976-2005 hindcasted mean wind directions are black. The colors correspond to the magnitude of change in modeled mean wind direction standard deviation during 2081-2100 from those hindcasted for 19762005. Angular standard deviation units are in degrees. Mean wind directions are "heading towards". 


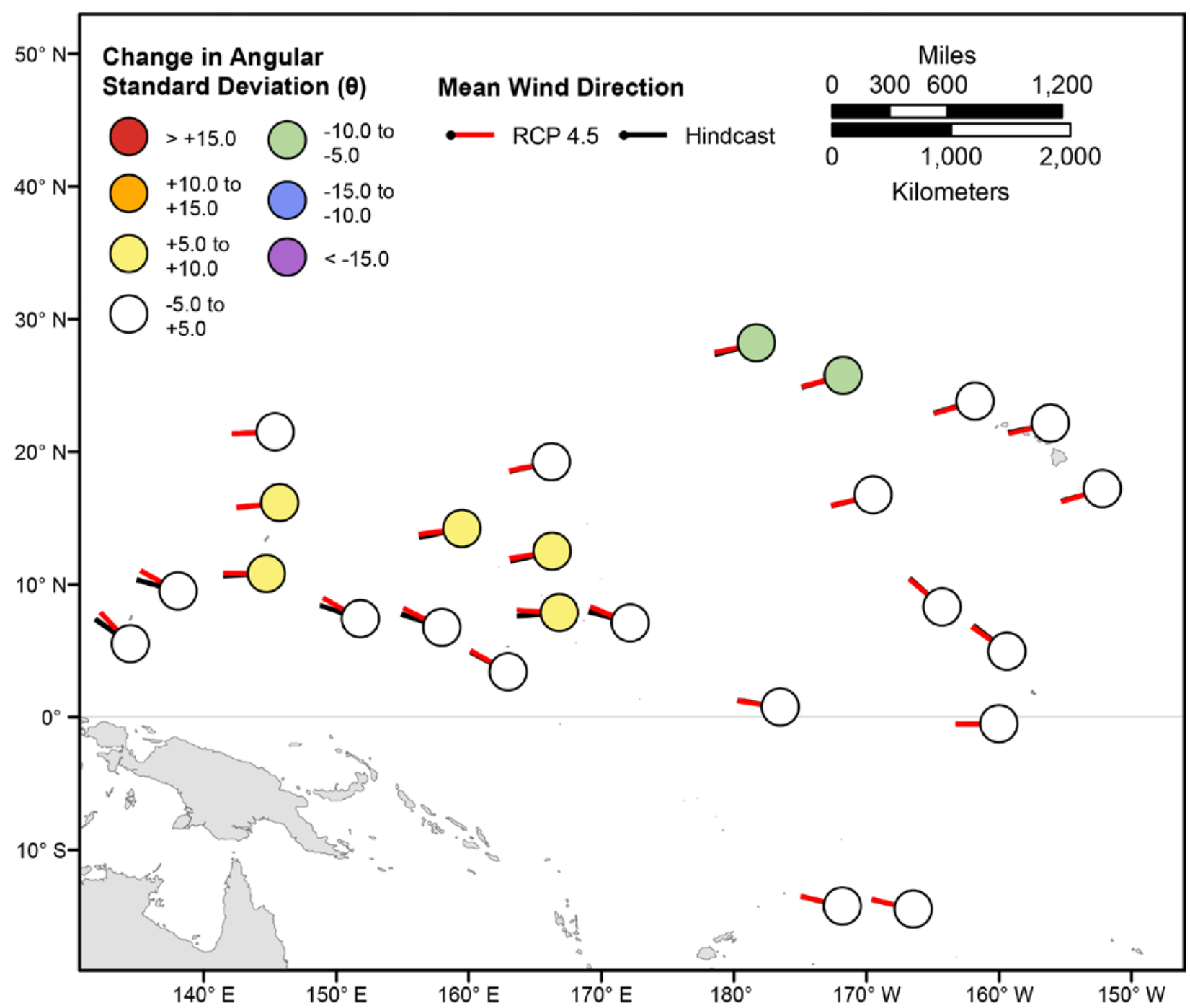

Figure 153. Map showing forecasted differences in mean wind directions and the standard deviation of wind directions for the years 2081-2100 from hindcasted values during the September-November season under the RCP4.5 future climatic scenario. Mean wind directions at each point are indicated by lines radiating from the center of each point where RCP4.5 2081-2100 mean wind directions are red and 1976-2005 hindcasted mean wind directions are black. The colors correspond to the magnitude of change in modeled mean wind direction standard deviation during 2081-2100 from those hindcasted for 1976-2005. Angular standard deviation units are in degrees. Mean wind directions are "heading towards". 


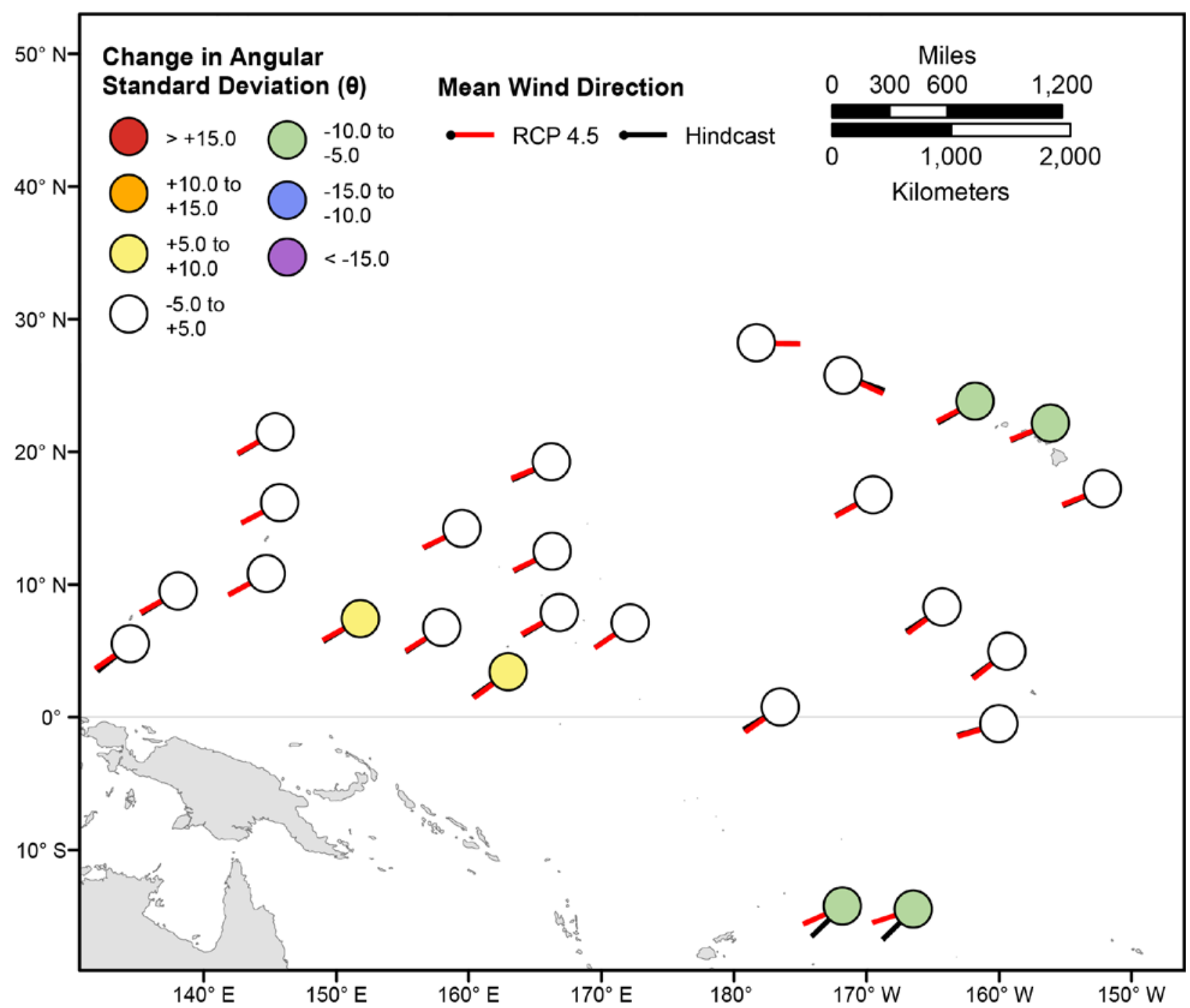

Figure 154. Map showing forecasted differences in the mean wind directions of the top 5 percent of wind speeds and the standard deviation of wind directions of the top 5 percent of wind speeds for the years 2081-2100 from hindcasted values during the December-February season under the RCP4.5 future climatic scenario. Mean wind directions at each point are indicated by lines radiating from the center of each point where RCP4.5 2081-2100 mean wind directions are red and 1976-2005 hindcasted mean wind directions are black. The colors correspond to the magnitude of change in modeled mean wind direction standard deviation during 2081-2100 from those hindcasted for 1976-2005. Angular standard deviation units are in degrees. Mean wind directions are "heading towards". 


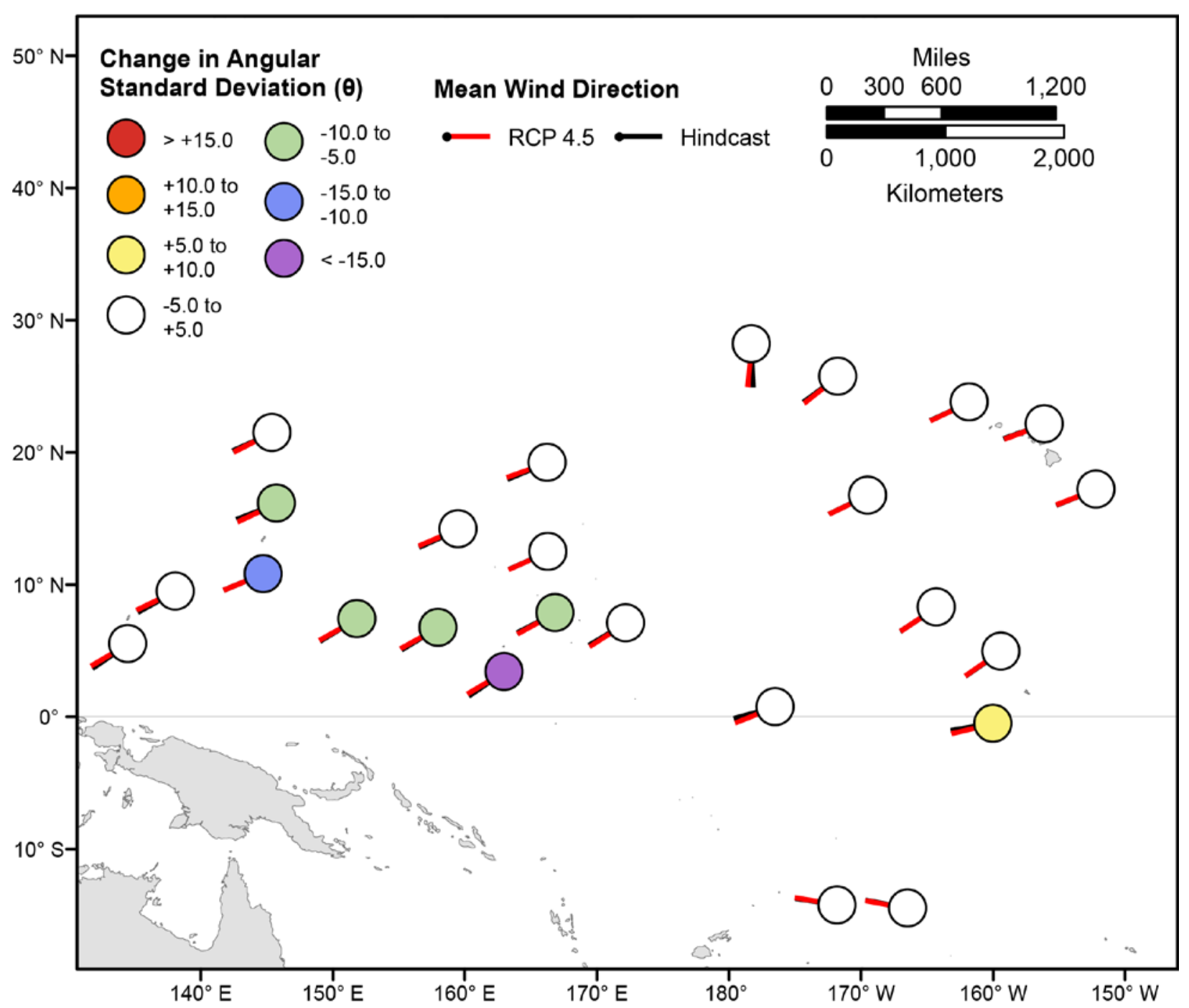

Figure 155. Map showing forecasted differences in the mean wind directions of the top 5 percent of wind speeds and the standard deviation of wind directions of the top 5 percent of wind speeds for the years 2081-2100 from hindcasted values during the March-May season under the RCP4.5 future climatic scenario. Mean wind directions at each point are indicated by lines radiating from the center of each point where RCP4.5 2081-2100 mean wind directions are red and 1976-2005 hindcasted mean wind directions are black. The colors correspond to the magnitude of change in modeled mean wind direction standard deviation during 2081-2100 from those hindcasted for 1976-2005. Angular standard deviation units are in degrees. Mean wind directions are "heading towards". 


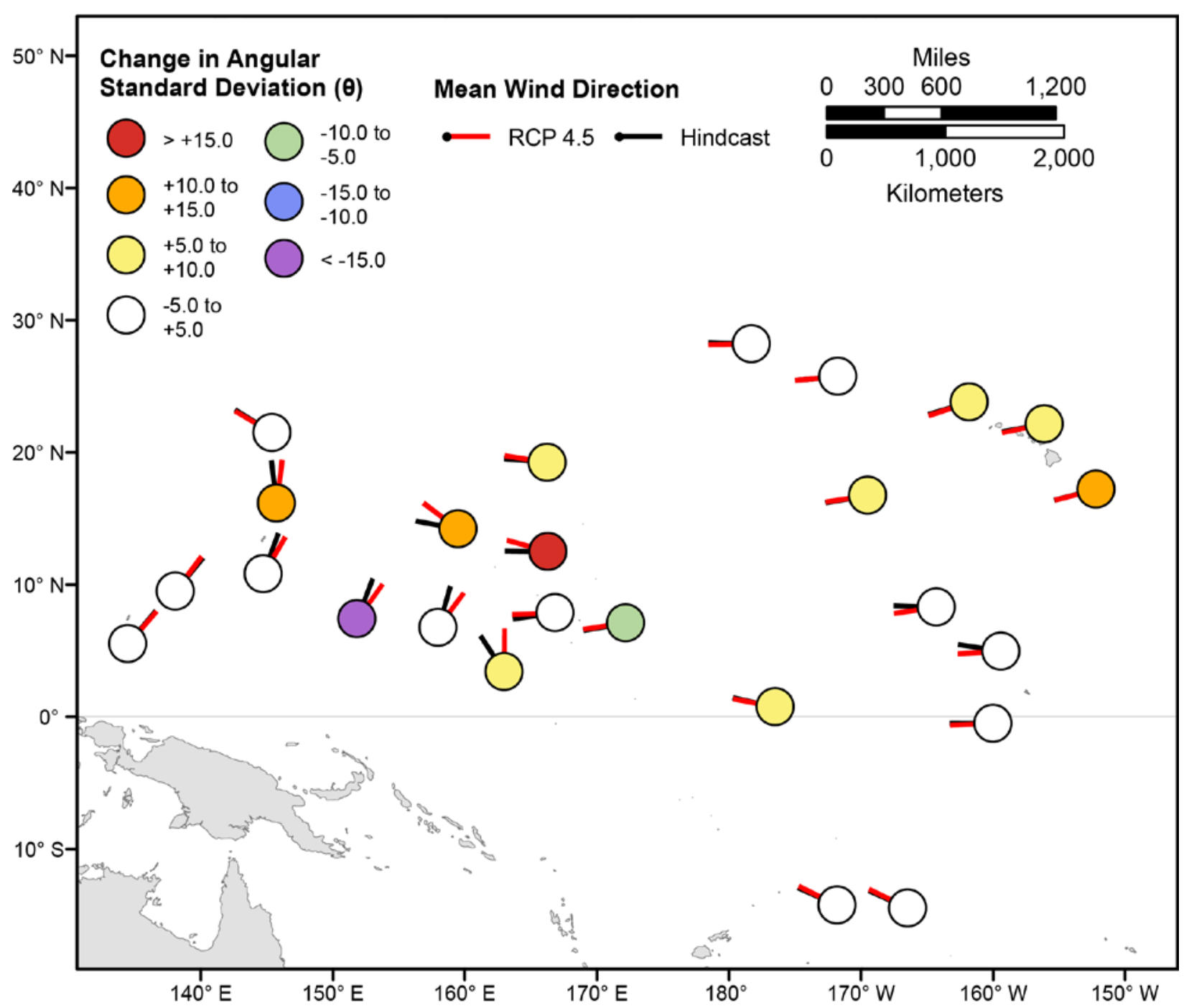

Figure 156. Map showing forecasted differences in the mean wind directions of the top 5 percent of wind speeds and the standard deviation of wind directions of the top 5 percent of wind speeds for the years 2081-2100 from hindcasted values during the June-August season under the RCP4.5 future climatic scenario. Mean wind directions at each point are indicated by lines radiating from the center of each point where RCP4.5 2081-2100 mean wind directions are red and 1976-2005 hindcasted mean wind directions are black. The colors correspond to the magnitude of change in modeled mean wind direction standard deviation during 2081-2100 from those hindcasted for 1976-2005. Angular standard deviation units are in degrees. Mean wind directions are "heading towards". 


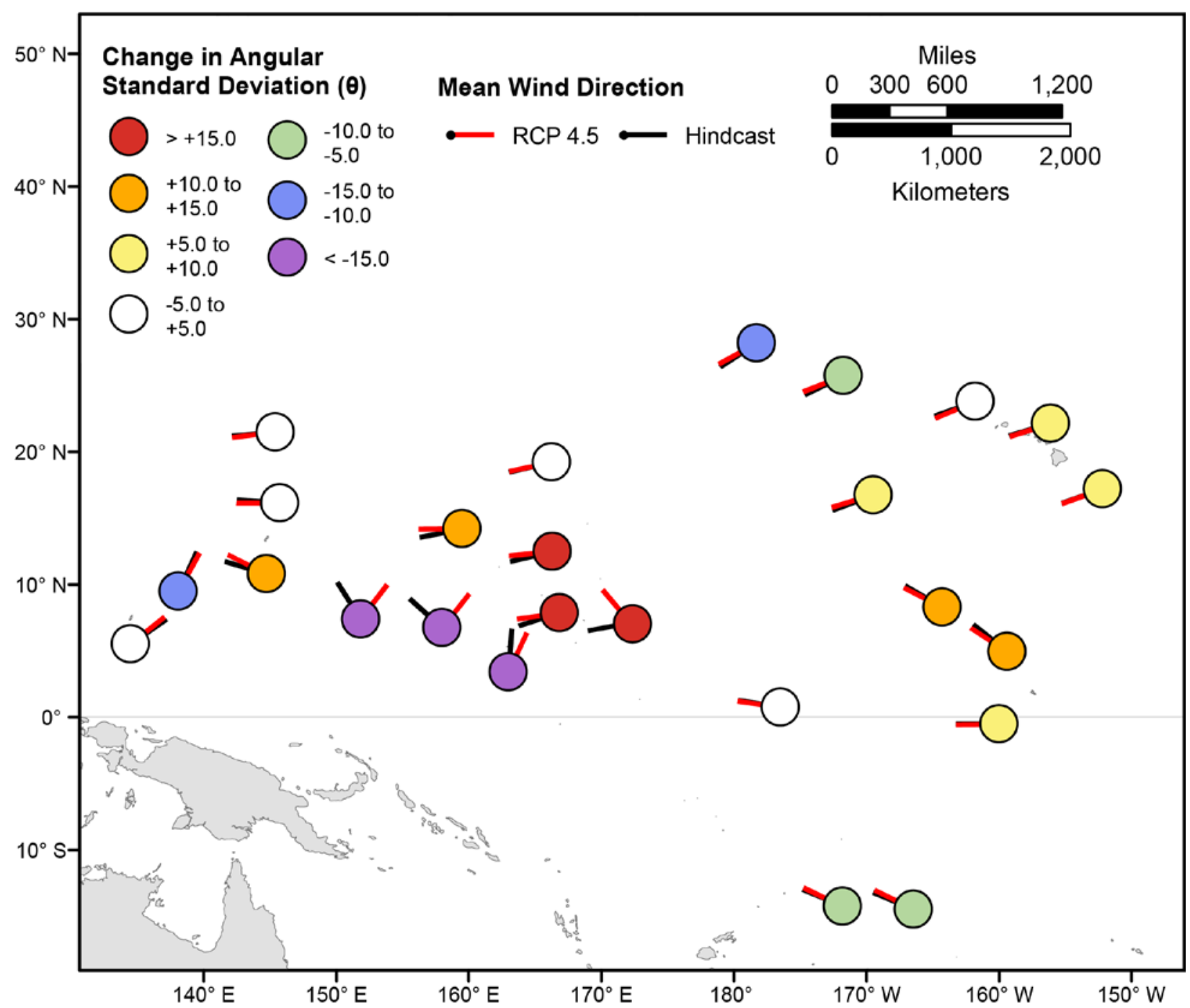

Figure 157. Map showing forecasted differences in the mean wind directions of the top 5 percent of wind speeds and the standard deviation of wind directions of the top 5 percent of wind speeds for the years 2081-2100 from hindcasted values during the September-November season under the RCP4.5 future climatic scenario. Mean wind directions at each point are indicated by lines radiating from the center of each point where RCP4.5 2081-2100 mean wind directions are red and 1976-2005 hindcasted mean wind directions are black. The colors correspond to the magnitude of change in modeled mean wind direction standard deviation during 2081-2100 from those hindcasted for 1976-2005. Angular standard deviation units are in degrees. Mean wind directions are "heading towards". 


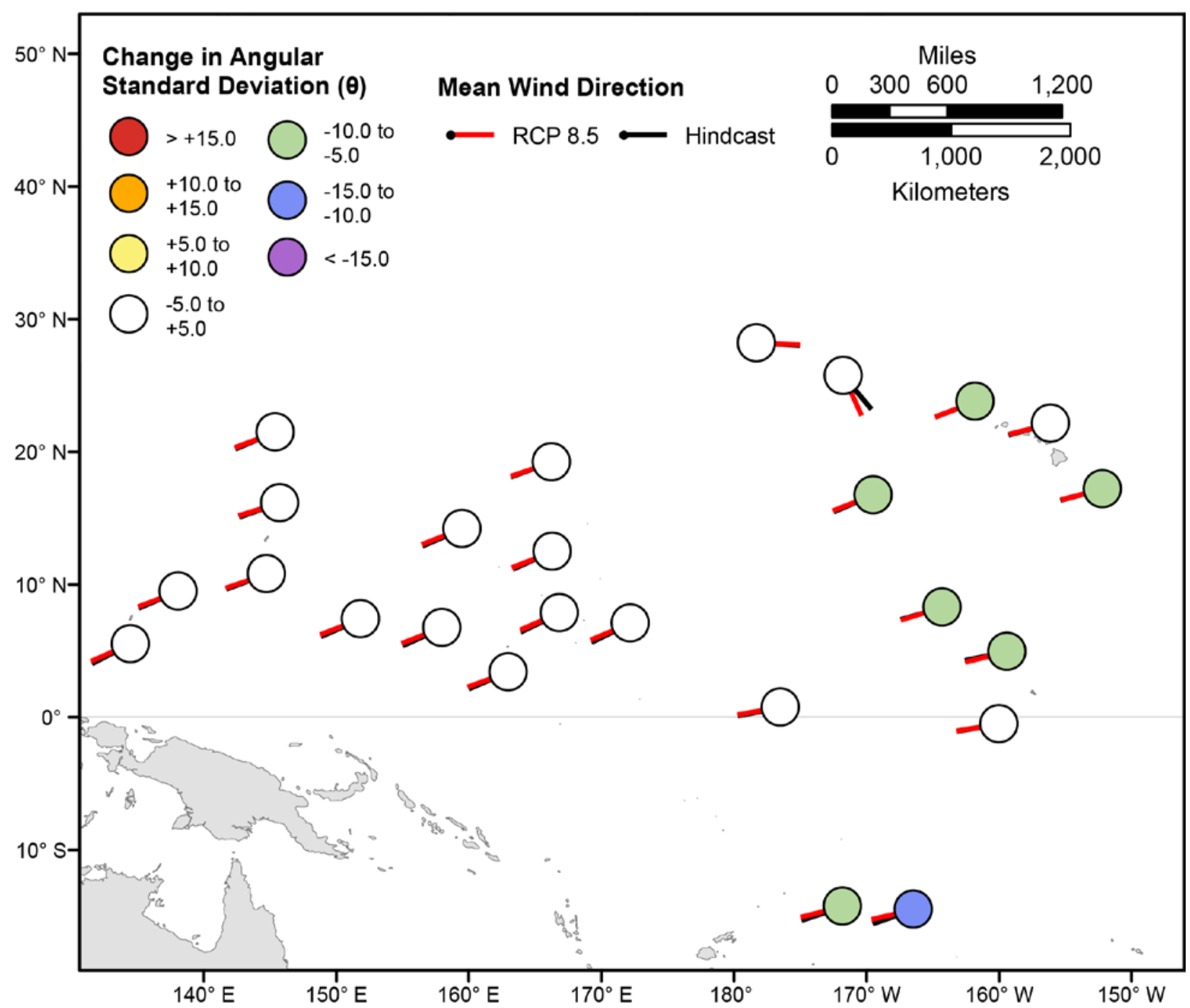

Figure 158. Map showing forecasted differences in mean wind directions and the standard deviation of wind directions for the years 2081-2100 from hindcasted values during the December-February season under the RCP8.5 future climatic scenario. Mean wind directions at each point are indicated by lines radiating from the center of each point where RCP8.5 2081-2100 mean wind directions are red and 1976-2005 hindcasted mean wind directions are black. The colors correspond to the magnitude of change in modeled mean wind direction standard deviation during 2081-2100 from those hindcasted for 19762005. Angular standard deviation units are in degrees. Mean wind directions are "heading towards". 


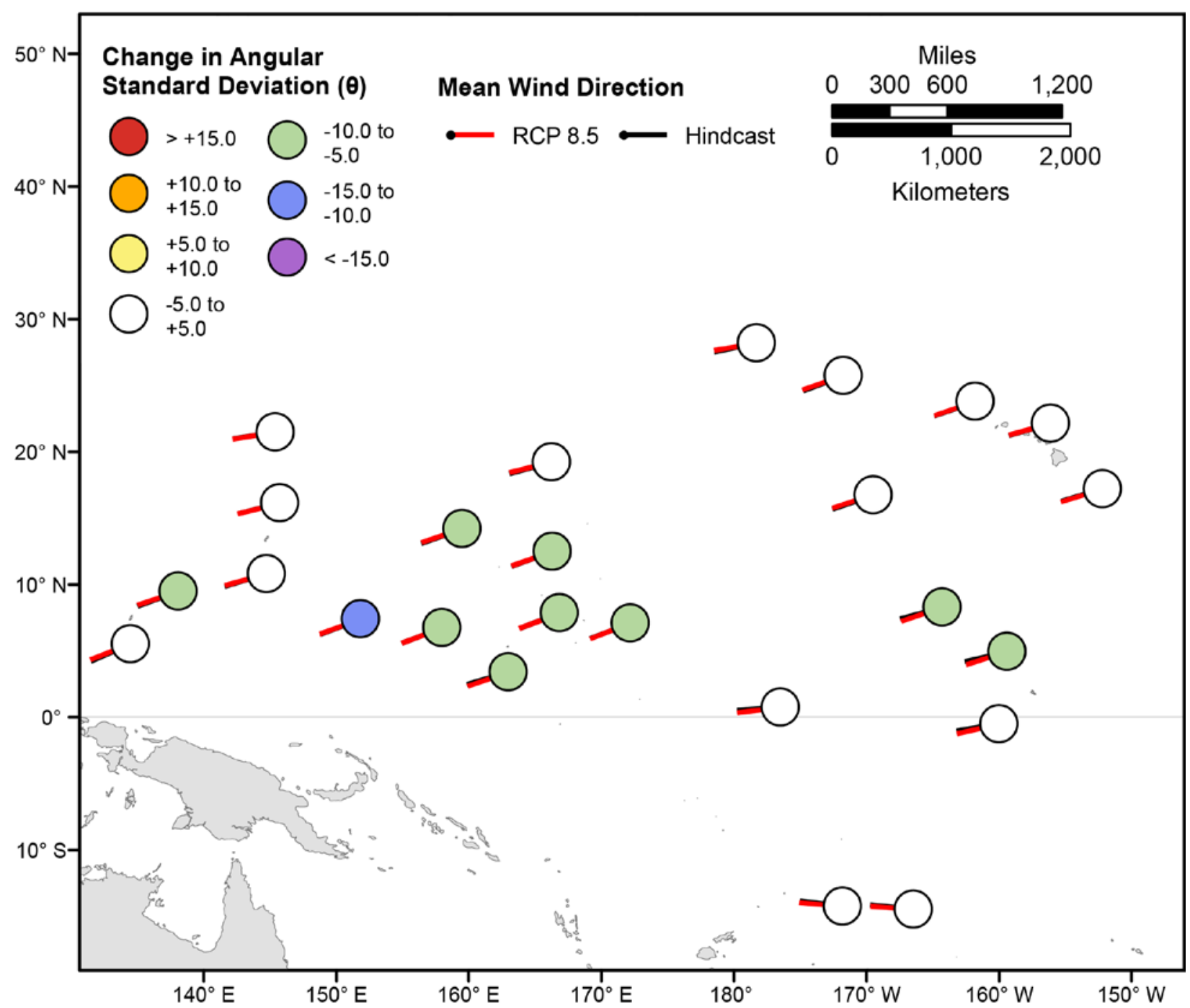

Figure 159. Map showing forecasted differences in mean wind directions and the standard deviation of wind directions for the years 2081-2100 from hindcasted values during the March-May season under the RCP8.5 future climatic scenario. Mean wind directions at each point are indicated by lines radiating from the center of each point where RCP8.5 2081-2100 mean wind directions are red and 1976-2005 hindcasted mean wind directions are black. The colors correspond to the magnitude of change in modeled mean wind direction standard deviation during 2081-2100 from those hindcasted for 19762005. Angular standard deviation units are in degrees. Mean wind directions are "heading towards". 


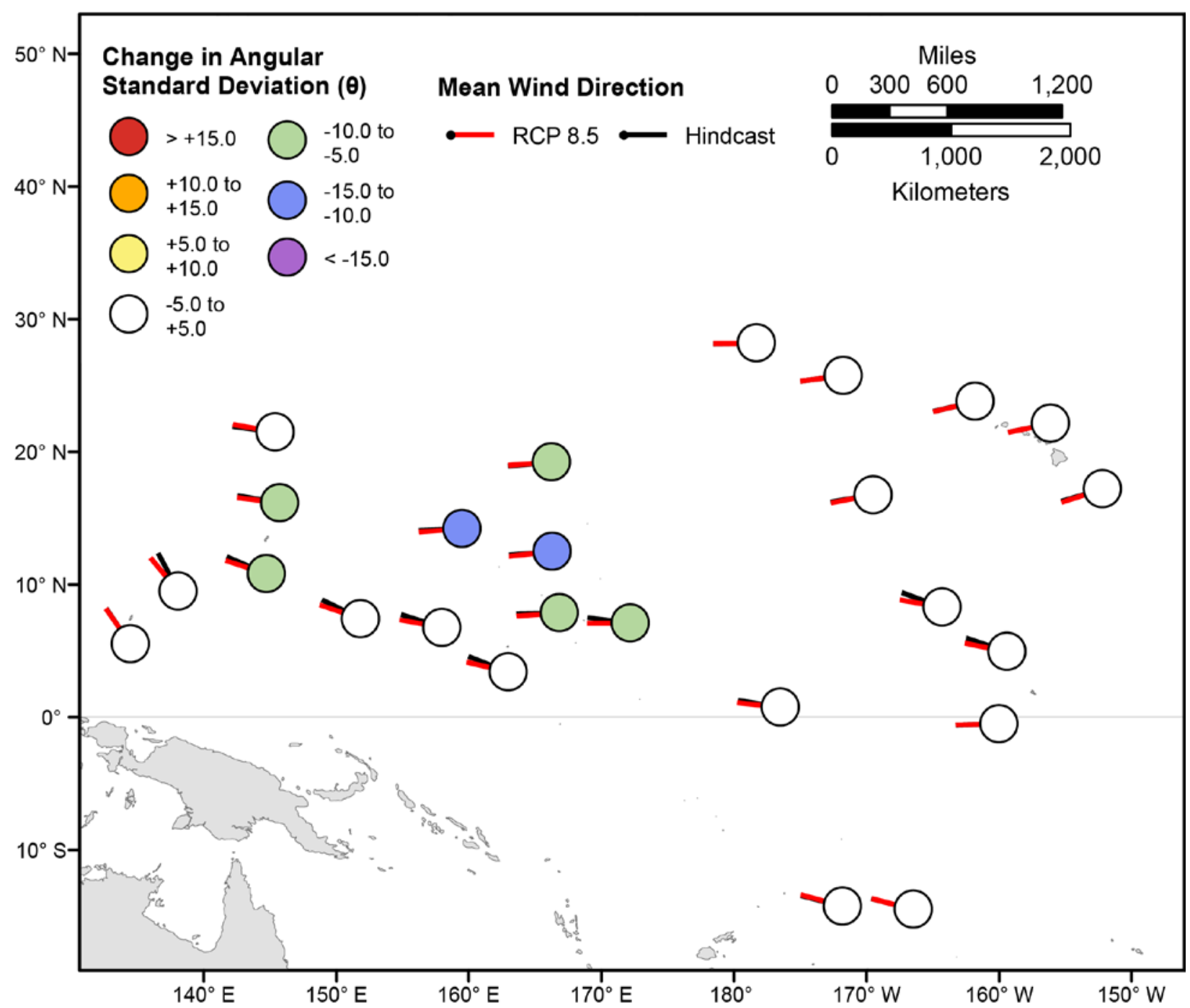

Figure 160. Map showing forecasted differences in mean wind directions and the standard deviation of wind directions for the years 2081-2100 from hindcasted values during the June-August season under the RCP8.5 future climatic scenario. Mean wind directions at each point are indicated by lines radiating from the center of each point where RCP8.5 2081-2100 mean wind directions are red and 1976-2005 hindcasted mean wind directions are black. The colors correspond to the magnitude of change in modeled mean wind direction standard deviation during 2081-2100 from those hindcasted for 19762005. Angular standard deviation units are in degrees. Mean wind directions are "heading towards". 


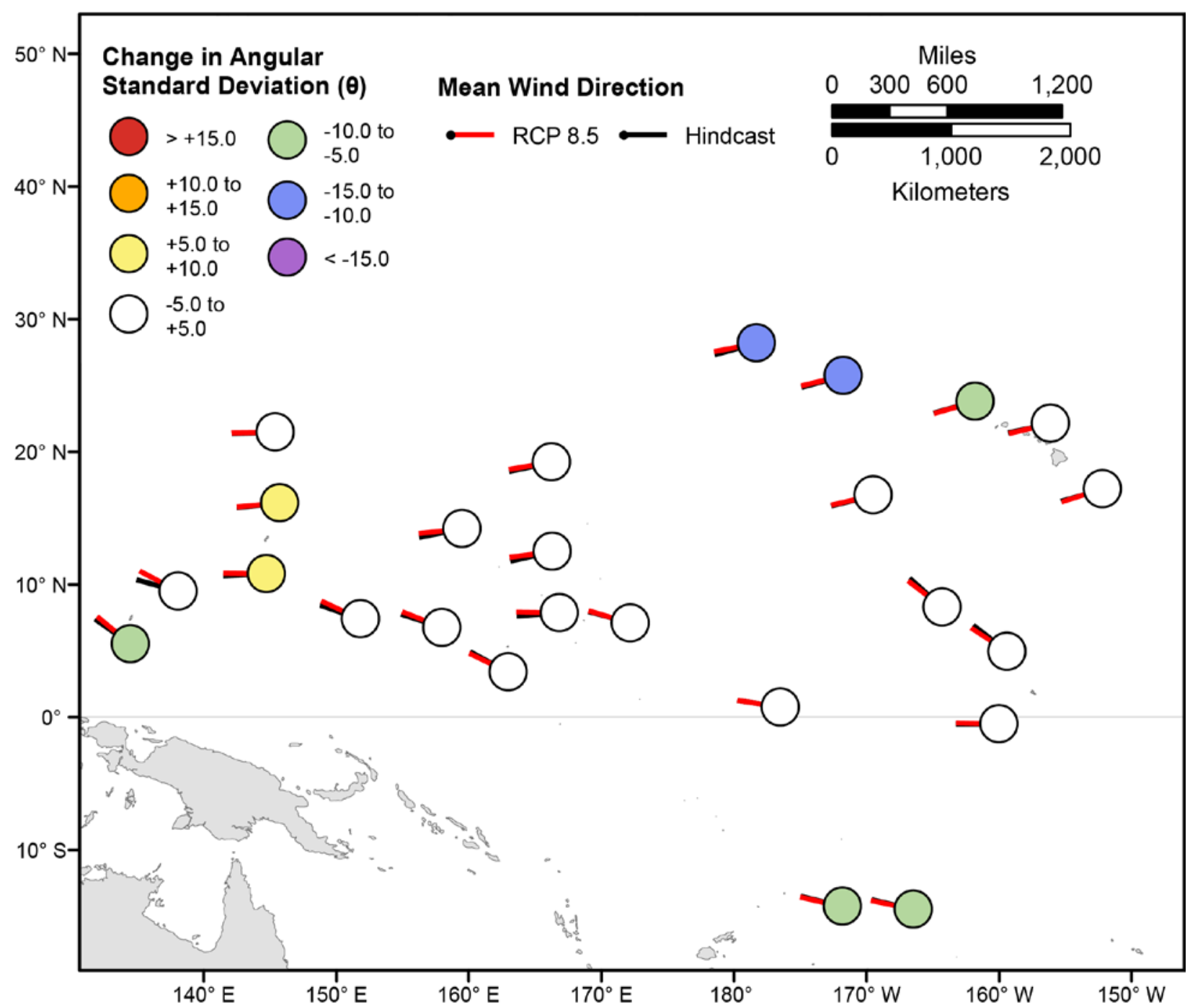

Figure 161. Map showing forecasted differences in mean wind directions and the standard deviation of wind directions for the years 2081-2100 from hindcasted values during the September-November season under the RCP8.5 future climatic scenario. Mean wind directions at each point are indicated by lines radiating from the center of each point where RCP8.5 2081-2100 mean wind directions are red and 1976-2005 hindcasted mean wind directions are black. The colors correspond to the magnitude of change in modeled mean wind direction standard deviation during 2081-2100 from those hindcasted for 1976-2005. Angular standard deviation units are in degrees. Mean wind directions are "heading towards". 


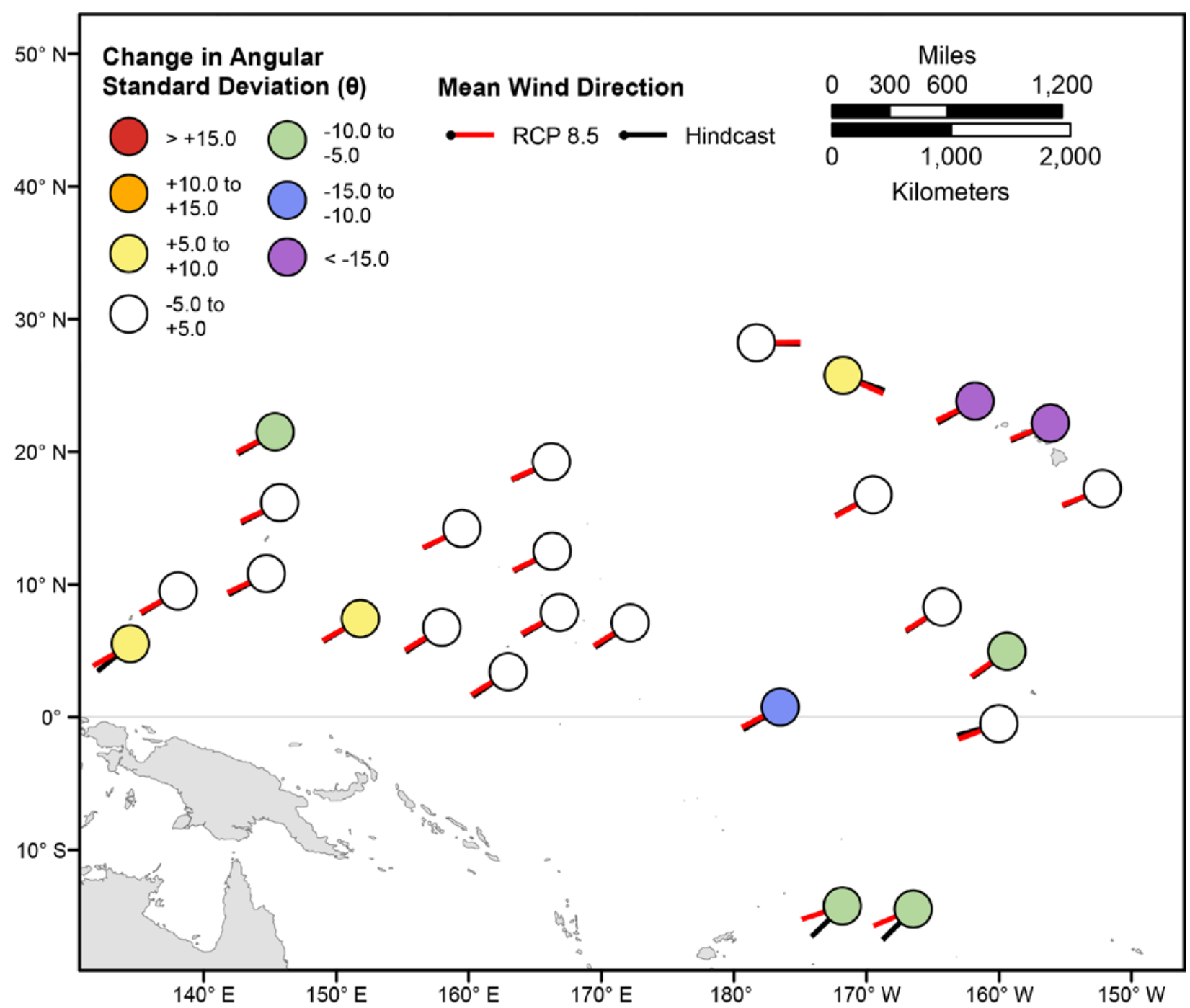

Figure 162. Map showing forecasted differences in the mean wind directions of the top 5 percent of wind speeds and the standard deviation of wind directions of the top 5 percent of wind speeds for the years 2081-2100 from hindcasted values during the December-February season under the RCP8.5 future climatic scenario. Mean wind directions at each point are indicated by lines radiating from the center of each point where RCP8.5 2081-2100 mean wind directions are red and 1976-2005 hindcasted mean wind directions are black. The colors correspond to the magnitude of change in modeled mean wind direction standard deviation during 2081-2100 from those hindcasted for 1976-2005. Angular standard deviation units are in degrees. Mean wind directions are "heading towards". 


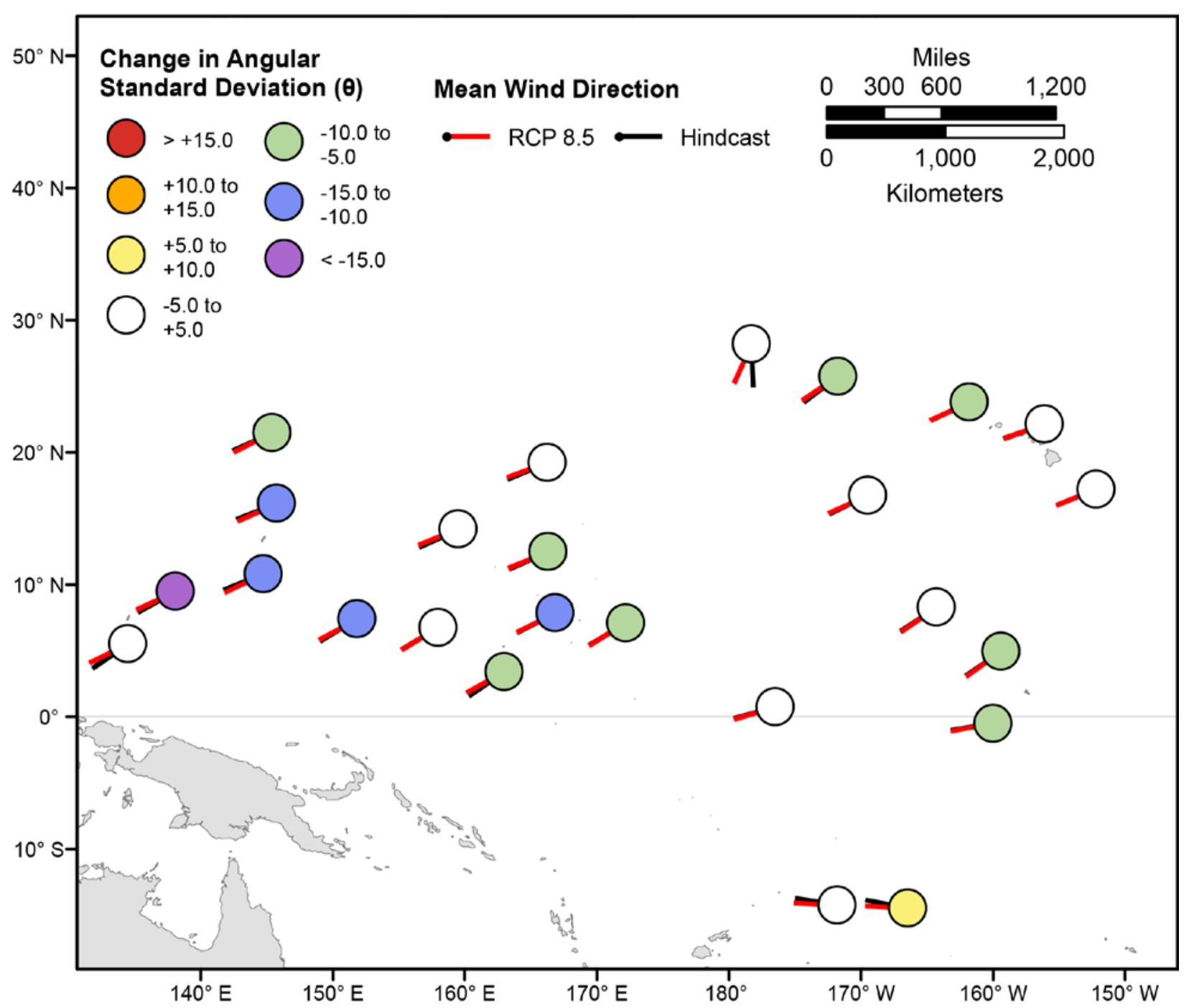

Figure 163. Map showing forecasted differences in the mean wind directions of the top 5 percent of wind speeds and the standard deviation of wind directions of the top 5 percent of wind speeds for the years 2081-2100 from hindcasted values during the March-May season under the RCP8.5 future climatic scenario. Mean wind directions at each point are indicated by lines radiating from the center of each point where RCP8.5 2081-2100 mean wind directions are red and 1976-2005 hindcasted mean wind directions are black. The colors correspond to the magnitude of change in modeled mean wind direction standard deviation during 2081-2100 from those hindcasted for 1976-2005. Angular standard deviation units are in degrees. Mean wind directions are "heading towards". 


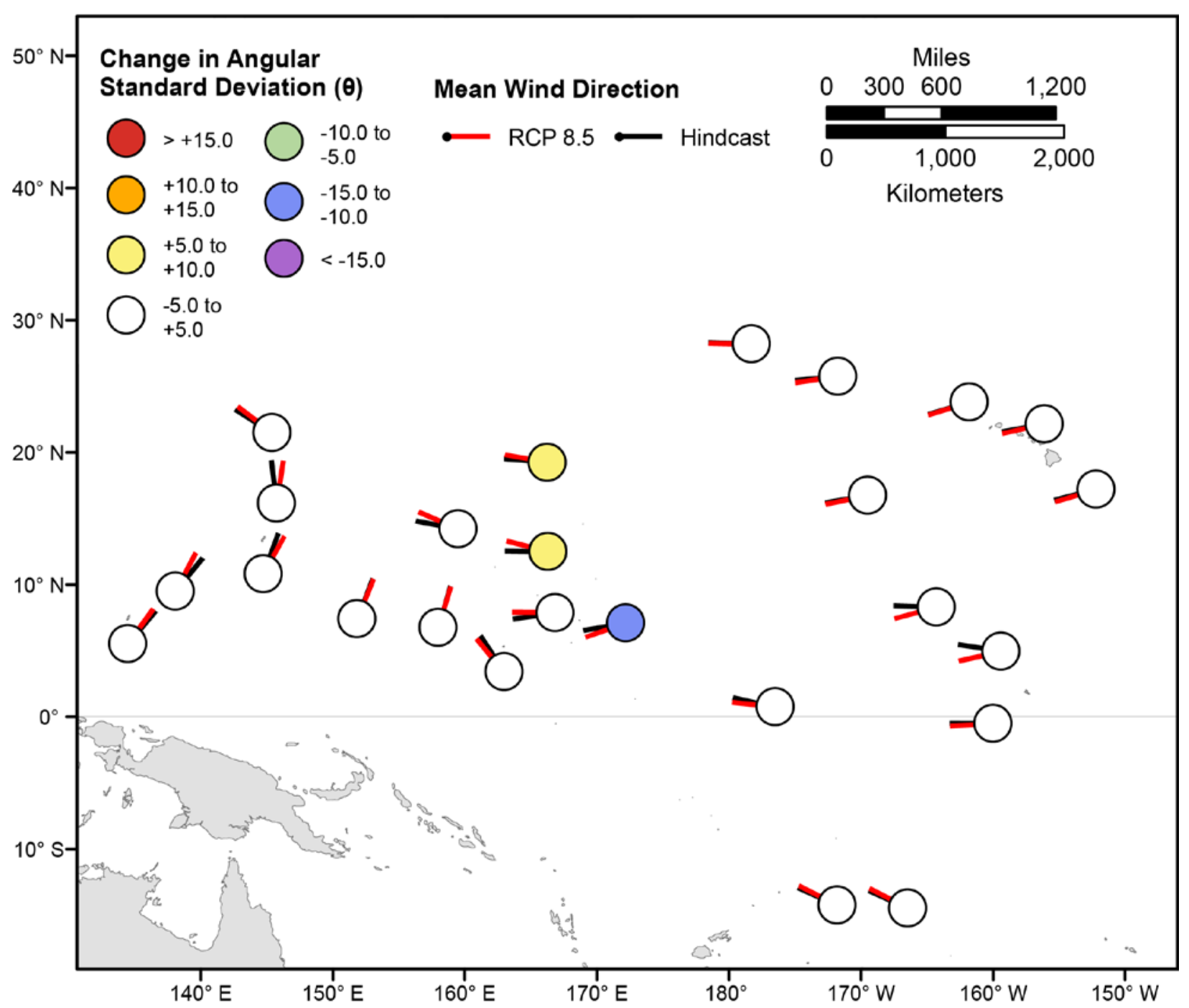

Figure 164. Map showing forecasted differences in the mean wind directions of the top 5 percent of wind speeds and the standard deviation of wind directions of the top 5 percent of wind speeds for the years 2081-2100 from hindcasted values during the June-August season under the RCP8.5 future climatic scenario. Mean wind directions at each point are indicated by lines radiating from the center of each point where RCP8.5 2081-2100 mean wind directions are red and 1976-2005 hindcasted mean wind directions are black. The colors correspond to the magnitude of change in modeled mean wind direction standard deviation during 2081-2100 from those hindcasted for 1976-2005. Angular standard deviation units are in degrees. Mean wind directions are "heading towards". 


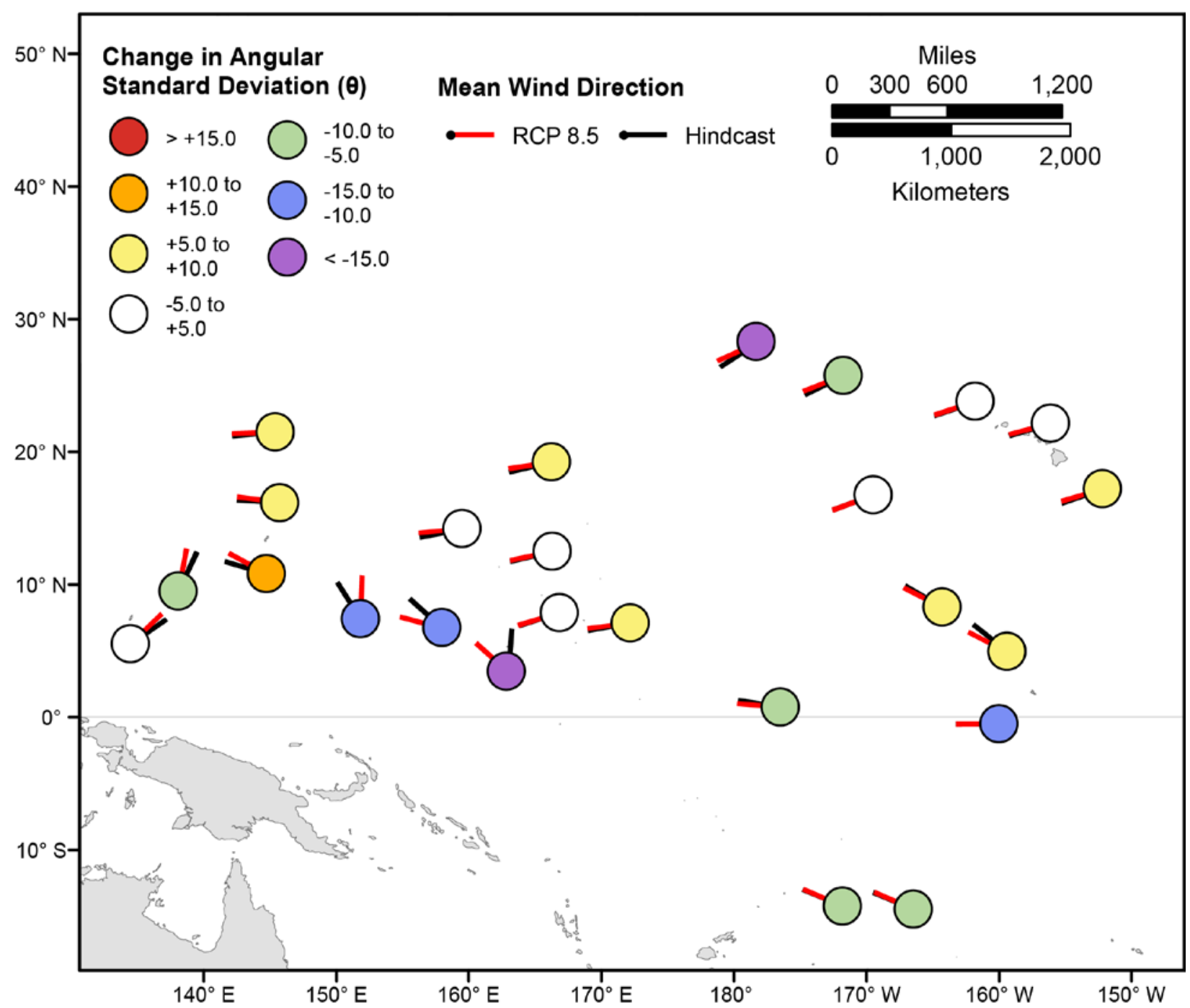

Figure 165. Map showing forecasted differences in the mean wind directions of the top 5 percent of wind speeds and the standard deviation of wind directions of the top 5 percent of wind speeds for the years 2081-2100 from hindcasted values during the September-November season under the RCP8.5 future climatic scenario. Mean wind directions at each point are indicated by lines radiating from the center of each point where RCP8.5 2081-2100 mean wind directions are red and 1976-2005 hindcasted mean wind directions are black. The colors correspond to the magnitude of change in modeled mean wind direction standard deviation during 2081-2100 from those hindcasted for 1976-2005. Angular standard deviation units are in degrees. Mean wind directions are "heading towards". 


\section{Appendix A. Wave Height, Wave Period, and Wave Direction Statistics}

Appendix A1. Table showing American Samoa monthly means and mean of the top 5 percent for significant wave height, peak wave period, and peak wave direction.

[Years: Hindcast $=1976-2005 ;$ RCP mid $=$ 2026-2045; RCP end $=$ 2081-2100. Wave directions are “coming from”]

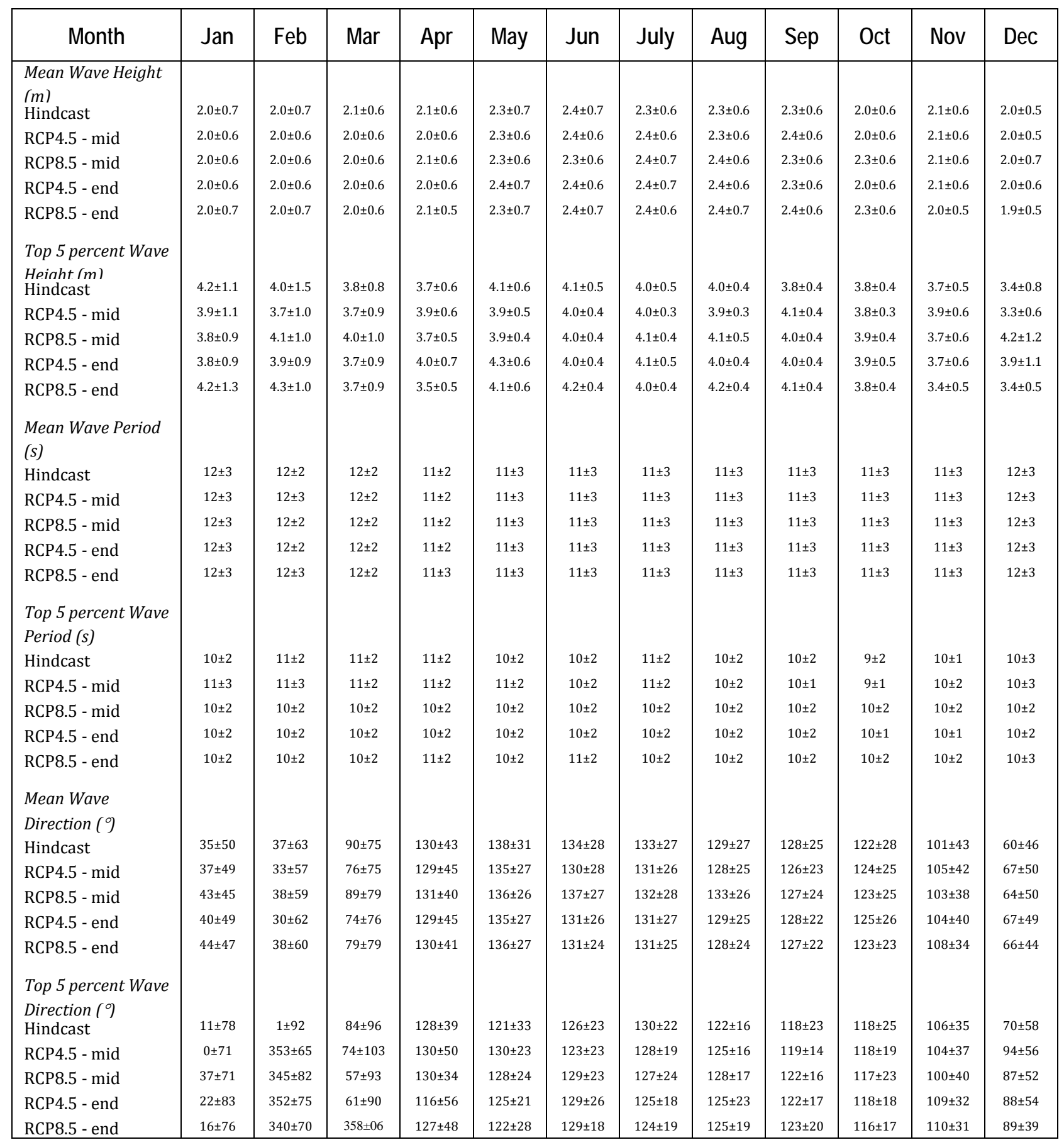


Appendix A2. Table showing return values of ensemble-average significant wave heights of hindcast and forecast scenarios, including lower and higher 95 percent confidence intervals, at the American Samoa location.

[Years: Hindcast $=1976-2005 ;$ RCP mid $=$ 2026-2045; RCP end $=2081-2100$. Wave height values are in meters]

\begin{tabular}{|c|c|c|c|c|c|c|c|c|c|c|c|c|c|c|c|}
\hline \multirow{2}{*}{ Scenario } & \multicolumn{3}{|c|}{ Hindcast } & \multicolumn{3}{c|}{ RCP4.5 - mid } & \multicolumn{3}{c|}{ RCP8.5 - mid } & \multicolumn{3}{c|}{ RCP4.5 - end } & \multicolumn{3}{c|}{ RCP8.5 - end } \\
\cline { 2 - 13 } & Low & $\boldsymbol{R}_{\mathbf{V}}$ & High & Low & $\boldsymbol{R}_{\mathbf{V}}$ & High & Low & $\boldsymbol{R}_{\mathbf{V}}$ & High & Low & $\boldsymbol{R}_{\mathbf{V}}$ & High & Low & $\boldsymbol{R}_{\mathbf{V}}$ & High \\
\hline 2-year & 5.92 & 6.86 & 8.56 & 5.67 & 6.69 & 8.76 & 5.91 & 6.91 & 8.90 & 5.78 & 6.94 & 9.50 & 5.90 & 6.79 & 8.47 \\
5-year & 6.50 & 8.27 & 12.03 & 6.10 & 7.94 & 12.42 & 6.32 & 8.07 & 12.16 & 6.15 & 8.18 & 13.59 & 6.30 & 7.83 & 11.26 \\
10-year & 6.97 & 9.64 & 16.02 & 6.43 & 9.10 & 16.61 & 6.62 & 9.09 & 15.68 & 6.41 & 9.27 & 18.18 & 6.58 & 8.74 & 14.21 \\
20-year & 7.46 & 11.32 & 21.77 & 6.74 & 10.48 & 22.60 & 6.90 & 10.24 & 20.47 & 6.66 & 10.51 & 24.65 & 6.85 & 9.78 & 18.16 \\
50-year & 8.15 & 14.16 & 33.45 & 7.16 & 12.72 & 34.68 & 7.25 & 12.03 & 29.55 & 6.95 & 12.44 & 37.44 & 7.20 & 11.37 & 25.52 \\
100-year & 8.71 & 16.89 & 46.89 & 7.47 & 14.79 & 48.48 & 7.50 & 13.59 & 39.35 & 7.15 & 14.14 & 51.80 & 7.45 & 12.76 & 33.32 \\
\hline
\end{tabular}


Appendix A3. Table showing Kauai monthly means and mean of the top 5 percent for significant wave height, peak wave period, and peak wave direction.

[Years: Hindcast $=1976-2005 ;$ RCP mid $=$ 2026-2045; RCP end $=$ 2081-2100. Wave directions are "coming from”]

\begin{tabular}{|c|c|c|c|c|c|c|c|c|c|c|c|c|}
\hline Month & Jan & Feb & Mar & Apr & May & Jun & July & Aug & Sep & Oct & Nov & Dec \\
\hline \multicolumn{13}{|c|}{$\begin{array}{l}\text { Mean Wave Height } \\
\text { (m) }\end{array}$} \\
\hline Hindcast & $3.5 \pm 1.0$ & $3.4 \pm 1.0$ & $2.9 \pm 0.8$ & $2.5 \pm 0.7$ & $2.1 \pm 0.6$ & $2.1 \pm 0.5$ & $2.1 \pm 0.5$ & $2.1 \pm 0.5$ & $2.2 \pm 0.6$ & $2.6 \pm 0.7$ & $3.0 \pm 0.8$ & $3.3 \pm 0.9$ \\
\hline RCP4.5 - mid & $3.5 \pm 1.0$ & $3.4 \pm 1.0$ & $3.0 \pm 0.8$ & $2.5 \pm 0.7$ & $2.1 \pm 0.6$ & $2.0 \pm 0.5$ & $2.1 \pm 0.5$ & $2.0 \pm 0.5$ & $2.0 \pm 0.6$ & $2.5 \pm 0.7$ & $3.0 \pm 0.8$ & $3.3 \pm 0.9$ \\
\hline RCP8.5 - mid & $3.4 \pm 1.0$ & $3.3 \pm 1.0$ & $2.9 \pm 0.8$ & $2.5 \pm 0.7$ & $2.1 \pm 0.5$ & $2.0 \pm 0.5$ & $2.0 \pm 0.5$ & $2.1 \pm 0.5$ & $2.1 \pm 0.6$ & $2.5 \pm 0.8$ & $3.0 \pm 0.8$ & $3.3 \pm 0.9$ \\
\hline RCP4.5 - end & $3.4 \pm 1.0$ & $3.4 \pm 1.0$ & $3.0 \pm 0.8$ & $2.5 \pm 0.7$ & $2.1 \pm 0.5$ & $2.0 \pm 0.5$ & $2.0 \pm 0.5$ & $2.0 \pm 0.6$ & $2.1 \pm 0.6$ & $2.5 \pm 0.7$ & $3.0 \pm 0.8$ & $3.3 \pm 0.9$ \\
\hline RCP8.5 - end & $3.3 \pm 1.0$ & $3.2 \pm 1.0$ & $2.8 \pm 0.8$ & $2.4 \pm 0.6$ & $2.0 \pm 0.5$ & $1.9 \pm 0.4$ & $2.0 \pm 0.5$ & $1.9 \pm 0.5$ & $2.0 \pm 0.5$ & $2.4 \pm 0.7$ & $2.8 \pm 0.8$ & $3.2 \pm 0.9$ \\
\hline \multicolumn{13}{|c|}{$\begin{array}{l}\text { Top } 5 \text { percent Wave } \\
\text { Height }(\mathrm{m})\end{array}$} \\
\hline Hindcast & $6.1 \pm 0.7$ & $6.0 \pm 0.7$ & $5.0 \pm 0.5$ & $4.2 \pm 0.5$ & $3.5 \pm 0.3$ & $3.4 \pm 0.4$ & $3.3 \pm 0.4$ & $3.4 \pm 0.5$ & $3.6 \pm 0.3$ & $4.5 \pm 0.5$ & $5.1 \pm 0.6$ & $5.5 \pm 0.6$ \\
\hline RCP4.5 - mid & $6.2 \pm 0.8$ & $5.9 \pm 0.6$ & $5.0 \pm 0.6$ & $4.2 \pm 0.3$ & $3.6 \pm 0.6$ & $3.1 \pm 0.2$ & $3.3 \pm 0.5$ & $3.4 \pm 0.4$ & $3.5 \pm 0.5$ & $4.5 \pm 0.7$ & $5.0 \pm 0.4$ & $5.8 \pm 0.7$ \\
\hline RCP8.5 - mid & $5.9 \pm 0.7$ & $5.8 \pm 0.6$ & $5.0 \pm 0.6$ & $4.1 \pm 0.4$ & $3.3 \pm 0.3$ & $3.2 \pm 0.3$ & $3.2 \pm 0.5$ & $3.4 \pm 0.4$ & $3.5 \pm 0.4$ & $4.6 \pm 0.6$ & $5.0 \pm 0.5$ & $5.6 \pm 0.5$ \\
\hline RCP4.5 - end & $5.9 \pm 0.6$ & $6.1 \pm 0.7$ & $5.0 \pm 0.5$ & $4.2 \pm 0.5$ & $3.4 \pm 0.3$ & $3.1 \pm 0.3$ & $3.3 \pm 0.7$ & $3.9 \pm 0.9$ & $3.6 \pm 0.6$ & $4.4 \pm 0.7$ & $4.8 \pm 0.4$ & $5.5 \pm 0.6$ \\
\hline \multicolumn{13}{|c|}{$\begin{array}{l}\text { Mean Wave Period } \\
\text { (s) }\end{array}$} \\
\hline Hindcast & $13 \pm 2$ & $12 \pm 2$ & $12 \pm 2$ & $10 \pm 3$ & $10 \pm 3$ & $10 \pm 3$ & $10 \pm 3$ & $10 \pm 3$ & $10 \pm 3$ & $11 \pm 3$ & $12 \pm 2$ & $12 \pm 2$ \\
\hline RCP4.5 - mid & $13 \pm 2$ & $13 \pm 2$ & $12 \pm 2$ & $11 \pm 3$ & $11 \pm 3$ & $10 \pm 3$ & $9 \pm 3$ & $10 \pm 3$ & $10 \pm 3$ & $11 \pm 3$ & $12 \pm 2$ & $12 \pm 2$ \\
\hline RCP8.5 - mid & $13 \pm 2$ & $12 \pm 2$ & $12 \pm 2$ & $11 \pm 3$ & $10 \pm 3$ & $10 \pm 3$ & $10 \pm 3$ & $10 \pm 3$ & $10 \pm 3$ & $11 \pm 3$ & $12 \pm 2$ & $12 \pm 2$ \\
\hline RCP4.5 - end & $13 \pm 2$ & $13 \pm 2$ & $12 \pm 2$ & $11 \pm 3$ & $11 \pm 3$ & $10 \pm 3$ & $9.8 \pm 3$ & $9.9 \pm 3$ & $10 \pm 3$ & $11 \pm 3$ & $12 \pm 2$ & $12 \pm 2$ \\
\hline \multicolumn{13}{|c|}{$\begin{array}{l}\text { Top } 5 \text { percent Wave } \\
\text { Period (s) }\end{array}$} \\
\hline Hindcast & $15 \pm 2$ & $15 \pm 2$ & $13 \pm 3$ & $11 \pm 3$ & $10 \pm 3$ & $8 \pm 1$ & $9 \pm 2$ & $9 \pm 2$ & $10 \pm 2$ & $11 \pm 2$ & $13 \pm 3$ & $14 \pm 2$ \\
\hline RCP4.5 - mid & $15 \pm 2$ & $15 \pm 2$ & $14 \pm 3$ & $11 \pm 3$ & $10 \pm 3$ & $8 \pm 2$ & $9 \pm 2$ & $10 \pm 2$ & $9 \pm 2$ & $11 \pm 2$ & $13 \pm 3$ & $15 \pm 2$ \\
\hline RCP8.5 - mid & $15 \pm 2$ & $15 \pm 2$ & $13 \pm 3$ & $11 \pm 3$ & $9 \pm 3$ & $9 \pm 3$ & $9 \pm 2$ & $9 \pm 2$ & $10 \pm 2$ & $11 \pm 2$ & $14 \pm 3$ & $14 \pm 2$ \\
\hline RCP4.5 - end & $15 \pm 2$ & $15 \pm 2$ & $13 \pm 2$ & $12 \pm 3$ & $10 \pm 3$ & $9 \pm 3$ & $9 \pm 2$ & $10 \pm 2$ & $9 \pm 1$ & $11 \pm 2$ & $13 \pm 3$ & $15 \pm 2$ \\
\hline $\begin{array}{l}\text { RCP8.5 - end } \\
\text { Mean Wave } \\
\text { Direction ( } 9\end{array}$ & $16 \pm 2$ & $15 \pm 2$ & $13 \pm 3$ & $12 \pm 3$ & $10 \pm 3$ & $9 \pm 3$ & $9 \pm 2$ & $10 \pm 3$ & $10 \pm 2$ & $11 \pm 2$ & $12 \pm 3$ & $15 \pm 3$ \\
\hline Hindcast & $336 \pm 45$ & $335 \pm 47$ & $7 \pm 55$ & $45 \pm 50$ & $75 \pm 43$ & $91 \pm 25$ & $93 \pm 20$ & $92 \pm 29$ & $77 \pm 41$ & $51 \pm 42$ & $22 \pm 47$ & $356 \pm 5 c$ \\
\hline RCP4.5 - mid & $334 \pm 43$ & $340 \pm 48$ & $6 \pm 54$ & $49 \pm 47$ & $74 \pm 47$ & $92 \pm 27$ & $92 \pm 19$ & $93 \pm 28$ & $83 \pm 40$ & $51 \pm 46$ & $24 \pm 46$ & $354 \pm 47$ \\
\hline RCP8.5 - mid & $339 \pm 45$ & $339 \pm 45$ & $2 \pm 52$ & $42 \pm 49$ & $74 \pm 45$ & $92 \pm 29$ & $94 \pm 24$ & $95 \pm 31$ & $83 \pm 44$ & $49 \pm 45$ & $21 \pm 44$ & $357 \pm 4 \varepsilon$ \\
\hline RCP4.5 - end & $332 \pm 42$ & $336 \pm 47$ & $6 \pm 51$ & $36 \pm 50$ & $71 \pm 46$ & $94 \pm 28$ & $94 \pm 23$ & $96 \pm 29$ & $82 \pm 42$ & $54 \pm 43$ & $25 \pm 43$ & $356 \pm 4 c$ \\
\hline $\begin{array}{l}\text { RCP8.5 - end } \\
\text { Top } 5 \text { percent } \\
\text { Direction ( }{ }^{\circ} \text { ) }\end{array}$ & $336 \pm 42$ & $335 \pm 42$ & $1 \pm 52$ & $46 \pm 48$ & $73 \pm 49$ & $96 \pm 26$ & $96 \pm 22$ & $98 \pm 30$ & $86 \pm 41$ & $55 \pm 41$ & $31 \pm 43$ & $356 \pm 48$ \\
\hline Hindcast & $316 \pm 23$ & $317 \pm 25$ & $356 \pm 55$ & $37 \pm 46$ & $68 \pm 29$ & $82 \pm 14$ & $86 \pm 16$ & $88 \pm 36$ & $71 \pm 39$ & $44 \pm 54$ & $3 \pm 51$ & $328 \pm 43$ \\
\hline RCP4.5 - mid & $316 \pm 23$ & $321 \pm 33$ & $339 \pm 47$ & $52 \pm 41$ & $68 \pm 29$ & $81 \pm 16$ & $89 \pm 24$ & $87 \pm 35$ & $82 \pm 44$ & $55 \pm 51$ & $7 \pm 51$ & $328 \pm 34$ \\
\hline RCP8.5 - mid & $321 \pm 31$ & $323 \pm 34$ & $342 \pm 53$ & $40 \pm 44$ & $68 \pm 23$ & $80 \pm 12$ & $87 \pm 21$ & $91 \pm 38$ & $80 \pm 52$ & $53 \pm 43$ & $0 \pm 40$ & $335 \pm 47$ \\
\hline RCP4.5 - end & $314 \pm 17$ & $316 \pm 30$ & $343 \pm 45$ & $21 \pm 48$ & $72 \pm 26$ & $90 \pm 19$ & $85 \pm 23$ & $105 \pm 57$ & $82 \pm 48$ & $48 \pm 54$ & $18 \pm 49$ & $331 \pm 3 c$ \\
\hline RCP8.5 - end & $318 \pm 24$ & $318 \pm 26$ & $343 \pm 47$ & $33 \pm 42$ & $63 \pm 35$ & $87 \pm 17$ & $91 \pm 28$ & $91 \pm 39$ & $101 \pm 68$ & $58 \pm 53$ & $21 \pm 47$ & $345 \pm 51$ \\
\hline
\end{tabular}


Appendix A4. Table showing return values of ensemble-average significant wave heights of hindcast and forecast scenarios, including lower and higher 95 percent confidence intervals, at the Kauai location.

[Years: Hindcast $=1976-2005 ;$ RCP mid $=$ 2026-2045; RCP end $=2081-2100$. Wave height values are in meters]

\begin{tabular}{|c|c|c|c|c|c|c|c|c|c|c|c|c|c|c|c|}
\hline \multirow{2}{*}{ Scenario } & \multicolumn{3}{|c|}{ Hindcast } & \multicolumn{3}{c|}{ RCP4.5 - mid } & \multicolumn{3}{c|}{ RCP8.5 - mid } & \multicolumn{3}{|c|}{ RCP4.5 - end } & \multicolumn{3}{c|}{ RCP8.5 - end } \\
\cline { 2 - 13 } & Low & $\boldsymbol{R}_{\boldsymbol{V}}$ & High & Low & $\boldsymbol{R}_{\boldsymbol{V}}$ & High & Low & $\boldsymbol{R}_{\boldsymbol{V}}$ & High & Low & $\boldsymbol{R}_{\boldsymbol{V}}$ & High & Low & $\boldsymbol{R}_{\boldsymbol{V}}$ & High \\
\hline 2-year & 7.51 & 8.06 & 8.88 & 7.41 & 8.17 & 9.53 & 7.26 & 7.89 & 8.94 & 7.22 & 7.81 & 8.83 & 7.27 & 7.89 & 8.88 \\
5-year & 7.83 & 8.65 & 9.99 & 7.70 & 8.89 & 11.26 & 7.50 & 8.42 & 10.08 & 7.47 & 8.38 & 10.17 & 7.51 & 8.38 & 9.87 \\
10-year & 8.02 & 9.07 & 10.89 & 7.89 & 9.42 & 12.79 & 7.64 & 8.76 & 10.95 & 7.62 & 8.80 & 11.35 & 7.64 & 8.69 & 10.58 \\
20-year & 8.19 & 9.46 & 11.82 & 8.04 & 9.94 & 14.52 & 7.74 & 9.07 & 11.83 & 7.75 & 9.23 & 12.70 & 7.74 & 8.95 & 11.26 \\
50-year & 8.37 & 9.95 & 13.12 & 8.21 & 10.61 & 17.20 & 7.85 & 9.43 & 13.00 & 7.90 & 9.78 & 14.79 & 7.84 & 9.23 & 12.10 \\
100-year & 8.49 & 10.29 & 14.16 & 8.32 & 11.11 & 19.55 & 7.91 & 9.66 & 13.91 & 7.99 & 10.19 & 16.64 & 7.89 & 9.41 & 12.70 \\
\hline
\end{tabular}


Appendix A5. Table showing Big Island of Hawaii monthly means and mean of the top 5 percent for significant wave height, peak wave period, and peak wave direction.

[Years: Hindcast $=1976-2005 ;$ RCP mid $=$ 2026-2045; RCP end $=$ 2081-2100. Wave directions are "coming from”]

\begin{tabular}{|c|c|c|c|c|c|c|c|c|c|c|c|c|}
\hline Month & Jan & Feb & Mar & Apr & May & Jun & July & Aug & Sep & Oct & Nov & Dec \\
\hline \multicolumn{13}{|c|}{$\begin{array}{l}\text { Mean Wave Height } \\
\text { (m) }\end{array}$} \\
\hline Hindcast & $2.8 \pm 0.7$ & $2.7 \pm 0.7$ & $2.6 \pm 0.7$ & $2.4 \pm 0.6$ & $2.2 \pm 0.5$ & $2.2 \pm 0.5$ & $2.2 \pm 0.5$ & $2.1 \pm 0.5$ & $2.1 \pm 0.6$ & $2.4 \pm 0.7$ & $2.8 \pm 0.8$ & $2.9 \pm 0.8$ \\
\hline RCP4.5 - mid & $2.8 \pm 0.7$ & $2.8 \pm 0.7$ & $2.7 \pm 0.7$ & $2.5 \pm 0.7$ & $2.2 \pm 0.5$ & $2.2 \pm 0.4$ & $2.2 \pm 0.5$ & $2.1 \pm 0.5$ & $2.0 \pm 0.5$ & $2.3 \pm 0.7$ & $2.8 \pm 0.8$ & $2.9 \pm 0.7$ \\
\hline RCP8.5 - mid & $2.8 \pm 0.7$ & $2.7 \pm 0.7$ & $2.6 \pm 0.7$ & $2.4 \pm 0.6$ & $2.1 \pm 0.5$ & $2.1 \pm 0.4$ & $2.2 \pm 0.4$ & $2.1 \pm 0.5$ & $2.0 \pm 0.5$ & $2.3 \pm 0.7$ & $2.7 \pm 0.7$ & $2.9 \pm 0.8$ \\
\hline RCP4.5 - end & $2.7 \pm 0.6$ & $2.7 \pm 0.7$ & $2.6 \pm 0.7$ & $2.4 \pm 0.6$ & $2.2 \pm 0.5$ & $2.2 \pm 0.5$ & $2.2 \pm 0.5$ & $2.1 \pm 0.6$ & $2.1 \pm 0.5$ & $2.3 \pm 0.6$ & $2.7 \pm 0.8$ & $2.9 \pm 0.7$ \\
\hline RCP8.5 - end & $2.7 \pm 0.7$ & $2.6 \pm 0.6$ & $2.5 \pm 0.6$ & $2.3 \pm 0.6$ & $2.1 \pm 0.4$ & $2.1 \pm 0.4$ & $2.2 \pm 0.5$ & $2.0 \pm 0.5$ & $2.0 \pm 0.5$ & $2.2 \pm 0.6$ & $2.6 \pm 0.7$ & $2.8 \pm 0.8$ \\
\hline \multicolumn{13}{|c|}{$\begin{array}{l}\text { Top } 5 \text { percent Wave } \\
\text { Height }(\mathrm{m})\end{array}$} \\
\hline Hindcast & $4.6 \pm 0.5$ & $4.5 \pm 0.5$ & $4.5 \pm 0.4$ & $3.9 \pm 0.3$ & $3.4 \pm 0.3$ & $3.4 \pm 0.3$ & $3.5 \pm 0.4$ & $3.5 \pm 0.6$ & $3.7 \pm 0.6$ & $4.4 \pm 0.5$ & $4.9 \pm 0.5$ & $4.7 \pm 0.4$ \\
\hline RCP4.5 - mid & $4.5 \pm 0.5$ & $4.7 \pm 0.6$ & $4.3 \pm 0.3$ & $4.1 \pm 0.3$ & $3.4 \pm 0.4$ & $3.2 \pm 0.3$ & $3.4 \pm 0.5$ & $3.4 \pm 0.5$ & $3.4 \pm 0.5$ & $4.2 \pm 0.7$ & $4.8 \pm 0.4$ & $4.7 \pm 0.5$ \\
\hline RCP8.5 - mid & $4.7 \pm 0.5$ & $4.6 \pm 0.5$ & $4.3 \pm 0.5$ & $3.9 \pm 0.4$ & $3.3 \pm 0.2$ & $3.1 \pm 0.3$ & $3.3 \pm 0.4$ & $3.5 \pm 0.5$ & $3.4 \pm 0.6$ & $4.2 \pm 0.5$ & $4.5 \pm 0.4$ & $5.0 \pm 0.6$ \\
\hline RCP4.5 - end & $4.4 \pm 0.5$ & $4.5 \pm 0.5$ & $4.3 \pm 0.4$ & $3.9 \pm 0.3$ & $3.4 \pm 0.3$ & $3.2 \pm 0.3$ & $3.6 \pm 0.8$ & $3.8 \pm 1.0$ & $3.5 \pm 0.6$ & $4.1 \pm 0.7$ & $4.8 \pm 0.5$ & $4.9 \pm 0.6$ \\
\hline \multicolumn{13}{|c|}{$\begin{array}{l}\text { Mean Wave Period } \\
\text { (s) }\end{array}$} \\
\hline Hindcast & $12 \pm 3$ & $12 \pm 3$ & $11 \pm 3$ & $10 \pm 3$ & $10 \pm 3$ & $10 \pm 3$ & $10 \pm 3$ & $10 \pm 3$ & $10 \pm 3$ & $10 \pm 3$ & $11 \pm 3$ & $12 \pm 3$ \\
\hline RCP4.5 - mid & $12 \pm 3$ & $12 \pm 3$ & $11 \pm 3$ & $10 \pm 3$ & $10 \pm 3$ & $10 \pm 3$ & $9 \pm 3$ & $10 \pm 3$ & $10 \pm 3$ & $11 \pm 3$ & $11 \pm 3$ & $12 \pm 3$ \\
\hline RCP8.5 - mid & $12 \pm 3$ & $12 \pm 3$ & $11 \pm 3$ & $10 \pm 3$ & $10 \pm 3$ & $10 \pm 3$ & $10 \pm 3$ & $10 \pm 3$ & $10 \pm 3$ & $11 \pm 3$ & $11 \pm 3$ & $12 \pm 3$ \\
\hline RCP4.5 - end & $12 \pm 3$ & $12 \pm 3$ & $11 \pm 3$ & $11 \pm 3$ & $10 \pm 3$ & $9.9 \pm 3$ & $9.7 \pm 3$ & $9.7 \pm 3$ & $10 \pm 3$ & $11 \pm 3$ & $11 \pm 3$ & $12 \pm 3$ \\
\hline \multicolumn{12}{|c|}{$\begin{array}{l}\text { Top } 5 \text { percent Wave } \\
\text { Period (s) }\end{array}$} & $12 \pm 3$ \\
\hline Hindcast & $12 \pm 3$ & $12 \pm 3$ & $10 \pm 2$ & $10 \pm 2$ & $9 \pm 2$ & $9 \pm 1$ & $9 \pm 2$ & $9 \pm 1$ & $9 \pm 1$ & $10 \pm 1$ & $11 \pm 2$ & $11 \pm 2$ \\
\hline RCP4.5 - mid & $13 \pm 3$ & $11 \pm 3$ & $10 \pm 2$ & $10 \pm 1$ & $10 \pm 2$ & $9 \pm 2$ & $9 \pm 1$ & $10 \pm 2$ & $9 \pm 1$ & $10 \pm 1$ & $11 \pm 2$ & $12 \pm 3$ \\
\hline RCP8.5 - mid & $12 \pm 3$ & $11 \pm 3$ & $11 \pm 2$ & $10 \pm 2$ & $9 \pm 2$ & $9 \pm 2$ & $9 \pm 2$ & $9 \pm 1$ & $9 \pm 1$ & $10 \pm 2$ & $11 \pm 2$ & $11 \pm 2$ \\
\hline RCP4.5 - end & $12 \pm 3$ & $12 \pm 3$ & $10 \pm 2$ & $10 \pm 2$ & $9 \pm 2$ & $9 \pm 2$ & $10 \pm 2$ & $10 \pm 2$ & $9 \pm 2$ & $10 \pm 2$ & $11 \pm 2$ & $11 \pm 2$ \\
\hline $\begin{array}{l}\text { RCP8.5 - end } \\
\text { Mean Wave } \\
\text { Direction ( } 9\end{array}$ & $13 \pm 3$ & $12 \pm 3$ & $10 \pm 2$ & $10 \pm 2$ & $9 \pm 2$ & $10 \pm 3$ & $9 \pm 2$ & $9 \pm 2$ & $10 \pm 2$ & $10 \pm 2$ & $11 \pm 2$ & $11 \pm 2$ \\
\hline Hindcast & $29 \pm 53$ & $25 \pm 55$ & $55 \pm 47$ & $75 \pm 33$ & $91 \pm 27$ & $92 \pm 17$ & $92 \pm 18$ & $92 \pm 21$ & $85 \pm 31$ & $69 \pm 33$ & $57 \pm 36$ & $47 \pm 44$ \\
\hline RCP4.5 - mid & $28 \pm 51$ & $31 \pm 52$ & $55 \pm 45$ & $72 \pm 31$ & $89 \pm 26$ & $94 \pm 18$ & $92 \pm 16$ & $92 \pm 23$ & $90 \pm 29$ & $70 \pm 36$ & $53 \pm 35$ & $44 \pm 42$ \\
\hline RCP8.5 - mid & $31 \pm 50$ & $31 \pm 52$ & $50 \pm 48$ & $73 \pm 34$ & $90 \pm 25$ & $94 \pm 19$ & $93 \pm 19$ & $93 \pm 23$ & $88 \pm 31$ & $68 \pm 40$ & $51 \pm 37$ & $48 \pm 43$ \\
\hline RCP4.5 - end & $29 \pm 54$ & $23 \pm 54$ & $52 \pm 44$ & $72 \pm 34$ & $90 \pm 25$ & $95 \pm 18$ & $94 \pm 18$ & $95 \pm 24$ & $90 \pm 33$ & $70 \pm 37$ & $52 \pm 36$ & $45 \pm 43$ \\
\hline $\begin{array}{l}\text { RCP8.5 - end } \\
\text { Top } 5 \text { percent } \\
\text { Direction ( } 9\end{array}$ & $25 \pm 51$ & $21 \pm 53$ & $49 \pm 46$ & $73 \pm 32$ & $91 \pm 27$ & $97 \pm 18$ & $95 \pm 17$ & $97 \pm 24$ & $92 \pm 32$ & $72 \pm 37$ & $56 \pm 34$ & $43 \pm 44$ \\
\hline Hindcast & $56 \pm 46$ & $50 \pm 49$ & $69 \pm 23$ & $75 \pm 20$ & $84 \pm 15$ & $87 \pm 12$ & $86 \pm 18$ & $81 \pm 16$ & $76 \pm 28$ & $73 \pm 24$ & $69 \pm 26$ & $64 \pm 26$ \\
\hline RCP4.5 - mid & $33 \pm 54$ & $59 \pm 43$ & $70 \pm 28$ & $74 \pm 14$ & $84 \pm 14$ & $87 \pm 12$ & $92 \pm 22$ & $82 \pm 18$ & $87 \pm 29$ & $74 \pm 30$ & $68 \pm 20$ & $56 \pm 37$ \\
\hline RCP8.5 - mid & $57 \pm 40$ & $59 \pm 40$ & $67 \pm 28$ & $75 \pm 20$ & $86 \pm 15$ & $88 \pm 12$ & $86 \pm 20$ & $86 \pm 28$ & $81 \pm 27$ & $68 \pm 28$ & $65 \pm 33$ & $69 \pm 22$ \\
\hline RCP4.5 - end & $52 \pm 50$ & $44 \pm 49$ & $69 \pm 28$ & $75 \pm 23$ & $85 \pm 13$ & $92 \pm 18$ & $94 \pm 24$ & $97 \pm 41$ & $95 \pm 45$ & $73 \pm 36$ & $68 \pm 19$ & $73 \pm 28$ \\
\hline RCP8.5 - end & $49 \pm 48$ & $38 \pm 56$ & $63 \pm 31$ & $76 \pm 19$ & $81 \pm 17$ & $91 \pm 17$ & $94 \pm 20$ & $93 \pm 30$ & $88 \pm 40$ & $75 \pm 40$ & $66 \pm 21$ & $70 \pm 25$ \\
\hline
\end{tabular}


Appendix A6. Table showing return values of ensemble-average significant wave heights of hindcast and forecast scenarios, including lower and higher 95 percent confidence intervals, at the Big Island of Hawaii location.

[Years: Hindcast $=1976-2005 ;$ RCP mid $=$ 2026-2045; RCP end $=$ 2081-2100. Wave height values are in meters

\begin{tabular}{|c|c|c|c|c|c|c|c|c|c|c|c|c|c|c|c|c|}
\hline \multirow{2}{*}{ Scenario } & \multicolumn{3}{|c|}{ Hindcast } & \multicolumn{3}{c|}{ RCP4.5 - mid } & \multicolumn{3}{c|}{ RCP8.5 - mid } & \multicolumn{3}{c|}{ RCP4.5 - end } & \multicolumn{3}{c|}{ RCP8.5 - end } \\
\cline { 2 - 14 } & Low & $\boldsymbol{R}_{\mathbf{V}}$ & High & Low & $\boldsymbol{R}_{\mathbf{V}}$ & High & Low & $\boldsymbol{R}_{\mathbf{V}}$ & High & Low & $\boldsymbol{R}_{\mathbf{V}}$ & High & Low & $\boldsymbol{R}_{\mathbf{V}}$ & High \\
\hline 2-year & 5.83 & 6.27 & 6.95 & 5.79 & 6.32 & 7.21 & 5.70 & 6.20 & 7.04 & 5.95 & 6.72 & 8.11 & 5.64 & 6.45 & 8.11 \\
5-year & 6.04 & 6.68 & 7.77 & 6.05 & 6.88 & 8.47 & 5.91 & 6.66 & 8.08 & 6.14 & 7.22 & 9.42 & 5.87 & 7.15 & 10.27 \\
10-year & 6.16 & 6.95 & 8.40 & 6.22 & 7.32 & 9.60 & 6.04 & 7.00 & 8.95 & 6.25 & 7.54 & 10.42 & 6.01 & 7.70 & 12.38 \\
20-year & 6.25 & 7.20 & 9.04 & 6.37 & 7.77 & 10.92 & 6.14 & 7.32 & 9.90 & 6.32 & 7.81 & 11.43 & 6.13 & 8.26 & 15.00 \\
50-year & 6.35 & 7.49 & 9.90 & 6.54 & 8.37 & 12.99 & 6.26 & 7.72 & 11.29 & 6.39 & 8.11 & 12.77 & 6.26 & 9.02 & 19.48 \\
100-year & 6.41 & 7.69 & 10.57 & 6.66 & 8.83 & 14.86 & 6.33 & 8.00 & 12.46 & 6.42 & 8.30 & 13.79 & 6.35 & 9.61 & 23.84 \\
\hline
\end{tabular}


Appendix A7. Table showing Midway monthly means and mean of the top 5 percent for significant wave height, peak wave period, and peak wave direction.

[Years: Hindcast $=$ 1976-2005; RCP mid = 2026-2045; RCP end = 2081-2100. Wave directions are “coming from"]

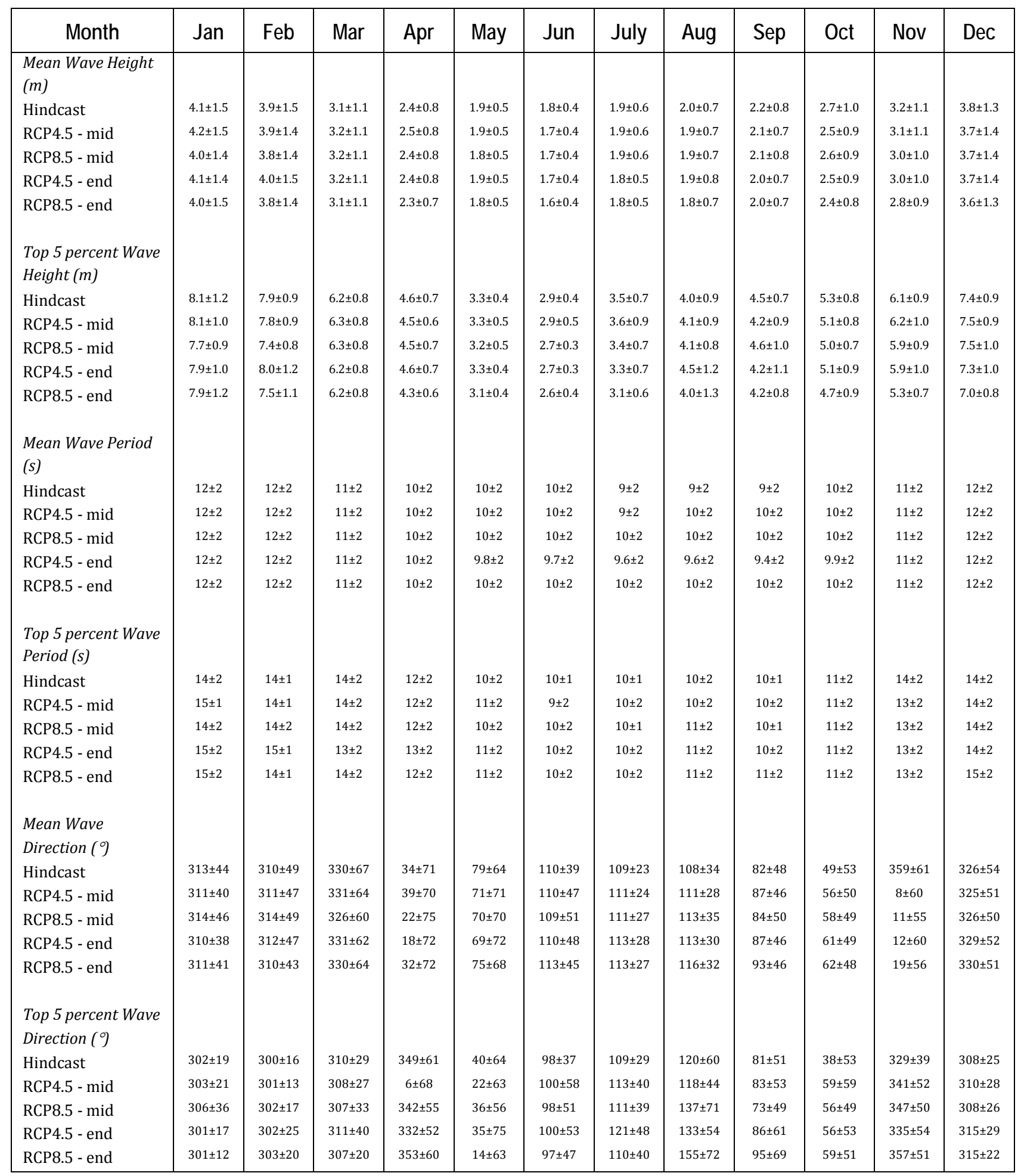


Appendix A8. Table showing return values of ensemble-average significant wave heights of hindcast and forecast scenarios, including lower and higher 95 percent confidence intervals, at the Midway location.

[Years: Hindcast $=1976-2005 ;$ RCP mid $=$ 2026-2045; RCP end $=$ 2081-2100. Wave height values are in meters $]$

\begin{tabular}{|c|c|c|c|c|c|c|c|c|c|c|c|c|c|c|c|}
\hline \multirow{2}{*}{ Scenario } & \multicolumn{3}{|c|}{ Hindcast } & \multicolumn{3}{c|}{ RCP4.5 - mid } & \multicolumn{3}{c|}{ RCP8.5 - mid } & \multicolumn{3}{c|}{ RCP4.5 - end } & \multicolumn{2}{c|}{ RCP8.5 - end } \\
\cline { 2 - 13 } & Low & $\boldsymbol{R}_{\boldsymbol{V}}$ & High & Low & $\boldsymbol{R}_{\boldsymbol{V}}$ & High & Low & $\boldsymbol{R}_{\boldsymbol{V}}$ & High & Low & $\boldsymbol{R}_{\boldsymbol{V}}$ & High & Low & $\boldsymbol{R}_{\boldsymbol{V}}$ & High \\
\hline 2-year & 10.33 & 11.17 & 12.46 & 10.03 & 10.93 & 12.50 & 9.54 & 10.49 & 12.27 & 10.49 & 11.57 & 13.28 & 9.69 & 10.84 & 12.90 \\
5-year & 10.85 & 12.18 & 14.42 & 10.26 & 11.49 & 13.86 & 9.81 & 11.21 & 14.19 & 11.00 & 12.61 & 15.41 & 10.10 & 11.85 & 15.41 \\
10-year & 11.20 & 12.95 & 16.12 & 10.37 & 11.82 & 14.84 & 9.96 & 11.70 & 15.79 & 11.32 & 13.34 & 17.11 & 10.35 & 12.58 & 17.58 \\
20-year & 11.52 & 13.72 & 18.02 & 10.45 & 12.10 & 15.77 & 10.08 & 12.15 & 17.54 & 11.58 & 14.02 & 18.91 & 10.55 & 13.29 & 20.02 \\
50-year & 11.88 & 14.74 & 20.89 & 10.53 & 12.39 & 16.93 & 10.20 & 12.70 & 20.08 & 11.86 & 14.86 & 21.42 & 10.77 & 14.18 & 23.72 \\
100-year & 12.13 & 15.52 & 23.37 & 10.56 & 12.56 & 17.76 & 10.26 & 13.07 & 22.21 & 12.03 & 15.45 & 23.43 & 10.90 & 14.83 & 26.93 \\
\hline
\end{tabular}


Appendix A9. Table showing Chuuk monthly means and mean of the top 5 percent for significant wave height, peak wave period, and peak wave direction.

[Years: Hindcast $=$ 1976-2005; RCP mid = 2026-2045; RCP end = 2081-2100. Wave directions are “coming from"]

\begin{tabular}{|c|c|c|c|c|c|c|c|c|c|c|c|c|}
\hline Month & Jan & Feb & Mar & Apr & May & Jun & July & Aug & Sep & Oct & Nov & Dec \\
\hline \multicolumn{13}{|c|}{$\begin{array}{l}\text { Mean Wave Height } \\
\text { (m) }\end{array}$} \\
\hline Hindcast & $2.7 \pm 0.6$ & $2.6 \pm 0.6$ & $2.5 \pm 0.6$ & $2.1 \pm 0.6$ & $1.6 \pm 0.5$ & $1.4 \pm 0.5$ & $1.4 \pm 0.6$ & $1.3 \pm 0.6$ & $1.3 \pm 0.6$ & $1.6 \pm 0.6$ & $2.1 \pm 0.7$ & $2.6 \pm 0.7$ \\
\hline RCP4.5 - mid & $2.7 \pm 0.7$ & $2.6 \pm 0.6$ & $2.5 \pm 0.6$ & $2.1 \pm 0.6$ & $1.6 \pm 0.5$ & $1.4 \pm 0.5$ & $1.4 \pm 0.6$ & $1.3 \pm 0.6$ & $1.3 \pm 0.5$ & $1.5 \pm 0.5$ & $2.0 \pm 0.6$ & $2.5 \pm 0.7$ \\
\hline RCP8.5 - mid & $2.6 \pm 0.6$ & $2.6 \pm 0.7$ & $2.4 \pm 0.6$ & $2.1 \pm 0.5$ & $1.6 \pm 0.5$ & $1.4 \pm 0.5$ & $1.4 \pm 0.5$ & $1.3 \pm 0.6$ & $1.3 \pm 0.5$ & $1.5 \pm 0.5$ & $2.0 \pm 0.7$ & $2.5 \pm 0.7$ \\
\hline RCP4.5 - end & $2.6 \pm 0.6$ & $2.6 \pm 0.6$ & $2.4 \pm 0.6$ & $2.0 \pm 0.5$ & $1.6 \pm 0.5$ & $1.4 \pm 0.6$ & $1.4 \pm 0.6$ & $1.4 \pm 0.6$ & $1.3 \pm 0.6$ & $1.5 \pm 0.6$ & $1.9 \pm 0.6$ & $2.4 \pm 0.7$ \\
\hline RCP8.5 - end & $2.5 \pm 0.6$ & $2.4 \pm 0.6$ & $2.3 \pm 0.6$ & $2.0 \pm 0.5$ & $1.6 \pm 0.5$ & $1.4 \pm 0.5$ & $1.4 \pm 0.6$ & $1.3 \pm 0.6$ & $1.3 \pm 0.6$ & $1.4 \pm 0.5$ & $1.8 \pm 0.6$ & $2.3 \pm 0.6$ \\
\hline \multicolumn{13}{|c|}{$\begin{array}{l}\text { Top } 5 \text { percent Wave } \\
\text { Height }(m)\end{array}$} \\
\hline Hindcast & $4.2 \pm 0.4$ & $4.1 \pm 0.5$ & $4.0 \pm 0.6$ & $3.6 \pm 0.5$ & $2.9 \pm 0.5$ & $2.9 \pm 0.7$ & $2.9 \pm 0.7$ & $3.2 \pm 0.9$ & $3.1 \pm 0.6$ & $3.4 \pm 0.5$ & $4.1 \pm 0.6$ & $4.3 \pm 0.6$ \\
\hline RCP4.5 - mid & $4.3 \pm 0.5$ & $4.0 \pm 0.4$ & $3.9 \pm 0.4$ & $3.5 \pm 0.5$ & $2.9 \pm 0.6$ & $2.6 \pm 0.5$ & $3.1 \pm 0.7$ & $3.3 \pm 1.0$ & $3.0 \pm 0.8$ & $3.0 \pm 0.5$ & $3.6 \pm 0.5$ & $4.3 \pm 0.4$ \\
\hline RCP8.5 - mid & $4.1 \pm 0.4$ & $4.3 \pm 0.6$ & $3.9 \pm 0.5$ & $3.4 \pm 0.4$ & $2.8 \pm 0.5$ & $2.8 \pm 0.7$ & $2.9 \pm 0.7$ & $3.2 \pm 0.8$ & $2.9 \pm 0.8$ & $3.0 \pm 0.5$ & $3.9 \pm 0.6$ & $4.4 \pm 0.6$ \\
\hline RCP4.5 - end & $3.9 \pm 0.3$ & $4.1 \pm 0.5$ & $3.9 \pm 0.5$ & $3.3 \pm 0.4$ & $2.7 \pm 0.3$ & $3.0 \pm 0.7$ & $3.1 \pm 0.7$ & $3.2 \pm 0.6$ & $3.1 \pm 0.7$ & $3.1 \pm 0.5$ & $3.6 \pm 0.6$ & $4.1 \pm 0.5$ \\
\hline RCP8.5 - end & $3.9 \pm 0.4$ & $3.9 \pm 0.5$ & $3.7 \pm 0.4$ & $3.2 \pm 0.4$ & $2.8 \pm 0.4$ & $2.7 \pm 0.5$ & $3.1 \pm 0.9$ & $3.2 \pm 0.7$ & $3.1 \pm 0.9$ & $2.9 \pm 0.8$ & $3.6 \pm 0.5$ & $3.9 \pm 0.5$ \\
\hline \multicolumn{13}{|c|}{$\begin{array}{l}\text { Mean Wave Period } \\
\text { (s) }\end{array}$} \\
\hline Hindcast & $10 \pm 2$ & $10 \pm 2$ & $9 \pm 2$ & $9 \pm 1$ & $9 \pm 1$ & $9 \pm 1$ & $9 \pm 1$ & $9 \pm 1$ & $9 \pm 1$ & $10 \pm 1$ & $10 \pm 2$ & $10 \pm 2$ \\
\hline RCP4.5 - mid & $10 \pm 2$ & $10 \pm 2$ & $9 \pm 2$ & $9 \pm 1$ & $9 \pm 1$ & $9 \pm 1$ & $9 \pm 1$ & $9 \pm 1$ & $9 \pm 1$ & $9 \pm 1$ & $10 \pm 1$ & $10 \pm 2$ \\
\hline RCP8.5 - mid & $10 \pm 2$ & $10 \pm 2$ & $9 \pm 2$ & $9 \pm 1$ & $9 \pm 1$ & $8 \pm 1$ & $9 \pm 1$ & $9 \pm 1$ & $9 \pm 1$ & $10 \pm 1$ & $10 \pm 2$ & $10 \pm 2$ \\
\hline RCP4.5 - end & $10 \pm 2$ & $10 \pm 2$ & $9 \pm 2$ & $9 \pm 1$ & $9 \pm 1$ & $9 \pm 1$ & $9 \pm 1$ & $9 \pm 1$ & $9 \pm 1$ & $10 \pm 1$ & $10 \pm 2$ & $10 \pm 2$ \\
\hline RCP8.5 - end & $10 \pm 2$ & $9 \pm 2$ & $9 \pm 2$ & $9 \pm 1$ & $8 \pm 1$ & $8 \pm 1$ & $9 \pm 1$ & $9 \pm 1$ & $9 \pm 1$ & $9 \pm 1$ & $10 \pm 1$ & $10 \pm 2$ \\
\hline \multicolumn{13}{|c|}{$\begin{array}{l}\text { Top } 5 \text { percent Wave } \\
\text { Period (s) }\end{array}$} \\
\hline Hindcast & $11 \pm 1$ & $11 \pm 2$ & $11 \pm 1$ & $10 \pm 1$ & $9 \pm 1$ & $9 \pm 1$ & $9 \pm 2$ & $9 \pm 2$ & $9 \pm 2$ & $11 \pm 2$ & $11 \pm 1$ & $11 \pm 1$ \\
\hline RCP4.5 - mid & $10 \pm 1$ & $10 \pm 1$ & $10 \pm 1$ & $10 \pm 1$ & $9 \pm 1$ & $9 \pm 1$ & $9 \pm 1$ & $9 \pm 1$ & $9 \pm 2$ & $10 \pm 2$ & $11 \pm 2$ & $11 \pm 1$ \\
\hline RCP8.5 - mid & $10 \pm 2$ & $10 \pm 1$ & $10 \pm 0.9$ & $10 \pm 1$ & $9 \pm 1$ & $9 \pm 1$ & $9 \pm 2$ & $9 \pm 2$ & $9 \pm 2$ & $10 \pm 2$ & $11 \pm 2$ & $10 \pm 1$ \\
\hline RCP4.5 - end & $10 \pm 1$ & $11 \pm 2$ & $10 \pm 1$ & $10 \pm 1$ & $9 \pm 0.8$ & $9 \pm 1$ & $9 \pm 2$ & $9 \pm 2$ & $9 \pm 2$ & $10 \pm 2$ & $11 \pm 2$ & $10 \pm 0.9$ \\
\hline RCP8.5 - end & $10 \pm 1$ & $10 \pm 1$ & $10 \pm 1$ & $9 \pm 0.8$ & $9 \pm 0.8$ & $9 \pm 1$ & $8 \pm 1$ & $9 \pm 2$ & $9 \pm 2$ & $10 \pm 2$ & $10 \pm 1$ & $10 \pm 0.9$ \\
\hline \multicolumn{13}{|l|}{$\begin{array}{l}\text { Mean Wave } \\
\text { Direction ( } 9\end{array}$} \\
\hline Hindcast & $54 \pm 14$ & $53 \pm 16$ & $55 \pm 17$ & $60 \pm 16$ & $66 \pm 22$ & $76 \pm 30$ & $87 \pm 43$ & $103 \pm 51$ & $80 \pm 48$ & $51 \pm 38$ & $50 \pm 26$ & $54 \pm 16$ \\
\hline RCP4.5 - mid & $53 \pm 18$ & $53 \pm 16$ & $56 \pm 14$ & $60 \pm 12$ & $66 \pm 18$ & $78 \pm 25$ & $94 \pm 45$ & $109 \pm 49$ & $93 \pm 47$ & $55 \pm 42$ & $49 \pm 24$ & $53 \pm 21$ \\
\hline RCP8.5 - mid & $53 \pm 15$ & $54 \pm 15$ & $56 \pm 16$ & $60 \pm 15$ & $67 \pm 17$ & $78 \pm 29$ & $92 \pm 46$ & $110 \pm 54$ & $94 \pm 52$ & $54 \pm 40$ & $51 \pm 22$ & $54 \pm 17$ \\
\hline RCP4.5 - end & $53 \pm 15$ & $51 \pm 17$ & $56 \pm 14$ & $59 \pm 15$ & $66 \pm 17$ & $77 \pm 28$ & $91 \pm 50$ & $108 \pm 53$ & $93 \pm 51$ & $54 \pm 43$ & $50 \pm 23$ & $54 \pm 18$ \\
\hline RCP8.5 - end & $52 \pm 17$ & $53 \pm 17$ & $56 \pm 14$ & $62 \pm 11$ & $68 \pm 14$ & $80 \pm 27$ & $91 \pm 44$ & $110 \pm 48$ & $101 \pm 50$ & $61 \pm 38$ & $53 \pm 21$ & $55 \pm 17$ \\
\hline \multicolumn{13}{|c|}{$\begin{array}{l}\text { Top } 5 \text { percent Wave } \\
\text { Direction }(9)\end{array}$} \\
\hline Hindcast & $54 \pm 13$ & $52 \pm 20$ & $48 \pm 34$ & $54 \pm 34$ & $62 \pm 58$ & $95 \pm 101$ & $220 \pm 102$ & $216 \pm 52$ & $258 \pm 90$ & $32 \pm 85$ & $54 \pm 52$ & $58 \pm 13$ \\
\hline RCP4.5 - mid & $56 \pm 23$ & $57 \pm 18$ & $55 \pm 21$ & $55 \pm 25$ & $67 \pm 43$ & $82 \pm 64$ & $212 \pm 69$ & $212 \pm 52$ & $228 \pm 61$ & $306 \pm 111$ & $46 \pm 53$ & $59 \pm 44$ \\
\hline RCP8.5 - mid & $56 \pm 12$ & $54 \pm 13$ & $52 \pm 25$ & $57 \pm 18$ & $66 \pm 46$ & $128 \pm 85$ & $222 \pm 80$ & $221 \pm 53$ & $226 \pm 47$ & $324 \pm 98$ & $51 \pm 57$ & $63 \pm 31$ \\
\hline RCP4.5 - end & $55 \pm 12$ & $49 \pm 33$ & $56 \pm 17$ & $57 \pm 29$ & $68 \pm 44$ & $96 \pm 96$ & $232 \pm 52$ & $229 \pm 41$ & $245 \pm 51$ & $298 \pm 104$ & $51 \pm 54$ & $60 \pm 30$ \\
\hline RCP8.5 - end & $53 \pm 22$ & $57 \pm 20$ & $53 \pm 28$ & $63 \pm 17$ & $68 \pm 38$ & $93 \pm 68$ & $204 \pm 84$ & $219 \pm 47$ & $219 \pm 59$ & 343103 & $63 \pm 49$ & $62 \pm 31$ \\
\hline
\end{tabular}


Appendix A10. Table showing return values of ensemble-average significant wave heights of hindcast and forecast scenarios, including lower and higher 95 percent confidence intervals, at the Chuuk location.

[Years: Hindcast $=1976-2005 ;$ RCP mid $=2026-2045 ;$ RCP end $=2081-2100$. Wave height values are in meters $]$

\begin{tabular}{|c|c|c|c|c|c|c|c|c|c|c|c|c|c|c|c|}
\hline \multirow{2}{*}{ Scenario } & \multicolumn{3}{|c|}{ Hindcast } & \multicolumn{3}{c|}{ RCP4.5 - mid } & \multicolumn{3}{c|}{ RCP8.5 - mid } & \multicolumn{3}{c|}{ RCP4.5 - end } & \multicolumn{2}{c|}{ RCP8.5 - end } \\
\cline { 2 - 12 } & Low & $R_{V}$ & High & Low & $R_{V}$ & High & Low & $R_{V}$ & High & Low & $R_{V}$ & High & Low & $R_{V}$ & High \\
\hline 2-year & 5.56 & 6.25 & 7.41 & 5.38 & 6.03 & 7.19 & 5.46 & 6.34 & 8.08 & 5.06 & 5.70 & 6.94 & 5.15 & 5.94 & 7.53 \\
5-year & 5.95 & 7.12 & 9.34 & 5.59 & 6.55 & 8.47 & 5.72 & 7.09 & 10.24 & 5.32 & 6.39 & 8.81 & 5.45 & 6.80 & 10.05 \\
10-year & 6.23 & 7.84 & 11.23 & 5.71 & 6.91 & 9.52 & 5.88 & 7.66 & 12.24 & 5.49 & 6.96 & 10.71 & 5.66 & 7.55 & 12.73 \\
20-year & 6.50 & 8.63 & 13.58 & 5.81 & 7.24 & 10.64 & 6.02 & 8.22 & 14.63 & 5.65 & 7.59 & 13.16 & 5.85 & 8.39 & 16.36 \\
50-year & 6.83 & 9.78 & 17.61 & 5.91 & 7.63 & 12.23 & 6.16 & 8.96 & 18.51 & 5.84 & 8.49 & 17.54 & 6.09 & 9.66 & 23.17 \\
100-year & 7.06 & 10.75 & 21.55 & 5.96 & 7.90 & 13.54 & 6.24 & 9.51 & 22.11 & 5.97 & 9.25 & 22.00 & 6.26 & 10.76 & 30.45 \\
\hline
\end{tabular}


Appendix A11. Table showing Saipan monthly means and mean of the top 5 percent for significant wave height, peak wave period, and peak wave direction.

[Years: Hindcast $=1976-2005 ;$ RCP mid $=$ 2026-2045; RCP end $=$ 2081-2100. Wave directions are “coming from”]

\begin{tabular}{|c|c|c|c|c|c|c|c|c|c|c|c|c|}
\hline Month & Jan & Feb & Mar & Apr & May & Jun & July & Aug & Sep & Oct & Nov & Dec \\
\hline \multicolumn{13}{|c|}{$\begin{array}{l}\text { Mean Wave Height } \\
\text { (m) }\end{array}$} \\
\hline Hindcast & $2.7 \pm 0.7$ & $2.6 \pm 0.7$ & $2.5 \pm 0.7$ & $2.2 \pm 0.6$ & $1.8 \pm 0.6$ & $1.7 \pm 0.7$ & $1.7 \pm 0.9$ & $1.7 \pm 1.0$ & $1.7 \pm 0.8$ & $2.1 \pm 0.9$ & $2.6 \pm 0.9$ & $2.8 \pm 0.7$ \\
\hline RCP4.5 - mid & $2.7 \pm 0.7$ & $2.6 \pm 0.6$ & $2.5 \pm 0.7$ & $2.2 \pm 0.6$ & $1.8 \pm 0.6$ & $1.7 \pm 0.6$ & $1.7 \pm 0.9$ & $1.6 \pm 0.8$ & $1.6 \pm 0.9$ & $1.9 \pm 0.7$ & $2.5 \pm 0.8$ & $2.7 \pm 0.8$ \\
\hline RCP8.5 - mid & $2.6 \pm 0.7$ & $2.6 \pm 0.7$ & $2.4 \pm 0.7$ & $2.2 \pm 0.6$ & $1.8 \pm 0.6$ & $1.8 \pm 0.8$ & $1.7 \pm 0.9$ & $1.7 \pm 1.0$ & $1.6 \pm 0.9$ & $1.9 \pm 0.7$ & $2.4 \pm 0.8$ & $2.8 \pm 0.7$ \\
\hline RCP4.5 - end & $2.6 \pm 0.7$ & $2.6 \pm 0.7$ & $2.5 \pm 0.7$ & $2.2 \pm 0.6$ & $1.8 \pm 0.5$ & $1.7 \pm 0.6$ & $1.7 \pm 0.9$ & $1.7 \pm 0.9$ & $1.6 \pm 0.7$ & $1.9 \pm 0.8$ & $2.5 \pm 0.8$ & $2.6 \pm 0.7$ \\
\hline RCP8.5 - end & $2.5 \pm 0.7$ & $2.4 \pm 0.7$ & $2.3 \pm 0.6$ & $2.1 \pm 0.5$ & $1.8 \pm 0.5$ & $1.7 \pm 0.6$ & $1.8 \pm 0.8$ & $1.6 \pm 1.0$ & $1.6 \pm 0.8$ & $1.8 \pm 0.7$ & $2.3 \pm 0.8$ & $2.6 \pm 0.7$ \\
\hline \multicolumn{13}{|c|}{$\begin{array}{l}\text { Top } 5 \text { percent Wave } \\
\text { Height }(\mathrm{m})\end{array}$} \\
\hline Hindcast & $4.3 \pm 0.4$ & $4.3 \pm 0.4$ & $4.4 \pm 0.8$ & $3.9 \pm 0.6$ & $3.5 \pm 0.8$ & $3.5 \pm 0.9$ & $4.5 \pm 1.0$ & $4.9 \pm 1.3$ & $4.3 \pm 1.0$ & $4.6 \pm 0.9$ & $5.0 \pm 1.0$ & $4.6 \pm 0.7$ \\
\hline RCP4.5 - mid & $4.3 \pm 0.4$ & $4.2 \pm 0.4$ & $4.2 \pm 0.6$ & $3.8 \pm 1.0$ & $3.3 \pm 0.6$ & $3.4 \pm 1.0$ & $4.4 \pm 1.0$ & $4.1 \pm 0.9$ & $4.5 \pm 1.3$ & $3.9 \pm 0.7$ & $4.5 \pm 0.8$ & $4.8 \pm 0.8$ \\
\hline RCP8.5 - mid & $4.3 \pm 0.5$ & $4.3 \pm 0.5$ & $4.2 \pm 0.6$ & $3.7 \pm 0.4$ & $3.2 \pm 0.5$ & $4.2 \pm 1.4$ & $4.5 \pm 1.0$ & $4.7 \pm 1.1$ & $4.4 \pm 1.3$ & $4.0 \pm 0.8$ & $4.7 \pm 1.0$ & $4.8 \pm 0.7$ \\
\hline RCP4.5 - end & $4.2 \pm 0.4$ & $4.4 \pm 0.5$ & $4.2 \pm 0.4$ & $3.7 \pm 0.4$ & $2.9 \pm 0.4$ & $3.4 \pm 1.0$ & $4.5 \pm 1.2$ & $4.7 \pm 1.1$ & $3.9 \pm 0.8$ & $4.0 \pm 0.9$ & $4.6 \pm 0.9$ & $4.4 \pm 0.6$ \\
\hline RCP8.5 - end & $4.3 \pm 0.4$ & $4.1 \pm 0.4$ & $3.9 \pm 0.6$ & $3.4 \pm 0.4$ & $3.1 \pm 0.5$ & $3.4 \pm 1.2$ & $4.3 \pm 0.9$ & $4.7 \pm 1.3$ & $4.3 \pm 0.9$ & $3.8 \pm 0.8$ & $4.5 \pm 0.7$ & $4.4 \pm 0.7$ \\
\hline \multicolumn{13}{|c|}{$\begin{array}{l}\text { Mean Wave Period } \\
\text { (s) }\end{array}$} \\
\hline Hindcast & $10 \pm 2$ & $10 \pm 2$ & $9 \pm 1$ & $9 \pm 1$ & $9 \pm 1$ & $9 \pm 1$ & $9 \pm 1$ & $9 \pm 1$ & $9 \pm 1$ & $10 \pm 1$ & $10 \pm 1$ & $10 \pm 2$ \\
\hline RCP4.5 - mid & $10 \pm 2$ & $10 \pm 2$ & $9 \pm 1$ & $9 \pm 1$ & $9 \pm 1$ & $8 \pm 1$ & $9 \pm 1$ & $9 \pm 1$ & $9 \pm 1$ & $9 \pm 1$ & $10 \pm 1$ & $10 \pm 2$ \\
\hline RCP8.5 - mid & $10 \pm 2$ & $10 \pm 2$ & $9 \pm 1$ & $9 \pm 1$ & $9 \pm 1$ & $9 \pm 1$ & $9 \pm 1$ & $9 \pm 1$ & $9 \pm 2$ & $9 \pm 1$ & $10 \pm 1$ & $10 \pm 2$ \\
\hline RCP4.5 - end & $10 \pm 2$ & $10 \pm 2$ & $9 \pm 1$ & $9 \pm 1$ & $9 \pm 1$ & $9 \pm 1$ & $9 \pm 1$ & $9 \pm 1$ & $9 \pm 1$ & $9 \pm 2$ & $10 \pm 2$ & $10 \pm 2$ \\
\hline RCP8.5 - end & $10 \pm 2$ & $9 \pm 2$ & $9 \pm 1$ & $9 \pm 1$ & $9 \pm 1$ & $8 \pm 1$ & $9 \pm 1$ & $9 \pm 1$ & $9 \pm 1$ & $9 \pm 1$ & $9 \pm 1$ & $9 \pm 1$ \\
\hline \multicolumn{13}{|c|}{$\begin{array}{l}\text { Top } 5 \text { percent Wave } \\
\text { Period (s) }\end{array}$} \\
\hline Hindcast & $10 \pm 1$ & $10 \pm 2$ & $10 \pm 1$ & $10 \pm 1$ & $10 \pm 1$ & $9 \pm 1$ & $10 \pm 2$ & $10 \pm 1$ & $10 \pm 1$ & $11 \pm 1$ & $11 \pm 1$ & $11 \pm 1$ \\
\hline RCP4.5 - mid & $10 \pm 1$ & $10 \pm 1$ & $10 \pm 1$ & $10 \pm 1$ & $9 \pm 0.9$ & $9 \pm 1$ & $10 \pm 1$ & $10 \pm 1$ & $10 \pm 1$ & $11 \pm 1$ & $11 \pm 1$ & $11 \pm 1$ \\
\hline RCP8.5 - mid & $10 \pm 2$ & $10 \pm 1$ & $10 \pm 1$ & $10 \pm 1$ & $10 \pm 0.9$ & $10 \pm 1$ & $10 \pm 1$ & $10 \pm 1$ & $10 \pm 1$ & $11 \pm 1$ & $11 \pm 1$ & $11 \pm 1$ \\
\hline RCP4.5 - end & $10 \pm 2$ & $10 \pm 2$ & $10 \pm 1$ & $10 \pm 1$ & $9 \pm 1$ & $10 \pm 1$ & $10 \pm 1$ & $10 \pm 1$ & $10 \pm 1$ & $11 \pm 2$ & $11 \pm 1$ & $10 \pm 1$ \\
\hline RCP8.5 - end & $10 \pm 1$ & $9 \pm 1$ & $10 \pm 1$ & $10 \pm 0.9$ & $9 \pm 0.8$ & $9 \pm 1$ & $10 \pm 1$ & $10 \pm 1$ & $10 \pm 1$ & $11 \pm 1$ & $11 \pm 1$ & $10 \pm 1$ \\
\hline \multicolumn{13}{|l|}{ Mean Wave } \\
\hline Hindcast & $64 \pm 23$ & $61 \pm 23$ & $69 \pm 20$ & $76 \pm 13$ & $81 \pm 15$ & $85 \pm 24$ & $92 \pm 50$ & $102 \pm 68$ & $75 \pm 59$ & $66 \pm 35$ & $72 \pm 23$ & $70 \pm 21$ \\
\hline RCP4.5 - mid & $64 \pm 24$ & $63 \pm 22$ & $69 \pm 18$ & $75 \pm 11$ & $80 \pm 13$ & $87 \pm 22$ & $94 \pm 43$ & $98 \pm 72$ & $77 \pm 65$ & $63 \pm 41$ & $69 \pm 22$ & $69 \pm 21$ \\
\hline RCP8.5 - mid & $64 \pm 23$ & $64 \pm 22$ & $70 \pm 18$ & $75 \pm 14$ & $81 \pm 10$ & $89 \pm 26$ & $93 \pm 47$ & $111 \pm 72$ & $77 \pm 73$ & $63 \pm 37$ & $70 \pm 21$ & $70 \pm 22$ \\
\hline RCP4.5 - end & $64 \pm 24$ & $62 \pm 23$ & $69 \pm 17$ & $75 \pm 13$ & $81 \pm 12$ & $87 \pm 20$ & $95 \pm 49$ & $108 \pm 69$ & $78 \pm 72$ & $63 \pm 37$ & $70 \pm 19$ & $70 \pm 18$ \\
\hline RCP8.5 - end & $63 \pm 24$ & $63 \pm 23$ & $68 \pm 17$ & $76 \pm 12$ & $82 \pm 11$ & $89 \pm 24$ & $93 \pm 43$ & $107 \pm 61$ & $83 \pm 73$ & $65 \pm 41$ & $71 \pm 18$ & $70 \pm 19$ \\
\hline \multicolumn{13}{|c|}{ Top 5 percent Wave } \\
\hline Hindcast & $63 \pm 18$ & $57 \pm 27$ & $67 \pm 23$ & $72 \pm 19$ & $85 \pm 28$ & $108 \pm 68$ & $204 \pm 64$ & $205 \pm 55$ & $194 \pm 93$ & $81 \pm 53$ & $81 \pm 40$ & $75 \pm 25$ \\
\hline RCP4.5 - mid & $64 \pm 23$ & $64 \pm 14$ & $69 \pm 19$ & $73 \pm 11$ & $81 \pm 22$ & $111 \pm 52$ & $205 \pm 76$ & $218 \pm 60$ & $218 \pm 84$ & $69 \pm 75$ & $74 \pm 34$ & $70 \pm 25$ \\
\hline RCP8.5 - mid & $62 \pm 18$ & $64 \pm 16$ & $68 \pm 15$ & $73 \pm 13$ & $82 \pm 14$ & $128 \pm 63$ & $210 \pm 64$ & $224 \pm 45$ & $229 \pm 52$ & $83 \pm 63$ & $82 \pm 35$ & $77 \pm 21$ \\
\hline RCP4.5 - end & $66 \pm 16$ & $54 \pm 26$ & $66 \pm 15$ & $71 \pm 11$ & $82 \pm 17$ & $103 \pm 55$ & $193 \pm 76$ & $224 \pm 53$ & $235 \pm 67$ & $78 \pm 70$ & $76 \pm 35$ & $73 \pm 21$ \\
\hline RCP8.5 - end & $65 \pm 18$ & $66 \pm 16$ & $68 \pm 14$ & $74 \pm 11$ & $82 \pm 12$ & $112 \pm 50$ & $202 \pm 73$ & $213 \pm 53$ & $209 \pm 68$ & $76 \pm 70$ & $82 \pm 28$ & $78 \pm 23$ \\
\hline
\end{tabular}


Appendix A12. Table showing return values of ensemble-average significant wave heights of hindcast and forecast scenarios, including lower and higher 95 percent confidence intervals, at the Saipan location.

[Years: Hindcast $=1976-2005 ;$ RCP mid $=$ 2026-2045; RCP end $=2081-2100$. Wave height values are in meters]

\begin{tabular}{|c|c|c|c|c|c|c|c|c|c|c|c|c|c|c|c|}
\hline \multirow{2}{*}{ Scenario } & \multicolumn{3}{|c|}{ Hindcast } & \multicolumn{3}{c|}{ RCP4.5 - mid } & \multicolumn{3}{c|}{ RCP8.5 - mid } & \multicolumn{3}{c|}{ RCP4.5 - end } & \multicolumn{3}{c|}{ RCP8.5 - end } \\
\cline { 2 - 14 } & Low & $\boldsymbol{R}_{\mathbf{V}}$ & High & Low & $\boldsymbol{R}_{\mathbf{V}}$ & High & Low & $\boldsymbol{R}_{\mathbf{V}}$ & High & Low & $\boldsymbol{R}_{\mathbf{V}}$ & High & Low & $\boldsymbol{R}_{\mathbf{V}}$ & High \\
\hline 2-year & 7.39 & 8.27 & 9.59 & 6.95 & 8.04 & 9.89 & 7.06 & 8.17 & 10.12 & 6.70 & 7.81 & 9.86 & 6.90 & 7.96 & 9.74 \\
5-year & 7.90 & 9.22 & 11.32 & 7.45 & 9.14 & 12.34 & 7.50 & 9.21 & 12.59 & 7.04 & 8.71 & 12.18 & 7.36 & 8.97 & 11.98 \\
10-year & 8.22 & 9.86 & 12.64 & 7.77 & 9.96 & 14.46 & 7.77 & 9.98 & 14.76 & 7.24 & 9.33 & 14.15 & 7.65 & 9.70 & 13.87 \\
20-year & 8.48 & 10.46 & 13.99 & 8.04 & 10.77 & 16.86 & 8.00 & 10.73 & 17.22 & 7.40 & 9.92 & 16.31 & 7.89 & 10.41 & 15.94 \\
50-year & 8.76 & 11.17 & 15.79 & 8.34 & 11.83 & 20.50 & 8.26 & 11.70 & 20.97 & 7.57 & 10.64 & 19.52 & 8.15 & 11.30 & 18.99 \\
100-year & 8.93 & 11.65 & 17.17 & 8.54 & 12.61 & 23.66 & 8.41 & 12.42 & 24.26 & 7.66 & 11.14 & 22.24 & 8.31 & 11.95 & 21.57 \\
\hline
\end{tabular}


Appendix A13. Table showing Asuncion monthly means and mean of the top 5 percent for significant wave height, peak wave period, and peak wave direction.

[Years: Hindcast $=$ 1976-2005; RCP mid = 2026-2045; RCP end = 2081-2100. Wave directions are "coming from”]

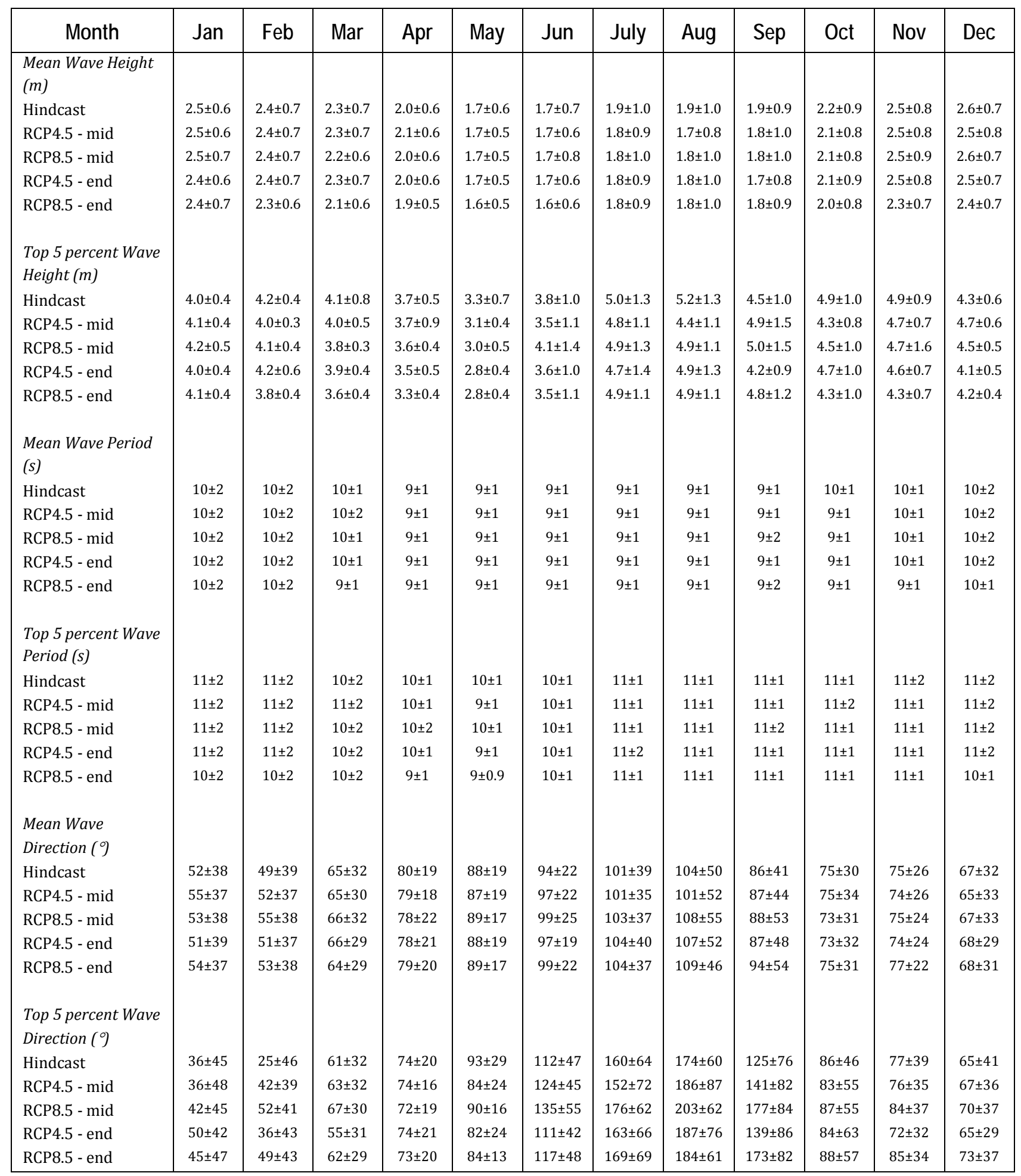


Appendix A14. Table showing return values of ensemble-average significant wave heights of hindcast and forecast scenarios, including lower and higher 95 percent confidence intervals, at the Asuncion location.

[Years: Hindcast $=1976-2005 ;$ RCP mid $=$ 2026-2045; RCP end $=2081-2100$. Wave height values are in meters]

\begin{tabular}{|c|c|c|c|c|c|c|c|c|c|c|c|c|c|c|c|}
\hline \multirow{2}{*}{ Scenario } & \multicolumn{3}{|c|}{ Hindcast } & \multicolumn{3}{c|}{ RCP4.5 - mid } & \multicolumn{3}{c|}{ RCP8.5 - mid } & \multicolumn{3}{c|}{ RCP4.5 - end } & \multicolumn{3}{c|}{ RCP8.5 - end } \\
\cline { 2 - 13 } & Low & $\boldsymbol{R}_{\mathbf{V}}$ & High & Low & $\boldsymbol{R}_{\mathbf{V}}$ & High & Low & $\boldsymbol{R}_{\mathbf{V}}$ & High & Low & $\boldsymbol{R}_{\mathbf{V}}$ & High & Low & $\boldsymbol{R}_{\mathbf{V}}$ & High \\
\hline 2-year & 7.86 & 8.86 & 10.32 & 7.29 & 8.62 & 10.93 & 7.58 & 9.11 & 11.79 & 7.01 & 8.31 & 10.64 & 7.05 & 8.29 & 10.41 \\
5-year & 8.37 & 9.79 & 12.02 & 7.81 & 9.83 & 13.74 & 8.22 & 10.62 & 15.36 & 7.45 & 9.39 & 13.32 & 7.48 & 9.28 & 12.67 \\
10-year & 8.67 & 10.39 & 13.23 & 8.12 & 10.69 & 16.11 & 8.63 & 11.77 & 18.53 & 7.70 & 10.15 & 15.58 & 7.72 & 9.93 & 14.43 \\
20-year & 8.89 & 10.90 & 14.39 & 8.38 & 11.52 & 18.73 & 8.99 & 12.91 & 22.20 & 7.91 & 10.86 & 18.07 & 7.91 & 10.51 & 16.23 \\
50-year & 9.12 & 11.47 & 15.83 & 8.66 & 12.55 & 22.59 & 9.39 & 14.42 & 27.92 & 8.12 & 11.74 & 21.73 & 8.10 & 11.18 & 18.69 \\
100-year & 9.25 & 11.84 & 16.86 & 8.83 & 13.29 & 25.85 & 9.65 & 15.56 & 33.02 & 8.25 & 12.36 & 24.81 & 8.20 & 11.63 & 20.61 \\
\hline
\end{tabular}


Appendix A15. Table showing Kosrae monthly means and mean of the top 5 percent for significant wave height, peak wave period, and peak wave direction.

[Years: Hindcast $=$ 1976-2005; RCP mid = 2026-2045; RCP end = 2081-2100. Wave directions are "coming from”]

\begin{tabular}{|c|c|c|c|c|c|c|c|c|c|c|c|c|}
\hline Month & Jan & Feb & Mar & Apr & May & Jun & July & Aug & Sep & Oct & Nov & Dec \\
\hline \multicolumn{13}{|c|}{$\begin{array}{l}\text { Mean Wave Height } \\
\text { (m) }\end{array}$} \\
\hline Hindcast & $2.3 \pm 0.5$ & $2.3 \pm 0.5$ & $2.1 \pm 0.5$ & $1.7 \pm 0.5$ & $1.4 \pm 0.4$ & $1.3 \pm 0.4$ & $1.3 \pm 0.4$ & $1.3 \pm 0.4$ & $1.3 \pm 0.5$ & $1.4 \pm 0.5$ & $1.7 \pm 0.6$ & $2.1 \pm 0.7$ \\
\hline RCP4.5 - mid & $2.3 \pm 0.5$ & $2.2 \pm 0.5$ & $2.1 \pm 0.5$ & $1.7 \pm 0.5$ & $1.4 \pm 0.4$ & $1.3 \pm 0.4$ & $1.4 \pm 0.4$ & $1.3 \pm 0.5$ & $1.3 \pm 0.4$ & $1.3 \pm 0.4$ & $1.6 \pm 0.5$ & $2.0 \pm 0.6$ \\
\hline RCP8.5 - mid & $2.2 \pm 0.5$ & $2.3 \pm 0.6$ & $2.1 \pm 0.5$ & $1.8 \pm 0.5$ & $1.4 \pm 0.5$ & $1.3 \pm 0.4$ & $1.4 \pm 0.4$ & $1.4 \pm 0.5$ & $1.3 \pm 0.4$ & $1.3 \pm 0.4$ & $1.6 \pm 0.5$ & $2.0 \pm 0.6$ \\
\hline RCP4.5 - end & $2.2 \pm 0.5$ & $2.2 \pm 0.5$ & $2.1 \pm 0.5$ & $1.7 \pm 0.5$ & $1.4 \pm 0.4$ & $1.3 \pm 0.5$ & $1.3 \pm 0.4$ & $1.4 \pm 0.5$ & $1.3 \pm 0.5$ & $1.3 \pm 0.5$ & $1.6 \pm 0.5$ & $2.0 \pm 0.6$ \\
\hline RCP8.5 - end & $2.1 \pm 0.5$ & $2.1 \pm 0.5$ & $2.0 \pm 0.5$ & $1.7 \pm 0.5$ & $1.4 \pm 0.5$ & $1.3 \pm 0.5$ & $1.4 \pm 0.4$ & $1.4 \pm 0.5$ & $1.3 \pm 0.4$ & $1.3 \pm 0.4$ & $1.4 \pm 0.5$ & $1.9 \pm 0.5$ \\
\hline \multicolumn{13}{|c|}{$\begin{array}{l}\text { Top } 5 \text { percent Wave } \\
\text { Height }(\mathrm{m})\end{array}$} \\
\hline Hindcast & $3.6 \pm 0.4$ & $3.5 \pm 0.3$ & $3.5 \pm 0.4$ & $3.1 \pm 0.5$ & $2.5 \pm 0.6$ & $2.4 \pm 0.4$ & $2.5 \pm 0.8$ & $2.6 \pm 0.7$ & $2.7 \pm 0.6$ & $2.8 \pm 0.6$ & $3.3 \pm 0.5$ & $3.8 \pm 0.5$ \\
\hline RCP4.5 - mid & $3.5 \pm 0.4$ & $3.5 \pm 0.5$ & $3.3 \pm 0.4$ & $3.0 \pm 0.3$ & $2.5 \pm 0.3$ & $2.5 \pm 0.5$ & $2.5 \pm 0.5$ & $2.7 \pm 0.7$ & $2.4 \pm 0.4$ & $2.6 \pm 0.6$ & $3.0 \pm 0.5$ & $3.7 \pm 0.4$ \\
\hline RCP8.5 - mid & $3.5 \pm 0.3$ & $3.8 \pm 0.5$ & $3.4 \pm 0.4$ & $3.2 \pm 0.6$ & $2.7 \pm 0.6$ & $2.4 \pm 0.3$ & $2.5 \pm 0.5$ & $2.8 \pm 0.6$ & $2.5 \pm 0.6$ & $2.6 \pm 0.6$ & $3.1 \pm 0.4$ & $3.5 \pm 0.4$ \\
\hline RCP 4.5 - end & $3.4 \pm 0.3$ & $3.4 \pm 0.3$ & $3.3 \pm 0.3$ & $2.8 \pm 0.3$ & $2.4 \pm 0.2$ & $2.6 \pm 0.7$ & $2.6 \pm 0.7$ & $2.8 \pm 0.6$ & $2.7 \pm 0.6$ & $2.6 \pm 0.5$ & $3.0 \pm 0.6$ & $3.6 \pm 0.4$ \\
\hline RCP8.5 - end & $3.2 \pm 0.2$ & $3.3 \pm 0.4$ & $3.2 \pm 0.3$ & $2.8 \pm 0.3$ & $2.7 \pm 0.4$ & $2.6 \pm 0.5$ & $2.5 \pm 0.6$ & $2.9 \pm 0.9$ & $2.4 \pm 0.5$ & $2.3 \pm 0.4$ & $2.9 \pm 0.6$ & $3.2 \pm 0.3$ \\
\hline \multicolumn{13}{|c|}{$\begin{array}{l}\text { Mean Wave Period } \\
\text { (s) }\end{array}$} \\
\hline Hindcast & $10 \pm 2$ & $10 \pm 2$ & $10 \pm 2$ & $9 \pm 2$ & $9 \pm 2$ & $9 \pm 2$ & $9 \pm 2$ & $9 \pm 2$ & $9 \pm 2$ & $10 \pm 2$ & $10 \pm 2$ & $10 \pm 2$ \\
\hline RCP4.5 - mid & $10 \pm 2$ & $10 \pm 2$ & $10 \pm 2$ & $9 \pm 2$ & $9 \pm 2$ & $9 \pm 2$ & $9 \pm 2$ & $9 \pm 2$ & $9 \pm 2$ & $10 \pm 2$ & $10 \pm 2$ & $10 \pm 2$ \\
\hline RCP8.5 - mid & $10 \pm 2$ & $10 \pm 2$ & $10 \pm 2$ & $9 \pm 2$ & $9 \pm 2$ & $9 \pm 2$ & $9 \pm 2$ & $9 \pm 2$ & $9 \pm 2$ & $10 \pm 2$ & $10 \pm 2$ & $10 \pm 2$ \\
\hline RCP 4.5 - end & $10 \pm 2$ & $10 \pm 2$ & $9.8 \pm 2$ & $9.5 \pm 2$ & $9.2 \pm 2$ & $9.1 \pm 2$ & $9.2 \pm 2$ & $9.3 \pm 2$ & $9.1 \pm 1$ & $9.6 \pm 2$ & $10 \pm 2$ & $10 \pm 2$ \\
\hline \multicolumn{13}{|c|}{$\begin{array}{l}\text { Top } 5 \text { percent Wave } \\
\text { Period (s) }\end{array}$} \\
\hline Hindcast & $10 \pm 2$ & $10 \pm 2$ & $10 \pm 2$ & $9 \pm 2$ & $9 \pm 1$ & $9 \pm 2$ & $9 \pm 2$ & $9 \pm 2$ & $9 \pm 2$ & $9 \pm 2$ & $10 \pm 2$ & $10 \pm 1$ \\
\hline RCP4.5 - mid & $10 \pm 2$ & $10 \pm 2$ & $10 \pm 2$ & $9 \pm 1$ & $9 \pm 1$ & $8 \pm 2$ & $9 \pm 2$ & $9 \pm 2$ & $10 \pm 2$ & $9 \pm 2$ & $10 \pm 2$ & $10 \pm 2$ \\
\hline RCP8.5 - mid & $10 \pm 2$ & $10 \pm 1$ & $9 \pm 1$ & $9 \pm 1$ & $8 \pm 1$ & $9 \pm 2$ & $9 \pm 2$ & $9 \pm 2$ & $9 \pm 2$ & $9 \pm 2$ & $10 \pm 2$ & $9 \pm 1$ \\
\hline RCP4.5 - end & $10 \pm 2$ & $10 \pm 2$ & $10 \pm 1$ & $9 \pm 1$ & $8 \pm 1$ & $9 \pm 1$ & $9 \pm 2$ & $9 \pm 1$ & $9 \pm 2$ & $9 \pm 2$ & $10 \pm 2$ & $9 \pm 1$ \\
\hline $\begin{array}{l}\text { RCP } 8.5 \text { - end } \\
\text { Mean Wave } \\
\text { Direction ( }{ }^{\circ} \text { ) }\end{array}$ & $10 \pm 3$ & $9 \pm 2$ & $9 \pm 2$ & $9 \pm 1$ & $8 \pm 0.9$ & $8 \pm 1$ & $9 \pm 2$ & $9 \pm 2$ & $9 \pm 2$ & $9 \pm 2$ & $9 \pm 2$ & $9 \pm 2$ \\
\hline Hindcast & $45 \pm 18$ & $45 \pm 19$ & $50 \pm 20$ & $61 \pm 23$ & $82 \pm 29$ & $104 \pm 32$ & $118 \pm 33$ & $128 \pm 38$ & $117 \pm 45$ & $79 \pm 49$ & $48 \pm 32$ & $44 \pm 20$ \\
\hline RCP4.5 - mid & $45 \pm 21$ & $45 \pm 19$ & $50 \pm 17$ & $61 \pm 21$ & $80 \pm 27$ & $101 \pm 27$ & $119 \pm 35$ & $128 \pm 36$ & $125 \pm 35$ & $90 \pm 48$ & $53 \pm 34$ & $45 \pm 26$ \\
\hline RCP8.5 - mid & $45 \pm 19$ & $47 \pm 20$ & $50 \pm 21$ & $59 \pm 22$ & $79 \pm 28$ & $102 \pm 30$ & $120 \pm 36$ & $134 \pm 40$ & $127 \pm 42$ & $90 \pm 45$ & $51 \pm 31$ & $45 \pm 21$ \\
\hline RCP4.5 - end & $44 \pm 20$ & $43 \pm 22$ & $50 \pm 18$ & $61 \pm 22$ & $78 \pm 25$ & $102 \pm 29$ & $119 \pm 38$ & $131 \pm 42$ & $127 \pm 41$ & $87 \pm 52$ & $50 \pm 34$ & $46 \pm 23$ \\
\hline $\begin{array}{l}\text { RCP8.5 - end } \\
\text { Top } 5 \text { percent } \\
\text { Direction ( } 9 \text { ) }\end{array}$ & $44 \pm 21$ & $46 \pm 20$ & $51 \pm 19$ & $61 \pm 16$ & $79 \pm 24$ & $101 \pm 29$ & $115 \pm 37$ & $129 \pm 35$ & $124 \pm 37$ & $95 \pm 43$ & $58 \pm 30$ & $48 \pm 22$ \\
\hline Hindcast & $49 \pm 15$ & $46 \pm 17$ & $44 \pm 30$ & $53 \pm 45$ & $77 \pm 68$ & $102 \pm 77$ & $181 \pm 87$ & $200 \pm 66$ & $235 \pm 76$ & $264 \pm 108$ & $39 \pm 68$ & $51 \pm 21$ \\
\hline RCP4.5 - mid & $49 \pm 21$ & $48 \pm 21$ & $53 \pm 15$ & $57 \pm 36$ & $69 \pm 57$ & $94 \pm 63$ & $164 \pm 78$ & $202 \pm 68$ & $197 \pm 68$ & $219 \pm 110$ & $48 \pm 62$ & $47 \pm 41$ \\
\hline RCP8.5 - mid & $52 \pm 18$ & $52 \pm 27$ & $54 \pm 31$ & $50 \pm 45$ & $71 \pm 66$ & $103 \pm 70$ & $193 \pm 80$ & $211 \pm 52$ & $223 \pm 67$ & $248 \pm 91$ & $28 \pm 64$ & $52 \pm 27$ \\
\hline RCP 4.5 - end & $45 \pm 15$ & $45 \pm 25$ & $52 \pm 27$ & $59 \pm 41$ & $66 \pm 19$ & $114 \pm 65$ & $210 \pm 75$ & $222 \pm 68$ & $235 \pm 58$ & $258 \pm 88$ & $352 \pm 84$ & $54 \pm 34$ \\
\hline RCP8.5 - end & $46 \pm 20$ & $53 \pm 15$ & $53 \pm 38$ & $61 \pm 26$ & $71 \pm 51$ & $100 \pm 71$ & $163 \pm 83$ & $210 \pm 59$ & $182 \pm 82$ & $19 \pm 126$ & $51 \pm 52$ & $51 \pm 14$ \\
\hline
\end{tabular}


Appendix A16. Table showing return values of ensemble-average significant wave heights of hindcast and forecast scenarios, including lower and higher 95 percent confidence intervals, at the Kosrae location.

[Years: Hindcast $=1976-2005 ;$ RCP mid $=$ 2026-2045; RCP end $=2081-2100$. Wave height values are in meters]

\begin{tabular}{|c|c|c|c|c|c|c|c|c|c|c|c|c|c|c|c|}
\hline \multirow{2}{*}{ Scenario } & \multicolumn{3}{|c|}{ Hindcast } & \multicolumn{3}{c|}{ RCP4.5 - mid } & \multicolumn{3}{c|}{ RCP8.5 - mid } & \multicolumn{3}{c|}{ RCP4.5 - end } & \multicolumn{3}{c|}{ RCP8.5 - end } \\
\cline { 2 - 13 } & Low & $\boldsymbol{R}_{\mathbf{V}}$ & High & Low & $\boldsymbol{R}_{\mathbf{V}}$ & High & Low & $\boldsymbol{R}_{\mathbf{V}}$ & High & Low & $\boldsymbol{R}_{\mathbf{V}}$ & High & Low & $\boldsymbol{R}_{\mathbf{V}}$ & High \\
\hline 2-year & 4.82 & 5.41 & 6.36 & 4.54 & 5.12 & 6.17 & 4.51 & 5.07 & 6.13 & 4.61 & 5.16 & 6.08 & 4.25 & 4.90 & 6.20 \\
5-year & 5.22 & 6.22 & 8.07 & 4.79 & 5.75 & 7.73 & 4.74 & 5.67 & 7.70 & 4.89 & 5.76 & 7.39 & 4.51 & 5.64 & 8.30 \\
10-year & 5.50 & 6.92 & 9.75 & 4.97 & 6.26 & 9.24 & 4.89 & 6.16 & 9.23 & 5.08 & 6.23 & 8.55 & 4.69 & 6.29 & 10.54 \\
20-year & 5.79 & 7.70 & 11.86 & 5.13 & 6.80 & 11.10 & 5.04 & 6.68 & 11.17 & 5.24 & 6.70 & 9.90 & 4.87 & 7.01 & 13.56 \\
50-year & 6.16 & 8.85 & 15.51 & 5.31 & 7.57 & 14.27 & 5.20 & 7.43 & 14.51 & 5.43 & 7.34 & 12.01 & 5.09 & 8.12 & 19.25 \\
100-year & 6.43 & 9.84 & 19.11 & 5.44 & 8.19 & 17.33 & 5.31 & 8.03 & 17.81 & 5.56 & 7.83 & 13.89 & 5.24 & 9.09 & 25.33 \\
\hline
\end{tabular}


Appendix A17. Table showing Palau monthly means and mean of the top 5 percent for significant wave height, peak wave period, and peak wave direction.

[Years: Hindcast $=$ 1976-2005; RCP mid = 2026-2045; RCP end = 2081-2100. Wave directions are "coming from”]

\begin{tabular}{|c|c|c|c|c|c|c|c|c|c|c|c|c|}
\hline Month & Jan & Feb & Mar & Apr & May & Jun & July & Aug & Sep & Oct & Nov & Dec \\
\hline \multicolumn{13}{|c|}{$\begin{array}{l}\text { Mean Wave Height } \\
\text { (m) }\end{array}$} \\
\hline Hindcast & $2.5 \pm 0.7$ & $2.5 \pm 0.7$ & $2.2 \pm 0.6$ & $1.7 \pm 0.5$ & $1.2 \pm 0.5$ & $1.0 \pm 0.5$ & $1.0 \pm 0.5$ & $1.0 \pm 0.6$ & $1.1 \pm 0.6$ & $1.4 \pm 0.6$ & $1.9 \pm 0.7$ & $2.3 \pm 0.7$ \\
\hline RCP4.5 - mid & $2.4 \pm 0.6$ & $2.4 \pm 0.6$ & $2.2 \pm 0.6$ & $1.8 \pm 0.5$ & $1.2 \pm 0.5$ & $1.0 \pm 0.5$ & $1.1 \pm 0.6$ & $0.98 \pm 0.5$ & $1.0 \pm 0.5$ & $1.3 \pm 0.6$ & $1.7 \pm 0.6$ & $2.2 \pm 0.7$ \\
\hline RCP8.5 - mid & $2.4 \pm 0.7$ & $2.4 \pm 0.6$ & $2.2 \pm 0.6$ & $1.7 \pm 0.5$ & $1.2 \pm 0.4$ & $1.0 \pm 0.5$ & $1.0 \pm 0.5$ & $1.0 \pm 0.6$ & $1.0 \pm 0.5$ & $1.3 \pm 0.6$ & $1.7 \pm 0.6$ & $2.2 \pm 0.7$ \\
\hline RCP4.5 - end & $2.4 \pm 0.7$ & $2.4 \pm 0.6$ & $2.2 \pm 0.6$ & $1.7 \pm 0.5$ & $1.2 \pm 0.4$ & $1.0 \pm 0.5$ & $1.1 \pm 0.5$ & $1.0 \pm 0.6$ & $1.0 \pm 0.6$ & $1.3 \pm 0.6$ & $1.7 \pm 0.6$ & $2.1 \pm 0.7$ \\
\hline RCP8.5 - end & $2.3 \pm 0.6$ & $2.3 \pm 0.6$ & $2.0 \pm 0.6$ & $1.6 \pm 0.4$ & $1.2 \pm 0.5$ & $1.0 \pm 0.5$ & $1.1 \pm 0.5$ & $1.0 \pm 0.6$ & $1.0 \pm 0.6$ & $1.2 \pm 0.5$ & $1.6 \pm 0.6$ & $2.0 \pm 0.6$ \\
\hline \multicolumn{13}{|c|}{$\begin{array}{l}\text { Top } 5 \text { percent Wave } \\
\text { Height }(\mathrm{m})\end{array}$} \\
\hline Hindcast & $4.3 \pm 0.5$ & $4.5 \pm 0.6$ & $3.9 \pm 0.6$ & $3.0 \pm 0.4$ & $2.4 \pm 0.4$ & $2.4 \pm 0.6$ & $2.7 \pm 0.5$ & $3.0 \pm 0.7$ & $3.1 \pm 0.6$ & $3.3 \pm 0.6$ & $4.0 \pm 0.8$ & $4.1 \pm 0.6$ \\
\hline RCP4.5 - mid & $4.1 \pm 0.4$ & $4.0 \pm 0.6$ & $3.8 \pm 0.6$ & $3.0 \pm 0.4$ & $2.3 \pm 0.5$ & $2.3 \pm 0.6$ & $3.0 \pm 0.6$ & $2.5 \pm 0.7$ & $2.6 \pm 0.7$ & $3.3 \pm 0.7$ & $3.5 \pm 0.7$ & $4.1 \pm 0.6$ \\
\hline RCP8.5 - mid & $4.2 \pm 0.4$ & $4.0 \pm 0.4$ & $3.8 \pm 0.5$ & $3.0 \pm 0.6$ & $2.3 \pm 0.4$ & $2.6 \pm 0.9$ & $2.7 \pm 0.6$ & $2.8 \pm 0.7$ & $2.8 \pm 0.8$ & $2.9 \pm 0.5$ & $3.3 \pm 0.4$ & $4.1 \pm 0.6$ \\
\hline RCP 4.5 - end & $4.2 \pm 0.5$ & $4.0 \pm 0.5$ & $3.6 \pm 0.6$ & $2.9 \pm 0.4$ & $2.3 \pm 0.4$ & $2.4 \pm 0.6$ & $2.8 \pm 0.7$ & $3.0 \pm 0.7$ & $3.0 \pm 0.7$ & $3.1 \pm 0.7$ & $3.3 \pm 0.4$ & $3.9 \pm 0.5$ \\
\hline \multicolumn{13}{|c|}{$\begin{array}{l}\text { Mean Wave Period } \\
(s)\end{array}$} \\
\hline Hindcast & $9 \pm 1$ & $9 \pm 1$ & $9 \pm 1$ & $9 \pm 1$ & $9 \pm 1$ & $9 \pm 1$ & $9 \pm 1$ & $9 \pm 1$ & $9 \pm 1$ & $10 \pm 2$ & $10 \pm 1$ & $10 \pm 1$ \\
\hline RCP4.5 - mid & $10 \pm 1$ & $9 \pm 1$ & $9 \pm 1$ & $9 \pm 1$ & $9 \pm 1$ & $8 \pm 1$ & $9 \pm 1$ & $9 \pm 1$ & $9 \pm 1$ & $9 \pm 1$ & $10 \pm 1$ & $10 \pm 1$ \\
\hline RCP8.5 - mid & $9 \pm 1$ & $9 \pm 2$ & $9 \pm 1$ & $9 \pm 1$ & $9 \pm 1$ & $8 \pm 1$ & $9 \pm 1$ & $9 \pm 1$ & $9 \pm 2$ & $10 \pm 2$ & $10 \pm 2$ & $10 \pm 2$ \\
\hline RCP4.5 - end & $9 \pm 1$ & $9 \pm 1$ & $9 \pm 1$ & $9 \pm 1$ & $9 \pm 1$ & $8 \pm 1$ & $8 \pm 1$ & $9 \pm 1$ & $9 \pm 1$ & $10 \pm 2$ & $10 \pm 2$ & $10 \pm 1$ \\
\hline $\begin{array}{l}\text { RCP8.5 - end } \\
\text { Top } 5 \text { percent } \\
\text { Period (s) }\end{array}$ & \multicolumn{11}{|c|}{$\begin{array}{l}\text { Top } 5 \text { percent Wave } \\
\text { Period (s) }\end{array}$} & $9 \pm 1$ \\
\hline Hindcast & $10 \pm 1$ & $10 \pm 1$ & $10 \pm 1$ & $10 \pm 1$ & $9 \pm 1$ & $8 \pm 1$ & $8 \pm 1$ & $8 \pm 1$ & $9 \pm 2$ & $10 \pm 2$ & $11 \pm 2$ & $11 \pm 2$ \\
\hline RCP4.5 - mid & $10 \pm 0.8$ & $10 \pm 0.9$ & $10 \pm 1$ & $10 \pm 1$ & $9 \pm 1$ & $7 \pm 1$ & $8 \pm 1$ & $8 \pm 1$ & $9 \pm 2$ & $9 \pm 2$ & $10 \pm 2$ & $10 \pm 1$ \\
\hline RCP8.5 - mid & $10 \pm 0.9$ & $10 \pm 1$ & $10 \pm 0.9$ & $10 \pm 1$ & $9 \pm 1$ & $8 \pm 1$ & $8 \pm 1$ & $8 \pm 1$ & $8 \pm 2$ & $9 \pm 2$ & $10 \pm 2$ & $10 \pm 1$ \\
\hline RCP4.5 - end & $10 \pm 1$ & $10 \pm 2$ & $10 \pm 0.9$ & $9 \pm 1$ & $8 \pm 1$ & $8 \pm 1$ & $8 \pm 1$ & $8 \pm 1$ & $8 \pm 2$ & $10 \pm 2$ & $10 \pm 2$ & $10 \pm 1$ \\
\hline $\begin{array}{l}\text { RCP } 8.5 \text { - end } \\
\text { Mean Wave } \\
\text { Direction ( }{ }^{\circ} \text { ) }\end{array}$ & $10 \pm 1$ & $10 \pm 0.8$ & $9 \pm 0.8$ & $9 \pm 1$ & $8 \pm 1$ & $8 \pm 1$ & $8 \pm 1$ & $8 \pm 1$ & $8 \pm 2$ & $10 \pm 2$ & $10 \pm 2$ & $10 \pm 1$ \\
\hline Hindcast & $51 \pm 18$ & $51 \pm 17$ & $57 \pm 16$ & $62 \pm 16$ & $66 \pm 24$ & $73 \pm 35$ & $84 \pm 47$ & $94 \pm 53$ & $64 \pm 52$ & $39 \pm 42$ & $43 \pm 30$ & $52 \pm 21$ \\
\hline RCP4.5 - mid & $50 \pm 19$ & $53 \pm 16$ & $57 \pm 15$ & $63 \pm 14$ & $66 \pm 18$ & $76 \pm 30$ & $92 \pm 51$ & $94 \pm 50$ & $79 \pm 51$ & $41 \pm 46$ & $43 \pm 32$ & $48 \pm 25$ \\
\hline RCP8.5 - mid & $52 \pm 18$ & $53 \pm 17$ & $58 \pm 16$ & $62 \pm 14$ & $67 \pm 17$ & $77 \pm 37$ & $88 \pm 50$ & $102 \pm 54$ & $78 \pm 55$ & $40 \pm 44$ & $43 \pm 31$ & $50 \pm 24$ \\
\hline RCP4.5 - end & $51 \pm 19$ & $52 \pm 15$ & $57 \pm 14$ & $62 \pm 16$ & $66 \pm 19$ & $76 \pm 32$ & $88 \pm 53$ & $102 \pm 55$ & $81 \pm 56$ & $38 \pm 44$ & $43 \pm 29$ & $51 \pm 21$ \\
\hline $\begin{array}{l}\text { RCP8.5 - end } \\
\text { Top } 5 \text { percent } \\
\text { Direction ( } 9 \text { ) }\end{array}$ & $51 \pm 18$ & $53 \pm 18$ & $57 \pm 15$ & $63 \pm 13$ & $68 \pm 21$ & $76 \pm 35$ & $89 \pm 49$ & $104 \pm 52$ & $86 \pm 55$ & $47 \pm 43$ & $46 \pm 30$ & $50 \pm 23$ \\
\hline Hindcast & $43 \pm 23$ & $35 \pm 27$ & $44 \pm 23$ & $57 \pm 33$ & $63 \pm 60$ & $250 \pm 109$ & $239 \pm 57$ & $235 \pm 50$ & $259 \pm 48$ & $284 \pm 73$ & $350 \pm 79$ & $46 \pm 37$ \\
\hline RCP4.5 - mid & $51 \pm 23$ & $52 \pm 25$ & $52 \pm 25$ & $64 \pm 30$ & $69 \pm 39$ & $168 \pm 97$ & $232 \pm 42$ & $229 \pm 66$ & $239 \pm 68$ & $260 \pm 75$ & $351 \pm 102$ & $43 \pm 56$ \\
\hline RCP8.5 - mid & $49 \pm 25$ & $48 \pm 27$ & $56 \pm 27$ & $57 \pm 29$ & $61 \pm 44$ & $216 \pm 71$ & $233 \pm 47$ & $231 \pm 33$ & $238 \pm 46$ & $278 \pm 71$ & $350 \pm 81$ & $51 \pm 54$ \\
\hline RCP 4.5 - end & $45 \pm 38$ & $46 \pm 17$ & $51 \pm 25$ & $59 \pm 41$ & $65 \pm 53$ & $169 \pm 99$ & $223 \pm 50$ & $235 \pm 37$ & $244 \pm 44$ & $276 \pm 76$ & $348 \pm 78$ & $48 \pm 48$ \\
\hline RCP8.5 - end & $49 \pm 22$ & $46 \pm 23$ & $53 \pm 29$ & $62 \pm 22$ & $73 \pm 53$ & $16 \pm 100$ & $220 \pm 52$ & $226 \pm 36$ & $234 \pm 46$ & $279 \pm 83$ & $349 \pm 94$ & $52 \pm 47$ \\
\hline
\end{tabular}


Appendix A18. Table showing return values of ensemble-average significant wave heights of hindcast and forecast scenarios, including lower and higher 95 percent confidence intervals, at the Palau location.

[Years: Hindcast $=1976-2005 ;$ RCP mid $=2026-2045 ;$ RCP end $=2081-2100$. Wave height values are in meters $]$

\begin{tabular}{|c|c|c|c|c|c|c|c|c|c|c|c|c|c|c|c|}
\hline \multirow{2}{*}{ Scenario } & \multicolumn{3}{|c|}{ Hindcast } & \multicolumn{3}{c|}{ RCP4.5 - mid } & \multicolumn{3}{c|}{ RCP8.5 - mid } & \multicolumn{3}{|c|}{ RCP4.5 - end } & \multicolumn{3}{c|}{ RCP8.5 - end } \\
\cline { 2 - 13 } & Low & $\boldsymbol{R}_{\boldsymbol{V}}$ & High & Low & $\boldsymbol{R}_{\boldsymbol{V}}$ & High & Low & $\boldsymbol{R}_{\boldsymbol{V}}$ & High & Low & $\boldsymbol{R}_{\boldsymbol{V}}$ & High & Low & $\boldsymbol{R}_{\boldsymbol{V}}$ & High \\
\hline 2-year & 5.61 & 6.16 & 6.98 & 5.27 & 5.85 & 6.80 & 5.20 & 6.01 & 7.62 & 5.16 & 5.74 & 6.73 & 5.17 & 5.75 & 6.75 \\
5-year & 5.96 & 6.81 & 8.19 & 5.50 & 6.35 & 7.91 & 5.44 & 6.71 & 9.63 & 5.41 & 6.31 & 8.00 & 5.38 & 6.24 & 7.89 \\
10-year & 6.19 & 7.28 & 9.19 & 5.64 & 6.69 & 8.79 & 5.59 & 7.23 & 11.51 & 5.57 & 6.72 & 9.08 & 5.50 & 6.58 & 8.82 \\
20-year & 6.39 & 7.75 & 10.26 & 5.75 & 7.01 & 9.72 & 5.71 & 7.75 & 13.75 & 5.70 & 7.12 & 10.28 & 5.60 & 6.89 & 9.82 \\
50-year & 6.62 & 8.35 & 11.82 & 5.86 & 7.39 & 11.01 & 5.84 & 8.44 & 17.41 & 5.85 & 7.63 & 12.07 & 5.70 & 7.27 & 11.24 \\
100-year & 6.77 & 8.78 & 13.10 & 5.93 & 7.66 & 12.04 & 5.92 & 8.96 & 20.82 & 5.94 & 8.01 & 13.59 & 5.76 & 7.53 & 12.39 \\
\hline
\end{tabular}


Appendix A19. Table showing Pohnpei monthly means and mean of the top 5 percent for significant wave height, peak wave period, and peak wave direction.

[Years: Hindcast $=$ 1976-2005; RCP mid = 2026-2045; RCP end = 2081-2100. Wave directions are "coming from”]

\begin{tabular}{|c|c|c|c|c|c|c|c|c|c|c|c|c|}
\hline Month & Jan & Feb & Mar & Apr & May & Jun & July & Aug & Sep & Oct & Nov & Dec \\
\hline \multicolumn{13}{|c|}{$\begin{array}{l}\text { Mean Wave Height } \\
\text { (m) }\end{array}$} \\
\hline Hindcast & $2.6 \pm 0.6$ & $2.6 \pm 0.6$ & $2.4 \pm 0.6$ & $2.0 \pm 0.6$ & $1.5 \pm 0.5$ & $1.4 \pm 0.5$ & $1.4 \pm 0.5$ & $1.3 \pm 0.5$ & $1.4 \pm 0.6$ & $1.6 \pm 0.6$ & $2.0 \pm 0.7$ & $2.5 \pm 0.7$ \\
\hline RCP4.5 - mid & $2.6 \pm 0.6$ & $2.5 \pm 0.6$ & $2.4 \pm 0.6$ & $2.0 \pm 0.5$ & $1.6 \pm 0.5$ & $1.4 \pm 0.5$ & $1.4 \pm 0.5$ & $1.3 \pm 0.6$ & $1.3 \pm 0.5$ & $1.5 \pm 0.6$ & $1.9 \pm 0.6$ & $2.4 \pm 0.7$ \\
\hline RCP8.5 - mid & $2.6 \pm 0.6$ & $2.6 \pm 0.7$ & $2.3 \pm 0.6$ & $2.0 \pm 0.6$ & $1.6 \pm 0.5$ & $1.4 \pm 0.5$ & $1.4 \pm 0.5$ & $1.4 \pm 0.6$ & $1.3 \pm 0.5$ & $1.4 \pm 0.5$ & $1.9 \pm 0.6$ & $2.4 \pm 0.7$ \\
\hline RCP4.5 - end & $2.5 \pm 0.5$ & $2.5 \pm 0.5$ & $2.4 \pm 0.6$ & $2.0 \pm 0.5$ & $1.6 \pm 0.5$ & $1.4 \pm 0.6$ & $1.4 \pm 0.5$ & $1.4 \pm 0.6$ & $1.3 \pm 0.6$ & $1.5 \pm 0.6$ & $1.8 \pm 0.6$ & $2.3 \pm 0.7$ \\
\hline RCP8.5 - end & $2.4 \pm 0.5$ & $2.4 \pm 0.5$ & $2.2 \pm 0.6$ & $1.9 \pm 0.5$ & $1.6 \pm 0.5$ & $1.4 \pm 0.5$ & $1.4 \pm 0.5$ & $1.4 \pm 0.6$ & $1.3 \pm 0.5$ & $1.4 \pm 0.5$ & $1.7 \pm 0.6$ & $2.2 \pm 0.6$ \\
\hline \multicolumn{13}{|c|}{$\begin{array}{l}\text { Top } 5 \text { percent Wave } \\
\text { Height }(\mathrm{m})\end{array}$} \\
\hline Hindcast & $4.1 \pm 0.4$ & $4.0 \pm 0.3$ & $3.9 \pm 0.4$ & $3.5 \pm 0.5$ & $2.8 \pm 0.6$ & $2.8 \pm 0.7$ & $2.9 \pm 0.8$ & $3.0 \pm 0.7$ & $3.2 \pm 0.8$ & $3.3 \pm 0.5$ & $3.8 \pm 0.4$ & $4.3 \pm 0.6$ \\
\hline RCP4.5 - mid & $4.0 \pm 0.5$ & $3.9 \pm 0.3$ & $3.7 \pm 0.4$ & $3.4 \pm 0.3$ & $2.8 \pm 0.4$ & $2.7 \pm 0.6$ & $2.9 \pm 0.6$ & $3.1 \pm 0.9$ & $2.9 \pm 0.7$ & $3.1 \pm 0.9$ & $3.6 \pm 0.6$ & $4.2 \pm 0.4$ \\
\hline RCP8.5 - mid & $3.9 \pm 0.4$ & $4.3 \pm 0.7$ & $3.8 \pm 0.5$ & $3.5 \pm 0.6$ & $2.9 \pm 0.5$ & $2.6 \pm 0.4$ & $2.9 \pm 0.6$ & $3.2 \pm 0.9$ & $2.8 \pm 0.7$ & $3.0 \pm 0.5$ & $3.7 \pm 0.5$ & $4.1 \pm 0.6$ \\
\hline RCP 4.5 - end & $3.8 \pm 0.3$ & $3.9 \pm 0.4$ & $3.7 \pm 0.4$ & $3.2 \pm 0.4$ & $2.7 \pm 0.3$ & $3.0 \pm 0.7$ & $2.9 \pm 0.7$ & $3.3 \pm 1.0$ & $3.1 \pm 0.7$ & $3.1 \pm 0.6$ & $3.6 \pm 0.6$ & $4.0 \pm 0.4$ \\
\hline \multicolumn{13}{|c|}{$\begin{array}{l}\text { Mean Wave Period } \\
(s)\end{array}$} \\
\hline Hindcast & $10 \pm 2$ & $10 \pm 2$ & $10 \pm 2$ & $9 \pm 1$ & $9 \pm 1$ & $9 \pm 1$ & $9 \pm 1$ & $9 \pm 1$ & $9 \pm 1$ & $10 \pm 2$ & $10 \pm 2$ & $10 \pm 2$ \\
\hline RCP4.5 - mid & $10 \pm 2$ & $10 \pm 2$ & $10 \pm 2$ & $9 \pm 1$ & $9 \pm 1$ & $9 \pm 1$ & $9 \pm 1$ & $9 \pm 1$ & $9 \pm 1$ & $9 \pm 1$ & $10 \pm 2$ & $10 \pm 2$ \\
\hline RCP8.5 - mid & $10 \pm 2$ & $10 \pm 2$ & $9 \pm 2$ & $9 \pm 1$ & $9 \pm 1$ & $9 \pm 1$ & $9 \pm 1$ & $9 \pm 2$ & $9 \pm 1$ & $10 \pm 1$ & $10 \pm 2$ & $10 \pm 2$ \\
\hline RCP 4.5 - end & $10 \pm 2$ & $9.9 \pm 2$ & $9.5 \pm 2$ & $9.1 \pm 1$ & $8.7 \pm 1$ & $8.7 \pm 1$ & $8.8 \pm 1$ & $9 \pm 1$ & $9 \pm 1$ & $9.6 \pm 1$ & $10 \pm 2$ & $10 \pm 2$ \\
\hline $\begin{array}{l}\text { RCP8.5 - end } \\
\text { Top } 5 \text { percent } \\
\text { Period (s) }\end{array}$ & \multicolumn{11}{|c|}{$\begin{array}{l}\text { Top } 5 \text { percent Wave } \\
\text { Period (s) }\end{array}$} & $10 \pm 2$ \\
\hline Hindcast & $10 \pm 2$ & $11 \pm 2$ & $10 \pm 1$ & $10 \pm 1$ & $9 \pm 1$ & $9 \pm 1$ & $9 \pm 2$ & $9 \pm 2$ & $9 \pm 2$ & $10 \pm 2$ & $10 \pm 1$ & $10 \pm 1$ \\
\hline RCP4.5 - mid & $10 \pm 2$ & $10 \pm 2$ & $10 \pm 1$ & $10 \pm 1$ & $9 \pm 1$ & $9 \pm 1$ & $9 \pm 1$ & $9 \pm 2$ & $9 \pm 2$ & $10 \pm 2$ & $10 \pm 2$ & $10 \pm 1$ \\
\hline RCP8.5 - mid & $10 \pm 2$ & $10 \pm 1$ & $10 \pm 1$ & $10 \pm 2$ & $9 \pm 1$ & $8 \pm 1$ & $8 \pm 1$ & $9 \pm 2$ & $9 \pm 2$ & $10 \pm 2$ & $11 \pm 2$ & $10 \pm 1$ \\
\hline RCP4.5 - end & $10 \pm 2$ & $11 \pm 2$ & $10 \pm 1$ & $9 \pm 1$ & $9 \pm 1$ & $9 \pm 1$ & $9 \pm 2$ & $9 \pm 1$ & $9 \pm 2$ & $10 \pm 2$ & $10 \pm 2$ & $10 \pm 1$ \\
\hline $\begin{array}{l}\text { RCP } 8.5 \text { - end } \\
\text { Mean Wave } \\
\text { Direction ( }{ }^{\circ} \text { ) }\end{array}$ & $10 \pm 2$ & $10 \pm 1$ & $10 \pm 1$ & $9 \pm 1$ & $8 \pm 0.8$ & $8 \pm 1$ & $9 \pm 1$ & $9 \pm 2$ & $9 \pm 2$ & $10 \pm 2$ & $10 \pm 2$ & $10 \pm 1$ \\
\hline Hindcast & $48 \pm 16$ & $47 \pm 18$ & $51 \pm 18$ & $58 \pm 18$ & $67 \pm 24$ & $84 \pm 33$ & $99 \pm 41$ & $113 \pm 47$ & $94 \pm 49$ & $55 \pm 43$ & $46 \pm 27$ & $48 \pm 17$ \\
\hline RCP4.5 - mid & $47 \pm 20$ & $48 \pm 17$ & $52 \pm 15$ & $58 \pm 15$ & $68 \pm 21$ & $83 \pm 27$ & $103 \pm 43$ & $118 \pm 46$ & $108 \pm 45$ & $63 \pm 47$ & $46 \pm 27$ & $48 \pm 23$ \\
\hline RCP8.5 - mid & $48 \pm 17$ & $49 \pm 17$ & $52 \pm 18$ & $57 \pm 17$ & $68 \pm 21$ & $84 \pm 31$ & $102 \pm 43$ & $122 \pm 51$ & $106 \pm 51$ & $62 \pm 43$ & $47 \pm 24$ & $48 \pm 18$ \\
\hline RCP4.5 - end & $47 \pm 17$ & $45 \pm 19$ & $51 \pm 16$ & $57 \pm 17$ & $67 \pm 19$ & $84 \pm 30$ & $100 \pm 46$ & $118 \pm 50$ & $107 \pm 49$ & $61 \pm 48$ & $46 \pm 26$ & $48 \pm 19$ \\
\hline $\begin{array}{l}\text { RCP8.5 - end } \\
\text { Top } 5 \text { percent } \\
\text { Direction ( } 9 \text { ) }\end{array}$ & $46 \pm 19$ & $48 \pm 19$ & $52 \pm 17$ & $59 \pm 12$ & $69 \pm 18$ & $84 \pm 27$ & $98 \pm 43$ & $121 \pm 45$ & $111 \pm 46$ & $69 \pm 41$ & $51 \pm 23$ & $50 \pm 18$ \\
\hline Hindcast & $51 \pm 15$ & $47 \pm 18$ & $47 \pm 33$ & $52 \pm 35$ & $57 \pm 54$ & $119 \pm 94$ & $223 \pm 106$ & $224 \pm 62$ & $255 \pm 80$ & $12 \pm 107$ & $50 \pm 49$ & $54 \pm 14$ \\
\hline RCP4.5 - mid & $52 \pm 24$ & $51 \pm 16$ & $53 \pm 19$ & $53 \pm 29$ & $67 \pm 49$ & $81 \pm 63$ & $213 \pm 82$ & $224 \pm 58$ & $223 \pm 61$ & $238 \pm 117$ & $46 \pm 51$ & $52 \pm 31$ \\
\hline RCP8.5 - mid & $53 \pm 15$ & $52 \pm 19$ & $55 \pm 28$ & $51 \pm 27$ & $66 \pm 53$ & $92 \pm 71$ & $219 \pm 83$ & $225 \pm 47$ & $229 \pm 53$ & $269 \pm 96$ & $43 \pm 54$ & $56 \pm 28$ \\
\hline RCP 4.5 - end & $49 \pm 14$ & $44 \pm 30$ & $54 \pm 21$ & $57 \pm 35$ & $65 \pm 26$ & $108 \pm 78$ & $234 \pm 68$ & $235 \pm 53$ & $245 \pm 48$ & $272 \pm 96$ & $36 \pm 64$ & $58 \pm 28$ \\
\hline RCP8.5 - end & $48 \pm 22$ & $53 \pm 17$ & $53 \pm 34$ & $62 \pm 16$ & $66 \pm 43$ & $79 \pm 58$ & $204 \pm 93$ & $229 \pm 46$ & $221 \pm 64$ & $28 \pm 102$ & $52 \pm 46$ & $56 \pm 17$ \\
\hline
\end{tabular}


Appendix A20. Table showing return values of ensemble-average significant wave heights of hindcast and forecast scenarios, including lower and higher 95 percent confidence intervals, at the Pohnpei location.

[Years: Hindcast $=1976-2005 ;$ RCP mid $=$ 2026-2045; RCP end $=2081-2100$. Wave height values are in meters]

\begin{tabular}{|c|c|c|c|c|c|c|c|c|c|c|c|c|c|c|c|}
\hline \multirow{2}{*}{ Scenario } & \multicolumn{3}{|c|}{ Hindcast } & \multicolumn{3}{c|}{ RCP4.5 - mid } & \multicolumn{3}{c|}{ RCP8.5 - mid } & \multicolumn{3}{c|}{ RCP4.5 - end } & \multicolumn{3}{c|}{ RCP8.5 - end } \\
\cline { 2 - 13 } & Low & $\boldsymbol{R}_{\mathbf{V}}$ & High & Low & $\boldsymbol{R}_{\mathbf{V}}$ & High & Low & $\boldsymbol{R}_{\mathbf{V}}$ & High & Low & $\boldsymbol{R}_{\mathbf{V}}$ & High & Low & $\boldsymbol{R}_{\mathbf{V}}$ & High \\
\hline 2-year & 5.41 & 5.88 & 6.60 & 5.36 & 6.04 & 7.20 & 5.12 & 5.83 & 7.24 & 5.11 & 5.86 & 7.28 & 4.97 & 5.70 & 7.11 \\
5-year & 5.79 & 6.59 & 7.90 & 5.62 & 6.64 & 8.56 & 5.42 & 6.66 & 9.54 & 5.41 & 6.65 & 9.42 & 5.20 & 6.34 & 8.88 \\
10-year & 6.07 & 7.17 & 9.11 & 5.78 & 7.06 & 9.67 & 5.63 & 7.38 & 12.01 & 5.61 & 7.31 & 11.58 & 5.34 & 6.82 & 10.51 \\
20-year & 6.34 & 7.80 & 10.54 & 5.91 & 7.45 & 10.85 & 5.83 & 8.21 & 15.36 & 5.79 & 8.02 & 14.35 & 5.46 & 7.29 & 12.45 \\
50-year & 6.70 & 8.70 & 12.86 & 6.04 & 7.92 & 12.53 & 6.09 & 9.47 & 21.69 & 6.01 & 9.05 & 19.26 & 5.59 & 7.92 & 15.58 \\
100-year & 6.95 & 9.44 & 15.01 & 6.12 & 8.25 & 13.91 & 6.27 & 10.58 & 28.49 & 6.16 & 9.91 & 24.21 & 5.67 & 8.39 & 18.47 \\
\hline
\end{tabular}


Appendix A21. Table showing Yap monthly means and mean of the top 5 percent for significant wave height, peak wave period, and peak wave direction.

[Years: Hindcast $=1976-2005 ;$ RCP mid $=$ 2026-2045; RCP end $=$ 2081-2100. Wave directions are “coming from”]

\begin{tabular}{|c|c|c|c|c|c|c|c|c|c|c|c|c|}
\hline Month & Jan & Feb & Mar & Apr & May & Jun & July & Aug & Sep & Oct & Nov & Dec \\
\hline \multicolumn{13}{|c|}{$\begin{array}{l}\text { Mean Wave Height } \\
\text { (m) }\end{array}$} \\
\hline Hindcast & $2.7 \pm 0.7$ & $2.7 \pm 0.7$ & $2.4 \pm 0.7$ & $2.0 \pm 0.6$ & $1.5 \pm 0.5$ & $1.3 \pm 0.6$ & $1.3 \pm 0.7$ & $1.3 \pm 0.8$ & $1.3 \pm 0.7$ & $1.6 \pm 0.7$ & $2.2 \pm 0.8$ & $2.6 \pm 0.7$ \\
\hline RCP4.5 - mid & $2.7 \pm 0.7$ & $2.7 \pm 0.7$ & $2.5 \pm 0.7$ & $2.0 \pm 0.6$ & $1.5 \pm 0.5$ & $1.3 \pm 0.6$ & $1.3 \pm 0.8$ & $1.2 \pm 0.6$ & $1.2 \pm 0.6$ & $1.5 \pm 0.7$ & $2.0 \pm 0.7$ & $2.5 \pm 0.8$ \\
\hline RCP8.5 - mid & $2.7 \pm 0.7$ & $2.7 \pm 0.7$ & $2.4 \pm 0.7$ & $2.0 \pm 0.5$ & $1.5 \pm 0.5$ & $1.3 \pm 0.7$ & $1.3 \pm 0.7$ & $1.3 \pm 0.8$ & $1.2 \pm 0.7$ & $1.4 \pm 0.6$ & $1.9 \pm 0.7$ & $2.5 \pm 0.7$ \\
\hline RCP4.5 - end & $2.6 \pm 0.7$ & $2.7 \pm 0.7$ & $2.4 \pm 0.7$ & $2.0 \pm 0.5$ & $1.5 \pm 0.5$ & $1.3 \pm 0.6$ & $1.3 \pm 0.7$ & $1.3 \pm 0.8$ & $1.2 \pm 0.7$ & $1.4 \pm 0.6$ & $2.0 \pm 0.7$ & $2.4 \pm 0.7$ \\
\hline RCP8.5 - end & $2.5 \pm 0.7$ & $2.5 \pm 0.7$ & $2.2 \pm 0.6$ & $1.9 \pm 0.5$ & $1.5 \pm 0.5$ & $1.3 \pm 0.5$ & $1.3 \pm 0.7$ & $1.3 \pm 0.8$ & $1.2 \pm 0.7$ & $1.3 \pm 0.5$ & $1.8 \pm 0.7$ & $2.3 \pm 0.7$ \\
\hline \multicolumn{13}{|c|}{$\begin{array}{l}\text { Top } 5 \text { percent Wave } \\
\text { Height }(\mathrm{m})\end{array}$} \\
\hline Hindcast & $4.5 \pm 0.5$ & $4.7 \pm 0.7$ & $4.3 \pm 0.7$ & $3.4 \pm 0.4$ & $2.9 \pm 0.6$ & $2.9 \pm 0.7$ & $3.5 \pm 0.7$ & $3.8 \pm 1.0$ & $3.5 \pm 0.8$ & $3.6 \pm 0.8$ & $4.4 \pm 0.9$ & $4.5 \pm 0.7$ \\
\hline RCP4.5 - mid & $4.4 \pm 0.5$ & $4.4 \pm 0.6$ & $4.2 \pm 0.6$ & $3.4 \pm 0.6$ & $2.7 \pm 0.4$ & $2.8 \pm 0.9$ & $3.7 \pm 0.8$ & $3.2 \pm 0.9$ & $3.2 \pm 0.8$ & $3.6 \pm 0.8$ & $3.9 \pm 0.7$ & $4.6 \pm 0.9$ \\
\hline RCP8.5 - mid & $4.5 \pm 0.5$ & $4.4 \pm 0.4$ & $4.2 \pm 0.7$ & $3.4 \pm 0.6$ & $2.8 \pm 0.6$ & $3.3 \pm 1.1$ & $3.4 \pm 0.7$ & $3.7 \pm 0.9$ & $3.4 \pm 0.9$ & $3.1 \pm 0.7$ & $3.7 \pm 0.6$ & $4.5 \pm 0.7$ \\
\hline RCP4.5 - end & $4.5 \pm 0.6$ & $4.3 \pm 0.5$ & $4.1 \pm 0.6$ & $3.3 \pm 0.5$ & $2.7 \pm 0.6$ & $2.8 \pm 0.7$ & $3.4 \pm 0.8$ & $3.7 \pm 0.9$ & $3.5 \pm 0.9$ & $3.4 \pm 0.8$ & $3.7 \pm 0.6$ & $4.3 \pm 0.6$ \\
\hline RCP8.5 - end & $4.4 \pm 0.7$ & $4.3 \pm 0.5$ & $3.7 \pm 0.3$ & $3.1 \pm 0.5$ & $2.7 \pm 0.5$ & $2.7 \pm 0.8$ & $3.3 \pm 0.9$ & $3.8 \pm 0.9$ & $3.3 \pm 0.9$ & $2.9 \pm 0.6$ & $3.9 \pm 0.9$ & $4.3 \pm 0.8$ \\
\hline \multicolumn{13}{|c|}{$\begin{array}{l}\text { Mean Wave Period } \\
\text { (s) }\end{array}$} \\
\hline Hindcast & $9 \pm 1$ & $9 \pm 1$ & $9 \pm 1$ & $9 \pm 1$ & $9 \pm 1$ & $9 \pm 1$ & $9 \pm 1$ & $9 \pm 1$ & $9 \pm 1$ & $10 \pm 1$ & $10 \pm 1$ & $10 \pm 1$ \\
\hline RCP4.5 - mid & $9 \pm 2$ & $9 \pm 1$ & $9 \pm 1$ & $9 \pm 1$ & $9 \pm 1$ & $9 \pm 1$ & $9 \pm 1$ & $9 \pm 1$ & $9 \pm 1$ & $9 \pm 1$ & $10 \pm 1$ & $10 \pm 1$ \\
\hline RCP8.5 - mid & $9 \pm 2$ & $9 \pm 2$ & $9 \pm 1$ & $9 \pm 1$ & $9 \pm 1$ & $8 \pm 1$ & $9 \pm 1$ & $9 \pm 1$ & $9 \pm 1$ & $10 \pm 2$ & $10 \pm 1$ & $10 \pm 2$ \\
\hline RCP4.5 - end & $9 \pm 1$ & $9 \pm 1$ & $9 \pm 1$ & $9 \pm 1$ & $9 \pm 1$ & $9 \pm 1$ & $9 \pm 1$ & $9 \pm 1$ & $9 \pm 1$ & $10 \pm 2$ & $10 \pm 2$ & $10 \pm 1$ \\
\hline \multicolumn{13}{|c|}{$\begin{array}{l}\text { Top } 5 \text { percent Wave } \\
\text { Period (s) }\end{array}$} \\
\hline Hindcast & $10 \pm 1$ & $10 \pm 1$ & $10 \pm 1$ & $10 \pm 1$ & $9 \pm 1$ & $9 \pm 1$ & $9 \pm 1$ & $9 \pm 1$ & $9 \pm 1$ & $10 \pm 2$ & $11 \pm 1$ & $10 \pm 1$ \\
\hline RCP4.5 - mid & $10 \pm 0.8$ & $10 \pm 0.7$ & $10 \pm 1$ & $10 \pm 1$ & $9 \pm 1$ & $8 \pm 1$ & $9 \pm 1$ & $8 \pm 1$ & $9 \pm 2$ & $10 \pm 2$ & $10 \pm 1$ & $10 \pm 1$ \\
\hline RCP8.5 - mid & $10 \pm 0.9$ & $10 \pm 0.9$ & $10 \pm 0.9$ & $10 \pm 1$ & $9 \pm 1$ & $8 \pm 1$ & $9 \pm 1$ & $9 \pm 1$ & $9 \pm 1$ & $10 \pm 2$ & $10 \pm 1$ & $11 \pm 1$ \\
\hline RCP4.5 - end & $10 \pm 1$ & $10 \pm 1$ & $10 \pm 0.8$ & $10 \pm 1$ & $9 \pm 0.9$ & $8 \pm 1$ & $9 \pm 1$ & $9 \pm 1$ & $9 \pm 1$ & $10 \pm 2$ & $10 \pm 2$ & $10 \pm 1$ \\
\hline $\begin{array}{l}\text { RCP8.5 - end } \\
\text { Mean Wave } \\
\text { Direction ( } 9 \text { ) }\end{array}$ & $10 \pm 1$ & $10 \pm 0.7$ & $9 \pm 0.9$ & $9 \pm 0.9$ & $9 \pm 0.8$ & $8 \pm 1$ & $8 \pm 1$ & $9 \pm 1$ & $9 \pm 1$ & $10 \pm 2$ & $10 \pm 1$ & $10 \pm 1$ \\
\hline Hindcast & $59 \pm 17$ & $59 \pm 16$ & $63 \pm 15$ & $68 \pm 15$ & $72 \pm 21$ & $77 \pm 34$ & $85 \pm 52$ & $98 \pm 61$ & $67 \pm 58$ & $48 \pm 42$ & $56 \pm 29$ & $61 \pm 19$ \\
\hline RCP4.5 - mid & $59 \pm 17$ & $60 \pm 16$ & $64 \pm 14$ & $68 \pm 11$ & $71 \pm 16$ & $81 \pm 30$ & $97 \pm 52$ & $95 \pm 62$ & $79 \pm 58$ & $48 \pm 48$ & $54 \pm 29$ & $59 \pm 23$ \\
\hline RCP8.5 - mid & $60 \pm 17$ & $61 \pm 15$ & $65 \pm 14$ & $68 \pm 13$ & $73 \pm 14$ & $81 \pm 34$ & $92 \pm 52$ & $113 \pm 64$ & $78 \pm 65$ & $47 \pm 44$ & $54 \pm 27$ & $60 \pm 22$ \\
\hline RCP4.5 - end & $60 \pm 19$ & $59 \pm 15$ & $63 \pm 13$ & $68 \pm 14$ & $72 \pm 16$ & $80 \pm 30$ & $90 \pm 56$ & $108 \pm 62$ & $83 \pm 68$ & $46 \pm 46$ & $54 \pm 28$ & $60 \pm 18$ \\
\hline $\begin{array}{l}\text { RCP8.5 - end } \\
\text { Top } 5 \text { percent } \\
\left.\text { Direction ( }{ }^{9}\right)\end{array}$ & $59 \pm 18$ & $60 \pm 16$ & $64 \pm 13$ & $69 \pm 11$ & $73 \pm 14$ & $81 \pm 34$ & $93 \pm 49$ & $111 \pm 60$ & $87 \pm 65$ & $53 \pm 43$ & $58 \pm 26$ & $61 \pm 18$ \\
\hline Hindcast & $56 \pm 20$ & $51 \pm 22$ & $52 \pm 22$ & $64 \pm 22$ & $72 \pm 54$ & $207 \pm 121$ & $237 \pm 51$ & $229 \pm 47$ & $244 \pm 57$ & $287 \pm 106$ & $50 \pm 80$ & $65 \pm 35$ \\
\hline RCP4.5 - mid & $62 \pm 16$ & $60 \pm 15$ & $58 \pm 28$ & $67 \pm 12$ & $70 \pm 37$ & $140 \pm 80$ & $224 \pm 42$ & $234 \pm 49$ & $231 \pm 66$ & $245 \pm 88$ & $52 \pm 76$ & $63 \pm 45$ \\
\hline RCP8.5 - mid & $59 \pm 16$ & $57 \pm 16$ & $61 \pm 23$ & $69 \pm 34$ & $74 \pm 35$ & $205 \pm 77$ & $232 \pm 46$ & $225 \pm 29$ & $231 \pm 40$ & $299 \pm 88$ & $55 \pm 69$ & $67 \pm 27$ \\
\hline RCP4.5 - end & $62 \pm 25$ & $53 \pm 16$ & $62 \pm 14$ & $67 \pm 35$ & $78 \pm 46$ & $142 \pm 83$ & $232 \pm 45$ & $229 \pm 40$ & $239 \pm 41$ & $267 \pm 87$ & $47 \pm 68$ & $57 \pm 30$ \\
\hline RCP8.5 - end & $60 \pm 25$ & $57 \pm 18$ & $61 \pm 18$ & $67 \pm 16$ & $77 \pm 26$ & $136 \pm 81$ & $217 \pm 42$ & $224 \pm 40$ & $227 \pm 47$ & $273 \pm 104$ & $73 \pm 76$ & $67 \pm 38$ \\
\hline
\end{tabular}


Appendix A22. Table showing return values of ensemble-average significant wave heights of hindcast and forecast scenarios, including lower and higher 95 percent confidence intervals, at the Yap location.

[Years: Hindcast $=1976-2005 ;$ RCP mid $=2026-2045 ;$ RCP end $=2081-2100$. Wave height values are in meters $]$

\begin{tabular}{|c|c|c|c|c|c|c|c|c|c|c|c|c|c|c|c|}
\hline \multirow{2}{*}{ Scenario } & \multicolumn{3}{|c|}{ Hindcast } & \multicolumn{3}{|c|}{ RCP4.5 - mid } & \multicolumn{3}{|c|}{ RCP8.5 - mid } & \multicolumn{3}{|c|}{ RCP4.5 - end } & \multicolumn{3}{|c|}{ RCP8.5 - end } \\
\hline & Low & $R_{V}$ & High & Low & $R_{v}$ & High & Low & $R_{V}$ & High & Low & $R_{v}$ & High & Low & $R_{V}$ & High \\
\hline 2-year & 6.22 & 7.05 & 8.44 & 5.85 & 6.80 & 8.74 & 5.98 & 6.84 & 8.46 & 5.88 & 6.69 & 8.12 & 6.11 & 7.05 & 8.71 \\
\hline 5-year & 6.64 & 7.98 & 10.53 & 6.15 & 7.70 & 11.47 & 6.29 & 7.65 & 10.59 & 6.20 & 7.44 & 9.93 & 6.39 & 7.73 & 10.37 \\
\hline 10-year & 6.92 & 8.71 & 12.45 & 6.35 & 8.43 & 14.23 & 6.49 & 8.28 & 12.57 & 6.39 & 7.99 & 11.52 & 6.54 & 8.17 & 11.67 \\
\hline 20-year & 7.17 & 9.47 & 14.74 & 6.52 & 9.21 & 17.79 & 6.65 & 8.91 & 14.93 & 6.55 & 8.54 & 13.33 & 6.65 & 8.56 & 13.00 \\
\hline 50-year & 7.47 & 10.51 & 18.45 & 6.71 & 10.31 & 24.10 & 6.84 & 9.75 & 18.76 & 6.73 & 9.24 & 16.10 & 6.76 & 9.00 & 14.80 \\
\hline 100-year & 7.66 & 11.33 & 21.87 & 6.84 & 11.21 & 30.48 & 6.96 & 10.40 & 22.32 & 6.84 & 9.76 & 18.54 & 6.82 & 9.28 & 16.20 \\
\hline
\end{tabular}


Appendix A23. Table showing Majuro monthly means and mean of the top 5 percent for significant wave height, peak wave period, and peak wave direction.

[Years: Hindcast $=$ 1976-2005; RCP mid $=$ 2026-2045; RCP end $=$ 2081-2100. Wave directions are “coming from”]

\begin{tabular}{|c|c|c|c|c|c|c|c|c|c|c|c|c|}
\hline Month & Jan & Feb & Mar & Apr & May & Jun & July & Aug & Sep & Oct & Nov & Dec \\
\hline \multicolumn{13}{|c|}{$\begin{array}{l}\text { Mean Wave Height } \\
\text { (m) }\end{array}$} \\
\hline Hindcast & $2.7 \pm 0.6$ & $2.6 \pm 0.6$ & $2.5 \pm 0.6$ & $2.1 \pm 0.6$ & $1.7 \pm 0.5$ & $1.6 \pm 0.4$ & $1.5 \pm 0.4$ & $1.4 \pm 0.4$ & $1.4 \pm 0.4$ & $1.6 \pm 0.5$ & $2.1 \pm 0.6$ & $2.6 \pm 0.7$ \\
\hline RCP4.5 - mid & $2.6 \pm 0.6$ & $2.6 \pm 0.6$ & $2.5 \pm 0.6$ & $2.2 \pm 0.5$ & $1.8 \pm 0.5$ & $1.6 \pm 0.5$ & $1.6 \pm 0.4$ & $1.4 \pm 0.4$ & $1.3 \pm 0.3$ & $1.5 \pm 0.4$ & $2.0 \pm 0.6$ & $2.5 \pm 0.6$ \\
\hline RCP8.5 - mid & $2.6 \pm 0.6$ & $2.6 \pm 0.6$ & $2.4 \pm 0.6$ & $2.2 \pm 0.6$ & $1.8 \pm 0.5$ & $1.6 \pm 0.5$ & $1.6 \pm 0.4$ & $1.4 \pm 0.4$ & $1.3 \pm 0.4$ & $1.5 \pm 0.4$ & $2.0 \pm 0.7$ & $2.5 \pm 0.6$ \\
\hline RCP4.5 - end & $2.5 \pm 0.5$ & $2.5 \pm 0.6$ & $2.5 \pm 0.6$ & $2.2 \pm 0.5$ & $1.8 \pm 0.5$ & $1.7 \pm 0.5$ & $1.5 \pm 0.4$ & $1.4 \pm 0.4$ & $1.3 \pm 0.4$ & $1.5 \pm 0.5$ & $1.9 \pm 0.6$ & $2.4 \pm 0.7$ \\
\hline RCP8.5 - end & $2.4 \pm 0.5$ & $2.4 \pm 0.6$ & $2.3 \pm 0.5$ & $2.1 \pm 0.5$ & $1.8 \pm 0.5$ & $1.7 \pm 0.5$ & $1.6 \pm 0.4$ & $1.4 \pm 0.4$ & $1.3 \pm 0.3$ & $1.4 \pm 0.4$ & $1.8 \pm 0.6$ & $2.3 \pm 0.6$ \\
\hline \multicolumn{13}{|c|}{$\begin{array}{l}\text { Top } 5 \text { percent Wave } \\
\text { Height }(\mathrm{m})\end{array}$} \\
\hline Hindcast & $4.1 \pm 0.5$ & $4.0 \pm 0.3$ & $3.9 \pm 0.4$ & $3.5 \pm 0.4$ & $2.8 \pm 0.3$ & $2.7 \pm 0.3$ & $2.6 \pm 0.4$ & $2.4 \pm 0.5$ & $2.4 \pm 0.5$ & $3.1 \pm 0.5$ & $3.8 \pm 0.4$ & $4.4 \pm 0.7$ \\
\hline RCP4.5 - mid & $4 \pm 0.3$ & $3.9 \pm 0.4$ & $3.9 \pm 0.4$ & $3.5 \pm 0.2$ & $2.8 \pm 0.2$ & $2.7 \pm 0.3$ & $2.6 \pm 0.3$ & $2.6 \pm 0.5$ & $2.2 \pm 0.4$ & $2.7 \pm 0.4$ & $3.5 \pm 0.5$ & $4.0 \pm 0.4$ \\
\hline RCP8.5 - mid & $4.1 \pm 0.5$ & $4.3 \pm 0.4$ & $3.9 \pm 0.3$ & $3.6 \pm 0.5$ & $2.9 \pm 0.3$ & $2.7 \pm 0.3$ & $2.7 \pm 0.5$ & $2.5 \pm 0.4$ & $2.4 \pm 0.7$ & $2.7 \pm 0.4$ & $3.8 \pm 0.6$ & $4.0 \pm 0.4$ \\
\hline RCP 4.5 - end & $3.8 \pm 0.3$ & $4.0 \pm 0.4$ & $3.9 \pm 0.4$ & $3.4 \pm 0.2$ & $2.9 \pm 0.2$ & $2.8 \pm 0.4$ & $2.7 \pm 0.5$ & $2.5 \pm 0.5$ & $2.4 \pm 0.5$ & $2.8 \pm 0.5$ & $3.5 \pm 0.7$ & $4.2 \pm 0.5$ \\
\hline RCP8.5 - end & $3.6 \pm 0.2$ & $3.8 \pm 0.4$ & $3.7 \pm 0.2$ & $3.3 \pm 0.3$ & $2.9 \pm 0.2$ & $2.8 \pm 0.3$ & $2.7 \pm 0.5$ & $2.5 \pm 0.7$ & $2.2 \pm 0.5$ & $2.7 \pm 0.5$ & $3.3 \pm 0.4$ & $3.7 \pm 0.3$ \\
\hline \multicolumn{13}{|c|}{$\begin{array}{l}\text { Mean Wave Period } \\
\text { (s) }\end{array}$} \\
\hline Hindcast & $10 \pm 2$ & $10 \pm 2$ & $10 \pm 2$ & $9 \pm 1$ & $9 \pm 1$ & $9 \pm 1$ & $9 \pm 1$ & $9 \pm 1$ & $9 \pm 1$ & $10 \pm 1$ & $10 \pm 2$ & $11 \pm 2$ \\
\hline RCP4.5 - mid & $10 \pm 2$ & $10 \pm 2$ & $10 \pm 2$ & $9 \pm 1$ & $9 \pm 1$ & $9 \pm 1$ & $9 \pm 1$ & $9 \pm 1$ & $9 \pm 1$ & $10 \pm 1$ & $10 \pm 2$ & $10 \pm 2$ \\
\hline RCP8.5 - mid & $10 \pm 2$ & $10 \pm 2$ & $10 \pm 2$ & $9 \pm 1$ & $9 \pm 1$ & $9 \pm 1$ & $9 \pm 1$ & $9 \pm 1$ & $9 \pm 2$ & $10 \pm 2$ & $10 \pm 2$ & $10 \pm 2$ \\
\hline RCP 4.5 - end & $11 \pm 2$ & $10 \pm 2$ & $9.9 \pm 2$ & $9.4 \pm 1$ & $8.9 \pm 1$ & $8.8 \pm 1$ & $8.8 \pm 1$ & $9 \pm 1$ & $9.1 \pm 1$ & $9.6 \pm 2$ & $10 \pm 2$ & $10 \pm 2$ \\
\hline \multicolumn{13}{|c|}{$\begin{array}{l}\text { Top } 5 \text { percent Wave } \\
\text { Period (s) }\end{array}$} \\
\hline Hindcast & $11 \pm 1$ & $11 \pm 2$ & $11 \pm 1$ & $10 \pm 1$ & $9 \pm 0.8$ & $9 \pm 0.8$ & $9 \pm 1$ & $9 \pm 1$ & $9 \pm 2$ & $10 \pm 2$ & $11 \pm 1$ & $11 \pm 1$ \\
\hline RCP4.5 - mid & $11 \pm 2$ & $11 \pm 2$ & $11 \pm 0.9$ & $10 \pm 0.9$ & $9 \pm 0.8$ & $9 \pm 0.8$ & $9 \pm 1$ & $9 \pm 2$ & $9 \pm 2$ & $10 \pm 2$ & $11 \pm 2$ & $10 \pm 1$ \\
\hline RCP8.5 - mid & $11 \pm 1$ & $11 \pm 1$ & $10 \pm 0.9$ & $10 \pm 0.9$ & $9 \pm 0.8$ & $9 \pm 0.7$ & $9 \pm 0.9$ & $9 \pm 1$ & $9 \pm 2$ & $11 \pm 2$ & $10 \pm 1$ & $11 \pm 1$ \\
\hline RCP4.5 - end & $10 \pm 1$ & $11 \pm 1$ & $11 \pm 1$ & $10 \pm 0.8$ & $9 \pm 0.6$ & $9 \pm 0.9$ & $9 \pm 1$ & $9 \pm 2$ & $9 \pm 2$ & $10 \pm 2$ & $11 \pm 2$ & $11 \pm 1$ \\
\hline $\begin{array}{l}\text { RCP } 8.5 \text { - end } \\
\text { Mean Wave } \\
\text { Direction ( }{ }^{\circ} \text { ) }\end{array}$ & $11 \pm 2$ & $11 \pm 1$ & $10 \pm 0.8$ & $10 \pm 0.9$ & $9 \pm 0.7$ & $9 \pm 0.8$ & $9 \pm 1$ & $9 \pm 1$ & $9 \pm 2$ & $10 \pm 2$ & $10 \pm 2$ & $11 \pm 1$ \\
\hline Hindcast & $52 \pm 15$ & $53 \pm 16$ & $57 \pm 15$ & $62 \pm 14$ & $71 \pm 15$ & $82 \pm 20$ & $90 \pm 23$ & $99 \pm 30$ & $93 \pm 36$ & $65 \pm 32$ & $52 \pm 21$ & $51 \pm 16$ \\
\hline RCP4.5 - mid & $52 \pm 15$ & $54 \pm 15$ & $57 \pm 13$ & $63 \pm 12$ & $72 \pm 14$ & $82 \pm 18$ & $90 \pm 21$ & $102 \pm 26$ & $100 \pm 27$ & $72 \pm 33$ & $53 \pm 20$ & $53 \pm 16$ \\
\hline RCP8.5 - mid & $53 \pm 15$ & $54 \pm 15$ & $57 \pm 14$ & $63 \pm 14$ & $72 \pm 14$ & $82 \pm 17$ & $91 \pm 23$ & $104 \pm 31$ & $101 \pm 34$ & $71 \pm 32$ & $51 \pm 22$ & $52 \pm 16$ \\
\hline RCP4.5 - end & $51 \pm 17$ & $51 \pm 17$ & $57 \pm 13$ & $62 \pm 12$ & $71 \pm 13$ & $81 \pm 15$ & $91 \pm 23$ & $102 \pm 33$ & $102 \pm 33$ & $70 \pm 37$ & $51 \pm 24$ & $53 \pm 17$ \\
\hline $\begin{array}{l}\text { RCP8.5 - end } \\
\text { Top } 5 \text { percent } \\
\text { Direction ( } 9 \text { ) }\end{array}$ & $52 \pm 16$ & $54 \pm 16$ & $58 \pm 13$ & $64 \pm 11$ & $73 \pm 12$ & $83 \pm 16$ & $90 \pm 24$ & $103 \pm 26$ & $103 \pm 30$ & $76 \pm 31$ & $57 \pm 20$ & $54 \pm 16$ \\
\hline Hindcast & $58 \pm 10$ & $58 \pm 12$ & $58 \pm 16$ & $61 \pm 22$ & $70 \pm 27$ & $77 \pm 33$ & $87 \pm 52$ & $117 \pm 73$ & $70 \pm 90$ & $55 \pm 62$ & $58 \pm 28$ & $56 \pm 16$ \\
\hline RCP4.5 - mid & $56 \pm 14$ & $58 \pm 13$ & $59 \pm 10$ & $62 \pm 8$ & $68 \pm 10$ & $76 \pm 37$ & $87 \pm 38$ & $128 \pm 67$ & $107 \pm 66$ & $55 \pm 76$ & $52 \pm 31$ & $57 \pm 11$ \\
\hline RCP8.5 - mid & $59 \pm 10$ & $58 \pm 10$ & $60 \pm 8$ & $66 \pm 35$ & $70 \pm 22$ & $77 \pm 30$ & $99 \pm 53$ & $128 \pm 72$ & $184 \pm 100$ & $40 \pm 69$ & $49 \pm 49$ & $56 \pm 11$ \\
\hline RCP 4.5 - end & $58 \pm 12$ & $58 \pm 9$ & $60 \pm 10$ & $64 \pm 9$ & $68 \pm 11$ & $78 \pm 28$ & $101 \pm 50$ & $139 \pm 86$ & $177 \pm 88$ & $24 \pm 103$ & $52 \pm 45$ & $58 \pm 19$ \\
\hline RCP8.5 - end & $56 \pm 14$ & $57 \pm 11$ & $63 \pm 7$ & $66 \pm 15$ & $69 \pm 7$ & $77 \pm 28$ & $86 \pm 58$ & $122 \pm 66$ & $115 \pm 88$ & $53 \pm 71$ & $55 \pm 34$ & $58 \pm 10$ \\
\hline
\end{tabular}


Appendix A24. Table showing return values of ensemble-average significant wave heights of hindcast and forecast scenarios, including lower and higher 95 percent confidence intervals, at the Majuro location.

[Years: Hindcast $=1976-2005 ;$ RCP mid $=2026-2045 ;$ RCP end $=2081-2100$. Wave height values are in meters $]$

\begin{tabular}{|c|c|c|c|c|c|c|c|c|c|c|c|c|c|c|c|}
\hline \multirow{2}{*}{ Scenario } & \multicolumn{3}{|c|}{ Hindcast } & \multicolumn{3}{c|}{ RCP4.5 - mid } & \multicolumn{3}{c|}{ RCP8.5 - mid } & \multicolumn{3}{c|}{ RCP4.5 - end } & \multicolumn{3}{c|}{ RCP8.5 - end } \\
\cline { 2 - 13 } & Low & $\boldsymbol{R}_{\boldsymbol{V}}$ & High & Low & $\boldsymbol{R}_{\boldsymbol{V}}$ & High & Low & $\boldsymbol{R}_{\boldsymbol{V}}$ & High & Low & $\boldsymbol{R}_{\boldsymbol{V}}$ & High & Low & $\boldsymbol{R}_{\boldsymbol{V}}$ & High \\
\hline 2-year & 5.06 & 5.56 & 6.38 & 4.71 & 5.14 & 5.89 & 4.94 & 5.41 & 6.23 & 4.91 & 5.54 & 6.68 & 4.48 & 4.96 & 5.88 \\
5-year & 5.39 & 6.25 & 7.85 & 4.92 & 5.62 & 6.99 & 5.16 & 5.92 & 7.39 & 5.13 & 6.11 & 8.13 & 4.68 & 5.49 & 7.33 \\
10-year & 5.63 & 6.84 & 9.31 & 5.06 & 6.00 & 8.03 & 5.31 & 6.31 & 8.45 & 5.27 & 6.53 & 9.40 & 4.81 & 5.94 & 8.83 \\
20-year & 5.87 & 7.51 & 11.16 & 5.18 & 6.40 & 9.27 & 5.44 & 6.72 & 9.70 & 5.39 & 6.94 & 10.87 & 4.94 & 6.44 & 10.82 \\
50-year & 6.17 & 8.50 & 14.41 & 5.34 & 6.95 & 11.31 & 5.59 & 7.27 & 11.69 & 5.52 & 7.48 & 13.15 & 5.10 & 7.19 & 14.44 \\
100-year & 6.40 & 9.36 & 17.64 & 5.44 & 7.39 & 13.22 & 5.69 & 7.70 & 13.52 & 5.59 & 7.88 & 15.17 & 5.20 & 7.83 & 18.20 \\
\hline
\end{tabular}


Appendix A25. Table showing Enewetak monthly means and mean of the top 5 percent for significant wave height, peak wave period, and peak wave direction.

[Years: Hindcast $=$ 1976-2005; RCP mid = 2026-2045; RCP end = 2081-2100. Wave directions are "coming from”]

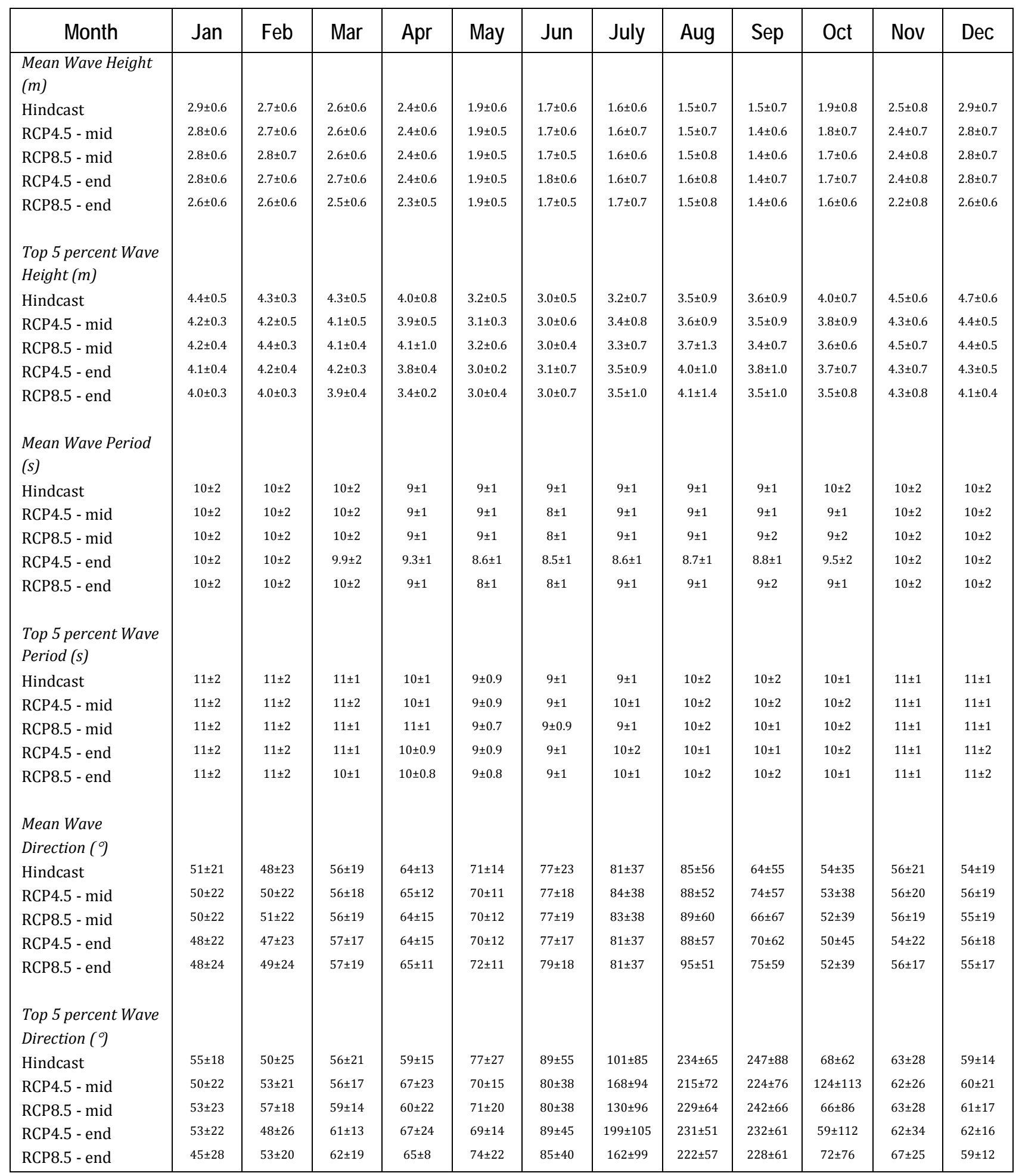


Appendix A26. Table showing return values of ensemble-average significant wave heights of hindcast and forecast scenarios, including lower and higher 95 percent confidence intervals, at the Enewetak location.

[Years: Hindcast $=1976-2005 ;$ RCP mid $=2026-2045 ;$ RCP end $=2081-2100$. Wave height values are in meters]

\begin{tabular}{|c|c|c|c|c|c|c|c|c|c|c|c|c|c|c|c|c|}
\hline \multirow{2}{*}{ Scenario } & \multicolumn{3}{|c|}{ Hindcast } & \multicolumn{3}{c|}{ RCP4.5 - mid } & \multicolumn{3}{c|}{ RCP8.5 - mid } & \multicolumn{3}{c|}{ RCP4.5 - end } & \multicolumn{3}{c|}{ RCP8.5 - end } \\
\cline { 2 - 14 } & Low & $\boldsymbol{R}_{\mathbf{V}}$ & High & Low & $\boldsymbol{R}_{\boldsymbol{V}}$ & High & Low & $\boldsymbol{R}_{\boldsymbol{V}}$ & High & Low & $\boldsymbol{R}_{\mathbf{V}}$ & High & Low & $\boldsymbol{R}_{\mathbf{V}}$ & High \\
\hline 2-year & 6.10 & 6.78 & 7.88 & 5.95 & 6.88 & 8.58 & 5.75 & 6.69 & 8.67 & 6.06 & 6.91 & 8.36 & 5.80 & 7.03 & 9.60 \\
5-year & 6.47 & 7.54 & 9.47 & 6.25 & 7.64 & 10.50 & 6.12 & 7.83 & 12.12 & 6.42 & 7.73 & 10.22 & 6.21 & 8.29 & 13.46 \\
10-year & 6.71 & 8.12 & 10.85 & 6.42 & 8.16 & 12.11 & 6.40 & 8.87 & 16.08 & 6.65 & 8.33 & 11.81 & 6.49 & 9.35 & 17.54 \\
20-year & 6.92 & 8.69 & 12.41 & 6.56 & 8.66 & 13.88 & 6.67 & 10.11 & 21.78 & 6.84 & 8.91 & 13.59 & 6.74 & 10.52 & 22.98 \\
50-year & 7.16 & 9.45 & 14.78 & 6.70 & 9.26 & 16.48 & 7.02 & 12.12 & 33.33 & 7.05 & 9.66 & 16.25 & 7.04 & 12.25 & 33.08 \\
100-year & 7.32 & 10.02 & 16.84 & 6.78 & 9.68 & 18.67 & 7.28 & 13.97 & 46.61 & 7.18 & 10.21 & 18.54 & 7.24 & 13.71 & 43.73 \\
\hline
\end{tabular}


Appendix A27. Table showing Bikini monthly means and mean of the top 5 percent for significant wave height, peak wave period, and peak wave direction.

[Years: Hindcast $=1976-2005 ;$ RCP mid $=$ 2026-2045; RCP end $=$ 2081-2100. Wave directions are “coming from”]

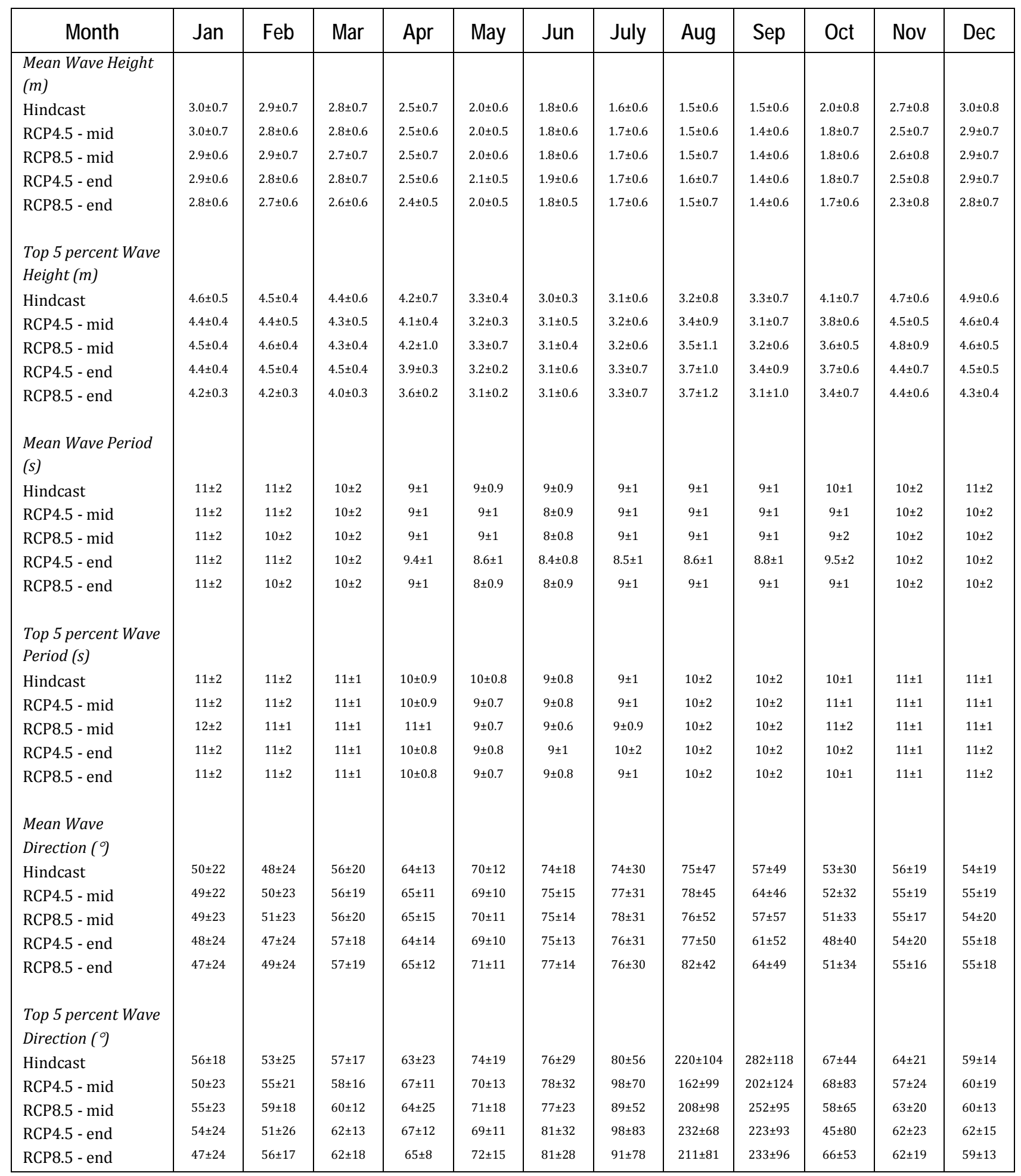


Appendix A28. Table showing return values of ensemble-average significant wave heights of hindcast and forecast scenarios, including lower and higher 95 percent confidence intervals, at the Bikini location.

[Years: Hindcast $=1976-2005 ;$ RCP mid $=2026-2045 ;$ RCP end $=2081-2100$. Wave height values are in meters $]$

\begin{tabular}{|c|c|c|c|c|c|c|c|c|c|c|c|c|c|c|c|}
\hline \multirow{2}{*}{ Scenario } & \multicolumn{3}{|c|}{ Hindcast } & \multicolumn{3}{c|}{ RCP4.5 - mid } & \multicolumn{3}{c|}{ RCP8.5 - mid } & \multicolumn{3}{|c|}{ RCP4.5 - end } & \multicolumn{3}{c|}{ RCP8.5 - end } \\
\cline { 2 - 13 } & Low & $\boldsymbol{R}_{\boldsymbol{V}}$ & High & Low & $\boldsymbol{R}_{\boldsymbol{V}}$ & High & Low & $\boldsymbol{R}_{\boldsymbol{V}}$ & High & Low & $\boldsymbol{R}_{\boldsymbol{V}}$ & High & Low & $\boldsymbol{R}_{\mathbf{V}}$ & High \\
\hline 2-year & 6.10 & 6.73 & 7.74 & 5.51 & 6.21 & 7.66 & 5.77 & 6.66 & 8.54 & 5.80 & 6.54 & 7.93 & 5.61 & 6.52 & 8.39 \\
5-year & 6.46 & 7.47 & 9.28 & 5.72 & 6.84 & 9.61 & 6.15 & 7.83 & 12.11 & 6.06 & 7.22 & 9.71 & 5.98 & 7.62 & 11.64 \\
10-year & 6.70 & 8.04 & 10.64 & 5.85 & 7.34 & 11.56 & 6.45 & 8.96 & 16.42 & 6.22 & 7.74 & 11.33 & 6.26 & 8.63 & 15.32 \\
20-year & 6.92 & 8.63 & 12.22 & 5.96 & 7.86 & 14.03 & 6.75 & 10.36 & 22.91 & 6.36 & 8.26 & 13.24 & 6.53 & 9.82 & 20.55 \\
50-year & 7.17 & 9.42 & 14.69 & 6.08 & 8.58 & 18.34 & 7.17 & 12.74 & 36.78 & 6.52 & 8.93 & 16.28 & 6.88 & 11.73 & 31.00 \\
100-year & 7.34 & 10.03 & 16.89 & 6.16 & 9.16 & 22.63 & 7.48 & 15.05 & 53.56 & 6.61 & 9.45 & 19.05 & 7.14 & 13.47 & 42.84 \\
\hline
\end{tabular}


Appendix A29. Table showing Molokai monthly means and mean of the top 5 percent for significant wave height, peak wave period, and peak wave direction.

[Years: Hindcast $=$ 1976-2005; RCP mid = 2026-2045; RCP end = 2081-2100. Wave directions are “coming from”]

\begin{tabular}{|c|c|c|c|c|c|c|c|c|c|c|c|c|}
\hline Month & Jan & Feb & Mar & Apr & May & Jun & July & Aug & Sep & Oct & Nov & Dec \\
\hline \multicolumn{13}{|c|}{$\begin{array}{l}\text { Mean Wave Height } \\
\text { (m) }\end{array}$} \\
\hline Hindcast & $3.2 \pm 0.9$ & $3.1 \pm 0.9$ & $2.7 \pm 0.8$ & $2.4 \pm 0.6$ & $2.0 \pm 0.5$ & $2.0 \pm 0.5$ & $2.0 \pm 0.5$ & $2.0 \pm 0.5$ & $2.1 \pm 0.6$ & $2.5 \pm 0.7$ & $2.9 \pm 0.8$ & $3.1 \pm 0.8$ \\
\hline RCP4.5 - mid & $3.2 \pm 0.9$ & $3.1 \pm 0.9$ & $2.8 \pm 0.7$ & $2.4 \pm 0.6$ & $2.0 \pm 0.5$ & $1.9 \pm 0.4$ & $2.0 \pm 0.4$ & $2.0 \pm 0.5$ & $1.9 \pm 0.5$ & $2.4 \pm 0.7$ & $2.9 \pm 0.8$ & $3.1 \pm 0.8$ \\
\hline RCP8.5 - mid & $3.2 \pm 0.9$ & $3.1 \pm 0.9$ & $2.8 \pm 0.7$ & $2.4 \pm 0.6$ & $2.0 \pm 0.5$ & $1.9 \pm 0.4$ & $1.9 \pm 0.4$ & $2.0 \pm 0.5$ & $2.0 \pm 0.5$ & $2.4 \pm 0.7$ & $2.9 \pm 0.8$ & $3.1 \pm 0.8$ \\
\hline RCP4.5 - end & $3.1 \pm 0.8$ & $3.1 \pm 0.9$ & $2.8 \pm 0.7$ & $2.4 \pm 0.6$ & $2.0 \pm 0.5$ & $2.0 \pm 0.4$ & $2.0 \pm 0.5$ & $2.0 \pm 0.6$ & $2.0 \pm 0.5$ & $2.4 \pm 0.7$ & $2.8 \pm 0.8$ & $3.1 \pm 0.8$ \\
\hline RCP8.5 - end & $3.0 \pm 0.9$ & $3.0 \pm 0.8$ & $2.7 \pm 0.7$ & $2.3 \pm 0.6$ & $1.9 \pm 0.4$ & $1.9 \pm 0.4$ & $1.9 \pm 0.5$ & $1.8 \pm 0.5$ & $1.9 \pm 0.5$ & $2.3 \pm 0.7$ & $2.7 \pm 0.7$ & $3.0 \pm 0.9$ \\
\hline \multicolumn{13}{|c|}{$\begin{array}{l}\text { Top } 5 \text { percent Wave } \\
\text { Height }(\mathrm{m})\end{array}$} \\
\hline Hindcast & $5.5 \pm 0.6$ & $5.4 \pm 0.6$ & $4.6 \pm 0.4$ & $3.9 \pm 0.4$ & $3.2 \pm 0.3$ & $3.1 \pm 0.4$ & $3.2 \pm 0.4$ & $3.3 \pm 0.4$ & $3.5 \pm 0.4$ & $4.3 \pm 0.5$ & $4.8 \pm 0.5$ & $5.0 \pm 0.5$ \\
\hline RCP4.5 - mid & $5.5 \pm 0.7$ & $5.3 \pm 0.5$ & $4.6 \pm 0.5$ & $4.0 \pm 0.4$ & $3.3 \pm 0.5$ & $2.9 \pm 0.3$ & $3.1 \pm 0.5$ & $3.2 \pm 0.4$ & $3.2 \pm 0.4$ & $4.3 \pm 0.6$ & $4.8 \pm 0.4$ & $5.3 \pm 0.6$ \\
\hline RCP8.5 - mid & $5.3 \pm 0.6$ & $5.3 \pm 0.6$ & $4.6 \pm 0.5$ & $3.8 \pm 0.4$ & $3.0 \pm 0.2$ & $2.9 \pm 0.3$ & $3.1 \pm 0.5$ & $3.3 \pm 0.5$ & $3.3 \pm 0.5$ & $4.4 \pm 0.6$ & $4.7 \pm 0.4$ & $5.1 \pm 0.4$ \\
\hline RCP4.5 - end & $5.2 \pm 0.5$ & $5.5 \pm 0.6$ & $4.5 \pm 0.4$ & $3.9 \pm 0.4$ & $3.1 \pm 0.3$ & $2.9 \pm 0.4$ & $3.3 \pm 0.9$ & $3.7 \pm 0.8$ & $3.4 \pm 0.6$ & $4.1 \pm 0.7$ & $4.6 \pm 0.4$ & $5.1 \pm 0.5$ \\
\hline RCP8.5 - end & $5.2 \pm 0.4$ & $5.1 \pm 0.7$ & $4.5 \pm 0.4$ & $3.6 \pm 0.3$ & $3.0 \pm 0.3$ & $2.7 \pm 0.2$ & $3.2 \pm 0.7$ & $3.4 \pm 0.7$ & $3.2 \pm 0.6$ & $4.0 \pm 0.6$ & $4.6 \pm 0.5$ & $5.2 \pm 0.6$ \\
\hline \multicolumn{13}{|c|}{$\begin{array}{l}\text { Mean Wave Period } \\
\text { (s) }\end{array}$} \\
\hline Hindcast & $13 \pm 2$ & $12 \pm 2$ & $11 \pm 2$ & $10 \pm 3$ & $10 \pm 3$ & $10 \pm 3$ & $10 \pm 3$ & $10 \pm 3$ & $10 \pm 3$ & $10 \pm 3$ & $12 \pm 2$ & $12 \pm 2$ \\
\hline RCP4.5 - mid & $13 \pm 2$ & $13 \pm 2$ & $12 \pm 2$ & $11 \pm 3$ & $10 \pm 3$ & $10 \pm 3$ & $9 \pm 3$ & $10 \pm 3$ & $10 \pm 3$ & $11 \pm 3$ & $12 \pm 2$ & $12 \pm 2$ \\
\hline RCP8.5 - mid & $13 \pm 2$ & $12 \pm 2$ & $12 \pm 2$ & $11 \pm 3$ & $10 \pm 3$ & $10 \pm 3$ & $10 \pm 3$ & $10 \pm 3$ & $10 \pm 3$ & $11 \pm 3$ & $12 \pm 2$ & $12 \pm 2$ \\
\hline RCP4.5 - end & $13 \pm 2$ & $13 \pm 2$ & $12 \pm 2$ & $11 \pm 3$ & $11 \pm 3$ & $10 \pm 3$ & $9.7 \pm 3$ & $9.7 \pm 3$ & $10 \pm 3$ & $11 \pm 3$ & $12 \pm 3$ & $12 \pm 2$ \\
\hline RCP8.5 - end & $13 \pm 2$ & $13 \pm 2$ & $12 \pm 2$ & $11 \pm 3$ & $11 \pm 3$ & $11 \pm 4$ & $10 \pm 3$ & $10 \pm 4$ & $11 \pm 3$ & $11 \pm 3$ & $11 \pm 3$ & $12 \pm 2$ \\
\hline \multicolumn{13}{|c|}{$\begin{array}{l}\text { Top } 5 \text { percent Wave } \\
\text { Period (s) }\end{array}$} \\
\hline Hindcast & $15 \pm 2$ & $15 \pm 2$ & $13 \pm 3$ & $11 \pm 2$ & $10 \pm 3$ & $9 \pm 2$ & $9 \pm 1$ & $9 \pm 1$ & $10 \pm 2$ & $11 \pm 2$ & $13 \pm 3$ & $14 \pm 3$ \\
\hline RCP4.5 - mid & $15 \pm 2$ & $14 \pm 2$ & $13 \pm 3$ & $11 \pm 2$ & $10 \pm 3$ & $9 \pm 2$ & $9 \pm 1$ & $10 \pm 2$ & $9 \pm 2$ & $11 \pm 2$ & $13 \pm 3$ & $15 \pm 3$ \\
\hline RCP8.5 - mid & $15 \pm 2$ & $14 \pm 2$ & $13 \pm 3$ & $11 \pm 3$ & $10 \pm 3$ & $9 \pm 2$ & $9 \pm 2$ & $9 \pm 1$ & $10 \pm 2$ & $11 \pm 2$ & $13 \pm 3$ & $14 \pm 3$ \\
\hline RCP4.5 - end & $15 \pm 2$ & $15 \pm 2$ & $13 \pm 3$ & $12 \pm 3$ & $10 \pm 3$ & $9 \pm 3$ & $10 \pm 2$ & $10 \pm 2$ & $9 \pm 1$ & $11 \pm 2$ & $12 \pm 3$ & $14 \pm 3$ \\
\hline RCP8.5 - end & $15 \pm 2$ & $15 \pm 2$ & $13 \pm 3$ & $12 \pm 3$ & $10 \pm 3$ & $10 \pm 3$ & $9 \pm 2$ & $10 \pm 3$ & $10 \pm 2$ & $11 \pm 2$ & $12 \pm 3$ & $14 \pm 3$ \\
\hline \multicolumn{13}{|l|}{ Mean Wave } \\
\hline \multicolumn{13}{|l|}{ Direction ( 9 ) } \\
\hline Hindcast & $345 \pm 49$ & $343 \pm 49$ & $18 \pm 54$ & $53 \pm 46$ & $78 \pm 39$ & $90 \pm 21$ & $91 \pm 18$ & $89 \pm 25$ & $77 \pm 37$ & $55 \pm 39$ & $31 \pm 45$ & $9 \pm 51$ \\
\hline RCP4.5 - mid & $343 \pm 47$ & $349 \pm 50$ & $17 \pm 54$ & $53 \pm 44$ & $77 \pm 41$ & $91 \pm 24$ & $90 \pm 17$ & $90 \pm 25$ & $81 \pm 35$ & $55 \pm 43$ & $30 \pm 44$ & $5 \pm 48$ \\
\hline RCP8.5 - mid & $348 \pm 47$ & $348 \pm 48$ & $12 \pm 53$ & $49 \pm 46$ & $78 \pm 39$ & $92 \pm 25$ & $92 \pm 21$ & $92 \pm 25$ & $81 \pm 38$ & $51 \pm 44$ & $27 \pm 43$ & $10 \pm 50$ \\
\hline RCP4.5 - end & $342 \pm 47$ & $343 \pm 49$ & $15 \pm 51$ & $44 \pm 48$ & $75 \pm 41$ & $93 \pm 24$ & $93 \pm 21$ & $93 \pm 26$ & $82 \pm 38$ & $56 \pm 41$ & $31 \pm 42$ & $7 \pm 50$ \\
\hline RCP8.5 - end & $344 \pm 46$ & $341 \pm 45$ & $10 \pm 52$ & $52 \pm 44$ & $77 \pm 43$ & $95 \pm 23$ & $94 \pm 19$ & $95 \pm 27$ & $84 \pm 38$ & $57 \pm 40$ & $37 \pm 41$ & $6 \pm 49$ \\
\hline \multirow{2}{*}{\multicolumn{13}{|c|}{$\begin{array}{l}\text { Top } 5 \text { percent Wave } \\
\text { Direction }(9)\end{array}$}} \\
\hline & & & & & & & & & & & & \\
\hline Hindcast & $323 \pm 35$ & $324 \pm 39$ & $21 \pm 55$ & $48 \pm 43$ & $70 \pm 31$ & $85 \pm 11$ & $85 \pm 15$ & $83 \pm 26$ & $70 \pm 37$ & $57 \pm 42$ & $30 \pm 52$ & $352 \pm 49$ \\
\hline RCP4.5 - mid & $321 \pm 30$ & $332 \pm 46$ & $359 \pm 56$ & $58 \pm 33$ & $71 \pm 30$ & $85 \pm 13$ & $88 \pm 19$ & $83 \pm 26$ & $81 \pm 34$ & $62 \pm 41$ & $28 \pm 51$ & $343 \pm 45$ \\
\hline RCP8.5 - mid & $331 \pm 45$ & $331 \pm 44$ & $2 \pm 55$ & $46 \pm 41$ & $72 \pm 26$ & $84 \pm 13$ & $87 \pm 20$ & $90 \pm 37$ & $77 \pm 37$ & $58 \pm 40$ & $19 \pm 44$ & $4 \pm 57$ \\
\hline RCP4.5 - end & $319 \pm 28$ & $326 \pm 43$ & $359 \pm 52$ & $29 \pm 47$ & $74 \pm 23$ & $93 \pm 20$ & $89 \pm 22$ & $101 \pm 52$ & $86 \pm 48$ & $56 \pm 49$ & $40 \pm 44$ & $349 \pm 54$ \\
\hline RCP8.5 - end & $327 \pm 39$ & $321 \pm 32$ & $353 \pm 50$ & $42 \pm 41$ & $65 \pm 37$ & $88 \pm 19$ & $92 \pm 24$ & $86 \pm 34$ & $84 \pm 57$ & $61 \pm 51$ & $36 \pm 46$ & $7 \pm 57$ \\
\hline
\end{tabular}


Appendix A30. Table showing return values of ensemble-average significant wave heights of hindcast and forecast scenarios, including lower and higher 95 percent confidence intervals, at the Molokai location.

[Years: Hindcast $=1976-2005 ;$ RCP mid $=$ 2026-2045; RCP end $=2081-2100$. Wave height values are in meters]

\begin{tabular}{|c|c|c|c|c|c|c|c|c|c|c|c|c|c|c|c|}
\hline \multirow{2}{*}{ Scenario } & \multicolumn{3}{|c|}{ Hindcast } & \multicolumn{3}{c|}{ RCP4.5 - mid } & \multicolumn{3}{c|}{ RCP8.5 - mid } & \multicolumn{3}{c|}{ RCP4.5 - end } & \multicolumn{3}{c|}{ RCP8.5 - end } \\
\cline { 2 - 14 } & Low & $\boldsymbol{R}_{\mathbf{V}}$ & High & Low & $\boldsymbol{R}_{\mathbf{V}}$ & High & Low & $\boldsymbol{R}_{\mathbf{V}}$ & High & Low & $\boldsymbol{R}_{\mathbf{V}}$ & High & Low & $\boldsymbol{R}_{\mathbf{V}}$ & High \\
\hline 2-year & 6.65 & 7.11 & 7.83 & 6.59 & 7.26 & 8.46 & 6.45 & 7.01 & 7.97 & 6.47 & 7.06 & 8.16 & 6.36 & 6.83 & 7.62 \\
5-year & 6.95 & 7.69 & 8.93 & 6.86 & 7.91 & 10.08 & 6.65 & 7.47 & 9.01 & 6.65 & 7.55 & 9.43 & 6.51 & 7.17 & 8.41 \\
10-year & 7.15 & 8.13 & 9.89 & 7.03 & 8.41 & 11.56 & 6.77 & 7.78 & 9.84 & 6.76 & 7.89 & 10.52 & 6.60 & 7.40 & 9.00 \\
20-year & 7.33 & 8.57 & 10.96 & 7.18 & 8.92 & 13.31 & 6.86 & 8.06 & 10.70 & 6.85 & 8.22 & 11.73 & 6.66 & 7.59 & 9.59 \\
50-year & 7.54 & 9.16 & 12.58 & 7.34 & 9.60 & 16.10 & 6.95 & 8.39 & 11.88 & 6.94 & 8.62 & 13.53 & 6.72 & 7.81 & 10.37 \\
100-year & 7.69 & 9.61 & 13.98 & 7.45 & 10.12 & 18.66 & 7.00 & 8.61 & 12.82 & 6.99 & 8.90 & 15.08 & 6.75 & 7.94 & 10.95 \\
\hline
\end{tabular}


Appendix A31. Table showing Northwest Hawaiian Islands monthly means and mean of the top 5 percent for significant wave height, peak wave period, and peak wave direction.

[Years: Hindcast $=1976-2005 ;$ RCP mid $=$ 2026-2045; RCP end = 2081-2100. Wave directions are "coming from”]

\begin{tabular}{|c|c|c|c|c|c|c|c|c|c|c|c|c|}
\hline Month & Jan & Feb & Mar & Apr & May & Jun & July & Aug & Sep & Oct & Nov & Dec \\
\hline \multicolumn{13}{|c|}{$\begin{array}{l}\text { Mean Wave Height } \\
\text { (m) }\end{array}$} \\
\hline Hindcast & $3.9 \pm 1.3$ & $3.7 \pm 1.3$ & $3.1 \pm 1.0$ & $2.5 \pm 0.8$ & $2.0 \pm 0.5$ & $2.0 \pm 0.5$ & $2.1 \pm 0.6$ & $2.1 \pm 0.6$ & $2.2 \pm 0.7$ & $2.7 \pm 0.9$ & $3.2 \pm 1.0$ & $3.6 \pm 1.1$ \\
\hline RCP4.5 - mid & $3.9 \pm 1.3$ & $3.7 \pm 1.2$ & $3.2 \pm 1.0$ & $2.6 \pm 0.7$ & $2.0 \pm 0.5$ & $1.9 \pm 0.5$ & $2.1 \pm 0.5$ & $2.0 \pm 0.7$ & $2.1 \pm 0.7$ & $2.6 \pm 0.9$ & $3.1 \pm 1.0$ & $3.6 \pm 1.2$ \\
\hline RCP8.5 - mid & $3.8 \pm 1.2$ & $3.6 \pm 1.2$ & $3.1 \pm 1.0$ & $2.4 \pm 0.7$ & $2.0 \pm 0.5$ & $1.9 \pm 0.5$ & $2.0 \pm 0.6$ & $2.1 \pm 0.7$ & $2.1 \pm 0.7$ & $2.7 \pm 0.9$ & $3.1 \pm 1.0$ & $3.5 \pm 1.2$ \\
\hline RCP4.5 - end & $3.8 \pm 1.2$ & $3.7 \pm 1.3$ & $3.1 \pm 1.0$ & $2.5 \pm 0.8$ & $2.0 \pm 0.5$ & $1.9 \pm 0.4$ & $2.0 \pm 0.5$ & $2.0 \pm 0.7$ & $2.1 \pm 0.6$ & $2.6 \pm 0.9$ & $3.0 \pm 0.9$ & $3.5 \pm 1.2$ \\
\hline RCP8.5 - end & $3.7 \pm 1.3$ & $3.5 \pm 1.2$ & $3.0 \pm 1.0$ & $2.4 \pm 0.7$ & $1.9 \pm 0.5$ & $1.8 \pm 0.4$ & $1.9 \pm 0.5$ & $1.9 \pm 0.6$ & $2.0 \pm 0.6$ & $2.5 \pm 0.8$ & $2.9 \pm 0.9$ & $3.4 \pm 1.1$ \\
\hline \multicolumn{13}{|c|}{$\begin{array}{l}\text { Top } 5 \text { percent Wave } \\
\text { Height }(\mathrm{m})\end{array}$} \\
\hline Hindcast & $7.4 \pm 1.0$ & $7.1 \pm 0.8$ & $5.6 \pm 0.6$ & $4.5 \pm 0.6$ & $3.4 \pm 0.4$ & $3.2 \pm 0.5$ & $3.7 \pm 0.7$ & $3.9 \pm 0.8$ & $4.2 \pm 0.6$ & $5.1 \pm 0.6$ & $5.7 \pm 0.7$ & $6.7 \pm 0.7$ \\
\hline RCP4.5 - mid & $7.3 \pm 0.9$ & $6.9 \pm 0.8$ & $5.8 \pm 0.7$ & $4.4 \pm 0.6$ & $3.4 \pm 0.4$ & $3.1 \pm 0.4$ & $3.5 \pm 0.7$ & $4.0 \pm 0.9$ & $4.2 \pm 1.0$ & $5.3 \pm 1.1$ & $5.7 \pm 0.8$ & $6.8 \pm 0.8$ \\
\hline RCP8.5 - mid & $6.9 \pm 0.8$ & $6.7 \pm 0.7$ & $5.6 \pm 0.7$ & $4.2 \pm 0.4$ & $3.4 \pm 0.6$ & $2.9 \pm 0.2$ & $3.4 \pm 0.7$ & $3.9 \pm 0.9$ & $4.4 \pm 1.1$ & $5.1 \pm 0.7$ & $5.6 \pm 0.7$ & $6.7 \pm 0.8$ \\
\hline RCP4.5 - end & $7.1 \pm 0.8$ & $7.2 \pm 1.0$ & $5.8 \pm 0.8$ & $4.5 \pm 0.5$ & $3.4 \pm 0.5$ & $2.9 \pm 0.3$ & $3.3 \pm 0.6$ & $4.3 \pm 1.2$ & $4.0 \pm 0.7$ & $5.0 \pm 0.9$ & $5.3 \pm 0.6$ & $6.6 \pm 0.8$ \\
\hline RCP8.5 - end & $7.1 \pm 0.9$ & $6.8 \pm 1.1$ & $5.6 \pm 0.7$ & $4.2 \pm 0.5$ & $3.2 \pm 0.3$ & $2.8 \pm 0.4$ & $3.2 \pm 0.6$ & $3.7 \pm 1.2$ & $3.9 \pm 0.8$ & $4.7 \pm 0.7$ & $5.2 \pm 0.6$ & $6.4 \pm 0.7$ \\
\hline \multicolumn{13}{|c|}{$\begin{array}{l}\text { Mean Wave Period } \\
\text { (s) }\end{array}$} \\
\hline Hindcast & $12 \pm 2$ & $12 \pm 2$ & $11 \pm 2$ & $10 \pm 2$ & $10 \pm 2$ & $9 \pm 2$ & $9 \pm 2$ & $9 \pm 2$ & $10 \pm 2$ & $10 \pm 2$ & $11 \pm 2$ & $12 \pm 2$ \\
\hline RCP4.5 - mid & $12 \pm 2$ & $12 \pm 2$ & $11 \pm 2$ & $10 \pm 2$ & $10 \pm 2$ & $10 \pm 2$ & $9 \pm 3$ & $9 \pm 2$ & $10 \pm 2$ & $10 \pm 2$ & $11 \pm 2$ & $12 \pm 2$ \\
\hline RCP8.5 - mid & $12 \pm 2$ & $12 \pm 2$ & $11 \pm 2$ & $10 \pm 2$ & $10 \pm 2$ & $10 \pm 2$ & $9 \pm 3$ & $10 \pm 3$ & $10 \pm 2$ & $10 \pm 2$ & $11 \pm 2$ & $12 \pm 2$ \\
\hline RCP4.5 - end & $12 \pm 2$ & $12 \pm 2$ & $11 \pm 2$ & $11 \pm 2$ & $10 \pm 2$ & $9.7 \pm 3$ & $9.5 \pm 3$ & $9.5 \pm 2$ & $9.5 \pm 2$ & $10 \pm 2$ & $11 \pm 2$ & $12 \pm 2$ \\
\hline RCP8.5 - end & $12 \pm 2$ & $12 \pm 2$ & $11 \pm 2$ & $10 \pm 2$ & $10 \pm 2$ & $10 \pm 3$ & $10 \pm 3$ & $10 \pm 3$ & $10 \pm 3$ & $10 \pm 2$ & $11 \pm 2$ & $12 \pm 2$ \\
\hline \multirow{2}{*}{\multicolumn{13}{|c|}{$\begin{array}{l}\text { Top } 5 \text { percent Wave } \\
\text { Period (s) }\end{array}$}} \\
\hline & & & & & & & & & & & & \\
\hline Hindcast & $14 \pm 2$ & $15 \pm 1$ & $14 \pm 2$ & $12 \pm 2$ & $10 \pm 2$ & $9 \pm 1$ & $9 \pm 1$ & $10 \pm 2$ & $10 \pm 1$ & $11 \pm 2$ & $13 \pm 2$ & $14 \pm 2$ \\
\hline RCP4.5 - mid & $15 \pm 2$ & $15 \pm 2$ & $14 \pm 2$ & $11 \pm 2$ & $10 \pm 2$ & $9 \pm 1$ & $9 \pm 1$ & $10 \pm 2$ & $10 \pm 2$ & $11 \pm 2$ & $13 \pm 2$ & $15 \pm 2$ \\
\hline RCP8.5 - mid & $15 \pm 2$ & $15 \pm 2$ & $14 \pm 2$ & $12 \pm 2$ & $10 \pm 2$ & $9 \pm 2$ & $9 \pm 1$ & $10 \pm 2$ & $10 \pm 1$ & $11 \pm 2$ & $13 \pm 2$ & $15 \pm 2$ \\
\hline RCP4.5 - end & $15 \pm 2$ & $15 \pm 2$ & $13 \pm 2$ & $12 \pm 2$ & $11 \pm 2$ & $9 \pm 2$ & $9 \pm 2$ & $11 \pm 2$ & $10 \pm 2$ & $11 \pm 2$ & $13 \pm 2$ & $14 \pm 2$ \\
\hline RCP8.5 - end & $15 \pm 2$ & $14 \pm 2$ & $14 \pm 2$ & $12 \pm 2$ & $10 \pm 2$ & $9 \pm 3$ & $10 \pm 2$ & $11 \pm 2$ & $10 \pm 2$ & $11 \pm 2$ & $12 \pm 2$ & $15 \pm 2$ \\
\hline \multicolumn{13}{|l|}{ Mean Wave } \\
\hline \multicolumn{13}{|l|}{ Direction ( 9 ) } \\
\hline Hindcast & $324 \pm 45$ & $322 \pm 49$ & $351 \pm 63$ & $47 \pm 58$ & $80 \pm 50$ & $102 \pm 29$ & $103 \pm 20$ & $102 \pm 30$ & $83 \pm 42$ & $53 \pm 46$ & $13 \pm 54$ & $341 \pm 53$ \\
\hline RCP4.5 - mid & $322 \pm 40$ & $325 \pm 50$ & $351 \pm 60$ & $51 \pm 56$ & $77 \pm 54$ & $103 \pm 35$ & $104 \pm 21$ & $107 \pm 26$ & $89 \pm 41$ & $56 \pm 44$ & $20 \pm 54$ & $339 \pm 50$ \\
\hline RCP8.5 - mid & $326 \pm 46$ & $327 \pm 49$ & $347 \pm 58$ & $41 \pm 61$ & $76 \pm 53$ & $102 \pm 36$ & $105 \pm 25$ & $108 \pm 33$ & $87 \pm 46$ & $57 \pm 43$ & $20 \pm 49$ & $340 \pm 50$ \\
\hline RCP4.5 - end & $320 \pm 40$ & $325 \pm 48$ & $352 \pm 59$ & $35 \pm 60$ & $74 \pm 55$ & $103 \pm 34$ & $106 \pm 23$ & $108 \pm 28$ & $87 \pm 41$ & $62 \pm 43$ & $23 \pm 52$ & $343 \pm 52$ \\
\hline RCP8.5 - end & $323 \pm 43$ & $323 \pm 44$ & $349 \pm 59$ & $49 \pm 57$ & $77 \pm 52$ & $105 \pm 31$ & $106 \pm 21$ & $110 \pm 29$ & $93 \pm 42$ & $63 \pm 42$ & $29 \pm 48$ & $344 \pm 50$ \\
\hline \multirow{2}{*}{\multicolumn{13}{|c|}{$\begin{array}{l}\text { Top } 5 \text { percent Wave } \\
\text { Direction }(9)\end{array}$}} \\
\hline & & & & & & & & & & & & \\
\hline Hindcast & $307 \pm 20$ & $306 \pm 18$ & $329 \pm 46$ & $24 \pm 58$ & $63 \pm 44$ & $94 \pm 21$ & $102 \pm 29$ & $104 \pm 54$ & $82 \pm 46$ & $43 \pm 50$ & $347 \pm 48$ & $314 \pm 26$ \\
\hline RCP4.5 - mid & $309 \pm 20$ & $309 \pm 24$ & $326 \pm 45$ & $46 \pm 59$ & $54 \pm 46$ & $93 \pm 24$ & $108 \pm 44$ & $122 \pm 48$ & $90 \pm 57$ & $53 \pm 54$ & $352 \pm 54$ & $316 \pm 28$ \\
\hline RCP8.5 - mid & $312 \pm 31$ & $311 \pm 25$ & $318 \pm 42$ & $13 \pm 61$ & $61 \pm 42$ & $93 \pm 20$ & $101 \pm 34$ & $124 \pm 63$ & $79 \pm 54$ & $51 \pm 41$ & $0 \pm 49$ & $313 \pm 25$ \\
\hline RCP4.5 - end & $307 \pm 17$ & $310 \pm 30$ & $330 \pm 48$ & $10 \pm 58$ & $66 \pm 50$ & $97 \pm 21$ & $104 \pm 41$ & $133 \pm 62$ & $93 \pm 66$ & $62 \pm 47$ & $352 \pm 56$ & $325 \pm 37$ \\
\hline RCP8.5 - end & $308 \pm 17$ & $310 \pm 23$ & $319 \pm 35$ & $23 \pm 58$ & $51 \pm 48$ & $96 \pm 23$ & $98 \pm 26$ & $132 \pm 66$ & $97 \pm 67$ & $62 \pm 52$ & $16 \pm 47$ & $328 \pm 41$ \\
\hline
\end{tabular}


Appendix A32. Table showing return values of ensemble-average significant wave heights of hindcast and forecast scenarios, including lower and higher 95 percent confidence intervals, at the Northwest Hawaiian Islands location.

[Years: Hindcast $=1976-2005 ;$ RCP mid $=$ 2026-2045; RCP end $=$ 2081-2100. Wave height values are in meters]

\begin{tabular}{|c|c|c|c|c|c|c|c|c|c|c|c|c|c|c|c|}
\hline \multirow{2}{*}{ Scenario } & \multicolumn{3}{|c|}{ Hindcast } & \multicolumn{3}{c|}{ RCP4.5 - mid } & \multicolumn{3}{c|}{ RCP8.5 - mid } & \multicolumn{3}{c|}{ RCP4.5 - end } & \multicolumn{3}{c|}{ RCP8.5 - end } \\
\cline { 2 - 14 } & Low & $\boldsymbol{R}_{\mathbf{V}}$ & High & Low & $\boldsymbol{R}_{\mathbf{V}}$ & High & Low & $\boldsymbol{R}_{\mathbf{V}}$ & High & Low & $\boldsymbol{R}_{\mathbf{V}}$ & High & Low & $\boldsymbol{R}_{\mathbf{V}}$ & High \\
\hline 2-year & 9.18 & 9.95 & 11.16 & 8.89 & 9.79 & 11.46 & 8.61 & 9.47 & 11.04 & 9.10 & 10.11 & 11.86 & 8.68 & 9.59 & 11.12 \\
5-year & 9.65 & 10.88 & 13.01 & 9.20 & 10.61 & 13.63 & 8.85 & 10.10 & 12.67 & 9.51 & 11.07 & 14.09 & 8.98 & 10.27 & 12.67 \\
10-year & 9.96 & 11.60 & 14.63 & 9.40 & 11.23 & 15.62 & 8.99 & 10.52 & 13.98 & 9.77 & 11.77 & 16.02 & 9.15 & 10.71 & 13.83 \\
20-year & 10.25 & 12.32 & 16.47 & 9.57 & 11.85 & 17.98 & 9.09 & 10.90 & 15.37 & 9.99 & 12.46 & 18.18 & 9.27 & 11.08 & 14.99 \\
50-year & 10.58 & 13.29 & 19.29 & 9.75 & 12.68 & 21.78 & 9.19 & 11.34 & 17.32 & 10.23 & 13.34 & 21.45 & 9.39 & 11.51 & 16.51 \\
100-year & 10.81 & 14.03 & 21.76 & 9.86 & 13.31 & 25.27 & 9.25 & 11.63 & 18.89 & 10.38 & 13.99 & 24.29 & 9.45 & 11.78 & 17.65 \\
\hline
\end{tabular}


Appendix A33. Table showing Guam monthly means and mean of the top 5 percent for significant wave height, peak wave period, and peak wave direction.

[Years: Hindcast $=$ 1976-2005; RCP mid = 2026-2045; RCP end = 2081-2100. Wave directions are “coming from"]

\begin{tabular}{|c|c|c|c|c|c|c|c|c|c|c|c|c|}
\hline Month & Jan & Feb & Mar & Apr & May & Jun & July & Aug & Sep & Oct & Nov & Dec \\
\hline \multicolumn{13}{|c|}{$\begin{array}{l}\text { Mean Wave Height } \\
\text { (m) }\end{array}$} \\
\hline Hindcast & $2.7 \pm 0.6$ & $2.6 \pm 0.7$ & $2.5 \pm 0.7$ & $2.2 \pm 0.6$ & $1.8 \pm 0.6$ & $1.6 \pm 0.6$ & $1.6 \pm 0.9$ & $1.6 \pm 1.0$ & $1.5 \pm 0.8$ & $1.9 \pm 0.8$ & $2.5 \pm 0.9$ & $2.7 \pm 0.7$ \\
\hline RCP4.5 - mid & $2.7 \pm 0.6$ & $2.5 \pm 0.6$ & $2.5 \pm 0.7$ & $2.2 \pm 0.6$ & $1.7 \pm 0.6$ & $1.6 \pm 0.6$ & $1.6 \pm 0.8$ & $1.5 \pm 0.8$ & $1.5 \pm 0.8$ & $1.7 \pm 0.7$ & $2.3 \pm 0.7$ & $2.6 \pm 0.7$ \\
\hline RCP8.5 - mid & $2.6 \pm 0.6$ & $2.6 \pm 0.7$ & $2.4 \pm 0.7$ & $2.1 \pm 0.6$ & $1.7 \pm 0.5$ & $1.6 \pm 0.8$ & $1.6 \pm 0.9$ & $1.6 \pm 1.0$ & $1.5 \pm 0.8$ & $1.7 \pm 0.7$ & $2.3 \pm 0.8$ & $2.7 \pm 0.7$ \\
\hline RCP4.5 - end & $2.6 \pm 0.6$ & $2.6 \pm 0.7$ & $2.5 \pm 0.6$ & $2.1 \pm 0.6$ & $1.7 \pm 0.5$ & $1.6 \pm 0.6$ & $1.6 \pm 0.9$ & $1.6 \pm 0.9$ & $1.5 \pm 0.7$ & $1.7 \pm 0.7$ & $2.3 \pm 0.8$ & $2.6 \pm 0.7$ \\
\hline RCP8.5 - end & $2.5 \pm 0.7$ & $2.4 \pm 0.6$ & $2.2 \pm 0.6$ & $2.0 \pm 0.5$ & $1.7 \pm 0.5$ & $1.6 \pm 0.7$ & $1.6 \pm 0.8$ & $1.6 \pm 1.0$ & $1.5 \pm 0.8$ & $1.6 \pm 0.6$ & $2.1 \pm 0.8$ & $2.5 \pm 0.7$ \\
\hline \multicolumn{13}{|c|}{$\begin{array}{l}\text { Top } 5 \text { percent Wave } \\
\text { Height }(\mathrm{m})\end{array}$} \\
\hline Hindcast & $4.3 \pm 0.5$ & $4.2 \pm 0.5$ & $4.3 \pm 0.9$ & $3.7 \pm 0.7$ & $3.4 \pm 0.9$ & $3.4 \pm 0.9$ & $4.4 \pm 1.0$ & $4.8 \pm 1.3$ & $4.2 \pm 1.1$ & $4.2 \pm 0.8$ & $4.9 \pm 0.9$ & $4.6 \pm 0.7$ \\
\hline RCP4.5 - mid & $4.2 \pm 0.3$ & $4.1 \pm 0.5$ & $4.1 \pm 0.8$ & $3.6 \pm 0.9$ & $3.2 \pm 0.7$ & $3.3 \pm 1.0$ & $4.2 \pm 0.9$ & $4.2 \pm 0.9$ & $4.2 \pm 1.3$ & $3.7 \pm 0.6$ & $4.3 \pm 1.0$ & $4.7 \pm 0.9$ \\
\hline RCP8.5 - mid & $4.2 \pm 0.5$ & $4.2 \pm 0.4$ & $4.1 \pm 0.6$ & $3.6 \pm 0.5$ & $3.0 \pm 0.5$ & $4.0 \pm 1.4$ & $4.3 \pm 0.9$ & $4.7 \pm 1.1$ & $4.2 \pm 1.3$ & $3.7 \pm 0.8$ & $4.3 \pm 0.8$ & $4.8 \pm 0.8$ \\
\hline RCP4.5 - end & $4.2 \pm 0.4$ & $4.3 \pm 0.4$ & $4.1 \pm 0.4$ & $3.6 \pm 0.5$ & $2.9 \pm 0.5$ & $3.2 \pm 0.9$ & $4.5 \pm 1.4$ & $4.6 \pm 1.1$ & $3.9 \pm 0.8$ & $3.7 \pm 0.8$ & $4.3 \pm 0.8$ & $4.3 \pm 0.7$ \\
\hline RCP8.5 - end & $4.2 \pm 0.5$ & $4.1 \pm 0.5$ & $3.8 \pm 0.5$ & $3.2 \pm 0.4$ & $3.0 \pm 0.6$ & $3.3 \pm 1.3$ & $4.0 \pm 0.8$ & $4.8 \pm 1.3$ & $4.1 \pm 0.9$ & $3.4 \pm 0.7$ & $4.4 \pm 0.9$ & $4.5 \pm 1.0$ \\
\hline \multicolumn{13}{|c|}{$\begin{array}{l}\text { Mean Wave Period } \\
\text { (s) }\end{array}$} \\
\hline Hindcast & $10 \pm 2$ & $10 \pm 2$ & $9 \pm 1$ & $9 \pm 1$ & $9 \pm 1$ & $9 \pm 1$ & $9 \pm 1$ & $9 \pm 1$ & $9 \pm 1$ & $10 \pm 1$ & $10 \pm 1$ & $10 \pm 2$ \\
\hline RCP4.5 - mid & $10 \pm 2$ & $9 \pm 2$ & $9 \pm 1$ & $9 \pm 1$ & $9 \pm 1$ & $8 \pm 1$ & $9 \pm 1$ & $9 \pm 1$ & $9 \pm 1$ & $9 \pm 1$ & $10 \pm 1$ & $10 \pm 2$ \\
\hline RCP8.5 - mid & $10 \pm 2$ & $10 \pm 2$ & $9 \pm 1$ & $9 \pm 1$ & $9 \pm 1$ & $9 \pm 1$ & $9 \pm 1$ & $9 \pm 1$ & $9 \pm 2$ & $9 \pm 1$ & $10 \pm 1$ & $10 \pm 2$ \\
\hline RCP4.5 - end & $9 \pm 2$ & $9 \pm 2$ & $9 \pm 1$ & $9 \pm 1$ & $9 \pm 1$ & $9 \pm 1$ & $9 \pm 1$ & $9 \pm 1$ & $9 \pm 1$ & $9 \pm 2$ & $10 \pm 2$ & $10 \pm 2$ \\
\hline RCP8.5 - end & $9 \pm 2$ & $9 \pm 2$ & $9 \pm 1$ & $9 \pm 1$ & $9 \pm 1$ & $8 \pm 1$ & $9 \pm 1$ & $9 \pm 1$ & $9 \pm 2$ & $9 \pm 1$ & $9 \pm 1$ & $9 \pm 1$ \\
\hline \multicolumn{13}{|c|}{$\begin{array}{l}\text { Top } 5 \text { percent Wave } \\
\text { Period (s) }\end{array}$} \\
\hline Hindcast & $10 \pm 1$ & $10 \pm 2$ & $10 \pm 1$ & $10 \pm 1$ & $10 \pm 1$ & $9 \pm 1$ & $10 \pm 1$ & $10 \pm 1$ & $10 \pm 1$ & $11 \pm 1$ & $11 \pm 1$ & $11 \pm 1$ \\
\hline RCP4.5 - mid & $10 \pm 1$ & $10 \pm 1$ & $10 \pm 1$ & $10 \pm 1$ & $9 \pm 0.9$ & $9 \pm 1$ & $10 \pm 1$ & $10 \pm 1$ & $10 \pm 2$ & $11 \pm 2$ & $11 \pm 1$ & $11 \pm 1$ \\
\hline RCP8.5 - mid & $10 \pm 1$ & $10 \pm 1$ & $10 \pm 1$ & $10 \pm 1$ & $9 \pm 0.9$ & $10 \pm 1$ & $10 \pm 1$ & $10 \pm 1$ & $10 \pm 1$ & $10 \pm 2$ & $11 \pm 1$ & $11 \pm 1$ \\
\hline RCP4.5 - end & $10 \pm 1$ & $10 \pm 2$ & $10 \pm 1$ & $10 \pm 0.9$ & $9 \pm 0.9$ & $9 \pm 1$ & $10 \pm 1$ & $10 \pm 1$ & $10 \pm 1$ & $10 \pm 2$ & $11 \pm 1$ & $10 \pm 1$ \\
\hline RCP8.5 - end & $10 \pm 1$ & $9 \pm 0.9$ & $10 \pm 1$ & $10 \pm 0.9$ & $9 \pm 0.8$ & $9 \pm 1$ & $10 \pm 1$ & $10 \pm 1$ & $10 \pm 1$ & $10 \pm 2$ & $11 \pm 1$ & $10 \pm 0.9$ \\
\hline \multicolumn{13}{|l|}{ Mean Wave } \\
\hline \multicolumn{13}{|l|}{ Direction ( 9 ) } \\
\hline Hindcast & $65 \pm 20$ & $63 \pm 20$ & $69 \pm 18$ & $74 \pm 13$ & $79 \pm 17$ & $84 \pm 28$ & $92 \pm 55$ & $111 \pm 76$ & $74 \pm 67$ & $62 \pm 39$ & $70 \pm 25$ & $69 \pm 19$ \\
\hline RCP4.5 - mid & $65 \pm 21$ & $64 \pm 19$ & $69 \pm 16$ & $74 \pm 10$ & $78 \pm 14$ & $85 \pm 27$ & $96 \pm 49$ & $107 \pm 82$ & $78 \pm 72$ & $59 \pm 48$ & $67 \pm 25$ & $68 \pm 21$ \\
\hline RCP8.5 - mid & $65 \pm 20$ & $65 \pm 19$ & $69 \pm 16$ & $74 \pm 13$ & $79 \pm 11$ & $88 \pm 29$ & $93 \pm 52$ & $126 \pm 78$ & $77 \pm 81$ & $59 \pm 42$ & $68 \pm 23$ & $69 \pm 20$ \\
\hline RCP4.5 - end & $65 \pm 21$ & $63 \pm 20$ & $69 \pm 15$ & $73 \pm 12$ & $80 \pm 13$ & $85 \pm 23$ & $95 \pm 54$ & $118 \pm 76$ & $77 \pm 81$ & $58 \pm 45$ & $68 \pm 21$ & $69 \pm 18$ \\
\hline RCP8.5 - end & $64 \pm 21$ & $64 \pm 20$ & $68 \pm 15$ & $75 \pm 11$ & $80 \pm 10$ & $88 \pm 28$ & $93 \pm 48$ & $116 \pm 68$ & $87 \pm 81$ & $60 \pm 46$ & $69 \pm 21$ & $70 \pm 17$ \\
\hline \multirow{2}{*}{\multicolumn{13}{|c|}{$\begin{array}{l}\text { Top } 5 \text { percent Wave } \\
\text { Direction }(9)\end{array}$}} \\
\hline & & & & & & & & & & & & \\
\hline Hindcast & $64 \pm 15$ & $57 \pm 23$ & $68 \pm 28$ & $71 \pm 24$ & $86 \pm 40$ & $134 \pm 94$ & $217 \pm 57$ & $214 \pm 50$ & $208 \pm 79$ & $83 \pm 79$ & $81 \pm 52$ & $76 \pm 23$ \\
\hline RCP4.5 - mid & $65 \pm 20$ & $63 \pm 12$ & $69 \pm 19$ & $70 \pm 14$ & $81 \pm 29$ & $116 \pm 64$ & $218 \pm 59$ & $224 \pm 48$ & $232 \pm 69$ & $88 \pm 122$ & $78 \pm 47$ & $72 \pm 28$ \\
\hline RCP8.5 - mid & $63 \pm 15$ & $64 \pm 14$ & $65 \pm 16$ & $74 \pm 17$ & $82 \pm 17$ & $154 \pm 77$ & $223 \pm 50$ & $223 \pm 34$ & $233 \pm 37$ & $89 \pm 83$ & $78 \pm 44$ & $78 \pm 25$ \\
\hline RCP4.5 - end & $67 \pm 14$ & $54 \pm 24$ & $66 \pm 13$ & $70 \pm 14$ & $85 \pm 27$ & $116 \pm 68$ & $218 \pm 60$ & $229 \pm 37$ & $239 \pm 49$ & $88 \pm 113$ & $76 \pm 41$ & $68 \pm 27$ \\
\hline RCP8.5 - end & $66 \pm 18$ & $65 \pm 16$ & $65 \pm 13$ & $73 \pm 12$ & $82 \pm 15$ & $128 \pm 68$ & $221 \pm 60$ & $220 \pm 41$ & $220 \pm 55$ & $84 \pm 96$ & $84 \pm 39$ & $79 \pm 29$ \\
\hline
\end{tabular}


Appendix A34. Table showing return values of ensemble-average significant wave heights of hindcast and forecast scenarios, including lower and higher 95 percent confidence intervals, at the Guam location.

[Years: Hindcast $=1976-2005 ;$ RCP mid $=2026-2045 ;$ RCP end $=2081-2100$. Wave height values are in meters $]$

\begin{tabular}{|c|c|c|c|c|c|c|c|c|c|c|c|c|c|c|c|}
\hline \multirow{2}{*}{ Scenario } & \multicolumn{3}{|c|}{ Hindcast } & \multicolumn{3}{c|}{ RCP4.5 - mid } & \multicolumn{3}{c|}{ RCP8.5 - mid } & \multicolumn{3}{c|}{ RCP4.5 - end } & \multicolumn{2}{c|}{ RCP8.5 - end } \\
\cline { 2 - 12 } & Low & $\boldsymbol{R}_{\boldsymbol{V}}$ & High & Low & $\boldsymbol{R}_{\boldsymbol{V}}$ & High & Low & $\boldsymbol{R}_{\boldsymbol{V}}$ & High & Low & $\boldsymbol{R}_{\boldsymbol{V}}$ & High & Low & $\boldsymbol{R}_{\boldsymbol{V}}$ & High \\
\hline 2-year & 7.34 & 8.32 & 9.84 & 6.83 & 8.05 & 10.19 & 6.73 & 7.88 & 10.04 & 6.58 & 7.80 & 10.09 & 6.67 & 8.09 & 11.01 \\
5-year & 7.89 & 9.38 & 11.88 & 7.31 & 9.20 & 12.90 & 7.19 & 9.07 & 13.11 & 6.97 & 8.82 & 12.78 & 7.09 & 9.35 & 14.83 \\
10-year & 8.22 & 10.13 & 13.54 & 7.62 & 10.04 & 15.26 & 7.49 & 10.01 & 16.07 & 7.20 & 9.56 & 15.12 & 7.35 & 10.34 & 18.54 \\
20-year & 8.51 & 10.84 & 15.30 & 7.87 & 10.87 & 17.91 & 7.76 & 11.01 & 19.73 & 7.38 & 10.26 & 17.77 & 7.57 & 11.34 & 23.14 \\
50-year & 8.83 & 11.72 & 17.78 & 8.15 & 11.92 & 21.95 & 8.08 & 12.40 & 25.91 & 7.58 & 11.14 & 21.80 & 7.81 & 12.71 & 30.98 \\
100-year & 9.03 & 12.35 & 19.79 & 8.32 & 12.70 & 25.45 & 8.28 & 13.52 & 31.86 & 7.69 & 11.77 & 25.30 & 7.96 & 13.78 & 38.60 \\
\hline
\end{tabular}


Appendix A35. Table showing Kwajalein monthly means and mean of the top 5 percent for significant wave height, peak wave period, and peak wave direction.

[Years: Hindcast $=$ 1976-2005; RCP mid = 2026-2045; RCP end = 2081-2100. Wave directions are “coming from”]

\begin{tabular}{|c|c|c|c|c|c|c|c|c|c|c|c|c|}
\hline Month & Jan & Feb & Mar & Apr & May & Jun & July & Aug & Sep & Oct & Nov & Dec \\
\hline \multicolumn{13}{|c|}{$\begin{array}{l}\text { Mean Wave Height } \\
\text { (m) }\end{array}$} \\
\hline Hindcast & $2.4 \pm 0.5$ & $2.3 \pm 0.5$ & $2.2 \pm 0.5$ & $1.9 \pm 0.5$ & $1.5 \pm 0.4$ & $1.3 \pm 0.4$ & $1.2 \pm 0.4$ & $1.1 \pm 0.4$ & $1.1 \pm 0.4$ & $1.4 \pm 0.5$ & $1.9 \pm 0.6$ & $2.3 \pm 0.6$ \\
\hline RCP4.5 - mid & $2.3 \pm 0.5$ & $2.3 \pm 0.5$ & $2.2 \pm 0.5$ & $1.9 \pm 0.5$ & $1.5 \pm 0.4$ & $1.3 \pm 0.4$ & $1.3 \pm 0.4$ & $1.1 \pm 0.5$ & $1.1 \pm 0.4$ & $1.3 \pm 0.5$ & $1.7 \pm 0.5$ & $2.2 \pm 0.6$ \\
\hline RCP8.5 - mid & $2.3 \pm 0.5$ & $2.3 \pm 0.5$ & $2.1 \pm 0.5$ & $1.9 \pm 0.5$ & $1.5 \pm 0.5$ & $1.3 \pm 0.4$ & $1.3 \pm 0.5$ & $1.2 \pm 0.5$ & $1.1 \pm 0.4$ & $1.3 \pm 0.4$ & $1.8 \pm 0.6$ & $2.2 \pm 0.6$ \\
\hline RCP4.5 - end & $2.3 \pm 0.5$ & $2.2 \pm 0.5$ & $2.2 \pm 0.5$ & $1.8 \pm 0.5$ & $1.5 \pm 0.4$ & $1.4 \pm 0.5$ & $1.2 \pm 0.4$ & $1.2 \pm 0.5$ & $1.1 \pm 0.4$ & $1.3 \pm 0.5$ & $1.7 \pm 0.6$ & $2.2 \pm 0.6$ \\
\hline RCP8.5 - end & $2.2 \pm 0.5$ & $2.2 \pm 0.5$ & $2.0 \pm 0.5$ & $1.8 \pm 0.4$ & $1.5 \pm 0.4$ & $1.4 \pm 0.4$ & $1.3 \pm 0.4$ & $1.2 \pm 0.5$ & $1.1 \pm 0.4$ & $1.2 \pm 0.4$ & $1.6 \pm 0.5$ & $2.1 \pm 0.5$ \\
\hline \multicolumn{13}{|c|}{$\begin{array}{l}\text { Top } 5 \text { percent Wave } \\
\text { Height }(\mathrm{m})\end{array}$} \\
\hline Hindcast & $3.6 \pm 0.4$ & $3.5 \pm 0.3$ & $3.4 \pm 0.5$ & $3.1 \pm 0.4$ & $2.5 \pm 0.3$ & $2.3 \pm 0.3$ & $2.4 \pm 0.4$ & $2.3 \pm 0.7$ & $2.3 \pm 0.6$ & $2.9 \pm 0.7$ & $3.5 \pm 0.5$ & $3.8 \pm 0.6$ \\
\hline RCP4.5 - mid & $3.5 \pm 0.3$ & $3.5 \pm 0.4$ & $3.3 \pm 0.3$ & $3.0 \pm 0.2$ & $2.5 \pm 0.3$ & $2.4 \pm 0.3$ & $2.4 \pm 0.4$ & $2.5 \pm 0.7$ & $2.2 \pm 0.5$ & $2.6 \pm 0.6$ & $3.2 \pm 0.4$ & $3.6 \pm 0.4$ \\
\hline RCP8.5 - mid & $3.5 \pm 0.3$ & $3.7 \pm 0.4$ & $3.3 \pm 0.3$ & $3.2 \pm 0.7$ & $2.6 \pm 0.5$ & $2.4 \pm 0.3$ & $2.5 \pm 0.5$ & $2.5 \pm 0.7$ & $2.3 \pm 0.5$ & $2.5 \pm 0.5$ & $3.5 \pm 0.6$ & $3.5 \pm 0.4$ \\
\hline RCP 4.5 - end & $3.4 \pm 0.3$ & $3.4 \pm 0.3$ & $3.4 \pm 0.3$ & $2.9 \pm 0.2$ & $2.5 \pm 0.2$ & $2.4 \pm 0.4$ & $2.4 \pm 0.7$ & $2.6 \pm 0.8$ & $2.5 \pm 0.6$ & $2.6 \pm 0.5$ & $3.2 \pm 0.6$ & $3.5 \pm 0.4$ \\
\hline \multicolumn{13}{|c|}{$\begin{array}{l}\text { Mean Wave Period } \\
\text { (s) }\end{array}$} \\
\hline Hindcast & $11 \pm 2$ & $10 \pm 2$ & $10 \pm 2$ & $9 \pm 2$ & $9 \pm 1$ & $9 \pm 2$ & $9 \pm 2$ & $9 \pm 2$ & $9 \pm 2$ & $10 \pm 2$ & $10 \pm 2$ & $11 \pm 2$ \\
\hline RCP4.5 - mid & $11 \pm 2$ & $10 \pm 2$ & $10 \pm 2$ & $9 \pm 1$ & $9 \pm 1$ & $9 \pm 2$ & $9 \pm 2$ & $9 \pm 2$ & $9 \pm 2$ & $10 \pm 2$ & $10 \pm 2$ & $10 \pm 2$ \\
\hline RCP8.5 - mid & $11 \pm 2$ & $10 \pm 2$ & $10 \pm 2$ & $9 \pm 2$ & $9 \pm 1$ & $9 \pm 2$ & $9 \pm 1$ & $9 \pm 2$ & $9 \pm 2$ & $10 \pm 2$ & $10 \pm 2$ & $11 \pm 2$ \\
\hline RCP 4.5 - end & $11 \pm 2$ & $11 \pm 2$ & $10 \pm 2$ & $9.5 \pm 2$ & $8.9 \pm 1$ & $8.7 \pm 1$ & $8.9 \pm 1$ & $9.1 \pm 2$ & $9.2 \pm 2$ & $9.8 \pm 2$ & $10 \pm 2$ & $11 \pm 2$ \\
\hline $\begin{array}{l}\text { RCP8.5 - end } \\
\text { Top } 5 \text { percent } \\
\text { Period (s) }\end{array}$ & \multicolumn{11}{|c|}{$\begin{array}{l}\text { Top } 5 \text { percent Wave } \\
\text { Period (s) }\end{array}$} & $10 \pm 2$ \\
\hline Hindcast & $11 \pm 2$ & $11 \pm 2$ & $10 \pm 2$ & $10 \pm 1$ & $9 \pm 1$ & $8 \pm 1$ & $9 \pm 1$ & $9 \pm 2$ & $9 \pm 2$ & $10 \pm 2$ & $10 \pm 1$ & $11 \pm 1$ \\
\hline RCP4.5 - mid & $11 \pm 3$ & $11 \pm 2$ & $10 \pm 2$ & $10 \pm 1$ & $9 \pm 1$ & $8 \pm 1$ & $8 \pm 1$ & $9 \pm 2$ & $9 \pm 2$ & $10 \pm 2$ & $10 \pm 2$ & $10 \pm 2$ \\
\hline RCP8.5 - mid & $11 \pm 2$ & $11 \pm 2$ & $10 \pm 1$ & $10 \pm 1$ & $9 \pm 1$ & $8 \pm 1$ & $8 \pm 1$ & $8 \pm 2$ & $9 \pm 2$ & $10 \pm 2$ & $10 \pm 2$ & $11 \pm 1$ \\
\hline RCP4.5 - end & $11 \pm 2$ & $11 \pm 2$ & $10 \pm 1$ & $10 \pm 1$ & $9 \pm 1$ & $8 \pm 1$ & $9 \pm 2$ & $9 \pm 2$ & $9 \pm 2$ & $10 \pm 2$ & $10 \pm 2$ & $10 \pm 2$ \\
\hline $\begin{array}{l}\text { RCP } 8.5 \text { - end } \\
\text { Mean Wave } \\
\text { Direction ( }{ }^{\circ} \text { ) }\end{array}$ & $11 \pm 3$ & $10 \pm 2$ & $10 \pm 2$ & $9 \pm 1$ & $9 \pm 1$ & $8 \pm 1$ & $8 \pm 1$ & $9 \pm 2$ & $9 \pm 2$ & $9 \pm 2$ & $10 \pm 2$ & $11 \pm 2$ \\
\hline Hindcast & $50 \pm 17$ & $49 \pm 18$ & $55 \pm 16$ & $61 \pm 15$ & $69 \pm 18$ & $80 \pm 25$ & $89 \pm 32$ & $100 \pm 43$ & $85 \pm 49$ & $56 \pm 37$ & $49 \pm 22$ & $49 \pm 17$ \\
\hline RCP4.5 - mid & $49 \pm 17$ & $50 \pm 18$ & $55 \pm 15$ & $62 \pm 14$ & $69 \pm 15$ & $80 \pm 21$ & $90 \pm 33$ & $104 \pm 39$ & $95 \pm 41$ & $60 \pm 39$ & $48 \pm 22$ & $51 \pm 18$ \\
\hline RCP8.5 - mid & $49 \pm 17$ & $51 \pm 17$ & $54 \pm 16$ & $62 \pm 16$ & $69 \pm 16$ & $79 \pm 20$ & $92 \pm 33$ & $108 \pm 46$ & $95 \pm 51$ & $60 \pm 38$ & $48 \pm 22$ & $50 \pm 17$ \\
\hline RCP4.5 - end & $48 \pm 19$ & $48 \pm 19$ & $55 \pm 15$ & $61 \pm 15$ & $68 \pm 14$ & $79 \pm 19$ & $90 \pm 33$ & $104 \pm 47$ & $98 \pm 49$ & $56 \pm 44$ & $47 \pm 24$ & $51 \pm 18$ \\
\hline $\begin{array}{l}\text { RCP8.5 - end } \\
\text { Top } 5 \text { percent } \\
\text { Direction ( } 9 \text { ) }\end{array}$ & $48 \pm 19$ & $50 \pm 19$ & $56 \pm 15$ & $62 \pm 11$ & $71 \pm 14$ & $81 \pm 20$ & $88 \pm 33$ & $105 \pm 38$ & $98 \pm 43$ & $63 \pm 38$ & $51 \pm 20$ & $51 \pm 16$ \\
\hline Hindcast & $55 \pm 14$ & $52 \pm 17$ & $54 \pm 19$ & $59 \pm 28$ & $69 \pm 31$ & $73 \pm 32$ & $90 \pm 67$ & $191 \pm 103$ & $267 \pm 110$ & $67 \pm 68$ & $59 \pm 32$ & $54 \pm 18$ \\
\hline RCP4.5 - mid & $49 \pm 18$ & $57 \pm 17$ & $56 \pm 12$ & $62 \pm 18$ & $69 \pm 18$ & $74 \pm 40$ & $93 \pm 56$ & $172 \pm 89$ & $194 \pm 127$ & $47 \pm 105$ & $49 \pm 28$ & $55 \pm 17$ \\
\hline RCP8.5 - mid & $54 \pm 16$ & $57 \pm 13$ & $57 \pm 11$ & $62 \pm 34$ & $73 \pm 26$ & $77 \pm 33$ & $111 \pm 65$ & $192 \pm 83$ & $239 \pm 94$ & $34 \pm 90$ & $52 \pm 45$ & $54 \pm 13$ \\
\hline RCP4.5 - end & $51 \pm 20$ & $53 \pm 16$ & $57 \pm 12$ & $62 \pm 17$ & $66 \pm 9$ & $79 \pm 37$ & $113 \pm 75$ & $209 \pm 74$ & $214 \pm 78$ & $355 \pm 109$ & $49 \pm 45$ & $57 \pm 18$ \\
\hline RCP8.5 - end & $49 \pm 17$ & $55 \pm 12$ & $60 \pm 15$ & $62 \pm 12$ & $70 \pm 15$ & $80 \pm 44$ & $86 \pm 75$ & $194 \pm 78$ & $170 \pm 133$ & $67 \pm 75$ & $55 \pm 27$ & $54 \pm 13$ \\
\hline
\end{tabular}


Appendix A36. Table showing return values of ensemble-average significant wave heights of hindcast and forecast scenarios, including lower and higher 95 percent confidence intervals, at the Kwajalein location.

[Years: Hindcast $=1976-2005 ;$ RCP mid $=$ 2026-2045; RCP end $=2081-2100$. Wave height values are in meters]

\begin{tabular}{|c|c|c|c|c|c|c|c|c|c|c|c|c|c|c|c|}
\hline \multirow{2}{*}{ Scenario } & \multicolumn{3}{|c|}{ Hindcast } & \multicolumn{3}{c|}{ RCP4.5 - mid } & \multicolumn{3}{c|}{ RCP8.5 - mid } & \multicolumn{3}{c|}{ RCP4.5 - end } & \multicolumn{3}{c|}{ RCP8.5 - end } \\
\cline { 2 - 14 } & Low & $\boldsymbol{R}_{\mathbf{V}}$ & High & Low & $\boldsymbol{R}_{\mathbf{V}}$ & High & Low & $\boldsymbol{R}_{\mathbf{V}}$ & High & Low & $\boldsymbol{R}_{\mathbf{V}}$ & High & Low & $\boldsymbol{R}_{\mathbf{V}}$ & High \\
\hline 2-year & 4.73 & 5.35 & 6.42 & 4.28 & 4.80 & 5.92 & 4.50 & 5.16 & 6.51 & 4.45 & 5.07 & 6.22 & 4.21 & 4.77 & 5.80 \\
5-year & 5.15 & 6.30 & 8.55 & 4.45 & 5.35 & 7.64 & 4.76 & 5.91 & 8.70 & 4.74 & 5.81 & 8.11 & 4.48 & 5.46 & 7.58 \\
10-year & 5.48 & 7.18 & 10.87 & 4.57 & 5.82 & 9.51 & 4.94 & 6.56 & 11.10 & 4.95 & 6.45 & 10.09 & 4.69 & 6.08 & 9.48 \\
20-year & 5.83 & 8.23 & 14.07 & 4.68 & 6.34 & 12.07 & 5.11 & 7.31 & 14.39 & 5.15 & 7.17 & 12.71 & 4.89 & 6.79 & 12.03 \\
50-year & 6.31 & 9.94 & 20.21 & 4.81 & 7.13 & 16.96 & 5.32 & 8.47 & 20.72 & 5.40 & 8.27 & 17.50 & 5.14 & 7.90 & 16.83 \\
100-year & 6.69 & 11.54 & 26.92 & 4.90 & 7.82 & 22.27 & 5.47 & 9.49 & 27.63 & 5.59 & 9.23 & 22.51 & 5.33 & 8.89 & 21.95 \\
\hline
\end{tabular}


Appendix A37. Table showing Wake monthly means and mean of the top 5 percent for significant wave height, peak wave period, and peak wave direction.

[Years: Hindcast $=$ 1976-2005; RCP mid = 2026-2045; RCP end = 2081-2100. Wave directions are "coming from”]

\begin{tabular}{|c|c|c|c|c|c|c|c|c|c|c|c|c|}
\hline Month & Jan & Feb & Mar & Apr & May & Jun & July & Aug & Sep & Oct & Nov & Dec \\
\hline $\begin{array}{l}\text { Mean Wave He } \\
\text { (m) }\end{array}$ & & & & & & & & & & & & \\
\hline Hindcast & $3.0 \pm 0.8$ & $2.9 \pm 0.8$ & $2.8 \pm 0.8$ & $2.6 \pm 0.7$ & $2.2 \pm 0.6$ & $2.1 \pm 0.6$ & $2.1 \pm 0.7$ & $2.1 \pm 0.7$ & $2.1 \pm 0.8$ & $2.7 \pm 0.9$ & $2.9 \pm 0.8$ & $3.1 \pm 0.8$ \\
\hline RCP4.5 - mid & $3.0 \pm 0.8$ & $2.9 \pm 0.7$ & $2.8 \pm 0.8$ & $2.6 \pm 0.7$ & $2.2 \pm 0.6$ & $2.0 \pm 0.6$ & $2.1 \pm 0.7$ & $2.0 \pm 0.7$ & $2.0 \pm 0.8$ & $2.6 \pm 0.9$ & $2.9 \pm 0.8$ & $3.0 \pm 0.8$ \\
\hline RCP8.5 - mid & $3.0 \pm 0.8$ & $2.9 \pm 0.8$ & $2.7 \pm 0.7$ & $2.6 \pm 0.7$ & $2.2 \pm 0.6$ & $2.0 \pm 0.5$ & $2.1 \pm 0.6$ & $2.1 \pm 0.8$ & $2.1 \pm 0.8$ & $2.5 \pm 0.9$ & $3.0 \pm 0.9$ & $3.0 \pm 0.8$ \\
\hline RCP4.5 - end & $3.0 \pm 0.8$ & $2.9 \pm 0.8$ & $2.8 \pm 0.7$ & $2.6 \pm 0.7$ & $2.2 \pm 0.5$ & $2.1 \pm 0.5$ & $2.1 \pm 0.7$ & $2.1 \pm 0.8$ & $2.0 \pm 0.8$ & $2.5 \pm 0.9$ & $2.9 \pm 0.9$ & $3.0 \pm 0.8$ \\
\hline RCP8.5 - end & $2.9 \pm 0.8$ & $2.8 \pm 0.7$ & $2.6 \pm 0.7$ & $2.5 \pm 0.6$ & $2.1 \pm 0.5$ & $2.0 \pm 0.5$ & $2.1 \pm 0.6$ & $2.0 \pm 0.9$ & $2.0 \pm 0.8$ & $2.4 \pm 0.8$ & $2.8 \pm 0.8$ & $2.9 \pm 0.8$ \\
\hline $\begin{array}{l}\text { Top } 5 \text { percent } \\
\text { Height }(\mathrm{m})\end{array}$ & & & & & & & & & & & & \\
\hline Hindcast & $5.0 \pm 0.6$ & $5.0 \pm 0.6$ & $4.7 \pm 0.5$ & $4.4 \pm 0.5$ & $3.6 \pm 0.3$ & $3.7 \pm 0.7$ & $3.9 \pm 0.8$ & $4.2 \pm 0.9$ & $4.6 \pm 1.0$ & $5.2 \pm 0.8$ & $5.0 \pm 0.6$ & $5.3 \pm 0.7$ \\
\hline RCP4.5 - mid & $5.0 \pm 0.5$ & $4.8 \pm 0.5$ & $4.8 \pm 0.6$ & $4.3 \pm 0.4$ & $3.6 \pm 0.4$ & $3.5 \pm 0.6$ & $4.0 \pm 0.7$ & $3.9 \pm 0.8$ & $4.4 \pm 0.9$ & $5.3 \pm 1.0$ & $5.1 \pm 0.6$ & $4.9 \pm 0.5$ \\
\hline RCP8.5 - mid & $.05 \pm 0.5$ & $4.9 \pm 0.5$ & $4.6 \pm 0.5$ & $4.5 \pm 0.9$ & $3.6 \pm 0.5$ & $3.3 \pm 0.4$ & $3.8 \pm 0.9$ & $4.6 \pm 1.3$ & $4.6 \pm 1.2$ & $5.0 \pm 0.9$ & $5.3 \pm 0.6$ & $5.0 \pm 0.5$ \\
\hline RCP 4.5 - end & $4.8 \pm 0.5$ & $4.9 \pm 0.5$ & $4.7 \pm 0.4$ & $4.4 \pm 0.5$ & $3.5 \pm 0.3$ & $3.4 \pm 0.7$ & $4.0 \pm 1.0$ & $4.6 \pm 0.9$ & $4.4 \pm 1.0$ & $4.9 \pm 0.9$ & $5.1 \pm 0.7$ & $4.8 \pm 0.5$ \\
\hline $\begin{array}{l}\text { RCP8.5 - end } \\
\text { Mean Wave Pe } \\
\text { (s) }\end{array}$ & $4.8 \pm 0.5$ & $4.6 \pm 0.3$ & $4.2 \pm 0.4$ & $4.0 \pm 0.4$ & $3.3 \pm 0.3$ & $3.3 \pm 0.8$ & $3.8 \pm 1.0$ & $4.9 \pm 1.4$ & $4.4 \pm 1.2$ & $4.6 \pm 0.9$ & $4.9 \pm 0.9$ & $4.8 \pm 0.4$ \\
\hline Hindcast & $11 \pm 2$ & $11 \pm 2$ & $10 \pm 2$ & $9 \pm 1$ & $9 \pm 1$ & $9 \pm 1$ & $9 \pm 1$ & $9 \pm 1$ & $9 \pm 1$ & $10 \pm 1$ & $10 \pm 2$ & $11 \pm 2$ \\
\hline RCP4.5 - mid & $11 \pm 2$ & $11 \pm 2$ & $10 \pm 2$ & $10 \pm 1$ & $9 \pm 1$ & $9 \pm 1$ & $9 \pm 1$ & $9 \pm 1$ & $9 \pm 1$ & $9 \pm 1$ & $10 \pm 2$ & $11 \pm 2$ \\
\hline RCP8.5 - mid & $11 \pm 2$ & $11 \pm 2$ & $10 \pm 2$ & $10 \pm 1$ & $9 \pm 1$ & $9 \pm 1$ & $9 \pm 1$ & $9 \pm 1$ & $9 \pm 1$ & $9 \pm 1$ & $10 \pm 2$ & $11 \pm 2$ \\
\hline RCP4.5 - end & $11 \pm 2$ & $11 \pm 2$ & $10 \pm 2$ & $9.6 \pm 1$ & $8.9 \pm 1$ & $8.7 \pm 1$ & $8.7 \pm 1$ & $8.8 \pm 1$ & $8.8 \pm 1$ & $9.4 \pm 1$ & $10 \pm 2$ & $11 \pm 2$ \\
\hline $\begin{array}{l}\text { RCP8.5 - end } \\
\text { Top } 5 \text { percent } \\
\text { Period (s) }\end{array}$ & $11 \pm 2$ & $11 \pm 2$ & $10 \pm 2$ & $9 \pm 1$ & $9 \pm 1$ & $9 \pm 1$ & $9 \pm 1$ & $9 \pm 1$ & $9 \pm 2$ & $9 \pm 1$ & $10 \pm 2$ & $11 \pm 2$ \\
\hline Hindcast & $13 \pm 2$ & $13 \pm 2$ & $11 \pm 2$ & $10 \pm 1$ & $10 \pm 0.8$ & $10 \pm 0.9$ & $10 \pm 1$ & $10 \pm 2$ & $10 \pm 1$ & $11 \pm 0.9$ & $11 \pm 1$ & $12 \pm 2$ \\
\hline RCP4.5 - mid & $13 \pm 2$ & $13 \pm 2$ & $12 \pm 2$ & $10 \pm 1$ & $10 \pm 1$ & $9 \pm 1$ & $10 \pm 1$ & $11 \pm 2$ & $11 \pm 2$ & $11 \pm 1$ & $11 \pm 2$ & $12 \pm 2$ \\
\hline RCP8.5 - mid & $13 \pm 2$ & $12 \pm 2$ & $11 \pm 2$ & $11 \pm 1$ & $9 \pm 0.7$ & $9 \pm 0.7$ & $10 \pm 1$ & $11 \pm 2$ & $10 \pm 1$ & $11 \pm 1$ & $11 \pm 1$ & $12 \pm 2$ \\
\hline RCP4.5 - end & $13 \pm 2$ & $13 \pm 2$ & $12 \pm 2$ & $10 \pm 1$ & $10 \pm 1$ & $9 \pm 1$ & $10 \pm 1$ & $11 \pm 1$ & $10 \pm 1$ & $11 \pm 1$ & $11 \pm 1$ & $12 \pm 2$ \\
\hline $\begin{array}{l}\text { RCP8.5 - end } \\
\text { Mean Wave } \\
\text { Direction ( }{ }^{9} \text { ) }\end{array}$ & $13 \pm 2$ & $12 \pm 2$ & $11 \pm 2$ & $10 \pm 1$ & $9 \pm 0.8$ & $9 \pm 1$ & $10 \pm 2$ & $11 \pm 2$ & $10 \pm 1$ & $10 \pm 1$ & $11 \pm 1$ & $12 \pm 2$ \\
\hline Hindcast & $29 \pm 43$ & $26 \pm 46$ & $51 \pm 39$ & $73 \pm 21$ & $83 \pm 15$ & $89 \pm 15$ & $90 \pm 25$ & $92 \pm 36$ & $77 \pm 36$ & $70 \pm 24$ & $62 \pm 28$ & $45 \pm 38$ \\
\hline RCP4.5 - mid & $27 \pm 43$ & $28 \pm 43$ & $50 \pm 39$ & $73 \pm 21$ & $82 \pm 16$ & $90 \pm 15$ & $93 \pm 27$ & $95 \pm 36$ & $82 \pm 35$ & $71 \pm 27$ & $63 \pm 25$ & $47 \pm 38$ \\
\hline RCP8.5 - mid & $29 \pm 43$ & $33 \pm 44$ & $49 \pm 41$ & $73 \pm 25$ & $83 \pm 15$ & $90 \pm 14$ & $93 \pm 27$ & $97 \pm 43$ & $83 \pm 41$ & $70 \pm 23$ & $66 \pm 23$ & $47 \pm 38$ \\
\hline RCP4.5 - end & $22 \pm 44$ & $24 \pm 44$ & $53 \pm 38$ & $73 \pm 25$ & $82 \pm 16$ & $91 \pm 12$ & $93 \pm 25$ & $96 \pm 37$ & $83 \pm 36$ & $69 \pm 26$ & $64 \pm 26$ & $49 \pm 37$ \\
\hline $\begin{array}{l}\text { RCP8.5 - end } \\
\text { Top } 5 \text { percent } \\
\text { Direction ( } 9 \text { ) }\end{array}$ & $26 \pm 43$ & $28 \pm 44$ & $50 \pm 39$ & $73 \pm 23$ & $84 \pm 15$ & $92 \pm 13$ & $94 \pm 24$ & $99 \pm 34$ & $86 \pm 39$ & $71 \pm 29$ & $66 \pm 22$ & $49 \pm 35$ \\
\hline Hindcast & $24 \pm 52$ & $6 \pm 52$ & $48 \pm 41$ & $70 \pm 17$ & $83 \pm 19$ & $91 \pm 25$ & $100 \pm 52$ & $153 \pm 78$ & $91 \pm 63$ & $79 \pm 35$ & $64 \pm 27$ & $54 \pm 37$ \\
\hline RCP4.5 - mid & $3 \pm 46$ & $17 \pm 49$ & $52 \pm 40$ & $73 \pm 18$ & $76 \pm 17$ & $89 \pm 26$ & $124 \pm 67$ & $133 \pm 70$ & $113 \pm 79$ & $83 \pm 50$ & $62 \pm 23$ & $43 \pm 43$ \\
\hline RCP8.5 - mid & $29 \pm 53$ & $40 \pm 52$ & $56 \pm 41$ & $70 \pm 24$ & $79 \pm 13$ & $90 \pm 15$ & $117 \pm 64$ & $180 \pm 79$ & $118 \pm 74$ & $74 \pm 36$ & $70 \pm 22$ & $45 \pm 40$ \\
\hline RCP 4.5 - end & $9 \pm 49$ & $11 \pm 54$ & $55 \pm 41$ & $73 \pm 25$ & $77 \pm 18$ & $92 \pm 17$ & $122 \pm 59$ & $190 \pm 75$ & $132 \pm 77$ & $73 \pm 41$ & $68 \pm 28$ & $49 \pm 40$ \\
\hline RCP8.5 - end & $3 \pm 45$ & $23 \pm 52$ & $52 \pm 43$ & $71 \pm 19$ & $76 \pm 13$ & $90 \pm 17$ & $112 \pm 58$ & $194 \pm 70$ & $152 \pm 86$ & $79 \pm 44$ & $69 \pm 22$ & $47 \pm 36$ \\
\hline
\end{tabular}


Appendix A38. Table showing return values of ensemble-average significant wave heights of hindcast and forecast scenarios, including lower and higher 95 percent confidence intervals, at the Wake location.

[Years: Hindcast $=1976-2005 ;$ RCP $\operatorname{mid}=2026-2045 ;$ RCP end $=2081-2100$. Wave height values are in meters $]$

\begin{tabular}{|c|c|c|c|c|c|c|c|c|c|c|c|c|c|c|c|}
\hline \multirow{2}{*}{ Scenario } & \multicolumn{3}{|c|}{ Hindcast } & \multicolumn{3}{c|}{ RCP4.5 - mid } & \multicolumn{3}{c|}{ RCP8.5 - mid } & \multicolumn{3}{c|}{ RCP4.5 - end } & \multicolumn{3}{c|}{ RCP8.5 - end } \\
\cline { 2 - 13 } & Low & $\boldsymbol{R}_{\boldsymbol{V}}$ & High & Low & $\boldsymbol{R}_{\boldsymbol{V}}$ & High & Low & $\boldsymbol{R}_{\boldsymbol{V}}$ & High & Low & $\boldsymbol{R}_{\boldsymbol{V}}$ & High & Low & $\boldsymbol{R}_{\boldsymbol{V}}$ & High \\
\hline 2-year & 7.14 & 7.90 & 9.11 & 6.68 & 7.37 & 8.58 & 6.96 & 7.89 & 9.54 & 6.76 & 7.75 & 9.73 & 6.73 & 8.09 & 11.02 \\
5-year & 7.54 & 8.72 & 10.77 & 6.97 & 8.07 & 10.27 & 7.37 & 8.87 & 11.89 & 7.08 & 8.70 & 12.48 & 7.24 & 9.69 & 16.11 \\
10-year & 7.80 & 9.32 & 12.15 & 7.17 & 8.61 & 11.81 & 7.64 & 9.64 & 14.09 & 7.30 & 9.46 & 15.21 & 7.61 & 11.17 & 22.03 \\
20-year & 8.02 & 9.89 & 13.67 & 7.33 & 9.17 & 13.63 & 7.88 & 10.43 & 16.73 & 7.48 & 10.25 & 18.66 & 7.97 & 12.93 & 30.65 \\
50-year & 8.26 & 10.62 & 15.87 & 7.53 & 9.92 & 16.56 & 8.16 & 11.53 & 21.05 & 7.69 & 11.37 & 24.64 & 8.43 & 15.78 & 48.34 \\
100-year & 8.42 & 11.16 & 17.72 & 7.65 & 10.50 & 19.25 & 8.35 & 12.40 & 25.10 & 7.83 & 12.27 & 30.56 & 8.77 & 18.42 & 68.94 \\
\hline
\end{tabular}


Appendix A39. Table showing Johnston Atoll monthly means and mean of the top 5 percent for significant wave height, peak wave period, and peak wave direction.

[Years: Hindcast $=1976-2005 ;$ RCP mid $=$ 2026-2045; RCP end $=$ 2081-2100. Wave directions are “coming from”]

\begin{tabular}{|c|c|c|c|c|c|c|c|c|c|c|c|c|}
\hline Month & Jan & Feb & Mar & Apr & May & Jun & July & Aug & Sep & Oct & Nov & Dec \\
\hline \multicolumn{13}{|c|}{$\begin{array}{l}\text { Mean Wave Height } \\
\text { (m) }\end{array}$} \\
\hline Hindcast & $3.4 \pm 0.8$ & $3.2 \pm 0.8$ & $3.1 \pm 0.8$ & $2.8 \pm 0.7$ & $2.4 \pm 0.6$ & $2.4 \pm 0.5$ & $2.3 \pm 0.5$ & $2.1 \pm 0.5$ & $2.1 \pm 0.5$ & $2.5 \pm 0.8$ & $3.1 \pm 0.8$ & $3.3 \pm 0.8$ \\
\hline RCP4.5 - mid & $3.3 \pm 0.8$ & $3.3 \pm 0.8$ & $3.1 \pm 0.7$ & $2.9 \pm 0.7$ & $2.4 \pm 0.5$ & $2.3 \pm 0.5$ & $2.3 \pm 0.5$ & $2.1 \pm 0.5$ & $2.0 \pm 0.5$ & $2.4 \pm 0.7$ & $3.0 \pm 0.8$ & $3.3 \pm 0.8$ \\
\hline RCP8.5 - mid & $3.3 \pm 0.8$ & $3.2 \pm 0.8$ & $3.0 \pm 0.8$ & $2.8 \pm 0.6$ & $2.3 \pm 0.5$ & $2.3 \pm 0.5$ & $2.3 \pm 0.5$ & $2.1 \pm 0.5$ & $2.0 \pm 0.5$ & $2.4 \pm 0.7$ & $3.0 \pm 0.8$ & $3.3 \pm 0.8$ \\
\hline RCP4.5 - end & $3.3 \pm 0.7$ & $3.2 \pm 0.8$ & $3.1 \pm 0.7$ & $2.8 \pm 0.6$ & $2.4 \pm 0.5$ & $2.3 \pm 0.5$ & $2.2 \pm 0.5$ & $2.2 \pm 0.6$ & $2.0 \pm 0.5$ & $2.4 \pm 0.8$ & $3.0 \pm 0.8$ & $3.3 \pm 0.8$ \\
\hline RCP8.5 - end & $3.2 \pm 0.8$ & $3.1 \pm 0.8$ & $2.9 \pm 0.7$ & $2.7 \pm 0.6$ & $2.3 \pm 0.5$ & $2.2 \pm 0.4$ & $2.3 \pm 0.5$ & $2.1 \pm 0.5$ & $2.0 \pm 0.5$ & $2.3 \pm 0.6$ & $2.8 \pm 0.7$ & $3.2 \pm 0.8$ \\
\hline \multicolumn{13}{|c|}{$\begin{array}{l}\text { Top } 5 \text { percent Wave } \\
\text { Height }(\mathrm{m})\end{array}$} \\
\hline Hindcast & $5.4 \pm 0.5$ & $5.2 \pm 0.5$ & $4.9 \pm 0.4$ & $4.4 \pm 0.4$ & $3.7 \pm 0.4$ & $3.6 \pm 0.5$ & $3.6 \pm 0.5$ & $3.4 \pm 0.7$ & $3.5 \pm 0.5$ & $4.7 \pm 0.7$ & $5.2 \pm 0.5$ & $5.3 \pm 0.5$ \\
\hline RCP4.5 - mid & $5.3 \pm 0.5$ & $5.2 \pm 0.4$ & $4.9 \pm 0.4$ & $4.7 \pm 0.4$ & $3.7 \pm 0.4$ & $3.4 \pm 0.4$ & $3.4 \pm 0.4$ & $3.6 \pm 0.9$ & $3.5 \pm 0.6$ & $4.3 \pm 0.6$ & $5.0 \pm 0.6$ & $5.2 \pm 0.4$ \\
\hline RCP8.5 - mid & $5.3 \pm 0.5$ & $5.3 \pm 0.4$ & $4.9 \pm 0.4$ & $4.3 \pm 0.4$ & $3.5 \pm 0.3$ & $3.3 \pm 0.3$ & $3.5 \pm 0.5$ & $3.5 \pm 0.8$ & $3.5 \pm 0.7$ & $4.5 \pm 0.7$ & $4.9 \pm 0.5$ & $5.4 \pm 0.4$ \\
\hline RCP4.5 - end & $5.1 \pm 0.4$ & $5.3 \pm 0.5$ & $4.8 \pm 0.5$ & $4.2 \pm 0.3$ & $3.7 \pm 0.5$ & $3.5 \pm 0.3$ & $3.5 \pm 0.5$ & $3.8 \pm 1.0$ & $3.3 \pm 0.6$ & $4.6 \pm 1.0$ & $5.1 \pm 0.5$ & $5.1 \pm 0.3$ \\
\hline RCP8.5 - end & $5.1 \pm 0.5$ & $5.1 \pm 0.6$ & $4.7 \pm 0.4$ & $4.1 \pm 0.3$ & $3.4 \pm 0.3$ & $3.3 \pm 0.4$ & $3.5 \pm 0.6$ & $3.4 \pm 0.6$ & $3.4 \pm 1.0$ & $4.0 \pm 0.9$ & $4.7 \pm 0.5$ & $5.1 \pm 0.4$ \\
\hline \multicolumn{13}{|c|}{$\begin{array}{l}\text { Mean Wave Period } \\
\text { (s) }\end{array}$} \\
\hline Hindcast & $12 \pm 2$ & $12 \pm 2$ & $11 \pm 2$ & $10 \pm 2$ & $9 \pm 2$ & $9 \pm 2$ & $9 \pm 2$ & $9 \pm 2$ & $10 \pm 2$ & $10 \pm 2$ & $11 \pm 2$ & $12 \pm 2$ \\
\hline RCP4.5 - mid & $12 \pm 2$ & $12 \pm 2$ & $11 \pm 2$ & $10 \pm 2$ & $9 \pm 2$ & $9 \pm 2$ & $9 \pm 2$ & $9 \pm 2$ & $10 \pm 3$ & $10 \pm 2$ & $11 \pm 2$ & $12 \pm 2$ \\
\hline RCP8.5 - mid & $12 \pm 2$ & $12 \pm 2$ & $11 \pm 2$ & $10 \pm 2$ & $9 \pm 2$ & $9 \pm 2$ & $9 \pm 3$ & $9 \pm 3$ & $10 \pm 3$ & $10 \pm 2$ & $11 \pm 2$ & $12 \pm 2$ \\
\hline RCP4.5 - end & $12 \pm 2$ & $12 \pm 2$ & $11 \pm 2$ & $10 \pm 2$ & $9.4 \pm 2$ & $9.2 \pm 2$ & $9.2 \pm 2$ & $9.3 \pm 2$ & $9.7 \pm 2$ & $10 \pm 2$ & $11 \pm 2$ & $12 \pm 2$ \\
\hline RCP8.5 - end & $12 \pm 2$ & $12 \pm 2$ & $11 \pm 2$ & $10 \pm 2$ & $9 \pm 2$ & $9 \pm 3$ & $9 \pm 2$ & $9 \pm 3$ & $10 \pm 3$ & $10 \pm 2$ & $11 \pm 2$ & $12 \pm 2$ \\
\hline \multicolumn{13}{|c|}{$\begin{array}{l}\text { Top } 5 \text { percent Wave } \\
\text { Period (s) }\end{array}$} \\
\hline Hindcast & $14 \pm 3$ & $14 \pm 3$ & $11 \pm 2$ & $10 \pm 2$ & $10 \pm 1$ & $9 \pm 0.6$ & $9 \pm 0.8$ & $9 \pm 2$ & $10 \pm 2$ & $11 \pm 2$ & $12 \pm 2$ & $13 \pm 3$ \\
\hline RCP4.5 - mid & $15 \pm 2$ & $14 \pm 3$ & $12 \pm 3$ & $10 \pm 1$ & $10 \pm 1$ & $9 \pm 0.6$ & $9 \pm 1$ & $9 \pm 2$ & $10 \pm 2$ & $11 \pm 2$ & $12 \pm 2$ & $13 \pm 3$ \\
\hline RCP8.5 - mid & $13 \pm 3$ & $13 \pm 3$ & $11 \pm 2$ & $10 \pm 2$ & $9 \pm 1$ & $9 \pm 1$ & $9 \pm 0.7$ & $9 \pm 2$ & $10 \pm 2$ & $11 \pm 2$ & $12 \pm 2$ & $13 \pm 3$ \\
\hline RCP4.5 - end & $14 \pm 3$ & $13 \pm 3$ & $12 \pm 2$ & $11 \pm 2$ & $10 \pm 2$ & $9 \pm 1$ & $9 \pm 0.8$ & $10 \pm 2$ & $10 \pm 2$ & $10 \pm 1$ & $11 \pm 2$ & $13 \pm 3$ \\
\hline RCP8.5 - end & $14 \pm 3$ & $13 \pm 3$ & $12 \pm 3$ & $10 \pm 2$ & $9 \pm 2$ & $9 \pm 1$ & $9 \pm 1$ & $10 \pm 2$ & $10 \pm 2$ & $10 \pm 2$ & $12 \pm 2$ & $13 \pm 3$ \\
\hline \multicolumn{13}{|l|}{ Mean Wave } \\
\hline Direction ( $\left.{ }^{\circ}\right)$ & & & & & & & & & & & & \\
\hline Hindcast & $14 \pm 41$ & $13 \pm 44$ & $43 \pm 39$ & $67 \pm 27$ & $83 \pm 18$ & $91 \pm 14$ & $94 \pm 19$ & $96 \pm 28$ & $85 \pm 39$ & $61 \pm 36$ & $44 \pm 33$ & $28 \pm 40$ \\
\hline RCP4.5 - mid & $9 \pm 39$ & $18 \pm 42$ & $40 \pm 39$ & $68 \pm 25$ & $84 \pm 20$ & $93 \pm 16$ & $94 \pm 18$ & $98 \pm 28$ & $92 \pm 36$ & $61 \pm 37$ & $47 \pm 33$ & $28 \pm 38$ \\
\hline RCP8.5 - mid & $16 \pm 41$ & $17 \pm 41$ & $38 \pm 41$ & $67 \pm 26$ & $84 \pm 19$ & $93 \pm 14$ & $95 \pm 20$ & $99 \pm 32$ & $91 \pm 41$ & $60 \pm 35$ & $45 \pm 31$ & $30 \pm 38$ \\
\hline RCP4.5 - end & $7 \pm 41$ & $11 \pm 43$ & $41 \pm 38$ & $63 \pm 28$ & $83 \pm 19$ & $93 \pm 14$ & $95 \pm 19$ & $98 \pm 32$ & $89 \pm 39$ & $63 \pm 41$ & $47 \pm 32$ & $30 \pm 38$ \\
\hline RCP8.5 - end & $11 \pm 40$ & $12 \pm 42$ & $38 \pm 40$ & $67 \pm 27$ & $85 \pm 19$ & $96 \pm 14$ & $95 \pm 16$ & $99 \pm 29$ & $95 \pm 39$ & $65 \pm 34$ & $49 \pm 29$ & $28 \pm 38$ \\
\hline \multirow{2}{*}{\multicolumn{13}{|c|}{$\begin{array}{l}\text { Top } 5 \text { percent Wave } \\
\text { Direction }(9)\end{array}$}} \\
\hline & & & & & & & & & & & & \\
\hline Hindcast & $6 \pm 43$ & $8 \pm 50$ & $47 \pm 35$ & $62 \pm 24$ & $74 \pm 14$ & $87 \pm 12$ & $89 \pm 22$ & $96 \pm 41$ & $78 \pm 50$ & $65 \pm 42$ & $52 \pm 39$ & $27 \pm 46$ \\
\hline RCP4.5 - mid & $352 \pm 36$ & $10 \pm 48$ & $46 \pm 39$ & $71 \pm 14$ & $77 \pm 15$ & $88 \pm 16$ & $88 \pm 32$ & $106 \pm 52$ & $102 \pm 70$ & $65 \pm 54$ & $48 \pm 42$ & $19 \pm 43$ \\
\hline RCP8.5 - mid & $26 \pm 45$ & $28 \pm 47$ & $49 \pm 36$ & $63 \pm 23$ & $79 \pm 14$ & $87 \pm 10$ & $90 \pm 27$ & $106 \pm 47$ & $100 \pm 76$ & $60 \pm 45$ & $45 \pm 36$ & $33 \pm 46$ \\
\hline RCP4.5 - end & $354 \pm 45$ & $10 \pm 50$ & $43 \pm 36$ & $60 \pm 26$ & $77 \pm 14$ & $87 \pm 14$ & $88 \pm 23$ & $102 \pm 71$ & $97 \pm 71$ & $71 \pm 63$ & $61 \pm 28$ & $23 \pm 44$ \\
\hline RCP8.5 - end & $353 \pm 40$ & $8 \pm 46$ & $40 \pm 44$ & $66 \pm 22$ & $76 \pm 17$ & $90 \pm 12$ & $89 \pm 25$ & $97 \pm 52$ & $113 \pm 71$ & $68 \pm 44$ & $47 \pm 34$ & $28 \pm 47$ \\
\hline
\end{tabular}


Appendix A40. Table showing return values of ensemble-average significant wave heights of hindcast and forecast scenarios, including lower and higher 95 percent confidence intervals, at the Johnston Atoll location.

[Years: Hindcast $=1976-2005 ;$ RCP mid $=$ 2026-2045; RCP end $=2081-2100$. Wave height values are in meters]

\begin{tabular}{|c|c|c|c|c|c|c|c|c|c|c|c|c|c|c|c|}
\hline \multirow{2}{*}{ Scenario } & \multicolumn{3}{|c|}{ Hindcast } & \multicolumn{3}{c|}{ RCP4.5 - mid } & \multicolumn{3}{c|}{ RCP8.5 - mid } & \multicolumn{3}{c|}{ RCP4.5 - end } & \multicolumn{3}{c|}{ RCP8.5 - end } \\
\cline { 2 - 13 } & Low & $\boldsymbol{R}_{\mathbf{V}}$ & High & Low & $\boldsymbol{R}_{\mathbf{V}}$ & High & Low & $\boldsymbol{R}_{\mathbf{V}}$ & High & Low & $\boldsymbol{R}_{\mathbf{V}}$ & High & Low & $\boldsymbol{R}_{\mathbf{V}}$ & High \\
\hline 2-year & 6.50 & 7.00 & 7.86 & 6.47 & 6.98 & 7.83 & 6.32 & 6.78 & 7.60 & 6.44 & 7.13 & 8.45 & 6.39 & 6.95 & 7.87 \\
5-year & 6.75 & 7.58 & 9.15 & 6.71 & 7.50 & 9.00 & 6.49 & 7.18 & 8.61 & 6.76 & 7.96 & 10.66 & 6.66 & 7.53 & 9.12 \\
10-year & 6.92 & 8.03 & 10.33 & 6.86 & 7.90 & 10.03 & 6.58 & 7.47 & 9.48 & 6.99 & 8.70 & 13.02 & 6.83 & 7.96 & 10.18 \\
20-year & 7.08 & 8.49 & 11.74 & 6.99 & 8.30 & 11.19 & 6.66 & 7.75 & 10.46 & 7.21 & 9.54 & 16.21 & 6.99 & 8.39 & 11.37 \\
50-year & 7.26 & 9.14 & 14.01 & 7.15 & 8.82 & 12.99 & 6.75 & 8.11 & 11.95 & 7.49 & 10.83 & 22.20 & 7.16 & 8.94 & 13.15 \\
100-year & 7.38 & 9.64 & 16.10 & 7.25 & 9.21 & 14.57 & 6.80 & 8.37 & 13.24 & 7.69 & 11.98 & 28.60 & 7.27 & 9.35 & 14.67 \\
\hline
\end{tabular}


Appendix A41. Table showing Kingman Reef monthly means and mean of the top 5 percent for significant wave height, peak wave period, and peak wave direction.

[Years: Hindcast $=1976-2005 ;$ RCP mid $=$ 2026-2045; RCP end $=$ 2081-2100. Wave directions are “coming from”]

\begin{tabular}{|c|c|c|c|c|c|c|c|c|c|c|c|c|}
\hline Month & Jan & Feb & Mar & Apr & May & Jun & July & Aug & Sep & Oct & Nov & Dec \\
\hline \multicolumn{13}{|c|}{$\begin{array}{l}\text { Mean Wave Height } \\
\text { (m) }\end{array}$} \\
\hline Hindcast & $2.9 \pm 0.6$ & $2.9 \pm 0.6$ & $2.7 \pm 0.6$ & $2.4 \pm 0.5$ & $2.1 \pm 0.5$ & $2.1 \pm 0.4$ & $2.0 \pm 0.4$ & $1.9 \pm 0.4$ & $1.8 \pm 0.4$ & $1.9 \pm 0.4$ & $2.3 \pm 0.6$ & $2.7 \pm 0.6$ \\
\hline RCP4.5 - mid & $2.9 \pm 0.6$ & $2.9 \pm 0.6$ & $2.8 \pm 0.6$ & $2.5 \pm 0.5$ & $2.2 \pm 0.5$ & $2.1 \pm 0.4$ & $2.0 \pm 0.4$ & $1.9 \pm 0.4$ & $1.8 \pm 0.3$ & $1.9 \pm 0.4$ & $2.3 \pm 0.5$ & $2.7 \pm 0.6$ \\
\hline RCP8.5 - mid & $2.9 \pm 0.6$ & $2.9 \pm 0.6$ & $2.7 \pm 0.6$ & $2.5 \pm 0.5$ & $2.2 \pm 0.5$ & $2.1 \pm 0.4$ & $2.0 \pm 0.4$ & $1.9 \pm 0.4$ & $1.8 \pm 0.3$ & $1.9 \pm 0.4$ & $2.2 \pm 0.5$ & $2.7 \pm 0.6$ \\
\hline RCP4.5 - end & $2.8 \pm 0.6$ & $2.9 \pm 0.6$ & $2.7 \pm 0.6$ & $2.4 \pm 0.5$ & $2.2 \pm 0.5$ & $2.1 \pm 0.4$ & $2.0 \pm 0.4$ & $1.9 \pm 0.4$ & $1.8 \pm 0.3$ & $1.9 \pm 0.4$ & $2.2 \pm 0.6$ & $2.7 \pm 0.6$ \\
\hline RCP8.5 - end & $2.8 \pm 0.6$ & $2.8 \pm 0.6$ & $2.6 \pm 0.6$ & $2.4 \pm 0.5$ & $2.2 \pm 0.4$ & $2.1 \pm 0.4$ & $2.0 \pm 0.3$ & $1.9 \pm 0.3$ & $1.8 \pm 0.3$ & $1.8 \pm 0.3$ & $2.1 \pm 0.5$ & $2.6 \pm 0.6$ \\
\hline \multicolumn{13}{|c|}{$\begin{array}{l}\text { Top } 5 \text { percent Wave } \\
\text { Height }(\mathrm{m})\end{array}$} \\
\hline Hindcast & $4.4 \pm 0.4$ & $4.3 \pm 0.3$ & $4.1 \pm 0.3$ & $3.6 \pm 0.3$ & $3.2 \pm 0.2$ & $3.1 \pm 0.2$ & $2.9 \pm 0.2$ & $2.8 \pm 0.2$ & $2.6 \pm 0.2$ & $3.1 \pm 0.4$ & $4.0 \pm 0.9$ & $4.2 \pm 0.4$ \\
\hline RCP4.5 - mid & $4.3 \pm 0.3$ & $4.3 \pm 0.3$ & $4.2 \pm 0.3$ & $3.8 \pm 0.3$ & $3.4 \pm 0.5$ & $3.1 \pm 0.2$ & $2.9 \pm 0.2$ & $2.8 \pm 0.3$ & $2.6 \pm 0.2$ & $2.8 \pm 0.2$ & $3.6 \pm 0.3$ & $4.2 \pm 0.3$ \\
\hline RCP8.5 - mid & $4.4 \pm 0.4$ & $4.3 \pm 0.3$ & $4.2 \pm 0.4$ & $3.6 \pm 0.3$ & $3.3 \pm 0.3$ & $3.0 \pm 0.2$ & $2.9 \pm 0.2$ & $2.7 \pm 0.2$ & $2.6 \pm 0.2$ & $2.9 \pm 0.3$ & $3.5 \pm 0.4$ & $4.2 \pm 0.3$ \\
\hline RCP4.5 - end & $4.3 \pm 0.3$ & $4.4 \pm 0.3$ & $4.0 \pm 0.2$ & $3.8 \pm 0.3$ & $3.3 \pm 0.4$ & $3.2 \pm 0.3$ & $2.8 \pm 0.2$ & $2.8 \pm 0.3$ & $2.5 \pm 0.2$ & $2.8 \pm 0.3$ & $3.7 \pm 0.7$ & $4.1 \pm 0.4$ \\
\hline RCP8.5 - end & $4.1 \pm 0.3$ & $4.2 \pm 0.4$ & $4.0 \pm 0.3$ & $3.6 \pm 0.2$ & $3.2 \pm 0.2$ & $3.1 \pm 0.2$ & $2.8 \pm 0.2$ & $2.8 \pm 0.4$ & $2.5 \pm 0.3$ & $2.7 \pm 0.3$ & $3.3 \pm 0.3$ & $4.2 \pm 0.4$ \\
\hline \multicolumn{13}{|c|}{$\begin{array}{l}\text { Mean Wave Period } \\
\text { (s) }\end{array}$} \\
\hline Hindcast & $12 \pm 3$ & $12 \pm 3$ & $11 \pm 2$ & $11 \pm 3$ & $11 \pm 3$ & $10 \pm 3$ & $10 \pm 3$ & $11 \pm 3$ & $11 \pm 3$ & $11 \pm 2$ & $12 \pm 2$ & $12 \pm 2$ \\
\hline RCP4.5 - mid & $13 \pm 3$ & $12 \pm 3$ & $11 \pm 3$ & $11 \pm 3$ & $10 \pm 3$ & $10 \pm 3$ & $10 \pm 3$ & $10 \pm 3$ & $11 \pm 3$ & $11 \pm 3$ & $12 \pm 2$ & $12 \pm 3$ \\
\hline RCP8.5 - mid & $12 \pm 3$ & $12 \pm 3$ & $11 \pm 3$ & $10 \pm 3$ & $10 \pm 3$ & $10 \pm 3$ & $10 \pm 3$ & $11 \pm 3$ & $11 \pm 3$ & $12 \pm 3$ & $12 \pm 2$ & $12 \pm 3$ \\
\hline RCP4.5 - end & $12 \pm 3$ & $12 \pm 3$ & $11 \pm 2$ & $11 \pm 3$ & $10 \pm 3$ & $10 \pm 3$ & $11 \pm 3$ & $11 \pm 3$ & $11 \pm 3$ & $11 \pm 3$ & $12 \pm 2$ & $12 \pm 3$ \\
\hline RCP8.5 - end & $12 \pm 3$ & $12 \pm 3$ & $11 \pm 3$ & $10 \pm 3$ & $10 \pm 3$ & $10 \pm 3$ & $10 \pm 3$ & $11 \pm 3$ & $11 \pm 3$ & $11 \pm 3$ & $11 \pm 3$ & $12 \pm 3$ \\
\hline \multirow{2}{*}{\multicolumn{13}{|c|}{$\begin{array}{l}\text { Top } 5 \text { percent Wave } \\
\text { Period (s) }\end{array}$}} \\
\hline & & & & & & & & & & & & \\
\hline Hindcast & $13 \pm 3$ & $13 \pm 3$ & $11 \pm 2$ & $10 \pm 2$ & $11 \pm 3$ & $11 \pm 3$ & $12 \pm 3$ & $12 \pm 3$ & $12 \pm 3$ & $12 \pm 2$ & $12 \pm 3$ & $13 \pm 3$ \\
\hline RCP4.5 - mid & $14 \pm 3$ & $13 \pm 3$ & $11 \pm 2$ & $11 \pm 2$ & $11 \pm 3$ & $10 \pm 3$ & $11 \pm 3$ & $12 \pm 3$ & $13 \pm 3$ & $13 \pm 3$ & $13 \pm 3$ & $14 \pm 3$ \\
\hline RCP8.5 - mid & $13 \pm 3$ & $13 \pm 3$ & $11 \pm 2$ & $10 \pm 2$ & $10 \pm 2$ & $11 \pm 3$ & $12 \pm 3$ & $12 \pm 3$ & $13 \pm 3$ & $13 \pm 3$ & $13 \pm 3$ & $13 \pm 3$ \\
\hline RCP4.5 - end & $14 \pm 3$ & $13 \pm 3$ & $11 \pm 2$ & $11 \pm 2$ & $10 \pm 3$ & $11 \pm 3$ & $12 \pm 4$ & $12 \pm 3$ & $12 \pm 3$ & $12 \pm 3$ & $12 \pm 2$ & $14 \pm 3$ \\
\hline RCP8.5 - end & $15 \pm 3$ & $13 \pm 3$ & $12 \pm 3$ & $10 \pm 2$ & $10 \pm 3$ & $11 \pm 4$ & $11 \pm 3$ & $12 \pm 3$ & $12 \pm 3$ & $13 \pm 3$ & $13 \pm 3$ & $14 \pm 3$ \\
\hline \multicolumn{13}{|l|}{ Mean Wave } \\
\hline Direction ( 9 ) & & & & & & & & & & & & \\
\hline Hindcast & $24 \pm 28$ & $25 \pm 31$ & $41 \pm 29$ & $62 \pm 29$ & $88 \pm 31$ & $101 \pm 28$ & $116 \pm 25$ & $124 \pm 25$ & $123 \pm 35$ & $82 \pm 50$ & $35 \pm 38$ & $27 \pm 29$ \\
\hline RCP4.5 - mid & $21 \pm 28$ & $28 \pm 27$ & $41 \pm 27$ & $61 \pm 26$ & $84 \pm 30$ & $100 \pm 27$ & $114 \pm 23$ & $124 \pm 23$ & $128 \pm 26$ & $92 \pm 51$ & $37 \pm 40$ & $25 \pm 29$ \\
\hline RCP8.5 - mid & $26 \pm 27$ & $28 \pm 28$ & $38 \pm 29$ & $60 \pm 26$ & $85 \pm 29$ & $100 \pm 26$ & $116 \pm 23$ & $126 \pm 24$ & $129 \pm 30$ & $96 \pm 49$ & $36 \pm 39$ & $26 \pm 28$ \\
\hline RCP4.5 - end & $22 \pm 29$ & $23 \pm 29$ & $39 \pm 27$ & $60 \pm 28$ & $85 \pm 29$ & $101 \pm 27$ & $117 \pm 24$ & $127 \pm 28$ & $130 \pm 29$ & $97 \pm 53$ & $36 \pm 39$ & $27 \pm 30$ \\
\hline RCP8.5 - end & $23 \pm 28$ & $27 \pm 29$ & $39 \pm 28$ & $62 \pm 26$ & $84 \pm 25$ & $99 \pm 25$ & $114 \pm 24$ & $127 \pm 25$ & $132 \pm 28$ & $105 \pm 47$ & $45 \pm 40$ & $26 \pm 29$ \\
\hline \multirow{2}{*}{\multicolumn{13}{|c|}{$\begin{array}{l}\text { Top } 5 \text { percent Wave } \\
\text { Direction }(9)\end{array}$}} \\
\hline & & & & & & & & & & & & \\
\hline Hindcast & $20 \pm 33$ & $27 \pm 32$ & $44 \pm 23$ & $54 \pm 21$ & $76 \pm 28$ & $91 \pm 29$ & $120 \pm 36$ & $127 \pm 25$ & $127 \pm 39$ & $42 \pm 51$ & $30 \pm 47$ & $21 \pm 36$ \\
\hline RCP4.5 - mid & $11 \pm 35$ & $25 \pm 32$ & $44 \pm 23$ & $57 \pm 16$ & $70 \pm 33$ & $90 \pm 25$ & $112 \pm 30$ & $129 \pm 30$ & $132 \pm 35$ & $55 \pm 67$ & $17 \pm 39$ & $21 \pm 32$ \\
\hline RCP8.5 - mid & $24 \pm 30$ & $27 \pm 32$ & $47 \pm 21$ & $56 \pm 11$ & $76 \pm 25$ & $97 \pm 32$ & $117 \pm 30$ & $124 \pm 27$ & $136 \pm 32$ & $65 \pm 68$ & $18 \pm 34$ & $21 \pm 39$ \\
\hline RCP4.5 - end & $20 \pm 35$ & $23 \pm 36$ & $45 \pm 23$ & $50 \pm 22$ & $76 \pm 32$ & $92 \pm 36$ & $123 \pm 32$ & $139 \pm 42$ & $142 \pm 42$ & $29 \pm 67$ & $30 \pm 37$ & $18 \pm 34$ \\
\hline RCP8.5 - end & $7 \pm 32$ & $25 \pm 35$ & $41 \pm 25$ & $59 \pm 17$ & $73 \pm 27$ & $94 \pm 39$ & $113 \pm 35$ & $137 \pm 43$ & $142 \pm 42$ & $59 \pm 85$ & $25 \pm 31$ & $26 \pm 33$ \\
\hline
\end{tabular}


Appendix A42. Table showing return values of ensemble-average significant wave heights of hindcast and forecast scenarios, including lower and higher 95 percent confidence intervals, at the Kingman Reef location.

[Years: Hindcast $=1976-2005 ;$ RCP mid $=$ 2026-2045; RCP end $=2081-2100$. Wave height values are in meters]

\begin{tabular}{|c|c|c|c|c|c|c|c|c|c|c|c|c|c|c|c|c|}
\hline \multirow{2}{*}{ Scenario } & \multicolumn{3}{|c|}{ Hindcast } & \multicolumn{3}{|c|}{ RCP4.5 - mid } & \multicolumn{3}{c|}{ RCP8.5 - mid } & \multicolumn{3}{|c|}{ RCP4.5 - end } & \multicolumn{3}{c|}{ RCP8.5 - end } \\
\cline { 2 - 13 } & Low & $\boldsymbol{R}_{\boldsymbol{V}}$ & High & Low & $\boldsymbol{R}_{\boldsymbol{V}}$ & High & Low & $\boldsymbol{R}_{\boldsymbol{V}}$ & High & Low & $\boldsymbol{R}_{\boldsymbol{V}}$ & High & Low & $\boldsymbol{R}_{\boldsymbol{V}}$ & High \\
\hline 2-year & 5.01 & 5.37 & 5.96 & 5.01 & 5.36 & 5.93 & 4.88 & 5.26 & 5.96 & 4.94 & 5.35 & 6.10 & 4.81 & 5.13 & 5.64 \\
5-year & 5.26 & 5.91 & 7.13 & 5.21 & 5.78 & 6.80 & 5.02 & 5.63 & 6.91 & 5.14 & 5.83 & 7.24 & 4.97 & 5.47 & 6.35 \\
10-year & 5.46 & 6.41 & 8.36 & 5.35 & 6.10 & 7.57 & 5.11 & 5.91 & 7.79 & 5.28 & 6.22 & 8.35 & 5.08 & 5.72 & 6.95 \\
20-year & 5.67 & 6.99 & 9.99 & 5.48 & 6.44 & 8.48 & 5.19 & 6.20 & 8.83 & 5.41 & 6.65 & 9.73 & 5.17 & 5.97 & 7.62 \\
50-year & 5.95 & 7.91 & 13.00 & 5.63 & 6.91 & 9.92 & 5.28 & 6.59 & 10.54 & 5.56 & 7.26 & 12.10 & 5.28 & 6.29 & 8.62 \\
100-year & 6.17 & 8.74 & 16.16 & 5.73 & 7.28 & 11.22 & 5.34 & 6.89 & 12.12 & 5.67 & 7.76 & 14.41 & 5.34 & 6.53 & 9.47 \\
\hline
\end{tabular}


Appendix A43. Table showing Palmyra monthly means and mean of the top 5 percent for significant wave height, peak wave period, and peak wave direction.

[Years: Hindcast $=$ 1976-2005; RCP mid = 2026-2045; RCP end = 2081-2100. Wave directions are "coming from”]

\begin{tabular}{|c|c|c|c|c|c|c|c|c|c|c|c|c|}
\hline Month & Jan & Feb & Mar & Apr & May & Jun & July & Aug & Sep & Oct & Nov & Dec \\
\hline \multicolumn{13}{|c|}{$\begin{array}{l}\text { Mean Wave Height } \\
\text { (m) }\end{array}$} \\
\hline Hindcast & $2.9 \pm 0.6$ & $2.8 \pm 0.6$ & $2.6 \pm 0.5$ & $2.3 \pm 0.5$ & $2.1 \pm 0.4$ & $2 \pm 0.4$ & $2.0 \pm 0.4$ & $1.9 \pm 0.4$ & $1.8 \pm 0.4$ & $1.9 \pm 0.4$ & $2.3 \pm 0.6$ & $2.7 \pm 0.6$ \\
\hline RCP4.5 - mid & $2.8 \pm 0.6$ & $2.9 \pm 0.6$ & $2.7 \pm 0.6$ & $2.4 \pm 0.5$ & $2.2 \pm 0.5$ & $2.1 \pm 0.4$ & $2.0 \pm 0.4$ & $1.9 \pm 0.4$ & $1.8 \pm 0.3$ & $1.9 \pm 0.4$ & $2.2 \pm 0.5$ & $2.6 \pm 0.6$ \\
\hline RCP8.5 - mid & $2.8 \pm 0.6$ & $2.8 \pm 0.6$ & $2.6 \pm 0.6$ & $2.4 \pm 0.5$ & $2.1 \pm 0.4$ & $2.0 \pm 0.4$ & $2.0 \pm 0.4$ & $1.9 \pm 0.4$ & $1.8 \pm 0.3$ & $1.9 \pm 0.4$ & $2.2 \pm 0.5$ & $2.6 \pm 0.6$ \\
\hline RCP4.5 - end & $2.8 \pm 0.6$ & $2.8 \pm 0.6$ & $2.7 \pm 0.5$ & $2.4 \pm 0.5$ & $2.2 \pm 0.5$ & $2.1 \pm 0.4$ & $2.0 \pm 0.4$ & $1.9 \pm 0.4$ & $1.8 \pm 0.3$ & $1.9 \pm 0.4$ & $2.2 \pm 0.6$ & $2.6 \pm 0.6$ \\
\hline RCP8.5 - end & $2.7 \pm 0.5$ & $2.7 \pm 0.6$ & $2.6 \pm 0.5$ & $2.4 \pm 0.5$ & $2.2 \pm 0.4$ & $2.1 \pm 0.4$ & $2.0 \pm 0.3$ & $1.9 \pm 0.3$ & $1.8 \pm 0.3$ & $1.8 \pm 0.3$ & $2.1 \pm 0.4$ & $2.6 \pm 0.6$ \\
\hline \multicolumn{13}{|c|}{$\begin{array}{l}\text { Top } 5 \text { percent Wave } \\
\text { Height }(\mathrm{m})\end{array}$} \\
\hline Hindcast & $4.3 \pm 0.4$ & $4.2 \pm 0.3$ & $4.0 \pm 0.3$ & $3.5 \pm 0.3$ & $3.1 \pm 0.2$ & $3.0 \pm 0.2$ & $2.9 \pm 0.2$ & $2.8 \pm 0.2$ & $2.6 \pm 0.2$ & $3.0 \pm 0.4$ & $3.9 \pm 0.9$ & $4.1 \pm 0.4$ \\
\hline RCP4.5 - mid & $4.2 \pm 0.3$ & $4.2 \pm 0.3$ & $4.1 \pm 0.3$ & $3.7 \pm 0.3$ & $3.3 \pm 0.4$ & $3.1 \pm 0.2$ & $2.9 \pm 0.2$ & $2.8 \pm 0.2$ & $2.6 \pm 0.2$ & $2.7 \pm 0.2$ & $3.5 \pm 0.3$ & $4.1 \pm 0.3$ \\
\hline RCP8.5 - mid & $4.2 \pm 0.4$ & $4.2 \pm 0.3$ & $4.1 \pm 0.4$ & $3.5 \pm 0.3$ & $3.2 \pm 0.3$ & $2.9 \pm 0.2$ & $2.9 \pm 0.2$ & $2.7 \pm 0.2$ & $2.6 \pm 0.2$ & $2.8 \pm 0.3$ & $3.4 \pm 0.4$ & $4.0 \pm 0.3$ \\
\hline RCP4.5 - end & $4.2 \pm 0.3$ & $4.3 \pm 0.3$ & $3.9 \pm 0.2$ & $3.7 \pm 0.3$ & $3.2 \pm 0.3$ & $3.1 \pm 0.3$ & $2.8 \pm 0.2$ & $2.8 \pm 0.3$ & $2.5 \pm 0.3$ & $2.8 \pm 0.4$ & $3.6 \pm 0.7$ & $4.0 \pm 0.4$ \\
\hline RCP8.5 - end & $4.0 \pm 0.3$ & $4.1 \pm 0.4$ & $3.9 \pm 0.3$ & $3.5 \pm 0.2$ & $3.1 \pm 0.2$ & $3.0 \pm 0.2$ & $2.8 \pm 0.2$ & $2.8 \pm 0.4$ & $2.5 \pm 0.2$ & $2.7 \pm 0.3$ & $3.2 \pm 0.3$ & $4.1 \pm 0.4$ \\
\hline \multicolumn{13}{|c|}{$\begin{array}{l}\text { Mean Wave Period } \\
\text { (s) }\end{array}$} \\
\hline Hindcast & $12 \pm 3$ & $12 \pm 3$ & $11 \pm 2$ & $11 \pm 3$ & $11 \pm 3$ & $10 \pm 3$ & $11 \pm 3$ & $11 \pm 3$ & $11 \pm 3$ & $11 \pm 3$ & $12 \pm 2$ & $12 \pm 2$ \\
\hline RCP4.5 - mid & $13 \pm 3$ & $12 \pm 3$ & $11 \pm 3$ & $11 \pm 3$ & $11 \pm 3$ & $10 \pm 3$ & $10 \pm 3$ & $11 \pm 3$ & $11 \pm 3$ & $11 \pm 3$ & $12 \pm 2$ & $12 \pm 3$ \\
\hline RCP8.5 - mid & $12 \pm 3$ & $12 \pm 3$ & $11 \pm 3$ & $11 \pm 3$ & $11 \pm 3$ & $10 \pm 3$ & $10 \pm 3$ & $11 \pm 3$ & $11 \pm 3$ & $12 \pm 3$ & $12 \pm 2$ & $12 \pm 2$ \\
\hline RCP 4.5 - end & $12 \pm 3$ & $12 \pm 3$ & $11 \pm 2$ & $11 \pm 3$ & $11 \pm 3$ & $11 \pm 3$ & $11 \pm 3$ & $11 \pm 3$ & $11 \pm 3$ & $12 \pm 3$ & $12 \pm 2$ & $12 \pm 3$ \\
\hline RCP8.5 - end & $12 \pm 3$ & $12 \pm 3$ & $11 \pm 3$ & $11 \pm 3$ & $11 \pm 3$ & $11 \pm 3$ & $10 \pm 3$ & $11 \pm 3$ & $11 \pm 3$ & $12 \pm 3$ & $12 \pm 3$ & $12 \pm 3$ \\
\hline \multicolumn{13}{|c|}{$\begin{array}{l}\text { Top } 5 \text { percent Wave } \\
\text { Period (s) }\end{array}$} \\
\hline Hindcast & $14 \pm 3$ & $13 \pm 3$ & $12 \pm 2$ & $11 \pm 2$ & $11 \pm 3$ & $11 \pm 3$ & $12 \pm 3$ & $12 \pm 3$ & $12 \pm 3$ & $12 \pm 2$ & $13 \pm 3$ & $13 \pm 3$ \\
\hline RCP4.5 - mid & $14 \pm 3$ & $13 \pm 3$ & $12 \pm 3$ & $11 \pm 2$ & $11 \pm 3$ & $11 \pm 3$ & $12 \pm 3$ & $12 \pm 3$ & $13 \pm 3$ & $13 \pm 3$ & $14 \pm 3$ & $14 \pm 3$ \\
\hline RCP8.5 - mid & $13 \pm 3$ & $13 \pm 3$ & $11 \pm 2$ & $10 \pm 2$ & $11 \pm 3$ & $12 \pm 3$ & $12 \pm 3$ & $12 \pm 3$ & $13 \pm 3$ & $13 \pm 3$ & $13 \pm 3$ & $14 \pm 3$ \\
\hline RCP4.5 - end & $14 \pm 3$ & $13 \pm 3$ & $11 \pm 2$ & $11 \pm 2$ & $11 \pm 3$ & $11 \pm 3$ & $12 \pm 4$ & $12 \pm 3$ & $12 \pm 3$ & $12 \pm 3$ & $12 \pm 2$ & $14 \pm 3$ \\
\hline RCP8.5 - end & $15 \pm 3$ & $13 \pm 3$ & $12 \pm 3$ & $10 \pm 2$ & $11 \pm 3$ & $12 \pm 4$ & $11 \pm 3$ & $12 \pm 3$ & $13 \pm 3$ & $13 \pm 3$ & $13 \pm 3$ & $14 \pm 3$ \\
\hline \multicolumn{13}{|l|}{ Mean Wave } \\
\hline \multicolumn{13}{|l|}{ Direction ( 9 ) } \\
\hline Hindcast & $23 \pm 28$ & $24 \pm 31$ & $40 \pm 29$ & $62 \pm 31$ & $91 \pm 32$ & $104 \pm 28$ & $118 \pm 24$ & $125 \pm 24$ & $124 \pm 33$ & $86 \pm 50$ & $36 \pm 39$ & $27 \pm 29$ \\
\hline RCP4.5 - mid & $20 \pm 28$ & $26 \pm 27$ & $39 \pm 28$ & $61 \pm 28$ & $87 \pm 32$ & $103 \pm 27$ & $116 \pm 22$ & $125 \pm 22$ & $130 \pm 25$ & $96 \pm 50$ & $38 \pm 42$ & $24 \pm 29$ \\
\hline RCP8.5 - mid & $24 \pm 27$ & $27 \pm 28$ & $37 \pm 30$ & $60 \pm 28$ & $87 \pm 31$ & $103 \pm 26$ & $118 \pm 22$ & $127 \pm 22$ & $130 \pm 28$ & $101 \pm 48$ & $37 \pm 41$ & $25 \pm 29$ \\
\hline RCP4.5 - end & $21 \pm 29$ & $22 \pm 29$ & $38 \pm 27$ & $60 \pm 30$ & $88 \pm 31$ & $104 \pm 27$ & $120 \pm 23$ & $129 \pm 26$ & $131 \pm 28$ & $101 \pm 52$ & $37 \pm 41$ & $26 \pm 31$ \\
\hline RCP8.5 - end & $22 \pm 29$ & $26 \pm 29$ & $37 \pm 28$ & $62 \pm 28$ & $86 \pm 27$ & $103 \pm 25$ & $117 \pm 23$ & $128 \pm 24$ & $133 \pm 26$ & $109 \pm 45$ & $47 \pm 41$ & $26 \pm 30$ \\
\hline \multirow{2}{*}{\multicolumn{13}{|c|}{$\begin{array}{l}\text { Top } 5 \text { percent Wave } \\
\text { Direction }(9)\end{array}$}} \\
\hline & & & & & & & & & & & & \\
\hline Hindcast & $17 \pm 34$ & $24 \pm 32$ & $41 \pm 23$ & $53 \pm 25$ & $80 \pm 34$ & $96 \pm 31$ & $125 \pm 34$ & $128 \pm 22$ & $129 \pm 34$ & $47 \pm 56$ & $25 \pm 47$ & $17 \pm 36$ \\
\hline RCP4.5 - mid & $7 \pm 36$ & $22 \pm 32$ & $41 \pm 24$ & $57 \pm 19$ & $75 \pm 40$ & $95 \pm 28$ & $118 \pm 27$ & $130 \pm 26$ & $135 \pm 32$ & $72 \pm 71$ & $15 \pm 38$ & $17 \pm 31$ \\
\hline RCP8.5 - mid & $20 \pm 31$ & $24 \pm 32$ & $45 \pm 21$ & $54 \pm 12$ & $79 \pm 28$ & $103 \pm 34$ & $123 \pm 28$ & $128 \pm 25$ & $136 \pm 28$ & $71 \pm 71$ & $15 \pm 34$ & $17 \pm 39$ \\
\hline RCP4.5 - end & $17 \pm 34$ & $20 \pm 35$ & $43 \pm 26$ & $48 \pm 24$ & $79 \pm 38$ & $100 \pm 39$ & $128 \pm 30$ & $140 \pm 37$ & $145 \pm 38$ & $34 \pm 70$ & $23 \pm 40$ & $13 \pm 34$ \\
\hline RCP8.5 - end & $5 \pm 32$ & $22 \pm 35$ & $38 \pm 26$ & $58 \pm 18$ & $75 \pm 31$ & $99 \pm 42$ & $121 \pm 32$ & $137 \pm 37$ & $142 \pm 36$ & $75 \pm 84$ & $23 \pm 32$ & $23 \pm 33$ \\
\hline
\end{tabular}


Appendix A44. Table showing return values of ensemble-average significant wave heights of hindcast and forecast scenarios, including lower and higher 95 percent confidence intervals, at the Palmyra location.

[Years: Hindcast $=1976-2005 ;$ RCP mid $=$ 2026-2045; RCP end $=2081-2100$. Wave height values are in meters]

\begin{tabular}{|c|c|c|c|c|c|c|c|c|c|c|c|c|c|c|c|}
\hline \multirow{2}{*}{ Scenario } & \multicolumn{3}{|c|}{ Hindcast } & \multicolumn{3}{|c|}{ RCP4.5 - mid } & \multicolumn{3}{c|}{ RCP8.5 - mid } & \multicolumn{3}{c|}{ RCP4.5 - end } & \multicolumn{3}{c|}{ RCP8.5 - end } \\
\cline { 2 - 13 } & Low & $\boldsymbol{R}_{\mathbf{V}}$ & High & Low & $\boldsymbol{R}_{\mathbf{V}}$ & High & Low & $\boldsymbol{R}_{\mathbf{V}}$ & High & Low & $\boldsymbol{R}_{\mathbf{V}}$ & High & Low & $\boldsymbol{R}_{\mathbf{V}}$ & High \\
\hline 2-year & 4.91 & 5.24 & 5.78 & 4.85 & 5.19 & 5.78 & 4.76 & 5.12 & 5.76 & 4.79 & 5.20 & 5.93 & 4.68 & 4.99 & 5.50 \\
5-year & 5.17 & 5.76 & 6.81 & 5.02 & 5.58 & 6.63 & 4.89 & 5.43 & 6.54 & 4.98 & 5.66 & 7.07 & 4.83 & 5.31 & 6.16 \\
10-year & 5.37 & 6.22 & 7.86 & 5.14 & 5.88 & 7.42 & 4.97 & 5.66 & 7.21 & 5.12 & 6.05 & 8.21 & 4.93 & 5.54 & 6.72 \\
20-year & 5.57 & 6.75 & 9.20 & 5.24 & 6.19 & 8.35 & 5.03 & 5.88 & 7.97 & 5.24 & 6.48 & 9.66 & 5.01 & 5.76 & 7.34 \\
50-year & 5.85 & 7.56 & 11.58 & 5.37 & 6.62 & 9.86 & 5.10 & 6.16 & 9.13 & 5.40 & 7.10 & 12.20 & 5.10 & 6.05 & 8.24 \\
100-year & 6.07 & 8.27 & 13.97 & 5.45 & 6.96 & 11.24 & 5.14 & 6.37 & 10.12 & 5.51 & 7.63 & 14.74 & 5.16 & 6.26 & 9.00 \\
\hline
\end{tabular}


Appendix A45. Table showing Rose Atoll monthly means and mean of the top 5 percent for significant wave height, peak wave period, and peak wave direction.

[Years: Hindcast $=$ 1976-2005; RCP mid = 2026-2045; RCP end = 2081-2100. Wave directions are “coming from"]

\begin{tabular}{|c|c|c|c|c|c|c|c|c|c|c|c|c|}
\hline Month & Jan & Feb & Mar & Apr & May & Jun & July & Aug & Sep & Oct & Nov & Dec \\
\hline \multicolumn{13}{|c|}{$\begin{array}{l}\text { Mean Wave Height } \\
\text { (m) }\end{array}$} \\
\hline Hindcast & $2.2 \pm 0.7$ & $2.2 \pm 0.7$ & $2.2 \pm 0.6$ & $2.2 \pm 0.6$ & $2.4 \pm 0.7$ & $2.5 \pm 0.7$ & $2.5 \pm 0.7$ & $2.4 \pm 0.7$ & $2.4 \pm 0.6$ & $2.3 \pm 0.6$ & $2.2 \pm 0.6$ & $2.1 \pm 0.5$ \\
\hline RCP4.5 - mid & $2.2 \pm 0.7$ & $2.1 \pm 0.6$ & $2.2 \pm 0.6$ & $2.3 \pm 0.6$ & $2.4 \pm 0.6$ & $2.5 \pm 0.7$ & $2.5 \pm 0.7$ & $2.4 \pm 0.6$ & $2.5 \pm 0.7$ & $2.3 \pm 0.6$ & $2.2 \pm 0.6$ & $2.1 \pm 0.5$ \\
\hline RCP8.5 - mid & $2.1 \pm 0.6$ & $2.2 \pm 0.7$ & $2.2 \pm 0.7$ & $2.3 \pm 0.6$ & $2.4 \pm 0.6$ & $2.4 \pm 0.7$ & $2.5 \pm 0.7$ & $2.5 \pm 0.7$ & $2.4 \pm 0.7$ & $2.4 \pm 0.6$ & $2.2 \pm 0.6$ & $2.2 \pm 0.7$ \\
\hline RCP4.5 - end & $2.1 \pm 0.6$ & $2.2 \pm 0.6$ & $2.1 \pm 0.6$ & $2.3 \pm 0.7$ & $2.5 \pm 0.7$ & $2.5 \pm 0.7$ & $2.5 \pm 0.7$ & $2.5 \pm 0.6$ & $2.4 \pm 0.6$ & $2.3 \pm 0.6$ & $2.2 \pm 0.6$ & $2.2 \pm 0.7$ \\
\hline RCP8.5 - end & $2.1 \pm 0.7$ & $2.2 \pm 0.7$ & $2.1 \pm 0.6$ & $2.2 \pm 0.5$ & $2.4 \pm 0.7$ & $2.5 \pm 0.7$ & $2.5 \pm 0.6$ & $2.5 \pm 0.7$ & $2.5 \pm 0.7$ & $2.4 \pm 0.6$ & $2.1 \pm 0.5$ & $2.1 \pm 0.5$ \\
\hline \multicolumn{13}{|c|}{$\begin{array}{l}\text { Top } 5 \text { percent Wave } \\
\text { Height }(\mathrm{m})\end{array}$} \\
\hline Hindcast & $4.4 \pm 1.0$ & $4.3 \pm 1.4$ & $4.0 \pm 0.7$ & $3.9 \pm 0.7$ & $4.3 \pm 0.6$ & $4.2 \pm 0.5$ & $4.1 \pm 0.5$ & $4.1 \pm 0.4$ & $3.9 \pm 0.4$ & $3.9 \pm 0.4$ & $3.9 \pm 0.6$ & $3.5 \pm 0.7$ \\
\hline RCP4.5 - mid & $4.1 \pm 1.3$ & $3.9 \pm 1.1$ & $3.9 \pm 1.0$ & $4.1 \pm 0.7$ & $4.1 \pm 0.5$ & $4.2 \pm 0.4$ & $4.2 \pm 0.3$ & $4.1 \pm 0.4$ & $4.2 \pm 0.4$ & $3.9 \pm 0.3$ & $4.0 \pm 0.6$ & $3.5 \pm 0.6$ \\
\hline RCP8.5 - mid & $4.0 \pm 1.0$ & $4.4 \pm 1.1$ & $4.2 \pm 1.0$ & $3.9 \pm 0.6$ & $4.1 \pm 0.4$ & $4.2 \pm 0.4$ & $4.2 \pm 0.4$ & $4.2 \pm 0.4$ & $4.2 \pm 0.4$ & $4.0 \pm 0.4$ & $3.8 \pm 0.6$ & $4.3 \pm 1.3$ \\
\hline RCP4.5 - end & $4.0 \pm 1.0$ & $4.1 \pm 0.9$ & $3.9 \pm 1.2$ & $4.2 \pm 0.7$ & $4.5 \pm 0.6$ & $4.2 \pm 0.4$ & $4.3 \pm 0.5$ & $4.1 \pm 0.4$ & $4.2 \pm 0.4$ & $4.0 \pm 0.5$ & $3.9 \pm 0.7$ & $4.1 \pm 1.1$ \\
\hline RCP8.5 - end & $4.4 \pm 1.6$ & $4.6 \pm 1.0$ & $3.9 \pm 1.0$ & $3.7 \pm 0.6$ & $4.3 \pm 0.5$ & $4.4 \pm 0.5$ & $4.2 \pm 0.4$ & $4.3 \pm 0.4$ & $4.2 \pm 0.4$ & $3.9 \pm 0.4$ & $3.5 \pm 0.5$ & $3.6 \pm 0.5$ \\
\hline \multicolumn{13}{|c|}{$\begin{array}{l}\text { Mean Wave Period } \\
\text { (s) }\end{array}$} \\
\hline Hindcast & $12 \pm 3$ & $12 \pm 2$ & $12 \pm 2$ & $11 \pm 2$ & $11 \pm 3$ & $11 \pm 3$ & $11 \pm 3$ & $11 \pm 3$ & $11 \pm 3$ & $11 \pm 3$ & $11 \pm 3$ & $12 \pm 3$ \\
\hline RCP4.5 - mid & $12 \pm 3$ & $12 \pm 3$ & $12 \pm 2$ & $12 \pm 2$ & $11 \pm 3$ & $11 \pm 3$ & $11 \pm 3$ & $11 \pm 3$ & $11 \pm 3$ & $11 \pm 3$ & $12 \pm 3$ & $12 \pm 3$ \\
\hline RCP8.5 - mid & $12 \pm 3$ & $12 \pm 2$ & $12 \pm 2$ & $11 \pm 2$ & $11 \pm 3$ & $11 \pm 3$ & $11 \pm 3$ & $11 \pm 3$ & $11 \pm 3$ & $11 \pm 3$ & $12 \pm 3$ & $12 \pm 3$ \\
\hline RCP4.5 - end & $12 \pm 3$ & $12 \pm 2$ & $12 \pm 2$ & $12 \pm 2$ & $11 \pm 3$ & $11 \pm 3$ & $11 \pm 3$ & $11 \pm 3$ & $11 \pm 3$ & $11 \pm 3$ & $11 \pm 3$ & $12 \pm 3$ \\
\hline RCP8.5 - end & $12 \pm 3$ & $12 \pm 3$ & $12 \pm 2$ & $12 \pm 3$ & $11 \pm 3$ & $11 \pm 3$ & $11 \pm 3$ & $11 \pm 3$ & $11 \pm 3$ & $11 \pm 3$ & $11 \pm 3$ & $12 \pm 3$ \\
\hline \multirow{2}{*}{\multicolumn{13}{|c|}{$\begin{array}{l}\text { Top } 5 \text { percent Wave } \\
\text { Period (s) }\end{array}$}} \\
\hline & & & & & & & & & & & & \\
\hline Hindcast & $11 \pm 2$ & $11 \pm 2$ & $11 \pm 2$ & $11 \pm 2$ & $11 \pm 2$ & $10 \pm 2$ & $11 \pm 3$ & $10 \pm 2$ & $10 \pm 2$ & $10 \pm 2$ & $10 \pm 2$ & $10 \pm 3$ \\
\hline RCP4.5 - mid & $11 \pm 3$ & $11 \pm 2$ & $11 \pm 2$ & $11 \pm 2$ & $11 \pm 2$ & $10 \pm 2$ & $11 \pm 2$ & $11 \pm 2$ & $10 \pm 1$ & $10 \pm 2$ & $10 \pm 2$ & $11 \pm 3$ \\
\hline RCP8.5 - mid & $11 \pm 2$ & $10 \pm 2$ & $11 \pm 2$ & $10 \pm 2$ & $11 \pm 2$ & $11 \pm 2$ & $11 \pm 2$ & $10 \pm 2$ & $10 \pm 2$ & $10 \pm 2$ & $10 \pm 2$ & $10 \pm 2$ \\
\hline RCP4.5 - end & $10 \pm 2$ & $11 \pm 2$ & $11 \pm 2$ & $11 \pm 2$ & $10 \pm 2$ & $11 \pm 2$ & $11 \pm 3$ & $10 \pm 2$ & $10 \pm 2$ & $10 \pm 2$ & $10 \pm 1$ & $10 \pm 2$ \\
\hline RCP8.5 - end & $10 \pm 2$ & $10 \pm 2$ & $10 \pm 2$ & $11 \pm 2$ & $10 \pm 2$ & $11 \pm 3$ & $10 \pm 2$ & $10 \pm 2$ & $10 \pm 2$ & $10 \pm 2$ & $10 \pm 2$ & $11 \pm 3$ \\
\hline \multicolumn{13}{|l|}{ Mean Wave } \\
\hline \multicolumn{13}{|l|}{ Direction ( $\left.{ }^{\circ}\right)$} \\
\hline Hindcast & $30 \pm 51$ & $31 \pm 65$ & $91 \pm 81$ & $134 \pm 45$ & $141 \pm 31$ & $137 \pm 29$ & $136 \pm 29$ & $132 \pm 28$ & $131 \pm 27$ & $124 \pm 30$ & $101 \pm 47$ & $56 \pm 49$ \\
\hline RCP4.5 - mid & $33 \pm 50$ & $28 \pm 59$ & $76 \pm 79$ & $133 \pm 48$ & $139 \pm 28$ & $134 \pm 30$ & $135 \pm 28$ & $131 \pm 26$ & $129 \pm 25$ & $126 \pm 28$ & $105 \pm 46$ & $62 \pm 53$ \\
\hline RCP8.5 - mid & $40 \pm 46$ & $34 \pm 61$ & $89 \pm 86$ & $135 \pm 43$ & $139 \pm 26$ & $140 \pm 29$ & $136 \pm 29$ & $136 \pm 27$ & $130 \pm 25$ & $126 \pm 27$ & $104 \pm 42$ & $60 \pm 52$ \\
\hline RCP4.5 - end & $36 \pm 50$ & $23 \pm 63$ & $72 \pm 82$ & $133 \pm 47$ & $138 \pm 28$ & $135 \pm 28$ & $135 \pm 28$ & $132 \pm 26$ & $130 \pm 24$ & $128 \pm 28$ & $106 \pm 43$ & $63 \pm 52$ \\
\hline RCP8.5 - end & $40 \pm 49$ & $32 \pm 61$ & $77 \pm 84$ & $134 \pm 42$ & $139 \pm 28$ & $134 \pm 26$ & $134 \pm 26$ & $131 \pm 26$ & $129 \pm 23$ & $126 \pm 24$ & $110 \pm 37$ & $63 \pm 47$ \\
\hline \multirow{2}{*}{\multicolumn{13}{|c|}{$\begin{array}{l}\text { Top } 5 \text { percent Wave } \\
\text { Direction }(9)\end{array}$}} \\
\hline & & & & & & & & & & & & \\
\hline Hindcast & $5 \pm 73$ & $357 \pm 88$ & $84 \pm 106$ & $132 \pm 41$ & $124 \pm 34$ & $127 \pm 29$ & $135 \pm 24$ & $125 \pm 17$ & $121 \pm 26$ & $120 \pm 29$ & $105 \pm 38$ & $60 \pm 60$ \\
\hline RCP4.5 - mid & $357 \pm 66$ & $350 \pm 65$ & $61 \pm 115$ & $132 \pm 49$ & $133 \pm 24$ & $125 \pm 27$ & $130 \pm 21$ & $128 \pm 17$ & $121 \pm 16$ & $121 \pm 20$ & $102 \pm 42$ & $89 \pm 66$ \\
\hline RCP8.5 - mid & $37 \pm 72$ & $345 \pm 79$ & $57 \pm 98$ & $133 \pm 39$ & $129 \pm 27$ & $133 \pm 24$ & $131 \pm 26$ & $130 \pm 19$ & $123 \pm 17$ & $120 \pm 24$ & $102 \pm 43$ & $75 \pm 57$ \\
\hline RCP4.5 - end & $24 \pm 80$ & $347 \pm 74$ & $47 \pm 100$ & $121 \pm 54$ & $128 \pm 22$ & $132 \pm 29$ & $128 \pm 19$ & $128 \pm 25$ & $123 \pm 19$ & $119 \pm 19$ & $111 \pm 33$ & $82 \pm 58$ \\
\hline RCP8.5 - end & $9 \pm 75$ & $338 \pm 61$ & $349 \pm 111$ & $134 \pm 50$ & $123 \pm 32$ & $131 \pm 20$ & $128 \pm 22$ & $128 \pm 21$ & $125 \pm 19$ & $119 \pm 20$ & $115 \pm 30$ & $87 \pm 44$ \\
\hline
\end{tabular}


Appendix A46. Table showing return values of ensemble-average significant wave heights of hindcast and forecast scenarios, including lower and higher 95 percent confidence intervals, at the Rose Atoll location.

[Years: Hindcast $=1976-2005 ;$ RCP mid $=$ 2026-2045; RCP end $=2081-2100$. Wave height values are in meters]

\begin{tabular}{|c|c|c|c|c|c|c|c|c|c|c|c|c|c|c|c|c|}
\hline \multirow{2}{*}{ Scenario } & \multicolumn{3}{|c|}{ Hindcast } & \multicolumn{3}{c|}{ RCP4.5 - mid } & \multicolumn{3}{c|}{ RCP8.5 - mid } & \multicolumn{3}{c|}{ RCP4.5 - end } & \multicolumn{3}{c|}{ RCP8.5 - end } \\
\cline { 2 - 13 } & Low & $\boldsymbol{R}_{\boldsymbol{V}}$ & High & Low & $\boldsymbol{R}_{\boldsymbol{V}}$ & High & Low & $\boldsymbol{R}_{\boldsymbol{V}}$ & High & Low & $\boldsymbol{R}_{\boldsymbol{V}}$ & High & Low & $\boldsymbol{R}_{\boldsymbol{V}}$ & High \\
\hline 2-year & 6.14 & 6.99 & 8.46 & 5.82 & 7.02 & 9.82 & 6.04 & 7.34 & 10.47 & 6.00 & 7.30 & 10.26 & 6.11 & 7.17 & 9.39 \\
5-year & 6.71 & 8.26 & 11.29 & 6.29 & 8.68 & 15.74 & 6.47 & 8.95 & 16.49 & 6.49 & 8.96 & 16.01 & 6.56 & 8.53 & 13.46 \\
10-year & 7.15 & 9.42 & 14.32 & 6.68 & 10.43 & 23.75 & 6.81 & 10.57 & 24.32 & 6.87 & 10.63 & 23.36 & 6.90 & 9.81 & 18.27 \\
20-year & 7.61 & 10.79 & 18.41 & 7.08 & 12.76 & 37.08 & 7.15 & 12.62 & 36.88 & 7.25 & 12.74 & 34.96 & 7.25 & 11.38 & 25.36 \\
50-year & 8.23 & 12.97 & 26.09 & 7.66 & 17.06 & 69.15 & 7.60 & 16.22 & 65.81 & 7.78 & 16.44 & 61.19 & 7.72 & 14.00 & 40.14 \\
100-year & 8.72 & 14.97 & 34.30 & 8.14 & 21.58 & 112.59 & 7.94 & 19.81 & 103.41 & 8.19 & 20.13 & 94.68 & 8.07 & 16.48 & 57.60 \\
\hline
\end{tabular}


Appendix A47. Table showing Howland monthly means and mean of the top 5 percent for significant wave height, peak wave period, and peak wave direction.

[Years: Hindcast $=1976-2005 ;$ RCP mid $=$ 2026-2045; RCP end $=$ 2081-2100. Wave directions are "coming from”]

\begin{tabular}{|c|c|c|c|c|c|c|c|c|c|c|c|c|}
\hline Month & Jan & Feb & Mar & Apr & May & Jun & July & Aug & Sep & Oct & Nov & Dec \\
\hline \multicolumn{13}{|c|}{$\begin{array}{l}\text { Mean Wave Height } \\
\text { (m) }\end{array}$} \\
\hline Hindcast & $2.4 \pm 0.5$ & $2.4 \pm 0.4$ & $2.2 \pm 0.4$ & $2.0 \pm 0.4$ & $1.8 \pm 0.4$ & $1.9 \pm 0.4$ & $1.9 \pm 0.4$ & $1.9 \pm 0.4$ & $1.8 \pm 0.4$ & $1.9 \pm 0.4$ & $2.1 \pm 0.5$ & $2.3 \pm 0.5$ \\
\hline RCP4.5 - mid & $2.4 \pm 0.5$ & $2.4 \pm 0.4$ & $2.3 \pm 0.4$ & $2.1 \pm 0.4$ & $1.8 \pm 0.3$ & $1.8 \pm 0.4$ & $2.0 \pm 0.4$ & $1.9 \pm 0.4$ & $1.8 \pm 0.4$ & $1.8 \pm 0.4$ & $2.0 \pm 0.5$ & $2.3 \pm 0.4$ \\
\hline RCP8.5 - mid & $2.4 \pm 0.4$ & $2.4 \pm 0.5$ & $2.2 \pm 0.4$ & $2.0 \pm 0.4$ & $1.8 \pm 0.3$ & $1.8 \pm 0.3$ & $1.9 \pm 0.4$ & $1.9 \pm 0.4$ & $1.8 \pm 0.3$ & $1.8 \pm 0.4$ & $2.0 \pm 0.4$ & $2.3 \pm 0.5$ \\
\hline RCP4.5 - end & $2.4 \pm 0.4$ & $2.4 \pm 0.5$ & $2.3 \pm 0.4$ & $2.0 \pm 0.4$ & $1.9 \pm 0.3$ & $1.9 \pm 0.4$ & $1.9 \pm 0.3$ & $1.9 \pm 0.4$ & $1.8 \pm 0.3$ & $1.8 \pm 0.4$ & $2.0 \pm 0.5$ & $2.3 \pm 0.5$ \\
\hline RCP8.5 - end & $2.3 \pm 0.4$ & $2.3 \pm 0.4$ & $2.2 \pm 0.4$ & $2.0 \pm 0.4$ & $1.8 \pm 0.3$ & $1.9 \pm 0.3$ & $2.0 \pm 0.3$ & $2.0 \pm 0.3$ & $1.9 \pm 0.3$ & $1.8 \pm 0.4$ & $1.9 \pm 0.4$ & $2.2 \pm 0.5$ \\
\hline \multicolumn{13}{|c|}{$\begin{array}{l}\text { Top } 5 \text { percent Wave } \\
\text { Height }(\mathrm{m})\end{array}$} \\
\hline Hindcast & $3.6 \pm 0.5$ & $3.5 \pm 0.2$ & $3.3 \pm 0.2$ & $2.9 \pm 0.2$ & $2.7 \pm 0.4$ & $2.6 \pm 0.2$ & $2.8 \pm 0.3$ & $2.8 \pm 0.2$ & $2.6 \pm 0.2$ & $2.9 \pm 0.5$ & $3.2 \pm 0.3$ & $3.5 \pm 0.4$ \\
\hline RCP4.5 - mid & $3.5 \pm 0.3$ & $3.5 \pm 0.3$ & $3.4 \pm 0.4$ & $3.0 \pm 0.2$ & $2.6 \pm 0.2$ & $2.7 \pm 0.2$ & $2.9 \pm 0.1$ & $2.8 \pm 0.2$ & $2.7 \pm 0.6$ & $2.7 \pm 0.1$ & $3.1 \pm 0.6$ & $3.3 \pm 0.2$ \\
\hline RCP8.5 - mid & $3.5 \pm 0.4$ & $3.6 \pm 0.3$ & $3.3 \pm 0.3$ & $2.9 \pm 0.2$ & $2.6 \pm 0.2$ & $2.6 \pm 0.2$ & $2.8 \pm 0.2$ & $2.8 \pm 0.2$ & $2.6 \pm 0.1$ & $2.7 \pm 0.3$ & $3.1 \pm 0.3$ & $3.5 \pm 0.4$ \\
\hline RCP4.5 - end & $3.5 \pm 0.3$ & $3.6 \pm 0.3$ & $3.2 \pm 0.2$ & $2.9 \pm 0.2$ & $2.6 \pm 0.2$ & $2.7 \pm 0.2$ & $2.7 \pm 0.2$ & $2.7 \pm 0.1$ & $2.6 \pm 0.2$ & $2.7 \pm 0.2$ & $3.3 \pm 0.6$ & $3.5 \pm 0.3$ \\
\hline RCP8.5 - end & $3.3 \pm 0.3$ & $3.4 \pm 0.3$ & $3.1 \pm 0.2$ & $2.9 \pm 0.4$ & $2.7 \pm 0.4$ & $2.7 \pm 0.2$ & $2.8 \pm 0.2$ & $2.8 \pm 0.2$ & $2.6 \pm 0.2$ & $2.8 \pm 0.6$ & $2.9 \pm 0.2$ & $3.3 \pm 0.3$ \\
\hline \multicolumn{13}{|c|}{$\begin{array}{l}\text { Mean Wave Period } \\
\text { (s) }\end{array}$} \\
\hline Hindcast & $12 \pm 2$ & $12 \pm 2$ & $11 \pm 2$ & $10 \pm 2$ & $11 \pm 3$ & $10 \pm 3$ & $10 \pm 3$ & $10 \pm 2$ & $10 \pm 2$ & $11 \pm 2$ & $11 \pm 2$ & $12 \pm 2$ \\
\hline RCP4.5 - mid & $12 \pm 2$ & $12 \pm 2$ & $11 \pm 2$ & $10 \pm 2$ & $10 \pm 2$ & $10 \pm 2$ & $10 \pm 2$ & $10 \pm 2$ & $10 \pm 3$ & $11 \pm 2$ & $11 \pm 2$ & $12 \pm 2$ \\
\hline RCP8.5 - mid & $12 \pm 2$ & $12 \pm 2$ & $11 \pm 2$ & $10 \pm 2$ & $10 \pm 2$ & $10 \pm 3$ & $10 \pm 3$ & $10 \pm 3$ & $11 \pm 3$ & $11 \pm 3$ & $11 \pm 2$ & $12 \pm 2$ \\
\hline RCP4.5 - end & $12 \pm 2$ & $12 \pm 2$ & $11 \pm 2$ & $11 \pm 2$ & $10 \pm 3$ & $10 \pm 3$ & $10 \pm 3$ & $10 \pm 3$ & $10 \pm 3$ & $11 \pm 3$ & $11 \pm 2$ & $12 \pm 2$ \\
\hline RCP8.5 - end & $12 \pm 2$ & $12 \pm 2$ & $11 \pm 2$ & $10 \pm 2$ & $10 \pm 3$ & $10 \pm 3$ & $10 \pm 2$ & $10 \pm 3$ & $10 \pm 3$ & $11 \pm 3$ & $11 \pm 2$ & $12 \pm 2$ \\
\hline \multicolumn{13}{|c|}{$\begin{array}{l}\text { Top } 5 \text { percent Wave } \\
\text { Period (s) }\end{array}$} \\
\hline Hindcast & $13 \pm 3$ & $13 \pm 3$ & $12 \pm 2$ & $11 \pm 2$ & $11 \pm 2$ & $11 \pm 3$ & $11 \pm 2$ & $10 \pm 2$ & $10 \pm 2$ & $12 \pm 2$ & $13 \pm 2$ & $13 \pm 2$ \\
\hline RCP4.5 - mid & $14 \pm 2$ & $13 \pm 3$ & $12 \pm 2$ & $11 \pm 1$ & $11 \pm 2$ & $10 \pm 2$ & $11 \pm 3$ & $10 \pm 2$ & $11 \pm 2$ & $11 \pm 3$ & $13 \pm 3$ & $13 \pm 2$ \\
\hline RCP8.5 - mid & $13 \pm 2$ & $13 \pm 2$ & $12 \pm 2$ & $11 \pm 2$ & $11 \pm 2$ & $11 \pm 2$ & $10 \pm 3$ & $11 \pm 2$ & $10 \pm 2$ & $12 \pm 3$ & $12 \pm 3$ & $13 \pm 2$ \\
\hline RCP4.5 - end & $14 \pm 3$ & $13 \pm 2$ & $12 \pm 2$ & $11 \pm 2$ & $11 \pm 2$ & $11 \pm 2$ & $11 \pm 2$ & $10 \pm 2$ & $10 \pm 3$ & $11 \pm 3$ & $12 \pm 2$ & $13 \pm 3$ \\
\hline RCP8.5 - end & $14 \pm 3$ & $13 \pm 2$ & $12 \pm 2$ & $11 \pm 2$ & $10 \pm 1$ & $11 \pm 2$ & $10 \pm 2$ & $10 \pm 3$ & $11 \pm 2$ & $11 \pm 2$ & $12 \pm 2$ & $13 \pm 3$ \\
\hline \multicolumn{13}{|l|}{ Mean Wave } \\
\hline \multicolumn{13}{|l|}{ Direction ( 9 ) } \\
\hline Hindcast & $27 \pm 23$ & $29 \pm 24$ & $41 \pm 26$ & $65 \pm 27$ & $97 \pm 30$ & $111 \pm 22$ & $114 \pm 19$ & $117 \pm 18$ & $114 \pm 26$ & $88 \pm 37$ & $48 \pm 34$ & $32 \pm 26$ \\
\hline RCP4.5 - mid & $27 \pm 25$ & $29 \pm 24$ & $39 \pm 23$ & $63 \pm 26$ & $95 \pm 28$ & $109 \pm 23$ & $113 \pm 17$ & $116 \pm 16$ & $118 \pm 20$ & $95 \pm 32$ & $53 \pm 34$ & $34 \pm 26$ \\
\hline RCP8.5 - mid & $29 \pm 23$ & $31 \pm 22$ & $38 \pm 25$ & $62 \pm 27$ & $96 \pm 26$ & $111 \pm 21$ & $114 \pm 18$ & $119 \pm 18$ & $119 \pm 21$ & $97 \pm 33$ & $51 \pm 35$ & $33 \pm 26$ \\
\hline RCP4.5 - end & $27 \pm 26$ & $26 \pm 25$ & $37 \pm 25$ & $62 \pm 28$ & $96 \pm 28$ & $110 \pm 22$ & $115 \pm 19$ & $119 \pm 19$ & $120 \pm 20$ & $99 \pm 37$ & $52 \pm 37$ & $33 \pm 29$ \\
\hline RCP8.5 - end & $28 \pm 25$ & $30 \pm 24$ & $40 \pm 25$ & $65 \pm 28$ & $96 \pm 27$ & $110 \pm 21$ & $114 \pm 19$ & $119 \pm 18$ & $120 \pm 21$ & $104 \pm 31$ & $64 \pm 32$ & $38 \pm 29$ \\
\hline \multirow{2}{*}{\multicolumn{13}{|c|}{$\begin{array}{l}\text { Top } 5 \text { percent Wave } \\
\text { Direction }(9)\end{array}$}} \\
\hline & & & & & & & & & & & & \\
\hline Hindcast & $18 \pm 23$ & $19 \pm 26$ & $32 \pm 26$ & $53 \pm 32$ & $98 \pm 60$ & $118 \pm 26$ & $122 \pm 28$ & $114 \pm 12$ & $110 \pm 22$ & $56 \pm 58$ & $23 \pm 38$ & $16 \pm 33$ \\
\hline RCP4.5 - mid & $11 \pm 24$ & $10 \pm 33$ & $32 \pm 25$ & $54 \pm 31$ & $94 \pm 38$ & $109 \pm 21$ & $114 \pm 12$ & $109 \pm 12$ & $117 \pm 34$ & $81 \pm 37$ & $29 \pm 42$ & $26 \pm 24$ \\
\hline RCP8.5 - mid & $23 \pm 24$ & $26 \pm 21$ & $37 \pm 21$ & $46 \pm 29$ & $101 \pm 27$ & $110 \pm 22$ & $110 \pm 15$ & $120 \pm 24$ & $117 \pm 17$ & $71 \pm 48$ & $4 \pm 58$ & $14 \pm 32$ \\
\hline RCP4.5 - end & $13 \pm 28$ & $18 \pm 28$ & $25 \pm 27$ & $52 \pm 30$ & $101 \pm 46$ & $107 \pm 33$ & $120 \pm 19$ & $118 \pm 26$ & $122 \pm 29$ & $82 \pm 52$ & $20 \pm 56$ & $8 \pm 40$ \\
\hline RCP8.5 - end & $8 \pm 23$ & $22 \pm 25$ & $35 \pm 24$ & $53 \pm 36$ & $91 \pm 42$ & $110 \pm 24$ & $113 \pm 22$ & $118 \pm 22$ & $125 \pm 36$ & $91 \pm 63$ & $40 \pm 33$ & $24 \pm 25$ \\
\hline
\end{tabular}


Appendix A48. Table showing return values of ensemble-average significant wave heights of hindcast and forecast scenarios, including lower and higher 95 percent confidence intervals, at the Howland location.

[Years: Hindcast $=1976-2005 ;$ RCP mid $=$ 2026-2045; RCP end $=2081-2100$. Wave height values are in meters]

\begin{tabular}{|c|c|c|c|c|c|c|c|c|c|c|c|c|c|c|c|}
\hline \multirow{2}{*}{ Scenario } & \multicolumn{3}{|c|}{ Hindcast } & \multicolumn{3}{c|}{ RCP4.5 - mid } & \multicolumn{3}{c|}{ RCP8.5 - mid } & \multicolumn{3}{c|}{ RCP4.5 - end } & \multicolumn{3}{c|}{ RCP8.5 - end } \\
\cline { 2 - 14 } & Low & $\boldsymbol{R}_{\mathbf{V}}$ & High & Low & $\boldsymbol{R}_{\mathbf{V}}$ & High & Low & $\boldsymbol{R}_{\mathbf{V}}$ & High & Low & $\boldsymbol{R}_{\mathbf{V}}$ & High & Low & $\boldsymbol{R}_{\mathbf{V}}$ & High \\
\hline 2-year & 4.10 & 4.40 & 4.88 & 4.05 & 4.40 & 5.03 & 4.06 & 4.41 & 5.01 & 4.01 & 4.36 & 4.99 & 3.88 & 4.34 & 5.26 \\
5-year & 4.32 & 4.84 & 5.79 & 4.26 & 4.90 & 6.18 & 4.22 & 4.78 & 5.83 & 4.17 & 4.75 & 5.97 & 4.07 & 4.88 & 6.82 \\
10-year & 4.48 & 5.22 & 6.70 & 4.43 & 5.35 & 7.39 & 4.33 & 5.06 & 6.56 & 4.28 & 5.08 & 6.96 & 4.21 & 5.37 & 8.54 \\
20-year & 4.64 & 5.66 & 7.88 & 4.60 & 5.88 & 9.01 & 4.42 & 5.34 & 7.39 & 4.38 & 5.44 & 8.21 & 4.34 & 5.93 & 10.94 \\
50-year & 4.86 & 6.33 & 9.96 & 4.83 & 6.71 & 12.01 & 4.53 & 5.71 & 8.67 & 4.51 & 5.97 & 10.41 & 4.51 & 6.82 & 15.60 \\
100-year & 5.02 & 6.92 & 12.05 & 5.01 & 7.45 & 15.19 & 4.60 & 5.99 & 9.81 & 4.60 & 6.41 & 12.62 & 4.64 & 7.62 & 20.75 \\
\hline
\end{tabular}


Appendix A49. Table showing Jarvis monthly means and mean of the top 5 percent for significant wave height, peak wave period, and peak wave direction.

[Years: Hindcast $=1976-2005 ;$ RCP mid $=$ 2026-2045; RCP end $=$ 2081-2100. Wave directions are “coming from”]

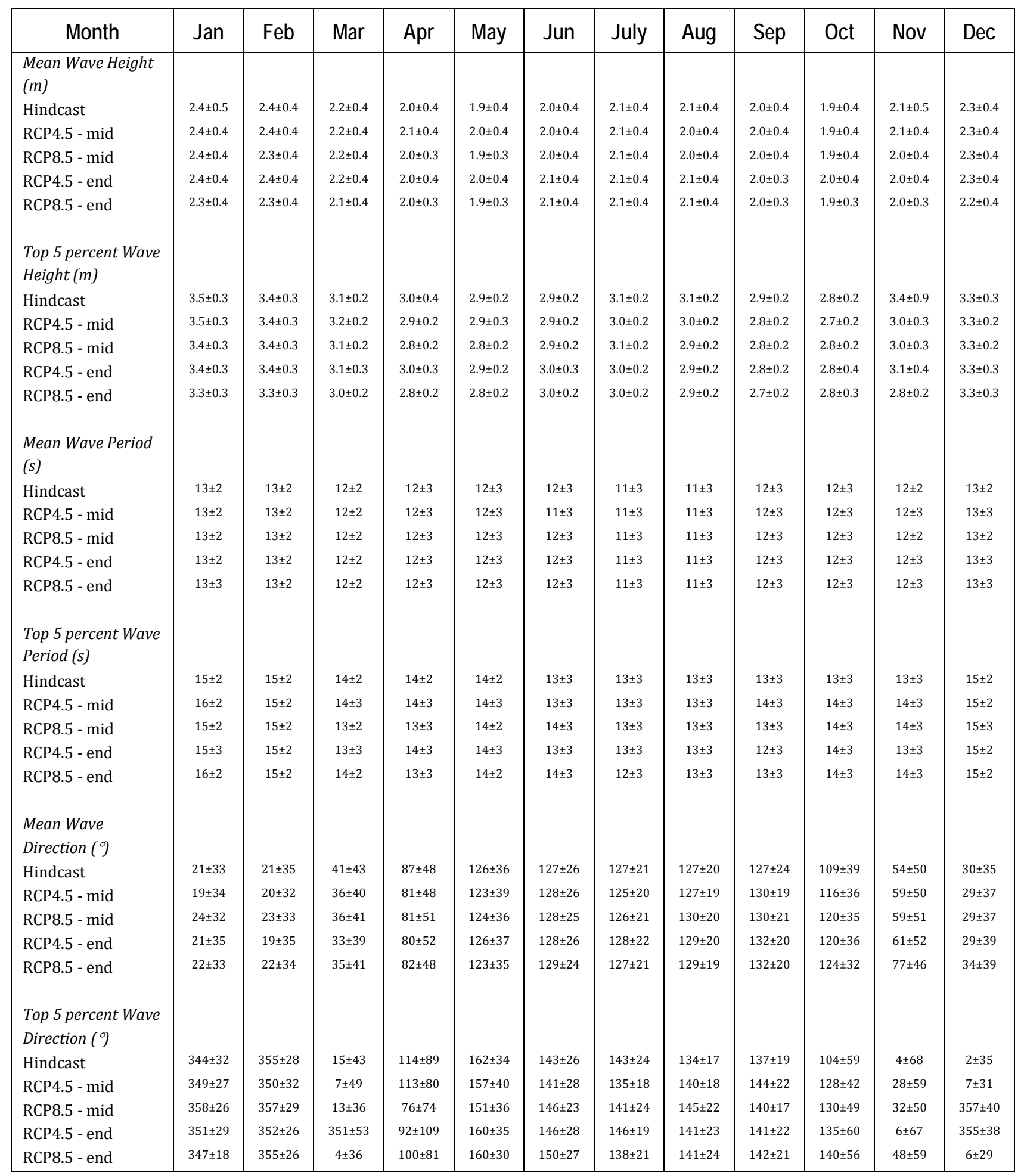


Appendix A50. Table showing return values of ensemble-average significant wave heights of hindcast and forecast scenarios, including lower and higher 95 percent confidence intervals, at the Jarvis location.

[Years: Hindcast $=1976-2005 ;$ RCP mid $=2026-2045 ;$ RCP end $=2081-2100$. Wave height values are in meters $]$

\begin{tabular}{|c|c|c|c|c|c|c|c|c|c|c|c|c|c|c|c|}
\hline \multirow{2}{*}{ Scenario } & \multicolumn{3}{|c|}{ Hindcast } & \multicolumn{3}{c|}{ RCP4.5 - mid } & \multicolumn{3}{c|}{ RCP8.5 - mid } & \multicolumn{3}{|c|}{ RCP4.5 - end } & \multicolumn{3}{c|}{ RCP8.5 - end } \\
\cline { 2 - 13 } & Low & $\boldsymbol{R}_{\boldsymbol{V}}$ & High & Low & $\boldsymbol{R}_{\boldsymbol{V}}$ & High & Low & $\boldsymbol{R}_{\boldsymbol{V}}$ & High & Low & $\boldsymbol{R}_{\boldsymbol{V}}$ & High & Low & $\boldsymbol{R}_{\boldsymbol{V}}$ & High \\
\hline 2-year & 4.05 & 4.34 & 4.81 & 3.96 & 4.24 & 4.75 & 3.86 & 4.09 & 4.47 & 3.97 & 4.30 & 4.91 & 3.84 & 4.07 & 4.47 \\
5-year & 4.28 & 4.80 & 5.74 & 4.06 & 4.49 & 5.32 & 3.96 & 4.31 & 4.95 & 4.11 & 4.66 & 5.81 & 3.94 & 4.29 & 4.94 \\
10-year & 4.46 & 5.22 & 6.72 & 4.12 & 4.66 & 5.79 & 4.02 & 4.47 & 5.36 & 4.21 & 4.96 & 6.68 & 4.00 & 4.44 & 5.32 \\
20-year & 4.65 & 5.71 & 8.01 & 4.17 & 4.81 & 6.29 & 4.08 & 4.62 & 5.80 & 4.30 & 5.26 & 7.76 & 4.04 & 4.58 & 5.72 \\
50-year & 4.91 & 6.49 & 10.36 & 4.22 & 5.00 & 7.01 & 4.14 & 4.82 & 6.46 & 4.41 & 5.70 & 9.59 & 4.09 & 4.75 & 6.29 \\
100-year & 5.11 & 7.20 & 12.82 & 4.25 & 5.13 & 7.60 & 4.17 & 4.96 & 7.00 & 4.48 & 6.06 & 11.37 & 4.12 & 4.87 & 6.76 \\
\hline
\end{tabular}




\section{Appendix B. Wind Speed and Wind Direction Statistics}

Appendix B1. Table showing American Samoa monthly means and mean of the top 5 percent for wind speed and mean wind direction.

[Years: Hindcast $=1976-2005 ;$ RCP mid $=$ 2026-2045; RCP end $=$ 2081-2100. Wind directions are “coming from”]

\begin{tabular}{|c|c|c|c|c|c|c|c|c|c|c|c|c|}
\hline Month & Jan & Feb & Mar & Apr & May & Jun & July & Aug & Sep & Oct & Nov & Dec \\
\hline \multicolumn{13}{|c|}{$\begin{array}{l}\text { Mean Wind Speed } \\
(\mathrm{m} / \mathrm{s})\end{array}$} \\
\hline Hindcast & $5.2 \pm 2.9$ & $5.0 \pm 2.8$ & $5.1 \pm 2.8$ & $5.4 \pm 2.7$ & $6.3 \pm 2.9$ & $6.9 \pm 2.7$ & $6.9 \pm 2.7$ & $7.1 \pm 2.7$ & $7.1 \pm 2.7$ & $6.8 \pm 2.7$ & $6.0 \pm 2.8$ & $5.3 \pm 2.6$ \\
\hline RCP4.5 - mid & $5.3 \pm 2.8$ & $5.1 \pm 2.8$ & $5.2 \pm 2.7$ & $5.5 \pm 2.7$ & $6.5 \pm 2.9$ & $7.0 \pm 2.7$ & $7.1 \pm 2.7$ & $7.1 \pm 2.6$ & $7.4 \pm 2.7$ & $7.0 \pm 2.7$ & $6.2 \pm 2.9$ & $5.4 \pm 2.6$ \\
\hline RCP8.5 - mid & $5.2 \pm 2.8$ & $5.1 \pm 2.8$ & $5.1 \pm 2.9$ & $5.5 \pm 2.8$ & $6.3 \pm 2.9$ & $6.8 \pm 2.7$ & $6.9 \pm 2.7$ & $7.1 \pm 2.8$ & $7.3 \pm 2.6$ & $6.9 \pm 2.7$ & $6.0 \pm 2.7$ & $5.4 \pm 3.0$ \\
\hline RCP4.5 - end & $5.2 \pm 2.7$ & $5.2 \pm 2.7$ & $4.8 \pm 2.7$ & $5.4 \pm 2.8$ & $6.6 \pm 3.0$ & $7.2 \pm 2.6$ & $6.9 \pm 2.8$ & $7.2 \pm 2.6$ & $7.3 \pm 2.6$ & $6.9 \pm 2.7$ & $6.1 \pm 2.7$ & $5.5 \pm 2.8$ \\
\hline $\begin{array}{l}\text { RCP8.5 - end } \\
\text { Top } 5 \text { percent } \\
\text { Speed }(\mathrm{m} / \mathrm{s})\end{array}$ & $5.4 \pm 2.8$ & $5.3 \pm 2.8$ & $5.2 \pm 2.8$ & $5.2 \pm 2.6$ & $6.2 \pm 2.9$ & $7.0 \pm 2.8$ & $7.0 \pm 2.7$ & $7.3 \pm 2.6$ & $7.3 \pm 2.6$ & $7 \pm 2.6$ & $6.1 \pm 2.7$ & $5.3 \pm 2.5$ \\
\hline Hindcast & $13.2 \pm 2.5$ & $12.5 \pm 2.9$ & $12.4 \pm 2.1$ & $11.7 \pm 1.3$ & $12.9 \pm 1.6$ & $12.8 \pm 1.5$ & $12.6 \pm 1.0$ & $12.8 \pm 1.1$ & $12.6 \pm 1.0$ & $12.6 \pm 1.2$ & $12.4 \pm 1.3$ & $11.7 \pm 2.0$ \\
\hline RCP4.5 - mid & $12.6 \pm 2.3$ & $12.6 \pm 2.6$ & $11.6 \pm 2.1$ & $11.9 \pm 1.3$ & $12.6 \pm 1.4$ & $12.5 \pm 1.0$ & $12.5 \pm 0.8$ & $12.4 \pm 0.9$ & $12.9 \pm 1.0$ & $12.6 \pm 0.8$ & $12.7 \pm 1.5$ & $11.5 \pm 1.7$ \\
\hline RCP8.5 - mid & $12.3 \pm 2.5$ & $12.5 \pm 2.3$ & $12.6 \pm 2.7$ & $12.1 \pm 1.2$ & $12.4 \pm 1.0$ & $12.4 \pm 1.2$ & $12.8 \pm 1.4$ & $12.8 \pm 1.0$ & $12.5 \pm 0.8$ & $12.6 \pm 1.1$ & $12.2 \pm 1.5$ & $13.5 \pm 2.7$ \\
\hline RCP4.5 - end & $12.2 \pm 2.3$ & $12.1 \pm 2.3$ & $11.9 \pm 2.4$ & $12.4 \pm 2.3$ & $12.8 \pm 1.8$ & $12.5 \pm 1.1$ & $12.6 \pm 1.1$ & $12.5 \pm 1.0$ & $12.6 \pm 1.0$ & $12.6 \pm 1.1$ & $11.9 \pm 1.3$ & $12.4 \pm 2.1$ \\
\hline $\begin{array}{l}\text { RCP8.5 - end } \\
\text { Mean Wind } \\
\left.\text { Direction ( }{ }^{\circ}\right)\end{array}$ & $12.7 \pm 2.9$ & $13.0 \pm 2.6$ & $12.6 \pm 2.8$ & $11.4 \pm 1.5$ & $12.7 \pm 1.6$ & $13.0 \pm 1.1$ & $12.6 \pm 0.9$ & $12.9 \pm 1.0$ & $12.7 \pm 1.0$ & $12.1 \pm 0.8$ & $11.8 \pm 1.2$ & $11.4 \pm 1.4$ \\
\hline Hindcast & $242 \pm 83$ & $217 \pm 98$ & $233 \pm 104$ & $281 \pm 68$ & $284 \pm 53$ & $285 \pm 43$ & $286 \pm 43$ & $283 \pm 39$ & $285 \pm 38$ & $285 \pm 39$ & $280 \pm 52$ & $268 \pm 66$ \\
\hline RCP4.5 - mid & $243 \pm 79$ & $207 \pm 97$ & $219 \pm 106$ & $283 \pm 72$ & $286 \pm 49$ & $284 \pm 43$ & $285 \pm 43$ & $285 \pm 38$ & $285 \pm 34$ & $286 \pm 37$ & $284 \pm 48$ & $271 \pm 67$ \\
\hline RCP8.5 - mid & $249 \pm 71$ & $214 \pm 96$ & $235 \pm 108$ & $285 \pm 70$ & $288 \pm 49$ & $287 \pm 44$ & $283 \pm 44$ & $286 \pm 41$ & $285 \pm 37$ & $283 \pm 38$ & $279 \pm 49$ & $266 \pm 70$ \\
\hline RCP4.5 - end & $250 \pm 76$ & $214 \pm 94$ & $233 \pm 106$ & $279 \pm 73$ & $286 \pm 48$ & $283 \pm 40$ & $284 \pm 43$ & $285 \pm 37$ & $286 \pm 33$ & $285 \pm 36$ & $280 \pm 48$ & $269 \pm 63$ \\
\hline $\begin{array}{l}\text { RCP8.5 - end } \\
\text { Top } 5 \text { percent } \\
\text { Direction ( } 9\end{array}$ & $250 \pm 70$ & $229 \pm 93$ & $232 \pm 97$ & $278 \pm 70$ & $283 \pm 50$ & $285 \pm 41$ & $286 \pm 39$ & $285 \pm 34$ & $286 \pm 31$ & $284 \pm 34$ & $277 \pm 45$ & $269 \pm 55$ \\
\hline Hindcast & $205 \pm 93$ & $182 \pm 89$ & $183 \pm 89$ & $289 \pm 54$ & $291 \pm 36$ & $296 \pm 31$ & $296 \pm 24$ & $292 \pm 24$ & $294 \pm 27$ & $295 \pm 24$ & $287 \pm 40$ & $280 \pm 63$ \\
\hline RCP4.5 - mid & $196 \pm 79$ & $164 \pm 67$ & $164 \pm 85$ & $292 \pm 53$ & $294 \pm 33$ & $290 \pm 32$ & $295 \pm 23$ & $296 \pm 23$ & $294 \pm 23$ & $293 \pm 19$ & $289 \pm 39$ & $281 \pm 57$ \\
\hline RCP8.5 - mid & $226 \pm 72$ & $152 \pm 76$ & $178 \pm 102$ & $294 \pm 45$ & $294 \pm 32$ & $297 \pm 33$ & $293 \pm 31$ & $298 \pm 23$ & $294 \pm 22$ & $294 \pm 25$ & $283 \pm 33$ & $284 \pm 57$ \\
\hline RCP 4.5 - end & $218 \pm 82$ & $169 \pm 84$ & $166 \pm 82$ & $266 \pm 76$ & $296 \pm 33$ & $295 \pm 25$ & $298 \pm 25$ & $296 \pm 22$ & $297 \pm 18$ & $296 \pm 17$ & $290 \pm 33$ & $286 \pm 53$ \\
\hline RCP8.5 - end & $234 \pm 85$ & $160 \pm 94$ & $173 \pm 78$ & $280 \pm 65$ & $292 \pm 40$ & $297 \pm 25$ & $297 \pm 23$ & $298 \pm 24$ & $297 \pm 18$ & $294 \pm 16$ & $286 \pm 33$ & $285 \pm 37$ \\
\hline
\end{tabular}


Appendix B2. Table showing return values of ensemble-average wind speeds of hindcast and forecast scenarios, including lower and higher 95 percent confidence intervals, at the American Samoa location.

[Years: Hindcast $=1976-2005 ;$ RCP mid $=$ 2026-2045; RCP end $=$ 2081-2100. Wind speeds are in meters per second]

\begin{tabular}{|c|c|c|c|c|c|c|c|c|c|c|c|c|c|c|c|}
\hline \multirow{2}{*}{ Scenario } & \multicolumn{3}{|c|}{ Hindcast } & \multicolumn{3}{|c|}{ RCP4.5 - mid } & \multicolumn{3}{c|}{ RCP8.5 - mid } & \multicolumn{3}{|c|}{ RCP4.5 - end } & \multicolumn{3}{c|}{ RCP8.5 - end } \\
\cline { 2 - 13 } & Low & $\boldsymbol{R}_{\mathbf{V}}$ & High & Low & $\boldsymbol{R}_{\mathbf{V}}$ & High & Low & $\boldsymbol{R}_{\mathbf{V}}$ & High & Low & $\boldsymbol{R}_{\mathbf{V}}$ & High & Low & $\boldsymbol{R}_{\mathbf{V}}$ & High \\
\hline 2-year & 18.63 & 20.53 & 23.67 & 18.06 & 19.99 & 23.26 & 18.84 & 20.96 & 24.46 & 17.77 & 20.54 & 26.43 & 18.16 & 20.54 & 25.11 \\
5-year & 19.71 & 22.87 & 28.72 & 18.90 & 21.87 & 27.47 & 19.79 & 22.99 & 28.82 & 18.62 & 23.23 & 34.98 & 18.99 & 22.82 & 31.32 \\
10-year & 20.47 & 24.76 & 33.47 & 19.43 & 23.25 & 31.07 & 20.37 & 24.43 & 32.42 & 19.18 & 25.46 & 43.94 & 19.53 & 24.60 & 37.24 \\
20-year & 21.18 & 26.77 & 39.22 & 19.88 & 24.59 & 35.09 & 20.85 & 25.81 & 36.31 & 19.68 & 27.89 & 55.82 & 20.00 & 26.44 & 44.50 \\
50-year & 22.04 & 29.63 & 48.71 & 20.38 & 26.31 & 41.09 & 21.38 & 27.52 & 41.92 & 20.25 & 31.41 & 77.66 & 20.53 & 28.96 & 56.65 \\
100-year & 22.63 & 31.94 & 57.64 & 20.69 & 27.57 & 46.23 & 21.70 & 28.75 & 46.56 & 20.62 & 34.33 & 100.54 & 20.86 & 30.94 & 68.25 \\
\hline
\end{tabular}


Appendix B3. Table showing Kauai monthly means and mean of the top 5 percent for wind speed and mean wind direction.

[Years: Hindcast = 1976-2005; RCP mid = 2026-2045; RCP end = 2081-2100. Wind directions are “coming from”]

\begin{tabular}{|c|c|c|c|c|c|c|c|c|c|c|c|c|}
\hline Month & Jan & Feb & Mar & Apr & May & Jun & July & Aug & Sep & Oct & Nov & Dec \\
\hline \multicolumn{13}{|c|}{$\begin{array}{l}\text { Mean Wind Speed } \\
(\mathrm{m} / \mathrm{s})\end{array}$} \\
\hline Hindcast & $6.9 \pm 3.1$ & $6.9 \pm 3.1$ & $7.5 \pm 3.1$ & $8.0 \pm 2.7$ & $8.1 \pm 2.4$ & $8.6 \pm 2.0$ & $8.5 \pm 1.9$ & $8.2 \pm 2.2$ & $7.8 \pm 2.5$ & $8.1 \pm 2.9$ & $7.9 \pm 3.1$ & $7.3 \pm 3.2$ \\
\hline RCP4.5 - mid & $6.8 \pm 3.1$ & $6.9 \pm 3.1$ & $7.6 \pm 3.1$ & $8.4 \pm 2.8$ & $7.9 \pm 2.4$ & $8.4 \pm 2.0$ & $8.8 \pm 1.7$ & $8.2 \pm 2.0$ & $7.7 \pm 2.4$ & $8.0 \pm 2.8$ & $8.1 \pm 3.0$ & $7.3 \pm 3.1$ \\
\hline RCP8.5 - mid & $6.9 \pm 3.2$ & $6.9 \pm 3.2$ & $7.5 \pm 3.1$ & $8.1 \pm 2.6$ & $7.8 \pm 2.3$ & $8.2 \pm 2.0$ & $8.5 \pm 1.8$ & $8.2 \pm 2.1$ & $7.6 \pm 2.4$ & $8.0 \pm 2.8$ & $8.1 \pm 2.9$ & $7.5 \pm 3.1$ \\
\hline RCP4.5 - end & $6.4 \pm 3.0$ & $6.9 \pm 3.2$ & $7.6 \pm 3.1$ & $8.0 \pm 2.7$ & $8.0 \pm 2.3$ & $8.4 \pm 1.9$ & $8.5 \pm 1.8$ & $8.2 \pm 2.1$ & $7.7 \pm 2.4$ & $8.1 \pm 2.8$ & $8.1 \pm 3.0$ & $7.3 \pm 3.1$ \\
\hline RCP8.5 - end & $6.4 \pm 3.1$ & $6.5 \pm 3.1$ & $7.4 \pm 3.0$ & $8.1 \pm 2.6$ & $7.6 \pm 2.3$ & $8.1 \pm 1.8$ & $8.5 \pm 1.7$ & $8.0 \pm 1.9$ & $7.6 \pm 2.2$ & $8.0 \pm 2.6$ & $8.2 \pm 2.8$ & $7.0 \pm 3.2$ \\
\hline \multicolumn{13}{|c|}{$\begin{array}{l}\text { Top } 5 \text { percent Wind } \\
\text { Speed }(\mathrm{m} / \mathrm{s})\end{array}$} \\
\hline Hindcast & $13.5 \pm 1.2$ & $13.5 \pm 1.2$ & $13.6 \pm 1.0$ & $13.0 \pm 0.8$ & $12.3 \pm 0.7$ & $12.5 \pm 1.0$ & $12.1 \pm 1.1$ & $12.4 \pm 1.4$ & $12.5 \pm 1.1$ & $13.7 \pm 1.4$ & $14.0 \pm 1.0$ & $13.8 \pm 1.2$ \\
\hline RCP4.5 - mid & $13.4 \pm 1.2$ & $13.5 \pm 1.0$ & $13.4 \pm 0.9$ & $13.4 \pm 0.8$ & $12.6 \pm 0.9$ & $12.0 \pm 0.8$ & $12.1 \pm 1.1$ & $11.9 \pm 0.9$ & $12.8 \pm 1.5$ & $13.6 \pm 1.4$ & $13.8 \pm 1.1$ & $13.6 \pm 1.1$ \\
\hline RCP8.5 - mid & $13.9 \pm 1.1$ & $13.7 \pm 1.2$ & $13.8 \pm 1.0$ & $13.0 \pm 0.8$ & $12.0 \pm 0.7$ & $11.9 \pm 0.6$ & $12.0 \pm 1.3$ & $12.4 \pm 1.2$ & $12.4 \pm 1.1$ & $13.9 \pm 1.3$ & $13.5 \pm 0.8$ & $13.9 \pm 1.0$ \\
\hline RCP4.5 - end & $12.8 \pm 1.1$ & $13.7 \pm 1.0$ & $13.5 \pm 0.7$ & $13.1 \pm 0.8$ & $12.4 \pm 0.8$ & $11.9 \pm 0.9$ & $12.2 \pm 1.3$ & $12.7 \pm 1.5$ & $12.5 \pm 1.3$ & $13.6 \pm 1.6$ & $13.8 \pm 0.8$ & $13.6 \pm 1.0$ \\
\hline RCP8.5 - end & $13.1 \pm 1.3$ & $13.5 \pm 1.2$ & $13.2 \pm 0.8$ & $12.7 \pm 0.6$ & $11.7 \pm 0.7$ & $11.5 \pm 0.7$ & $12.1 \pm 1.5$ & $12.0 \pm 1.6$ & $12.1 \pm 1.9$ & $13.1 \pm 1.7$ & $13.7 \pm 1.3$ & $13.7 \pm 1.3$ \\
\hline \multicolumn{13}{|l|}{$\begin{array}{l}\text { Mean Wind } \\
\text { Direction }(9)\end{array}$} \\
\hline Hindcast & $248 \pm 77$ & $243 \pm 78$ & $248 \pm 50$ & $250 \pm 35$ & $253 \pm 24$ & $256 \pm 17$ & $256 \pm 17$ & $258 \pm 24$ & $258 \pm 30$ & $255 \pm 34$ & $251 \pm 41$ & $254 \pm 59$ \\
\hline RCP4.5 - mid & $247 \pm 76$ & $249 \pm 69$ & $250 \pm 52$ & $251 \pm 32$ & $254 \pm 24$ & $256 \pm 16$ & $256 \pm 15$ & $257 \pm 22$ & $258 \pm 28$ & $254 \pm 35$ & $251 \pm 37$ & $251 \pm 54$ \\
\hline RCP8.5 - mid & $248 \pm 72$ & $244 \pm 73$ & $248 \pm 52$ & $249 \pm 29$ & $254 \pm 23$ & $256 \pm 16$ & $256 \pm 17$ & $257 \pm 24$ & $259 \pm 29$ & $253 \pm 35$ & $249 \pm 37$ & $252 \pm 55$ \\
\hline RCP4.5 - end & $252 \pm 78$ & $244 \pm 72$ & $247 \pm 50$ & $250 \pm 33$ & $254 \pm 22$ & $256 \pm 15$ & $256 \pm 18$ & $257 \pm 24$ & $257 \pm 32$ & $254 \pm 33$ & $250 \pm 37$ & $252 \pm 50$ \\
\hline RCP8.5 - end & $250 \pm 70$ & $243 \pm 75$ & $248 \pm 50$ & $250 \pm 31$ & $254 \pm 23$ & $257 \pm 14$ & $256 \pm 15$ & $257 \pm 21$ & $257 \pm 28$ & $253 \pm 30$ & $252 \pm 32$ & $253 \pm 53$ \\
\hline \multicolumn{13}{|c|}{$\begin{array}{l}\text { Top } 5 \text { percent Wind } \\
\text { Direction }(9)\end{array}$} \\
\hline Hindcast & $232 \pm 66$ & $238 \pm 51$ & $244 \pm 26$ & $245 \pm 17$ & $247 \pm 13$ & $251 \pm 15$ & $252 \pm 13$ & $258 \pm 31$ & $255 \pm 24$ & $253 \pm 31$ & $247 \pm 22$ & $246 \pm 39$ \\
\hline RCP4.5 - mid & $234 \pm 68$ & $246 \pm 39$ & $246 \pm 22$ & $246 \pm 16$ & $246 \pm 15$ & $252 \pm 18$ & $252 \pm 23$ & $251 \pm 26$ & $256 \pm 31$ & $255 \pm 34$ & $248 \pm 22$ & $241 \pm 38$ \\
\hline RCP8.5 - mid & $241 \pm 46$ & $240 \pm 41$ & $243 \pm 25$ & $244 \pm 12$ & $245 \pm 10$ & $247 \pm 7$ & $253 \pm 20$ & $257 \pm 33$ & $259 \pm 35$ & $250 \pm 28$ & $242 \pm 19$ & $245 \pm 32$ \\
\hline RCP4.5 - end & $243 \pm 65$ & $238 \pm 50$ & $243 \pm 22$ & $245 \pm 16$ & $246 \pm 12$ & $248 \pm 10$ & $250 \pm 20$ & $257 \pm 41$ & $252 \pm 37$ & $250 \pm 32$ & $244 \pm 19$ & $246 \pm 27$ \\
\hline RCP8.5 - end & $240 \pm 37$ & $236 \pm 42$ & $244 \pm 18$ & $246 \pm 11$ & $248 \pm 12$ & $249 \pm 10$ & $253 \pm 23$ & $255 \pm 25$ & $264 \pm 40$ & $251 \pm 28$ & $246 \pm 23$ & $250 \pm 26$ \\
\hline
\end{tabular}


Appendix B4. Table showing return values of ensemble-average wind speeds of hindcast and forecast scenarios, including lower and higher 95 percent confidence intervals, at the Kauai location.

[Years: Hindcast $=1976-2005 ;$ RCP mid $=$ 2026-2045; RCP end $=$ 2081-2100. Wind speeds are in meters per second]

\begin{tabular}{|c|c|c|c|c|c|c|c|c|c|c|c|c|c|c|c|}
\hline \multirow{2}{*}{ Scenario } & \multicolumn{3}{|c|}{ Hindcast } & \multicolumn{3}{c|}{ RCP4.5 - mid } & \multicolumn{3}{c|}{ RCP8.5 - mid } & \multicolumn{3}{c|}{ RCP4.5 - end } & \multicolumn{2}{c|}{ RCP8.5 - end } \\
\cline { 2 - 13 } & Low & $\boldsymbol{R}_{\boldsymbol{V}}$ & High & Low & $\boldsymbol{R}_{\boldsymbol{V}}$ & High & Low & $\boldsymbol{R}_{\boldsymbol{V}}$ & High & Low & $\boldsymbol{R}_{\boldsymbol{V}}$ & High & Low & $\boldsymbol{R}_{\boldsymbol{V}}$ & High \\
\hline 2-year & 17.80 & 18.92 & 20.66 & 17.25 & 18.57 & 20.94 & 16.99 & 18.05 & 20.04 & 17.24 & 18.45 & 20.50 & 17.54 & 19.22 & 22.16 \\
5-year & 18.44 & 20.16 & 23.07 & 17.67 & 19.62 & 23.58 & 17.42 & 19.17 & 22.95 & 17.66 & 19.41 & 22.68 & 18.31 & 21.02 & 26.41 \\
10-year & 18.85 & 21.06 & 25.05 & 17.91 & 20.34 & 25.76 & 17.71 & 20.08 & 25.81 & 17.90 & 20.05 & 24.37 & 18.82 & 22.45 & 30.38 \\
20-year & 19.20 & 21.92 & 27.17 & 18.10 & 21.01 & 28.11 & 17.97 & 21.05 & 29.39 & 18.08 & 20.61 & 26.09 & 19.29 & 23.94 & 35.14 \\
50-year & 19.59 & 23.01 & 30.21 & 18.29 & 21.82 & 31.50 & 18.28 & 22.43 & 35.53 & 18.27 & 21.26 & 28.42 & 19.83 & 25.99 & 42.92 \\
100-year & 19.83 & 23.80 & 32.71 & 18.40 & 22.38 & 34.30 & 18.49 & 23.55 & 41.56 & 18.37 & 21.69 & 30.22 & 20.20 & 27.62 & 50.18 \\
\hline
\end{tabular}


Appendix B5. Table showing Big Island of Hawaii monthly means and mean of the top 5 percent for wind speed and mean wind direction.

[Years: Hindcast $=1976-2005 ;$ RCP mid = 2026-2045; RCP end = 2081-2100. Wind directions are "coming from”]

\begin{tabular}{|c|c|c|c|c|c|c|c|c|c|c|c|c|}
\hline Month & Jan & Feb & Mar & Apr & May & Jun & July & Aug & Sep & Oct & Nov & Dec \\
\hline \multicolumn{13}{|c|}{$\begin{array}{l}\text { Mean Wind Speed } \\
(\mathrm{m} / \mathrm{s})\end{array}$} \\
\hline Hindcast & $7.0 \pm 3.0$ & $6.8 \pm 3.1$ & $7.7 \pm 2.9$ & $8.2 \pm 2.5$ & $8 \pm 2.1$ & $8.5 \pm 1.8$ & $8.2 \pm 1.8$ & $7.9 \pm 2.0$ & $7.4 \pm 2.3$ & $7.7 \pm 2.6$ & $8.1 \pm 2.8$ & $7.7 \pm 3.0$ \\
\hline RCP4.5 - mid & $6.9 \pm 3.0$ & $7.1 \pm 3.1$ & $7.7 \pm 3.0$ & $8.4 \pm 2.6$ & $8.0 \pm 2.0$ & $8.3 \pm 1.7$ & $8.4 \pm 1.7$ & $7.8 \pm 2.0$ & $7.2 \pm 2.2$ & $7.5 \pm 2.6$ & $8.2 \pm 2.8$ & $7.7 \pm 3.0$ \\
\hline RCP8.5 - mid & $7.0 \pm 3.1$ & $7.0 \pm 3.1$ & $7.6 \pm 2.9$ & $8.2 \pm 2.4$ & $7.9 \pm 2.0$ & $8.2 \pm 1.7$ & $8.2 \pm 1.7$ & $8.0 \pm 2.0$ & $7.1 \pm 2.2$ & $7.3 \pm 2.6$ & $8.0 \pm 2.6$ & $7.9 \pm 2.9$ \\
\hline RCP4.5 - end & $6.7 \pm 2.9$ & $6.9 \pm 3.0$ & $7.7 \pm 2.9$ & $8.1 \pm 2.4$ & $8.0 \pm 1.9$ & $8.5 \pm 1.7$ & $8.3 \pm 1.8$ & $7.9 \pm 2.0$ & $7.3 \pm 2.3$ & $7.5 \pm 2.6$ & $8.1 \pm 2.8$ & $7.8 \pm 2.9$ \\
\hline RCP8.5 - end & $6.6 \pm 2.9$ & $6.4 \pm 2.9$ & $7.5 \pm 2.8$ & $8.1 \pm 2.4$ & $7.6 \pm 1.9$ & $8.2 \pm 1.5$ & $8.4 \pm 1.6$ & $7.7 \pm 1.9$ & $7.1 \pm 2.1$ & $7.4 \pm 2.5$ & $8.0 \pm 2.7$ & $7.6 \pm 3.0$ \\
\hline \multicolumn{13}{|c|}{$\begin{array}{l}\text { Top } 5 \text { percent Wind } \\
\text { Speed }(\mathrm{m} / \mathrm{s})\end{array}$} \\
\hline Hindcast & $12.8 \pm 0.9$ & $12.9 \pm 0.8$ & $13.2 \pm 0.9$ & $12.5 \pm 0.7$ & $11.7 \pm 0.7$ & $11.7 \pm 0.9$ & $11.7 \pm 0.9$ & $11.8 \pm 1.1$ & $11.9 \pm 1.3$ & $13.0 \pm 1.1$ & $13.7 \pm 1.1$ & $13.5 \pm 0.9$ \\
\hline RCP4.5 - mid & $12.9 \pm 1.1$ & $13.3 \pm 1.0$ & $13.1 \pm 0.7$ & $12.9 \pm 0.7$ & $11.8 \pm 1.3$ & $11.3 \pm 0.7$ & $11.7 \pm 1.1$ & $11.4 \pm 1.0$ & $11.8 \pm 1.3$ & $13.0 \pm 1.4$ & $13.5 \pm 1.1$ & $13.2 \pm 1.0$ \\
\hline RCP8.5 - mid & $13.3 \pm 1.0$ & $13.2 \pm 1.0$ & $13.0 \pm 0.8$ & $12.3 \pm 0.8$ & $11.3 \pm 0.6$ & $11.1 \pm 0.5$ & $11.4 \pm 0.8$ & $12.1 \pm 1.2$ & $11.3 \pm 1.4$ & $12.8 \pm 1.3$ & $13.0 \pm 0.9$ & $13.7 \pm 1.0$ \\
\hline RCP 4.5 - end & $12.4 \pm 0.9$ & $13.0 \pm 1.0$ & $12.9 \pm 0.6$ & $12.3 \pm 0.6$ & $11.4 \pm 0.7$ & $11.6 \pm 1.2$ & $12.0 \pm 1.6$ & $12.1 \pm 1.8$ & $12.0 \pm 1.3$ & $12.9 \pm 1.7$ & $13.5 \pm 0.9$ & $13.4 \pm 1.1$ \\
\hline RCP8.5 - end & $12.6 \pm 1.2$ & $12.5 \pm 0.9$ & $12.7 \pm 0.7$ & $12.0 \pm 0.6$ & $10.9 \pm 0.5$ & $11.1 \pm 0.8$ & $11.9 \pm 1.7$ & $11.8 \pm 1.5$ & $11.3 \pm 1.6$ & $12.4 \pm 1.5$ & $13.3 \pm 1.4$ & $13.5 \pm 1.2$ \\
\hline \multicolumn{13}{|l|}{$\begin{array}{l}\text { Mean Wind } \\
\text { Direction }(9)\end{array}$} \\
\hline Hindcast & $256 \pm 51$ & $253 \pm 53$ & $251 \pm 35$ & $253 \pm 24$ & $254 \pm 19$ & $254 \pm 14$ & $253 \pm 16$ & $253 \pm 23$ & $255 \pm 29$ & $254 \pm 32$ & $253 \pm 31$ & $255 \pm 37$ \\
\hline RCP4.5 - mid & $256 \pm 50$ & $254 \pm 46$ & $254 \pm 36$ & $251 \pm 22$ & $254 \pm 17$ & $254 \pm 14$ & $252 \pm 16$ & $252 \pm 25$ & $255 \pm 28$ & $253 \pm 32$ & $252 \pm 28$ & $254 \pm 35$ \\
\hline RCP8.5 - mid & $255 \pm 46$ & $253 \pm 48$ & $252 \pm 37$ & $252 \pm 23$ & $255 \pm 17$ & $254 \pm 14$ & $252 \pm 16$ & $253 \pm 24$ & $253 \pm 31$ & $254 \pm 36$ & $252 \pm 30$ & $254 \pm 38$ \\
\hline RCP4.5 - end & $259 \pm 48$ & $255 \pm 51$ & $254 \pm 36$ & $253 \pm 23$ & $254 \pm 16$ & $254 \pm 13$ & $252 \pm 18$ & $253 \pm 27$ & $254 \pm 32$ & $253 \pm 32$ & $251 \pm 30$ & $254 \pm 31$ \\
\hline $\begin{array}{l}\text { RCP } 8.5 \text { - end } \\
\text { Top } 5 \text { percent } \\
\left.\text { Direction ( }{ }^{9}\right)\end{array}$ & $258 \pm 42$ & $254 \pm 50$ & $253 \pm 35$ & $252 \pm 22$ & $254 \pm 15$ & $253 \pm 12$ & $252 \pm 16$ & $251 \pm 23$ & $252 \pm 30$ & $252 \pm 33$ & $252 \pm 28$ & $255 \pm 32$ \\
\hline Hindcast & $249 \pm 30$ & $246 \pm 21$ & $246 \pm 14$ & $249 \pm 12$ & $252 \pm 11$ & $255 \pm 12$ & $255 \pm 19$ & $255 \pm 20$ & $256 \pm 31$ & $251 \pm 22$ & $249 \pm 20$ & $245 \pm 19$ \\
\hline RCP4.5 - mid & $248 \pm 32$ & $248 \pm 18$ & $246 \pm 13$ & $247 \pm 10$ & $252 \pm 11$ & $254 \pm 13$ & $253 \pm 15$ & $251 \pm 24$ & $255 \pm 29$ & $255 \pm 36$ & $249 \pm 16$ & $246 \pm 18$ \\
\hline RCP8.5 - mid & $245 \pm 23$ & $243 \pm 22$ & $245 \pm 16$ & $248 \pm 12$ & $253 \pm 10$ & $253 \pm 9$ & $254 \pm 20$ & $253 \pm 34$ & $254 \pm 30$ & $256 \pm 37$ & $247 \pm 20$ & $248 \pm 15$ \\
\hline RCP4.5 - end & $252 \pm 22$ & $244 \pm 28$ & $247 \pm 13$ & $250 \pm 12$ & $252 \pm 12$ & $257 \pm 22$ & $256 \pm 27$ & $255 \pm 40$ & $263 \pm 50$ & $253 \pm 31$ & $244 \pm 19$ & $251 \pm 18$ \\
\hline RCP8.5 - end & $251 \pm 22$ & $244 \pm 22$ & $246 \pm 16$ & $249 \pm 11$ & $250 \pm 7$ & $251 \pm 9$ & $257 \pm 21$ & $251 \pm 30$ & $261 \pm 41$ & $256 \pm 37$ & $249 \pm 14$ & $250 \pm 16$ \\
\hline
\end{tabular}


Appendix B6. Table showing return values of ensemble-average wind speeds of hindcast and forecast scenarios, including lower and higher 95 percent confidence intervals, at the Big Island of Hawaii location.

[Years: Hindcast $=1976-2005 ;$ RCP mid $=$ 2026-2045; RCP end $=$ 2081-2100. Wind speeds are in meters per second]

\begin{tabular}{|c|c|c|c|c|c|c|c|c|c|c|c|c|c|c|c|}
\hline \multirow{2}{*}{ Scenario } & \multicolumn{3}{|c|}{ Hindcast } & \multicolumn{3}{c|}{ RCP4.5 - mid } & \multicolumn{3}{c|}{ RCP8.5 - mid } & \multicolumn{3}{|c|}{ RCP4.5 - end } & \multicolumn{3}{c|}{ RCP8.5 - end } \\
\cline { 2 - 13 } & Low & $\boldsymbol{R}_{\boldsymbol{V}}$ & High & Low & $\boldsymbol{R}_{\boldsymbol{V}}$ & High & Low & $\boldsymbol{R}_{\boldsymbol{V}}$ & High & Low & $\boldsymbol{R}_{\boldsymbol{V}}$ & High & Low & $\boldsymbol{R}_{\boldsymbol{V}}$ & High \\
\hline 2-year & 16.30 & 17.25 & 18.75 & 16.40 & 17.73 & 20.24 & 16.14 & 17.16 & 19.03 & 16.76 & 18.15 & 20.62 & 16.68 & 18.14 & 20.63 \\
5-year & 16.83 & 18.33 & 20.96 & 16.82 & 18.85 & 23.24 & 16.56 & 18.22 & 21.68 & 17.33 & 19.53 & 23.95 & 17.22 & 19.37 & 23.46 \\
10-year & 17.19 & 19.15 & 22.89 & 17.07 & 19.66 & 25.90 & 16.85 & 19.06 & 24.20 & 17.70 & 20.59 & 26.99 & 17.53 & 20.22 & 25.75 \\
20-year & 17.50 & 19.98 & 25.05 & 17.27 & 20.45 & 28.94 & 17.10 & 19.94 & 27.26 & 18.02 & 21.66 & 30.55 & 17.78 & 20.99 & 28.17 \\
50-year & 17.86 & 21.06 & 28.35 & 17.49 & 21.45 & 33.65 & 17.39 & 21.16 & 32.35 & 18.39 & 23.09 & 36.23 & 18.04 & 21.93 & 31.58 \\
100-year & 18.10 & 21.89 & 31.21 & 17.61 & 22.18 & 37.81 & 17.58 & 22.13 & 37.19 & 18.62 & 24.19 & 41.39 & 18.19 & 22.57 & 34.33 \\
\hline
\end{tabular}


Appendix B7. Table showing Midway monthly means and mean of the top 5 percent for wind speed and mean wind direction.

[Years: Hindcast = 1976-2005; RCP mid = 2026-2045; RCP end = 2081-2100. Wind directions are “coming from”]

\begin{tabular}{|c|c|c|c|c|c|c|c|c|c|c|c|c|}
\hline Month & Jan & Feb & Mar & Apr & May & Jun & July & Aug & Sep & Oct & Nov & Dec \\
\hline \multicolumn{13}{|c|}{$\begin{array}{l}\text { Mean Wind Speed } \\
(\mathrm{m} / \mathrm{s})\end{array}$} \\
\hline Hindcast & $8.7 \pm 4.0$ & $8.3 \pm 3.8$ & $7.0 \pm 3.1$ & $6.4 \pm 2.7$ & $5.7 \pm 2.4$ & $5.9 \pm 2.2$ & $6.8 \pm 2.3$ & $6.8 \pm 2.5$ & $6.8 \pm 2.9$ & $7.3 \pm 3.2$ & $7.2 \pm 3.3$ & $8.0 \pm 3.7$ \\
\hline RCP4.5 - mid & $8.6 \pm 4.0$ & $8.1 \pm 3.7$ & $7.1 \pm 3.1$ & $6.5 \pm 2.8$ & $5.5 \pm 2.4$ & $5.6 \pm 2.2$ & $6.9 \pm 2.2$ & $6.5 \pm 2.4$ & $6.6 \pm 2.7$ & $7.2 \pm 3.1$ & $7.1 \pm 3.4$ & $7.8 \pm 3.7$ \\
\hline RCP8.5 - mid & $8.5 \pm 3.9$ & $8.2 \pm 3.6$ & $7.0 \pm 3.2$ & $6.1 \pm 2.7$ & $5.5 \pm 2.5$ & $5.4 \pm 2.2$ & $6.9 \pm 2.2$ & $6.7 \pm 2.5$ & $6.6 \pm 2.9$ & $7.4 \pm 3.1$ & $7.1 \pm 3.4$ & $7.6 \pm 3.8$ \\
\hline RCP4.5 - end & $8.6 \pm 3.8$ & $8.2 \pm 3.7$ & $7.1 \pm 3.2$ & $6.2 \pm 2.8$ & $5.7 \pm 2.4$ & $5.5 \pm 2.1$ & $6.6 \pm 2.2$ & $6.5 \pm 2.5$ & $6.5 \pm 2.6$ & $7.3 \pm 3.0$ & $7.0 \pm 3.3$ & $7.6 \pm 3.6$ \\
\hline RCP8.5 - end & $8.2 \pm 3.8$ & $8.0 \pm 3.5$ & $6.8 \pm 3.0$ & $6.2 \pm 2.6$ & $5.4 \pm 2.4$ & $5.5 \pm 2.1$ & $6.5 \pm 2.1$ & $6.3 \pm 2.2$ & $6.5 \pm 2.6$ & $7.1 \pm 2.9$ & $6.8 \pm 3.1$ & $7.3 \pm 3.5$ \\
\hline \multicolumn{13}{|c|}{$\begin{array}{l}\text { Top } 5 \text { percent Wind } \\
\text { Speed }(\mathrm{m} / \mathrm{s})\end{array}$} \\
\hline Hindcast & $17.9 \pm 1.9$ & $16.9 \pm 1.5$ & $14.3 \pm 1.6$ & $12.4 \pm 1.5$ & $10.7 \pm 1.0$ & $10.1 \pm 0.9$ & $11.5 \pm 1.5$ & $12.1 \pm 1.8$ & $13.5 \pm 1.7$ & $14.4 \pm 1.7$ & $14.5 \pm 1.6$ & $16.6 \pm 1.8$ \\
\hline RCP4.5 - mid & $17.4 \pm 1.6$ & $16.6 \pm 1.8$ & $14.1 \pm 1.5$ & $12.5 \pm 1.3$ & $10.5 \pm 1.2$ & $10.1 \pm 1.3$ & $11.6 \pm 1.6$ & $12.2 \pm 2.1$ & $12.5 \pm 1.7$ & $13.9 \pm 1.7$ & $15.0 \pm 1.7$ & $16.6 \pm 2.0$ \\
\hline RCP8.5 - mid & $17.4 \pm 1.8$ & $16.4 \pm 1.7$ & $14.4 \pm 1.7$ & $12.2 \pm 1.6$ & $10.9 \pm 1.2$ & $9.9 \pm 1.1$ & $11.2 \pm 1.5$ & $12.4 \pm 2.0$ & $13.5 \pm 2.0$ & $14.0 \pm 1.6$ & $14.6 \pm 1.6$ & $16.5 \pm 1.9$ \\
\hline RCP4.5 - end & $17.1 \pm 1.7$ & $16.8 \pm 2.0$ & $14.5 \pm 1.7$ & $12.1 \pm 1.4$ & $10.8 \pm 1.0$ & $9.8 \pm 0.8$ & $11.0 \pm 1.5$ & $12.4 \pm 2.7$ & $12.4 \pm 2.0$ & $14.0 \pm 1.7$ & $14.4 \pm 1.8$ & $16.0 \pm 1.9$ \\
\hline RCP8.5 - end & $16.8 \pm 1.8$ & $16.4 \pm 1.7$ & $13.5 \pm 1.4$ & $12.0 \pm 1.3$ & $10.4 \pm 1.0$ & $9.7 \pm 1.1$ & $10.7 \pm 1.3$ & $11.4 \pm 2.2$ & $12.7 \pm 2.0$ & $13.2 \pm 1.4$ & $13.8 \pm 1.6$ & $15.4 \pm 1.7$ \\
\hline \multicolumn{13}{|l|}{$\begin{array}{l}\text { Mean Wind } \\
\text { Direction ( } 9\end{array}$} \\
\hline Hindcast & $92 \pm 86$ & $87 \pm 89$ & $283 \pm 134$ & $255 \pm 67$ & $261 \pm 54$ & $270 \pm 43$ & $267 \pm 32$ & $269 \pm 37$ & $262 \pm 49$ & $252 \pm 57$ & $241 \pm 92$ & $108 \pm 117$ \\
\hline RCP4.5 - mid & $92 \pm 85$ & $84 \pm 93$ & $206 \pm 136$ & $256 \pm 65$ & $260 \pm 60$ & $270 \pm 47$ & $268 \pm 29$ & $270 \pm 34$ & $262 \pm 46$ & $255 \pm 50$ & $250 \pm 88$ & $99 \pm 117$ \\
\hline RCP8.5 - mid & $88 \pm 92$ & $88 \pm 97$ & $203 \pm 139$ & $257 \pm 71$ & $258 \pm 58$ & $270 \pm 49$ & $268 \pm 33$ & $271 \pm 39$ & $262 \pm 49$ & $256 \pm 50$ & $245 \pm 78$ & $106 \pm 129$ \\
\hline RCP4.5 - end & $88 \pm 81$ & $87 \pm 94$ & $227 \pm 126$ & $257 \pm 69$ & $262 \pm 55$ & $269 \pm 46$ & $268 \pm 33$ & $270 \pm 37$ & $264 \pm 43$ & $256 \pm 48$ & $250 \pm 78$ & $105 \pm 130$ \\
\hline RCP8.5 - end & $88 \pm 87$ & $86 \pm 88$ & $257 \pm 121$ & $259 \pm 63$ & $262 \pm 55$ & $271 \pm 45$ & $268 \pm 29$ & $271 \pm 34$ & $266 \pm 45$ & $258 \pm 45$ & $251 \pm 71$ & $122 \pm 125$ \\
\hline \multicolumn{13}{|c|}{$\begin{array}{l}\text { Top } 5 \text { percent Wind } \\
\text { Direction ( } 9\end{array}$} \\
\hline Hindcast & $88 \pm 42$ & $90 \pm 43$ & $107 \pm 82$ & $232 \pm 58$ & $249 \pm 32$ & $266 \pm 32$ & $268 \pm 25$ & $279 \pm 39$ & $260 \pm 37$ & $236 \pm 42$ & $190 \pm 96$ & $97 \pm 60$ \\
\hline RCP4.5 - mid & $100 \pm 55$ & $95 \pm 48$ & $109 \pm 84$ & $239 \pm 45$ & $248 \pm 39$ & $265 \pm 43$ & $268 \pm 27$ & $269 \pm 30$ & $256 \pm 32$ & $252 \pm 39$ & $219 \pm 80$ & $100 \pm 63$ \\
\hline RCP8.5 - mid & $89 \pm 57$ & $89 \pm 47$ & $113 \pm 82$ & $236 \pm 54$ & $243 \pm 37$ & $263 \pm 32$ & $270 \pm 32$ & $277 \pm 44$ & $252 \pm 38$ & $244 \pm 30$ & $218 \pm 68$ & $95 \pm 57$ \\
\hline RCP4.5 - end & $86 \pm 42$ & $93 \pm 49$ & $117 \pm 87$ & $242 \pm 53$ & $255 \pm 39$ & $261 \pm 28$ & $270 \pm 27$ & $274 \pm 39$ & $260 \pm 38$ & $243 \pm 36$ & $225 \pm 68$ & $98 \pm 64$ \\
\hline RCP8.5 - end & $87 \pm 38$ & $88 \pm 45$ & $111 \pm 90$ & $240 \pm 51$ & $251 \pm 38$ & $262 \pm 28$ & $269 \pm 25$ & $282 \pm 49$ & $257 \pm 43$ & $248 \pm 32$ & $237 \pm 51$ & $99 \pm 75$ \\
\hline
\end{tabular}


Appendix B8. Table showing return values of ensemble-average wind speeds of hindcast and forecast scenarios, including lower and higher 95 percent confidence intervals, at the Midway location.

[Years: Hindcast $=1976-2005 ;$ RCP mid $=$ 2026-2045; RCP end $=$ 2081-2100. Wind speeds are in meters per second]

\begin{tabular}{|c|c|c|c|c|c|c|c|c|c|c|c|c|c|c|c|c|}
\hline \multirow{2}{*}{ Scenario } & \multicolumn{3}{|c|}{ Hindcast } & \multicolumn{3}{c|}{ RCP4.5 - mid } & \multicolumn{3}{c|}{ RCP8.5 - mid } & \multicolumn{3}{c|}{ RCP4.5 - end } & \multicolumn{2}{c|}{ RCP8.5 - end } \\
\cline { 2 - 13 } & Low & $\boldsymbol{R}_{\boldsymbol{V}}$ & High & Low & $\boldsymbol{R}_{\boldsymbol{V}}$ & High & Low & $\boldsymbol{R}_{\boldsymbol{V}}$ & High & Low & $\boldsymbol{R}_{\boldsymbol{V}}$ & High & Low & $\boldsymbol{R}_{\boldsymbol{V}}$ & High \\
\hline 2-year & 22.76 & 24.25 & 26.65 & 22.56 & 24.09 & 26.51 & 22.07 & 23.70 & 26.72 & 22.39 & 24.33 & 27.82 & 21.44 & 23.30 & 26.62 \\
5-year & 23.54 & 25.87 & 30.01 & 23.16 & 25.30 & 28.96 & 22.46 & 24.76 & 29.57 & 23.10 & 26.10 & 32.21 & 22.09 & 24.89 & 30.56 \\
10-year & 24.04 & 27.07 & 32.88 & 23.48 & 26.06 & 30.72 & 22.67 & 25.43 & 31.78 & 23.55 & 27.41 & 36.10 & 22.47 & 26.02 & 33.92 \\
20-year & 24.48 & 28.25 & 36.07 & 23.73 & 26.71 & 32.39 & 22.82 & 26.01 & 34.04 & 23.92 & 28.70 & 40.53 & 22.78 & 27.10 & 37.64 \\
50-year & 24.97 & 29.77 & 40.84 & 23.97 & 27.41 & 34.49 & 22.95 & 26.65 & 37.09 & 24.33 & 30.36 & 47.38 & 23.11 & 28.44 & 43.19 \\
100-year & 25.28 & 30.91 & 44.91 & 24.10 & 27.86 & 35.99 & 23.03 & 27.06 & 39.46 & 24.58 & 31.59 & 53.42 & 23.30 & 29.40 & 47.92 \\
\end{tabular}


Appendix B9. Table showing Chuuk monthly means and mean of the top 5 percent for wind speed and mean wind direction.

[Years: Hindcast = 1976-2005; RCP mid = 2026-2045; RCP end = 2081-2100. Wind directions are “coming from”]

\begin{tabular}{|c|c|c|c|c|c|c|c|c|c|c|c|c|}
\hline Month & Jan & Feb & Mar & Apr & May & Jun & July & Aug & Sep & Oct & Nov & Dec \\
\hline \multicolumn{13}{|c|}{$\begin{array}{l}\text { Mean Wind Speed } \\
(\mathrm{m} / \mathrm{s})\end{array}$} \\
\hline Hindcast & $8.5 \pm 2.0$ & $8.5 \pm 1.9$ & $8.0 \pm 2.0$ & $6.8 \pm 2.4$ & $5.2 \pm 2.4$ & $4.4 \pm 2.4$ & $4.2 \pm 2.4$ & $4.3 \pm 2.5$ & $4.0 \pm 2.3$ & $4.2 \pm 2.4$ & $5.3 \pm 2.8$ & $7.5 \pm 2.6$ \\
\hline RCP4.5 - mid & $8.4 \pm 2.2$ & $8.3 \pm 2.0$ & $8.0 \pm 2.0$ & $6.9 \pm 2.2$ & $5.2 \pm 2.4$ & $4.4 \pm 2.4$ & $4.5 \pm 2.6$ & $4.3 \pm 2.5$ & $4.1 \pm 2.3$ & $4.1 \pm 2.3$ & $4.9 \pm 2.5$ & $7.1 \pm 2.6$ \\
\hline RCP8.5 - mid & $8.3 \pm 2.1$ & $8.4 \pm 1.9$ & $7.9 \pm 1.9$ & $7.0 \pm 2.0$ & $5.2 \pm 2.4$ & $4.5 \pm 2.4$ & $4.4 \pm 2.4$ & $4.4 \pm 2.5$ & $4.2 \pm 2.3$ & $4.1 \pm 2.4$ & $4.9 \pm 2.6$ & $7.1 \pm 2.7$ \\
\hline RCP4.5 - end & $8.2 \pm 2.0$ & $8.3 \pm 1.9$ & $7.9 \pm 2.0$ & $6.7 \pm 2.1$ & $5.2 \pm 2.4$ & $4.7 \pm 2.5$ & $4.5 \pm 2.5$ & $4.5 \pm 2.6$ & $4.2 \pm 2.4$ & $4.1 \pm 2.4$ & $4.9 \pm 2.5$ & $6.9 \pm 2.6$ \\
\hline RCP8.5 - end & $8.1 \pm 2.0$ & $8.0 \pm 1.9$ & $7.9 \pm 1.7$ & $7.0 \pm 2.0$ & $5.5 \pm 2.4$ & $4.7 \pm 2.5$ & $4.7 \pm 2.6$ & $4.5 \pm 2.5$ & $4.2 \pm 2.5$ & $3.8 \pm 2.2$ & $4.6 \pm 2.5$ & $7.0 \pm 2.5$ \\
\hline \multicolumn{13}{|c|}{$\begin{array}{l}\text { Top } 5 \text { percent Wind } \\
\text { Speed }(\mathrm{m} / \mathrm{s})\end{array}$} \\
\hline Hindcast & $12.2 \pm 0.8$ & $12.3 \pm 1.2$ & $11.7 \pm 1.3$ & $11.2 \pm 1.3$ & $10.3 \pm 1.2$ & $10.2 \pm 1.7$ & $10.7 \pm 2.1$ & $11.3 \pm 2.2$ & $10.7 \pm 1.9$ & $10.5 \pm 1.9$ & $11.7 \pm 1.6$ & $12.4 \pm 1.0$ \\
\hline RCP4.5 - mid & $12.7 \pm 1.1$ & $12.1 \pm 0.8$ & $11.6 \pm 1.3$ & $10.9 \pm 0.8$ & $10.2 \pm 1.2$ & $10.1 \pm 1.6$ & $11.3 \pm 2.0$ & $11.8 \pm 2.3$ & $11.0 \pm 2.1$ & $10.5 \pm 1.6$ & $11.1 \pm 1.7$ & $12.5 \pm 1.6$ \\
\hline RCP8.5 - mid & $12.5 \pm 1.2$ & $12.2 \pm 0.9$ & $11.6 \pm 1.0$ & $10.9 \pm 0.7$ & $10.1 \pm 1.3$ & $10.5 \pm 2.2$ & $10.6 \pm 2.0$ & $11.5 \pm 2.2$ & $10.9 \pm 2.1$ & $10.5 \pm 1.8$ & $11.5 \pm 1.6$ & $12.9 \pm 1.5$ \\
\hline RCP 4.5 - end & $11.7 \pm 0.6$ & $11.9 \pm 1.0$ & $11.6 \pm 1.4$ & $10.6 \pm 0.8$ & $10.2 \pm 1.0$ & $10.6 \pm 1.8$ & $11.2 \pm 1.8$ & $11.7 \pm 1.6$ & $11.0 \pm 2.0$ & $10.8 \pm 1.7$ & $10.7 \pm 1.7$ & $12.3 \pm 1.3$ \\
\hline RCP8.5 - end & $12.2 \pm 1.3$ & $11.7 \pm 1.0$ & $11.3 \pm 1.0$ & $10.9 \pm 0.9$ & $10.5 \pm 1.6$ & $10.3 \pm 1.5$ & $11.5 \pm 2.3$ & $11.6 \pm 1.8$ & $11.4 \pm 2.5$ & $9.9 \pm 2.3$ & $10.8 \pm 1.7$ & $12.1 \pm 1.5$ \\
\hline \multicolumn{13}{|l|}{$\begin{array}{l}\text { Mean Wind } \\
\text { Direction }(9)\end{array}$} \\
\hline Hindcast & $245 \pm 18$ & $244 \pm 18$ & $245 \pm 23$ & $250 \pm 31$ & $257 \pm 53$ & $269 \pm 71$ & $299 \pm 73$ & $324 \pm 68$ & $324 \pm 74$ & $317 \pm 90$ & $263 \pm 63$ & $249 \pm 30$ \\
\hline RCP4.5 - mid & $247 \pm 23$ & $245 \pm 19$ & $246 \pm 19$ & $248 \pm 26$ & $255 \pm 45$ & $268 \pm 69$ & $300 \pm 73$ & $321 \pm 72$ & $322 \pm 73$ & $318 \pm 85$ & $265 \pm 70$ & $252 \pm 37$ \\
\hline RCP8.5 - mid & $247 \pm 17$ & $244 \pm 16$ & $247 \pm 21$ & $249 \pm 24$ & $254 \pm 44$ & $269 \pm 69$ & $302 \pm 79$ & $328 \pm 72$ & $325 \pm 71$ & $328 \pm 87$ & $264 \pm 68$ & $252 \pm 33$ \\
\hline RCP4.5 - end & $248 \pm 17$ & $245 \pm 19$ & $247 \pm 20$ & $250 \pm 25$ & $256 \pm 42$ & $265 \pm 74$ & $301 \pm 76$ & $325 \pm 71$ & $327 \pm 73$ & $332 \pm 87$ & $267 \pm 67$ & $253 \pm 35$ \\
\hline $\begin{array}{l}\text { RCP } 8.5 \text { - end } \\
\text { Top } 5 \text { percent } \\
\left.\text { Direction ( }{ }^{9}\right)\end{array}$ & $248 \pm 19$ & $246 \pm 17$ & $246 \pm 17$ & $250 \pm 19$ & $256 \pm 40$ & $267 \pm 68$ & $290 \pm 71$ & $317 \pm 70$ & $325 \pm 70$ & $311 \pm 82$ & $267 \pm 65$ & $253 \pm 31$ \\
\hline Hindcast & $239 \pm 16$ & $237 \pm 18$ & $237 \pm 30$ & $239 \pm 37$ & $249 \pm 64$ & $275 \pm 97$ & $24 \pm 80$ & $28 \pm 47$ & $41 \pm 62$ & $48 \pm 73$ & $258 \pm 72$ & $240 \pm 25$ \\
\hline RCP4.5 - mid & $237 \pm 32$ & $236 \pm 16$ & $239 \pm 27$ & $240 \pm 30$ & $244 \pm 33$ & $262 \pm 73$ & $33 \pm 64$ & $30 \pm 54$ & $34 \pm 53$ & $55 \pm 57$ & $242 \pm 77$ & $243 \pm 46$ \\
\hline RCP8.5 - mid & $241 \pm 19$ & $233 \pm 20$ & $238 \pm 28$ & $241 \pm 18$ & $247 \pm 52$ & $300 \pm 82$ & $43 \pm 72$ & $37 \pm 45$ & $34 \pm 43$ & $57 \pm 61$ & $251 \pm 74$ & $242 \pm 39$ \\
\hline RCP4.5 - end & $239 \pm 11$ & $241 \pm 29$ & $238 \pm 21$ & $241 \pm 34$ & $246 \pm 47$ & $263 \pm 94$ & $41 \pm 49$ & $35 \pm 35$ & $46 \pm 40$ & $50 \pm 54$ & $254 \pm 79$ & $243 \pm 36$ \\
\hline RCP8.5 - end & $239 \pm 24$ & $240 \pm 23$ & $238 \pm 28$ & $242 \pm 16$ & $247 \pm 35$ & $274 \pm 70$ & $34 \pm 67$ & $35 \pm 39$ & $29 \pm 39$ & $47 \pm 80$ & $250 \pm 58$ & $245 \pm 35$ \\
\hline
\end{tabular}


Appendix B10. Table showing return values of ensemble-average wind speeds of hindcast and forecast scenarios, including lower and higher 95 percent confidence intervals, at the Chuuk location.

[Years: Hindcast $=1976-2005 ;$ RCP mid $=$ 2026-2045; RCP end $=$ 2081-2100. Wind speeds are in meters per second]

\begin{tabular}{|c|c|c|c|c|c|c|c|c|c|c|c|c|c|c|c|c|}
\hline \multirow{2}{*}{ Scenario } & \multicolumn{3}{|c|}{ Hindcast } & \multicolumn{3}{c|}{ RCP4.5 - mid } & \multicolumn{3}{c|}{ RCP8.5 - mid } & \multicolumn{3}{c|}{ RCP4.5 - end } & \multicolumn{2}{c|}{ RCP8.5 - end } \\
\cline { 2 - 13 } & Low & $\boldsymbol{R}_{\boldsymbol{V}}$ & High & Low & $\boldsymbol{R}_{\boldsymbol{V}}$ & High & Low & $\boldsymbol{R}_{\boldsymbol{V}}$ & High & Low & $\boldsymbol{R}_{\boldsymbol{V}}$ & High & Low & $\boldsymbol{R}_{\boldsymbol{V}}$ & High \\
\hline 2-year & 16.86 & 18.81 & 22.23 & 17.32 & 19.05 & 21.90 & 16.86 & 18.91 & 22.75 & 16.13 & 17.76 & 20.62 & 17.63 & 19.65 & 22.95 \\
5-year & 17.82 & 21.07 & 27.64 & 18.04 & 20.60 & 25.22 & 17.44 & 20.45 & 26.90 & 16.74 & 19.20 & 24.03 & 18.33 & 21.15 & 26.21 \\
10-year & 18.48 & 22.94 & 32.94 & 18.47 & 21.66 & 27.86 & 17.76 & 21.51 & 30.38 & 17.10 & 20.22 & 26.91 & 18.70 & 22.10 & 28.58 \\
20-year & 19.09 & 24.96 & 39.57 & 18.82 & 22.64 & 30.60 & 18.01 & 22.48 & 34.17 & 17.40 & 21.19 & 30.07 & 18.98 & 22.90 & 30.87 \\
50-year & 19.83 & 27.86 & 50.97 & 19.18 & 23.81 & 34.41 & 18.26 & 23.65 & 39.71 & 17.71 & 22.40 & 34.74 & 19.25 & 23.78 & 33.78 \\
100-year & 20.35 & 30.25 & 62.13 & 19.39 & 24.62 & 37.42 & 18.40 & 24.46 & 44.35 & 17.90 & 23.26 & 38.67 & 19.39 & 24.33 & 35.90 \\
\end{tabular}


Appendix B11. Table showing Saipan monthly means and mean of the top 5 percent for wind speed and mean wind direction.

[Years: Hindcast = 1976-2005; RCP mid = 2026-2045; RCP end = 2081-2100. Wind directions are “coming from”]

\begin{tabular}{|c|c|c|c|c|c|c|c|c|c|c|c|c|}
\hline Month & Jan & Feb & Mar & Apr & May & Jun & July & Aug & Sep & Oct & Nov & Dec \\
\hline \multicolumn{13}{|c|}{$\begin{array}{l}\text { Mean Wind Speed } \\
(\mathrm{m} / \mathrm{s})\end{array}$} \\
\hline Hindcast & $8.7 \pm 2.3$ & $8.6 \pm 2.4$ & $8.4 \pm 2.3$ & $7.9 \pm 2.1$ & $7.1 \pm 2.2$ & $6.4 \pm 2.5$ & $5.9 \pm 3.2$ & $5.8 \pm 3.3$ & $5.5 \pm 3.0$ & $6.2 \pm 3.0$ & $8.4 \pm 2.6$ & $8.9 \pm 2.2$ \\
\hline RCP4.5 - mid & $8.8 \pm 2.3$ & $8.5 \pm 2.4$ & $8.5 \pm 2.2$ & $8.0 \pm 1.9$ & $7.1 \pm 2.2$ & $6.7 \pm 2.5$ & $5.9 \pm 3.1$ & $5.6 \pm 3.0$ & $5.4 \pm 3.0$ & $5.7 \pm 2.8$ & $7.9 \pm 2.5$ & $8.7 \pm 2.4$ \\
\hline RCP8.5 - mid & $8.7 \pm 2.3$ & $8.6 \pm 2.3$ & $8.4 \pm 2.2$ & $7.9 \pm 1.8$ & $7.0 \pm 2.0$ & $6.8 \pm 2.6$ & $6.1 \pm 3.2$ & $5.7 \pm 3.3$ & $5.6 \pm 3.0$ & $5.7 \pm 2.8$ & $7.8 \pm 2.6$ & $8.9 \pm 2.4$ \\
\hline RCP4.5 - end & $8.6 \pm 2.3$ & $8.7 \pm 2.2$ & $8.4 \pm 2.2$ & $7.8 \pm 2.0$ & $7.2 \pm 2.0$ & $6.7 \pm 2.4$ & $6.2 \pm 3.0$ & $5.9 \pm 3.3$ & $5.5 \pm 2.7$ & $5.7 \pm 2.8$ & $8.0 \pm 2.6$ & $8.7 \pm 2.2$ \\
\hline RCP8.5 - end & $8.5 \pm 2.4$ & $8.3 \pm 2.4$ & $8.1 \pm 2.1$ & $7.8 \pm 1.8$ & $7.1 \pm 2.0$ & $6.6 \pm 2.5$ & $6.2 \pm 3.1$ & $5.7 \pm 3.3$ & $5.6 \pm 3.0$ & $5.7 \pm 2.7$ & $7.8 \pm 2.6$ & $8.6 \pm 2.3$ \\
\hline \multicolumn{13}{|c|}{$\begin{array}{l}\text { Top } 5 \text { percent Wind } \\
\text { Speed }(\mathrm{m} / \mathrm{s})\end{array}$} \\
\hline Hindcast & $13.2 \pm 0.8$ & $13.2 \pm 1.0$ & $13.2 \pm 1.7$ & $12.0 \pm 1.2$ & $11.8 \pm 1.7$ & $12.0 \pm 2.1$ & $14.0 \pm 2.4$ & $14.6 \pm 2.5$ & $13.4 \pm 2.8$ & $13.5 \pm 2.5$ & $14.1 \pm 1.8$ & $13.3 \pm 1.2$ \\
\hline RCP4.5 - mid & $13.1 \pm 0.7$ & $13.2 \pm 0.8$ & $13.0 \pm 1.3$ & $11.9 \pm 1.8$ & $11.3 \pm 1.3$ & $12.4 \pm 2.5$ & $13.6 \pm 2.4$ & $13.3 \pm 2.3$ & $13.8 \pm 3.2$ & $12.2 \pm 1.9$ & $13.2 \pm 1.9$ & $13.8 \pm 1.4$ \\
\hline RCP8.5 - mid & $13.3 \pm 0.9$ & $13.2 \pm 0.7$ & $13.0 \pm 1.2$ & $11.7 \pm 1.0$ & $10.8 \pm 1.1$ & $13.2 \pm 3.1$ & $14.2 \pm 2.5$ & $14.3 \pm 2.3$ & $14.0 \pm 3.0$ & $12.2 \pm 2.2$ & $13.5 \pm 2.1$ & $13.8 \pm 1.7$ \\
\hline RCP 4.5 - end & $13.0 \pm 0.8$ & $13.2 \pm 1.0$ & $12.9 \pm 0.8$ & $11.8 \pm 0.8$ & $10.7 \pm 0.7$ & $11.6 \pm 2.0$ & $13.6 \pm 2.9$ & $14.4 \pm 2.5$ & $12.3 \pm 2.1$ & $12.4 \pm 2.2$ & $13.5 \pm 1.8$ & $13.2 \pm 1.4$ \\
\hline RCP8.5 - end & $13.3 \pm 0.9$ & $13.0 \pm 0.8$ & $12.3 \pm 0.9$ & $11.2 \pm 0.8$ & $11.0 \pm 1.3$ & $11.9 \pm 2.3$ & $13.6 \pm 2.4$ & $14.3 \pm 2.6$ & $13.8 \pm 2.4$ & $11.8 \pm 1.9$ & $13.3 \pm 1.8$ & $13.3 \pm 1.6$ \\
\hline \multicolumn{13}{|l|}{$\begin{array}{l}\text { Mean Wind } \\
\text { Direction ( } 9 \text { ) }\end{array}$} \\
\hline Hindcast & $251 \pm 21$ & $247 \pm 21$ & $251 \pm 20$ & $256 \pm 20$ & $262 \pm 25$ & $266 \pm 40$ & $283 \pm 79$ & $330 \pm 108$ & $294 \pm 103$ & $263 \pm 63$ & $260 \pm 32$ & $255 \pm 22$ \\
\hline RCP4.5 - mid & $251 \pm 22$ & $249 \pm 21$ & $251 \pm 18$ & $255 \pm 15$ & $262 \pm 27$ & $268 \pm 39$ & $281 \pm 72$ & $330 \pm 115$ & $323 \pm 119$ & $263 \pm 74$ & $259 \pm 34$ & $256 \pm 22$ \\
\hline RCP8.5 - mid & $251 \pm 21$ & $250 \pm 20$ & $251 \pm 18$ & $256 \pm 16$ & $261 \pm 23$ & $269 \pm 37$ & $282 \pm 76$ & $342 \pm 110$ & $355 \pm 125$ & $265 \pm 74$ & $260 \pm 33$ & $257 \pm 22$ \\
\hline RCP4.5 - end & $251 \pm 21$ & $248 \pm 20$ & $251 \pm 19$ & $256 \pm 18$ & $262 \pm 24$ & $266 \pm 37$ & $280 \pm 77$ & $319 \pm 105$ & $318 \pm 110$ & $265 \pm 76$ & $258 \pm 31$ & $256 \pm 20$ \\
\hline $\begin{array}{l}\text { RCP } 8.5 \text { - end } \\
\text { Top } 5 \text { percent } \\
\left.\text { Direction ( }{ }^{9}\right)\end{array}$ & $252 \pm 21$ & $249 \pm 22$ & $251 \pm 17$ & $256 \pm 14$ & $262 \pm 21$ & $268 \pm 43$ & $278 \pm 69$ & $308 \pm 97$ & $338 \pm 115$ & $264 \pm 74$ & $258 \pm 29$ & $257 \pm 20$ \\
\hline Hindcast & $240 \pm 14$ & $238 \pm 12$ & $245 \pm 26$ & $248 \pm 22$ & $260 \pm 33$ & $278 \pm 52$ & $357 \pm 70$ & $12 \pm 57$ & $8 \pm 79$ & $278 \pm 64$ & $258 \pm 39$ & $249 \pm 20$ \\
\hline RCP4.5 - mid & $242 \pm 13$ & $239 \pm 11$ & $243 \pm 19$ & $247 \pm 18$ & $260 \pm 25$ & $279 \pm 51$ & $356 \pm 80$ & $28 \pm 66$ & $30 \pm 76$ & $259 \pm 68$ & $256 \pm 37$ & $247 \pm 27$ \\
\hline RCP8.5 - mid & $243 \pm 12$ & $241 \pm 11$ & $243 \pm 17$ & $250 \pm 12$ & $258 \pm 21$ & $282 \pm 59$ & $15 \pm 63$ & $29 \pm 55$ & $40 \pm 47$ & $285 \pm 70$ & $257 \pm 34$ & $251 \pm 28$ \\
\hline RCP4.5 - end & $243 \pm 14$ & $237 \pm 15$ & $241 \pm 12$ & $248 \pm 20$ & $259 \pm 18$ & $272 \pm 51$ & $344 \pm 84$ & $36 \pm 51$ & $40 \pm 57$ & $275 \pm 66$ & $255 \pm 33$ & $246 \pm 19$ \\
\hline RCP8.5 - end & $243 \pm 14$ & $241 \pm 13$ & $240 \pm 11$ & $251 \pm 13$ & $257 \pm 14$ & $283 \pm 56$ & $12 \pm 77$ & $27 \pm 55$ & $27 \pm 66$ & $269 \pm 76$ & $256 \pm 24$ & $251 \pm 19$ \\
\hline
\end{tabular}


Appendix B12. Table showing return values of ensemble-average wind speeds of hindcast and forecast scenarios, including lower and higher 95 percent confidence intervals, at the Saipan location.

[Years: Hindcast $=1976-2005 ;$ RCP mid $=$ 2026-2045; RCP end $=$ 2081-2100. Wind speeds are in meters per second]

\begin{tabular}{|c|c|c|c|c|c|c|c|c|c|c|c|c|c|c|c|c|}
\hline \multirow{2}{*}{ Scenario } & \multicolumn{3}{|c|}{ Hindcast } & \multicolumn{3}{c|}{ RCP4.5 - mid } & \multicolumn{3}{c|}{ RCP8.5 - mid } & \multicolumn{3}{c|}{ RCP4.5 - end } & \multicolumn{2}{c|}{ RCP8.5 - end } \\
\cline { 2 - 13 } & Low & $\boldsymbol{R}_{\boldsymbol{V}}$ & High & Low & $\boldsymbol{R}_{\boldsymbol{V}}$ & High & Low & $\boldsymbol{R}_{\boldsymbol{V}}$ & High & Low & $\boldsymbol{R}_{\boldsymbol{V}}$ & High & Low & $\boldsymbol{R}_{\boldsymbol{V}}$ & High \\
\hline 2-year & 20.87 & 22.67 & 25.25 & 20.13 & 22.46 & 26.21 & 20.39 & 22.84 & 26.87 & 19.76 & 21.64 & 24.46 & 19.87 & 22.11 & 25.83 \\
5-year & 21.91 & 24.49 & 28.42 & 21.15 & 24.58 & 30.63 & 21.51 & 25.23 & 32.02 & 20.56 & 23.13 & 27.25 & 20.67 & 23.87 & 29.74 \\
10-year & 22.52 & 25.66 & 30.70 & 21.76 & 26.03 & 34.10 & 22.21 & 26.97 & 36.32 & 20.98 & 24.02 & 29.13 & 21.11 & 25.02 & 32.71 \\
20-year & 23.01 & 26.69 & 32.86 & 22.25 & 27.36 & 37.69 & 22.80 & 28.63 & 41.00 & 21.30 & 24.76 & 30.83 & 21.45 & 26.03 & 35.70 \\
50-year & 23.50 & 27.85 & 35.58 & 22.77 & 28.96 & 42.63 & 23.45 & 30.74 & 47.84 & 21.60 & 25.54 & 32.85 & 21.79 & 27.18 & 39.67 \\
100-year & 23.79 & 28.60 & 37.52 & 23.08 & 30.05 & 46.52 & 23.86 & 32.26 & 53.55 & 21.76 & 26.00 & 34.21 & 21.98 & 27.93 & 42.69 \\
\hline
\end{tabular}


Appendix B13. Table showing Asuncion monthly means and mean of the top 5 percent for wind speed and mean wind direction.

[Years: Hindcast $=1976-2005 ;$ RCP mid $=$ 2026-2045; RCP end = 2081-2100. Wind directions are "coming from”]

\begin{tabular}{|c|c|c|c|c|c|c|c|c|c|c|c|c|}
\hline Month & Jan & Feb & Mar & Apr & May & Jun & July & Aug & Sep & Oct & Nov & Dec \\
\hline \multicolumn{13}{|c|}{$\begin{array}{l}\text { Mean Wind Speed } \\
(\mathrm{m} / \mathrm{s})\end{array}$} \\
\hline Hindcast & $7.3 \pm 2.8$ & $7.1 \pm 2.8$ & $6.9 \pm 2.8$ & $6.6 \pm 2.4$ & $6.4 \pm 2.3$ & $6.4 \pm 2.4$ & $6.3 \pm 3.0$ & $6.1 \pm 3.0$ & $6.1 \pm 2.8$ & $6.9 \pm 3.0$ & $7.8 \pm 2.7$ & $7.7 \pm 2.8$ \\
\hline RCP4.5 - mid & $7.5 \pm 2.7$ & $7.2 \pm 2.9$ & $7.0 \pm 2.7$ & $6.7 \pm 2.5$ & $6.3 \pm 2.2$ & $6.6 \pm 2.3$ & $6.4 \pm 2.7$ & $5.8 \pm 2.8$ & $5.8 \pm 2.9$ & $6.6 \pm 2.7$ & $7.7 \pm 2.6$ & $7.5 \pm 2.8$ \\
\hline RCP8.5 - mid & $7.4 \pm 2.8$ & $7.2 \pm 2.8$ & $6.9 \pm 2.7$ & $6.7 \pm 2.3$ & $6.2 \pm 2.1$ & $6.5 \pm 2.4$ & $6.4 \pm 2.8$ & $5.8 \pm 3.0$ & $6.0 \pm 3.0$ & $6.5 \pm 2.8$ & $7.6 \pm 2.7$ & $7.8 \pm 2.7$ \\
\hline RCP4.5 - end & $7.2 \pm 2.8$ & $7.3 \pm 2.8$ & $7.0 \pm 2.7$ & $6.7 \pm 2.4$ & $6.2 \pm 2.1$ & $6.5 \pm 2.2$ & $6.4 \pm 2.8$ & $5.9 \pm 2.9$ & $5.7 \pm 2.7$ & $6.5 \pm 2.9$ & $7.7 \pm 2.6$ & $7.4 \pm 2.7$ \\
\hline RCP8.5 - end & $7.3 \pm 2.9$ & $7.2 \pm 2.8$ & $6.9 \pm 2.7$ & $6.4 \pm 2.3$ & $6.0 \pm 2.0$ & $6.3 \pm 2.3$ & $6.5 \pm 2.7$ & $6.0 \pm 2.8$ & $5.9 \pm 2.9$ & $6.5 \pm 2.6$ & $7.5 \pm 2.5$ & $7.4 \pm 2.8$ \\
\hline \multicolumn{13}{|c|}{$\begin{array}{l}\text { Top } 5 \text { percent Wind } \\
\text { Speed }(\mathrm{m} / \mathrm{s})\end{array}$} \\
\hline Hindcast & $13.0 \pm 1.0$ & $12.7 \pm 0.9$ & $12.8 \pm 1.4$ & $11.7 \pm 1.1$ & $11.3 \pm 1.6$ & $12.1 \pm 2.1$ & $14.2 \pm 2.8$ & $14.4 \pm 2.7$ & $12.9 \pm 2.2$ & $14.0 \pm 2.5$ & $13.9 \pm 1.6$ & $13.3 \pm 1.2$ \\
\hline RCP4.5 - mid & $12.8 \pm 0.9$ & $13.0 \pm 0.9$ & $12.6 \pm 1.1$ & $12.0 \pm 1.5$ & $10.7 \pm 1.0$ & $12.2 \pm 2.5$ & $13.2 \pm 2.5$ & $13.1 \pm 2.5$ & $13.6 \pm 2.9$ & $12.7 \pm 1.8$ & $13.4 \pm 1.6$ & $13.7 \pm 1.2$ \\
\hline RCP8.5 - mid & $13.0 \pm 0.9$ & $13.0 \pm 1.1$ & $12.3 \pm 0.9$ & $11.5 \pm 0.9$ & $10.4 \pm 1.1$ & $12.2 \pm 2.6$ & $13.7 \pm 2.8$ & $13.5 \pm 2.5$ & $14.1 \pm 3.1$ & $13.1 \pm 2.0$ & $13.2 \pm 2.5$ & $13.4 \pm 1.2$ \\
\hline RCP 4.5 - end & $12.8 \pm 0.9$ & $12.7 \pm 0.8$ & $12.6 \pm 0.8$ & $11.5 \pm 1.1$ & $10.3 \pm 1.2$ & $11.5 \pm 2.0$ & $13.3 \pm 2.7$ & $13.4 \pm 2.7$ & $12.2 \pm 2.4$ & $13.3 \pm 2.1$ & $13.2 \pm 1.6$ & $12.9 \pm 1.1$ \\
\hline RCP8.5 - end & $13.2 \pm 0.8$ & $12.7 \pm 0.8$ & $12.1 \pm 0.8$ & $11.2 \pm 0.9$ & $9.9 \pm 0.8$ & $11.8 \pm 2.3$ & $13.7 \pm 2.5$ & $13.7 \pm 2.6$ & $13.6 \pm 2.7$ & $12.4 \pm 2.4$ & $12.9 \pm 1.5$ & $13.1 \pm 1.0$ \\
\hline \multicolumn{13}{|l|}{$\begin{array}{l}\text { Mean Wind } \\
\text { Direction ( } 9 \text { ) }\end{array}$} \\
\hline Hindcast & $245 \pm 51$ & $240 \pm 52$ & $251 \pm 43$ & $262 \pm 34$ & $270 \pm 33$ & $273 \pm 35$ & $279 \pm 52$ & $284 \pm 67$ & $274 \pm 59$ & $268 \pm 46$ & $265 \pm 37$ & $255 \pm 41$ \\
\hline RCP4.5 - mid & $246 \pm 49$ & $243 \pm 50$ & $250 \pm 40$ & $260 \pm 30$ & $271 \pm 35$ & $276 \pm 31$ & $277 \pm 47$ & $284 \pm 67$ & $275 \pm 63$ & $268 \pm 49$ & $264 \pm 36$ & $255 \pm 43$ \\
\hline RCP8.5 - mid & $245 \pm 48$ & $244 \pm 49$ & $251 \pm 42$ & $261 \pm 32$ & $271 \pm 31$ & $277 \pm 33$ & $278 \pm 50$ & $288 \pm 73$ & $279 \pm 71$ & $267 \pm 50$ & $265 \pm 35$ & $256 \pm 43$ \\
\hline RCP4.5 - end & $246 \pm 51$ & $242 \pm 45$ & $251 \pm 42$ & $260 \pm 32$ & $272 \pm 33$ & $275 \pm 30$ & $279 \pm 51$ & $287 \pm 67$ & $275 \pm 62$ & $268 \pm 49$ & $264 \pm 34$ & $259 \pm 41$ \\
\hline $\begin{array}{l}\text { RCP } 8.5 \text { - end } \\
\text { Top } 5 \text { percent } \\
\left.\text { Direction ( }{ }^{9}\right)\end{array}$ & $246 \pm 49$ & $242 \pm 48$ & $249 \pm 40$ & $261 \pm 31$ & $272 \pm 30$ & $277 \pm 33$ & $280 \pm 47$ & $286 \pm 61$ & $282 \pm 68$ & $268 \pm 48$ & $264 \pm 32$ & $259 \pm 39$ \\
\hline Hindcast & $236 \pm 29$ & $236 \pm 26$ & $239 \pm 21$ & $245 \pm 21$ & $265 \pm 30$ & $276 \pm 40$ & $307 \pm 66$ & $320 \pm 70$ & $289 \pm 70$ & $268 \pm 48$ & $255 \pm 38$ & $244 \pm 26$ \\
\hline RCP4.5 - mid & $240 \pm 24$ & $236 \pm 18$ & $239 \pm 19$ & $244 \pm 17$ & $261 \pm 26$ & $287 \pm 37$ & $287 \pm 66$ & $344 \pm 86$ & $290 \pm 77$ & $264 \pm 54$ & $250 \pm 36$ & $244 \pm 26$ \\
\hline RCP8.5 - mid & $242 \pm 27$ & $241 \pm 25$ & $241 \pm 22$ & $247 \pm 20$ & $264 \pm 25$ & $284 \pm 40$ & $313 \pm 67$ & $346 \pm 86$ & $342 \pm 84$ & $268 \pm 50$ & $259 \pm 36$ & $246 \pm 27$ \\
\hline RCP4.5 - end & $240 \pm 25$ & $237 \pm 24$ & $239 \pm 19$ & $247 \pm 18$ & $260 \pm 25$ & $277 \pm 32$ & $304 \pm 64$ & $328 \pm 78$ & $295 \pm 75$ & $267 \pm 54$ & $251 \pm 35$ & $244 \pm 22$ \\
\hline RCP8.5 - end & $240 \pm 21$ & $238 \pm 20$ & $238 \pm 15$ & $249 \pm 15$ & $263 \pm 20$ & $282 \pm 40$ & $312 \pm 64$ & $327 \pm 75$ & $343 \pm 92$ & $263 \pm 49$ & $255 \pm 29$ & $248 \pm 24$ \\
\hline
\end{tabular}


Appendix B14. Table showing return values of ensemble-average wind speeds of hindcast and forecast scenarios, including lower and higher 95 percent confidence intervals, at the Asuncion location.

[Years: Hindcast $=1976-2005 ;$ RCP mid $=$ 2026-2045; RCP end $=$ 2081-2100. Wind speeds are in meters per second]

\begin{tabular}{|c|c|c|c|c|c|c|c|c|c|c|c|c|c|c|c|}
\hline \multirow{2}{*}{ Scenario } & \multicolumn{3}{|c|}{ Hindcast } & \multicolumn{3}{c|}{ RCP4.5 - mid } & \multicolumn{3}{c|}{ RCP8.5 - mid } & \multicolumn{3}{c|}{ RCP4.5 - end } & \multicolumn{2}{c|}{ RCP8.5 - end } \\
\cline { 2 - 13 } & Low & $\boldsymbol{R}_{\boldsymbol{V}}$ & High & Low & $\boldsymbol{R}_{\boldsymbol{V}}$ & High & Low & $\boldsymbol{R}_{\boldsymbol{V}}$ & High & Low & $\boldsymbol{R}_{\boldsymbol{V}}$ & High & Low & $\boldsymbol{R}_{\boldsymbol{V}}$ & High \\
\hline 2-year & 20.83 & 22.81 & 25.78 & 19.32 & 21.91 & 26.53 & 20.13 & 22.84 & 27.40 & 19.02 & 21.50 & 25.87 & 18.95 & 21.21 & 25.18 \\
5-year & 22.00 & 24.98 & 29.81 & 20.23 & 24.13 & 32.01 & 21.35 & 25.53 & 33.36 & 19.84 & 23.48 & 30.67 & 19.55 & 22.69 & 28.85 \\
10-year & 22.73 & 26.51 & 33.00 & 20.77 & 25.72 & 36.66 & 22.12 & 27.52 & 38.49 & 20.31 & 24.84 & 34.56 & 19.87 & 23.62 & 31.56 \\
20-year & 23.35 & 27.94 & 36.30 & 21.21 & 27.24 & 41.82 & 22.79 & 29.47 & 44.20 & 20.68 & 26.07 & 38.70 & 20.10 & 24.40 & 34.23 \\
50-year & 24.03 & 29.70 & 40.86 & 21.68 & 29.12 & 49.48 & 23.52 & 31.98 & 52.78 & 21.06 & 27.55 & 44.56 & 20.30 & 25.24 & 37.69 \\
100-year & 24.45 & 30.93 & 44.45 & 21.96 & 30.46 & 56.00 & 23.99 & 33.83 & 60.15 & 21.27 & 28.55 & 49.31 & 20.41 & 25.76 & 40.25 \\
\hline
\end{tabular}


Appendix B15. Table showing Kosrae monthly means and mean of the top 5 percent for wind speed and mean wind direction.

[Years: Hindcast = 1976-2005; RCP mid = 2026-2045; RCP end = 2081-2100. Wind directions are “coming from”]

\begin{tabular}{|c|c|c|c|c|c|c|c|c|c|c|c|c|}
\hline Month & Jan & Feb & Mar & Apr & May & Jun & July & Aug & Sep & Oct & Nov & Dec \\
\hline \multicolumn{13}{|c|}{$\begin{array}{l}\text { Mean Wind Speed } \\
(\mathrm{m} / \mathrm{s})\end{array}$} \\
\hline Hindcast & $7.5 \pm 2.1$ & $7.4 \pm 2.0$ & $6.8 \pm 2.0$ & $5.7 \pm 2.1$ & $4.4 \pm 2.0$ & $4.1 \pm 2.0$ & $4.2 \pm 1.9$ & $4.4 \pm 1.9$ & $4.4 \pm 2.0$ & $4.3 \pm 2.1$ & $4.6 \pm 2.4$ & $6.3 \pm 2.6$ \\
\hline RCP4.5 - mid & $7.5 \pm 2.0$ & $7.4 \pm 2.0$ & $7.0 \pm 2.0$ & $5.8 \pm 2.1$ & $4.5 \pm 2.0$ & $4.2 \pm 2.1$ & $4.3 \pm 1.9$ & $4.5 \pm 2.0$ & $4.5 \pm 2.0$ & $4.3 \pm 2.1$ & $4.5 \pm 2.2$ & $6.1 \pm 2.4$ \\
\hline RCP8.5 - mid & $7.3 \pm 2.1$ & $7.5 \pm 2.1$ & $7.0 \pm 2.0$ & $5.9 \pm 2.1$ & $4.5 \pm 2.2$ & $4.1 \pm 2.0$ & $4.4 \pm 2.0$ & $4.5 \pm 2.0$ & $4.6 \pm 1.9$ & $4.3 \pm 2.1$ & $4.4 \pm 2.2$ & $5.9 \pm 2.5$ \\
\hline RCP4.5 - end & $7.2 \pm 2.0$ & $7.3 \pm 1.9$ & $6.9 \pm 1.9$ & $5.7 \pm 2.0$ & $4.5 \pm 2.0$ & $4.2 \pm 2.1$ & $4.3 \pm 2.0$ & $4.5 \pm 2.1$ & $4.5 \pm 2.1$ & $4.2 \pm 2.1$ & $4.5 \pm 2.2$ & $5.9 \pm 2.4$ \\
\hline RCP8.5 - end & $7.1 \pm 2.0$ & $7.2 \pm 1.9$ & $6.9 \pm 1.8$ & $5.9 \pm 1.9$ & $4.7 \pm 2.1$ & $4.3 \pm 2.1$ & $4.4 \pm 1.9$ & $4.8 \pm 2.0$ & $4.6 \pm 2.0$ & $4.2 \pm 2.0$ & $4.3 \pm 2.0$ & $5.9 \pm 2.3$ \\
\hline \multicolumn{13}{|c|}{$\begin{array}{l}\text { Top } 5 \text { percent Wind } \\
\text { Speed }(\mathrm{m} / \mathrm{s})\end{array}$} \\
\hline Hindcast & $11.3 \pm 0.8$ & $10.9 \pm 0.6$ & $10.9 \pm 1.1$ & $10.1 \pm 1.2$ & $9.0 \pm 1.4$ & $8.8 \pm 1.3$ & $9.0 \pm 2.1$ & $9.3 \pm 2.0$ & $9.6 \pm 1.7$ & $9.7 \pm 1.7$ & $10.4 \pm 1.7$ & $11.5 \pm 1.2$ \\
\hline RCP4.5 - mid & $11.3 \pm 0.9$ & $11.0 \pm 1.2$ & $10.6 \pm 0.7$ & $10.0 \pm 0.8$ & $9.0 \pm 1.0$ & $9.3 \pm 1.7$ & $9.1 \pm 1.8$ & $9.7 \pm 1.9$ & $9.2 \pm 1.5$ & $9.6 \pm 1.7$ & $9.9 \pm 1.7$ & $11.2 \pm 1.3$ \\
\hline RCP8.5 - mid & $11.2 \pm 0.7$ & $11.6 \pm 1.5$ & $10.8 \pm 0.8$ & $10.5 \pm 1.4$ & $9.6 \pm 1.6$ & $8.7 \pm 1.2$ & $9.5 \pm 1.8$ & $9.8 \pm 1.7$ & $9.4 \pm 1.5$ & $9.5 \pm 1.8$ & $10.0 \pm 1.3$ & $11.0 \pm 0.9$ \\
\hline RCP4.5 - end & $10.7 \pm 0.6$ & $10.6 \pm 0.6$ & $10.4 \pm 0.8$ & $9.7 \pm 0.8$ & $9.1 \pm 0.8$ & $9.2 \pm 1.9$ & $9.4 \pm 2.0$ & $10.1 \pm 1.7$ & $9.9 \pm 1.5$ & $9.3 \pm 1.4$ & $9.9 \pm 1.7$ & $11.1 \pm 1.2$ \\
\hline RCP8.5 - end & $10.8 \pm 0.7$ & $10.9 \pm 1.0$ & $10.4 \pm 0.8$ & $9.8 \pm 0.6$ & $9.6 \pm 1.4$ & $9.5 \pm 1.9$ & $9.2 \pm 1.9$ & $10.0 \pm 2.2$ & $9.2 \pm 1.5$ & $9.1 \pm 1.5$ & $9.2 \pm 1.4$ & $10.6 \pm 1.0$ \\
\hline \multicolumn{13}{|l|}{$\begin{array}{l}\text { Mean Wind } \\
\text { Direction ( } 9 \text { ) }\end{array}$} \\
\hline Hindcast & $245 \pm 21$ & $245 \pm 19$ & $247 \pm 25$ & $253 \pm 33$ & $267 \pm 53$ & $278 \pm 60$ & $292 \pm 55$ & $301 \pm 51$ & $306 \pm 55$ & $311 \pm 65$ & $278 \pm 60$ & $251 \pm 38$ \\
\hline RCP4.5 - mid & $246 \pm 23$ & $244 \pm 22$ & $246 \pm 21$ & $253 \pm 31$ & $261 \pm 44$ & $272 \pm 59$ & $288 \pm 55$ & $303 \pm 53$ & $304 \pm 54$ & $309 \pm 62$ & $284 \pm 64$ & $256 \pm 42$ \\
\hline RCP8.5 - mid & $246 \pm 22$ & $245 \pm 21$ & $247 \pm 20$ & $250 \pm 31$ & $258 \pm 47$ & $275 \pm 59$ & $289 \pm 59$ & $304 \pm 57$ & $305 \pm 54$ & $309 \pm 66$ & $281 \pm 64$ & $255 \pm 39$ \\
\hline RCP4.5 - end & $247 \pm 23$ & $245 \pm 21$ & $247 \pm 21$ & $252 \pm 31$ & $258 \pm 45$ & $273 \pm 58$ & $288 \pm 57$ & $304 \pm 57$ & $307 \pm 59$ & $308 \pm 66$ & $283 \pm 64$ & $256 \pm 40$ \\
\hline RCP8.5 - end & $248 \pm 22$ & $247 \pm 18$ & $248 \pm 19$ & $251 \pm 24$ & $259 \pm 40$ & $267 \pm 55$ & $282 \pm 54$ & $299 \pm 52$ & $302 \pm 52$ & $303 \pm 60$ & $281 \pm 58$ & $257 \pm 35$ \\
\hline \multicolumn{13}{|c|}{$\begin{array}{l}\text { Top } 5 \text { percent Wind } \\
\text { Direction ( } 9\end{array}$} \\
\hline Hindcast & $236 \pm 14$ & $235 \pm 12$ & $232 \pm 29$ & $236 \pm 31$ & $246 \pm 63$ & $262 \pm 82$ & $346 \pm 92$ & $355 \pm 75$ & $18 \pm 82$ & $38 \pm 80$ & $261 \pm 91$ & $233 \pm 27$ \\
\hline RCP4.5 - mid & $234 \pm 17$ & $235 \pm 25$ & $238 \pm 13$ & $238 \pm 29$ & $238 \pm 43$ & $258 \pm 74$ & $330 \pm 96$ & $3 \pm 71$ & $337 \pm 73$ & $8 \pm 68$ & $282 \pm 105$ & $235 \pm 43$ \\
\hline RCP8.5 - mid & $238 \pm 14$ & $237 \pm 29$ & $236 \pm 17$ & $233 \pm 49$ & $233 \pm 57$ & $258 \pm 89$ & $30 \pm 100$ & $30 \pm 68$ & $30 \pm 82$ & $33 \pm 83$ & $226 \pm 85$ & $234 \pm 25$ \\
\hline RCP4.5 - end & $232 \pm 14$ & $236 \pm 20$ & $236 \pm 17$ & $242 \pm 26$ & $240 \pm 24$ & $274 \pm 67$ & $30 \pm 98$ & $31 \pm 74$ & $30 \pm 76$ & $33 \pm 83$ & $319 \pm 114$ & $232 \pm 41$ \\
\hline RCP8.5 - end & $238 \pm 16$ & $242 \pm 21$ & $240 \pm 28$ & $241 \pm 20$ & $242 \pm 42$ & $251 \pm 79$ & $322 \pm 99$ & $3 \pm 79$ & $329 \pm 83$ & $355 \pm 91$ & $261 \pm 74$ & $235 \pm 24$ \\
\hline
\end{tabular}


Appendix B16. Table showing return values of ensemble-average wind speeds of hindcast and forecast scenarios, including lower and higher 95 percent confidence intervals, at the Kosrae location.

[Years: Hindcast $=1976-2005 ;$ RCP mid $=$ 2026-2045; RCP end $=$ 2081-2100. Wind speeds are in meters per second]

\begin{tabular}{|c|c|c|c|c|c|c|c|c|c|c|c|c|c|c|c|c|}
\hline \multirow{2}{*}{ Scenario } & \multicolumn{3}{|c|}{ Hindcast } & \multicolumn{3}{c|}{ RCP4.5 - mid } & \multicolumn{3}{c|}{ RCP8.5 - mid } & \multicolumn{3}{c|}{ RCP4.5 - end } & \multicolumn{2}{c|}{ RCP8.5 - end } \\
\cline { 2 - 13 } & Low & $\boldsymbol{R}_{\boldsymbol{V}}$ & High & Low & $\boldsymbol{R}_{\boldsymbol{V}}$ & High & Low & $\boldsymbol{R}_{\boldsymbol{V}}$ & High & Low & $\boldsymbol{R}_{\boldsymbol{V}}$ & High & Low & $\boldsymbol{R}_{\boldsymbol{V}}$ & High \\
\hline 2-year & 15.25 & 17.04 & 20.24 & 15.02 & 16.86 & 20.33 & 14.77 & 16.36 & 19.19 & 14.70 & 16.54 & 19.99 & 14.45 & 16.35 & 20.20 \\
5-year & 16.20 & 19.32 & 25.71 & 15.67 & 18.58 & 24.92 & 15.43 & 17.94 & 23.03 & 15.35 & 18.26 & 24.51 & 15.19 & 18.49 & 26.46 \\
10-year & 16.89 & 21.30 & 31.37 & 16.08 & 19.91 & 29.21 & 15.85 & 19.16 & 26.52 & 15.77 & 19.58 & 28.70 & 15.71 & 20.38 & 33.25 \\
20-year & 17.56 & 23.52 & 38.77 & 16.43 & 21.25 & 34.35 & 16.22 & 20.38 & 30.61 & 16.13 & 20.91 & 33.67 & 16.21 & 22.52 & 42.55 \\
50-year & 18.42 & 26.90 & 52.19 & 16.83 & 23.06 & 42.75 & 16.63 & 22.03 & 37.15 & 16.52 & 22.68 & 41.72 & 16.82 & 25.82 & 60.30 \\
100-year & 19.04 & 29.83 & 66.03 & 17.07 & 24.45 & 50.58 & 16.90 & 23.29 & 43.10 & 16.77 & 24.04 & 49.15 & 17.26 & 28.72 & 79.58 \\
\hline
\end{tabular}


Appendix B17. Table showing Palau monthly means and mean of the top 5 percent for wind speed and mean wind direction.

[Years: Hindcast $=1976-2005 ;$ RCP mid $=$ 2026-2045; RCP end = 2081-2100. Wind directions are "coming from”]

\begin{tabular}{|c|c|c|c|c|c|c|c|c|c|c|c|c|}
\hline Month & Jan & Feb & Mar & Apr & May & Jun & July & Aug & Sep & Oct & Nov & Dec \\
\hline \multicolumn{13}{|c|}{$\begin{array}{l}\text { Mean Wind Speed } \\
(\mathrm{m} / \mathrm{s})\end{array}$} \\
\hline Hindcast & $7.7 \pm 2.5$ & $8.1 \pm 2.3$ & $7.3 \pm 2.2$ & $6.2 \pm 2.2$ & $4.7 \pm 2.3$ & $4.0 \pm 2.3$ & $4.0 \pm 2.5$ & $4.1 \pm 2.7$ & $4.0 \pm 2.6$ & $4.2 \pm 2.6$ & $4.9 \pm 2.7$ & $6.5 \pm 2.8$ \\
\hline RCP4.5 - mid & $7.3 \pm 2.6$ & $7.7 \pm 2.3$ & $7.3 \pm 2.1$ & $6.3 \pm 2.1$ & $4.7 \pm 2.2$ & $4.1 \pm 2.3$ & $4.3 \pm 2.7$ & $4.0 \pm 2.3$ & $3.9 \pm 2.3$ & $4.2 \pm 2.6$ & $4.7 \pm 2.7$ & $6.0 \pm 2.8$ \\
\hline RCP8.5 - mid & $7.5 \pm 2.5$ & $7.6 \pm 2.3$ & $7.4 \pm 2.1$ & $6.3 \pm 2.0$ & $4.6 \pm 2.2$ & $4.2 \pm 2.4$ & $4.2 \pm 2.6$ & $4.3 \pm 2.6$ & $4.0 \pm 2.5$ & $4.1 \pm 2.5$ & $4.7 \pm 2.6$ & $6.1 \pm 2.8$ \\
\hline RCP4.5 - end & $7.4 \pm 2.5$ & $7.8 \pm 2.1$ & $7.2 \pm 2.2$ & $6.1 \pm 2.2$ & $4.7 \pm 2.2$ & $4.1 \pm 2.3$ & $4.4 \pm 2.5$ & $4.3 \pm 2.7$ & $4.1 \pm 2.6$ & $4.0 \pm 2.4$ & $4.5 \pm 2.5$ & $5.9 \pm 2.7$ \\
\hline RCP8.5 - end & $7.1 \pm 2.4$ & $7.4 \pm 2.2$ & $7.2 \pm 2.0$ & $6.2 \pm 1.9$ & $4.6 \pm 2.3$ & $4.2 \pm 2.3$ & $4.4 \pm 2.6$ & $4.3 \pm 2.6$ & $4.2 \pm 2.5$ & $4.0 \pm 2.3$ & $4.5 \pm 2.6$ & $5.8 \pm 2.6$ \\
\hline \multicolumn{13}{|c|}{$\begin{array}{l}\text { Top } 5 \text { percent Wind } \\
\text { Speed }(\mathrm{m} / \mathrm{s})\end{array}$} \\
\hline Hindcast & $12.6 \pm 1.1$ & $13.0 \pm 1.3$ & $11.8 \pm 1.2$ & $10.5 \pm 1.3$ & $9.7 \pm 1.3$ & $9.9 \pm 1.6$ & $10.9 \pm 1.4$ & $11.8 \pm 1.8$ & $11.5 \pm 1.6$ & $11.2 \pm 1.5$ & $11.9 \pm 2.1$ & $12.3 \pm 1.5$ \\
\hline RCP4.5 - mid & $12.7 \pm 1.5$ & $12.2 \pm 1.1$ & $11.5 \pm 1.1$ & $10.3 \pm 1.2$ & $9.6 \pm 1.4$ & $10.2 \pm 1.8$ & $11.7 \pm 1.5$ & $10.3 \pm 1.7$ & $10.4 \pm 2.0$ & $11.7 \pm 2.1$ & $11.8 \pm 2.0$ & $12.4 \pm 1.9$ \\
\hline RCP8.5 - mid & $12.3 \pm 1.2$ & $12.1 \pm 0.9$ & $11.8 \pm 1.3$ & $10.5 \pm 1.3$ & $9.3 \pm 1.0$ & $10.6 \pm 2.1$ & $11.0 \pm 1.5$ & $11.4 \pm 1.7$ & $11.0 \pm 2.0$ & $10.9 \pm 1.3$ & $11.1 \pm 1.5$ & $12.2 \pm 1.7$ \\
\hline RCP4.5 - end & $12.4 \pm 1.5$ & $12.1 \pm 1.4$ & $11.4 \pm 1.2$ & $10.7 \pm 1.3$ & $9.5 \pm 1.4$ & $10.2 \pm 1.8$ & $11.1 \pm 1.8$ & $11.6 \pm 1.7$ & $11.6 \pm 1.8$ & $11.0 \pm 2.2$ & $10.8 \pm 1.7$ & $11.9 \pm 1.6$ \\
\hline RCP8.5 - end & $11.9 \pm 1.1$ & $12.1 \pm 1.1$ & $11.7 \pm 1.3$ & $10.0 \pm 1.0$ & $9.6 \pm 1.3$ & $9.9 \pm 1.7$ & $11.0 \pm 1.8$ & $11.6 \pm 1.9$ & $11.5 \pm 2.3$ & $10.1 \pm 1.5$ & $11.3 \pm 2.1$ & $11.8 \pm 1.7$ \\
\hline \multicolumn{13}{|l|}{$\begin{array}{l}\text { Mean Wind } \\
\text { Direction }(9)\end{array}$} \\
\hline Hindcast & $243 \pm 30$ & $239 \pm 27$ & $241 \pm 33$ & $246 \pm 36$ & $257 \pm 62$ & $283 \pm 85$ & $333 \pm 82$ & $350 \pm 75$ & $354 \pm 86$ & $341 \pm 105$ & $255 \pm 81$ & $249 \pm 47$ \\
\hline RCP4.5 - mid & $244 \pm 37$ & $242 \pm 29$ & $243 \pm 27$ & $248 \pm 33$ & $256 \pm 52$ & $287 \pm 86$ & $345 \pm 83$ & $330 \pm 74$ & $339 \pm 80$ & $358 \pm 103$ & $259 \pm 84$ & $248 \pm 50$ \\
\hline RCP8.5 - mid & $244 \pm 32$ & $243 \pm 28$ & $244 \pm 29$ & $248 \pm 32$ & $255 \pm 52$ & $295 \pm 91$ & $345 \pm 88$ & $343 \pm 77$ & $342 \pm 77$ & $348 \pm 100$ & $261 \pm 87$ & $249 \pm 54$ \\
\hline RCP4.5 - end & $246 \pm 32$ & $243 \pm 24$ & $244 \pm 27$ & $248 \pm 37$ & $258 \pm 61$ & $288 \pm 92$ & $340 \pm 87$ & $348 \pm 76$ & $347 \pm 80$ & $354 \pm 103$ & $258 \pm 87$ & $250 \pm 51$ \\
\hline RCP8.5 - end & $245 \pm 35$ & $243 \pm 27$ & $245 \pm 26$ & $248 \pm 27$ & $258 \pm 61$ & $287 \pm 91$ & $337 \pm 88$ & $342 \pm 77$ & $344 \pm 77$ & $319 \pm 93$ & $264 \pm 82$ & $254 \pm 51$ \\
\hline \multicolumn{13}{|c|}{$\begin{array}{l}\text { Top } 5 \text { percent Wind } \\
\text { Direction }(9)\end{array}$} \\
\hline Hindcast & $233 \pm 29$ & $222 \pm 38$ & $232 \pm 37$ & $241 \pm 39$ & $247 \pm 74$ & $44 \pm 97$ & $38 \pm 51$ & $36 \pm 34$ & $53 \pm 36$ & $54 \pm 46$ & $69 \pm 115$ & $241 \pm 48$ \\
\hline RCP4.5 - mid & $236 \pm 34$ & $236 \pm 31$ & $236 \pm 34$ & $246 \pm 42$ & $250 \pm 50$ & $8 \pm 102$ & $45 \pm 34$ & $40 \pm 44$ & $37 \pm 47$ & $55 \pm 45$ & $55 \pm 119$ & $234 \pm 80$ \\
\hline RCP8.5 - mid & $238 \pm 36$ & $242 \pm 33$ & $239 \pm 40$ & $236 \pm 41$ & $245 \pm 45$ & $33 \pm 61$ & $41 \pm 40$ & $37 \pm 32$ & $47 \pm 35$ & $55 \pm 48$ & $55 \pm 93$ & $246 \pm 68$ \\
\hline RCP4.5 - end & $235 \pm 48$ & $235 \pm 26$ & $236 \pm 33$ & $244 \pm 48$ & $250 \pm 63$ & $22 \pm 97$ & $37 \pm 40$ & $44 \pm 35$ & $45 \pm 36$ & $55 \pm 52$ & $78 \pm 130$ & $240 \pm 52$ \\
\hline RCP8.5 - end & $239 \pm 36$ & $236 \pm 40$ & $244 \pm 38$ & $246 \pm 31$ & $256 \pm 60$ & $10 \pm 90$ & $36 \pm 42$ & $37 \pm 30$ & $41 \pm 40$ & $49 \pm 63$ & $58 \pm 91$ & $253 \pm 70$ \\
\hline
\end{tabular}


Appendix B18. Table showing return values of ensemble-average wind speeds of hindcast and forecast scenarios, including lower and higher 95 percent confidence intervals, at the Palau location.

[Years: Hindcast $=1976-2005 ;$ RCP mid $=$ 2026-2045; RCP end $=$ 2081-2100. Wind speeds are in meters per second]

\begin{tabular}{|c|c|c|c|c|c|c|c|c|c|c|c|c|c|c|c|}
\hline \multirow{2}{*}{ Scenario } & \multicolumn{3}{|c|}{ Hindcast } & \multicolumn{3}{c|}{ RCP4.5 - mid } & \multicolumn{3}{c|}{ RCP8.5 - mid } & \multicolumn{3}{c|}{ RCP4.5 - end } & \multicolumn{2}{c|}{ RCP8.5 - end } \\
\cline { 2 - 13 } & Low & $\boldsymbol{R}_{\boldsymbol{V}}$ & High & Low & $\boldsymbol{R}_{\boldsymbol{V}}$ & High & Low & $\boldsymbol{R}_{\boldsymbol{V}}$ & High & Low & $\boldsymbol{R}_{\boldsymbol{V}}$ & High & Low & $\boldsymbol{R}_{\boldsymbol{V}}$ & High \\
\hline 2-year & 17.15 & 18.57 & 20.79 & 17.09 & 18.94 & 22.18 & 16.67 & 18.62 & 22.31 & 17.31 & 18.94 & 21.49 & 17.04 & 18.55 & 21.18 \\
5-year & 17.95 & 20.16 & 23.94 & 17.66 & 20.31 & 25.51 & 17.49 & 20.76 & 27.93 & 18.03 & 20.38 & 24.37 & 17.57 & 19.80 & 24.09 \\
10-year & 18.46 & 21.32 & 26.59 & 17.98 & 21.22 & 28.10 & 18.05 & 22.55 & 33.59 & 18.45 & 21.33 & 26.53 & 17.88 & 20.65 & 26.42 \\
20-year & 18.91 & 22.46 & 29.48 & 18.22 & 22.02 & 30.78 & 18.57 & 24.49 & 40.86 & 18.79 & 22.18 & 28.68 & 18.12 & 21.42 & 28.88 \\
50-year & 19.41 & 23.92 & 33.74 & 18.45 & 22.93 & 34.42 & 19.20 & 27.33 & 53.70 & 19.13 & 23.15 & 31.50 & 18.37 & 22.34 & 32.34 \\
100-year & 19.74 & 25.00 & 37.32 & 18.57 & 23.53 & 37.27 & 19.63 & 29.69 & 66.64 & 19.32 & 23.80 & 33.62 & 18.51 & 22.97 & 35.12 \\
\end{tabular}


Appendix B19. Table showing Pohnpei monthly means and mean of the top 5 percent for wind speed and mean wind direction.

[Years: Hindcast $=1976-2005 ;$ RCP mid $=$ 2026-2045; RCP end = 2081-2100. Wind directions are "coming from”]

\begin{tabular}{|c|c|c|c|c|c|c|c|c|c|c|c|c|}
\hline Month & Jan & Feb & Mar & Apr & May & Jun & July & Aug & Sep & Oct & Nov & Dec \\
\hline \multicolumn{13}{|c|}{$\begin{array}{l}\text { Mean Wind Speed } \\
(\mathrm{m} / \mathrm{s})\end{array}$} \\
\hline Hindcast & $8.5 \pm 2.0$ & $8.4 \pm 2.0$ & $7.8 \pm 2.1$ & $6.7 \pm 2.4$ & $5.0 \pm 2.4$ & $4.3 \pm 2.4$ & $4.1 \pm 2.3$ & $4.2 \pm 2.3$ & $4.1 \pm 2.4$ & $4.2 \pm 2.4$ & $5.2 \pm 2.8$ & $7.5 \pm 2.7$ \\
\hline RCP4.5 - mid & $8.4 \pm 2.2$ & $8.3 \pm 2.0$ & $8.0 \pm 2.0$ & $6.8 \pm 2.3$ & $5.2 \pm 2.4$ & $4.5 \pm 2.4$ & $4.3 \pm 2.4$ & $4.3 \pm 2.4$ & $4.1 \pm 2.3$ & $4.1 \pm 2.3$ & $4.9 \pm 2.5$ & $7.2 \pm 2.7$ \\
\hline RCP8.5 - mid & $8.3 \pm 2.1$ & $8.4 \pm 2.2$ & $7.9 \pm 2.1$ & $6.9 \pm 2.2$ & $5.2 \pm 2.5$ & $4.5 \pm 2.3$ & $4.4 \pm 2.4$ & $4.3 \pm 2.4$ & $4.2 \pm 2.2$ & $4.1 \pm 2.3$ & $4.8 \pm 2.6$ & $7.1 \pm 2.7$ \\
\hline RCP4.5 - end & $8.2 \pm 2.0$ & $8.2 \pm 1.9$ & $7.8 \pm 2.0$ & $6.6 \pm 2.2$ & $5.2 \pm 2.4$ & $4.7 \pm 2.5$ & $4.3 \pm 2.2$ & $4.4 \pm 2.5$ & $4.2 \pm 2.4$ & $4.1 \pm 2.3$ & $4.9 \pm 2.6$ & $6.9 \pm 2.7$ \\
\hline RCP8.5 - end & $8.0 \pm 2.1$ & $8.0 \pm 1.9$ & $7.9 \pm 1.8$ & $6.9 \pm 2.0$ & $5.5 \pm 2.4$ & $4.6 \pm 2.4$ & $4.5 \pm 2.4$ & $4.4 \pm 2.4$ & $4.1 \pm 2.3$ & $3.9 \pm 2.1$ & $4.6 \pm 2.5$ & $7.0 \pm 2.5$ \\
\hline \multicolumn{13}{|c|}{$\begin{array}{l}\text { Top } 5 \text { percent Wind } \\
\text { Speed }(\mathrm{m} / \mathrm{s})\end{array}$} \\
\hline Hindcast & $12.2 \pm 0.8$ & $12.1 \pm 0.7$ & $11.6 \pm 0.7$ & $11.0 \pm 0.9$ & $10.3 \pm 1.6$ & $10.3 \pm 1.9$ & $10.4 \pm 2.1$ & $10.8 \pm 2.0$ & $11.1 \pm 2.2$ & $11.0 \pm 1.9$ & $11.8 \pm 1.4$ & $12.6 \pm 1.2$ \\
\hline RCP4.5 - mid & $12.6 \pm 1.0$ & $11.9 \pm 0.6$ & $11.6 \pm 0.8$ & $10.8 \pm 0.5$ & $10.1 \pm 0.9$ & $10.2 \pm 1.7$ & $10.9 \pm 2.2$ & $11.4 \pm 2.2$ & $10.8 \pm 2.2$ & $10.5 \pm 2.3$ & $11.0 \pm 1.6$ & $12.7 \pm 1.1$ \\
\hline RCP8.5 - mid & $12.2 \pm 0.9$ & $12.6 \pm 1.6$ & $11.7 \pm 1.1$ & $11.1 \pm 1.0$ & $10.3 \pm 1.3$ & $10.0 \pm 1.5$ & $10.7 \pm 2.1$ & $11.6 \pm 2.2$ & $10.6 \pm 2.0$ & $10.5 \pm 1.8$ & $11.3 \pm 1.4$ & $12.6 \pm 1.2$ \\
\hline RCP4.5 - end & $11.8 \pm 0.7$ & $11.6 \pm 0.6$ & $11.4 \pm 0.9$ & $10.6 \pm 1.1$ & $10.3 \pm 1.1$ & $10.5 \pm 1.7$ & $10.2 \pm 1.9$ & $11.8 \pm 2.5$ & $11.3 \pm 1.9$ & $10.6 \pm 1.9$ & $11.2 \pm 1.8$ & $12.3 \pm 1.1$ \\
\hline RCP8.5 - end & $12.0 \pm 0.9$ & $11.7 \pm 1.0$ & $11.4 \pm 1.3$ & $10.8 \pm 0.7$ & $10.6 \pm 1.3$ & $10.4 \pm 1.7$ & $11.0 \pm 2.3$ & $11.6 \pm 2.3$ & $10.9 \pm 2.4$ & $9.6 \pm 2.2$ & $11.1 \pm 1.7$ & $12.0 \pm 1.2$ \\
\hline \multicolumn{13}{|l|}{$\begin{array}{l}\text { Mean Wind } \\
\text { Direction }(9)\end{array}$} \\
\hline Hindcast & $245 \pm 17$ & $244 \pm 16$ & $246 \pm 21$ & $250 \pm 30$ & $255 \pm 51$ & $266 \pm 66$ & $290 \pm 68$ & $310 \pm 66$ & $319 \pm 72$ & $312 \pm 83$ & $263 \pm 59$ & $248 \pm 28$ \\
\hline RCP4.5 - mid & $247 \pm 21$ & $245 \pm 18$ & $246 \pm 17$ & $249 \pm 27$ & $255 \pm 41$ & $263 \pm 62$ & $285 \pm 65$ & $312 \pm 70$ & $316 \pm 70$ & $317 \pm 81$ & $266 \pm 65$ & $252 \pm 35$ \\
\hline RCP8.5 - mid & $246 \pm 16$ & $244 \pm 17$ & $247 \pm 18$ & $248 \pm 25$ & $253 \pm 42$ & $264 \pm 61$ & $287 \pm 72$ & $317 \pm 74$ & $316 \pm 69$ & $320 \pm 83$ & $265 \pm 66$ & $251 \pm 31$ \\
\hline RCP4.5 - end & $248 \pm 17$ & $245 \pm 18$ & $247 \pm 19$ & $251 \pm 25$ & $253 \pm 41$ & $263 \pm 64$ & $285 \pm 67$ & $318 \pm 72$ & $320 \pm 73$ & $324 \pm 82$ & $269 \pm 65$ & $252 \pm 34$ \\
\hline RCP8.5 - end & $248 \pm 18$ & $246 \pm 15$ & $247 \pm 17$ & $249 \pm 18$ & $253 \pm 37$ & $261 \pm 57$ & $281 \pm 64$ & $309 \pm 66$ & $315 \pm 66$ & $308 \pm 77$ & $267 \pm 61$ & $252 \pm 27$ \\
\hline \multicolumn{13}{|c|}{$\begin{array}{l}\text { Top } 5 \text { percent Wind } \\
\text { Direction }(9)\end{array}$} \\
\hline Hindcast & $238 \pm 13$ & $236 \pm 13$ & $239 \pm 18$ & $239 \pm 22$ & $240 \pm 47$ & $273 \pm 92$ & $20 \pm 101$ & $36 \pm 65$ & $49 \pm 63$ & $43 \pm 83$ & $256 \pm 62$ & $235 \pm 20$ \\
\hline RCP4.5 - mid & $239 \pm 21$ & $238 \pm 12$ & $240 \pm 16$ & $241 \pm 27$ & $239 \pm 28$ & $250 \pm 64$ & $35 \pm 90$ & $37 \pm 64$ & $32 \pm 64$ & $44 \pm 64$ & $230 \pm 67$ & $237 \pm 31$ \\
\hline RCP8.5 - mid & $238 \pm 12$ & $234 \pm 27$ & $241 \pm 21$ & $237 \pm 29$ & $245 \pm 41$ & $254 \pm 75$ & $53 \pm 78$ & $47 \pm 50$ & $41 \pm 60$ & $56 \pm 68$ & $239 \pm 62$ & $240 \pm 25$ \\
\hline RCP4.5 - end & $238 \pm 11$ & $239 \pm 12$ & $239 \pm 18$ & $242 \pm 25$ & $241 \pm 23$ & $255 \pm 77$ & $40 \pm 85$ & $49 \pm 54$ & $50 \pm 46$ & $44 \pm 68$ & $251 \pm 87$ & $237 \pm 29$ \\
\hline RCP8.5 - end & $238 \pm 19$ & $241 \pm 14$ & $236 \pm 26$ & $241 \pm 10$ & $238 \pm 37$ & $247 \pm 63$ & $15 \pm 95$ & $46 \pm 50$ & $30 \pm 64$ & $23 \pm 98$ & $237 \pm 52$ & $238 \pm 21$ \\
\hline
\end{tabular}


Appendix B20. Table showing return values of ensemble-average wind speeds of hindcast and forecast scenarios, including lower and higher 95 percent confidence intervals, at the Pohnpei location.

[Years: Hindcast $=1976-2005 ;$ RCP mid $=$ 2026-2045; RCP end $=$ 2081-2100. Wind speeds are in meters per second]

\begin{tabular}{|c|c|c|c|c|c|c|c|c|c|c|c|c|c|c|c|}
\hline \multirow{2}{*}{ Scenario } & \multicolumn{3}{|c|}{ Hindcast } & \multicolumn{3}{c|}{ RCP4.5 - mid } & \multicolumn{3}{c|}{ RCP8.5 - mid } & \multicolumn{3}{c|}{ RCP4.5 - end } & \multicolumn{2}{c|}{ RCP8.5 - end } \\
\cline { 2 - 13 } & Low & $\boldsymbol{R}_{\boldsymbol{V}}$ & High & Low & $\boldsymbol{R}_{\boldsymbol{V}}$ & High & Low & $\boldsymbol{R}_{\boldsymbol{V}}$ & High & Low & $\boldsymbol{R}_{\boldsymbol{V}}$ & High & Low & $\boldsymbol{R}_{\boldsymbol{V}}$ & High \\
\hline 2-year & 17.09 & 18.67 & 21.14 & 17.24 & 19.07 & 22.05 & 16.66 & 18.63 & 22.34 & 16.50 & 18.44 & 21.85 & 17.26 & 18.87 & 21.44 \\
5-year & 17.92 & 20.32 & 24.40 & 18.09 & 20.85 & 25.80 & 17.38 & 20.54 & 27.35 & 17.16 & 20.03 & 25.68 & 17.92 & 20.23 & 24.23 \\
10-year & 18.44 & 21.49 & 27.03 & 18.61 & 22.12 & 28.89 & 17.85 & 22.02 & 32.06 & 17.54 & 21.12 & 28.81 & 18.30 & 21.12 & 26.32 \\
20-year & 18.87 & 22.59 & 29.80 & 19.05 & 23.33 & 32.20 & 18.26 & 23.54 & 37.75 & 17.84 & 22.13 & 32.15 & 18.60 & 21.90 & 28.39 \\
50-year & 19.35 & 23.94 & 33.71 & 19.53 & 24.84 & 36.96 & 18.72 & 25.61 & 47.13 & 18.15 & 23.34 & 36.94 & 18.89 & 22.80 & 31.09 \\
100-year & 19.64 & 24.90 & 36.86 & 19.83 & 25.91 & 40.87 & 19.01 & 27.21 & 55.95 & 18.33 & 24.17 & 40.84 & 19.06 & 23.38 & 33.11 \\
\hline
\end{tabular}


Appendix B21. Table showing Yap monthly means and mean of the top 5 percent for wind speed and mean wind direction.

[Years: Hindcast $=1976-2005 ;$ RCP mid $=$ 2026-2045; RCP end $=$ 2081-2100. Wind directions are “coming from”]

\begin{tabular}{|c|c|c|c|c|c|c|c|c|c|c|c|c|}
\hline Month & Jan & Feb & Mar & Apr & May & Jun & July & Aug & Sep & Oct & Nov & Dec \\
\hline \multicolumn{13}{|c|}{$\begin{array}{l}\text { Mean Wind Speed } \\
(\mathrm{m} / \mathrm{s})\end{array}$} \\
\hline Hindcast & $8.6 \pm 2.4$ & $8.9 \pm 2.2$ & $8.2 \pm 2.1$ & $7.1 \pm 2.1$ & $5.5 \pm 2.3$ & $4.6 \pm 2.5$ & $4.5 \pm 2.9$ & $4.6 \pm 3.1$ & $4.4 \pm 2.8$ & $4.6 \pm 2.6$ & $6.0 \pm 3.0$ & $7.8 \pm 2.7$ \\
\hline RCP4.5 - mid & $8.3 \pm 2.5$ & $8.6 \pm 2.2$ & $8.3 \pm 2.1$ & $7.2 \pm 2.0$ & $5.5 \pm 2.3$ & $4.7 \pm 2.6$ & $4.7 \pm 3.1$ & $4.5 \pm 2.7$ & $4.4 \pm 2.6$ & $4.7 \pm 2.7$ & $5.7 \pm 2.8$ & $7.5 \pm 2.7$ \\
\hline RCP8.5 - mid & $8.5 \pm 2.3$ & $8.5 \pm 2.1$ & $8.2 \pm 2.1$ & $7.2 \pm 1.9$ & $5.4 \pm 2.3$ & $4.8 \pm 2.7$ & $4.6 \pm 2.9$ & $4.7 \pm 3.0$ & $4.4 \pm 2.8$ & $4.4 \pm 2.4$ & $5.4 \pm 2.7$ & $7.5 \pm 2.8$ \\
\hline RCP4.5 - end & $8.4 \pm 2.4$ & $8.7 \pm 2.0$ & $8.1 \pm 2.0$ & $7.0 \pm 2.1$ & $5.6 \pm 2.2$ & $4.7 \pm 2.6$ & $4.9 \pm 2.9$ & $4.8 \pm 3.0$ & $4.5 \pm 2.8$ & $4.4 \pm 2.5$ & $5.5 \pm 2.6$ & $7.3 \pm 2.6$ \\
\hline RCP8.5 - end & $8.2 \pm 2.3$ & $8.3 \pm 2.0$ & $7.9 \pm 1.9$ & $7.1 \pm 1.7$ & $5.6 \pm 2.3$ & $4.8 \pm 2.5$ & $4.8 \pm 2.9$ & $4.8 \pm 3.0$ & $4.6 \pm 2.7$ & $4.2 \pm 2.3$ & $5.3 \pm 2.8$ & $7.2 \pm 2.6$ \\
\hline \multicolumn{13}{|c|}{$\begin{array}{l}\text { Top } 5 \text { percent Wind } \\
\text { Speed }(\mathrm{m} / \mathrm{s})\end{array}$} \\
\hline Hindcast & $13.2 \pm 1.2$ & $13.4 \pm 1.3$ & $12.3 \pm 1.4$ & $11.0 \pm 0.9$ & $10.3 \pm 1.4$ & $10.8 \pm 2.0$ & $12.5 \pm 2.1$ & $13.4 \pm 1.9$ & $12.5 \pm 2.1$ & $11.4 \pm 1.8$ & $12.8 \pm 2.2$ & $13.1 \pm 1.6$ \\
\hline RCP4.5 - mid & $12.8 \pm 1.0$ & $12.6 \pm 1.2$ & $12.2 \pm 1.3$ & $10.9 \pm 1.0$ & $10.2 \pm 1.3$ & $11.1 \pm 2.2$ & $13.4 \pm 1.9$ & $11.8 \pm 2.3$ & $11.9 \pm 2.5$ & $12.1 \pm 2.2$ & $12.3 \pm 1.9$ & $13.1 \pm 1.6$ \\
\hline RCP8.5 - mid & $13.1 \pm 1.2$ & $12.5 \pm 0.8$ & $12.5 \pm 1.4$ & $11.0 \pm 1.4$ & $10.0 \pm 1.3$ & $12.2 \pm 2.6$ & $12.5 \pm 1.7$ & $13.1 \pm 2.2$ & $12.5 \pm 2.3$ & $10.8 \pm 1.9$ & $11.5 \pm 1.6$ & $13.0 \pm 1.6$ \\
\hline RCP4.5 - end & $13.2 \pm 1.4$ & $12.4 \pm 1.0$ & $12.0 \pm 1.3$ & $11.1 \pm 1.4$ & $10.1 \pm 1.4$ & $10.8 \pm 1.9$ & $12.7 \pm 2.1$ & $12.9 \pm 1.9$ & $12.4 \pm 2.1$ & $11.5 \pm 2.5$ & $11.5 \pm 1.8$ & $12.6 \pm 1.4$ \\
\hline RCP8.5 - end & $12.8 \pm 1.2$ & $12.4 \pm 0.9$ & $11.7 \pm 0.9$ & $10.5 \pm 1.1$ & $10.0 \pm 1.3$ & $10.7 \pm 1.9$ & $12.7 \pm 2.5$ & $13.3 \pm 2.1$ & $12.1 \pm 2.7$ & $10.4 \pm 2.1$ & $12.1 \pm 2.1$ & $12.5 \pm 1.7$ \\
\hline \multicolumn{13}{|l|}{$\begin{array}{l}\text { Mean Wind } \\
\text { Direction }(9)\end{array}$} \\
\hline Hindcast & $246 \pm 24$ & $243 \pm 22$ & $246 \pm 25$ & $250 \pm 29$ & $259 \pm 49$ & $276 \pm 83$ & $345 \pm 93$ & $10 \pm 85$ & $14 \pm 95$ & $308 \pm 107$ & $260 \pm 63$ & $251 \pm 33$ \\
\hline RCP4.5 - mid & $247 \pm 28$ & $245 \pm 21$ & $246 \pm 22$ & $251 \pm 24$ & $257 \pm 46$ & $279 \pm 83$ & $346 \pm 95$ & $358 \pm 87$ & $5 \pm 93$ & $339 \pm 108$ & $257 \pm 65$ & $252 \pm 37$ \\
\hline RCP8.5 - mid & $247 \pm 23$ & $246 \pm 21$ & $247 \pm 23$ & $252 \pm 25$ & $258 \pm 44$ & $283 \pm 86$ & $349 \pm 93$ & $8 \pm 86$ & $8 \pm 91$ & $345 \pm 115$ & $260 \pm 70$ & $252 \pm 38$ \\
\hline RCP4.5 - end & $249 \pm 23$ & $245 \pm 19$ & $247 \pm 21$ & $252 \pm 26$ & $260 \pm 43$ & $275 \pm 84$ & $343 \pm 95$ & $5 \pm 86$ & $6 \pm 89$ & $352 \pm 112$ & $259 \pm 65$ & $253 \pm 35$ \\
\hline RCP8.5 - end & $248 \pm 25$ & $246 \pm 21$ & $248 \pm 19$ & $251 \pm 20$ & $258 \pm 47$ & $277 \pm 86$ & $328 \pm 91$ & $359 \pm 86$ & $8 \pm 87$ & $320 \pm 103$ & $262 \pm 64$ & $255 \pm 35$ \\
\hline \multicolumn{13}{|c|}{$\begin{array}{l}\text { Top } 5 \text { percent Wind } \\
\text { Direction }(9)\end{array}$} \\
\hline Hindcast & $237 \pm 22$ & $233 \pm 27$ & $236 \pm 35$ & $249 \pm 38$ & $253 \pm 63$ & $12 \pm 110$ & $44 \pm 44$ & $34 \pm 38$ & $48 \pm 51$ & $45 \pm 74$ & $285 \pm 92$ & $248 \pm 41$ \\
\hline RCP4.5 - mid & $239 \pm 21$ & $238 \pm 17$ & $244 \pm 32$ & $248 \pm 16$ & $252 \pm 42$ & $341 \pm 84$ & $36 \pm 42$ & $38 \pm 47$ & $31 \pm 48$ & $21 \pm 79$ & $249 \pm 80$ & $249 \pm 50$ \\
\hline RCP8.5 - mid & $238 \pm 29$ & $242 \pm 24$ & $243 \pm 33$ & $245 \pm 40$ & $249 \pm 41$ & $22 \pm 67$ & $38 \pm 44$ & $35 \pm 30$ & $38 \pm 40$ & $51 \pm 77$ & $252 \pm 83$ & $249 \pm 46$ \\
\hline RCP4.5 - end & $245 \pm 32$ & $236 \pm 17$ & $240 \pm 19$ & $253 \pm 44$ & $257 \pm 47$ & $335 \pm 92$ & $37 \pm 45$ & $40 \pm 32$ & $38 \pm 43$ & $38 \pm 72$ & $247 \pm 77$ & $242 \pm 34$ \\
\hline RCP8.5 - end & $238 \pm 32$ & $237 \pm 22$ & $242 \pm 19$ & $246 \pm 20$ & $255 \pm 31$ & $336 \pm 90$ & $25 \pm 32$ & $33 \pm 36$ & $35 \pm 45$ & $32 \pm 77$ & $282 \pm 93$ & $253 \pm 44$ \\
\hline
\end{tabular}


Appendix B22. Table showing return values of ensemble-average wind speeds of hindcast and forecast scenarios, including lower and higher 95 percent confidence intervals, at the Yap location.

[Years: Hindcast $=1976-2005 ;$ RCP mid $=$ 2026-2045; RCP end $=$ 2081-2100. Wind speeds are in meters per second]

\begin{tabular}{|c|c|c|c|c|c|c|c|c|c|c|c|c|c|c|c|c|}
\hline \multirow{2}{*}{ Scenario } & \multicolumn{3}{|c|}{ Hindcast } & \multicolumn{3}{c|}{ RCP4.5 - mid } & \multicolumn{3}{c|}{ RCP8.5 - mid } & \multicolumn{3}{c|}{ RCP4.5 - end } & \multicolumn{2}{c|}{ RCP8.5 - end } \\
\cline { 2 - 13 } & Low & $\boldsymbol{R}_{\boldsymbol{V}}$ & High & Low & $\boldsymbol{R}_{\boldsymbol{V}}$ & High & Low & $\boldsymbol{R}_{\boldsymbol{V}}$ & High & Low & $\boldsymbol{R}_{\boldsymbol{V}}$ & High & Low & $\boldsymbol{R}_{\boldsymbol{V}}$ & High \\
\hline 2-year & 18.96 & 20.51 & 22.80 & 18.72 & 20.38 & 22.89 & 18.46 & 20.32 & 23.40 & 18.17 & 19.98 & 23.03 & 18.83 & 20.67 & 23.60 \\
5-year & 20.01 & 22.41 & 26.26 & 19.40 & 21.68 & 25.37 & 19.04 & 21.62 & 26.31 & 18.83 & 21.46 & 26.33 & 19.45 & 21.96 & 26.29 \\
10-year & 20.69 & 23.81 & 29.10 & 19.77 & 22.46 & 27.06 & 19.35 & 22.42 & 28.41 & 19.20 & 22.44 & 28.90 & 19.77 & 22.72 & 28.13 \\
20-year & 21.30 & 25.17 & 32.16 & 20.04 & 23.11 & 28.60 & 19.57 & 23.09 & 30.42 & 19.50 & 23.32 & 31.53 & 20.00 & 23.35 & 29.83 \\
50-year & 22.01 & 26.93 & 36.57 & 20.30 & 23.80 & 30.44 & 19.78 & 23.81 & 32.95 & 19.79 & 24.34 & 35.11 & 20.20 & 24.00 & 31.87 \\
100-year & 22.47 & 28.23 & 40.20 & 20.44 & 24.22 & 31.70 & 19.89 & 24.25 & 34.77 & 19.96 & 25.03 & 37.90 & 20.31 & 24.39 & 33.27 \\
\hline
\end{tabular}


Appendix B23. Table showing Majuro monthly means and mean of the top 5 percent for wind speed and mean wind direction.

[Years: Hindcast $=1976-2005 ;$ RCP mid $=$ 2026-2045; RCP end = 2081-2100. Wind directions are "coming from”]

\begin{tabular}{|c|c|c|c|c|c|c|c|c|c|c|c|c|}
\hline Month & Jan & Feb & Mar & Apr & May & Jun & July & Aug & Sep & Oct & Nov & Dec \\
\hline \multicolumn{13}{|c|}{$\begin{array}{l}\text { Mean Wind Speed } \\
(\mathrm{m} / \mathrm{s})\end{array}$} \\
\hline Hindcast & $8.7 \pm 1.9$ & $8.6 \pm 1.9$ & $8.1 \pm 2.1$ & $6.8 \pm 2.4$ & $5.2 \pm 2.5$ & $4.8 \pm 2.4$ & $4.5 \pm 2.2$ & $4.3 \pm 2.1$ & $4.1 \pm 2.0$ & $4.2 \pm 2.3$ & $5.2 \pm 2.7$ & $7.6 \pm 2.8$ \\
\hline RCP4.5 - mid & $8.6 \pm 1.9$ & $8.5 \pm 1.9$ & $8.3 \pm 2.0$ & $6.9 \pm 2.3$ & $5.6 \pm 2.5$ & $5.1 \pm 2.5$ & $4.7 \pm 2.3$ & $4.3 \pm 2.2$ & $4 \pm 1.9$ & $4.2 \pm 2.2$ & $4.9 \pm 2.6$ & $7.4 \pm 2.7$ \\
\hline RCP8.5 - mid & $8.6 \pm 1.9$ & $8.6 \pm 2.0$ & $8.1 \pm 2.0$ & $7.2 \pm 2.3$ & $5.6 \pm 2.5$ & $4.9 \pm 2.4$ & $4.7 \pm 2.3$ & $4.3 \pm 2.1$ & $4.1 \pm 2.0$ & $4.1 \pm 2.0$ & $5.0 \pm 2.7$ & $7.2 \pm 2.7$ \\
\hline RCP4.5 - end & $8.4 \pm 1.8$ & $8.4 \pm 1.8$ & $8.1 \pm 1.9$ & $6.9 \pm 2.3$ & $5.7 \pm 2.5$ & $5.1 \pm 2.5$ & $4.6 \pm 2.2$ & $4.4 \pm 2.1$ & $4.2 \pm 2.1$ & $4.1 \pm 2.2$ & $4.9 \pm 2.5$ & $7.1 \pm 2.6$ \\
\hline RCP8.5 - end & $8.3 \pm 1.8$ & $8.4 \pm 1.8$ & $8.1 \pm 1.7$ & $7.3 \pm 2.2$ & $6.1 \pm 2.5$ & $5.3 \pm 2.5$ & $4.9 \pm 2.3$ & $4.4 \pm 2.0$ & $4.1 \pm 1.9$ & $4.0 \pm 2.2$ & $4.8 \pm 2.5$ & $7.2 \pm 2.5$ \\
\hline \multicolumn{13}{|c|}{$\begin{array}{l}\text { Top } 5 \text { percent Wind } \\
\text { Speed }(\mathrm{m} / \mathrm{s})\end{array}$} \\
\hline Hindcast & $12.3 \pm 0.7$ & $12.2 \pm 0.6$ & $12.0 \pm 0.7$ & $11.3 \pm 1.0$ & $10.2 \pm 1.1$ & $10.0 \pm 0.9$ & $9.9 \pm 1.4$ & $9.8 \pm 1.8$ & $9.3 \pm 1.6$ & $10.3 \pm 2.1$ & $11.6 \pm 1.4$ & $12.7 \pm 1.4$ \\
\hline RCP4.5 - mid & $12.2 \pm 0.6$ & $12.1 \pm 0.9$ & $11.8 \pm 0.7$ & $11.1 \pm 0.6$ & $10.5 \pm 0.8$ & $10.5 \pm 1.1$ & $10.1 \pm 1.2$ & $10.1 \pm 1.9$ & $8.8 \pm 1.7$ & $9.9 \pm 2.0$ & $11.2 \pm 1.6$ & $12.5 \pm 0.9$ \\
\hline RCP8.5 - mid & $12.2 \pm 0.6$ & $12.5 \pm 0.7$ & $11.9 \pm 0.7$ & $11.8 \pm 1.4$ & $10.6 \pm 0.9$ & $10.2 \pm 1.0$ & $10.4 \pm 1.7$ & $10.0 \pm 1.8$ & $9.7 \pm 2.3$ & $9.4 \pm 1.7$ & $11.9 \pm 2.0$ & $12.1 \pm 0.7$ \\
\hline RCP4.5 - end & $11.9 \pm 0.8$ & $11.9 \pm 0.7$ & $11.7 \pm 0.8$ & $11.1 \pm 0.7$ & $10.6 \pm 1.0$ & $10.4 \pm 1.2$ & $10.1 \pm 1.9$ & $10.2 \pm 2.1$ & $9.9 \pm 1.9$ & $10.3 \pm 2.0$ & $11.1 \pm 2.1$ & $12.5 \pm 1.1$ \\
\hline RCP8.5 - end & $11.6 \pm 0.6$ & $11.8 \pm 0.7$ & $11.6 \pm 0.7$ & $11.2 \pm 0.8$ & $10.8 \pm 0.8$ & $10.6 \pm 1.1$ & $10.3 \pm 1.6$ & $9.6 \pm 2.0$ & $9.2 \pm 1.9$ & $10.1 \pm 2.1$ & $10.8 \pm 1.3$ & $11.7 \pm 0.9$ \\
\hline \multicolumn{13}{|l|}{$\begin{array}{l}\text { Mean Wind } \\
\text { Direction }(9)\end{array}$} \\
\hline Hindcast & $242 \pm 16$ & $243 \pm 14$ & $246 \pm 17$ & $250 \pm 25$ & $256 \pm 45$ & $263 \pm 53$ & $278 \pm 56$ & $296 \pm 58$ & $308 \pm 65$ & $302 \pm 70$ & $262 \pm 58$ & $246 \pm 31$ \\
\hline RCP4.5 - mid & $244 \pm 16$ & $244 \pm 15$ & $244 \pm 14$ & $250 \pm 22$ & $253 \pm 36$ & $261 \pm 49$ & $273 \pm 51$ & $295 \pm 56$ & $302 \pm 61$ & $305 \pm 67$ & $267 \pm 62$ & $250 \pm 32$ \\
\hline RCP8.5 - mid & $244 \pm 16$ & $243 \pm 14$ & $245 \pm 15$ & $249 \pm 23$ & $253 \pm 37$ & $261 \pm 50$ & $277 \pm 51$ & $296 \pm 57$ & $308 \pm 62$ & $305 \pm 70$ & $266 \pm 64$ & $249 \pm 31$ \\
\hline RCP4.5 - end & $245 \pm 16$ & $245 \pm 14$ & $246 \pm 15$ & $250 \pm 22$ & $253 \pm 38$ & $260 \pm 51$ & $273 \pm 52$ & $297 \pm 58$ & $307 \pm 63$ & $310 \pm 73$ & $270 \pm 62$ & $250 \pm 31$ \\
\hline RCP8.5 - end & $246 \pm 15$ & $246 \pm 13$ & $246 \pm 13$ & $249 \pm 19$ & $252 \pm 29$ & $258 \pm 45$ & $270 \pm 51$ & $289 \pm 55$ & $303 \pm 61$ & $301 \pm 67$ & $266 \pm 54$ & $251 \pm 28$ \\
\hline \multicolumn{13}{|c|}{$\begin{array}{l}\text { Top } 5 \text { percent Wind } \\
\text { Direction }(9)\end{array}$} \\
\hline Hindcast & $235 \pm 11$ & $236 \pm 9$ & $238 \pm 15$ & $239 \pm 20$ & $243 \pm 31$ & $244 \pm 43$ & $262 \pm 77$ & $349 \pm 99$ & $21 \pm 100$ & $335 \pm 89$ & $246 \pm 47$ & $235 \pm 24$ \\
\hline RCP4.5 - mid & $237 \pm 10$ & $239 \pm 17$ & $236 \pm 9$ & $240 \pm 13$ & $238 \pm 23$ & $243 \pm 45$ & $256 \pm 64$ & $325 \pm 82$ & $317 \pm 87$ & $359 \pm 86$ & $239 \pm 48$ & $236 \pm 15$ \\
\hline RCP8.5 - mid & $236 \pm 9$ & $236 \pm 10$ & $236 \pm 9$ & $239 \pm 30$ & $238 \pm 19$ & $244 \pm 42$ & $284 \pm 74$ & $337 \pm 98$ & $28 \pm 78$ & $14 \pm 91$ & $238 \pm 69$ & $234 \pm 14$ \\
\hline RCP4.5 - end & $235 \pm 14$ & $238 \pm 9$ & $238 \pm 16$ & $236 \pm 14$ & $237 \pm 23$ & $245 \pm 39$ & $269 \pm 64$ & $346 \pm 96$ & $19 \pm 78$ & $27 \pm 83$ & $246 \pm 75$ & $234 \pm 28$ \\
\hline RCP8.5 - end & $239 \pm 10$ & $239 \pm 11$ & $238 \pm 10$ & $238 \pm 19$ & $238 \pm 15$ & $241 \pm 35$ & $251 \pm 77$ & $321 \pm 90$ & $356 \pm 113$ & $5 \pm 101$ & $249 \pm 50$ & $236 \pm 14$ \\
\hline
\end{tabular}


Appendix B24. Table showing return values of ensemble-average wind speeds of hindcast and forecast scenarios, including lower and higher 95 percent confidence intervals, at the Majuro location.

[Years: Hindcast $=1976-2005 ;$ RCP $\mathrm{mid}=$ 2026-2045; RCP end $=$ 2081-2100. Wind speeds are in meters per second]

\begin{tabular}{|c|c|c|c|c|c|c|c|c|c|c|c|c|c|c|c|}
\hline \multirow{2}{*}{ Scenario } & \multicolumn{3}{|c|}{ Hindcast } & \multicolumn{3}{c|}{ RCP4.5 - mid } & \multicolumn{3}{c|}{ RCP8.5 - mid } & \multicolumn{3}{c|}{ RCP4.5 - end } & \multicolumn{2}{c|}{ RCP8.5 - end } \\
\cline { 2 - 13 } & Low & $\boldsymbol{R}_{\boldsymbol{V}}$ & High & Low & $\boldsymbol{R}_{\boldsymbol{V}}$ & High & Low & $\boldsymbol{R}_{\boldsymbol{V}}$ & High & Low & $\boldsymbol{R}_{\boldsymbol{V}}$ & High & Low & $\boldsymbol{R}_{\boldsymbol{V}}$ & High \\
\hline 2-year & 15.90 & 17.74 & 21.17 & 15.48 & 16.59 & 18.40 & 15.75 & 17.26 & 19.96 & 16.11 & 18.06 & 21.61 & 15.08 & 16.46 & 19.06 \\
5-year & 16.96 & 20.41 & 27.93 & 15.93 & 17.54 & 20.41 & 16.51 & 19.07 & 24.26 & 16.84 & 19.90 & 26.23 & 15.63 & 17.89 & 22.81 \\
10-year & 17.79 & 22.95 & 35.70 & 16.18 & 18.17 & 21.96 & 17.05 & 20.59 & 28.55 & 17.31 & 21.29 & 30.41 & 15.99 & 19.05 & 26.46 \\
20-year & 18.66 & 26.06 & 46.88 & 16.39 & 18.73 & 23.54 & 17.56 & 22.26 & 33.97 & 17.70 & 22.68 & 35.30 & 16.32 & 20.27 & 31.01 \\
50-year & 19.86 & 31.26 & 69.54 & 16.59 & 19.39 & 25.66 & 18.20 & 24.71 & 43.44 & 18.14 & 24.52 & 43.03 & 16.70 & 21.99 & 38.77 \\
100-year & 20.81 & 36.23 & 95.58 & 16.71 & 19.83 & 27.30 & 18.65 & 26.77 & 52.86 & 18.41 & 25.90 & 50.03 & 16.96 & 23.38 & 46.34 \\
\hline
\end{tabular}


Appendix B25. Table showing Enewetak monthly means and mean of the top 5 percent for wind speed and mean wind direction.

[Years: Hindcast $=1976-2005 ;$ RCP mid $=$ 2026-2045; RCP end $=$ 2081-2100. Wind directions are “coming from”]

\begin{tabular}{|c|c|c|c|c|c|c|c|c|c|c|c|c|}
\hline Month & Jan & Feb & Mar & Apr & May & Jun & July & Aug & Sep & Oct & Nov & Dec \\
\hline \multicolumn{13}{|c|}{$\begin{array}{l}\text { Mean Wind Speed } \\
(\mathrm{m} / \mathrm{s})\end{array}$} \\
\hline Hindcast & $9.4 \pm 1.8$ & $9.1 \pm 1.9$ & $9.0 \pm 1.8$ & $8.5 \pm 2.0$ & $7.1 \pm 2.5$ & $6.0 \pm 2.8$ & $5.4 \pm 2.9$ & $5.2 \pm 2.9$ & $5.0 \pm 2.6$ & $5.7 \pm 3.0$ & $8.0 \pm 2.8$ & $9.4 \pm 2.1$ \\
\hline RCP4.5 - mid & $9.4 \pm 1.9$ & $9.0 \pm 1.9$ & $9.0 \pm 1.8$ & $8.5 \pm 2.0$ & $7.3 \pm 2.3$ & $6.3 \pm 2.8$ & $5.5 \pm 3.0$ & $5.0 \pm 2.8$ & $4.9 \pm 2.7$ & $5.3 \pm 2.9$ & $7.5 \pm 2.9$ & $9.2 \pm 2.0$ \\
\hline RCP8.5 - mid & $9.3 \pm 1.9$ & $9.2 \pm 1.9$ & $8.9 \pm 1.8$ & $8.5 \pm 1.9$ & $7.4 \pm 2.3$ & $6.4 \pm 2.7$ & $5.6 \pm 2.9$ & $5.1 \pm 2.9$ & $4.8 \pm 2.7$ & $5.2 \pm 2.7$ & $7.3 \pm 3.0$ & $9.2 \pm 2.1$ \\
\hline RCP4.5 - end & $9.2 \pm 1.8$ & $9.1 \pm 1.8$ & $9.1 \pm 1.8$ & $8.4 \pm 2.0$ & $7.4 \pm 2.3$ & $6.4 \pm 2.8$ & $5.7 \pm 3.0$ & $5.5 \pm 3.0$ & $4.9 \pm 2.8$ & $5.3 \pm 2.7$ & $7.3 \pm 2.9$ & $9.2 \pm 2.0$ \\
\hline RCP8.5 - end & $9.0 \pm 1.9$ & $8.8 \pm 1.9$ & $8.7 \pm 1.7$ & $8.4 \pm 1.7$ & $7.6 \pm 2.2$ & $6.5 \pm 2.7$ & $5.7 \pm 3.0$ & $5.2 \pm 3.1$ & $4.7 \pm 2.7$ & $4.9 \pm 2.6$ & $7.0 \pm 2.9$ & $9.0 \pm 1.9$ \\
\hline \multicolumn{13}{|c|}{$\begin{array}{l}\text { Top } 5 \text { percent Wind } \\
\text { Speed }(\mathrm{m} / \mathrm{s})\end{array}$} \\
\hline Hindcast & $12.8 \pm 0.7$ & $12.7 \pm 0.7$ & $12.6 \pm 1.0$ & $12.3 \pm 1.4$ & $11.6 \pm 1.4$ & $11.1 \pm 1.3$ & $11.4 \pm 1.7$ & $12.4 \pm 2.5$ & $11.8 \pm 2.3$ & $12.7 \pm 1.8$ & $13.4 \pm 1.5$ & $13.3 \pm 1.0$ \\
\hline RCP4.5 - mid & $12.7 \pm 0.6$ & $12.6 \pm 0.7$ & $12.2 \pm 0.5$ & $12.2 \pm 1.1$ & $11.0 \pm 0.6$ & $11.2 \pm 1.5$ & $12.2 \pm 2.0$ & $12.0 \pm 2.2$ & $11.9 \pm 2.3$ & $12.6 \pm 2.4$ & $13.3 \pm 1.8$ & $13.2 \pm 1.1$ \\
\hline RCP8.5 - mid & $12.6 \pm 0.6$ & $12.9 \pm 0.7$ & $12.4 \pm 0.7$ & $12.1 \pm 1.5$ & $11.5 \pm 1.2$ & $11.2 \pm 1.1$ & $11.8 \pm 1.8$ & $12.2 \pm 2.7$ & $12.0 \pm 2.3$ & $12.1 \pm 2.1$ & $13.2 \pm 1.4$ & $13.1 \pm 0.9$ \\
\hline RCP 4.5 - end & $12.5 \pm 0.5$ & $12.5 \pm 0.7$ & $12.6 \pm 0.7$ & $11.9 \pm 1.2$ & $11.0 \pm 0.6$ & $11.3 \pm 1.6$ & $11.9 \pm 2.3$ & $13.2 \pm 2.3$ & $12.6 \pm 2.6$ & $12.1 \pm 2.1$ & $12.9 \pm 1.7$ & $13.1 \pm 1.3$ \\
\hline RCP8.5 - end & $12.4 \pm 0.6$ & $12.3 \pm 0.7$ & $12.3 \pm 1.1$ & $11.4 \pm 0.5$ & $11.1 \pm 1.2$ & $11.0 \pm 1.3$ & $12.2 \pm 2.3$ & $13.2 \pm 2.9$ & $11.7 \pm 2.6$ & $11.8 \pm 2.7$ & $13.0 \pm 2.1$ & $12.5 \pm 0.8$ \\
\hline \multicolumn{13}{|l|}{$\begin{array}{l}\text { Mean Wind } \\
\text { Direction ( } 9 \text { ) }\end{array}$} \\
\hline Hindcast & $246 \pm 12$ & $246 \pm 14$ & $248 \pm 13$ & $250 \pm 16$ & $254 \pm 29$ & $259 \pm 48$ & $268 \pm 71$ & $294 \pm 99$ & $294 \pm 111$ & $263 \pm 78$ & $252 \pm 34$ & $248 \pm 15$ \\
\hline RCP4.5 - mid & $248 \pm 13$ & $247 \pm 13$ & $248 \pm 12$ & $251 \pm 15$ & $254 \pm 24$ & $258 \pm 43$ & $270 \pm 67$ & $286 \pm 94$ & $301 \pm 102$ & $271 \pm 89$ & $254 \pm 40$ & $250 \pm 15$ \\
\hline RCP8.5 - mid & $247 \pm 11$ & $246 \pm 12$ & $248 \pm 11$ & $250 \pm 16$ & $254 \pm 22$ & $258 \pm 38$ & $268 \pm 67$ & $293 \pm 97$ & $312 \pm 109$ & $271 \pm 89$ & $253 \pm 39$ & $249 \pm 15$ \\
\hline RCP4.5 - end & $247 \pm 12$ & $246 \pm 13$ & $248 \pm 11$ & $252 \pm 15$ & $253 \pm 23$ & $258 \pm 38$ & $267 \pm 64$ & $290 \pm 95$ & $307 \pm 105$ & $274 \pm 92$ & $253 \pm 41$ & $250 \pm 15$ \\
\hline $\begin{array}{l}\text { RCP } 8.5 \text { - end } \\
\text { Top } 5 \text { percent } \\
\left.\text { Direction ( }{ }^{9}\right)\end{array}$ & $248 \pm 13$ & $248 \pm 13$ & $249 \pm 10$ & $251 \pm 11$ & $255 \pm 21$ & $258 \pm 38$ & $265 \pm 58$ & $285 \pm 81$ & $305 \pm 102$ & $271 \pm 89$ & $254 \pm 39$ & $250 \pm 14$ \\
\hline Hindcast & $244 \pm 9$ & $243 \pm 9$ & $242 \pm 11$ & $244 \pm 16$ & $254 \pm 24$ & $260 \pm 39$ & $273 \pm 60$ & $9 \pm 91$ & $11 \pm 95$ & $265 \pm 62$ & $250 \pm 30$ & $244 \pm 12$ \\
\hline RCP4.5 - mid & $245 \pm 8$ & $243 \pm 10$ & $244 \pm 7$ & $246 \pm 17$ & $250 \pm 14$ & $252 \pm 27$ & $311 \pm 83$ & $322 \pm 84$ & $336 \pm 79$ & $321 \pm 84$ & $249 \pm 28$ & $246 \pm 14$ \\
\hline RCP8.5 - mid & $245 \pm 8$ & $243 \pm 9$ & $245 \pm 9$ & $243 \pm 23$ & $250 \pm 22$ & $255 \pm 32$ & $288 \pm 78$ & $351 \pm 100$ & $36 \pm 75$ & $288 \pm 85$ & $247 \pm 32$ & $246 \pm 13$ \\
\hline RCP4.5 - end & $245 \pm 8$ & $243 \pm 10$ & $244 \pm 8$ & $250 \pm 16$ & $251 \pm 17$ & $259 \pm 27$ & $300 \pm 73$ & $33 \pm 69$ & $16 \pm 76$ & $308 \pm 101$ & $246 \pm 31$ & $245 \pm 16$ \\
\hline RCP8.5 - end & $245 \pm 14$ & $244 \pm 14$ & $246 \pm 12$ & $249 \pm 7$ & $252 \pm 16$ & $256 \pm 32$ & $289 \pm 70$ & $9 \pm 75$ & $11 \pm 70$ & $273 \pm 81$ & $250 \pm 25$ & $246 \pm 9$ \\
\hline
\end{tabular}


Appendix B26. Table showing return values of ensemble-average wind speeds of hindcast and forecast scenarios, including lower and higher 95 percent confidence intervals, at the Enewetak location.

[Years: Hindcast $=1976-2005 ;$ RCP mid $=$ 2026-2045; RCP end $=$ 2081-2100. Wind speeds are in meters per second]

\begin{tabular}{|c|c|c|c|c|c|c|c|c|c|c|c|c|c|c|c|}
\hline \multirow{2}{*}{ Scenario } & \multicolumn{3}{|c|}{ Hindcast } & \multicolumn{3}{c|}{ RCP4.5 - mid } & \multicolumn{3}{c|}{ RCP8.5 - mid } & \multicolumn{3}{c|}{ RCP4.5 - end } & \multicolumn{2}{c|}{ RCP8.5 - end } \\
\cline { 2 - 13 } & Low & $\boldsymbol{R}_{\boldsymbol{V}}$ & High & Low & $\boldsymbol{R}_{\boldsymbol{V}}$ & High & Low & $\boldsymbol{R}_{\boldsymbol{V}}$ & High & Low & $\boldsymbol{R}_{\boldsymbol{V}}$ & High & Low & $\boldsymbol{R}_{\boldsymbol{V}}$ & High \\
\hline 2-year & 18.19 & 19.96 & 22.81 & 18.27 & 20.17 & 23.31 & 17.46 & 19.63 & 23.74 & 18.26 & 20.28 & 23.64 & 18.51 & 21.32 & 26.33 \\
5-year & 18.95 & 21.57 & 26.18 & 18.87 & 21.50 & 26.22 & 18.23 & 21.69 & 29.26 & 19.05 & 22.01 & 27.45 & 19.25 & 23.16 & 31.01 \\
10-year & 19.40 & 22.65 & 28.82 & 19.19 & 22.30 & 28.28 & 18.73 & 23.30 & 34.47 & 19.51 & 23.19 & 30.46 & 19.63 & 24.32 & 34.53 \\
20-year & 19.76 & 23.64 & 31.55 & 19.42 & 22.97 & 30.24 & 19.16 & 24.95 & 40.78 & 19.88 & 24.26 & 33.59 & 19.90 & 25.30 & 38.04 \\
50-year & 20.12 & 24.80 & 35.27 & 19.63 & 23.68 & 32.66 & 19.64 & 27.18 & 51.22 & 20.25 & 25.54 & 37.92 & 20.15 & 26.38 & 42.65 \\
100-year & 20.33 & 25.59 & 38.19 & 19.73 & 24.12 & 34.38 & 19.95 & 28.92 & 61.06 & 20.47 & 26.40 & 41.35 & 20.28 & 27.05 & 46.13 \\
\hline
\end{tabular}


Appendix B27. Table showing Bikini monthly means and mean of the top 5 percent for wind speed and mean wind direction.

[Years: Hindcast $=1976-2005 ;$ RCP mid $=$ 2026-2045; RCP end = 2081-2100. Wind directions are "coming from”]

\begin{tabular}{|c|c|c|c|c|c|c|c|c|c|c|c|c|}
\hline Month & Jan & Feb & Mar & Apr & May & Jun & July & Aug & Sep & Oct & Nov & Dec \\
\hline \multicolumn{13}{|c|}{$\begin{array}{l}\text { Mean Wind Speed } \\
(\mathrm{m} / \mathrm{s})\end{array}$} \\
\hline Hindcast & $9.4 \pm 1.9$ & $9.1 \pm 1.9$ & $9.0 \pm 1.9$ & $8.5 \pm 2.1$ & $7.2 \pm 2.5$ & $6.2 \pm 2.8$ & $5.5 \pm 2.9$ & $5.2 \pm 2.9$ & $4.9 \pm 2.6$ & $5.7 \pm 3$ & $8.0 \pm 2.9$ & $9.4 \pm 2.1$ \\
\hline RCP4.5 - mid & $9.4 \pm 1.9$ & $9.1 \pm 1.9$ & $9.1 \pm 1.8$ & $8.6 \pm 2.0$ & $7.5 \pm 2.3$ & $6.4 \pm 2.8$ & $5.6 \pm 3.0$ & $5.1 \pm 2.8$ & $4.8 \pm 2.6$ & $5.3 \pm 2.8$ & $7.5 \pm 2.9$ & $9.2 \pm 2.1$ \\
\hline RCP8.5 - mid & $9.2 \pm 1.9$ & $9.2 \pm 2.0$ & $9.0 \pm 1.8$ & $8.6 \pm 1.9$ & $7.5 \pm 2.3$ & $6.6 \pm 2.8$ & $5.7 \pm 2.9$ & $5.1 \pm 2.9$ & $4.8 \pm 2.6$ & $5.3 \pm 2.7$ & $7.3 \pm 3.0$ & $9.2 \pm 2.1$ \\
\hline RCP4.5 - end & $9.2 \pm 1.8$ & $9.1 \pm 1.8$ & $9.1 \pm 1.8$ & $8.4 \pm 2.0$ & $7.5 \pm 2.3$ & $6.6 \pm 2.7$ & $5.7 \pm 2.9$ & $5.5 \pm 3.0$ & $4.9 \pm 2.7$ & $5.3 \pm 2.7$ & $7.3 \pm 3.0$ & $9.2 \pm 2.1$ \\
\hline RCP8.5 - end & $8.9 \pm 1.9$ & $8.8 \pm 1.9$ & $8.8 \pm 1.7$ & $8.5 \pm 1.7$ & $7.7 \pm 2.1$ & $6.7 \pm 2.6$ & $5.9 \pm 2.9$ & $5.3 \pm 3.1$ & $4.6 \pm 2.6$ & $4.9 \pm 2.6$ & $7.0 \pm 3.0$ & $9.0 \pm 1.9$ \\
\hline \multicolumn{13}{|c|}{$\begin{array}{l}\text { Top } 5 \text { percent Wind } \\
\text { Speed }(\mathrm{m} / \mathrm{s})\end{array}$} \\
\hline Hindcast & $12.9 \pm 0.7$ & $12.8 \pm 0.7$ & $12.7 \pm 1.2$ & $12.5 \pm 1.9$ & $11.4 \pm 0.8$ & $11.1 \pm 1.1$ & $11.1 \pm 1.5$ & $12.0 \pm 2.4$ & $11.3 \pm 2.1$ & $12.7 \pm 1.8$ & $13.5 \pm 1.5$ & $13.4 \pm 1.1$ \\
\hline RCP4.5 - mid & $12.7 \pm 0.5$ & $12.8 \pm 1.0$ & $12.3 \pm 0.7$ & $12.3 \pm 1.1$ & $11.2 \pm 0.8$ & $11.3 \pm 1.4$ & $11.7 \pm 1.8$ & $12.0 \pm 2.1$ & $11.3 \pm 2.2$ & $12.5 \pm 2.3$ & $13.2 \pm 1.5$ & $13.2 \pm 0.9$ \\
\hline RCP8.5 - mid & $12.7 \pm 0.6$ & $12.9 \pm 0.7$ & $12.4 \pm 0.6$ & $12.4 \pm 1.9$ & $11.4 \pm 1.6$ & $11.4 \pm 1.2$ & $11.7 \pm 1.5$ & $12.1 \pm 2.7$ & $11.7 \pm 2.2$ & $11.8 \pm 2.0$ & $13.3 \pm 1.5$ & $12.9 \pm 0.7$ \\
\hline RCP4.5 - end & $12.6 \pm 0.6$ & $12.6 \pm 0.7$ & $12.7 \pm 0.7$ & $11.9 \pm 0.6$ & $11.2 \pm 0.9$ & $11.2 \pm 1.5$ & $11.8 \pm 2.0$ & $13.0 \pm 2.3$ & $12.5 \pm 2.5$ & $12.0 \pm 2.0$ & $13.2 \pm 1.6$ & $13.1 \pm 1.0$ \\
\hline RCP8.5 - end & $12.3 \pm 0.6$ & $12.3 \pm 0.6$ & $12.2 \pm 0.7$ & $11.5 \pm 0.5$ & $11.0 \pm 0.7$ & $11.3 \pm 1.5$ & $11.9 \pm 1.7$ & $13.1 \pm 3.1$ & $11.2 \pm 2.9$ & $11.8 \pm 2.4$ & $13.0 \pm 1.8$ & $12.6 \pm 0.8$ \\
\hline \multicolumn{13}{|l|}{$\begin{array}{l}\text { Mean Wind } \\
\text { Direction ( } 9 \text { ) }\end{array}$} \\
\hline Hindcast & $246 \pm 12$ & $246 \pm 13$ & $248 \pm 12$ & $250 \pm 15$ & $254 \pm 28$ & $258 \pm 47$ & $265 \pm 67$ & $286 \pm 94$ & $287 \pm 108$ & $262 \pm 75$ & $251 \pm 34$ & $247 \pm 15$ \\
\hline RCP4.5 - mid & $247 \pm 13$ & $246 \pm 13$ & $247 \pm 12$ & $250 \pm 15$ & $253 \pm 23$ & $257 \pm 41$ & $267 \pm 61$ & $280 \pm 86$ & $291 \pm 98$ & $269 \pm 86$ & $252 \pm 38$ & $249 \pm 15$ \\
\hline RCP8.5 - mid & $246 \pm 11$ & $246 \pm 12$ & $248 \pm 11$ & $250 \pm 15$ & $253 \pm 21$ & $257 \pm 37$ & $266 \pm 62$ & $287 \pm 90$ & $299 \pm 106$ & $268 \pm 86$ & $252 \pm 39$ & $248 \pm 15$ \\
\hline RCP4.5 - end & $246 \pm 11$ & $246 \pm 12$ & $248 \pm 11$ & $251 \pm 15$ & $253 \pm 23$ & $257 \pm 37$ & $264 \pm 60$ & $282 \pm 89$ & $297 \pm 101$ & $270 \pm 91$ & $252 \pm 43$ & $249 \pm 15$ \\
\hline RCP8.5 - end & $248 \pm 13$ & $248 \pm 13$ & $249 \pm 10$ & $251 \pm 11$ & $254 \pm 20$ & $257 \pm 36$ & $263 \pm 52$ & $280 \pm 74$ & $293 \pm 98$ & $269 \pm 86$ & $253 \pm 39$ & $249 \pm 14$ \\
\hline \multicolumn{13}{|c|}{$\begin{array}{l}\text { Top } 5 \text { percent Wind } \\
\text { Direction ( } 9\end{array}$} \\
\hline Hindcast & $244 \pm 8$ & $244 \pm 8$ & $244 \pm 9$ & $247 \pm 23$ & $250 \pm 17$ & $256 \pm 29$ & $265 \pm 55$ & $339 \pm 89$ & $340 \pm 100$ & $265 \pm 59$ & $249 \pm 28$ & $243 \pm 12$ \\
\hline RCP4.5 - mid & $245 \pm 7$ & $243 \pm 10$ & $244 \pm 7$ & $248 \pm 13$ & $249 \pm 11$ & $252 \pm 26$ & $282 \pm 65$ & $312 \pm 77$ & $324 \pm 77$ & $295 \pm 74$ & $247 \pm 25$ & $246 \pm 11$ \\
\hline RCP8.5 - mid & $245 \pm 8$ & $244 \pm 8$ & $245 \pm 8$ & $248 \pm 27$ & $250 \pm 17$ & $254 \pm 29$ & $270 \pm 51$ & $334 \pm 87$ & $17 \pm 92$ & $275 \pm 74$ & $246 \pm 25$ & $245 \pm 10$ \\
\hline RCP4.5 - end & $245 \pm 8$ & $245 \pm 9$ & $244 \pm 11$ & $248 \pm 10$ & $249 \pm 18$ & $254 \pm 24$ & $279 \pm 62$ & $26 \pm 76$ & $2 \pm 81$ & $286 \pm 92$ & $246 \pm 31$ & $244 \pm 16$ \\
\hline RCP8.5 - end & $244 \pm 8$ & $244 \pm 8$ & $246 \pm 8$ & $247 \pm 7$ & $251 \pm 17$ & $256 \pm 29$ & $278 \pm 70$ & $348 \pm 75$ & $358 \pm 87$ & $272 \pm 67$ & $247 \pm 23$ & $245 \pm 9$ \\
\hline
\end{tabular}


Appendix B28. Table showing return values of ensemble-average wind speeds of hindcast and forecast scenarios, including lower and higher 95 percent confidence intervals, at the Bikini location.

[Years: Hindcast $=1976-2005 ;$ RCP mid $=$ 2026-2045; RCP end $=$ 2081-2100. Wind speeds are in meters per second]

\begin{tabular}{|c|c|c|c|c|c|c|c|c|c|c|c|c|c|c|c|}
\hline \multirow{2}{*}{ Scenario } & \multicolumn{3}{|c|}{ Hindcast } & \multicolumn{3}{c|}{ RCP4.5 - mid } & \multicolumn{3}{c|}{ RCP8.5 - mid } & \multicolumn{3}{c|}{ RCP4.5 - end } & \multicolumn{2}{c|}{ RCP8.5 - end } \\
\cline { 2 - 13 } & Low & $\boldsymbol{R}_{\boldsymbol{V}}$ & High & Low & $\boldsymbol{R}_{\boldsymbol{V}}$ & High & Low & $\boldsymbol{R}_{\boldsymbol{V}}$ & High & Low & $\boldsymbol{R}_{\boldsymbol{V}}$ & High & Low & $\boldsymbol{R}_{\boldsymbol{V}}$ & High \\
\hline 2-year & 17.81 & 19.69 & 22.82 & 17.76 & 19.39 & 21.89 & 17.66 & 19.86 & 23.85 & 17.72 & 19.62 & 22.93 & 17.81 & 20.54 & 25.90 \\
5-year & 18.86 & 21.95 & 27.70 & 18.30 & 20.46 & 24.01 & 18.65 & 22.29 & 29.79 & 18.29 & 20.98 & 26.23 & 18.68 & 23.00 & 32.82 \\
10-year & 19.57 & 23.75 & 32.26 & 18.57 & 21.06 & 25.37 & 19.33 & 24.26 & 35.55 & 18.60 & 21.86 & 28.75 & 19.22 & 24.88 & 39.34 \\
20-year & 20.23 & 25.65 & 37.71 & 18.76 & 21.53 & 26.56 & 19.95 & 26.36 & 42.67 & 18.83 & 22.63 & 31.30 & 19.68 & 26.78 & 47.21 \\
50-year & 21.02 & 28.30 & 46.61 & 18.92 & 21.99 & 27.90 & 20.69 & 29.34 & 54.75 & 19.04 & 23.49 & 34.72 & 20.18 & 29.32 & 60.20 \\
100-year & 21.57 & 30.42 & 54.90 & 19.00 & 22.26 & 28.76 & 21.19 & 31.77 & 66.44 & 19.16 & 24.04 & 37.34 & 20.49 & 31.27 & 72.43 \\
\hline
\end{tabular}


Appendix B29. Table showing Molokai monthly means and mean of the top 5 percent for wind speed and mean wind direction.

[Years: Hindcast $=1976-2005 ;$ RCP mid $=$ 2026-2045; RCP end = 2081-2100. Wind directions are "coming from”]

\begin{tabular}{|c|c|c|c|c|c|c|c|c|c|c|c|c|}
\hline Month & Jan & Feb & Mar & Apr & May & Jun & July & Aug & Sep & Oct & Nov & Dec \\
\hline \multicolumn{13}{|c|}{$\begin{array}{l}\text { Mean Wind Speed } \\
(\mathrm{m} / \mathrm{s})\end{array}$} \\
\hline Hindcast & $6.8 \pm 3.0$ & $6.7 \pm 3.1$ & $7.3 \pm 3.1$ & $7.8 \pm 2.7$ & $7.9 \pm 2.3$ & $8.5 \pm 1.9$ & $8.3 \pm 1.8$ & $8.1 \pm 2.1$ & $7.7 \pm 2.4$ & $7.9 \pm 2.8$ & $7.8 \pm 3.0$ & $7.1 \pm 3.1$ \\
\hline RCP4.5 - mid & $6.7 \pm 3.1$ & $6.9 \pm 3.1$ & $7.4 \pm 3.1$ & $8.2 \pm 2.8$ & $7.7 \pm 2.3$ & $8.2 \pm 1.9$ & $8.5 \pm 1.7$ & $8.0 \pm 1.9$ & $7.6 \pm 2.3$ & $7.9 \pm 2.7$ & $8.0 \pm 3.0$ & $7.1 \pm 3.1$ \\
\hline RCP8.5 - mid & $6.7 \pm 3.2$ & $6.8 \pm 3.1$ & $7.3 \pm 3.1$ & $7.9 \pm 2.6$ & $7.6 \pm 2.2$ & $8.0 \pm 2.0$ & $8.2 \pm 1.7$ & $8.0 \pm 2.0$ & $7.5 \pm 2.4$ & $7.8 \pm 2.8$ & $7.9 \pm 2.9$ & $7.3 \pm 3.0$ \\
\hline RCP4.5 - end & $6.3 \pm 3.0$ & $6.7 \pm 3.1$ & $7.4 \pm 3.0$ & $7.8 \pm 2.6$ & $7.8 \pm 2.2$ & $8.3 \pm 1.9$ & $8.3 \pm 1.8$ & $8.0 \pm 2.0$ & $7.6 \pm 2.4$ & $7.8 \pm 2.6$ & $7.9 \pm 2.9$ & $7.3 \pm 3.1$ \\
\hline RCP8.5 - end & $6.3 \pm 3.0$ & $6.4 \pm 3.1$ & $7.2 \pm 2.9$ & $7.9 \pm 2.6$ & $7.4 \pm 2.2$ & $7.9 \pm 1.8$ & $8.3 \pm 1.7$ & $7.8 \pm 1.9$ & $7.4 \pm 2.1$ & $7.8 \pm 2.5$ & $8.0 \pm 2.7$ & $6.9 \pm 3.1$ \\
\hline \multicolumn{13}{|c|}{$\begin{array}{l}\text { Top } 5 \text { percent Wind } \\
\text { Speed }(\mathrm{m} / \mathrm{s})\end{array}$} \\
\hline Hindcast & $13.1 \pm 1.2$ & $13.2 \pm 1.1$ & $13.6 \pm 1.1$ & $13.1 \pm 1.0$ & $12.4 \pm 0.7$ & $12.4 \pm 1.0$ & $12.0 \pm 0.9$ & $12.3 \pm 1.4$ & $12.3 \pm 1.0$ & $13.5 \pm 1.5$ & $13.7 \pm 1.1$ & $13.4 \pm 1.1$ \\
\hline RCP4.5 - mid & $13.2 \pm 1.2$ & $13.4 \pm 1.1$ & $13.0 \pm 0.9$ & $13.3 \pm 0.9$ & $12.6 \pm 1.1$ & $11.8 \pm 0.6$ & $12.1 \pm 1.1$ & $11.8 \pm 0.8$ & $12.5 \pm 1.1$ & $13.6 \pm 1.6$ & $13.7 \pm 1.2$ & $13.4 \pm 1.1$ \\
\hline RCP8.5 - mid & $13.6 \pm 1.1$ & $13.3 \pm 1.2$ & $13.6 \pm 1.1$ & $13.0 \pm 1.0$ & $12.0 \pm 0.7$ & $11.9 \pm 0.7$ & $11.8 \pm 1.0$ & $12.1 \pm 0.8$ & $12.1 \pm 0.9$ & $13.7 \pm 1.4$ & $13.3 \pm 0.8$ & $13.5 \pm 1.0$ \\
\hline RCP 4.5 - end & $12.5 \pm 1.0$ & $13.3 \pm 1.1$ & $13.1 \pm 0.7$ & $12.9 \pm 0.9$ & $12.2 \pm 0.7$ & $11.8 \pm 0.8$ & $12.1 \pm 1.2$ & $12.6 \pm 1.5$ & $12.7 \pm 1.4$ & $13.2 \pm 1.6$ & $13.2 \pm 0.9$ & $13.4 \pm 1.3$ \\
\hline RCP8.5 - end & $13.0 \pm 1.3$ & $13.3 \pm 1.2$ & $12.9 \pm 0.8$ & $12.6 \pm 0.7$ & $11.7 \pm 0.7$ & $11.5 \pm 0.7$ & $12.3 \pm 1.7$ & $12.0 \pm 1.7$ & $11.7 \pm 1.5$ & $12.8 \pm 1.4$ & $13.6 \pm 1.3$ & $13.4 \pm 1.1$ \\
\hline \multicolumn{13}{|l|}{$\begin{array}{l}\text { Mean Wind } \\
\text { Direction }(9)\end{array}$} \\
\hline Hindcast & $255 \pm 76$ & $247 \pm 79$ & $251 \pm 51$ & $254 \pm 34$ & $256 \pm 25$ & $259 \pm 15$ & $258 \pm 16$ & $259 \pm 22$ & $260 \pm 28$ & $258 \pm 32$ & $255 \pm 40$ & $258 \pm 57$ \\
\hline RCP4.5 - mid & $254 \pm 75$ & $253 \pm 69$ & $253 \pm 52$ & $253 \pm 32$ & $257 \pm 23$ & $259 \pm 16$ & $257 \pm 13$ & $258 \pm 21$ & $260 \pm 26$ & $257 \pm 33$ & $255 \pm 36$ & $255 \pm 53$ \\
\hline RCP8.5 - mid & $253 \pm 72$ & $249 \pm 73$ & $251 \pm 53$ & $252 \pm 30$ & $257 \pm 23$ & $259 \pm 15$ & $258 \pm 16$ & $259 \pm 23$ & $260 \pm 27$ & $256 \pm 35$ & $252 \pm 37$ & $256 \pm 54$ \\
\hline RCP4.5 - end & $260 \pm 77$ & $249 \pm 73$ & $252 \pm 50$ & $253 \pm 33$ & $257 \pm 21$ & $259 \pm 14$ & $258 \pm 17$ & $259 \pm 23$ & $259 \pm 30$ & $257 \pm 32$ & $253 \pm 37$ & $256 \pm 49$ \\
\hline $\begin{array}{l}\text { RCP } 8.5 \text { - end } \\
\text { Top } 5 \text { percent } \\
\left.\text { Direction ( }{ }^{9}\right)\end{array}$ & $258 \pm 69$ & $250 \pm 77$ & $252 \pm 50$ & $253 \pm 31$ & $258 \pm 23$ & $259 \pm 13$ & $258 \pm 14$ & $258 \pm 20$ & $259 \pm 27$ & $256 \pm 30$ & $255 \pm 33$ & $258 \pm 52$ \\
\hline Hindcast & $244 \pm 73$ & $244 \pm 59$ & $249 \pm 25$ & $251 \pm 17$ & $254 \pm 14$ & $258 \pm 13$ & $257 \pm 14$ & $263 \pm 26$ & $261 \pm 22$ & $255 \pm 27$ & $250 \pm 24$ & $251 \pm 38$ \\
\hline RCP4.5 - mid & $244 \pm 73$ & $249 \pm 42$ & $247 \pm 21$ & $249 \pm 19$ & $255 \pm 14$ & $257 \pm 11$ & $258 \pm 18$ & $258 \pm 25$ & $258 \pm 19$ & $256 \pm 32$ & $250 \pm 22$ & $246 \pm 42$ \\
\hline RCP8.5 - mid & $246 \pm 52$ & $240 \pm 47$ & $248 \pm 27$ & $250 \pm 13$ & $253 \pm 9$ & $255 \pm 8$ & $258 \pm 20$ & $260 \pm 34$ & $261 \pm 29$ & $254 \pm 31$ & $245 \pm 16$ & $248 \pm 40$ \\
\hline RCP4.5 - end & $256 \pm 67$ & $238 \pm 56$ & $247 \pm 25$ & $250 \pm 18$ & $254 \pm 10$ & $257 \pm 13$ & $256 \pm 19$ & $262 \pm 35$ & $262 \pm 37$ & $252 \pm 33$ & $248 \pm 24$ & $251 \pm 30$ \\
\hline RCP8.5 - end & $248 \pm 39$ & $244 \pm 47$ & $249 \pm 24$ & $251 \pm 11$ & $258 \pm 12$ & $257 \pm 9$ & $260 \pm 20$ & $255 \pm 21$ & $267 \pm 34$ & $257 \pm 32$ & $250 \pm 18$ & $252 \pm 27$ \\
\hline
\end{tabular}


Appendix B30. Table showing return values of ensemble-average wind speeds of hindcast and forecast scenarios, including lower and higher 95 percent confidence intervals, at the Molokai location.

[Years: Hindcast $=1976-2005 ;$ RCP mid $=$ 2026-2045; RCP end $=$ 2081-2100. Wind speeds are in meters per second]

\begin{tabular}{|c|c|c|c|c|c|c|c|c|c|c|c|c|c|c|c|}
\hline \multirow{2}{*}{ Scenario } & \multicolumn{3}{|c|}{ Hindcast } & \multicolumn{3}{c|}{ RCP4.5 - mid } & \multicolumn{3}{c|}{ RCP8.5 - mid } & \multicolumn{3}{c|}{ RCP4.5 - end } & \multicolumn{2}{c|}{ RCP8.5 - end } \\
\cline { 2 - 13 } & Low & $\boldsymbol{R}_{\boldsymbol{V}}$ & High & Low & $\boldsymbol{R}_{\boldsymbol{V}}$ & High & Low & $\boldsymbol{R}_{\boldsymbol{V}}$ & High & Low & $\boldsymbol{R}_{\boldsymbol{V}}$ & High & Low & $\boldsymbol{R}_{\boldsymbol{V}}$ & High \\
\hline 2-year & 17.66 & 18.80 & 20.56 & 17.57 & 18.87 & 21.03 & 16.89 & 18.04 & 20.24 & 17.01 & 18.48 & 21.21 & 17.30 & 18.64 & 20.87 \\
5-year & 18.41 & 20.25 & 23.36 & 18.26 & 20.35 & 24.16 & 17.25 & 19.01 & 22.86 & 17.49 & 19.75 & 24.57 & 17.83 & 19.80 & 23.40 \\
10-year & 18.92 & 21.37 & 25.84 & 18.73 & 21.49 & 26.97 & 17.46 & 19.72 & 25.19 & 17.79 & 20.68 & 27.56 & 18.14 & 20.59 & 25.39 \\
20-year & 19.39 & 22.53 & 28.66 & 19.15 & 22.66 & 30.21 & 17.63 & 20.40 & 27.88 & 18.03 & 21.59 & 31.00 & 18.39 & 21.31 & 27.45 \\
50-year & 19.96 & 24.10 & 33.03 & 19.65 & 24.25 & 35.29 & 17.82 & 21.27 & 32.05 & 18.29 & 22.76 & 36.37 & 18.65 & 22.16 & 30.29 \\
100-year & 20.35 & 25.32 & 36.88 & 19.99 & 25.49 & 39.84 & 17.93 & 21.90 & 35.76 & 18.44 & 23.63 & 41.15 & 18.80 & 22.74 & 32.53 \\
\hline
\end{tabular}


Appendix B31. Table showing Northwest Hawaiian Islands monthly means and mean of the top 5 percent for wind speed and mean wind direction.

[Years: Hindcast $=$ 1976-2005; RCP mid = 2026-2045; RCP end $=$ 2081-2100. Wind directions are “coming from”]

\begin{tabular}{|c|c|c|c|c|c|c|c|c|c|c|c|c|}
\hline Month & Jan & Feb & Mar & Apr & May & Jun & July & Aug & Sep & Oct & Nov & Dec \\
\hline \multicolumn{13}{|c|}{$\begin{array}{l}\text { Mean Wind Speed } \\
(\mathrm{m} / \mathrm{s})\end{array}$} \\
\hline Hindcast & $7.7 \pm 3.7$ & $7.5 \pm 3.5$ & $6.9 \pm 3.0$ & $7.1 \pm 2.8$ & $6.7 \pm 2.3$ & $7.0 \pm 2.2$ & $7.6 \pm 2.2$ & $7.3 \pm 2.4$ & $7.3 \pm 2.7$ & $7.7 \pm 3.1$ & $7.4 \pm 3.2$ & $7.4 \pm 3.5$ \\
\hline RCP4.5 - mid & $7.6 \pm 3.6$ & $7.3 \pm 3.3$ & $7.1 \pm 3.1$ & $7.2 \pm 2.8$ & $6.5 \pm 2.4$ & $6.8 \pm 2.2$ & $7.7 \pm 2.0$ & $7.3 \pm 2.3$ & $7.1 \pm 2.7$ & $7.7 \pm 3.0$ & $7.2 \pm 3.1$ & $7.1 \pm 3.4$ \\
\hline RCP8.5 - mid & $7.6 \pm 3.6$ & $7.5 \pm 3.3$ & $6.9 \pm 3.1$ & $6.7 \pm 2.6$ & $6.4 \pm 2.4$ & $6.5 \pm 2.2$ & $7.5 \pm 2.1$ & $7.3 \pm 2.4$ & $6.9 \pm 2.7$ & $8.0 \pm 3.0$ & $7.5 \pm 3.2$ & $7.1 \pm 3.4$ \\
\hline RCP4.5 - end & $7.5 \pm 3.5$ & $7.4 \pm 3.4$ & $7.1 \pm 3.0$ & $7.0 \pm 2.8$ & $6.7 \pm 2.4$ & $6.7 \pm 2.2$ & $7.4 \pm 2.0$ & $7.2 \pm 2.4$ & $7.1 \pm 2.4$ & $7.7 \pm 2.9$ & $7.3 \pm 3.1$ & $7.1 \pm 3.3$ \\
\hline RCP8.5 - end & $7.2 \pm 3.4$ & $7.2 \pm 3.2$ & $6.9 \pm 2.9$ & $6.9 \pm 2.6$ & $6.4 \pm 2.3$ & $6.6 \pm 2.0$ & $7.3 \pm 1.8$ & $6.9 \pm 2.1$ & $7.0 \pm 2.4$ & $7.7 \pm 2.7$ & $7.1 \pm 3.0$ & $6.8 \pm 3.3$ \\
\hline \multicolumn{13}{|c|}{$\begin{array}{l}\text { Top } 5 \text { percent Wind } \\
\text { Speed }(\mathrm{m} / \mathrm{s})\end{array}$} \\
\hline Hindcast & $16.4 \pm 1.9$ & $15.3 \pm 1.5$ & $13.4 \pm 1.2$ & $12.8 \pm 1.2$ & $11.3 \pm 1.0$ & $10.9 \pm 1.0$ & $11.9 \pm 1.4$ & $12.3 \pm 2.0$ & $13.4 \pm 1.6$ & $14.3 \pm 1.6$ & $14.1 \pm 1.3$ & $15.3 \pm 1.7$ \\
\hline RCP4.5 - mid & $16.1 \pm 1.7$ & $14.7 \pm 1.6$ & $13.8 \pm 1.4$ & $12.8 \pm 1.2$ & $11.1 \pm 1.0$ & $10.7 \pm 1.0$ & $11.7 \pm 1.5$ & $12.7 \pm 2.2$ & $13.0 \pm 2.1$ & $14.3 \pm 2.0$ & $14.2 \pm 1.6$ & $15.0 \pm 1.6$ \\
\hline RCP8.5 - mid & $15.7 \pm 1.8$ & $14.8 \pm 1.5$ & $13.6 \pm 1.4$ & $12.1 \pm 1.0$ & $11.3 \pm 1.2$ & $10.4 \pm 0.5$ & $11.6 \pm 1.6$ & $12.4 \pm 2.0$ & $13.0 \pm 2.2$ & $14.2 \pm 1.6$ & $14.2 \pm 1.3$ & $14.9 \pm 1.6$ \\
\hline RCP4.5 - end & $15.4 \pm 1.6$ & $15.3 \pm 1.6$ & $13.9 \pm 1.4$ & $12.9 \pm 1.1$ & $11.2 \pm 0.9$ & $10.6 \pm 0.8$ & $11.2 \pm 1.2$ & $12.5 \pm 2.5$ & $12.5 \pm 2.0$ & $14.2 \pm 1.7$ & $13.8 \pm 1.2$ & $14.7 \pm 1.8$ \\
\hline RCP8.5 - end & $15.1 \pm 1.8$ & $14.6 \pm 1.6$ & $12.9 \pm 1.4$ & $12.2 \pm 1.0$ & $10.9 \pm 0.9$ & $10.3 \pm 1.0$ & $10.6 \pm 0.9$ & $11.4 \pm 2.2$ & $12.5 \pm 1.8$ & $13.6 \pm 1.7$ & $13.8 \pm 1.3$ & $14.3 \pm 1.5$ \\
\hline \multicolumn{13}{|l|}{$\begin{array}{l}\text { Mean Wind } \\
\text { Direction ( } 9\end{array}$} \\
\hline Hindcast & $117 \pm 113$ & $119 \pm 118$ & $242 \pm 87$ & $248 \pm 49$ & $254 \pm 36$ & $262 \pm 27$ & $262 \pm 25$ & $265 \pm 31$ & $263 \pm 40$ & $253 \pm 46$ & $244 \pm 68$ & $207 \pm 114$ \\
\hline RCP4.5 - mid & $115 \pm 112$ & $137 \pm 134$ & $238 \pm 87$ & $251 \pm 47$ & $254 \pm 42$ & $261 \pm 31$ & $263 \pm 24$ & $266 \pm 29$ & $261 \pm 36$ & $253 \pm 39$ & $251 \pm 64$ & $217 \pm 117$ \\
\hline RCP8.5 - mid & $118 \pm 130$ & $145 \pm 128$ & $242 \pm 87$ & $251 \pm 49$ & $254 \pm 39$ & $261 \pm 30$ & $262 \pm 25$ & $266 \pm 32$ & $262 \pm 40$ & $252 \pm 40$ & $245 \pm 59$ & $222 \pm 107$ \\
\hline RCP4.5 - end & $108 \pm 108$ & $132 \pm 130$ & $238 \pm 84$ & $249 \pm 48$ & $254 \pm 37$ & $261 \pm 31$ & $262 \pm 23$ & $265 \pm 29$ & $261 \pm 35$ & $255 \pm 38$ & $249 \pm 59$ & $228 \pm 105$ \\
\hline $\begin{array}{l}\text { RCP8.5 - end } \\
\text { Top } 5 \text { percent } \\
\text { Direction ( }{ }^{9} \text { ) }\end{array}$ & $111 \pm 121$ & $122 \pm 118$ & $244 \pm 81$ & $252 \pm 44$ & $254 \pm 35$ & $262 \pm 27$ & $262 \pm 22$ & $264 \pm 27$ & $263 \pm 35$ & $256 \pm 36$ & $250 \pm 53$ & $224 \pm 101$ \\
\hline Hindcast & $103 \pm 62$ & $104 \pm 66$ & $220 \pm 76$ & $237 \pm 39$ & $247 \pm 20$ & $259 \pm 19$ & $265 \pm 25$ & $269 \pm 39$ & $266 \pm 36$ & $244 \pm 34$ & $227 \pm 55$ & $144 \pm 90$ \\
\hline RCP4.5 - mid & $108 \pm 65$ & $108 \pm 76$ & $216 \pm 71$ & $246 \pm 24$ & $246 \pm 24$ & $256 \pm 24$ & $265 \pm 33$ & $273 \pm 36$ & $259 \pm 42$ & $243 \pm 37$ & $240 \pm 55$ & $134 \pm 90$ \\
\hline RCP8.5 - mid & $106 \pm 76$ & $119 \pm 78$ & $216 \pm 75$ & $244 \pm 31$ & $245 \pm 24$ & $257 \pm 15$ & $262 \pm 30$ & $273 \pm 44$ & $256 \pm 35$ & $240 \pm 29$ & $234 \pm 43$ & $126 \pm 91$ \\
\hline RCP4.5 - end & $103 \pm 61$ & $114 \pm 66$ & $205 \pm 81$ & $239 \pm 31$ & $249 \pm 21$ & $255 \pm 14$ & $262 \pm 29$ & $277 \pm 46$ & $263 \pm 40$ & $249 \pm 30$ & $240 \pm 49$ & $167 \pm 99$ \\
\hline RCP8.5 - end & $94 \pm 57$ & $106 \pm 67$ & $219 \pm 68$ & $246 \pm 26$ & $246 \pm 21$ & $256 \pm 19$ & $258 \pm 23$ & $272 \pm 47$ & $264 \pm 39$ & $253 \pm 32$ & $236 \pm 35$ & $207 \pm 87$ \\
\hline
\end{tabular}


Appendix B32. Table showing return values of ensemble-average wind speeds of hindcast and forecast scenarios, including lower and higher 95 percent confidence intervals, at the Northwest Hawaiian Islands location.

[Years: Hindcast $=1976-2005 ;$ RCP mid $=$ 2026-2045; RCP end $=$ 2081-2100. Wind speeds are in meters per second]

\begin{tabular}{|c|c|c|c|c|c|c|c|c|c|c|c|c|c|c|c|}
\hline \multirow{2}{*}{ Scenario } & \multicolumn{3}{|c|}{ Hindcast } & \multicolumn{3}{c|}{ RCP4.5 - mid } & \multicolumn{3}{c|}{ RCP8.5 - mid } & \multicolumn{2}{|c|}{ RCP4.5 - end } & \multicolumn{3}{c|}{ RCP8.5 - end } \\
\cline { 2 - 13 } & Low & $\boldsymbol{R}_{\boldsymbol{V}}$ & High & Low & $\boldsymbol{R}_{\boldsymbol{V}}$ & High & Low & $\boldsymbol{R}_{\boldsymbol{V}}$ & High & Low & $\boldsymbol{R}_{\boldsymbol{V}}$ & High & Low & $\boldsymbol{R}_{\boldsymbol{V}}$ & High \\
\hline 2-year & 21.36 & 22.74 & 24.76 & 20.60 & 22.14 & 24.67 & 20.61 & 22.20 & 24.88 & 20.28 & 21.94 & 24.79 & 19.73 & 21.34 & 24.04 \\
5-year & 22.20 & 24.24 & 27.46 & 21.00 & 23.03 & 26.68 & 21.11 & 23.35 & 27.51 & 20.85 & 23.26 & 27.89 & 20.42 & 22.86 & 27.40 \\
10-year & 22.71 & 25.27 & 29.52 & 21.19 & 23.54 & 27.99 & 21.38 & 24.07 & 29.45 & 21.17 & 24.15 & 30.36 & 20.84 & 23.95 & 30.22 \\
20-year & 23.14 & 26.21 & 31.60 & 21.32 & 23.92 & 29.16 & 21.58 & 24.69 & 31.35 & 21.42 & 24.96 & 32.94 & 21.20 & 25.00 & 33.29 \\
50-year & 23.60 & 27.34 & 34.38 & 21.43 & 24.30 & 30.50 & 21.77 & 25.37 & 33.82 & 21.68 & 25.91 & 36.52 & 21.58 & 26.31 & 37.78 \\
100-year & 23.88 & 28.12 & 36.51 & 21.48 & 24.51 & 31.38 & 21.87 & 25.80 & 35.65 & 21.82 & 26.55 & 39.36 & 21.82 & 27.25 & 41.53 \\
\hline
\end{tabular}


Appendix B33. Table showing Guam monthly means and mean of the top 5 percent for wind speed and mean wind direction.

[Years: Hindcast $=$ 1976-2005; RCP mid $=$ 2026-2045; RCP end = 2081-2100. Wind directions are "coming from”]

\begin{tabular}{|c|c|c|c|c|c|c|c|c|c|c|c|c|}
\hline Month & Jan & Feb & Mar & Apr & May & Jun & July & Aug & Sep & Oct & Nov & Dec \\
\hline \multicolumn{13}{|c|}{$\begin{array}{l}\text { Mean Wind Speed } \\
(\mathrm{m} / \mathrm{s})\end{array}$} \\
\hline Hindcast & $9.1 \pm 2.1$ & $9.0 \pm 2.1$ & $8.8 \pm 2.0$ & $8.0 \pm 2.0$ & $6.8 \pm 2.4$ & $5.7 \pm 2.7$ & $5.5 \pm 3.2$ & $5.6 \pm 3.2$ & $5.3 \pm 2.9$ & $5.6 \pm 2.9$ & $7.8 \pm 2.8$ & $8.9 \pm 2.3$ \\
\hline RCP4.5 - mid & $9.0 \pm 2.2$ & $8.9 \pm 2.1$ & $8.8 \pm 2.0$ & $8.0 \pm 1.8$ & $6.6 \pm 2.3$ & $6.1 \pm 2.7$ & $5.5 \pm 3.2$ & $5.5 \pm 3.0$ & $5.3 \pm 2.8$ & $5.2 \pm 2.6$ & $7.1 \pm 2.8$ & $8.6 \pm 2.4$ \\
\hline RCP8.5 - mid & $9.0 \pm 2.2$ & $8.9 \pm 2.1$ & $8.7 \pm 1.9$ & $8.0 \pm 1.7$ & $6.6 \pm 2.2$ & $6.0 \pm 2.7$ & $5.6 \pm 3.2$ & $5.6 \pm 3.3$ & $5.3 \pm 2.9$ & $5.1 \pm 2.6$ & $6.9 \pm 2.8$ & $8.8 \pm 2.4$ \\
\hline RCP4.5 - end & $9.0 \pm 2.1$ & $9.1 \pm 2.0$ & $8.7 \pm 2.0$ & $7.9 \pm 1.9$ & $6.9 \pm 2.2$ & $6.0 \pm 2.6$ & $5.8 \pm 3.1$ & $5.7 \pm 3.2$ & $5.4 \pm 2.8$ & $5.3 \pm 2.6$ & $7.1 \pm 2.7$ & $8.6 \pm 2.3$ \\
\hline RCP8.5 - end & $8.8 \pm 2.2$ & $8.6 \pm 2.2$ & $8.4 \pm 1.9$ & $7.8 \pm 1.6$ & $6.8 \pm 2.2$ & $6.0 \pm 2.7$ & $5.8 \pm 3.2$ & $5.6 \pm 3.3$ & $5.3 \pm 2.9$ & $5.1 \pm 2.6$ & $6.9 \pm 2.9$ & $8.4 \pm 2.4$ \\
\hline \multicolumn{13}{|c|}{$\begin{array}{l}\text { Top } 5 \text { percent Wind } \\
\text { Speed }(\mathrm{m} / \mathrm{s})\end{array}$} \\
\hline Hindcast & $13.2 \pm 0.8$ & $13.3 \pm 1.0$ & $13.2 \pm 1.9$ & $11.9 \pm 1.6$ & $11.8 \pm 2.5$ & $11.7 \pm 1.9$ & $13.9 \pm 2.3$ & $14.5 \pm 2.4$ & $13.2 \pm 2.8$ & $12.8 \pm 2.6$ & $13.9 \pm 2.0$ & $13.4 \pm 1.5$ \\
\hline RCP4.5 - mid & $13.1 \pm 0.9$ & $13.2 \pm 1.1$ & $13.0 \pm 1.8$ & $11.7 \pm 1.7$ & $11.0 \pm 1.4$ & $12.1 \pm 2.2$ & $13.8 \pm 2.4$ & $13.4 \pm 2.2$ & $12.9 \pm 2.5$ & $11.5 \pm 2.3$ & $12.9 \pm 2.4$ & $13.6 \pm 1.7$ \\
\hline RCP8.5 - mid & $13.4 \pm 1.2$ & $12.9 \pm 0.7$ & $12.9 \pm 1.2$ & $11.5 \pm 1.1$ & $10.8 \pm 1.7$ & $12.6 \pm 2.9$ & $13.8 \pm 2.3$ & $14.4 \pm 2.3$ & $13.3 \pm 2.9$ & $11.8 \pm 2.6$ & $12.9 \pm 2.1$ & $13.7 \pm 1.7$ \\
\hline RCP 4.5 - end & $13.1 \pm 0.8$ & $13.1 \pm 0.9$ & $12.7 \pm 0.9$ & $11.8 \pm 1.4$ & $11.0 \pm 1.3$ & $11.3 \pm 1.7$ & $13.5 \pm 2.6$ & $14.2 \pm 2.3$ & $12.6 \pm 1.9$ & $11.8 \pm 2.5$ & $12.6 \pm 1.6$ & $13.1 \pm 1.5$ \\
\hline RCP8.5 - end & $13.3 \pm 1.3$ & $13.0 \pm 0.9$ & $12.2 \pm 0.8$ & $11.0 \pm 0.8$ & $10.7 \pm 1.0$ & $11.8 \pm 2.4$ & $13.3 \pm 2.4$ & $14.5 \pm 2.5$ & $13.3 \pm 2.4$ & $11.5 \pm 2.1$ & $13.2 \pm 2.5$ & $13.5 \pm 2.5$ \\
\hline \multicolumn{13}{|l|}{$\begin{array}{l}\text { Mean Wind } \\
\text { Direction ( } 9 \text { ) }\end{array}$} \\
\hline Hindcast & $249 \pm 18$ & $246 \pm 18$ & $249 \pm 18$ & $253 \pm 21$ & $260 \pm 33$ & $266 \pm 58$ & $301 \pm 100$ & $12 \pm 103$ & $355 \pm 117$ & $267 \pm 85$ & $259 \pm 39$ & $253 \pm 23$ \\
\hline RCP4.5 - mid & $249 \pm 20$ & $247 \pm 18$ & $250 \pm 17$ & $253 \pm 15$ & $260 \pm 31$ & $269 \pm 56$ & $299 \pm 90$ & $13 \pm 107$ & $20 \pm 111$ & $278 \pm 102$ & $259 \pm 43$ & $255 \pm 24$ \\
\hline RCP8.5 - mid & $249 \pm 18$ & $248 \pm 17$ & $249 \pm 16$ & $254 \pm 16$ & $260 \pm 29$ & $270 \pm 54$ & $301 \pm 95$ & $16 \pm 103$ & $33 \pm 107$ & $274 \pm 102$ & $259 \pm 43$ & $255 \pm 24$ \\
\hline RCP4.5 - end & $250 \pm 18$ & $246 \pm 17$ & $249 \pm 16$ & $254 \pm 18$ & $261 \pm 29$ & $267 \pm 55$ & $295 \pm 93$ & $3 \pm 105$ & $13 \pm 110$ & $279 \pm 102$ & $258 \pm 40$ & $255 \pm 21$ \\
\hline $\begin{array}{l}\text { RCP } 8.5 \text { - end } \\
\text { Top } 5 \text { percent } \\
\left.\text { Direction ( }{ }^{9}\right)\end{array}$ & $250 \pm 19$ & $248 \pm 18$ & $250 \pm 15$ & $254 \pm 13$ & $260 \pm 28$ & $269 \pm 59$ & $289 \pm 86$ & $357 \pm 105$ & $26 \pm 105$ & $277 \pm 98$ & $258 \pm 41$ & $256 \pm 22$ \\
\hline Hindcast & $241 \pm 15$ & $236 \pm 14$ & $244 \pm 28$ & $248 \pm 26$ & $263 \pm 44$ & $290 \pm 76$ & $24 \pm 67$ & $26 \pm 47$ & $14 \pm 65$ & $302 \pm 91$ & $263 \pm 52$ & $248 \pm 23$ \\
\hline RCP4.5 - mid & $242 \pm 16$ & $240 \pm 11$ & $243 \pm 21$ & $247 \pm 21$ & $255 \pm 31$ & $296 \pm 71$ & $16 \pm 57$ & $35 \pm 49$ & $41 \pm 56$ & $303 \pm 94$ & $264 \pm 49$ & $246 \pm 32$ \\
\hline RCP8.5 - mid & $243 \pm 15$ & $242 \pm 11$ & $241 \pm 19$ & $251 \pm 15$ & $259 \pm 19$ & $322 \pm 81$ & $27 \pm 52$ & $29 \pm 37$ & $42 \pm 35$ & $318 \pm 104$ & $259 \pm 51$ & $250 \pm 30$ \\
\hline RCP4.5 - end & $242 \pm 14$ & $238 \pm 17$ & $242 \pm 11$ & $251 \pm 22$ & $263 \pm 26$ & $284 \pm 68$ & $29 \pm 65$ & $36 \pm 36$ & $40 \pm 49$ & $333 \pm 94$ & $257 \pm 43$ & $245 \pm 27$ \\
\hline RCP8.5 - end & $245 \pm 18$ & $239 \pm 14$ & $240 \pm 12$ & $250 \pm 16$ & $256 \pm 21$ & $291 \pm 83$ & $24 \pm 59$ & $36 \pm 42$ & $39 \pm 49$ & $328 \pm 103$ & $256 \pm 39$ & $251 \pm 27$ \\
\hline
\end{tabular}


Appendix B34. Table showing return values of ensemble-average wind speeds of hindcast and forecast scenarios, including lower and higher 95 percent confidence intervals, at the Guam location.

[Years: Hindcast $=1976-2005 ;$ RCP mid $=$ 2026-2045; RCP end $=$ 2081-2100. Wind speeds are in meters per second]

\begin{tabular}{|c|c|c|c|c|c|c|c|c|c|c|c|c|c|c|c|c|}
\hline \multirow{2}{*}{ Scenario } & \multicolumn{3}{|c|}{ Hindcast } & \multicolumn{3}{c|}{ RCP4.5 - mid } & \multicolumn{3}{c|}{ RCP8.5 - mid } & \multicolumn{3}{c|}{ RCP4.5 - end } & \multicolumn{2}{c|}{ RCP8.5 - end } \\
\cline { 2 - 13 } & Low & $\boldsymbol{R}_{\boldsymbol{V}}$ & High & Low & $\boldsymbol{R}_{\boldsymbol{V}}$ & High & Low & $\boldsymbol{R}_{\boldsymbol{V}}$ & High & Low & $\boldsymbol{R}_{\boldsymbol{V}}$ & High & Low & $\boldsymbol{R}_{\boldsymbol{V}}$ & High \\
\hline 2-year & 20.45 & 22.85 & 26.86 & 19.96 & 22.16 & 25.73 & 19.81 & 22.05 & 26.02 & 19.00 & 20.97 & 24.13 & 20.16 & 22.60 & 26.68 \\
5-year & 21.71 & 25.61 & 32.92 & 20.90 & 24.12 & 29.82 & 20.81 & 24.43 & 31.69 & 19.94 & 22.88 & 28.03 & 21.09 & 24.66 & 31.27 \\
10-year & 22.56 & 27.78 & 38.48 & 21.45 & 25.45 & 33.02 & 21.48 & 26.31 & 36.98 & 20.51 & 24.22 & 31.15 & 21.63 & 26.05 & 34.90 \\
20-year & 23.33 & 30.03 & 45.05 & 21.89 & 26.65 & 36.29 & 22.08 & 28.26 & 43.32 & 20.99 & 25.47 & 34.43 & 22.06 & 27.33 & 38.68 \\
50-year & 24.23 & 33.12 & 55.61 & 22.35 & 28.08 & 40.75 & 22.78 & 30.95 & 53.68 & 21.50 & 27.01 & 39.02 & 22.49 & 28.84 & 43.92 \\
100-year & 24.84 & 35.54 & 65.29 & 22.62 & 29.05 & 44.23 & 23.25 & 33.08 & 63.35 & 21.82 & 28.08 & 42.70 & 22.75 & 29.86 & 48.07 \\
\hline
\end{tabular}


Appendix B35. Table showing Kwajalein monthly means and mean of the top 5 percent for wind speed and mean wind direction.

[Years: Hindcast $=1976-2005 ;$ RCP mid $=$ 2026-2045; RCP end $=$ 2081-2100. Wind directions are “coming from”]

\begin{tabular}{|c|c|c|c|c|c|c|c|c|c|c|c|c|}
\hline Month & Jan & Feb & Mar & Apr & May & Jun & July & Aug & Sep & Oct & Nov & Dec \\
\hline \multicolumn{13}{|c|}{$\begin{array}{l}\text { Mean Wind Speed } \\
(\mathrm{m} / \mathrm{s})\end{array}$} \\
\hline Hindcast & $9.3 \pm 1.8$ & $9.1 \pm 1.8$ & $8.8 \pm 1.9$ & $7.7 \pm 2.4$ & $6.1 \pm 2.7$ & $5.3 \pm 2.7$ & $4.8 \pm 2.6$ & $4.4 \pm 2.5$ & $4.2 \pm 2.3$ & $4.7 \pm 2.6$ & $6.6 \pm 3.1$ & $8.7 \pm 2.5$ \\
\hline RCP4.5 - mid & $9.3 \pm 1.9$ & $9.0 \pm 1.9$ & $8.9 \pm 1.8$ & $7.8 \pm 2.2$ & $6.4 \pm 2.7$ & $5.6 \pm 2.7$ & $4.9 \pm 2.7$ & $4.4 \pm 2.6$ & $4.2 \pm 2.3$ & $4.5 \pm 2.4$ & $5.9 \pm 3.0$ & $8.5 \pm 2.5$ \\
\hline RCP8.5 - mid & $9.1 \pm 1.8$ & $9.1 \pm 1.9$ & $8.7 \pm 1.8$ & $8.0 \pm 2.2$ & $6.4 \pm 2.6$ & $5.6 \pm 2.7$ & $5.0 \pm 2.7$ & $4.5 \pm 2.6$ & $4.1 \pm 2.3$ & $4.4 \pm 2.4$ & $6.0 \pm 3.0$ & $8.5 \pm 2.4$ \\
\hline RCP4.5 - end & $9.1 \pm 1.7$ & $9.0 \pm 1.7$ & $8.8 \pm 1.8$ & $7.7 \pm 2.2$ & $6.4 \pm 2.6$ & $5.6 \pm 2.8$ & $4.9 \pm 2.6$ & $4.6 \pm 2.6$ & $4.2 \pm 2.4$ & $4.3 \pm 2.5$ & $5.9 \pm 2.9$ & $8.4 \pm 2.4$ \\
\hline RCP8.5 - end & $8.8 \pm 1.8$ & $8.8 \pm 1.8$ & $8.7 \pm 1.7$ & $8.0 \pm 2.1$ & $6.9 \pm 2.4$ & $5.9 \pm 2.8$ & $5.1 \pm 2.7$ & $4.4 \pm 2.6$ & $4.0 \pm 2.2$ & $4.1 \pm 2.4$ & $5.7 \pm 2.9$ & $8.2 \pm 2.3$ \\
\hline \multicolumn{13}{|c|}{$\begin{array}{l}\text { Top } 5 \text { percent Wind } \\
\text { Speed }(\mathrm{m} / \mathrm{s})\end{array}$} \\
\hline Hindcast & $12.7 \pm 0.6$ & $12.6 \pm 0.8$ & $12.4 \pm 0.8$ & $11.9 \pm 1.0$ & $10.9 \pm 0.9$ & $10.6 \pm 1.0$ & $10.6 \pm 1.4$ & $10.9 \pm 2.0$ & $10.4 \pm 2.1$ & $11.5 \pm 2.1$ & $13.1 \pm 1.5$ & $13.2 \pm 1.3$ \\
\hline RCP4.5 - mid & $12.6 \pm 0.5$ & $12.7 \pm 1.2$ & $12.1 \pm 0.5$ & $11.7 \pm 0.7$ & $11.1 \pm 1.0$ & $11.0 \pm 1.3$ & $11.0 \pm 1.4$ & $11.4 \pm 2.2$ & $10.6 \pm 2.1$ & $11.0 \pm 2.3$ & $12.5 \pm 1.4$ & $13.1 \pm 1.2$ \\
\hline RCP8.5 - mid & $12.5 \pm 0.7$ & $12.9 \pm 0.9$ & $12.2 \pm 0.6$ & $12.1 \pm 1.6$ & $11.3 \pm 1.4$ & $10.9 \pm 1.0$ & $11.2 \pm 1.8$ & $11.5 \pm 2.3$ & $10.6 \pm 2.0$ & $10.7 \pm 1.9$ & $12.7 \pm 1.3$ & $12.8 \pm 0.8$ \\
\hline RCP 4.5 - end & $12.4 \pm 0.8$ & $12.4 \pm 0.7$ & $12.2 \pm 0.7$ & $11.5 \pm 0.5$ & $10.9 \pm 0.8$ & $10.9 \pm 1.1$ & $10.9 \pm 2.0$ & $11.7 \pm 2.3$ & $11.1 \pm 2.1$ & $11.0 \pm 2.0$ & $12.4 \pm 1.6$ & $13.0 \pm 1.1$ \\
\hline RCP8.5 - end & $12.1 \pm 0.5$ & $12.2 \pm 0.7$ & $12.0 \pm 0.7$ & $11.5 \pm 0.9$ & $10.9 \pm 0.8$ & $11.0 \pm 1.3$ & $11.1 \pm 1.6$ & $11.3 \pm 2.6$ & $9.9 \pm 2.6$ & $10.9 \pm 2.1$ & $12.2 \pm 1.3$ & $12.3 \pm 0.9$ \\
\hline \multicolumn{13}{|l|}{$\begin{array}{l}\text { Mean Wind } \\
\text { Direction }(9)\end{array}$} \\
\hline Hindcast & $243 \pm 12$ & $243 \pm 12$ & $246 \pm 13$ & $249 \pm 21$ & $253 \pm 39$ & $258 \pm 51$ & $269 \pm 64$ & $291 \pm 74$ & $302 \pm 85$ & $278 \pm 81$ & $252 \pm 46$ & $245 \pm 21$ \\
\hline RCP4.5 - mid & $244 \pm 13$ & $244 \pm 13$ & $245 \pm 11$ & $249 \pm 19$ & $252 \pm 31$ & $258 \pm 49$ & $268 \pm 58$ & $288 \pm 73$ & $301 \pm 79$ & $289 \pm 83$ & $255 \pm 52$ & $248 \pm 23$ \\
\hline RCP8.5 - mid & $244 \pm 12$ & $244 \pm 12$ & $246 \pm 12$ & $249 \pm 19$ & $252 \pm 30$ & $257 \pm 45$ & $271 \pm 59$ & $295 \pm 74$ & $311 \pm 82$ & $290 \pm 85$ & $254 \pm 56$ & $247 \pm 22$ \\
\hline RCP4.5 - end & $245 \pm 12$ & $245 \pm 12$ & $246 \pm 12$ & $250 \pm 19$ & $252 \pm 32$ & $258 \pm 47$ & $267 \pm 57$ & $294 \pm 77$ & $307 \pm 83$ & $295 \pm 89$ & $255 \pm 57$ & $248 \pm 21$ \\
\hline $\begin{array}{l}\text { RCP } 8.5 \text { - end } \\
\text { Top } 5 \text { percent } \\
\left.\text { Direction ( }{ }^{9}\right)\end{array}$ & $246 \pm 13$ & $246 \pm 12$ & $247 \pm 11$ & $249 \pm 15$ & $253 \pm 26$ & $257 \pm 43$ & $266 \pm 53$ & $288 \pm 67$ & $300 \pm 81$ & $288 \pm 84$ & $255 \pm 52$ & $248 \pm 20$ \\
\hline Hindcast & $241 \pm 9$ & $240 \pm 9$ & $242 \pm 16$ & $244 \pm 27$ & $245 \pm 25$ & $247 \pm 28$ & $260 \pm 60$ & $350 \pm 95$ & $13 \pm 109$ & $274 \pm 70$ & $245 \pm 37$ & $238 \pm 16$ \\
\hline RCP4.5 - mid & $240 \pm 9$ & $242 \pm 13$ & $240 \pm 8$ & $243 \pm 15$ & $244 \pm 15$ & $248 \pm 35$ & $258 \pm 56$ & $325 \pm 89$ & $325 \pm 92$ & $318 \pm 94$ & $240 \pm 36$ & $241 \pm 12$ \\
\hline RCP8.5 - mid & $241 \pm 8$ & $240 \pm 9$ & $241 \pm 7$ & $243 \pm 31$ & $247 \pm 20$ & $250 \pm 33$ & $273 \pm 67$ & $339 \pm 85$ & $28 \pm 93$ & $318 \pm 105$ & $241 \pm 43$ & $239 \pm 10$ \\
\hline RCP4.5 - end & $241 \pm 10$ & $242 \pm 10$ & $240 \pm 11$ & $244 \pm 8$ & $243 \pm 16$ & $247 \pm 28$ & $264 \pm 60$ & $7 \pm 84$ & $5 \pm 81$ & $354 \pm 104$ & $239 \pm 48$ & $240 \pm 20$ \\
\hline RCP8.5 - end & $242 \pm 8$ & $241 \pm 8$ & $243 \pm 8$ & $244 \pm 8$ & $245 \pm 14$ & $252 \pm 40$ & $267 \pm 75$ & $340 \pm 82$ & $328 \pm 96$ & $294 \pm 90$ & $242 \pm 32$ & $242 \pm 11$ \\
\hline
\end{tabular}


Appendix B36. Table showing return values of ensemble-average wind speeds of hindcast and forecast scenarios, including lower and higher 95 percent confidence intervals, at the Kwajalein location.

[Years: Hindcast $=1976-2005 ;$ RCP mid $=$ 2026-2045; RCP end $=$ 2081-2100. Wind speeds are in meters per second]

\begin{tabular}{|c|c|c|c|c|c|c|c|c|c|c|c|c|c|c|c|}
\hline \multirow{2}{*}{ Scenario } & \multicolumn{3}{|c|}{ Hindcast } & \multicolumn{3}{c|}{ RCP4.5 - mid } & \multicolumn{3}{c|}{ RCP8.5 - mid } & \multicolumn{3}{c|}{ RCP4.5 - end } & \multicolumn{2}{c|}{ RCP8.5 - end } \\
\cline { 2 - 13 } & Low & $\boldsymbol{R}_{\boldsymbol{V}}$ & High & Low & $\boldsymbol{R}_{\boldsymbol{V}}$ & High & Low & $\boldsymbol{R}_{\boldsymbol{V}}$ & High & Low & $\boldsymbol{R}_{\boldsymbol{V}}$ & High & Low & $\boldsymbol{R}_{\boldsymbol{V}}$ & High \\
\hline 2-year & 16.70 & 18.88 & 23.14 & 16.37 & 18.28 & 22.23 & 16.81 & 18.82 & 22.50 & 16.70 & 18.37 & 21.29 & 16.61 & 18.55 & 22.08 \\
5-year & 17.75 & 21.75 & 30.99 & 16.83 & 19.71 & 26.67 & 17.66 & 20.95 & 27.86 & 17.31 & 19.83 & 24.80 & 17.39 & 20.50 & 26.93 \\
10-year & 18.55 & 24.42 & 39.95 & 17.09 & 20.73 & 30.65 & 18.23 & 22.67 & 33.01 & 17.68 & 20.87 & 27.76 & 17.90 & 22.01 & 31.44 \\
20-year & 19.35 & 27.62 & 52.73 & 17.29 & 21.70 & 35.27 & 18.75 & 24.49 & 39.37 & 17.99 & 21.87 & 31.03 & 18.35 & 23.57 & 36.85 \\
50-year & 20.42 & 32.83 & 78.40 & 17.50 & 22.91 & 42.52 & 19.36 & 27.04 & 50.08 & 18.30 & 23.11 & 35.88 & 18.86 & 25.68 & 45.66 \\
100-year & 21.22 & 37.69 & 97.64 & 17.61 & 23.78 & 49.03 & 19.77 & 29.09 & 60.40 & 18.50 & 23.99 & 39.98 & 19.19 & 27.33 & 53.86 \\
\hline
\end{tabular}


Appendix B37. Table showing Wake monthly means and mean of the top 5 percent for wind speed and mean wind direction.

[Years: Hindcast $=1976-2005 ;$ RCP mid $=$ 2026-2045; RCP end $=$ 2081-2100. Wind directions are “coming from”]

\begin{tabular}{|c|c|c|c|c|c|c|c|c|c|c|c|c|}
\hline Month & Jan & Feb & Mar & Apr & May & Jun & July & Aug & Sep & Oct & Nov & Dec \\
\hline \multicolumn{13}{|c|}{$\begin{array}{l}\text { Mean Wind Speed } \\
(\mathrm{m} / \mathrm{s})\end{array}$} \\
\hline Hindcast & $7.3 \pm 2.8$ & $7.2 \pm 2.8$ & $7.7 \pm 2.6$ & $8.3 \pm 2.3$ & $8.0 \pm 1.9$ & $7.6 \pm 2.1$ & $7.2 \pm 2.4$ & $6.8 \pm 2.7$ & $6.7 \pm 2.8$ & $7.8 \pm 2.9$ & $8.6 \pm 2.6$ & $8.2 \pm 2.8$ \\
\hline RCP4.5 - mid & $7.4 \pm 2.8$ & $7.2 \pm 2.7$ & $7.8 \pm 2.6$ & $8.3 \pm 2.2$ & $7.9 \pm 1.9$ & $7.6 \pm 2.0$ & $7.4 \pm 2.3$ & $6.7 \pm 2.6$ & $6.6 \pm 2.8$ & $7.8 \pm 2.8$ & $8.6 \pm 2.6$ & $8.0 \pm 2.7$ \\
\hline RCP8.5 - mid & $7.3 \pm 2.8$ & $7.4 \pm 2.9$ & $7.7 \pm 2.6$ & $8.2 \pm 2.3$ & $8.0 \pm 1.9$ & $7.7 \pm 2.0$ & $7.4 \pm 2.5$ & $6.7 \pm 2.8$ & $6.6 \pm 2.9$ & $7.5 \pm 2.8$ & $8.8 \pm 2.5$ & $8.0 \pm 2.8$ \\
\hline RCP4.5 - end & $7.1 \pm 2.7$ & $7.2 \pm 2.7$ & $7.8 \pm 2.7$ & $8.2 \pm 2.3$ & $7.9 \pm 1.9$ & $7.7 \pm 1.8$ & $7.4 \pm 2.4$ & $6.9 \pm 2.6$ & $6.7 \pm 2.8$ & $7.5 \pm 2.8$ & $8.6 \pm 2.5$ & $8.1 \pm 2.6$ \\
\hline RCP8.5 - end & $7.2 \pm 2.8$ & $7.0 \pm 2.8$ & $7.5 \pm 2.5$ & $8.2 \pm 2.0$ & $7.8 \pm 1.8$ & $7.5 \pm 1.8$ & $7.5 \pm 2.2$ & $7.2 \pm 2.5$ & $6.6 \pm 2.6$ & $7.5 \pm 2.7$ & $8.6 \pm 2.4$ & $8.0 \pm 2.5$ \\
\hline \multicolumn{13}{|c|}{$\begin{array}{l}\text { Top } 5 \text { percent Wind } \\
\text { Speed }(\mathrm{m} / \mathrm{s})\end{array}$} \\
\hline Hindcast & $12.9 \pm 1.2$ & $12.7 \pm 0.8$ & $12.9 \pm 1.0$ & $12.9 \pm 1.3$ & $11.6 \pm 0.9$ & $11.8 \pm 1.3$ & $12.0 \pm 1.7$ & $12.8 \pm 1.8$ & $13.0 \pm 2.1$ & $14.1 \pm 2.1$ & $13.9 \pm 1.4$ & $13.5 \pm 0.9$ \\
\hline RCP4.5 - mid & $12.7 \pm 0.8$ & $12.6 \pm 0.8$ & $13.0 \pm 0.9$ & $12.5 \pm 0.8$ & $11.5 \pm 0.7$ & $11.5 \pm 1.6$ & $12.2 \pm 1.7$ & $12.3 \pm 1.8$ & $13.0 \pm 2.2$ & $14.1 \pm 2.1$ & $13.8 \pm 1.4$ & $13.2 \pm 0.9$ \\
\hline RCP8.5 - mid & $12.8 \pm 0.8$ & $13.0 \pm 0.8$ & $12.7 \pm 0.9$ & $12.7 \pm 1.7$ & $11.6 \pm 1.2$ & $11.3 \pm 0.9$ & $12.5 \pm 1.9$ & $13.5 \pm 2.6$ & $13.6 \pm 2.8$ & $13.3 \pm 1.5$ & $14.0 \pm 1.3$ & $13.2 \pm 1.0$ \\
\hline RCP4.5 - end & $12.5 \pm 0.9$ & $12.6 \pm 0.9$ & $12.7 \pm 0.7$ & $12.4 \pm 0.9$ & $11.3 \pm 0.8$ & $11.2 \pm 1.4$ & $12.3 \pm 2.1$ & $12.8 \pm 2.2$ & $13.2 \pm 2.4$ & $13.4 \pm 1.8$ & $13.6 \pm 1.2$ & $12.9 \pm 0.8$ \\
\hline RCP8.5 - end & $12.6 \pm 0.8$ & $12.7 \pm 0.9$ & $12.3 \pm 0.8$ & $11.9 \pm 0.6$ & $11.1 \pm 0.6$ & $11.1 \pm 1.4$ & $11.8 \pm 1.8$ & $13.5 \pm 2.7$ & $12.7 \pm 2.6$ & $13.4 \pm 2.3$ & $13.4 \pm 1.2$ & $12.8 \pm 0.6$ \\
\hline \multicolumn{13}{|l|}{$\begin{array}{l}\text { Mean Wind } \\
\text { Direction }(9)\end{array}$} \\
\hline Hindcast & $249 \pm 39$ & $249 \pm 43$ & $252 \pm 32$ & $254 \pm 21$ & $257 \pm 17$ & $261 \pm 24$ & $265 \pm 36$ & $270 \pm 48$ & $262 \pm 48$ & $258 \pm 35$ & $254 \pm 24$ & $251 \pm 29$ \\
\hline RCP4.5 - mid & $250 \pm 39$ & $249 \pm 40$ & $252 \pm 29$ & $255 \pm 18$ & $257 \pm 17$ & $260 \pm 22$ & $267 \pm 33$ & $269 \pm 46$ & $267 \pm 48$ & $260 \pm 35$ & $255 \pm 26$ & $254 \pm 30$ \\
\hline RCP8.5 - mid & $249 \pm 40$ & $248 \pm 41$ & $252 \pm 29$ & $255 \pm 20$ & $257 \pm 15$ & $260 \pm 21$ & $268 \pm 35$ & $273 \pm 50$ & $267 \pm 50$ & $260 \pm 36$ & $256 \pm 22$ & $253 \pm 30$ \\
\hline RCP4.5 - end & $250 \pm 40$ & $249 \pm 38$ & $253 \pm 30$ & $256 \pm 20$ & $257 \pm 15$ & $261 \pm 17$ & $266 \pm 34$ & $270 \pm 47$ & $266 \pm 48$ & $259 \pm 39$ & $255 \pm 24$ & $253 \pm 25$ \\
\hline RCP8.5 - end & $250 \pm 39$ & $250 \pm 41$ & $254 \pm 28$ & $256 \pm 16$ & $259 \pm 15$ & $262 \pm 20$ & $266 \pm 32$ & $270 \pm 40$ & $269 \pm 46$ & $260 \pm 37$ & $255 \pm 21$ & $253 \pm 25$ \\
\hline \multicolumn{13}{|c|}{$\begin{array}{l}\text { Top } 5 \text { percent Wind } \\
\text { Direction }(9)\end{array}$} \\
\hline Hindcast & $246 \pm 16$ & $245 \pm 20$ & $244 \pm 15$ & $246 \pm 11$ & $255 \pm 16$ & $264 \pm 24$ & $268 \pm 44$ & $300 \pm 66$ & $271 \pm 51$ & $259 \pm 38$ & $250 \pm 23$ & $245 \pm 13$ \\
\hline RCP4.5 - mid & $246 \pm 16$ & $243 \pm 17$ & $246 \pm 12$ & $250 \pm 11$ & $250 \pm 11$ & $258 \pm 24$ & $274 \pm 45$ & $279 \pm 55$ & $285 \pm 59$ & $267 \pm 52$ & $247 \pm 16$ & $247 \pm 13$ \\
\hline RCP8.5 - mid & $247 \pm 14$ & $246 \pm 15$ & $245 \pm 13$ & $247 \pm 15$ & $253 \pm 10$ & $260 \pm 18$ & $283 \pm 55$ & $311 \pm 78$ & $291 \pm 71$ & $259 \pm 42$ & $251 \pm 17$ & $245 \pm 13$ \\
\hline RCP4.5 - end & $247 \pm 14$ & $248 \pm 17$ & $247 \pm 12$ & $253 \pm 15$ & $253 \pm 9$ & $262 \pm 19$ & $281 \pm 47$ & $314 \pm 84$ & $282 \pm 61$ & $257 \pm 35$ & $250 \pm 22$ & $249 \pm 11$ \\
\hline RCP8.5 - end & $245 \pm 16$ & $247 \pm 15$ & $249 \pm 11$ & $250 \pm 10$ & $252 \pm 8$ & $259 \pm 22$ & $277 \pm 48$ & $329 \pm 80$ & $304 \pm 73$ & $263 \pm 39$ & $248 \pm 18$ & $247 \pm 10$ \\
\hline
\end{tabular}


Appendix B38. Table showing return values of ensemble-average wind speeds of hindcast and forecast scenarios, including lower and higher 95 percent confidence intervals, at the Wake location.

[Years: Hindcast $=1976-2005 ;$ RCP mid $=$ 2026-2045; RCP end $=$ 2081-2100. Wind speeds are in meters per second]

\begin{tabular}{|c|c|c|c|c|c|c|c|c|c|c|c|c|c|c|c|}
\hline \multirow{2}{*}{ Scenario } & \multicolumn{3}{|c|}{ Hindcast } & \multicolumn{3}{c|}{ RCP4.5 - mid } & \multicolumn{3}{c|}{ RCP8.5 - mid } & \multicolumn{3}{c|}{ RCP4.5 - end } & \multicolumn{2}{c|}{ RCP8.5 - end } \\
\cline { 2 - 13 } & Low & $\boldsymbol{R}_{\boldsymbol{V}}$ & High & Low & $\boldsymbol{R}_{\boldsymbol{V}}$ & High & Low & $\boldsymbol{R}_{\boldsymbol{V}}$ & High & Low & $\boldsymbol{R}_{\boldsymbol{V}}$ & High & Low & $\boldsymbol{R}_{\boldsymbol{V}}$ & High \\
\hline 2-year & 18.43 & 20.05 & 22.61 & 17.80 & 19.47 & 22.40 & 17.75 & 20.52 & 26.74 & 17.85 & 19.85 & 23.45 & 17.78 & 20.34 & 25.35 \\
5-year & 19.41 & 22.01 & 26.53 & 18.44 & 20.99 & 26.00 & 18.60 & 23.42 & 36.73 & 18.40 & 21.25 & 27.02 & 18.63 & 22.70 & 32.02 \\
10-year & 20.07 & 23.52 & 30.00 & 18.83 & 22.08 & 29.09 & 19.19 & 26.00 & 48.10 & 18.70 & 22.16 & 29.79 & 19.16 & 24.55 & 38.41 \\
20-year & 20.67 & 25.06 & 33.95 & 19.15 & 23.14 & 32.52 & 19.73 & 28.97 & 64.32 & 18.92 & 22.95 & 32.63 & 19.62 & 26.44 & 46.25 \\
50-year & 21.37 & 27.13 & 40.07 & 19.50 & 24.47 & 37.66 & 20.39 & 33.58 & 96.82 & 19.13 & 23.85 & 36.50 & 20.12 & 29.01 & 59.41 \\
100-year & 21.85 & 28.73 & 45.46 & 19.71 & 25.44 & 42.06 & 20.85 & 37.67 & 133.79 & 19.24 & 24.43 & 39.51 & 20.44 & 31.02 & 72.01 \\
\hline
\end{tabular}


Appendix B39. Table showing Johnston Atoll monthly means and mean of the top 5 percent for wind speed and mean wind direction.

[Years: Hindcast $=1976-2005 ;$ RCP mid $=$ 2026-2045; RCP end $=$ 2081-2100. Wind directions are “coming from”]

\begin{tabular}{|c|c|c|c|c|c|c|c|c|c|c|c|c|}
\hline Month & Jan & Feb & Mar & Apr & May & Jun & July & Aug & Sep & Oct & Nov & Dec \\
\hline \multicolumn{13}{|c|}{$\begin{array}{l}\text { Mean Wind Speed } \\
(\mathrm{m} / \mathrm{s})\end{array}$} \\
\hline Hindcast & $7.6 \pm 2.9$ & $7.5 \pm 2.9$ & $8.3 \pm 2.7$ & $8.7 \pm 2.3$ & $8.4 \pm 1.9$ & $8.3 \pm 1.9$ & $7.8 \pm 2.1$ & $7.2 \pm 2.2$ & $6.5 \pm 2.5$ & $7.5 \pm 2.9$ & $8.5 \pm 2.7$ & $8.1 \pm 2.9$ \\
\hline RCP4.5 - mid & $7.6 \pm 2.7$ & $7.6 \pm 2.8$ & $8.2 \pm 2.7$ & $8.8 \pm 2.4$ & $8.2 \pm 1.9$ & $8.2 \pm 1.8$ & $8.1 \pm 1.8$ & $7.4 \pm 2.3$ & $6.6 \pm 2.4$ & $7.3 \pm 2.7$ & $8.6 \pm 2.6$ & $8.3 \pm 2.6$ \\
\hline RCP8.5 - mid & $7.6 \pm 2.9$ & $7.6 \pm 2.9$ & $8.1 \pm 2.8$ & $8.8 \pm 2.1$ & $8.2 \pm 1.9$ & $8.2 \pm 1.7$ & $8.1 \pm 1.9$ & $7.2 \pm 2.2$ & $6.3 \pm 2.5$ & $7.2 \pm 2.8$ & $8.5 \pm 2.6$ & $8.3 \pm 2.8$ \\
\hline RCP4.5 - end & $7.3 \pm 2.7$ & $7.5 \pm 2.9$ & $8.3 \pm 2.6$ & $8.7 \pm 2.1$ & $8.3 \pm 1.8$ & $8.3 \pm 1.7$ & $8.0 \pm 1.9$ & $7.4 \pm 2.2$ & $6.6 \pm 2.4$ & $7.2 \pm 2.8$ & $8.5 \pm 2.7$ & $8.3 \pm 2.6$ \\
\hline \multicolumn{13}{|c|}{$\begin{array}{l}\text { Top } 5 \text { percent Wind } \\
\text { Speed }(\mathrm{m} / \mathrm{s})\end{array}$} \\
\hline Hindcast & $13.3 \pm 0.9$ & $13.3 \pm 0.9$ & $13.4 \pm 0.8$ & $12.9 \pm 0.8$ & $12.0 \pm 1.0$ & $11.8 \pm 1.2$ & $11.9 \pm 1.4$ & $11.8 \pm 1.6$ & $11.8 \pm 1.5$ & $13.5 \pm 1.6$ & $13.8 \pm 1.2$ & $13.6 \pm 1.0$ \\
\hline RCP4.5 - mid & $13.0 \pm 0.9$ & $13.2 \pm 0.9$ & $13.2 \pm 0.7$ & $13.2 \pm 0.7$ & $11.6 \pm 0.8$ & $11.5 \pm 1.1$ & $11.6 \pm 1.0$ & $12.6 \pm 2.0$ & $11.7 \pm 1.7$ & $13.2 \pm 1.7$ & $13.6 \pm 1.3$ & $13.5 \pm 1.0$ \\
\hline RCP8.5 - mid & $13.5 \pm 1.0$ & $13.5 \pm 0.9$ & $13.4 \pm 0.8$ & $12.5 \pm 0.7$ & $11.5 \pm 0.7$ & $11.4 \pm 1.2$ & $11.8 \pm 1.3$ & $11.7 \pm 1.8$ & $11.7 \pm 2.0$ & $13.2 \pm 1.5$ & $13.5 \pm 1.0$ & $13.6 \pm 1.0$ \\
\hline RCP4.5 - end & $12.8 \pm 0.8$ & $13.4 \pm 1.0$ & $13.2 \pm 0.9$ & $12.5 \pm 0.6$ & $11.7 \pm 1.0$ & $11.4 \pm 0.9$ & $11.6 \pm 1.4$ & $12.3 \pm 2.0$ & $11.7 \pm 1.8$ & $13.4 \pm 2.0$ & $13.8 \pm 1.1$ & $13.2 \pm 0.7$ \\
\hline $\begin{array}{l}\text { RCP8.5 - end } \\
\text { Mean Wind } \\
\text { Direction ( }{ }^{9} \text { ) }\end{array}$ & \multicolumn{11}{|c|}{$\begin{array}{l}\text { Mean Wind } \\
\left.\text { Direction ( }{ }^{\circ}\right)\end{array}$} & $13.0 \pm 0.9$ \\
\hline Hindcast & $246 \pm 41$ & $246 \pm 41$ & $249 \pm 27$ & $251 \pm 21$ & $254 \pm 17$ & $259 \pm 17$ & $261 \pm 25$ & $262 \pm 33$ & $263 \pm 43$ & $256 \pm 40$ & $251 \pm 29$ & $249 \pm 35$ \\
\hline RCP4.5 - mid & $245 \pm 40$ & $248 \pm 36$ & $249 \pm 29$ & $252 \pm 21$ & $255 \pm 18$ & $258 \pm 17$ & $260 \pm 21$ & $264 \pm 31$ & $264 \pm 43$ & $257 \pm 38$ & $252 \pm 27$ & $249 \pm 29$ \\
\hline RCP8.5 - mid & $247 \pm 38$ & $246 \pm 36$ & $248 \pm 29$ & $251 \pm 19$ & $255 \pm 18$ & $258 \pm 13$ & $260 \pm 21$ & $264 \pm 33$ & $263 \pm 44$ & $256 \pm 39$ & $250 \pm 28$ & $248 \pm 35$ \\
\hline RCP4.5 - end & $246 \pm 41$ & $246 \pm 39$ & $248 \pm 27$ & $251 \pm 19$ & $255 \pm 16$ & $258 \pm 14$ & $260 \pm 22$ & $262 \pm 32$ & $262 \pm 38$ & $258 \pm 43$ & $251 \pm 28$ & $249 \pm 29$ \\
\hline $\begin{array}{l}\text { RCP8.5 - end } \\
\text { Top } 5 \text { percent } \\
\left.\text { Direction ( }{ }^{9}\right)\end{array}$ & $249 \pm 36$ & $248 \pm 36$ & $249 \pm 25$ & $251 \pm 17$ & $256 \pm 15$ & $259 \pm 15$ & $259 \pm 19$ & $260 \pm 29$ & $263 \pm 37$ & $257 \pm 36$ & $252 \pm 28$ & $250 \pm 27$ \\
\hline Hindcast & $241 \pm 24$ & $240 \pm 19$ & $242 \pm 12$ & $245 \pm 10$ & $249 \pm 10$ & $257 \pm 13$ & $259 \pm 23$ & $267 \pm 40$ & $263 \pm 44$ & $252 \pm 33$ & $245 \pm 21$ & $243 \pm 22$ \\
\hline RCP4.5 - mid & $239 \pm 26$ & $244 \pm 15$ & $245 \pm 11$ & $247 \pm 9$ & $249 \pm 12$ & $256 \pm 19$ & $259 \pm 27$ & $282 \pm 47$ & $274 \pm 52$ & $259 \pm 44$ & $249 \pm 21$ & $241 \pm 19$ \\
\hline RCP8.5 - mid & $240 \pm 18$ & $242 \pm 14$ & $242 \pm 11$ & $245 \pm 9$ & $249 \pm 12$ & $255 \pm 9$ & $261 \pm 25$ & $272 \pm 42$ & $282 \pm 63$ & $257 \pm 35$ & $245 \pm 20$ & $243 \pm 15$ \\
\hline RCP4.5 - end & $240 \pm 23$ & $241 \pm 15$ & $242 \pm 11$ & $246 \pm 9$ & $251 \pm 13$ & $256 \pm 13$ & $257 \pm 15$ & $275 \pm 53$ & $268 \pm 48$ & $259 \pm 48$ & $246 \pm 18$ & $242 \pm 16$ \\
\hline RCP8.5 - end & $240 \pm 18$ & $240 \pm 16$ & $244 \pm 10$ & $247 \pm 10$ & $250 \pm 9$ & $257 \pm 12$ & $258 \pm 20$ & $262 \pm 36$ & $269 \pm 50$ & $256 \pm 34$ & $244 \pm 21$ & $244 \pm 19$ \\
\hline
\end{tabular}


Appendix B40. Table showing return values of ensemble-average wind speeds of hindcast and forecast scenarios, including lower and higher 95 percent confidence intervals, at the Johnston Atoll location.

[Years: Hindcast $=1976-2005 ;$ RCP mid $=$ 2026-2045; RCP end $=$ 2081-2100. Wind speeds are in meters per second]

\begin{tabular}{|c|c|c|c|c|c|c|c|c|c|c|c|c|c|c|c|}
\hline \multirow{2}{*}{ Scenario } & \multicolumn{3}{|c|}{ Hindcast } & \multicolumn{3}{c|}{ RCP4.5 - mid } & \multicolumn{3}{c|}{ RCP8.5 - mid } & \multicolumn{3}{c|}{ RCP4.5 - end } & \multicolumn{3}{c|}{ RCP8.5 - end } \\
\cline { 2 - 14 } & Low & $\boldsymbol{R}_{\mathbf{V}}$ & High & Low & $\boldsymbol{R}_{\mathbf{V}}$ & High & Low & $\boldsymbol{R}_{\mathbf{V}}$ & High & Low & $\boldsymbol{R}_{\mathbf{V}}$ & High & Low & $\boldsymbol{R}_{\mathbf{V}}$ & High \\
\hline 2-year & 17.02 & 18.20 & 20.17 & 16.95 & 18.47 & 21.45 & 16.98 & 18.16 & 20.13 & 16.73 & 18.60 & 22.47 & 16.53 & 18.00 & 20.75 \\
5-year & 17.60 & 19.48 & 23.02 & 17.52 & 20.04 & 25.82 & 17.41 & 19.12 & 22.24 & 17.44 & 20.72 & 28.90 & 17.19 & 19.69 & 25.10 \\
10-year & 17.99 & 20.47 & 25.59 & 17.90 & 21.34 & 30.22 & 17.66 & 19.75 & 23.86 & 17.95 & 22.63 & 36.10 & 17.66 & 21.13 & 29.54 \\
20-year & 18.32 & 21.47 & 28.58 & 18.24 & 22.73 & 35.86 & 17.85 & 20.31 & 25.50 & 18.43 & 24.83 & 46.18 & 18.09 & 22.71 & 35.31 \\
50-year & 18.71 & 22.82 & 33.29 & 18.64 & 24.73 & 45.82 & 18.04 & 20.95 & 27.71 & 19.04 & 28.29 & 66.02 & 18.63 & 25.07 & 45.67 \\
100-year & 18.97 & 23.86 & 37.53 & 18.91 & 26.37 & 55.85 & 18.15 & 21.38 & 29.40 & 19.47 & 31.40 & 88.16 & 19.01 & 27.08 & 56.25 \\
\hline
\end{tabular}


Appendix B41. Table showing Kingman Reef monthly means and mean of the top 5 percent for wind speed and mean wind direction.

[Years: Hindcast $=1976-2005 ;$ RCP mid $=$ 2026-2045; RCP end $=$ 2081-2100. Wind directions are “coming from”]

\begin{tabular}{|c|c|c|c|c|c|c|c|c|c|c|c|c|}
\hline Month & Jan & Feb & Mar & Apr & May & Jun & July & Aug & Sep & Oct & Nov & Dec \\
\hline \multicolumn{13}{|c|}{$\begin{array}{l}\text { Mean Wind Speed } \\
(\mathrm{m} / \mathrm{s})\end{array}$} \\
\hline Hindcast & $6.9 \pm 2.6$ & $7.2 \pm 2.4$ & $7.1 \pm 2.5$ & $6.4 \pm 2.4$ & $5.5 \pm 2.3$ & $5.0 \pm 2.1$ & $4.8 \pm 1.8$ & $4.7 \pm 1.7$ & $4.5 \pm 1.8$ & $4.3 \pm 1.9$ & $4.4 \pm 2.2$ & $5.7 \pm 2.4$ \\
\hline RCP4.5 - mid & $6.8 \pm 2.4$ & $7.5 \pm 2.3$ & $7.4 \pm 2.5$ & $6.9 \pm 2.3$ & $6.0 \pm 2.3$ & $5.4 \pm 2.2$ & $4.9 \pm 1.9$ & $4.7 \pm 1.8$ & $4.5 \pm 1.8$ & $4.3 \pm 1.9$ & $4.4 \pm 2.0$ & $5.6 \pm 2.5$ \\
\hline RCP8.5 - mid & $7.0 \pm 2.4$ & $7.3 \pm 2.4$ & $7.2 \pm 2.4$ & $6.9 \pm 2.3$ & $6.1 \pm 2.3$ & $5.4 \pm 2.1$ & $4.9 \pm 1.7$ & $4.6 \pm 1.7$ & $4.5 \pm 1.7$ & $4.3 \pm 1.9$ & $4.4 \pm 2.0$ & $5.5 \pm 2.4$ \\
\hline RCP 4.5 - end & $6.8 \pm 2.4$ & $7.3 \pm 2.3$ & $7.3 \pm 2.3$ & $6.8 \pm 2.3$ & $6.2 \pm 2.3$ & $5.4 \pm 2.1$ & $4.8 \pm 1.7$ & $4.6 \pm 1.8$ & $4.5 \pm 1.8$ & $4.3 \pm 1.9$ & $4.3 \pm 2.1$ & $5.7 \pm 2.6$ \\
\hline RCP8.5 - end & $7.0 \pm 2.3$ & $7.6 \pm 2.1$ & $7.4 \pm 2.2$ & $7.2 \pm 2.2$ & $6.7 \pm 2.1$ & $5.7 \pm 2.2$ & $4.9 \pm 1.8$ & $4.8 \pm 1.8$ & $4.4 \pm 1.7$ & $4.1 \pm 1.8$ & $4.1 \pm 1.9$ & $5.6 \pm 2.3$ \\
\hline \multicolumn{13}{|c|}{$\begin{array}{l}\text { Top } 5 \text { percent Wind } \\
\text { Speed }(\mathrm{m} / \mathrm{s})\end{array}$} \\
\hline Hindcast & $11.8 \pm 1.0$ & $11.7 \pm 0.7$ & $11.7 \pm 0.7$ & $11.0 \pm 0.6$ & $10.4 \pm 0.7$ & $9.9 \pm 1.0$ & $8.8 \pm 1.0$ & $8.8 \pm 1.4$ & $8.5 \pm 1.1$ & $8.6 \pm 1.3$ & $10.0 \pm 1.9$ & $11.0 \pm 1.2$ \\
\hline RCP4.5 - mid & $11.5 \pm 0.9$ & $11.6 \pm 0.6$ & $12.1 \pm 0.7$ & $11.3 \pm 0.6$ & $10.5 \pm 1.1$ & $10.4 \pm 0.9$ & $9.2 \pm 1.0$ & $8.9 \pm 1.5$ & $8.3 \pm 1.0$ & $8.5 \pm 1.0$ & $9.2 \pm 1.2$ & $11.0 \pm 1$. \\
\hline RCP8.5 - mid & $11.7 \pm 1.1$ & $11.9 \pm 0.8$ & $11.9 \pm 0.7$ & $11.3 \pm 0.6$ & $10.7 \pm 0.9$ & $9.9 \pm 0.7$ & $8.9 \pm 0.9$ & $8.4 \pm 0.9$ & $8.3 \pm 1.1$ & $8.6 \pm 1.3$ & $9.4 \pm 1.2$ & $10.8 \pm 0$. \\
\hline RCP4.5 - end & $11.5 \pm 0.8$ & $11.8 \pm 0.6$ & $11.5 \pm 0.6$ & $11.3 \pm 0.8$ & $11.0 \pm 0.9$ & $10.3 \pm 1.0$ & $9.0 \pm 1.3$ & $9.1 \pm 1.6$ & $8.6 \pm 1.2$ & $8.9 \pm 1.5$ & $9.6 \pm 1.8$ & $11.4 \pm 1$. \\
\hline RCP8.5 - end & $11.3 \pm 0.6$ & $11.5 \pm 0.7$ & $11.7 \pm 0.6$ & $11.3 \pm 0.7$ & $10.8 \pm 0.7$ & $10.5 \pm 0.9$ & $9.1 \pm 1.2$ & $8.9 \pm 1.3$ & $8.3 \pm 1.2$ & $8.4 \pm 1.2$ & $8.9 \pm 1.3$ & $10.8 \pm 1$. \\
\hline \multicolumn{13}{|l|}{$\begin{array}{l}\text { Mean Wind } \\
\left.\text { Direction ( }{ }^{\circ}\right)\end{array}$} \\
\hline Hindcast & $251 \pm 30$ & $249 \pm 26$ & $251 \pm 25$ & $254 \pm 29$ & $261 \pm 33$ & $273 \pm 41$ & $291 \pm 41$ & $307 \pm 39$ & $317 \pm 41$ & $319 \pm 48$ & $297 \pm 57$ & $264 \pm 42$ \\
\hline RCP4.5 - mid & $251 \pm 28$ & $248 \pm 22$ & $249 \pm 22$ & $251 \pm 23$ & $256 \pm 30$ & $266 \pm 35$ & $287 \pm 39$ & $305 \pm 39$ & $314 \pm 42$ & $318 \pm 49$ & $298 \pm 59$ & $263 \pm 42$ \\
\hline RCP8.5 - mid & $250 \pm 28$ & $249 \pm 24$ & $249 \pm 21$ & $251 \pm 22$ & $257 \pm 27$ & $265 \pm 34$ & $286 \pm 37$ & $307 \pm 39$ & $316 \pm 42$ & $320 \pm 49$ & $297 \pm 57$ & $263 \pm 42$ \\
\hline RCP4.5 - end & $251 \pm 28$ & $249 \pm 26$ & $250 \pm 21$ & $252 \pm 21$ & $256 \pm 26$ & $266 \pm 35$ & $287 \pm 39$ & $308 \pm 42$ & $315 \pm 43$ & $318 \pm 50$ & $295 \pm 58$ & $261 \pm 42$ \\
\hline RCP8.5 - end & $251 \pm 25$ & $249 \pm 18$ & $249 \pm 18$ & $250 \pm 18$ & $254 \pm 23$ & $261 \pm 31$ & $282 \pm 37$ & $301 \pm 41$ & $315 \pm 43$ & $317 \pm 49$ & $289 \pm 54$ & $260 \pm 38$ \\
\hline \multicolumn{13}{|c|}{$\begin{array}{l}\text { Top } 5 \text { percent Wind } \\
\text { Direction ( } 9 \text { ) }\end{array}$} \\
\hline Hindcast & $237 \pm 12$ & $234 \pm 14$ & $234 \pm 10$ & $237 \pm 13$ & $244 \pm 21$ & $254 \pm 44$ & $283 \pm 48$ & $317 \pm 47$ & $324 \pm 44$ & $321 \pm 44$ & $270 \pm 58$ & $240 \pm 25$ \\
\hline RCP4.5 - mid & $238 \pm 17$ & $237 \pm 10$ & $233 \pm 10$ & $237 \pm 10$ & $237 \pm 28$ & $244 \pm 29$ & $272 \pm 49$ & $313 \pm 47$ & $317 \pm 42$ & $329 \pm 48$ & $271 \pm 57$ & $238 \pm 24$ \\
\hline RCP8.5 - mid & $234 \pm 14$ & $233 \pm 10$ & $235 \pm 9$ & $237 \pm 10$ & $241 \pm 18$ & $246 \pm 21$ & $271 \pm 47$ & $309 \pm 41$ & $322 \pm 49$ & $322 \pm 54$ & $255 \pm 54$ & $234 \pm 18$ \\
\hline RCP4.5 - end & $233 \pm 13$ & $234 \pm 12$ & $235 \pm 11$ & $237 \pm 10$ & $239 \pm 20$ & $245 \pm 28$ & $278 \pm 53$ & $326 \pm 58$ & $324 \pm 52$ & $335 \pm 68$ & $247 \pm 57$ & $233 \pm 25$ \\
\hline RCP8.5 - end & $239 \pm 12$ & $238 \pm 11$ & $234 \pm 10$ & $236 \pm 11$ & $239 \pm 13$ & $240 \pm 26$ & $266 \pm 51$ & $321 \pm 57$ & $324 \pm 51$ & $333 \pm 70$ & $258 \pm 48$ & $237 \pm 15$ \\
\hline
\end{tabular}


Appendix B42. Table showing return values of ensemble-average wind speeds of hindcast and forecast scenarios, including lower and higher 95 percent confidence intervals, at the Kingman Reef location.

[Years: Hindcast $=1976-2005 ;$ RCP mid $=$ 2026-2045; RCP end $=$ 2081-2100. Wind speeds are in meters per second]

\begin{tabular}{|c|c|c|c|c|c|c|c|c|c|c|c|c|c|c|c|}
\hline \multirow{2}{*}{ Scenario } & \multicolumn{3}{|c|}{ Hindcast } & \multicolumn{3}{c|}{ RCP4.5 - mid } & \multicolumn{3}{c|}{ RCP8.5 - mid } & \multicolumn{3}{c|}{ RCP4.5 - end } & \multicolumn{3}{c|}{ RCP8.5 - end } \\
\cline { 2 - 13 } & Low & $\boldsymbol{R}_{\mathbf{V}}$ & High & Low & $\boldsymbol{R}_{\mathbf{V}}$ & High & Low & $\boldsymbol{R}_{\mathbf{V}}$ & High & Low & $\boldsymbol{R}_{\mathbf{V}}$ & High & Low & $\boldsymbol{R}_{\mathbf{V}}$ & High \\
\hline 2-year & 14.32 & 15.32 & 16.96 & 13.94 & 14.87 & 16.55 & 13.89 & 14.76 & 16.28 & 14.09 & 15.28 & 17.65 & 13.62 & 14.11 & 14.87 \\
5-year & 15.03 & 16.80 & 20.04 & 14.41 & 16.02 & 19.38 & 14.32 & 15.75 & 18.59 & 14.62 & 16.76 & 21.81 & 13.87 & 14.59 & 15.84 \\
10-year & 15.57 & 18.10 & 23.18 & 14.75 & 17.02 & 22.31 & 14.61 & 16.55 & 20.80 & 15.01 & 18.12 & 26.47 & 14.02 & 14.94 & 16.62 \\
20-year & 16.12 & 19.59 & 27.24 & 15.09 & 18.15 & 26.14 & 14.88 & 17.41 & 23.50 & 15.40 & 19.72 & 33.04 & 14.15 & 15.26 & 17.44 \\
50-year & 16.85 & 21.89 & 34.48 & 15.51 & 19.87 & 33.12 & 15.21 & 18.62 & 28.02 & 15.91 & 22.28 & 46.03 & 14.28 & 15.66 & 18.59 \\
100-year & 17.41 & 23.91 & 41.84 & 15.82 & 21.37 & 40.34 & 15.43 & 19.61 & 32.35 & 16.29 & 24.63 & 60.60 & 14.37 & 15.94 & 19.51 \\
\hline
\end{tabular}


Appendix B43. Table showing Palmyra monthly means and mean of the top 5 percent for wind speed and mean wind direction.

[Years: Hindcast $=1976-2005 ;$ RCP mid $=$ 2026-2045; RCP end $=$ 2081-2100. Wind directions are “coming from”]

\begin{tabular}{|c|c|c|c|c|c|c|c|c|c|c|c|c|}
\hline Month & Jan & Feb & Mar & Apr & May & Jun & July & Aug & Sep & Oct & Nov & Dec \\
\hline \multicolumn{13}{|c|}{$\begin{array}{l}\text { Mean Wind Speed } \\
(\mathrm{m} / \mathrm{s})\end{array}$} \\
\hline Hindcast & $6.4 \pm 2.4$ & $6.7 \pm 2.3$ & $6.8 \pm 2.4$ & $6.1 \pm 2.3$ & $5.5 \pm 2.1$ & $5.3 \pm 2.0$ & $5.3 \pm 1.8$ & $5.2 \pm 1.7$ & $5.0 \pm 1.7$ & $4.7 \pm 1.8$ & $4.6 \pm 2.1$ & $5.3 \pm 2.2$ \\
\hline RCP4.5 - mid & $6.2 \pm 2.3$ & $6.9 \pm 2.3$ & $7.1 \pm 2.4$ & $6.6 \pm 2.3$ & $5.9 \pm 2.2$ & $5.5 \pm 2.0$ & $5.5 \pm 1.8$ & $5.3 \pm 1.8$ & $5.1 \pm 1.8$ & $4.8 \pm 1.8$ & $4.6 \pm 2.0$ & $5.2 \pm 2.3$ \\
\hline RCP8.5 - mid & $6.4 \pm 2.3$ & $6.9 \pm 2.4$ & $6.9 \pm 2.4$ & $6.6 \pm 2.3$ & $5.9 \pm 2.2$ & $5.6 \pm 1.9$ & $5.5 \pm 1.7$ & $5.2 \pm 1.8$ & $5.1 \pm 1.8$ & $4.7 \pm 1.8$ & $4.6 \pm 2.0$ & $5.2 \pm 2.3$ \\
\hline RCP 4.5 - end & $6.4 \pm 2.3$ & $6.8 \pm 2.3$ & $7.0 \pm 2.2$ & $6.6 \pm 2.2$ & $6.1 \pm 2.1$ & $5.5 \pm 2.0$ & $5.4 \pm 1.7$ & $5.2 \pm 1.8$ & $5.1 \pm 1.8$ & $4.8 \pm 1.8$ & $4.5 \pm 2.1$ & $5.4 \pm 2.4$ \\
\hline RCP8.5 - end & $6.5 \pm 2.2$ & $7.2 \pm 2.0$ & $7.1 \pm 2.2$ & $6.9 \pm 2.1$ & $6.4 \pm 2.1$ & $5.7 \pm 2.0$ & $5.4 \pm 1.8$ & $5.4 \pm 1.8$ & $5.1 \pm 1.7$ & $4.6 \pm 1.8$ & $4.4 \pm 1.9$ & $5.2 \pm 2.2$ \\
\hline \multicolumn{13}{|c|}{$\begin{array}{l}\text { Top } 5 \text { percent Wind } \\
\text { Speed }(\mathrm{m} / \mathrm{s})\end{array}$} \\
\hline Hindcast & $11.5 \pm 1.2$ & $11.5 \pm 0.8$ & $11.6 \pm 0.7$ & $10.9 \pm 0.7$ & $10.3 \pm 0.8$ & $9.8 \pm 1.1$ & $8.9 \pm 1.0$ & $8.9 \pm 1.3$ & $8.6 \pm 1.0$ & $8.6 \pm 1.2$ & $9.9 \pm 2.2$ & $10.4 \pm 1$ \\
\hline RCP4.5 - mid & $11.2 \pm 0.9$ & $11.4 \pm 0.6$ & $11.9 \pm 0.6$ & $11.2 \pm 0.7$ & $10.6 \pm 1.2$ & $10.3 \pm 1.0$ & $9.2 \pm 1.0$ & $9.0 \pm 1.5$ & $8.6 \pm 0.9$ & $8.5 \pm 1.0$ & $9.1 \pm 1.3$ & $10.6 \pm 1$. \\
\hline RCP8.5 - mid & $11.3 \pm 0.9$ & $11.7 \pm 0.9$ & $11.8 \pm 0.8$ & $11.2 \pm 0.7$ & $10.6 \pm 0.9$ & $9.9 \pm 0.7$ & $9.0 \pm 0.8$ & $8.7 \pm 0.8$ & $8.6 \pm 1.0$ & $8.7 \pm 1.3$ & $9.2 \pm 1.3$ & $10.6 \pm 1$. \\
\hline RCP4.5 - end & $11.3 \pm 0.9$ & $11.5 \pm 0.7$ & $11.4 \pm 0.7$ & $11.1 \pm 0.8$ & $10.9 \pm 0.9$ & $10.3 \pm 1.0$ & $9.0 \pm 1.2$ & $9.2 \pm 1.5$ & $8.8 \pm 1.2$ & $8.9 \pm 1.3$ & $9.5 \pm 2.0$ & $11.2 \pm 1$. \\
\hline RCP8.5 - end & $11.0 \pm 0.8$ & $11.2 \pm 0.7$ & $11.6 \pm 0.7$ & $11.3 \pm 0.7$ & $10.8 \pm 0.8$ & $10.4 \pm 0.9$ & $9.1 \pm 1.1$ & $9.1 \pm 1.2$ & $8.5 \pm 1.0$ & $8.5 \pm 1.1$ & $8.9 \pm 1.3$ & $10.7 \pm 1$. \\
\hline \multicolumn{13}{|l|}{$\begin{array}{l}\text { Mean Wind } \\
\text { Direction ( } 9\end{array}$} \\
\hline Hindcast & $255 \pm 32$ & $252 \pm 27$ & $253 \pm 25$ & $256 \pm 30$ & $264 \pm 32$ & $275 \pm 38$ & $289 \pm 37$ & $301 \pm 34$ & $310 \pm 35$ & $312 \pm 40$ & $299 \pm 48$ & $270 \pm 42$ \\
\hline RCP4.5 - mid & $256 \pm 31$ & $252 \pm 24$ & $251 \pm 22$ & $253 \pm 23$ & $258 \pm 30$ & $269 \pm 32$ & $287 \pm 35$ & $300 \pm 34$ & $307 \pm 38$ & $310 \pm 41$ & $297 \pm 49$ & $270 \pm 43$ \\
\hline RCP8.5 - mid & $255 \pm 31$ & $251 \pm 26$ & $251 \pm 22$ & $253 \pm 22$ & $258 \pm 26$ & $268 \pm 31$ & $286 \pm 33$ & $302 \pm 35$ & $308 \pm 38$ & $312 \pm 42$ & $298 \pm 48$ & $270 \pm 43$ \\
\hline RCP4.5 - end & $254 \pm 29$ & $252 \pm 26$ & $251 \pm 22$ & $253 \pm 21$ & $257 \pm 26$ & $269 \pm 33$ & $287 \pm 35$ & $303 \pm 38$ & $307 \pm 38$ & $309 \pm 42$ & $297 \pm 49$ & $267 \pm 42$ \\
\hline RCP8.5 - end & $254 \pm 26$ & $251 \pm 18$ & $250 \pm 18$ & $251 \pm 18$ & $255 \pm 23$ & $263 \pm 30$ & $284 \pm 35$ & $299 \pm 37$ & $308 \pm 39$ & $309 \pm 42$ & $291 \pm 45$ & $266 \pm 38$ \\
\hline \multicolumn{13}{|c|}{$\begin{array}{l}\text { Top } 5 \text { percent Wind } \\
\text { Direction ( } 9 \text { ) }\end{array}$} \\
\hline Hindcast & $234 \pm 20$ & $232 \pm 17$ & $233 \pm 11$ & $236 \pm 16$ & $244 \pm 23$ & $257 \pm 47$ & $286 \pm 44$ & $311 \pm 43$ & $316 \pm 41$ & $319 \pm 43$ & $289 \pm 66$ & $238 \pm 31$ \\
\hline RCP4.5 - mid & $234 \pm 18$ & $234 \pm 10$ & $232 \pm 10$ & $235 \pm 9$ & $235 \pm 29$ & $244 \pm 31$ & $276 \pm 47$ & $310 \pm 44$ & $307 \pm 38$ & $321 \pm 47$ & $274 \pm 55$ & $236 \pm 25$ \\
\hline RCP8.5 - mid & $230 \pm 16$ & $232 \pm 10$ & $233 \pm 9$ & $236 \pm 9$ & $240 \pm 19$ & $246 \pm 22$ & $276 \pm 44$ & $305 \pm 35$ & $312 \pm 43$ & $313 \pm 48$ & $261 \pm 58$ & $232 \pm 19$ \\
\hline RCP4.5 - end & $230 \pm 16$ & $231 \pm 11$ & $234 \pm 19$ & $235 \pm 10$ & $239 \pm 19$ & $245 \pm 27$ & $282 \pm 48$ & $319 \pm 53$ & $314 \pm 49$ & $328 \pm 65$ & $259 \pm 63$ & $231 \pm 28$ \\
\hline RCP8.5 - end & $235 \pm 12$ & $235 \pm 10$ & $232 \pm 9$ & $235 \pm 10$ & $238 \pm 11$ & $239 \pm 26$ & $273 \pm 49$ & $317 \pm 51$ & $312 \pm 45$ & $322 \pm 65$ & $261 \pm 48$ & $234 \pm 16$ \\
\hline
\end{tabular}


Appendix B44. Table showing return values of ensemble-average wind speeds of hindcast and forecast scenarios, including lower and higher 95 percent confidence intervals, at the Palmyra location.

[Years: Hindcast $=1976-2005 ;$ RCP mid $=$ 2026-2045; RCP end $=$ 2081-2100. Wind speeds are in meters per second]

\begin{tabular}{|c|c|c|c|c|c|c|c|c|c|c|c|c|c|c|c|}
\hline \multirow{2}{*}{ Scenario } & \multicolumn{3}{|c|}{ Hindcast } & \multicolumn{3}{c|}{ RCP4.5 - mid } & \multicolumn{3}{c|}{ RCP8.5 - mid } & \multicolumn{3}{c|}{ RCP4.5 - end } & \multicolumn{3}{c|}{ RCP8.5 - end } \\
\cline { 2 - 14 } & Low & $\boldsymbol{R}_{\mathbf{V}}$ & High & Low & $\boldsymbol{R}_{\mathbf{V}}$ & High & Low & $\boldsymbol{R}_{\mathbf{V}}$ & High & Low & $\boldsymbol{R}_{\mathbf{V}}$ & High & Low & $\boldsymbol{R}_{\mathbf{V}}$ & High \\
\hline 2-year & 14.40 & 15.43 & 17.07 & 13.95 & 14.98 & 16.91 & 13.84 & 14.78 & 16.45 & 14.07 & 15.21 & 17.39 & 13.63 & 14.09 & 14.79 \\
5-year & 15.17 & 16.96 & 20.11 & 14.48 & 16.32 & 20.30 & 14.22 & 15.68 & 18.60 & 14.61 & 16.64 & 21.14 & 13.88 & 14.57 & 15.69 \\
10-year & 15.75 & 18.28 & 23.10 & 14.88 & 17.53 & 23.96 & 14.45 & 16.35 & 20.49 & 15.02 & 17.92 & 25.20 & 14.04 & 14.90 & 16.38 \\
20-year & 16.33 & 19.75 & 26.86 & 15.28 & 18.94 & 28.95 & 14.65 & 17.01 & 22.67 & 15.41 & 19.40 & 30.75 & 14.17 & 15.21 & 17.08 \\
50-year & 17.09 & 21.96 & 33.32 & 15.80 & 21.16 & 38.42 & 14.88 & 17.87 & 26.02 & 15.93 & 21.73 & 41.31 & 14.32 & 15.58 & 18.03 \\
100-year & 17.66 & 23.87 & 39.67 & 16.19 & 23.17 & 48.64 & 15.02 & 18.51 & 28.99 & 16.31 & 23.81 & 52.73 & 14.41 & 15.83 & 18.76 \\
\hline
\end{tabular}


Appendix B45. Table showing Rose Atoll monthly means and mean of the top 5 percent for wind speed and mean wind direction.

[Years: Hindcast $=1976-2005 ;$ RCP mid $=$ 2026-2045; RCP end $=$ 2081-2100. Wind directions are “coming from”]

\begin{tabular}{|c|c|c|c|c|c|c|c|c|c|c|c|c|}
\hline Month & Jan & Feb & Mar & Apr & May & Jun & July & Aug & Sep & Oct & Nov & Dec \\
\hline \multicolumn{13}{|c|}{$\begin{array}{l}\text { Mean Wind Speed } \\
(\mathrm{m} / \mathrm{s})\end{array}$} \\
\hline Hindcast & $5.2 \pm 2.9$ & $4.9 \pm 2.8$ & $5.1 \pm 2.8$ & $5.4 \pm 2.7$ & $6.3 \pm 2.9$ & $6.9 \pm 2.7$ & $6.9 \pm 2.7$ & $7.1 \pm 2.7$ & $7.0 \pm 2.7$ & $6.7 \pm 2.7$ & $6.0 \pm 2.8$ & $5.3 \pm 2.6$ \\
\hline RCP4.5 - mid & $5.3 \pm 2.8$ & $5.1 \pm 2.8$ & $5.3 \pm 2.7$ & $5.5 \pm 2.7$ & $6.5 \pm 2.9$ & $7.0 \pm 2.7$ & $7.0 \pm 2.7$ & $7.1 \pm 2.6$ & $7.3 \pm 2.7$ & $7.0 \pm 2.7$ & $6.2 \pm 2.9$ & $5.3 \pm 2.6$ \\
\hline RCP8.5 - mid & $5.3 \pm 2.8$ & $5.1 \pm 2.7$ & $5.1 \pm 2.9$ & $5.5 \pm 2.8$ & $6.3 \pm 2.8$ & $6.7 \pm 2.7$ & $6.9 \pm 2.7$ & $7.0 \pm 2.7$ & $7.2 \pm 2.6$ & $7.0 \pm 2.7$ & $6.0 \pm 2.7$ & $5.4 \pm 3.0$ \\
\hline RCP 4.5 - end & $5.3 \pm 2.7$ & $5.2 \pm 2.7$ & $4.8 \pm 2.7$ & $5.4 \pm 2.8$ & $6.6 \pm 2.9$ & $7.2 \pm 2.6$ & $6.9 \pm 2.7$ & $7.2 \pm 2.6$ & $7.3 \pm 2.6$ & $6.9 \pm 2.7$ & $6.1 \pm 2.7$ & $5.5 \pm 2.8$ \\
\hline RCP8.5 - end & $5.5 \pm 2.7$ & $5.3 \pm 2.9$ & $5.2 \pm 2.7$ & $5.2 \pm 2.6$ & $6.2 \pm 2.8$ & $7.0 \pm 2.8$ & $7.0 \pm 2.7$ & $7.3 \pm 2.6$ & $7.3 \pm 2.6$ & $7.0 \pm 2.6$ & $6.0 \pm 2.6$ & $5.4 \pm 2.5$ \\
\hline \multicolumn{13}{|c|}{$\begin{array}{l}\text { Top } 5 \text { percent Wind } \\
\text { Speed }(\mathrm{m} / \mathrm{s})\end{array}$} \\
\hline Hindcast & $13.2 \pm 2.6$ & $12.5 \pm 2.9$ & $12.2 \pm 2.2$ & $11.6 \pm 1.3$ & $12.9 \pm 1.6$ & $12.9 \pm 1.6$ & $12.6 \pm 1.1$ & $12.8 \pm 1.1$ & $12.5 \pm 1.1$ & $12.5 \pm 1.2$ & $12.4 \pm 1.3$ & $11.8 \pm 2$. \\
\hline RCP4.5 - mid & $12.6 \pm 2.4$ & $12.6 \pm 2.8$ & $11.8 \pm 2.1$ & $11.9 \pm 1.5$ & $12.6 \pm 1.4$ & $12.6 \pm 1.0$ & $12.5 \pm 0.8$ & $12.4 \pm 1.0$ & $12.8 \pm 1.0$ & $12.5 \pm 0.8$ & $12.6 \pm 1.5$ & $11.6 \pm 1.7$ \\
\hline RCP8.5 - mid & $12.4 \pm 2.5$ & $12.3 \pm 2.3$ & $12.8 \pm 2.9$ & $12.2 \pm 1.5$ & $12.4 \pm 1.0$ & $12.3 \pm 1.2$ & $12.8 \pm 1.4$ & $12.7 \pm 1.0$ & $12.6 \pm 0.8$ & $12.5 \pm 1.0$ & $12.0 \pm 1.5$ & $13.5 \pm 2.7$ \\
\hline RCP4.5 - end & $12.2 \pm 2.2$ & $12.0 \pm 2.3$ & $11.9 \pm 2.4$ & $12.3 \pm 2.2$ & $12.9 \pm 1.5$ & $12.6 \pm 1.1$ & $12.7 \pm 1.1$ & $12.5 \pm 1.0$ & $12.6 \pm 1.0$ & $12.6 \pm 1.1$ & $11.9 \pm 1.3$ & $12.5 \pm 2$. \\
\hline RCP8.5 - end & $12.7 \pm 2.9$ & $13.2 \pm 2.5$ & $12.5 \pm 2.7$ & $11.3 \pm 1.7$ & $12.7 \pm 1.7$ & $13.0 \pm 1.2$ & $12.6 \pm 0.9$ & $12.9 \pm 1.0$ & $12.6 \pm 1.0$ & $12.1 \pm 0.7$ & $11.7 \pm 1.2$ & $11.4 \pm 1$. \\
\hline \multicolumn{13}{|l|}{$\begin{array}{l}\text { Mean Wind } \\
\left.\text { Direction ( }{ }^{\circ}\right)\end{array}$} \\
\hline Hindcast & $242 \pm 80$ & $222 \pm 94$ & $233 \pm 99$ & $280 \pm 69$ & $284 \pm 53$ & $285 \pm 42$ & $285 \pm 43$ & $283 \pm 39$ & $285 \pm 38$ & $284 \pm 39$ & $280 \pm 51$ & $267 \pm 65$ \\
\hline RCP4.5 - mid & $244 \pm 76$ & $217 \pm 92$ & $221 \pm 10$ & $283 \pm 72$ & $286 \pm 48$ & $284 \pm 42$ & $284 \pm 43$ & $284 \pm 38$ & $285 \pm 34$ & $285 \pm 37$ & $283 \pm 49$ & $270 \pm 66$ \\
\hline RCP8.5 - mid & $250 \pm 68$ & $223 \pm 92$ & $240 \pm 10$ & $284 \pm 70$ & $287 \pm 49$ & $286 \pm 44$ & $283 \pm 43$ & $286 \pm 41$ & $284 \pm 36$ & $283 \pm 36$ & $279 \pm 48$ & $264 \pm 69$ \\
\hline RCP4.5 - end & $252 \pm 73$ & $222 \pm 90$ & $233 \pm 10$ & $278 \pm 73$ & $286 \pm 48$ & $283 \pm 39$ & $284 \pm 43$ & $285 \pm 37$ & $285 \pm 33$ & $284 \pm 35$ & $280 \pm 47$ & $267 \pm 62$ \\
\hline RCP8.5 - end & $253 \pm 66$ & $234 \pm 88$ & $235^{2} \pm 95$ & $278 \pm 69$ & $283 \pm 49$ & $285 \pm 40$ & $285 \pm 39$ & $285 \pm 34$ & $285 \pm 31$ & $283 \pm 34$ & $277 \pm 43$ & $269 \pm 54$ \\
\hline \multicolumn{13}{|c|}{$\begin{array}{l}\text { Top } 5 \text { percent Wind } \\
\text { Direction ( } 9 \text { ) }\end{array}$} \\
\hline Hindcast & $208 \pm 87$ & $179 \pm 90$ & $188 \pm 92$ & $289 \pm 57$ & $292 \pm 34$ & $295 \pm 31$ & $295 \pm 24$ & $292 \pm 24$ & $293 \pm 28$ & $296 \pm 24$ & $288 \pm 40$ & $275 \pm 65$ \\
\hline RCP4.5 - mid & $195 \pm 78$ & $169 \pm 68$ & $162 \pm 85$ & $294 \pm 55$ & $294 \pm 32$ & $290 \pm 32$ & $296 \pm 23$ & $297 \pm 22$ & $294 \pm 23$ & $294 \pm 20$ & $289 \pm 40$ & $281 \pm 57$ \\
\hline RCP8.5 - mid & $226 \pm 72$ & $152 \pm 78$ & $180 \pm 10$ & $296 \pm 46$ & $294 \pm 33$ & $295 \pm 33$ & $293 \pm 31$ & $298 \pm 23$ & $295 \pm 21$ & $293 \pm 25$ & $284 \pm 33$ & $279 \pm 59$ \\
\hline RCP4.5 - end & $229 \pm 84$ & $171 \pm 92$ & $167 \pm 83$ & $267 \pm 74$ & $297 \pm 32$ & $295 \pm 25$ & $297 \pm 26$ & $296 \pm 22$ & $298 \pm 18$ & $297 \pm 17$ & $292 \pm 32$ & $284 \pm 52$ \\
\hline RCP8.5 - end & $233 \pm 85$ & $160 \pm 89$ & $171 \pm 79$ & $285 \pm 61$ & $290 \pm 40$ & $297 \pm 25$ & $297 \pm 23$ & $298 \pm 24$ & $296 \pm 19$ & $294 \pm 16$ & $288 \pm 33$ & $286 \pm 37$ \\
\hline
\end{tabular}


Appendix B46. Table showing return values of ensemble-average wind speeds of hindcast and forecast scenarios, including lower and higher 95 percent confidence intervals, at the Rose Atoll location.

[Years: Hindcast $=1976-2005 ;$ RCP mid $=$ 2026-2045; RCP end $=$ 2081-2100. Wind speeds are in meters per second]

\begin{tabular}{|c|c|c|c|c|c|c|c|c|c|c|c|c|c|c|c|c|}
\hline \multirow{2}{*}{ Scenario } & \multicolumn{3}{|c|}{ Hindcast } & \multicolumn{3}{c|}{ RCP4.5 - mid } & \multicolumn{3}{c|}{ RCP8.5 - mid } & \multicolumn{3}{c|}{ RCP4.5 - end } & \multicolumn{2}{c|}{ RCP8.5 - end } \\
\cline { 2 - 13 } & Low & $R_{V}$ & High & Low & $\boldsymbol{R}_{\boldsymbol{V}}$ & High & Low & $\boldsymbol{R}_{\boldsymbol{V}}$ & High & Low & $\boldsymbol{R}_{\boldsymbol{V}}$ & High & Low & $\boldsymbol{R}_{\boldsymbol{V}}$ & High \\
\hline 2-year & 18.65 & 20.55 & 23.72 & 18.11 & 20.16 & 23.75 & 18.97 & 21.22 & 25.02 & 17.60 & 20.34 & 26.35 & 18.34 & 20.72 & 25.21 \\
5-year & 19.76 & 22.95 & 28.88 & 18.98 & 22.21 & 28.61 & 19.91 & 23.31 & 29.68 & 18.46 & 23.17 & 35.74 & 19.16 & 22.91 & 31.03 \\
10-year & 20.54 & 24.91 & 33.80 & 19.54 & 23.77 & 32.99 & 20.48 & 24.80 & 33.57 & 19.05 & 25.65 & 46.14 & 19.68 & 24.57 & 36.39 \\
20-year & 21.28 & 27.01 & 39.79 & 20.03 & 25.35 & 38.09 & 20.96 & 26.22 & 37.78 & 19.59 & 28.44 & 60.62 & 20.12 & 26.25 & 42.76 \\
50-year & 22.18 & 30.01 & 49.78 & 20.58 & 27.46 & 46.14 & 21.47 & 27.99 & 43.91 & 20.24 & 32.70 & 88.84 & 20.61 & 28.47 & 53.04 \\
100-year & 22.82 & 32.47 & 59.29 & 20.94 & 29.06 & 53.39 & 21.78 & 29.25 & 49.02 & 20.68 & 36.41 & 120.09 & 20.91 & 30.16 & 62.51 \\
\hline
\end{tabular}


Appendix B47. Table showing Howland monthly means and mean of the top 5 percent for wind speed and mean wind direction.

[Years: Hindcast $=1976-2005 ;$ RCP mid $=$ 2026-2045; RCP end $=$ 2081-2100. Wind directions are “coming from”]

\begin{tabular}{|c|c|c|c|c|c|c|c|c|c|c|c|c|}
\hline Month & Jan & Feb & Mar & Apr & May & Jun & July & Aug & Sep & Oct & Nov & Dec \\
\hline \multicolumn{13}{|c|}{$\begin{array}{l}\text { Mean Wind Speed } \\
(\mathrm{m} / \mathrm{s})\end{array}$} \\
\hline Hindcast & $5.7 \pm 1.9$ & $5.9 \pm 1.7$ & $5.5 \pm 1.6$ & $5.1 \pm 1.6$ & $4.8 \pm 1.8$ & $5.2 \pm 1.9$ & $6.0 \pm 1.8$ & $6.1 \pm 1.8$ & $6.0 \pm 1.9$ & $5.5 \pm 1.9$ & $5.3 \pm 2.0$ & $5.3 \pm 2.1$ \\
\hline RCP4.5 - mid & $5.6 \pm 2.0$ & $5.9 \pm 1.7$ & $5.5 \pm 1.7$ & $5.2 \pm 1.7$ & $4.8 \pm 1.8$ & $5.1 \pm 1.9$ & $6.0 \pm 1.9$ & $6.1 \pm 1.8$ & $6.0 \pm 1.8$ & $5.6 \pm 2.0$ & $5.1 \pm 2.1$ & $5.2 \pm 2.0$ \\
\hline RCP8.5 - mid & $5.8 \pm 1.9$ & $6.0 \pm 1.7$ & $5.5 \pm 1.7$ & $5.0 \pm 1.6$ & $4.6 \pm 1.7$ & $5.1 \pm 1.9$ & $6.0 \pm 1.9$ & $6.1 \pm 1.8$ & $6.0 \pm 1.8$ & $5.6 \pm 1.9$ & $5.4 \pm 2.0$ & $5.4 \pm 2.0$ \\
\hline RCP 4.5 - end & $5.8 \pm 1.9$ & $5.9 \pm 1.8$ & $5.6 \pm 1.7$ & $5.0 \pm 1.7$ & $4.8 \pm 1.8$ & $5.2 \pm 1.9$ & $5.9 \pm 1.9$ & $6.1 \pm 1.8$ & $6.0 \pm 1.8$ & $5.6 \pm 1.9$ & $5.3 \pm 2.0$ & $5.4 \pm 2.2$ \\
\hline RCP8.5 - end & $5.8 \pm 1.8$ & $6.0 \pm 1.7$ & $5.6 \pm 1.7$ & $5.1 \pm 1.8$ & $4.9 \pm 1.8$ & $5.2 \pm 1.9$ & $6.2 \pm 2.0$ & $6.5 \pm 1.9$ & $6.3 \pm 1.8$ & $5.9 \pm 1.9$ & $5.5 \pm 1.8$ & $5.5 \pm 1.9$ \\
\hline \multicolumn{13}{|c|}{$\begin{array}{l}\text { Top } 5 \text { percent Wind } \\
\text { Speed }(\mathrm{m} / \mathrm{s})\end{array}$} \\
\hline Hindcast & $9.6 \pm 0.9$ & $9.5 \pm 0.8$ & $8.7 \pm 0.6$ & $8.2 \pm 0.8$ & $8.6 \pm 1.1$ & $8.9 \pm 0.8$ & $9.3 \pm 0.8$ & $9.4 \pm 0.6$ & $9.4 \pm 0.7$ & $9.2 \pm 0.8$ & $9.4 \pm 1.1$ & $9.8 \pm 1.4$ \\
\hline RCP4.5 - mid & $9.5 \pm 0.7$ & $9.5 \pm 0.8$ & $9.0 \pm 0.9$ & $8.3 \pm 0.6$ & $8.4 \pm 0.9$ & $8.8 \pm 0.6$ & $9.5 \pm 0.6$ & $9.7 \pm 0.6$ & $9.6 \pm 1.2$ & $9.4 \pm 0.6$ & $9.3 \pm 1.3$ & $9.1 \pm 0.7$ \\
\hline RCP8.5 - mid & $9.5 \pm 0.6$ & $9.6 \pm 0.8$ & $8.8 \pm 0.6$ & $8.2 \pm 0.6$ & $7.9 \pm 0.6$ & $8.9 \pm 0.7$ & $9.6 \pm 0.7$ & $9.6 \pm 0.8$ & $9.5 \pm 0.7$ & $9.2 \pm 0.7$ & $9.6 \pm 1.1$ & $9.4 \pm 1.2$ \\
\hline RCP4.5 - end & $9.7 \pm 1.0$ & $9.5 \pm 0.7$ & $8.8 \pm 0.6$ & $8.1 \pm 0.5$ & $8.4 \pm 0.9$ & $9.1 \pm 0.8$ & $9.6 \pm 1.0$ & $9.5 \pm 0.7$ & $9.4 \pm 0.7$ & $9.4 \pm 0.7$ & $9.5 \pm 1.3$ & $10.1 \pm 1.3$ \\
\hline RCP8.5 - end & $9.5 \pm 0.8$ & $9.3 \pm 0.7$ & $8.9 \pm 0.8$ & $8.5 \pm 0.7$ & $8.5 \pm 1.4$ & $9.0 \pm 0.8$ & $9.7 \pm 0.7$ & $9.7 \pm 0.6$ & $9.5 \pm 0.7$ & $9.6 \pm 1.5$ & $8.9 \pm 0.6$ & $9.2 \pm 0.8$ \\
\hline \multicolumn{13}{|l|}{$\begin{array}{l}\text { Mean Wind } \\
\left.\text { Direction ( }{ }^{\circ}\right)\end{array}$} \\
\hline Hindcast & $256 \pm 33$ & $254 \pm 28$ & $258 \pm 29$ & $268 \pm 33$ & $275 \pm 38$ & $278 \pm 36$ & $279 \pm 28$ & $280 \pm 27$ & $280 \pm 32$ & $280 \pm 35$ & $277 \pm 41$ & $268 \pm 37$ \\
\hline RCP4.5 - mid & $258 \pm 32$ & $254 \pm 34$ & $255 \pm 35$ & $265 \pm 35$ & $272 \pm 40$ & $275 \pm 37$ & $278 \pm 31$ & $278 \pm 26$ & $279 \pm 27$ & $278 \pm 32$ & $277 \pm 38$ & $268 \pm 36$ \\
\hline RCP8.5 - mid & $258 \pm 32$ & $255 \pm 28$ & $258 \pm 30$ & $266 \pm 31$ & $272 \pm 36$ & $276 \pm 34$ & $277 \pm 29$ & $279 \pm 27$ & $279 \pm 31$ & $279 \pm 37$ & $277 \pm 40$ & $266 \pm 37$ \\
\hline RCP4.5 - end & $255 \pm 33$ & $253 \pm 29$ & $254 \pm 31$ & $264 \pm 33$ & $270 \pm 42$ & $274 \pm 37$ & $276 \pm 30$ & $279 \pm 30$ & $279 \pm 32$ & $279 \pm 38$ & $276 \pm 41$ & $266 \pm 39$ \\
\hline RCP8.5 - end & $258 \pm 31$ & $254 \pm 25$ & $258 \pm 29$ & $263 \pm 33$ & $268 \pm 37$ & $271 \pm 35$ & $277 \pm 35$ & $278 \pm 31$ & $280 \pm 31$ & $280 \pm 34$ & $277 \pm 34$ & $268 \pm 31$ \\
\hline \multicolumn{13}{|c|}{$\begin{array}{l}\text { Top } 5 \text { percent Wind } \\
\text { Direction ( } 9\end{array}$} \\
\hline Hindcast & $234 \pm 40$ & $232 \pm 30$ & $238 \pm 27$ & $260 \pm 31$ & $282 \pm 53$ & $286 \pm 35$ & $283 \pm 28$ & $280 \pm 17$ & $279 \pm 23$ & $276 \pm 36$ & $284 \pm 72$ & $258 \pm 56$ \\
\hline RCP4.5 - mid & $251 \pm 28$ & $235 \pm 48$ & $235 \pm 41$ & $260 \pm 34$ & $282 \pm 49$ & $284 \pm 30$ & $281 \pm 22$ & $277 \pm 12$ & $280 \pm 27$ & $275 \pm 19$ & $280 \pm 43$ & $264 \pm 29$ \\
\hline RCP8.5 - mid & $238 \pm 30$ & $235 \pm 25$ & $241 \pm 23$ & $262 \pm 31$ & $279 \pm 24$ & $281 \pm 22$ & $278 \pm 17$ & $279 \pm 21$ & $279 \pm 18$ & $277 \pm 33$ & $284 \pm 72$ & $240 \pm 55$ \\
\hline RCP4.5 - end & $232 \pm 35$ & $229 \pm 26$ & $235 \pm 26$ & $260 \pm 23$ & $273 \pm 54$ & $283 \pm 40$ & $281 \pm 29$ & $279 \pm 28$ & $277 \pm 31$ & $276 \pm 40$ & $284 \pm 68$ & $247 \pm 66$ \\
\hline RCP8.5 - end & $239 \pm 31$ & $236 \pm 26$ & $243 \pm 21$ & $260 \pm 33$ & $272 \pm 53$ & $276 \pm 30$ & $277 \pm 32$ & $274 \pm 21$ & $276 \pm 39$ & $277 \pm 46$ & $273 \pm 32$ & $254 \pm 36$ \\
\hline
\end{tabular}


Appendix B48. Table showing return values of ensemble-average wind speeds of hindcast and forecast scenarios, including lower and higher 95 percent confidence intervals, at the Howland location.

[Years: Hindcast $=1976-2005 ;$ RCP mid $=$ 2026-2045; RCP end $=$ 2081-2100. Wind speeds are in meters per second]

\begin{tabular}{|c|c|c|c|c|c|c|c|c|c|c|c|c|c|c|c|}
\hline \multirow{2}{*}{ Scenario } & \multicolumn{3}{|c|}{ Hindcast } & \multicolumn{3}{c|}{ RCP4.5 - mid } & \multicolumn{3}{c|}{ RCP8.5 - mid } & \multicolumn{3}{c|}{ RCP4.5 - end } & \multicolumn{3}{c|}{ RCP8.5 - end } \\
\cline { 2 - 14 } & Low & $\boldsymbol{R}_{\mathbf{V}}$ & High & Low & $\boldsymbol{R}_{\mathbf{V}}$ & High & Low & $\boldsymbol{R}_{\mathbf{V}}$ & High & Low & $\boldsymbol{R}_{\mathbf{V}}$ & High & Low & $\boldsymbol{R}_{\mathbf{V}}$ & High \\
\hline 2-year & 12.32 & 13.36 & 15.21 & 12.22 & 13.15 & 14.77 & 11.93 & 12.83 & 14.64 & 12.63 & 13.84 & 16.07 & 12.09 & 13.17 & 15.36 \\
5-year & 12.93 & 14.81 & 18.74 & 12.78 & 14.43 & 17.70 & 12.32 & 13.95 & 17.82 & 13.15 & 15.16 & 19.40 & 12.54 & 14.48 & 19.19 \\
10-year & 13.39 & 16.16 & 22.60 & 13.21 & 15.58 & 20.77 & 12.62 & 14.98 & 21.41 & 13.51 & 16.24 & 22.66 & 12.88 & 15.69 & 23.52 \\
20-year & 13.87 & 17.75 & 27.94 & 13.64 & 16.90 & 24.85 & 12.91 & 16.19 & 26.49 & 13.84 & 17.39 & 26.74 & 13.21 & 17.11 & 29.69 \\
50-year & 14.51 & 20.32 & 38.23 & 14.22 & 18.98 & 32.35 & 13.29 & 18.14 & 36.57 & 14.23 & 19.04 & 33.76 & 13.64 & 19.40 & 41.98 \\
100-year & 15.01 & 22.68 & 49.51 & 14.66 & 20.84 & 40.20 & 13.57 & 19.93 & 47.95 & 14.49 & 20.40 & 40.63 & 13.96 & 21.51 & 55.91 \\
\hline
\end{tabular}


Appendix B49. Table showing Jarvis monthly means and mean of the top 5 percent for wind speed and mean wind direction.

[Years: Hindcast $=1976-2005 ;$ RCP mid $=$ 2026-2045; RCP end $=$ 2081-2100. Wind directions are “coming from”]

\begin{tabular}{|c|c|c|c|c|c|c|c|c|c|c|c|c|}
\hline Month & Jan & Feb & Mar & Apr & May & Jun & July & Aug & Sep & Oct & Nov & Dec \\
\hline \multicolumn{13}{|c|}{$\begin{array}{l}\text { Mean Wind Speed } \\
(\mathrm{m} / \mathrm{s})\end{array}$} \\
\hline Hindcast & $6.3 \pm 1.9$ & $6.3 \pm 1.7$ & $6.0 \pm 1.6$ & $5.7 \pm 1.7$ & $5.5 \pm 1.7$ & $6.1 \pm 1.8$ & $6.7 \pm 1.8$ & $6.7 \pm 1.7$ & $6.5 \pm 1.7$ & $6.2 \pm 1.7$ & $6.0 \pm 1.8$ & $6.0 \pm 1.9$ \\
\hline RCP4.5 - mid & $6.1 \pm 1.9$ & $6.0 \pm 1.8$ & $5.8 \pm 1.7$ & $5.6 \pm 1.7$ & $5.3 \pm 1.9$ & $6.0 \pm 1.8$ & $6.7 \pm 1.8$ & $6.7 \pm 1.6$ & $6.4 \pm 1.5$ & $6.0 \pm 1.7$ & $5.9 \pm 1.8$ & $5.9 \pm 1.8$ \\
\hline RCP8.5 - mid & $6.3 \pm 1.8$ & $6.2 \pm 1.7$ & $5.8 \pm 1.7$ & $5.5 \pm 1.7$ & $5.3 \pm 1.7$ & $6.0 \pm 1.8$ & $6.7 \pm 1.8$ & $6.6 \pm 1.7$ & $6.4 \pm 1.7$ & $6.1 \pm 1.7$ & $5.8 \pm 1.8$ & $5.8 \pm 1.9$ \\
\hline RCP4.5 - end & $6.2 \pm 1.8$ & $6.1 \pm 1.7$ & $5.8 \pm 1.7$ & $5.5 \pm 1.8$ & $5.3 \pm 1.7$ & $6.0 \pm 1.8$ & $6.6 \pm 1.7$ & $6.7 \pm 1.7$ & $6.5 \pm 1.6$ & $6.2 \pm 1.7$ & $5.8 \pm 1.8$ & $5.8 \pm 1.9$ \\
\hline \multicolumn{13}{|c|}{$\begin{array}{l}\text { Top } 5 \text { percent Wind } \\
\text { Speed }(\mathrm{m} / \mathrm{s})\end{array}$} \\
\hline Hindcast & $10.1 \pm 1.0$ & $9.6 \pm 0.8$ & $9.1 \pm 0.7$ & $9.0 \pm 1.0$ & $8.8 \pm 0.7$ & $9.4 \pm 0.7$ & $9.7 \pm 0.5$ & $9.8 \pm 0.5$ & $9.5 \pm 0.6$ & $9.4 \pm 0.5$ & $10.0 \pm 1.7$ & $9.8 \pm 0.7$ \\
\hline RCP4.5 - mid & $9.6 \pm 0.7$ & $9.4 \pm 0.8$ & $8.9 \pm 0.6$ & $8.7 \pm 0.6$ & $8.9 \pm 1.0$ & $9.5 \pm 0.7$ & $9.8 \pm 0.6$ & $9.6 \pm 0.5$ & $9.0 \pm 0.4$ & $9.2 \pm 0.6$ & $9.6 \pm 0.7$ & $9.7 \pm 0.8$ \\
\hline RCP8.5 - mid & $9.7 \pm 0.7$ & $9.3 \pm 0.8$ & $8.9 \pm 0.8$ & $8.6 \pm 0.7$ & $8.6 \pm 0.7$ & $9.4 \pm 0.6$ & $9.9 \pm 0.8$ & $9.5 \pm 0.6$ & $9.3 \pm 0.6$ & $9.2 \pm 0.6$ & $9.4 \pm 0.6$ & $9.5 \pm 0.7$ \\
\hline RCP4.5 - end & $9.8 \pm 0.8$ & $9.5 \pm 0.7$ & $9.2 \pm 1.0$ & $8.8 \pm 0.7$ & $8.6 \pm 0.7$ & $9.5 \pm 0.8$ & $9.6 \pm 0.5$ & $9.7 \pm 0.6$ & $9.2 \pm 0.6$ & $9.3 \pm 1.0$ & $9.4 \pm 0.7$ & $9.7 \pm 0.8$ \\
\hline \multicolumn{13}{|l|}{$\begin{array}{l}\text { Mean Wind } \\
\left.\text { Direction ( }{ }^{\circ}\right)\end{array}$} \\
\hline Hindcast & $259 \pm 29$ & $255 \pm 28$ & $258 \pm 27$ & $261 \pm 26$ & $263 \pm 27$ & $266 \pm 24$ & $268 \pm 23$ & $270 \pm 21$ & $270 \pm 21$ & $270 \pm 22$ & $270 \pm 28$ & $265 \pm 28$ \\
\hline RCP4.5 - mid & $261 \pm 24$ & $258 \pm 29$ & $257 \pm 33$ & $257 \pm 34$ & $259 \pm 34$ & $265 \pm 23$ & $267 \pm 21$ & $269 \pm 20$ & $270 \pm 21$ & $269 \pm 22$ & $269 \pm 25$ & $266 \pm 22$ \\
\hline RCP8.5 - mid & $259 \pm 31$ & $256 \pm 31$ & $256 \pm 30$ & $258 \pm 30$ & $262 \pm 28$ & $264 \pm 23$ & $268 \pm 22$ & $269 \pm 21$ & $270 \pm 20$ & $270 \pm 23$ & $269 \pm 27$ & $265 \pm 30$ \\
\hline RCP4.5 - end & $259 \pm 33$ & $256 \pm 29$ & $256 \pm 29$ & $257 \pm 30$ & $260 \pm 31$ & $265 \pm 24$ & $268 \pm 24$ & $271 \pm 23$ & $271 \pm 23$ & $271 \pm 26$ & $269 \pm 30$ & $265 \pm 30$ \\
\hline $\begin{array}{l}\text { RCP8.5 - end } \\
\text { Top } 5 \text { percent } \\
\left.\text { Direction ( }{ }^{9}\right)\end{array}$ & $259 \pm 26$ & $256 \pm 27$ & $256 \pm 27$ & $257 \pm 28$ & $259 \pm 29$ & $264 \pm 23$ & $270 \pm 23$ & $271 \pm 20$ & $272 \pm 21$ & $271 \pm 26$ & $272 \pm 26$ & $267 \pm 27$ \\
\hline Hindcast & $252 \pm 40$ & $251 \pm 33$ & $255 \pm 29$ & $261 \pm 30$ & $266 \pm 18$ & $266 \pm 15$ & $271 \pm 16$ & $273 \pm 16$ & $271 \pm 17$ & $269 \pm 15$ & $269 \pm 50$ & $261 \pm 30$ \\
\hline RCP4.5 - mid & $260 \pm 26$ & $256 \pm 33$ & $258 \pm 39$ & $263 \pm 29$ & $266 \pm 33$ & $268 \pm 13$ & $268 \pm 12$ & $268 \pm 14$ & $270 \pm 14$ & $268 \pm 16$ & $266 \pm 26$ & $262 \pm 15$ \\
\hline RCP8.5 - mid & $252 \pm 38$ & $250 \pm 37$ & $251 \pm 28$ & $261 \pm 37$ & $267 \pm 20$ & $265 \pm 10$ & $268 \pm 11$ & $269 \pm 13$ & $271 \pm 13$ & $269 \pm 15$ & $267 \pm 17$ & $259 \pm 30$ \\
\hline RCP4.5 - end & $251 \pm 44$ & $246 \pm 32$ & $249 \pm 41$ & $257 \pm 25$ & $264 \pm 26$ & $266 \pm 11$ & $268 \pm 11$ & $269 \pm 17$ & $270 \pm 17$ & $268 \pm 34$ & $271 \pm 56$ & $262 \pm 28$ \\
\hline RCP8.5 - end & $244 \pm 38$ & $240 \pm 32$ & $252 \pm 22$ & $262 \pm 16$ & $266 \pm 19$ & $264 \pm 12$ & $267 \pm 11$ & $270 \pm 14$ & $269 \pm 14$ & $270 \pm 30$ & $270 \pm 13$ & $262 \pm 25$ \\
\hline
\end{tabular}


Appendix B50. Table showing return values of ensemble-average wind speeds of hindcast and forecast scenarios, including lower and higher 95 percent confidence intervals, at the Jarvis location.

[Years: Hindcast $=1976-2005 ;$ RCP mid $=$ 2026-2045; RCP end $=$ 2081-2100. Wind speeds are in meters per second]

\begin{tabular}{|c|c|c|c|c|c|c|c|c|c|c|c|c|c|c|c|}
\hline \multirow{2}{*}{ Scenario } & \multicolumn{3}{|c|}{ Hindcast } & \multicolumn{3}{c|}{ RCP4.5 - mid } & \multicolumn{3}{c|}{ RCP8.5 - mid } & \multicolumn{3}{c|}{ RCP4.5 - end } & \multicolumn{3}{c|}{ RCP8.5 - end } \\
\cline { 2 - 14 } & Low & $\boldsymbol{R}_{\mathbf{V}}$ & High & Low & $\boldsymbol{R}_{\mathbf{V}}$ & High & Low & $\boldsymbol{R}_{\mathbf{V}}$ & High & Low & $\boldsymbol{R}_{\mathbf{V}}$ & High & Low & $\boldsymbol{R}_{\mathbf{V}}$ & High \\
\hline 2-year & 12.25 & 13.09 & 14.64 & 11.93 & 12.75 & 14.49 & 11.92 & 12.58 & 13.71 & 12.09 & 12.84 & 14.24 & 11.75 & 12.41 & 13.66 \\
5-year & 12.81 & 14.47 & 18.08 & 12.21 & 13.60 & 17.16 & 12.21 & 13.25 & 15.23 & 12.40 & 13.62 & 16.23 & 12.04 & 13.15 & 15.58 \\
10-year & 13.29 & 15.89 & 22.28 & 12.39 & 14.33 & 20.06 & 12.40 & 13.75 & 16.58 & 12.60 & 14.24 & 18.14 & 12.23 & 13.77 & 17.53 \\
20-year & 13.81 & 17.72 & 28.65 & 12.56 & 15.15 & 24.00 & 12.57 & 14.26 & 18.13 & 12.78 & 14.90 & 20.51 & 12.42 & 14.45 & 20.05 \\
50-year & 14.60 & 21.00 & 42.39 & 12.76 & 16.38 & 31.50 & 12.76 & 14.93 & 20.52 & 12.99 & 15.81 & 24.49 & 12.64 & 15.44 & 24.53 \\
100-year & 15.27 & 24.35 & 59.17 & 12.90 & 17.44 & 39.61 & 12.88 & 15.43 & 22.64 & 13.13 & 16.55 & 28.33 & 12.79 & 16.28 & 29.06 \\
\hline
\end{tabular}




\section{Appendix C. Monthly Trends in Wave Height}

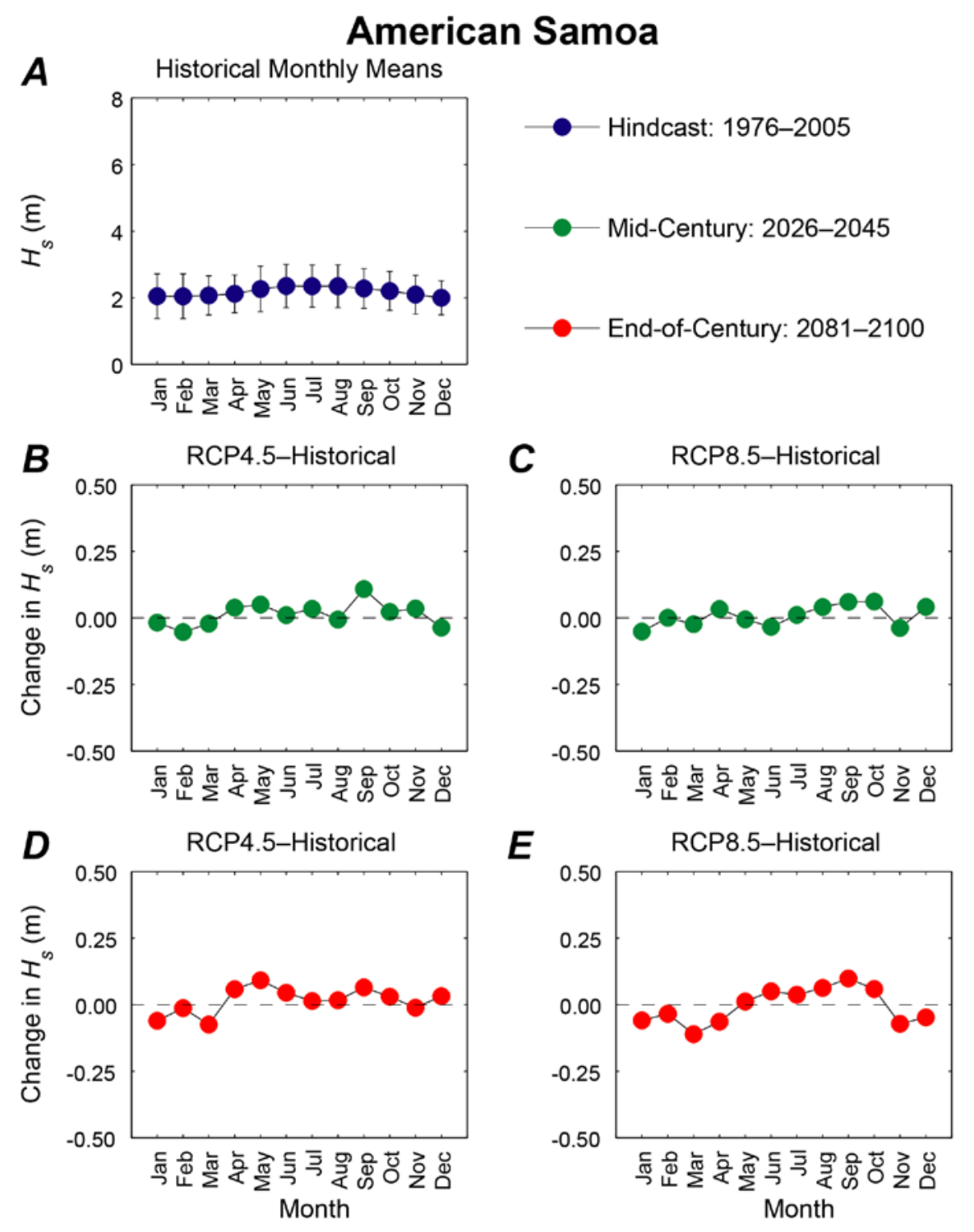

Appendix C1. Plots showing trends in monthly mean significant wave height $\left(H_{s}\right)$, in meters, at the American Samoa location. A. Hindcasted (1976-2005) mean significant wave heights by month with associated error bars. $B$. Plot of the change in mean 2026-2045 significant wave heights for the RCP4.5 scenario from hindcasted monthly significant wave height means. C. Plot of the change in mean 2026-2045 significant wave heights for the RCP8.5 scenario from hindcasted monthly significant wave height means. D. Plot of the change in mean 2081-2100 significant wave heights for the RCP4.5 scenario from hindcasted monthly significant wave height means. E. Plot of the change in mean 2081-2100 significant wave heights for the RCP8.5 scenario from hindcasted monthly significant wave height means. 

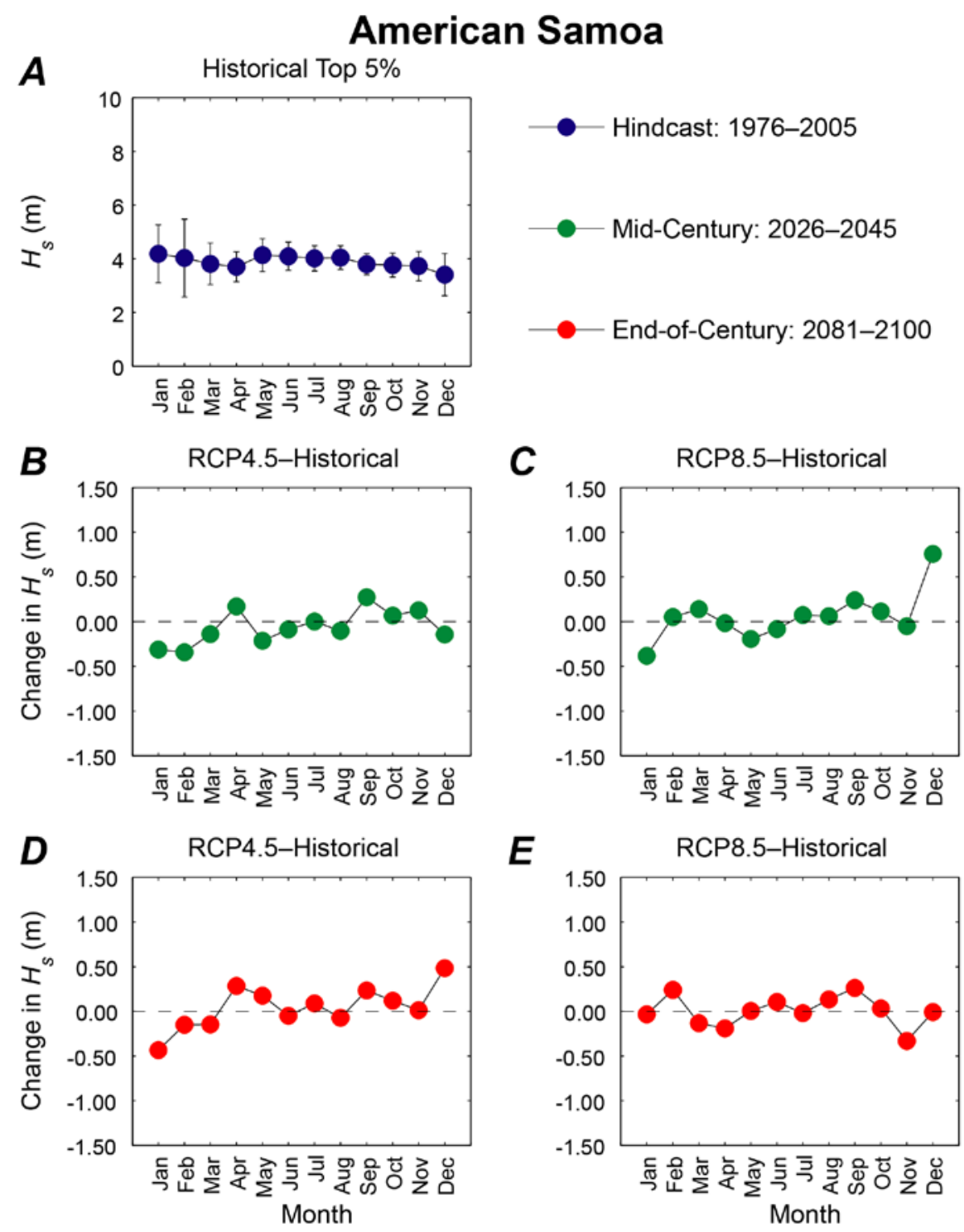

Appendix C2. Plots showing trends in monthly mean of the top 5 percent of significant wave heights $\left(H_{s}\right)$, in meters, at the American Samoa location. A. Hindcasted (1976-2005) mean of the top 5 percent of significant wave heights by month with associated error bars. $B$. Plot of the change in mean of the top 5 percent of 2026-2045 significant wave heights for the RCP4.5 scenario from hindcasted top 5 percent of monthly significant wave height means. $C$. Plot of the change in mean of the top 5 percent of 2026-2045 significant wave heights for the RCP8.5 scenario from hindcasted top 5 percent of monthly significant wave height means. D. Plot of the change in mean of the top 5 percent of 20812100 significant wave heights for the RCP4.5 scenario from hindcasted top 5 percent of monthly significant wave height means. E. Plot of the change in mean of the top 5 percent of 2081-2100 significant wave heights for the RCP8.5 scenario from hindcasted top 5 percent of monthly significant wave height means. 


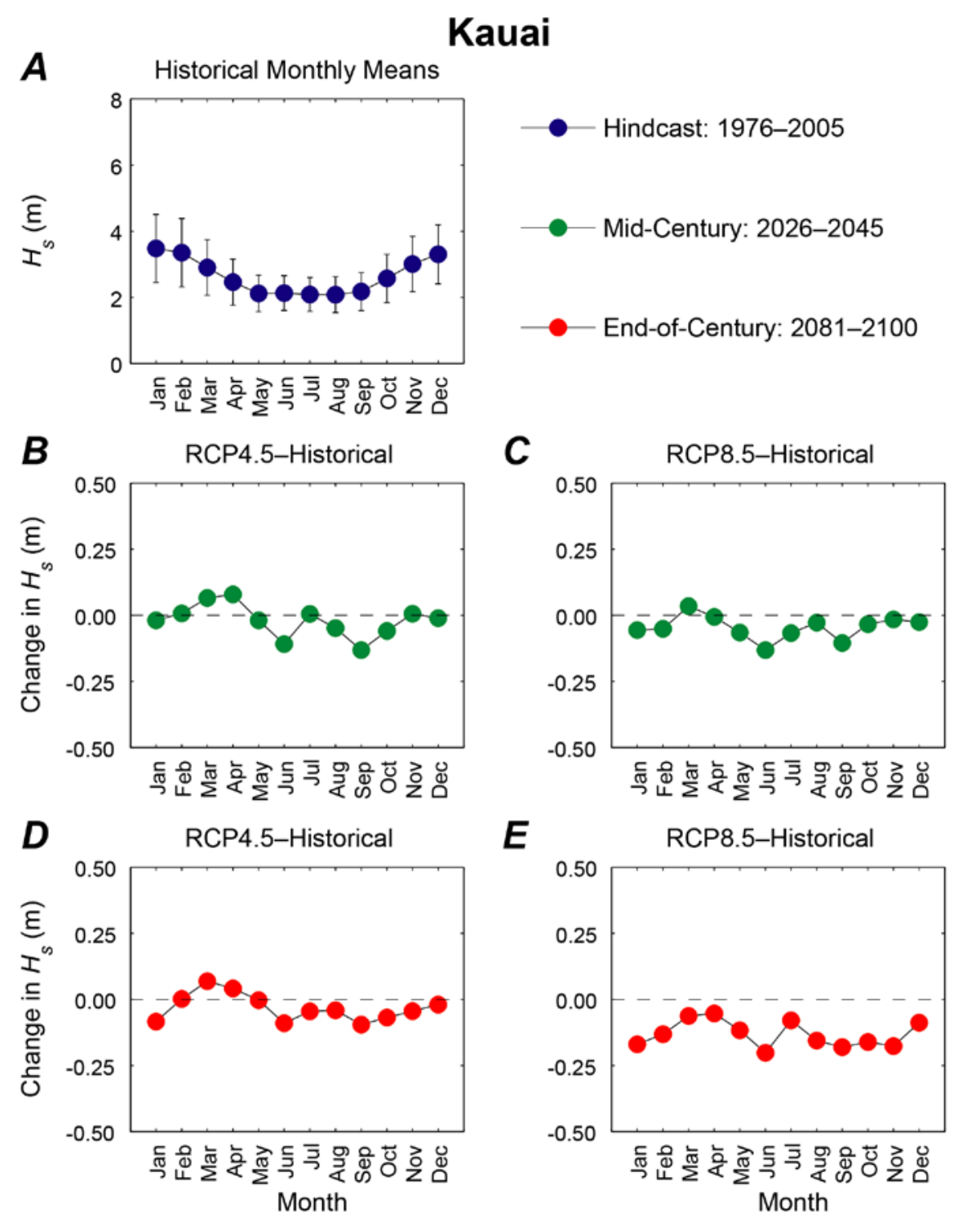

Appendix C3. Plots showing trends in monthly mean significant wave height $\left(H_{s}\right)$, in meters, at the Kauai location. A. Hindcasted (1976-2005) mean significant wave heights by month with associated error bars. $B$. Plot of the change in mean 2026-2045 significant wave heights for the RCP4.5 scenario from hindcasted monthly significant wave height means. C. Plot of the change in mean 2026-2045 significant wave heights for the RCP8.5 scenario from hindcasted monthly significant wave height means. D. Plot of the change in mean 2081-2100 significant wave heights for the RCP4.5 scenario from hindcasted monthly significant wave height means. E. Plot of the change in mean 2081-2100 significant wave heights for the RCP8.5 scenario from hindcasted monthly significant wave height means. 


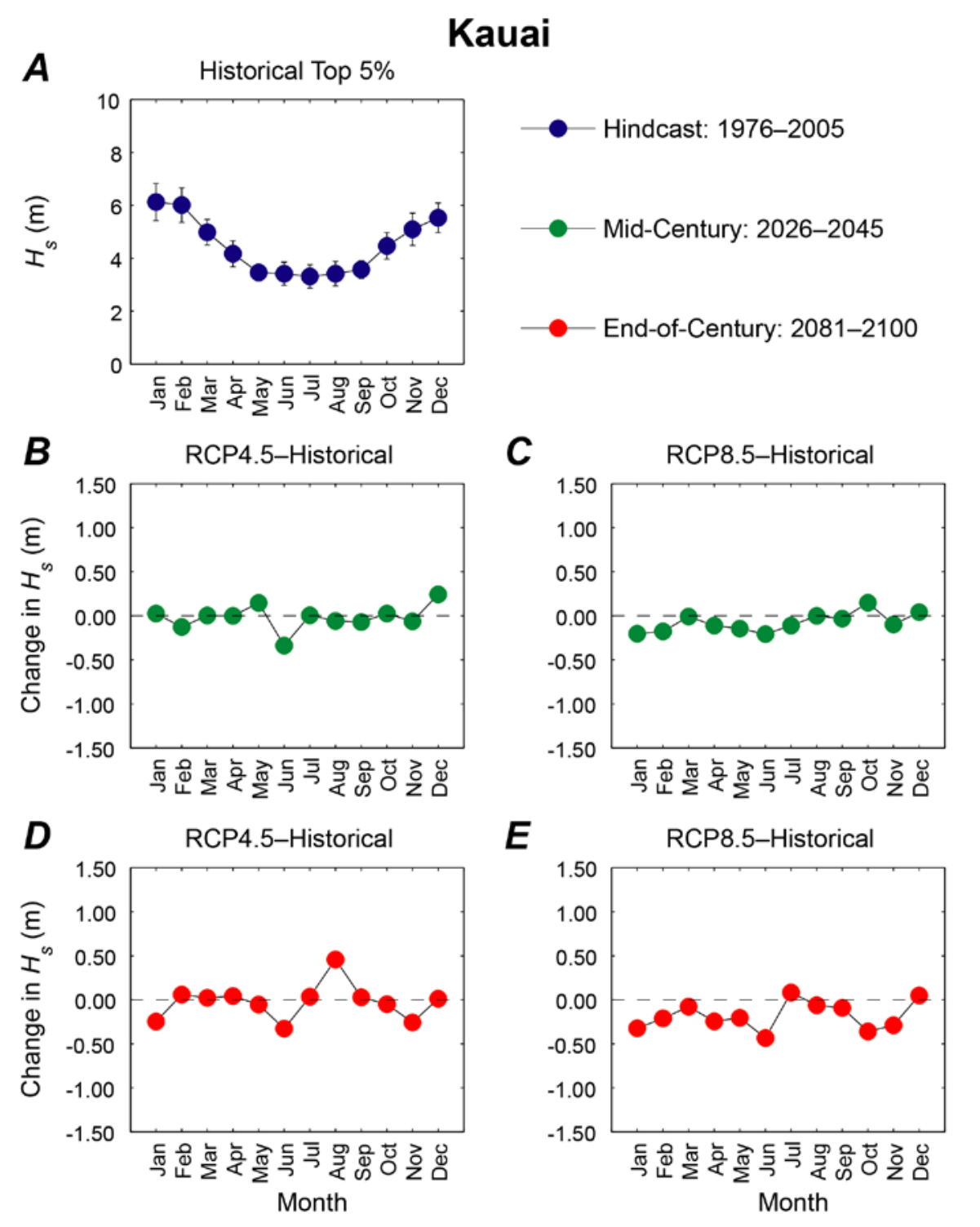

Appendix C4. Plots showing trends in monthly mean of the top 5 percent of significant wave heights $\left(H_{s}\right)$, in meters, at the Kauai location. A. Hindcasted (1976-2005) mean of the top 5 percent of significant wave heights by month with associated error bars. $B$. Plot of the change in mean of the top 5 percent of 2026-2045 significant wave heights for the RCP4.5 scenario from hindcasted top 5 percent of monthly significant wave height means. C. Plot of the change in mean of the top 5 percent of 20262045 significant wave heights for the RCP8.5 scenario from hindcasted top 5 percent of monthly significant wave height means. D. Plot of the change in mean of the top 5 percent of 2081-2100 significant wave heights for the RCP4.5 scenario from hindcasted top 5 percent of monthly significant wave height means. E. Plot of the change in mean of the top 5 percent of 2081-2100 significant wave heights for the RCP8.5 scenario from hindcasted top 5 percent of monthly significant wave height means. 


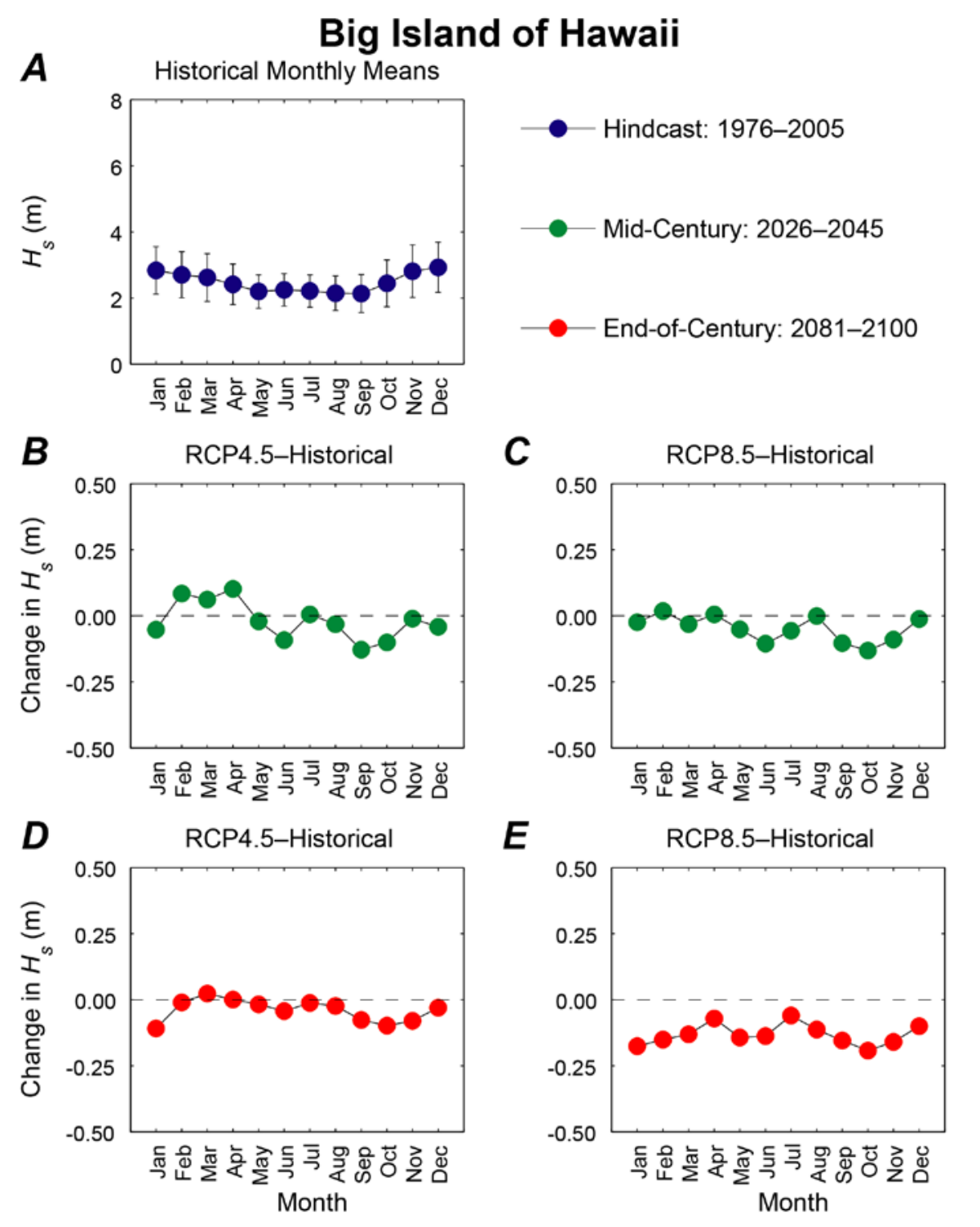

Appendix C5. Plots showing trends in monthly mean significant wave height $\left(H_{s}\right)$, in meters, at the Big Island of Hawaii location. A. Hindcasted (1976-2005) mean significant wave heights by month with associated error bars. $B$. Plot of the change in mean 2026-2045 significant wave heights for the RCP4.5 scenario from hindcasted monthly significant wave height means. C. Plot of the change in mean 2026-2045 significant wave heights for the RCP8.5 scenario from hindcasted monthly significant wave height means. D. Plot of the change in mean 2081-2100 significant wave heights for the RCP4.5 scenario from hindcasted monthly significant wave height means. E. Plot of the change in mean 2081-2100 significant wave heights for the RCP8.5 scenario from hindcasted monthly significant wave height means. 


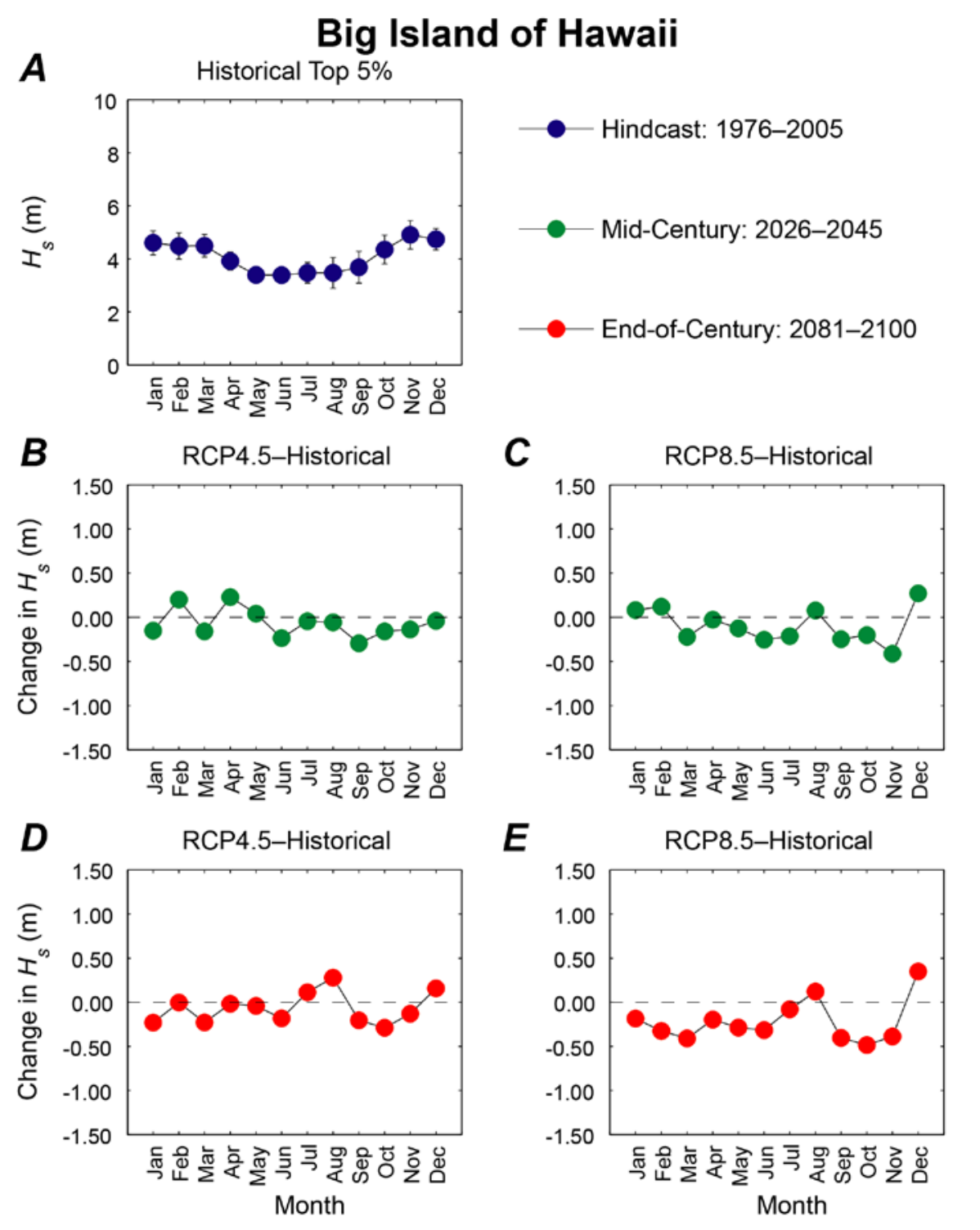

Appendix C6. Plots showing trends in monthly mean of the top 5 percent of significant wave heights $\left(H_{s}\right)$, in meters, at the Big Island of Hawaii location. A. Hindcasted (1976-2005) mean of the top 5 percent of significant wave heights by month with associated error bars. $B$. Plot of the change in mean of the top 5 percent of 2026-2045 significant wave heights for the RCP4.5 scenario from hindcasted top 5 percent of monthly significant wave height means. $C$. Plot of the change in mean of the top 5 percent of 2026-2045 significant wave heights for the RCP8.5 scenario from hindcasted top 5 percent of monthly significant wave height means. $D$. Plot of the change in mean of the top 5 percent of 20812100 significant wave heights for the RCP4.5 scenario from hindcasted top 5 percent of monthly significant wave height means. E. Plot of the change in mean of the top 5 percent of 2081-2100 significant wave heights for the RCP8.5 scenario from hindcasted top 5 percent of monthly significant wave height means. 

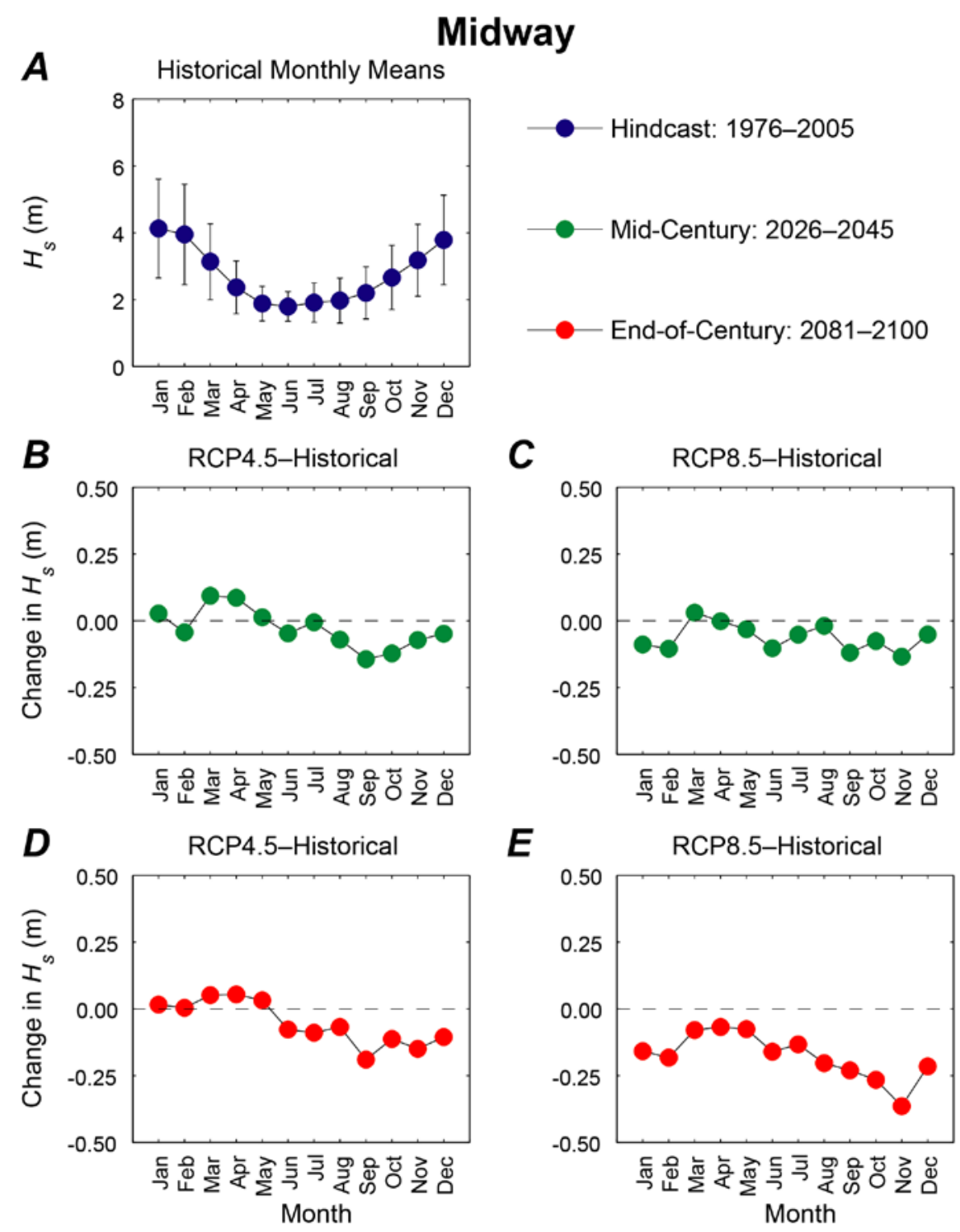

Appendix C7. Plots showing trends in monthly mean significant wave height $\left(H_{s}\right)$, in meters, at the Midway location. A. Hindcasted (1976-2005) mean significant wave heights by month with associated error bars. $B$. Plot of the change in mean 2026-2045 significant wave heights for the RCP4.5 scenario from hindcasted monthly significant wave height means. C. Plot of the change in mean 2026-2045 significant wave heights for the RCP8.5 scenario from hindcasted monthly significant wave height means. D. Plot of the change in mean 2081-2100 significant wave heights for the RCP4.5 scenario from hindcasted monthly significant wave height means. E. Plot of the change in mean 2081-2100 significant wave heights for the RCP8.5 scenario from hindcasted monthly significant wave height means. 

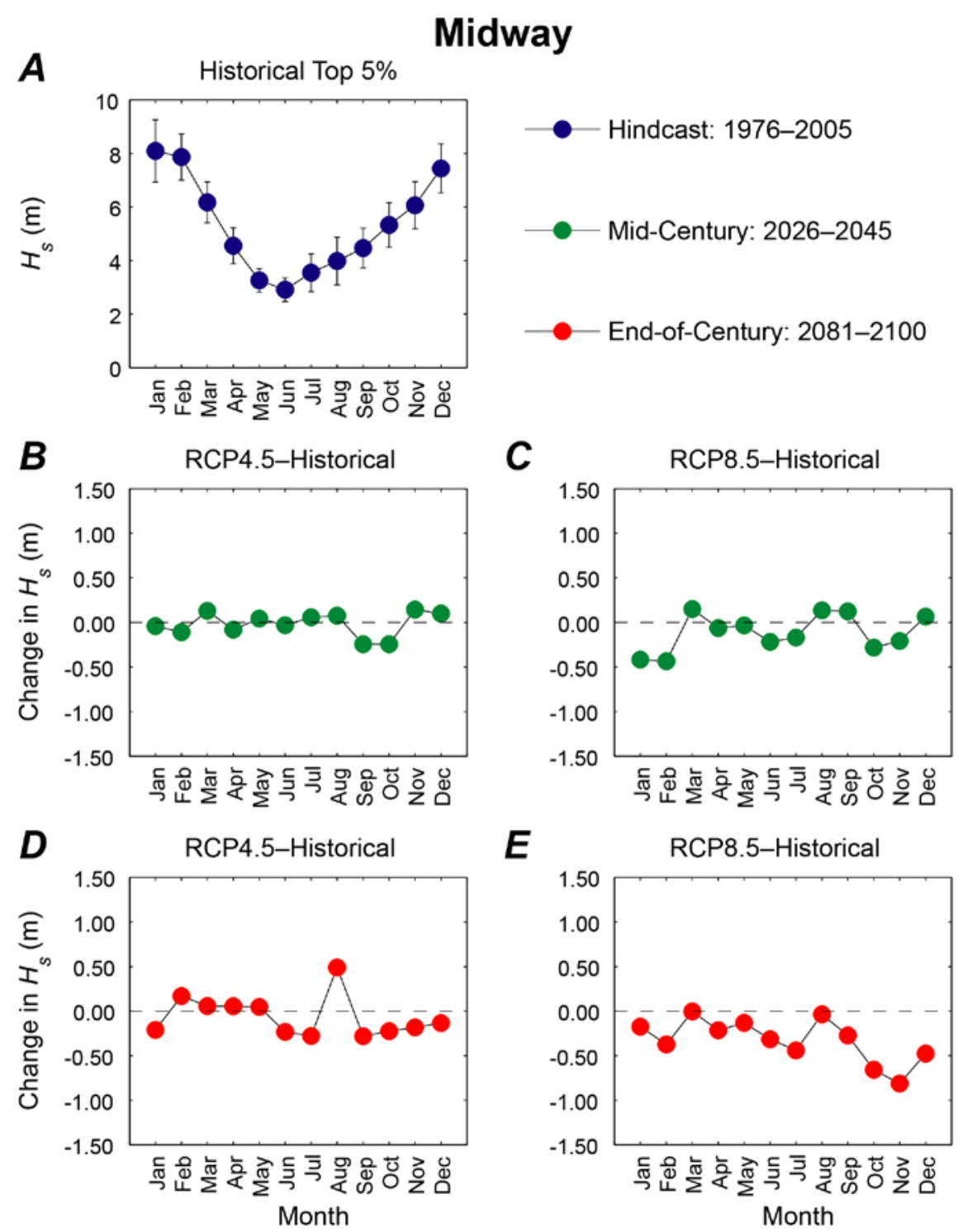

Appendix C8. Plots showing trends in monthly mean of the top 5 percent of significant wave heights $\left(H_{s}\right)$, in meters, at the Midway location. A. Hindcasted (1976-2005) mean of the top 5 percent of significant wave heights by month with associated error bars. $B$. Plot of the change in mean of the top 5 percent of 2026-2045 significant wave heights for the RCP4.5 scenario from hindcasted top 5 percent of monthly significant wave height means. C. Plot of the change in mean of the top 5 percent of 20262045 significant wave heights for the RCP8.5 scenario from hindcasted top 5 percent of monthly significant wave height means. D. Plot of the change in mean of the top 5 percent of 2081-2100 significant wave heights for the RCP4.5 scenario from hindcasted top 5 percent of monthly significant wave height means. E. Plot of the change in mean of the top 5 percent of 2081-2100 significant wave heights for the RCP8.5 scenario from hindcasted top 5 percent of monthly significant wave height means. 


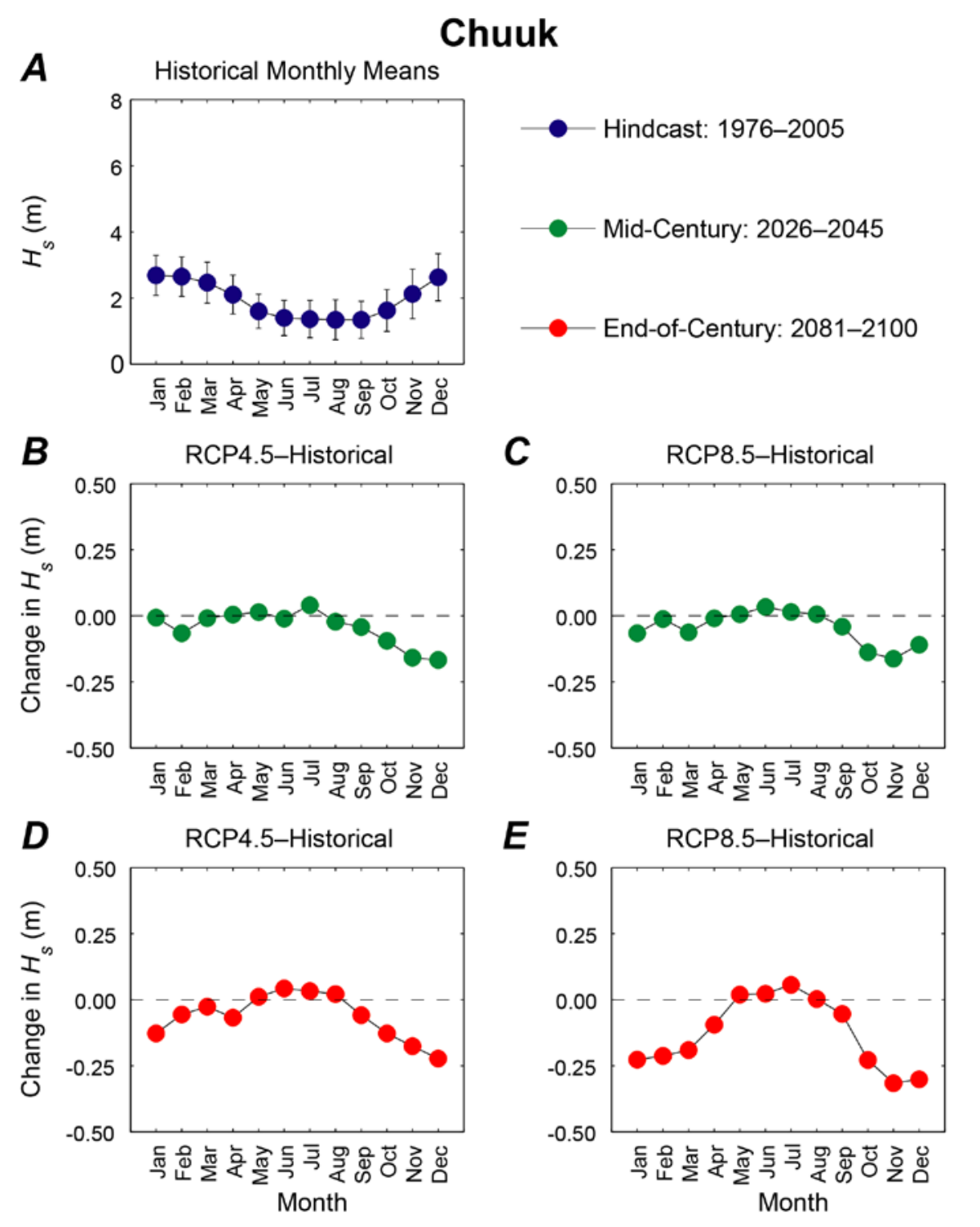

Appendix C9. Plots showing trends in monthly mean significant wave height $\left(H_{s}\right)$, in meters, at the Chuuk location. A. Hindcasted (1976-2005) mean significant wave heights by month with associated error bars. $B$. Plot of the change in mean 2026-2045 significant wave heights for the RCP4.5 scenario from hindcasted monthly significant wave height means. C. Plot of the change in mean 2026-2045 significant wave heights for the RCP8.5 scenario from hindcasted monthly significant wave height means. $D$. Plot of the change in mean 2081-2100 significant wave heights for the RCP4.5 scenario from hindcasted monthly significant wave height means. E. Plot of the change in mean 2081-2100 significant wave heights for the RCP8.5 scenario from hindcasted monthly significant wave height means. 

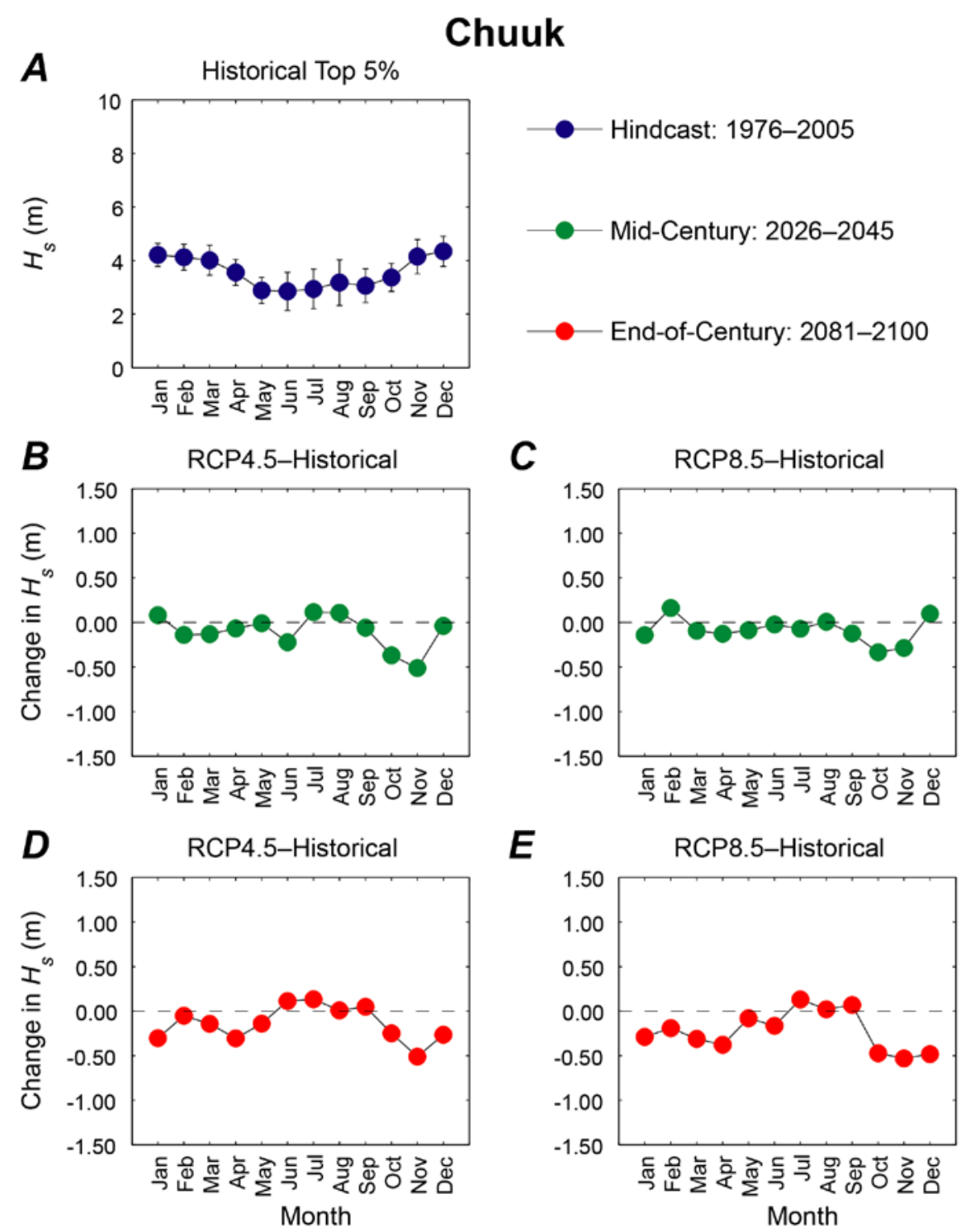

Appendix C10. Plots showing trends in monthly mean of the top 5 percent of significant wave heights $\left(H_{s}\right)$, in meters, at the Chuuk location. A. Hindcasted (1976-2005) mean of the top 5 percent of significant wave heights by month with associated error bars. $B$. Plot of the change in mean of the top 5 percent of 2026-2045 significant wave heights for the RCP4.5 scenario from hindcasted top 5 percent of monthly significant wave height means. C. Plot of the change in mean of the top 5 percent of 20262045 significant wave heights for the RCP8.5 scenario from hindcasted top 5 percent of monthly significant wave height means. D. Plot of the change in mean of the top 5 percent of 2081-2100 significant wave heights for the RCP4.5 scenario from hindcasted top 5 percent of monthly significant wave height means. E. Plot of the change in mean of the top 5 percent of 2081-2100 significant wave heights for the RCP8.5 scenario from hindcasted top 5 percent of monthly significant wave height means. 


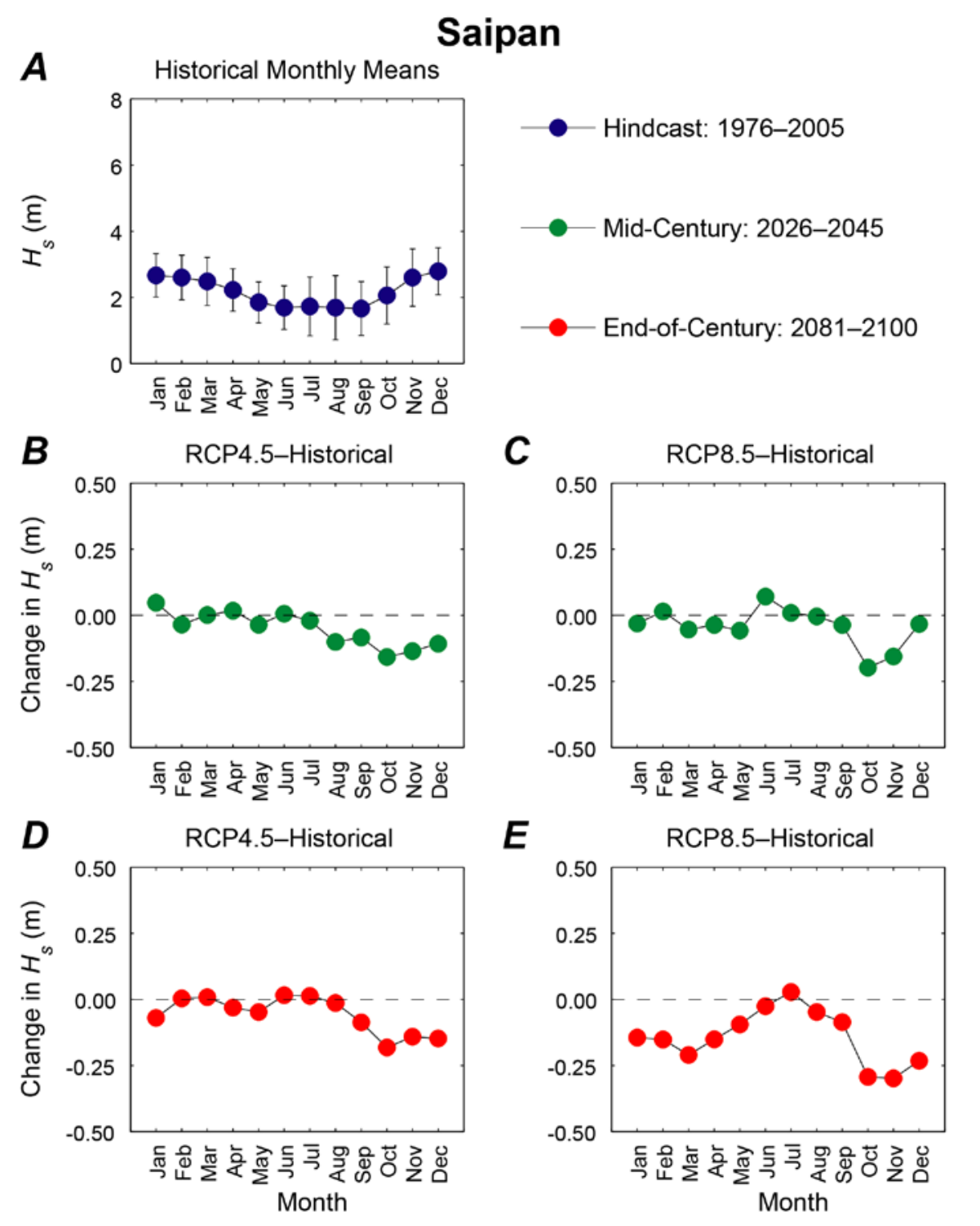

Appendix C11. Plots showing trends in monthly mean significant wave height $\left(H_{s}\right)$, in meters, at the Saipan location. A. Hindcasted (1976-2005) mean significant wave heights by month with associated error bars. $B$. Plot of the change in mean 2026-2045 significant wave heights for the RCP4.5 scenario from hindcasted monthly significant wave height means. C. Plot of the change in mean 2026-2045 significant wave heights for the RCP8.5 scenario from hindcasted monthly significant wave height means. $D$. Plot of the change in mean 2081-2100 significant wave heights for the RCP4.5 scenario from hindcasted monthly significant wave height means. E. Plot of the change in mean 2081-2100 significant wave heights for the RCP8.5 scenario from hindcasted monthly significant wave height means. 

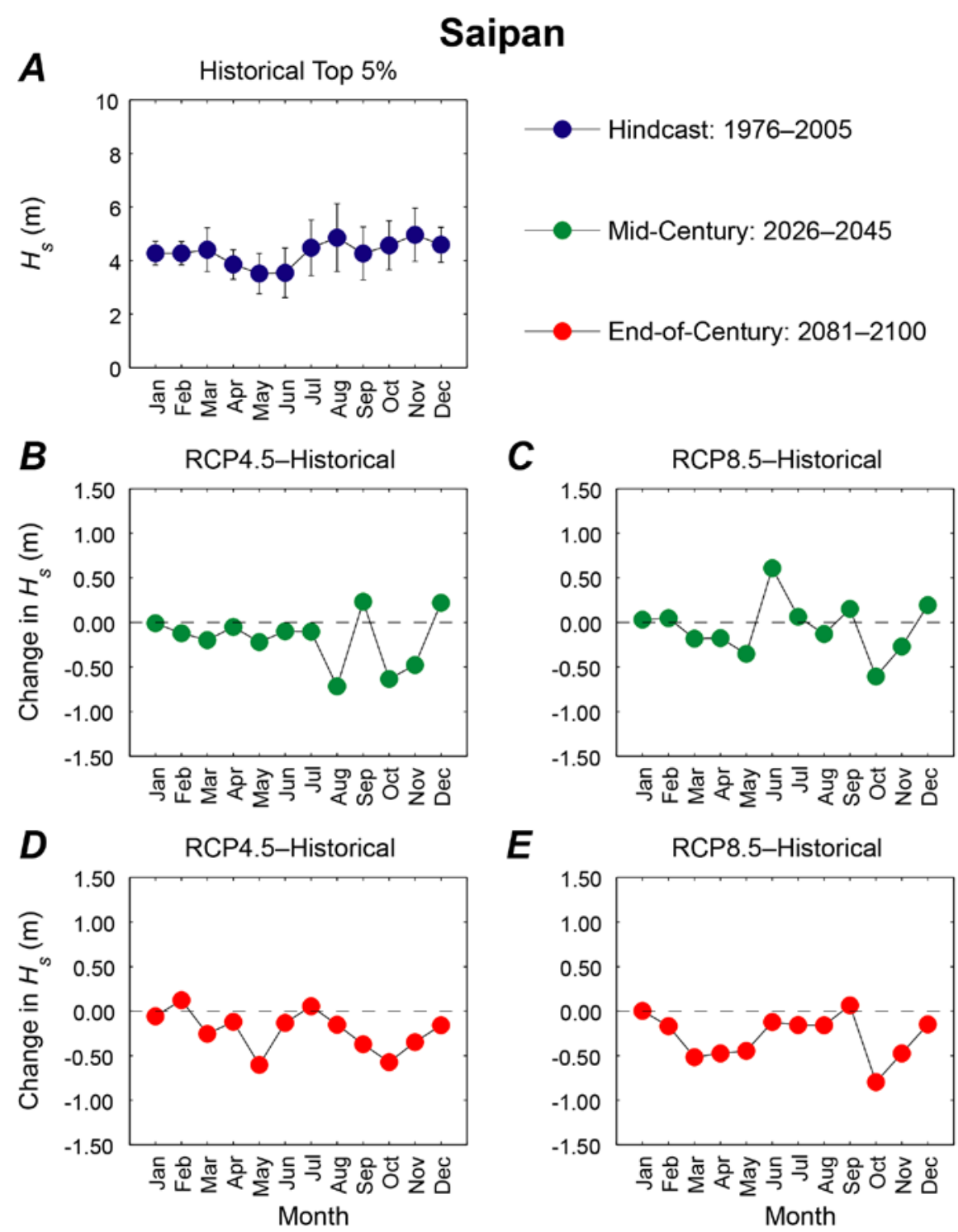

Appendix C12. Plots showing trends in monthly mean of the top 5 percent of significant wave heights $\left(H_{s}\right)$, in meters, at the Saipan location. A. Hindcasted (1976-2005) mean of the top 5 percent of significant wave heights by month with associated error bars. $B$. Plot of the change in mean of the top 5 percent of 2026-2045 significant wave heights for the RCP4.5 scenario from hindcasted top 5 percent of monthly significant wave height means. C. Plot of the change in mean of the top 5 percent of 20262045 significant wave heights for the RCP8.5 scenario from hindcasted top 5 percent of monthly significant wave height means. D. Plot of the change in mean of the top 5 percent of 2081-2100 significant wave heights for the RCP4.5 scenario from hindcasted top 5 percent of monthly significant wave height means. E. Plot of the change in mean of the top 5 percent of 2081-2100 significant wave heights for the RCP8.5 scenario from hindcasted top 5 percent of monthly significant wave height means. 


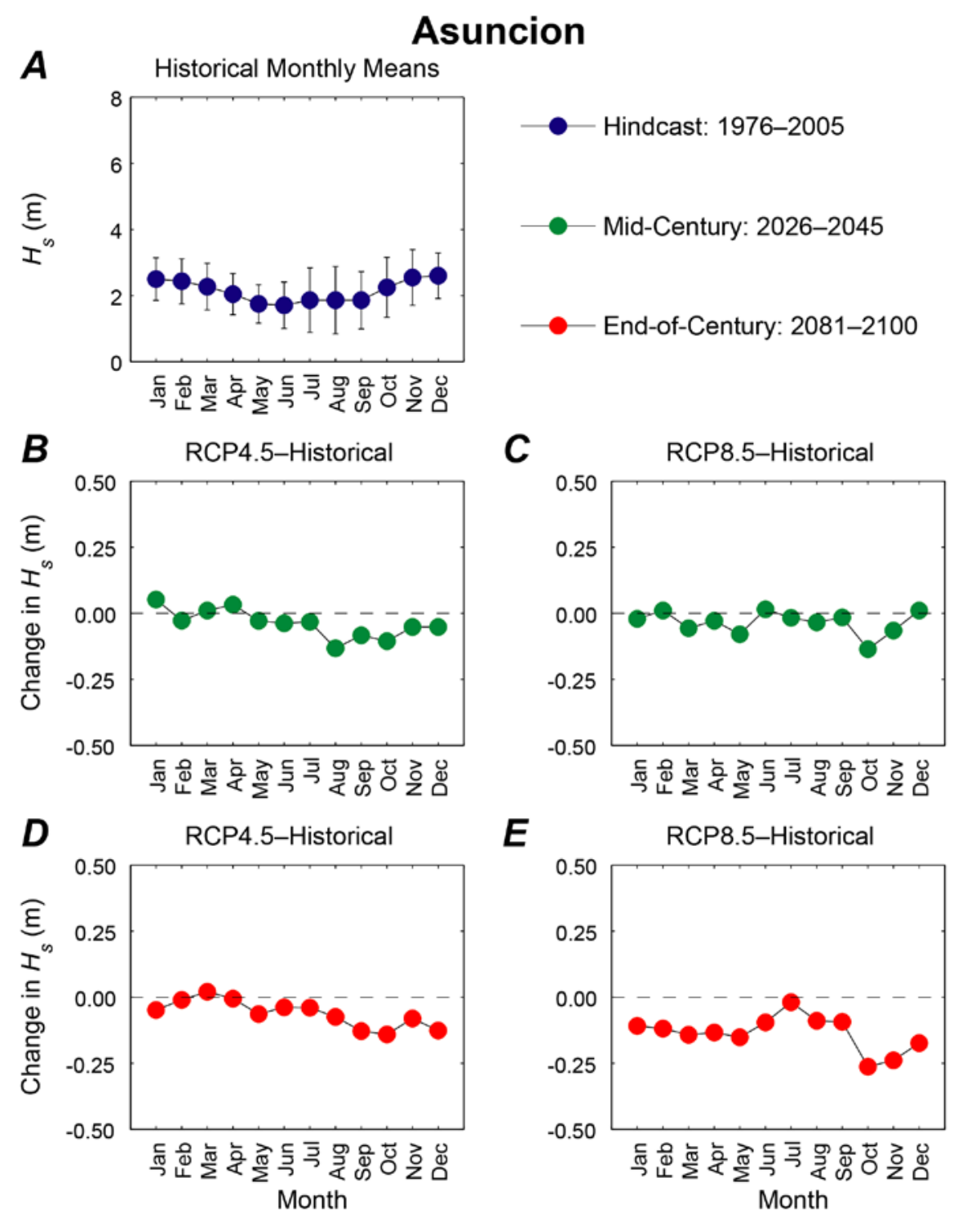

Appendix C13. Plots showing trends in monthly mean significant wave height $\left(H_{s}\right)$, in meters, at the Asuncion location. A. Hindcasted (1976-2005) mean significant wave heights by month with associated error bars. $B$. Plot of the change in mean 2026-2045 significant wave heights for the RCP4.5 scenario from hindcasted monthly significant wave height means. C. Plot of the change in mean 2026-2045 significant wave heights for the RCP8.5 scenario from hindcasted monthly significant wave height means. D. Plot of the change in mean 2081-2100 significant wave heights for the RCP4.5 scenario from hindcasted monthly significant wave height means. E. Plot of the change in mean 2081-2100 significant wave heights for the RCP8.5 scenario from hindcasted monthly significant wave height means. 

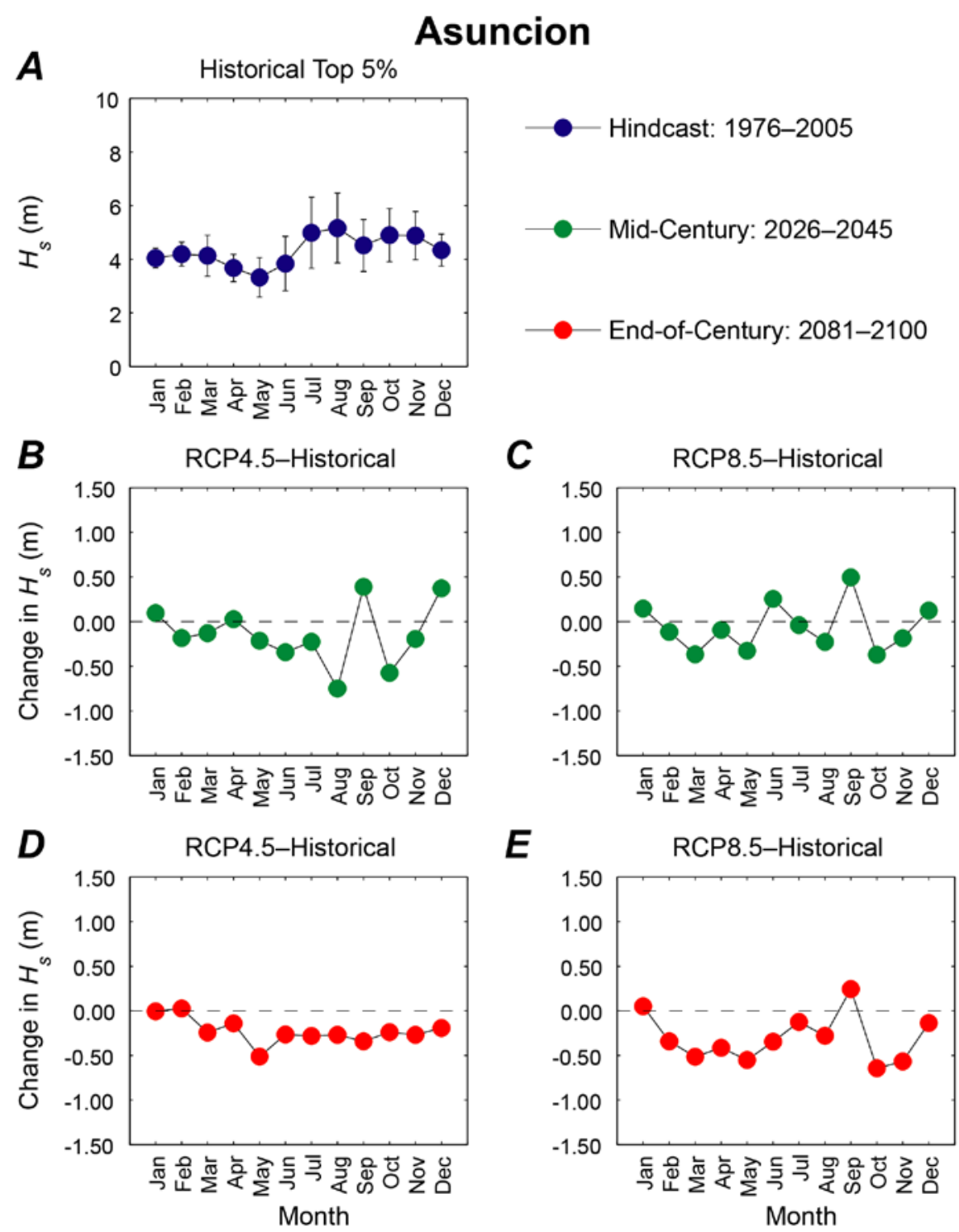

Appendix C14. Plots showing trends in monthly mean of the top 5 percent of significant wave heights $\left(H_{s}\right)$, in meters, at the Asuncion location. A. Hindcasted (1976-2005) mean of the top 5 percent of significant wave heights by month with associated error bars. $B$. Plot of the change in mean of the top 5 percent of 2026-2045 significant wave heights for the RCP4.5 scenario from hindcasted top 5 percent of monthly significant wave height means. $C$. Plot of the change in mean of the top 5 percent of 2026-2045 significant wave heights for the RCP8.5 scenario from hindcasted top 5 percent of monthly significant wave height means. $D$. Plot of the change in mean of the top 5 percent of 20812100 significant wave heights for the RCP4.5 scenario from hindcasted top 5 percent of monthly significant wave height means. E. Plot of the change in mean of the top 5 percent of 2081-2100 significant wave heights for the RCP8.5 scenario from hindcasted top 5 percent of monthly significant wave height means. 

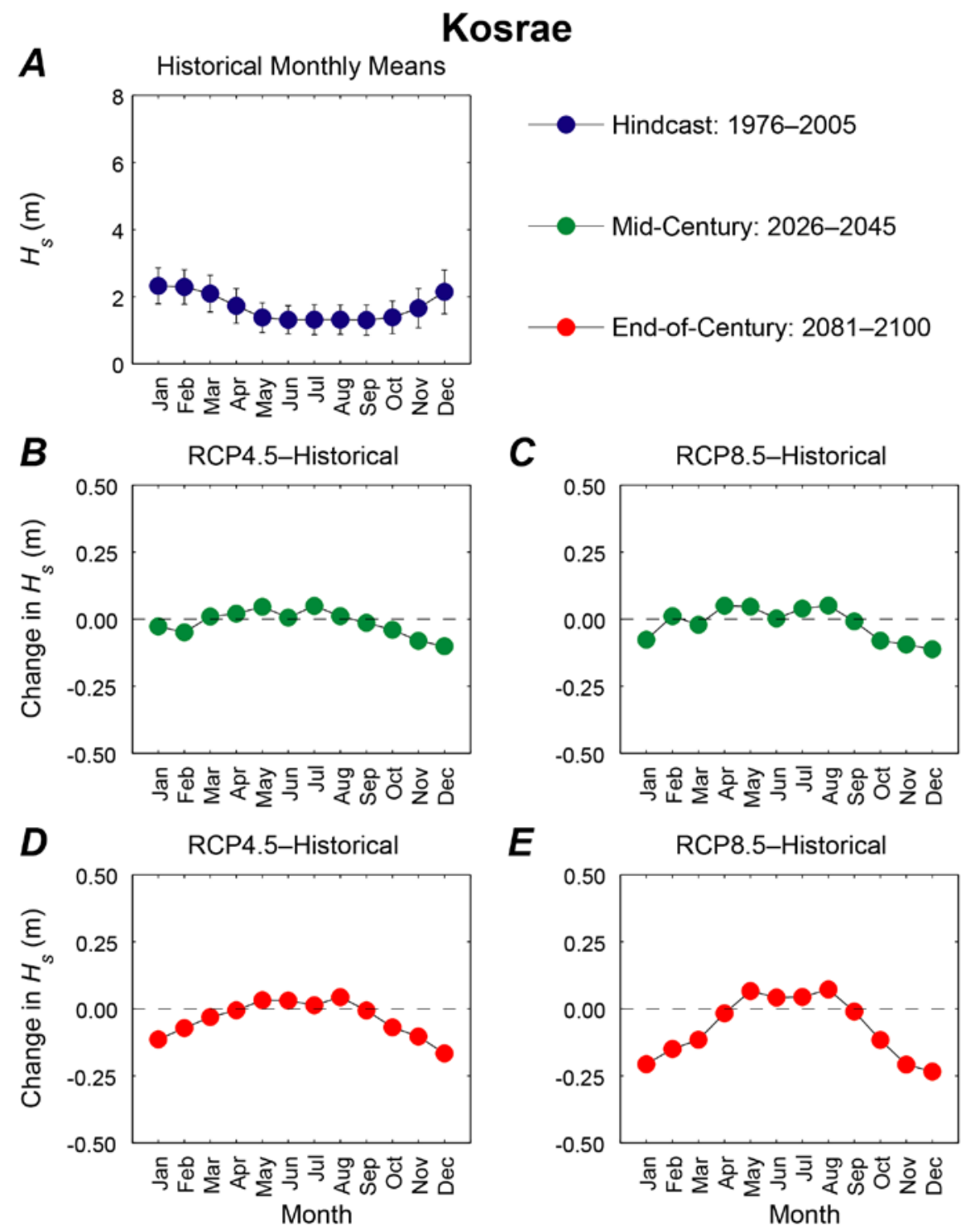

Appendix C15. Plots showing trends in monthly mean significant wave height $\left(H_{s}\right)$, in meters, at the Kosrae location. A. Hindcasted (1976-2005) mean significant wave heights by month with associated error bars. $B$. Plot of the change in mean 2026-2045 significant wave heights for the RCP4.5 scenario from hindcasted monthly significant wave height means. C. Plot of the change in mean 2026-2045 significant wave heights for the RCP8.5 scenario from hindcasted monthly significant wave height means. $D$. Plot of the change in mean 2081-2100 significant wave heights for the RCP4.5 scenario from hindcasted monthly significant wave height means. E. Plot of the change in mean 2081-2100 significant wave heights for the RCP8.5 scenario from hindcasted monthly significant wave height means. 

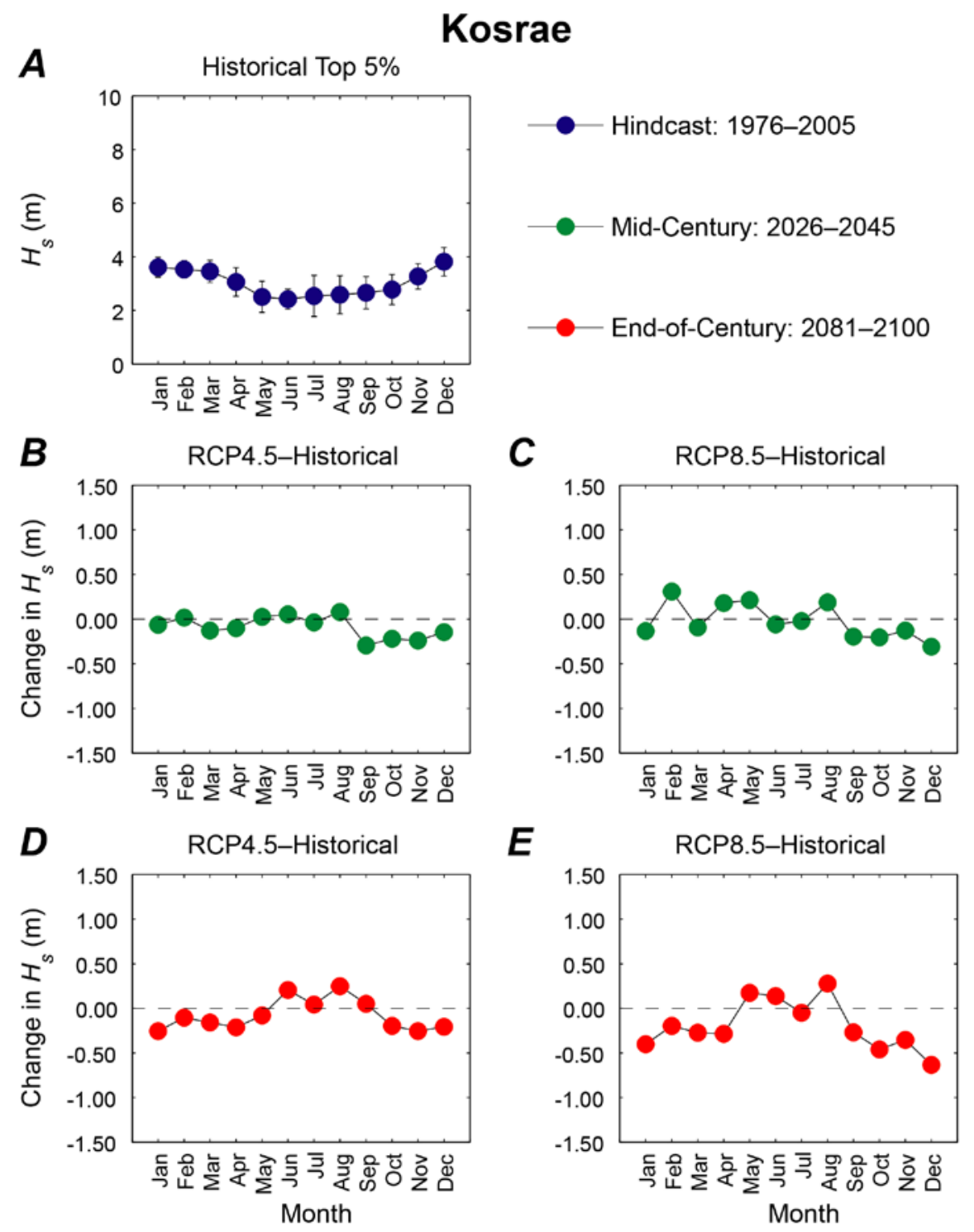

Appendix C16. Plots showing trends in monthly mean of the top 5 percent of significant wave heights $\left(H_{s}\right)$, in meters, at the Kosrae location. A. Hindcasted (1976-2005) mean of the top 5 percent of significant wave heights by month with associated error bars. $B$. Plot of the change in mean of the top 5 percent of 2026-2045 significant wave heights for the RCP4.5 scenario from hindcasted top 5 percent of monthly significant wave height means. $C$. Plot of the change in mean of the top 5 percent of 2026 2045 significant wave heights for the RCP8.5 scenario from hindcasted top 5 percent of monthly significant wave height means. D. Plot of the change in mean of the top 5 percent of 2081-2100 significant wave heights for the RCP4.5 scenario from hindcasted top 5 percent of monthly significant wave height means. E. Plot of the change in mean of the top 5 percent of 2081-2100 significant wave heights for the RCP8.5 scenario from hindcasted top 5 percent of monthly significant wave height means. 

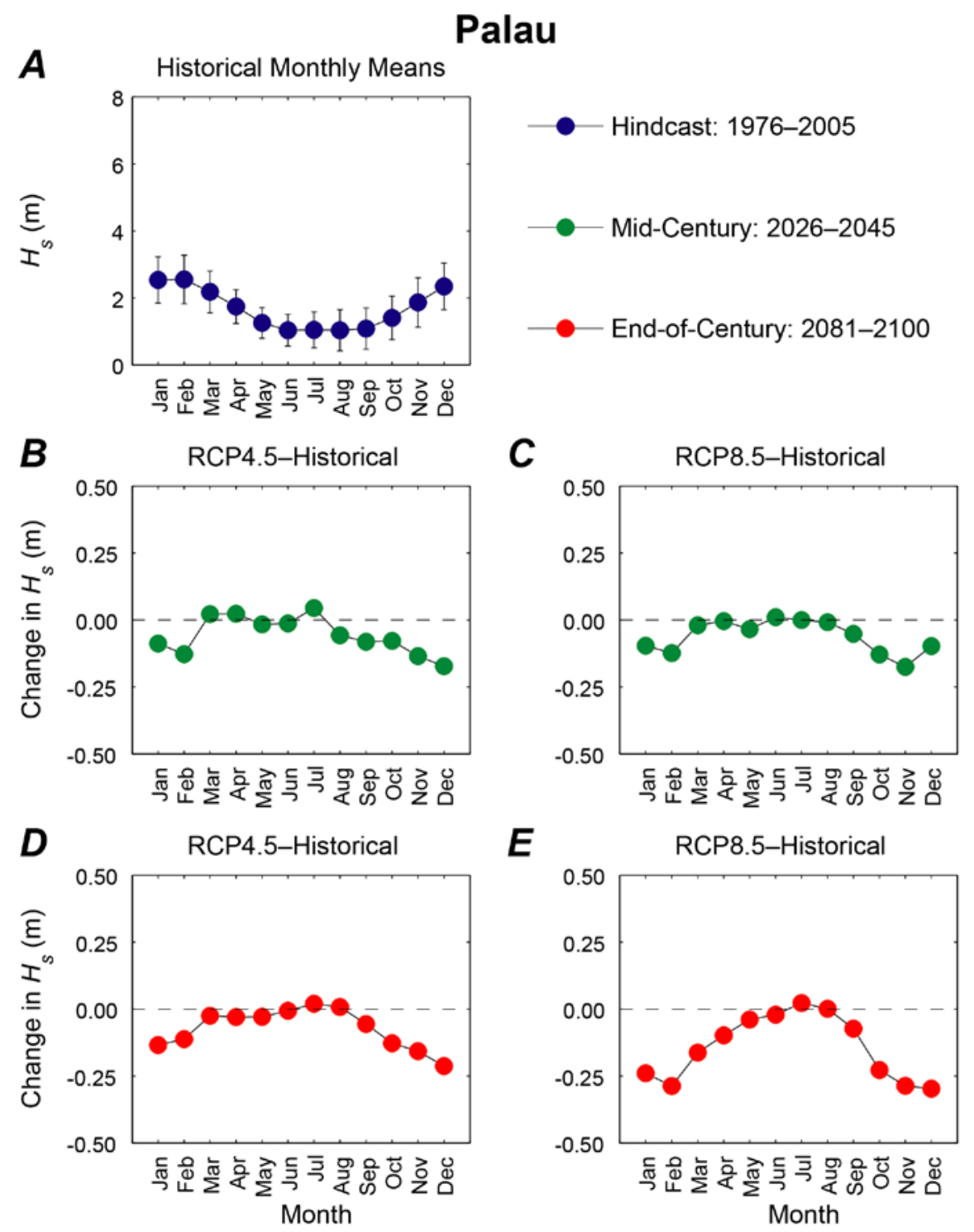

Appendix C17. Plots showing trends in monthly mean significant wave height $\left(H_{s}\right)$, in meters, at the Palau location. A. Hindcasted (1976-2005) mean significant wave heights by month with associated error bars. $B$. Plot of the change in mean 2026-2045 significant wave heights for the RCP4.5 scenario from hindcasted monthly significant wave height means. C. Plot of the change in mean 2026-2045 significant wave heights for the RCP8.5 scenario from hindcasted monthly significant wave height means. $D$. Plot of the change in mean 2081-2100 significant wave heights for the RCP4.5 scenario from hindcasted monthly significant wave height means. E. Plot of the change in mean 2081-2100 significant wave heights for the RCP8.5 scenario from hindcasted monthly significant wave height means. 

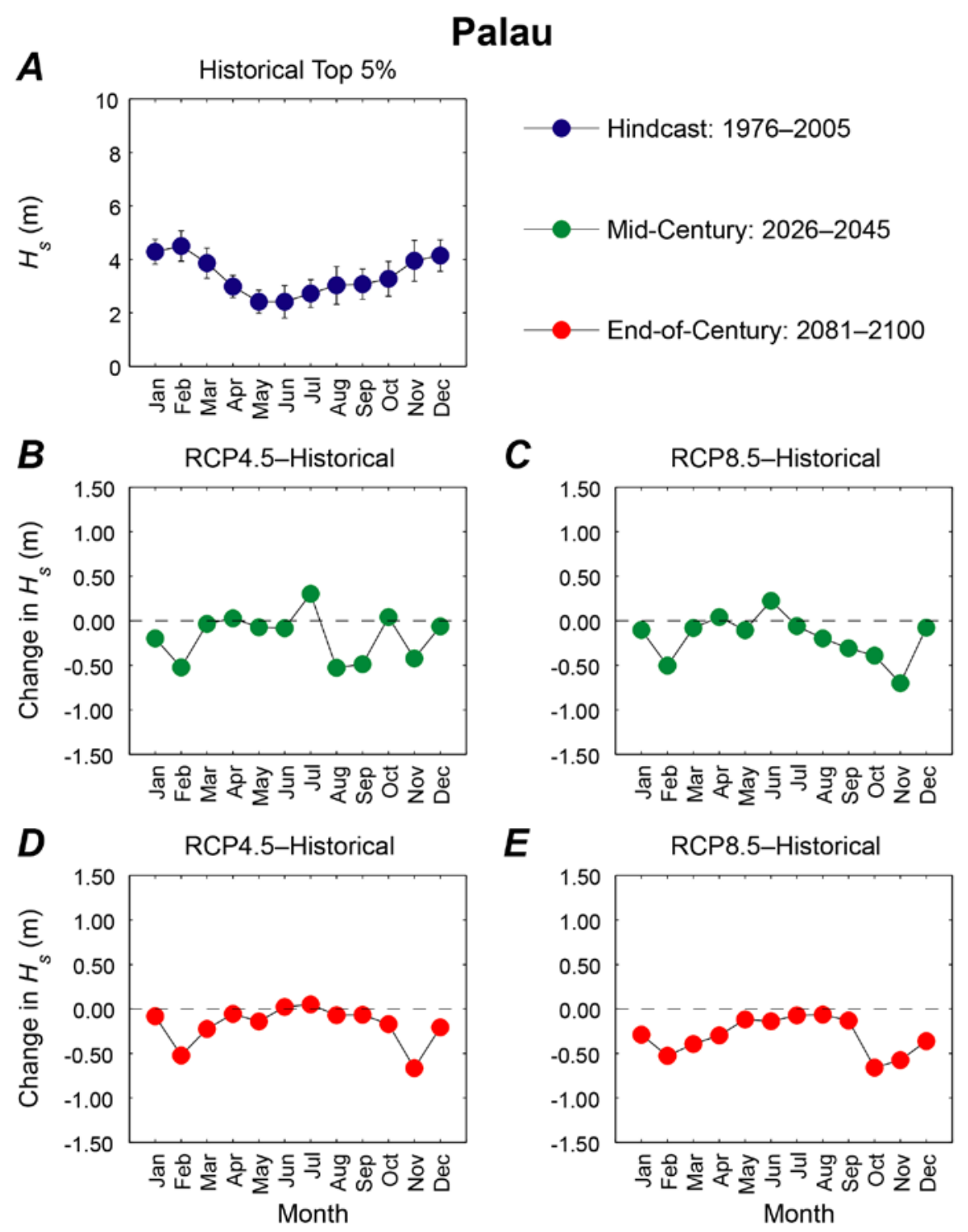

Appendix C18. Plots showing trends in monthly mean of the top 5 percent of significant wave heights $\left(H_{s}\right)$, in meters, at the Palau location. A. Hindcasted (1976-2005) mean of the top 5 percent of significant wave heights by month with associated error bars. $B$. Plot of the change in mean of the top 5 percent of 2026-2045 significant wave heights for the RCP4.5 scenario from hindcasted top 5 percent of monthly significant wave height means. $C$. Plot of the change in mean of the top 5 percent of 2026 2045 significant wave heights for the RCP8.5 scenario from hindcasted top 5 percent of monthly significant wave height means. D. Plot of the change in mean of the top 5 percent of 2081-2100 significant wave heights for the RCP4.5 scenario from hindcasted top 5 percent of monthly significant wave height means. E. Plot of the change in mean of the top 5 percent of 2081-2100 significant wave heights for the RCP8.5 scenario from hindcasted top 5 percent of monthly significant wave height means. 

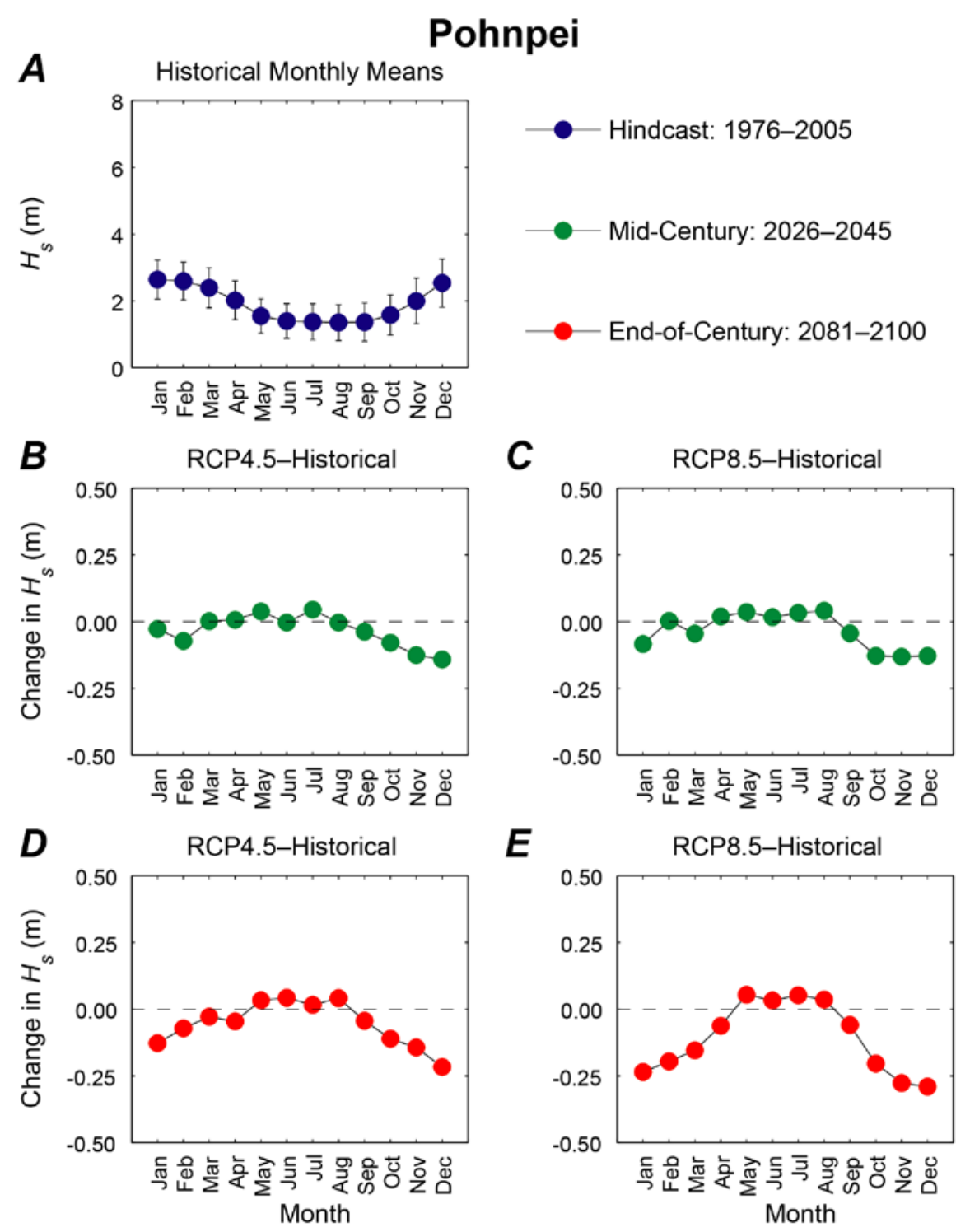

Appendix C19. Plots showing trends in monthly mean significant wave height $\left(H_{s}\right)$, in meters, at the Pohnpei location. A. Hindcasted (1976-2005) mean significant wave heights by month with associated error bars. $B$. Plot of the change in mean 2026-2045 significant wave heights for the RCP4.5 scenario from hindcasted monthly significant wave height means. C. Plot of the change in mean 2026-2045 significant wave heights for the RCP8.5 scenario from hindcasted monthly significant wave height means. D. Plot of the change in mean 2081-2100 significant wave heights for the RCP4.5 scenario from hindcasted monthly significant wave height means. E. Plot of the change in mean 2081-2100 significant wave heights for the RCP8.5 scenario from hindcasted monthly significant wave height means. 

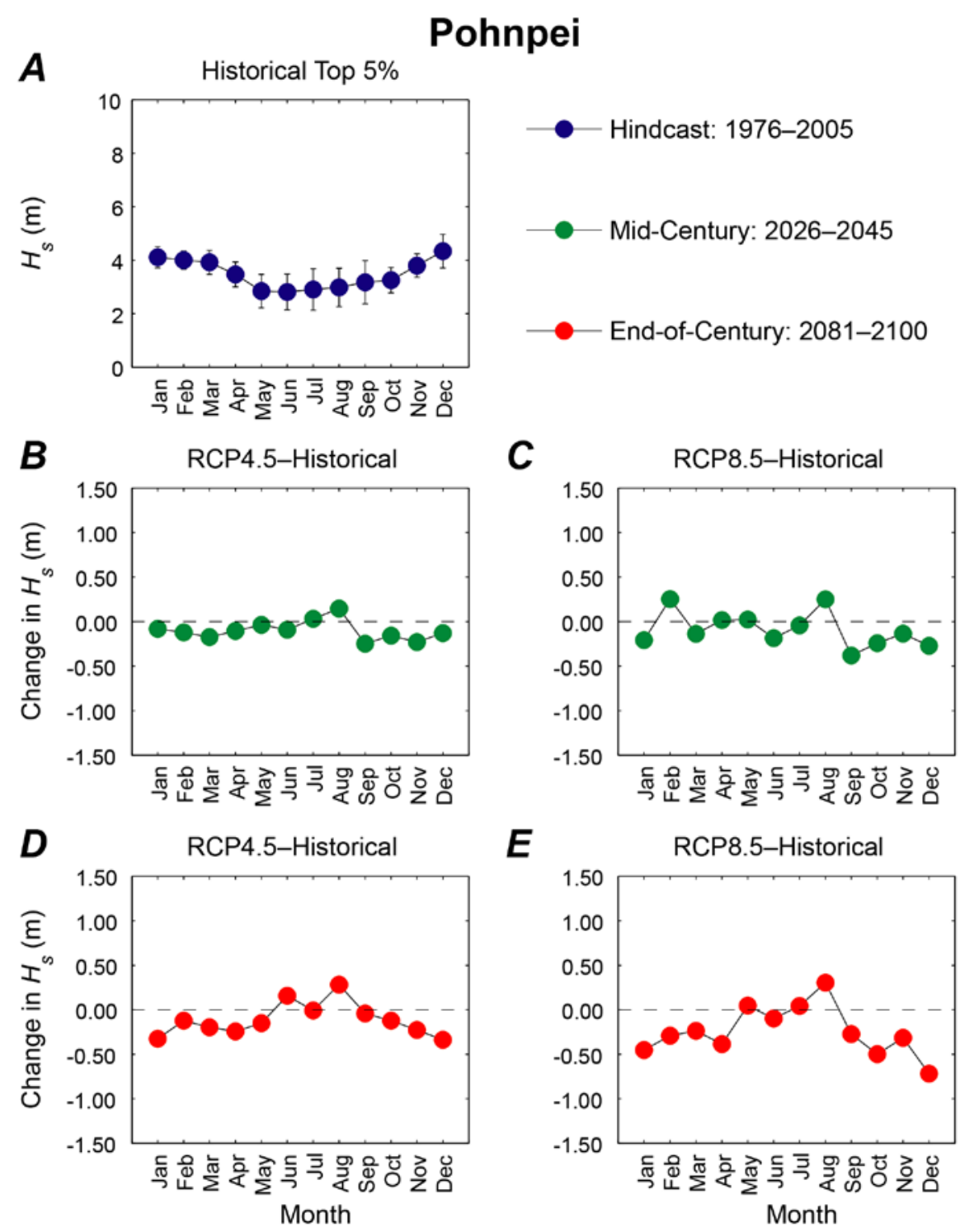

Appendix C20. Plots showing trends in monthly mean of the top 5 percent of significant wave heights $\left(H_{s}\right)$, in meters, at the Pohnpei location. A. Hindcasted (1976-2005) mean of the top 5 percent of significant wave heights by month with associated error bars. $B$. Plot of the change in mean of the top 5 percent of 2026-2045 significant wave heights for the RCP4.5 scenario from hindcasted top 5 percent of monthly significant wave height means. $C$. Plot of the change in mean of the top 5 percent of 2026-2045 significant wave heights for the RCP8.5 scenario from hindcasted top 5 percent of monthly significant wave height means. $D$. Plot of the change in mean of the top 5 percent of 20812100 significant wave heights for the RCP4.5 scenario from hindcasted top 5 percent of monthly significant wave height means. E. Plot of the change in mean of the top 5 percent of 2081-2100 significant wave heights for the RCP8.5 scenario from hindcasted top 5 percent of monthly significant wave height means. 

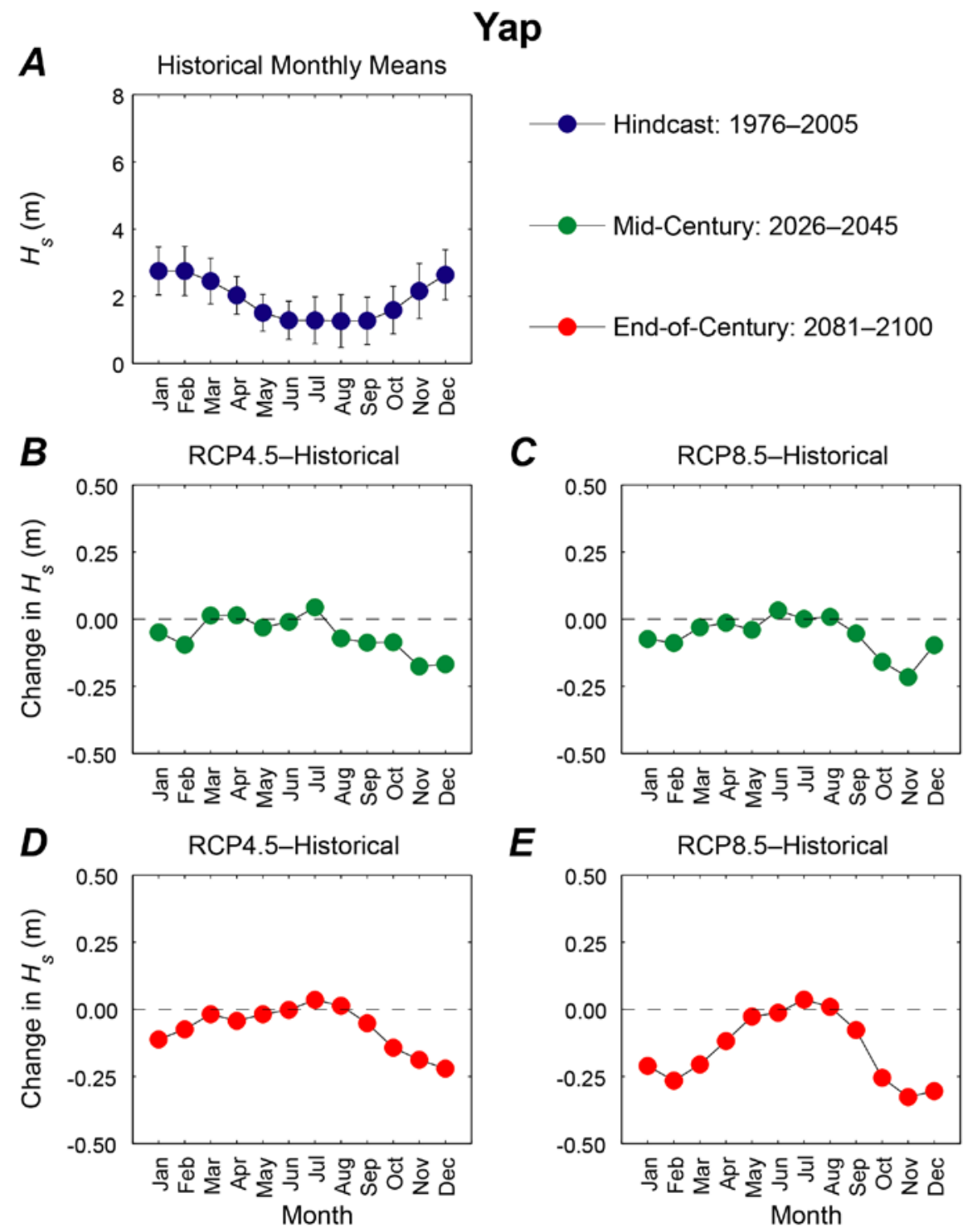

Appendix C21. Plots showing trends in monthly mean significant wave height $\left(H_{s}\right)$, in meters, at the Yap location. A. Hindcasted (1976-2005) mean significant wave heights by month with associated error bars. $B$. Plot of the change in mean 2026-2045 significant wave heights for the RCP4.5 scenario from hindcasted monthly significant wave height means. C. Plot of the change in mean 2026-2045 significant wave heights for the RCP8.5 scenario from hindcasted monthly significant wave height means. $D$. Plot of the change in mean 2081-2100 significant wave heights for the RCP4.5 scenario from hindcasted monthly significant wave height means. E. Plot of the change in mean 2081-2100 significant wave heights for the RCP8.5 scenario from hindcasted monthly significant wave height means. 

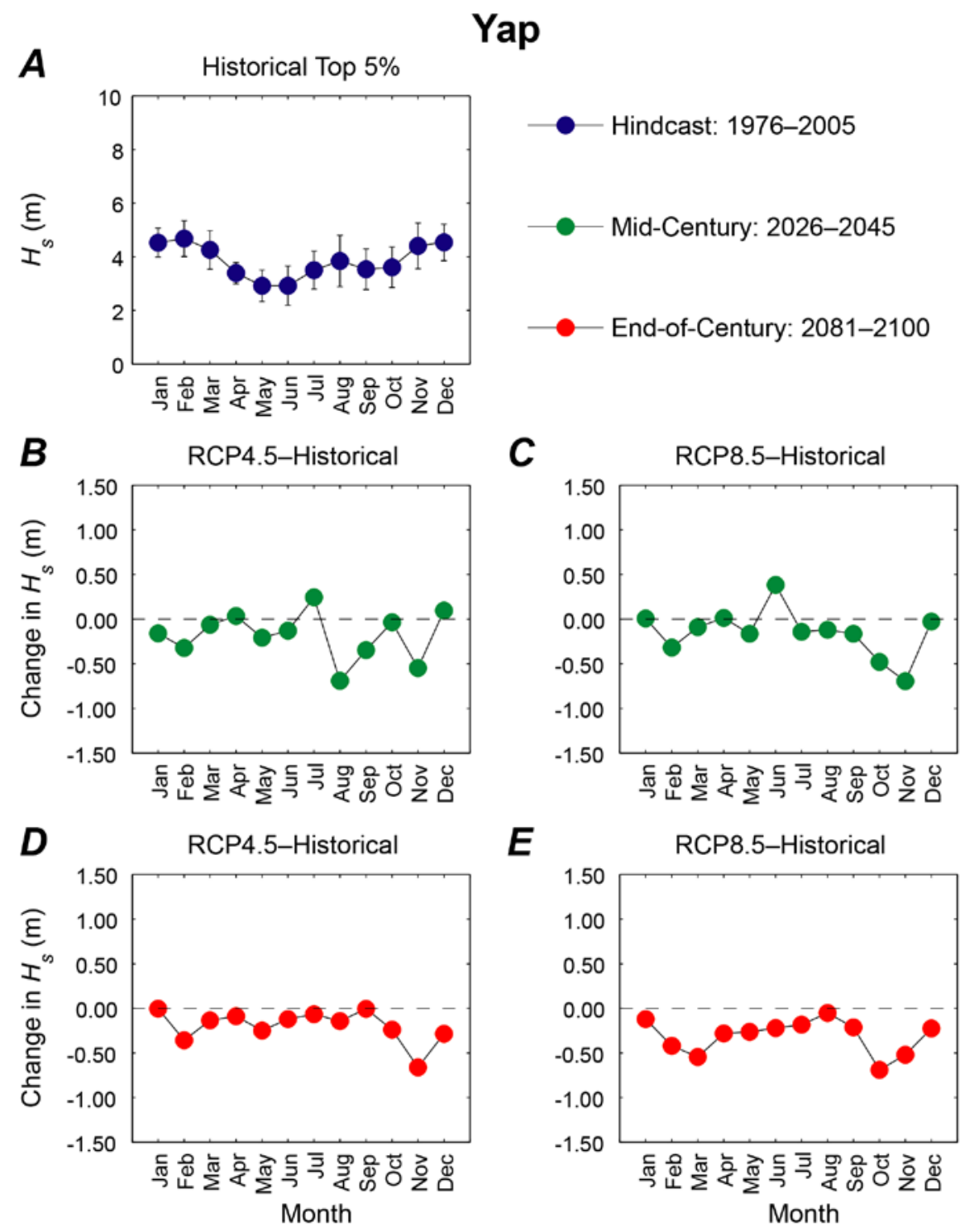

Appendix C22. Plots showing trends in monthly mean of the top 5 percent of significant wave heights $\left(H_{s}\right)$, in meters, at the Yap location. A. Hindcasted (1976-2005) mean of the top 5 percent of significant wave heights by month with associated error bars. $B$. Plot of the change in mean of the top 5 percent of 2026-2045 significant wave heights for the RCP4.5 scenario from hindcasted top 5 percent of monthly significant wave height means. $C$. Plot of the change in mean of the top 5 percent of 2026 2045 significant wave heights for the RCP8.5 scenario from hindcasted top 5 percent of monthly significant wave height means. D. Plot of the change in mean of the top 5 percent of 2081-2100 significant wave heights for the RCP4.5 scenario from hindcasted top 5 percent of monthly significant wave height means. E. Plot of the change in mean of the top 5 percent of 2081-2100 significant wave heights for the RCP8.5 scenario from hindcasted top 5 percent of monthly significant wave height means. 


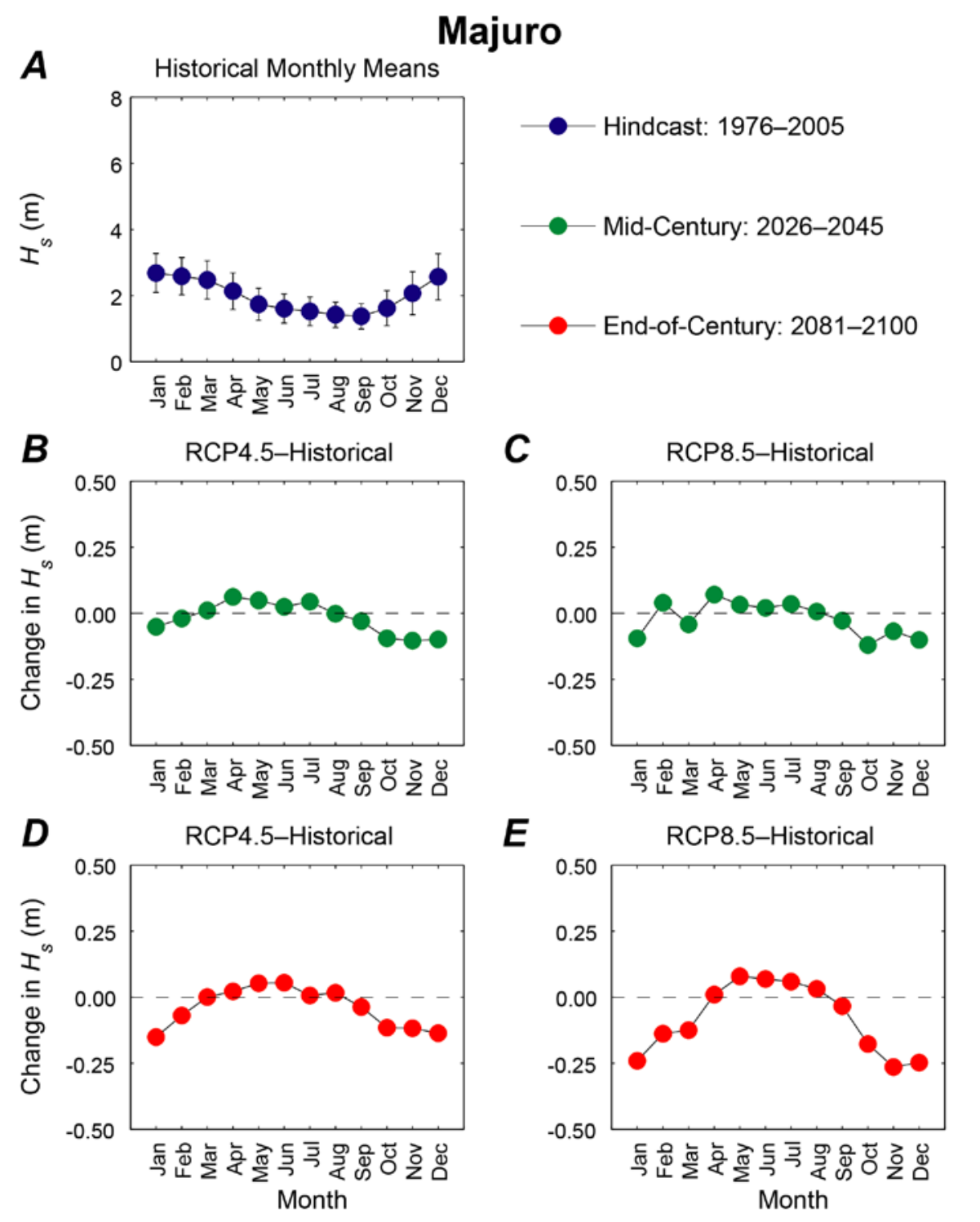

Appendix C23. Plots showing trends in monthly mean significant wave height $\left(H_{s}\right)$, in meters, at the Majuro location. A. Hindcasted (1976-2005) mean significant wave heights by month with associated error bars. $B$. Plot of the change in mean 2026-2045 significant wave heights for the RCP4.5 scenario from hindcasted monthly significant wave height means. C. Plot of the change in mean 2026-2045 significant wave heights for the RCP8.5 scenario from hindcasted monthly significant wave height means. $D$. Plot of the change in mean 2081-2100 significant wave heights for the RCP4.5 scenario from hindcasted monthly significant wave height means. E. Plot of the change in mean 2081-2100 significant wave heights for the RCP8.5 scenario from hindcasted monthly significant wave height means. 

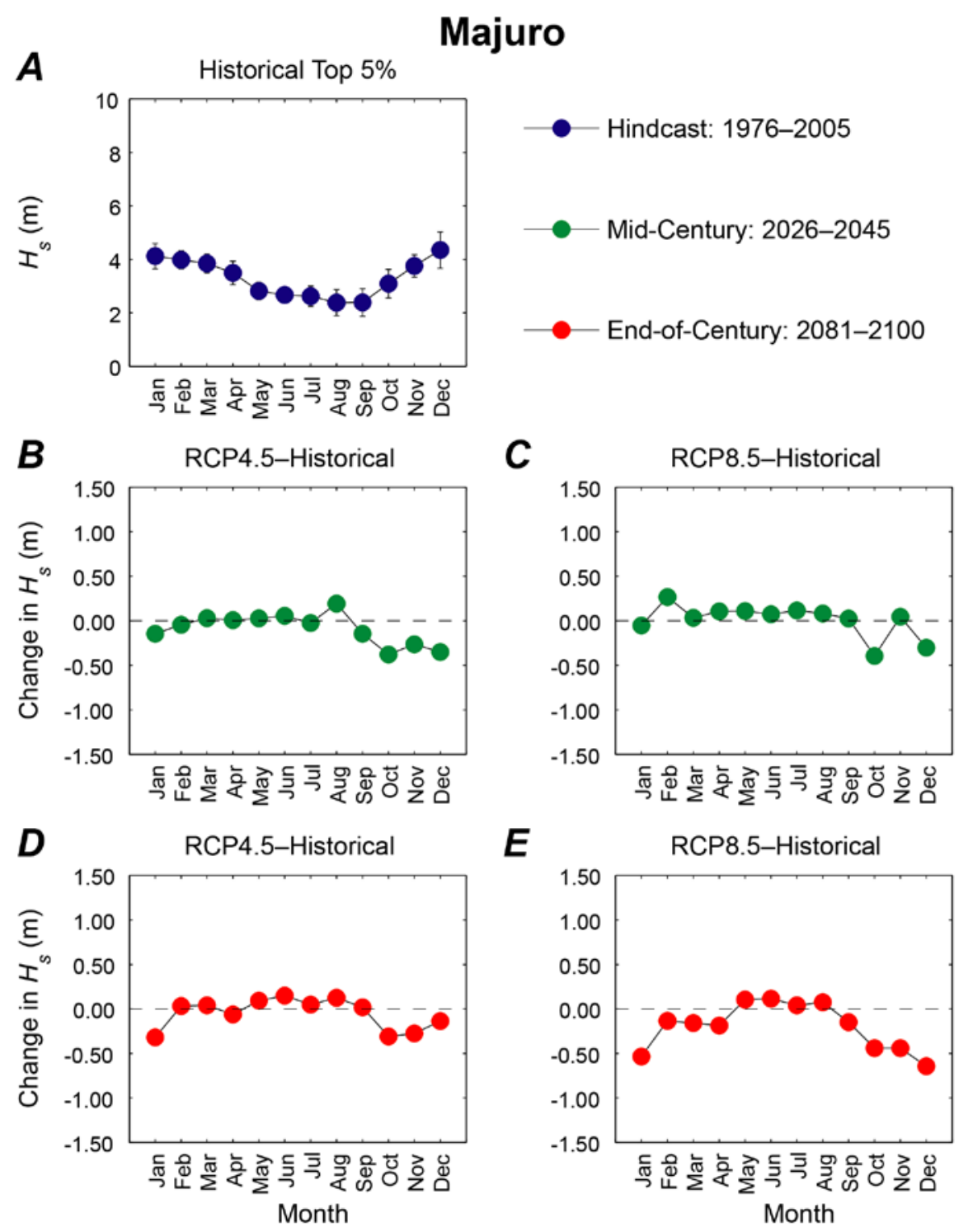

Appendix C24. Plots showing trends in monthly mean of the top 5 percent of significant wave heights $\left(H_{s}\right)$, in meters, at the Majuro location. A. Hindcasted (1976-2005) mean of the top 5 percent of significant wave heights by month with associated error bars. $B$. Plot of the change in mean of the top 5 percent of 2026-2045 significant wave heights for the RCP4.5 scenario from hindcasted top 5 percent of monthly significant wave height means. $C$. Plot of the change in mean of the top 5 percent of 2026 2045 significant wave heights for the RCP8.5 scenario from hindcasted top 5 percent of monthly significant wave height means. D. Plot of the change in mean of the top 5 percent of 2081-2100 significant wave heights for the RCP4.5 scenario from hindcasted top 5 percent of monthly significant wave height means. E. Plot of the change in mean of the top 5 percent of 2081-2100 significant wave heights for the RCP8.5 scenario from hindcasted top 5 percent of monthly significant wave height means. 

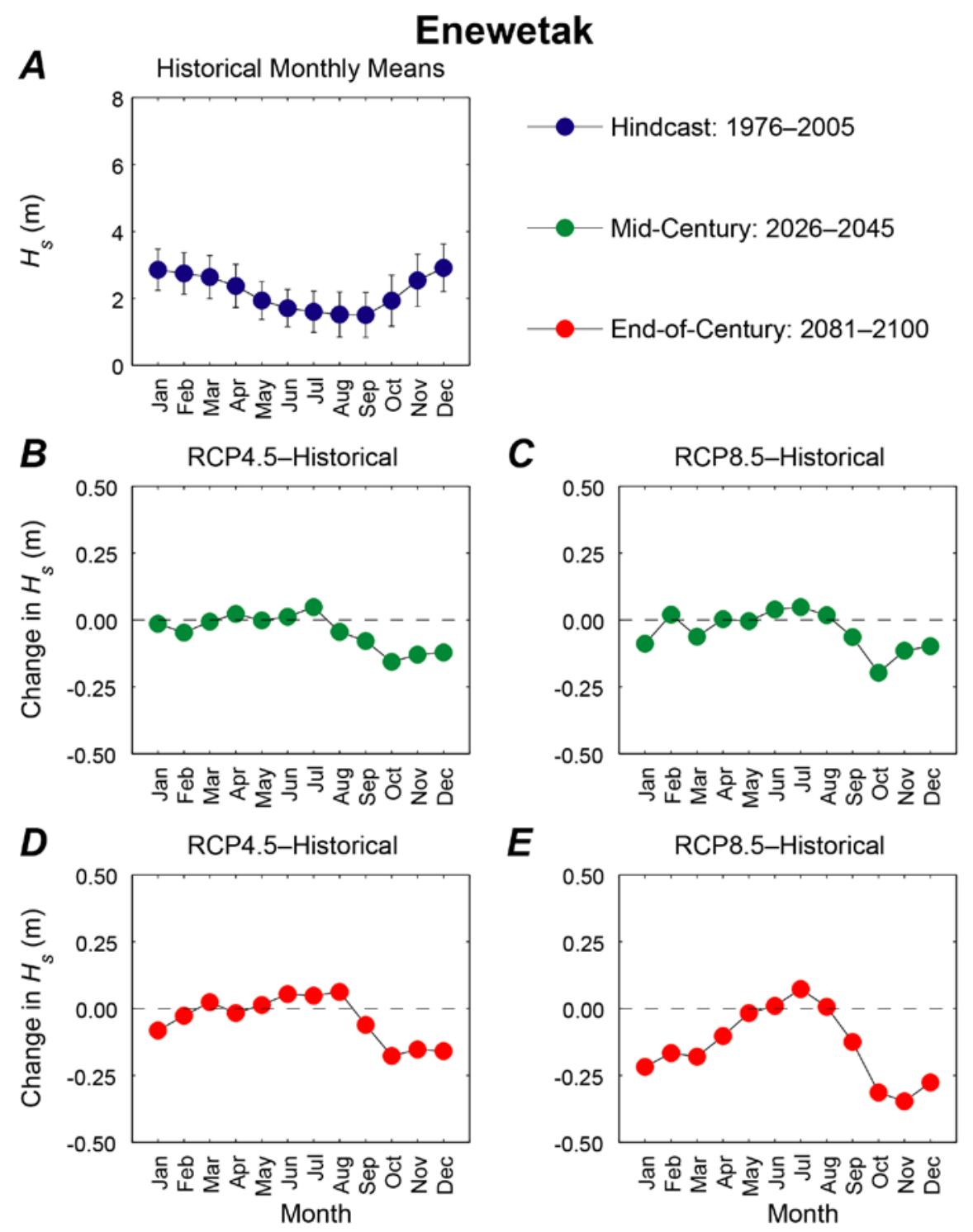

Appendix C25. Plots showing trends in monthly mean significant wave height $\left(H_{s}\right)$, in meters, at the Enewetak location. A. Hindcasted (1976-2005) mean significant wave heights by month with associated error bars. $B$. Plot of the change in mean 2026-2045 significant wave heights for the RCP4.5 scenario from hindcasted monthly significant wave height means. C. Plot of the change in mean 2026-2045 significant wave heights for the RCP8.5 scenario from hindcasted monthly significant wave height means. D. Plot of the change in mean 2081-2100 significant wave heights for the RCP4.5 scenario from hindcasted monthly significant wave height means. E. Plot of the change in mean 2081-2100 significant wave heights for the RCP8.5 scenario from hindcasted monthly significant wave height means. 

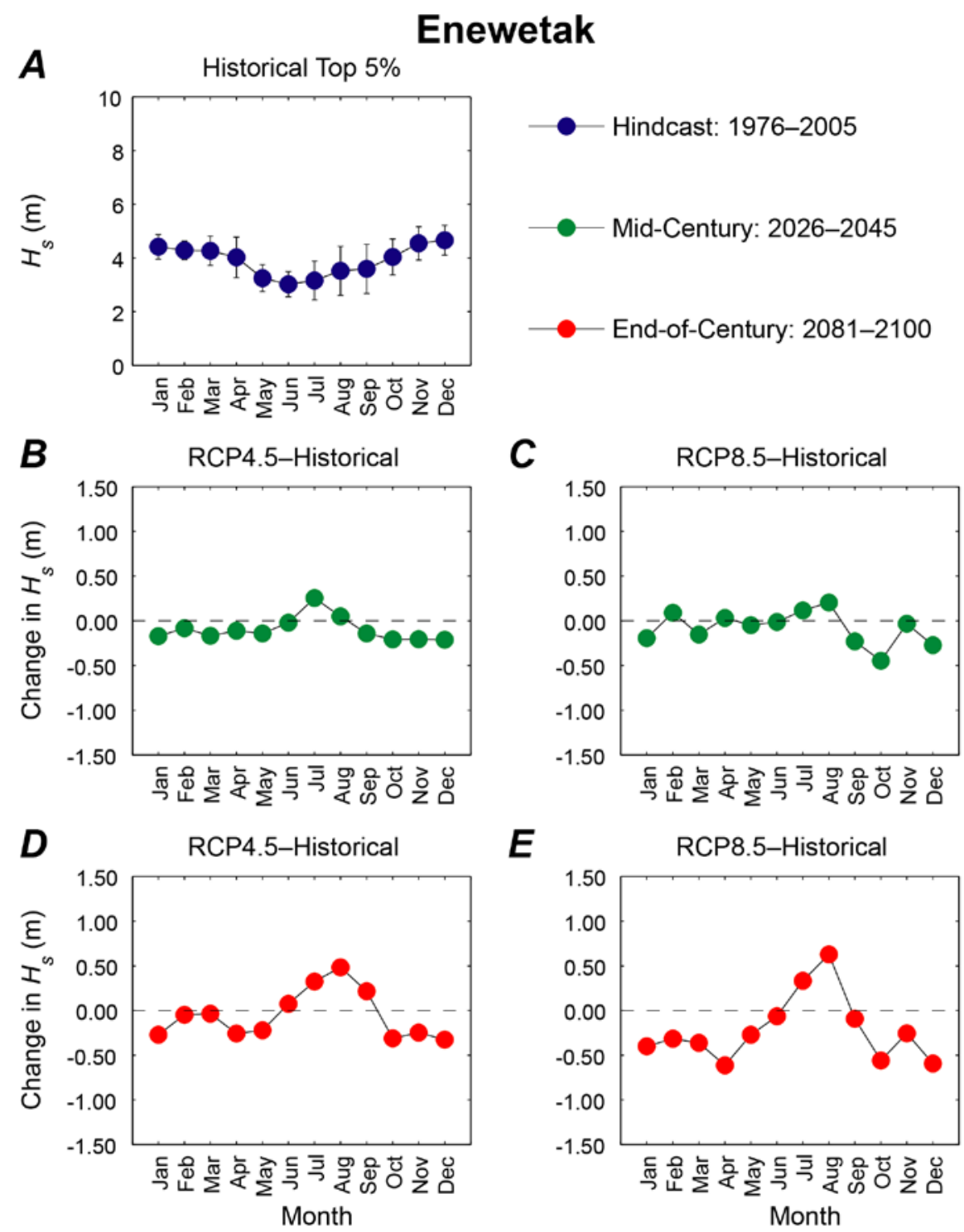

Appendix C26. Plots showing trends in monthly mean of the top 5 percent of significant wave heights $\left(H_{s}\right)$, in meters, at the Enewetak location. A. Hindcasted (1976-2005) mean of the top 5 percent of significant wave heights by month with associated error bars. $B$. Plot of the change in mean of the top 5 percent of 2026-2045 significant wave heights for the RCP4.5 scenario from hindcasted top 5 percent of monthly significant wave height means. $C$. Plot of the change in mean of the top 5 percent of 2026-2045 significant wave heights for the RCP8.5 scenario from hindcasted top 5 percent of monthly significant wave height means. $D$. Plot of the change in mean of the top 5 percent of 20812100 significant wave heights for the RCP4.5 scenario from hindcasted top 5 percent of monthly significant wave height means. E. Plot of the change in mean of the top 5 percent of 2081-2100 significant wave heights for the RCP8.5 scenario from hindcasted top 5 percent of monthly significant wave height means. 

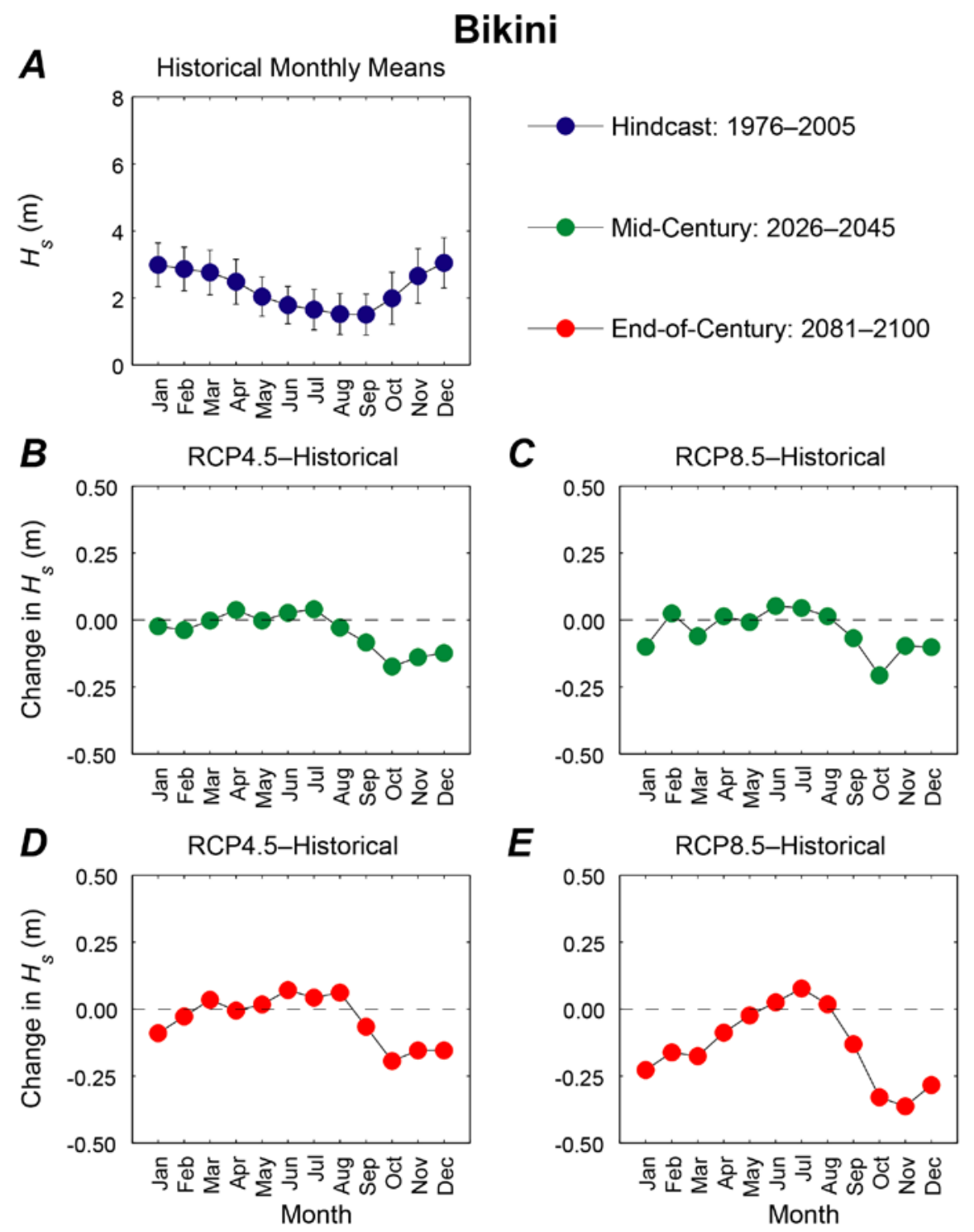

Appendix C27. Plots showing trends in monthly mean significant wave height $\left(H_{s}\right)$, in meters, at the Bikini location. A. Hindcasted (1976-2005) mean significant wave heights by month with associated error bars. $B$. Plot of the change in mean 2026-2045 significant wave heights for the RCP4.5 scenario from hindcasted monthly significant wave height means. C. Plot of the change in mean 2026-2045 significant wave heights for the RCP8.5 scenario from hindcasted monthly significant wave height means. $D$. Plot of the change in mean 2081-2100 significant wave heights for the RCP4.5 scenario from hindcasted monthly significant wave height means. E. Plot of the change in mean 2081-2100 significant wave heights for the RCP8.5 scenario from hindcasted monthly significant wave height means. 

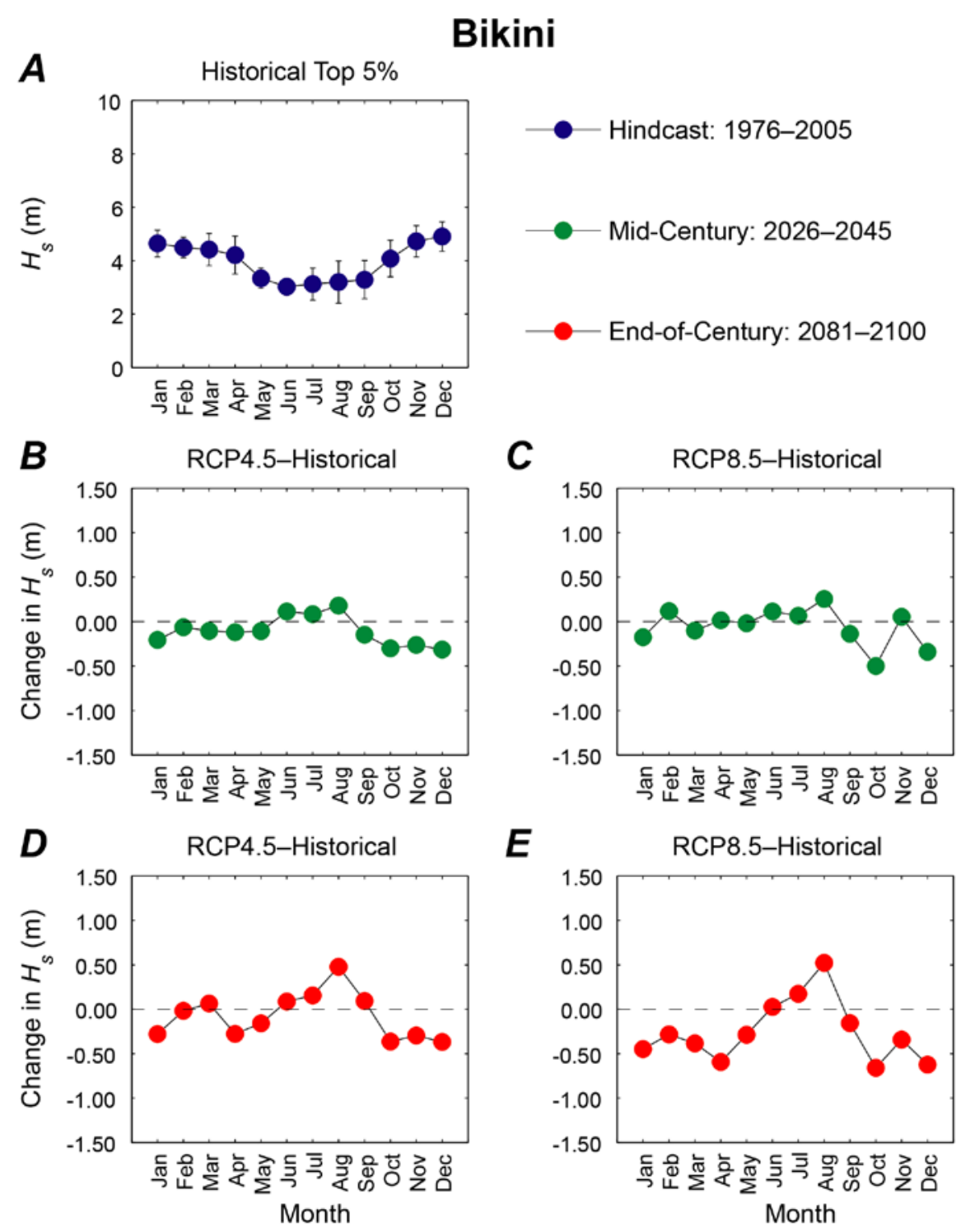

Appendix C28. Plots showing trends in monthly mean of the top 5 percent of significant wave heights $\left(H_{s}\right)$, in meters, at the Bikini location. A. Hindcasted (1976-2005) mean of the top 5 percent of significant wave heights by month with associated error bars. $B$. Plot of the change in mean of the top 5 percent of 2026-2045 significant wave heights for the RCP4.5 scenario from hindcasted top 5 percent of monthly significant wave height means. $C$. Plot of the change in mean of the top 5 percent of 2026 2045 significant wave heights for the RCP8.5 scenario from hindcasted top 5 percent of monthly significant wave height means. D. Plot of the change in mean of the top 5 percent of 2081-2100 significant wave heights for the RCP4.5 scenario from hindcasted top 5 percent of monthly significant wave height means. E. Plot of the change in mean of the top 5 percent of 2081-2100 significant wave heights for the RCP8.5 scenario from hindcasted top 5 percent of monthly significant wave height means. 

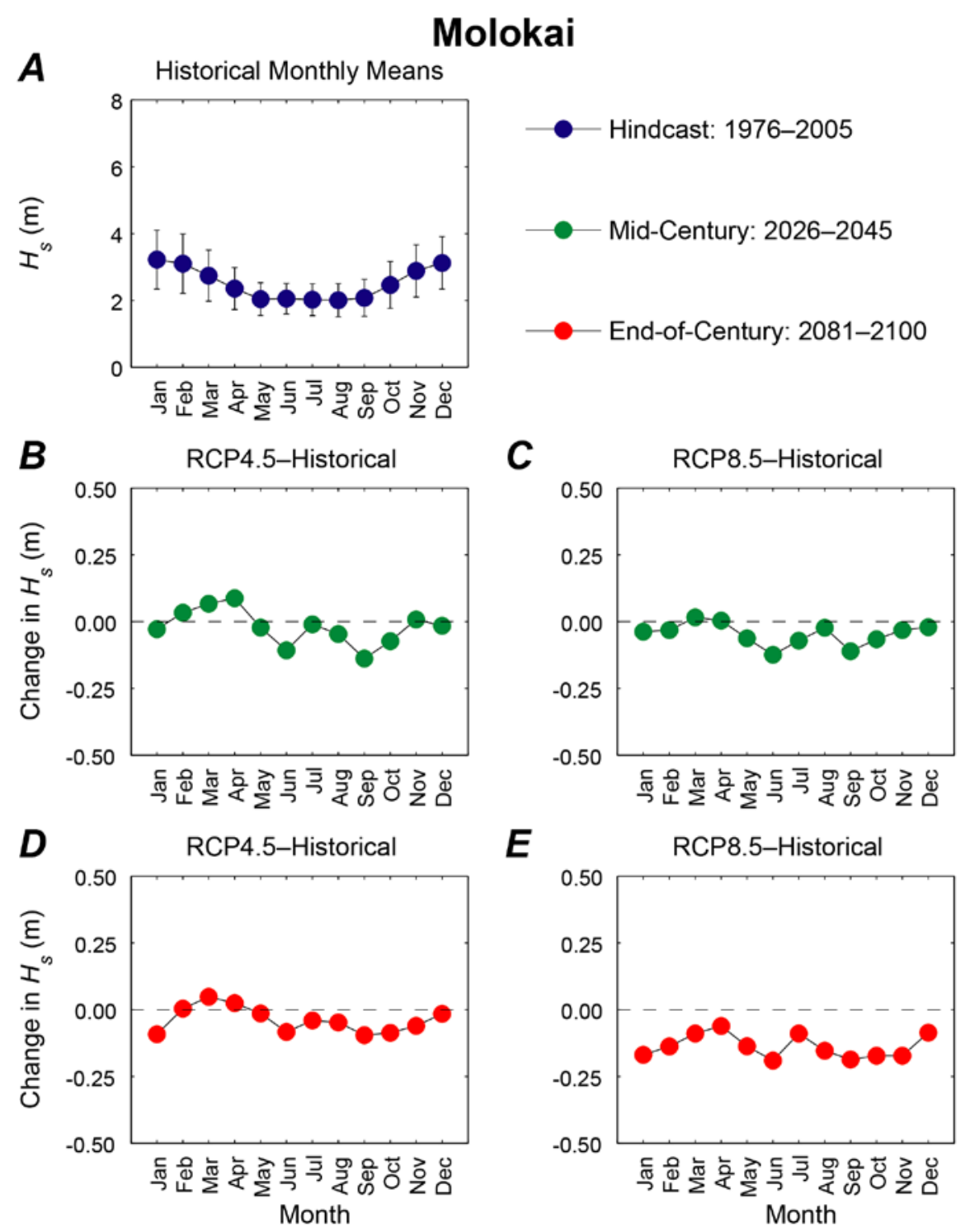

Appendix C29. Plots showing trends in monthly mean significant wave height $\left(H_{s}\right)$, in meters, at the Molokai location. A. Hindcasted (1976-2005) mean significant wave heights by month with associated error bars. $B$. Plot of the change in mean 2026-2045 significant wave heights for the RCP4.5 scenario from hindcasted monthly significant wave height means. C. Plot of the change in mean 2026-2045 significant wave heights for the RCP8.5 scenario from hindcasted monthly significant wave height means. D. Plot of the change in mean 2081-2100 significant wave heights for the RCP4.5 scenario from hindcasted monthly significant wave height means. E. Plot of the change in mean 2081-2100 significant wave heights for the RCP8.5 scenario from hindcasted monthly significant wave height means. 

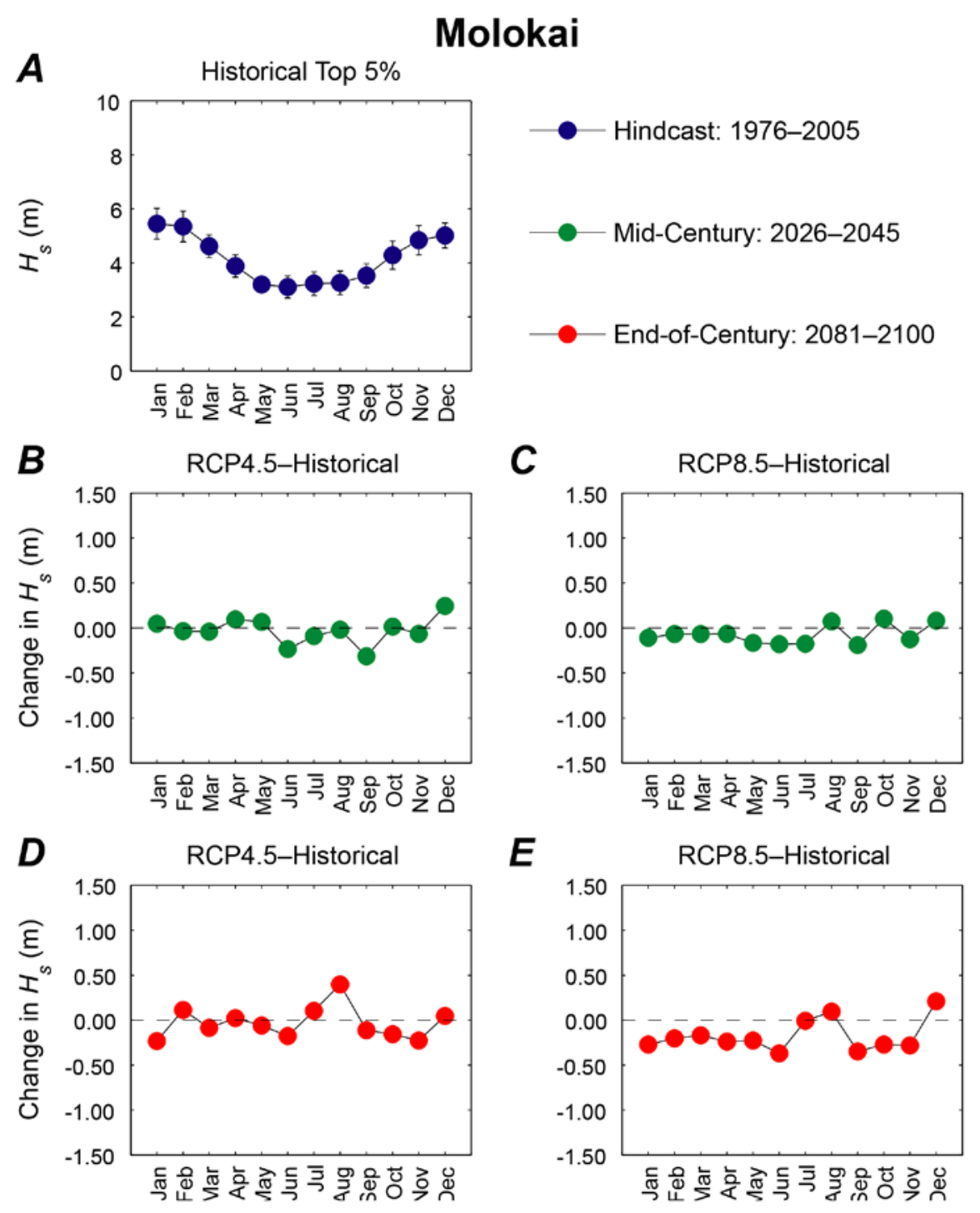

Appendix C30. Plots showing trends in monthly mean of the top 5 percent of significant wave heights $\left(H_{s}\right)$, in meters, at the Molokai location. A. Hindcasted (1976-2005) mean of the top 5 percent of significant wave heights by month with associated error bars. $B$. Plot of the change in mean of the top 5 percent of 2026-2045 significant wave heights for the RCP4.5 scenario from hindcasted top 5 percent of monthly significant wave height means. $C$. Plot of the change in mean of the top 5 percent of 2026-2045 significant wave heights for the RCP8.5 scenario from hindcasted top 5 percent of monthly significant wave height means. $D$. Plot of the change in mean of the top 5 percent of 20812100 significant wave heights for the RCP4.5 scenario from hindcasted top 5 percent of monthly significant wave height means. E. Plot of the change in mean of the top 5 percent of 2081-2100 significant wave heights for the RCP8.5 scenario from hindcasted top 5 percent of monthly significant wave height means. 

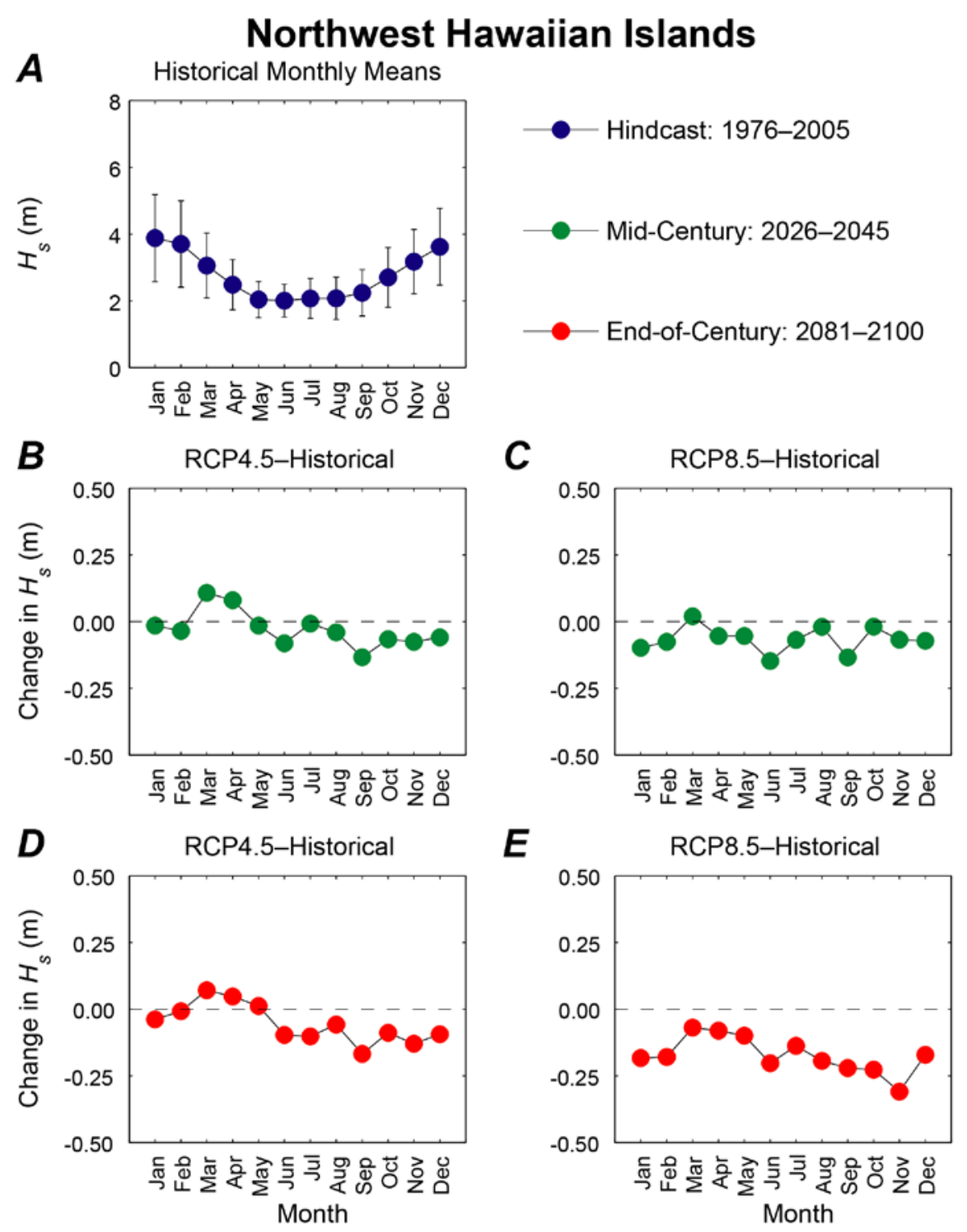

Appendix C31. Plots showing trends in monthly mean significant wave height $\left(H_{s}\right)$, in meters, at the Northwest Hawaiian Islands location. A. Hindcasted (1976-2005) mean significant wave heights by month with associated error bars. B. Plot of the change in mean 2026-2045 significant wave heights for the RCP4.5 scenario from hindcasted monthly significant wave height means. C. Plot of the change in mean 2026-2045 significant wave heights for the RCP8.5 scenario from hindcasted monthly significant wave height means. $D$. Plot of the change in mean 2081-2100 significant wave heights for the RCP4.5 scenario from hindcasted monthly significant wave height means. E. Plot of the change in mean 2081-2100 significant wave heights for the RCP8.5 scenario from hindcasted monthly significant wave height means. 

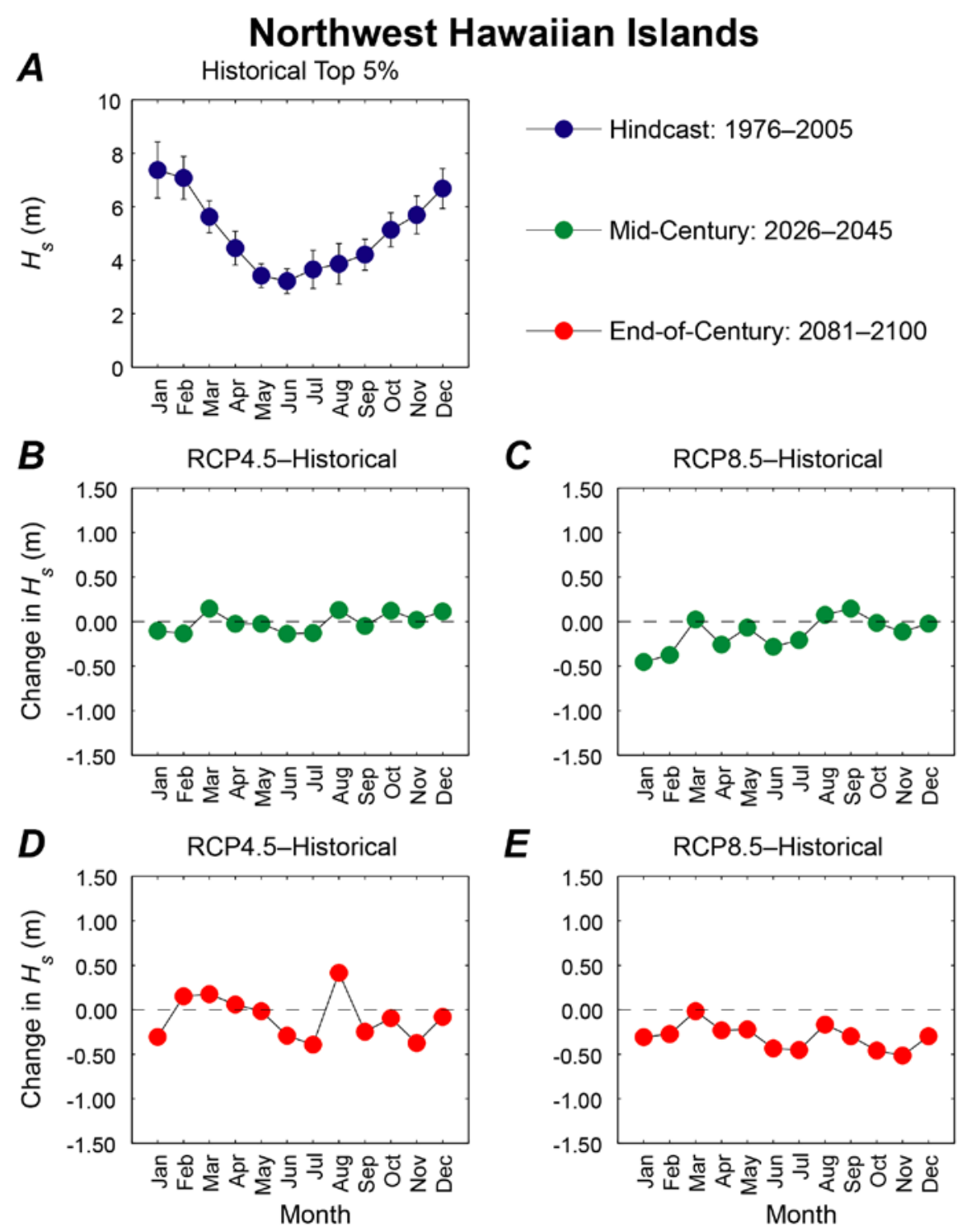

Appendix C32. Plots showing trends in monthly mean of the top 5 percent of significant wave heights $\left(H_{s}\right)$, in meters, at the Northwest Hawaiian Islands location. A. Hindcasted (1976-2005) mean of the top 5 percent of significant wave heights by month with associated error bars. $B$. Plot of the change in mean of the top 5 percent of 2026-2045 significant wave heights for the RCP4.5 scenario from hindcasted top 5 percent of monthly significant wave height means. $C$. Plot of the change in mean of the top 5 percent of 2026-2045 significant wave heights for the RCP8.5 scenario from hindcasted top 5 percent of monthly significant wave height means. $D$. Plot of the change in mean of the top 5 percent of 2081-2100 significant wave heights for the RCP4.5 scenario from hindcasted top 5 percent of monthly significant wave height means. $E$. Plot of the change in mean of the top 5 percent of 2081-2100 significant wave heights for the RCP8.5 scenario from hindcasted top 5 percent of monthly significant wave height means. 

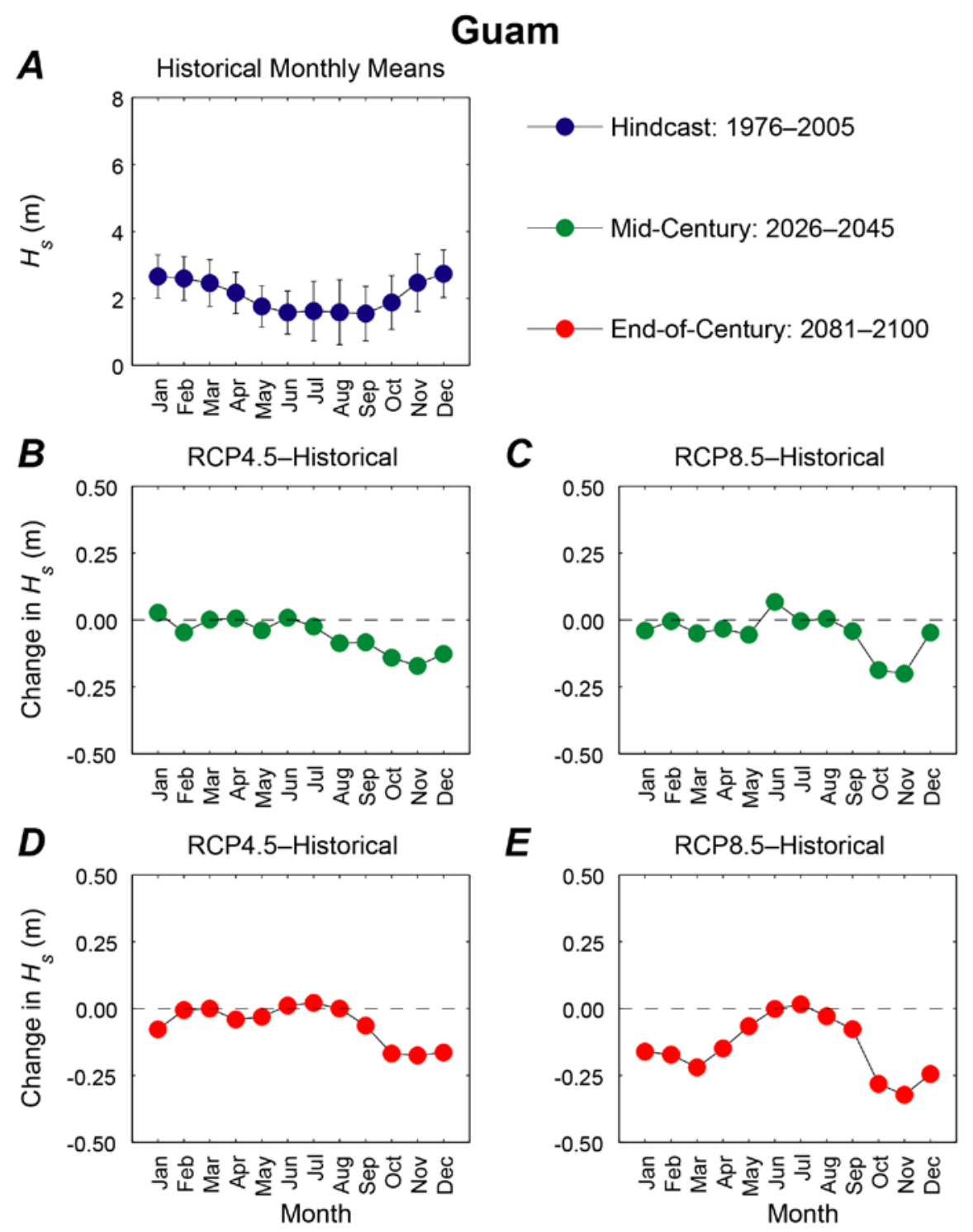

Appendix C33. Plots showing trends in monthly mean significant wave height $\left(H_{s}\right)$, in meters, at the Guam location. A. Hindcasted (1976-2005) mean significant wave heights by month with associated error bars. $B$. Plot of the change in mean 2026-2045 significant wave heights for the RCP4.5 scenario from hindcasted monthly significant wave height means. C. Plot of the change in mean 2026-2045 significant wave heights for the RCP8.5 scenario from hindcasted monthly significant wave height means. D. Plot of the change in mean 2081-2100 significant wave heights for the RCP4.5 scenario from hindcasted monthly significant wave height means. E. Plot of the change in mean 2081-2100 significant wave heights for the RCP8.5 scenario from hindcasted monthly significant wave height means. 

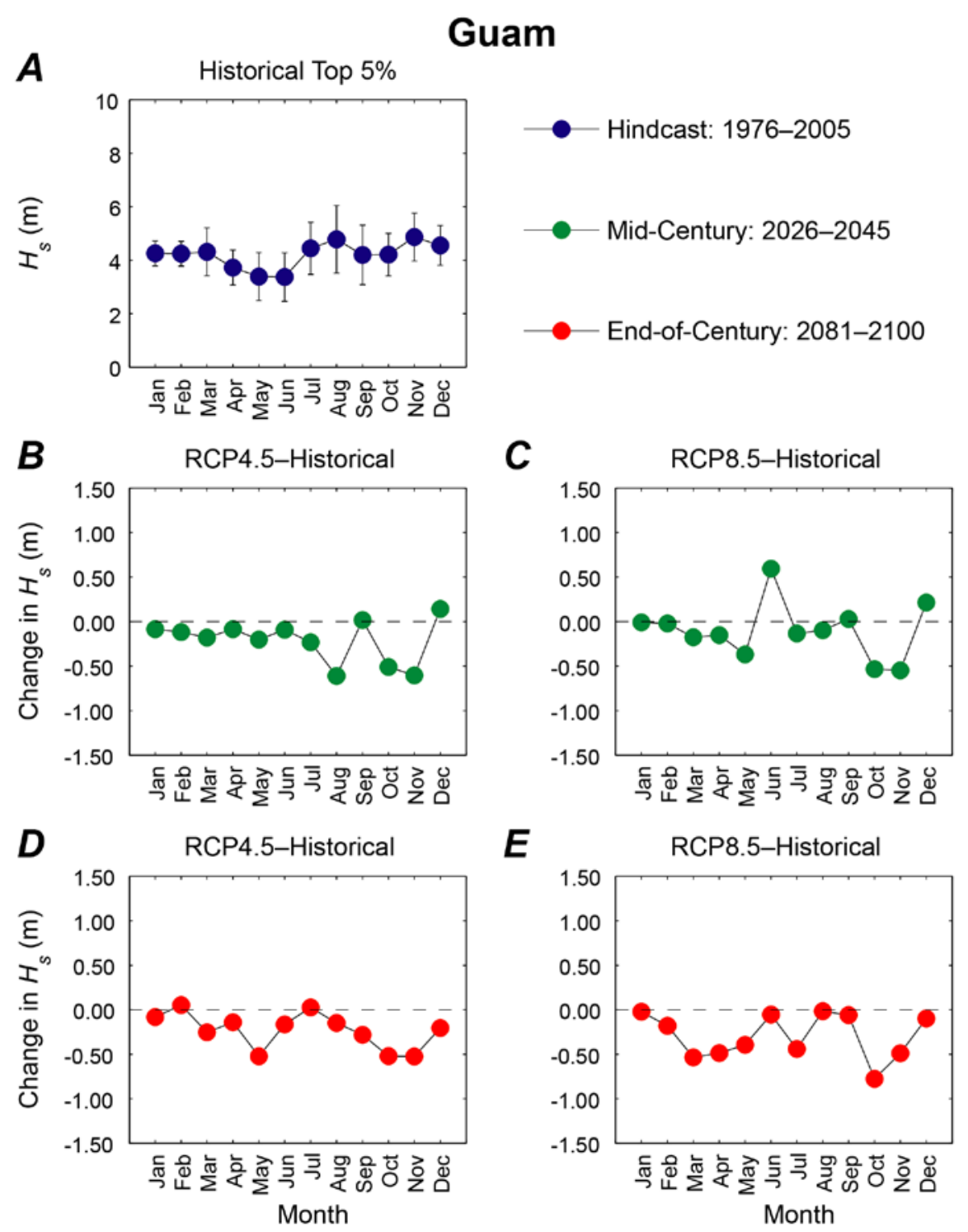

Appendix C34. Plots showing trends in monthly mean of the top 5 percent of significant wave heights $\left(H_{s}\right)$, in meters, at the Guam location. A. Hindcasted (1976-2005) mean of the top 5 percent of significant wave heights by month with associated error bars. $B$. Plot of the change in mean of the top 5 percent of 2026-2045 significant wave heights for the RCP4.5 scenario from hindcasted top 5 percent of monthly significant wave height means. $C$. Plot of the change in mean of the top 5 percent of 2026 2045 significant wave heights for the RCP8.5 scenario from hindcasted top 5 percent of monthly significant wave height means. D. Plot of the change in mean of the top 5 percent of 2081-2100 significant wave heights for the RCP4.5 scenario from hindcasted top 5 percent of monthly significant wave height means. E. Plot of the change in mean of the top 5 percent of 2081-2100 significant wave heights for the RCP8.5 scenario from hindcasted top 5 percent of monthly significant wave height means. 

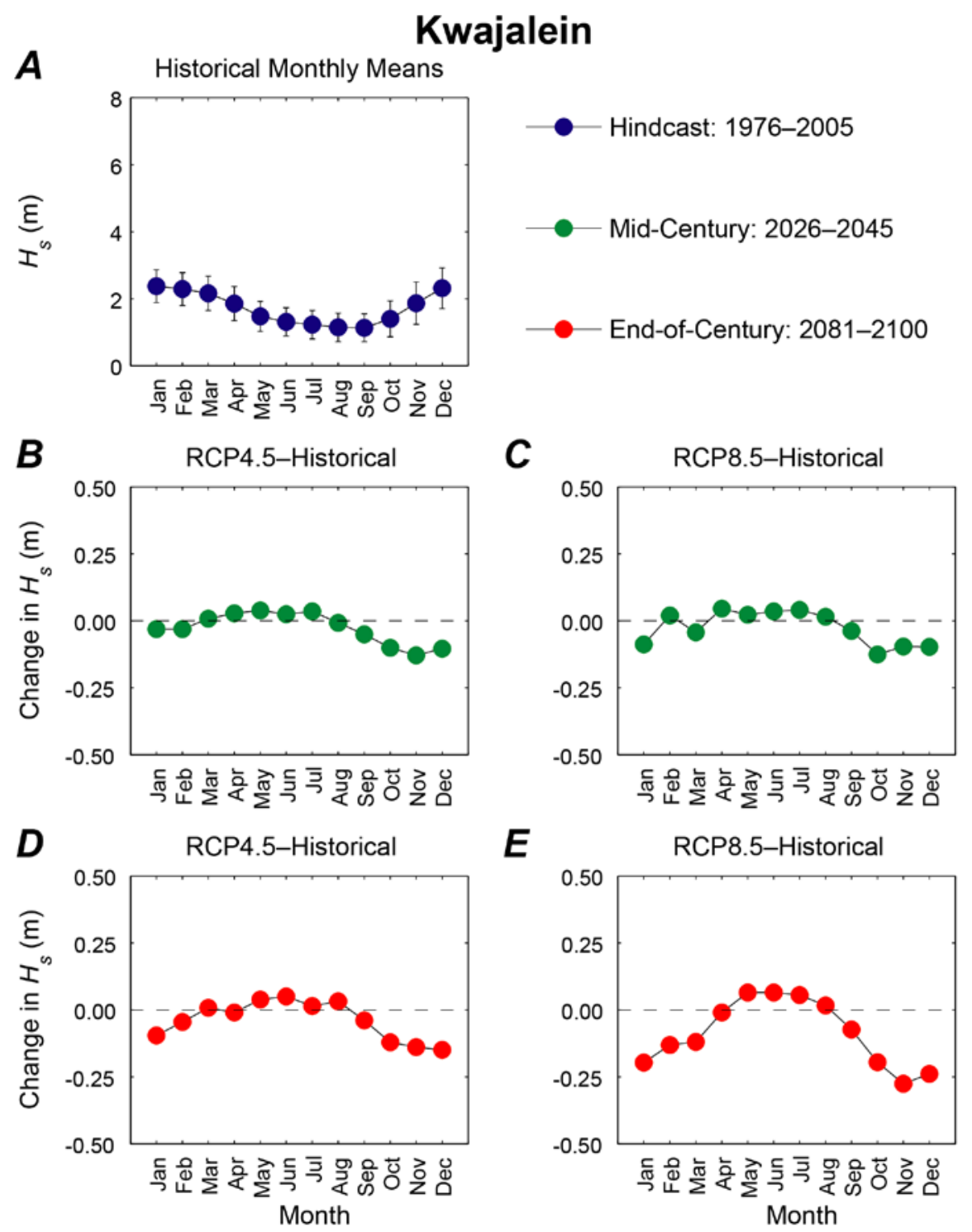

Appendix C35. Plots showing trends in monthly mean significant wave height $\left(H_{s}\right)$, in meters, at the Kwajalein location. A. Hindcasted (1976-2005) mean significant wave heights by month with associated error bars. $B$. Plot of the change in mean 2026-2045 significant wave heights for the RCP4.5 scenario from hindcasted monthly significant wave height means. C. Plot of the change in mean 2026-2045 significant wave heights for the RCP8.5 scenario from hindcasted monthly significant wave height means. D. Plot of the change in mean 2081-2100 significant wave heights for the RCP4.5 scenario from hindcasted monthly significant wave height means. E. Plot of the change in mean 2081-2100 significant wave heights for the RCP8.5 scenario from hindcasted monthly significant wave height means. 

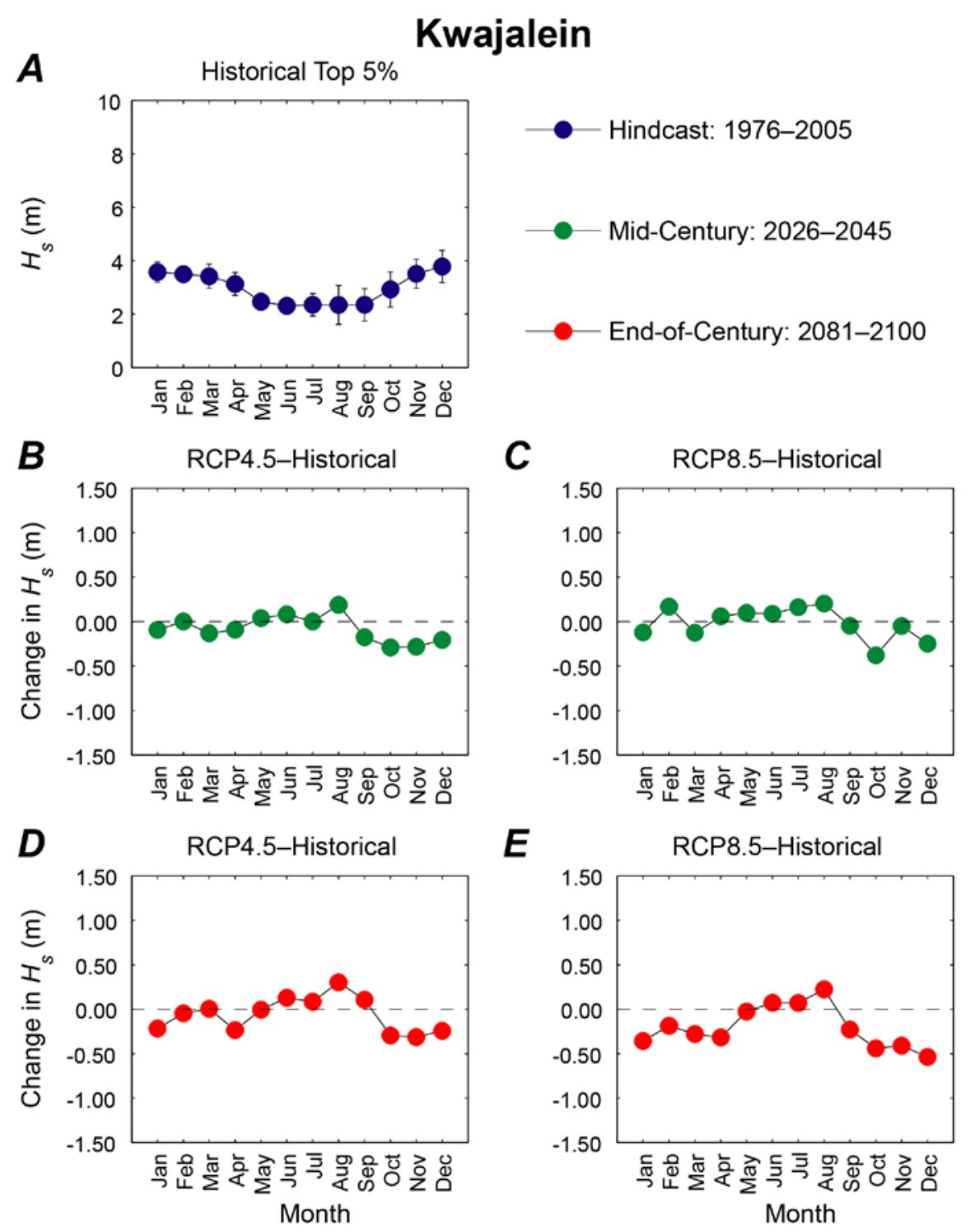

Appendix C36. Plots showing trends in monthly mean of the top 5 percent of significant wave heights $\left(H_{s}\right)$, in meters, at the Kwajalein location. A. Hindcasted (1976-2005) mean of the top 5 percent of significant wave heights by month with associated error bars. $B$. Plot of the change in mean of the top 5 percent of 2026-2045 significant wave heights for the RCP4.5 scenario from hindcasted top 5 percent of monthly significant wave height means. $C$. Plot of the change in mean of the top 5 percent of 2026-2045 significant wave heights for the RCP8.5 scenario from hindcasted top 5 percent of monthly significant wave height means. $D$. Plot of the change in mean of the top 5 percent of 20812100 significant wave heights for the RCP4.5 scenario from hindcasted top 5 percent of monthly significant wave height means. E. Plot of the change in mean of the top 5 percent of 2081-2100 significant wave heights for the RCP8.5 scenario from hindcasted top 5 percent of monthly significant wave height means. 

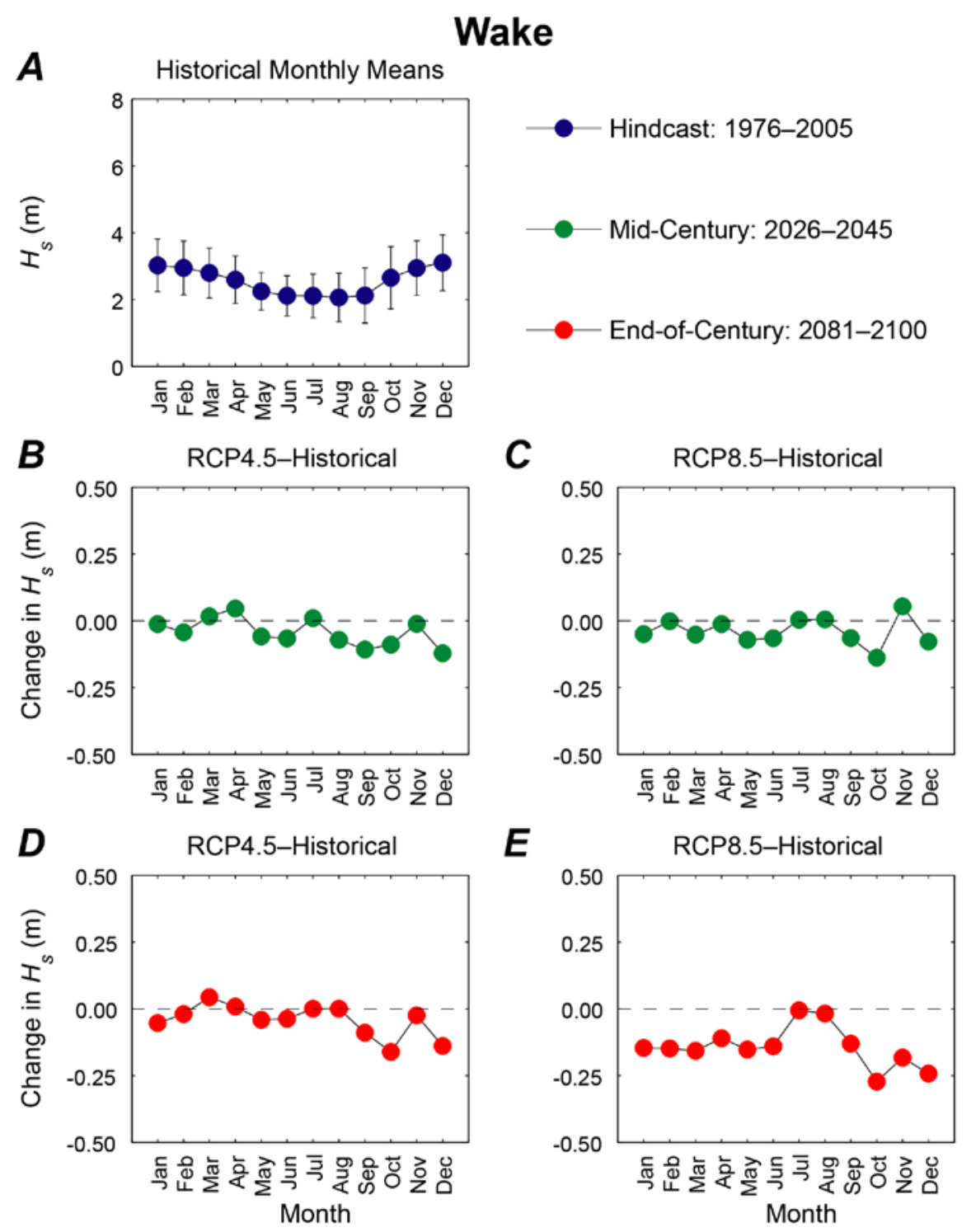

Appendix C37. Plots showing trends in monthly mean significant wave height $\left(H_{s}\right)$, in meters, at the Wake location. A. Hindcasted (1976-2005) mean significant wave heights by month with associated error bars. $B$. Plot of the change in mean 2026-2045 significant wave heights for the RCP4.5 scenario from hindcasted monthly significant wave height means. C. Plot of the change in mean 2026-2045 significant wave heights for the RCP8.5 scenario from hindcasted monthly significant wave height means. D. Plot of the change in mean 2081-2100 significant wave heights for the RCP4.5 scenario from hindcasted monthly significant wave height means. E. Plot of the change in mean 2081-2100 significant wave heights for the RCP8.5 scenario from hindcasted monthly significant wave height means. 

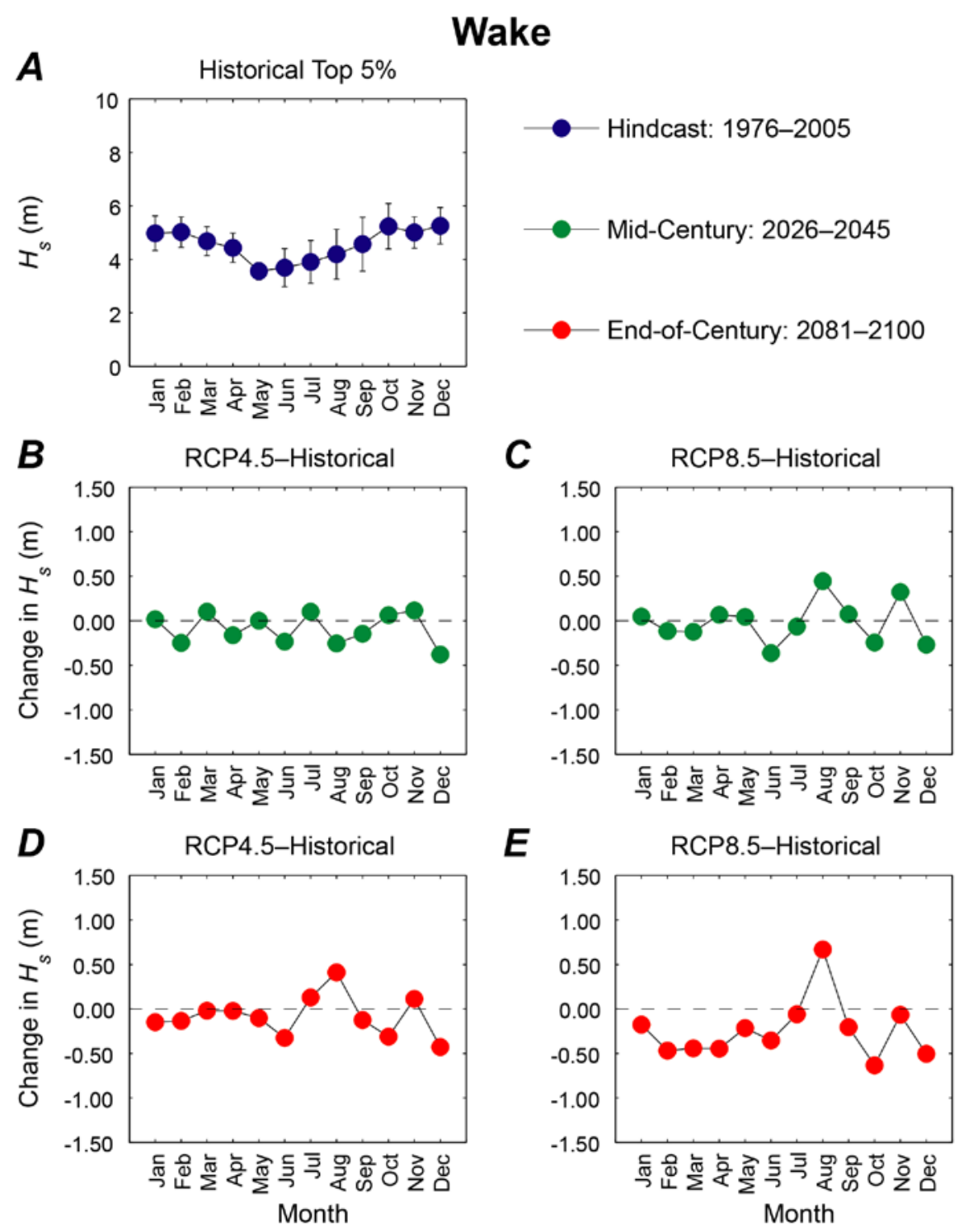

Appendix C38. Plots showing trends in monthly mean of the top 5 percent of significant wave heights $\left(H_{s}\right)$, in meters, at the Wake location. $A$. Hindcasted (1976-2005) mean of the top 5 percent of significant wave heights by month with associated error bars. $B$. Plot of the change in mean of the top 5 percent of 2026-2045 significant wave heights for the RCP4.5 scenario from hindcasted top 5 percent of monthly significant wave height means. $C$. Plot of the change in mean of the top 5 percent of 2026 2045 significant wave heights for the RCP8.5 scenario from hindcasted top 5 percent of monthly significant wave height means. D. Plot of the change in mean of the top 5 percent of 2081-2100 significant wave heights for the RCP4.5 scenario from hindcasted top 5 percent of monthly significant wave height means. E. Plot of the change in mean of the top 5 percent of 2081-2100 significant wave heights for the RCP8.5 scenario from hindcasted top 5 percent of monthly significant wave height means. 


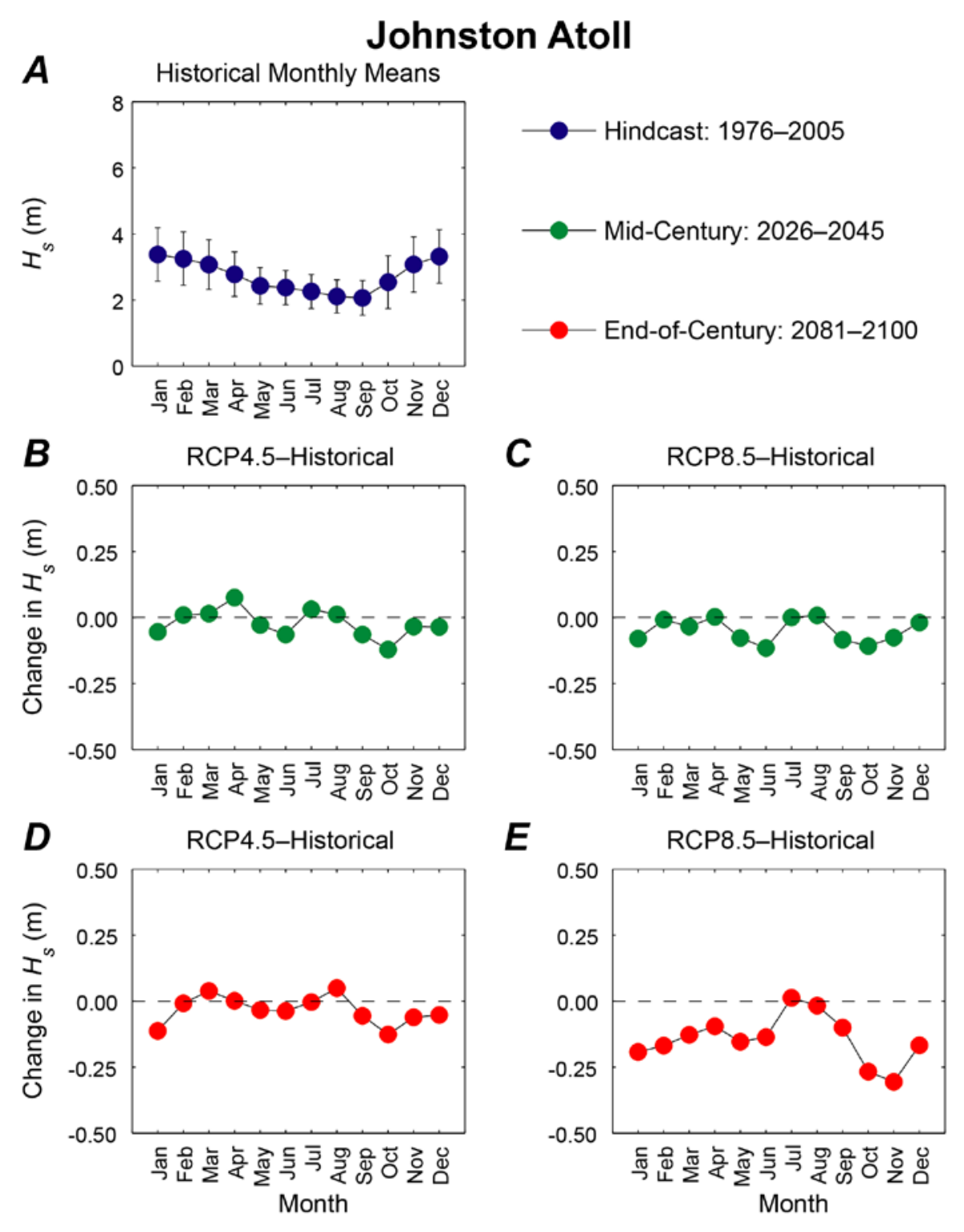

Appendix C39. Plots showing trends in monthly mean significant wave height $\left(H_{s}\right)$, in meters, at the Johnston Atoll location. A. Hindcasted (1976-2005) mean significant wave heights by month with associated error bars. B. Plot of the change in mean 2026-2045 significant wave heights for the RCP4.5 scenario from hindcasted monthly significant wave height means. C. Plot of the change in mean 2026-2045 significant wave heights for the RCP8.5 scenario from hindcasted monthly significant wave height means. D. Plot of the change in mean 2081-2100 significant wave heights for the RCP4.5 scenario from hindcasted monthly significant wave height means. E. Plot of the change in mean 2081-2100 significant wave heights for the RCP8.5 scenario from hindcasted monthly significant wave height means. 

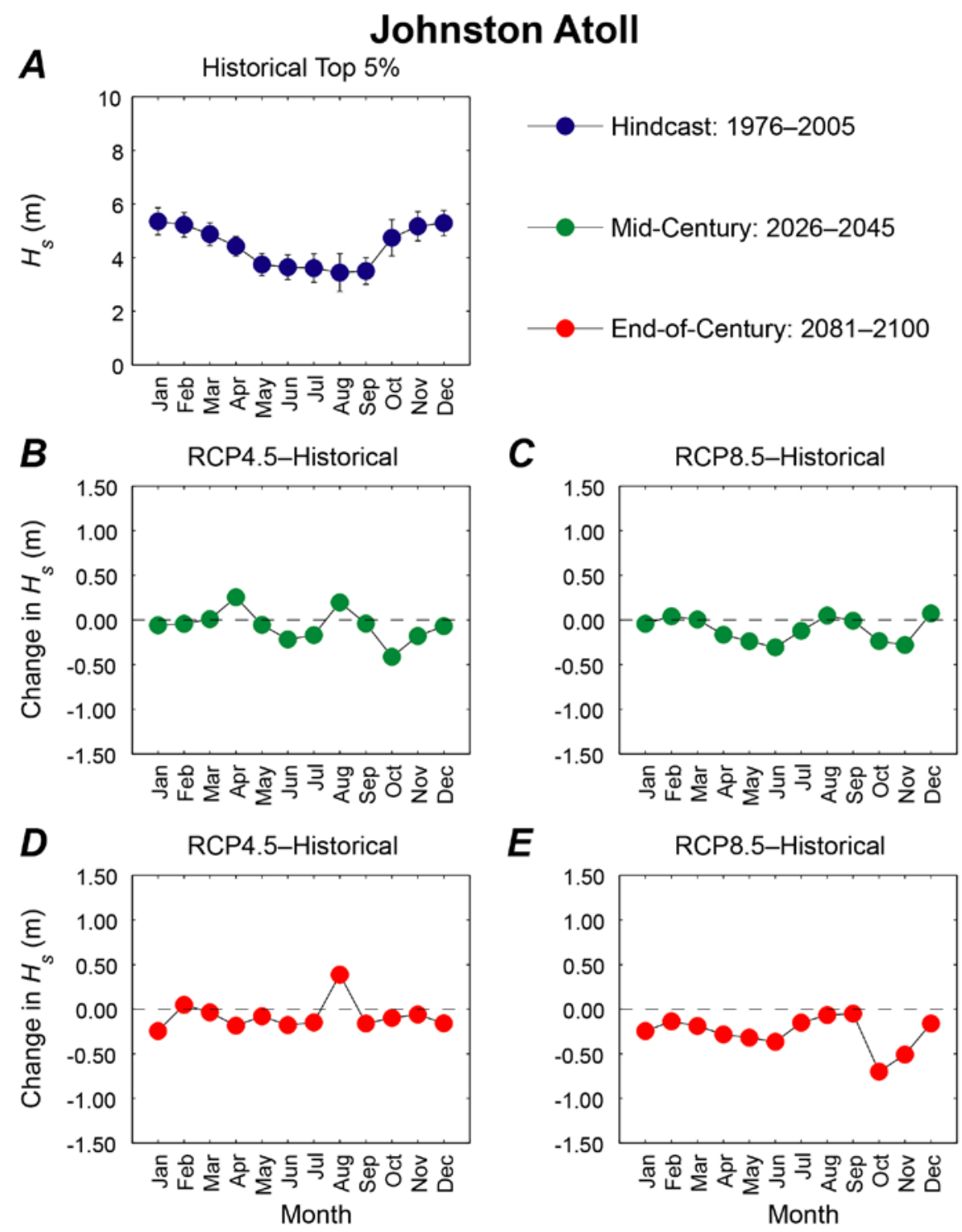

Appendix $\mathbf{C 4 0 . P l o t s ~ s h o w i n g ~ t r e n d s ~ i n ~ m o n t h l y ~ m e a n ~ o f ~ t h e ~ t o p ~} 5$ percent of significant wave heights $\left(H_{s}\right)$, in meters, at the Johnston Atoll location. A. Hindcasted (1976-2005) mean of the top 5 percent of significant wave heights by month with associated error bars. $B$. Plot of the change in mean of the top 5 percent of 2026-2045 significant wave heights for the RCP4.5 scenario from hindcasted top 5 percent of monthly significant wave height means. $C$. Plot of the change in mean of the top 5 percent of 2026-2045 significant wave heights for the RCP8.5 scenario from hindcasted top 5 percent of monthly significant wave height means. $D$. Plot of the change in mean of the top 5 percent of 20812100 significant wave heights for the RCP4.5 scenario from hindcasted top 5 percent of monthly significant wave height means. E. Plot of the change in mean of the top 5 percent of 2081-2100 significant wave heights for the RCP8.5 scenario from hindcasted top 5 percent of monthly significant wave height means. 

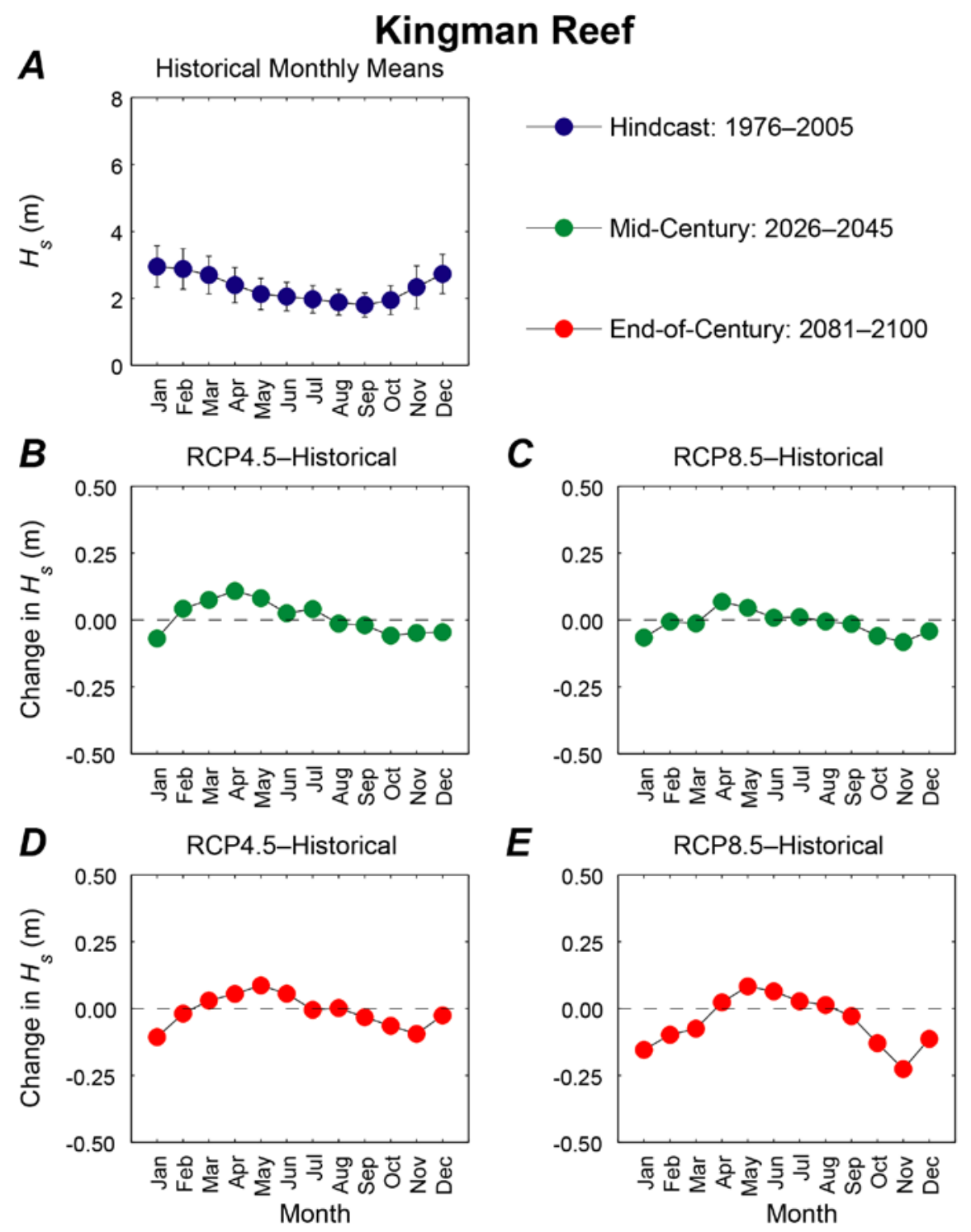

Appendix C41. Plots showing trends in monthly mean significant wave height $\left(H_{s}\right)$, in meters, at the Kingman Reef location. A. Hindcasted (1976-2005) mean significant wave heights by month with associated error bars. B. Plot of the change in mean 2026-2045 significant wave heights for the RCP4.5 scenario from hindcasted monthly significant wave height means. C. Plot of the change in mean 2026-2045 significant wave heights for the RCP8.5 scenario from hindcasted monthly significant wave height means. D. Plot of the change in mean 2081-2100 significant wave heights for the RCP4.5 scenario from hindcasted monthly significant wave height means. E. Plot of the change in mean 2081-2100 significant wave heights for the RCP8.5 scenario from hindcasted monthly significant wave height means. 

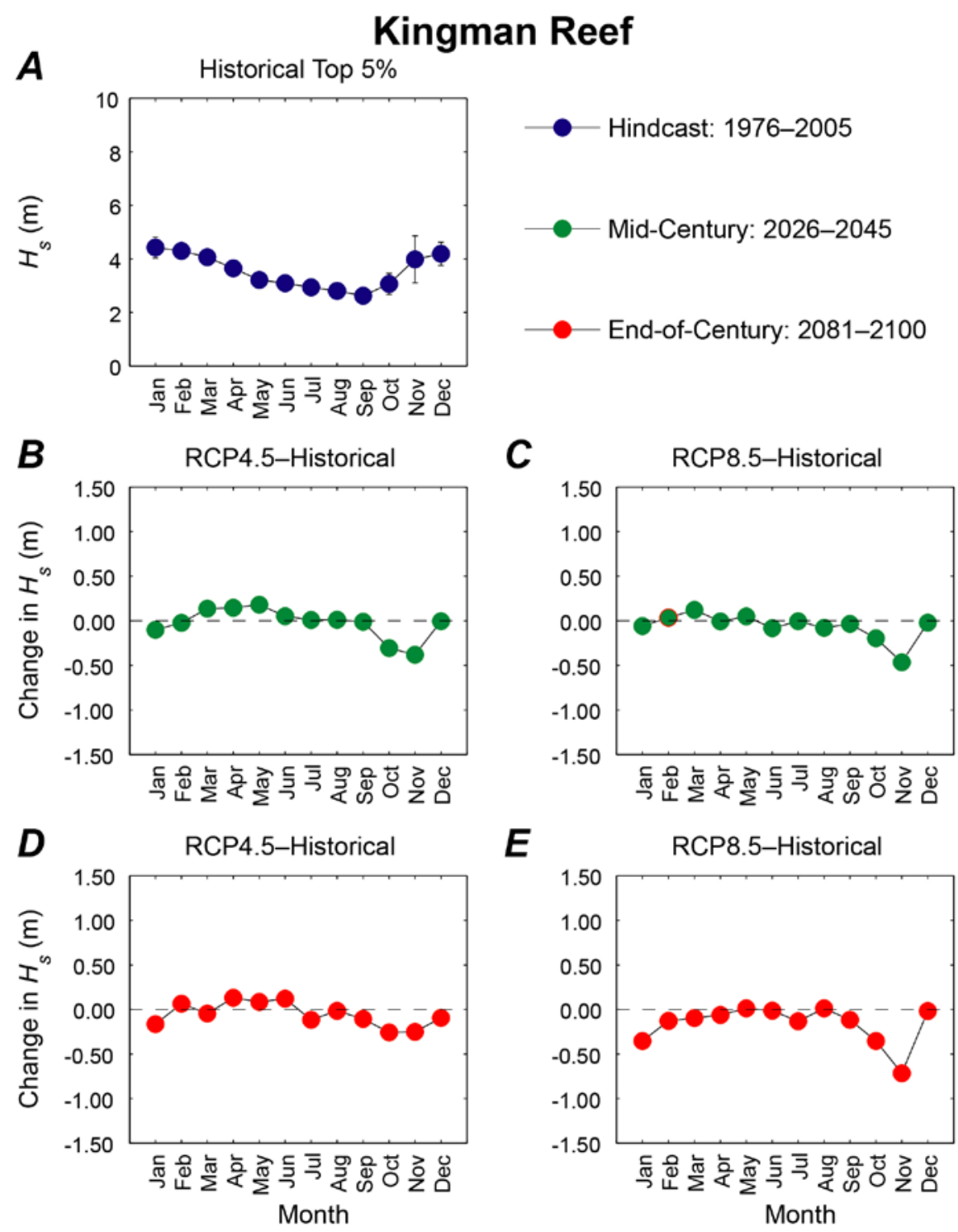

Appendix C42. Plots showing trends in monthly mean of the top 5 percent of significant wave heights $\left(H_{s}\right)$, in meters, at the Kingman Reef location. A. Hindcasted (1976-2005) mean of the top 5 percent of significant wave heights by month with associated error bars. $B$. Plot of the change in mean of the top 5 percent of 2026-2045 significant wave heights for the RCP4.5 scenario from hindcasted top 5 percent of monthly significant wave height means. $C$. Plot of the change in mean of the top 5 percent of 2026-2045 significant wave heights for the RCP8.5 scenario from hindcasted top 5 percent of monthly significant wave height means. D. Plot of the change in mean of the top 5 percent of 20812100 significant wave heights for the RCP4.5 scenario from hindcasted top 5 percent of monthly significant wave height means. E. Plot of the change in mean of the top 5 percent of 2081-2100 significant wave heights for the RCP8.5 scenario from hindcasted top 5 percent of monthly significant wave height means. 

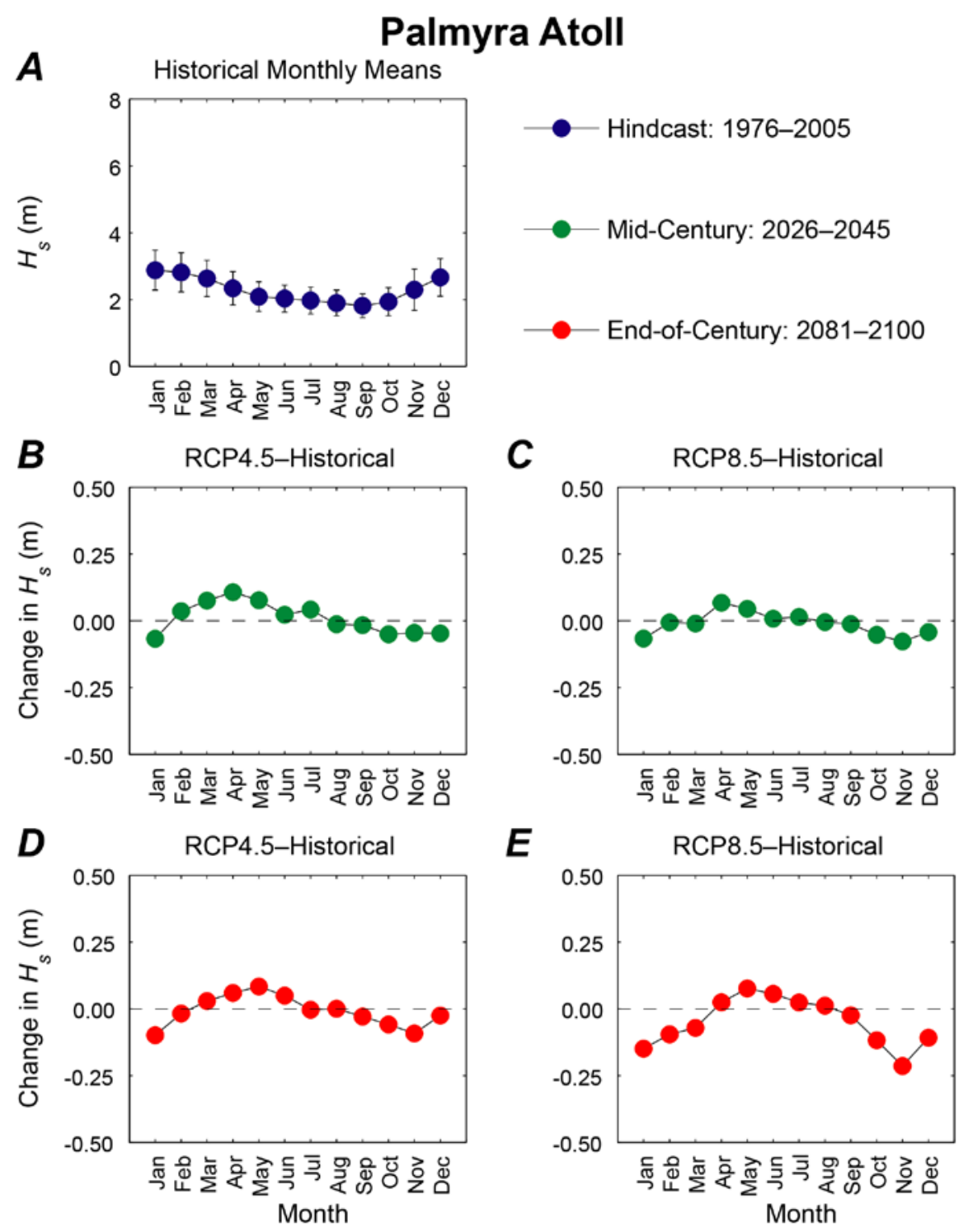

Appendix C43. Plots showing trends in monthly mean significant wave height $\left(H_{s}\right)$, in meters, at the Palmyra Atoll location. A. Hindcasted (1976-2005) mean significant wave heights by month with associated error bars. $B$. Plot of the change in mean 2026-2045 significant wave heights for the RCP4.5 scenario from hindcasted monthly significant wave height means. C. Plot of the change in mean 2026-2045 significant wave heights for the RCP8.5 scenario from hindcasted monthly significant wave height means. D. Plot of the change in mean 2081-2100 significant wave heights for the RCP4.5 scenario from hindcasted monthly significant wave height means. E. Plot of the change in mean 2081-2100 significant wave heights for the RCP8.5 scenario from hindcasted monthly significant wave height means. 

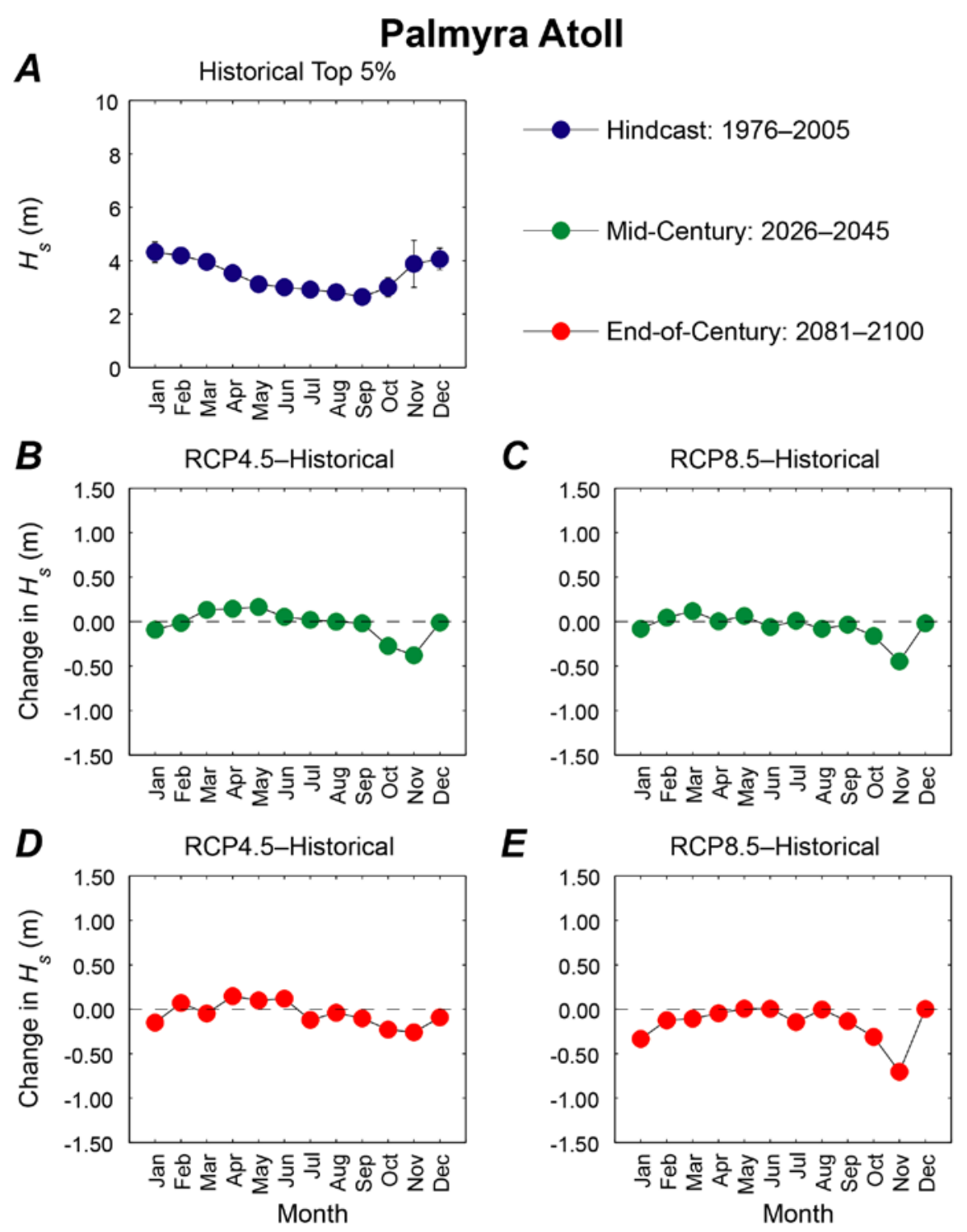

Appendix C44. Plots showing trends in monthly mean of the top 5 percent of significant wave heights $\left(H_{s}\right)$, in meters, at the Palmyra Atoll location. A. Hindcasted (1976-2005) mean of the top 5 percent of significant wave heights by month with associated error bars. $B$. Plot of the change in mean of the top 5 percent of 2026-2045 significant wave heights for the RCP4.5 scenario from hindcasted top 5 percent of monthly significant wave height means. $C$. Plot of the change in mean of the top 5 percent of 2026-2045 significant wave heights for the RCP8.5 scenario from hindcasted top 5 percent of monthly significant wave height means. $D$. Plot of the change in mean of the top 5 percent of 20812100 significant wave heights for the RCP4.5 scenario from hindcasted top 5 percent of monthly significant wave height means. E. Plot of the change in mean of the top 5 percent of 2081-2100 significant wave heights for the RCP8.5 scenario from hindcasted top 5 percent of monthly significant wave height means. 

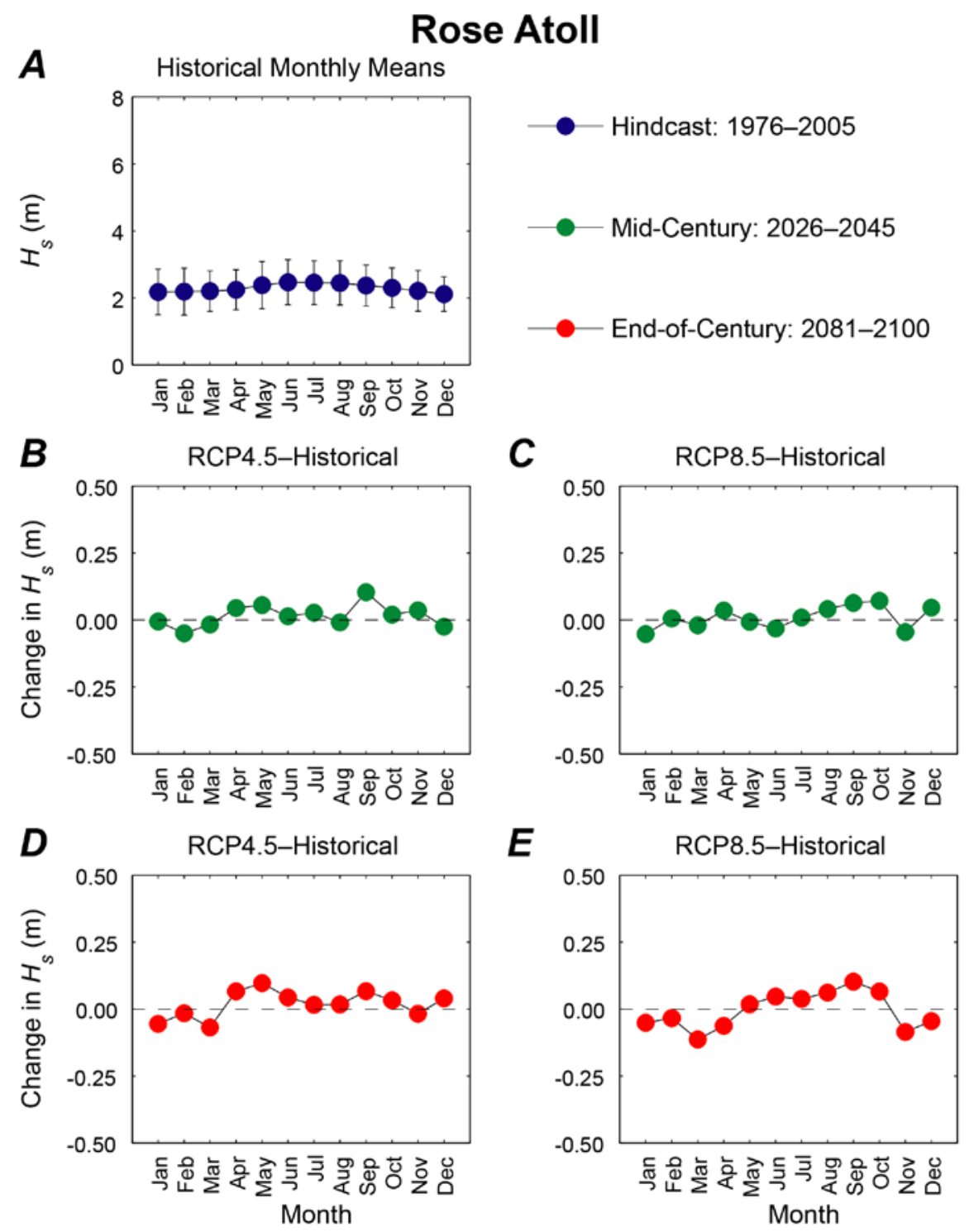

Appendix C45. Plots showing trends in monthly mean significant wave height $\left(H_{s}\right)$, in meters, at the Rose Atoll location. A. Hindcasted (1976-2005) mean significant wave heights by month with associated error bars. B. Plot of the change in mean 2026-2045 significant wave heights for the RCP4.5 scenario from hindcasted monthly significant wave height means. $C$. Plot of the change in mean 2026-2045 significant wave heights for the RCP8.5 scenario from hindcasted monthly significant wave height means. D. Plot of the change in mean 2081-2100 significant wave heights for the RCP4.5 scenario from hindcasted monthly significant wave height means. $E$. Plot of the change in mean 2081-2100 significant wave heights for the RCP8.5 scenario from hindcasted monthly significant wave height means. 

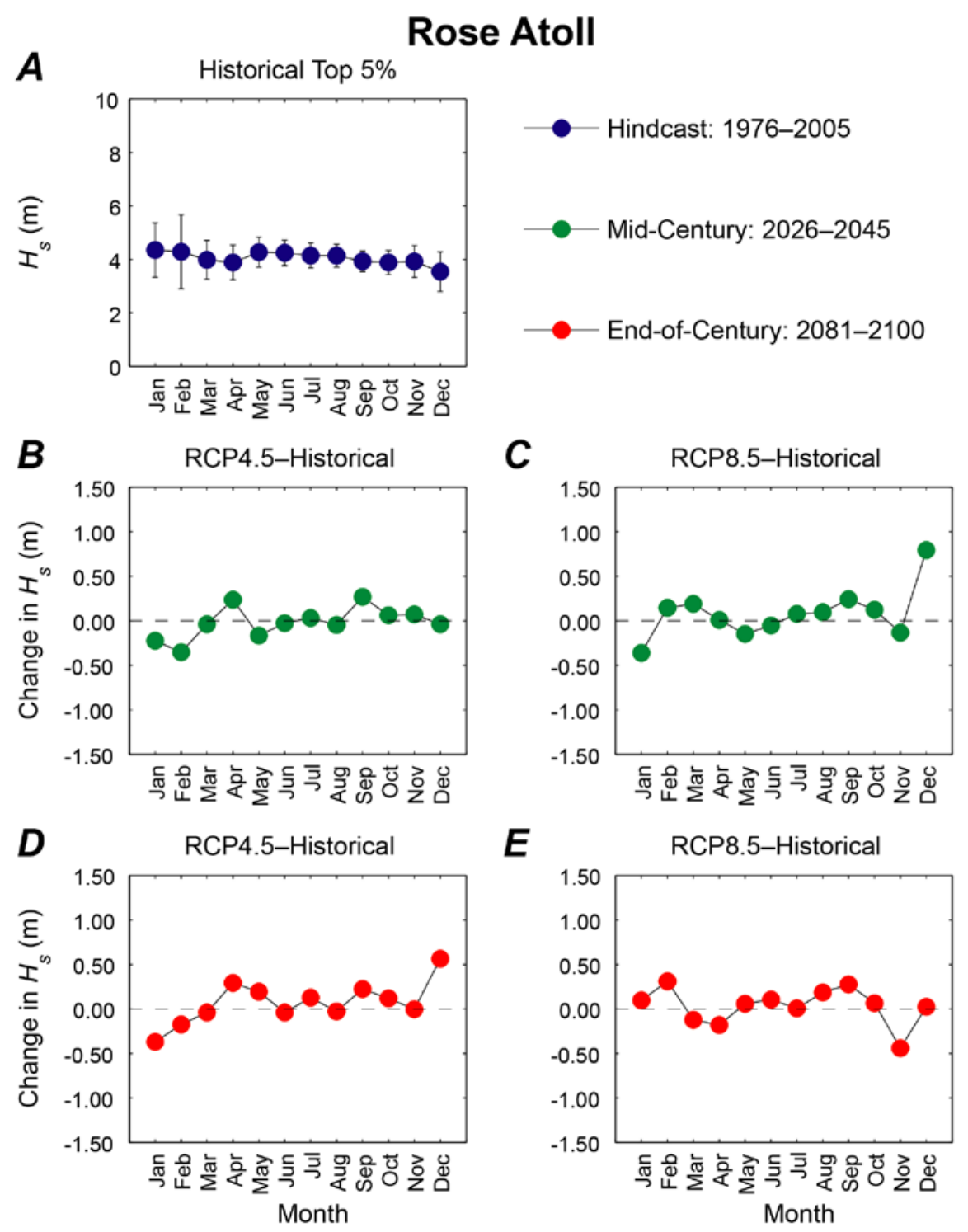

Appendix C46. Plots showing trends in monthly mean of the top 5 percent of significant wave heights $\left(H_{s}\right)$, in meters, at the Rose Atoll location. A. Hindcasted (1976-2005) mean of the top 5 percent of significant wave heights by month with associated error bars. $B$. Plot of the change in mean of the top 5 percent of 2026-2045 significant wave heights for the RCP4.5 scenario from hindcasted top 5 percent of monthly significant wave height means. $C$. Plot of the change in mean of the top 5 percent of 2026-2045 significant wave heights for the RCP8.5 scenario from hindcasted top 5 percent of monthly significant wave height means. $D$. Plot of the change in mean of the top 5 percent of 20812100 significant wave heights for the RCP4.5 scenario from hindcasted top 5 percent of monthly significant wave height means. E. Plot of the change in mean of the top 5 percent of 2081-2100 significant wave heights for the RCP8.5 scenario from hindcasted top 5 percent of monthly significant wave height means. 

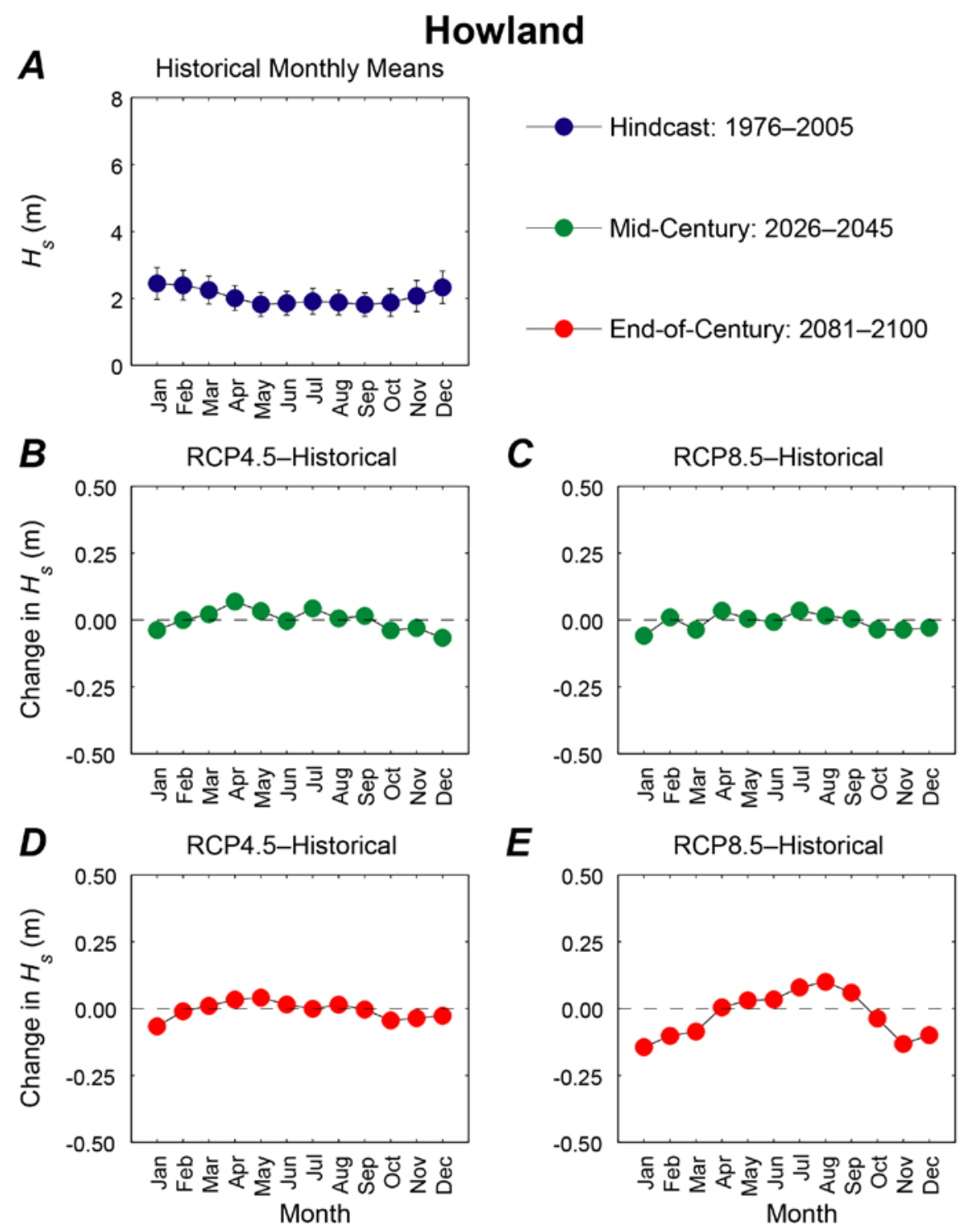

Appendix C47. Plots showing trends in monthly mean significant wave height $\left(H_{s}\right)$, in meters, at the Howland location. A. Hindcasted (1976-2005) mean significant wave heights by month with associated error bars. $B$. Plot of the change in mean 2026-2045 significant wave heights for the RCP4.5 scenario from hindcasted monthly significant wave height means. C. Plot of the change in mean 2026-2045 significant wave heights for the RCP8.5 scenario from hindcasted monthly significant wave height means. D. Plot of the change in mean 2081-2100 significant wave heights for the RCP4.5 scenario from hindcasted monthly significant wave height means. E. Plot of the change in mean 2081-2100 significant wave heights for the RCP8.5 scenario from hindcasted monthly significant wave height means. 

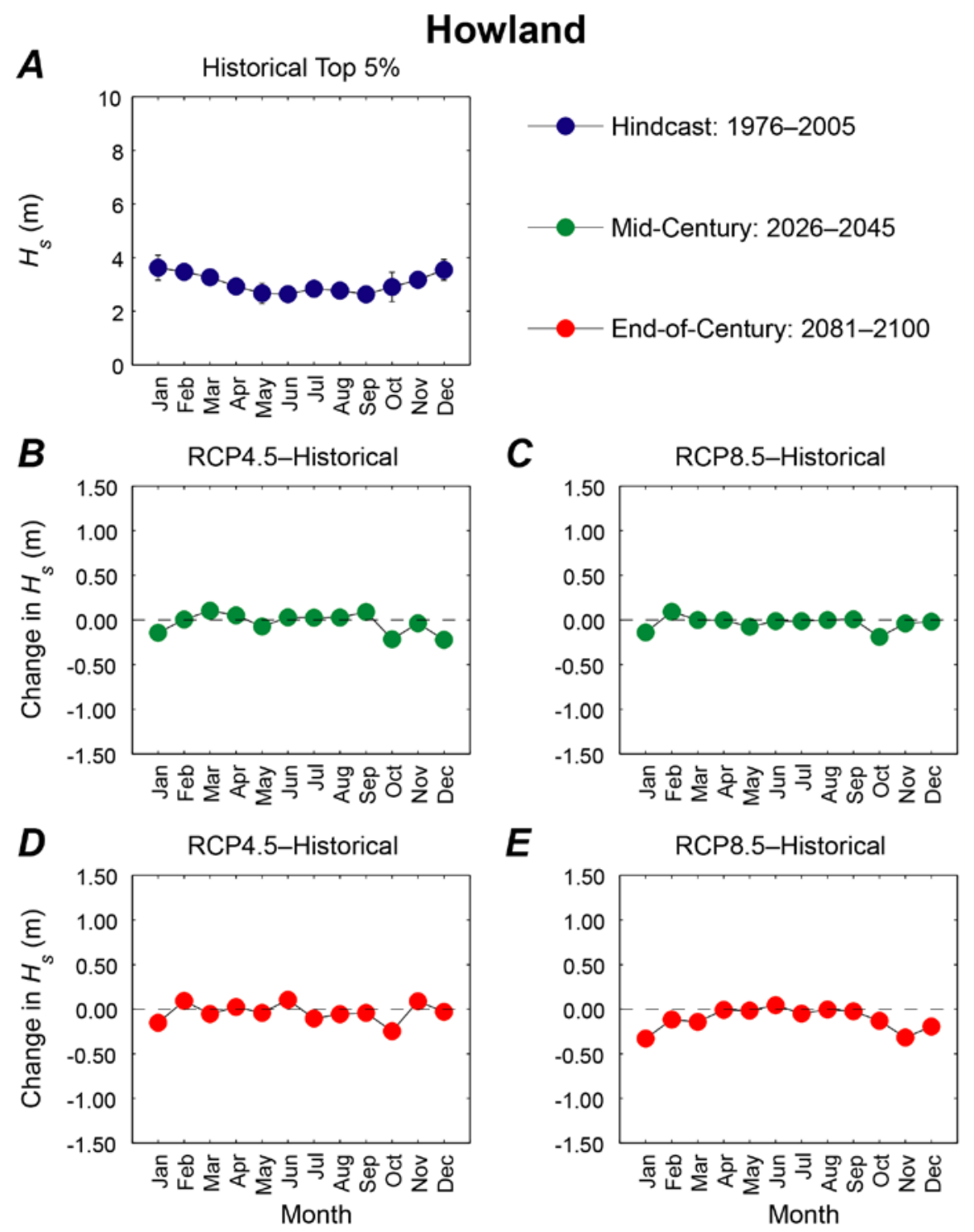

Appendix C48. Plots showing trends in monthly mean of the top 5 percent of significant wave heights $\left(H_{s}\right)$, in meters, at the Howland location. A. Hindcasted (1976-2005) mean of the top 5 percent of significant wave heights by month with associated error bars. $B$. Plot of the change in mean of the top 5 percent of 2026-2045 significant wave heights for the RCP4.5 scenario from hindcasted top 5 percent of monthly significant wave height means. $C$. Plot of the change in mean of the top 5 percent of 2026-2045 significant wave heights for the RCP8.5 scenario from hindcasted top 5 percent of monthly significant wave height means. $D$. Plot of the change in mean of the top 5 percent of 20812100 significant wave heights for the RCP4.5 scenario from hindcasted top 5 percent of monthly significant wave height means. E. Plot of the change in mean of the top 5 percent of 2081-2100 significant wave heights for the RCP8.5 scenario from hindcasted top 5 percent of monthly significant wave height means. 

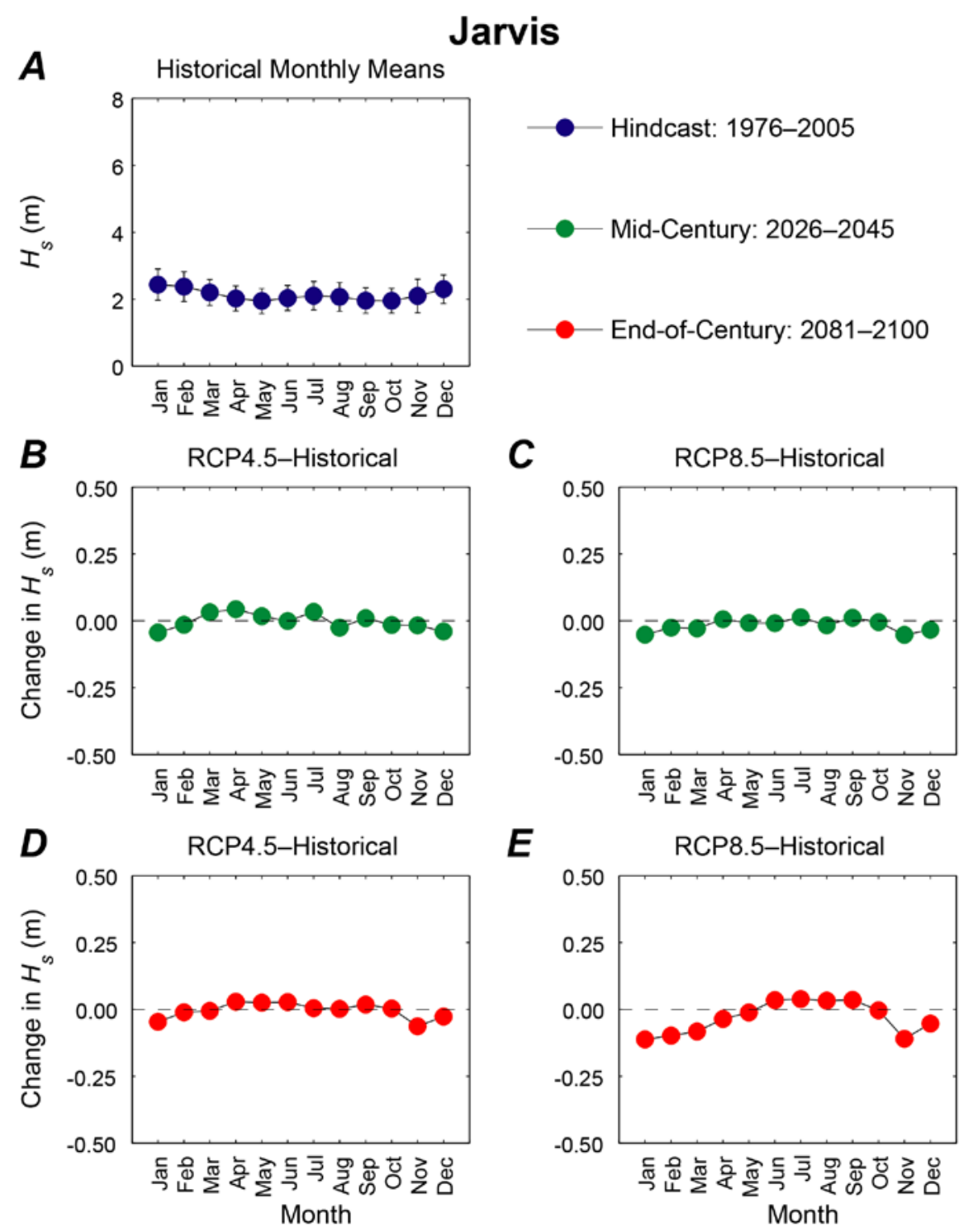

Appendix C49. Plots showing trends in monthly mean significant wave height $\left(H_{s}\right)$, in meters, at the Jarvis location. A. Hindcasted (1976-2005) mean significant wave heights by month with associated error bars. $B$. Plot of the change in mean 2026-2045 significant wave heights for the RCP4.5 scenario from hindcasted monthly significant wave height means. C. Plot of the change in mean 2026-2045 significant wave heights for the RCP8.5 scenario from hindcasted monthly significant wave height means. $D$. Plot of the change in mean 2081-2100 significant wave heights for the RCP4.5 scenario from hindcasted monthly significant wave height means. E. Plot of the change in mean 2081-2100 significant wave heights for the RCP8.5 scenario from hindcasted monthly significant wave height means. 

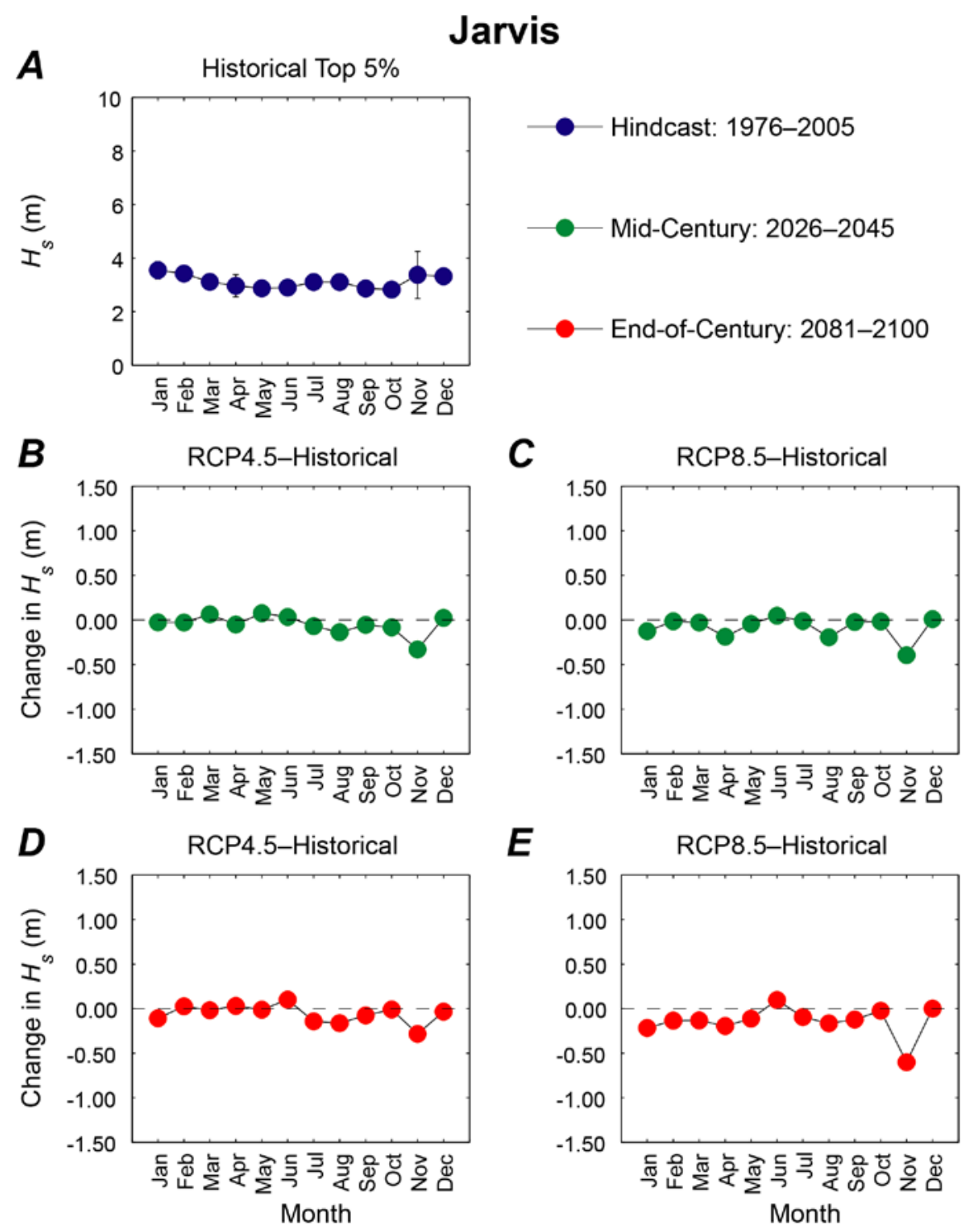

Appendix C50. Plots showing trends in monthly mean of the top 5 percent of significant wave heights $\left(H_{s}\right)$, in meters, at the Jarvis location. A. Hindcasted (1976-2005) mean of the top 5 percent of significant wave heights by month with associated error bars. $B$. Plot of the change in mean of the top 5 percent of 2026-2045 significant wave heights for the RCP4.5 scenario from hindcasted top 5 percent of monthly significant wave height means. $C$. Plot of the change in mean of the top 5 percent of 2026 2045 significant wave heights for the RCP8.5 scenario from hindcasted top 5 percent of monthly significant wave height means. D. Plot of the change in mean of the top 5 percent of 2081-2100 significant wave heights for the RCP4.5 scenario from hindcasted top 5 percent of monthly significant wave height means. E. Plot of the change in mean of the top 5 percent of 2081-2100 significant wave heights for the RCP8.5 scenario from hindcasted top 5 percent of monthly significant wave height means. 


\section{Appendix D. Monthly Trends in Wind Speeds}
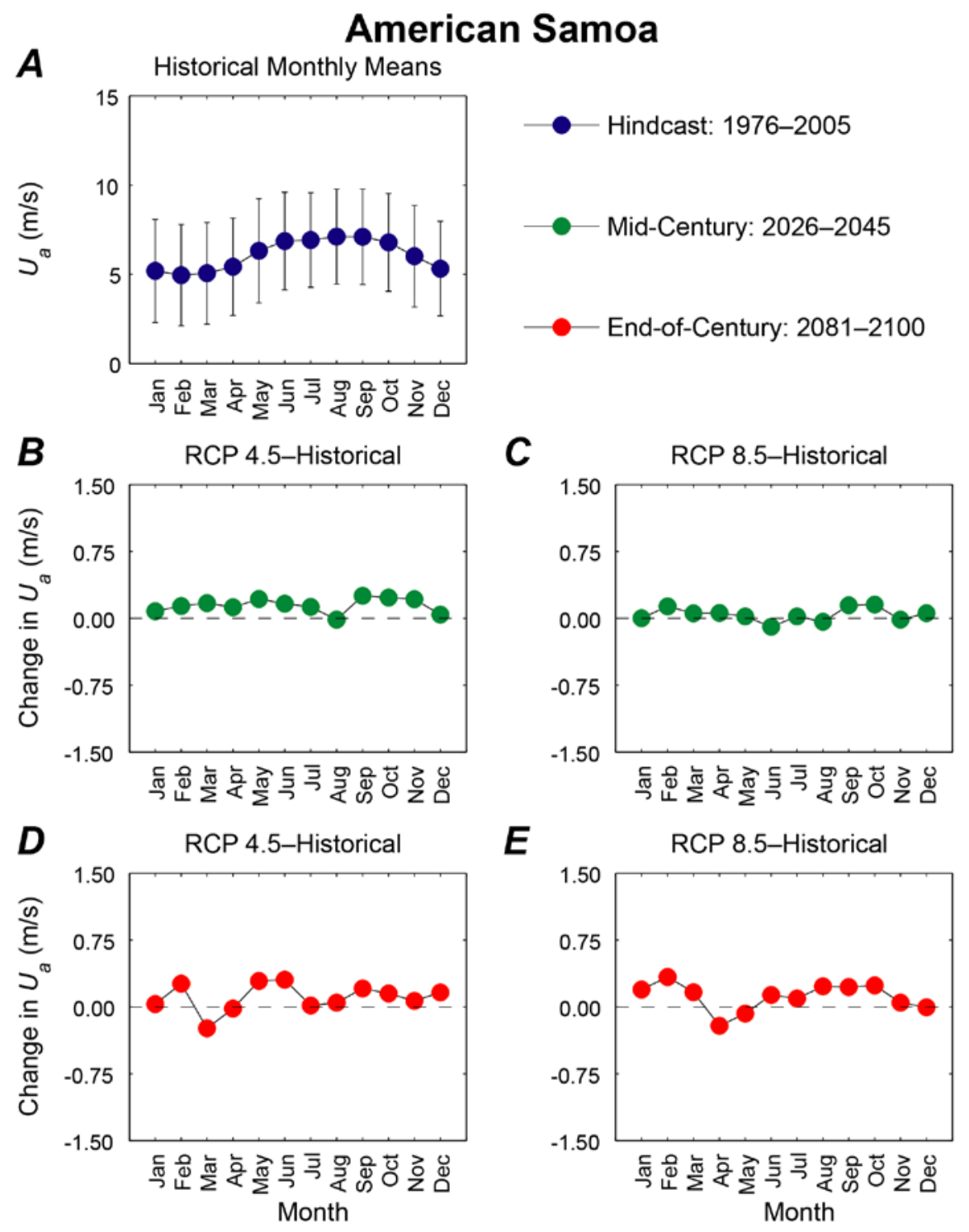

Appendix D1. Plots showing trends in monthly mean wind speed, in meters per second, at the American Samoa location. A. Hindcasted (1976-2005) mean wind speeds by month with associated error bars. $B$. Plot of the change in mean 2026-2045 wind speeds for the RCP4.5 scenario from hindcasted monthly wind speed means. C. Plot of the change in mean 2026-2045 wind speeds for the RCP8.5 scenario from hindcasted monthly wind speed means. D. Plot of the change in mean 2081-2100 wind speeds for the RCP4.5 scenario from hindcasted monthly wind speed means. E. Plot of the change in mean 2081-2100 wind speeds for the RCP8.5 scenario from hindcasted monthly wind speed means. 

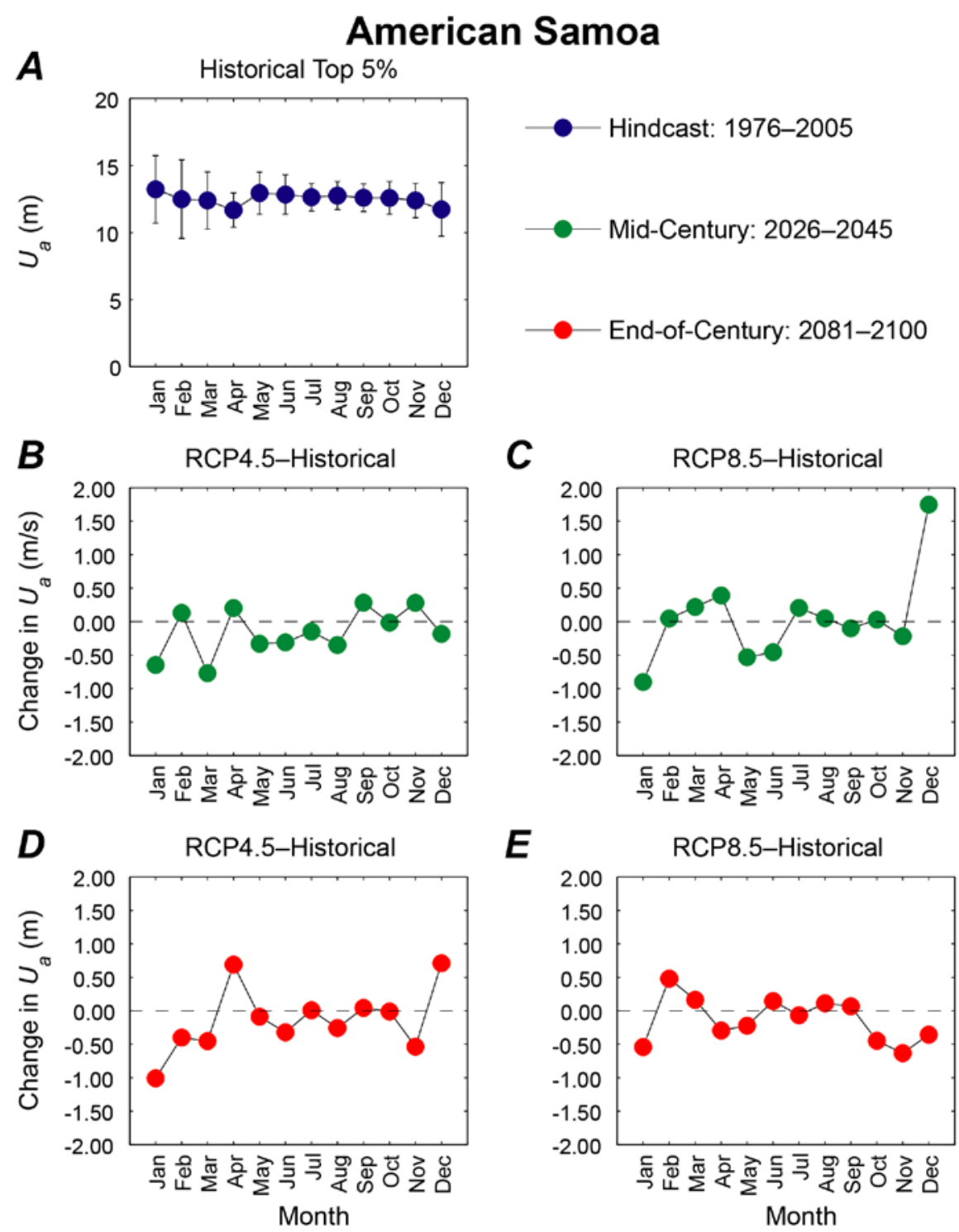

Appendix D2. Plots showing trends in monthly mean of the top 5 percent of wind speeds, in meters, at the American Samoa location. A. Hindcasted (1976-2005) mean of the top 5 percent of wind speeds by month with associated error bars. $B$. Plot of the change in mean of the top 5 percent of 2026-2045 wind speeds for the RCP4.5 scenario from hindcasted top 5 percent of monthly wind speed means. C. Plot of the change in mean of the top 5 percent of 2026-2045 wind speeds for the RCP8.5 scenario from hindcasted top 5 percent of monthly wind speed means. $D$. Plot of the change in mean of the top 5 percent of 2081-2100 wind speeds for the RCP4.5 scenario from hindcasted top 5 percent of monthly wind speed means. E. Plot of the change in mean of the top 5 percent of 20812100 wind speeds for the RCP 8.5 scenario from hindcasted top 5 percent of monthly wind speed means. 

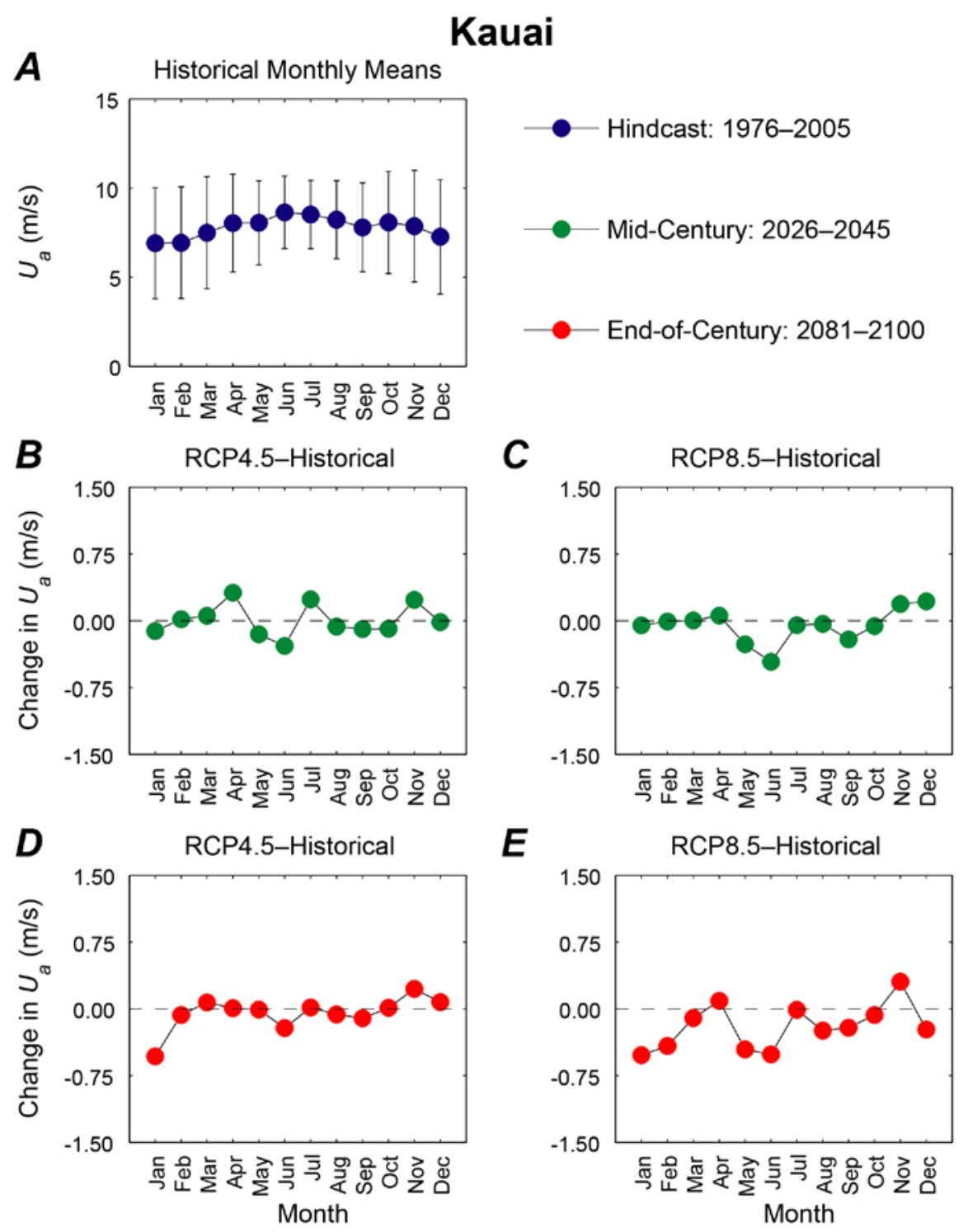

Appendix D3. Plots showing trends in monthly mean wind speed, in meters per second, at the Kauai location. A. Hindcasted (1976-2005) mean wind speeds by month with associated error bars. B. Plot of the change in mean 2026-2045 wind speeds for the RCP4.5 scenario from hindcasted monthly wind speed means. C. Plot of the change in mean 2026-2045 wind speeds for the RCP8.5 scenario from hindcasted monthly wind speed means. $D$. Plot of the change in mean 2081-2100 wind speeds for the RCP4.5 scenario from hindcasted monthly wind speed means. E. Plot of the change in mean 2081-2100 wind speeds for the RCP8.5 scenario from hindcasted monthly wind speed means. 


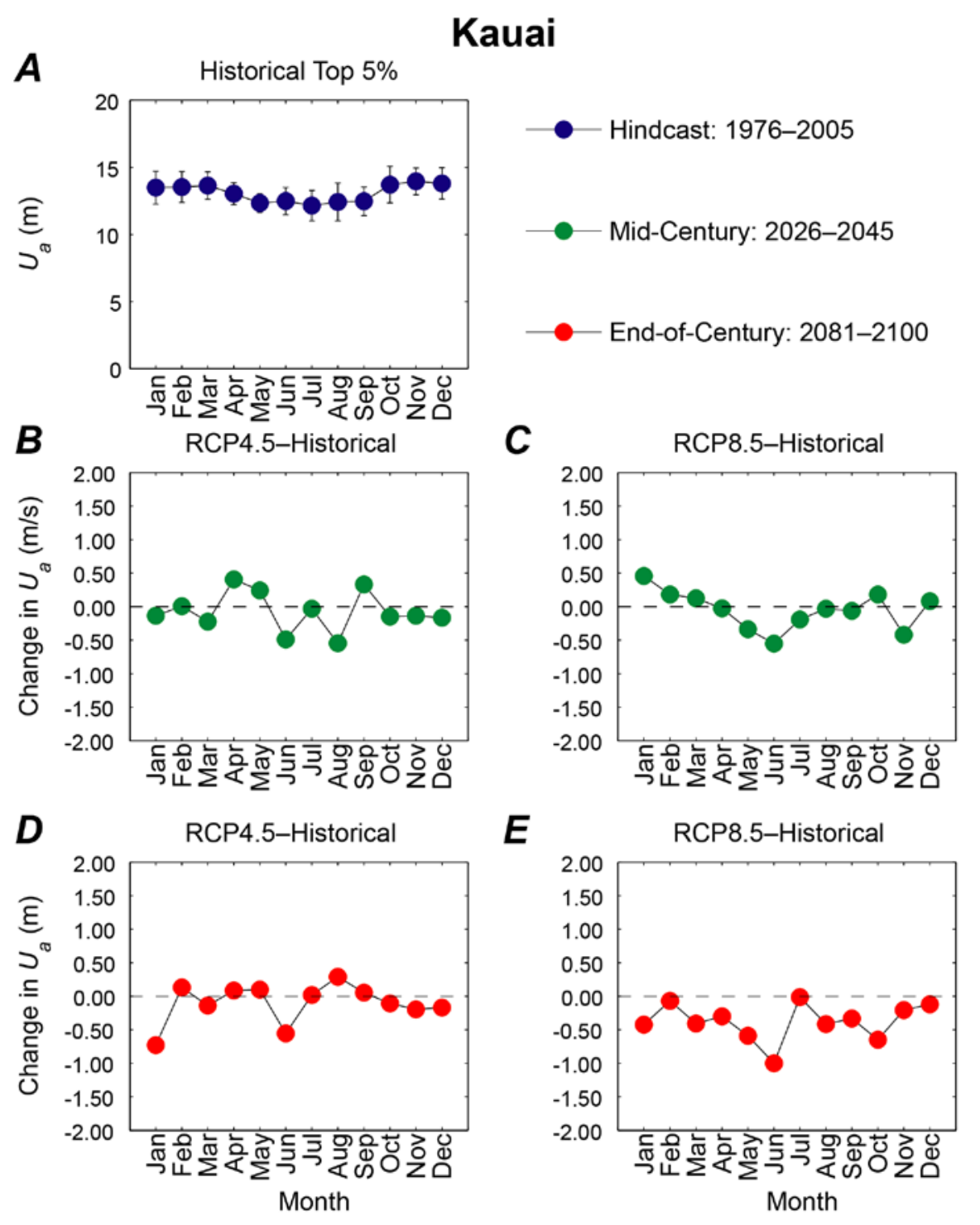

Appendix D4. Plots showing trends in monthly mean of the top 5 percent of wind speeds, in meters, at the Kauai location. A. Hindcasted (1976-2005) mean of the top 5 percent of wind speeds by month with associated error bars. $B$. Plot of the change in mean of the top 5 percent of 2026-2045 wind speeds for the RCP4.5 scenario from hindcasted top 5 percent of monthly wind speed means. $C$. Plot of the change in mean of the top 5 percent of 2026-2045 wind speeds for the RCP8.5 scenario from hindcasted top 5 percent of monthly wind speed means. $D$. Plot of the change in mean of the top 5 percent of 2081-2100 wind speeds for the RCP4.5 scenario from hindcasted top 5 percent of monthly wind speed means. E. Plot of the change in mean of the top 5 percent of 2081-2100 wind speeds for the RCP8.5 scenario from hindcasted top 5 percent of monthly wind speed means. 

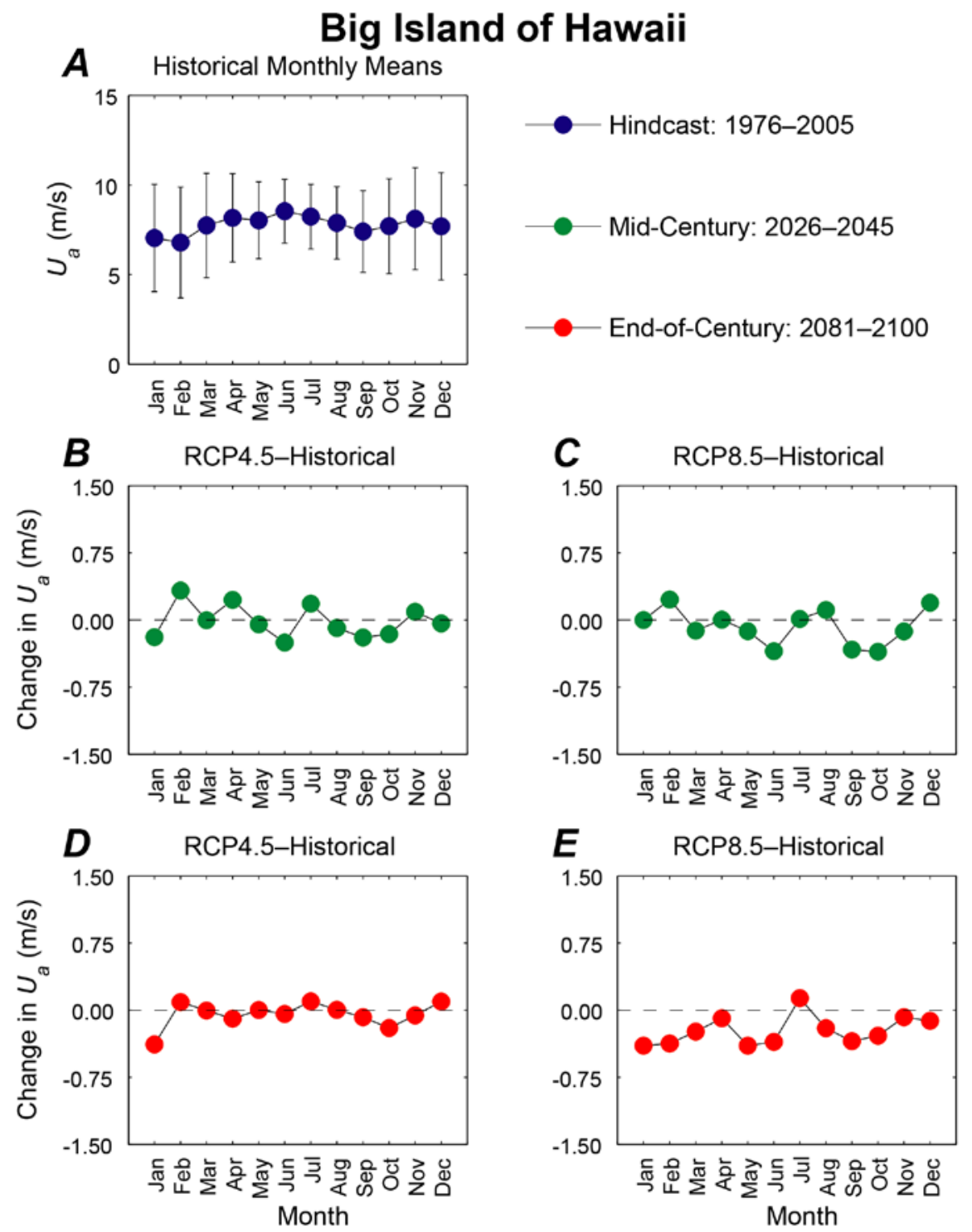

Appendix D5. Plots showing trends in monthly mean wind speed, in meters per second, at the Big Island of Hawaii location. A. Hindcasted (1976-2005) mean wind speeds by month with associated error bars. $B$. Plot of the change in mean 2026-2045 wind speeds for the RCP4.5 scenario from hindcasted monthly wind speed means. C. Plot of the change in mean 2026-2045 wind speeds for the RCP8.5 scenario from hindcasted monthly wind speed means. $D$. Plot of the change in mean 2081-2100 wind speeds for the RCP4.5 scenario from hindcasted monthly wind speed means. $E$. Plot of the change in mean 2081-2100 wind speeds for the RCP8.5 scenario from hindcasted monthly wind speed means. 

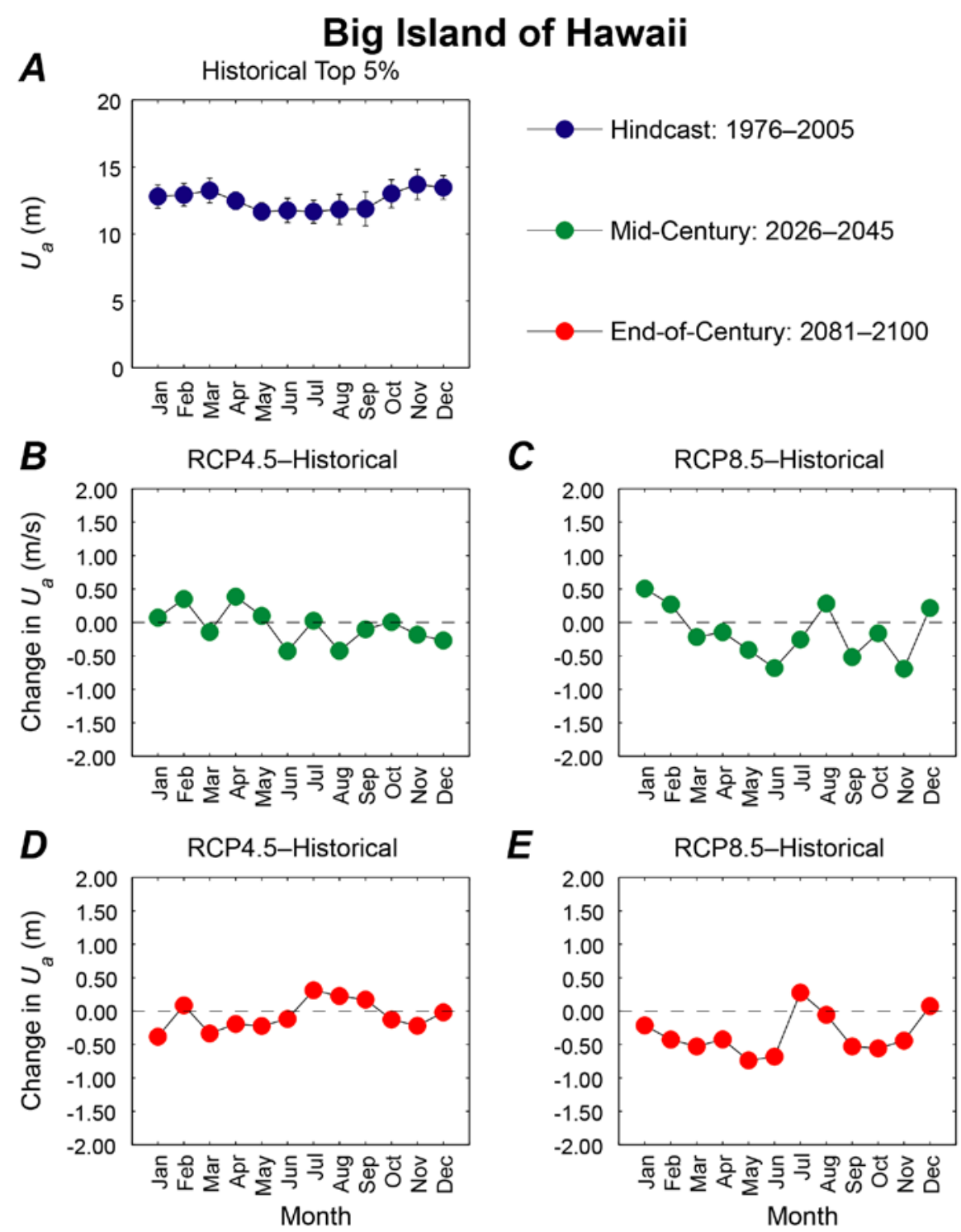

Appendix D6. Plots showing trends in monthly mean of the top 5 percent of wind speeds, in meters, at the Big Island of Hawaii location. A. Hindcasted (1976-2005) mean of the top 5 percent of wind speeds by month with associated error bars. $B$. Plot of the change in mean of the top 5 percent of 2026-2045 wind speeds for the RCP4.5 scenario from hindcasted top 5 percent of monthly wind speed means. C. Plot of the change in mean of the top 5 percent of 2026-2045 wind speeds for the RCP 8.5 scenario from hindcasted top 5 percent of monthly wind speed means. $D$. Plot of the change in mean of the top 5 percent of 2081-2100 wind speeds for the RCP4.5 scenario from hindcasted top 5 percent of monthly wind speed means. E. Plot of the change in mean of the top 5 percent of 20812100 wind speeds for the RCP 8.5 scenario from hindcasted top 5 percent of monthly wind speed means. 

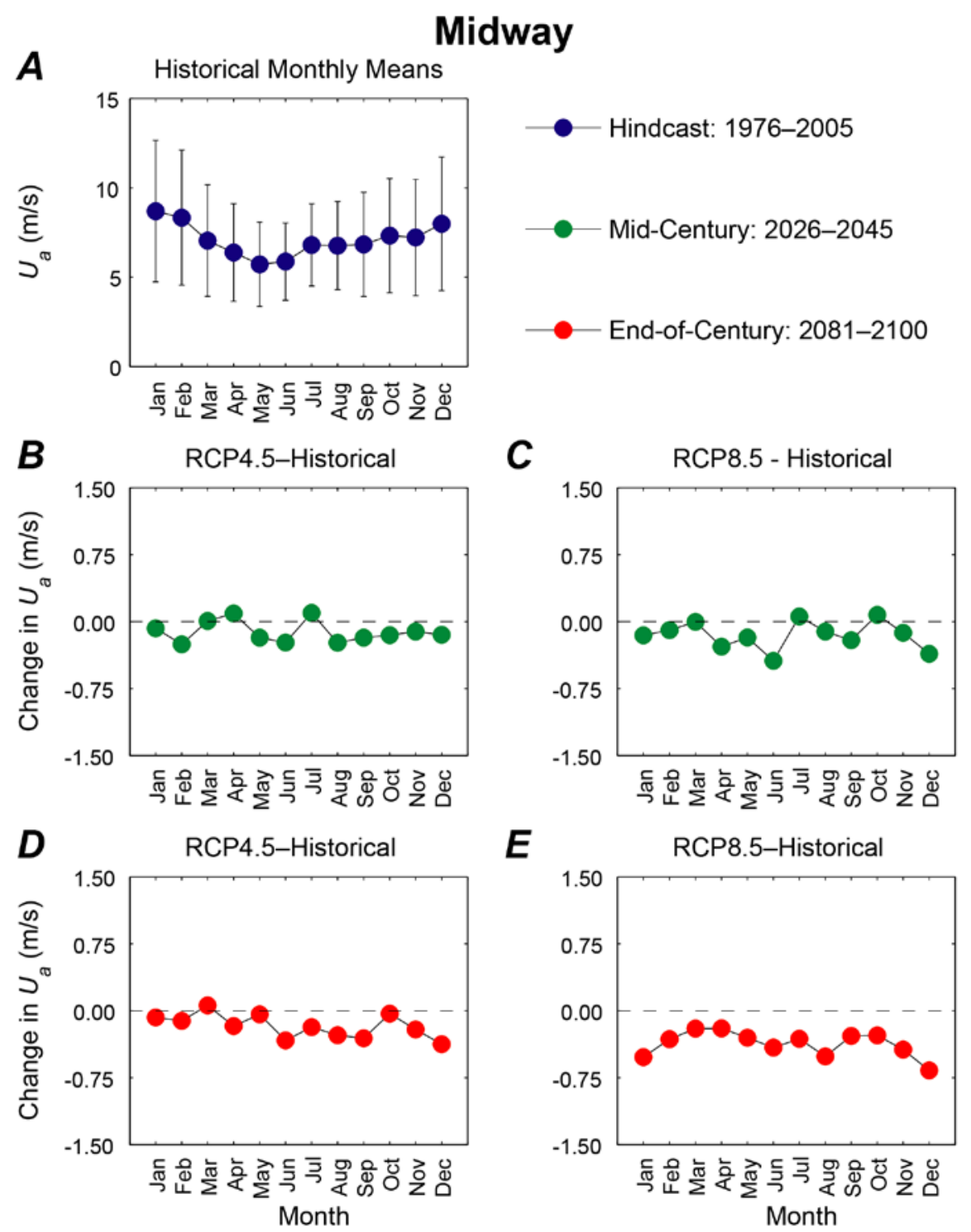

Appendix D7. Plots showing trends in monthly mean wind speed, in meters per second, at the Midway location. A. Hindcasted (1976-2005) mean wind speeds by month with associated error bars. B. Plot of the change in mean 2026-2045 wind speeds for the RCP4.5 scenario from hindcasted monthly wind speed means. C. Plot of the change in mean 2026-2045 wind speeds for the RCP8.5 scenario from hindcasted monthly wind speed means. $D$. Plot of the change in mean 2081-2100 wind speeds for the RCP4.5 scenario from hindcasted monthly wind speed means. E. Plot of the change in mean 2081-2100 wind speeds for the RCP8.5 scenario from hindcasted monthly wind speed means. 

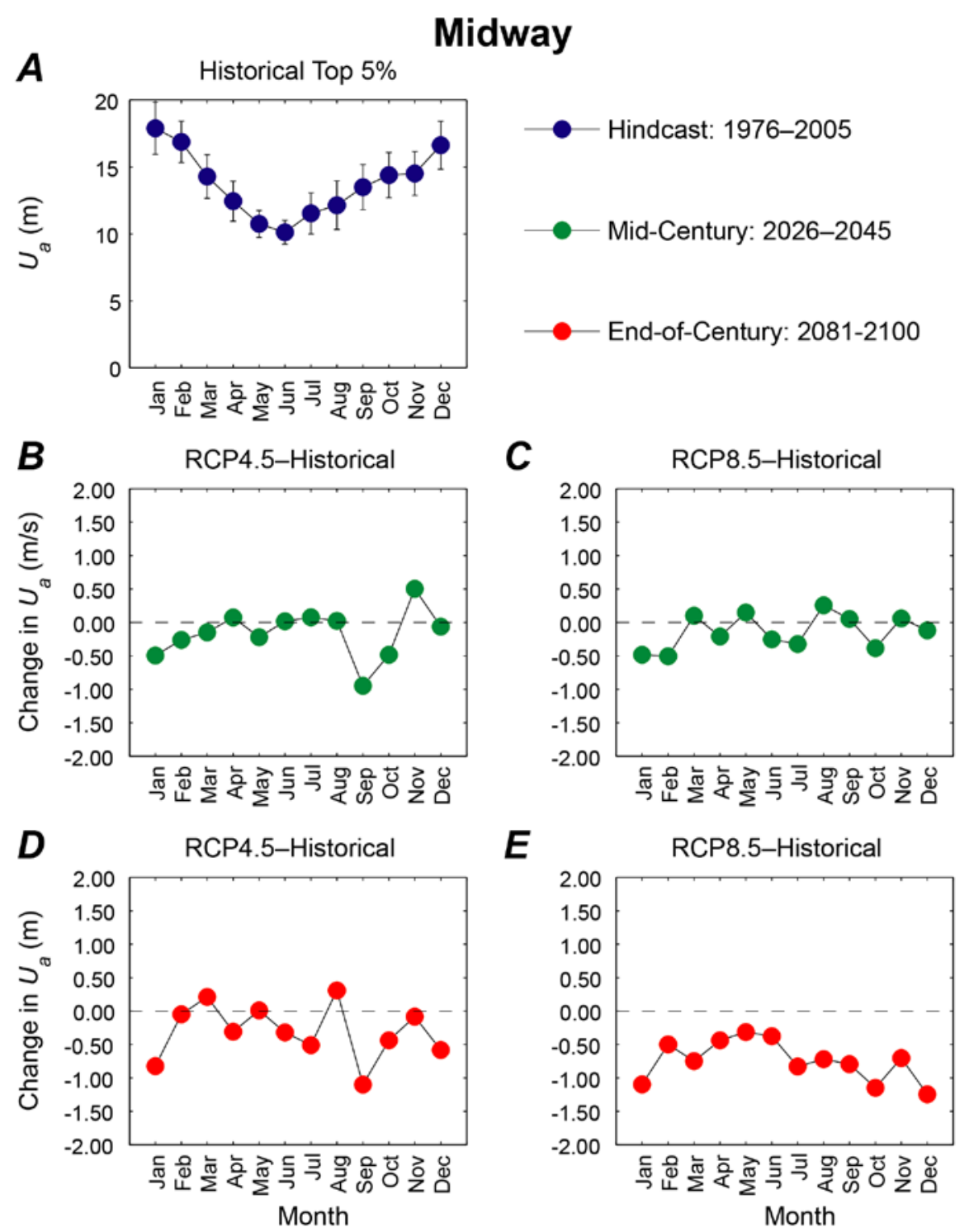

Appendix D8. Plots showing trends in monthly mean of the top 5 percent of wind speeds, in meters, at the Midway location. A. Hindcasted (1976-2005) mean of the top 5 percent of wind speeds by month with associated error bars. $B$. Plot of the change in mean of the top 5 percent of 2026-2045 wind speeds for the RCP4.5 scenario from hindcasted top 5 percent of monthly wind speed means. $C$. Plot of the change in mean of the top 5 percent of 2026-2045 wind speeds for the RCP8.5 scenario from hindcasted top 5 percent of monthly wind speed means. $D$. Plot of the change in mean of the top 5 percent of 2081-2100 wind speeds for the RCP4.5 scenario from hindcasted top 5 percent of monthly wind speed means. E. Plot of the change in mean of the top 5 percent of 2081-2100 wind speeds for the RCP8.5 scenario from hindcasted top 5 percent of monthly wind speed means. 

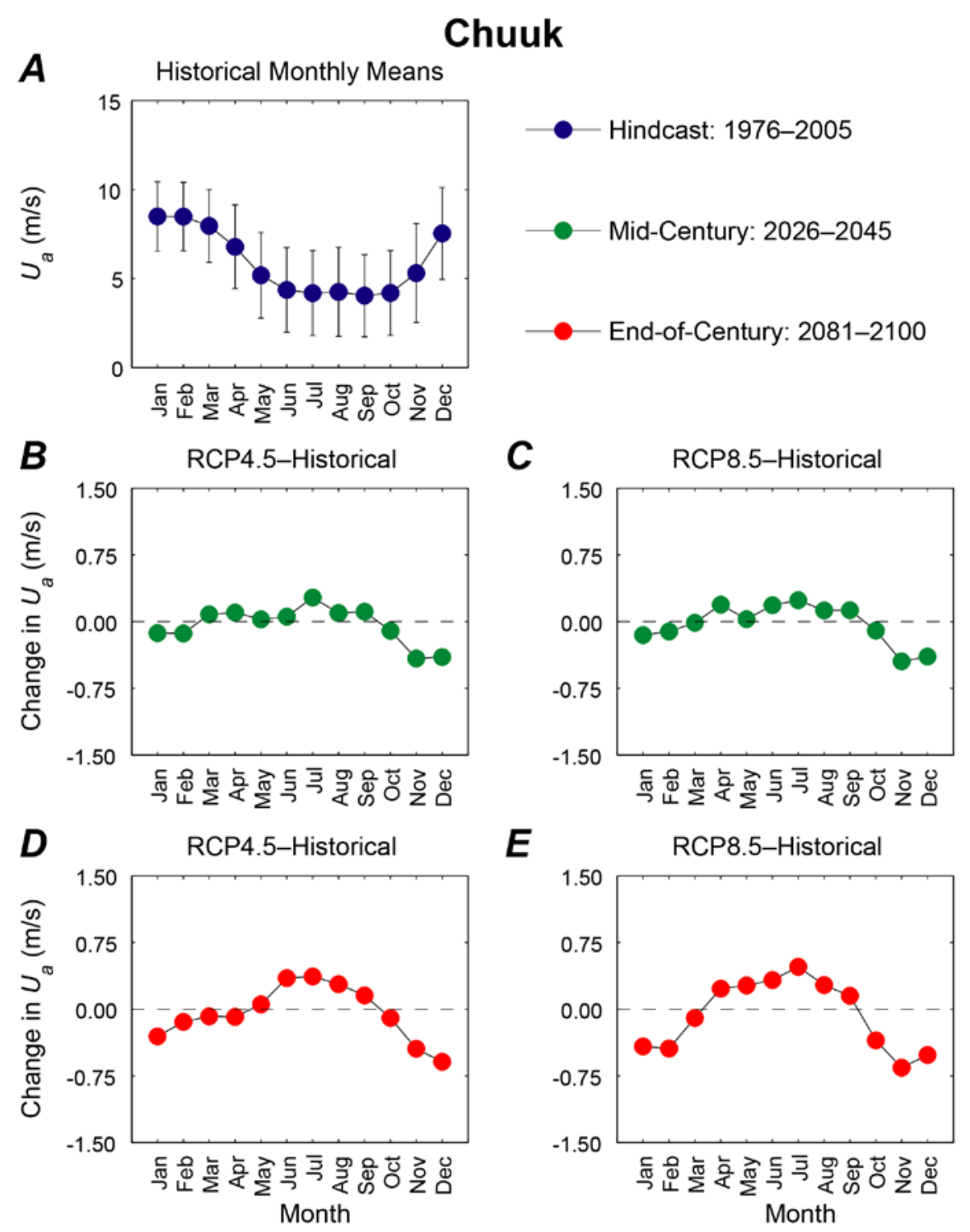

Appendix D9. Plots showing trends in monthly mean wind speed, in meters per second, at the Chuuk location. A. Hindcasted (1976-2005) mean wind speeds by month with associated error bars. B. Plot of the change in mean 2026-2045 wind speeds for the RCP4.5 scenario from hindcasted monthly wind speed means. C. Plot of the change in mean 2026-2045 wind speeds for the RCP8.5 scenario from hindcasted monthly wind speed means. $D$. Plot of the change in mean 2081-2100 wind speeds for the RCP4.5 scenario from hindcasted monthly wind speed means. E. Plot of the change in mean 2081-2100 wind speeds for the RCP8.5 scenario from hindcasted monthly wind speed means. 

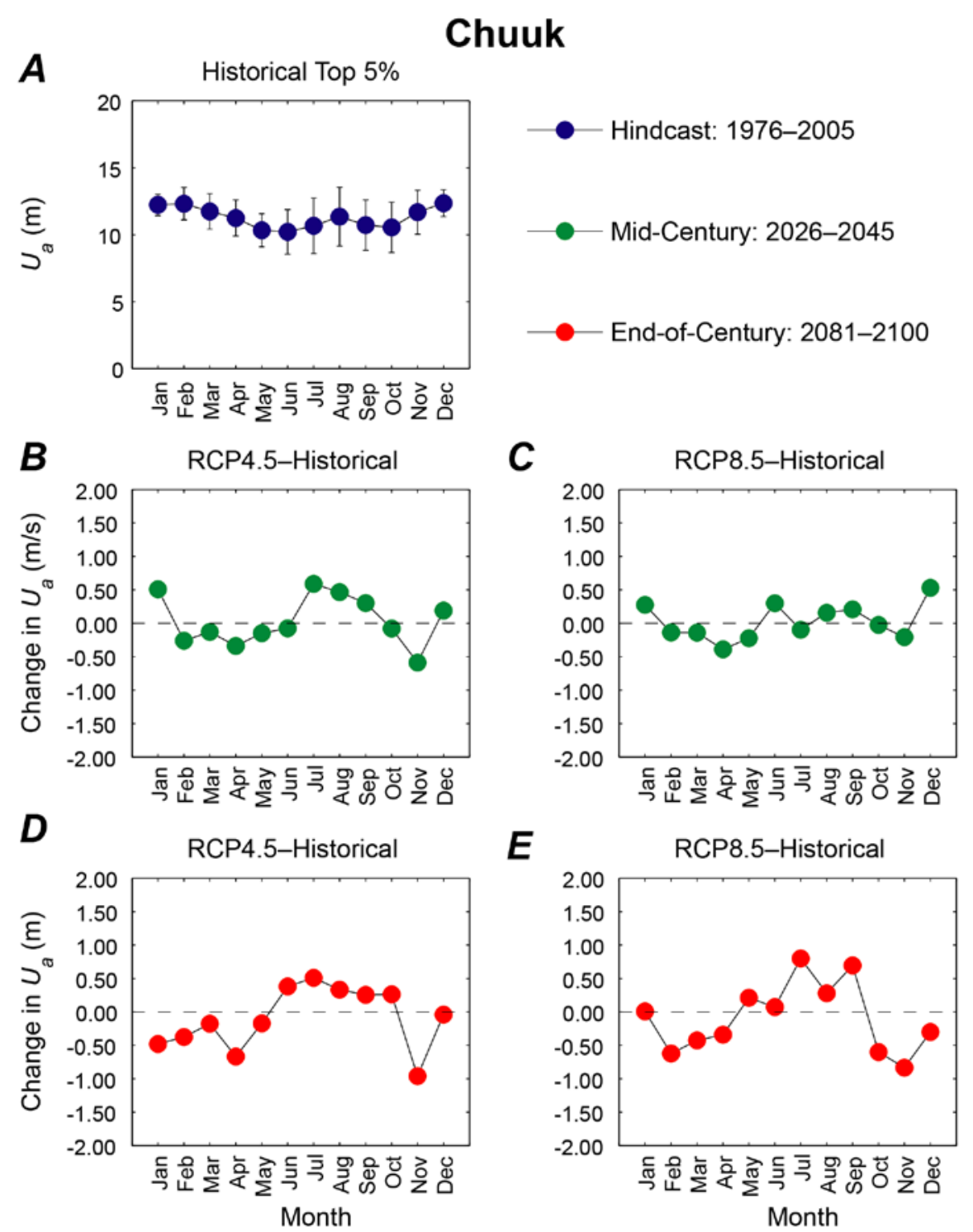

Appendix D10. Plots showing trends in monthly mean of the top 5 percent of wind speeds, in meters, at the Chuuk location. A. Hindcasted (1976-2005) mean of the top 5 percent of wind speeds by month with associated error bars. $B$. Plot of the change in mean of the top 5 percent of 2026-2045 wind speeds for the RCP4.5 scenario from hindcasted top 5 percent of monthly wind speed means. $C$. Plot of the change in mean of the top 5 percent of 2026-2045 wind speeds for the RCP8.5 scenario from hindcasted top 5 percent of monthly wind speed means. $D$. Plot of the change in mean of the top 5 percent of 2081-2100 wind speeds for the RCP4.5 scenario from hindcasted top 5 percent of monthly wind speed means. E. Plot of the change in mean of the top 5 percent of 2081-2100 wind speeds for the RCP8.5 scenario from hindcasted top 5 percent of monthly wind speed means. 

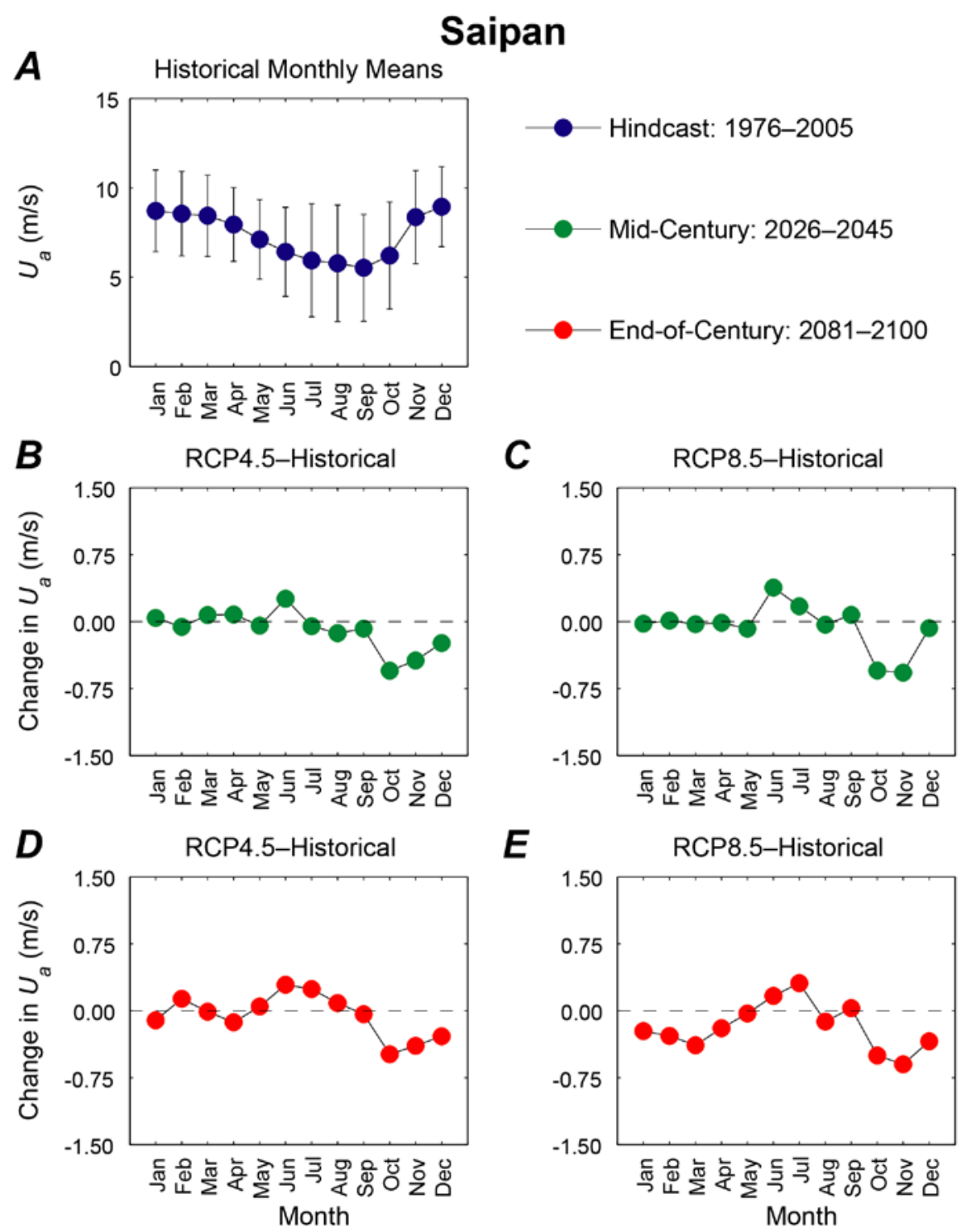

Appendix D11. Plots showing trends in monthly mean wind speed, in meters per second, at the Saipan location. A. Hindcasted (1976-2005) mean wind speeds by month with associated error bars. B. Plot of the change in mean 2026-2045 wind speeds for the RCP4.5 scenario from hindcasted monthly wind speed means. C. Plot of the change in mean 2026-2045 wind speeds for the RCP8.5 scenario from hindcasted monthly wind speed means. $D$. Plot of the change in mean 2081-2100 wind speeds for the RCP4.5 scenario from hindcasted monthly wind speed means. E. Plot of the change in mean 2081-2100 wind speeds for the RCP8.5 scenario from hindcasted monthly wind speed means. 

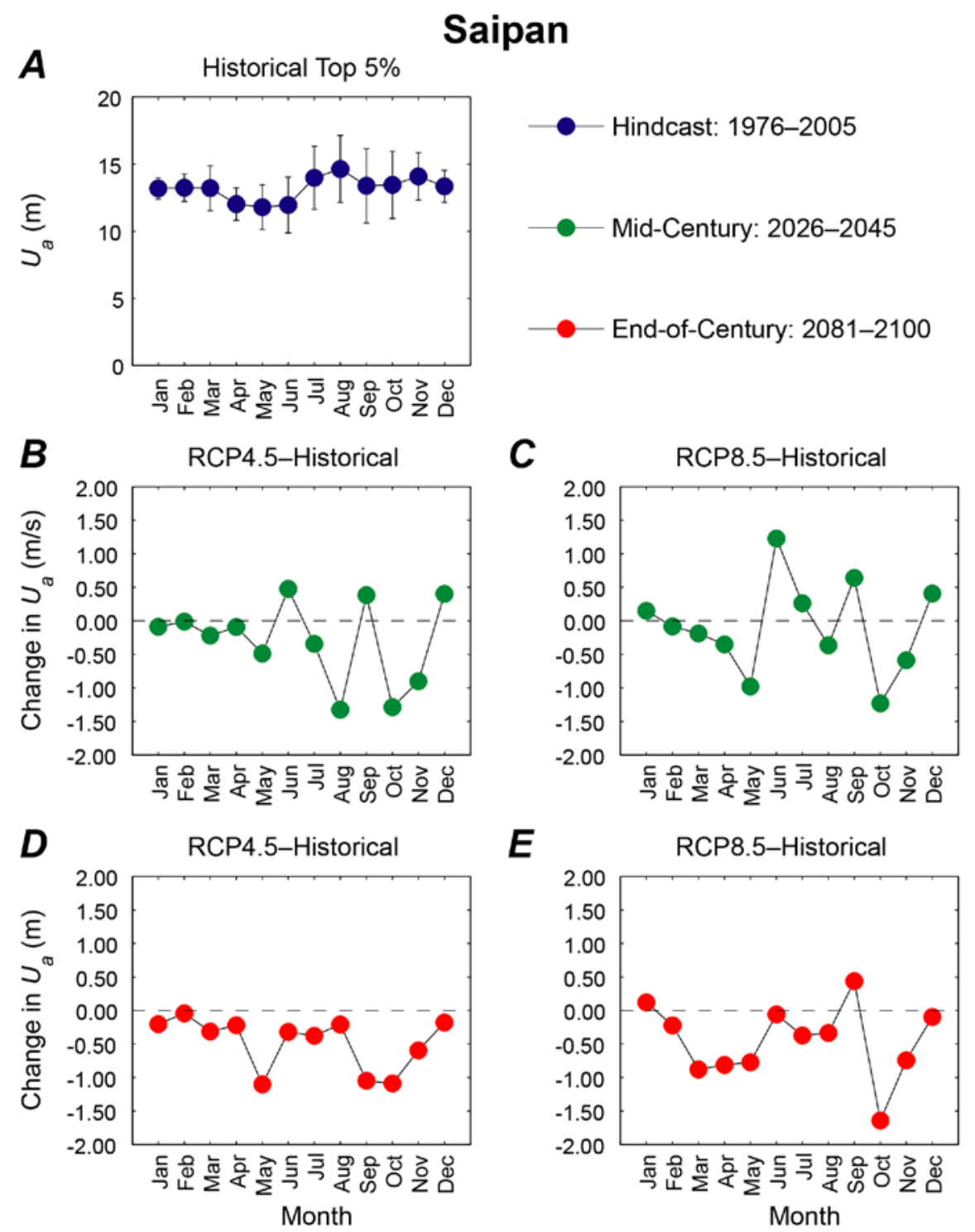

Appendix D12. Plots showing trends in monthly mean of the top 5 percent of wind speeds, in meters, at the Saipan location. A. Hindcasted (1976-2005) mean of the top 5 percent of wind speeds by month with associated error bars. $B$. Plot of the change in mean of the top 5 percent of 2026-2045 wind speeds for the RCP4.5 scenario from hindcasted top 5 percent of monthly wind speed means. C. Plot of the change in mean of the top 5 percent of 2026-2045 wind speeds for the RCP8.5 scenario from hindcasted top 5 percent of monthly wind speed means. $D$. Plot of the change in mean of the top 5 percent of 2081-2100 wind speeds for the RCP4.5 scenario from hindcasted top 5 percent of monthly wind speed means. E. Plot of the change in mean of the top 5 percent of 2081-2100 wind speeds for the RCP8.5 scenario from hindcasted top 5 percent of monthly wind speed means. 

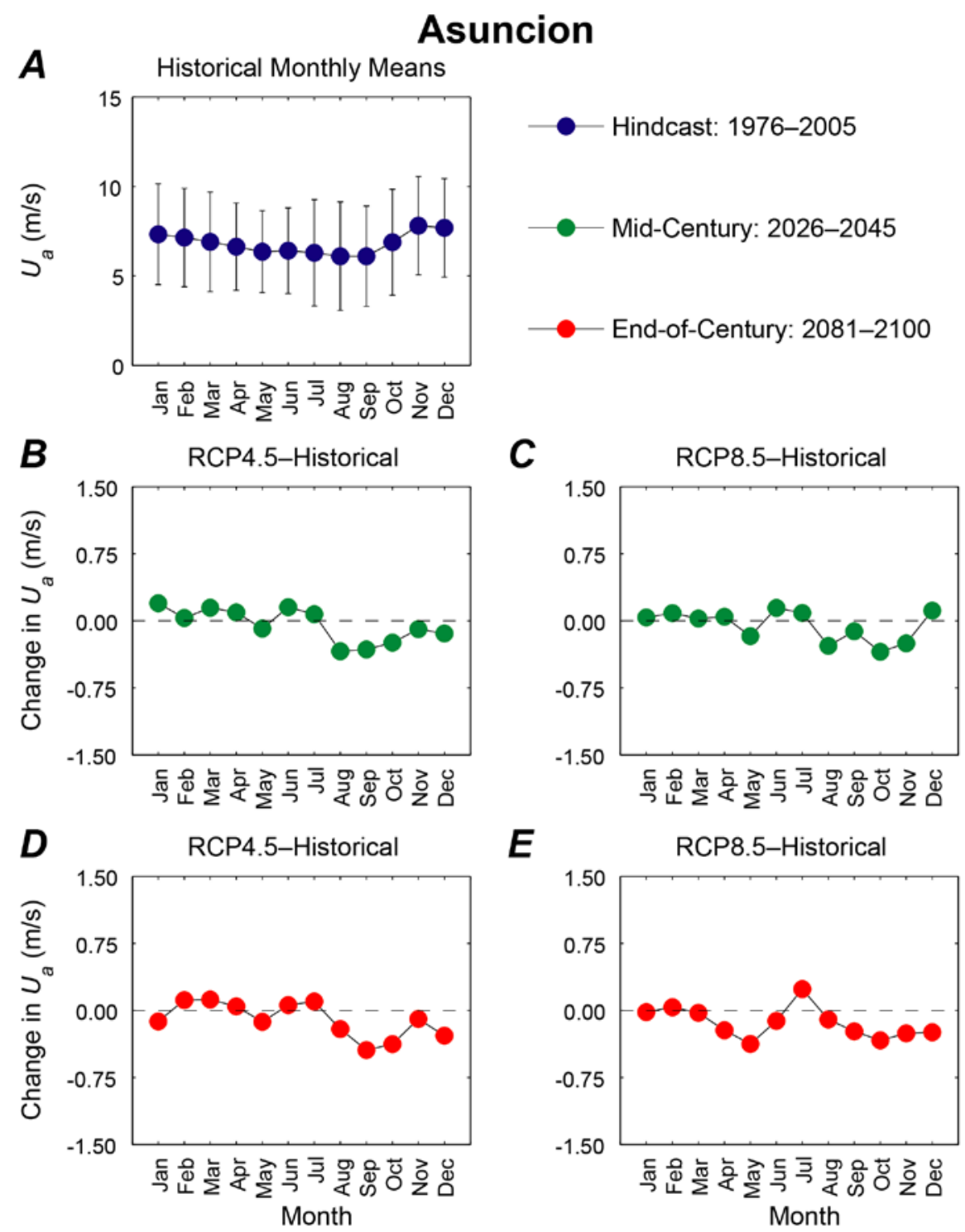

Appendix D13. Plots showing trends in monthly mean wind speed, in meters per second, at the Asuncion location. A. Hindcasted (1976-2005) mean wind speeds by month with associated error bars. B. Plot of the change in mean 2026-2045 wind speeds for the RCP4.5 scenario from hindcasted monthly wind speed means. C. Plot of the change in mean 2026-2045 wind speeds for the RCP8.5 scenario from hindcasted monthly wind speed means. $D$. Plot of the change in mean 2081-2100 wind speeds for the RCP4.5 scenario from hindcasted monthly wind speed means. E. Plot of the change in mean 2081-2100 wind speeds for the RCP8.5 scenario from hindcasted monthly wind speed means. 

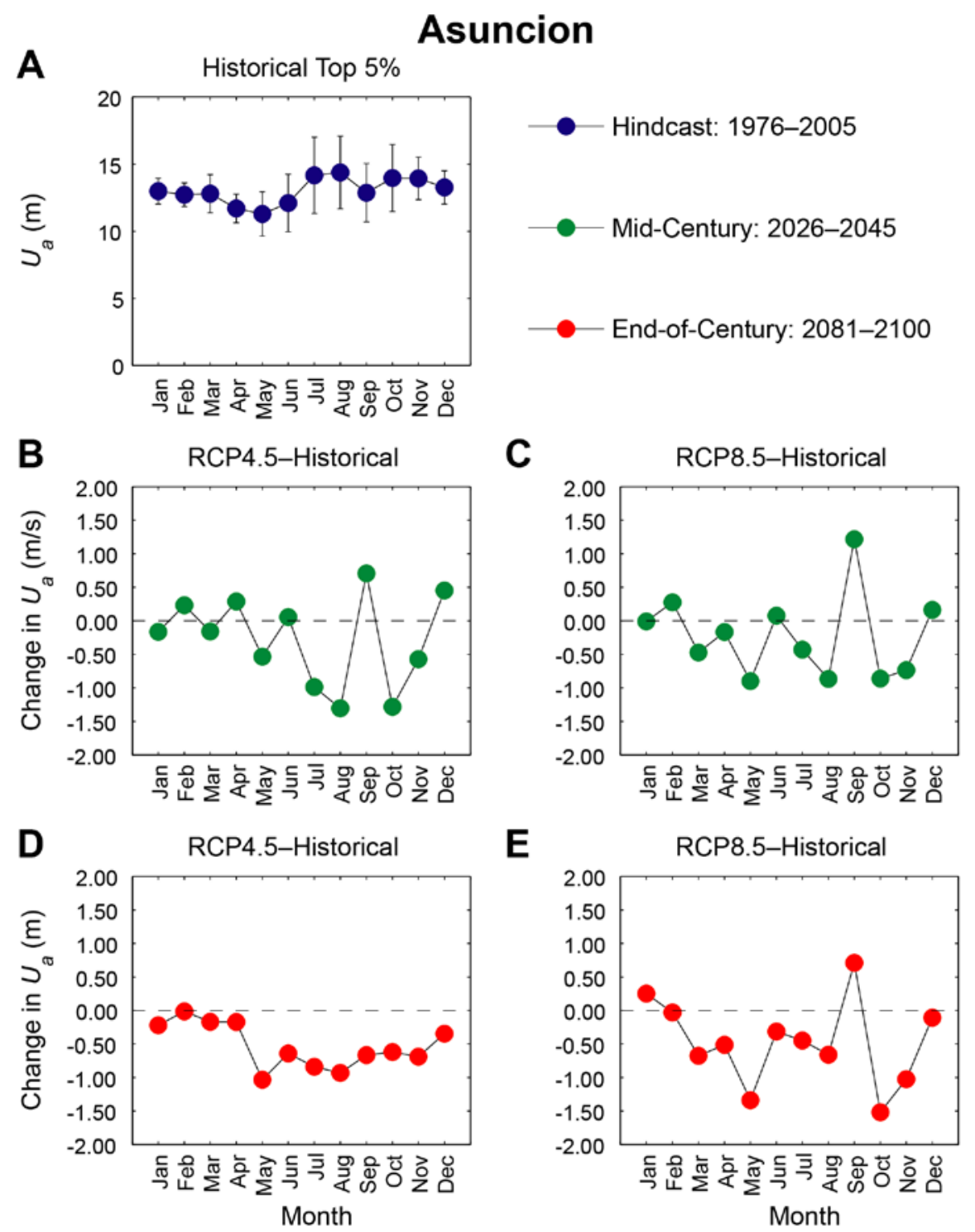

Appendix D14. Plots showing trends in monthly mean of the top 5 percent of wind speeds, in meters, at the Asuncion location. A. Hindcasted (1976-2005) mean of the top 5 percent of wind speeds by month with associated error bars. $B$. Plot of the change in mean of the top 5 percent of 2026-2045 wind speeds for the RCP4.5 scenario from hindcasted top 5 percent of monthly wind speed means. $C$. Plot of the change in mean of the top 5 percent of 2026-2045 wind speeds for the RCP8.5 scenario from hindcasted top 5 percent of monthly wind speed means. $D$. Plot of the change in mean of the top 5 percent of 2081-2100 wind speeds for the RCP4.5 scenario from hindcasted top 5 percent of monthly wind speed means. E. Plot of the change in mean of the top 5 percent of 2081-2100 wind speeds for the RCP8.5 scenario from hindcasted top 5 percent of monthly wind speed means. 

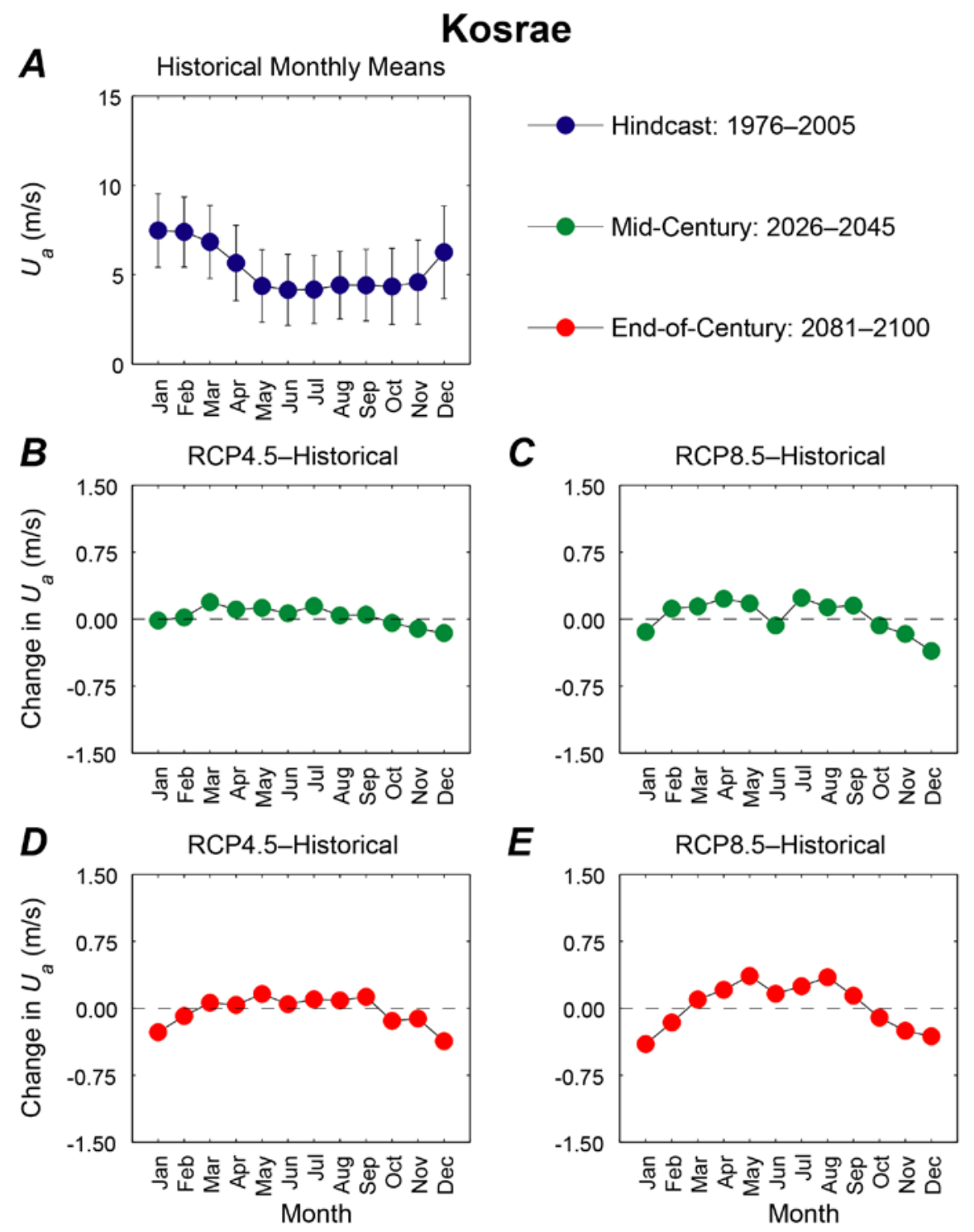

Appendix D15. Plots showing trends in monthly mean wind speed, in meters per second, at the Kosrae location. A. Hindcasted (1976-2005) mean wind speeds by month with associated error bars. B. Plot of the change in mean 2026-2045 wind speeds for the RCP4.5 scenario from hindcasted monthly wind speed means. C. Plot of the change in mean 2026-2045 wind speeds for the RCP8.5 scenario from hindcasted monthly wind speed means. $D$. Plot of the change in mean 2081-2100 wind speeds for the RCP4.5 scenario from hindcasted monthly wind speed means. E. Plot of the change in mean 2081-2100 wind speeds for the RCP8.5 scenario from hindcasted monthly wind speed means. 

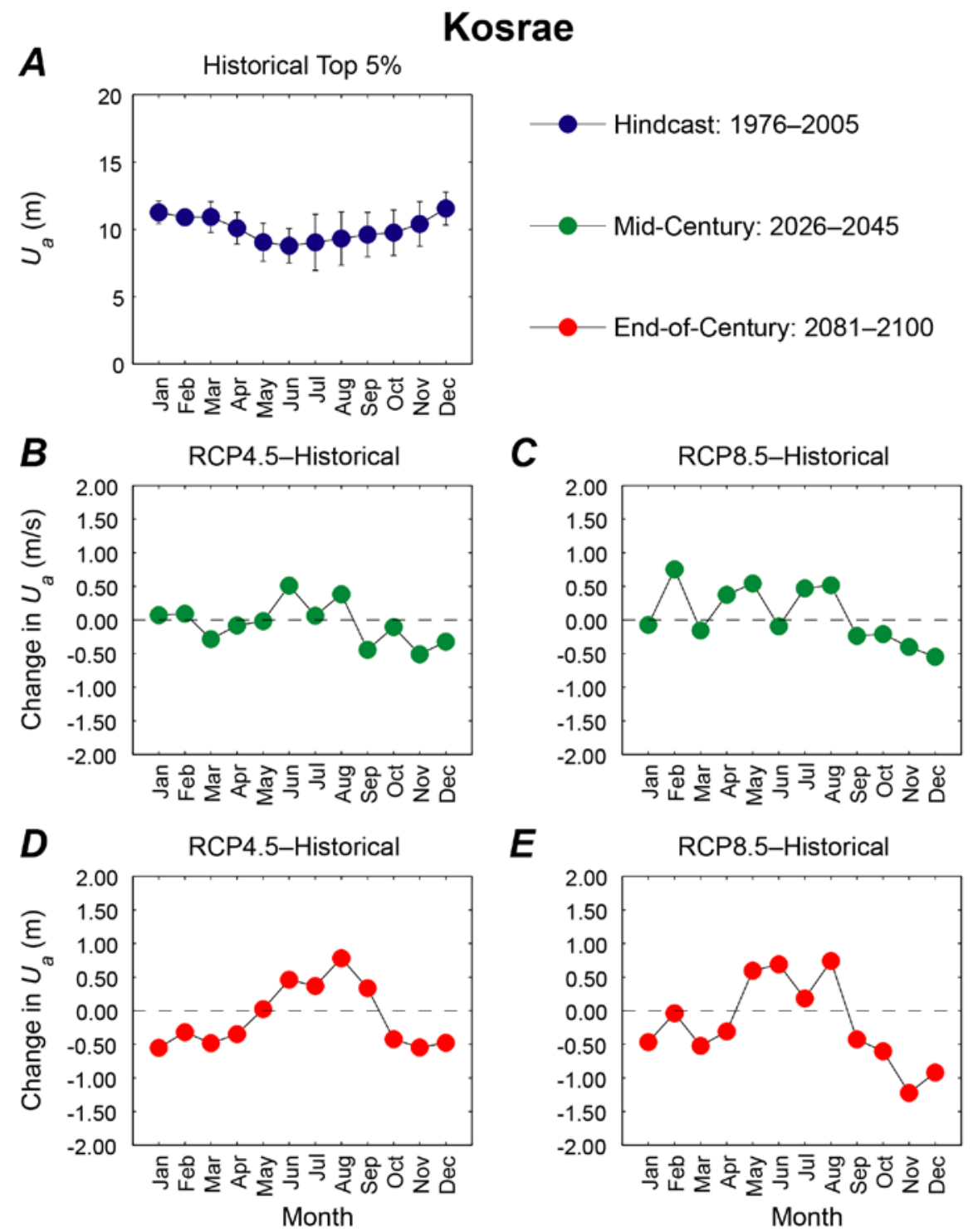

Appendix D16. Plots showing trends in monthly mean of the top 5 percent of wind speeds, in meters, at the Kosrae location. A. Hindcasted (1976-2005) mean of the top 5 percent of wind speeds by month with associated error bars. $B$. Plot of the change in mean of the top 5 percent of 2026-2045 wind speeds for the RCP4.5 scenario from hindcasted top 5 percent of monthly wind speed means. C. Plot of the change in mean of the top 5 percent of 2026-2045 wind speeds for the RCP8.5 scenario from hindcasted top 5 percent of monthly wind speed means. $D$. Plot of the change in mean of the top 5 percent of 2081-2100 wind speeds for the RCP4.5 scenario from hindcasted top 5 percent of monthly wind speed means. E. Plot of the change in mean of the top 5 percent of 2081-2100 wind speeds for the RCP8.5 scenario from hindcasted top 5 percent of monthly wind speed means. 

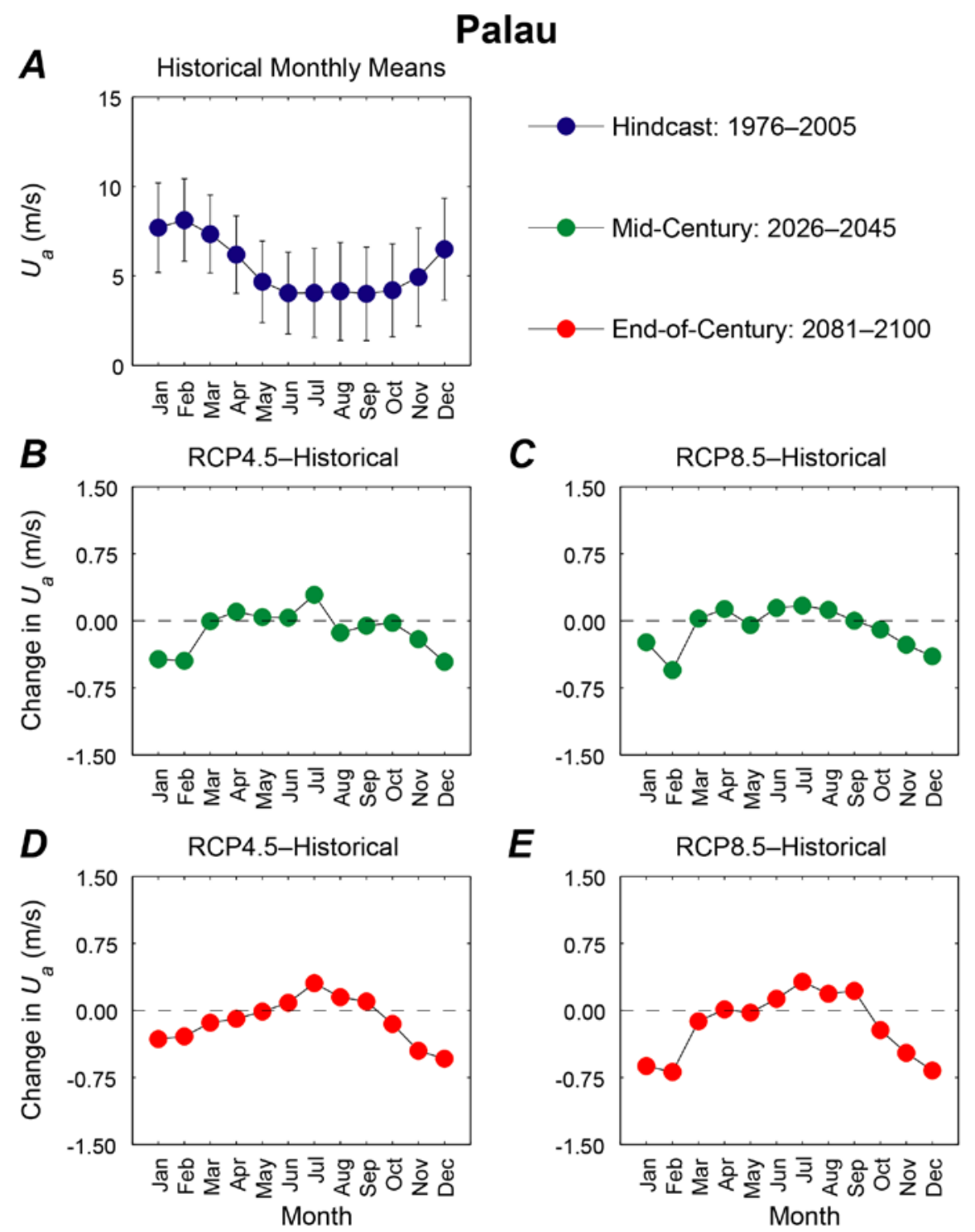

Appendix D17. Plots showing trends in monthly mean wind speed, in meters per second, at the Palau location. A. Hindcasted (1976-2005) mean wind speeds by month with associated error bars. B. Plot of the change in mean 2026-2045 wind speeds for the RCP4.5 scenario from hindcasted monthly wind speed means. C. Plot of the change in mean 2026-2045 wind speeds for the RCP8.5 scenario from hindcasted monthly wind speed means. $D$. Plot of the change in mean 2081-2100 wind speeds for the RCP4.5 scenario from hindcasted monthly wind speed means. E. Plot of the change in mean 2081-2100 wind speeds for the RCP8.5 scenario from hindcasted monthly wind speed means. 

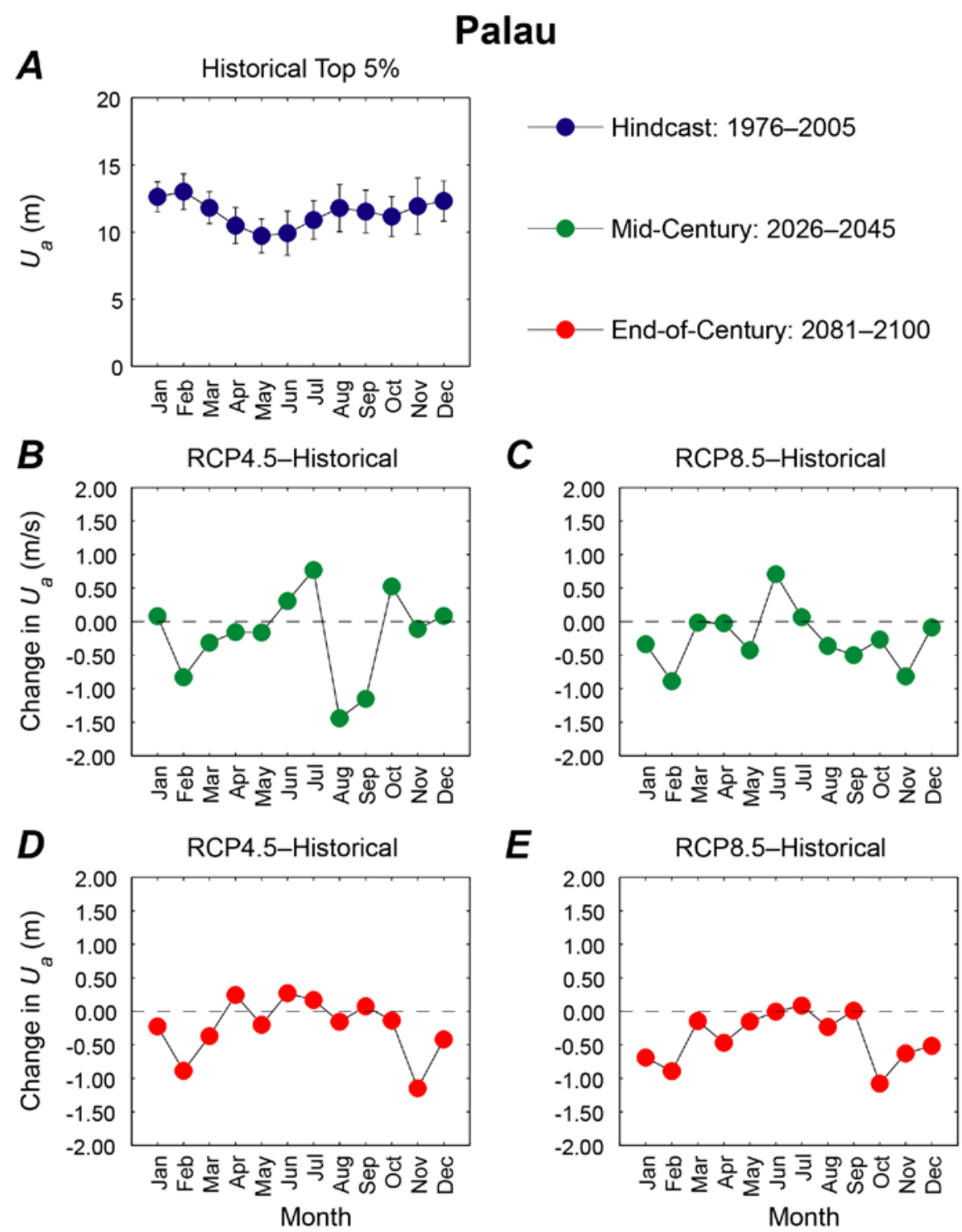

Appendix D18. Plots showing trends in monthly mean of the top 5 percent of wind speeds, in meters, at the Palau location. A. Hindcasted (1976-2005) mean of the top 5 percent of wind speeds by month with associated error bars. $B$. Plot of the change in mean of the top 5 percent of 2026-2045 wind speeds for the RCP4.5 scenario from hindcasted top 5 percent of monthly wind speed means. $C$. Plot of the change in mean of the top 5 percent of 2026-2045 wind speeds for the RCP8.5 scenario from hindcasted top 5 percent of monthly wind speed means. $D$. Plot of the change in mean of the top 5 percent of 2081-2100 wind speeds for the RCP4.5 scenario from hindcasted top 5 percent of monthly wind speed means. E. Plot of the change in mean of the top 5 percent of 2081-2100 wind speeds for the RCP8.5 scenario from hindcasted top 5 percent of monthly wind speed means. 

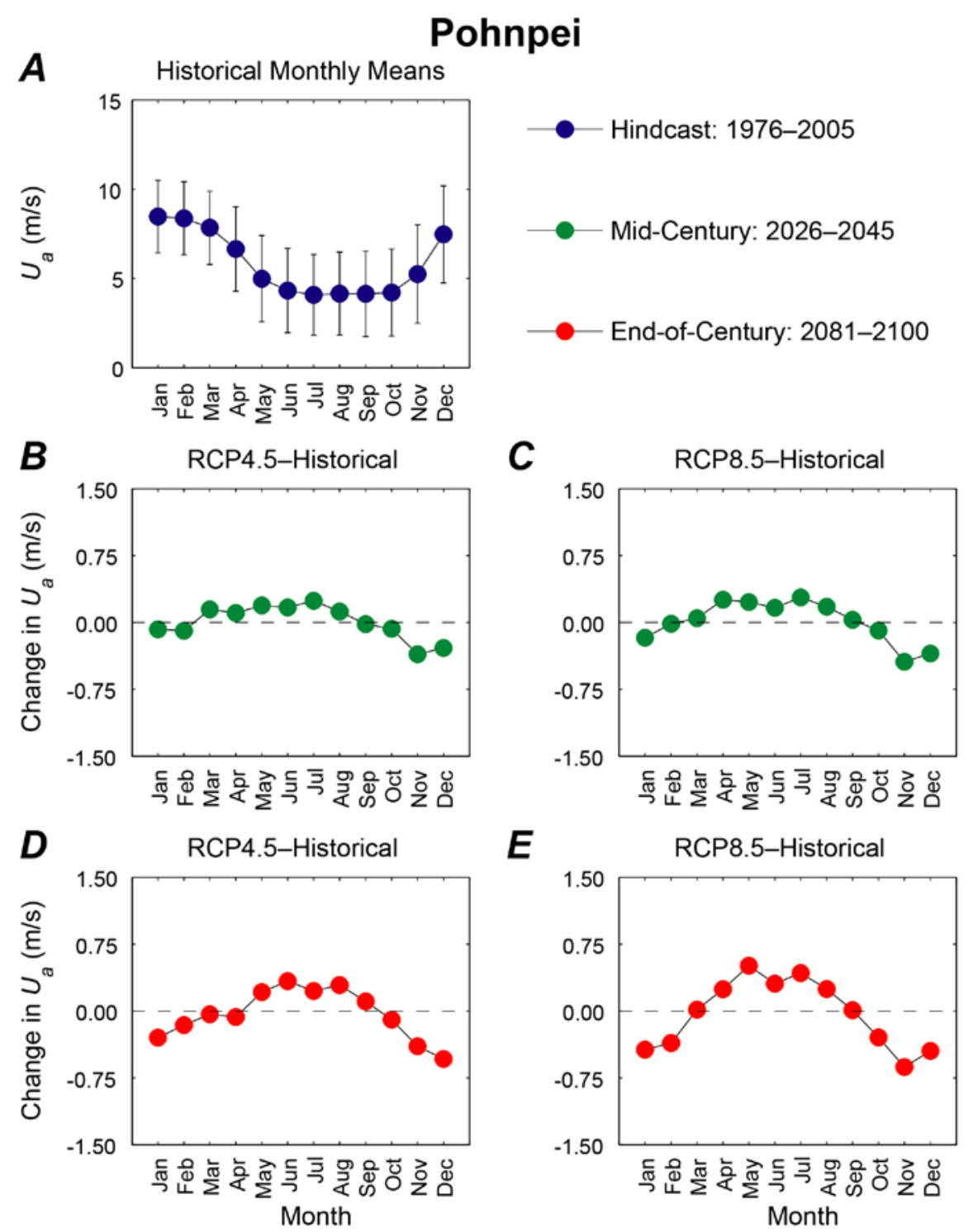

Appendix D19. Plots showing trends in monthly mean wind speed, in meters per second, at the Pohnpei location. A. Hindcasted (1976-2005) mean wind speeds by month with associated error bars. B. Plot of the change in mean 2026-2045 wind speeds for the RCP4.5 scenario from hindcasted monthly wind speed means. C. Plot of the change in mean 2026-2045 wind speeds for the RCP8.5 scenario from hindcasted monthly wind speed means. $D$. Plot of the change in mean 2081-2100 wind speeds for the RCP4.5 scenario from hindcasted monthly wind speed means. E. Plot of the change in mean 2081-2100 wind speeds for the RCP8.5 scenario from hindcasted monthly wind speed means. 

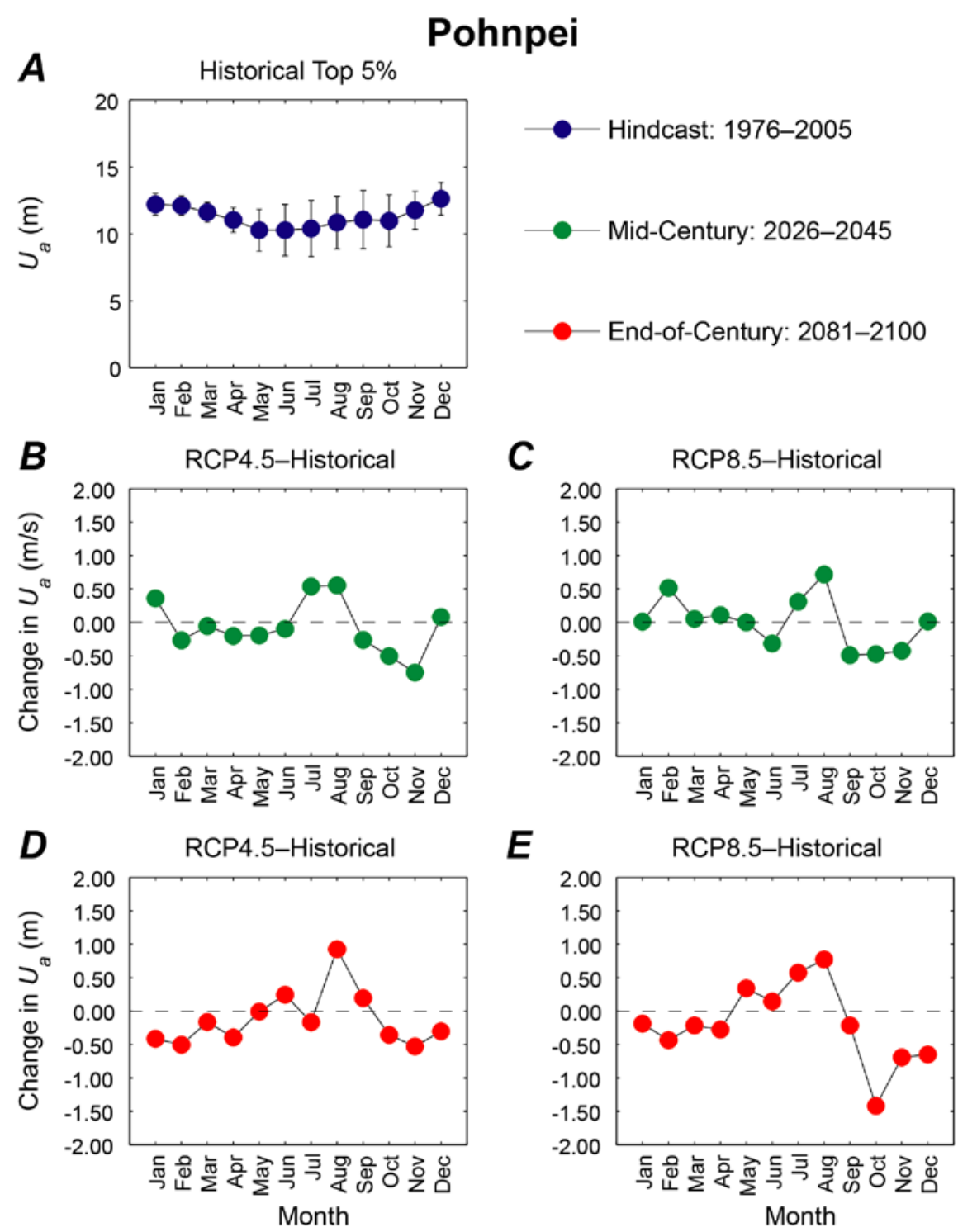

Appendix D20. Plots showing trends in monthly mean of the top 5 percent of wind speeds, in meters, at the Pohnpei location. A. Hindcasted (1976-2005) mean of the top 5 percent of wind speeds by month with associated error bars. $B$. Plot of the change in mean of the top 5 percent of 2026-2045 wind speeds for the RCP4.5 scenario from hindcasted top 5 percent of monthly wind speed means. $C$. Plot of the change in mean of the top 5 percent of 2026-2045 wind speeds for the RCP8.5 scenario from hindcasted top 5 percent of monthly wind speed means. $D$. Plot of the change in mean of the top 5 percent of 2081-2100 wind speeds for the RCP4.5 scenario from hindcasted top 5 percent of monthly wind speed means. E. Plot of the change in mean of the top 5 percent of 2081-2100 wind speeds for the RCP8.5 scenario from hindcasted top 5 percent of monthly wind speed means. 

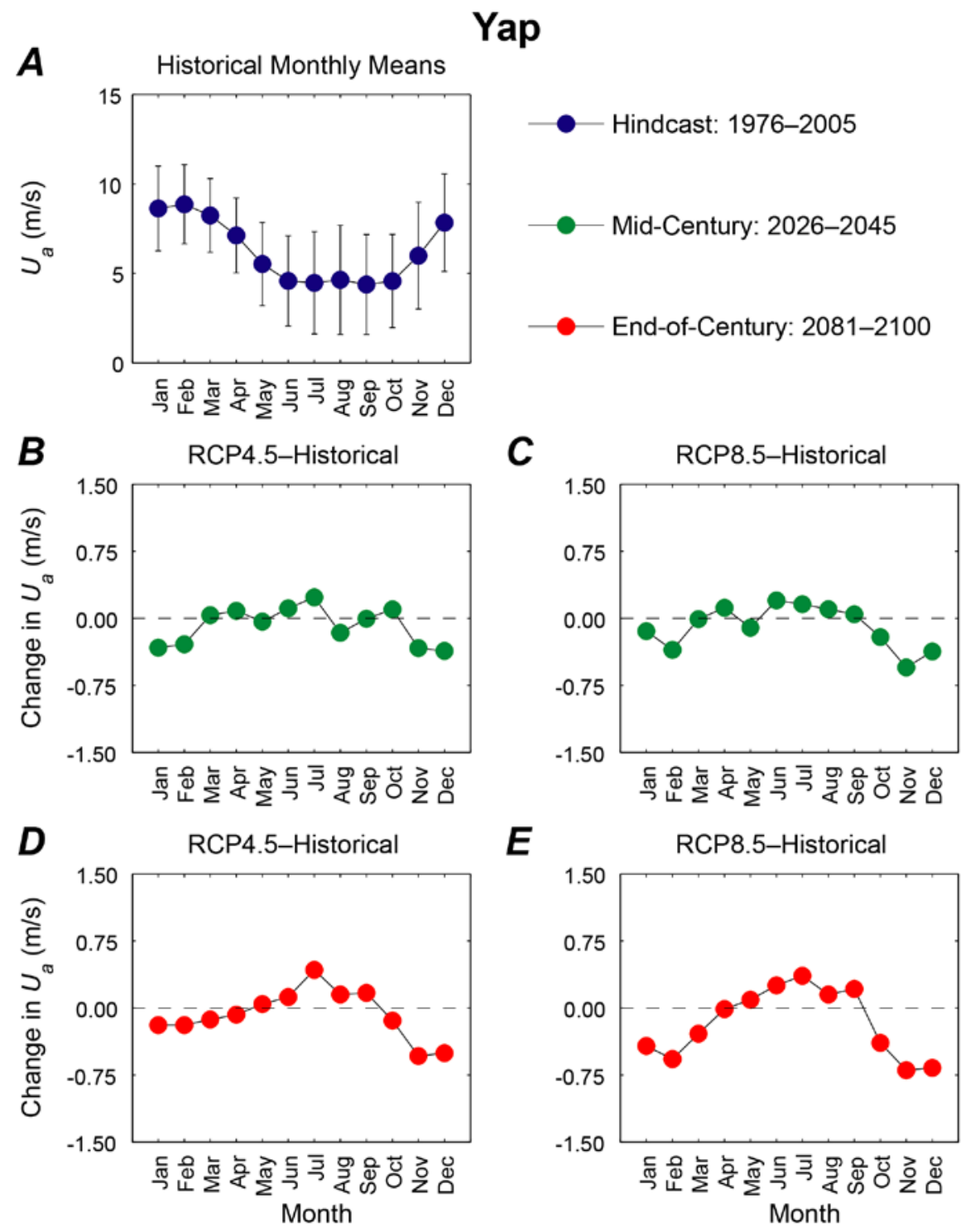

Appendix D21. Plots showing trends in monthly mean wind speed, in meters per second, at the Yap location. A. Hindcasted (1976-2005) mean wind speeds by month with associated error bars. B. Plot of the change in mean 2026-2045 wind speeds for the RCP4.5 scenario from hindcasted monthly wind speed means. C. Plot of the change in mean 2026-2045 wind speeds for the RCP8.5 scenario from hindcasted monthly wind speed means. $D$. Plot of the change in mean 2081-2100 wind speeds for the RCP4.5 scenario from hindcasted monthly wind speed means. E. Plot of the change in mean 2081-2100 wind speeds for the RCP8.5 scenario from hindcasted monthly wind speed means. 

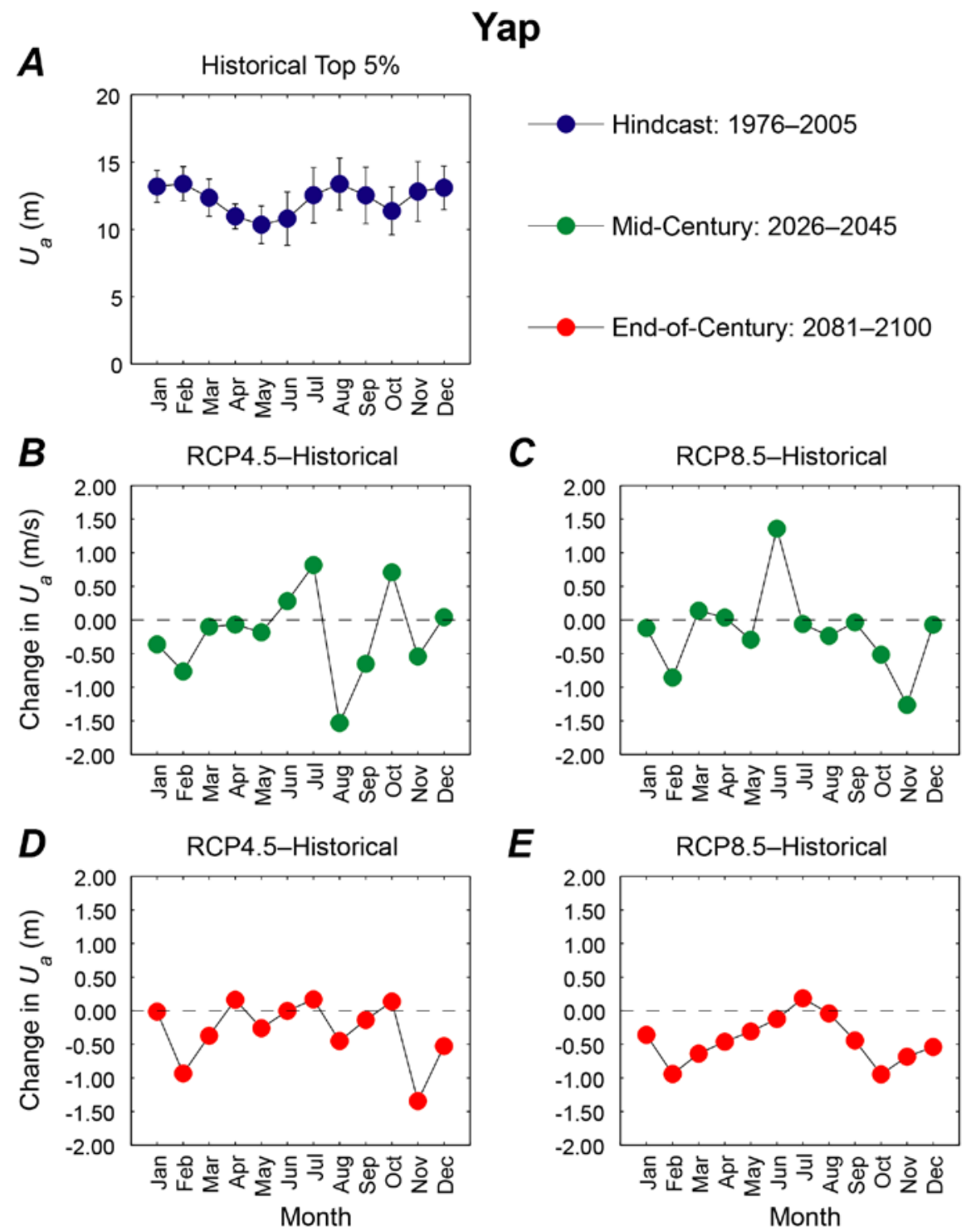

Appendix D22. Plots showing trends in monthly mean of the top 5 percent of wind speeds, in meters, at the Yap location. A. Hindcasted (1976-2005) mean of the top 5 percent of wind speeds by month with associated error bars. $B$. Plot of the change in mean of the top 5 percent of 2026-2045 wind speeds for the RCP4.5 scenario from hindcasted top 5 percent of monthly wind speed means. $C$. Plot of the change in mean of the top 5 percent of 2026-2045 wind speeds for the RCP8.5 scenario from hindcasted top 5 percent of monthly wind speed means. $D$. Plot of the change in mean of the top 5 percent of 2081-2100 wind speeds for the RCP4.5 scenario from hindcasted top 5 percent of monthly wind speed means. E. Plot of the change in mean of the top 5 percent of 2081-2100 wind speeds for the RCP8.5 scenario from hindcasted top 5 percent of monthly wind speed means. 

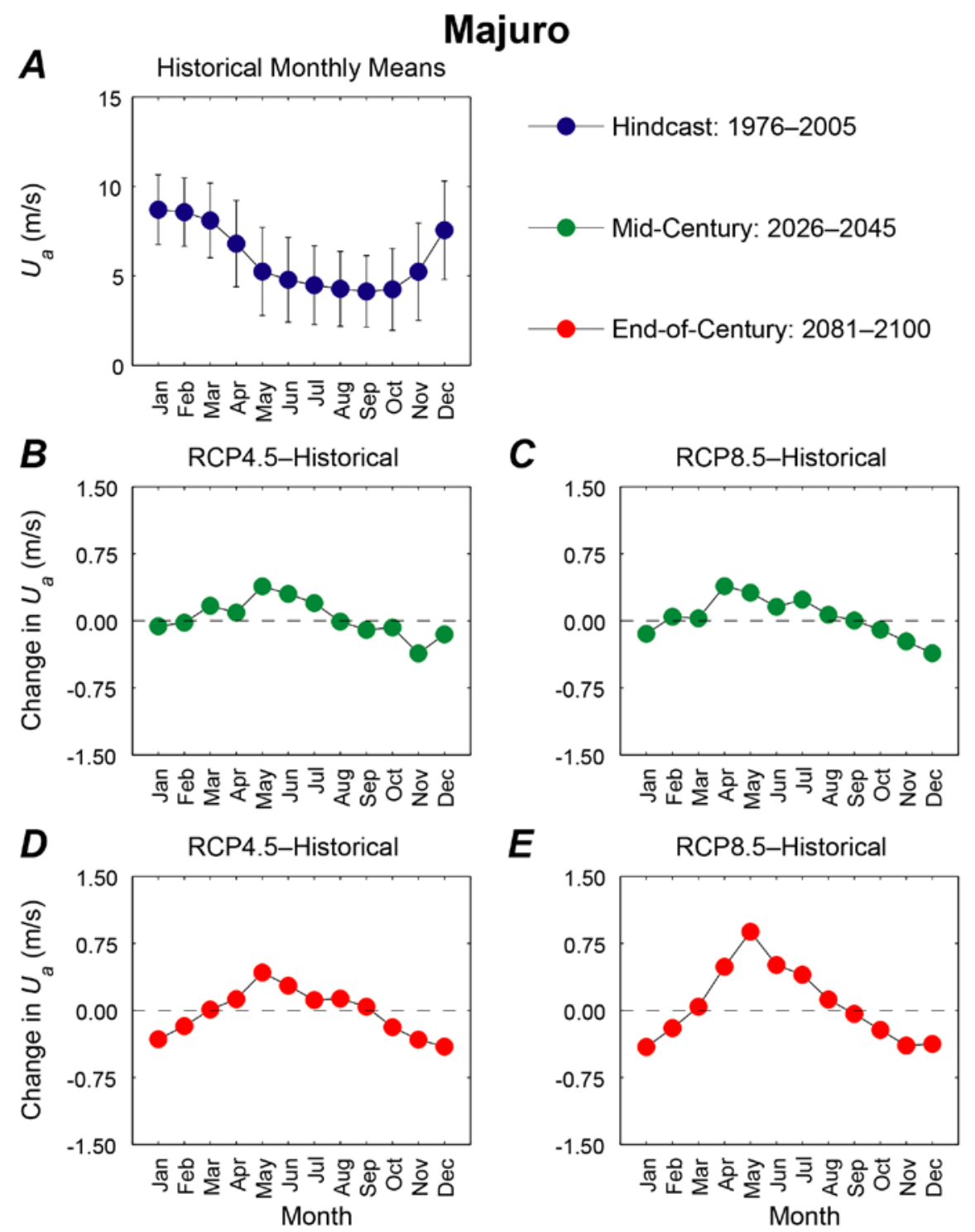

Appendix D23. Plots showing trends in monthly mean wind speed, in meters per second, at the Majuro location. A. Hindcasted (1976-2005) mean wind speeds by month with associated error bars. B. Plot of the change in mean 2026-2045 wind speeds for the RCP4.5 scenario from hindcasted monthly wind speed means. C. Plot of the change in mean 2026-2045 wind speeds for the RCP8.5 scenario from hindcasted monthly wind speed means. $D$. Plot of the change in mean 2081-2100 wind speeds for the RCP4.5 scenario from hindcasted monthly wind speed means. E. Plot of the change in mean 2081-2100 wind speeds for the RCP8.5 scenario from hindcasted monthly wind speed means. 

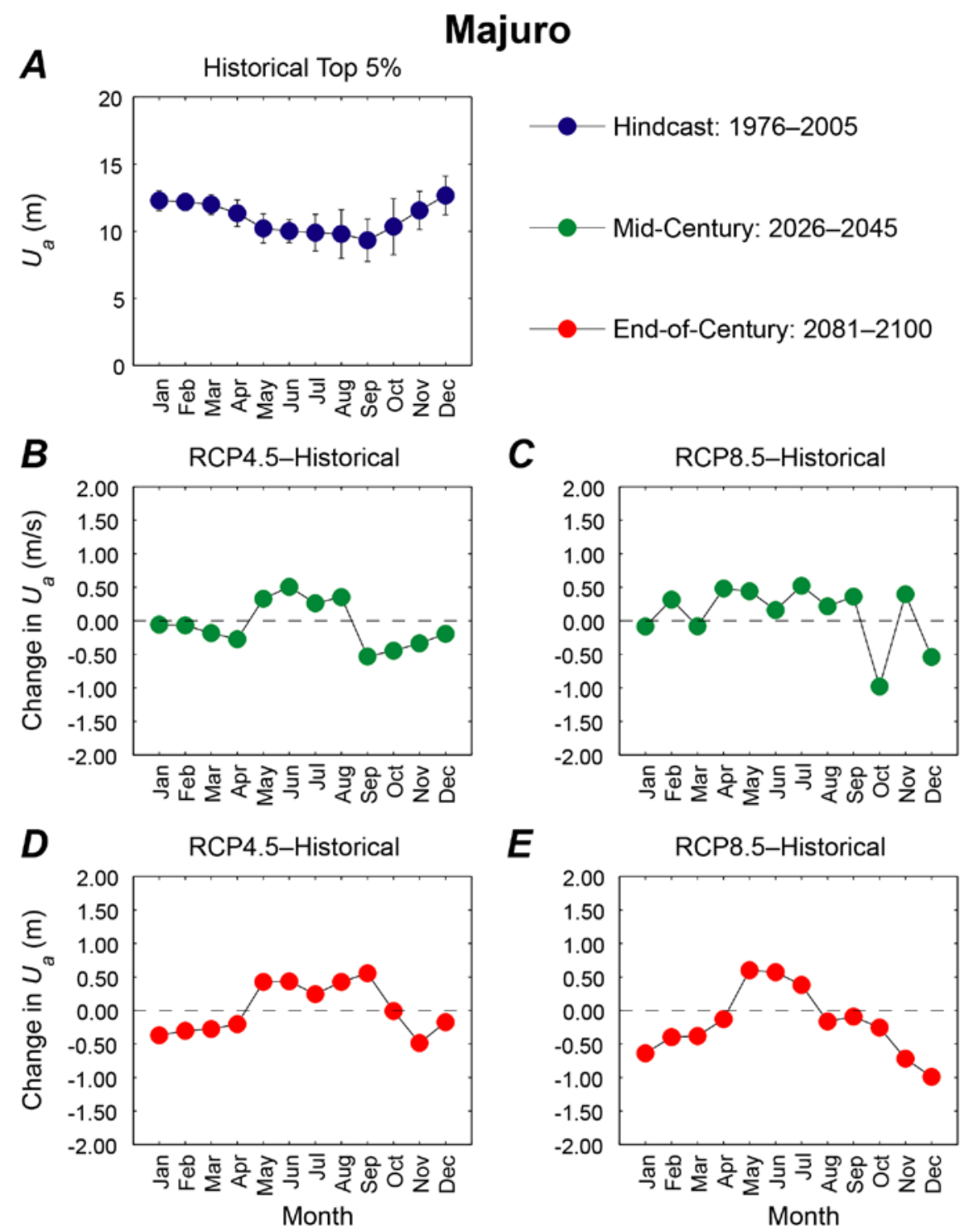

Appendix D24. Plots showing trends in monthly mean of the top 5 percent of wind speeds, in meters, at the Majuro location. A. Hindcasted (1976-2005) mean of the top 5 percent of wind speeds by month with associated error bars. $B$. Plot of the change in mean of the top 5 percent of 2026-2045 wind speeds for the RCP4.5 scenario from hindcasted top 5 percent of monthly wind speed means. $C$. Plot of the change in mean of the top 5 percent of 2026-2045 wind speeds for the RCP8.5 scenario from hindcasted top 5 percent of monthly wind speed means. $D$. Plot of the change in mean of the top 5 percent of 2081-2100 wind speeds for the RCP4.5 scenario from hindcasted top 5 percent of monthly wind speed means. E. Plot of the change in mean of the top 5 percent of 2081-2100 wind speeds for the RCP8.5 scenario from hindcasted top 5 percent of monthly wind speed means. 

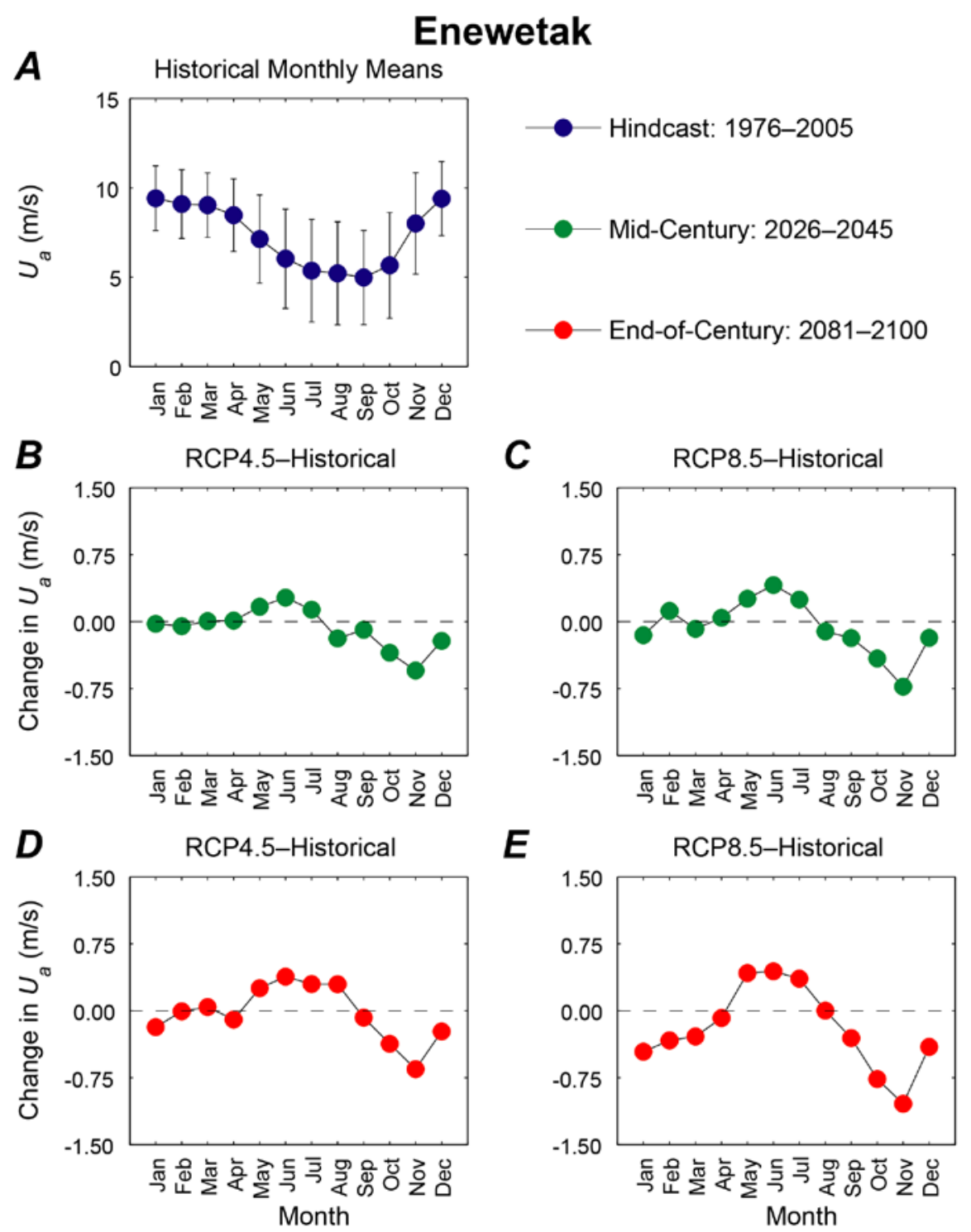

Appendix D25. Plots showing trends in monthly mean wind speed, in meters per second, at the Enewetak location. A. Hindcasted (1976-2005) mean wind speeds by month with associated error bars. B. Plot of the change in mean 2026-2045 wind speeds for the RCP4.5 scenario from hindcasted monthly wind speed means. C. Plot of the change in mean 2026-2045 wind speeds for the RCP8.5 scenario from hindcasted monthly wind speed means. $D$. Plot of the change in mean 2081-2100 wind speeds for the RCP4.5 scenario from hindcasted monthly wind speed means. E. Plot of the change in mean 2081-2100 wind speeds for the RCP8.5 scenario from hindcasted monthly wind speed means. 

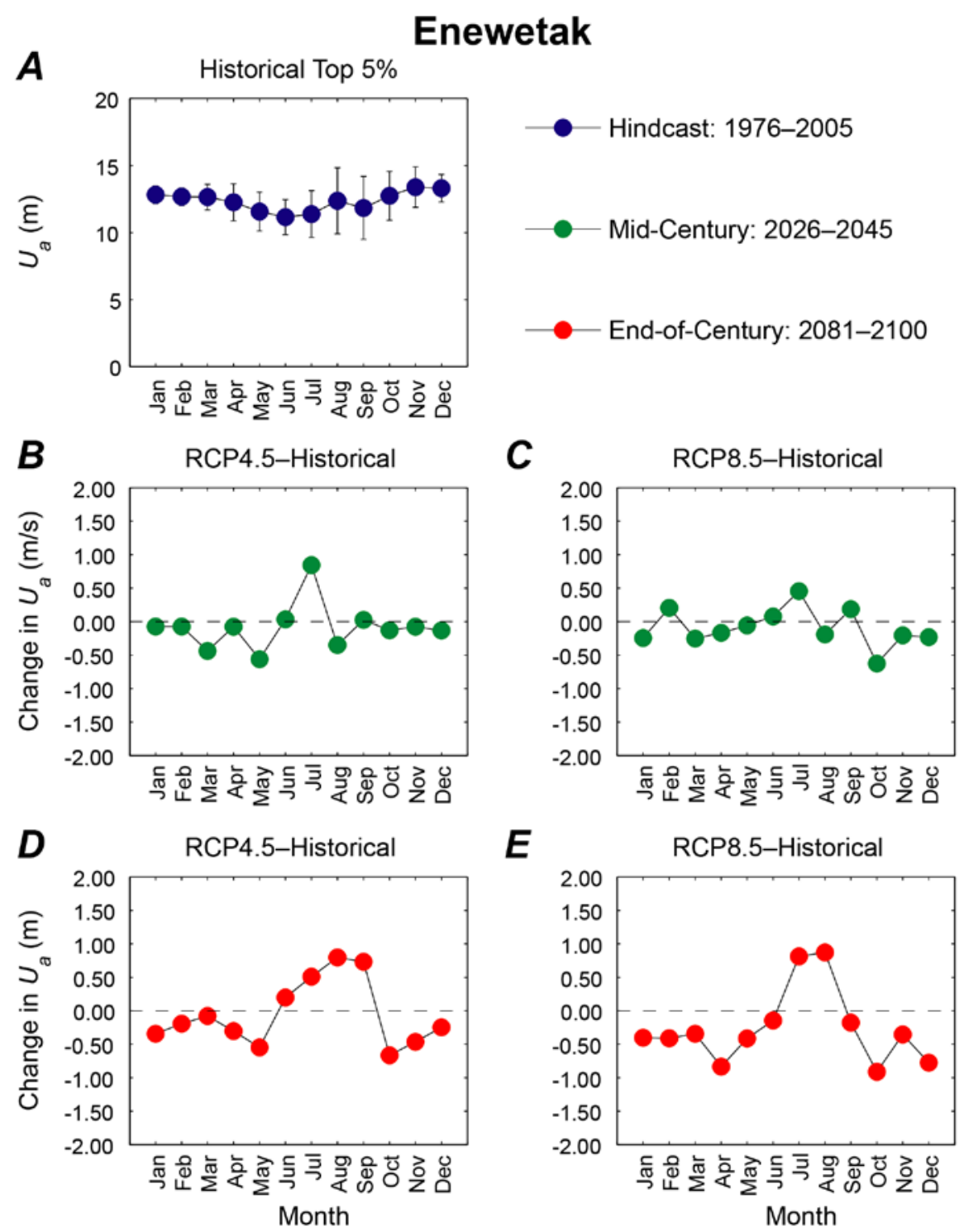

Appendix D26. Plots showing trends in monthly mean of the top 5 percent of wind speeds, in meters, at the Enewetak location. A. Hindcasted (1976-2005) mean of the top 5 percent of wind speeds by month with associated error bars. $B$. Plot of the change in mean of the top 5 percent of 2026-2045 wind speeds for the RCP4.5 scenario from hindcasted top 5 percent of monthly wind speed means. $C$. Plot of the change in mean of the top 5 percent of 2026-2045 wind speeds for the RCP8.5 scenario from hindcasted top 5 percent of monthly wind speed means. $D$. Plot of the change in mean of the top 5 percent of 2081-2100 wind speeds for the RCP4.5 scenario from hindcasted top 5 percent of monthly wind speed means. E. Plot of the change in mean of the top 5 percent of 2081-2100 wind speeds for the RCP8.5 scenario from hindcasted top 5 percent of monthly wind speed means. 

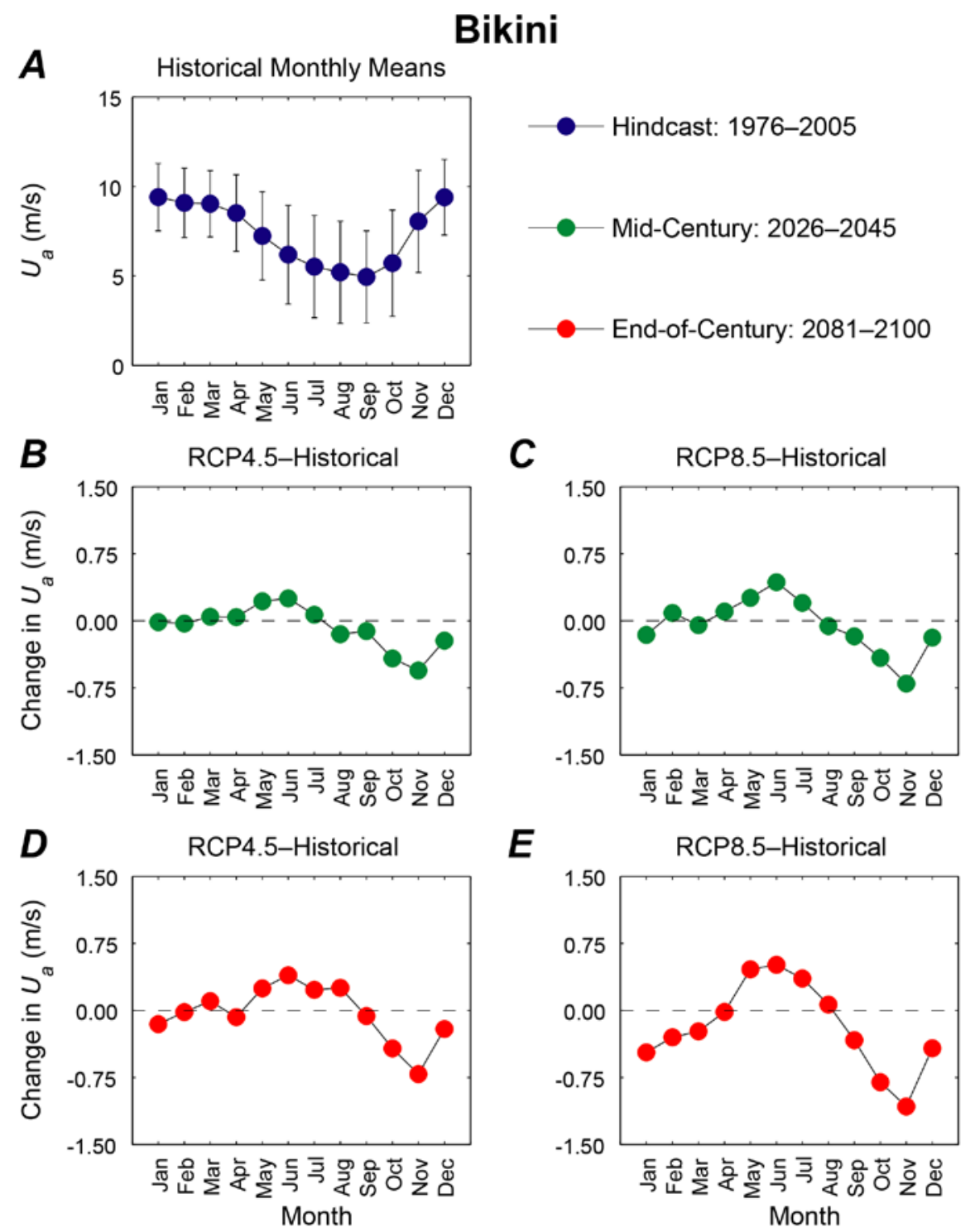

Appendix D27. Plots showing trends in monthly mean wind speed, in meters per second, at the Bikini location. A. Hindcasted (1976-2005) mean wind speeds by month with associated error bars. B. Plot of the change in mean 2026-2045 wind speeds for the RCP4.5 scenario from hindcasted monthly wind speed means. C. Plot of the change in mean 2026-2045 wind speeds for the RCP8.5 scenario from hindcasted monthly wind speed means. $D$. Plot of the change in mean 2081-2100 wind speeds for the RCP4.5 scenario from hindcasted monthly wind speed means. E. Plot of the change in mean 2081-2100 wind speeds for the RCP8.5 scenario from hindcasted monthly wind speed means. 

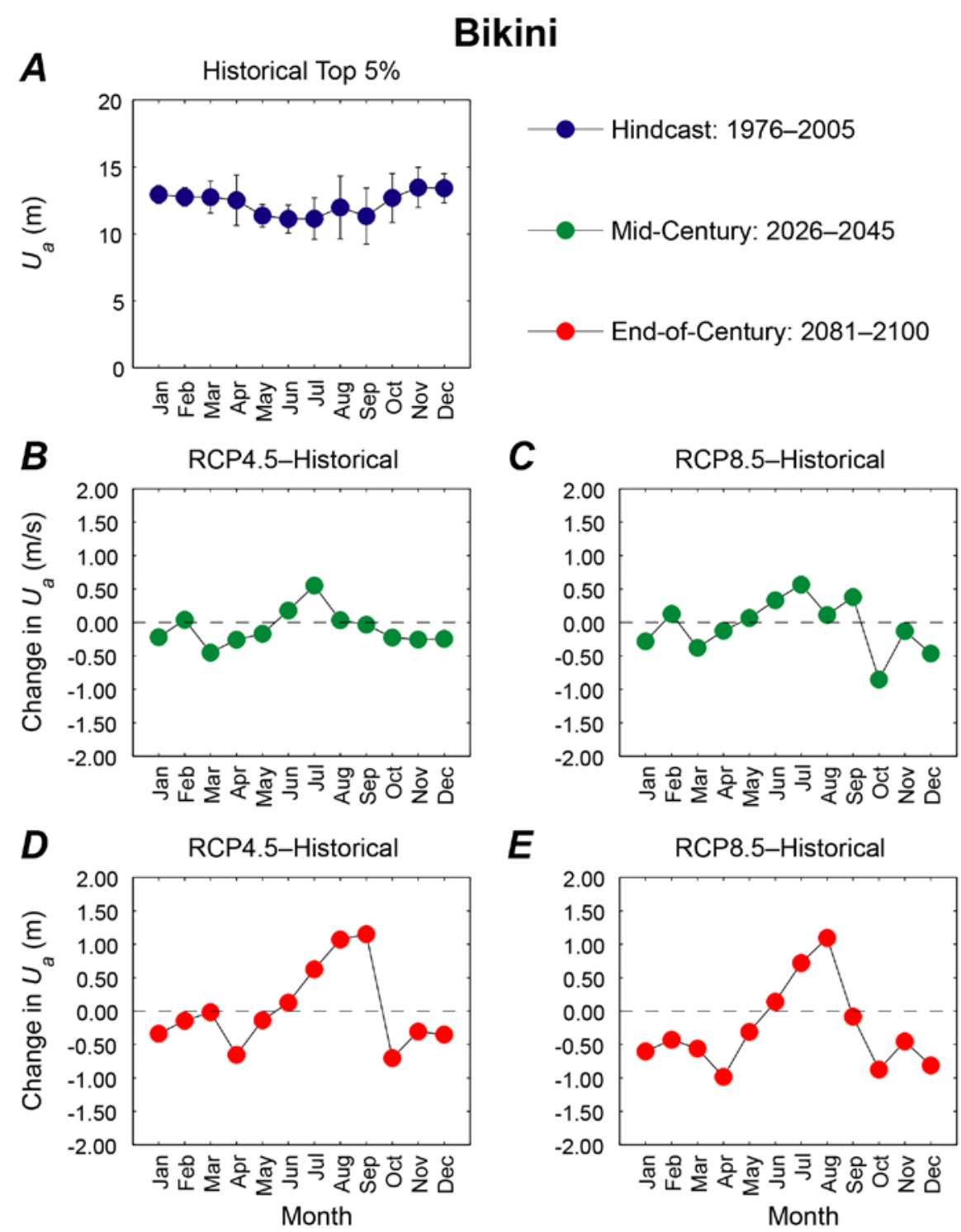

Appendix D28. Plots showing trends in monthly mean of the top 5 percent of wind speeds, in meters, at the Bikini location. A. Hindcasted (1976-2005) mean of the top 5 percent of wind speeds by month with associated error bars. $B$. Plot of the change in mean of the top 5 percent of 2026-2045 wind speeds for the RCP4.5 scenario from hindcasted top 5 percent of monthly wind speed means. C. Plot of the change in mean of the top 5 percent of 2026-2045 wind speeds for the RCP8.5 scenario from hindcasted top 5 percent of monthly wind speed means. $D$. Plot of the change in mean of the top 5 percent of 2081-2100 wind speeds for the RCP4.5 scenario from hindcasted top 5 percent of monthly wind speed means. E. Plot of the change in mean of the top 5 percent of 2081-2100 wind speeds for the RCP8.5 scenario from hindcasted top 5 percent of monthly wind speed means. 

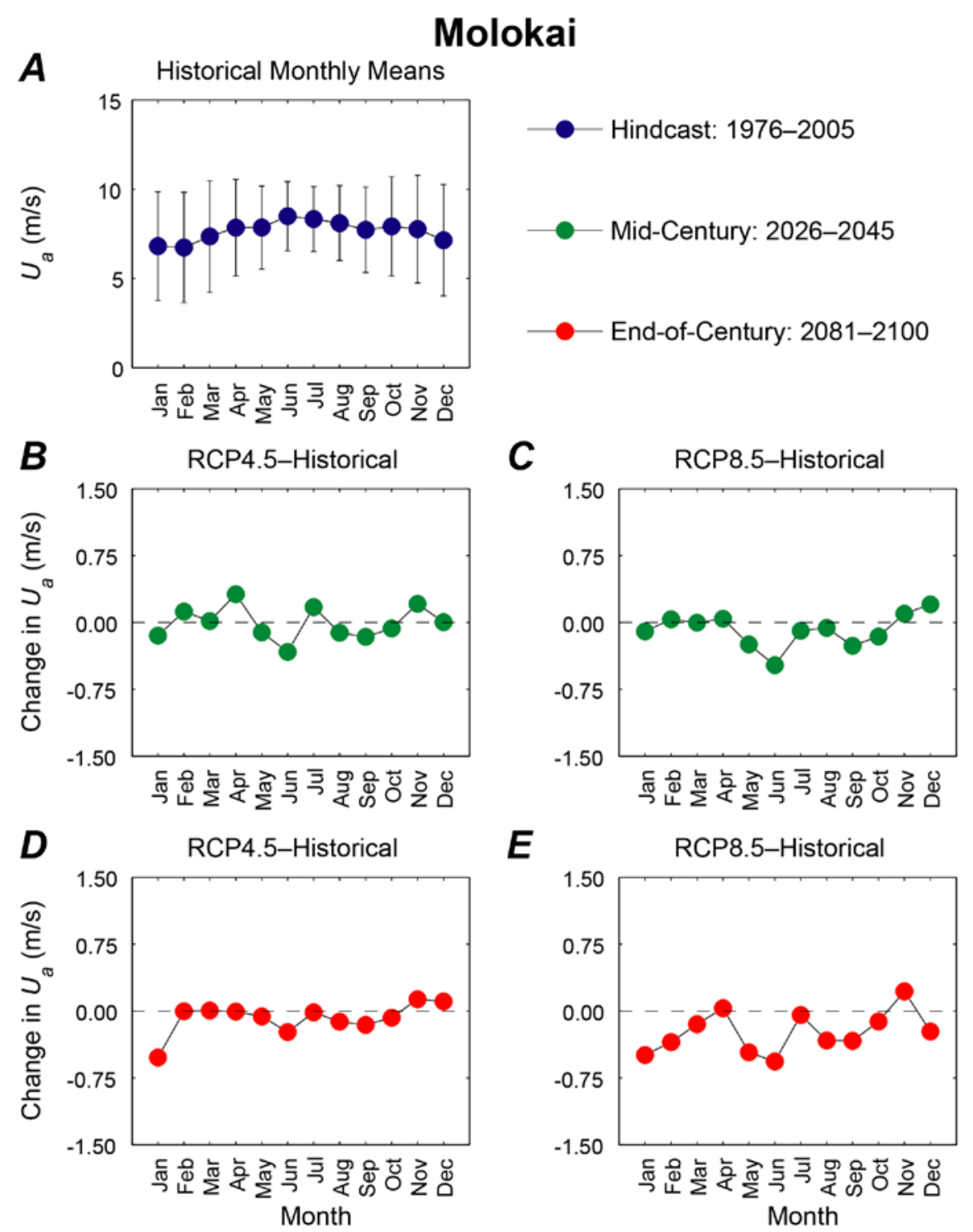

Appendix D29. Plots showing trends in monthly mean wind speed, in meters per second, at the Molokai location. A. Hindcasted (1976-2005) mean wind speeds by month with associated error bars. B. Plot of the change in mean 2026-2045 wind speeds for the RCP4.5 scenario from hindcasted monthly wind speed means. C. Plot of the change in mean 2026-2045 wind speeds for the RCP8.5 scenario from hindcasted monthly wind speed means. $D$. Plot of the change in mean 2081-2100 wind speeds for the RCP4.5 scenario from hindcasted monthly wind speed means. E. Plot of the change in mean 2081-2100 wind speeds for the RCP8.5 scenario from hindcasted monthly wind speed means. 

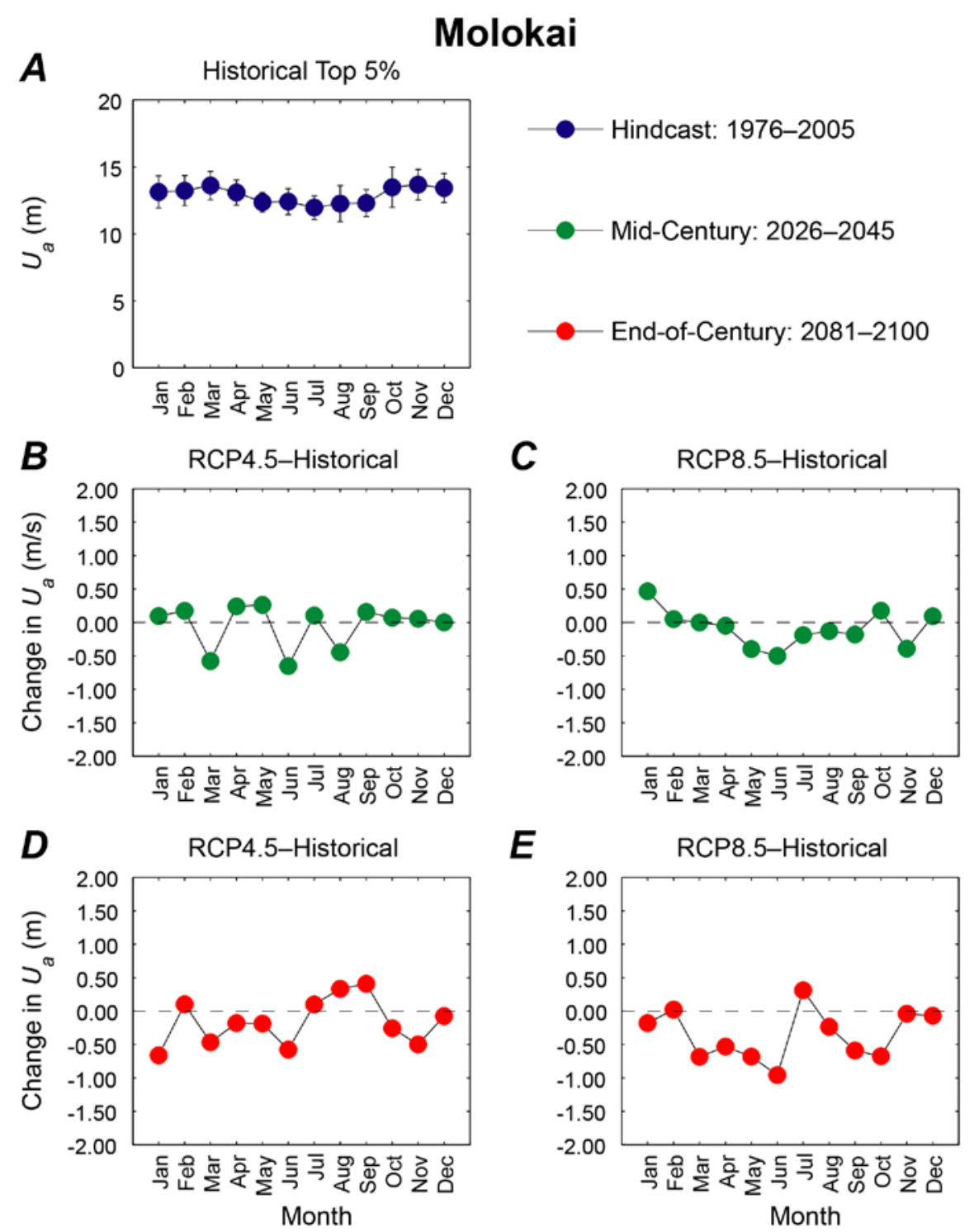

Appendix D30. Plots showing trends in monthly mean of the top 5 percent of wind speeds, in meters, at the Molokai location. A. Hindcasted (1976-2005) mean of the top 5 percent of wind speeds by month with associated error bars. $B$. Plot of the change in mean of the top 5 percent of 2026-2045 wind speeds for the RCP4.5 scenario from hindcasted top 5 percent of monthly wind speed means. $C$. Plot of the change in mean of the top 5 percent of 2026-2045 wind speeds for the RCP8.5 scenario from hindcasted top 5 percent of monthly wind speed means. $D$. Plot of the change in mean of the top 5 percent of 2081-2100 wind speeds for the RCP4.5 scenario from hindcasted top 5 percent of monthly wind speed means. E. Plot of the change in mean of the top 5 percent of 2081-2100 wind speeds for the RCP8.5 scenario from hindcasted top 5 percent of monthly wind speed means. 

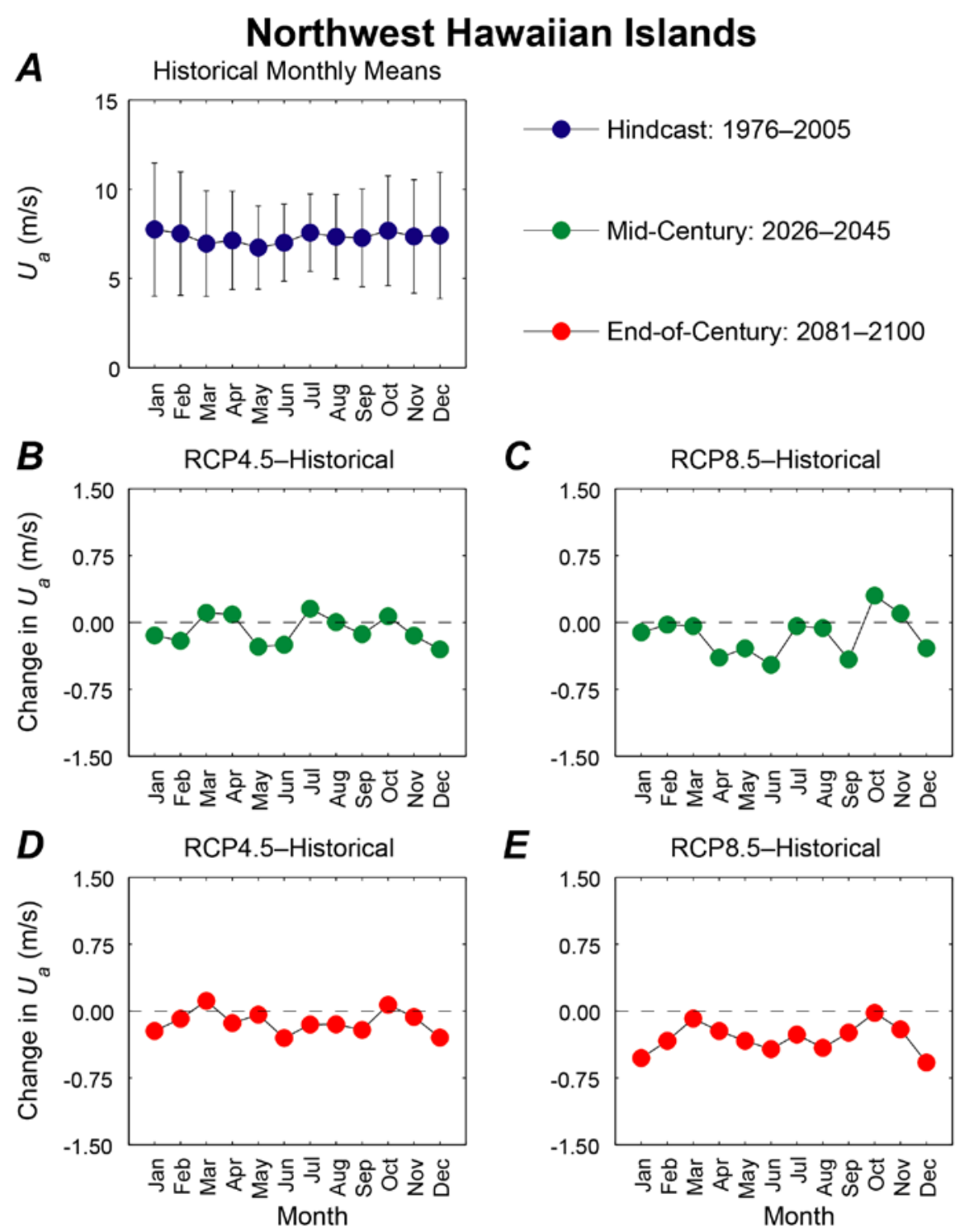

Appendix D31. Plots showing trends in monthly mean wind speed, in meters per second, at the Northwest Hawaiian Islands location. A. Hindcasted (1976-2005) mean wind speeds by month with associated error bars. B. Plot of the change in mean 2026-2045 wind speeds for the RCP4.5 scenario from hindcasted monthly wind speed means. C. Plot of the change in mean 2026-2045 wind speeds for the RCP8.5 scenario from hindcasted monthly wind speed means. $D$. Plot of the change in mean 2081-2100 wind speeds for the RCP4.5 scenario from hindcasted monthly wind speed means. $E$. Plot of the change in mean 2081-2100 wind speeds for the RCP8.5 scenario from hindcasted monthly wind speed means. 

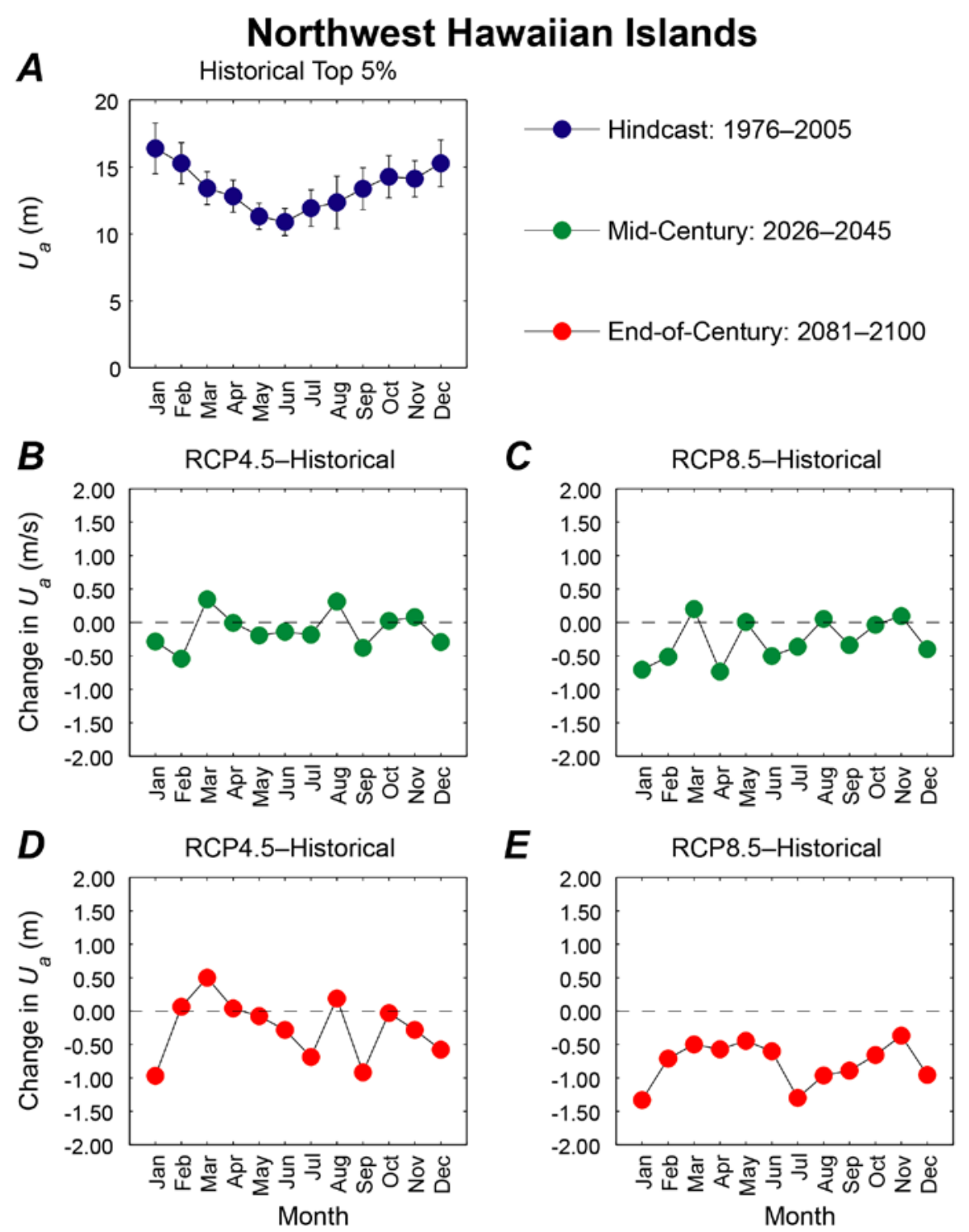

Appendix D32. Plots showing trends in monthly mean of the top 5 percent of wind speeds, in meters, at the Northwest Hawaiian Islands location. A. Hindcasted (1976-2005) mean of the top 5 percent of wind speeds by month with associated error bars. $B$. Plot of the change in mean of the top 5 percent of 2026-2045 wind speeds for the RCP4.5 scenario from hindcasted top 5 percent of monthly wind speed means. C. Plot of the change in mean of the top 5 percent of 2026-2045 wind speeds for the RCP8.5 scenario from hindcasted top 5 percent of monthly wind speed means. $D$. Plot of the change in mean of the top 5 percent of 2081-2100 wind speeds for the RCP4.5 scenario from hindcasted top 5 percent of monthly wind speed means. E. Plot of the change in mean of the top 5 percent of 20812100 wind speeds for the RCP 8.5 scenario from hindcasted top 5 percent of monthly wind speed means. 

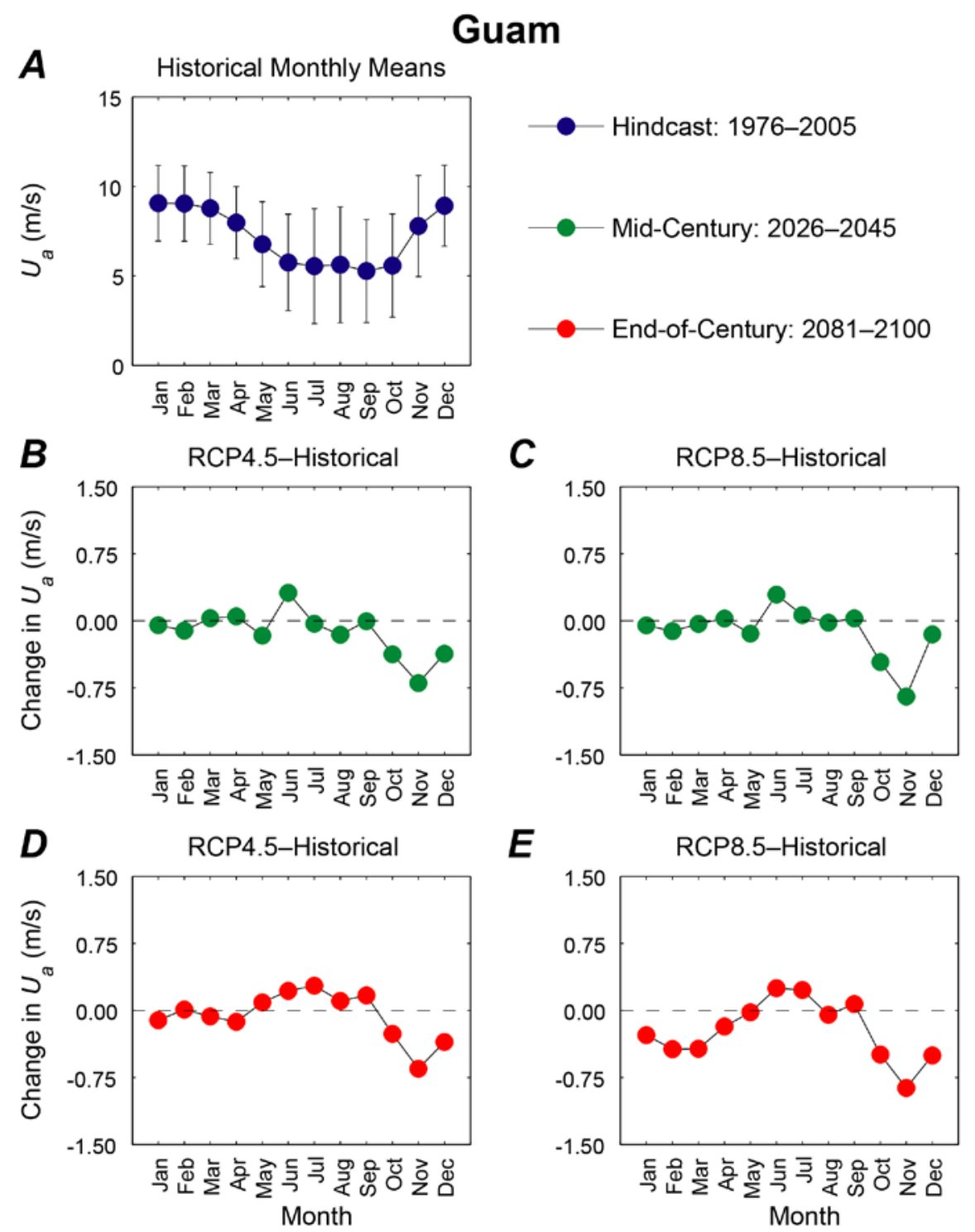

Appendix D33. Plots showing trends in monthly mean wind speed, in meters per second, at the Guam location. A. Hindcasted (1976-2005) mean wind speeds by month with associated error bars. B. Plot of the change in mean 2026-2045 wind speeds for the RCP4.5 scenario from hindcasted monthly wind speed means. C. Plot of the change in mean 2026-2045 wind speeds for the RCP8.5 scenario from hindcasted monthly wind speed means. $D$. Plot of the change in mean 2081-2100 wind speeds for the RCP4.5 scenario from hindcasted monthly wind speed means. E. Plot of the change in mean 2081-2100 wind speeds for the RCP8.5 scenario from hindcasted monthly wind speed means. 

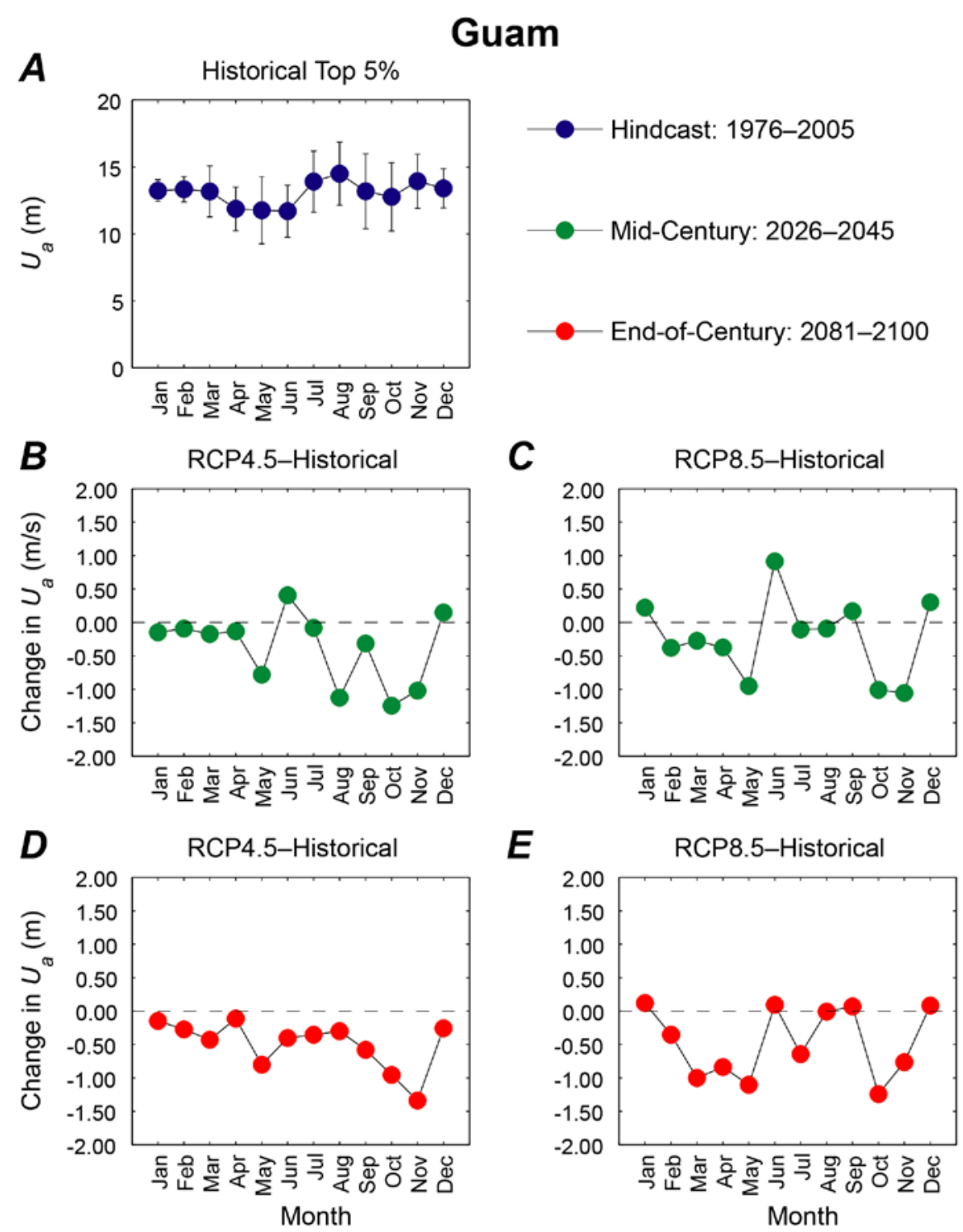

Appendix D34. Plots showing trends in monthly mean of the top 5 percent of wind speeds, in meters, at the Guam location. A. Hindcasted (1976-2005) mean of the top 5 percent of wind speeds by month with associated error bars. $B$. Plot of the change in mean of the top 5 percent of 2026-2045 wind speeds for the RCP4.5 scenario from hindcasted top 5 percent of monthly wind speed means. C. Plot of the change in mean of the top 5 percent of 2026-2045 wind speeds for the RCP8.5 scenario from hindcasted top 5 percent of monthly wind speed means. $D$. Plot of the change in mean of the top 5 percent of 2081-2100 wind speeds for the RCP4.5 scenario from hindcasted top 5 percent of monthly wind speed means. E. Plot of the change in mean of the top 5 percent of 2081-2100 wind speeds for the RCP8.5 scenario from hindcasted top 5 percent of monthly wind speed means. 

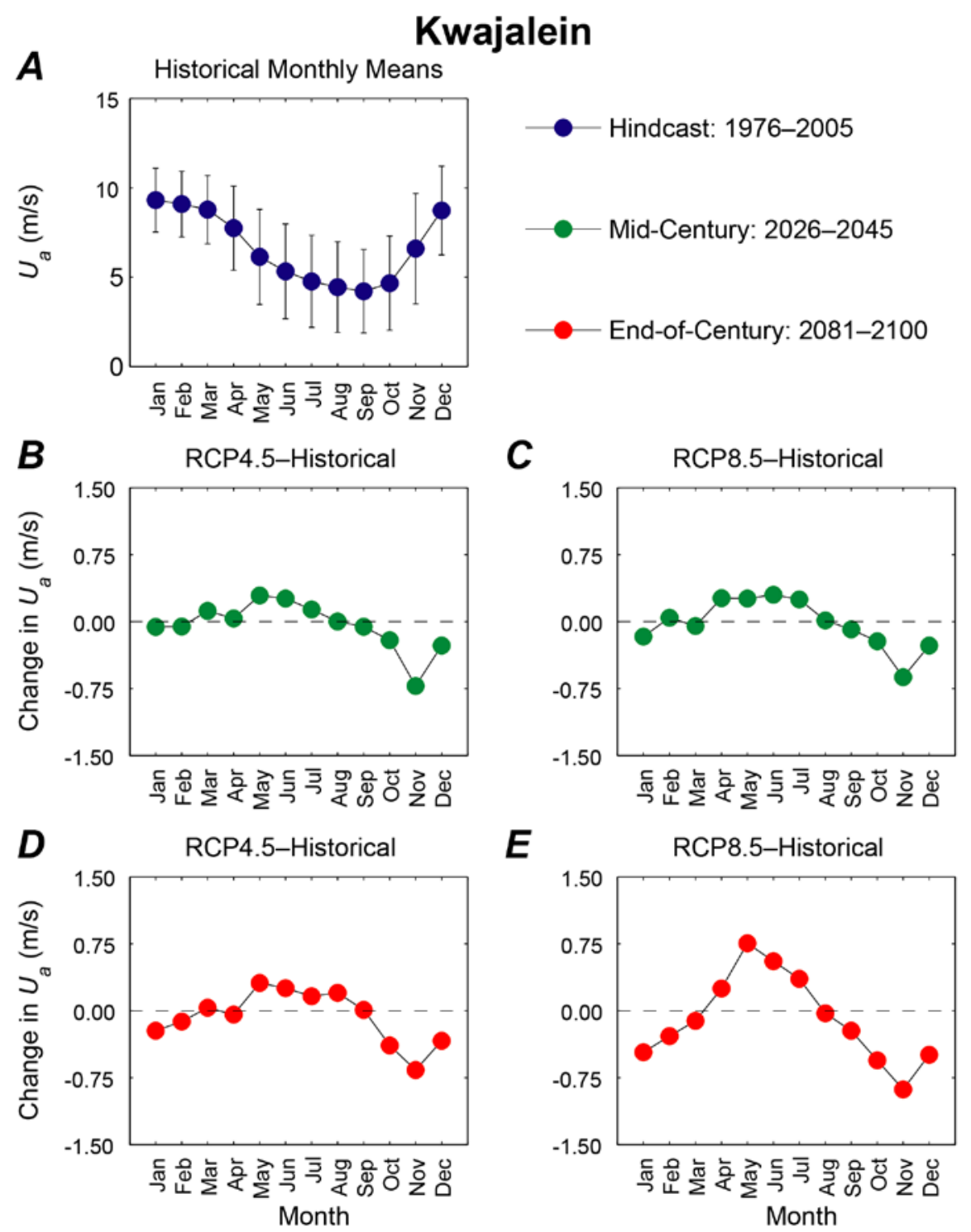

Appendix D35. Plots showing trends in monthly mean wind speed, in meters per second, at the Kwajalein location. A. Hindcasted (1976-2005) mean wind speeds by month with associated error bars. B. Plot of the change in mean 2026-2045 wind speeds for the RCP4.5 scenario from hindcasted monthly wind speed means. C. Plot of the change in mean 2026-2045 wind speeds for the RCP8.5 scenario from hindcasted monthly wind speed means. $D$. Plot of the change in mean 2081-2100 wind speeds for the RCP4.5 scenario from hindcasted monthly wind speed means. E. Plot of the change in mean 2081-2100 wind speeds for the RCP8.5 scenario from hindcasted monthly wind speed means. 

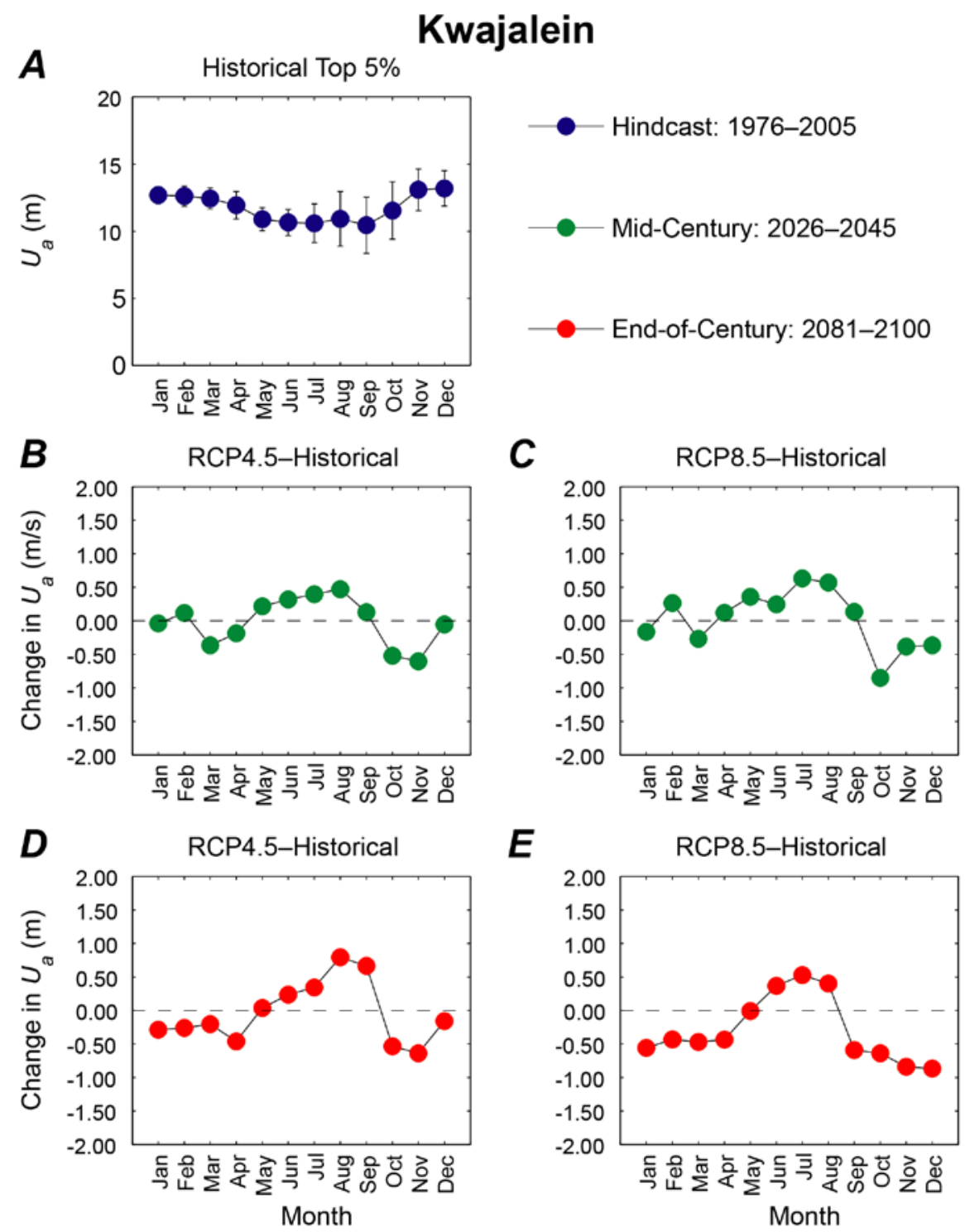

Appendix D36. Plots showing trends in monthly mean of the top 5 percent of wind speeds, in meters, at the Kwajalein location. A. Hindcasted (1976-2005) mean of the top 5 percent of wind speeds by month with associated error bars. $B$. Plot of the change in mean of the top 5 percent of 2026-2045 wind speeds for the RCP4.5 scenario from hindcasted top 5 percent of monthly wind speed means. $C$. Plot of the change in mean of the top 5 percent of 2026-2045 wind speeds for the RCP8.5 scenario from hindcasted top 5 percent of monthly wind speed means. $D$. Plot of the change in mean of the top 5 percent of 2081-2100 wind speeds for the RCP4.5 scenario from hindcasted top 5 percent of monthly wind speed means. E. Plot of the change in mean of the top 5 percent of 2081-2100 wind speeds for the RCP8.5 scenario from hindcasted top 5 percent of monthly wind speed means. 

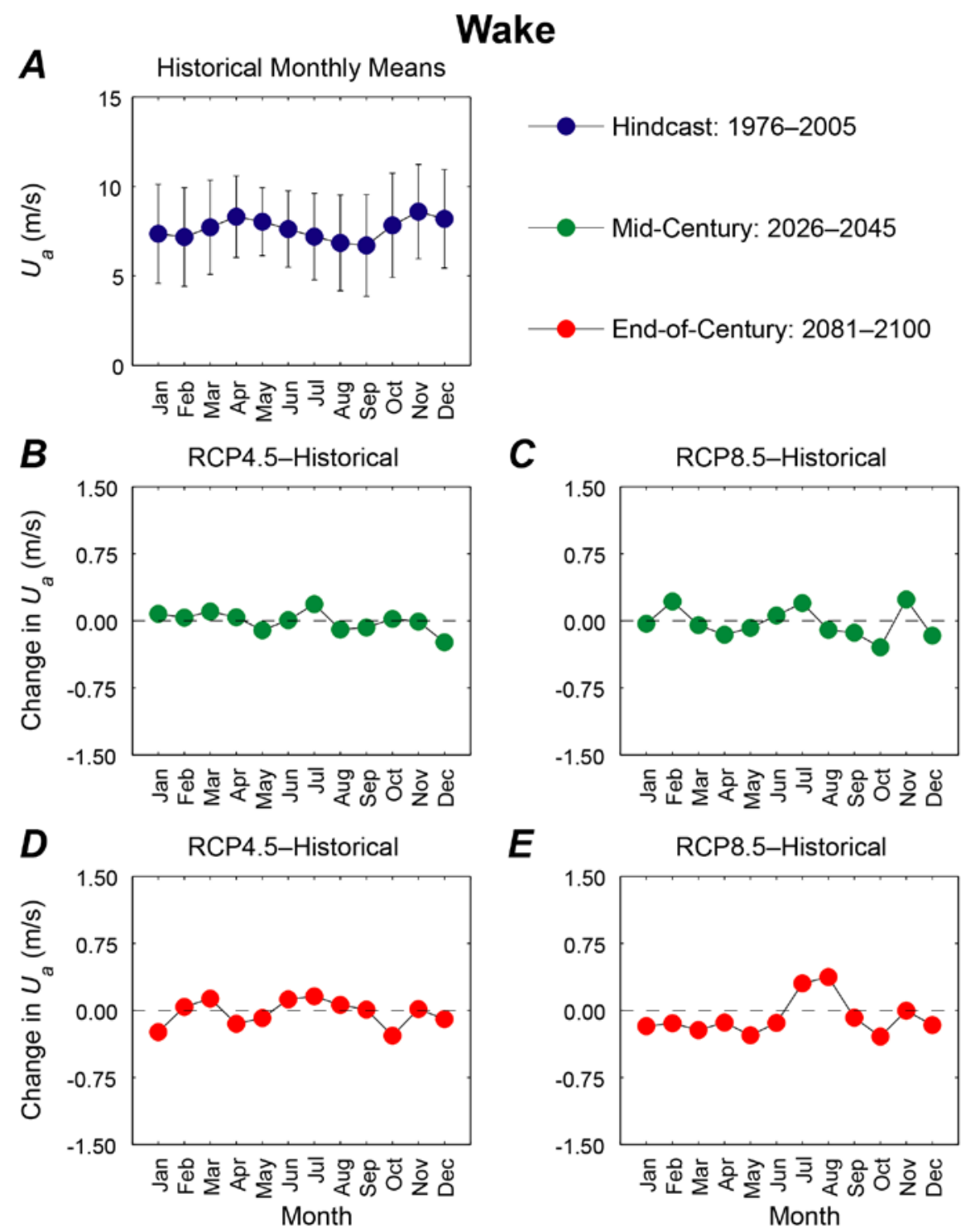

Appendix D37. Plots showing trends in monthly mean wind speed, in meters per second, at the Wake location. A. Hindcasted (1976-2005) mean wind speeds by month with associated error bars. B. Plot of the change in mean 2026-2045 wind speeds for the RCP4.5 scenario from hindcasted monthly wind speed means. C. Plot of the change in mean 2026-2045 wind speeds for the RCP8.5 scenario from hindcasted monthly wind speed means. $D$. Plot of the change in mean 2081-2100 wind speeds for the RCP4.5 scenario from hindcasted monthly wind speed means. E. Plot of the change in mean 2081-2100 wind speeds for the RCP8.5 scenario from hindcasted monthly wind speed means. 

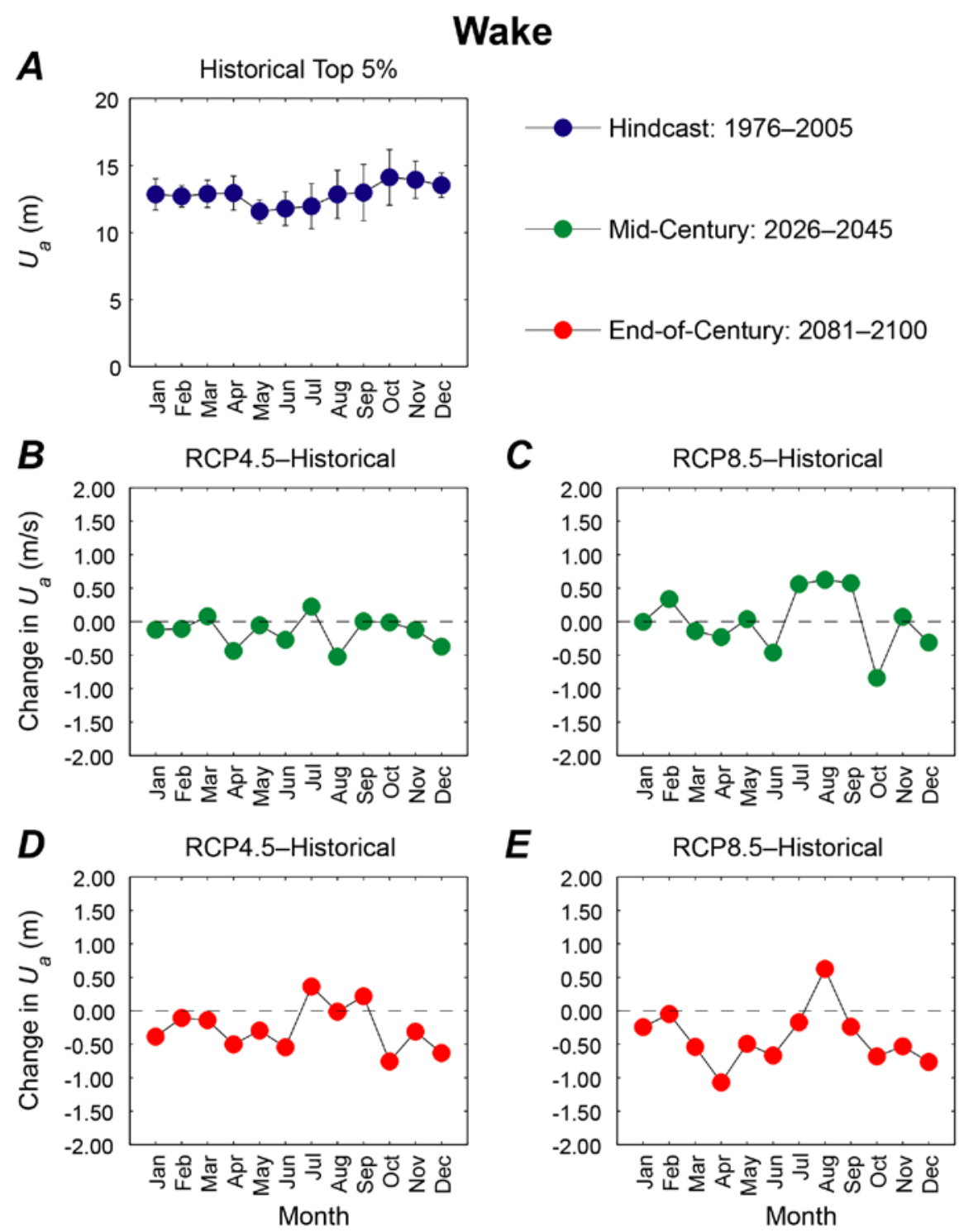

Appendix D38. Plots showing trends in monthly mean of the top 5 percent of wind speeds, in meters, at the Wake location. A. Hindcasted (1976-2005) mean of the top 5 percent of wind speeds by month with associated error bars. $B$. Plot of the change in mean of the top 5 percent of 2026-2045 wind speeds for the RCP4.5 scenario from hindcasted top 5 percent of monthly wind speed means. C. Plot of the change in mean of the top 5 percent of 2026-2045 wind speeds for the RCP8.5 scenario from hindcasted top 5 percent of monthly wind speed means. $D$. Plot of the change in mean of the top 5 percent of 2081-2100 wind speeds for the RCP4.5 scenario from hindcasted top 5 percent of monthly wind speed means. E. Plot of the change in mean of the top 5 percent of 2081-2100 wind speeds for the RCP8.5 scenario from hindcasted top 5 percent of monthly wind speed means. 

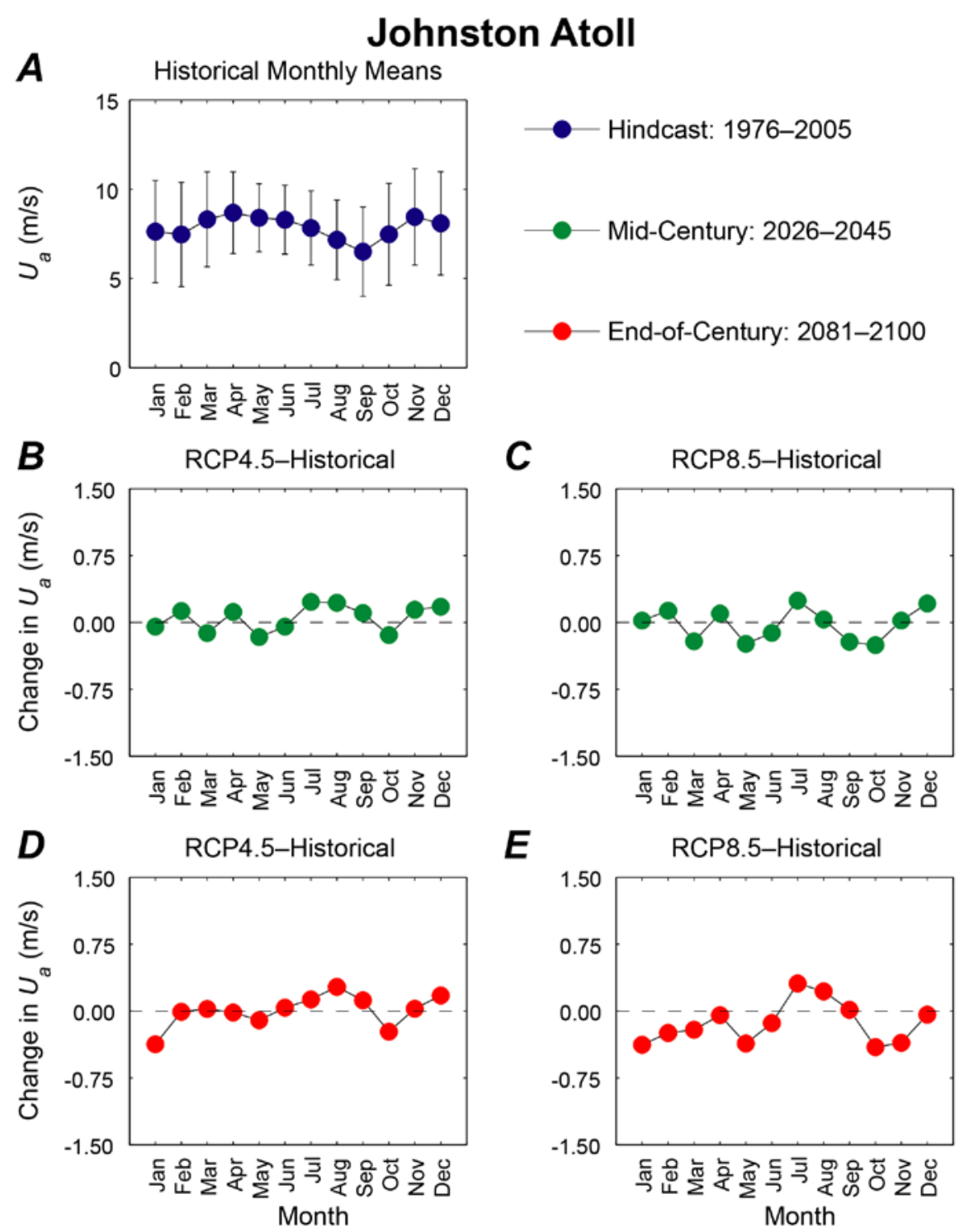

Appendix D39. Plots showing trends in monthly mean wind speed, in meters per second, at the Johnston Atoll location. A. Hindcasted (1976-2005) mean wind speeds by month with associated error bars. $B$. Plot of the change in mean 2026-2045 wind speeds for the RCP4.5 scenario from hindcasted monthly wind speed means. C. Plot of the change in mean 2026-2045 wind speeds for the RCP8.5 scenario from hindcasted monthly wind speed means. D. Plot of the change in mean 2081-2100 wind speeds for the RCP4.5 scenario from hindcasted monthly wind speed means. E. Plot of the change in mean 2081-2100 wind speeds for the RCP8.5 scenario from hindcasted monthly wind speed means. 

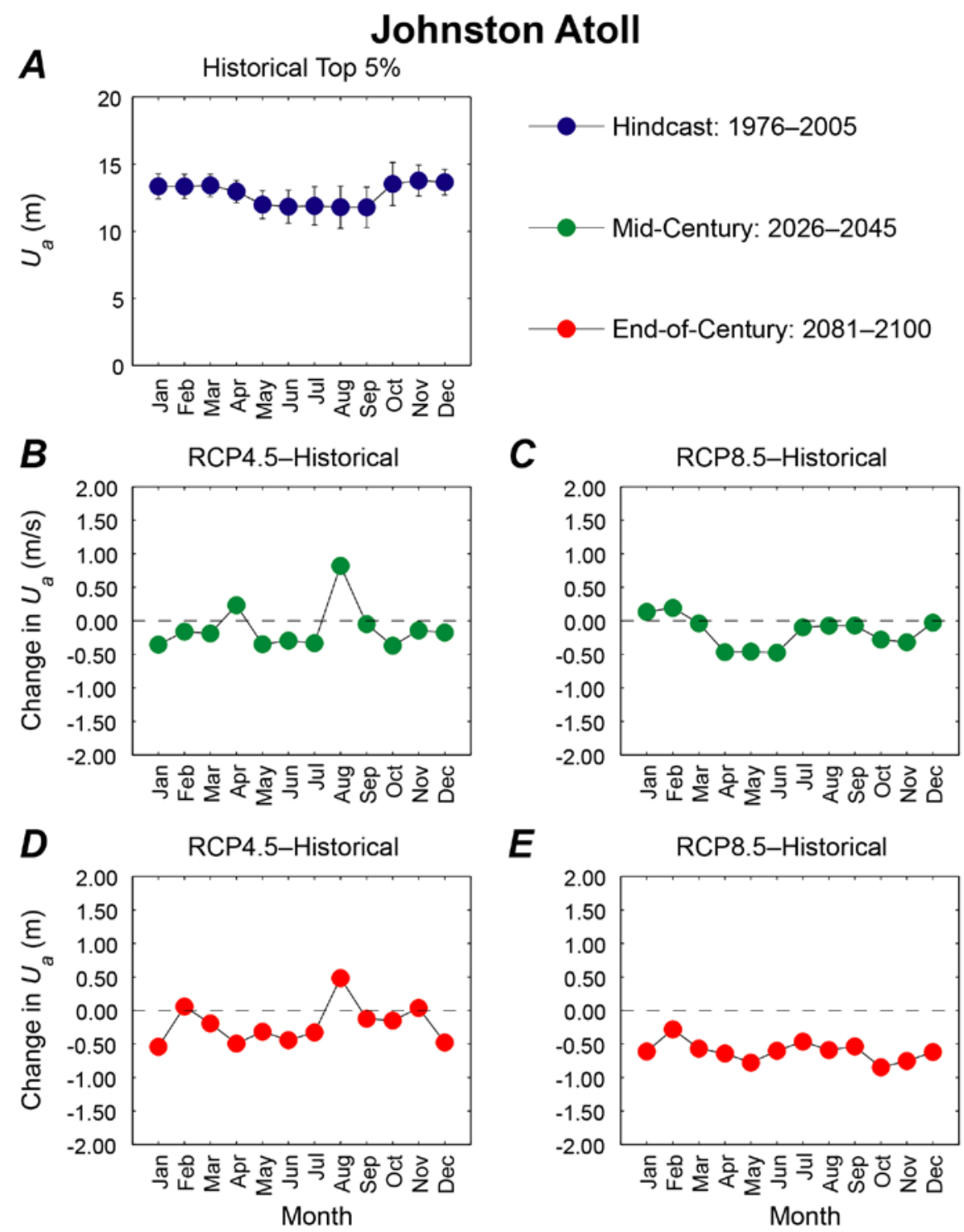

Appendix D40. Plots showing trends in monthly mean of the top 5 percent of wind speeds, in meters, at the Johnston Atoll location. A. Hindcasted (1976-2005) mean of the top 5 percent of wind speeds by month with associated error bars. $B$. Plot of the change in mean of the top 5 percent of 2026-2045 wind speeds for the RCP4.5 scenario from hindcasted top 5 percent of monthly wind speed means. C. Plot of the change in mean of the top 5 percent of 2026-2045 wind speeds for the RCP8.5 scenario from hindcasted top 5 percent of monthly wind speed means. $D$. Plot of the change in mean of the top 5 percent of 2081-2100 wind speeds for the RCP4.5 scenario from hindcasted top 5 percent of monthly wind speed means. E. Plot of the change in mean of the top 5 percent of 20812100 wind speeds for the RCP 8.5 scenario from hindcasted top 5 percent of monthly wind speed means. 

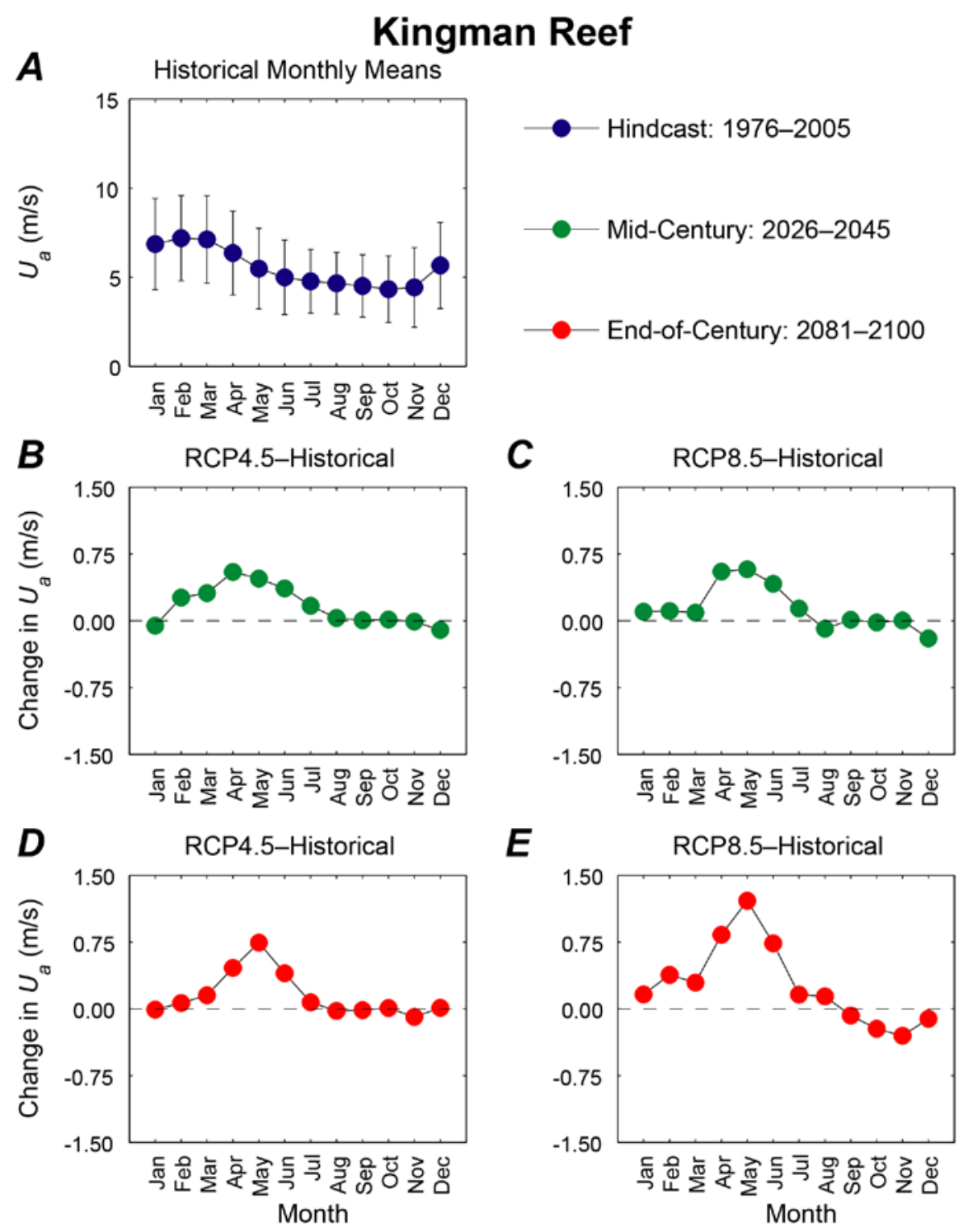

Appendix D41. Plots showing trends in monthly mean wind speed, in meters per second, at the Kingman Reef location. A. Hindcasted (1976-2005) mean wind speeds by month with associated error bars. $B$. Plot of the change in mean 2026-2045 wind speeds for the RCP4.5 scenario from hindcasted monthly wind speed means. C. Plot of the change in mean 2026-2045 wind speeds for the RCP8.5 scenario from hindcasted monthly wind speed means. D. Plot of the change in mean 2081-2100 wind speeds for the RCP4.5 scenario from hindcasted monthly wind speed means. E. Plot of the change in mean 2081-2100 wind speeds for the RCP8.5 scenario from hindcasted monthly wind speed means. 

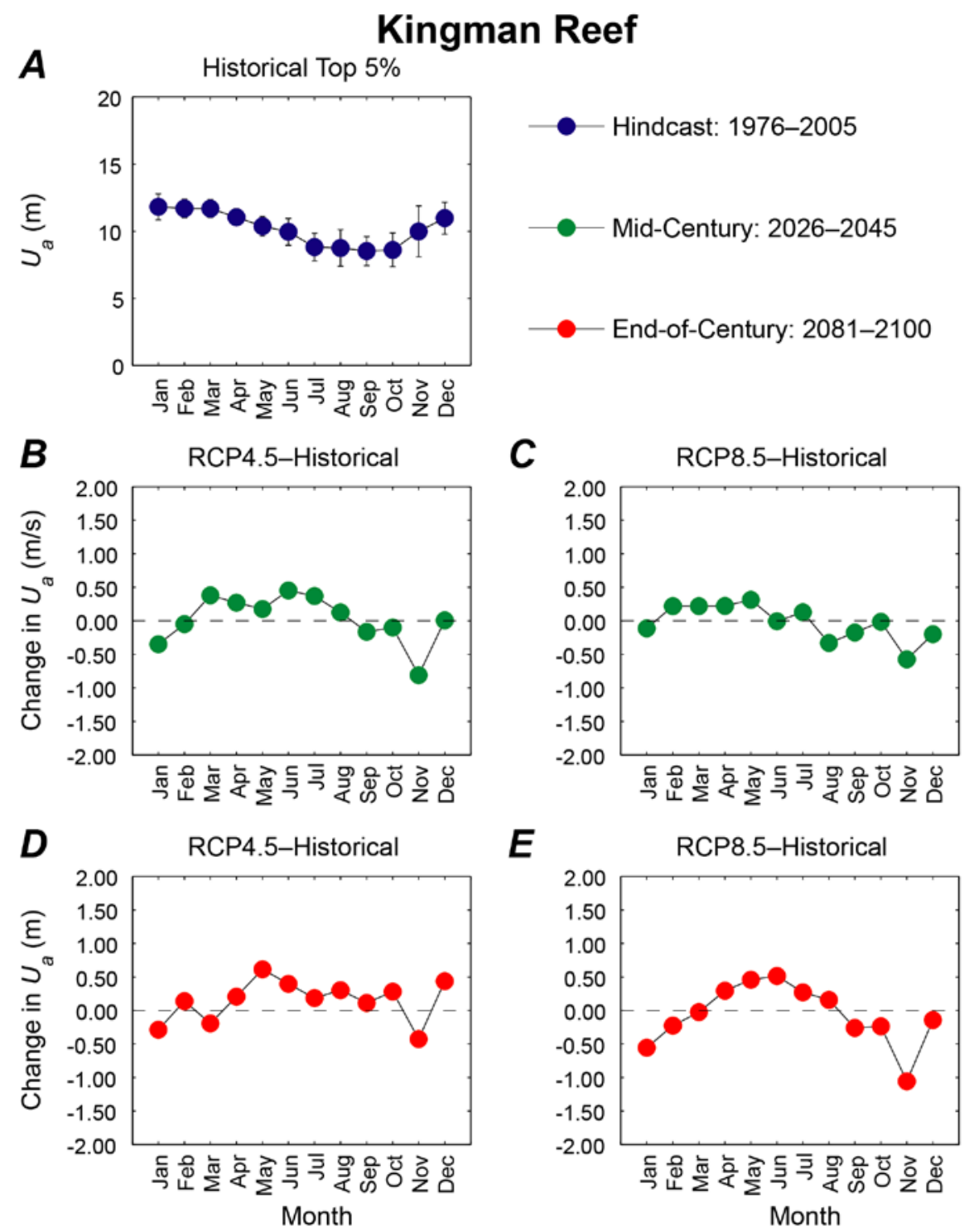

Appendix D42. Plots showing trends in monthly mean of the top 5 percent of wind speeds, in meters, at the Kingman Reef location. A. Hindcasted (1976-2005) mean of the top 5 percent of wind speeds by month with associated error bars. $B$. Plot of the change in mean of the top 5 percent of 2026-2045 wind speeds for the RCP4.5 scenario from hindcasted top 5 percent of monthly wind speed means. C. Plot of the change in mean of the top 5 percent of 2026-2045 wind speeds for the RCP8.5 scenario from hindcasted top 5 percent of monthly wind speed means. $D$. Plot of the change in mean of the top 5 percent of 2081-2100 wind speeds for the RCP4.5 scenario from hindcasted top 5 percent of monthly wind speed means. E. Plot of the change in mean of the top 5 percent of 20812100 wind speeds for the RCP 8.5 scenario from hindcasted top 5 percent of monthly wind speed means. 

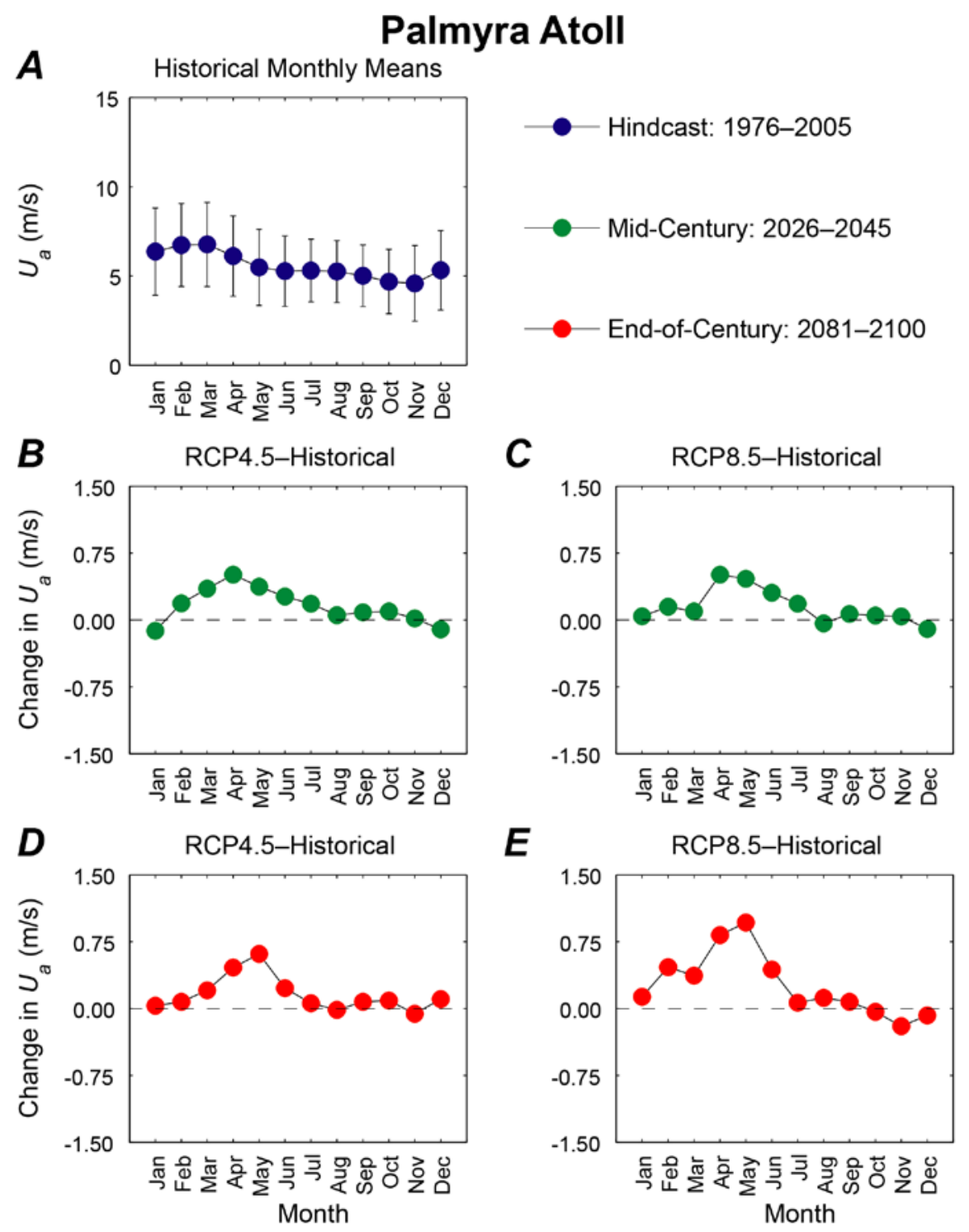

Appendix D43. Plots showing trends in monthly mean wind speed, in meters per second, at the Palmyra Atoll location. A. Hindcasted (1976-2005) mean wind speeds by month with associated error bars. $B$. Plot of the change in mean 2026-2045 wind speeds for the RCP4.5 scenario from hindcasted monthly wind speed means. C. Plot of the change in mean 2026-2045 wind speeds for the RCP8.5 scenario from hindcasted monthly wind speed means. D. Plot of the change in mean 2081-2100 wind speeds for the RCP4.5 scenario from hindcasted monthly wind speed means. E. Plot of the change in mean 2081-2100 wind speeds for the RCP8.5 scenario from hindcasted monthly wind speed means. 

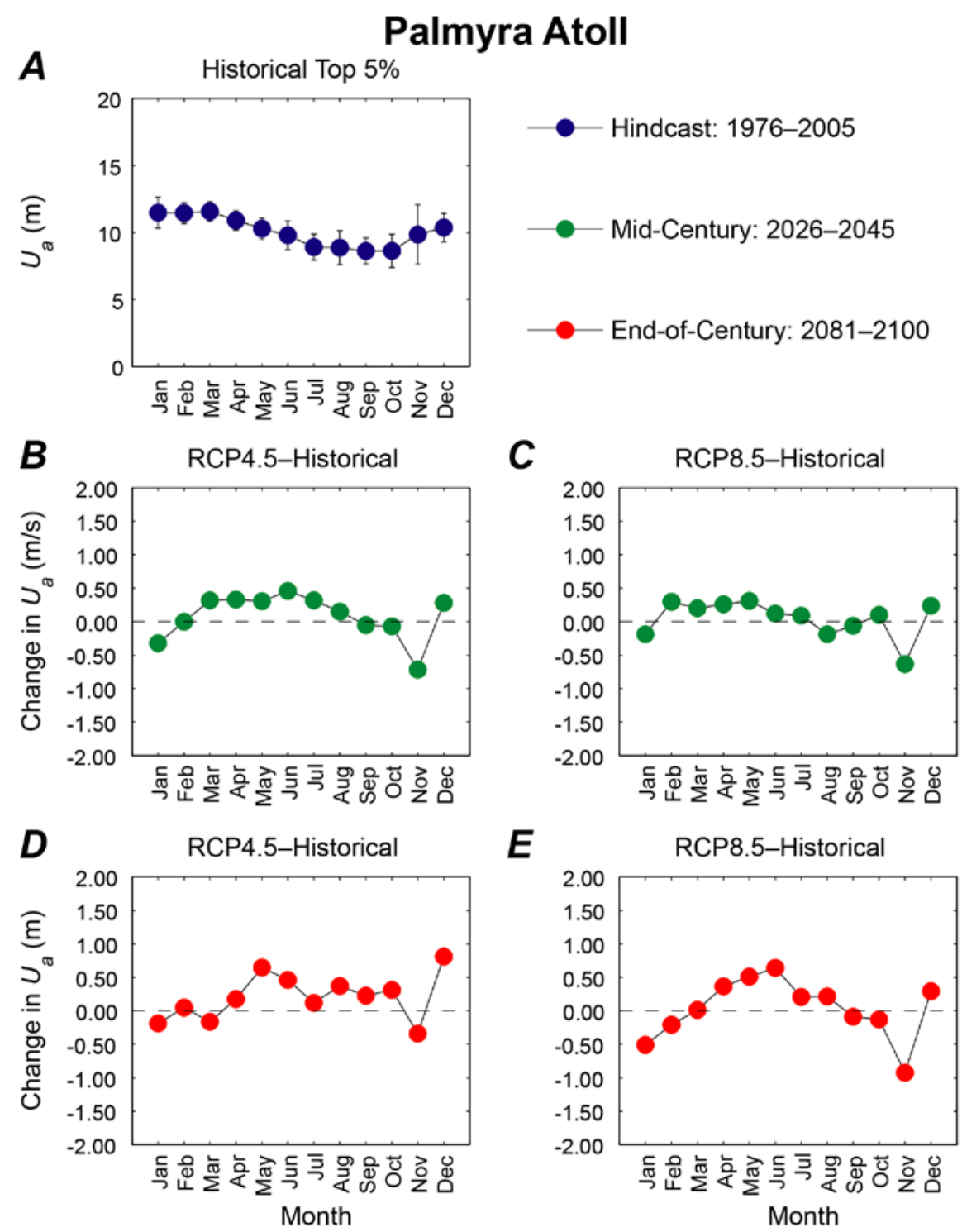

Appendix D44. Plots showing trends in monthly mean of the top 5 percent of wind speeds, in meters, at the Palmyra Atoll location. A. Hindcasted (1976-2005) mean of the top 5 percent of wind speeds by month with associated error bars. $B$. Plot of the change in mean of the top 5 percent of 2026-2045 wind speeds for the RCP4.5 scenario from hindcasted top 5 percent of monthly wind speed means. C. Plot of the change in mean of the top 5 percent of 2026-2045 wind speeds for the RCP8.5 scenario from hindcasted top 5 percent of monthly wind speed means. $D$. Plot of the change in mean of the top 5 percent of 2081-2100 wind speeds for the RCP4.5 scenario from hindcasted top 5 percent of monthly wind speed means. E. Plot of the change in mean of the top 5 percent of 20812100 wind speeds for the RCP 8.5 scenario from hindcasted top 5 percent of monthly wind speed means. 

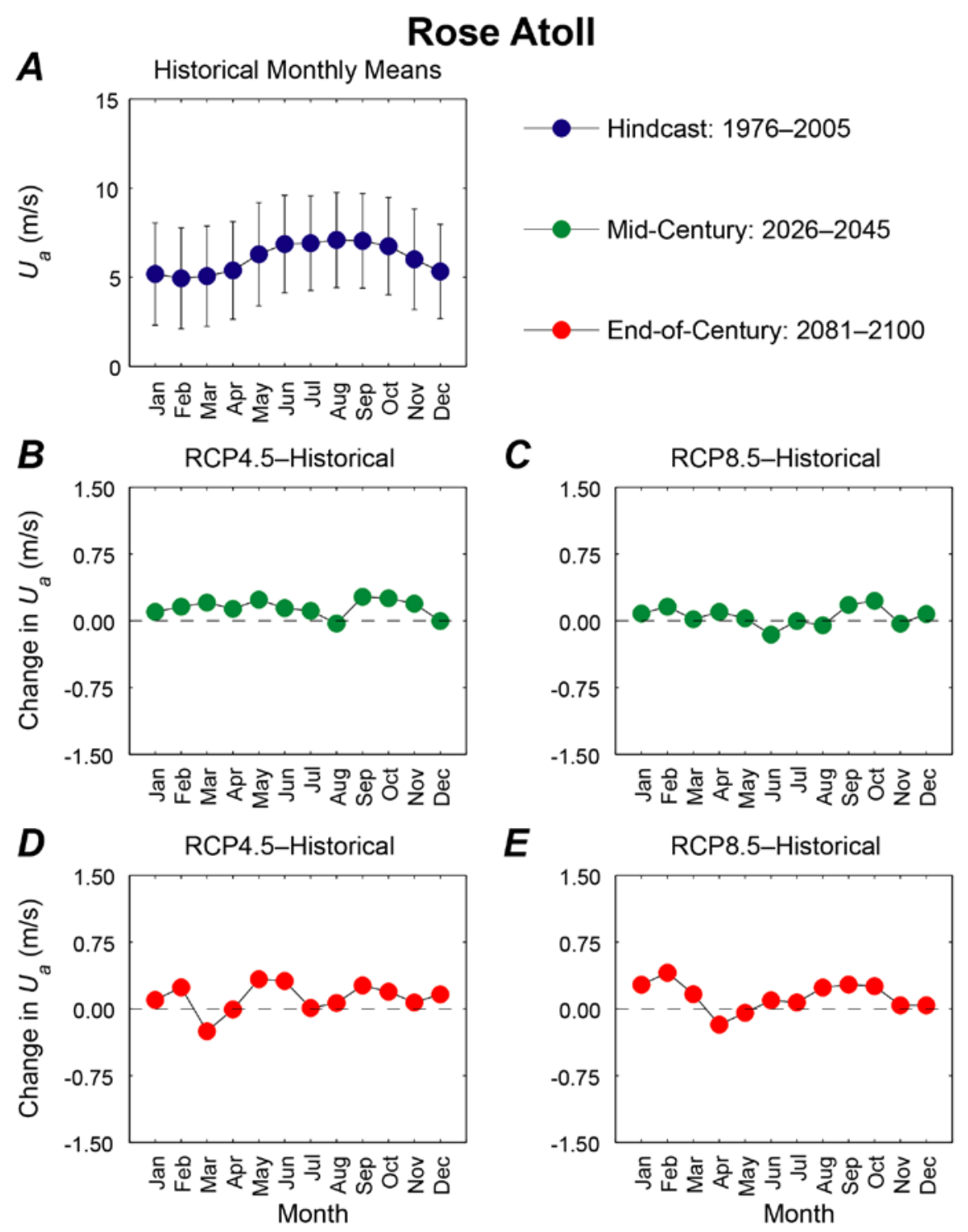

Appendix D45. Plots showing trends in monthly mean wind speed, in meters per second, at the Rose Atoll location. A. Hindcasted (1976-2005) mean wind speeds by month with associated error bars. B. Plot of the change in mean 2026-2045 wind speeds for the RCP4.5 scenario from hindcasted monthly wind speed means. C. Plot of the change in mean 2026-2045 wind speeds for the RCP8.5 scenario from hindcasted monthly wind speed means. $D$. Plot of the change in mean 2081-2100 wind speeds for the RCP4.5 scenario from hindcasted monthly wind speed means. E. Plot of the change in mean 2081-2100 wind speeds for the RCP8.5 scenario from hindcasted monthly wind speed means. 

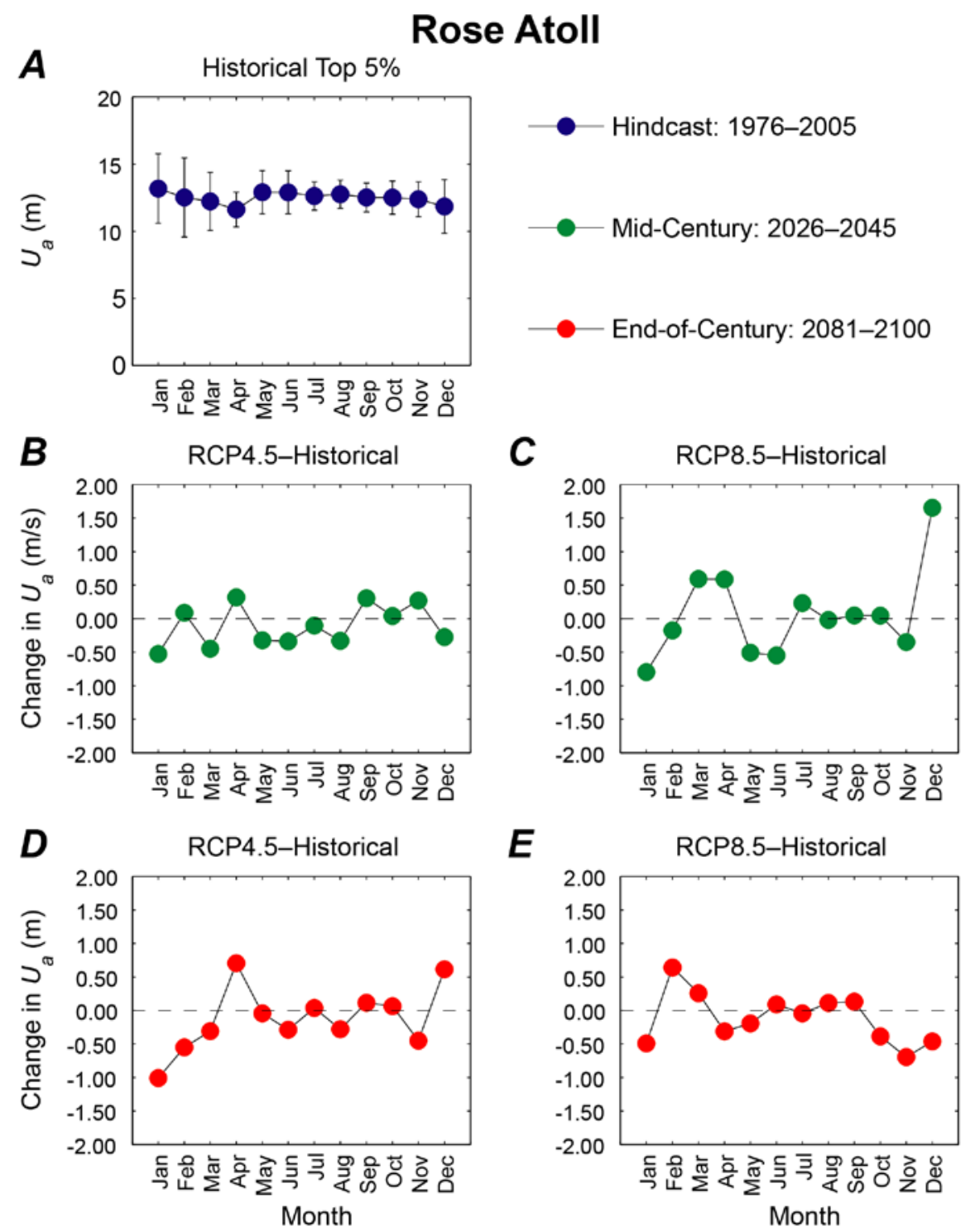

Appendix D46. Plots showing trends in monthly mean of the top 5 percent of wind speeds, in meters, at the Rose Atoll location. A. Hindcasted (1976-2005) mean of the top 5 percent of wind speeds by month with associated error bars. $B$. Plot of the change in mean of the top 5 percent of 2026-2045 wind speeds for the RCP4.5 scenario from hindcasted top 5 percent of monthly wind speed means. $C$. Plot of the change in mean of the top 5 percent of 2026-2045 wind speeds for the RCP8.5 scenario from hindcasted top 5 percent of monthly wind speed means. $D$. Plot of the change in mean of the top 5 percent of 2081-2100 wind speeds for the RCP4.5 scenario from hindcasted top 5 percent of monthly wind speed means. E. Plot of the change in mean of the top 5 percent of 2081-2100 wind speeds for the RCP8.5 scenario from hindcasted top 5 percent of monthly wind speed means. 

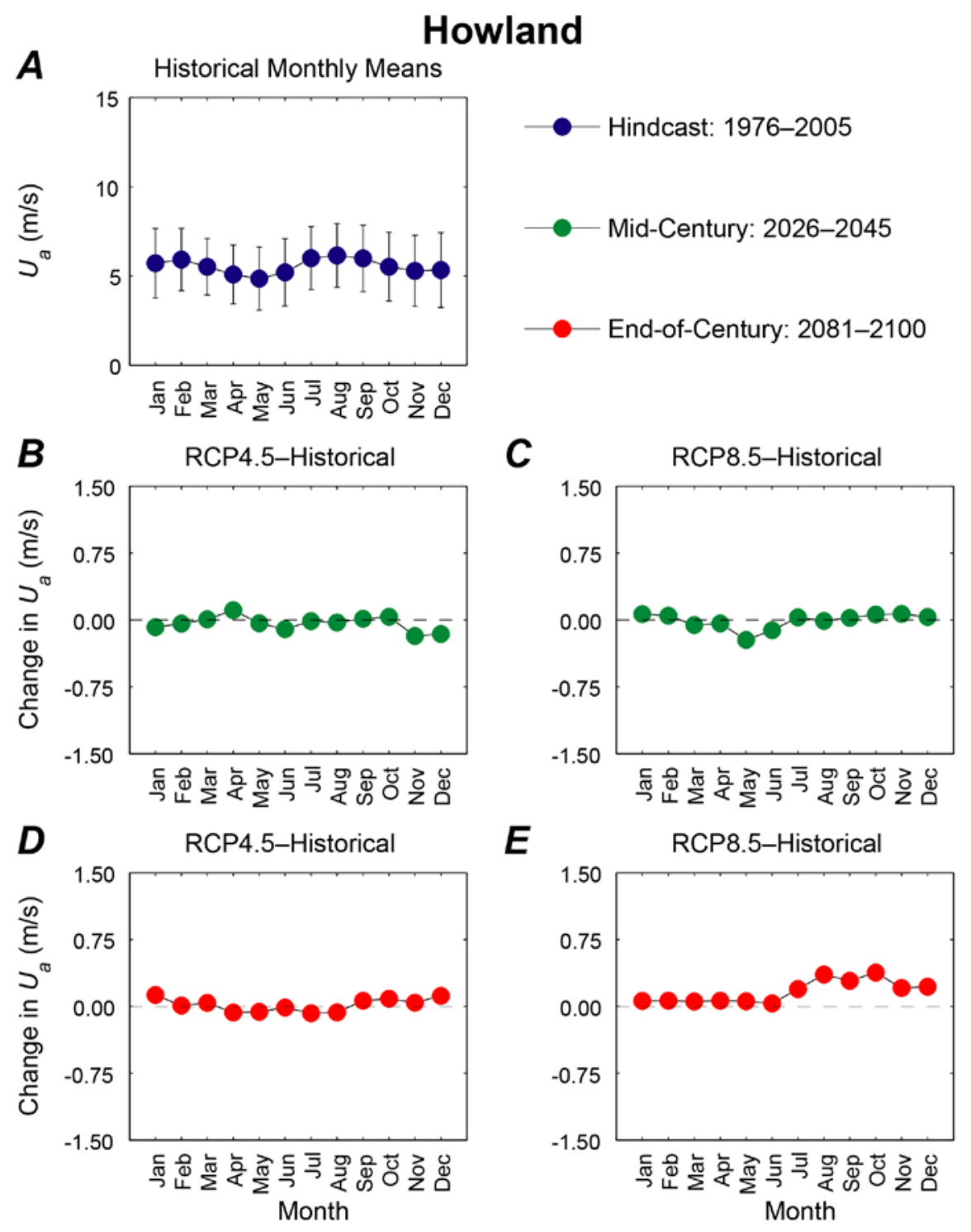

Appendix D47. Plots showing trends in monthly mean wind speed, in meters per second, at the Howland location. A. Hindcasted (1976-2005) mean wind speeds by month with associated error bars. B. Plot of the change in mean 2026-2045 wind speeds for the RCP4.5 scenario from hindcasted monthly wind speed means. C. Plot of the change in mean 2026-2045 wind speeds for the RCP8.5 scenario from hindcasted monthly wind speed means. $D$. Plot of the change in mean 2081-2100 wind speeds for the RCP4.5 scenario from hindcasted monthly wind speed means. E. Plot of the change in mean 2081-2100 wind speeds for the RCP8.5 scenario from hindcasted monthly wind speed means. 

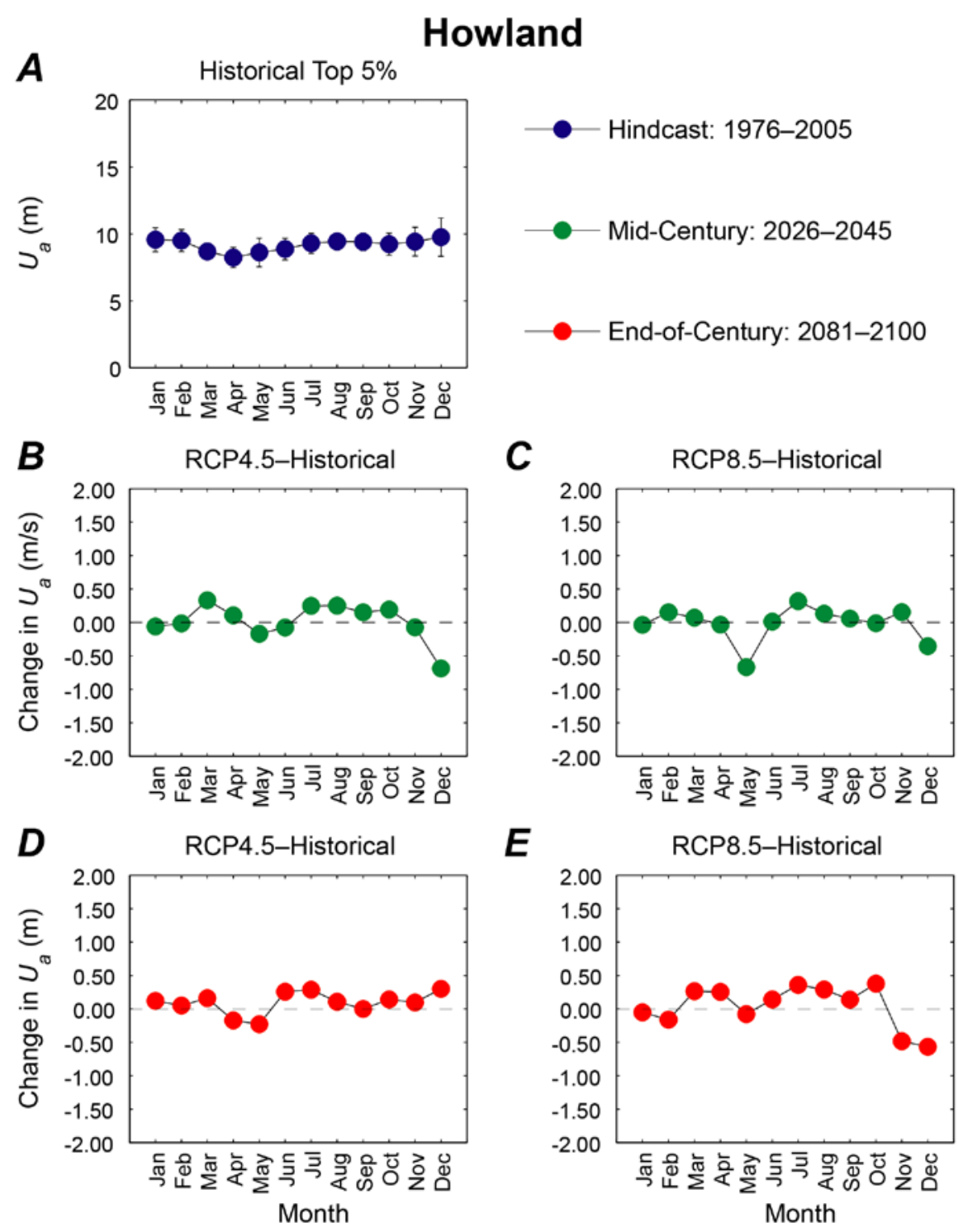

Appendix D48. Plots showing trends in monthly mean of the top 5 percent of wind speeds, in meters, at the Howland location. A. Hindcasted (1976-2005) mean of the top 5 percent of wind speeds by month with associated error bars. $B$. Plot of the change in mean of the top 5 percent of 2026-2045 wind speeds for the RCP4.5 scenario from hindcasted top 5 percent of monthly wind speed means. $C$. Plot of the change in mean of the top 5 percent of 2026-2045 wind speeds for the RCP8.5 scenario from hindcasted top 5 percent of monthly wind speed means. $D$. Plot of the change in mean of the top 5 percent of 2081-2100 wind speeds for the RCP4.5 scenario from hindcasted top 5 percent of monthly wind speed means. E. Plot of the change in mean of the top 5 percent of 2081-2100 wind speeds for the RCP8.5 scenario from hindcasted top 5 percent of monthly wind speed means. 

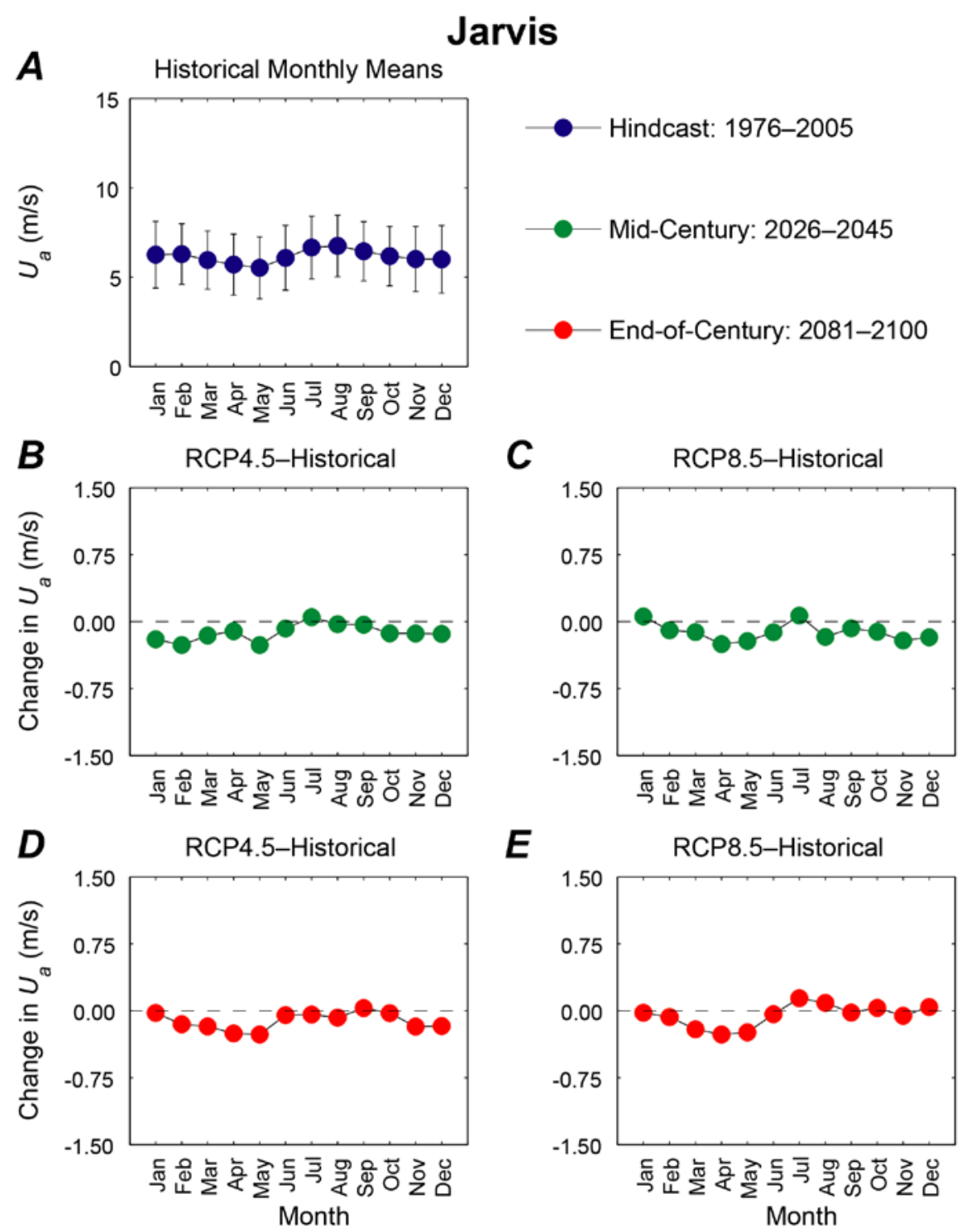

Appendix D49. Plots showing trends in monthly mean wind speed, in meters per second, at the Jarvis location. A. Hindcasted (1976-2005) mean wind speeds by month with associated error bars. B. Plot of the change in mean 2026-2045 wind speeds for the RCP4.5 scenario from hindcasted monthly wind speed means. C. Plot of the change in mean 2026-2045 wind speeds for the RCP8.5 scenario from hindcasted monthly wind speed means. $D$. Plot of the change in mean 2081-2100 wind speeds for the RCP4.5 scenario from hindcasted monthly wind speed means. E. Plot of the change in mean 2081-2100 wind speeds for the RCP8.5 scenario from hindcasted monthly wind speed means. 

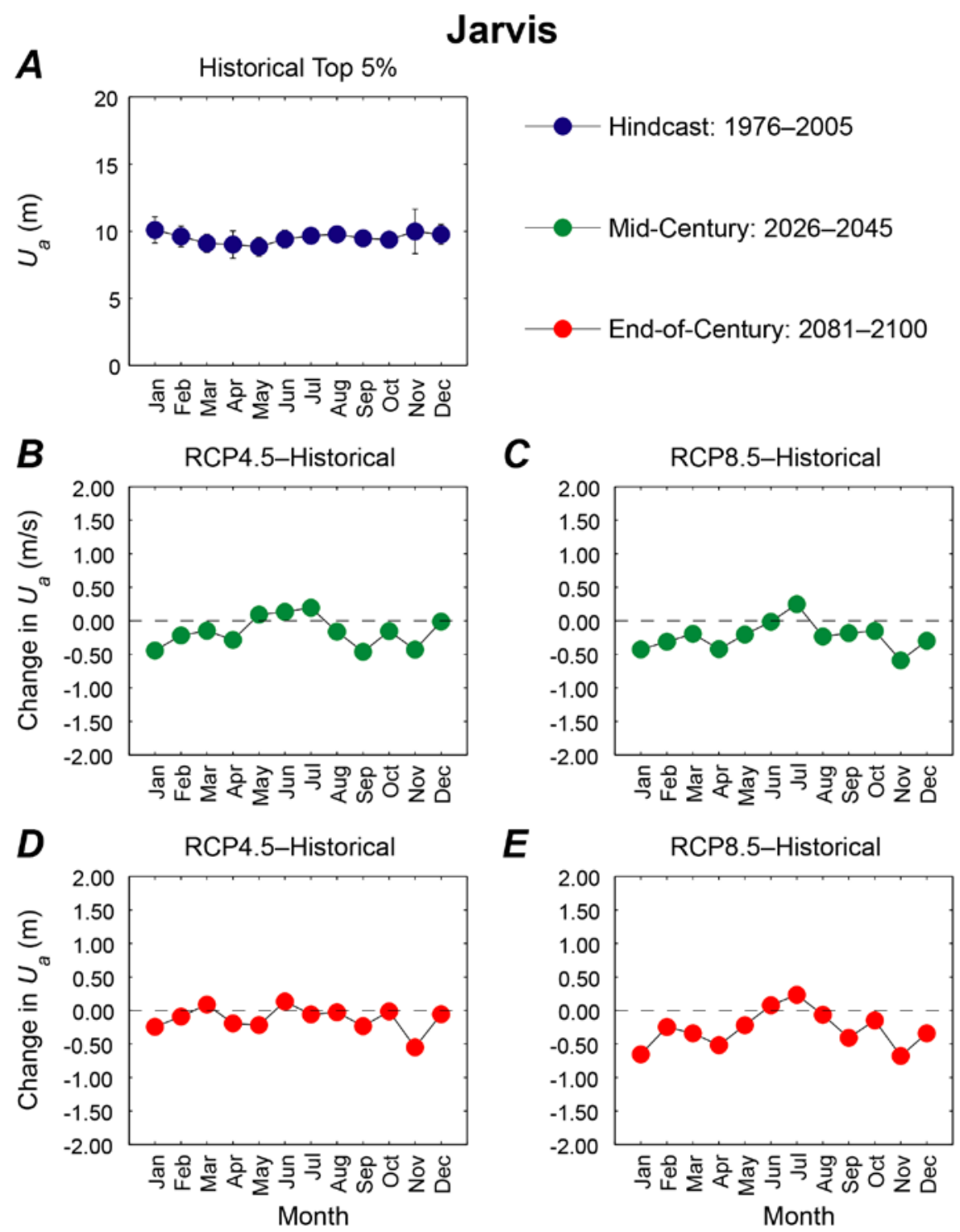

Appendix D50. Plots showing trends in monthly mean of the top 5 percent of wind speeds, in meters, at the Jarvis location. A. Hindcasted (1976-2005) mean of the top 5 percent of wind speeds by month with associated error bars. $B$. Plot of the change in mean of the top 5 percent of 2026-2045 wind speeds for the RCP4.5 scenario from hindcasted top 5 percent of monthly wind speed means. C. Plot of the change in mean of the top 5 percent of 2026-2045 wind speeds for the RCP8.5 scenario from hindcasted top 5 percent of monthly wind speed means. $D$. Plot of the change in mean of the top 5 percent of 2081-2100 wind speeds for the RCP4.5 scenario from hindcasted top 5 percent of monthly wind speed means. E. Plot of the change in mean of the top 5 percent of 2081-2100 wind speeds for the RCP8.5 scenario from hindcasted top 5 percent of monthly wind speed means. 